\title{
Analysis of Radioactive Releases During Proposed Demolition Activities for the 224-U and 224-UA Buildings
}

Report to CH2M HILL Plateau Remediation Company (CHPRC)

\author{
BA Napier \\ JP Rishel \\ JG Droppo Jr.
}

March 2009

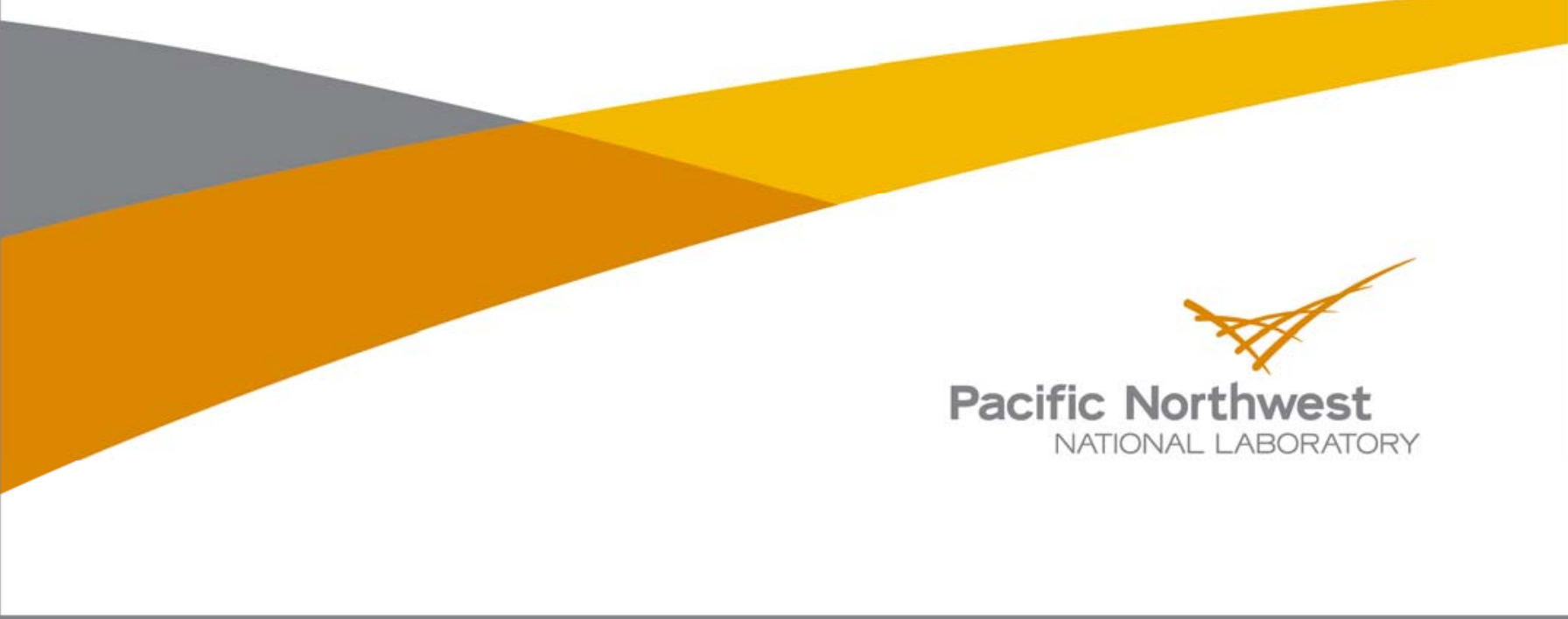




\title{
Analysis of Radioactive Releases During Proposed Demolition Activities for the 224-U and 224-UA Buildings
}

Report to CH2M HILL Plateau Remediation Company (CHPRC)

\author{
BA Napier \\ JP Rishel \\ JG Droppo Jr.
}

March 2009

Prepared for

the U.S. Department of Energy

under Contract DE-AC05-76RL01830

Pacific Northwest National Laboratory

Richland, Washington 99352 



\section{Acknowledgments}

The authors would like to extend their appreciation to Earl Lloyd and Alex Nazarali of the CH2M HILL Plateau Plateau Remediation Company for their financial and technical support of this work. Thanks are also extended to Wayne Cosby of PNNL for the final edit of the document. This work was funded by CH2M HILL Plateau Plateau Remediation Company under Contract DE-AC05-76RL01830. 



\section{Acronyms and Abbreviations}

\begin{tabular}{|c|c|}
\hline AED & aerodynamic equivalent diameter \\
\hline AERMOD & American Meteorological Society/Environmental Protection Agency Regulatory Model \\
\hline AMAD & activity median aerodynamic diameter \\
\hline ARF & airborne release fraction \\
\hline $\mathrm{Bq}$ & becquerel \\
\hline CFR & Code of Federal Regulations \\
\hline CHPRC & CH2M HILL Plateau Radiation Contract \\
\hline $\mathrm{Ci}$ & Curie(s) \\
\hline $\mathrm{DAC}$ & derived air concentration \\
\hline DOE & U.S. Department of Energy \\
\hline dpm & disintegrations per minute \\
\hline DR & damage ratio \\
\hline EF & emission factor \\
\hline EPA & U.S. Environmental Protection Agency \\
\hline ERDF & Environmental Restoration Disposal Facility \\
\hline FSL & Forecast Systems Laboratory \\
\hline $\mathrm{Ft}$ & foot (feet) \\
\hline $\mathrm{g}$ & gram \\
\hline HEPA & high-efficiency particulate air (filter) \\
\hline HMS & Hanford Meteorological Station \\
\hline in. & inch(es) \\
\hline lb. & pound \\
\hline LPF & leak path factor \\
\hline $\mathrm{m}$ & meter(s) \\
\hline MAR & material-at-risk \\
\hline NCDC & National Climatic Data Center \\
\hline PNNL & Pacific Northwest National Laboratory \\
\hline $\mathrm{Pu}$ & plutonium \\
\hline PUREX & plutonium-uranium extraction \\
\hline RF & respirable fraction \\
\hline
\end{tabular}


source term

UNH uranyl nitrate hexahydrate

$\mathrm{UO}_{3} \quad$ uranium tri-oxide

WS wind speed 


\section{Contents}

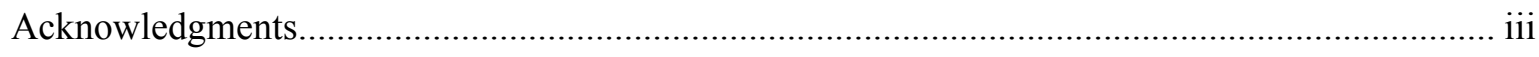

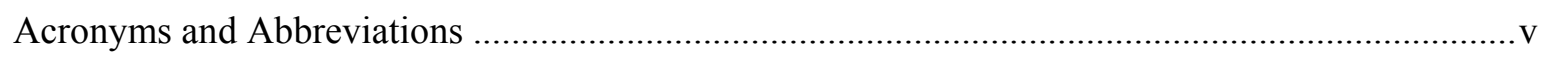

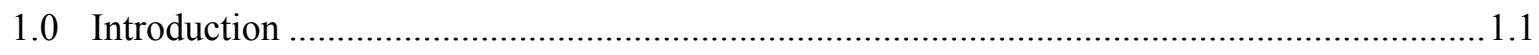

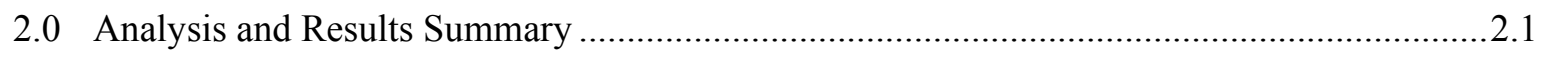

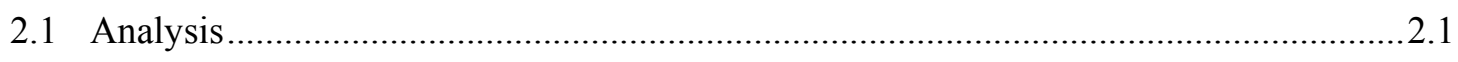

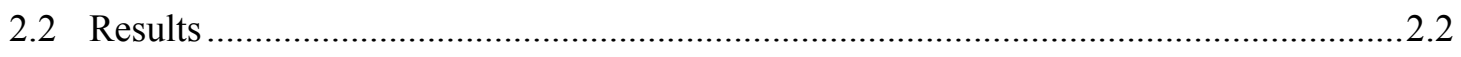

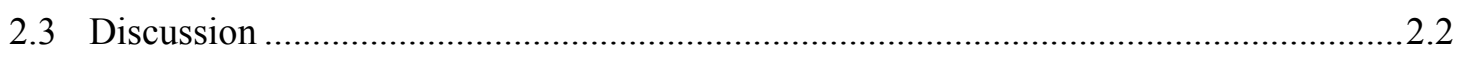

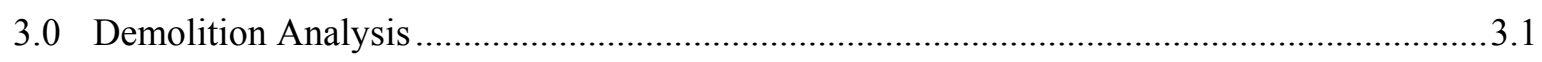

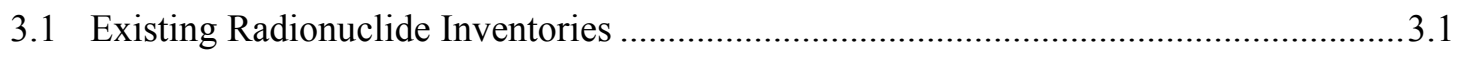

3.2 Demolition of the $\mathrm{UO}_{3}$ Powder-Handling Systems with Multiprocesssor ........................

3.3 Demolition of the Main Building Structures ............................................................... 3.3

3.4 Demolition of the Calciners and Associated Equipment...............................................3.3

3.5 Demolition Schedule Assumed for Modeling .............................................................. 3.3

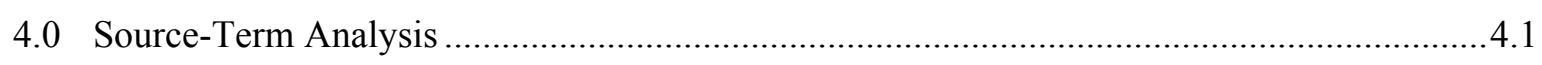

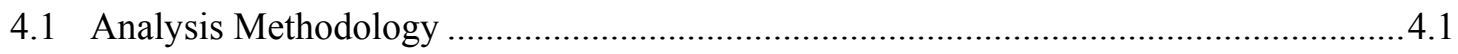

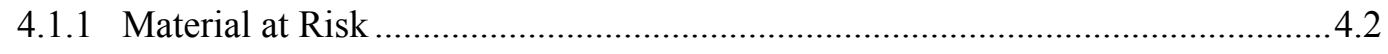

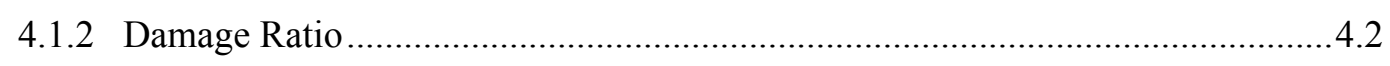

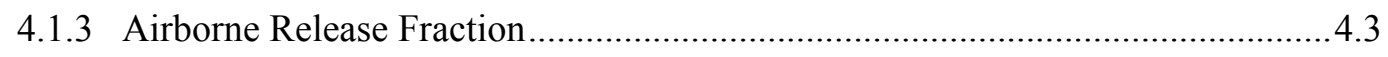

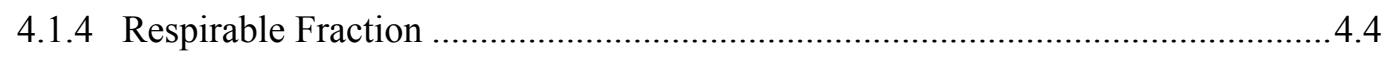

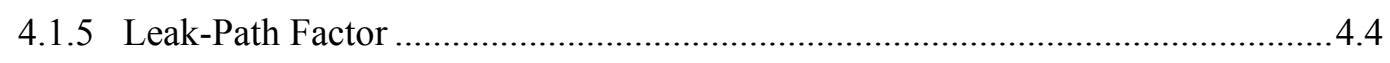

4.1.6 Limitation of Airborne Contamination............................................................ 4.5

4.2 Unit Operations/Release Mechanisms................................................................... 4.5

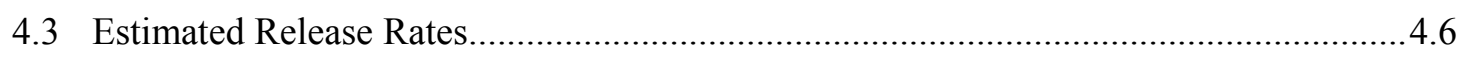

5.0 Atmospheric Dispersion and Deposition Modeling …....................................................... 5.1

5.1 Air-Dispersion Modeling Assumptions and Data .......................................................

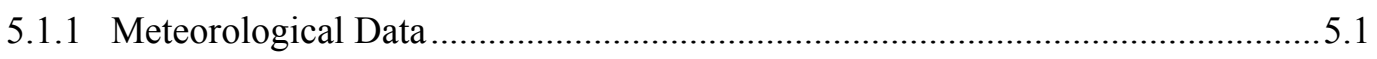

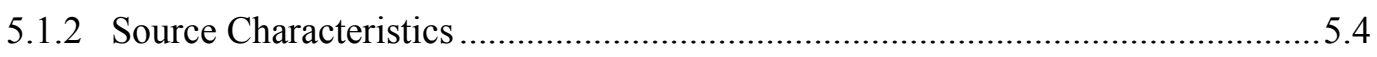

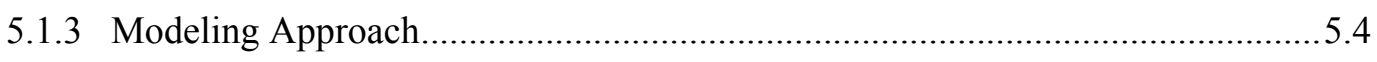

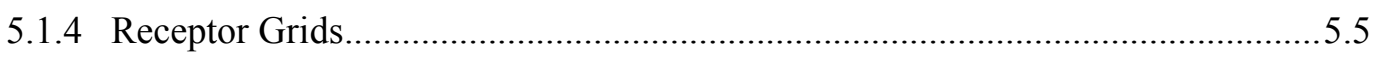

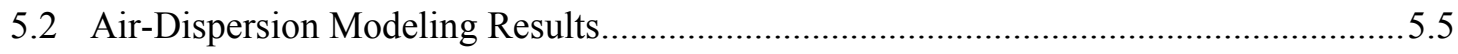

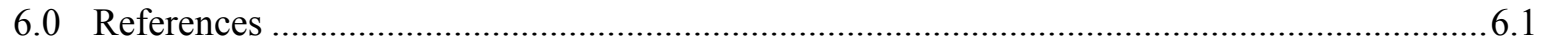

Appendix A Source Computation Worksheet.......................................................................... 1

Appendix B AERMOD Emission Rates ............................................................................... B.1

Appendix C Output AERMOD Listing for Combined 224-U and 224-UA Building Analysis ..... C.1 


\section{Figures}

1.1. The 224-U and 224-UA Buildings, Which Are in a Location Surrounded by Other Large Structures, Complicating Atmospheric Dispersion Modeling

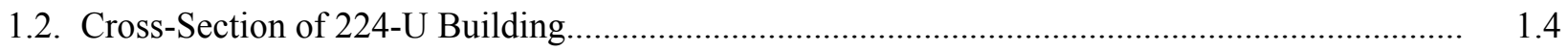

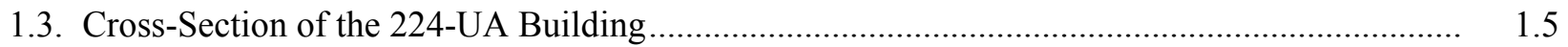

5.1. Five-Year Annual Wind Rose Hanford Station \#19 (2002-2006).......................................... 5.2

5.2. Five-Year Annual Wind Rose Hanford Station \#19 (2002-2006 July through September, hours 8:00 am to 4:00 pm)

5.3. Five-Year Annual Wind Rose Hanford Station \#19 (2002-2006 Oct. through Dec., hours 8:00 am to $4: 00 \mathrm{pm})$

5.4. Predicted Peak Hourly Air Concentrations for the 224-U Building Demolition; Values Less Than $341 \mu \mathrm{g} / \mathrm{m}^{3}$ Correspond to Concentrations Below 12 DAC-hrs.

5.5. Predicted Peak Deposition Amounts for the 224-U Building Demolition; Values Less Than $0.05 \mu \mathrm{g} / \mathrm{m}^{2}$ Correspond to Uranium Deposition Rates of Less Than $1000 \mathrm{dpm} / 100 \mathrm{~cm}^{2}$

5.6. Predicted Peak Hourly Air Concentrations for the 224-UA Building Demolition; Values Less Than $341 \mu \mathrm{g} / \mathrm{m}^{3}$ Correspond to Concentrations Below 12 DAC-hrs

5.7. Predicted Peak Deposition Amounts for the 224-UA Building Demolition; Values Less Than $0.05 \mu \mathrm{g} / \mathrm{m}^{2}$ Correspond to uranium Deposition Rates of Less Than $1000 \mathrm{dpm} / 100 \mathrm{~cm}^{2}$

5.8. Predicted Peak Deposition Amounts for the 224A and 224-UA Building Demolition; Values Less Than $0.05 \mu \mathrm{g} / \mathrm{m}^{2}$ Correspond to Uranium Deposition Rates of Less Than $1000 \mathrm{dpm} /$ $100 \mathrm{~cm}^{2}$

\section{Tables}

3.1. Conservative Inventory of Radionuclides in $\mathrm{UO}_{3}$ Complex, Grams ..........................................

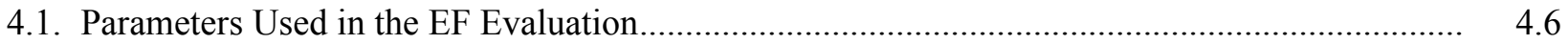




\subsection{Introduction}

An emission and air dispersion modeling effort was conducted relative to proposed demolition activities for the 224-U and 224-UA Buildings. The 224-U and 224-UA Buildings (Figure 1.1, 1.2, and 1.3) are located in the U-Plant $\mathrm{UO}_{3}$ complex in the 200 West Area of the Hanford Site. These facilities converted uranyl nitrate hexahydrate (UNH), a product of Hanford's Plutonium-Uranium Extraction (PUREX) Plant, into uranium trioxide $\left(\mathrm{UO}_{3}\right)$. The $\mathrm{UO}_{3}$ powder was shipped offsite for conversion to uranium metal for nuclear reactors. The $\mathrm{UO}_{3}$ Plant consists of two major buildings: the 224-U building (built in 1943 as part of the plutonium processing construction, but converted to uranyl nitrate concentration, conversion processing); and the 224-UA building built in 1956 to house new continuous calciners. The 224-U building houses the concentrating and nitric acid recovery equipment. In addition to the calciners, the 224-UA building houses the powder-handling tower and the $\mathrm{UO}_{3}$ powder packaging machinery (used to load T-hoppers). During deactivation in 1993, most of the radioactive material was removed; the remaining is primarily residual uranium tri-oxide dust in the calciners and powder handling systems. The U.S. Department of Energy (DOE) and CH2M HILL Plateau Plateau Remediation Company (CHPRC) plans call for demolishing the structure in late 2009.

Pacific Northwest National Laboratory (PNNL) supports the demolition planning effort by making engineering estimates of potential releases for various demolition alternatives. Air-dispersion modeling has been conducted using those release rates to provide information on the location and levels of radioactivity. Similar efforts for planning the demolition of a highly contaminated structure are reported by Droppo et al. (2007). The $\mathrm{UO}_{3}$ plant demolition being modeled in this analysis differs from the previous efforts in that this facility only has uranium contamination versus the previous efforts for facilities where plutonium was the contamination of concern.

The facilities surrounding the $\mathrm{UO}_{3}$ complex, particularly the 221-U canyon building, have the potential of affecting dispersion patterns through various meteorological phenomena, including building wake effects. Atmospheric dispersion calculations have been run using the American Meteorological Society/Environmental Protection Agency Regulatory Model (AERMOD) (EPA 2008), a computer code developed by the U.S. Environmental Protection Agency (EPA). This code calculates dispersion patterns considering building wake effects and other meteorological phenomena. The results from the AERMOD calculations are being used to plan demolition activities that will keep potential contamination within the boundaries established for the project contamination area.

The 224-U building is 197 feet long, 60 feet wide, and 40 feet tall. The building is divided along its length by a 12 -inch-thick concrete shield wall into a gallery side and a canyon side. The gallery side is a three-story reinforced concrete frame structure with concrete floor and roof slabs. Exterior and interior walls are non-reinforced, 8-inch concrete blocks. The roof, supported by concrete beams, is a flat reinforced concrete slab between 5 and 12 inches thick. The canyon side, constructed of 12-inch-thick reinforced concrete walls, is also three stories high. The canyon is divided into six cells and the Luckey pot room. Each cell is separated by a concrete wall that extends part way ( 15 feet) toward the ceiling. A portion of C-cell is the only part of the 224-U building that extends below grade. In 1956, the 224-UA building (housing six electrically heated agitated-trough calciners designed for continuous operation) was constructed to process the uranyl nitrate product from the PUREX Plant. The 224 UA Building is approximately 75 feet long and 67 feet wide. The floor slab and footing are reinforced concrete. Equipment footing and supports are reinforced concrete pier columns with steel "I" beam framing. The 
outside walls consist of insulated metallic-coated steel panels. The ground floor consists of six two-story cells. The continuous calciners are located on the second floor of these cells. The five-floor-high tower section of the building located over the load-out room was used to handle the $\mathrm{UO}_{3}$ powder. The tower contained two primary bag filters, two cyclones, and a storage bin. The 224 UA Building roof consists of steel panels covered with insulation and built-up roofing material. Numerous pieces of equipment and ducting, including the 296 U-2 and 296 U-14 exhausters, are located on the roof.

$\mathrm{UO}_{3}$ powder handling was conducted using high-volume air vacuum transport. The vacuum systems had substantial ducting and bag and high-efficiency particulate air (HEPA) filters at the exhaust end to retain any $\mathrm{UO}_{3}$ dust that escaped the powder-handling system. The old powder-handling system consists of a tower and a pair of bag filter housings, with associated ducting, on the south-west end of the 224-U building. There is a pair of bag filter housings on the north-west side of the 224-UA building, with associated ducting and a large HEPA filter on the roof.

Debris will be disposed of at the Environmental Restoration Disposal Facility (ERDF) in the 200 Areas. 


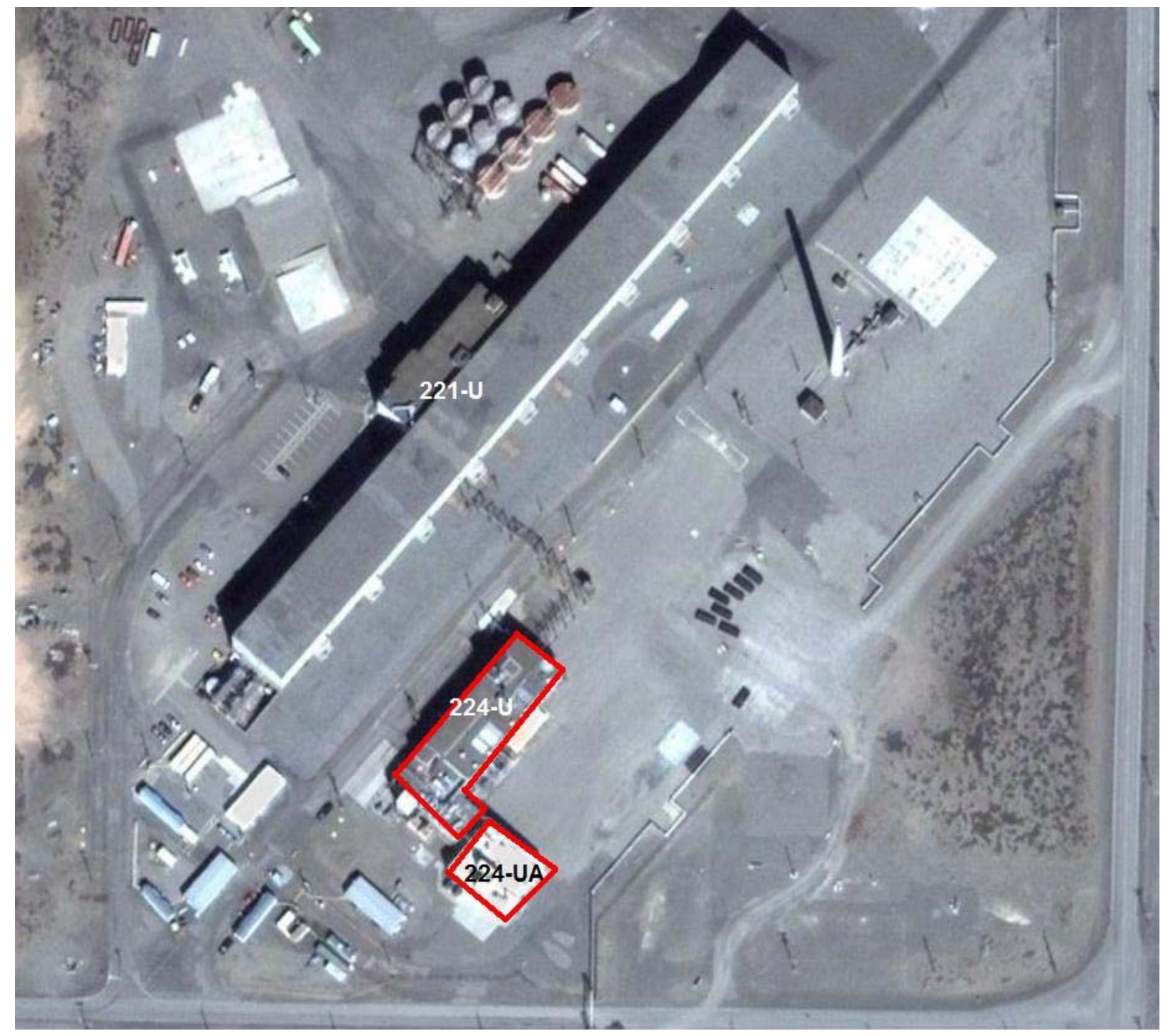

Figure 1.1. The 224-U and 224-UA Buildings, Which Are in a Location Surrounded by Other Large Structures, Complicating Atmospheric Dispersion Modeling. The location of the $\mathrm{UO}_{3}$ complex within the buildings is highlighted. 


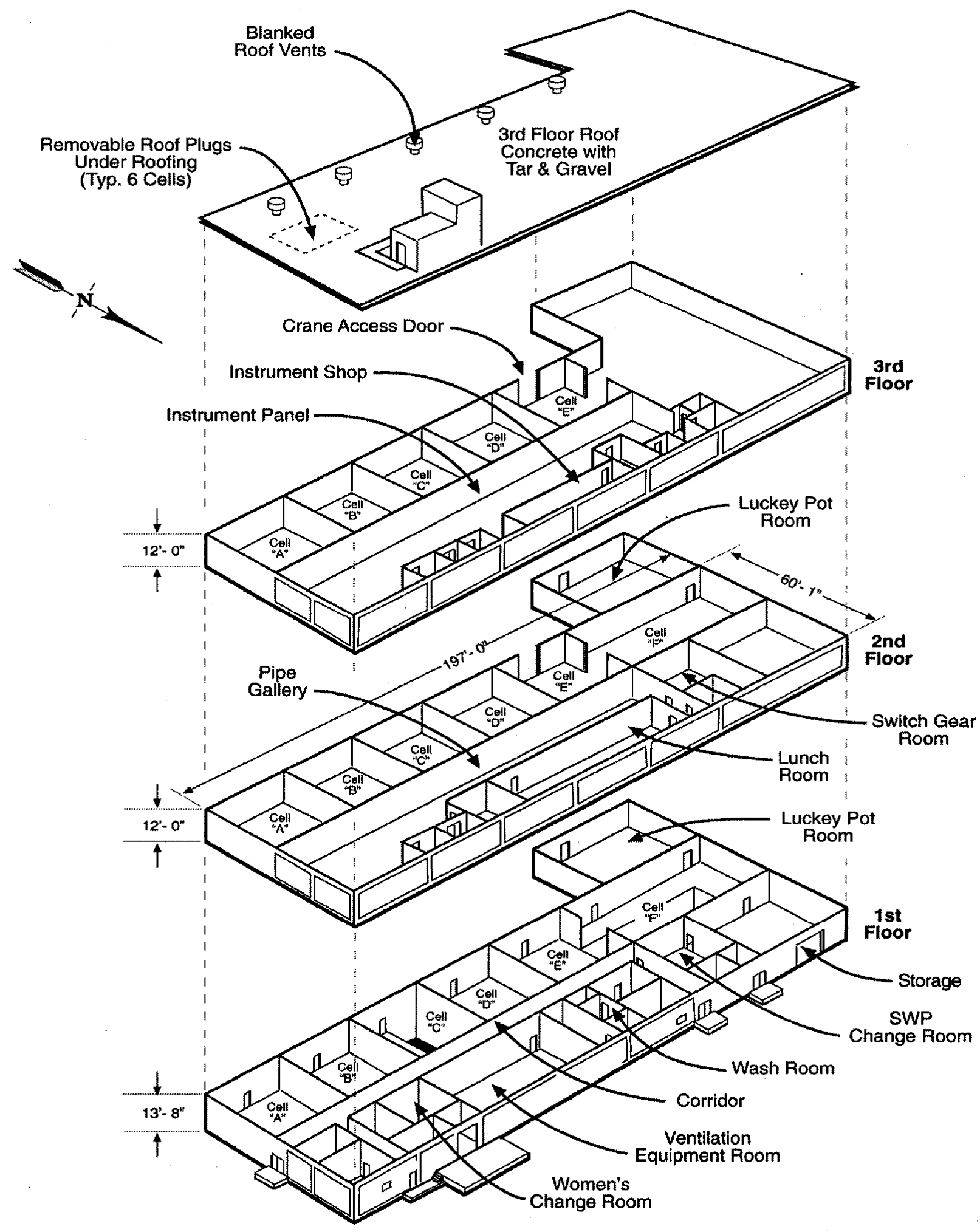

Figure 1.2. Cross-Section of 224-U Building 


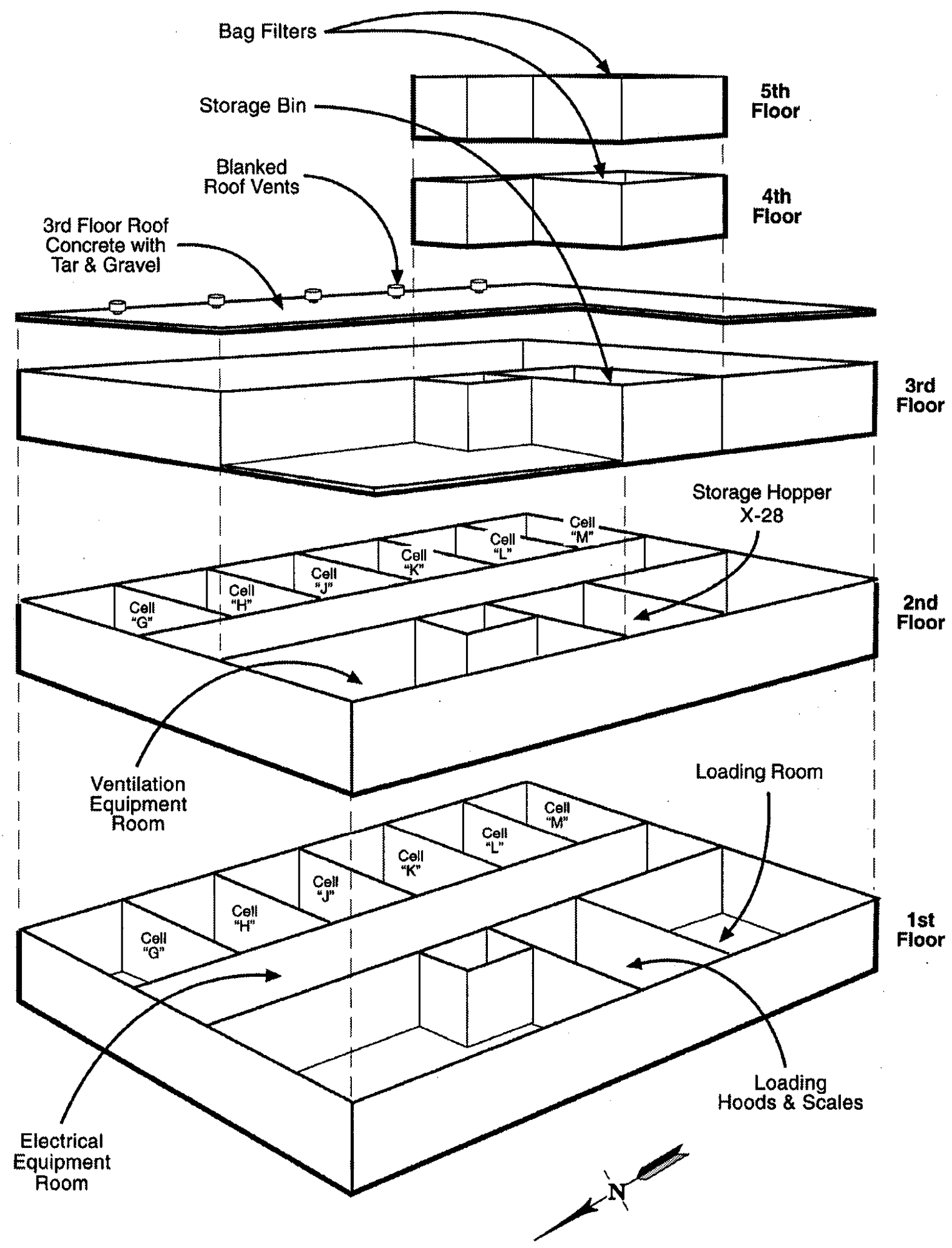

Figure 1.3. Cross-Section of the 224-UA Building 
The most-contaminated equipment associated with $224-\mathrm{U}$ is a pair of bag filter housings and a powder storage bin outside the southwest side of the building, and their associated ducting. The mostcontaminated equipment associated with the 224-UA building is a pair of bag filter housings on the northwest side of the building, in the narrow gap between 224-U and 224-UA, a HEPA filter on the roof, and their associated ducting. The calciners in 224-UA also contain a substantial quantity of $\mathrm{UO}_{3}$ powder.

There is a minimal working distance between the 224-U building and the $221-\mathrm{U}$ canyon building of about 150 feet $(50 \mathrm{~m})$.

This report covers the summary of analysis and results of modeling scenarios (Chapter 2.0), a description of the demolition operations considered (Chapter 3.0), a description of the source-term analysis (Chapter 4.0), and a summary of the atmospheric dispersion and deposition modeling used (Chapter 5.0). The references are in Chapter 6.0.

Computation details and model input/output listings are in the Appendices. The source term computation is in Appendix A, the emission rate computation in Appendix B, and the AERMOD input/output listings for the combined demolition of both buildings are in Appendix C. 


\subsection{Analysis and Results Summary}

Atmospheric dispersion modeling has been conducted in support of the demolition of the 224-U and 224-UA buildings using estimated release rates to provide information on the location and levels of radioactive contamination that may be expected. The facilities surrounding the $\mathrm{UO}_{3}$ plant have the potential to affect dispersion patterns through various meteorological phenomena, including building wake effects. Hourly meteorological data collected over a 5-year period were used to examine the effects of wind speed, direction, and stability on projected concentrations of contaminants in air and deposited on nearby surfaces.

The radioactive contamination of concern for $\mathrm{UO}_{3}$ plant demolition is largely located in powder handling systems associated with, and largely outside of, both buildings. A radionuclide inventory was provided by CHPRC (2008).

CHPRC (2008) also indicates that the demolition activities will occur in two distinct phases. Initially, the 224-U building will be completely demolished and removed. Then the 224-UA building will be demolished and removed. The modeling is thus conducted for two time periods: a 20 -working-day period for 224-U, and a separate 20 -working-day period for 224-UA. The overall period is anticipated to fall within the periods of July-September and /or October-December 2009.

\subsection{Analysis}

EPA's AERMOD computer code is used to estimate atmospheric dispersion and deposition of the released radioactive materials in the immediate vicinity of the demolition activities. These hour-by-hour estimates for the time periods that demolition is planned included the effect of the nearby buildings on the air flow. These estimates are made for weather conditions that are representative of the expected demolition period. The air concentrations and deposition rates are modeled for an array of receptors covering the demolition site and surrounding area. Both average and peak values of air concentrations are evaluated with modeling results reported as DAC for air concentrations and dpm per $100 \mathrm{~cm}^{2}$ for deposition.

The modeling analysis defines the potential levels of air and soil exposures from the proposed demolition activities. Potential air exposures are defined in terms of 1 ) the distribution of occurrences of peak concentrations at a control boundary and 2) spatial patterns of average and peak concentrations. The potential soil exposures are defined in terms of spatial patterns of total deposited concentrations of alpha and beta/gamma emitting materials.

Using these methods, emission and air-transport computations were made to assess the potential concentrations from different sets of demolition assumptions. The analysis process consisted of three steps:

- Step 1. Estimate the emission rates for the proposed demolition activities - Step 1 starts with an estimate of the amount of contamination in the structure, what form it is in, and where it is located. Demolition methods and associated activities are identified in this step. These data are combined to generate estimates of emissions during the demolition activities. 
- Step 2. Compute the airborne and deposited concentrations-Step 2 takes the emission rate estimates from Step 1 and produces estimates of environmental concentrations. An assumed 1-hour release is used to define potential peak exposures. The main products are hourly peak air concentrations and hourly peak (equivalent to total) deposition.

- Step 3. Determine if the potential concentration levels are acceptable-Step 3 uses standards to evaluate the viability of the demolition option that has been modeled. For air exposures, a derived air concentration limit of 12 (DAC)-hours per week is used. For deposition, a limit $1000 \mathrm{dpm} / 100 \mathrm{~cm}^{2}$ of uranium is used. If none of the locations within the selected areas show values that exceed these limits, then the demolition is deemed clearly viable.

\subsection{Results}

The worst case for the occurrence of the highest air concentrations is for the majority of the release to occur over a short time period. To address this possibility, the potential peak hourly concentrations over the planned work periods were modeled using the estimated emission rates.

To evaluate the potential exposure levels from the planned demolition activities, the local patterns of air concentrations and soil deposition were computed for each hour of the appropriate time periods using 5 years of recent meteorological data. Although the demolition is planned to occur either in the July to September or the October to December 2009 time frame, the potential peak concentrations were computed for entire years of data to provide an analysis that will produce bounding values no matter when the demolition occurs at this site. On each day, the activities are assumed to occur during the day shift.

Because none of the modeled values exceeded the air and soil concentration limits, it was unnecessary to generate frequency of occurrence plots for air and soil concentrations. That is, the gridded modeling results show that frequency of occurrence of exceedances will be zero at all control boundaries.

The analysis showed that open-air demolition of the $\mathrm{UO}_{3}$ Plant buildings is a viable option. The planning for open-air demolition includes definition of the measures that will be taken to minimize air emissions during the demolition activities. The modeling results presented here are closely tied to the details of how the demolition is to be conducted.

\subsection{Discussion}

The results indicate that the radiological exposures from the planned demolition efforts will be below the designated limits for air and soil exposures. 


\subsection{Demolition Analysis}

The potential emission rates associated with proposed demolition activities are estimated based on specific methods of execution. This section provides a definition or description of those activities including the assumptions and approximations that are required to provide a context for the demolition.

\subsection{Existing Radionuclide Inventories}

The radioactive contamination of concern for the $\mathrm{UO}_{3}$ complex building demolition is located within process equipment remaining in and adjacent to the buildings.

A conservatively estimated inventory provided by CHPRC (2008) is given in Table 3.1. The inventory is assumed to consist of residual dust adhering to the inner surfaces of the machinery, ducts, and filter housings. The dust is assumed to be $\mathrm{UO}_{3}$ powder, with a density of $7.29 \mathrm{grams} / \mathrm{mL}$ and 5-micron particle size.

Demolition of the buildings, although different in specifics, is assumed to be similar with three main components: removal of the external $\mathrm{UO}_{3}$ powder-handling systems, demolition of the main parts of the structures, and demolition of the calciners and their associated equipment.

For this analysis, the uranium is assumed to be enriched to $1 \%{ }^{235} \mathrm{U}$. The material has been separated for nearly 20 years; therefore, it is assumed that the short-lived uranium progeny are in equilibrium with the uranium isotopes. As a result, 1 gram of $\mathrm{UO}_{3}$ powder is assumed to contain:
${ }^{238} \mathrm{U},{ }^{234} \mathrm{Th},{ }^{234 \mathrm{~m}} \mathrm{~Pa}$
3.33E-7 Ci
${ }^{235} \mathrm{U},{ }^{231} \mathrm{Th}$
2.14E-8 Ci
${ }^{234} \mathrm{U}$
5.24E-7 Ci (Slightly enriched from equilibrium with ${ }^{238} \mathrm{U}$.)

The uranium isotopes all emit alpha particles; the progeny are all beta/gamma emitters. Thus, one gram of $\mathrm{UO}_{3}$ powder/dust will contain 20,180 dpm.

\subsection{Demolition of the $\mathrm{UO}_{3}$ Powder-Handling Systems with Multiprocesssor}

At the initiation of work, the most-accessible equipment on the outsides of the buildings is assumed to be removed first. For both buildings, this includes the ducting, bag filter housings, and for 224-U, the storage hopper.

The modeling analysis requires a definition of representative demolition activities and a plan for performing those activities. The equipment is removed using a large excavator to manipulate a multiprocessor. This device will separate the components into manageable sizes and lower them to the 
ground. It is anticipated that one working day will be required to dismantle the external equipment and a second working day to package it into ERDF boxes.

Misting, water, and fixatives will be used throughout the demolition process and load-out to minimize airborne contamination spread. All demolition and load-out will only occur when sustained wind speeds are less than 12 miles per hour.

Table 3.1. Conservative Inventory of Radionuclides in $\mathrm{UO}_{3}$ Complex, Grams

\begin{tabular}{lcc}
\hline 224-U Building & & \\
Location & Quantity (lbs) & Quantity (g) \\
\hline Primary Bag Filters X-3 & 85 & 38600 \\
Hopper X-3 & 20 & 36300 \\
Powder Handling Cyclone X-5 & 180 & 9070 \\
Storage Bin X-7 & 40 & 181600 \\
Secondary Bag Filters X-11 & 50 & 22700 \\
Hopper X-11 & 18 & 8170 \\
Glass Wool Filter X-12 & 473 & 215000 \\
Total & & \\
\hline 224-UA Building & 12 & 5440 \\
Location & 300 & 136000 \\
\hline Zero ft Level Pickup Bins G-M & 36 & 16300 \\
Calciners G-M & 28 & 12700 \\
Powder Handling Cyclones, X-22-1 \& -2 & 140 & 63500 \\
Storage Bin X-26-1 & 50 & 22700 \\
Primary Bag Filters and Pipes X-28-1 \& -2 & 140 & 63500 \\
X-28's Hopper & 50 & 22700 \\
Secondary Bag Filters and Pipes X-29-1 \& -2 & 130 & 59000 \\
X-29's Hopper & & 402000 \\
Discharge to HEPA Filters and HEPA Filters & & \\
Total & 140 \\
\hline
\end{tabular}

Rubble produced during demolition will be loaded out with front-end loaders or the thumb-andbucket on the excavator. The rubble will be picked up and placed into transfer boxes at various locations within the excavation. No additional containment structures (tents, etc.) are assumed for this activity; however, like the demolition itself, misting, water, and fixatives will be used to minimize airborne contamination spread. All loading will only occur when sustained wind speeds are less than 15 miles per hour. The 12-mph wind speed only applies when disturbing the $\mathrm{UO}_{3}$-contaminated equipment and debris. 


\subsection{Demolition of the Main Building Structures}

The walls and roofs are to be demolished from the top down with a large excavator to manipulate a multiprocessor. This device will crush the concrete into roughly 1 -foot pieces. (The ERDF acceptance criteria include requirements that the pieces be sized into less than 1-foot cubes.) Misting, water, and/or fixatives will be used throughout the demolition process and load-out to minimize airborne contamination spread. It is assumed that demolition of the 224-U and 224-UA building superstructures will take 8 working days each. All demolition and load out will only occur when sustained wind speeds are less than 12 miles per hour.

Rubble produced during demolition will be loaded out with front-end loaders and the thumb-andbucket on the excavator. The rubble will be picked up and placed into transfer boxes at various locations within the demolition zone. No additional containment structures (tents, etc.) are assumed for this activity; however, like the demolition itself, misting, water, and fixatives will be used to minimize airborne contamination spread. All loading will only occur when sustained wind speeds are less than 15 miles per hour.

\subsection{Demolition of the Calciners and Associated Equipment}

The continuous calciners are located on the second floor of the 224-UA cells. The calciners are large pieces of mechanical equipment developed and designed at Hanford. They had large troughs with paddle agitators that essentially turned and mixed the UNH throughout the process. The calciners used air as an agitant and maintained a seal between the calciner and the powder pickup bin, while passing a continuous stream of $\mathrm{UO}_{3}$. The calciners produced a pebbly product that consisted of spheres with an average diameter of 200 microns; the residual is assumed to be smaller (about 5-micron activity median aerodynamic diameter [AMAD]) dust particles.

It is assumed that the demolition of the calciners, because they are large, robust pieces of concrete and steel, will take 10 working days. They will be removed in large sections using a large excavator to manipulate a multiprocessor, with minimal disruption of the internal components of the calciners, although they may have to be broken into large segments for handling and disposal.

Rubble produced during demolition will be loaded out with front-end loaders and/or the thumb-andbucket on the excavator. The rubble will be picked up and placed into transfer boxes at various locations within the demolition zone. No additional containment structures (tents, etc.) are assumed for this activity; however, like the demolition itself, misting, water, and fixatives will be used to minimize airborne contamination spread. All sorting of contaminated debris will only occur when sustained wind speeds are less than 15 miles per hour.

\subsection{Demolition Schedule Assumed for Modeling}

The basic plan is that all demolition will occur between October and December 2009.

A generic daily schedule was provided by CHPRC staff. This generic schedule provides an indication of the hours of each day in which activities occur and the approximate fractions of time spent in demolition, load-out, and other procedures that result in emissions. This general schedule will be run 
5 days per week, with one 10-hour shift per work day. It is assumed that actual demolition activities will take place during only about 8 hours per work day.

The demolition activities are thus assumed to be conducted for two time periods: a 20-working-day period for 224-U and a second 20 -working-day period for 224-UA. It is assumed that the 224-U building outside equipment will be removed first, and then the relatively-uncontaminated remainder of the 224-U building. The external equipment on the 224-UA building will be removed, followed by the building superstructure, and then the calciners and supporting foundations and associated equipment underneath. 


\subsection{Source-Term Analysis}

The radiological consequences have been established using the methods discussed in DOE-HDBK3010-94 (DOE 1994). This approach was successfully used for the 233-S Building (AlphaTRAC 2003a, 2003b), 232-Z Building (Droppo and Napier 2006), and 105 KE Basin (Napier et al. 2008) and is particularly appropriate for facilities such as the $\mathrm{UO}_{3}$ Plant buildings, where the dose from the inhalation pathway will dominate the overall dose.

\subsection{Analysis Methodology}

The source term may be quantified using the five-factor formula. ${ }^{\text {(a) }}$

$$
\begin{gathered}
\mathrm{ST}=\mathrm{MAR} * \mathrm{DR} * \mathrm{ARF} * \mathrm{RF} * \mathrm{LPF} \\
\mathrm{ST}=\mathrm{MAR} * \mathrm{EF}
\end{gathered}
$$

where $\quad$ Source term $(\mathrm{ST})=$ the total quantity of respirable material released to the atmosphere during the demolition

$$
\begin{aligned}
\text { Material-at-risk }(\mathrm{MAR})= & \begin{array}{l}
\text { the total quantity of radionuclides (in grams or curies of activity } \\
\text { for each radionuclide) available to be acted on by a given } \\
\text { physical stress }
\end{array} \\
\text { Damage ratio }(\mathrm{DR})= & \begin{array}{l}
\text { the fraction of the MAR actually impacted by the demolition } \\
\text { conditions }
\end{array} \\
\text { Airborne release fraction }(\mathrm{ARF})= & \text { the fraction of a radioactive material suspended in air as an } \\
& \text { aerosol and thus available for transport due to a physical stress } \\
& \text { from a specific activity }
\end{aligned}
$$

The last four factors are sometimes combined into an emission factor (EF) to be multiplied with the MAR, where $\mathrm{EF}=\mathrm{DR} * \mathrm{ARF} * \mathrm{RF} * \mathrm{LPF}$.

For these analyses, the MAR is defined as the inventory that is on the surface area being demolished. While it is permissible to exclude material forms that are considered to be unaffected from the MAR, experience suggests that for these forms, the DR is usually best set to zero for the release mechanism. The overall result using either approach is the same. However, by assigning DR values to each combination of the inventory form and release mechanism, there is the expectation that each credited form is also reviewed against secondary events and, therefore, less likely to be overlooked.

(a) The following discussion is adapted from GENII Computer Code Application Guidance for Documented Safety Analysis, DOE-EH-4.2.1.4-Interim-GENII, Rev. 1, U.S Department of Energy, Washington, D.C. 
Radioactive contamination emissions (STs) have been calculated by release mechanism and demolition area for on-shift and off-shift activities. The emissions from the applicable sources have been combined to provide emission estimates for each day from each demolition area.

In the following sections, each term is discussed in the order seen in the source-term equation.

\subsubsection{Material at Risk}

The material at risk for any unit operation is the quantity of the radioactive inventory that is exposed for demolition over a 1-day period. MAR values are calculated assuming no fixatives are used.

The building structural materials demolished using the multiprocessor will be crushed or torn into rubble pieces during removal. The contamination is largely dust and particulates from the $\mathrm{UO}_{3}$ calcining and powder-handling operations. The particle-size distribution of the $\mathrm{UO}_{3}$ dust will be assumed to be 5 microns for evaluating transport, dispersion, deposition, and dosimetry.

Some contamination that could be subject to resuspension during off-shift periods is calculated for each demolition area. However, the planned procedure is to have the working surfaces covered with fixatives sprays, etc. Given these assumptions, there is no removable MAR considered subject to resuspension during each off-shift period.

\subsubsection{Damage Ratio}

The main components containing $\mathrm{UO}_{3}$ powder are the powder-handling ducts, cyclones, hoppers, and filter (bag and HEPA) housings. It is assumed that these components are contaminated with dust on their inner surfaces. Removal with a multiprocessor will result in tears and holes in these pieces. It is assumed that $10 \%$ of the contained $\mathrm{UO}_{3}$ powder will be released through holes and tears in the metal.

A multiprocessor will be used to demolish the buildings and to break the calciners into large parts. The MAR is assumed to be evenly distributed over the entire contaminated area being worked on (wall segment, floor area, etc.). The DR is that portion or percentage of the contaminated area acted on by the shear force, or the portion or percentage of the contamination acted on by shear forces. Jaws are assumed to fracture, crush, spall, or otherwise impact the surface being sheared. Because the buildings are of concrete block and reinforced concrete construction, the equipment is assumed to reduce essentially the entire portion of wall or floor being worked on to small pieces. Half of the surfaces are assumed to be rubblized during demolition operations (factor of 0.5 ). This value is greater than that used for the $232-Z$ Building, which was based on pulling down a block wall (0.05-Droppo and Napier 2006), and slightly smaller than that used for the analysis of the reinforced concrete walls of the 233-S Building (0.6AlphaTRAC 2003a). Until placed in the ERDF boxes, all of the material rubblized will remain piled on the ground and moistened with water and routinely sprayed with fixatives.

The calciners are a special case. These machines are large, solid pieces with contamination on the inside surfaces. Because they are too large to simply remove, they must be broken into smaller pieces, but they will not be completely rubblized. Therefore, it is assumed that $10 \%$ of the surface contaminated with $\mathrm{UO}_{3}$ will be damaged. 


\subsubsection{Airborne Release Fraction}

Powders released from the ducts, cyclones, hoppers, and filter housings will be released into the air; they will fall to the ground under the work areas. The DOE handbook (DOE 1994) discusses several sets of experimental observations directly related to airborne releases from falling powders. Sutter et al. (1981) observed $\mathrm{UO}_{3}$ and $\mathrm{TiO}_{2}$ powders freely falling into moving air from a height of 3 meters. They report a release fraction of about $2 \times 10^{-3}$. Ballinger et al. (1988) developed a model for releases from falling powders, wherein

$$
\operatorname{ARF}=0.1064\left(\mathrm{M}^{0.125}\right)\left(\mathrm{H}^{2.37}\right) / \rho^{1.02}
$$

where $\mathrm{M}=$ mass spilled, $\mathrm{kg}$

$\mathrm{H}=$ height of spill, $\mathrm{m}$

$\rho=$ density, $\mathrm{kg} / \mathrm{m}^{3}$.

For a 1-kg release of $\mathrm{UO}_{3}$ powder, of density $7.29 \mathrm{~g} / \mathrm{mL}$ (CRC Handbook 1986), from a height of 3 meters, the estimated ARF using this equation is $1.6 \times 10^{-4}$. Plinke et al. (1991) reports an ARF approaching $10^{-3}$ for applicable powders; this reference also indicates a large decrease in ARF with increasing moisture content - thus confirming that misting will be helpful in reducing emissions. Once the powders fall to the ground, the ongoing demolition activities will result in rubble falling onto it. The DOE Handbook (ref., page 4-85) indicates an ARF of about $10^{-3}$ for suspension caused by objects falling into powder, with release fractions of up to $10^{-2}$ (but corrected for burial by fallen rubble). Based on these observations, an airborne release fraction of 0.01 is selected for application to falling powders released from contaminated equipment punctured by demolition operations.

For demolition of walls and floors, DOE's factors for impaction stress due to vibration shock were selected as the most representative release fractions for the crushing processes; the factor selected was 0.001 for removable contaminants (DOE 1994). EPA's (EPA 1995) compilation of airborne release fractions includes a range of uncontrolled release fractions for crushing of ores and rocks that range from 0.012 to 6 pounds per ton of ore, which relates to an ARF of $6 \times 10^{-6}$ to $3 \times 10^{-3}$-these ranges overlap, supporting the selection of the DOE values.

Load-out activities include picking up rubble with either a front-end loader or thumb and bucket on the excavator and dumping of rubble and larger pieces into transfer containers. Similar activities may also occur at a sorting/sizing station. As the material falls into the container from the elevated bucket of the loader where it originates, it will be subject to entrainment in the air. EPA considers its emission factor equation for aggregate-handling and storage piles to be applicable to the drop of bulk material onto piles (adapted from EPA 1995; available at http://www.epa.gov/ttn/chief/ap42/):

$$
\mathrm{ARF}_{\mathrm{DROP}}=1.6 \times 10^{-6}\left[(\mathrm{WS} / 2.2)^{1.3}\right] /\left[(\mathrm{M} / 2)^{1.4}\right]
$$

where WS $=$ characteristic wind speed over drop of material $(\mathrm{m} / \mathrm{s})$-A characteristic wind speed for rubble drop was calculated using a characteristic wind speed for the site estimated by examining a wind climatology from the Hanford Site (Mantooth 2003). A compilation of average wind speeds was provided in the climatology. The ARF is more influenced by periods of higher winds (such as wind 
gusts). The characteristic wind speed for rubble-handling was estimated to be $3.2 \mathrm{~m} / \mathrm{s}$ (AphaTRAC 2003a); the result is not sensitive to this assumption.

$\mathrm{M}=$ moisture value associated with dry material (control effectiveness of water spray handled separately) (\%). Because water spray and mist are applied to the pile, a moisture value of $2 \%$ for a wet construction aggregate was chosen, based on past experience. Small changes in assumed moisture content result in large variation of the resulting ARF, and the ARF decreases more than exponentially with $\mathrm{M}$; the $2 \%$ value selected is believed to be conservatively low, resulting in a calculated ARF that should overestimate the releases via this route.

The EPA equation includes a particle-size multiplier ranging from about 0.1 to 0.8 . For this analysis, this was conservatively set to 1.0 for all particle sizes. Using these values, the ARF for rubble-handling is estimated to be $2.3 \times 10^{-6}$.

\subsubsection{Respirable Fraction}

The RF is the fraction of airborne radionuclides as particles that can be transported through air and inhaled into the human respiratory system. The RF is assumed to include particles $10-\mu \mathrm{m}$ AED and less. In this study, all of the suspendable material is addressed (not just the respirable portion). It is estimated that most radioactive particles in the contamination are respirable in size. In this study, the radioactive particles are considered to be all 5 microns. This particle size is an AERMOD input parameter that is used for transport and deposition computations.

A respirable fraction of 1.0 is applied in the ST equation because the removal of nonrespirable particles from the plume is treated separately as a transport and dispersion function within the AERMOD modeling.

\subsubsection{Leak-Path Factor}

The LPF is the fraction of the radionuclides in the aerosol transported through some confinement deposition or filtration mechanism. For the purpose of this study, the LPF is used to address any controls applied during and after the demolition process. This includes the effects of water mists, sprays, and fixatives applied to surfaces and rubble after demolition.

The application of a water mist to contaminated surfaces during demolition serves to reduce the percentage of airborne particulates in the respirable size range. The efficiency of the mist varies with each application and depends on, among other variables, mist particle size, water flow rate, and the size of potential airborne particles. For the purpose of this analysis, the water-mist application is assumed to reduce the quantity of airborne particulates by $90 \%$. The efficiency of the water-mist process must be weighed in light of the generated waste stream and the need to confine and capture runoff from the misting process. Thus, the LPF for concrete crushing is assumed to be 0.1 . This value is slightly lower than that used for the 233-S Building (0.3), based on observations of the effectiveness of the misting on that facility ${ }^{(a)}$ and during demolition of 232-Z.

(a) Conversation with Dan Mantooth, 26 May 2004. A continuous application of water from an atomizer (e.g., a "fog cannon") is assumed. 


\subsubsection{Limitation of Airborne Contamination}

The dosimetry depends on the mixture of radioisotopes present. The inventory listed in Table 3.1 is assumed to represent the $\mathrm{UO}_{3}$ present in the 224-U and 224-UA buildings and equipment. The spectrum of radionuclides is assumed to be constant.

DOE (1998) regulations specify in 10 CFR 835.2, "Definitions," that an airborne radioactivity area means any area accessible to individuals where the concentration of airborne radioactivity above natural background exceeds or is likely to exceed the DAC, or an individual present in the area without respiratory protection could receive an intake exceeding 12 DAC-hours in a week. If radionuclides "A," " $\mathrm{B}$," and " $\mathrm{C}$ " are present in concentrations $\mathrm{C}_{\mathrm{A}}, \mathrm{C}_{\mathrm{B}}$, and $\mathrm{C}_{\mathrm{C}}$, and if the applicable DACs are $\mathrm{DAC} \mathrm{C}_{\mathrm{A}}, \mathrm{DAC}_{\mathrm{B}}$, and $\mathrm{DAC}_{\mathrm{C}}$, respectively, then the concentrations shall be limited so that the following relationship exists:

$$
\frac{\mathrm{C}_{\mathrm{A}}}{\mathrm{DAC}_{\mathrm{A}}}+\frac{\mathrm{C}_{\mathrm{B}}}{\mathrm{DAC}_{\mathrm{B}}}+\frac{\mathrm{C}_{\mathrm{C}}}{\mathrm{DAC}_{\mathrm{C}}} \leq 1
$$

For a mixture of radionuclides where the concentrations of each are expressed in terms of a fraction, $\mathrm{f}$, of a total, $\mathrm{DAC}_{\mathrm{T}}$, this can be written as:

$$
\frac{f_{\mathrm{A}} * \mathrm{DAC}_{\mathrm{T}}}{\mathrm{DAC}_{\mathrm{A}}}+\frac{\mathrm{f}_{\mathrm{B}} * \mathrm{DAC}_{\mathrm{T}}}{\mathrm{DAC}_{\mathrm{B}}}+\frac{\mathrm{f}_{\mathrm{C}} * \mathrm{DAC}_{\mathrm{T}}}{\mathrm{DAC}_{\mathrm{C}}} \leq 1
$$

This relationship can be used to determine a maximum total concentration that meets the requirements as:

$$
\frac{f_{A}}{\mathrm{DAC}_{\mathrm{A}}}+\frac{\mathrm{f}_{\mathrm{B}}}{\mathrm{DAC}_{\mathrm{B}}}+\frac{\mathrm{f}_{\mathrm{C}}}{\mathrm{DAC}_{\mathrm{C}}}=\frac{1}{\mathrm{DAC}_{\mathrm{T}}}
$$

This sum-of-fractions approach has been used to determine a compound DAC for the mixture in the $\mathrm{UO}_{3}$ complex using the individual radionuclide DACs listed in the Appendix of 10 CFR 835. Using all of the reported radionuclides in the inventory, the DAC is $5.34 \times 10^{-10} \mu \mathrm{Ci} / \mathrm{mL}$.

The $20 \mathrm{dpm} / 100 \mathrm{~cm} 2$ alpha-emitter limit is equivalent to $1.03 \times 10-3$ grams $/ \mathrm{m} 2$; a $100 \mathrm{dpm} / 100 \mathrm{~cm} 2$ alpha-emitter limit is equivalent to $0.0513 \mathrm{~g} / \mathrm{m} 2 . "$.

\subsection{Unit Operations/Release Mechanisms}

ST release rates have been estimated for seven unit operations:

- Demolition of building segments with an excavator/multiprocessor

- Load-out of building segment rubble with a front-end loader

- Demolition of calciners and equipment with multiprocessor

- Pick-up of calciners and equipment segment rubble with front-end loader and excavator

- Demolition of ducting, cyclones, and filter housings with multiprocessor

- Pick-up of ducting, cyclones, and filter housings with front-end loader and excavator

- Resuspension during off-shift periods. 
A summary of the various parameters and their application in the source-term equation is provided in Table 4.1.

\subsection{Estimated Release Rates}

A series of spreadsheets were prepared to implement the models described above for the release rates for unit operations that define the steps of the demolition. The atmospheric transport analysis applied these on the appropriate number of days to complete the full demolition. These results are provided as the spreadsheet output listings presented in Appendix A.

The listings in Appendix A provide the results for unit operations demolishing external dust-handling equipment (ducts, cyclones, filter housings), buildings, calciners and associated equipment, and load-out of rubble from each in the immediate vicinity of the excavation.

The total emissions over the entire demolition procedure are also presented in the spreadsheet in Appendix A. It is interesting to note that the total amount of contaminated, respirable dust generated over the entire operation is dominated by about 20 grams from the dust-handling equipment of each building. These releases will occur over a brief period (less than one working day per building). The external filter housings dominate the source term and the releases; the emissions during the removal of these components will dominate the entire deposition footprint of the whole activity.

Table 4.1. Parameters Used in the EF Evaluation

\begin{tabular}{|c|c|c|c|c|c|c|c|}
\hline \multirow{2}{*}{$\begin{array}{c}\text { Parameter: } \\
\text { Term in Equation: }\end{array}$} & \multirow{2}{*}{$\begin{array}{c}\text { Damage } \\
\text { Ratio } \\
D R \\
\end{array}$} & $\begin{array}{l}\text { Airborne } \\
\text { Release } \\
\text { Fraction }\end{array}$ & $\begin{array}{c}\text { Size } \\
\text { Fraction } \\
\text { in Size } \\
\text { Range }\end{array}$ & $\begin{array}{l}\text { Leak } \\
\text { Path } \\
\text { Factor }\end{array}$ & \multirow{2}{*}{$\begin{array}{c}\text { Emission } \\
\text { Factor } \\
E F \\
\end{array}$} & \multicolumn{2}{|c|}{ Source Extent } \\
\hline & & $A R F$ & $R F$ & $L P F$ & & Applied to & Units \\
\hline Dust handling-demolition & 0.10 & $1.0 \mathrm{E}-02$ & 1.00 & 0.10 & $1.000 \mathrm{E}-04$ & Removable & $\mathrm{lb}$ \\
\hline Dust handling_loading & 0.10 & $2.3 \mathrm{E}-06$ & 1.00 & 0.10 & $2.300 \mathrm{E}-08$ & Removable & $\mathrm{lb}$ \\
\hline Main-demolition & 0.50 & $1.0 \mathrm{E}-03$ & 1.00 & 0.10 & $5.000 \mathrm{E}-05$ & Removable & $\mathrm{lb}$ \\
\hline Main—loading & 0.50 & $2.3 \mathrm{E}-06$ & 1.00 & 0.10 & $1.150 \mathrm{E}-07$ & Removable & $\mathrm{lb}$ \\
\hline Calciner-demolition & 0.10 & $1.0 \mathrm{E}-03$ & 1.00 & 0.10 & $1.000 \mathrm{E}-05$ & Removable & $\mathrm{lb}$ \\
\hline Calciner-loading & 0.10 & $2.3 \mathrm{E}-06$ & 1.00 & 0.10 & $2.300 \mathrm{E}-08$ & Removable & $\mathrm{lb}$ \\
\hline Resuspension-Between Shifts & 1.00 & $0.0 \mathrm{E}+00$ & 1.00 & 0.10 & $0.000 \mathrm{E}+00$ & Removable & $\mathrm{lb}$ \\
\hline
\end{tabular}




\subsection{Atmospheric Dispersion and Deposition Modeling}

Releases of contaminants to the air during the demolition activities described above potentially can have impacts in terms of the resulting increases in air and soil concentrations. An atmospheric dispersion modeling analysis has been conducted to generate estimates of these concentrations.

Potential air and soil exposures are modeled for the proposed demolition of the 224-U and 224-UA Buildings using the air dispersion model, AERMOD. AERMOD was developed by EPA and is the recommended dispersion model for regulatory air-quality computations. AERMOD includes formulations for addressing air dispersion in the immediate vicinity of air-emission sources. The model has dry deposition algorithms that account for the particle-size distribution and density as well as local surface and meteorological conditions. Important in the selection of AERMOD for this application is its capability to address building wake effects; the current version of AERMOD incorporates the building wake formulations developed by EPRI. The most recently released version of AERMOD (Version 07026 as implemented in Lakes ${ }^{\mathrm{a}}$ ISC/AERMOD Version: 5.9.0) was used.

AERMOD information and documentation is available at EPA's website for regulatory air models (EPA 2008). AERMOD is considered a commercial model. For Hanford Site applications, such a model must be tested to verify that it is operating correctly in its current implementation (Project Hanford Management System 2002). A series of test cases distributed with AERMOD obtained from the EPA website (EPA 2008) were run before and after the computations reported here. The AERMOD runs were conducted using two computers (PNNL property numbers WE13827 and WE13623) with the Windows XP operating system with recent updates installed on both computers. Test case results which are documented in the project files shows the code to be working correctly before and after the production runs.

\subsection{Air-Dispersion Modeling Assumptions and Data}

AERMOD is used to model the air concentrations and soil deposition associated with the planned demolition activities for 224-U and 224-UA Buildings

\subsubsection{Meteorological Data}

The air-dispersion analysis used multiple years of local meteorological data to define the local dispersion climatology. Five years of meteorological data records (calendar years 2002 to 2006) were obtained from the Hanford Meteorological Station (HMS) database for the analysis. ${ }^{(b)}$ Surface meteorological input data to AERMOD consisted of a merged dataset containing surface data incorporating wind speed and direction data from the Hanford telemetry station number 19 located in the 200W area combined with meteorological surface observations from the central HMS station. Vertical

(a) Lakes Environmental Software, Waterloo, Ontario, Canada, http://www.weblakes.com/lakecont.html.

(b) Ken Burk, Hanford Meteorological Station, Email dated 12/13/2007 defining link for 1997 to 2006 for Hanford meteorological data. 
structure input data to AERMOD consisted of radiosonde data from the meteorological station at the Spokane airport. ${ }^{(a)}$

Figure 5.1 shows a wind rose plot $^{(\mathrm{b})}$ for all hours for the 5 years from Hanford Station \#19. Figure 5.2 and Figure 5.3 show wind rose plots ${ }^{(\mathrm{a})}$ for the months and hours that the demolition activities are planned. A description of the Hanford observation network along with an annual summary of the local climatology can be found in Hoitink et al. (2004).

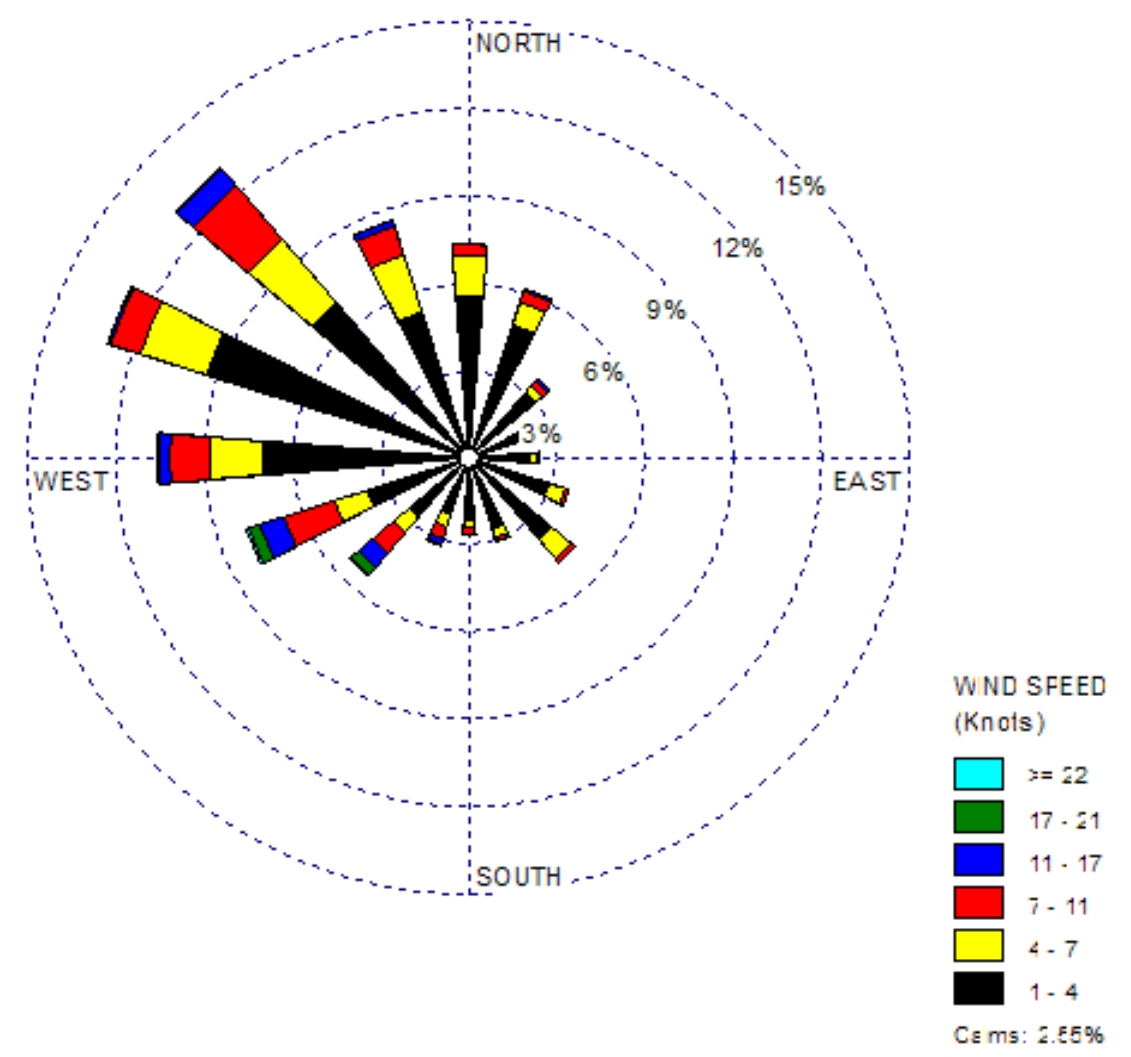

Figure 5.1. Five-Year Annual Wind Rose Hanford Station \#19 (2002-2006)

(a) Forecast Systems Laboratory (FSL)/National Climatic Data Center (NCDC) Radiosonde Database Access, http://raob.fsl.noaa.gov/ for radiosonde data for Spokane Washington.

(b) Meteorological convention is used, which defines winds by direction from which they come. 


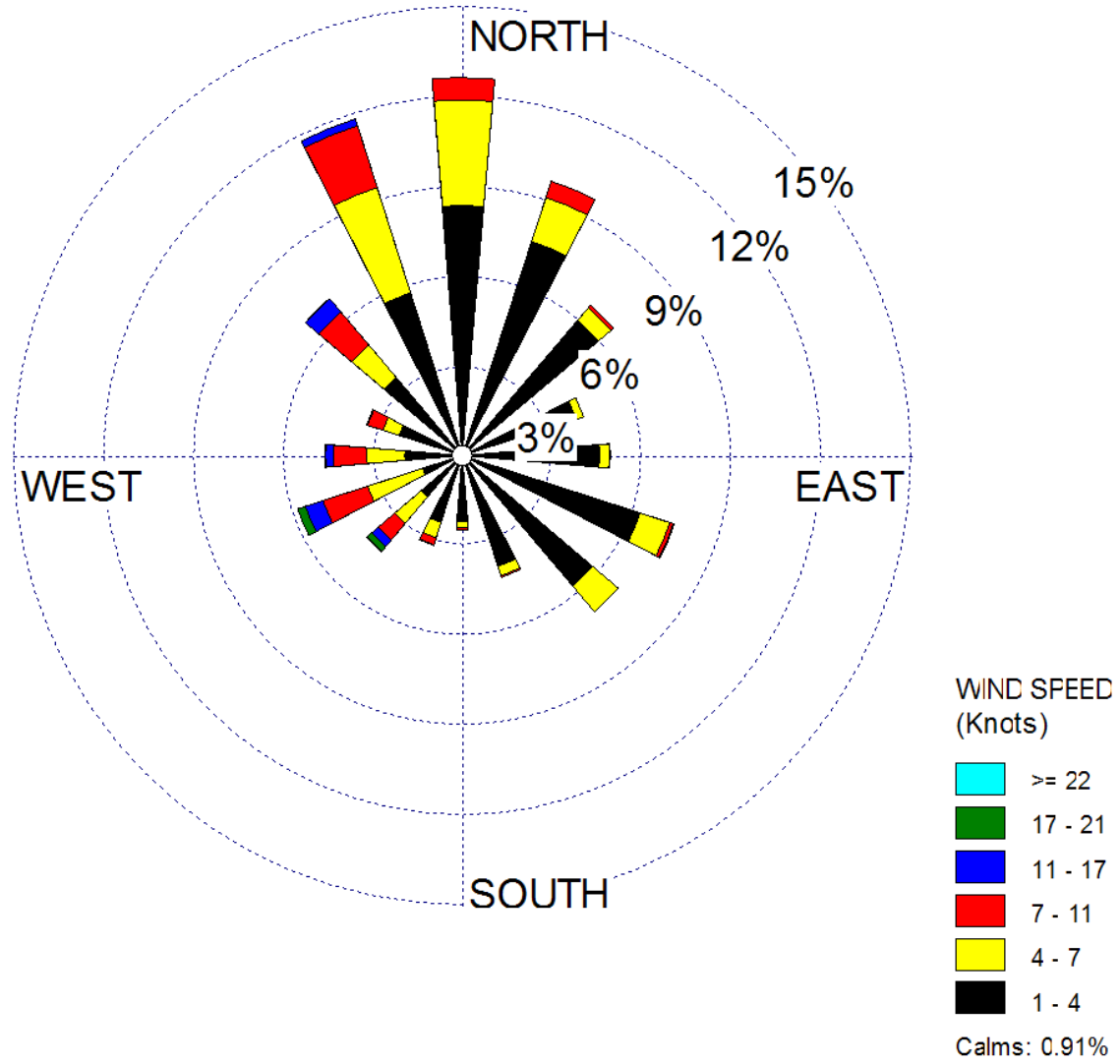

Figure 5.2. Five-Year Annual Wind Rose Hanford Station \#19 (2002-2006 July through September, hours 8:00 am to 4:00 pm) 


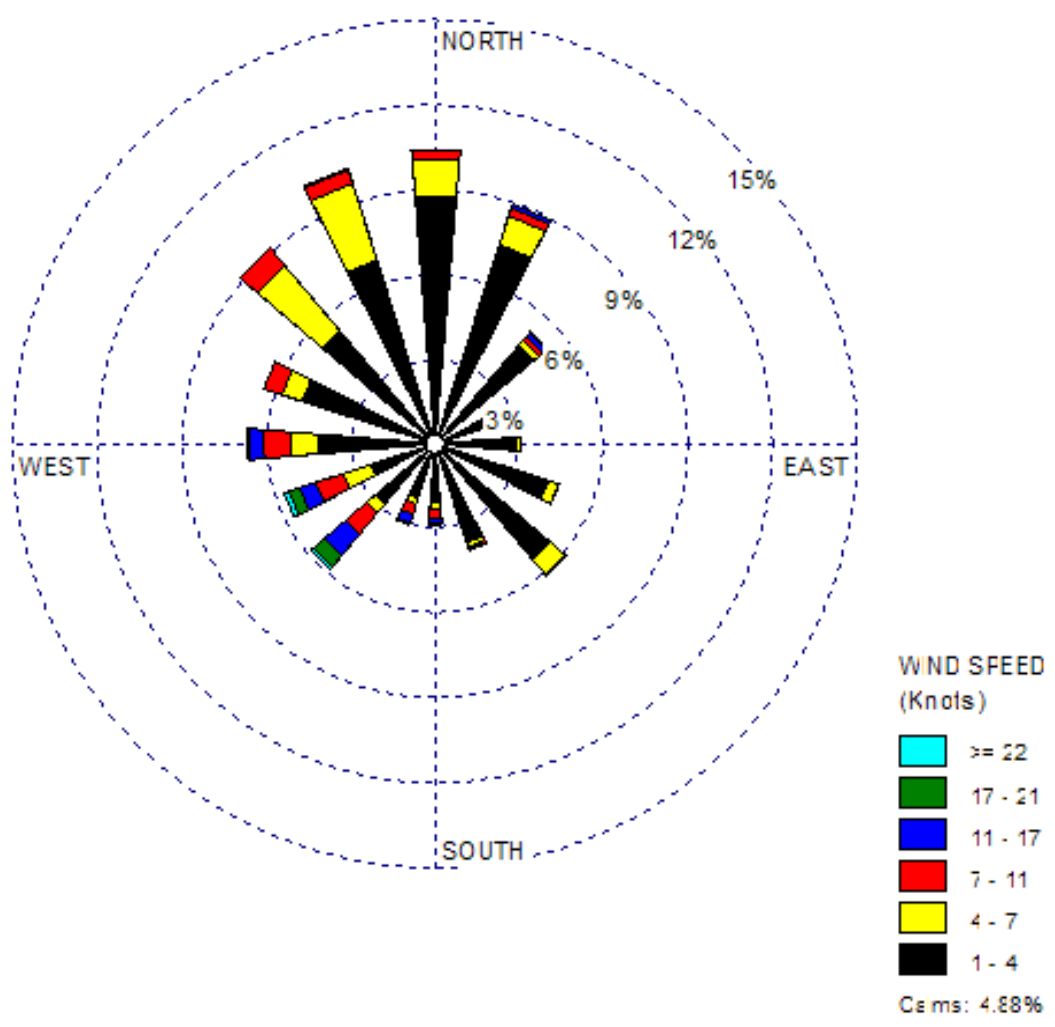

Figure 5.3. Five-Year Annual Wind Rose Hanford Station \#19 (2002-2006 Oct. through Dec., hours $8: 00$ am to $4: 00 \mathrm{pm})$

\subsubsection{Source Characteristics}

The main sources for air emissions will be the removal of the bag houses. These bag houses were modeled as a matrix of point sources, with each bag structure modeled as three vertical points (top, middle, and near ground level). The AERMOD runs were configured to directly produce maximum hourly concentration and deposition values for the days associated with the demolition activities.

\subsubsection{Modeling Approach}

The demolition is planned to occur in either the July to September or the October to December time frames. To fully bracket the possible peak exposures when the demolition occurs, the peak values of air and soil concentrations were modeled for an entire year of meteorological observations.

The activities are to occur during the day shift. The worst case for the occurrence of the highest air concentrations is for the majority of the release to occur over a short time period. To evaluate the potential exposure levels from the planned demolition activities, the local patterns of potential peak air concentrations and soil deposition were computed as though the estimated release from each of the buildings occurred during 1 hour. By looking at the potential peaks for all the hours during the planned 
work periods, the worst-case values are defined. The two proposed 3-month time periods for the demolition activities are included in this annual bounding computation.

\subsubsection{Receptor Grids}

Initial computations were made for a rectangular grid appropriate for defining the spatial patterns of the locations of the maximum air concentrations and deposition amounts.

\subsection{Air-Dispersion Modeling Results}

The air-dispersion results are presented as maps of potential peak air concentrations and deposition amounts. All demolition emissions were conservatively assumed to occur in 1 hour during the removal of the bag house structures. The maps are prepared as a composite of the peak values that were modeled for each hour over a five-year period. The results thus include the planned work periods but provide bounding information based on conditions that occur over at any time of the year. The values at each point on these maps represent the worst exposure that could occur at that point during demolition - no matter what time of year is selected for the demolition activities. The actual exposures that will occur during demolition will be defined by the ambient meteorological conditions that occur during the actual demolition process. The results of the modeling bound the values for the actual exposures.

The 224-U building demolition will occur first. Figure 5.4 is a map of the predicted peak hourly air concentrations for the 224-U building demolition. On this and following plots of air concentrations, isopleths are plotted at intervals to indicate conformance to the DAC-hour exposure limit given above;

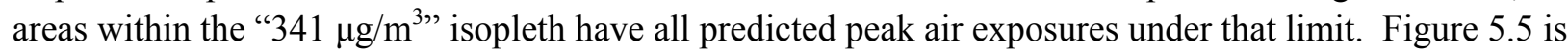
a map of the predicted peak deposition amounts for the 224-U building demolition. On this and following plots of deposition, isopleths are plotted at intervals to indicate conformance to the $1000 \mathrm{dpm} / 100 \mathrm{~cm}^{2}$ exposure limit given above; areas within the " $0.05 \mu \mathrm{g} / \mathrm{m}^{2 "}$ " isopleth have all predicted peak soil uranium depositions under the limit.

After the 224-U Building is removed, the demolition of 224-UA will start. The air-dispersion modeling accounts for the fact that the 224-U Building is gone. Figure 5.6 is a map of the predicted peak hourly air concentrations for the 224-UA Building demolition. Figure 5.7 is a map of the predicted peak deposition amounts for the 224-UA Building demolition by itself, and Figure 5.8 is a map of the predicted peak deposition amounts for both the 224-UA and 224-UA Building demolitions. 

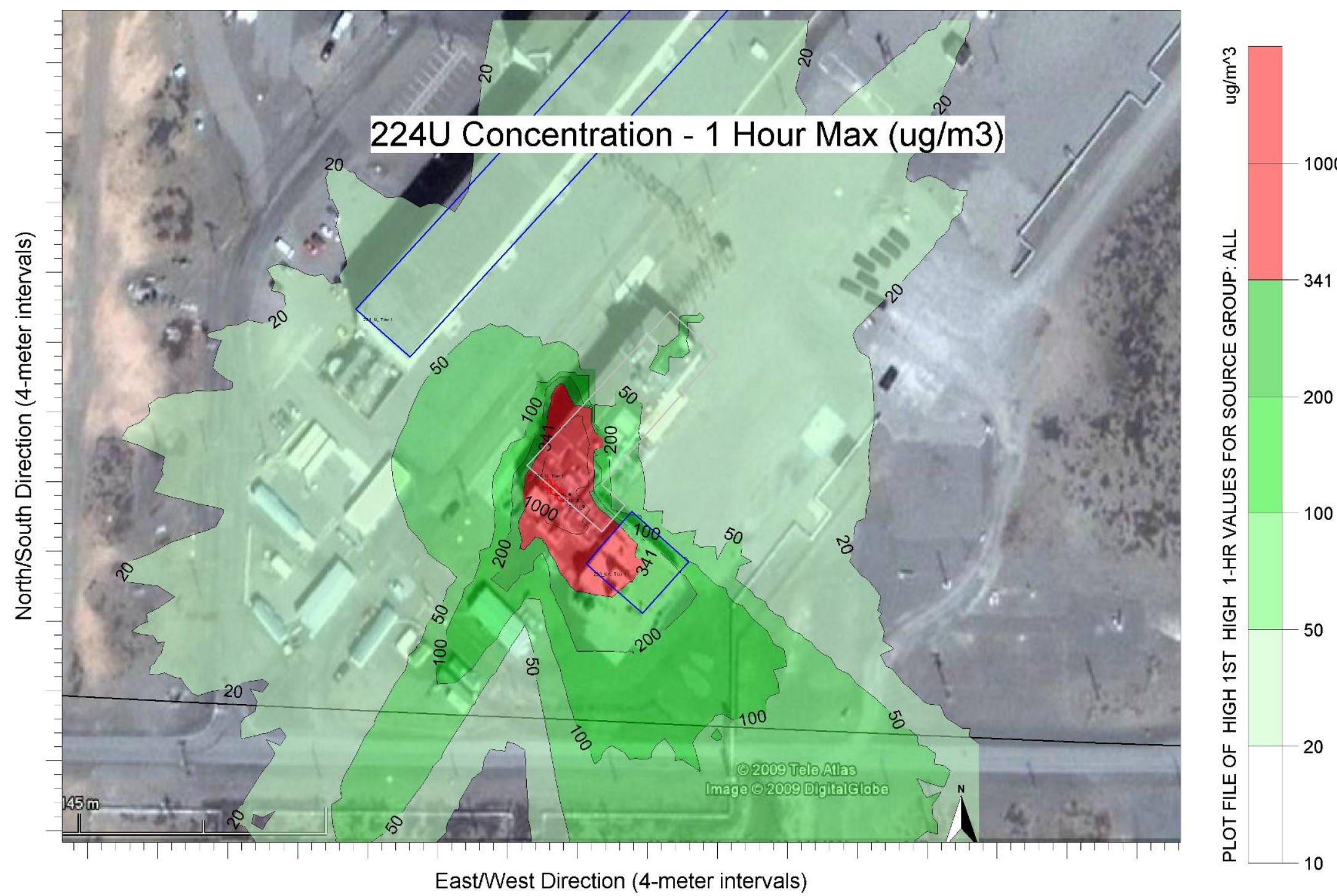

Figure 5.4. Predicted Peak Hourly Air Concentrations for the 224-U Building Demolition; Values Less Than $341 \mu \mathrm{g} / \mathrm{m}^{3}$ Correspond to Concentrations Below 12 DAC-hrs 

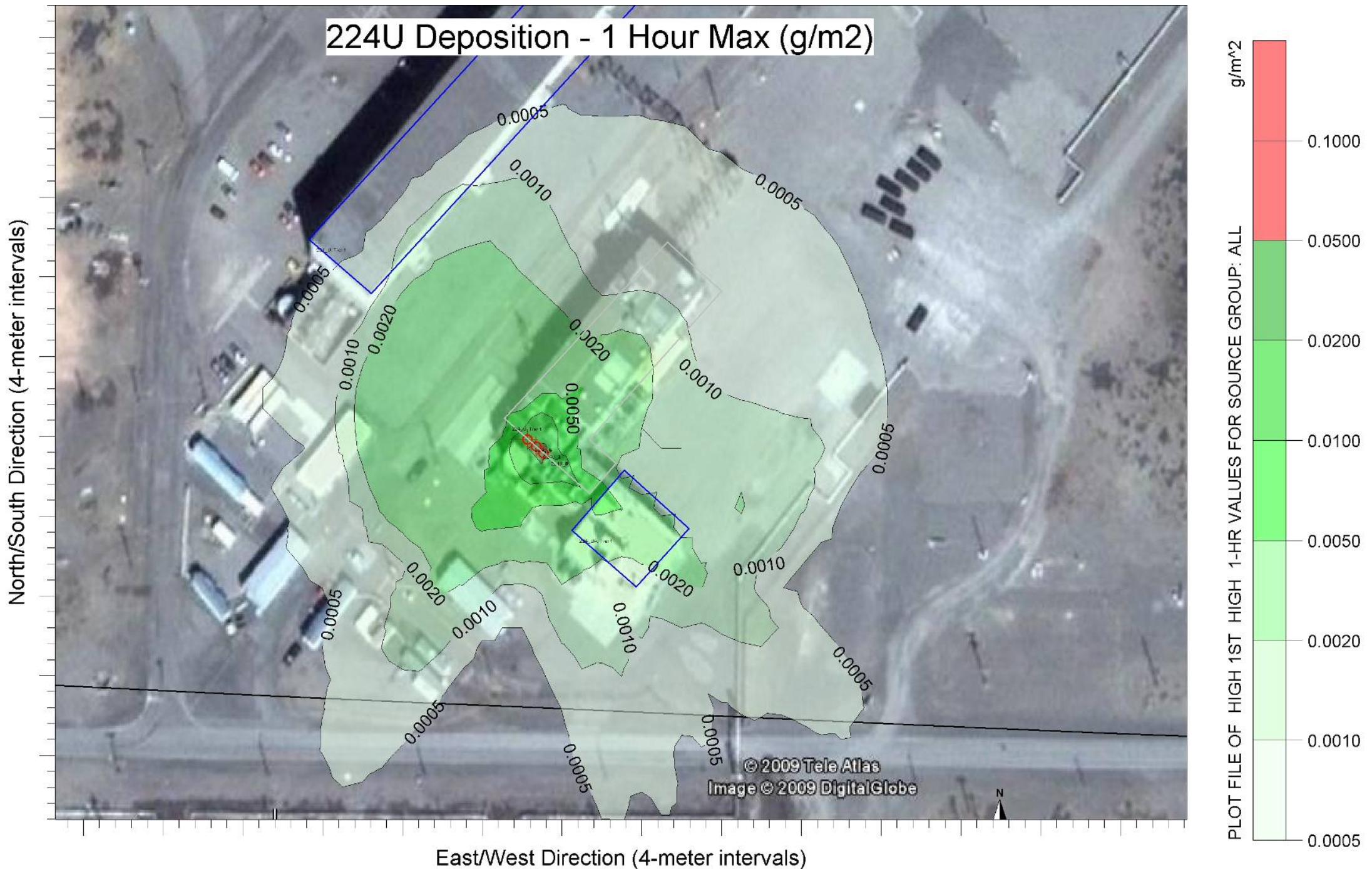

Figure 5.5. Predicted Peak Deposition Amounts for the 224-U Building Demolition; Values Less Than $0.05 \mu \mathrm{g} / \mathrm{m}^{2}$ Correspond to Uranium Deposition Rates of Less Than $1000 \mathrm{dpm} / 100 \mathrm{~cm}^{2}$ 


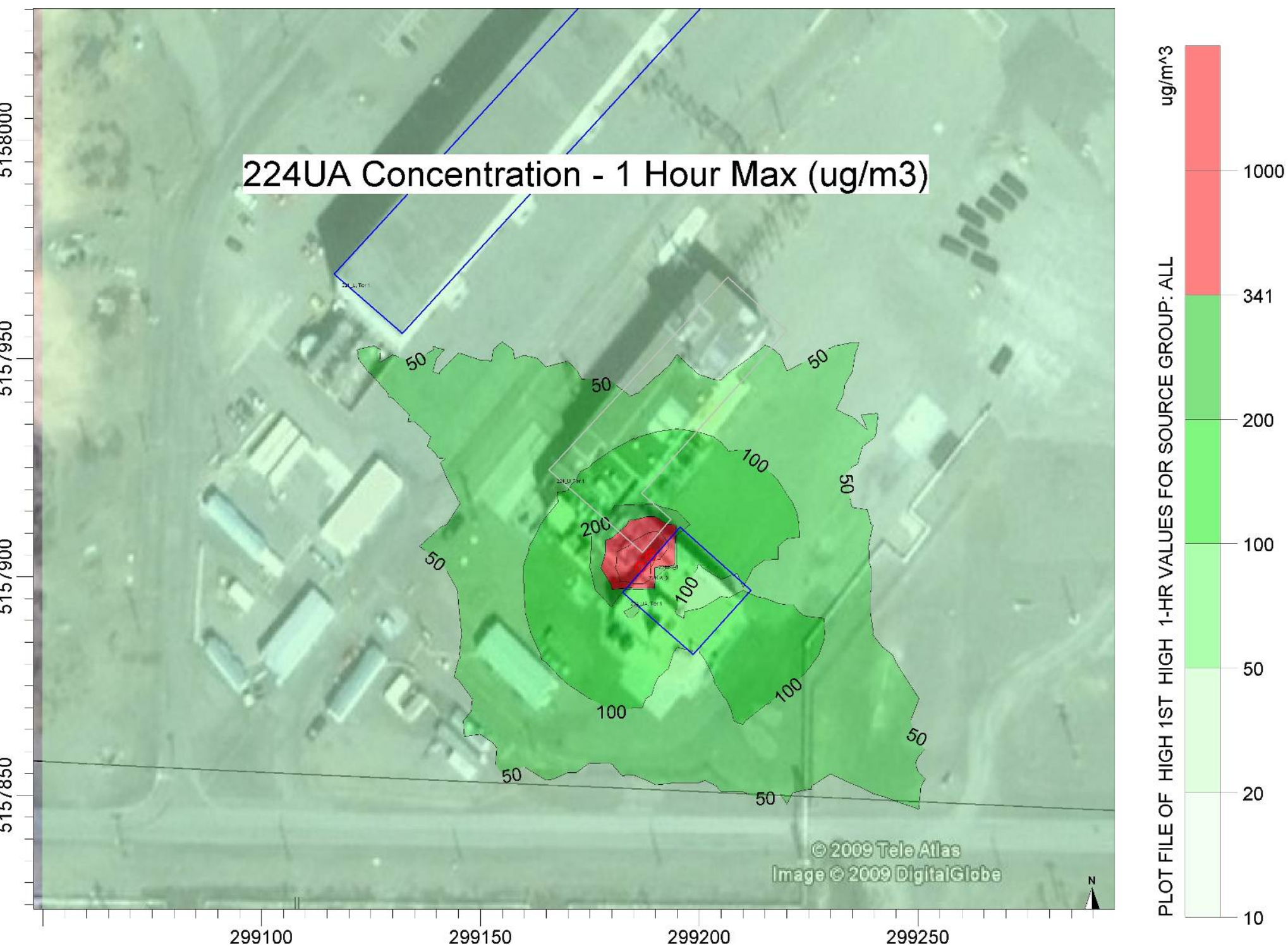

Figure 5.6. Predicted Peak Hourly Air Concentrations for the 224-UA Building Demolition; Values Less Than $341 \mu \mathrm{g} / \mathrm{m}^{3}$ Correspond to Concentrations Below 12 DAC-hrs 

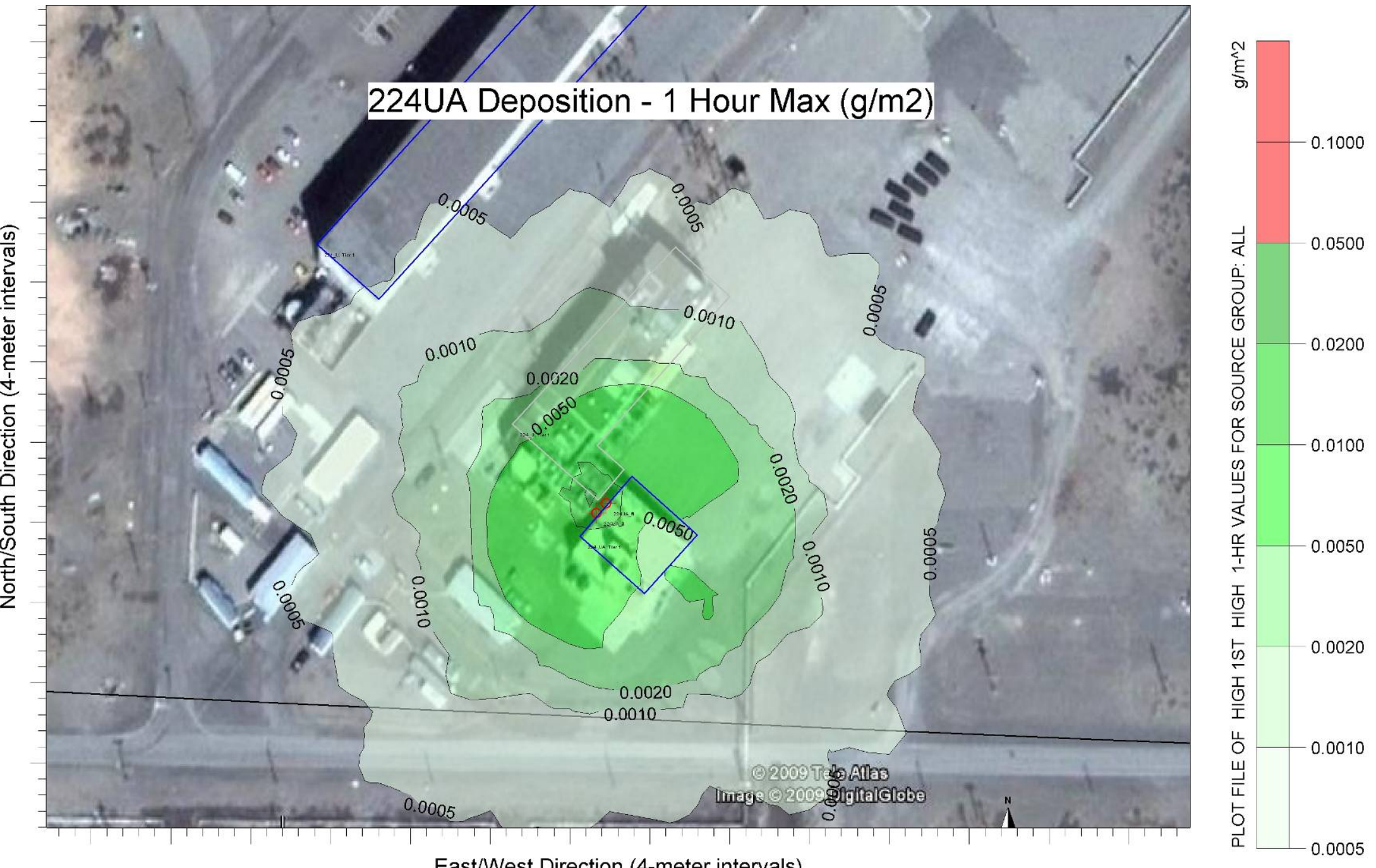

Figure 5.7. Predicted Peak Deposition Amounts for the 224-UA Building Demolition; Values Less Than $0.05 \mu \mathrm{g} / \mathrm{m}^{2}$ Correspond to uranium Deposition Rates of Less Than $1000 \mathrm{dpm} / 100 \mathrm{~cm}^{2}$ 


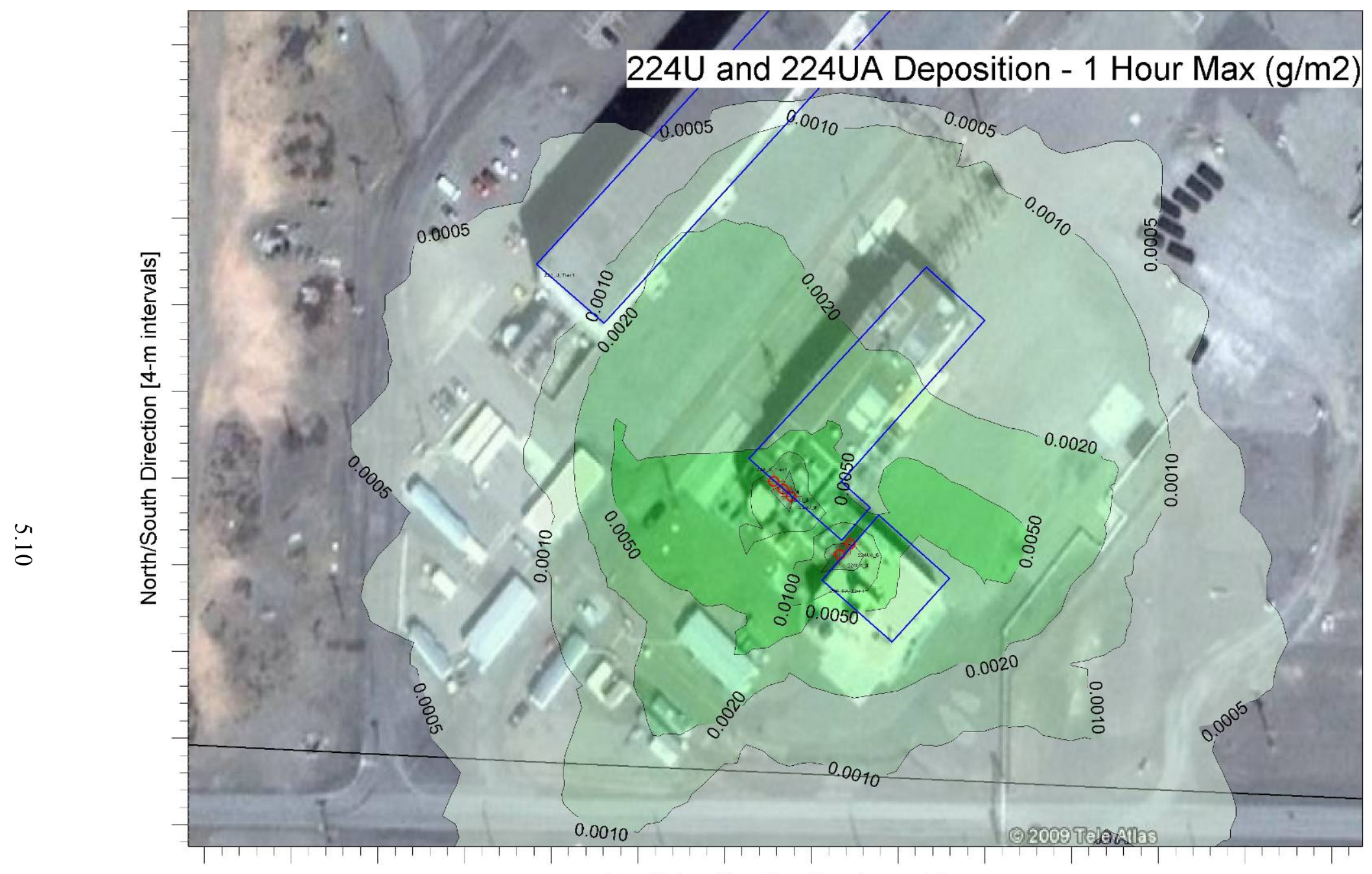

East।West Direction [4-m intervals]

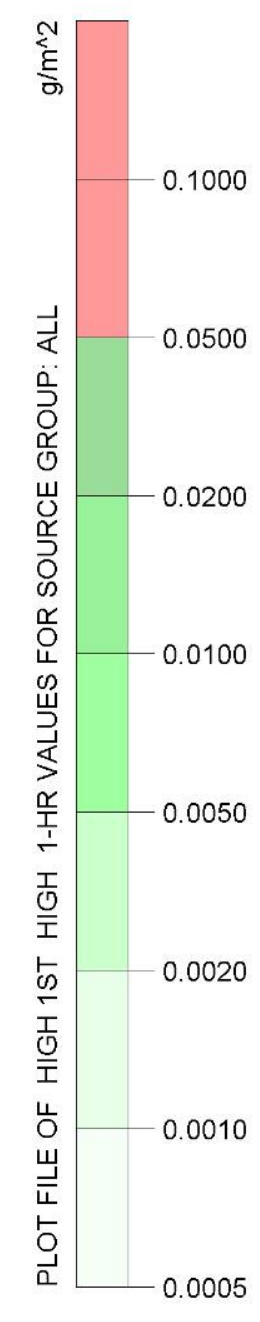

Figure 5.8. Predicted Peak Deposition Amounts for the 224A and 224-UA Building Demolition; Values Less Than $0.05 \mu \mathrm{g} / \mathrm{m}^{2} \mathrm{Correspond} \mathrm{to}$ Uranium Deposition Rates of Less Than $1000 \mathrm{dpm} / 100 \mathrm{~cm}^{2}$ 


\subsection{References}

AlphaTRAC. 2003a. Demolition Scenarios and Source Terms for Atmospheric Modeling. AlphaTRAC, Inc., 8670 Wolff Court, Suite 120, Westminster, Colorado.

AlphaTRAC. 2003b. Technical Review of Atmospheric Modeling for the 233-S Demolition Project at the Hanford Site. AlphaTRAC, Inc., 8670 Wolff Court, Suite 120, Westminster, Colorado.

Ballinger MY, JW Buck, PC Owczarski, and JE Ayer. 1988. Methods for Describing Airborne Fractions of Free Fall Spills of Powders and Liquids. NUREG/CR-4997 (PNL-6300), Pacific Northwest Laboratory, Richland, WA.

CH2M Hill Plateau Remediation Company (CHPRC). 2008. Statement of Work, Inter-Contractor Work Orders, , Agreement No: 36402-59, Release 00036402, Atmospheric Modeling for U Ancillary Demolition (Revision 4 12/16/2008).

CRC (Chemical Rubber Company). 1986. CRC Handbook of Chemistry and Physics, $67^{\text {th }}$ Edition. CRC Press, Boca Raton, Florida.

DOE (see U.S. Department of Energy)

Droppo JG, and Napier BA. 2006. Analysis of Radioactive Releases During Proposed Demolition Activities for the 232-Z Building. PNNL-15851, Pacific Northwest National Laboratory, Richland, Washington.

Droppo JG, Jr, BA Napier, ER Lloyd, DS Mantooth, MJ Minette, and EM Mattlin. 2007. “Operational Limitations for Demolition of a Highly Alpha-Contaminated Building - Modeled Versus Measured Air and Surface Activity Concentrations.” In 2007 Midyear Topical Meeting - Decontamination, Decommissioning, and Environmental Cleanup, January 21 - 24, 2007, Knoxville, Tennessee. PNNL-SA-52608, Pacific Northwest National Laboratory, Richland, Washington.

EPA (see U.S. Environmental Protection Agency)

Hoitink DK, KW Burk, JV Ramsdell Jr., and WJ Shaw. 2004. Hanford Site Climatological Data Summary 2003 with Historical Data. PNNL-14616, Pacific Northwest National Laboratory, Richland, Washington.

Mantooth D.S. 2003. Demolition Specifics, Fluor Hanford, Richland, Washington.

Napier BA, Droppo JG, and Rishel JP. 2008. Analysis of Radioactive Releases During Proposed Demolition Activities for the 105-KE Basin, PNNL-17631, Pacific Northwest National Laboratory, Richland, Washington.

Plinke MAE, D Leith, DB Holstein, and MG Boundy. 1991. "Experimental Examination of Factors That Affect Dust Generation.” American Industrial Hygiene Association Journal 52(12)521-528. 
Project Hanford Management System. 2002. Computer Software Management. HNF-PRO-309. October 24, 2002. Fluor Hanford, Inc. Richland, Washington.

Sutter SL, JW Johnston, and J Mishima. 1981. Aerosols Generated by Free Fall Spills of Powders and Solutions in Static Air. NUREG/CR-2139 (PNL-3786), Pacific Northwest Laboratory, Richland, Washington.

U.S. Department of Energy (DOE). 1994. DOE Handbook, Airborne Release Fractions/Rates and Respirable Fractions for Nonreactor Nuclear Facilities, Volume 1-Analysis of Experimental Data. DOEHDBK-3010-94.

U.S. Department of Energy (DOE). 1998. Title 10, U.S. Code of Federal Regulations, Part 835. Occupational Radiation Protection. FR 63:213, pp. 59662-59689.

U.S. Environmental Protection Agency (EPA). 1995. Compilation of Air Pollutant Emission Factors AP-42, Fifth Edition, Volume 1: Stationary Point and Area Sources. Research Triangle Park, North Carolina.

U.S. Environmental Protection Agency (EPA). 2008. Technology Transfer Network Support Center for Regulatory Air Models, http://www.epa.gov/scram001/(Access last checked 6/5/2008)). 
Appendix A

Source Computation Worksheet 


\section{Appendix A}

\section{Source Computation Worksheet}

\begin{tabular}{|c|c|c|c|c|c|c|c|c|c|c|c|}
\hline Location & Operation & $\begin{array}{c}\text { MAR } \\
\text { for Day } \\
1 b\end{array}$ & $\begin{array}{l}\text { Emission } \\
\text { Factor }\end{array}$ & $\begin{array}{l}\text { Duration of } \\
\text { Activity } \\
\text { (Hrs) }\end{array}$ & $\begin{array}{c}\text { Time of } \\
\text { day }\end{array}$ & $\begin{array}{c}\text { Daily } \\
\text { Release } \\
\text { lb }\end{array}$ & $\begin{array}{c}\text { Daily } \\
\text { Release } \\
\text { grams }\end{array}$ & $\begin{array}{c}\text { Duration of } \\
\begin{array}{c}\text { Demolition } \\
\text { days }\end{array} \\
\end{array}$ & $\begin{array}{c}\text { Total } \\
\text { Release } \\
\text { grams }\end{array}$ & \multicolumn{2}{|c|}{$\begin{array}{l}\text { Release Schedule } \\
\text { grams }\end{array}$} \\
\hline \multicolumn{12}{|c|}{ 224-U DEMOLITION AREA } \\
\hline & Dust handling- demolition & 473.0 & $1.00 \mathrm{E}-04$ & 8.0 & $8: 00-3: 00$ & $4.73 \mathrm{E}-02$ & $2.15 \mathrm{E}+01$ & 1.0 & 21.5 & Day 1 & 21.5 \\
\hline & Dust handling - loading & 473.0 & $2.30 \mathrm{E}-08$ & 8.0 & $8: 00-3: 00$ & $1.09 \mathrm{E}-05$ & $4.94 \mathrm{E}-03$ & 1.0 & $4.9 \mathrm{E}-03$ & Day 2 & $4.9 \mathrm{E}-03$ \\
\hline & Main - demolition & 0.0 & $5.00 \mathrm{E}-05$ & 4.0 & am & $0.00 \mathrm{E}+00$ & 0.0 & 8.0 & 0.0 & Days 3-20 & 0 \\
\hline & Main - loading & 0.0 & $1.15 \mathrm{E}-07$ & 4.0 & $\mathrm{pm}$ & $0.00 \mathrm{E}+00$ & 0.0 & 8.0 & 0.0 & & \\
\hline & Calciner - demolition & 0.0 & $1.00 \mathrm{E}-05$ & 4.0 & am & $0.00 \mathrm{E}+00$ & 0.0 & 10.0 & 0.0 & & \\
\hline & Calciner - loading & 0.0 & $2.30 \mathrm{E}-08$ & 4.0 & $\mathrm{pm}$ & $0.00 \mathrm{E}+00$ & 0.0 & 10.0 & 0.0 & & \\
\hline & $\begin{array}{l}\text { Resuspension - Between } \\
\text { Shifts }\end{array}$ & & $0.00 \mathrm{E}+00$ & 16.0 & off & $0.00 \mathrm{E}+00$ & 0.0 & 20.0 & 0.0 & & \\
\hline & & & & & & & & & 21.5 & & \\
\hline \multicolumn{12}{|c|}{ 224-U DEMOLITION AREA } \\
\hline & Dust handling- demolition & 558.0 & $1.00 \mathrm{E}-04$ & 8.0 & $8: 00-3: 00$ & $5.58 \mathrm{E}-02$ & $2.53 \mathrm{E}+01$ & 1.0 & 25.3 & Day 1 & 25.3 \\
\hline & Dust handling - loading & 558.0 & $2.30 \mathrm{E}-08$ & 8.0 & $8: 00-3: 00$ & $1.28 \mathrm{E}-05$ & $5.83 \mathrm{E}-03$ & 1.0 & $5.8 \mathrm{E}-03$ & Day 2 & $5.8 \mathrm{E}-03$ \\
\hline & Main - demolition & 3.5 & $5.00 \mathrm{E}-05$ & 4.0 & am & $1.75 \mathrm{E}-04$ & $7.95 \mathrm{E}-02$ & 8.0 & 0.6 & Days 3-10 am & $7.95 \mathrm{E}-02$ \\
\hline & Main - loading & 3.5 & $1.15 \mathrm{E}-07$ & 4.0 & $\mathrm{pm}$ & $4.03 \mathrm{E}-07$ & $1.83 \mathrm{E}-04$ & 8.0 & $1.5 \mathrm{E}-03$ & Days 3-10 pm & $1.83 \mathrm{E}-04$ \\
\hline & Calciner - demolition & 30.0 & $1.00 \mathrm{E}-05$ & 4.0 & am & $3.00 \mathrm{E}-04$ & $1.36 \mathrm{E}-01$ & 10.0 & 1.4 & Days $11-20 \mathrm{am}$ & $1.36 \mathrm{E}-01$ \\
\hline & Calciner - loading & 30.0 & $2.30 \mathrm{E}-08$ & 4.0 & $\mathrm{pm}$ & $6.90 \mathrm{E}-07$ & $3.13 \mathrm{E}-04$ & 10.0 & $3.13 \mathrm{E}-03$ & Days $11-20 \mathrm{pm}$ & $3.13 \mathrm{E}-04$ \\
\hline & $\begin{array}{l}\text { Resuspension - Between } \\
\text { Shifts }\end{array}$ & & $0.00 \mathrm{E}+00$ & 16.0 & off & $0.00 \mathrm{E}+00$ & 0.0 & 20.0 & 0.0 & & \\
\hline & & & & & & & & & 27.3 & & \\
\hline
\end{tabular}


Appendix B

\section{AERMOD Emission Rates}




\section{Appendix B}

\section{AERMOD Emission Rates}

Emission rates for input to the AERMOD model were computed for the demolition of the two sets of filter bags by assuming that the estimated mass suspended by demolition given in Appendix A occurred in a one hour period. The release from each filter bag was modeled as three points of release located from the surface up to the height of the filter structure. The suspended mass was assumed to be equally distributed over the each of the matrix of assumed release points. Emission rates used in the modeling for the 224-U and 224-UA buildings and associated structures listed in the table below are computed by dividing the estimated suspended mass by the number of modeled release points and the number of seconds in an hour (i.e., 3600 seconds).

Computation of AERMOD Emission Rate Inputs

\begin{tabular}{|c|c|c|c|c|c|c|}
\hline $\begin{array}{l}\text { Source } \\
\text { Name }\end{array}$ & $\begin{array}{c}\text { Number } \\
\text { of } \\
\text { Release } \\
\text { Points }\end{array}$ & $\begin{array}{l}\text { Max. Mass } \\
\text { Suspended } \\
\text { In One Hour } \\
\text { (g) }\end{array}$ & $\begin{array}{l}\text { Source } \\
\text { Id }\end{array}$ & $\begin{array}{l}\text { Release } \\
\text { Time } \\
\text { (s) }\end{array}$ & $\begin{array}{l}\text { AERMOD } \\
\text { Emission } \\
\text { Rate } \\
(\mathrm{g} / \mathrm{s})\end{array}$ & $\begin{array}{c}\begin{array}{c}\text { Release } \\
\text { Height }\end{array} \\
(\mathrm{m})\end{array}$ \\
\hline \multirow[t]{9}{*}{$224 U$} & \multirow[t]{9}{*}{9} & \multirow[t]{9}{*}{21.4742} & 224U_1 & 3600 & 6.64E-04 & 6.1 \\
\hline & & & 224U_2 & 3600 & $6.64 \mathrm{E}-04$ & 3.05 \\
\hline & & & 224U_3 & 3600 & $6.64 \mathrm{E}-04$ & 0 \\
\hline & & & $224 U_{-} 4$ & 3600 & $6.64 \mathrm{E}-04$ & 6.1 \\
\hline & & & $224 U \_5$ & 3600 & 6.64E-04 & 3.05 \\
\hline & & & 224U_6 & 3600 & 6.64E-04 & 0 \\
\hline & & & $224 U \_7$ & 3600 & 6.64E-04 & 6.1 \\
\hline & & & $224 U \_8$ & 3600 & 6.64E-04 & 3.05 \\
\hline & & & 224U_9 & 3600 & $6.64 \mathrm{E}-04$ & 0 \\
\hline \multirow[t]{6}{*}{$224 U A$} & \multirow[t]{6}{*}{6} & \multirow[t]{6}{*}{24.7884} & 224UA_1 & 3600 & 1.17E-03 & 6.1 \\
\hline & & & 224UA_2 & 3600 & 1.17E-03 & 3.05 \\
\hline & & & 224UA_3 & 3600 & 1.17E-03 & 0 \\
\hline & & & 224UA_4 & 3600 & 1.17E-03 & 6.1 \\
\hline & & & 224UA_5 & 3600 & 1.17E-03 & 3.05 \\
\hline & & & 224UA_6 & 3600 & 1.17E-03 & 0 \\
\hline
\end{tabular}


Appendix C

Output AERMOD Listing for Combined 224-U and 224-UA Building Analysis 
** AERMOD Input Produced by:

* * ISC-AERMOD View Ver. 5.9.0

** Lakes Environmental Software Inc.

** Lakes Environment

** File: C: \Jim\Udispersion \u_ua\u_ua_bd3.ADI

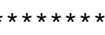

******

** AERMOD Control Pathway

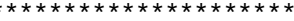

CO

TITLEONE U/UA Building Demolition

TITLETWO Unit Release - Worst Cas

MODELOPT CONC DDEP FLAT TOXICS

AVERTIME 1

POLLUTID UO3

CO FINISHED

** AERMOD Source Pathway

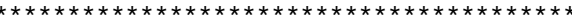

SO STARTING

* * Source Location **

** Source ID - Type - X Coord. - Y Coord. **

LOCATION 224U_1 POINT 299173.590 5157917.380 0.0

* DESCRSRC 224U Upper

LOCATION 224U_2 POINT 299173.590 5157917.380 0.0

* DESCRSRC 224U Middle

LOCATION 224U_3 POINT $299173.5905157917 .380 \quad 0.0$

* DESCRSRC 224U Lower

LOCATION 224U 4 POINT 299171.399 5157919.188 0.0

* * DESCRSRC 224U Upper

LOCATION 224U 5 POINT 299171.399 5157919.188 0.0

* * DESCRSRC $224 \mathrm{U}$ Upper

LOCATION 224U_6 POINT 299171.399 5157919.188 0.0

** DESCRSRC 224U Upper

LOCATION 224U_7 POINT 299175.4505157915 .6300 .0

* DESCRSRC 224U Uppe

LOCATION 224U_8 POINT 299175.450 $5157915.630 \quad 0.0$

* DESCRSRC 224U Upper

LOCATION 224U_9 POINT 299175.450 $5157915.630 \quad 0.0$

LOCATION 224UA I POINT 299186.653 5157902.299 0.0 
* * DESCRSRC 224U Upper

LOCATION 224UA_2 POINT 299186.653 5157902.299 0.0

* * DESCRSRC 224U Üpper

LOCATION 224UA_3 POINT 299186.653 5157902.299 0.0

* * DESCRSRC $224 \mathrm{U}$ Upper

LOCATION 224UA_4 POINT 299189.010 $5157904.760 \quad 0.0$

* DESCRSRC 224U Upper

LOCATION 224UA_5 POINT 299189.010 5157904.7600 .0

* * DESCRSRC 224U Upper

LOCATION 224UA_6 POINT $299189.0105157904 .760 \quad 0.0$

* * DESCRSRC 224U Upper

* Source Parameters **

SRCPARAM 224U_1 $0.00066358 \quad 6.096 \quad 0.000 \quad 0.00000 \quad 1.524$ $\begin{array}{llllllll}\text { SRCPARAM } & 224 \mathrm{U} 2 & 0.00066358 & 3.048 & 0.000 & 0.00000 & 1.524\end{array}$ $\begin{array}{lllllllll}\text { SRCPARAM 224U_3 } & 0.00066358 & 0.000 & 0.000 & 0.00000 & 1.524\end{array}$ $\begin{array}{llllllll}\text { SRCPARAM 224U_4 } & 0.00066358 & 6.096 & 0.000 & 0.00000 & 1.524\end{array}$ $\begin{array}{llllllll}\text { SRCPARAM } 224 U^{5} & 0.00066358 & 3.048 & 0.000 & 0.00000 & 1.524\end{array}$ $\begin{array}{lllllll}\text { SRCPARAM 224U_6 } & 0.00066358 & 0.000 & 0.000 & 0.00000 & 1.524\end{array}$ $\begin{array}{lllllll}\text { SRCPARAM 224U } 7 & 0.00066358 & 6.096 & 0.000 & 0.00000 & 1.524\end{array}$ SRCPARAM 224U $80.00066358 \quad 3.048 \quad 0.000 \quad 0.00000 \quad 1.524$ $\begin{array}{llllllll}\text { SRCPARAM 224U-9 } & 0.00066358 & 0.000 & 0.000 & 0.00000 & 1.524\end{array}$ SRCPARAM 224UA_1 $0.001171296 \quad 6.096 \quad 0.000 \quad 0.00000 \quad 1.524$ $\begin{array}{llllllll}\text { SRCPARAM 224UA_2 } & 0.001171296 & 3.048 & 0.000 & 0.00000 & 1.524\end{array}$ $\begin{array}{lllllllll}\text { SRCPARAM 224UA_3 } & 0.001171296 & 0.000 & 0.000 & 0.00000 & 1.524\end{array}$ $\begin{array}{lllllllll}\text { SRCPARAM 224UA_4 } & 0.001171296 & 6.096 & 0.000 & 0.00000 & 1.524\end{array}$ $\begin{array}{lllllllll}\text { SRCPARAM } & 224 \mathrm{UA} & 5 & 0.001171296 & 3.052 & 0.000 & 0.00000 & 1.524\end{array}$ $\begin{array}{lllllllll}\text { SRCPARAM } 224 \mathrm{UA} & 6 & 0.001171296 & 0.000 & 0.000 & 0.00000 & 1.524\end{array}$

** Building Downwash **

$\begin{array}{lllllll}\text { BUILDHGT 224U_1 } & 12.19 & 15.24 & 15.24 & 12.19 & 12.19 & 12.19\end{array}$

BUILDHGT 224U-1 12.19

$\begin{array}{lllllll}\text { BUILDHGT 224U_1 } & 15.24 & 15.24 & 15.24 & 12.19 & 12.19 & 12.19\end{array}$

$\begin{array}{lllllll}\text { BUILDHGT 224U_1 } & 12.19 & 12.19 & 12.19 & 12.19 & 12.19 & 12.19 \\ \text { BUILDHGT 224U_1 } & 12.19 & 12.19 & 12.19 & 12.19 & 12.19 & 12.19\end{array}$

$\begin{array}{lllllll}\text { BUILDHGT 224U_2 } & 12.19 & 15.24 & 15.24 & 12.19 & 12.19 & 12.19\end{array}$

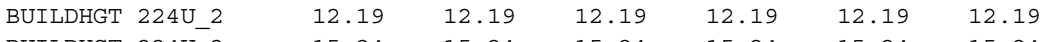

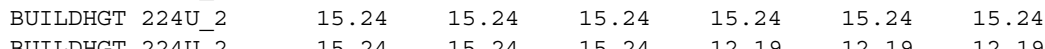

$\begin{array}{lllll}15.24 & 15.24 & 12.19 & 12.19 & 12.19 \\ 12.19 & 12.19 & 12.19 & 12.19 & 12.19\end{array}$

$\begin{array}{lllll}12.19 & 12.19 & 12.19 & 12.19 & 12.19\end{array}$

$\begin{array}{lllllll}\text { BUILDHGT } 224 \mathrm{U}-3 & 12.19 & 15.24 & 15.24 & 12.19 & 12.19 & 12.19\end{array}$

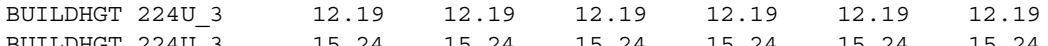

$\begin{array}{lllllll}\text { BUILDHGT 224U_3 } & 15.24 & 15.24 & 15.24 & 15.24 & 15.24 & 15.24\end{array}$

$\begin{array}{lllllll}\text { BUILDHGT 224U_3 } & 15.24 & 15.24 & 15.24 & 12.19 & 12.19 & 12.19\end{array}$

$\begin{array}{lllllll}\text { BUILDHGT 224U_3 } & 12.19 & 12.19 & 12.19 & 12.19 & 12.19 & 12.19 \\ \text { BUILDHGT 224U_3 } & 12.19 & 12.19 & 12.19 & 12.19 & 12.19 & 12.19\end{array}$

$\begin{array}{lllllll}\text { BUILDHGT 224U_4 } & 15.24 & 15.24 & 15.24 & 12.19 & 12.19 & 12.19\end{array}$

$\begin{array}{lllllll}\text { BUILDHGT 224U_4 } & 12.19 & 12.19 & 12.19 & 12.19 & 12.19 & 12.19\end{array}$

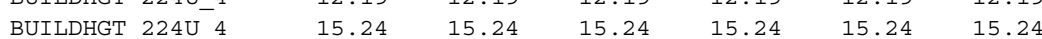




\begin{tabular}{|c|c|c|c|c|c|c|c|}
\hline BUILDHGT & $224 \mathrm{U} \_4$ & 15.24 & 15.24 & 15.24 & 12.19 & 12.19 & 12.19 \\
\hline BUILDHGT & $224 \mathrm{U} \_4$ & 12.19 & 12.19 & 12.19 & 12.19 & 12.19 & 12.19 \\
\hline BUILDHGT & $224 \mathrm{U}_{-}^{-} 4$ & 12.19 & 12.19 & 12.19 & 12.19 & 12.19 & 12.19 \\
\hline BUILDHGT & $224 \mathrm{U} \_5$ & 15.24 & 15.24 & 15.24 & 12.19 & 12.19 & 12.19 \\
\hline BUILDHGT & $224 \mathrm{U}_{-} 5$ & 12.19 & 12.19 & 12.19 & 12.19 & 12.19 & 12.19 \\
\hline BUILDHGT & $224 \mathrm{U} \_5$ & 15.24 & 15.24 & 15.24 & 15.24 & 15.24 & 15.24 \\
\hline BUILDHGT & $224 \mathrm{U} \_5$ & 15.24 & 15.24 & 15.24 & 12.19 & 12.19 & 12.19 \\
\hline BUILDHGT & $224 \mathrm{U} \_5$ & 12.19 & 12.19 & 12.19 & 12.19 & 12.19 & 12.19 \\
\hline BUILDHGT & $224 \mathrm{U}_{-} 5$ & 12.19 & 12.19 & 12.19 & 12.19 & 12.19 & 12.19 \\
\hline BUILDHGT & $224 \mathrm{U} \_6$ & 15.24 & 15.24 & 15.24 & 12.19 & 12.19 & 12.19 \\
\hline BUILDHGT & $224 \mathrm{U} \_6$ & 12.19 & 12.19 & 12.19 & 12.19 & 12.19 & 12.19 \\
\hline BUILDHGT & $224 \mathrm{U} \_6$ & 15.24 & 15.24 & 15.24 & 15.24 & 15.24 & 15.24 \\
\hline BUILDHGT & $224 \mathrm{U} \_6$ & 15.24 & 15.24 & 15.24 & 12.19 & 12.19 & 12.19 \\
\hline BUILDHGT & $224 \mathrm{U} \_6$ & 12.19 & 12.19 & 12.19 & 12.19 & 12.19 & 12.19 \\
\hline BUILDHGT & $224 \mathrm{U} \_6$ & 12.19 & 12.19 & 12.19 & 12.19 & 12.19 & 12.19 \\
\hline BUILDHGT & $224 \mathrm{U} \_7$ & 12.19 & 15.24 & 15.24 & 12.19 & 12.19 & 12.19 \\
\hline BUILDHGT & $224 U_{-}^{-} 7$ & 12.19 & 12.19 & 12.19 & 12.19 & 12.19 & 12.19 \\
\hline BUILDHGT & $224 \mathrm{U}_{-}^{-7}$ & 15.24 & 15.24 & 15.24 & 15.24 & 15.24 & 15.24 \\
\hline BUILDHGT & 224U_7 & 15.24 & 15.24 & 15.24 & 12.19 & 12.19 & 12.19 \\
\hline BUILDHGT & $224 \mathrm{U} \_7$ & 12.19 & 12.19 & 12.19 & 12.19 & 12.19 & 12.19 \\
\hline BUILDHGT & $224 \mathrm{U}_{-}^{-7}$ & 12.19 & 12.19 & 12.19 & 12.19 & 12.19 & 12.19 \\
\hline BUILDHGT & $224 \mathrm{U} \_8$ & 12.19 & 15.24 & 15.24 & 12.19 & 12.19 & 12.19 \\
\hline BUILDHGT & $224 \mathrm{U} \_8$ & 12.19 & 12.19 & 12.19 & 12.19 & 12.19 & 12.19 \\
\hline BUILDHGT & $224 U_{-}^{-} 8$ & 15.24 & 15.24 & 15.24 & 15.24 & 15.24 & 15.24 \\
\hline BUILDHGT & $224 U_{-}^{-} 8$ & 15.24 & 15.24 & 15.24 & 12.19 & 12.19 & 12.19 \\
\hline BUILDHGT & $224 \mathrm{U}^{-} 8$ & 12.19 & 12.19 & 12.19 & 12.19 & 12.19 & 12.19 \\
\hline BUILDHGT & $224 \mathrm{U} \_8$ & 12.19 & 12.19 & 12.19 & 12.19 & 12.19 & 12.19 \\
\hline BUILDHGT & 224U_9 & 12.19 & 15.24 & 15.24 & 12.19 & 12.19 & 12.19 \\
\hline BUILDHGT & $224 U_{-}^{-} 9$ & 12.19 & 12.19 & 12.19 & 12.19 & 12.19 & 12.19 \\
\hline BUILDHGT & $224 \mathrm{U}_{-} 9$ & 15.24 & 15.24 & 15.24 & 15.24 & 15.24 & 15.24 \\
\hline BUILDHGT & $224 \mathrm{U}_{-}^{-} 9$ & 15.24 & 15.24 & 15.24 & 12.19 & 12.19 & 12.19 \\
\hline BUILDHGT & $224 \mathrm{U} \_9$ & 12.19 & 12.19 & 12.19 & 12.19 & 12.19 & 12.19 \\
\hline BUILDHGT & $224 \mathrm{U}_{-}^{-} 9$ & 12.19 & 12.19 & 12.19 & 12.19 & 12.19 & 12.19 \\
\hline BUILDHGT & $224 \mathrm{UA} \_1$ & 12.19 & 12.19 & 12.19 & 12.19 & 12.19 & 12.19 \\
\hline BUILDHGT & $224 \mathrm{UA} \_1$ & 12.19 & 12.19 & 12.19 & 12.19 & 12.19 & 12.19 \\
\hline BUILDHGT & 224UA_1 & 12.19 & 12.19 & 12.19 & 12.19 & 12.19 & 12.19 \\
\hline BUILDHGT & $224 \mathrm{UA} \_1$ & 12.19 & 12.19 & 12.19 & 12.19 & 12.19 & 12.19 \\
\hline BUILDHGT & $224 \mathrm{UA} \_1$ & 12.19 & 12.19 & 12.19 & 12.19 & 12.19 & 12.19 \\
\hline BUILDHGT & $224 \mathrm{UA} \_1$ & 12.19 & 12.19 & 12.19 & 12.19 & 12.19 & 12.19 \\
\hline BUILDHGT & $224 \mathrm{UA} \_2$ & 12.19 & 12.19 & 12.19 & 12.19 & 12.19 & 12.19 \\
\hline BUILDHGT & $224 \mathrm{UA} \_2$ & 12.19 & 12.19 & 12.19 & 12.19 & 12.19 & 12.19 \\
\hline BUILDHGT & $224 \mathrm{UA} \_2$ & 12.19 & 12.19 & 12.19 & 12.19 & 12.19 & 12.19 \\
\hline BUILDHGT & $224 \mathrm{UA}^{-} 2$ & 12.19 & 12.19 & 12.19 & 12.19 & 12.19 & 12.19 \\
\hline BUILDHGT & $224 \mathrm{UA}^{-} 2$ & 12.19 & 12.19 & 12.19 & 12.19 & 12.19 & 12.19 \\
\hline BUILDHGT & $24 \mathrm{UA} 2$ & 12.19 & 12.19 & 12.19 & 12.19 & 12.19 & 12.19 \\
\hline
\end{tabular}




\begin{tabular}{|c|c|c|c|c|c|c|c|}
\hline BUILDHGT & $224 \mathrm{UA} 33$ & 12.19 & 12.19 & 12.19 & 12.19 & 12.19 & 12.19 \\
\hline BUILDHGT & $224 \mathrm{UA}^{-} 3$ & 12.19 & 12.19 & 12.19 & 12.19 & 12.19 & 12.19 \\
\hline BUILDHGT & $224 \mathrm{UA}^{-} 3$ & 12.19 & 12.19 & 12.19 & 12.19 & 12.19 & 12.19 \\
\hline BUILDHGT & $224 \mathrm{UA}_{-} 3$ & 12.19 & 12.19 & 12.19 & 12.19 & 12.19 & 12.19 \\
\hline BUILDHGT & $224 \mathrm{UA}^{-} 3$ & 12.19 & 12.19 & 12.19 & 12.19 & 12.19 & 12.19 \\
\hline BUILDHGT & $224 \mathrm{UA}^{-3}$ & 12.19 & 12.19 & 12.19 & 12.19 & 12.19 & 12.19 \\
\hline BUILDHGT & 224UA_4 & 12.19 & 12.19 & 12.19 & 12.19 & 12.19 & 12.19 \\
\hline BUILDHGT & 224UA_4 & 12.19 & 12.19 & 12.19 & 12.19 & 12.19 & 12.19 \\
\hline BUILDHGT & $224 \mathrm{UA}^{-} 4$ & 12.19 & 12.19 & 12.19 & 12.19 & 12.19 & 12.19 \\
\hline BUILDHGT & $224 \mathrm{UA}^{-} 4$ & 12.19 & 12.19 & 12.19 & 12.19 & 12.19 & 12.19 \\
\hline BUILDHGT & 224UA_4 & 12.19 & 12.19 & 12.19 & 12.19 & 12.19 & 12.19 \\
\hline BUILDHGT & $224 \mathrm{UA}^{-} 4$ & 12.19 & 12.19 & 12.19 & 12.19 & 12.19 & 12.19 \\
\hline BUILDHGT & 224UA_5 & 12.19 & 12.19 & 12.19 & 12.19 & 12.19 & 12.19 \\
\hline BUILDHGT & $224 \mathrm{UA}^{-} 5$ & 12.19 & 12.19 & 12.19 & 12.19 & 12.19 & 12.19 \\
\hline BUILDHGT & 224UA_5 & 12.19 & 12.19 & 12.19 & 12.19 & 12.19 & 12.19 \\
\hline BUILDHGT & $224 \mathrm{UA}^{-} 5$ & 12.19 & 12.19 & 12.19 & 12.19 & 12.19 & 12.19 \\
\hline BUILDHGT & 224UA_5 & 12.19 & 12.19 & 12.19 & 12.19 & 12.19 & 12.19 \\
\hline BUILDHGT & 224UA_5 & 12.19 & 12.19 & 12.19 & 12.19 & 12.19 & 12.19 \\
\hline BUILDHGT & 224UA_6 & 12.19 & 12.19 & 12.19 & 12.19 & 12.19 & 12.19 \\
\hline BUILDHGT & $224 \mathrm{UA}^{-6} 6$ & 12.19 & 12.19 & 12.19 & 12.19 & 12.19 & 12.19 \\
\hline BUILDHGT & $224 \mathrm{UA}^{-} 6$ & 12.19 & 12.19 & 12.19 & 12.19 & 12.19 & 12.19 \\
\hline BUILDHGT & $224 \mathrm{UA}^{-} 6$ & 12.19 & 12.19 & 12.19 & 12.19 & 12.19 & 12.19 \\
\hline BUILDHGT & $224 \mathrm{UA}^{-} 6$ & 12.19 & 12.19 & 12.19 & 12.19 & 12.19 & 12.19 \\
\hline BUILDHGT & $224 \mathrm{UA}^{-} 6$ & 12.19 & 12.19 & 12.19 & 12.19 & 12.19 & 12.19 \\
\hline BUILDWID & $224 \mathrm{U} \_1$ & 48.01 & 113.08 & 73.22 & 28.67 & 35.80 & 44.89 \\
\hline BUILDWID & $224 \mathrm{U}_{1}$ & 52.62 & 58.74 & 63.08 & 65.50 & 65.94 & 64.37 \\
\hline BUILDWID & $224 \mathrm{U}^{-} 1$ & 246.56 & 246.81 & 240.99 & 227.85 & 207.78 & 181.41 \\
\hline BUILDWID & $224 \mathrm{U}^{-} 1$ & 149.52 & 113.08 & 73.22 & 28.67 & 35.80 & 44.89 \\
\hline BUILDWID & $224 \mathrm{U}_{-1} 1$ & 52.62 & 58.74 & 63.08 & 65.50 & 65.94 & 64.37 \\
\hline BUILDWID & $224 \mathrm{U}^{-1} 1$ & 60.84 & 62.10 & 62.99 & 61.97 & 59.06 & 54.36 \\
\hline BUILDWID & 224U_2 & 48.01 & 113.08 & 73.22 & 28.67 & 35.80 & 44.89 \\
\hline BUILDWID & $224 \mathrm{U}^{-} 2$ & 52.62 & 58.74 & 63.08 & 65.50 & 65.94 & 64.37 \\
\hline BUILDWID & $224 \mathrm{U}^{2} 2$ & 246.56 & 246.81 & 240.99 & 227.85 & 207.78 & 181.41 \\
\hline BUILDWID & $224 \mathrm{U}^{-} 2$ & 149.52 & 113.08 & 73.22 & 28.67 & 35.80 & 44.89 \\
\hline BUILDWID & $224 \mathrm{U}^{-} 2$ & 52.62 & 58.74 & 63.08 & 65.50 & 65.94 & 64.37 \\
\hline BUILDWID & $224 \mathrm{U}^{2} 2$ & 60.84 & 62.10 & 62.99 & 61.97 & 59.06 & 54.36 \\
\hline BUILDWID & $224 \mathrm{U} \_3$ & 48.01 & 113.08 & 73.22 & 28.67 & 35.80 & 44.89 \\
\hline BUILDWID & $224 \mathrm{U}^{-} 3$ & 52.62 & 58.74 & 63.08 & 65.50 & 65.94 & 64.37 \\
\hline BUILDWID & $224 \mathrm{U}^{-} 3$ & 246.56 & 246.81 & 240.99 & 227.85 & 207.78 & 181.41 \\
\hline BUILDWID & $224 \mathrm{U} 3$ & 149.52 & 113.08 & 73.22 & 28.67 & 35.80 & 44.89 \\
\hline BUILDWID & $224 \mathrm{U}^{-} 3$ & 52.62 & 58.74 & 63.08 & 65.50 & 65.94 & 64.37 \\
\hline BUILDWID & $224 \mathrm{U}^{-} 3$ & 60.84 & 62.10 & 62.99 & 61.97 & 59.06 & 54.36 \\
\hline BUILDWID & $224 \mathrm{U} \_4$ & 9.52 & 08 & 73.22 & 28.67 & 35.80 & 44.89 \\
\hline BUILDWID & $224 \mathrm{U} \_4$ & 52.62 & 58.74 & 63.08 & 65.50 & 65.94 & 64.37 \\
\hline BUILDWID & $224 \mathrm{U}^{-} 4$ & 246.56 & 246.81 & 240.99 & 227.85 & 207.78 & 181.41 \\
\hline BUILDWID & $224 \mathrm{U}^{-} 4$ & 149.52 & 113.08 & 73.22 & 28.67 & 35.80 & 44.89 \\
\hline
\end{tabular}




\begin{tabular}{|c|c|c|c|c|c|c|c|}
\hline BUILDWID & $224 \mathrm{U} \_4$ & 52.62 & 58.74 & 63.08 & 65.50 & 65.94 & 64.37 \\
\hline BUILDWID & $224 \mathrm{U}_{-} 4$ & 60.84 & 62.10 & 62.99 & 61.97 & 59.06 & 54.36 \\
\hline BUILDWID & $224 \mathrm{U} \_5$ & 149.52 & 113.08 & 73.22 & 28.67 & 35.80 & 44.89 \\
\hline BUILDWID & $224 \mathrm{U} \_5$ & 52.62 & 58.74 & 63.08 & 65.50 & 65.94 & 64.37 \\
\hline BUILDWID & $224 \mathrm{U} \_5$ & 246.56 & 246.81 & 240.99 & 227.85 & 207.78 & 181.41 \\
\hline BUILDWID & $224 \mathrm{U} \_5$ & 149.52 & 113.08 & 73.22 & 28.67 & 35.80 & 44.89 \\
\hline BUILDWID & $224 \mathrm{U}^{-5} 5$ & 52.62 & 58.74 & 63.08 & 65.50 & 65.94 & 64.37 \\
\hline BUILDWID & $224 \mathrm{U} \_5$ & 60.84 & 62.10 & 62.99 & 61.97 & 59.06 & 54.36 \\
\hline BUILDWID & $224 \mathrm{U} \_6$ & 149.52 & 113.08 & 73.22 & 28.67 & 35.80 & 44.89 \\
\hline BUILDWID & $224 \mathrm{U} \_6$ & 52.62 & 58.74 & 63.08 & 65.50 & 65.94 & 64.37 \\
\hline BUILDWID & $224 \mathrm{U} \_6$ & 246.56 & 246.81 & 240.99 & 227.85 & 207.78 & 181.41 \\
\hline BUILDWID & $224 \mathrm{U} \_6$ & 149.52 & 113.08 & 73.22 & 28.67 & 35.80 & 44.89 \\
\hline BUILDWID & $224 \mathrm{U}^{-} 6$ & 52.62 & 58.74 & 63.08 & 65.50 & 65.94 & 64.37 \\
\hline BUILDWID & $224 \mathrm{U}_{-} 6$ & 60.84 & 62.10 & 62.99 & 61.97 & 59.06 & 54.36 \\
\hline BUILDWID & $224 \mathrm{U} \_7$ & 48.01 & 113.08 & 73.22 & 28.67 & 35.80 & 44.89 \\
\hline BUILDWID & $224 \mathrm{U} \_7$ & 52.62 & 58.74 & 63.08 & 65.50 & 65.94 & 64.37 \\
\hline BUILDWID & $224 \mathrm{U}_{-}^{-} 7$ & 246.56 & 246.81 & 240.99 & 227.85 & 207.78 & 181.41 \\
\hline BUILDWID & $224 \mathrm{U}^{-} 7$ & 149.52 & 113.08 & 73.22 & 28.67 & 35.80 & 44.89 \\
\hline BUILDWID & $224 \mathrm{U} \_7$ & 52.62 & 58.74 & 63.08 & 65.50 & 65.94 & 64.37 \\
\hline BUILDWID & $224 \mathrm{U} \_7$ & 60.84 & 62.10 & 62.99 & 61.97 & 59.06 & 54.36 \\
\hline BUILDWID & $224 \mathrm{U} \_8$ & 48.01 & 113.08 & 73.22 & 28.67 & 35.80 & 44.89 \\
\hline BUILDWID & $224 \mathrm{U}^{-} 8$ & 52.62 & 58.74 & 63.08 & 65.50 & 65.94 & 64.37 \\
\hline BUILDWID & $224 \mathrm{U} \_8$ & 246.56 & 246.81 & 240.99 & 227.85 & 207.78 & 181.41 \\
\hline BUILDWID & $224 \mathrm{U} \_8$ & 149.52 & 113.08 & 73.22 & 28.67 & 35.80 & 44.89 \\
\hline BUILDWID & $224 \mathrm{U}_{-} 8$ & 52.62 & 58.74 & 63.08 & 65.50 & 65.94 & 64.37 \\
\hline BUILDWID & $224 \mathrm{U} \_8$ & 60.84 & 62.10 & 62.99 & 61.97 & 59.06 & 54.36 \\
\hline BUILDWID & $224 \mathrm{U} \_9$ & 48.01 & 113.08 & 73.22 & 28.67 & 35.80 & 44.89 \\
\hline BUILDWID & $224 \mathrm{U} \_9$ & 52.62 & 58.74 & 63.08 & 65.50 & 65.94 & 64.37 \\
\hline BUILDWID & $224 \mathrm{U}_{-} 9$ & 246.56 & 246.81 & 240.99 & 227.85 & 207.78 & 181.41 \\
\hline BUILDWID & $224 \mathrm{U}_{-} 9$ & 149.52 & 113.08 & 73.22 & 28.67 & 35.80 & 44.89 \\
\hline BUILDWID & $224 \mathrm{U}^{-} 9$ & 52.62 & 58.74 & 63.08 & 65.50 & 65.94 & 64.37 \\
\hline BUILDWID & $224 \mathrm{U} \_9$ & 60.84 & 62.10 & 62.99 & 61.97 & 59.06 & 54.36 \\
\hline BUILDWID & 224UA_1 & 48.01 & 40.19 & 31.16 & 28.67 & 35.80 & 44.89 \\
\hline BUILDWID & 224UA_1 & 52.62 & 58.74 & 63.08 & 65.50 & 65.94 & 64.37 \\
\hline BUILDWID & $224 \mathrm{UA}^{-1}$ & 60.84 & 62.10 & 62.99 & 61.97 & 59.06 & 54.36 \\
\hline BUILDWID & $224 \mathrm{UA}^{-} 1$ & 48.01 & 40.19 & 31.16 & 28.67 & 35.80 & 44.89 \\
\hline BUILDWID & $224 \mathrm{UA}^{-} \mathrm{I}$ & 52.62 & 58.74 & 63.08 & 65.50 & 65.94 & 64.37 \\
\hline BUILDWID & 224UA_1 & 60.84 & 62.10 & 62.99 & 61.97 & 59.06 & 54.36 \\
\hline BUILDWID & 224UA_2 & 48.01 & 40.19 & 31.16 & 28.67 & 35.80 & 44.89 \\
\hline BUILDWID & 224UA_2 & 52.62 & 58.74 & 63.08 & 65.50 & 65.94 & 64.37 \\
\hline BUILDWID & 224UA_2 & 60.84 & 62.10 & 62.99 & 61.97 & 59.06 & 54.36 \\
\hline BUILDWID & 224UA_2 & 48.01 & 40.19 & 31.16 & 28.67 & 35.80 & 44.89 \\
\hline BUILDWID & 224UA_2 & 52.62 & 58.74 & 63.08 & 65.50 & 65.94 & 64.37 \\
\hline BUILDWID & 224UA_2 & 60.84 & 62.10 & 62.99 & 61.97 & 59.06 & 54.36 \\
\hline & 24IJA 3 & 8.01 & 40.19 & 31.16 & 28.67 & 35.80 & \\
\hline
\end{tabular}




\begin{tabular}{|c|c|c|c|c|c|c|c|}
\hline BUILDWID & 224UA_3 & 52.62 & 58.74 & 63.08 & 65.50 & 65.94 & 64.37 \\
\hline BUILDWID & 224UA_3 & 60.84 & 62.10 & 62.99 & 61.97 & 59.06 & 54.36 \\
\hline BUILDWID & $224 \mathrm{UA}^{-} 3$ & 48.01 & 40.19 & 31.16 & 28.67 & 35.80 & 44.89 \\
\hline BUILDWID & $224 \mathrm{UA}^{-} 3$ & 52.62 & 58.74 & 63.08 & 65.50 & 65.94 & 64.37 \\
\hline BUILDWID & 224UA_3 & 60.84 & 62.10 & 62.99 & 61.97 & 59.06 & 54.36 \\
\hline BUILDWID & 224UA_4 & 48.01 & 40.19 & 31.16 & 28.67 & 35.80 & 44.89 \\
\hline BUILDWID & 224UA_4 & 52.62 & 58.74 & 63.08 & 65.50 & 65.94 & 64.37 \\
\hline BUILDWID & 224UA_4 & 60.84 & 62.10 & 62.99 & 61.97 & 59.06 & 54.36 \\
\hline BUILDWID & $224 \mathrm{UA}^{-} 4$ & 48.01 & 40.19 & 31.16 & 28.67 & 35.80 & 44.89 \\
\hline BUILDWID & 224UA_4 & 52.62 & 58.74 & 63.08 & 65.50 & 65.94 & 64.37 \\
\hline BUILDWID & $224 \mathrm{UA}^{-} 4$ & 60.84 & 62.10 & 62.99 & 61.97 & 59.06 & 54.36 \\
\hline BUILDWID & 224UA_5 & 48.01 & 40.19 & 31.16 & 28.67 & 35.80 & 44.89 \\
\hline BUILDWID & $224 \mathrm{UA}^{-} 5$ & 52.62 & 58.74 & 63.08 & 65.50 & 65.94 & 64.37 \\
\hline BUILDWID & 224UA_5 & 60.84 & 62.10 & 62.99 & 61.97 & 59.06 & 54.36 \\
\hline BUILDWID & 224UA_5 & 48.01 & 40.19 & 31.16 & 28.67 & 35.80 & 44.89 \\
\hline BUILDWID & $224 \mathrm{UA}^{-} 5$ & 52.62 & 58.74 & 63.08 & 65.50 & 65.94 & 64.37 \\
\hline BUILDWID & 224UA_5 & 60.84 & 62.10 & 62.99 & 61.97 & 59.06 & 54.36 \\
\hline BUILDWID & $224 \mathrm{UA} \_6$ & 48.01 & 40.19 & 31.16 & 28.67 & 35.80 & 44.89 \\
\hline BUILDWID & $224 \mathrm{UA}^{-} 6$ & 52.62 & 58.74 & 63.08 & 65.50 & 65.94 & 64.37 \\
\hline BUILDWID & $224 \mathrm{UA}^{-} 6$ & 60.84 & 62.10 & 62.99 & 61.97 & 59.06 & 54.36 \\
\hline BUILDWID & 224UA_6 & 48.01 & 40.19 & 31.16 & 28.67 & 35.80 & 44.89 \\
\hline BUILDWID & 224UA_6 & 52.62 & 58.74 & 63.08 & 65.50 & 65.94 & 64.37 \\
\hline BUILDWID & 224UA_6 & 60.84 & 62.10 & 62.99 & 61.97 & 59.06 & 54.36 \\
\hline BUILDLEN & $224 \mathrm{U}_{-} 1$ & 65.50 & 235.12 & 244.55 & 60.84 & 62.10 & 62.99 \\
\hline BUILDLEN & $224 \mathrm{U}_{-1} 1$ & 61.97 & 59.06 & 54.36 & 48.01 & 40.19 & 31.16 \\
\hline BUILDLEN & $224 \mathrm{U}_{-1} 1$ & 31.12 & 52.69 & 93.75 & 131.96 & 166.16 & 195.32 \\
\hline BUILDLEN & $224 \mathrm{U}^{-} 1$ & 218.54 & 235.12 & 244.55 & 60.84 & 62.10 & 62.99 \\
\hline BUILDLEN & $224 \mathrm{U}^{-} 1$ & 61.97 & 59.06 & 54.36 & 48.01 & 40.19 & 31.16 \\
\hline BUILDLEN & $224 \mathrm{U}^{-1} 1$ & 28.67 & 35.80 & 44.89 & 52.62 & 58.74 & 63.08 \\
\hline BUILDLEN & $224 \mathrm{U} \_2$ & 65.50 & 235.12 & 244.55 & 60.84 & 62.10 & 62.99 \\
\hline BUILDLEN & $224 \mathrm{U}^{-} 2$ & 61.97 & 59.06 & 54.36 & 48.01 & 40.19 & 31.16 \\
\hline BUILDLEN & $224 \mathrm{U}^{-} 2$ & 31.12 & 52.69 & 93.75 & 131.96 & 166.16 & 195.32 \\
\hline BUILDLEN & $224 \mathrm{U}^{-} 2$ & 218.54 & 235.12 & 244.55 & 60.84 & 62.10 & 62.99 \\
\hline BUILDLEN & $224 \mathrm{U} \_2$ & 61.97 & 59.06 & 54.36 & 48.01 & 40.19 & 31.16 \\
\hline BUILDLEN & $224 \mathrm{U}^{-} 2$ & 28.67 & 35.80 & 44.89 & 52.62 & 58.74 & 63.08 \\
\hline BUILDLEN & $224 \mathrm{U}$ & 65.50 & 235.12 & 244.55 & 60.84 & 62.10 & 62.99 \\
\hline BUILDLEN & $224 \mathrm{U}^{-} 3$ & 61.97 & 59.06 & 54.36 & 48.01 & 40.19 & 31.16 \\
\hline BUILDLEN & $224 \mathrm{U}^{-} 3$ & 31.12 & 52.69 & 93.75 & 131.96 & 166.16 & 195.32 \\
\hline BUILDLEN & $224 \mathrm{U}^{-} 3$ & 218.54 & 235.12 & 244.55 & 60.84 & 62.10 & 62.99 \\
\hline BUILDLEN & $224 \mathrm{U}^{3} 3$ & 61.97 & 59.06 & 54.36 & 48.01 & 40.19 & 31.16 \\
\hline BUILDLEN & $224 \mathrm{U}^{-} 3$ & 28.67 & 35.80 & 44.89 & 52.62 & 58.74 & 63.08 \\
\hline BUILDLEN & $224 \mathrm{U} \_4$ & 218.54 & 235.12 & 244.55 & 60.84 & 62.10 & 62.99 \\
\hline BUILDLEN & $224 \mathrm{U}^{-} 4$ & 61.97 & 59.06 & 54.36 & 48.01 & 40.19 & 31.16 \\
\hline BUILDLEN & $224 \mathrm{U} \_4$ & 31.12 & 52.69 & 93.75 & 131.96 & 166.16 & 195.32 \\
\hline BUILDLEN & $224 \mathrm{U}_{-}^{-} 4$ & 218.54 & 235.12 & 244.55 & 60.84 & 62.10 & 62.99 \\
\hline BUILDLEN & $224 \mathrm{U}^{-} 4$ & 61.97 & 59.06 & 54.36 & 48.01 & 40.19 & 31.16 \\
\hline
\end{tabular}




\begin{tabular}{|c|c|c|c|c|c|c|c|}
\hline BUILDLEN & $224 \mathrm{U} \_4$ & 28.67 & 35.80 & 44.89 & 52.62 & 58.74 & 63.08 \\
\hline BUILDLEN & $224 \mathrm{U} \_5$ & 218.54 & 235.12 & 244.55 & 60.84 & 62.10 & 62.99 \\
\hline BUILDLEN & $224 \mathrm{U}^{-} 5$ & 61.97 & 59.06 & 54.36 & 48.01 & 40.19 & 31.16 \\
\hline BUILDLEN & $224 \mathrm{U}_{-} 5$ & 31.12 & 52.69 & 93.75 & 131.96 & 166.16 & 195.32 \\
\hline BUILDLEN & $224 \mathrm{U}^{-} 5$ & 218.54 & 235.12 & 244.55 & 60.84 & 62.10 & 62.99 \\
\hline BUILDLEN & $224 \mathrm{U}^{-} 5$ & 61.97 & 59.06 & 54.36 & 48.01 & 40.19 & 31.16 \\
\hline BUILDLEN & $224 \mathrm{U}^{-} 5$ & 28.67 & 35.80 & 44.89 & 52.62 & 58.74 & 63.08 \\
\hline BUILDLEN & $224 \mathrm{U} 6$ & 218.54 & 235.12 & 244.55 & 60.84 & 62.10 & 62.99 \\
\hline BUILDLEN & $224 \mathrm{U}^{-} 6$ & 61.97 & 59.06 & 54.36 & 48.01 & 40.19 & 31.16 \\
\hline BUILDLEN & $224 \mathrm{U}^{-} 6$ & 31.12 & 52.69 & 93.75 & 131.96 & 166.16 & 195.32 \\
\hline BUILDLEN & $224 \mathrm{U}^{-} 6$ & 218.54 & 235.12 & 244.55 & 60.84 & 62.10 & 62.99 \\
\hline BUILDLEN & $224 \mathrm{U}_{-} 6$ & 61.97 & 59.06 & 54.36 & 48.01 & 40.19 & 31.16 \\
\hline BUILDLEN & $224 \mathrm{U}^{-} 6$ & 28.67 & 35.80 & 44.89 & 52.62 & 58.74 & 63.08 \\
\hline BUILDLEN & $224 \mathrm{U} \_7$ & 65.50 & 235.12 & 244.55 & 60.84 & 62.10 & 62.99 \\
\hline BUILDLEN & $224 \mathrm{U}_{-}^{-} 7$ & 61.97 & 59.06 & 54.36 & 48.01 & 40.19 & 31.16 \\
\hline BUILDLEN & $224 \mathrm{U}^{-} 7$ & 31.12 & 52.69 & 93.75 & 131.96 & 166.16 & 195.32 \\
\hline BUILDLEN & $224 \mathrm{U}^{-} 7$ & 218.54 & 235.12 & 244.55 & 60.84 & 62.10 & 62.99 \\
\hline BUILDLEN & $224 \mathrm{U}_{-}^{-} 7$ & 61.97 & 59.06 & 54.36 & 48.01 & 40.19 & 31.16 \\
\hline BUILDLEN & $224 \mathrm{U}_{-}^{-7}$ & 28.67 & 35.80 & 44.89 & 52.62 & 58.74 & 63.08 \\
\hline BUILDLEN & $224 \mathrm{U} \_8$ & 65.50 & 235.12 & 244.55 & 60.84 & 62.10 & 62.99 \\
\hline BUILDLEN & $224 \mathrm{U}^{-} 8$ & 61.97 & 59.06 & 54.36 & 48.01 & 40.19 & 31.16 \\
\hline BUILDLEN & $224 \mathrm{U}^{-} 8$ & 31.12 & 52.69 & 93.75 & 131.96 & 166.16 & 195.32 \\
\hline BUILDLEN & $224 \mathrm{U}^{-} 8$ & 218.54 & 235.12 & 244.55 & 60.84 & 62.10 & 62.99 \\
\hline BUILDLEN & $224 \mathrm{U}^{-} 8$ & 61.97 & 59.06 & 54.36 & 48.01 & 40.19 & 31.16 \\
\hline BUILDLEN & $224 \mathrm{U}^{-} 8$ & 28.67 & 35.80 & 44.89 & 52.62 & 58.74 & 63.08 \\
\hline BUILDLEN & $224 \mathrm{U} \_9$ & 65.50 & 235.12 & 244.55 & 60.84 & 62.10 & 62.99 \\
\hline BUILDLEN & $224 \mathrm{U}^{-} 9$ & 61.97 & 59.06 & 54.36 & 48.01 & 40.19 & 31.16 \\
\hline BUILDLEN & $224 \mathrm{U}_{-} 9$ & 31.12 & 52.69 & 93.75 & 131.96 & 166.16 & 195.32 \\
\hline BUILDLEN & $224 \mathrm{U}^{-} 9$ & 218.54 & 235.12 & 244.55 & 60.84 & 62.10 & 62.99 \\
\hline BUILDLEN & $224 \mathrm{U}^{-} 9$ & 61.97 & 59.06 & 54.36 & 48.01 & 40.19 & 31.16 \\
\hline BUILDLEN & $224 \mathrm{U}_{-} 9$ & 28.67 & 35.80 & 44.89 & 52.62 & 58.74 & 63.08 \\
\hline BUILDLEN & 224UA_1 & 65.50 & 65.94 & 64.37 & 60.84 & 62.10 & 62.99 \\
\hline BUILDLEN & $224 \mathrm{UA}^{-} 1$ & 61.97 & 59.06 & 54.36 & 48.01 & 40.19 & 31.16 \\
\hline BUILDLEN & $224 \mathrm{UA}^{-} \mathrm{I}$ & 28.67 & 35.80 & 44.89 & 52.62 & 58.74 & 63.08 \\
\hline BUILDLEN & $224 \mathrm{UA}$ & 65.50 & 65.94 & 64.37 & 60.84 & 62.10 & 62.99 \\
\hline BUILDLEN & $224 \mathrm{UA}$ & 61.97 & 59.06 & 54.36 & 48.01 & 40.19 & 31.16 \\
\hline BUILDLEN & $224 \mathrm{UA}^{-} \mathrm{I}$ & 28.67 & 35.80 & 44.89 & 52.62 & 58.74 & 63.08 \\
\hline BUILDLEN & 224UA_2 & 65.50 & 65.94 & 64.37 & 60.84 & 62.10 & 62.99 \\
\hline BUILDLEN & $224 \mathrm{UA}^{-} 2$ & 61.97 & 59.06 & 54.36 & 48.01 & 40.19 & 31.16 \\
\hline BUILDLEN & 224UA_2 & 28.67 & 35.80 & 44.89 & 52.62 & 58.74 & 63.08 \\
\hline BUILDLEN & 224UA_2 & 65.50 & 65.94 & 64.37 & 60.84 & 62.10 & 62.99 \\
\hline BUILDLEN & 224UA_2 & 61.97 & 59.06 & 54.36 & 48.01 & 40.19 & 31.16 \\
\hline BUILDLEN & 224UA_2 & 28.67 & 35.80 & 44.89 & 52.62 & 58.74 & 63.08 \\
\hline & & 5.50 & & 64.37 & 60.84 & 62.10 & 62.99 \\
\hline BUILDLEN & $224 \mathrm{UA} 3$ & 61.97 & 59.06 & 54.36 & 48.01 & 40.19 & 31.16 \\
\hline
\end{tabular}




\begin{tabular}{|c|c|c|c|c|c|c|c|}
\hline BUILDLEN & 224UA_3 & 28.67 & 35.80 & 44.89 & 52.62 & 58.74 & 63.08 \\
\hline BUILDLEN & 224UA_3 & 65.50 & 65.94 & 64.37 & 60.84 & 62.10 & 62.99 \\
\hline BUILDLEN & $224 \mathrm{UA}^{-} 3$ & 61.97 & 59.06 & 54.36 & 48.01 & 40.19 & 31.16 \\
\hline BUILDLEN & 224UA_3 & 28.67 & 35.80 & 44.89 & 52.62 & 58.74 & 63.08 \\
\hline BUILDLEN & 224UA_4 & 65.50 & 65.94 & 64.37 & 60.84 & 62.10 & 62.99 \\
\hline BUILDLEN & 224UA_4 & 61.97 & 59.06 & 54.36 & 48.01 & 40.19 & 31.16 \\
\hline BUILDLEN & 224UA_4 & 28.67 & 35.80 & 44.89 & 52.62 & 58.74 & 63.08 \\
\hline BUILDLEN & 224UA_4 & 65.50 & 65.94 & 64.37 & 60.84 & 62.10 & 62.99 \\
\hline BUILDLEN & 224UA_4 & 61.97 & 59.06 & 54.36 & 48.01 & 40.19 & 31.16 \\
\hline BUILDLEN & 224UA_4 & 28.67 & 35.80 & 44.89 & 52.62 & 58.74 & 63.08 \\
\hline BUILDLEN & 224UA_5 & 65.50 & 65.94 & 64.37 & 60.84 & 62.10 & 62.99 \\
\hline BUILDLEN & 224UA_5 & 61.97 & 59.06 & 54.36 & 48.01 & 40.19 & 31.16 \\
\hline BUILDLEN & 224UA_5 & 28.67 & 35.80 & 44.89 & 52.62 & 58.74 & 63.08 \\
\hline BUILDLEN & $224 \mathrm{UA}^{-}$5 & 65.50 & 65.94 & 64.37 & 60.84 & 62.10 & 62.99 \\
\hline BUILDLEN & 224UA_5 & 61.97 & 59.06 & 54.36 & 48.01 & 40.19 & 31.16 \\
\hline BUILDLEN & 224UA_5 & 28.67 & 35.80 & 44.89 & 52.62 & 58.74 & 63.08 \\
\hline JILDLEN & $224 \mathrm{UA} \_6$ & 65.50 & 65.94 & 64.37 & 60.84 & 62.10 & 62.99 \\
\hline BUILDLEN & $224 \mathrm{UA}^{-} 6$ & 61.97 & 59.06 & 54.36 & 48.01 & 40.19 & 31.16 \\
\hline BUILDLEN & $224 \mathrm{UA}^{-} 6$ & 28.67 & 35.80 & 44.89 & 52.62 & 58.74 & 63.08 \\
\hline BUILDLEN & $224 \mathrm{UA}^{-} 6$ & 65.50 & 65.94 & 64.37 & 60.84 & 62.10 & 62.99 \\
\hline BUILDLEN & 224UA_6 & 61.97 & 59.06 & 54.36 & 48.01 & 40.19 & 31.16 \\
\hline BUILDLEN & 224UA_6 & 28.67 & 35.80 & 44.89 & 52.62 & 58.74 & 63.08 \\
\hline XBADJ & $224 \mathrm{U} \_1$ & -9.30 & 21.85 & 12.50 & -0.41 & -1.51 & -3.32 \\
\hline XBADJ & 224U_1 & -5.02 & -6.57 & -7.92 & -9.03 & -9.86 & -10.40 \\
\hline XBADJ & $224 \mathrm{U}_{-} 1$ & -76.99 & -108.60 & -147.59 & -182.09 & -211.06 & -233.62 \\
\hline XBADJ & $224 \mathrm{U} \_1$ & -249.08 & -256.97 & -257.05 & -60.44 & -60.59 & -59.68 \\
\hline XBADJ & 224U_1 & -56.95 & -52.49 & -46.44 & -38.98 & -30.33 & -20.76 \\
\hline XBADJ & $224 \mathrm{U}_{-} 1$ & -18.05 & -17.69 & -16.95 & -15.70 & -13.97 & -11.81 \\
\hline XBADJ & 224U_2 & -9.30 & 21.85 & 12.50 & -0.41 & -1.51 & -3.32 \\
\hline XBADJ & $224 \mathrm{U}^{-} 2$ & -5.02 & -6.57 & -7.92 & -9.03 & -9.86 & -10.40 \\
\hline XBADJ & $224 \mathrm{U}^{-} 2$ & -76.99 & -108.60 & -147.59 & -182.09 & -211.06 & -233.62 \\
\hline XBADJ & $224 \mathrm{U} \_2$ & -249.08 & -256.97 & -257.05 & -60.44 & -60.59 & -59.68 \\
\hline XBADJ & $224 \mathrm{U}_{-} 2$ & -56.95 & -52.49 & -46.44 & -38.98 & -30.33 & -20.76 \\
\hline XBADJ & $224 \mathrm{U}^{-} 2$ & -18.05 & -17.69 & -16.95 & -15.70 & -13.97 & -11.81 \\
\hline XBADJ & & & & 12.50 & -0.41 & -1.51 & -3.32 \\
\hline XBADJ & $24 \mathrm{U}^{-} 3$ & -5.02 & -6.57 & -7.92 & -9.03 & -9.86 & -10.40 \\
\hline XBADJ & $224 \mathrm{U}^{-} 3$ & -76.99 & -108.60 & -147.59 & -182.09 & -211.06 & -233.62 \\
\hline XBADJ & $224 \mathrm{U}^{-} 3$ & -249.08 & -256.97 & -257.05 & -60.44 & -60.59 & -59.68 \\
\hline XBADJ & $224 \mathrm{U}^{-} 3$ & -56.95 & -52.49 & -46.44 & -38.98 & -30.33 & -20.76 \\
\hline XBADJ & $224 U_{-} 3$ & -18.05 & -17.69 & -16.95 & -15.70 & -13.97 & -11.81 \\
\hline XBADJ & 224U_4 & 29. & 20.90 & 12.03 & -0.38 & -1.00 & -2.32 \\
\hline XBADJ & $224 \mathrm{U}_{-} 4$ & -3.58 & -4.73 & -5.73 & -6.56 & -7.19 & -7.60 \\
\hline XBADJ & $224 \mathrm{U}^{-} 4$ & -74.15 & -105.81 & -144.93 & -179.64 & -208.90 & -231.81 \\
\hline XBADJ & $224 \mathrm{U} \_4$ & -247.68 & -256.02 & -256.58 & -60.46 & -61.10 & -60.67 \\
\hline XBADJ & $224 \mathrm{U}_{-} 4$ & -58.39 & -54.34 & -48.63 & -41.45 & -33.01 & -23.56 \\
\hline XBADJ & $224 \mathrm{U}^{-} 4$ & -20.89 & -20.48 & -19.61 & -18.15 & -16.13 & -13.62 \\
\hline
\end{tabular}




\begin{tabular}{|c|c|c|c|c|c|c|c|}
\hline XBADJ & $224 \mathrm{U} \_5$ & 29.14 & 20.90 & 12.03 & -0.38 & -1.00 & -2.32 \\
\hline XBADJ & $224 U_{-}^{-} 5$ & -3.58 & -4.73 & -5.73 & -6.56 & -7.19 & -7.60 \\
\hline XBADJ & $224 \mathrm{U}^{-} 5$ & -74.15 & -105.81 & -144.93 & -179.64 & -208.90 & -231.81 \\
\hline XBADJ & $224 \mathrm{U}^{-} 5$ & -247.68 & -256.02 & -256.58 & -60.46 & -61.10 & -60.67 \\
\hline XBADJ & $224 U_{-}^{-} 5$ & -58.39 & -54.34 & -48.63 & -41.45 & -33.01 & -23.56 \\
\hline XBADJ & $224 \mathrm{U}^{-} 5$ & -20.89 & -20.48 & -19.61 & -18.15 & -16.13 & -13.62 \\
\hline XBADJ & $224 \mathrm{U} 6$ & 29.14 & 20.90 & 12.03 & -0.38 & -1.00 & -2.32 \\
\hline XBADJ & $224 \mathrm{U}^{-} 6$ & -3.58 & -4.73 & -5.73 & -6.56 & -7.19 & -7.60 \\
\hline XBADJ & $224 \mathrm{U}^{-} 6$ & -74.15 & -105.81 & -144.93 & -179.64 & -208.90 & -231.81 \\
\hline XBADJ & $224 U_{-}^{-} 6$ & -247.68 & -256.02 & -256.58 & -60.46 & -61.10 & -60.67 \\
\hline XBADJ & $224 \mathrm{U}_{-}^{-} 6$ & -58.39 & -54.34 & -48.63 & -41.45 & -33.01 & -23.56 \\
\hline XBADJ & $224 U_{-}^{-} 6$ & -20.89 & -20.48 & -19.61 & -18.15 & -16.13 & -13.62 \\
\hline XBADJ & $224 \mathrm{U} \_7$ & -7.90 & 22.86 & 13.08 & -0.26 & -1.81 & -4.05 \\
\hline XBADJ & 224U_7 & -6.17 & -8.10 & -9.78 & -11.17 & -12.21 & -12.89 \\
\hline XBADJ & $224 \mathrm{U}_{-}^{-} 7$ & -79.54 & -111.14 & -150.04 & -184.37 & -213.11 & -235.37 \\
\hline XBADJ & $224 \mathrm{U}^{-} 7$ & -250.48 & -257.97 & -257.63 & -60.58 & -60.29 & -58.94 \\
\hline XBADJ & $224 \mathrm{U}_{-}^{-} 7$ & -55.80 & -50.96 & -44.58 & -36.84 & -27.98 & -18.28 \\
\hline XBADJ & $224 \mathrm{U}_{-}^{-} 7$ & -15.50 & -15.16 & -14.51 & -13.42 & -11.92 & -10.06 \\
\hline XBADJ & $224 \mathrm{U} 8$ & -7.90 & 22.86 & 13.08 & -0.26 & -1.81 & -4.05 \\
\hline XBADJ & $224 \mathrm{U}_{-}^{-} 8$ & -6.17 & -8.10 & -9.78 & -11.17 & -12.21 & -12.89 \\
\hline XBADJ & $224 \mathrm{U}_{-}^{-} 8$ & -79.54 & -111.14 & -150.04 & -184.37 & -213.11 & -235.37 \\
\hline XBADJ & $224 \mathrm{U}_{-}^{-} 8$ & -250.48 & -257.97 & -257.63 & -60.58 & -60.29 & -58.94 \\
\hline XBADJ & 224U_8 & -55.80 & -50.96 & -44.58 & -36.84 & -27.98 & -18.28 \\
\hline XBADJ & $224 \mathrm{U}_{-}^{-} 8$ & -15.50 & -15.16 & -14.51 & -13.42 & -11.92 & -10.06 \\
\hline XBADJ & $224 \mathrm{U} 9$ & -7.90 & 22.86 & 13.08 & -0.26 & -1.81 & -4.05 \\
\hline XBADJ & $224 U_{-}^{-} 9$ & -6.17 & -8.10 & -9.78 & -11.17 & -12.21 & -12.89 \\
\hline XBADJ & $224 \mathrm{U}_{-}^{-} 9$ & -79.54 & -111.14 & -150.04 & -184.37 & -213.11 & -235.37 \\
\hline XBADJ & 224U_9 & -250.48 & -257.97 & -257.63 & -60.58 & -60.29 & -58.94 \\
\hline XBADJ & $224 \mathrm{U} 9$ & -55.80 & -50.96 & -44.58 & -36.84 & -27.98 & -18.28 \\
\hline XBADJ & $224 \mathrm{U}_{-} 9$ & -15.50 & -15.16 & -14.51 & -13.42 & -11.92 & -10.06 \\
\hline XBADJ & 224UA_1 & 3.29 & 3.20 & 3.02 & 2.75 & -1.83 & -7.09 \\
\hline XBADJ & $224 \mathrm{UA}^{-} 1$ & -12.14 & -16.82 & -20.98 & -24.51 & -27.30 & -29.25 \\
\hline XBADJ & $224 \mathrm{UA}$ & -30.32 & -38.06 & -47.53 & -55.56 & -61.89 & -66.35 \\
\hline XBADJ & $224 \mathrm{UA}^{-} \mathrm{I}$ & -68.79 & -69.14 & -67.39 & -63.59 & -60.28 & -55.90 \\
\hline XBADJ & $224 \mathrm{UA}^{-} 1$ & -49.83 & -42.25 & -33.38 & -23.49 & -12.90 & -1.91 \\
\hline XBADJ & $224 \mathrm{UA}^{-} 1$ & 1.66 & 2.26 & 2.64 & 2.94 & 3.15 & 3.27 \\
\hline XBADJ & 224UA_2 & 3.29 & 3.20 & 3.02 & 2.75 & -1.83 & -7.09 \\
\hline XBADJ & 224 UA_2 & -12.14 & -16.82 & -20.98 & -24.51 & -27.30 & -29.25 \\
\hline XBADJ & $224 \mathrm{UA}^{-} 2$ & -30.32 & -38.06 & -47.53 & -55.56 & -61.89 & -66.35 \\
\hline XBADJ & 224UA_2 & -68.79 & -69.14 & -67.39 & -63.59 & -60.28 & -55.90 \\
\hline XBADJ & 224UA_2 & -49.83 & -42.25 & -33.38 & -23.49 & -12.90 & -1.91 \\
\hline XBADJ & $224 \mathrm{UA}^{-}$2 & 1.66 & 2.26 & 2.64 & 2.94 & 3.15 & 3.27 \\
\hline XBADJ & 224 UA_3 & 3.29 & 3.20 & 3.02 & 2.75 & -1.83 & -7.09 \\
\hline XBADJ & $224 \mathrm{UA} 3$ & -12.14 & -16.82 & -20.98 & -24.51 & -27.30 & -29.25 \\
\hline XBADJ & $224 \mathrm{UA}_{-} 3$ & -30.32 & -38.06 & -47.53 & -55.56 & -61.89 & -66.35 \\
\hline
\end{tabular}




\begin{tabular}{|c|c|c|c|c|c|c|c|}
\hline XBADJ & 224UA_3 & -68.79 & -69.14 & -67.39 & -63.59 & -60.28 & -55.90 \\
\hline XBADJ & 224UA_3 & -49.83 & -42.25 & -33.38 & -23.49 & -12.90 & -1.91 \\
\hline XBADJ & $224 \mathrm{UA} \_3$ & 1.66 & 2.26 & 2.64 & 2.94 & 3.15 & 3.27 \\
\hline XBADJ & 224UA_4 & 0.45 & 0.08 & -0.29 & -0.65 & -5.21 & -10.36 \\
\hline XBADJ & 224UA_4 & -15.19 & -19.56 & -23.34 & -26.41 & -28.67 & -30.06 \\
\hline XBADJ & 224UA_4 & -30.54 & -37.69 & -46.58 & -54.05 & -59.88 & -63.89 \\
\hline XBADJ & 224UA_4 & -65.96 & -66.02 & -64.08 & -60.19 & -56.89 & -52.63 \\
\hline XBADJ & $224 \mathrm{UA}_{-} 4$ & -46.77 & -39.50 & -31.02 & -21.60 & -11.52 & -1.10 \\
\hline XBADJ & 224UA_4 & 1.88 & 1.89 & 1.69 & 1.43 & 1.14 & 0.81 \\
\hline XBADJ & 224UA_5 & 0.45 & 0.08 & -0.29 & -0.65 & -5.21 & -10.36 \\
\hline XBADJ & 224UA_5 & -15.19 & -19.56 & -23.34 & -26.41 & -28.67 & -30.06 \\
\hline XBADJ & $224 \mathrm{UA}^{-} 5$ & -30.54 & -37.69 & -46.58 & -54.05 & -59.88 & -63.89 \\
\hline XBADJ & 224UA_5 & -65.96 & -66.02 & -64.08 & -60.19 & -56.89 & -52.63 \\
\hline XBADJ & 224UA_5 & -46.77 & -39.50 & -31.02 & -21.60 & -11.52 & -1.10 \\
\hline XBADJ & 224UA_5 & 1.88 & 1.89 & 1.69 & 1.43 & 1.14 & 0.81 \\
\hline XBADJ & 224UA_6 & 0.45 & 0.08 & -0.29 & -0.65 & -5.21 & -10.36 \\
\hline XBADJ & 224UA_6 & -15.19 & -19.56 & -23.34 & -26.41 & -28.67 & -30.06 \\
\hline XBADJ & $224 \mathrm{UA}^{-} 6$ & -30.54 & -37.69 & -46.58 & -54.05 & -59.88 & -63.89 \\
\hline XBADJ & 224 UA_6 6 & -65.96 & -66.02 & -64.08 & -60.19 & -56.89 & -52.63 \\
\hline XBADJ & 224UA_6 & -46.77 & -39.50 & -31.02 & -21.60 & -11.52 & -1.10 \\
\hline XBADJ & 224UA_6 & 1.88 & 1.89 & 1.69 & 1.43 & 1.14 & 0.81 \\
\hline YBADJ & $224 \mathrm{U} \_1$ & -14.97 & 14.71 & 38.66 & -3.71 & 0.21 & 5.49 \\
\hline YBADJ & $224 \mathrm{U}_{-} 1$ & 10.61 & 15.40 & 19.73 & 23.45 & 26.47 & 28.68 \\
\hline YBADJ & $224 \mathrm{U}^{-} 1$ & 126.04 & 113.18 & 97.16 & 78.19 & 56.85 & 33.78 \\
\hline YBADJ & $224 \mathrm{U} \_1$ & 9.68 & -14.71 & -38.66 & 3.71 & -0.21 & -5.49 \\
\hline YBADJ & $224 \mathrm{U} \_1$ & -10.61 & -15.40 & -19.73 & -23.45 & -26.47 & -28.68 \\
\hline YBADJ & $224 \mathrm{U} \_1$ & -30.02 & -29.54 & -28.18 & -25.96 & -22.96 & -19.26 \\
\hline YBADJ & $224 \mathrm{U} \_2$ & -14.97 & 14.71 & 38.66 & -3.71 & 0.21 & 5.49 \\
\hline YBADJ & 224U_2 & 10.61 & 15.40 & 19.73 & 23.45 & 26.47 & 28.68 \\
\hline YBADJ & $224 \mathrm{U}^{-} 2$ & 126.04 & 113.18 & 97.16 & 78.19 & 56.85 & 33.78 \\
\hline YBADJ & $224 \mathrm{U}^{-} 2$ & 9.68 & -14.71 & -38.66 & 3.71 & -0.21 & -5.49 \\
\hline YBADJ & $224 \mathrm{U} \_2$ & -10.61 & -15.40 & -19.73 & -23.45 & -26.47 & -28.68 \\
\hline YBADJ & 224U_2 & -30.02 & -29.54 & -28.18 & -25.96 & -22.96 & -19.26 \\
\hline YBADJ & $224 \mathrm{U} \_3$ & -14.97 & 14.71 & 38.66 & -3.71 & 0.21 & 5.49 \\
\hline YBADJ & $224 \mathrm{U}^{-} 3$ & 10.61 & 15.40 & 19.73 & 23.45 & 26.47 & 28.68 \\
\hline YBADJ & $224 \mathrm{U}^{-} 3$ & 126.04 & 113.18 & 97.16 & 78.19 & 56.85 & 33.78 \\
\hline YBADJ & $224 \mathrm{U}^{-} 3$ & 9.68 & -14.71 & -38.66 & 3.71 & -0.21 & -5.49 \\
\hline YBADJ & $224 \mathrm{U}^{-3}$ & -10.61 & -15.40 & -19.73 & -23.45 & -26.47 & -28.68 \\
\hline YBADJ & $224 U_{-}^{-} 3$ & -30.02 & -29.54 & -28.18 & -25.96 & -22.96 & -19.26 \\
\hline YBADJ & $224 \mathrm{U} \_4$ & -12.15 & 12.04 & 35.86 & -6.55 & -2.58 & 2.83 \\
\hline YBADJ & 224U_4 & 8.16 & 13.24 & 17.92 & 22.05 & 25.52 & 28.21 \\
\hline YBADJ & $224 \mathrm{U}_{-} 4$ & 126.06 & 113.69 & 98.16 & 79.63 & 58.69 & 35.97 \\
\hline YBADJ & 224U_4 & 12.15 & -12.04 & -35.86 & 6.55 & 2.58 & -2.83 \\
\hline YBADJ & $224 \mathrm{U} 4$ & -8.16 & -13.24 & -17.92 & -22.05 & -25.52 & -28.21 \\
\hline YBADJ & 224U_4 & -30.04 & -30.05 & -29.17 & -27.40 & -24.80 & -21.45 \\
\hline
\end{tabular}




\begin{tabular}{|c|c|c|c|c|c|c|c|}
\hline YBADJ & 224U_5 & -12.15 & 12.04 & 35.86 & -6.55 & -2.58 & 2.83 \\
\hline YBADJ & 224U_5 & 8.16 & 13.24 & 17.92 & 22.05 & 25.52 & 28.21 \\
\hline YBADJ & $224 \mathrm{U} \_5$ & 126.06 & 113.69 & 98.16 & 79.63 & 58.69 & 35.97 \\
\hline YBADJ & $224 \mathrm{U} 5$ & 12.15 & -12.04 & -35.86 & 6.55 & 2.58 & -2.83 \\
\hline YBADJ & $224 \mathrm{U}_{-} 5$ & -8.16 & -13.24 & -17.92 & -22.05 & -25.52 & -28.21 \\
\hline YBADJ & $224 \mathrm{U}^{-} 5$ & -30.04 & -30.05 & -29.17 & -27.40 & -24.80 & -21.45 \\
\hline YBADJ & $224 \mathrm{U} \_6$ & -12.15 & 12.04 & 35.86 & -6.55 & -2.58 & 2.83 \\
\hline YBADJ & $224 \mathrm{U}_{-} 6$ & 8.16 & 13.24 & 17.92 & 22.05 & 25.52 & 28.21 \\
\hline YBADJ & $224 \mathrm{U}_{-} 6$ & 126.06 & 113.69 & 98.16 & 79.63 & 58.69 & 35.97 \\
\hline YBADJ & $224 \mathrm{U}^{-} 6$ & 12.15 & -12.04 & -35.86 & 6.55 & 2.58 & -2.83 \\
\hline YBADJ & $224 \mathrm{U}^{-} 6$ & -8.16 & -13.24 & -17.92 & -22.05 & -25.52 & -28.21 \\
\hline YBADJ & $224 \mathrm{U} \_6$ & -30.04 & -30.05 & -29.17 & -27.40 & -24.80 & -21.45 \\
\hline YBADJ & $224 U_{-} 7$ & -12.84 & 17.06 & 41.14 & -1.16 & 2.75 & 7.94 \\
\hline YBADJ & 224U_7 & 12.89 & 17.45 & 21.48 & 24.86 & 27.48 & 29.26 \\
\hline YBADJ & $224 \mathrm{U}^{-} 7$ & 126.19 & 112.88 & 96.43 & 77.04 & 55.32 & 31.92 \\
\hline YBADJ & $224 \mathrm{U}_{-}^{-} 7$ & 7.54 & -17.06 & -41.14 & 1.16 & -2.75 & -7.94 \\
\hline YBADJ & 224U_7 & -12.89 & -17.45 & -21.48 & -24.86 & -27.48 & -29.26 \\
\hline YBADJ & $224 \mathrm{U}_{-} 7$ & -30.16 & -29.24 & -27.44 & -24.82 & -21.43 & -17.40 \\
\hline YBADJ & $224 \mathrm{U} \_8$ & -12.84 & 17.06 & 41.14 & -1.16 & 2.75 & 7.94 \\
\hline YBADJ & $224 \mathrm{U} \_8$ & 12.89 & 17.45 & 21.48 & 24.86 & 27.48 & 29.26 \\
\hline YBADJ & 224U_-8 & 126.19 & 112.88 & 96.43 & 77.04 & 55.32 & 31.92 \\
\hline YBADJ & $224 \mathrm{U}_{-} 8$ & 7.54 & -17.06 & -41.14 & 1.16 & -2.75 & -7.94 \\
\hline YBADJ & 224U_8 & -12.89 & -17.45 & -21.48 & -24.86 & -27.48 & -29.26 \\
\hline YBADJ & $224 \mathrm{U} \_8$ & -30.16 & -29.24 & -27.44 & -24.82 & -21.43 & -17.40 \\
\hline YBADJ & 224U_9 & -12.84 & 17.06 & 41.14 & -1.16 & 2.75 & 7.94 \\
\hline YBADJ & $224 \mathrm{U}_{-}^{-} 9$ & 12.89 & 17.45 & 21.48 & 24.86 & 27.48 & 29.26 \\
\hline YBADJ & 224U_9 & 126.19 & 112.88 & 96.43 & 77.04 & 55.32 & 31.92 \\
\hline YBADJ & 224U_9 & 7.54 & -17.06 & -41.14 & 1.16 & -2.75 & -7.94 \\
\hline YBADJ & 224U_9 & -12.89 & -17.45 & -21.48 & -24.86 & -27.48 & -29.26 \\
\hline YBADJ & 224U_9 & -30.16 & -29.24 & -27.44 & -24.82 & -21.43 & -17.40 \\
\hline YBADJ & $224 \mathrm{UA} \_1$ & 0.51 & 7.20 & 13.67 & 15.99 & 20.16 & 25.09 \\
\hline YBADJ & $224 \mathrm{UA}^{-} 1$ & 29.25 & 32.52 & 34.81 & 36.04 & 36.17 & 35.21 \\
\hline YBADJ & 224UA_1 & 33.17 & 29.22 & 24.41 & 18.85 & 12.72 & 6.20 \\
\hline YBADJ & $224 \mathrm{UA}^{-} 1$ & -0.51 & -7.20 & -13.67 & -15.99 & -20.16 & -25.09 \\
\hline YBADJ & $224 \mathrm{UA}^{-} 1$ & -29.25 & -32.52 & -34.81 & -36.04 & -36.17 & -35.21 \\
\hline YBADJ & 224UA_1 & -33.17 & -29.22 & -24.41 & -18.85 & -12.72 & -6.20 \\
\hline YBADJ & 224UA_2 & 0.51 & 7.20 & 13.67 & 15.99 & 20.16 & 25.09 \\
\hline YBADJ & 224 UA_2 & 29.25 & 32.52 & 34.81 & 36.04 & 36.17 & 35.21 \\
\hline YBADJ & $224 \mathrm{UA}^{-} 2$ & 33.17 & 29.22 & 24.41 & 18.85 & 12.72 & 6.20 \\
\hline YBADJ & $224 \mathrm{UA}^{-} 2$ & -0.51 & -7.20 & -13.67 & -15.99 & -20.16 & -25.09 \\
\hline YBADJ & 224UA_2 & -29.25 & -32.52 & -34.81 & -36.04 & -36.17 & -35.21 \\
\hline YBADJ & $224 \mathrm{UA}^{-} 2$ & -33.17 & -29.22 & -24.41 & -18.85 & -12.72 & -6.20 \\
\hline YBADJ & 224UA_3 & 0.51 & 7.20 & 13.67 & 15.99 & 20.16 & 25.09 \\
\hline YBADJ & $224 \mathrm{UA}^{-} 3$ & 29.25 & 32.52 & 34.81 & 36.04 & 36.17 & 35.21 \\
\hline YBADJ & 224UA_3 & 33.17 & 29.22 & 24.41 & 18.85 & 12.72 & 6.20 \\
\hline YBADJ & $224 \mathrm{UA}^{-} 3$ & -0.51 & -7.20 & -13.67 & -15.99 & -20.16 & -25.09 \\
\hline
\end{tabular}




$\begin{array}{llrrrrrr}\text { YBADJ } & \text { 224UA_3 } & -29.25 & -32.52 & -34.81 & -36.04 & -36.17 & -35.21 \\ \text { YBADJ } & \text { 224UA_3 } & -33.17 & -29.22 & -24.41 & -18.85 & -12.72 & -6.20 \\ & & & & & & & \\ \text { YBADJ } & \text { 224UA_4 } & 2.40 & 8.57 & 14.48 & 16.21 & 19.79 & 24.13 \\ \text { YBADJ } & \text { 224UA_4 } & 27.74 & 30.51 & 32.35 & 33.21 & 33.05 & 31.90 \\ \text { YBADJ } & \text { 224UA_4 } & 29.77 & 25.84 & 21.13 & 15.79 & 9.97 & 3.84 \\ \text { YBADJ } & \text { 224UA_4 } & -2.40 & -8.57 & -14.48 & -16.21 & -19.79 & -24.13 \\ \text { YBADJ } & \text { 224UA_4 } & -27.74 & -30.51 & -32.35 & -33.21 & -33.05 & -31.90 \\ \text { YBADJ } & \text { 224UA_4 } & -29.77 & -25.84 & -21.13 & -15.79 & -9.97 & -3.84 \\ & & & & & & & \\ \text { YBADJ } & \text { 224UA_5 } & 2.40 & 8.57 & 14.48 & 16.21 & 19.79 & 24.13 \\ \text { YBADJ } & \text { 224UA_5 } & 27.74 & 30.51 & 32.35 & 33.21 & 33.05 & 31.90 \\ \text { YBADJ } & \text { 224UA_5 } & 29.77 & 25.84 & 21.13 & 15.79 & 9.97 & 3.84 \\ \text { YBADJ } & \text { 224UA_5 } & -2.40 & -8.57 & -14.48 & -16.21 & -19.79 & -24.13 \\ \text { YBADJ } & \text { 224UA_5 } & -27.74 & -30.51 & -32.35 & -33.21 & -33.05 & -31.90 \\ \text { YBADJ } & \text { 224UA_5 } & -29.77 & -25.84 & -21.13 & -15.79 & -9.97 & -3.84 \\ & & & & & & & \\ \text { YBADJ } & \text { 224UA_6 } & 2.40 & 8.57 & 14.48 & 16.21 & 19.79 & 24.13 \\ \text { YBADJ } & \text { 224UA_6 } & 27.74 & 30.51 & 32.35 & 33.21 & 33.05 & 31.90 \\ \text { YBADJ } & \text { 224UA_6 } & 29.77 & 25.84 & 21.13 & 15.79 & 9.97 & 3.84 \\ \text { YBADJ } & \text { 224UA_6 } & -2.40 & -8.57 & -14.48 & -16.21 & -19.79 & -24.13 \\ \text { YBADJ } & \text { 224UA_6 } & -27.74 & -30.51 & -32.35 & -33.21 & -33.05 & -31.90 \\ \text { YBADJ } & \text { 224UA_6 } & -29.77 & -25.84 & -21.13 & -15.79 & -9.97 & -3.84\end{array}$

** Variable Emissions Type: "By Month / Hour / Seven Days"

** Variable Emission Scenario: "Scenario 2"

** Month = January; Day of Week = Monday EMISFACT 224U 3 MHRDOW7 0000000000000 EMISFACT 224U 3 MHRDOW7 $000 \begin{array}{lllllll}-3 & 0 & 0 & 0 & 0 & 0 & 0\end{array}$ EMISFACT 224U 3 MHRDOW7 $00 \begin{array}{llllllllll} & 0 & 0 & 0 & 0 & 0 & 0 & 0\end{array}$

** Month = February; Day of week = Monday EMISFACT 224U_3 MHRDOW7 00000000000

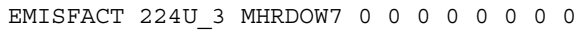
EMISFACT 224U_3 MHRDOW7 $00 \begin{array}{lllllllll} & 0 & 0 & 0 & 0 & 0 & 0 & 0\end{array}$

** Month $=$ March; Day of week = Monday

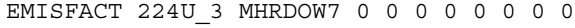
EMISFACT 224U_3 MHRDOW7 $0 \begin{array}{lllllll}0 & 0 & 0 & 0 & 0 & 0 & 0\end{array}$ EMISFACT 224U_3 MHRDOW7 $00 \begin{array}{lllllll}0 & 0 & 0 & 0 & 0 & 0 & 0\end{array}$

** Month $=$ April; Day of Week = Monday EMISFACT 224U_3 MHRDOW7 00000000000 EMISFACT 224U_3 MHRDOW7 000000 EMISFACT 224U 3 MHRDOW7 0000000

** Month = May; Day of Week = Monday EMISFACT 2240_3 MHRDOW7 00000000000

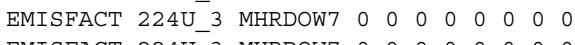
EMISFACT 224U_3 MHRDOW7 00000000000

$* *$ Month $=$ June; Day of Week = Monday

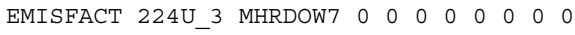

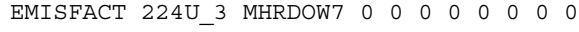
EMISFACT 224U 3 MHRDOW7 $00 \begin{array}{lllllll}0 & 0 & 0 & 0 & 0 & 0 & 0\end{array}$

$* *$ Month $=\mathrm{July} ;$ Day of Week $=$ Monday

EMISFACT 224U_3 MHRDOW7 0000000000 
EMISFACT 224U_3 MHRDOW7 001000000000

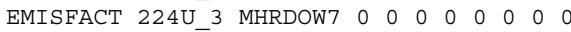

** Month $=$ August $; \quad$ Day of Week = Monday

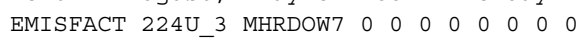
EMISFACT 224U 3 MHRDOW7 000000000000 EMISFACT 2240.

EMISFACT 2240_3 MHRDOW7 00000000000

** Month = September $;$ Day of Week = Monda EMISFACT 224U_3 MHRDOW7 00000000000 EMISFACT 224U_3 MHRDOW7 000000000000

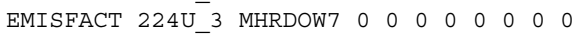

* Month = October; $;$ Day of Week = Monday

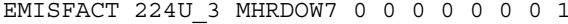

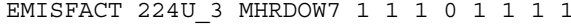
EMISFACT 224U 3 MHRDOW7 $00 \begin{array}{lllllll}0 & 0 & 0 & 0 & 0 & 0 & 0\end{array}$

** Month $=$ November; Day of Week = Monday FMISFACT 224U 3 MHRDOW7 000000000

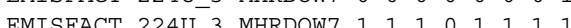
EMTSACT 2240

EMISEACT 2240_3 MHRDOW $\begin{array}{lllllllll}0 & 0 & 0 & 0 & 0 & 0 & 0 & 0\end{array}$ * * Month = December; Day of Week = Monday

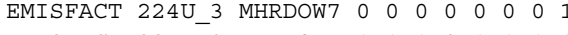

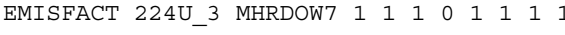
EMISFACT 224U_3 MHRDOW7 00000000000

* Month $=$ January; Day of Week $=$ Tuesday EMISFACT 224U_3 MHRDOW7 $000 \begin{array}{llllll}0 & 0 & 0 & 0 & 0 & 0\end{array}$ EMISFACT 224U_3 MHRDOW7 000000000000

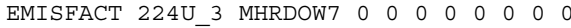

** Month $=$ February; Day of Week $=$ Tuesday

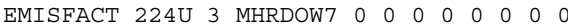

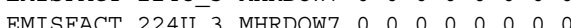

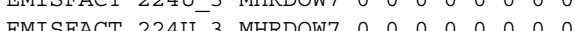
EMISFACT 2240_3 MHRDOW $\begin{array}{llllllllllll}0 & 0 & 0 & 0 & 0 & 0 & 0 & 0\end{array}$ * Month $=$ March; Day of Week = Tuesday EMISFACT 224__3 MHRDOW7 0 C 000000000

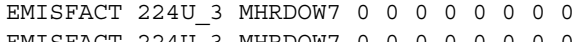
EMISFACT 224U_3 MHRDOW7 0000000000

* Month = April; Day of Week = Tuesday EMISFACT 224U_3 MHRDOW7 0 C $00 \begin{array}{lllllllll} & 0 & 0 & 0 & 0 & 0\end{array}$ EMISFACT 224U_3 MHRDOW7 $00 \begin{array}{lllllll}0 & 0 & 0 & 0 & 0 & 0 & 0\end{array}$ EMISFACT 224U_3 MHRDOW7 00000000000

* Month $=$ May; Day of Week $=$ Tuesday EMISFACT 224U_3 MHRDOW7 00000000000 EMISFACT 224__3 MHRDOW7 $00 \begin{array}{llllllll}0 & 0 & 0 & 0 & 0 & 0 & 0\end{array}$ EMISFACT 224U_3 MHRDOW7 0000000000 * Month $=$ June; Day of Week = Tuesday EMISFACT 2240_3 MHRDOW7 $0 \begin{array}{llllllll}3 & 0 & 0 & 0 & 0 & 0 & 0\end{array}$

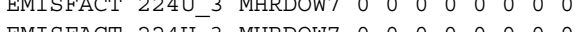
EMISFACT 224U_3 MHRDOW7 $000 \begin{array}{lllllll}0 & 0 & 0 & 0 & 0 & 0 & 0\end{array}$ * Month $=$ July; Day of week = Tuesday EMISFACT 224U_3 MHRDOW7 0000000000 EMISFACT 224U_3 MHRDOW7 $00 \begin{array}{lllllll}0 & 0 & 0 & 0 & 0 & 0 & 0\end{array}$

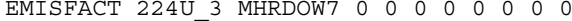

* Month = August; Day of Week = Tuesday

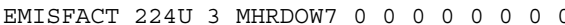
EMISFACT 224U 3 MHRDOW7 0 
EMISFACT 224U_3 MHRDOW7 0000000000

** Month $=$ September; Day of Week = Tuesday EMISFACT 224U_3 MHRDOW7 0000000000

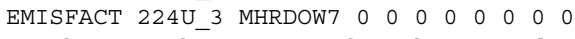
* Month = October; Day of Week = Tuesday EMISFACT 2240_3 MHRDOW7 0 C $0 \begin{array}{lllllll}0 & 0 & 0 & 0 & 0 & 1\end{array}$ EMISFACT 224__ MHRDOW7 $1 \begin{array}{llllllll}1 & 1 & 1 & 0 & 1 & 1 & 1 & 1\end{array}$ EMISFACT 224U_3 MHRDOW7 $000 \begin{array}{llllll}-3 & 0 & 0 & 0 & 0 & 0\end{array}$

* Month = November; Day of Week = Tuesday EMISFACT 224U_3 MHRDOW7 00000000001

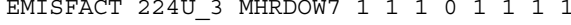
EMISFACT 224U_3 MHRDOW7 $00 \begin{array}{lllllll}0 & 0 & 0 & 0 & 0 & 0 & 0\end{array}$

** Month $=$ December; Day of Week $=$ Tuesday

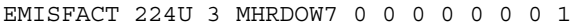
EMISFACT 224U 3 MHRDOW7 1101001 EMISFACT 224U-3 MHRDOW7 000000000

* Month - January. Day of Week * Month = January; Day of Week = Wednesday EMISFACT 224U_3 MHRDOW7 000100000 EMISFACT 224__3 MURDOW7 0000000000000 EMISFACT 224U_3 MHRDOW7 000000000000

$*$ Month $=$ February; Day of Week $=$ Wednesday EMISFACT 224U_3 MHRDOW7 0000000000

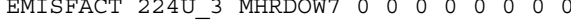
EMISFACT 224U_3 MHRDOW7 $000 \begin{array}{llllll}0 & 0 & 0 & 0 & 0 & 0\end{array}$

** Month $=$ March; Day of Week = Wednesday EMISFACT 224U 3 MHRDOW7 $00 \begin{array}{lllllll}0 & 0 & 0 & 0 & 0 & 0 & 0\end{array}$

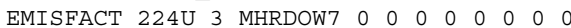

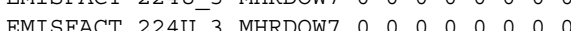

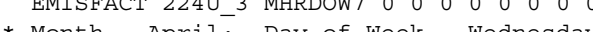

$* *$ Month $=$ Aprili $;$ Day of Week $=$ Wednesday

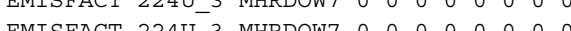
EMISFACT 2240_- MHRDOW 000000000000 EMISFACT 224U_3 MHRDOW7 $000 \begin{array}{lllllll}0 & 0 & 0 & 0 & 0 & 0 & 0\end{array}$

* Month = May; Day of Week = Wednesday EMISFACT 224U_3 MHRDOW7 00000000000 EMISFACT 224U_3 MHRDOW7 00000000000

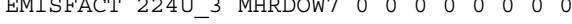

* Month $=$ June; Day of Week $=$ Wednesday EMISFACT 224U_3 MHRDOW7 0000000000

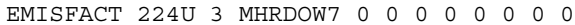
EMISFACT 224U 3 MHRDOW7 0 a 000000

* Month = July; _ Day of Wer Month = July; Day of week = Wednesday

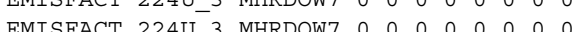
EMISFACT 2240. MTRD EMISFACT 2240_3 MHRDOW7 $0 \begin{array}{llllllll}0 & 0 & 0 & 0 & 0 & 0 & 0\end{array}$

* Month = August $;$ Day of Week = Wednesda EMISFACT 224U_3 MHRDOW7 0000000000 EMISFACT 224U_3 MHRDOW7 $000 \begin{array}{llllll}0 & 0 & 0 & 0 & 0 & 0\end{array}$

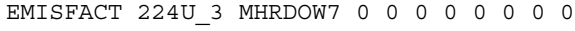

** Month $=$ September; Day of Week = Wednesday EMISFACT 224U_3 MHRDOW7 00000000000

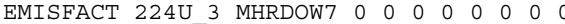


** Month $=$ October; Day of Week = Wednesday EMISFACT 224U_3 MHRDOW7 0000000001 EMISFACT 224U_3 MHRDOW7 $1 \begin{array}{lllllllll}1 & 1 & 0 & 1 & 1 & 1 & 1\end{array}$ EMISFACT 224U_3 MHRDOW7 000000000000

** Month = November; Day of Week = Wednesda

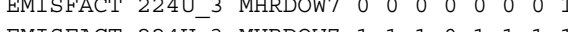
BMISFACT 224_-3 MHRDOW7 $\begin{array}{llllllll}1 & 1 & 1 & 0 & 1 & 1 & 1 & 1\end{array}$

Month = December; Day of Week = Wednesday

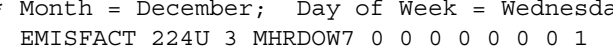
$\begin{array}{llllllllllll}\text { EMISFACT } & 224 \mathrm{U} & 3 & \text { MHRDOW7 } & 0 & 0 & 0 & 0 & 0 & 0 & 0 & 1 \\ \text { EMISFACT } & 224 \mathrm{U} & 3 & \text { MHRDOW7 } & 1 & 1 & 1 & 0 & 1 & 1 & 1 & 1\end{array}$

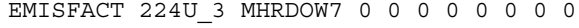

** Month = January; Day of Week = Thursday EMISFACT 224U_3 MHRDOW7 00000000000

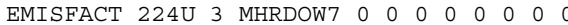
EMISFACT 224U 3 MHRDOW7 0 O 0 (2) 0

** Month = February; Day of Week = Thursday EMISFACT 224U 3 MHRDOW7 000000000000 EMISFACT 224U 3 MHRDOW7 001000000000 EMISFACT 224U 3 MHRDOW7 00000000000

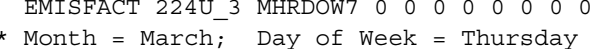
Month $=$ March; $; \quad$ Day of week $=$ Thursday
EMISFACT 224U_3 MHRDOW7 000000000 EMISFACT 224U_3 MHRDOW7 $0 \begin{array}{lllllll}0 & 0 & 0 & 0 & 0 & 0 & 0\end{array}$

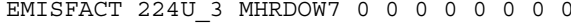

* Month $=$ April; $;$ Day of Week = Thursday

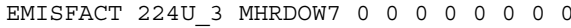
EMISFACT 224U 3 MHRDOW7 $00 \begin{array}{lllllll}3 & 0 & 0 & 0 & 0 & 0 & 0\end{array}$

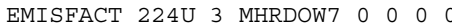

* Month $=$ May; Day of Week = Thursday

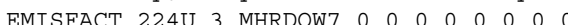

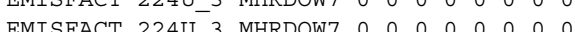
EMISFACT 224.

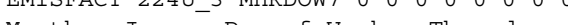
* Month = June; Day of Week = Thursday EMISFACT 224U_3 MHRDOW7 00000000000

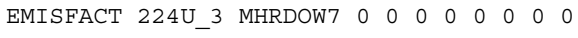
EMISFACT 224U_3 MHRDOW7 00000000000

** Month $=$ July; Day of Week = Thursday EMISFACT 224U_3 MHRDOW7 0000000000

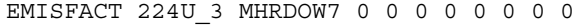
EMISFACT 224U 3 MHRDOW7 0 C 00 C

* Month = August; Day of Week = Thursday IMISFACT $224 \mathrm{U}$, Day of Week = Thursday

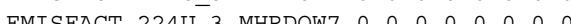

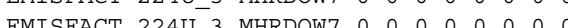

* Month = September; Day of Week = Thursday EMISFACT 224U_3 MHRDOW7 0000000000 EMISFACT 224U_3 MHRDOW7 0000000000

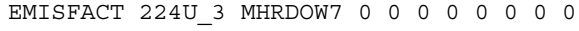

** Month $=$ October; Day of Week = Thursday

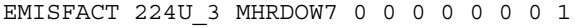

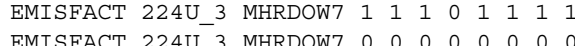

* Month $=$ November; Day of Week $=$ Thursday 


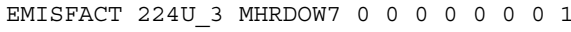
EMISFACT 224U_3 MHRDOW7 $1 \begin{array}{lllllllll}1 & 1 & 0 & 1 & 1 & 1 & 1\end{array}$

* MOISFACT 224U_3 MHRDOW7 0000000000

Month = December; Day of Week = Thursday

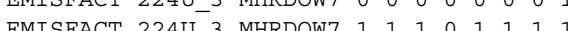
EMISFAC 224_- MHRDOW7 $\begin{array}{llllllll}1 & 1 & 1 & 0 & 1 & 1 & 1 & 1\end{array}$

EMISFACI 2240_3 MHRDOW7 0000000000

* Month = January; Day of Week = Friday EMISFACT 224U_3 MHRDOW7 0000000000 EMISFACT 224U_3 MHRDOW7 $00 \begin{array}{ccccccc}0 & 0 & 0 & 0 & 0 & 0 & 0\end{array}$ EMISFACT 224U_3 MHRDOW7 $000 \begin{array}{lllllll}0 & 0 & 0 & 0 & 0 & 0 & 0\end{array}$

* Month $=$ February; Day of Week = Friday EMISFACT 224U_3 MHRDOW7 0000000000 EMISFACT 224U 3 MHRDOW7 $00 \begin{array}{lllllll}0 & 0 & 0 & 0 & 0 & 0 & 0\end{array}$ EMISFACT 224U 3 MURDOW7 0 O 000

** Month $=$ March; Day of Week = Friday

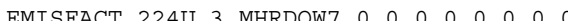
EMISFACT 224U 3 MHRDOW7 $00 \begin{array}{lllllllllllll}0 & 0 & 0 & 0 & 0 & 0 & 0\end{array}$ EMTSEACT 224U

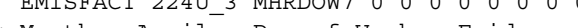

* Month = April; Day of Week = Friday

EMISFACT 224U_3 MHRDOW7 0000000000 EMISFACT 224U_3 MHRDOW7 000000000000 EMISFACT 224U_3 MHRDOW7 000000000

* Month = May; Day of Week = Friday

EMISFACT 224U_3 MHRDOW7 0000000000

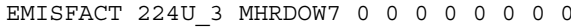
EMISFACT 224U 3 MHRDOW7 0 C

** Month $=$ June; Day of Week = Friday

EMISFACT 224U_3 MHRDOW7 0000000000

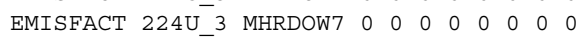
EMISFACT 224U 3 MHRDOW7 $000 \begin{array}{llllllllll}-3 & 0 & 0 & 0 & 0 & 0 & 0\end{array}$

** Month $=$ July; Day of Week = Friday

EMISFACT 224U_3 MHRDOW7 00000000000 EMISFACT 224U_3 MHRDOW7 000000000000 EMISFACT 224U_3 MHRDOW7 0000000000

** Month $=$ August $;$ Day of week = Friday EMISFACT 224U_3 MHRDOW7 0000000000 EMISFACT 224U_3 MHRDOW7 $0 \begin{array}{lllllll}0 & 0 & 0 & 0 & 0 & 0 & 0\end{array}$ EMISFACT 224U_3 MHRDOW7 00000000000

** Month $=$ September; Day of Week = Friday EMISFACT 224U_3 MHRDOW7 000000000000 EMISFACT 224U 3 MHRDOW7 $00 \begin{array}{lllllllll}0 & 0 & 0 & 0 & 0 & 0 & 0\end{array}$

$* *$ Month $=$ October; Day of Week = Friday EMISFACT 224U_3 MHRDOW7 000000001

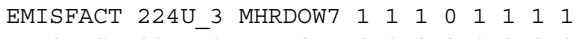
EMISFACT 224U_3 MHRDOW7 0000000000

$* *$ Month $=$ November; Day of Week = Friday EMISFACT 224U_3 MHRDOW7 $000 \begin{array}{llllll}0 & 0 & 0 & 0 & 0 & 1\end{array}$ EMISFACT 224U 3 MHRDOW7 $1 \begin{array}{lllllll}1 & 1 & 0 & 1 & 1 & 1 & 1\end{array}$

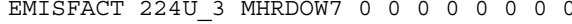

* Month $=$ December; $;$ Day of Week = Friday

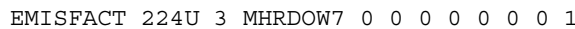




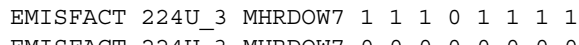
EMISFACT 224U_3 MHRDOW7 0000000000

** Month $=$ January; Day of Week = Saturday EMISFACT 224U_3 MHRDOW7 0000000000 EMISFACT 2240_3 MRDOW7 0 O 00000000

EMISFACT 2240_3 MHRDOW7 $0 \begin{array}{lllllllllll}0 & 0 & 0 & 0 & 0 & 0 & 0\end{array}$

** Month = February; Day of Week = Saturday EMISFACT 224U_3 MHRDOW7 00000000000 EMISFACT 224U_3 MHRDOW7 $00 \begin{array}{llllllll}0 & 0 & 0 & 0 & 0 & 0 & 0\end{array}$ EMISFACT 224U_3 MHRDOW7 00000000000

** Month $=$ March; Day of Week = Saturday EMISFACT 224U_3 MHRDOW7 00000000000 EMISFACT 224U_3 MHRDOW7 $000 \begin{array}{llllll}0 & 0 & 0 & 0 & 0 & 0\end{array}$ EMISFACT 224U 3 MHRDOW7 $00 \begin{array}{lllllllllll}0 & 0 & 0 & 0 & 0 & 0 & 0\end{array}$

** Month $=$ April $;$ Day of Week = Saturday EMISFACT 224U_3 MHRDOW7 00000000000

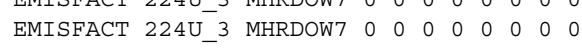
FMISFACT 224U 3 MHRDOW7 $0 \begin{array}{llllllllllll}-3 & 0 & 0 & 0 & 0 & 0 & 0 & 0\end{array}$

* Month = May; Day of Week = Saturday EMISFACT 224U 3 MHRDOW7 0000000000000 EMISFACT 224U 3 MHRDOW7 000000000000 EMISFACT 224U_3 MHRDOW7 00000000000

* Month $=$ June; Day of Week = Saturday EMISFACT 224U 3 MHRDOW7 000000000 EMISFACT 224U_3 MHRDOW7 $0 \begin{array}{lllllll}0 & 0 & 0 & 0 & 0 & 0 & 0\end{array}$ EMISFACT 224U_3 MHRDOW7 00000000000

** Month $=$ July; Day of Week = Saturday EMISFACT 224U 3 MHRDOW7 000000000

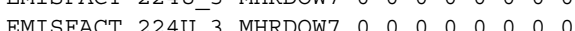

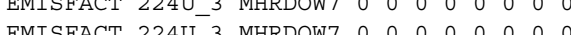

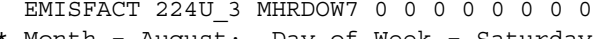
* Month = August; Day of Week = Saturday EMISFACT 224U_3 MHRDOW7 0 O 0000000 EMISFACT 224U_3 MHRDOW7 $00 \begin{array}{llllllll}0 & 0 & 0 & 0 & 0 & 0 & 0\end{array}$ EMISFACT 224U_3 MHRDOW7 00000000000

* Month $=$ September; Day of Week = Saturday EMISFACT 224U_3 MHRDOW7 0000000000 EMISFACT 224U_3 MHRDOW7 0000000000000 EMISFACT 224U_3 MHRDOW7 00000000000

** Month $=$ October; Day of Week = Saturday EMISFACT 224U 3 MHRDOW7 00000000000 EMISFACT 224U 3 MHRDOW7 0 O 000000

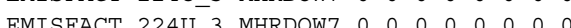

*Mish = 2240-3

* Month = November; Day of Week = Saturday EMISFACT 224U_3 MHRDOW7 00000000 EMISFACT 224_

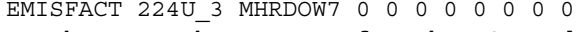

* Month $=$ December; Day of Week = Saturday

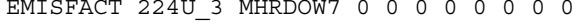
EMISFACT 224U_3 MHRDOW7 $00 \begin{array}{lllllll}0 & 0 & 0 & 0 & 0 & 0 & 0\end{array}$

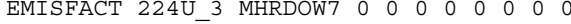

** Month = January; Day of Week = Sunday EMISFACT 224U 3 MHRDOW7 0000000 EMISFACT 224U_3 MHRDOW7 0 


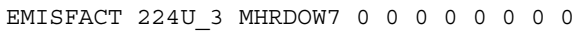

** Month $=$ February; Day of Week $=$ Sunday EMISFACT 224U_3 MHRDOW7 0000000000000

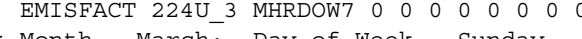
* Month $=$ March; Day of Week = Sunday EMISFACT 224U 3 MHRDOW7 $0 \begin{array}{lllllll}0 & 0 & 0 & 0 & 0 & 0 & 0\end{array}$ EMISFACI 2240_3 MHRDOW7 00000000000 EMISFACT 224U_3 MHRDOW7 00000000000

* Month $=$ April; Day of Week = Sunday

EMISFACT 224U_3 MHRDOW7 00000000000 EMISFACT 224U_3 MHRDOW7 $0 \begin{array}{lllllll}0 & 0 & 0 & 0 & 0 & 0 & 0\end{array}$

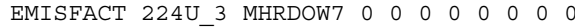

** Month $=$ May; Day of Week = Sunday

EMISFACT 224U_3 MHRDOW7 0000000000

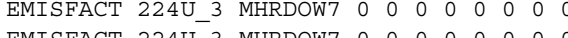

* Month = June; Day of week = Sunday

* Month = June; Day of week = Sunday EMISFACT 224U_3 MHRDOW7 000000000000 EMISFACT 224U_3 MHRDOW7 000000000

* Month $=$ July; Day of week = Sunday

EMISFACT 224U_3 MHRDOW7 00000000000 EMISFACT 224U_3 MHRDOW7 $0 \begin{array}{lllllll}0 & 0 & 0 & 0 & 0 & 0 & 0\end{array}$ EMISFACT 224U_3 MHRDOW7 $000 \begin{array}{lllllll}0 & 0 & 0 & 0 & 0 & 0 & 0\end{array}$

** Month $=$ August; Day of Week $=$ Sunday EMISFACT 224U_3 MHRDOW7 00000000000

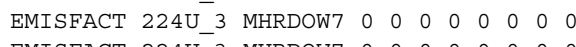
EMISFACT 224U_3 MHRDOW7 00000000000

** Month $=$ September; Day of week = Sunday EMISFACT 2240_3 MHRDOW7 $0 \begin{array}{llllllllllllllllll}0 & 0 & 0 & 0 & 0 & 0 & 0\end{array}$ EMISFACT 224__3 MHRDOW7 0000000000000 EMISFACT 2240_3 MHRDOW7 $0 \begin{array}{llllllllll}0 & 0 & 0 & 0 & 0 & 0 & 0\end{array}$

** Month $=$ October; Day of Week = Sunday EMISFACT 224U_3 MHRDOW7 000000000 EMISFACT 224U_3 MHRDOW7 $00 \begin{array}{lllllll}0 & 0 & 0 & 0 & 0 & 0 & 0\end{array}$ EMISFACT 224U_3 MHRDOW7 $00 \begin{array}{lllllllll}0 & 0 & 0 & 0 & 0 & 0 & 0 & 0\end{array}$

* Month $=$ November; Day of Week $=$ Sunday EMISFACT 224U_3 MHRDOW7 0000000000

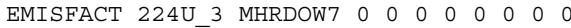

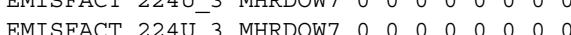

* Month Month = December; Day of Week = Sunday INTSCT 224_MMISFACT 2240 3 MHROW7 $0 \begin{array}{llllllllllllll}0 & 0 & 0 & 0 & 0 & 0 & 0\end{array}$

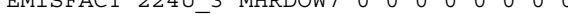
* Month $=$ January; Day of Week = Monday EMISFACT 224U_2 MHRDOW7 0000000000 EMISFACT 224U_2 MHRDOW7 $0 \begin{array}{lllllll}0 & 0 & 0 & 0 & 0 & 0 & 0\end{array}$

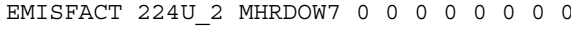

** Month $=$ February; Day of Week = Monday EMISFACT 224U 2 MHRDOW7 00010000000

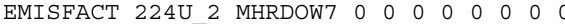
EMISFACT 224U 2 MHRDOW7 
** Month $=$ March; Day of Week = Monday EMISFACT 224U_2 MHRDOW7 000000000000 EMISFACT 224U_2 MHRDOW7 000000000000 EMISFACT 224U 2 MHRDOW7 00000000000

* Month = April; Day of Week = Monday

EMISFACT 224U 2 MHRDOW7 00000000000 EMISFACT 224U 2 MHRDOW7 0000000000000 EMISFACT 224U_2 MHRDOW7 000000000000

** Month $=$ May; Day of week = Monday

EMISFACT 224U_2 MHRDOW7 000000000 EMISFACT 224U_2 MHRDOW7 $00 \begin{array}{llllllll}0 & 0 & 0 & 0 & 0 & 0 & 0\end{array}$

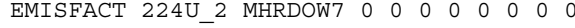

** Month $=$ June; Day of Week = Monday

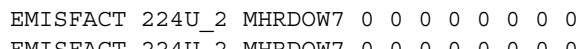

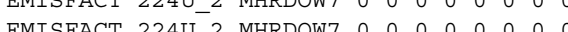

$*$ Month $=$ July; $;$ Day of Week = Monday

$*$ Month = July; Day of Week = Monday
EMISFACT 224U 2 MHRDOW7 0000000

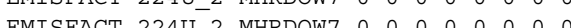
EMISFACT 224U_2 MHRDOW7 $0 \begin{array}{lllllllllllllll}0 & 0 & 0 & 0 & 0 & 0 & 0\end{array}$

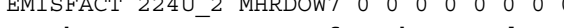
* Month = August; Day of Week = Monday EMISFACT 224U_2 MHRDOW7 000000000

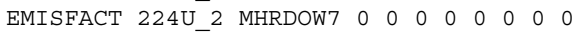
EMISFACT 224U_2 MHRDOW7 0000000000

** Month $=$ September $;$ Day of Week $=$ Monday EMISFACT 224U_2 MHRDOW7 00000000000 EMISFACT 224U 2 MHRDOW7 $00 \begin{array}{lllllllllll}0 & 0 & 0 & 0 & 0 & 0 & 0 & 0\end{array}$ EMISFACT 224U_2 MHRDOW7 00000

** Month = October, Day of Week = Monday TMTSEACT 224U_2 MHRDOW7 000000

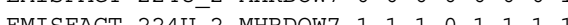

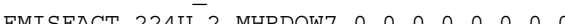

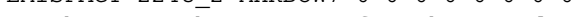
* Month $=$ November; Day of Week = Monday EMISFACT 224U_2 MHRDOW7 0000000001

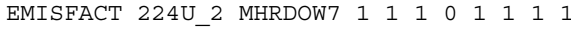
EMISFACT 224U_2 MHRDOW7 0000000000

* Month $=$ December; Day of Week = Monday EMISFACT 224U_2 MHRDOW7 00000000001

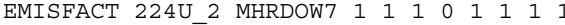

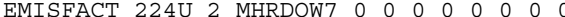

* * Month = January, Day of Week = Tuesday TMISFACT $224 U$ - MHRDOW7 000000

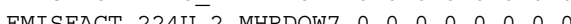
DMTSAC 224

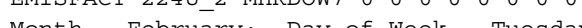

* Month = February; Day of Week = Tuesday EMISFACT 224U_2 MHRDOW7 0000000000 EMISFACT 224U_2 MHRDOW7 000000000000 EMISFACT 224U_2 MHRDOW7 00000000000

** Month $=$ March; Day of Week $=$ Tuesday EMISFACT 224U_2 MHRDOW7 $000 \begin{array}{llllll}0 & 0 & 0 & 0 & 0 & 0\end{array}$ EMISFACT 224U_2 MHRDOW7 $00 \begin{array}{lllllll}0 & 0 & 0 & 0 & 0 & 0 & 0\end{array}$ EMISFACT 224U 2 MHRDOW7 0000000000

** Month $=$ April $;$ Day of Week $=$ Tuesday 
EMISFACT 224U_2 MHRDOW7 0000000000

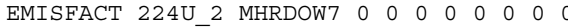
EMISFACT 224U 2 MHRDOW7 00000 * Month $=$ May; Day of Week $=$ Tuesday EMISFACT 224U_2 MHRDOW7 000000000

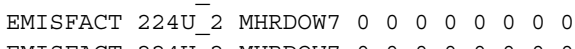
EMISFACT 224U_2 MHRDOW7 00020000000

** Month $=$ June; Day of Week = Tuesday

EMISFACT 224U_2 MHRDOW7 00000000000

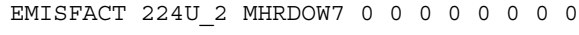
EMISFACT 224U_2 MHRDOW7 00000000000

** Month $=$ July; Day of Week = Tuesday

EMISFACT 224U_2 MHRDOW7 $00 \begin{array}{lllllll}0 & 0 & 0 & 0 & 0 & 0 & 0\end{array}$ EMISFACT 224U 2 MHRDOW7 $00 \begin{array}{lllllll}0 & 0 & 0 & 0 & 0 & 0 & 0\end{array}$

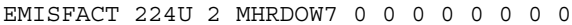

* * Month = August, Day of Week = Tuesday MMISFA

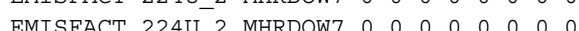
(1)

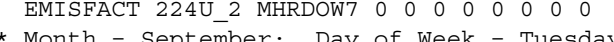

** Month = September; Day of Week = Tuesday EMISFACT 224U_2 MHRDOW7 00000000000 EMISFACT 224U_2 MHRDOW7 000000000000 EMISFACT 224U_2 MHRDOW7 $00 \begin{array}{llllllll}0 & 0 & 0 & 0 & 0 & 0 & 0\end{array}$

* Month = October; Day of Week = Tuesday

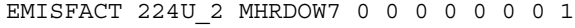

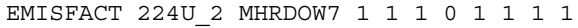

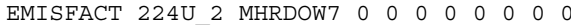
을 $\quad * *$ Month $=$ November; Day of Week = Tuesday

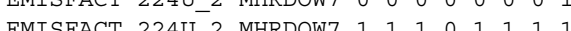
BMISTCT 2240_2 MHDOW7 $1 \begin{array}{lllllll}1 & 1 & 0 & 1 & 1 & 1 & 1\end{array}$

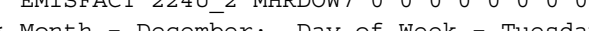

* Month = December; Day of Week = Tuesday

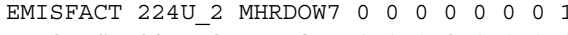

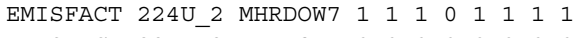
EMISFACT 224U_2 MHRDOW7 00000000000

** Month = January; Day of Week = Wednesday EMISFACT 224U_2 MHRDOW7 000000000000 EMISFACT 224U_2 MHRDOW7 $00 \begin{array}{lllllll}0 & 0 & 0 & 0 & 0 & 0 & 0\end{array}$

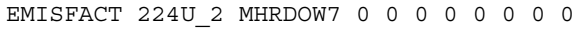

** Month $=$ February; Day of Week $=$ Wednesday EMISFACT 224U_2 MHRDOW7 00000000000 $\begin{array}{llllllllll} & 0 & & \end{array}$ EMISFACT 224U 2 MHRDOW7 $000 \begin{array}{lllllllll}-2 & 0 & 0 & 0 & 0 & 0 & 0\end{array}$

** Month $=$ March; Day of Week $=$ Wednesday EMISFACT 224U 2 MHRDOW7 000000000000

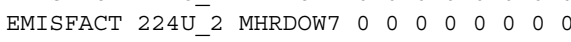
EMISFACT 224U_2 MHRDOW7 0000000000

* Month = April; Day of Week = Wednesday EMISFACT 224U_2 MHRDOW7 000000000 EMISFACT 224U 2 MHRDOW7 $00 \begin{array}{lllllll}0 & 0 & 0 & 0 & 0 & 0 & 0\end{array}$

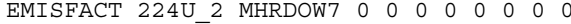

* Month $=$ May; Day of Week = Wednesday EMISFACT 224U 2 MHRDOW7 0 O 
EMISFACT 224U_2 MHRDOW7 000000000000 EMISFACT 224U 2 MHRDOW7 $00 \begin{array}{llllllllllll}0 & 0 & 0 & 0 & 0 & 0 & 0\end{array}$

** Month $=$ June; Day of Week $=$ Wednesday

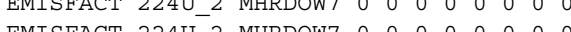

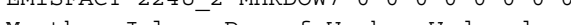

** Month $=$ July; Day of Week = Wednesday EMISFACT 224U_2 MHRDOW7 0000000000 EMISFACT 224U_2 MHRDOW7 $00 \begin{array}{llllllllll}0 & 0 & 0 & 0 & 0 & 0 & 0\end{array}$ EMISFACT 224U_2 MHRDOW7 0000000000

** Month $=$ August; Day of Week $=$ Wednesday EMISFACT 224U_2 MHRDOW7 00000000000 EMISFACT 224U_2 MHRDOW7 $00 \begin{array}{lllllll}0 & 0 & 0 & 0 & 0 & 0 & 0\end{array}$ EMISFACT 224U_2 MHRDOW7 00000000000

** Month $=$ September; Day of Week = Wednesday EMISFACT 224U_2 MHRDOW7 00000000000 $\begin{array}{lllllllllllll}2 & \text { MHRDOW7 } & 0 & 0 & 0 & 0 & 0 & 0 & 0 & 0\end{array}$ 224U 2 MHRDOW7 00000000000

** Month = October; Day of Week = Wednesday

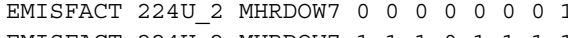

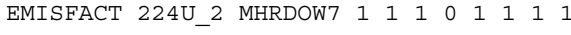
EMISFACT 224U_2 MHRDOW7 0000000000

** Month $=$ November; Day of Week = Wednesday

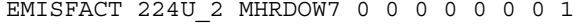

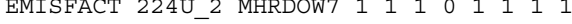

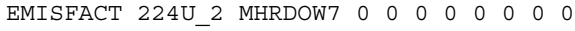

** Month $=$ December; Day of Week $=$ Wednesday

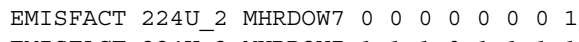
2 MHRDOW7 $1 \begin{array}{llllllllll}1 & 1 & 1 & 0 & 1 & 1 & 1 & 1\end{array}$ EMISFACT 224U_2 MHRDOW7 0000000000 * Month = January; Day of Week = Thursda EMISFACT 224U_2 MHRDOW7 0000000000 EMISFACT 224U_2 MHRDOW7 0000000000000

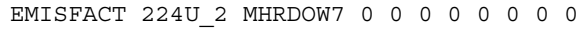

* Month $=$ February; Day of Week = Thursday EMISFACT 224U_2 MHRDOW7 0000000000

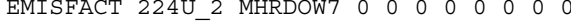
EMISFACT 224U 2 MHRDOW7 $00 \begin{array}{lllllll}0 & 0 & 0 & 0 & 0 & 0 & 0\end{array}$

* Month $=$ March; Day of Week = Thursday EMISFACT 224U_ 2 MHRDOW7 00000 O

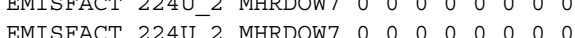
EMISFACT 224U_ MHRDOW7 $0 \begin{array}{llllllllllll}0 & 0 & 0 & 0 & 0 & 0 & 0\end{array}$

EMISFACT 224_-2 MHRDOW $\begin{array}{llllllllll}0 & 0 & 0 & 0 & 0 & 0 & 0 & 0\end{array}$ * Month = April;

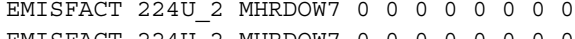
EMISFACT 2240_2 MRDOW7 $0 \begin{array}{llllllllllll}0 & 0 & 0 & 0 & 0 & 0 & 0\end{array}$ EMISFACT 224U_2 MHRDOW7 $000 \begin{array}{lllllll}0 & 0 & 0 & 0 & 0 & 0 & 0\end{array}$

* Month $=$ May; Day of Week = Thursday EMISFACT 224U_2 MHRDOW7 00000000000 EMISFACT 224U_2 MHRDOW7 $0 \begin{array}{llllllll}0 & 0 & 0 & 0 & 0 & 0 & 0 & 0\end{array}$

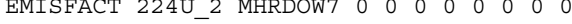

** Month $=$ June; Day of Week = Thursday EMISFACT 224U_2 MHRDOW7 0000000000 EMISFACT 224U 2 MHRDOW7 00000 
EMISFACT 224U_2 MHRDOW7 000000000

** Month $=$ July; Day of Week = Thursday

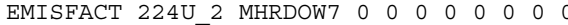
FMISFACT 224U-2 MHRDOW7 00000000000

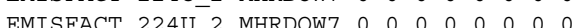

** Month = August; Day of Week

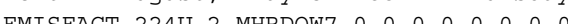

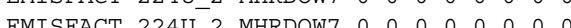
EMISFACT 224U_2 MHRDOW7 00000000000

* Month $=$ September; Day of Week = Thursday EMISFACT 224U_2 MHRDOW7 00000000000

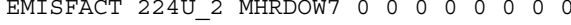

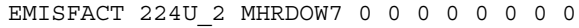

** Month = October; Day of Week = Thursday

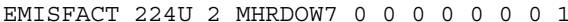
EMISFACT 224U_

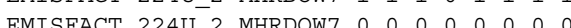

* Month = November. Day of Week Thura

* Month = November; Day of Week = Thursday

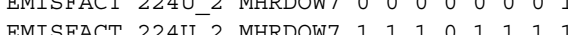

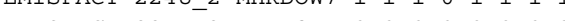

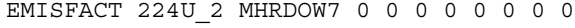

* Month $=$ December; Day of Week = Thursday

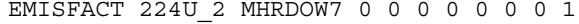
EMISFACT 224U_2 MHRDOW7 $1 \begin{array}{llllllll}1 & 1 & 0 & 1 & 1 & 1 & 1\end{array}$

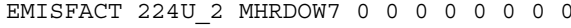

** Month = January; Day of week = Friday EMISFACT 224U 2 MHRDOW7 $00 \begin{array}{lllllll}0 & 0 & 0 & 0 & 0 & 0 & 0\end{array}$

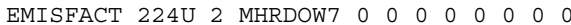
EMISFACT 224U_

2240 Month = February; Day of Week = Friday

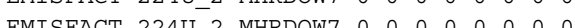

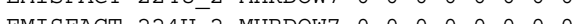
EMISFACT 224U_2 MHRDOW7 $\begin{array}{llllllll}0 & 0 & 0 & 0 & 0 & 0 & 0 & 0\end{array}$

* Month $=$ March; Day of Week = Friday EMISFACT 224U_2 MHRDOW7 $000 \begin{array}{lllllll}0 & 0 & 0 & 0 & 0 & 0\end{array}$ EMISFACT 224U_2 MHRDOW7 $000 \begin{array}{lllllll}0 & 0 & 0 & 0 & 0 & 0 & 0\end{array}$ EMISFACT 224U_2 MHRDOW7 $00 \begin{array}{lllllll}0 & 0 & 0 & 0 & 0 & 0 & 0\end{array}$

** Month $=$ April; Day of Week = Friday

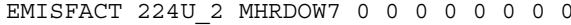
EMISFACT 224U 2 MHRDOW7 $00 \begin{array}{lllllllllll}0 & 0 & 0 & 0 & 0 & 0 & 0 & 0\end{array}$

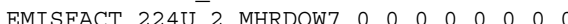

* Month = May; Day of Week = Friday

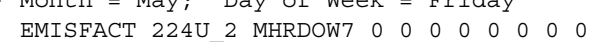

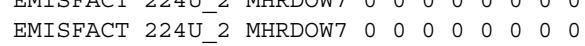
EMISFACT 224U 2 MHRDOW7 $00 \begin{array}{ccccccc}0 & 0 & 0 & 0 & 0 & 0 & 0\end{array}$

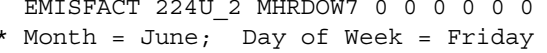

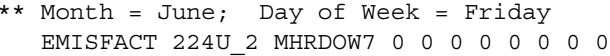

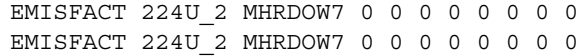
EMISFACT 224U_2 MHRDOW7 $0 \begin{array}{lllllll}0 & 0 & 0 & 0 & 0 & 0 & 0\end{array}$

** Month $=$ July; Day of Week = Friday

EMISFACT 224U_2 MHRDOW7 $00 \begin{array}{lllllll}0 & 0 & 0 & 0 & 0 & 0 & 0\end{array}$ FMISFACT 224U_2 MHRDOW7 
** Month $=$ August; Day of Week = Friday EMISFACT 224U_2 MHRDOW7 0000000000 EMISFACT 224U_2

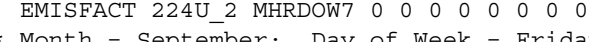

* Month = September; Day of Week = Friday EMISFACT 224U_2 MHRDOW7 $0 \begin{array}{llllllllll}0 & 0 & 0 & 0 & 0 & 0\end{array}$ EMISFACT 224_EMISFACT 2240_2 MHRDOW7 $0 \begin{array}{llllllll}0 & 0 & 0 & 0 & 0 & 0 & 0 & 0\end{array}$

** Month = October; Day of Week = Friday EMISFACT 224U_2 MHRDOW7 0 O $00 \begin{array}{lllll}0 & 0 & 0 & 0 & 1\end{array}$ EMISFACT 224U_2 MHRDOW7 11111001011

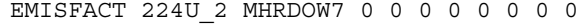

* Month $=$ November; Day of Week = Friday EMISFACT 224U_2 MHRDOW7 0000000001

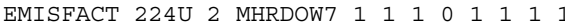

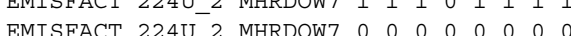

* Month Month = December; Day of Week = Friday

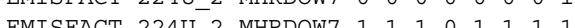

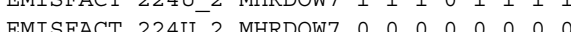

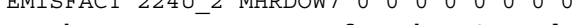

* Month = January; Day of Week = Saturday EMISFACT 224U_2 MHRDOW7 00000000000 EMISFACT 224U_2 MHRDOW7 $000 \begin{array}{llllll}0 & 0 & 0 & 0 & 0 & 0\end{array}$ EMISFACT 224U_2 MHRDOW7 00000000000

** Month $=$ February; Day of Week = Saturday EMISFACT 224U_2 MHRDOW7 000100000000 EMISFACT 224U 2 MHRDOW7 $00 \begin{array}{llllllllllllll}0 & 0 & 0 & 0 & 0 & 0 & 0\end{array}$ EMISFACT 224U 2 MRDDW7 00000

* Month Month March; Day of week = Saturday EMISFACT 224U

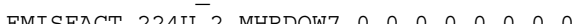

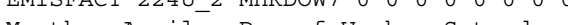
* Month = April; Day of Week = Saturday EMISFACT 224U_2 MHRDOW7 0000000000 EMISFACT 224U_2 MHRDOW7 00000000000 EMISFACT 224U_2 MHRDOW7 00000000000

** Month = May; Day of Week = Saturday EMISFACT 224U_2 MHRDOW7 0000000000 EMISFACT 224U_2 MHRDOW7 $00 \begin{array}{lllllll}0 & 0 & 0 & 0 & 0 & 0 & 0\end{array}$ EMISFACT 224U 2 MHRDOW7 0 - 0

* Month = June, Day of Week = Saturday Month = June, Day or Week = Saturday

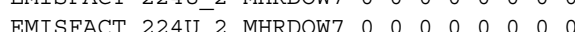
MISFAC EMISFACT 2240_2 MHRDOW7 $0 \begin{array}{llllllllll}0 & 0 & 0 & 0 & 0 & 0 & 0\end{array}$ * Month = July; Day of Week = Saturday EMISFACT 2240_2 MHRDOW7 00000000000 EMISFACT 224U_2 MHRDOW7 $00 \begin{array}{llllllll}0 & 0 & 0 & 0 & 0 & 0 & 0\end{array}$ EMISFACT 224U_2 MHRDOW7 $000 \begin{array}{lllllll}0 & 0 & 0 & 0 & 0 & 0 & 0\end{array}$

* Month = August; Day of Week = Saturday EMISFACT 224U_2 MHRDOW7 00000000000 EMISFACT 224U_2 MHRDOW7 $00 \begin{array}{lllllll}0 & 0 & 0 & 0 & 0 & 0 & 0\end{array}$ EMISFACT 224U 2 MHRDOW7 $00 \begin{array}{lllllll}0 & 0 & 0 & 0 & 0 & 0 & 0\end{array}$

** Month $=$ September; Day of Week = Saturday 
EMISFACT 224U_2 MHRDOW7 0000000000

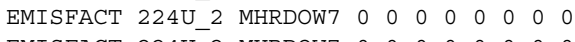

EMISFACT 224U_2 MHRDOW7 $00 \begin{array}{llllllll}0 & 0 & 0 & 0 & 0 & 0 & 0\end{array}$

Month = October; Day of Week = Saturday EMISFACT 224U-2 MHRDOW7 000000000

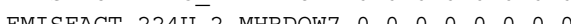

EMISFACT 2240_2 MHRDOW7 $0 \begin{array}{lllllllllllll}0 & 0 & 0 & 0 & 0 & 0 & 0\end{array}$

* Month $=$ November; Day of Week = Saturday

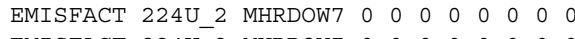
EMISFACT 224U_2 MHRDOW7 $00 \begin{array}{lllllll}0 & 0 & 0 & 0 & 0 & 0 & 0\end{array}$

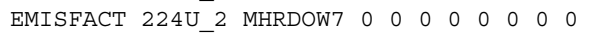

** Month $=$ December $;$ Day of Week = Saturday EMISFACT 224U_2 MHRDOW7 00000000000 EMISFACT 224U 2 MHRDOW7 $00 \begin{array}{lllllll}0 & 0 & 0 & 0 & 0 & 0 & 0\end{array}$

** Month = Januaryi Day of Week TMISFACT 224U 2 MHRDOw7 0000000 EMTSEACT 224U_2 MHRDOW7 000000000000 MMTSEACT 2240

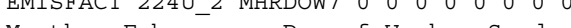

** Month = February; Day of Week = Sunday EMISFACT 224U_2 MHRDOW7 000000000 EMISFACT 224U_2 MHRDOW7 00000000000

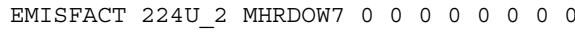

* Month $=$ March; Day of Week = Sunday EMISFACT 224U_2 MHRDOW7 000000000 EMISFACT 224U_2 MHRDOW7 $000 \begin{array}{cccccc}0 & 0 & 0 & 0 & 0 & 0\end{array}$ EMISFACT 224U 2 MHRDOW7 0 C

** Month $=$ April $;$ Day of Week = Sunday EMISFACT 224U 2 MHRDOW7 00000000000 EMISFACT 224U 2 MHRDOW7 00000000000

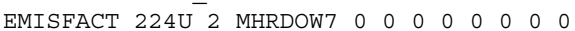

** Month = May; Day of Week = Sunday

EMISFACT 224U_2 MHRDOW7 0000000000 EMISFACT 224U_2 MHRDOW7 000000000000 EMISFACT 224U_2 MHRDOW7 0000000000

** Month $=$ June; ${ }^{2}$ Day of Week = Sunday

EMISFACT 224U_2 MHRDOW7 000000000

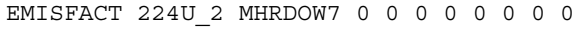
EMISFACT 224U_2 MHRDOW7 $00 \begin{array}{ccccccc}0 & 0 & 0 & 0 & 0 & 0 & 0\end{array}$

** Month $=$ July; Day of Week = Sunday

EMISFACT 224U_2 MHRDOW7 0000000000

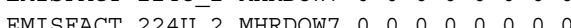
DMTSEACT 224U_2 MHRDOW7 00000000000

EMISFACT 224__ MHRDOW7 $0 \begin{array}{lllllll}0 & 0 & 0 & 0 & 0 & 0 & 0\end{array}$ * Month $=$ August $;$ Day of Week = Sunday EMISFACT 2240_2 MHRDOW7 0 0 0 0 0 0 0 0 0 0 EMISFACT 224U_2 MHRDOW7 00000000000 EMISFACT 224U_2 MHRDOW7 0000000000

* Month $=$ September; Day of Week = Sunda EMISFACT 224U_2 MHRDOW7 00000000000

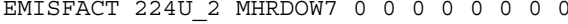
EMISFACT 224U 2 MHRDOW7 $00 \begin{array}{lllllll}0 & 0 & 0 & 0 & 0 & 0 & 0\end{array}$

** Month $=$ October; Day of Week = Sunday EMISFACT 224U_ 2 MHRDOW7 0 - $000-0$ 
EMISFACT 224U_2 MHRDOW7 000000000000 EMISFACT 224U_2 MHRDOW7 $000 \begin{array}{llllllll}0 & 0 & 0 & 0 & 0 & 0\end{array}$

** Month $=$ November; Day of Week = Sunday $\begin{array}{llll}0 & 0 & 0\end{array}$ EMISFACT 2240_2 MHRDOW7 0 0 000000000

EMISEACT 2240_2 MHRDOW7 $\begin{array}{ccccccccc}0 & 0 & 0 & 0 & 0 & 0 & 0\end{array}$

** Month = December; Day of Week = Sunday EMISFACT 224U_2 MHRDOW7 0000000000 EMISFACT 224U_2 MHRDOW7 000000000000

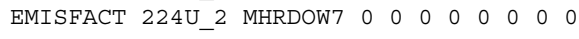

$*$ Month $=$ January; Day of Week $=$ Monday EMISFACT 224U_1 MHRDOW7 00000000000 EMISFACT 224U_1 MHRDOW7 $00 \begin{array}{llllllll}0 & 0 & 0 & 0 & 0 & 0 & 0\end{array}$ EMISFACT 224U 1 MHRDOW7 $00 \begin{array}{llllllllll}1 & 0 & 0 & 0 & 0 & 0 & 0\end{array}$

** Month = February; Day of Week = Monday EMISFACT 224U_1 MHRDOW7 00000000000

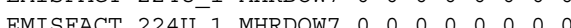

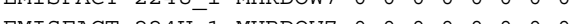

Month $=$ March; Day of Week = Monday

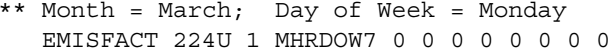

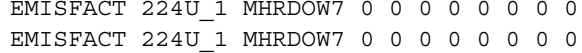

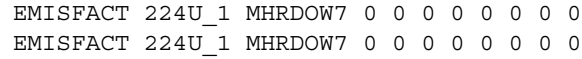

* Month $=$ April; Day of Week = Monday

EMISFACT 224U_1 MHRDOW7 000000000 EMISFACT 224U_1 MHRDOW7 $00 \begin{array}{lllllll}0 & 0 & 0 & 0 & 0 & 0 & 0\end{array}$ EMISFACT 224U 1 MHRDOW7 $00 \begin{array}{lllllllll}1 & 0 & 0 & 0 & 0 & 0 & 0 & 0\end{array}$

** Month $=$ May; Day of Week = Monday

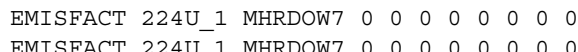

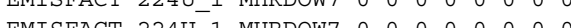

* Month $=$ June; Day of Week = Monday

* Month = June; Day of Week $=$ Monday EMISFACT 224U_1 MHRDOW7 000000000000 EMISFACT 224U_1 MHRDOW7 00000000000

* Month $=$ July; Day of Week = Monday

EMISFACT 224U_1 MHRDOW7 00000000000

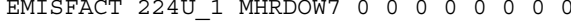
EMISFACT 224U_1 MHRDOW7 000000000000

** Month $=$ August; $; \quad$ Day of Week = Monday

EMISFACT 224U_1 MHRDOW7 00000000000 EMISFACT 224U_1 MHRDOW7 00000000000

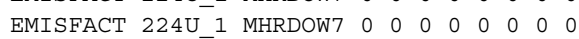

** Month = September; Day of Week = Monday EMISFACT 224U 1 MHRDOW7 000000000000

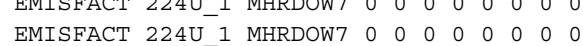
* Month = October; Day of Week = Monday Month = October; Day of week = Monday
EMISFACT 224U 1 MHRDOW7 000000001

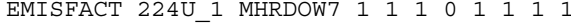

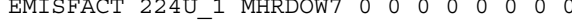

$* *$ Month $=$ November; Day of Week $=$ Monday EMISFACT 224U 1 MHRDOW7 $00 \begin{array}{lllllll}0 & 0 & 0 & 0 & 0 & 0 & 1\end{array}$ EMISFACT 224U 1 MHRDOW7 111010 
EMISFACT 224U_1 MHRDOW7 0000000000

** Month $=$ December; Day of Week $=$ Monday EMISFACT 224U_1 MHRDOW7 001000000001 EMISEACT 224_-1 MHRDOW7 1111 EMISFACT 2240_1 MHRDOW7 $00 \begin{array}{lllllll}0 & 0 & 0 & 0 & 0 & 0 & 0\end{array}$ * Month = January; Day of Week = Tuesday EMISFACT 224U_1 MHRDOW7 000000000

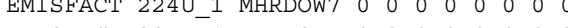
EMISFACT 224U_1 MHRDOW7 0000000000

* Month = February; Day of Week = Tuesday EMISFACT 224U_1 MHRDOW7 00000000000

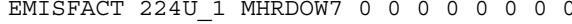
EMISFACT 224U_1 MHRDOW7 $00 \begin{array}{lllllll}0 & 0 & 0 & 0 & 0 & 0 & 0\end{array}$

** Month $=$ March; Day of Week = Tuesday EMISFACT 224U 1 MHRDOW7 000 C

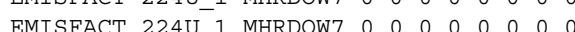

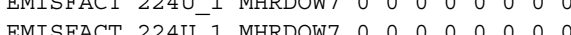

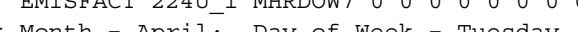
* Month $=$ April; $i$ Day of Week = Tuesday

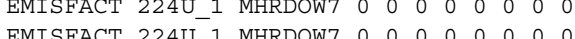

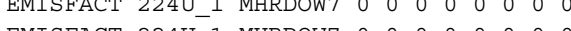
EMISFACT 224U_1 MHRDOW7 000000000 $*$ Month $=$ May; Day of Week $=$ Tuesday EMISFACT 224U_1 MHRDOW7 00000000000

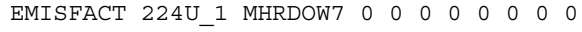

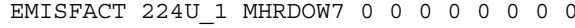

** Month $=$ June; Day of Week $=$ Tuesday

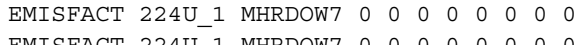
EMISFACT 224_-1 MHRDW7 $\begin{array}{cccccccccc}0 & 0 & 0 & 0 & 0 & 0 & 0 & 0\end{array}$

EMISFACT 2240_1 MHRDOW7 0000000000000

* Month = July; Day of Week = Tuesday EMISFACT 2240_1 MHRDOW7 0 C $\begin{array}{cccccc}0 & 0 & 0 & 0 & 0 & 0\end{array}$

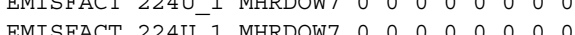

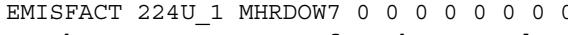

** Month $=$ August; Day of Week $=$ Tuesday EMISFACT 224U_1 MHRDOW7 000000000 EMISFACT 224U_1 MHRDOW7 $000 \begin{array}{lllllll}0 & 0 & 0 & 0 & 0 & 0 & 0\end{array}$

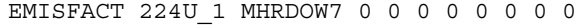

** Month $=$ September; Day of Week = Tuesday EMISFACT 224U_1 MHRDOW7 00010000000

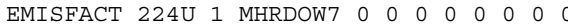

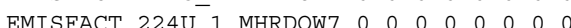

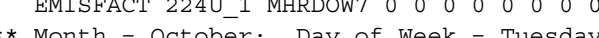
MMITAC MISTC EMISFACT 22401 MHRDOW7 $\begin{array}{llllllll}1 & 1 & 1 & 0 & 1 & 1 & 1 & 1\end{array}$

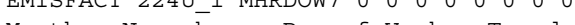
* Month = November; Day of week = Tuesday EMISFACT 224U_1 MHRDOW7 0000000001

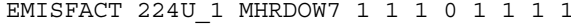
EMISFACT 224U_1 MHRDOW7 $00 \begin{array}{llllllll}0 & 0 & 0 & 0 & 0 & 0 & 0\end{array}$

** Month $=$ December; Day of Week = Tuesday EMISFACT 224U_1 MHRDOW7 $000 \begin{array}{llllll}0 & 0 & 0 & 0 & 0 & 1\end{array}$

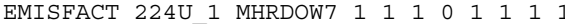
EMISFACT 224U 1 MHRDOW7 
** Month = January; Day of Week = Wednesday

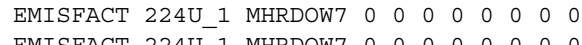

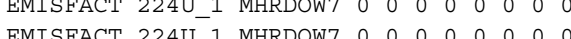

** Month = February; Day of week = Wednesday EMISFACT 224U_1 MHRDOW7 0000000000000

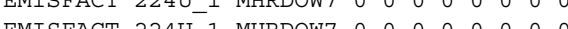
EMISFACT 224_-1 MHRDOW7 0000000000

** Month $=$ March; Day of Week $=$ Wednesday EMISFACT 224U_1 MHRDOW7 00000000000 EMISFACT 224U_1 MHRDOW7 00000000000

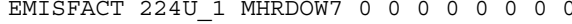

* Month $=$ April; Day of Week $=$ Wednesday

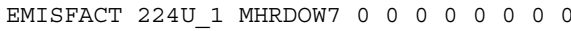
EMISFACT 224U 1 MHRDOW7 001000010000

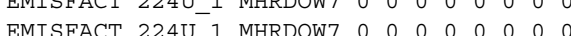

* EMISAC Month = May; $;$ Day of Week = Wednesday

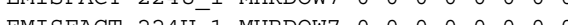
EMISFACT 2240-1 MHRDOW7 $0 \begin{array}{llllllllllllllll}0 & 0 & 0 & 0 & 0 & 0\end{array}$

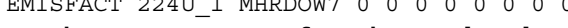
* Month $=$ June; Day of Week = Wednesday EMISFACT 224U_1 MHRDOW7 0000000000 EMISFACT 224U_1 MHRDOW7 00000000000 EMISFACT 224U_1 MHRDOW7 $00 \begin{array}{lllllll}0 & 0 & 0 & 0 & 0 & 0 & 0\end{array}$

** Month $=$ July; Day of Week = Wednesday

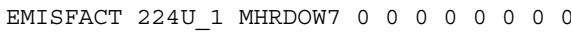

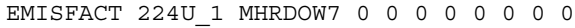

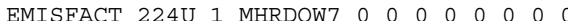
** Month = August; Day of week = Wednesday EMISFACT 224U_1 MHRDOW7 00000000000 EMISFACT 224U 1 MHRDOW7 000000000000

* Month = September; Day of Week = Wednesday EMISFACT 224U_1 MHRDOW7 0000000000 EMISFACT 224U_1 MHRDOW7 00000000000 EMISFACT 224U_1 MHRDOW7 0000000000

** Month $=$ October; Day of Week = Wednesday EMISFACT 224U_1 MHRDOW7 00000000001 EMISFACT 224U_1 MHRDOW7 $11110 \begin{array}{lllllll}1 & 0 & 1 & 1 & 1 & 1\end{array}$

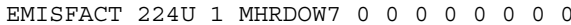

** Month $=$ November; $;$ Day of Week $=$ Wednesday EMISFACT 224U 1 MHRDOW7 00000000

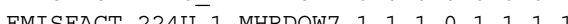

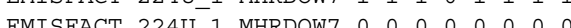

* Month = December; Day of Week = Wednesday EMISFACT 224U_1 MHRDOW7 00000000001

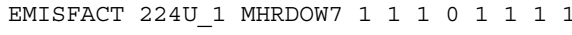

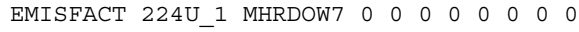

$* *$ Month $=$ January; Day of Week $=$ Thursday EMISFACT 224U_1 MHRDOW7 00010000000 EMISFACT 224U_1 MHRDOW7 $00 \begin{array}{lllllll}0 & 0 & 0 & 0 & 0 & 0 & 0\end{array}$ EMISFACT 224U 1 MHRDOW7 $00 \begin{array}{llllllll}0 & 0 & 0 & 0 & 0 & 0 & 0\end{array}$

** Month $=$ February; Day of Week = Thursday 
EMISFACT 224U_1 MHRDOW7 0000000000 EMISFACT 224U 1 MHRDOW7 $000 \begin{array}{lllllllll}0 & 0 & 0 & 0 & 0 & 0 & 0 & 0\end{array}$

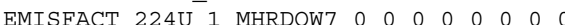

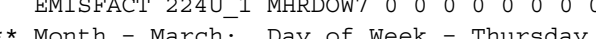
Month = March; Day of Week = Thursday EMISFACT 2240_-1 MHRDW7 $0 \begin{array}{lllllllll}0 & 0 & 0 & 0 & 0 & 0 & 0\end{array}$ EMISFAC 2240

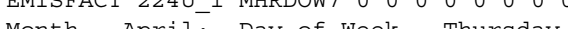
** Month $=$ April; Day of Week = Thursday EMISFACT 224U_1 MHRDOW7 000000000000 EMISFACT 224U_1 MHRDOW7 $00 \begin{array}{lllllll}0 & 0 & 0 & 0 & 0 & 0 & 0\end{array}$ EMISFACT 224U_1 MHRDOW7 0000000000

$* *$ Month $=$ May; Day of Week $=$ Thursday

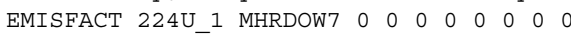
EMISFACT 224U 1 MHRDOW7 $00 \begin{array}{lllllll}0 & 0 & 0 & 0 & 0 & 0 & 0\end{array}$ EMISFACT 224U

** Month = June; Day of Week = Thursday TMISFA

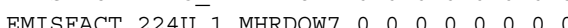

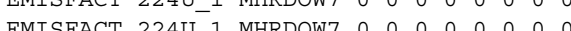
EMISFACT 2240_1 MHRDOW7 $0 \begin{array}{llllllllllllllll}0 & 0 & 0 & 0 & 0 & 0 & 0\end{array}$ * Month = July; Day of Week = Thursday EMISFACT 224U_1 MHRDOW7 000000000 EMISFACT 224U_1 MHRDOW7 00000000000 EMISFACT 224U_1 MHRDOW7 0000000000

** Month = August; Day of Week = Thursday EMISFACT 224U_1 MHRDOW7 000000000 EMISFACT 224U_1 MHRDOW7 $00 \begin{array}{lllllll}0 & 0 & 0 & 0 & 0 & 0 & 0\end{array}$ EMISFACT 224U_1 MHRDOW7 $00 \begin{array}{llllllll}0 & 0 & 0 & 0 & 0 & 0 & 0\end{array}$

N $\quad * *$ Month $=$ September; Day of Week = Thursday EMISFACT 224U 1 MHRDOW7 000000000000

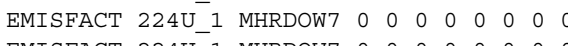

EMISFACT 224U_1

* Month = October; Day of Week = Thursda

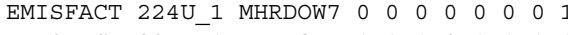

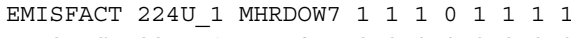

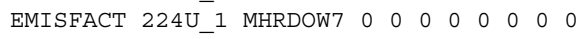

** Month = November; Day of Week = Thursday

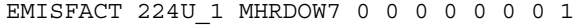

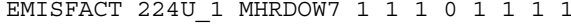
EMISFACT 224U_1 MHRDOW7 00000000000

** Month $=$ December; Day of Week = Thursday EMISFACT 224U_1 MHRDOW7 000000000001 EMISFACT 224U_1 MHRDOW7 000000000000 * Month = January; Day of Week = Friday EMISFACT 2240_1 MHRDOW7 0000000000

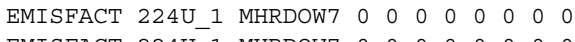
EMISFACT 224U_1 MHRDOW7 $00 \begin{array}{lllllll}-1 & 0 & 0 & 0 & 0 & 0 & 0\end{array}$

* Month = February; Day of Week = Friday EMISFACT 224U_1 MHRDOW7 000000000

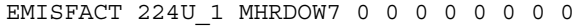
EMISFACT 224U_1 MHRDOW7 $00 \begin{array}{llllllllll}0 & 0 & 0 & 0 & 0 & 0 & 0\end{array}$

$* *$ Month $=$ March; Day of Week = Friday

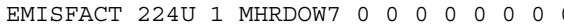


EMISFACT 224U_1 MHRDOW7 000000000000

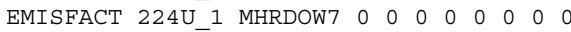

** Month $=$ April; Day of Week = Friday

EMISFACT 224U 1 MHRDOW7 $00 \begin{array}{lllllll}0 & 0 & 0 & 0 & 0 & 0 & 0\end{array}$ EMISFACT 224U 1 MHRDOW7 000000000000

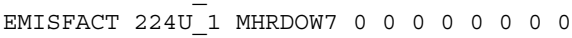

** Month = May; Day of Week = Friday

EMISFACT 224U_1 MHRDOW7 0000000000 EMISFACT 224U_1 MHRDOW7 000000000000 EMISFACT 224U_1 MHRDOW7 00000000000

** Month $=$ June; Day of week = Friday

EMISFACT 224U_1 MHRDOW7 000000000

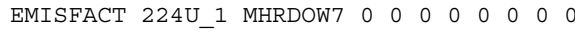
EMISFACT 224U 1 MHRDOW7 $00 \begin{array}{lllllllllllll}0 & 0 & 0 & 0 & 0 & 0 & 0\end{array}$

** Month $=$ July; $^{-}$Day of week $=$Friday

EMISFACT 224U_1 MHRDOW7 00000000000

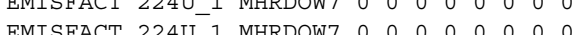

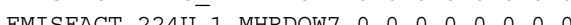

EMISFACT 224_-1 MHRDOW7 $0 \begin{array}{llllllll}0 & 0 & 0 & 0 & 0 & 0 & 0\end{array}$

* Month = August; Day of Week = Friday EMISFACT 2240_1 MHRDOW7 00000000000

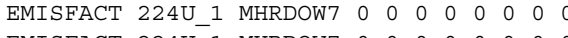
EMISFACT 224U_1 MHRDOW7 $000 \begin{array}{lllllll}-1 & 0 & 0 & 0 & 0 & 0\end{array}$

* Month $=$ September; Day of Week = Frida EMISFACT 224U_1 MHRDOW7 00000000000 EMISFACT 224U_1 MHRDOW7 $00 \begin{array}{lllllll}0 & 0 & 0 & 0 & 0 & 0 & 0\end{array}$ EMISFACT 224U 1 MHRDOW7 $00 \begin{array}{llllllllll}1 & 0 & 0 & 0 & 0 & 0 & 0\end{array}$

* Month = October; Day of Week = Friday

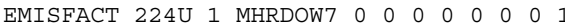
EMISFACT 224U_ EMTSFACT 224U_ MHRDOW7 000000

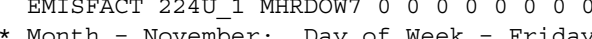
* Month $=$ November $;$ Day of Week = Friday

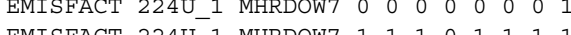
EMISFACT 224U_1 MHRDOW7 $1 \begin{array}{llllllll}1 & 1 & 0 & 1 & 1 & 1 & 1\end{array}$ EMISFACT 224U_1 MHRDOW7 000000000

* Month = December; Day of Week = Friday EMISFACT 224U_1 MHRDOW7 00000000001

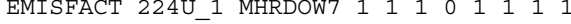

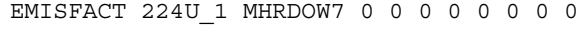

* Month = January; Day of Week = Saturday EMISFACT 224U 1 MHRDOW7 00000000000

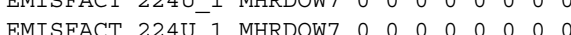

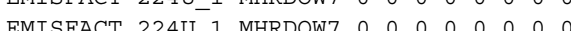

* Month Month = February; Day of week = Saturday

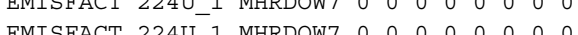
EMISFACT 224_- MHRDOW7 000000000000

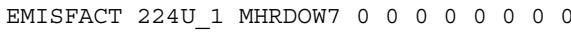

* Month $=$ March; Day of Week = Saturday EMISFACT 224U_1 MHRDOW7 00000000000 EMISFACT 224U_1 MHRDOW7 $0 \begin{array}{lllllll}0 & 0 & 0 & 0 & 0 & 0 & 0\end{array}$

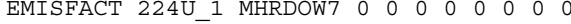

** Month $=$ April; Day of Week = Saturday EMISFACT 224U 1 MHRDOW7 $00 \begin{array}{lllllll}0 & 0 & 0 & 0 & 0 & 0 & 0\end{array}$ EMISFACT 224U 1 MHRDOW7 00 
EMISFACT 224U_1 MHRDOW7 000000000

** Month = May; Day of Week = Saturday

EMISFACT 224U 1 MHRDOW7 000000000000 EMISFACT 224U-1 MHRDOW7 0000000000

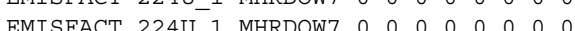
* Month = June,- Day of Week = Saturday

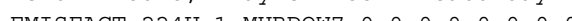
EMISFACT 224U MHRDOW7 000000000 EMISFACT 224U_1 MHRDOW7 $00 \begin{array}{llllllll}0 & 0 & 0 & 0 & 0 & 0 & 0\end{array}$

* Month $=$ July; Day of Week = Saturday EMISFACT 224U_1 MHRDOW7 000000000 EMISFACT 224U_1 MHRDOW7 $00 \begin{array}{lllllll}0 & 0 & 0 & 0 & 0 & 0 & 0\end{array}$ EMISFACT 224U_1 MHRDOW7 00000000000

** Month $=$ August; Day of Week = Saturday EMISFACT 224U 1 MHRDOW7 000000000000 FMISFACT 224U 1 MHRDOW7 000000000 EMTSFACT 224U

* Month = September; Day of Week = Saturday EMISFACT 224U_1 MHRDOW7 0000000000000 EMISFACT 2240-1 MHRD

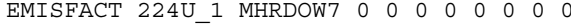

* Month = October; Day of Week = Saturday EMISFACT 224U_1 MHRDOW7 00000000000

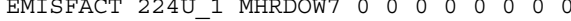

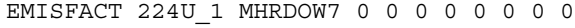

** Month $=$ November; Day of Week = Saturday

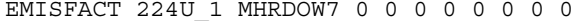

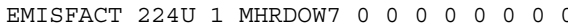
EMISEACT $224 U$

* Month = Dece- MHer Day of Week = Saturday Month = December; $;$ Day of Week = Saturday

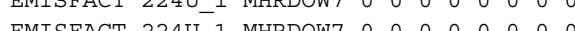

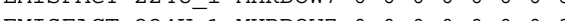
EMISFACT 2240_1 MHRDOW7 0000000000

** Month = January; Day of Week = Sunday EMISFACT 224U 1 MHRDOW7 0 O $00 \begin{array}{lllll}0 & 0 & 0 & 0 & 0\end{array}$ EMISFACT 224U_1 MHRDOW7 000100000000 EMISFACT 224U_1 MHRDOW7 $00 \begin{array}{lllllll}0 & 0 & 0 & 0 & 0 & 0 & 0\end{array}$

* Month $=$ February; Day of Week $=$ Sunday

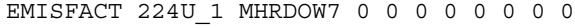

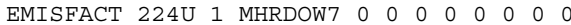

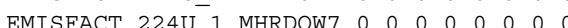

* Month $=$ March; Day of Week = Sunday

$\begin{array}{llllllllll}\text { MMISFACT } 224 \mathrm{U} & 1 \text { MHRDOW7 } 0 & 0 & 0 & 0 & 0 & 0 & 0 & 0\end{array}$

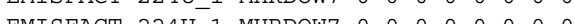

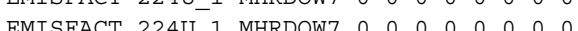
EMISFACT 2240_1 MURDOW7 000000000000 * Month $=$ April; Day of Week = Sunday EMISFACT 224U_1 MHRDOW7 00000000000 EMISFACT 224U_1 MHRDOW7 $0 \begin{array}{lllllll}0 & 0 & 0 & 0 & 0 & 0 & 0\end{array}$

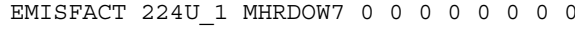

** Month $=$ May; Day of Week = Sunday EMISFACT 224U_1 MHRDOW7 0000000000 
** Month $=$ June; Day of Week $=$ Sunday EMISFACT 224U_1 MHRDOW7 00000000000 EMISFACT 224U_1 MHRDOW7 $000 \begin{array}{lllllll}1 & 0 & 0 & 0 & 0 & 0 & 0\end{array}$ * Month $=$ July; Day of Week = Sunday

* Month = July; Day of week = Sunday EMISFACT 2240_1 MRRDOW7 0 c $\begin{array}{cccccccc}0 & 0 & 0 & 0 & 0 & 0\end{array}$ EMISFACT 2240.1 MHRDOW7 $\begin{array}{llllllllllllllll}0 & 0 & 0 & 0 & 0 & 0 & 0\end{array}$ EMISFACT 2240_1 MHRDOW7 $\begin{array}{lllllllll}0 & 0 & 0 & 0 & 0 & 0 & 0 & 0\end{array}$

** Month $=$ August $; \quad$ Day of week = Sunday EMISFACT 224U_1 MHRDOW7 00000000000 EMISFACT 224U_1 MHRDOW7 00000000000

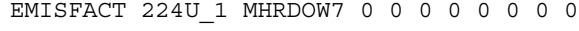

** Month $=$ September; Day of Week $=$ Sunday EMISFACT 224U 1 MHRDOW7 $000 \begin{array}{llllll}0 & 0 & 0 & 0 & 0 & 0\end{array}$

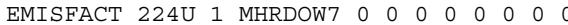
EMISFACT 224U 1 MHRDOW7 0.000000

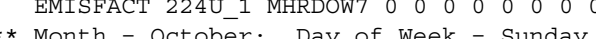
Month = October; Day of week = Sunday

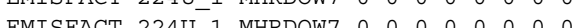

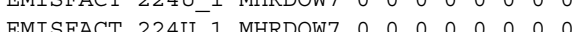

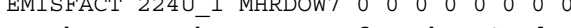
* Month $=$ November $; \quad$ Day of Week = Sunday EMISFACT 224U_1 MHRDOW7 00000000000 EMISFACT 224U_1 MHRDOW7 $00 \begin{array}{lllllll}0 & 0 & 0 & 0 & 0 & 0 & 0\end{array}$ EMISFACT 224U_1 MHRDOW7 00000000000

** Month $=$ December; Day of Week = Sunday EMISFACT 224U_1 MHRDOW7 000000000000 EMISFACT 224U 1 MHRDOW7 $00 \begin{array}{lllllll}0 & 0 & 0 & 0 & 0 & 0 & 0\end{array}$ EMISFACT 224U 1 MHRDOW7 0 O 000

* Mont * Month = January; Day or Week = Monday

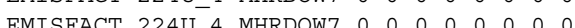

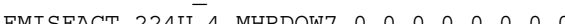
EMISFACT 224_-4 MHRDOW7 000000000000

* Month = February; Day of Week = Monday EMISFACT 224U_4 MHRDOW7 0000000000 EMISFACT 224U_4 MHRDOW7 00000000000 EMISFACT 224U_4 MHRDOW7 00000000000

** Month $=$ March; Day of Week = Monday EMISFACT 224U_4 MHRDOW7 0000000000 EMISFACT 224U_4 MHRDOW7 $00 \begin{array}{lllllll}0 & 0 & 0 & 0 & 0 & 0 & 0\end{array}$ EMISFACT 224U 4 MHRDOW7 0 C

** Month $=$ April; Day of Week = Monday EMISFACT 224U 4 MHRDOW7 00000000 EMISFACT 224U 4 MHRDOW7 $0 \begin{array}{lllllllll}0 & 0 & 0 & 0 & 0 & 0 & 0\end{array}$ EMISFACT 224U 4 MHRDOW7 000000000000

* Month = May; Day of Week = Monday

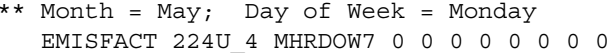

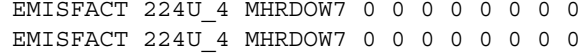
EMISFACT 224U_4 MHRDOW7 $000 \begin{array}{lllllll}0 & 0 & 0 & 0 & 0 & 0 & 0\end{array}$

$* *$ Month $=$ June; Day of Week $=$ Monday

EMISFACT 224U_4 MHRDOW7 000000000 EMISFACT 224U_4 MHRDOW7 $000 \begin{array}{llllll}0 & 0 & 0 & 0 & 0 & 0\end{array}$ EMISFACT 224U 4 MHRDOW7 $00 \begin{array}{lllllll}0 & 0 & 0 & 0 & 0 & 0 & 0\end{array}$

$* *$ Month $=$ July; ${ }^{-}$Day of Week $=$Monday 
EMISFACT 224U_4 MHRDOW7 0000000000

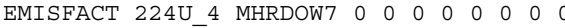
EMISFACT $224 \mathrm{U}$

** Month = August; Day of Week = Monday EMTSEAT 224U 4 May or Week = Monday

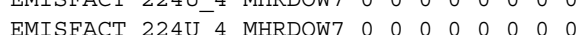

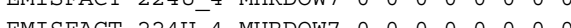

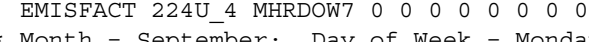

* Month $=$ September; Day of Week = Monday EMISFACT 224U_4 MHRDOW7 00000000000 EMISFACT 224U_4 MHRDOW7 $000 \begin{array}{llllll}0 & 0 & 0 & 0 & 0 & 0\end{array}$ EMISFACT 224U_4 MHRDOW7 00000000000

** Month $=$ October; Day of Week = Monday

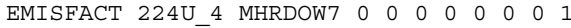
EMISFACT 224U 4 MHRDOW7 $1 \begin{array}{llllllll}1 & 1 & 1 & 0 & 1 & 1 & 1 & 1\end{array}$ EMISFACT 224U 4 MHRDOW7 0 - 0 - 00

** Month = November; Day of Week = Monday EMISFACT $224 \mathrm{U} 4$ MHRDOW7 00000

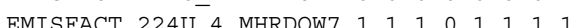

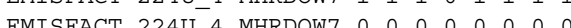

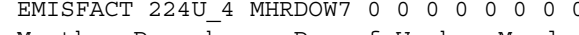

* Month $=$ December; Day of Week = Monday

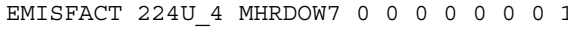

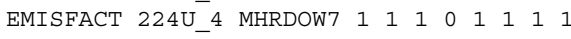
EMISFACT 224U_4 MHRDOW7 0000000000

* Month = January; Day of Week = Tuesday EMISFACT 224U_4 MHRDOW7 00000000000 EMISFACT 224U_4 MHRDOW7 $00 \begin{array}{lllllll}0 & 0 & 0 & 0 & 0 & 0 & 0\end{array}$

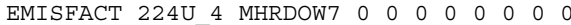

** Month $=\mathrm{F}$ EMISFACT 224U 4 MHRDOW7 0000000000

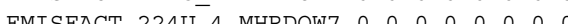

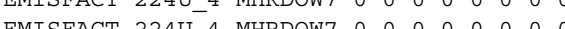

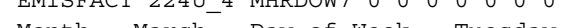
* Month = March; Day of Week = Tuesday EMISFACT 224U_4 MHRDOW7 000000000000

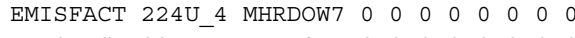
EMISFACT 224U_4 MHRDOW7 0000000000

** Month $=$ April $;$ Day of Week = Tuesday

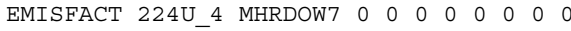

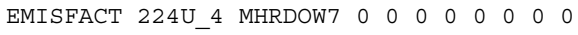
EMISFACT 224U_4 MHRDOW7 000000000000

** Month $=$ May; Day of Week $=$ Tuesday EMISFACT 224U_4 MHRDOW7 00000000000

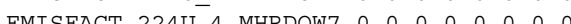

* Month = June; Day of Week = Tuesday

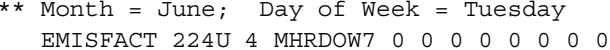

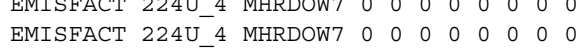

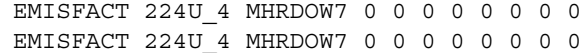

* Month $=$ July; Day of Week = Tuesday

EMISFACT 224U_4 MHRDOW7 000000000

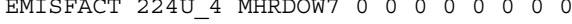
EMISFACT 224U 4 MHRDOW7 $00 \begin{array}{lllllll}0 & 0 & 0 & 0 & 0 & 0 & 0\end{array}$

** Month $=$ August; Day of Week = Tuesday

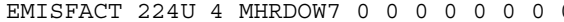


EMISFACT 224U_4 MHRDOW7 00000000000 EMISFACT 224U_4 MHRDOW7 000000000000

** Month = September; Day of Week = Tuesday EMTSFACT 224U 4 MHRDOW7 0

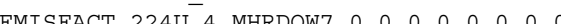

** Month $=0$. EMISFACT

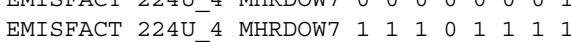
EMISFACT 224U_4 MHRDOW7 000000000000

$* *$ Month $=$ November; Day of week $=$ Tuesday

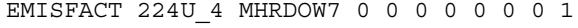

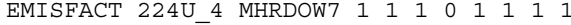
EMISFACT 224U 4 MHRDOW7 $00 \begin{array}{llllllll}0 & 0 & 0 & 0 & 0 & 0 & 0\end{array}$

** Month $=$ December; Day of week = Tuesday EMISFACT 224U_4 MHRDOW7 000000000 EMISFACT 224- 4 MHRDOW7 111

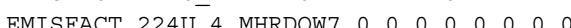

EMISFACT 2240_4 MAO * Month = January; Day of Week = Wednesday EMISFACT 224U_4 MHRDOW7 000100000000

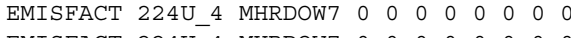

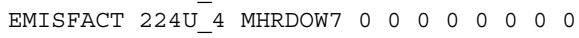

** Month = February; Day of Week = Wednesday

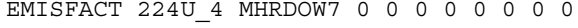
EMISFACT 224U_4 MHRDOW7 $000 \begin{array}{lllllllll}0 & 0 & 0 & 0 & 0 & 0 & 0\end{array}$ EMISFACT 224U 4 MHRDOW7 $00 \begin{array}{lllllll}0 & 0 & 0 & 0 & 0 & 0 & 0\end{array}$

** Month $=$ March; Day of Week = Wednesday

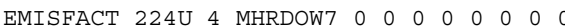

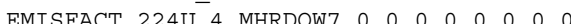
EMTSFACT 224U-4 MHRDOW7 0000000000

EMISFACT 2240_4 MHRDOW7 $00 \begin{array}{ccccccc}0 & 0 & 0 & 0 & 0 & 0 & 0\end{array}$ * Month = April; Day of Week = Wednesday EMISFACT 2240_4 MHRDOW7 0 O 000000000 EMISFACT 224U_4 MHRDOW7 000000000000

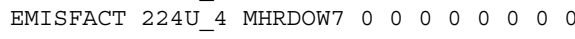

* Month = May; Day of Week = Wednesday EMISFACT 224U_4 MHRDOW7 0 C $00 \begin{array}{llllllll} & 0 & 0 & 0 & 0 & 0\end{array}$

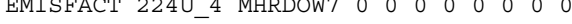
EMISFACT 224U 4 MHRDOW7 $00 \begin{array}{lllllllll}0 & 0 & 0 & 0 & 0 & 0 & 0\end{array}$

* Month $=$ June; Day of Week $=$ Wednesday EMISFACT 224U 4 MHRDOW7 0000000000

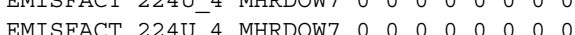
EMISFACT 224U_

EMISEACT 2240_4 MHRDOW7 $0 \begin{array}{lllllllll}0 & 0 & 0 & 0 & 0 & 0 & 0 & 0\end{array}$ ** Month = July; $;$ Day of Week = Wednesday

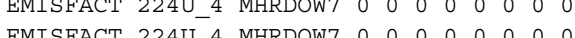
EMISFACT 224_- MHRDOW7 00000000000

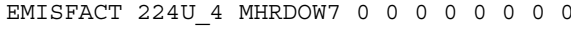

** Month = August; Day of Week $=$ Wednesday EMISFACT 224U_4 MHRDOW7 00000000000 EMISFACT 224U_4 MHRDOW7 0000000000000

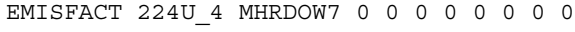

** Month = September; Day of Week = Wednesday EMISFACT 224U_4 MHRDOW7 00000000000 EMISFACT 224U 4 MHRDOW7 00100000 
EMISFACT 224U_4 MHRDOW7 0000000000

** Month $=$ October; Day of Week $=$ Wednesday EMISFACT 224U_4 MHRDOW7 0000000000001

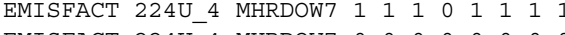

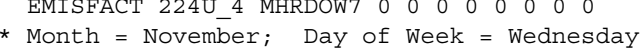
EMISFACT 224U_4 MHRDOW7 0000000001

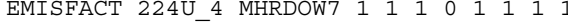
EMISFACT 224U_4 MHRDOW7 0000000000

* Month $=$ December; Day of Week = Wednesday EMISFACT 224U_4 MHRDOW7 0000000001

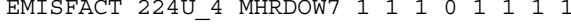

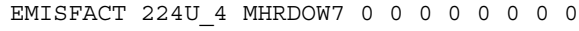

* Month $=$ January; Day of Week = Thursday EMISFACT 224U 4 MHRDOW7 $00 \begin{array}{lllllll}0 & 0 & 0 & 0 & 0 & 0 & 0\end{array}$

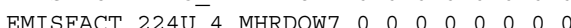

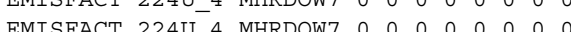

* Month = February. Day of Week = Thura

* Month = February; Day of Week = Thursday EMISFACT 224U_4 MHRDOW7 0000000000

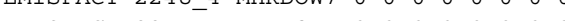

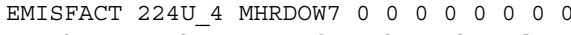

* Month $=$ March; Day of Week = Thursday EMISFACT 224U_4 MHRDOW7 000000000

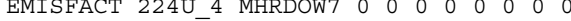

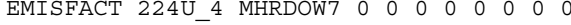

* Month $=$ April; Day of Week = Thursday EMISFACT 224U 4 MHRDOW7 0 O $00 \begin{array}{lllll}0 & 0 & 0 & 0 & 0\end{array}$

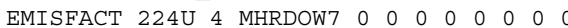
EMTSFACT 224U 4 MHRDOW7 00000000

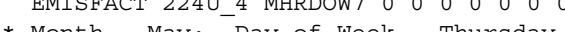
Month = May; Day of Week = Thursday

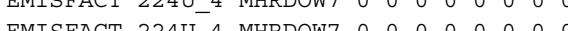

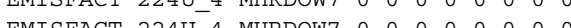

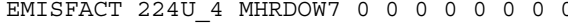

* Month $=$ June; Day of Week = Thursday EMISFACT 224U_4 MHRDOW7 000000000

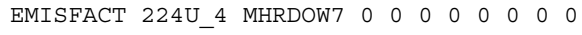
EMISFACT 224U_4 MHRDOW7 000000000000

** Month $=$ July; Day of Week = Thursday EMISFACT 224U 4 MHRDOW7 000000000

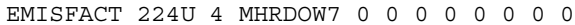

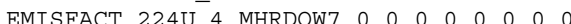

* Month Month

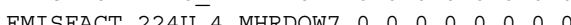

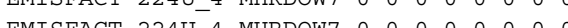

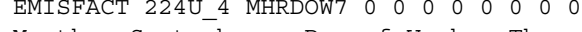

* Month = September; Day of Week = Thursday EMISFACT 224U_4 MHRDOW7 0000000000

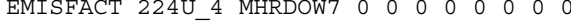

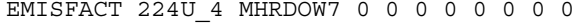

** Month $=$ October; Day of Week $=$ Thursday EMISFACT 224U 4 MHRDOW7 $00 \begin{array}{lllllll}0 & 0 & 0 & 0 & 0 & 0 & 1\end{array}$ EMISFACT 224U 4 MHRDOW7 $1 \begin{array}{lllllllllll}1 & 1 & 0 & 1 & 1 & 1 & 1\end{array}$ EMISFACT 224U 4 MHRDOW7 001000 
** Month $=$ November $;$ Day of Week = Thursday EMISFACT 224U_4 MHRDOW7 0000000001

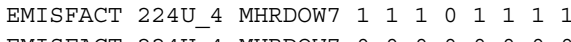

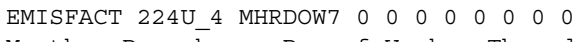

** Month = December; Day of Week = Thursday

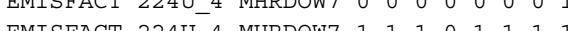
EMISFACT 224_-4 MHRDOW7 $\begin{array}{llllllll}1 & 1 & 1 & 0 & 1 & 1 & 1 & 1\end{array}$

** Month = January; Day of Week = Friday EMISFACT 224U_4 MHRDOW7 0 O $00 \begin{array}{lllll}0 & 0 & 0 & 0 & 0\end{array}$

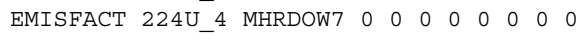
EMISFACT 224U_4 MHRDOW7 0010000000000

** Month $=$ February; Day of week = Friday EMISFACT 224U 4 MHRDOW7 00000000000

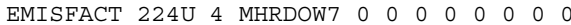

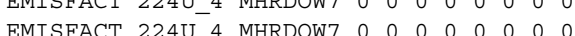

** Month = March; Day of Week = Friday

$\begin{array}{lllllllll}\text { Month }=\text { March; Day of Week } & \text { Friday } \\ \text { EMISFACT 224U 4 MHRDOW7 } 000 & 0 & 0 & 0 & 0 & 0 & 0\end{array}$ EMISFACT 224U 4 MHRDOW7 00000000000 EMISFACT 224U 4 MHRDOW7 $000 \begin{array}{llllllll}- & 0 & 0 & 0 & 0 & 0 & 0 & 0\end{array}$

* Month = April; Day of Week = Friday

EMISFACT 224U_4 MHRDOW7 0000000000 EMISFACT 224U_4 MHRDOW7 00000000000 EMISFACT 224U_4 MHRDOW7 $00 \begin{array}{lllllll}0 & 0 & 0 & 0 & 0 & 0 & 0\end{array}$

** Month $=$ May; $;$ Day of Week = Friday

EMISFACT 224U 4 MHRDOW7 $000 \begin{array}{lllllll}0 & 0 & 0 & 0 & 0 & 0\end{array}$

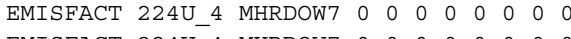

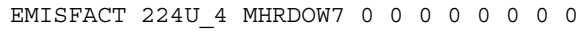
** Month $=$ June; $;$ Day of Week = Friday EMISFACT 224U_4 MHRDOW7 00000000000 EMISFACT 224U_4 MHRDOW7 000000000000 EMISFACT 2240_4 MHRDOW7 0 a 000 o 00 o * Month $=$ July; Day of week $=$ Friday EMISFACT 224U_4 MHRDOW7 000000000 EMISFACT 224U_4 MHRDOW7 00000000000 EMISFACT 224U_4 MHRDOW7 00000000000

** Month $=$ August $;$ Day of Week = Friday EMISFACT 224U_4 MHRDOW7 0000000000 EMISFACT 224U_4 MHRDOW7 $0 \begin{array}{lllllll}0 & 0 & 0 & 0 & 0 & 0 & 0\end{array}$

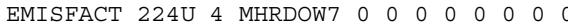

** Month = September; Day of Week = Friday EMISFACT 224U 4 MHRDOW7 000000000

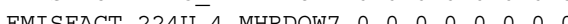
BMISFAC 224MonshC 2240_4 MHROW7 00000000000

* Month = October; Day of Week = Friday EMISFACT 224U_4 MHRDOW7 00000000001

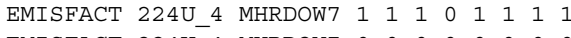
EMISFACT 224U_4 MHRDOW7 00000000000

* Month $=$ November; Day of Week = Friday EMISFACT 224U_4 MHRDOW7 00000000001

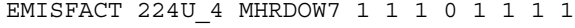
EMISFACT 224U 4 MHRDOW7 000 C

* Month $=$ December; Day of Week $=$ Friday 


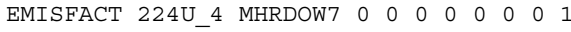

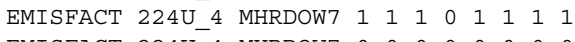
EMISFACT 224U_4 MHRDOW7 $00 \begin{array}{lllllll}0 & 0 & 0 & 0 & 0 & 0 & 0\end{array}$

** Month = January; Day of Week = Saturday EMISFACT 224U_4 MHRDOW7 000 O

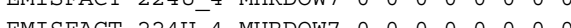

EMISFACT 2240_4 MHRDOW7 $00 \begin{array}{llllllll}0 & 0 & 0 & 0 & 0 & 0 & 0\end{array}$

* Month = February; Day of Week = Saturday EMISFACT 224U_4 MHRDOW7 0000000000 EMISFACT 224U_4 MHRDOW7 000000000000 EMISFACT 224U_4 MHRDOW7 00000000000

** Month $=$ March; Day of Week = Saturday EMISFACT 224U_4 MHRDOW7 0 O $00 \begin{array}{lllll}0 & 0 & 0 & 0 & 0\end{array}$

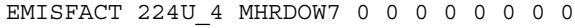
EMISFACT 224U 4 MHRDOW7 0 O 0 000

** Month = April; Day of Week = Saturday TMISFACT $224 U$ U MHRDOW7 0 o 0 o 0000

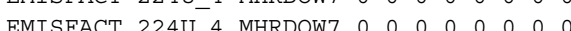

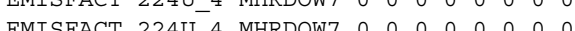

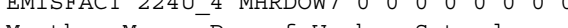
* Month = May; Day of Week = Saturday EMISFACT 224U_4 MHRDOW7 000000000 EMISFACT 224U_4 MHRDOW7 000000000000 EMISFACT 224U_4 MHRDOW7 00000000000

** Month $=$ June; Day of Week = Saturday EMISFACT 224U_4 MHRDOW7 000000000

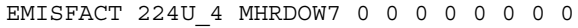

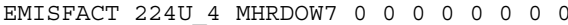
in $\quad *$ Month $=$ July; Day of Week = Saturday

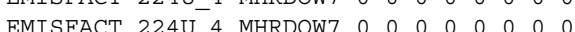

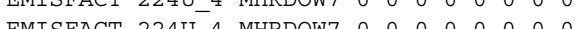
EMISFACT 224__4 MHRDOW7 $0 \begin{array}{lllllllllllll}0 & 0 & 0 & 0 & 0 & 0 & 0\end{array}$

** Month = August; Day of Week = Saturday EMISFACT 224U_4 MHRDOW7 0000000000 EMISFACT 224U_4 MHRDOW7 $00 \begin{array}{cccccccc}0 & 0 & 0 & 0 & 0 & 0 & 0\end{array}$ EMISFACT 224U_4 MHRDOW7 00000000000

** Month $=$ September; Day of Week = Saturday EMISFACT 224U_4 MHRDOW7 $000 \begin{array}{llllll}0 & 0 & 0 & 0 & 0 & 0\end{array}$ EMISFACT 224U_4 MHRDOW7 0000000000000 EMISFACT 224U_4 MHRDOW7 000000000000

** Month $=$ October $; \quad$ Day of Week = Saturday EMISFACT 224U_4 MHRDOW7 00000000000

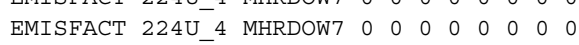
EMISFACT 224U 4 MHRDOW7 00000000000

** Month = November; Day of Week = Saturday EMISFACT 224U_4 MHRDOW7 000000000000

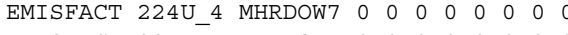
EMISFACT 224U_4 MHRDOW7 00000000000

* Month $=$ December; Day of Week = Saturday EMISFACT 224U_4 MHRDOW7 00000000000 EMISFACT 224U_4 MHRDOW7 $00 \begin{array}{lllllll}0 & 0 & 0 & 0 & 0 & 0 & 0\end{array}$ EMISFACT 224U 4 MHRDOW7 $00 \begin{array}{lllllll}0 & 0 & 0 & 0 & 0 & 0 & 0\end{array}$

** Month = January; Day of Week = Sunday EMISFACT 224U 4 MHRDOW7 0 0 0 - 0 
EMISFACT 224U_4 MHRDOW7 00000000000 EMISFACT 224U_4 MHRDOW7 $00 \begin{array}{lllllll}0 & 0 & 0 & 0 & 0 & 0 & 0\end{array}$

** Month = February; Day of Week = Sunday

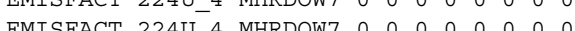

EMISFACT 2240_4 MHRDOW7 $0 \begin{array}{llllllll}0 & 0 & 0 & 0 & 0 & 0 & 0\end{array}$

* Month $=$ March; Day of Week = Sunday

EMISFACT 2240 4 MarDOW7 00000 EMISFACT 224U_4 MHRDOW7 $0 \begin{array}{llllllllll}0 & 0 & 0 & 0 & 0 & 0 & 0\end{array}$ EMISFACT 224U_4 MHRDOW7 00000000000

$*$ Month $=$ April; Day of Week = Sunday

EMISFACT 224U_4 MHRDOW7 00000000000 EMISFACT 224U_4 MHRDOW7 $000 \begin{array}{llllll}0 & 0 & 0 & 0 & 0 & 0\end{array}$ EMISFACT 224U 4 MHRDOW7 $00 \begin{array}{lllllllllll}0 & 0 & 0 & 0 & 0 & 0 & 0\end{array}$

** Month $=$ May; Day of Week $=$ Sunday

EMISFACT 224U 4 MHRDOW7 000000000 EMISFACT 224-4 MRDW7 $0 \begin{array}{llllllll}0 & 0 & 0 & 0 & 0 & 0 & 0\end{array}$ EMTSFACT 224U- MHRDOW7 000000000

* Month = June; Day of Week = Sunday

Month = June; Day of Week = Sunday
EMISFACT 224U 4 MHRDOW7 000000000

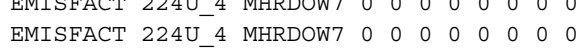

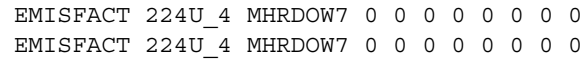

* Month $=$ July; Day of Week = Sunday

EMISFACT 224U_4 MHRDOW7 000000000 EMISFACT 224U_4 MHRDOW7 $0 \begin{array}{lllllll}0 & 0 & 0 & 0 & 0 & 0 & 0\end{array}$

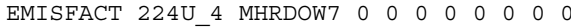

** Month = August; Day of Week $=$ Sunday FMISFACT 224U_ 4 MHRDOW7 0000000000

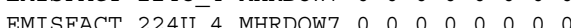

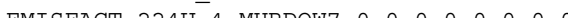

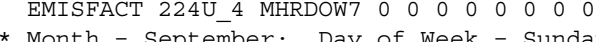
* Month = September; Day of Week = Sunda EMISFACT 2240_4 MHRDOW7 0 o $00 \begin{array}{ccccccc}0 & 0 & 0 & 0 & 0\end{array}$ EMISFACT 224__4 MHRDOW7 00000000000 EMISFACT 224U_4 MHRDOW7 00000000000

* Month = October; Day of Week = Sunday EMISFACT 224U_4 MHRDOW7 000000000

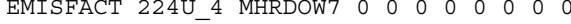

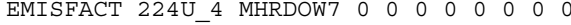

** Month $=$ November; Day of Week = Sunday EMISFACT 224U 4 MHRDOW7 0 O $00 \begin{array}{lllll}0 & 0 & 0 & 0 & 0\end{array}$

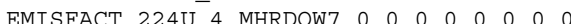

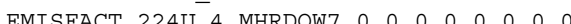

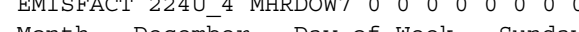

* Month = December; Day of Week = Sunday EMISFACT 2240_4 MHRDOW7 0000000000

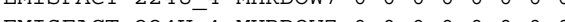
EMISFACT 224U_4 MHRDOW7 $000 \begin{array}{llllll}0 & 0 & 0 & 0 & 0 & 0\end{array}$

* Month $=$ January; Day of Week = Monday EMISFACT 224U_5 MHRDOW7 00000000000 EMISFACT 224U_5 MHRDOW7 $00 \begin{array}{lllllll}0 & 0 & 0 & 0 & 0 & 0 & 0\end{array}$

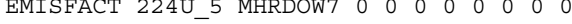

$*$ Month $=$ February; Day of Week $=$ Monday EMISFACT 224U 5 MHRDOW7 $000 \begin{array}{lllllll}0 & 0 & 0 & 0 & 0 & 0\end{array}$ EMISFACT 224U 5 MHRDOW7 0 - 000 
EMISFACT 224U_5 MHRDOW7 0000000000

** Month $=$ March; Day of Week = Monday

EMISFACT 224U_5 MHRDOW7 00000000000 BMISEACT 2240_5 MHRI EMISFACT 224_5 MHRDOW7 $00 \begin{array}{llllllll}0 & 0 & 0 & 0 & 0 & 0 & 0\end{array}$ * Month = April; Day of Week = Monday

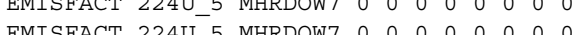
EMISFACT 2240_5 MHRDOW7 0000000000000 EMISFACT 224U_5 MHRDOW7 001000000000

* Month = May; Day of Week = Monday

EMISFACT 224U_5 MHRDOW7 00000000000

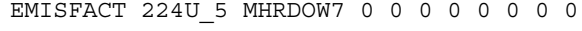
EMISFACT 224U_5 MHRDOW7 $00 \begin{array}{lllllll}0 & 0 & 0 & 0 & 0 & 0 & 0\end{array}$

** Month $=$ June; Day of Week = Monday

EMISFACT 224U_5 MHRDOW7 00000000000 EMISFACI 224__5 MHRDOW7 $0 \begin{array}{llllllllll}0 & 0 & 0 & 0 & 0 & 0 & 0 & 0\end{array}$

* Month $=$ July; Day of Week = Monday

* * Month = July; Day of week = Monday

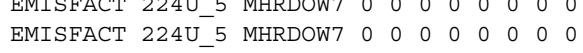
EMISFACT 224U_5 MHRDOW7 000000000

** Month $=$ August $; \quad$ Day of Week = Monday EMISFACT 224U_5 MHRDOW7 00000000000

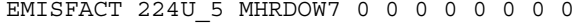

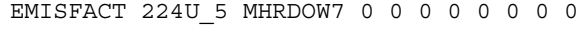

** Month $=$ September $;$ Day of Week $=$ Monday EMISFACT 224U 5 MHRDOW7 00000000000 EMISFACT 224U 5 MHRDOW7 0000000000 EMISFACT 224U

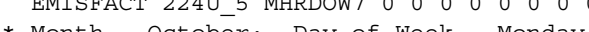
* Month = October; Day of Week = Monday

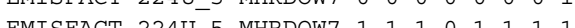
EMISFACT 2240_5 MHRDW7 $\begin{array}{llllllll}1 & 1 & 1 & 0 & 1 & 1 & 1 & 1\end{array}$ EMISFACT 2240_5 MHRDOW7 $0 \begin{array}{llllllll}1 & 1 & 0 & 0 & 1 & 1 & 1 \\ 0 & 0 & 0 & 0 & 0 & 0\end{array}$

** Month $=$ November; Day of Week = Monday EMISFACT 224U_5 MHRDOW7 0000000001 EMISFACT 224U_5 MHRDOW7 $1 \begin{array}{llllllll}1 & 1 & 0 & 1 & 1 & 1 & 1\end{array}$ EMISFACT 224U_5 MHRDOW7 00100000000

** Month $=$ December; Day of Week = Monday EMISFACT 224U_5 MHRDOW7 00000000001 EMISFACT 224U 5 MHRDOW7 $1 \begin{array}{llllllllll}1 & 1 & 1 & 0 & 1 & 1 & 1 & 1\end{array}$ EMISFACT 224U 5 MHRDOW7 0 O 00000

* Month = January Day of Month - January, Day or Week = Tuesday

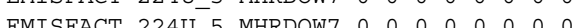
EMISFACT 2240 EMISFACT 2240_5 MHRDOW7 0 O 000000000 * Month = February; Day of week = Tuesda EMISFACT 224U_5 MHRDOW7 00000000000 EMISFACT 224U_5 MHRDOW7 0000000000000

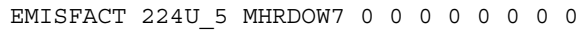

** Month $=$ March; Day of Week = Tuesday EMISFACT 224U 5 MHRDOW7 $00 \begin{array}{lllllll}0 & 0 & 0 & 0 & 0 & 0 & 0\end{array}$ EMISFACT 224U 5 MHRDOW7 $00 \begin{array}{lllllll}0 & 0 & 0 & 0 & 0 & 0 & 0\end{array}$ EMISFACT 224U 5 MHRDOW7 
** Month $=$ April; Day of Week = Tuesday EMISFACT 224U_5 MHRDOW7 00000000000 EMISFACT 224U_5 MHR

* Month = May; Day of Week = Tuesday

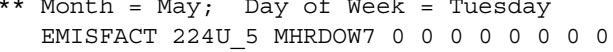
EMISFACT 224U_5 MHRDOW7 000000000000 EMISFACT 224U 5 MHRDOW7 $00 \begin{array}{lllllll}0 & 0 & 0 & 0 & 0 & 0 & 0\end{array}$

** Month $=$ June; $;$ Day of Week = Tuesday

EMISFACT 224U_5 MHRDOW7 000000000 EMISFACT 224U_5 MHRDOW7 00000000000 EMISFACT 224U_5 MHRDOW7 001000000000

** Month $=$ July; Day of week = Tuesday EMISFACT 224U_5 MHRDOW7 00000000000 EMISFACT 224U_5 MHRDOW7 0 C $0 \begin{array}{lllllllllll}0 & 0 & 0 & 0 & 0 & 0\end{array}$

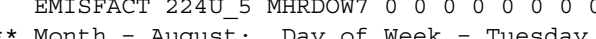

* Month = August; $;$ Day of Week = Tuesday

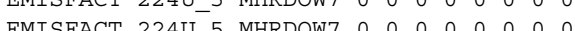

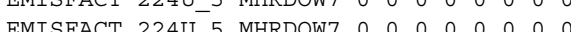

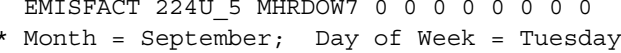
* Month = September; Day of Week $=$ Tuesday
EMISFACT 224U_5 MHRDOW7 0000000000 EMISFACT 224U_5 MHRDOW7 $000 \begin{array}{llllll}0 & 0 & 0 & 0 & 0 & 0\end{array}$ EMISFACT 224U_5 MHRDOW7 0000000000

** Month $=$ October; Day of Week = Tuesday

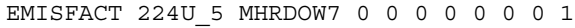

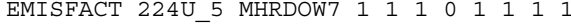
EMISFACT 224U 5 MHRDOW7 0

** Month = November; Day of Week = Tuesday EMISFACT 224U 5 MHRDOW7 00000000001

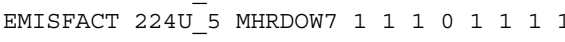

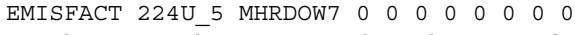

** Month = December; Day of Week = Tuesday EMISFACT 224U_5 MHRDOW7 0000000001 EMISFACT 224U_5 MHRDOW7 1111001101 EMISFACT 224U_5 MHRDOW7 0000000000

** Month $=$ January; Day of Week $=$ Wednesday EMISFACT 224U_5 MHRDOW7 000000000000 EMISFACT 224U_5 MHRDOW7 $00 \begin{array}{lllllll}0 & 0 & 0 & 0 & 0 & 0 & 0\end{array}$

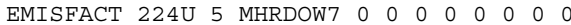

** Month $=$ February; Day of Week = Wednesday EMISFACT 224U_5 MHRDOW7 0 DO 0000000

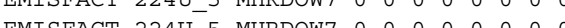

EMISFAC 2240_5 MHRDOW7 $00 \begin{array}{llllllll}0 & 0 & 0 & 0 & 0 & 0 & 0 & 0\end{array}$

** Month $=$ March; Day of Week $=$ Wednesday EMISFACT 224U_5 MHRDOW7 00000000000 EMISFACT 224U_5 MHRDOW7 000000000000 EMISFACT 224U_5 MHRDOW7 0000000000

* Month $=$ April $;$ Day of Week $=$ Wednesday EMISFACT 224U 5 MHRDOW7 $000 \begin{array}{llllll}0 & 0 & 0 & 0 & 0 & 0\end{array}$ EMISFACT 224U_5 MHRDOW7 $000 \begin{array}{cccccc}0 & 0 & 0 & 0 & 0 & 0\end{array}$ EMISFACT 224U 5 MHRDOW7 0 H 0000000

** Month $=$ May; Day of Week $=$ Wednesday 
EMISFACT 224U_5 MHRDOW7 0000000000 EMISFACT 224U 5 MHRDOW7 $00 \begin{array}{lllllll}0 & 0 & 0 & 0 & 0 & 0 & 0\end{array}$ EMISFACT 224U 5 MHRDOW7 0 O 00000000

** Month = June, EMTSEAT $2240-5$ MHRDow7 00000000

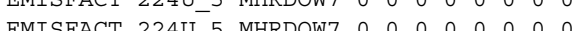
MMISFCT 224U EMISFACT 2240_5 MHRDOW7 $0 \begin{array}{llllllllllllll}0 & 0 & 0 & 0 & 0 & 0 & 0\end{array}$

** Month $=$ July; Day of Week = Wednesday EMISFACT 224U_5 MHRDOW7 0000000000 EMISFACT 224U_5 MHRDOW7 $00 \begin{array}{lllllll}0 & 0 & 0 & 0 & 0 & 0 & 0\end{array}$ EMISFACT 224U_5 MHRDOW7 000000000

** Month $=$ August; Day of Week $=$ Wednesday EMISFACT 224U_5 MHRDOW7 00000000000

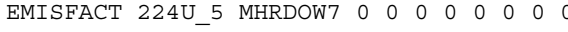
EMISFACT 224U 5 MHRDOW7 0000000000

** Month $=$ September; Day of Week = Wednesday EMISFACT 224U_5 MHRDOW7 0 EMISFACT 2240_5 MHRDOW7 $0 \begin{array}{llllllllllllll}0 & 0 & 0 & 0 & 0 & 0\end{array}$

EMISFACT 2240_5 MHRDOW7 $0 \begin{array}{lllllllll}0 & 0 & 0 & 0 & 0 & 0 & 0\end{array}$

* Month = October; Day of Week = Wednesday EMISFACT 224U_5 MHRDOW7 00000000001 EMISFACT 224U_5 MHRDOW7 $11110 \begin{array}{lllllll}1 & 0 & 1 & 1 & 1 & 1\end{array}$ EMISFACT 224U_5 MHRDOW7 00000000000

** Month $=$ November; Day of Week $=$ Wednesday

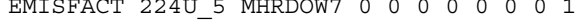
EMISFACT 224U 5 MHRDOW7 $11110 \begin{array}{lllllll}1 & 0 & 1 & 1 & 1 & 1\end{array}$ EMISFACT 224U 5 MHRDOW7 $00 \begin{array}{llllllll}0 & 0 & 0 & 0 & 0 & 0 & 0\end{array}$ it ** Month = December; Day of week = Wednesday

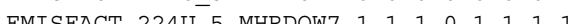

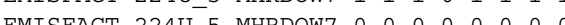

EMISFAC 2240_5 Mrown

* Month = January; Day of Week = Thursda EMISFACT 224U_5 MHRDOW7 000000000000 EMISFACT 224U_5 MHRDOW7 001000 C EMISFACT 224U_5 MHRDOW7 $000 \begin{array}{lllllll}- & 0 & 0 & 0 & 0 & 0 & 0\end{array}$

** Month = February; Day of Week = Thursday

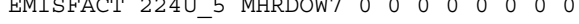
EMISFACT 224U_5 MHRDOW7 0000000000000 EMISFACT 224U 5 MHRDOW7 $00 \begin{array}{llllllllll}0 & 0 & 0 & 0 & 0 & 0 & 0\end{array}$

** Month $=$ March; Day of Week = Thursday EMISFACT 224U_5 MHRDOW7 00000000000 EMISFACT 224U 5 MHRDOW7 000000000000 EMISFACT 224U 5 MHRDOW7 00000000000

* Month = Aprili $;$ Day of Week = Thursday $\begin{array}{llllll}\text { Month = April; } & \text { Day of Week = Thursday } \\ \text { EMISFACT 224U 5 MHRDOW7 } 000 & 0 & 0 & 0 & 0 & 0\end{array}$

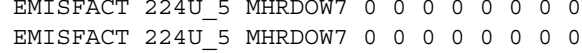

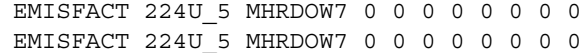

* Month = May; Day of Week = Thursday EMISFACT 224U 5 MHRDOW7 0000000000 EMISFACT 224U_5 MHRDOW7 $000 \begin{array}{llllll}0 & 0 & 0 & 0 & 0 & 0\end{array}$

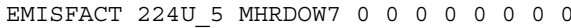

* Month $=$ June; Day of Week $=$ Thursday

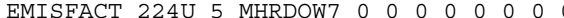


EMISFACT 224U_5 MHRDOW7 00000000000

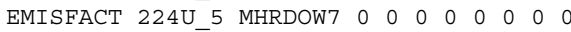

** Month $=$ July; ${ }^{-}$Day of week = Thursday EMISFACT 224U 5 MHRDOW7 0000000000

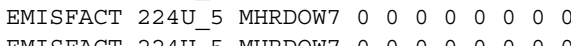

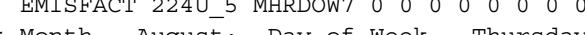
* Month = August; Day of Week = Thursday EMISFACT 224U_5 MHRDOW7 0000000000

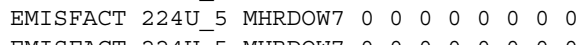
EMISFACT 224U_5 MHRDOW7 000000000

** Month $=$ September; Day of Week = Thursday EMISFACT 224U_5 MHRDOW7 00000000000 EMISFACT 224U_5 MHRDOW7 0000000000000

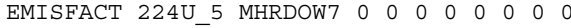

** Month $=$ October; Day of Week $=$ Thursday EMISFACT 224U_5 MHRDOW7 00000000001 $\begin{array}{llllllllll} & & & \end{array}$ C 224U_5 MHRDOW7 00000000000

** Month = November; Day of Week = Thursday

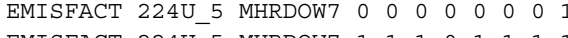

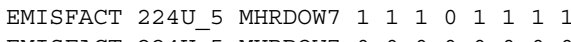
EMISFACT 224U_5 MHRDOW7 0000000000

* Month $=$ December; Day of week = Thursday

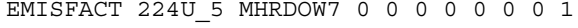

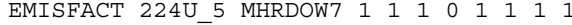

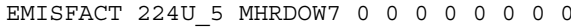

* Month = January; Day of Week = Friday EMISFACT 224U 5 MHRDOW7 000000000000

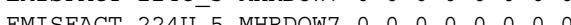
EMTSFACT 224U-5 MHRDOW7 0000000000

EMISFACT 224U_5 MHRDOW7 $00 \begin{array}{llllllll}0 & 0 & 0 & 0 & 0 & 0 & 0 & 0\end{array}$ * Month = February; Day of Week = Friday EMISFACT 2240_5 MHRDOW7 0 O 000000000

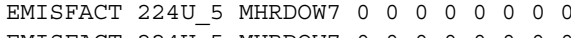
EMISFACT 224U_5 MHRDOW7 000000000

* Month $=$ March; Day of Week = Friday EMISFACT 224U_5 MHRDOW7 0000000000

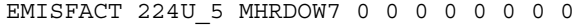

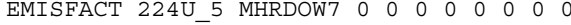

* Month $=$ April; Day of Week = Friday EMISFACT 224U_5 MHRDOW7 00000000000 EMISFACT 224U_5 MHRDOW7 00000000000

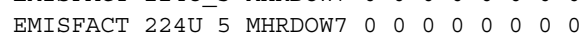

* Month = May; Day of Week = Friday

$\begin{array}{llllllllll}\text { * Month = May; Day of Week } & \text { Friday } & & \\ \text { EMISFACT 224U_5 MHRDOW7 } & 0 & 0 & 0 & 0 & 0 & 0 & 0 & 0\end{array}$

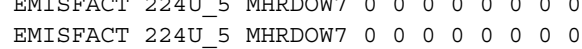
EMISFACT 224U 5 MHRDOW7 001000000000 EMISFACT 224U_5 MHRDOW7 0000000
$*$ Month $=$ June; EMISFACT 224U_5 MHRDOW7 $00 \begin{array}{llllllll} & 0 & 0 & 0 & 0 & 0 & 0\end{array}$ EMISFACT 224U_5 MHRDOW7 001000000000

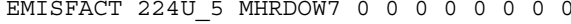

** Month $=$ July; Day of week = Friday

EMISFACT 224U_5 MHRDOW7 0000000000 EMISFACT 224U 5 MHRDOW7 0 C 
EMISFACT 224U_5 MHRDOW7 0000000000

** Month $=$ August; $;$ Day of Week = Friday

EMISFACT 224U 5 MHRDOW7 0000000000 EMISFACT 224U_5 MHRDOW7 000000000

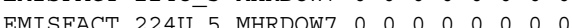

** Month = September; Day of Week DMTSEAC $224 \mathrm{U} 5$ MHRDOW7 000000

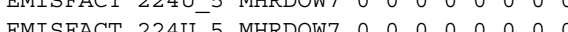
EMISFACT 224U_5 MHRDOW7 00000000000

* Month = October; Day of Week = Friday

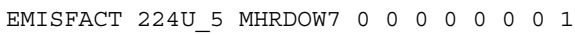

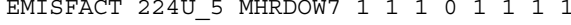

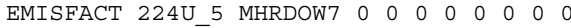

$* *$ Month $=$ November; $;$ Day of Week = Friday

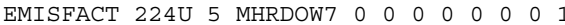

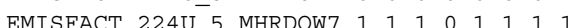
FMTSFACT 224U 5 MHRDOW7 0 o 00000

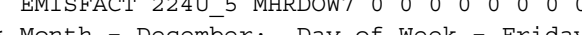

* Month = December; Day of Week = Friday EMISFACT 224__5 MHRDOW7 $0 \begin{array}{llllllll}0 & 0 & 0 & 0 & 0 & 0 & 1\end{array}$

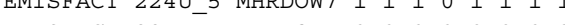
EMISFACT 224U_5 MHRDOW7 $000 \begin{array}{llllll}0 & 0 & 0 & 0 & 0 & 0\end{array}$

* Month = January; Day of Week = Saturda EMISFACT 224U_5 MHRDOW7 00000000000

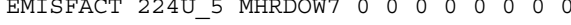

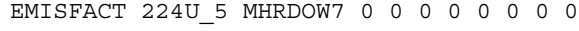

** Month $=$ February; Day of week = Saturday EMISFACT 224U 5 MHRDOW7 00000000000

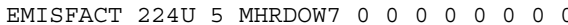

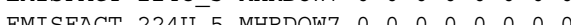

* Month = March * Month = Marchi Day of Week = Saturday EMISFACT 224U_- MRDOW7 $0 \begin{array}{lllllll}0 & 0 & 0 & 0 & 0 & 0 & 0\end{array}$

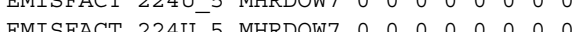
EMISFACT 224U_5 MHRDOW7 $000 \begin{array}{lllllll}0 & 0 & 0 & 0 & 0 & 0 & 0\end{array}$

* Month $=$ April; Day of Week = Saturday EMISFACT 224U_5 MHRDOW7 0 C $00 \begin{array}{llllll} & 0 & 0 & 0 & 0 & 0\end{array}$ EMISFACT 224U_5 MHRDOW7 $000 \begin{array}{lllllll}0 & 0 & 0 & 0 & 0 & 0\end{array}$ EMISFACT 224U_5 MHRDOW7 000000000000

** Month $=$ May; Day of Week = Saturday EMISFACT 224U 5 MHRDOW7 $000 \begin{array}{lllllll}0 & 0 & 0 & 0 & 0 & 0 & 0\end{array}$

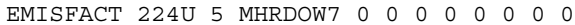
EMISFACT 224U 5 MHRDOW7 0000000

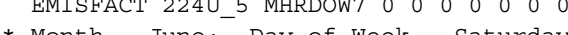

Month = June $;$ Day of week = Saturday

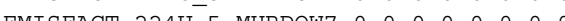
EMISFACT 2240. MHROW7 $0 \begin{array}{llllllllllll}0 & 0 & 0 & 0 & 0 & 0 & 0\end{array}$ EMISFACT 2240_5 MHRDOW7 000000000000 * Month $=$ July; Day of Week = Saturday EMISFACT 224U_5 MHRDOW7 000000000 EMISFACT 224U_5 MHRDOW7 $0 \begin{array}{llllllll}0 & 0 & 0 & 0 & 0 & 0 & 0\end{array}$

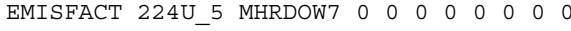

** Month $=$ August; Day of Week = Saturday EMISFACT 224U 5 MHRDOW7 0 O $00 \begin{array}{lllll}0 & 0 & 0 & 0 & 0\end{array}$ EMISFACT 224U 5 MHRDOW7 $00 \begin{array}{lllllll}0 & 0 & 0 & 0 & 0 & 0 & 0\end{array}$ EMISFACT 224U 5 MHRDOW7 
** Month $=$ September $;$ Day of Week = Saturday EMISFACT 224U_5 MHRDOW7 000000000000 EMISFACT 224U_5 MHRDOW7 0000000000000 EMISFACT 224U 5 MHRDOW7 000000000000

** Month = October; Day of Week = Saturday EMISFACT 224U_5 MHRDOW7 0000000000

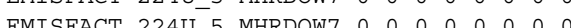
$\begin{array}{lllllll}0 & 0 & 0 & 0 & 0 & 0\end{array}$

* Month $=$ November; Day of Week = Saturday EMISFACT 224U_5 MHRDOW7 000000000000 EMISFACT 224U_5 MHRDOW7 00000000000

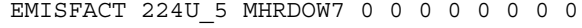

** Month $=$ December; Day of Week = Saturday EMISFACT 224U_5 MHRDOW7 00000000000 EMISFACT 224U 5 MHRDOW7 00000000000

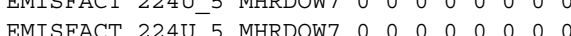

* EMISEACT 2240_5 MHRDOW7 000000000000 Month $=$ January; Day of week $=$ Sunday

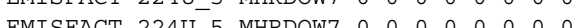

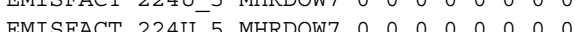
EMISFACT 2240_5 MHRDOW7 000000000000 * Month $=$ February; Day of Week = Sunday EMISFACT 224U_5 MHRDOW7 0000000000 EMISFACT 224U_5 MHRDOW7 $0 \begin{array}{lllllll}0 & 0 & 0 & 0 & 0 & 0 & 0\end{array}$ EMISFACT 224U_5 MHRDOW7 00000000000

** Month $=$ March; Day of Week = Sunday EMISFACT 224U_5 MHRDOW7 00000000000

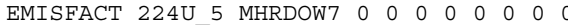

$\begin{array}{lllllllll} & \text { EMISFACT 224U_5 MHRDOW7 } 0 & 0 & 0 & 0 & 0 & 0 & 0 \\ * * & \text { Month }=\text { April; } & 0\end{array}$ EMISFACT 224U_5 MHRDOW7 $000 \begin{array}{lllllll}0 & 0 & 0 & 0 & 0 & 0 & 0\end{array}$ EMISFACT 224U_5 MHRDOW7 000000000000

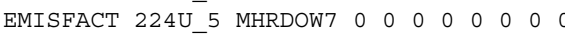

* Month = May; Day of Week = Sunday

EMISFACT 224U_5 MHRDOW7 0000000000 EMISFACT 224U_5 MHRDOW7 00000000000 EMISFACT 224U_5 MHRDOW7 0000000000

** Month $=$ June; Day of Week = Sunday EMISFACT 224U_5 MHRDOW7 000000000 EMISFACT 224U_5 MHRDOW7 $00 \begin{array}{lllllll}0 & 0 & 0 & 0 & 0 & 0 & 0\end{array}$ EMISFACT 224U 5 MHRDOW7 0 C 0000000000

** Month $=$ July; Day of week = Sunday EMISFACT 224U 5 MHRDOW7 0000000000000

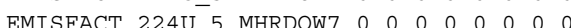

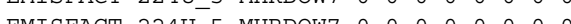

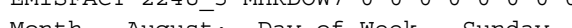
** Month = August $;$ Day of Week = Sunday EMISFACT 2240_5 MHRDOW7 0 0 0000000 EMISFACT 224U_5 MHRDOW7 000000000000 EMISFACT 224U_5 MHRDOW7 0000000000

$*$ Month $=$ September $;$ Day of Week $=$ Sunday EMISFACT 224U_5 MHRDOW7 000000000000 EMISFACT 224U_5 MHRDOW7 $00 \begin{array}{lllllll}0 & 0 & 0 & 0 & 0 & 0 & 0\end{array}$ EMISFACT 224U 5 MHRDOW7 00000000000

* Month $=$ October; Day of week = Sunday 
EMISFACT 224U_5 MHRDOW7 0000000000 EMISFACT 224U 5 MHRDOW7 $00 \begin{array}{lllllll}0 & 0 & 0 & 0 & 0 & 0 & 0\end{array}$ EMISFACT 224U 5 MHRDOW7 0 O 00000000

** Month $=$ Noventer; Day of Month = November; Day of Week = Sunday

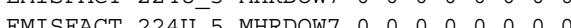

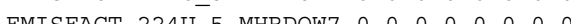

EMISFACT 2240_5 MHRDOW7 $000 \begin{array}{llllllllll}0 & 0 & 0 & 0 & 0 & 0 & 0\end{array}$

* Month $=$ December; Day of Week = Sunday EMISFACT 224U_5 MHRDOW7 00000000000

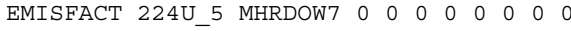

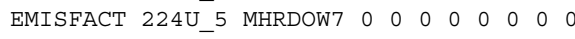

** Month = January; Day of Week = Monday EMISFACT 224U_6 MHRDOW7 0000000000 EMISFACT 224U_6 MHRDOW7 $00 \begin{array}{lllllll}0 & 0 & 0 & 0 & 0 & 0 & 0\end{array}$ EMISFACT 224U 6 MHRDOW7 0 0 000

** Month = Februaryi Day of Week = Monday EMISFACT $224 \mathrm{U} 6$ MHRDOW7 0 o 000000

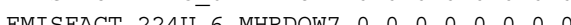
BMISFACT 2240 EMISFACT 2240_6 MHRDOW7 $0 \begin{array}{llllllllllllll}0 & 0 & 0 & 0 & 0 & 0 & 0\end{array}$ * Month $=$ March; Day of Week = Monday EMISFACT 224U_6 MHRDOW7 0 o 0000000 EMISFACT 224U_6 MHRDOW7 000000000000 EMISFACT 224U_6 MHRDOW7 0000000000

$* *$ Month $=$ April; Day of Week = Monday

EMISFACT 224U_6 MHRDOW7 $000 \begin{array}{lllllll}0 & 0 & 0 & 0 & 0 & 0 & 0\end{array}$

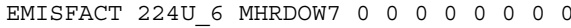
EMISFACT 224U 6 MHRDOW7 $0 \begin{array}{llllllll}0 & 0 & 0 & 0 & 0 & 0 & 0\end{array}$

\section{A $\quad * *$ Month $=$ May; Day of Week = Monday}

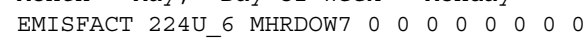
EMTSFACT 224U-6 MHRDOW7 0000000000

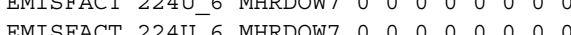

* Month $=$ June; $;$ Day of Week = Monday

* Month $=$ June; Day of Week = Monday

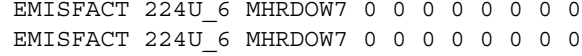
EMISFACT 224U_6 MHRDOW7 0000000000

** Month $=\mathrm{July}_{\mathrm{i}}$; Day of Week = Monday

EMISFACT 224U 6 MHRDOW7 000000000

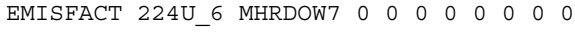
EMISFACT 224U 6 MHRDOW7 $0 \begin{array}{llllllllllllll}0 & 0 & 0 & 0 & 0 & 0 & 0\end{array}$

** Month = August; $;$ Day of Week = Monday EMISFACT 224U_6 MHRDOW7 00000000000

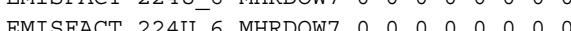

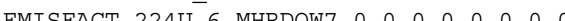

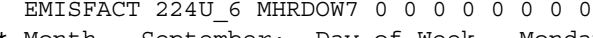
* Month = September; Day of Week = Monda EMISFACT 224U_6 MHRDOW7 00000000000

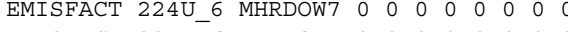
EMISFACT 224U_6 MHRDOW7 0000000000

* Month = October; Day of Week = Monday EMISFACT 224U_6 MHRDOW7 0000000001

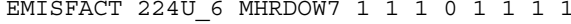

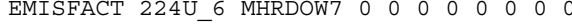

$*$ Month $=$ November; Day of Week = Monday

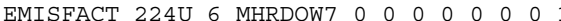




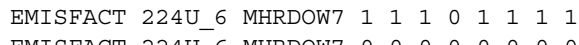
EMISFACT 224U_6 MHRDOW7 00000000000

** Month $=$ December $;$ Day of Week = Monday

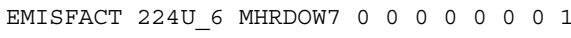
EMISFACT 224U 6 MHRDOW7 $1 \begin{array}{llllllll}-6 & 1 & 0 & 1 & 1 & 1 & 1\end{array}$

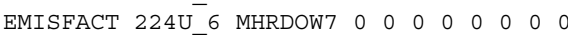

** Month = January; Day of Week = Tuesday EMISFACT 224U_6 MHRDOW7 00000000000

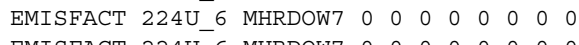

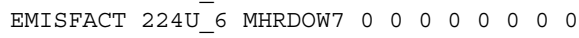

$* *$ Month $=$ February; Day of Week $=$ Tuesday EMISFACT 224U_6 MHRDOW7 000000000000 EMISFACT 224U_6 MHRDOW7 0000000000000

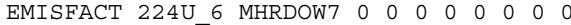

** Month $=$ March; $\overline{\text { Day }}$ of Week = Tuesday EMISFACT 224U_6 MHRDOW7 00000000000

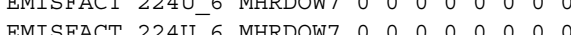

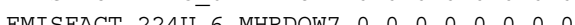

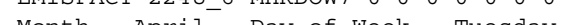

* Month = April; $;$ Day of Week = Tuesday

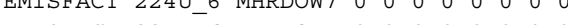
EMISFACT 224U_6 MHRDOW7 0000000000 EMISFACT 224U_6 MHRDOW7 000000000000

* Month $=$ May; Day of Week = Tuesday

EMISFACT 224U_6 MHRDOW7 000000000

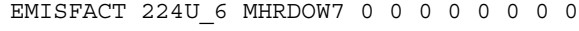

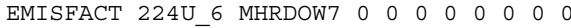

** Month $=$ June; Day of Week $=$ Tuesday EMISFACT 224U_6 MHRDOW7 00000000000000

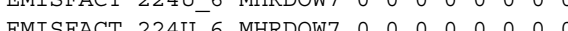

* Month = July; Day of Week = Tuesday

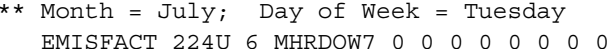

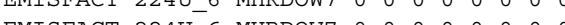

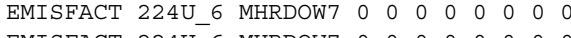

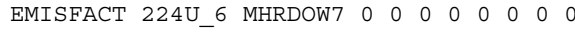

* Month = August; Day of Week = Tuesday EMISFACT 224U_6 MHRDOW7 0000000000 EMISFACT 224U_6 MHRDOW7 $00 \begin{array}{lllllll}0 & 0 & 0 & 0 & 0 & 0 & 0\end{array}$ EMISFACT 224U_6 MHRDOW7 00000000000

** Month $=$ September; Day of Week = Tuesday EMISFACT 224U_6 MHRDOW7 000000000000 EMISFACT 224U_6 MHRDOW7 000000000000 EMISFACT 224U 6 MHRDOW7 000000000000

* Month = October; Day of Week = Tuesday EMISFACT 224U_6 MHRDOW7 00000000001

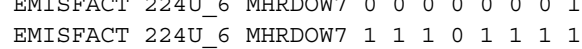

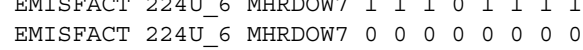

* Month $=$ November; Day of Week $=$ Tuesday EMISFACT 224U_6 MHRDOW7 0000000001

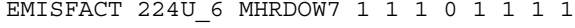

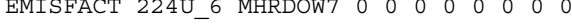

** Month = December; Day of Week = Tuesday EMISFACT $224 \mathrm{U} 6$ MHRDOW7 
EMISFACT 224U_6 MHRDOW7 0000000000

** Month $=$ January; Day of Week $=$ Wednesday EMISFACT 224U_6 MHRDOW7 000000000000 EMISFACT 2240_6 MHRDOW7 00000000000

* Month = February; Day of Week = Wednesday EMISFACT 224U_6 MHRDOW7 000000000000 EMISFACT 224_6 MHRDOW7 000000000000 EMISFACT 224U_6 MHRDOW7 $000 \begin{array}{lllllll}0 & 0 & 0 & 0 & 0 & 0 & 0\end{array}$

* Month $=$ March; Day of Week = Wednesday EMISFACT 224U_6 MHRDOW7 00000000000 EMISFACT 224U_6 MHRDOW7 $00 \begin{array}{lllllll}0 & 0 & 0 & 0 & 0 & 0 & 0\end{array}$

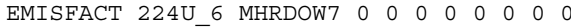

$* *$ Month $=$ April $;$ Day of Week $=$ Wednesday EMISFACT 224U 6 MHRDOW7 00000000000

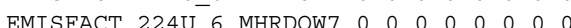
EMISFACT 224U-6 MHRDOW7 0000000000

EMISEACT 2240 - 6 May

* Month = May; Day of Week = Wednesday EMISFACT 224U_6 MHRDOW7 $0 \begin{array}{llllllllllll}0 & 0 & 0 & 0 & 0 & 0 & 0\end{array}$

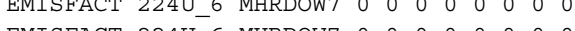

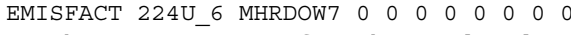

** Month $=$ June; Day of Week $=$ Wednesday EMISFACT 224U_6 MHRDOW7 00000000000

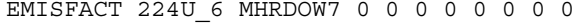
EMISFACT 224U_6 MHRDOW7 $00 \begin{array}{lllllll}0 & 0 & 0 & 0 & 0 & 0 & 0\end{array}$

** Month $=$ July; Day of Week = Wednesday

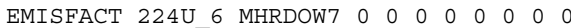

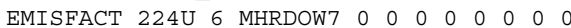
EMTSFACT 224U-6 MHRDOW7 00000000

* Month = 2240 - M May Month = August; Day or Week = Wednesday

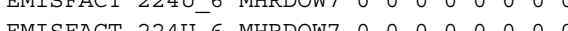
EMISFACT 224_-6 MHRDOW7 00000000000

EMISFACT 224U_6 MHRDOW7 000000000000

** Month $=$ September; Day of Week $=$ Wednesday EMISFACT 224U_6 MHRDOW7 0000000000

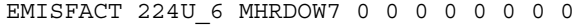

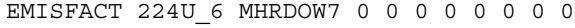

** Month $=$ October; Day of Week $=$ Wednesday

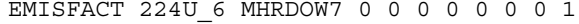
EMISFACT 224U 6 MHRDOW7 $1 \begin{array}{llllllllllll}1 & 1 & 0 & 1 & 1 & 1 & 1\end{array}$ EMISFACT 224U 6 MHRDOW7 000000

** Month = November; Day of Week = Wednesday EMISFACT 224U_6 $\begin{array}{llllllllll}\text { EMISFACT } 224 U-6 & \text { MHRDOW7 } & 1 & 1 & 1 & 0 & 1 & 1 & 1 & 1 \\ \text { EMISFACT } 224 \mathrm{U} 6 & \text { MHRDOW7 } & 0 & 0 & 0 & 0 & 0 & 0 & 0 & 0\end{array}$

** Month $=$ December; Day of Week $=$ Wednesday EMISFACT 224U_6 MHRDOW7 000000000001

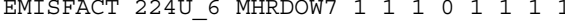

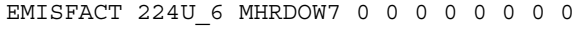

** Month $=$ January; Day of Week = Thursday EMISFACT 224U 6 MHRDOW7 $00 \begin{array}{lllllll}0 & 0 & 0 & 0 & 0 & 0 & 0\end{array}$

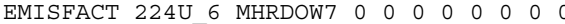


** Month = February; Day of Week = Thursday EMISFACT 224U_6 MHRDOW7 000000000000 EMISFACT 224U_6 MHRDOW7 0000000000000 EMISFACT 224U 6 MHRDOW7 00000000000

** Month $=$ March; Day of Week = Thursday EMISFACT 224U 6 MHRDOW7 00000000000

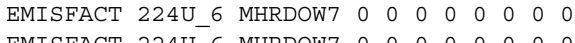

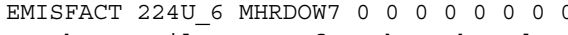

** Month $=$ April; Day of Week = Thursday EMISFACT 224U_6 MHRDOW7 0000000000 EMISFACT 224U_6 MHRDOW7 $00 \begin{array}{lllllllllll}0 & 0 & 0 & 0 & 0 & 0 & 0\end{array}$

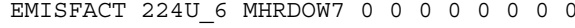

$* *$ Month $=$ May; Day of Week $=$ Thursday EMISFACT 224U 6 MHRDOW7 $000 \begin{array}{lllllll}0 & 0 & 0 & 0 & 0 & 0\end{array}$

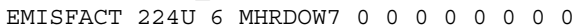

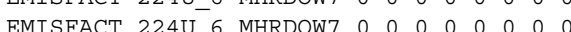

* EMISACT 224U_6 MHRDOW7 $00 \begin{array}{lllllll}0 & 0 & 0 & 0 & 0 & 0 & 0\end{array}$

* Month = June; Day of Week = Thursday (1) EMISFACT $2240-6$ MRDOW7 $\begin{array}{lllllllllllllllll}6 & 0 & 0 & 0 & 0 & 0 & 0\end{array}$ EMISFACT 224U_6 MHRDOW7 $0 \begin{array}{lllllllllllllllll}0 & 0 & 0 & 0 & 0 & 0 & 0 & 0 & 0\end{array}$ * Month $=$ July; Day of Week = Thursday EMISFACT 224U_6 MHRDOW7 00000000000 EMISFACT 224U_6 MHRDOW7 00000000000 EMISFACT 224U_6 MHRDOW7 $00 \begin{array}{llllllll}0 & 0 & 0 & 0 & 0 & 0 & 0\end{array}$

** Month $=$ August; Day of Week = Thursday EMISFACT 224U_6 MHRDOW7 00000000000 EMISFACT 224U 6 MHRDOW7 $00 \begin{array}{lllllll}0 & 0 & 0 & 0 & 0 & 0 & 0\end{array}$

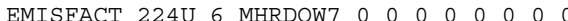

** Month $=$ September; Day of Week = Thursday EMISFACT 224U_6 MHRDOW7 00000000000 EMISFACT 2240_6 MHRDOW7 $0 \begin{array}{llllllllllllll}0 & 0 & 0 & 0 & 0 & 0 & 0\end{array}$

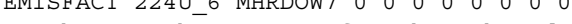

* Month = October; Day of Week = Thursday EMISFACT 224U_6 MHRDOW7 000000000001

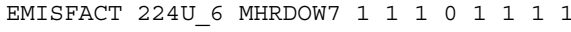
EMISFACT 224U_6 MHRDOW7 00000000000

** Month $=$ November $;$ Day of Week $=$ Thursday EMISFACT 224U_6 MHRDOW7 000000000001

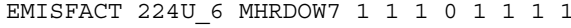

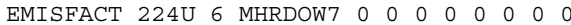

** Month $=$ December; $;$ Day of week = Thursday

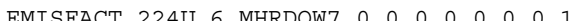

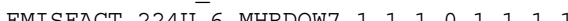
EMTSEAC 2240

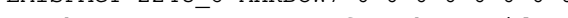
* Month = January; Day of Week = Friday EMISFACT 224U_6 MHRDOW7 00000000000 EMISFACT 224U_6 MHRDOW7 000000000000 EMISFACT 224U_6 MHRDOW7 00000000000

$* *$ Month $=$ February; Day of Week = Friday EMISFACT 224U_6 MHRDOW7 0000000000 EMISFACT 224U_6 MHRDOW7 $000 \begin{array}{cccccc}0 & 0 & 0 & 0 & 0 & 0\end{array}$ EMISFACT 224U 6 MHRDOW7 0000000000

** Month $=$ March; Day of Week = Friday 
EMISFACT 224U_6 MHRDOW7 0000000000 EMISFACT 224U 6 MHRDOW7 0000000000 EMISFACT 224U 6 MHRDOW7 000

* Month = April; Day of Week = Friday

EMISFACT 224U_6 MHRDOW7 00000000000 EMISFACT 224U_6 MHRDOW7 00000000000

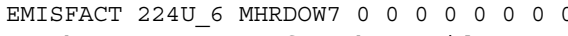
* Month $=$ May; Day of Week = Friday

EMISFACT 224U_6 MHRDOW7 00000000000

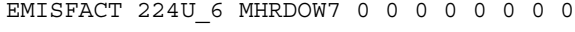
EMISFACT 224U_6 MHRDOW7 000000000000

** Month $=$ June $;$ Day of Week = Friday

EMISFACT 224U_6 MHRDOW7 0 O $00 \begin{array}{llllll}0 & 0 & 0 & 0 & 0\end{array}$ EMISFACT 224U 6 MHRDOW7 $00 \begin{array}{lllllll}0 & 0 & 0 & 0 & 0 & 0 & 0\end{array}$

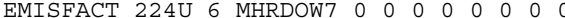

* Month $=\mathrm{July} ;$ Day of week = Friday

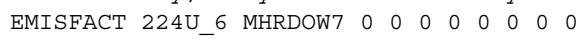
EMISFACT 224U_6 MHRDOW7 000000000000 EMISFACT 224U-6 MHRDOW7 0000000000

** Month = August $;$ Day of week = Friday EMISFACT 224U_6 MHRDOW7 000000000000 EMISFACT 224U_6 MHRDOW7 00000000000 EMISFACT 224U_6 MHRDOW7 00000000000

* Month $=$ September $;$ Day of Week $=$ Frida EMISFACT 224U_6 MHRDOW7 00000000000

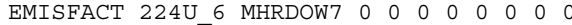
EMISFACT 224U 6 MHRDOW7 $00 \begin{array}{llllllllll}0 & 0 & 0 & 0 & 0 & 0 & 0\end{array}$

** Month = October; Day of Week = Friday EMISFACT 224U 6 MHRDOW7 000000 EMISFACT 224U

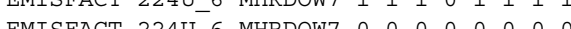

EMISEACT 2240_6 MHRDOW7 $\begin{array}{lllllllllllllllllll}0 & 0 & 0 & 0 & 0 & 0 & 0\end{array}$ * Month = November $;$ Day of Week = Friday

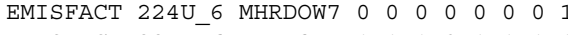

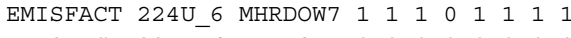
EMISFACT 224U_6 MHRDOW7 $00 \begin{array}{lllllll}- & 0 & 0 & 0 & 0 & 0 & 0\end{array}$

** Month $=$ December; $;$ Day of week = Friday

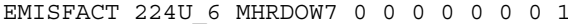

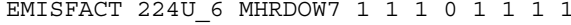

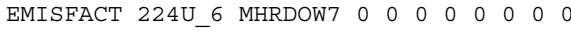

** Month = January; Day of Week = Saturday EMISFACT 224U_6 MHRDOW7 000000000000

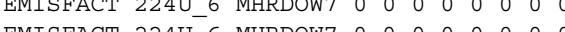

* Month = February; Day of Week = Saturday EMISFACT 224U_6 MHRDOW7 000000000000 EMISFACT 224U_6 MHRDOW7 $000 \begin{array}{lllllll}0 & 0 & 0 & 0 & 0 & 0 & 0\end{array}$ EMISFACT 224U_6 MHRDOW7 00000000000

* Month = March; Day of Week = Saturday

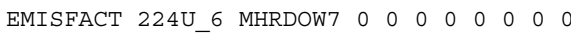
EMISFACT 224U_6 MHRDOW7 $000 \begin{array}{llllll}0 & 0 & 0 & 0 & 0 & 0\end{array}$ EMISFACT 224U 6 MHRDOW7 $00 \begin{array}{lllllll}0 & 0 & 0 & 0 & 0 & 0 & 0\end{array}$

** Month $=$ April; Day of Week = Saturday

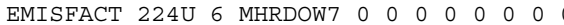


EMISFACT 224U_6 MHRDOW7 000000000000 EMISFACT 224U 6 MHRDOW7 0 C $00 \begin{array}{ccccc}0 & 0 & 0 & 0 & 0\end{array}$

** Month = May; Day of Week = Saturday

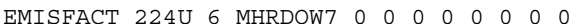

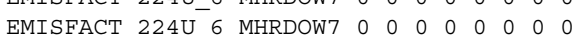
EMISFACT 224U 6 MHRDOW7 00000000000

** Month EMISFACT 224 ( EMISFACT 224U_6 MHRDOW7 00000000000 EMISFACT 224U 6 MHRDOW7 $000 \begin{array}{lllllllll}-0 & 0 & 0 & 0 & 0 & 0\end{array}$

** Month $=$ July; $;$ Day of Week = Saturday EMISFACT 224U_6 MHRDOW7 0000000000 EMISFACT 224U_6 MHRDOW7 $000 \begin{array}{cccccc}0 & 0 & 0 & 0 & 0 & 0\end{array}$ EMISFACT 224U 6 MHRDOW7 $00 \begin{array}{llllllll}0 & 0 & 0 & 0 & 0 & 0 & 0\end{array}$

** Month = August; $;$ Day of Week = Saturday EMISFACT 224U_6 MHRDOW7 0000000000000 EMISFACT 2240-6 MHRDOW7 00000000000

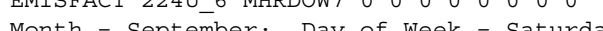

** Month = September; Day of Week = Saturday EMISFACT 224U_6 MHRDOW7 00000000000 EMISFACT 224U_6 MHRDOW7 $000 \begin{array}{lllllll}0 & 0 & 0 & 0 & 0 & 0 & 0\end{array}$ EMISFACT 224U_6 MHRDOW7 00000000000

* Month = October; Day of Week = Saturday EMISFACT 224U_6 MHRDOW7 000000000000 EMISFACT 224U_6 MHRDOW7 000000000000

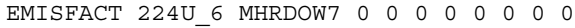

** Month $=$ November; Day of Week = Saturday EMISFACT 224U 6 MHRDOW7 00000000000 EMISFACT 224U-6 MHRDOW7 000000000000

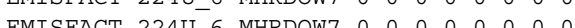

* Month = December. Day of Week ** Month = December $;$ Day of Week = Saturday EMISFACT 224U_6 MHRDOW7 00000000000 EMISFACT 224U_6 MHRDOW7 0000000000000 EMISFACT 224U_6 MHRDOW7 0000000000

* Month = January; Day of Week = Sunday EMISFACT 224U_6 MHRDOW7 00000000000

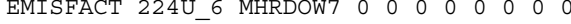
EMISFACT 224U_6 MHRDOW7 $00 \begin{array}{lllllllll}0 & 0 & 0 & 0 & 0 & 0 & 0\end{array}$

* Month $=$ February; Day of Week $=$ Sunday EMISFACT 224U 6 MHRDOW7 000000000

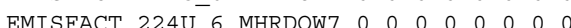

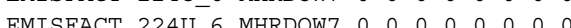

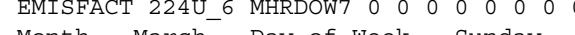
* Month = March; $;$ Day of Week = Sunday EMISFACT 224U_6 MHROW7 $0 \begin{array}{lllllll}0 & 0 & 0 & 0 & 0 & 0 & 0\end{array}$ EMISFACT 2240_6 MHRDOW7 $0 \begin{array}{lllllllll}0 & 0 & 0 & 0 & 0 & 0 & 0\end{array}$ EMISFACT 224U_6 MHRDOW7 0000000000

* Month $=$ April; $;$ Day of Week = Sunday EMISFACT 224U_6 MHRDOW7 00000000000 EMISFACT 224U_6 MHRDOW7 $0 \begin{array}{lllllll}0 & 0 & 0 & 0 & 0 & 0 & 0\end{array}$

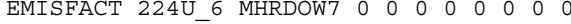

** Month $=$ May; Day of Week = Sunday

EMISFACT 224U_6 MHRDOW7 00000000000

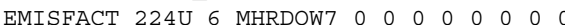


EMISFACT 224U 6 MHRDOW7 0000000000

** Month $=$ June; Day of Week = Sunday

EMISFACT 224U_6 MHRDOW7 00000000000 $\begin{array}{lllll}0 & 0 & 0\end{array}$ EMISFACT 224U 6 MHRDOW7 $000 \begin{array}{llllll}0 & 0 & 0 & 0 & 0 & 0\end{array}$

** Month $=$ July; Day of Week = Sunday

EMISFACT 2240_6 MHRDOW7 0000000000000 EMISFACT 2240_6 MHRDOW7 $\begin{array}{lllllllllll}0 & 0 & 0 & 0 & 0 & 0 & 0 & 0 & 0\end{array}$ EMISFACT 224U_6 MHRDOW7 00000000000

* Month = August $;$ Day of Week = Sunday EMISFACT 224U_6 MHRDOW7 00000000000

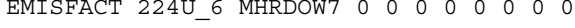

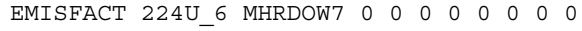

** Month $=$ September $; \quad$ Day of Week = Sunday EMISFACT 224U_6 MHRDOW7 00000000000 EMISFACT 2240-6 MHRDOW7 $0 \begin{array}{llllllllllllll}0 & 0 & 0 & 0 & 0 & 0 & 0\end{array}$

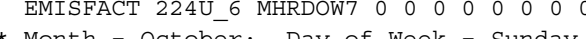
* Month = October; Day of week = Sunday EMISFACT 2240_6 MHRDOW7 $0 \begin{array}{ccccccccc}0 & 0 & 0 & 0 & 0 & 0 & 0\end{array}$ EMISFACT 224_-6 MHRDW7 0000000000000 EMISFACT 224U_6 MHRDOW7 000000000

* Month $=$ November; Day of Week = Sunday EMISFACT 224U_6 MHRDOW7 00000000000

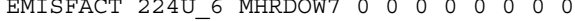

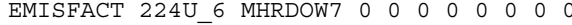

* Month $=$ December; $;$ Day of Week = Sunday EMISFACT 224U 6 MHRDOW7 $000 \begin{array}{llllll}0 & 0 & 0 & 0 & 0 & 0\end{array}$ EMISFACT 224U 6 MHRDOW7 00000010000 EMISFACT 224U_6 MHRDOW7 000000000

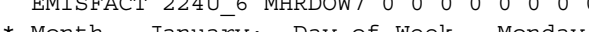
* Month = January; Day of Week = Monday EMISFACT 224U_

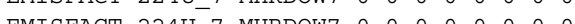

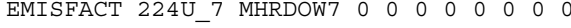

* Month = February; Day of week = Monday EMISFACT 224U_7 MHRDOW7 000000000 EMISFACT 224U_7 MHRDOW7 $00 \begin{array}{lllllll}0 & 0 & 0 & 0 & 0 & 0 & 0\end{array}$ EMISFACT 224U_7 MHRDOW7 $00 \begin{array}{lllllll}0 & 0 & 0 & 0 & 0 & 0 & 0\end{array}$

** Month $=$ March; Day of Week = Monday EMISFACT 224U 7 MHRDOW7 000000000 EMISFACT 224U 7 MHRDOW7 $00 \begin{array}{llllllllll}0 & 0 & 0 & 0 & 0 & 0 & 0 & 0\end{array}$ EMISFACT 224U 7 MHRDOW7 0 O 0 C 0

$* *$ Month = April; Day of Week = Monday

$\begin{array}{lllllllll}\text { Month }=\text { April; Day of week } & = & \text { Monday } \\ \text { EMISFACT 224U } 7 \text { MHRDOW7 } 000 & 0 & 0 & 0 & 0 & 0 & 0\end{array}$

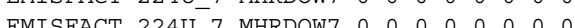
EMISFACT 2240-7 MHROW7 $0 \begin{array}{lllllllllllll}0 & 0 & 0 & 0 & 0 & 0 & 0\end{array}$ EMISFACT 2240_7 MHRDOW7 0 o 000 O 000 * Month = May; Day of Week = Monday

EMISFACT 224U_7 MHRDOW7 0000000000 EMISFACT 224U_7 MHRDOW7 $0 \begin{array}{llllllll}0 & 0 & 0 & 0 & 0 & 0 & 0\end{array}$

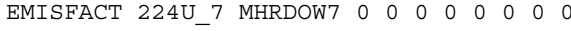

** Month $=$ June; Day of Week = Monday

EMISFACT 224U_7 MHRDOW7 $00 \begin{array}{lllllll}0 & 0 & 0 & 0 & 0 & 0 & 0\end{array}$ EMISFCT 22407 MHRDOW 70 
** Month $=$ July; Day of Week $=$ Monday EMISFACT 224U_7 MHRDOW7 00000000000 EMISFACT 224U_7 MHRDOW7 000000000000 EMISFACT 224U_7 MHRDOW7 000000000000 * Month = August; Day of Week = Monday EMISFACT 2240_7 MHRDOW7 $0 \begin{array}{lllllllllll}0 & 0 & 0 & 0 & 0 & 0 & 0\end{array}$

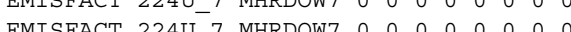

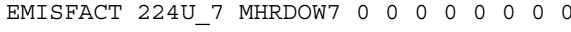

** Month $=$ September $;$ Day of Week $=$ Monday EMISFACT 224U_7 MHRDOW7 000 O 000000

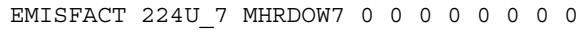
EMISFACT 224U_7 MHRDOW7 0010000000000

* Month $=$ October; Day of Week = Monday

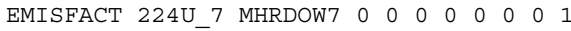

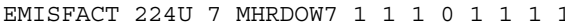

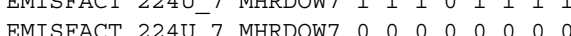

* MOn Month = November; Day of Week = Monday BMISFACT 2240_7. EMISFACI 224_- MHRDOW7 $\begin{array}{llllllll}1 & 1 & 1 & 0 & 1 & 1 & 1 & 1\end{array}$ EMISFACT 2240_7 MHRDOW7 001000000000 * Month $=$ December; Day of Week = Monday EMISFACT 224U_7 MHRDOW7 0000000001

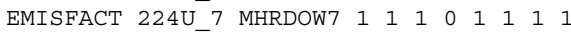
EMISFACT 224U_7 MHRDOW7 0000000000

* Month = January; Day of week = Tuesday EMISFACT 224U_7 MHRDOW7 00000000000 EMISFACT 224U 7 MHRDOW7 $00 \begin{array}{lllllll}0 & 0 & 0 & 0 & 0 & 0 & 0\end{array}$ EMISFACT 224U 7 MHRDOW7 0 O 000

** Month = February; Day of week = Tuesday EMISFACT 224U 7 MHRDOW7 00000000000 EMISFACT 224U_7 MHRDOW7 000000000000

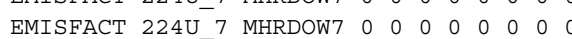

Month $=$ March; Day of week = Tuesday EMISFACT 224U_7 MHRDOW7 000000000 EMISFACT 224U_7 MHRDOW7 $000 \begin{array}{lllllll}-7 & 0 & 0 & 0 & 0 & 0 & 0\end{array}$ EMISFACT 224U_7 MHRDOW7 00000000000

* Month $=$ April; Day of Week = Tuesday EMISFACT 224U_7 MHRDOW7 000000000 EMISFACT 224U_7 MHRDOW7 $00 \begin{array}{lllllll}0 & 0 & 0 & 0 & 0 & 0 & 0\end{array}$

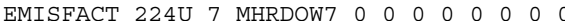

* Month = May; Day of Week = Tuesday EMISFACT 224U_7 MHRDOW7 $00 \begin{array}{lllllll}0 & 0 & 0 & 0 & 0 & 0 & 0\end{array}$

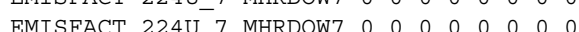

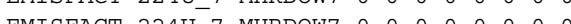

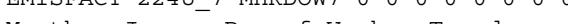
* Month $=$ June; Day of Week = Tuesday EMISFACT 2240_7 MHRDOW7 00000000000 EMISFACT 224U_7 MHRDOW7 000000000000

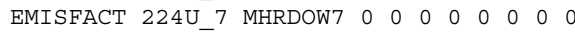
** Month $=$ July; Day of Week = Tuesday EMISFACT 224U_7 MHRDOW7 0000000000 EMISFACT 224U_7 MHRDOW7 $00 \begin{array}{ccccccc}0 & 0 & 0 & 0 & 0 & 0 & 0\end{array}$

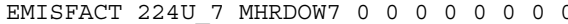

$* *$ Month $=$ August $;$ Day of Week $=$ Tuesday 
EMISFACT 224U_7 MHRDOW7 000000000000

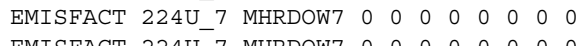

* MonsACT 224U_7 MHRDOW7 000000000000

Month = September; Day of Week = Tuesday

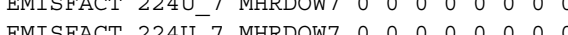
EMISFACT 2240 7 MHRDOW7 $0 \begin{array}{llllllllllllll}0 & 0 & 0 & 0 & 0 & 0\end{array}$

EMISFACT 2240_7 MHRDOW7 $0 \begin{array}{lllllllll}0 & 0 & 0 & 0 & 0 & 0 & 0 & 0\end{array}$ * Month = October; Day of Week = Tuesday EMISFACT 224U_7 MHRDOW7 00000000001

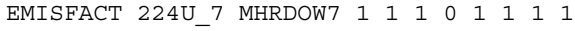
EMISFACT 224U_7 MHRDOW7 $00 \begin{array}{lllllll}0 & 0 & 0 & 0 & 0 & 0 & 0\end{array}$

** Month $=$ November $;$ Day of Week $=$ Tuesday

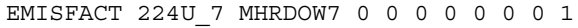
EMISFACT 224U 7 MHRDOW7 $11110 \begin{array}{lllllll}1 & 0 & 1 & 1 & 1 & 1\end{array}$

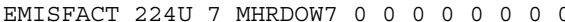

** Month = December; Day of Week = Tuesday EMISFACT 22407 MHRDOW7 000000

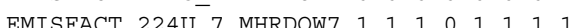

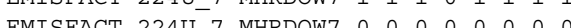

* Month = January; Day of Week = Wednesday Month = January; Day of Week $=$ Wednesday
EMISFACT 224U_7 MHRDOW7 0000000000 EMISFACT 224U_7 MHRDOW7 00000000000

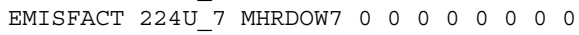

** Month $=$ February; Day of Week $=$ Wednesday EMISFACT 224U_7 MHRDOW7 00000000000 EMISFACT 224U 7 MHRDOW7 $00 \begin{array}{lllllll}0 & 0 & 0 & 0 & 0 & 0 & 0\end{array}$

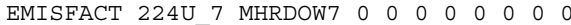

** Month = March; Day of Week = Wednesday EMTSFACT 224U 7 MHRDOH7 000000000

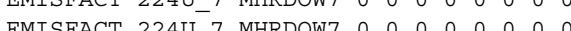
BMISFACT 224 7 MHRDOH 0 O 000000000

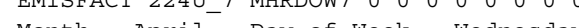
* Month $=$ April $;$ Day of Week $=$ Wednesday EMISFACT 224U_7 MHRDOW7 000000000 EMISFACT 224U_7 MHRDOW7 000000000000 EMISFACT 224U_7 MHRDOW7 $00 \begin{array}{lllllllll}-7 & 0 & 0 & 0 & 0 & 0 & 0\end{array}$

* Month $=$ May; Day of Week $=$ Wednesday EMISFACT 224U_7 MHRDOW7 0000000000 EMISFACT 224U_7 MHRDOW7 $0 \begin{array}{lllllll}0 & 0 & 0 & 0 & 0 & 0 & 0\end{array}$ EMISFACT 224U 7 MHRDOW7 $00 \begin{array}{ccccccc}0 & 0 & 0 & 0 & 0 & 0 & 0\end{array}$

** Month $=$ June; Day of Week = Wednesday EMISFACT 224U_7 MHRDOW7 00000000000 EMISFACT 224U 7 MHRDOW7 000000000000

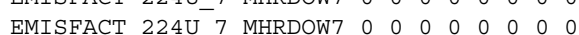

* Month = July; Day of Week = Wednesday Month = July; Day of Week = Wednesday
EMISFACT 224U 7 MHRDOW7 000000000

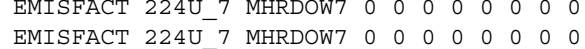
$\begin{array}{lllllllllll}\text { EMISFACT } & 224 \mathrm{U}-7 & \text { MHRDOW7 } & 0 & 0 & 0 & 0 & 0 & 0 & 0 & 0 \\ \text { EMISFACT } & 224 \mathrm{U} 7 & \text { MHRDOW7 } & 0 & 0 & 0 & 0 & 0 & 0 & 0 & 0\end{array}$

* Month = August $;$ Day of Week = Wednesda EMISFACT 224U_7 MHRDOW7 0000000000 EMISFACT 224U_7 MHRDOW7 000000000000

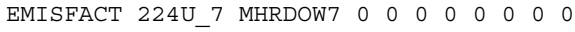

** Month $=$ September; Day of Week = Wednesday

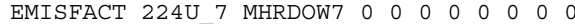




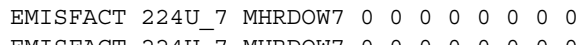
EMISFACT 224U_7 MHRDOW7 000000000000

** Month $=$ October; Day of Week $=$ Wednesday EMISFACT 224U_7 MHRDOW7 000000000001 EMISFACT 224U-7 MHRDOW7 $1 \begin{array}{llllllll}-1 & 1 & 0 & 1 & 1 & 1 & 1\end{array}$ EMISFACT 224U 7 MHRDOW7 00000000000

** Month = November; Day of Week = Wednesday EMISFACT 224U_7 MHRDOW7 0000000000 EMISFACT 224U_7 MHRDOW7 $11110 \begin{array}{llllll}1 & 1 & 1 & 1 & 1 & 1\end{array}$ EMISFACT 224U_7 MHRDOW7 $00 \begin{array}{llllllll}0 & 0 & 0 & 0 & 0 & 0 & 0\end{array}$

** Month $=$ December; Day of Week $=$ Wednesday EMISFACT 224U_7 MHRDOW7 0000000000001

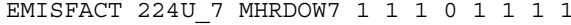
EMISFACT 224U_7 MHRDOW7 $00 \begin{array}{lllllll}0 & 0 & 0 & 0 & 0 & 0 & 0\end{array}$

** Month = January; Day of Week = Thursday

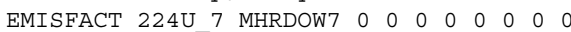
EMISFACT 224U 7 MHRDOW7 $00 \begin{array}{llllllllllllll}0 & 0 & 0 & 0 & 0 & 0 & 0 & 0\end{array}$ EMTSEACT 224U-7 MHRDOW7 0 O 000000000

* MomiseacT 2240_7 MHRDOW7 0 o 00000 Month = February; Day of Week = Thursday EMISFACT 224U_7 MHRDOW7 00000000000

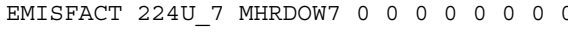
EMISFACT 224U_7 MHRDOW7 00000000000

* Month $=$ March; Day of Week = Thursday EMISFACT 224U_7 MHRDOW7 000000000 EMISFACT 224U_7 MHRDOW7 0000000000000 EMISFACT 224U 7 MHRDOW7 $00 \begin{array}{llllllllll}0 & 0 & 0 & 0 & 0 & 0 & 0\end{array}$

* Month = April; Day of Week = Thursday

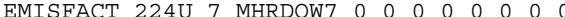

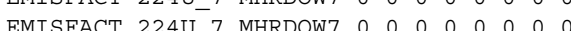

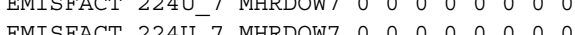

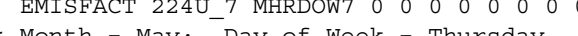
* Month = May; Day of Week = Thursday

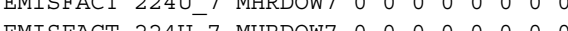

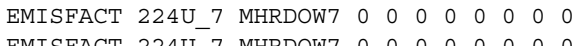

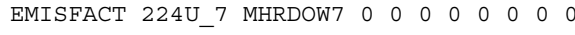
* Month = June; Day of Week = Thursday EMISFACT 224U_7 MHRDOW7 00000000000

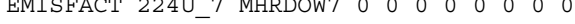
EMISFACT 224U_7 MHRDOW7 00000000000

** Month = July; Day of Week = Thursday

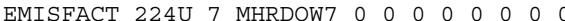
EMISFACT 224U 7 MHRDOW7 0000000000

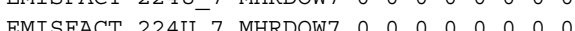

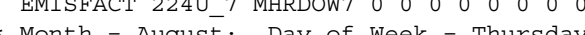
* Month EMISFACT 2240_7 MHROW7 0000000000

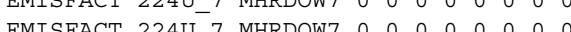
EMISFACT 224U_7 MHRDOW7 $00 \begin{array}{lllllll}0 & 0 & 0 & 0 & 0 & 0 & 0\end{array}$

** Month $=$ September; Day of week = Thursday EMISFACT 224U_7 MHRDOW7 00000000000 EMISFACT 224U_7 MHRDOW7 $00 \begin{array}{lllllll}0 & 0 & 0 & 0 & 0 & 0 & 0\end{array}$ EMISFACT 224U_7 MHRDOW7 $000 \begin{array}{llllll}0 & 0 & 0 & 0 & 0 & 0\end{array}$

** Month = October $;$ Day of Week = Thursday EMISFACT 224U 7 MHRDOW7 
EMISFACT 224U_7 MHRDOW7 0000000000

** Month $=$ November; Day of Week = Thursday EMISFACT 224U_7 MHRDOW7 0000000000001 EMr

EMISFACT 224U_7 MHRDOW7 $0 \begin{array}{llllllllll}0 & 0 & 0 & 0 & 0 & 0 & 0 & 0\end{array}$ * Month $=$ December; Day of Week = Thursday EMISFACT 224U_7 MHRDOW $70 \begin{array}{llllllll}0 & 0 & 0 & 0 & 0 & 0 & 0 & 1\end{array}$

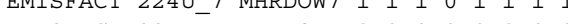

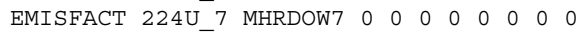

* Month = January; Day of Week = Friday EMISFACT 224U_7 MHRDOW7 $000 \begin{array}{llllll}0 & 0 & 0 & 0 & 0 & 0\end{array}$

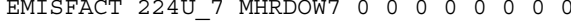
EMISFACT 224U_7 MHRDOW7 000000000000

$* *$ Month $=$ February; Day of Week = Friday EMISFACT 224U 7 MHRDOW7 0000000000

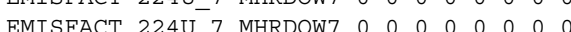
EMISFACT 224U-7 MHRDOW7 $0 \begin{array}{llllllll}0 & 0 & 0 & 0 & 0 & 0 & 0\end{array}$

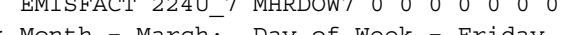

* Month = March; Day of Week = Friday EMISFACT 2240_7 MHRDOW7 $0 \begin{array}{llllllll}0 & 0 & 0 & 0 & 0 & 0 & 0\end{array}$

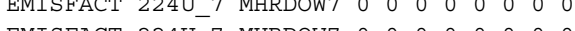
EMISFACT 224U_7 MHRDOW7 0000000000

$* *$ Month $=$ April; Day of Week = Friday EMISFACT 224U_7 MHRDOW7 00000000000

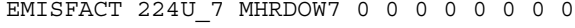

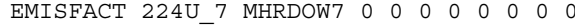

** Month $=$ May; Day of Week = Friday

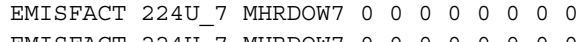
EMISFACT 224U_7 MHRDOW7 000000000000 EMISFACT 224U 7 MHRDOW7 $000 \begin{array}{lllllll}-7 & 0 & 0 & 0 & 0 & 0 & 0\end{array}$

* Month = June; Day of Week = Friday

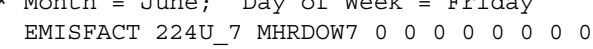

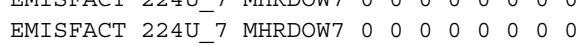
EMISFACT 224U 7 MHRDOW7 00000000000 $* *$ Month $=$ July; ${ }^{-}$Day of week $=$Friday

EMISFACT 224U_7 MHRDOW7 000000000 EMISFACT 224U_7 MHRDOW7 $000 \begin{array}{lllllll}0 & 0 & 0 & 0 & 0 & 0\end{array}$

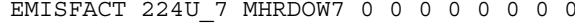

* Month $=$ August; Day of Week = Friday EMISFACT 224U 7 MHRDOW7 $000 \begin{array}{llllll}0 & 0 & 0 & 0 & 0 & 0\end{array}$ EMISFACT 224U 7 MHRDOW7 $00 \begin{array}{llllllllllll}0 & 0 & 0 & 0 & 0 & 0 & 0\end{array}$ EMISFACT 224U 7 MHRDOW7 0000000

* Month = Septen Month = September; Day of Week = Frida

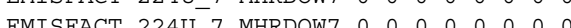
EMISFACT 224_- MAR

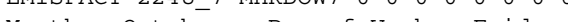
* Month = October; Day of Week = Friday EMISFACT 224U_7 MHRDOW7 0000000001

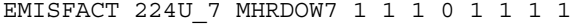
EMISFACT 224U_7 MHRDOW7 $000 \begin{array}{llllll}0 & 0 & 0 & 0 & 0 & 0\end{array}$

** Month $=$ November; Day of Week = Friday

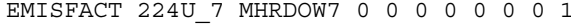
EMISFACT 224U 7 MHRDOW7 $1110 \begin{array}{llllllll}1 & 1 & 0 & 1 & 1 & 1 & 1\end{array}$ EMISFACT 224U 7 MHRDOW7 00 
** Month $=$ December $;$ Day of Week = Friday EMISFACT 224U 7 MHRDOW7 0000000000

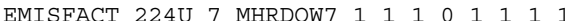
EMISFACT 224U-7 MHRDOW7 $0 \begin{array}{lllllll}1 & 1 & 0 & 1 & 1 & 1 & 1\end{array}$

* EMISFACT 2240_7 MHRDOW7 0000000 * Month = January; Day of Week = Saturda

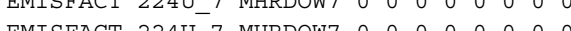

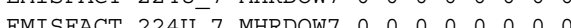
$\begin{array}{lllllll}0 & 0 & 0 & 0 & 0 & 0\end{array}$

* Month = February; Day of week = Saturday EMISFACT 224U_7 MHRDOW7 $000 \begin{array}{llllll}0 & 0 & 0 & 0 & 0 & 0\end{array}$ EMISFACT 224U_7 MHRDOW7 $000 \begin{array}{cccccc}0 & 0 & 0 & 0 & 0 & 0\end{array}$ EMISFACT 224U_7 MHRDOW7 $0 \begin{array}{lllllll}0 & 0 & 0 & 0 & 0 & 0 & 0\end{array}$

* Month $=$ March; Day of Week = Saturday EMISFACT 224U 7 MHRDOW7 $000 \begin{array}{llllll}0 & 0 & 0 & 0 & 0 & 0\end{array}$ EMISFACT 224U 7 MHRDOW7 $00 \begin{array}{llllllll}0 & 0 & 0 & 0 & 0 & 0 & 0\end{array}$

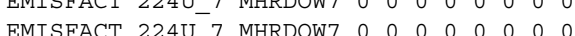

* Morth * Month = April; Day of Week = Saturday

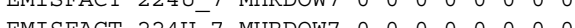

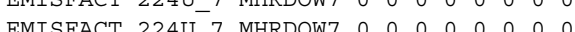
EMISFACT 2240_7 MHRDOW7 00000000000 * Month = May; Day of Week = Saturday EMISFACT 224U_7 MHRDOW7 0000000000

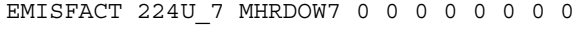
EMISFACT 224U_7 MHRDOW7 $00 \begin{array}{llllllll}0 & 0 & 0 & 0 & 0 & 0 & 0\end{array}$

** Month $=$ June; Day of Week = Saturday EMISFACT 224U 7 MHRDOW7 0 M $00 \begin{array}{lllllll} & 0 & 0 & 0 & 0\end{array}$ EMISFACT 224U 7 MHRDOW7 $00 \begin{array}{lllllll}0 & 0 & 0 & 0 & 0 & 0 & 0\end{array}$ EMISFACT 224U 7 MHRDOW7 0 O 0

* Month = Julyi Day OA Month = July; Day of week = Saturday

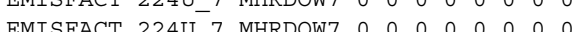
MIAC 2240 EMISFACT 2240_ MRRDOW7 $0 \begin{array}{lllllll}0 & 0 & 0 & 0 & 0 & 0 & 0\end{array}$

* Month = August; Day of Week = Saturday EMISFACT 224U_7 MHRDOW7 00000000000 EMISFACT 224U_7 MHRDOW7 $000 \begin{array}{llllll}0 & 0 & 0 & 0 & 0 & 0\end{array}$ EMISFACT 224U_7 MHRDOW7 $000 \begin{array}{lllllll}-7 & 0 & 0 & 0 & 0 & 0 & 0\end{array}$

** Month $=$ September $; \quad$ Day of Week = Saturday EMISFACT 224U_7 MHRDOW7 000000000000 EMISFACT 224U_7 MHRDOW7 $00 \begin{array}{lllllll}0 & 0 & 0 & 0 & 0 & 0 & 0\end{array}$

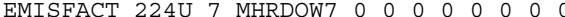

** Month = October; Day of Week = Saturday EMISFACT 224U 7 MHRDOW7 000000000000

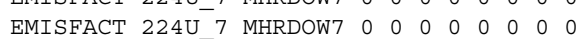
EMISFACT 224U 7 MHRDOW7 00000000000

* Month = November; Day of Week = Saturday EMISFACT 224U_7 MHRDOW7 00000000000

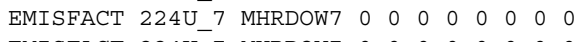

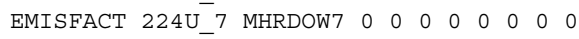

** Month $=$ December; Day of Week = Saturday EMISFACT 224U_7 MHRDOW7 000000000000 EMISFACT 224U 7 MHRDOW7 $00 \begin{array}{lllllll}0 & 0 & 0 & 0 & 0 & 0 & 0\end{array}$

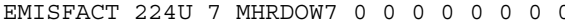

* Month $=$ January; Day of week = Sunday 
EMISFACT 224U_7 MHRDOW7 0000000000 EMISFACT 224U 7 MHRDOW7 $00 \begin{array}{lllllll}0 & 0 & 0 & 0 & 0 & 0 & 0\end{array}$ EMISFACT 224U 7 MHRDOW7 00000

* * Month = Februaryi Day of Week = Sunday Month a Pebruary; Day or week = sunday

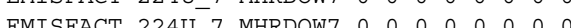
EMISFACT 224_- MHRW

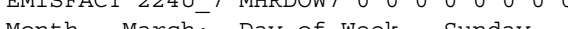
* Month $=$ March; Day of Week = Sunday EMISFACT 224U_7 MHRDOW7 00000000000 EMISFACT 224U_7 MHRDOW7 $000 \begin{array}{lllllllll}0 & 0 & 0 & 0 & 0 & 0 & 0\end{array}$ EMISFACT 224U_7 MHRDOW7 $000 \begin{array}{lllllll}0 & 0 & 0 & 0 & 0 & 0 & 0\end{array}$

** Month $=$ April; Day of Week = Sunday EMISFACT 224U_7 MHRDOW7 0 O $00 \begin{array}{lllllll} & 0 & 0 & 0 & 0\end{array}$ EMISFACT 224U 7 MHRDOW7 $00 \begin{array}{lllllll}0 & 0 & 0 & 0 & 0 & 0 & 0\end{array}$ EMISFACT 224U 7 MHRDOW7 000 O

** Month = May; Day of Week = Sunday

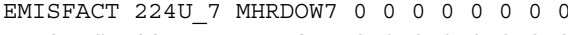

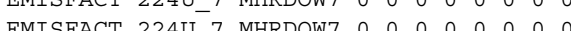
EMISFACT 2240_7 MHRDOW7 $0 \begin{array}{lllllllllllllllll}0 & 0 & 0 & 0 & 0 & 0 & 0\end{array}$ * Month $=$ June; Day of Week = Sunday EMISFACT 224U_7 MHRDOW7 0000000000 EMISFACT 224U_7 MHRDOW7 000000000000 EMISFACT 224U_7 MHRDOW7 000000000000

** Month $=$ July; Day of week = Sunday

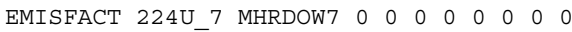
EMISFACT 224U_7 MHRDOW7 $00 \begin{array}{lllllll}0 & 0 & 0 & 0 & 0 & 0 & 0\end{array}$ EMISFACT 224U 7 MHRDOW7 0 C

** Month = August, Day of Week = Sunday

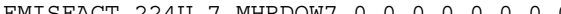
EMISFACT 224U-7 MHRDOW7 0000000000 BMISFAC 224 7 -

EMISFACT 224__ ** Month $=$ September $;$ Day of Week = Sunday EMISFACT 224U_7 MHRDOW7 000100000000

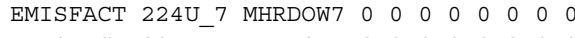
EMISFACT 224U_7 MHRDOW7 00000000000

* Month $=$ October; Day of Week = Sunday EMISFACT 224U_7 MHRDOW7 00000000000 EMISFACT 224U_7 MHRDOW7 $00 \begin{array}{lllllll}0 & 0 & 0 & 0 & 0 & 0 & 0\end{array}$ EMISFACT 224U 7 MHRDOW7 $00 \begin{array}{ccccccc}0 & 0 & 0 & 0 & 0 & 0 & 0\end{array}$

** Month $=$ November; Day of Week = Sunday EMISFACT 224U_7 MHRDOW7 00000000000

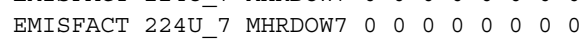

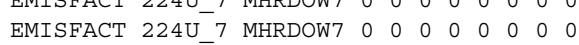

* Month $=$ December; $;$ Day of Week = Sunday

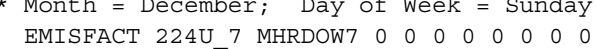

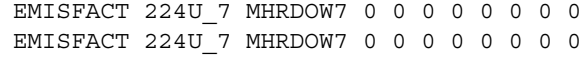

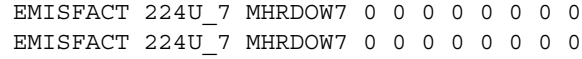

* Month = January; Day of Week = Monday EMISFACT 224U_8 MHRDOW7 0000000000 EMISFACT 224U_8 MHRDOW7 0000000000000

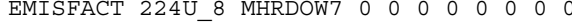
$*$ Month $=$ February; Day of Week = Monday

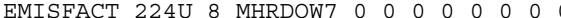


EMISFACT 224U_8 MHRDOW7 000000000000

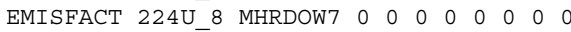

** Month $=$ March; Day of Week = Monday

EMISFACT 224U_8 MHRDOW7 000000000000 EMTSFACT 224U-8 MHRDOW7 000000000 EMISFACT 224U 8 MHRDOW7 $000 \begin{array}{lllllll}-8 & 0 & 0 & 0 & 0 & 0 & 0\end{array}$

** Month = April; $;$ Day of Week = Monday

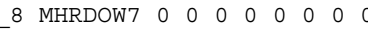
EMISFACT 224U_8 MHRDOW7 000000000000 EMISFACT 224U_8 MHRDOW7 00000000000

** Month $=$ May; Day of Week = Monday

EMISFACT 224U_8 MHRDOW7 0000000000

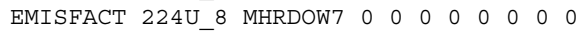
EMISFACT 224U_8 MHRDOW7 00000000000

** Month $=$ June; Day of Week $=$ Monday

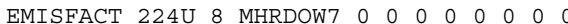
EMTSFACT 224U_ 8 MHRDOW7 00000000000 EMISFCT 224.

* Month = July; Day of Week = Monday

* Month $=$ July; Day of Week = Monday

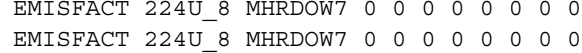

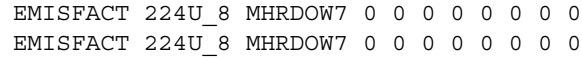

* Month $=$ August; Day of Week = Monday EMISFACT 224U_8 MHRDOW7 000000000

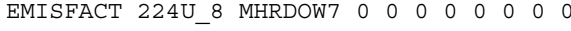
EMISFACT 224U_8 MHRDOW7 $000 \begin{array}{lllllll}0 & 0 & 0 & 0 & 0 & 0\end{array}$

* Month $=$ September $;$ Day of Week $=$ Monday EMISFACT 224U 8 MHRDOW7 00000000000

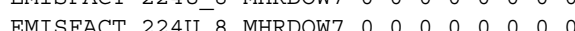

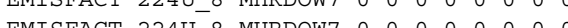

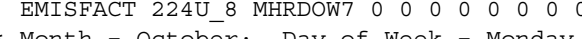
* Month = October; Day of Week = Monday

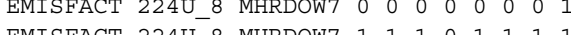

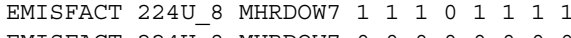
EMISFACT 224U_8 MHRDOW7 000000000

* Month $=$ November; Day of Week = Monday EMISFACT 224U_8 MHRDOW7 $00 \begin{array}{lllllll}0 & 0 & 0 & 0 & 0 & 0 & 1\end{array}$

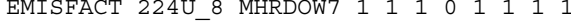
EMISFACT 224U_8 MHRDOW7 $000 \begin{array}{llllll}0 & 0 & 0 & 0 & 0 & 0\end{array}$

$* *$ Month $=$ December; $;$ Day of Week = Monday EMISFACT 224U 8 MHRDOW7 $000 \begin{array}{lllllll}0 & 0 & 0 & 0 & 0 & 0 & 1\end{array}$

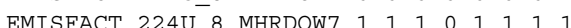
FMISFACT 224U_ MHRDOW7 0 o 00000

* EMISFACT 2240_8 MHRDOW7 0 O 000000 * Month = January; Day of Week = Tuesday EMISFACT 224U_8 MHRDOW7 $0 \begin{array}{ccccccc}0 & 0 & 0 & 0 & 0 & 0 & 0\end{array}$

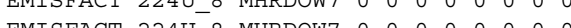

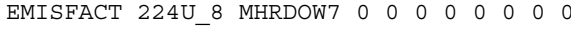

* Month $=$ February; Day of Week = Tuesday EMISFACT 224U_8 MHRDOW7 00000000000 EMISFACT 224U_8 MHRDOW7 $00 \begin{array}{lllllll}0 & 0 & 0 & 0 & 0 & 0 & 0\end{array}$

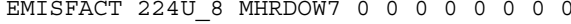

$*$ Month $=$ March; Day of Week = Tuesday

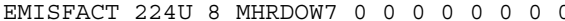

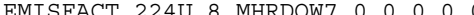


EMISFACT 224U_8 MHRDOW7 000000000

** Month $=$ April; Day of Week = Tuesday

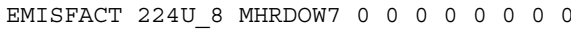

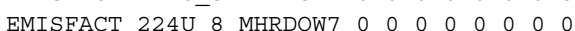

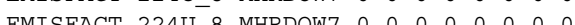

** Month = May; Day of Week $=$ Tuesday

EMISFACT 224U_8 MHRDOW7 0000000000

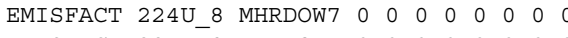
EMISFACT 224U_8 MHRDOW7 00000000000

* Month $=$ June; Day of Week = Tuesday

EMISFACT 224U_8 MHRDOW7 0000000000

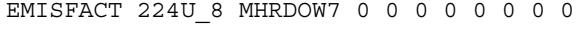

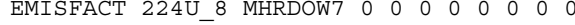

** Month $=$ July; Day of Week = Tuesday EMISFACT 224U_8 MHRDOW7 000000000000 EMISFACT 224U_8 MHRDOW7 00000000000 EMISFACT 224U 8 MHRDOW7 00000000000

* Month = August; Day of Week = Tuesday EMISFACT 224U_8 MHRDOW7 00000000000 EMISFACT 224__8 MHRDOW7 0000000000000 EMISFACT 224U_8 MHRDOW7 00000000000

* Month $=$ September; Day of Week = Tuesday EMISFACT 224U_8 MHRDOW7 00000000000 EMISFACT 224U_8 MHRDOW7 0000000000000 EMISFACT 224U_8 MHRDOW7 00000000000

** Month $=$ October; Day of Week $=$ Tuesday

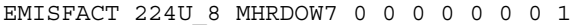
EMISFACT 224U 8 MHRDOW7 $110 \begin{array}{llllllllllll}1 & 1 & 0 & 1 & 1 & 1 & 1\end{array}$ EMISFACT 224U-8 MHRDOW7 00000000

* Month = Novembi Day of Week = Tuesa Month = November; Day of Week Tuesday EMISFACT 224UBMTSACT 2240_8 MHRDOW7 $\begin{array}{lllllll}1 & 1 & 0 & 1 & 1 & 1 & 1\end{array}$

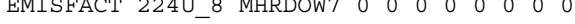

** Month $=$ December; Day of Week = Tuesday

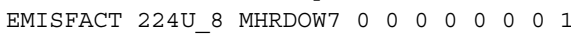

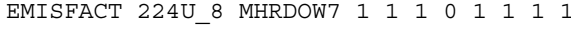

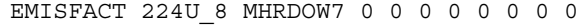

** Month $=$ January; Day of Week $=$ Wednesday EMISFACT 224U_8 MHRDOW7 00000000000

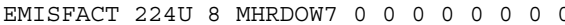
EMISFACT 224U 8 MHRDOW7 000000000

** Month $=$ February; Day of Week $=$ Wednesday EMISFACT $224 \mathrm{U}$

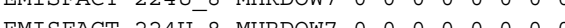
EMISFACT 224__ * Month $=$ March; Day of Week $=$ Wednesday EMISFACT 224U_8 MHRDOW7 0000000000 EMISFACT 224U_8 MHRDOW7 $0 \begin{array}{lllllll}0 & 0 & 0 & 0 & 0 & 0 & 0\end{array}$ EMISFACT 224U_8 MHRDOW7 $0 \begin{array}{llllllll}0 & 0 & 0 & 0 & 0 & 0 & 0\end{array}$

** Month $=$ April; Day of Week = Wednesday EMISFACT 224U 8 MHRDOW7 $000 \begin{array}{lllllll}0 & 0 & 0 & 0 & 0 & 0\end{array}$ EMISFACT 224U 8 MHRDOW7 000 C EMISFACT 224U 8 MHRDO 
** Month $=$ May; Day of Week = Wednesday EMISFACT 224U_8 MHRDOW7 00000000000 EMISFCT 224U EMISFACT 2240_8 MHRDOW7 $00 \begin{array}{ccccccc}0 & 0 & 0 & 0 & 0 & 0 & 0\end{array}$ ** Month $=$ June; $;$ Day of Week = Wednesday EMISFACT 224U_8 MHRDOW7 00000000000 EMISFACT 224_-8 MHRDW EMISFACT 224U_8 MHRDOW7 000000000

** Month $=$ July; Day of Week = Wednesday EMISFACT 224U_8 MHRDOW7 00000000000 EMISFACT 224U_8 MHRDOW7 000000000000

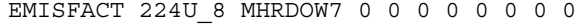

** Month $=$ August; Day of Week $=$ Wednesday EMISFACT 224U 8 MHRDOW7 00000000000 EMISFACT 224U 8 MHRDOW7 0001000100000

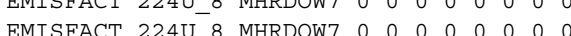

* Month = September; Day of Week = Wednesday EMISFACT 224U 8 MHRDOW7 000000000000 EMISFACT 224U 8 MHRDOW7 001000000000 EMISFACT 224U 8 MHRDOW7 000000000000

* Month = October; Day of Week = Wednesday EMISFACT 224U_8 MHRDOW7 0000000001 EMISFACT 224U_8 MHRDOW7 $1 \begin{array}{lllllll}1 & 1 & 0 & 1 & 1 & 1 & 1\end{array}$ EMISFACT 224U_8 MHRDOW7 001000000000

$* *$ Month $=$ November $;$ Day of Week $=$ Wednesday EMISFACT 224U_8 MHRDOW7 $000 \begin{array}{llllll}0 & 0 & 0 & 0 & 0 & 1\end{array}$

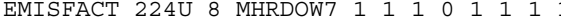

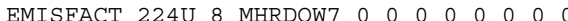

** Month $=$ December; Day of Week $=$ Wednesday EMISFACT 224U_8 MHRDOW7 000000000001

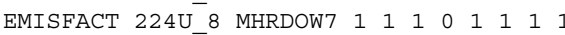
EMISFACT 224U 8 MHRDOW7 $00 \begin{array}{llllllll}-0 & 0 & 0 & 0 & 0 & 0 & 0\end{array}$

** Month = January; Day of Week = Thursday EMISFACT 224U_8 MHRDOW7 00000000000

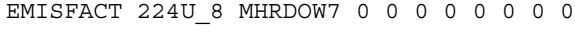
EMISFACT 224U_8 MHRDOW7 00000000000

** Month $=$ February; Day of Week $=$ Thursday EMISFACT 224U_8 MHRDOW7 0000000000000 EMISFACT 224U_8 MHRDOW7 $00 \begin{array}{lllllll}0 & 0 & 0 & 0 & 0 & 0 & 0\end{array}$

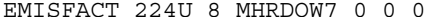

** Month = MarchTMTSFACT 224U 8 MHRDOH7 00000000 DMTSEACT 224U-

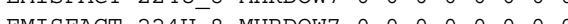

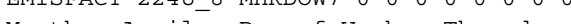

** Month = April; Day of Week = Thursday EMISFACT 224U_8 MHRDOW7 000000000 EMISFACT 224U_8 MHRDOW7 000000000000 EMISFACT 224U_8 MHRDOW7 00000000000

$* *$ Month $=$ May; Day of Week $=$ Thursday EMISFACT 224U_8 MHRDOW7 00000000000 EMISFACT 224U_8 MHRDOW7 $00 \begin{array}{lllllll}0 & 0 & 0 & 0 & 0 & 0 & 0\end{array}$ EMISFACT 224U 8 MHRDOW7 000 O

* Month $=$ June; Day of Week $=$ Thursday 
EMISFACT 224U_8 MHRDOW7 0000000000 EMISFACT 224U 8 MHRDOW7 $000 \begin{array}{lllllll}0 & 0 & 0 & 0 & 0 & 0 & 0\end{array}$ EMISFACT 224U 8 MHRDOW7 000

** Month = Julyi Day of Week = Thursday Month = July; Day or Week = Thursday EMISFACT 2240

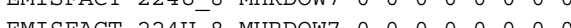
EMISFACT 2240_8 MHRDOW7 000000000000

** Month = August; Day of Week = Thursday EMISFACT 224U_8 MHRDOW7 00000000000 EMISFACT 224U_8 MHRDOW7 000000000000 EMISFACT 224U_8 MHRDOW7 $000 \begin{array}{lllllll}- & 0 & 0 & 0 & 0 & 0 & 0\end{array}$

** Month $=$ September $;$ Day of Week $=$ Thursday EMISFACT 224U_8 MHRDOW7 000000000000

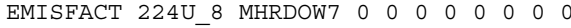

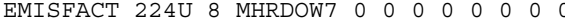

* Month = October; Day of Week = Thursday EMISFACT 2240 , MHRD 700000

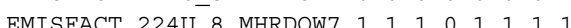

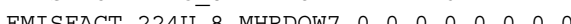

EMISFACT 2240_8 MHRDOW7 $0 \begin{array}{llllllll}0 & 0 & 0 & 0 & 0 & 0 & 0\end{array}$

* Month = November; Day of week = Thursday EMISFACT 224U_8 MHRDOW7 000000001 EMISFACT 224U_8 MHRDOW7 $11110 \begin{array}{lllllll}1 & 0 & 1 & 1 & 1 & 1\end{array}$ EMISFACT 224U_8 MHRDOW7 00000000000

** Month $=$ December; Day of Week = Thursday EMISFACT 224U_8 MHRDOW7 $00 \begin{array}{lllllll}0 & 0 & 0 & 0 & 0 & 0 & 1\end{array}$

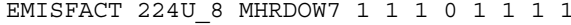

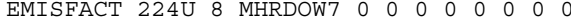

** Month = January; Day of Week = Friday TMTSEACT 224U EMTSFACT 224U_ 8 MHRDOW7 0000000000 BMISFACT 224

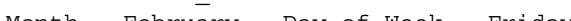

** Month = February; Day of Week = Friday EMISFACT 224U_8 MHRDOW7 $000 \begin{array}{llllll}0 & 0 & 0 & 0 & 0 & 0\end{array}$ EMISFACT 224U_8 MHRDOW7 00000000000 EMISFACT 224U_8 MHRDOW7 0000000000

** Month $=$ March; Day of Week = Friday EMISFACT 224U_8 MHRDOW7 0000000000

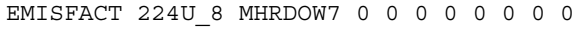
EMISFACT 224U_8 MHRDOW7 $00 \begin{array}{llllllll}0 & 0 & 0 & 0 & 0 & 0 & 0\end{array}$

** Month $=$ April; Day of Week = Friday EMISFACT 224U_8 MHRDOW7 0000000000 EMISFACT 224U-8 MHRDOW7 000000000000 EMISFACT 224U 8 MHRDOW7 0000000000 ** Month = May; Day of week = Friday EMISFACT 224U_8 MHRDOW7 00000000000 EMISFACT 224U_8 MHRDOW7 $000 \begin{array}{lllllll}0 & 0 & 0 & 0 & 0 & 0 & 0\end{array}$ EMISFACT 224U_8 MHRDOW7 0000000000

** Month $=$ June; Day of Week = Friday EMISFACT 224U_8 MHRDOW7 00000000000 EMISFACT 224U_8 MHRDOW7 $00 \begin{array}{lllllll}0 & 0 & 0 & 0 & 0 & 0 & 0\end{array}$ EMISFACT 224U 8 MHRDOW7 $00 \begin{array}{llllllllll}0 & 0 & 0 & 0 & 0 & 0 & 0\end{array}$ ** Month $=\mathrm{July} ;$ Day of Week = Friday EMISFACT 224U 8 MHRDOW7 0000000000 
EMISFACT 224U_8 MHRDOW7 000000000000

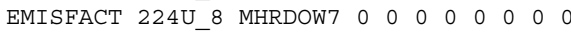

** Month $=$ August $; \quad$ Day of Week = Friday

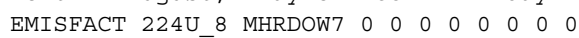

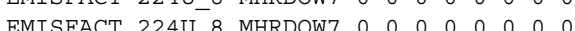
MISTCT 2240

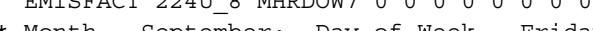

** Month = September $;$ Day of Week = Frida EMISFACT 224U_8 MHRDOW7 00000000000 EMISFACT 224U_8 MHRDOW7 000000000000 EMISFACT 224U_8 MHRDOW7 $000 \begin{array}{llllll}0 & 0 & 0 & 0 & 0 & 0\end{array}$

** Month $=$ October; Day of Week = Friday EMISFACT 224U_8 MHRDOW7 00000000001

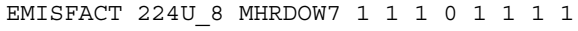
EMISFACT 224U 8 MHRDOW7 $00 \begin{array}{lllllllll}0 & 0 & 0 & 0 & 0 & 0 & 0\end{array}$

** Month $=$ November; Day of Week = Friday EMISFACT 224U_8 MHRDOW7 000000000001 224U 8 MHRDOW7 111110011111 224U 8 MHRDOW7 00000000000

** Month $=$ December; Day of Week = Friday EMISFACT 224U_8 MHRDOW7 00000000000

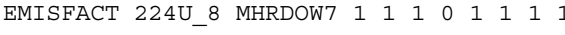
EMISFACT 224U_8 MHRDOW7 00000000000

* Month = January; Day of Week = Saturday EMISFACT 224U_8 MHRDOW7 000000000000 EMISFACT 224U_8 MHRDOW7 000000000000

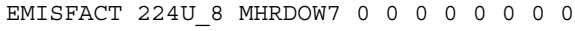

** Month $=$ February; Day of Week = Saturday EMISFACT 224U 8 MHRDOW7 00000000000

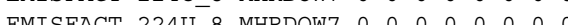

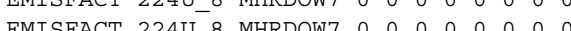

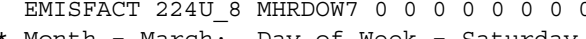
* Month = March; Day of Week = Saturday EMISFACT 2240_8 MHRDOW7 $0 \begin{array}{lllllll}0 & 0 & 0 & 0 & 0 & 0 & 0\end{array}$

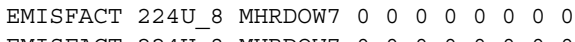
EMISFACT 224U_8 MHRDOW7 0000000000

* Month = April; Day of Week = Saturday EMISFACT 224U_8 MHRDOW7 $000 \begin{array}{lllllll}0 & 0 & 0 & 0 & 0 & 0\end{array}$

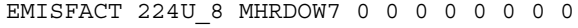

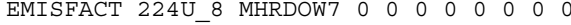

* Month = May; Day of Week = Saturday

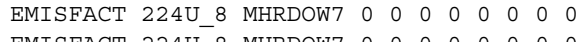
EMISTACT $2240-8$ M EMISEACT 2240_8 MHRDOW7 $0 \begin{array}{lllllllll}0 & 0 & 0 & 0 & 0 & 0 & 0\end{array}$ * Month = June, Day of Week = Saturday EMISFACT 2240_8 MHRDOW7 0000000000 EMISFACT 2240_8 MURDOW7 $0 \begin{array}{llllllll}0 & 0 & 0 & 0 & 0 & 0 & 0\end{array}$ EMISFACT 224U_8 MHRDOW7 $000 \begin{array}{llllll}0 & 0 & 0 & 0 & 0 & 0\end{array}$ * Month $=$ July; Day of Week = Saturday EMISFACT 224U_8 MHRDOW7 0000000000

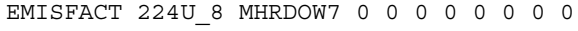

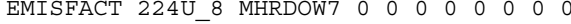

* Month = August; Day of Week = Saturday

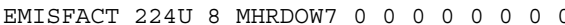
EMISFACT 224U 8 MHRDOW7 0 
EMISFACT 224U_8 MHRDOW7 000000000000

** Month $=$ September; Day of Week = Saturday EMISFACT 224U_8 MHRDOW7 0000000000 4U 8 MHRDOW7 00 EMISFACT 224U_8 MHRDOW7 000000000000

** Month = October; Day of Week = Saturday

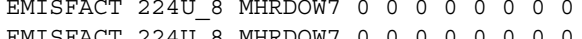
EMISFACT 2240_8 MHRDOW7 000000000000 EMISFACT 224U_8 MHRDOW7 $00 \begin{array}{lllllll}0 & 0 & 0 & 0 & 0 & 0 & 0\end{array}$

* Month = November; Day of Week = Saturday EMISFACT 224U_8 MHRDOW7 00000000000

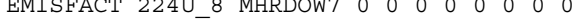
EMISFACT 224U_8 MHRDOW7 $00 \begin{array}{llllllll}0 & 0 & 0 & 0 & 0 & 0 & 0\end{array}$

** Month $=$ December; Day of Week = Saturday EMISFACT 224U_8 MHRDOW7 000000000000

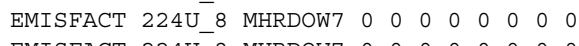
EMISFACT 224U 8 MHRDOW7 00000000000

* Month = January; Day of Week = Sunday EMISFACT 224U_8 MHRDOW7 00000000000

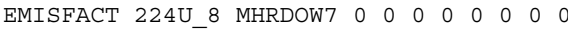

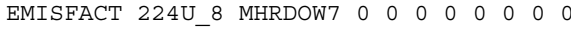

* Month = February; Day of Week = Sunday EMISFACT 224U 8 MHRDOW7 00000000000 EMISFACT 224U_8 MHRDOW7 $0 \begin{array}{llllllll}0 & 0 & 0 & 0 & 0 & 0 & 0 & 0\end{array}$ EMISFACT 224U_8 MHRDOW7 $000 \begin{array}{lllllll}0 & 0 & 0 & 0 & 0 & 0 & 0\end{array}$

** Month $=$ March; Day of Week = Sunday EMISFACT 224U_8 MHRDOW7 000000000000 EMISFACT 224U_8 MHRDOW7 000000000000 EMISFACT 224U 8 MHRDOW7 00000000000

* Month = April; Day of Week = Sunday EMISFACT 224U 8 MHRDOW7 00000000000

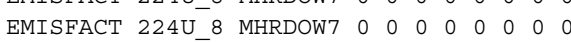
EMISFACT 224U_8 MHRDOW7 $000 \begin{array}{lllllll}- & 0 & 0 & 0 & 0 & 0 & 0\end{array}$

** Month $=$ May; Day of Week = Sunday EMISFACT 224U_8 MHRDOW7 000000000 EMISFACT 224U_8 MHRDOW7 00000000000 EMISFACT 224U_8 MHRDOW7 $00 \begin{array}{lllllll}0 & 0 & 0 & 0 & 0 & 0 & 0\end{array}$

** Month $=$ June; Day of Week = Sunday EMISFACT 224U_8 MHRDOW7 0000000000

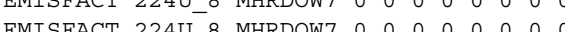

* Month $=$ July; Day of Week = Sunday

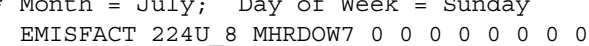
EMISFACT 224U_8 MHRDOW7 000000000000 EMISFACT 224U 8 MHRDOW7 00000000000 EMISFACT 224U_8 MHRDOW7 00000000
Month = Auqust; Month = August $; \quad$ Day of Week = Sunday
EMISFACT 224U 8 MHRDOW7 000000000

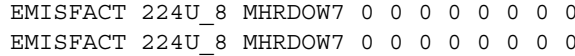
EMISFACT 224U_8 MHRDOW7 00000000000

** Month $=$ September; Day of Week = Sunday EMISFACT 224U 8 MHRDOW7 $00 \begin{array}{llllllll}0 & 0 & 0 & 0 & 0 & 0 & 0\end{array}$

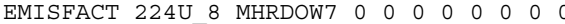
EMISFACT 224U 8 MHRDOW 
** Month $=$ October; Day of Week = Sunday EMISFACT 224U 8 MHRDOW7 0 O $00 \begin{array}{lllll}0 & 0 & 0 & 0 & 0\end{array}$ EMISFACT 224U_8 MHRDOW7 0 C

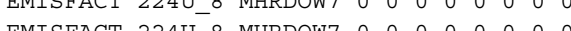

EMISAC 2240_8 * * Month = November; Day of Week = Sunday EMISFACT 2240_-8 MHROW7 000000000 EMISFACT 224U_- MRDOW7 $0 \begin{array}{lllllllll}0 & 0 & 0 & 0 & 0 & 0 & 0\end{array}$ EMISFACT 2240_8 MHRDOW7 $0 \begin{array}{lllllll}0 & 0 & 0 & 0 & 0 & 0 & 0\end{array}$

* Month $=$ December; Day of Week = Sunday EMISFACT 224U_8 MHRDOW7 000000000

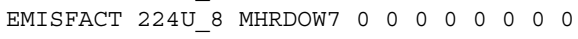

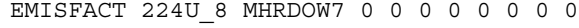

* Month $=$ January; Day of Week = Monday EMISFACT 224U 9 MHRDOW7 0 O $00 \begin{array}{llllll}0 & 0 & 0 & 0 & 0\end{array}$ EMISFACT 224U 9 MHRDOW7 $00 \begin{array}{lllllllllllll}0 & 0 & 0 & 0 & 0 & 0 & 0 & 0\end{array}$

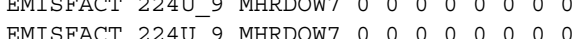

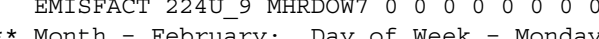
Month = February; Day of Week = Monday

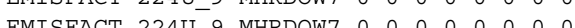
EMISFACT 2240-9 MHRDOW7 $0 \begin{array}{llllllllllllll}0 & 0 & 0 & 0 & 0 & 0 & 0\end{array}$

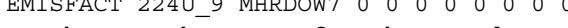
* Month $=$ March; Day of Week = Monday

EMISFACT 224U_9 MHRDOW7 0000000000 EMISFACT 224U_9 MHRDOW7 $00 \begin{array}{lllllllllllll}0 & 0 & 0 & 0 & 0 & 0 & 0\end{array}$ EMISFACT 224U_9 MHRDOW7 $00 \begin{array}{llllllll}0 & 0 & 0 & 0 & 0 & 0 & 0\end{array}$

$*$ Month $=$ April $;$ Day of Week = Monday EMISFACT 224U_9 MHRDOW7 0000000000

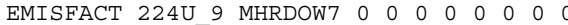
EMISFACT 224U

** Month = May; Day of Week = Monday

EMISFACT 224U_9 MHRDOW7 00000000000 EMISFACT 224U_9 MHRDOW7 00000000000

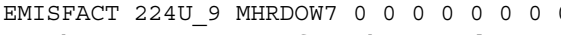

** Month $=$ June; Day of Week = Monday

EMISFACT 224U_9 MHRDOW7 0000000000 EMISFACT 224U_9 MHRDOW7 00000000000 EMISFACT 224U_9 MHRDOW7 00000000000

** Month $=$ July; Day of Week = Monday

EMISFACT 224U_9 MHRDOW7 0000000000 EMISFACT 224U_9 MHRDOW7 $00 \begin{array}{lllllll}0 & 0 & 0 & 0 & 0 & 0 & 0\end{array}$ EMISFACT 224U_9 MHRDOW7 0 o 00

** Month = August; $;$ Day of Week = Monday

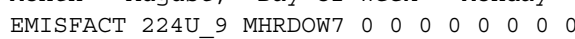

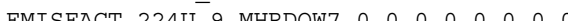

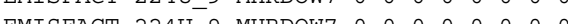
EMISFACI 2240_9 MRDOW7 $0 \begin{array}{llllllllllll}0 & 0 & 0 & 0 & 0 & 0\end{array}$

* Month = September; Day of Week = Monday EMISFACT 224U_9 MHRDOW7 00000000000 EMISFACT 224U_9 MHRDOW7 000000000000 EMISFACT 224U_9 MHRDOW7 0000000000

** Month = October; Day of Week = Monday EMISFACT 224U 9 MHRDOW7 000000000001 EMISFACT 224U_9 MHRDOW7 $1 \begin{array}{lllllllll}1 & 1 & 1 & 0 & 1 & 1 & 1 & 1\end{array}$ EMISFACT 224U 9 MHRDOW7 00010010000

* Month $=$ November; Day of Week $=$ Monday 


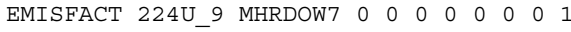

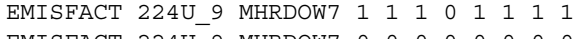
EMISFACT 224U_9 MHRDOW7 $00 \begin{array}{lllllll}0 & 0 & 0 & 0 & 0 & 0 & 0\end{array}$

** Month $=$ December; Day of Week = Monday EMISFACT 224U_9 MHRDOW7 000000000001 EMISFACI 224U_9 MHRDOW7 $\begin{array}{cccccccc}1 & 1 & 1 & 0 & 1 & 1 & 1 & 1\end{array}$

EMISFACT 2240_9 MRDOW7 00000000000

* Month = January; Day of Week = Tuesday EMISFACT 224U_9 MHRDOW7 00000000000

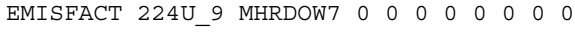
EMISFACT 224U_9 MHRDOW7 $000 \begin{array}{lllllll}0 & 0 & 0 & 0 & 0 & 0 & 0\end{array}$

** Month $=$ February; Day of Week $=$ Tuesday EMISFACT 224U_9 MHRDOW7 00000000000 EMISFACT 224U 9 MHRDOW7 $000 \begin{array}{llllllll}0 & 0 & 0 & 0 & 0 & 0 & 0 & 0\end{array}$ EMISFACT 224U 9 M

** Month = March; Day of Week = Tuesday EMISFACT $224 \mathrm{U}$ ( MHRDOW7 000000000

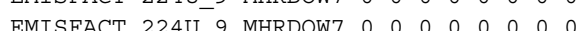
MISFC EMISFACT 2240_9 MHRDOW7 $0 \begin{array}{lllllllllll}0 & 0 & 0 & 0 & 0 & 0 & 0\end{array}$

** Month = April; Day of Week = Tuesday EMISFACT 224U_9 MHRDOW7 00000000000 EMISFACT 224U_9 MHRDOW7 00000000000 EMISFACT 224U_9 MHRDOW7 0000000000

$* *$ Month $=$ May; Day of Week $=$ Tuesday

EMISFACT 224U_9 MHRDOW7 000000000

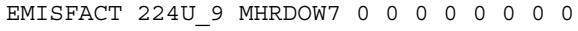
EMISFACT 224U 9 MHRDOW7 0 C

i $\quad * *$ Month $=$ June; Day of Week $=$ Tuesday EMISFACT 224UMISFACT 2240

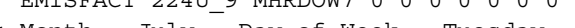
* Month = July; Day of Week = Tuesday EMISFACT 224U_9 MHRDOW7 0000000000000 EMISFACT 224U_9 MHRDOW7 000000000000 EMISFACT 224U_9 MHRDOW7 0000000000

** Month = August; $;$ Day of week = Tuesday EMISFACT 224U_9 MHRDOW7 00000000000

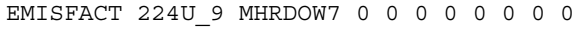

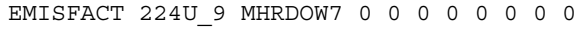

** Month $=$ September; Day of Week = Tuesday

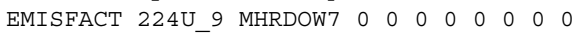
EMISFACT 224U-9 MHRDOW7 0000000000 EMTSEACT 224U-9 MHRDOW7 00000

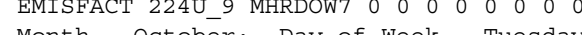
* Month = October; Day of Week = Tuesday

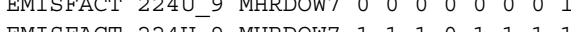
EMISFACT 224U_9 MHRDOW7 $1 \begin{array}{lllllllll}1 & 1 & 1 & 0 & 1 & 1 & 1 & 1\end{array}$ EMISFACT 224U_9 MHRDOW7 0000000000

* Month = November; Day of Week = Tuesday EMISFACT 224U_9 MHRDOW7 00000000001 $\begin{array}{llllllllll}\text { EMISFACT } 224 U-9 & \text { MHRDOW7 } & 1 & 1 & 1 & 0 & 1 & 1 & 1 & 1 \\ \text { EMISFACT } 224 U-9 & \text { MHRDOW7 } & 0 & 0 & 0 & 0 & 0 & 0 & 0 & 0\end{array}$

Month = December $;$ Day of Week = Tuesday

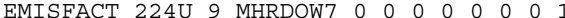




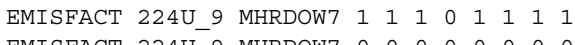
EMISFACT 224U_9 MHRDOW7 000000100000

** Month = January; Day of Week = Wednesday EMISPCT 224U_- MHRDOW7 000000000000 EMTSFACT 224U

* Month = February; Day of Week = Wednesday EMISFACT 224U_9 MHRDOW7 000000000 EMISFACT 224U_9 MHRDOW7 000000000000 EMISFACT 224U_9 MHRDOW7 $000 \begin{array}{lllllll}- & 0 & 0 & 0 & 0 & 0 & 0\end{array}$

* Month $=$ March; Day of Week $=$ Wednesday EMISFACT 224U_9 MHRDOW7 00000000000

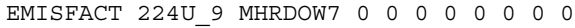
EMISFACT 224U 9 MHRDOW7 $00 \begin{array}{llllllllll}0 & 0 & 0 & 0 & 0 & 0 & 0\end{array}$

** Month $=$ April; Day of Week $=$ Wednesday EMISFACT 224U_9 MHRDOW7 00000000000 EMISFACT 224U_9 MHRDOW7 000000000000 EMISFACT 224U

* Month = May; Day of Week = Wednesday

Month = May; Day of Week = Wednesday
EMISFACT 224U 9 MHRDOW7 0000000000 $\begin{array}{llllllllll}\text { EMISFACT } 224 U-9 & \text { MHRDOW7 } & 0 & 0 & 0 & 0 & 0 & 0 & 0 & 0 \\ \text { EMISFACT } 224 \mathrm{U} 9 & \text { MHRDOW7 } & 0 & 0 & 0 & 0 & 0 & 0 & 0 & 0\end{array}$ $\begin{array}{lllllllllll}\text { EMISFACT } & 224 U_{-9} 9 & \text { MHRDOW7 } & 0 & 0 & 0 & 0 & 0 & 0 & 0 & 0 \\ \text { EMISFACT } & 224 U_{-} 9 & \text { MHRDOW7 } & 0 & 0 & 0 & 0 & 0 & 0 & 0 & 0\end{array}$

* Month $=$ June; Day of Week $=$ Wednesday EMISFACT 224U_9 MHRDOW7 000000000 EMISFACT 224U_9 MHRDOW7 $00 \begin{array}{lllllll}0 & 0 & 0 & 0 & 0 & 0 & 0\end{array}$ EMISFACT 224U_9 MHRDOW7 00000000000

* Month = July; Day of Week = Wednesday EMISFACT 224U 9 MHRDOW7 0000000 EMISFACT $224 U_{-}$ EMISFCC 2240_-

EMISFACT 224U_9 MHRDOW7 $00 \begin{array}{ccccccc}0 & 0 & 0 & 0 & 0 & 0 & 0\end{array}$ * Month = August; Day of Week = Wednesda EMISFACT 224U_9 MHRDOW7 000000000 EMISFACT 224U_9 MHRDOW7 00000000000 EMISFACT 224U_9 MHRDOW7 00000000000

* Month $=$ September $;$ Day of Week $=$ Wednesday EMISFACT 224U_9 MHRDOW7 0000000000 EMISFACT 224U_9 MHRDOW7 $00 \begin{array}{lllllll}0 & 0 & 0 & 0 & 0 & 0 & 0\end{array}$ EMISFACT 224U_9 MHRDOW7 00000000000

** Month $=$ October; Day of Week = Wednesday

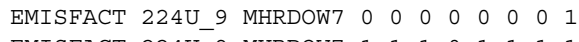
EMISFACT 224U_9 MHRDOW7 $11110 \begin{array}{llllllll}1 & 1 & 1 & 1 & 1 & 1\end{array}$ EMISFACT 224U 9 MHRDOW7 0000000000

** Month $=$ November; Day of Week = Wednesday

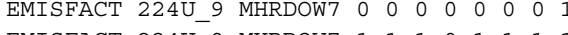
EMISFACT 224U-9 MHRDOW7 $00 \begin{array}{llllllll}0 & 0 & 0 & 0 & 0 & 0 & 0\end{array}$

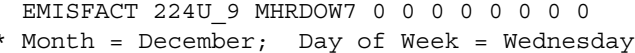
EMISFACT 224U_9 MHRDOW7 $0 \begin{array}{lllllll}0 & 0 & 0 & 0 & 0 & 0 & 1\end{array}$

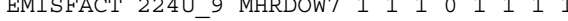
EMISFACT 224U_9 MHRDOW7 $000 \begin{array}{llllll}0 & 0 & 0 & 0 & 0 & 0\end{array}$

** Month = January; Day of Week = Thursday 
EMISFACT 224U_9 MHRDOW7 00000000000

** Month $=$ February; Day of week $=$ Thursday EMISFACT 224U_9 MHRDOW7 00000000000

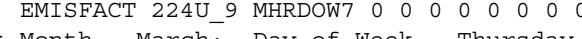
* Month $=$ March; $;$ Day of Week = Thursday EMISFACT 2240 - MHRDOW7 $0 \begin{array}{lllllll}0 & 0 & 0 & 0 & 0 & 0 & 0\end{array}$ EMISFACT 2240_9 MHRDOW7 000000000000

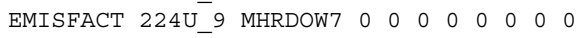

* Month = April; Day of Week = Thursday EMISFACT 224U_9 MHRDOW7 00000000000

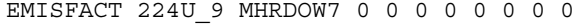

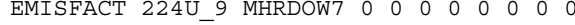

** Month = May; Day of Week = Thursday EMISFACT 224U_9 MHRDOW7 00000000000 EMISFACT 224U_9 MHRDOW7 00000000000 EMISFACT 224U_9 MHRDOW7 000000000000

* Month = June; Day of Week = Thursday

Month = June; Day of Week = Thursday
EMISFACT 224U 9 MHRDOW7 00000000000 EMISFACT 224U_9 MHRDOW7 000000000000 EMISFACT 224U_9 MHRDOW7 0000000000

** Month $=$ July; Day of Week $=$ Thursday EMISFACT 224U_9 MHRDOW7 00000000000

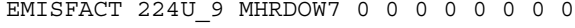
EMISFACT 224U_9 MHRDOW7 001000000000

** Month $=$ August; Day of Week = Thursday EMISFACT 224U 9 MHRDOW7 00000000000

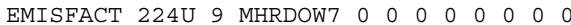
EMISFACT 224U-

** Month = September; Day of Week = Thursday EMISFACT 224U_9 MHRDOW7 000000000000

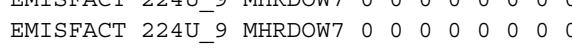
EMISFACT 224U 9 MHRDOW7 $00 \begin{array}{ccccccc}0 & 0 & 0 & 0 & 0 & 0 & 0\end{array}$

** Month $=$ October; Day of Week = Thursday EMISFACT 224U_9 MHRDOW7 $000 \begin{array}{llllll}0 & 0 & 0 & 0 & 0 & 1\end{array}$

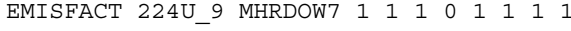

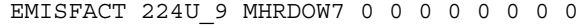

** Month $=$ November; Day of Week = Thursday

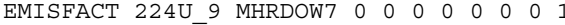
EMISFACT 224U_9 MHRDOW7 $1 \begin{array}{llllllll}1 & 1 & 0 & 1 & 1 & 1 & 1\end{array}$

* Monthe Month = Deceris DMTSFACT 2240EMISFACT 2240_9 MRRDW $\begin{array}{llllllll}1 & 1 & 1 & 0 & 1 & 1 & 1 & 1\end{array}$ EMISFACT 2240 * Month = January; Day of Week = Friday EMISFACT 224U_9 MHRDOW7 0000000000 EMISFACT 224U_9 MHRDOW7 $0 \begin{array}{lllllll}0 & 0 & 0 & 0 & 0 & 0 & 0\end{array}$

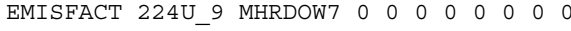

** Month = February; Day of Week = Friday

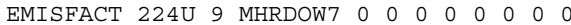
EMISFACT 224U 9 MHRDOW7 $00 \begin{array}{llllllllll}0 & 0 & 0 & 0 & 0 & 0 & 0 & 0\end{array}$ EMISFACT 224U 9 MHRDOW 
** Month $=$ March; Day of Week = Friday EMISFACT 224U_9 MHRDOW7 000000000000 EMISFACT 224U_9 MHRDOW7 $000 \begin{array}{lllllllll}0 & 0 & 0 & 0 & 0 & 0 & 0\end{array}$ EMISFACT 224U 9 MHRDOW7 00000000000

** Month $=$ April; $;$ Day of Week = Friday EMISFACT 224U_9 MHRDOW7 00000000000

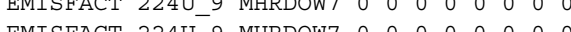
EMISFACT 2240_9 MHRDOW7 0000000000

** Month = May; Day of Week = Friday

EMISFACT 224U_9 MHRDOW7 0000000000 EMISFACT 224U_9 MHRDOW7 $000 \begin{array}{llllllll}- & 0 & 0 & 0 & 0 & 0 & 0\end{array}$

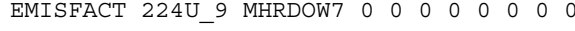

** Month $=$ June; Day of Week = Friday EMISFACT 224U_9 MHRDOW7 00000000000

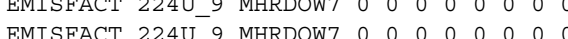
** Month $=$ July; Day of Week = Friday

* Month = July; Day of Week = Friday
EMISFACT 224U 9 MHRDOW7 000000000 EMISFACT 2240_- MHRDOW7 $0 \begin{array}{cccccccc}0 & 0 & 0 & 0 & 0 & 0 & 0\end{array}$ EMISFACT 2240_9 MHRDOW7 $\begin{array}{llllllllll}0 & 0 & 0 & 0 & 0 & 0 & 0 & 0\end{array}$ EMISFACT 2240_9 MHRDOW7 $\begin{array}{llllllllllll}0 & 0 & 0 & 0 & 0 & 0 & 0 & 0 & 0\end{array}$ * Month $=$ August; Day of Week = Friday EMISFACT 224U_9 MHRDOW7 0000000000 EMISFACT 224U_9 MHRDOW7 00000000000 EMISFACT 224U_9 MHRDOW7 00000000000

** Month $=$ September $;$ Day of Week $=$ Friday EMISFACT 224U_9 MHRDOW7 00000000000

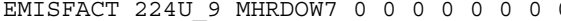
EMISFACT 224U 9 MHRDOW7 000

* Month = October; Day of Week = Friday DMTSEACT 224U 9 MHRDOW7 000000000 TMISFACT 224U INTSACT 224U

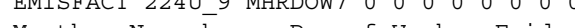

* Month $=$ November; Day of Week = Friday EMISFACT 224U_9 MHRDOW7 0000000001 EMISFACT 224U_9 MHRDOW7 $1 \begin{array}{llllllll}1 & 1 & 0 & 1 & 1 & 1 & 1\end{array}$ EMISFACT 224U_9 MHRDOW7 0000000000

* Month $=$ December; Day of Week = Friday

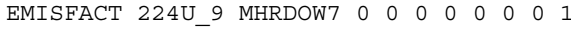

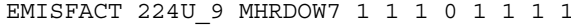

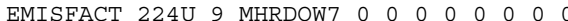

** Month = January; Day of Week = Saturday EMISFACT 224U

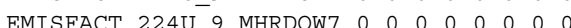
EMTSEACT 2240

* Month = February; Day of Week = Saturday EMISFACT 224U_9 MHRDOW7 0000000000 EMISFACT 224U_9 MHRDOW7 00000000000 EMISFACT 224U_9 MHRDOW7 $00 \begin{array}{llllllll}0 & 0 & 0 & 0 & 0 & 0 & 0\end{array}$

* Month $=$ March; Day of Week = Saturday EMISFACT 224U_9 MHRDOW7 $000 \begin{array}{llllll}0 & 0 & 0 & 0 & 0 & 0\end{array}$ EMISFACT 224U_9 MHRDOW7 $000 \begin{array}{cccccc}0 & 0 & 0 & 0 & 0 & 0\end{array}$

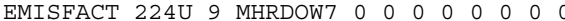

** Month $=$ April $;$ Day of Week = Saturday 
EMISFACT 224U_9 MHRDOW7 0000000000 EMISFACT 224U_9 MHRDOW7 $00 \begin{array}{llllllllll}0 & 0 & 0 & 0 & 0 & 0 & 0\end{array}$ EMISFACT 224U

** Month = May; Day of Week = Saturday

Month = May; $i$ Day of Week = Saturday EMISFACT 224U-9 MHRDOW7 0000000000000 EMISFACT 2240.9 MRDOW7 $0 \begin{array}{llllllllll}0 & 0 & 0 & 0 & 0 & 0 & 0\end{array}$

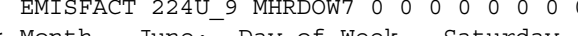

* Month = June; Day of Week = Saturday

EMISFACT 224U_9 MHRDOW7 000000000000

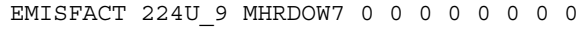
EMISFACT 224U_9 MHRDOW7 00000000000

** Month $=$ July; Day of Week = Saturday

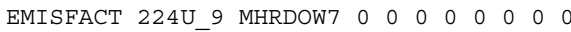

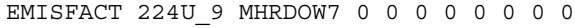
EMISFACT 224U 9 MHRDOW7 000000000

** Month = August; Day of week = Saturday EMISFACT 224U_9 MHRDOW7 00000000000

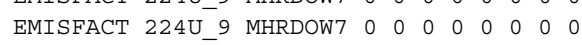
EMISFACT 224U_9 MHRDOW7 0000000000

** Month = September; Day of Week = Saturday EMISFACT 224U_9 MHRDOW7 0000000000 EMISFACT 224U_9 MHRDOW7 $000 \begin{array}{lllllll}- & 0 & 0 & 0 & 0 & 0 & 0\end{array}$ EMISFACT 224U_9 MHRDOW7 00000000000

** Month = October; Day of Week = Saturday EMISFACT 224U_9 MHRDOW7 00000000000 EMISFACT 224U_9 MHRDOW7 $00 \begin{array}{lllllll}0 & 0 & 0 & 0 & 0 & 0 & 0\end{array}$ EMISFACT 224U 9 MHRDOW7 00000000000 क $\quad *$ Month = November; Day of Week = Saturda

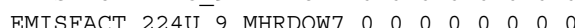
MISFAC 2240

Month

** Month = December $;$ Day of Week = Saturday EMISFACT 224U_9 MHRDOW7 000000000000 EMISFACT 224U_9 MHRDOW7 000000000000 EMISFACT 224U_9 MHRDOW7 00000000000

** Month = January; Day of week = Sunday EMISFACT 224U 9 MHRDOW7 $00 \begin{array}{lllllll}0 & 0 & 0 & 0 & 0 & 0 & 0\end{array}$

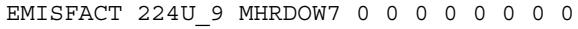
EMISFACT 224U_9 MHRDOW7 $00 \begin{array}{ccccccc}0 & 0 & 0 & 0 & 0 & 0 & 0\end{array}$

** Month = February; Day of Week = Sunday

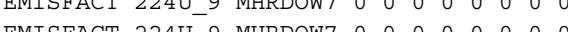

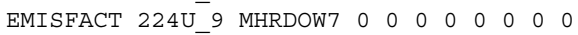

** Month $=$ March; Day of Week = Sunday EMISFACT 224U_9 MHRDOW7 00000000000 EMISFACT 224U_9 MHRDOW7 000000000000 EMISFACT 224U_9 MHRDOW7 00000000000

* Month $=$ April; Day of Week = Sunday EMISFACT 224U_9 MHRDOW7 00000000000

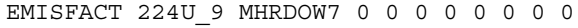
EMISFACT 224U 9 MHRDOW7 $00 \begin{array}{lllllll}0 & 0 & 0 & 0 & 0 & 0 & 0\end{array}$

* Month $=$ May; Day of Week $=$ Sunday

EMISFACT 224U 9 MHRDOW7 0000000000 
EMISFACT 224U_9 MHRDOW7 000000000000 EMISFACT 224U 9 MHRDOW7 00000000000

** Month $=$ June; ${ }^{-}$Day of Week = Sunday

EMISFACT 224U_9 MHRDOW7 000000000000 EMISFACT 2240_9 MHRDOW7 $0 \begin{array}{llllllllllllll}0 & 0 & 0 & 0 & 0 & 0 & 0\end{array}$

EMISFACT 2240_9 MHRDOW7 $0 \begin{array}{llllllll}0 & 0 & 0 & 0 & 0 & 0 & 0 & 0\end{array}$ ** Month = July; Day of Week = Sunday

EMISFACT 224U_9 MHRDOW7 0000000000 EMISFACT 224U_9 MHRDOW7 000000000000 EMISFACT 224U_9 MHRDOW7 0000000000

** Month $=$ August; Day of Week $=$ Sunday EMISFACT 224U_9 MHRDOW7 000000000

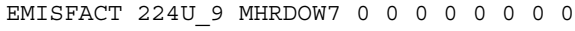
EMISFACT 224U_9 MHRDOW7 00000000000

** Month $=$ September; Day of Week = Sunday EMISFACT 224U_9 MHRDOW7 000000000000 EMISFACT $224 \mathrm{U}$

EMISEACT 224__

* Month = October; Day of Week = Sunday

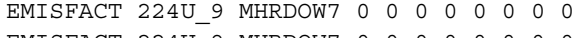
EMISFACT 224U_9 MHRDOW7 $00 \begin{array}{llllllll}0 & 0 & 0 & 0 & 0 & 0 & 0 & 0\end{array}$

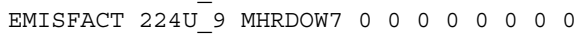

* Month $=$ November; Day of Week = Sunday EMISFACT 224U_9 MHRDOW7 00000000000 EMISFACT 224U_9 MHRDOW7 $000 \begin{array}{lllllllll}0 & 0 & 0 & 0 & 0 & 0 & 0\end{array}$

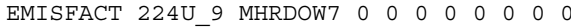

* Month $=$ December; Day of Week = Sunday EMISFACT 224U 9 MHRDOW7 0000000000

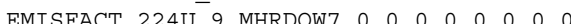

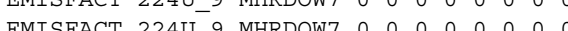

EMISFACT 224U_9 MHRDOW7 $00 \begin{array}{llllllll}0 & 0 & 0 & 0 & 0 & 0 & 0\end{array}$

** Month $=$ January $;$ Day of Week = Monday

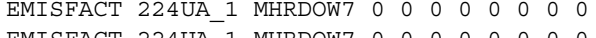
EMISFACT 224UA_1 MHRDOW7 000000000000 EMISFACT 224UA_1 MHRDOW7 00000000000

* Month = February; Day of Week = Monday EMISFACT 224UA_1 MHRDOW7 00000000000 EMISFACT 224UA_1 MHRDOW7 $00 \begin{array}{llllllll}0 & 0 & 0 & 0 & 0 & 0 & 0\end{array}$ EMISFACT 224UA_1 MHRDOW7 00000000000

** Month $=$ March; Day of Week = Monday

EMISFACT 224UA_1 MHRDOW7 0000000000000

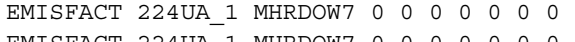

* Month = April; Day of Week = Monday

* * Month = April; Day of Week = Monday

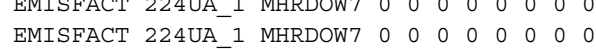
EMISFACT 224UA 1 MHRDOW7 $00 \begin{array}{ccccccc}0 & 0 & 0 & 0 & 0 & 0\end{array}$

** Month = May; Day of Week = Monday

EMISFACT 224UA_1 MHRDOW7 000000000000 EMISFACT 224UA 1 MHRDOW7 $00 \begin{array}{lllllll}0 & 0 & 0 & 0 & 0 & 0 & 0\end{array}$ EMISFACT 224UA_1 MHRDOW7 $00 \begin{array}{lllllll}0 & 0 & 0 & 0 & 0 & 0 & 0\end{array}$

** Month $=$ June; Day of Week = Monday

EMISFACT 224UA_1 MHRDOW7 00000000000 EMISFACT 224UA 1 MHRDOW7 0 C 
EMISFACT 224UA_1 MHRDOW7 00000000000

** Month $=$ July; Day of Week = Monday

EMISFACT 224UA_1 MHRDOW7 $00 \begin{array}{lllllll}0 & 0 & 0 & 0 & 0 & 0 & 0\end{array}$ EMISFACT 224UA_1 MHRDOW7 000000000000

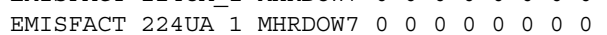

** Month = August $;$ Day of Week = Monday EMISFACT 224UA_1 MHRDOW7 $\begin{array}{lllllllll}0 & 0 & 0 & 0 & 0 & 0 & 0 & 0\end{array}$

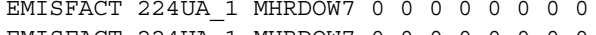
EMISFACT 224UA_1 MHRDOW7 $00 \begin{array}{lllllll}0 & 0 & 0 & 0 & 0 & 0 & 0\end{array}$

* Month = September; Day of Week = Monday EMISFACT 224UA_1 MHRDOW7 00000000000 EMISFACT 224UA_1 MHRDOW7 $00 \begin{array}{lllllll}0 & 0 & 0 & 0 & 0 & 0 & 0\end{array}$

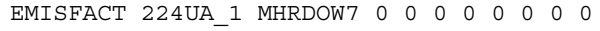

** Month = October; Day of Week = Monday EMISFACT 224UA 1 MHRDOW7 0000000001

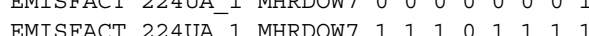
EMTSFACT 224UA 1 MHRDOW7 0000000

EMISEACT 2240A-1. MHRDOW7 00000000 * Month = November; Day of Week = Monday EMISFACT 224UA_1 MHRDOW7 0 ONO 000000 EMISFACI 224UA_1 MHRDOW7 $1 \begin{array}{llllllll}1 & 1 & 1 & 0 & 1 & 1 & 1\end{array}$ EMISFACT 224UA_1 MHRDOW7 $00 \begin{array}{lllllll}0 & 0 & 0 & 0 & 0 & 0\end{array}$

* Month $=$ December; Day of Week = Monday EMISFACT 224UA_1 MHRDOW7 0000000000

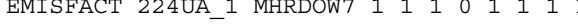

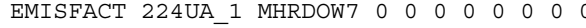

** Month $=$ January; Day of Week $=$ Tuesday EMISFACT 224UA 1 MHRDOW7 000100000000

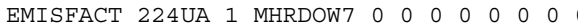
EMISFACT 224UA 1 MHRDOW7 000000000

* Month = Februaryi' Day of Week = Tuesday * Month = February; , Day of Week = Tuesday EMISFACT 224UA-1 MRDOW7 $0 \begin{array}{lllllll}0 & 0 & 0 & 0 & 0 & 0 & 0\end{array}$ EMISFACT 224UA_1 MHRDOW7 $0 \begin{array}{lllllllll}0 & 0 & 0 & 0 & 0 & 0 & 0\end{array}$

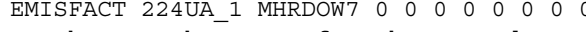
* Month $=$ March; Day of Week = Tuesday EMISFACT 224UA_1 MHRDOW7 000000000000 EMISFACT 224UA_1 MHRDOW7 $00 \begin{array}{lllllll}0 & 0 & 0 & 0 & 0 & 0 & 0\end{array}$

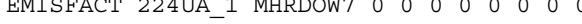

** Month $=$ April; Day of Week = Tuesday EMISFACT 224UA 1 MHRDOW7 00010000000 EMISFACT 224UA 1 MHRDOW7 $00 \begin{array}{lllllll}0 & 0 & 0 & 0 & 0 & 0\end{array}$ EMISFACT 224UA 1 MHRDOW7 00000

** Month = May; Day of Week = Tuesday

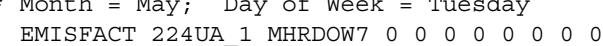
EMISFACT 224UA_1 MHRDOW7 000000000000 EMISFACT 224UA_1 MHRDOW7 00100000000 EMISFACT 224UA_1 MHRDOW7 $00 \begin{array}{llllll}0 & 0 & 0 & 0 & 0 & 0 \\ * & \text { Month }=\text { June; } & \text { Day of Week }=\text { Tuesday }\end{array}$

$\begin{array}{llllllllll}\text { * } & \text { Month }=\text { June; Day of Week = Tuesday } \\ \text { EMISFACT 224UA_1 MHRDOW7 } 0 & 0 & 0 & 0 & 0 & 0 & 0 & 0\end{array}$

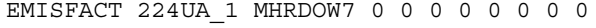

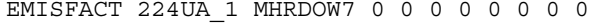

** Month = July; Day of Week = Tuesday

EMISFACT 224UA_1 MHRDOW7 000000000000 EMISFACT 224UA_ 1 MHRDOW7 
** Month $=$ August; $;$ Day of Week = Tuesday

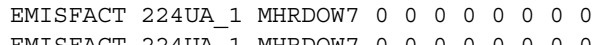
EMISEACT 224UA-1 MHRDOW7 $0 \begin{array}{llllllllll}0 & 0 & 0 & 0 & 0 & 0 & 0 & 0\end{array}$

* Month = September; Day of Week = Tuesday * Month = September;

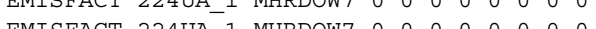

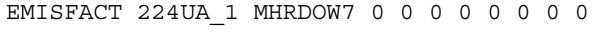

** Month = October; Day of Week = Tuesday EMISFACT 224UA_1 MHRDOW7 000000000001 EMISFACT 224UA_1 MHRDOW7 1111100111

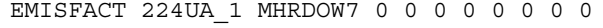

** Month $=$ November; Day of Week $=$ Tuesday EMISFACT 224UA_1 MHRDOW7 $00 \begin{array}{lllllll}0 & 0 & 0 & 0 & 0 & 0 & 1\end{array}$ EMISFACT 224UA 1 MHRDOW7 $1 \begin{array}{lllllllllll}1 & 1 & 1 & 0 & 1 & 1 & 1 & 1\end{array}$ EMISFACT 224UA 1 MHRDOW7 $0 \begin{array}{llllllll}1 & 0 & 0 & 1 & 1 & 1 & 1\end{array}$

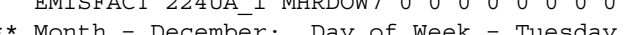
* Month $=$ December $;$ Day of Week = Tuesday

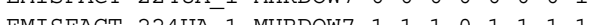

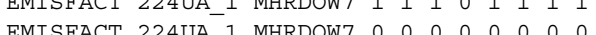

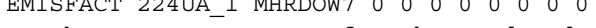

** Month = January; Day of Week = Wednesday EMISFACT 224UA_1 MHRDOW7 00000000000

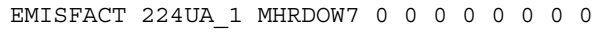
EMISFACT 224UA_1 MHRDOW7 $0 \begin{array}{lllllll}0 & 0 & 0 & 0 & 0 & 0 & 0\end{array}$

** Month = February; Day of Week = Wednesday

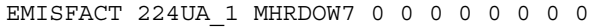

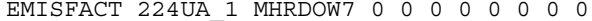

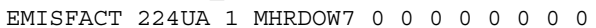

** Month = Marchi Day of Week = Wednesday MMTS = Machi Day of Week = wednesday

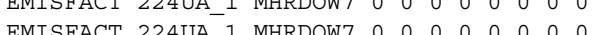
EMISFACT 224AEMISFACT 2240A_1 MHRDOW7 000000000000 * Month = April; Day of Week = Wednesday EMISFACT 224UA_1 MHRDOW7 000000000000 EMISFACT 224UA_1 MHRDOW7 000000000000 EMISFACT 224UA_1 MHRDOW7 00000000000

** Month $=$ May; Day of Week $=$ Wednesday EMISFACT 224UA_1 MHRDOW7 $00 \begin{array}{lllllll}0 & 0 & 0 & 0 & 0 & 0 & 0\end{array}$

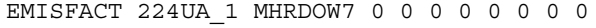

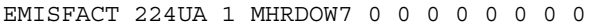

* Month = June; Day of Week = Wednesday MMISFACT 224UA 1 MHRDOW7 0 lo 000 a

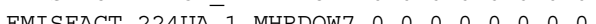

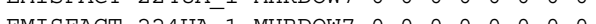
EMISFACT 2240A-1 MHRDOW7 $0 \begin{array}{lllllllllllllll}0 & 0 & 0 & 0 & 0 & 0 & 0\end{array}$ ** Month $=$ July; Day of Week = Wednesday EMISFACT 224UA_1 MHRDOW7 00000000000 EMISFACT 224UA_1 MHRDOW7 000000000000

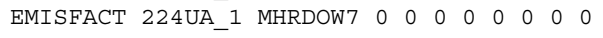

** Month $=$ August; Day of Week $=$ Wednesday

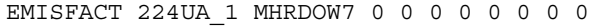

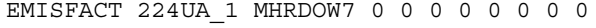

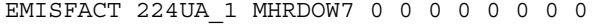

* Month $=$ September; Day of Week $=$ Wednesday 
EMISFACT 224UA_1 MHRDOW7 000000000000

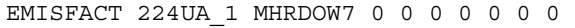

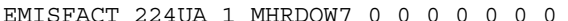

** Month = October; Day of Week = Wednesday EMISFACT 224UA 1 MHRDOW7 0000000001

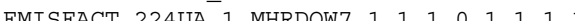
FMISFACT 224UA 1 MHRDOW7 $00 \begin{array}{llllllllll}1 & 1 & 1 & 0 & 1 & 1 & 1 & 1\end{array}$

** Month $=$ November; $;$ Day of Week $=$ Wednesday EMISFACT 224UA_1 MHRDOW7 0000000000 EMISFACT 224UA 1 MHRDOW7 $1 \begin{array}{llllllll}1 & 1 & 0 & 1 & 1 & 1 & 1\end{array}$ EMISFACT 224UA_1 MHRDOW7 001000010000

** Month $=$ December; Day of Week $=$ Wednesday EMISFACT 224UA_1 MHRDOW7 $00 \begin{array}{lllllll}0 & 0 & 0 & 0 & 0 & 0 & 1\end{array}$

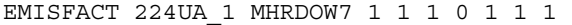

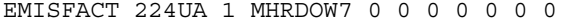

* Month = January; Day of Week = Thursday FMISFACT 224 M D

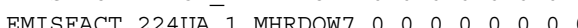

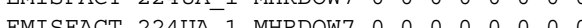
EMISFACT 224UA_1 MHRDOW7 000000000000

** Month = February; Day of Week = Thursday

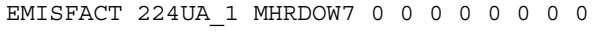
EMISFACT 224UA_1 MHRDOW7 $0 \begin{array}{lllllllll}0 & 0 & 0 & 0 & 0 & 0 & 0 & 0\end{array}$

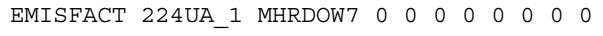

** Month $=$ March; Day of Week = Thursday EMISFACT 224UA_1 MHRDOW7 00000000000

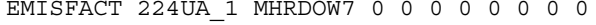

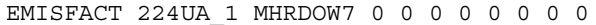

** Month = April; Day of Week = Thursday EMISFAT 224 ; 1 Mardow7 0 o 0 Thursday

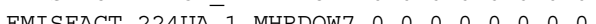
EMISFACT 224UA-1 MHRDOW7 $0 \begin{array}{llllllllllllllllll}0 & 0 & 0 & 0 & 0 & 0 & 0\end{array}$ month = Mayi Day of Week $=$ Thursday * Month = May; Day of Week = Thursday

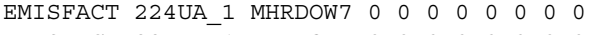
EMISFACT 224UA_1 MHRDOW7 $0 \begin{array}{llllllll}0 & 0 & 0 & 0 & 0 & 0 & 0 & 0\end{array}$ EMISFACT 224UA_1 MHRDOW7 00010000000

** Month $=$ June; Day of Week $=$ Thursday EMISFACT 224UA_1 MHRDOW7 $00 \begin{array}{lllllll}0 & 0 & 0 & 0 & 0 & 0 & 0\end{array}$

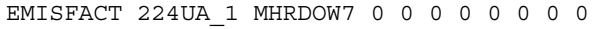

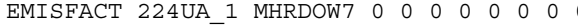

$* *$ Month $=$ July; Day of Week $=$ Thursday

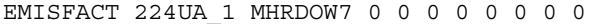
FMISFACT 224UA

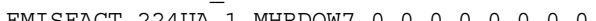

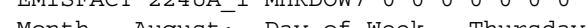
* Month = August $;$ Day of Week $=$ Thursday EMISFACT 224UA_1 MHRDOW7 00000000000 EMISFACT 224UA_1 MHRDOW7 $\begin{array}{lllllllll}0 & 0 & 0 & 0 & 0 & 0 & 0 & 0\end{array}$ EMISFACT 224UA_1 MHRDOW7 000000000000

* Month = September; Day of Week = Thursday EMISFACT 224UA_1 MHRDOW7 00000000000 EMISFACT 224UA 1 MHRDOW7 $00 \begin{array}{lllllll}0 & 0 & 0 & 0 & 0 & 0 & 0\end{array}$

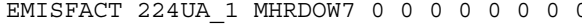
* Month $=$ October; Day of Week = Thursday

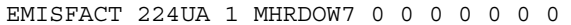


EMISFACT 224UA_1 MHRDOW7 $1 \begin{array}{llllllll}1 & 1 & 0 & 1 & 1 & 1 & 1\end{array}$ EMISFACT 224UA_1 MHRDOW7 00000000000

** Month $=$ November; $;$ Day of Week = Thursday EMISFACT 224UA 1 MHRDOW7 0000000001 EMISFACT 224UA 1 MHRDOW7 $1 \begin{array}{llllllll}1 & 1 & 1 & 0 & 1 & 1 & 1 & 1\end{array}$ EMISFACT 224UA 1 MHRDOW7 00000000000

** Month = December; Day of Week = Thursday EMISFACT 224UA_1 MHRDOW7 00000000

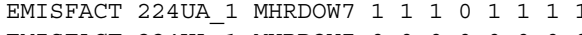
EMISFACT 224UA_1 MHRDOW7 00000000000

** Month = January; Day of Week = Friday EMISFACT 224UA_1 MHRDOW7 001000000000

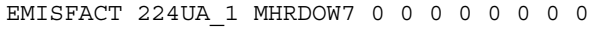
EMISFACT 224UA_1 MHRDOW7 000000000000

** Month = February; Day of week = Friday EMISFACT 224UA_1 MHRDOW7 00000000000 EMISFACT 224UA_1 MHRDOW7 000000000000 EMISFACT 224UA 1 MHRDOW7 00000000000

Month = March; Day of Week = Friday

** Month = March; Day of Week = Friday EMISFACT 224UA 1 MHRDOW7 00000000000 EMISFACT 224UA_1 MHRDOW7 000000000000

** Month $=$ April; Day of Week = Friday EMISFACT 224UA_1 MHRDOW7 00000000000

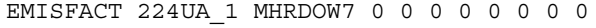

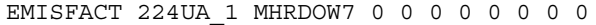

** Month = May; Day of Week = Friday

EMISFACT 224UA_1 MHRDOW7 00000000000

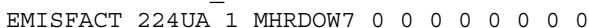

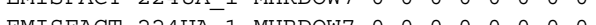

* Month = June; Day of Week = Friday

$\begin{array}{llllllllll}\text { * } & \text { Month = June } ; \quad \text { Day of Week }= & \text { Friday } & & \\ \text { EMISFACT 224UA } 1 \text { MHRDOW7 } & 0 & 0 & 0 & 0 & 0 & 0 & 0\end{array}$ EMISFACT 224UA_1 MHRDOW7 000000000000

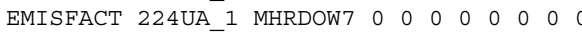
* Month $=$ July; Day of Week = Friday EMISFACT 224UA_1 MHRDOW7 0000000000 EMISFACT 224UA_1 MHRDOW7 $00 \begin{array}{llllllll}0 & 0 & 0 & 0 & 0 & 0 & 0\end{array}$

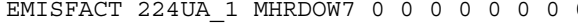

** Month $=$ August; Day of Week = Friday EMISFACT 224UA_1 MHRDOW7 0000000000000 EMISFACT 224UA-

EMISEACT 2240_-1 MHRDOW7 0 0 00000000 * Month = Septenber; Day or Week = Friday EMISFACT 224UA_- MHRDOW7 000000000

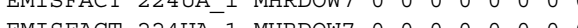
EMISFACT 224UA_1 MHRDOW7 00000000000 * Month $=$ October; Day of Week = Friday EMISFACT 224UA_1 MHRDOW7 0000000000 EMISFACT 224UA_1 MHRDOW7 $1 \begin{array}{llllllllll}1 & 1 & 1 & 0 & 1 & 1 & 1 & 1\end{array}$ EMISFACT 224UA_1 MHRDOW7 $00 \begin{array}{llllllllll}0 & 0 & 0 & 0 & 0 & 0 & 0\end{array}$

* Month $=$ November $;$ Day of Week $=$ Friday EMISFACT 224UA 1 MHRDOW7 00100000001 EMISFACT 224UA 1 MHRDOW7 1 
EMISFACT 224UA_1 MHRDOW7 0000000000

** Month $=$ December $;$ Day of Week = Friday

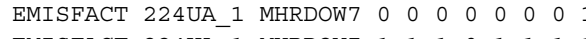
EMISFCC 2240_1 MHRDOW7, 1111001011

EMISFACI 2240A_1 MHRDOW7 0 * Month = January; Day of Week = Saturday EMISFACT 224UA_1 MHRDOW7 000000000 EMISFACT 2240A_1 MHRDOW7 00000000000000 EMISFACT 224UA_1 MHRDOW7 00000000000

* Month = February; Day of week = Saturday EMISFACT 224UA_1 MHRDOW7 000000000 EMISFACT 224UA_1 MHRDOW7 $00 \begin{array}{llllllll}0 & 0 & 0 & 0 & 0 & 0 & 0\end{array}$

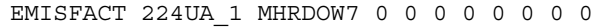

* Month $=$ March; Day of Week = Saturday EMISFACT 224UA 1 MHRDOW7 00000000000

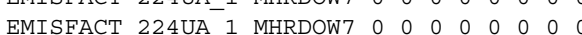

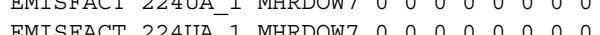

EMISEACT 2240_1 MHRDOW 0 o 0 o 0 o 00

* Month = April; $;$ Day of Week = Saturday EMISFACT 224UA_1 MHRDOW7 00000000000

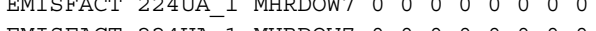
EMISFACT 224UA_1 MHRDOW7 $00 \begin{array}{lllllllll}0 & 0 & 0 & 0 & 0 & 0 & 0\end{array}$

* Month = May; Day of Week = Saturday

EMISFACT 224UA_1 MHRDOW7 000000000000

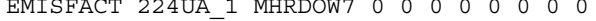

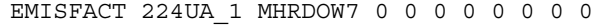

** Month $=$ June; Day of Week = Saturday

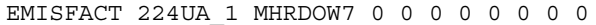

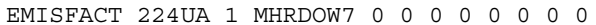
EMTSEACT 224UA_1 MHRDOW7 0000000000

* Month = Julyi - Day of Week = Saturday

Month = July; $;$ Day of Week = Saturday EMISFACT 224UA_. MHRDOW7 $0 \begin{array}{cccccccc}0 & 0 & 0 & 0 & 0 & 0 & 0\end{array}$ EMISFACT 224UA_1 MHRDOW7 0 O 00000000 EMISFACT 224UA_1 MHRDOW7 0000000000 * Month = August; Day of Week = Saturday EMISFACT 224UA_1 MHRDOW7 000000000000 EMISFACT 224UA_1 MHRDOW7 $00 \begin{array}{lllllll}0 & 0 & 0 & 0 & 0 & 0 & 0\end{array}$

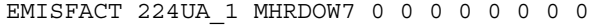

** Month $=$ September; Day of Week = Saturday EMISFACT 224UA_1 MHRDOW7 000000000000

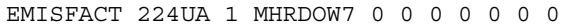
EMISFACT 224UA 1 MHRDOW7 00000

* Month = October, Day of Week = Saturday Month = October; Day or Week = saturday

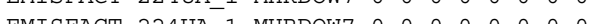
EMISFAC 2240A-1 MHRDOW $0 \begin{array}{lllllllll}0 & 0 & 0 & 0 & 0\end{array}$ EMISFACT 2240A-1 MHRDOW7 0 O 0000000000 * Month = November; Day of week = Saturday EMISFACT 224UA_1 MHRDOW7 00000000000 EMISFACT 224UA_1 MHRDOW7 $0 \begin{array}{llllllllll}0 & 0 & 0 & 0 & 0 & 0 & 0\end{array}$ EMISFACT 224UA_1 MHRDOW7 $00 \begin{array}{lllllll}0 & 0 & 0 & 0 & 0 & 0 & 0\end{array}$

** Month $=$ December; Day of Week = Saturday EMISFACT 224UA_1 MHRDOW7 $00 \begin{array}{lllllll}0 & 0 & 0 & 0 & 0 & 0 & 0\end{array}$

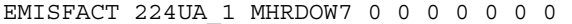
EMISFACT 224UA 1 MHRDOW7 0010 
** Month = January; Day of Week = Sunday EMISFACT 224UA_1 MHRDOW7 0000000000000

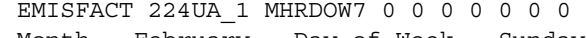
* Month = February; Day of Week = Sunday EMISFACT 224UA_1 MHRDOW7 $00 \begin{array}{llllll}0 & 0 & 0 & 0 & 0 & 0\end{array}$

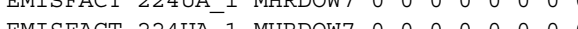

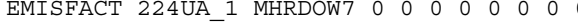

** Month $=$ March; Day of Week = Sunday

EMISFACT 224UA_1 MHRDOW7 00000000000 EMISFACT 224UA_1 MHRDOW7 $00 \begin{array}{llllllll}-1 & 0 & 0 & 0 & 0 & 0 & 0\end{array}$

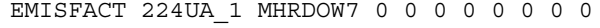

** Month $=$ April; Day of Week = Sunday

EMISFACT 224UA 1 MHRDOW7 00000000000

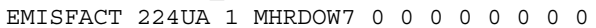

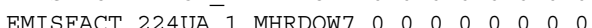

** Month = May; Day of Week = Sunday

$\begin{array}{llllllllll}\text { Month }=\text { May; Day of week } & \text { Sunday } & & \\ \text { EMISFACT 224UA 1 MHRDOW7 } 0 & 0 & 0 & 0 & 0 & 0 & 0 & 0\end{array}$ EMISFACT 224UA_1 MHRDOW7 000000000000 EMISFACT 224UA_1 MHRDOW7 $\begin{array}{lllllllllllll}0 & 0 & 0 & 0 & 0 & 0 & 0 & 0 \\ \text { EMISFACT 224UA_1 MHRDOW7 } & 0 & 0 & 0 & 0 & 0 & 0 & 0 & 0\end{array}$ ** Month $=$ June; Day of Week = Sunday

EMISFACT 224UA_1 MHRDOW7 $00 \begin{array}{llllllll}0 & 0 & 0 & 0 & 0 & 0 & 0\end{array}$ EMISFACT 224UA_1 MHRDOW7 00000000000

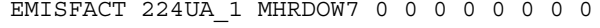

** Month $=$ July; Day of Week = Sunday

EMISFACT 224UA_1 MHRDOW7 00000000000

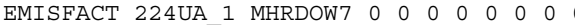
EMISFACT 224UA 1 MHRDOW7 0 l

* Month $=$ August $;$ Day of week = Sunday EMISFACT 224UA_1 MHRDOW7 000000000000 EMISFACT 224UA 1 MHRDOW7 $00 \begin{array}{lllllllll}0 & 0 & 0 & 0 & 0 & 0 & 0\end{array}$ EMISFACT 224UA 1 MHRDOW7 $00 \begin{array}{lllllll}0 & 0 & 0 & 0 & 0 & 0 & 0\end{array}$

** Month = September; Day of Week = Sunday EMISFACT 224UA_1 MHRDOW7 00000000000

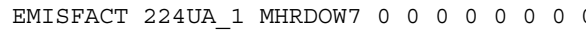
EMISFACT 224UA_1 MHRDOW7 $00 \begin{array}{lllllll}0 & 0 & 0 & 0 & 0 & 0 & 0\end{array}$

* Month $=$ October; Day of Week = Sunday EMISFACT 224UA_1 MHRDOW7 00000000000

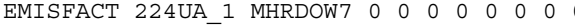

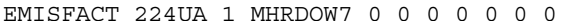

** Month = November EMISFACT 224UA 1 MHRDOW7 000000 EMTSFACT 224UA_ MHRDOW7 0000000000 0

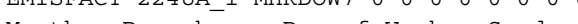
* Month $=$ December; Day of week = Sunday EMISFACT 224UA_1 MHRDOW7 000000000 EMISFACT 224UA_1 MHRDOW7 000000000000 EMISFACT 224UA_1 MHRDOW7 00000000000

* Month $=$ January; Day of Week = Monday EMISFACT 224UA_2 MHRDOW7 00000000000

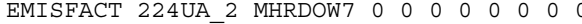
EMISFACT 224UA 2 MHRDOW7 $00 \begin{array}{lllllll}0 & 0 & 0 & 0 & 0 & 0 & 0\end{array}$

$* *$ Month $=$ February; Day of week = Monday 
EMISFACT 224UA_2 MHRDOW7 000000000000 EMISFACT 224UA_2 MHRDOW7 0000000000000 EMISFACT 224UA_2 MHRDOW7 00000000000

* Month $=$ March; Day of Week = Monday

EMISFACT 224UA 2 2 MHRDOW7 000000000000

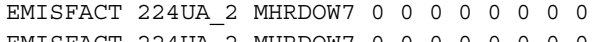
EMISFACT 2240A_2 MHRDOW7 $0 \begin{array}{lllllllllllllll}0 & 0 & 0 & 0 & 0 & 0 & 0\end{array}$

* Month = April; Day of Week = Monday

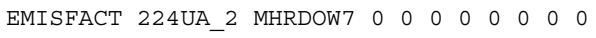

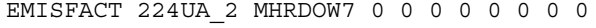

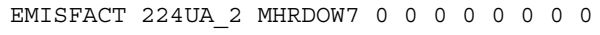

** Month = May; Day of Week = Monday

EMISFACT 224UA 2 MHRDOW7 00010000000 EMISFACT 224UA 2 MHRDOW7 $00 \begin{array}{lllllll}0 & 0 & 0 & 0 & 0 & 0 & 0\end{array}$

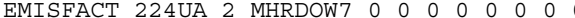

** Month $=$ June; Day of Week = Monday EMISFACT 224UA_2 MHRDOW7 000000000000 TMISFACT 224UA_

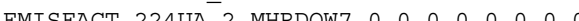

EMISFACT 2240A_2 MHRDOW7 $\begin{array}{lllllllll}0 & 0 & 0 & 0 & 0 & 0 & 0\end{array}$ * Month $=$ July; Day of Week = Monday EMISFACT 224UA_2 MHRDOW7 00000000000

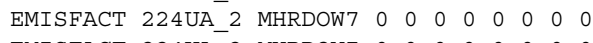
EMISFACT 224UA_2 MHRDOW7 000000000000

* Month $=$ August; Day of Week $=$ Monday EMISFACT 224UA_2 MHRDOW7 00000000000 EMISFACT 224UA_2 MHRDOW7 $00 \begin{array}{lllllll}0 & 0 & 0 & 0 & 0 & 0 & 0\end{array}$ EMISFACT 224UA 2 MHRDOW7 0000000000

** Month = September, Day of Week = Monday EMTSFACT 224UA 2 MHRDOW7 0 o 0 = Monday

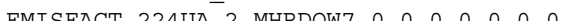
MISFCT 2240A

EMISEACT 2240_- MHRDOW 0 O 0 o 00 o 0 * Month = October; Day of Week = Monday EMISFACT 224UA_2 MHRDOW7 0000000000 EMISFACT 224UA_2 MHRDOW7 $1 \begin{array}{cccccccc}1 & 1 & 0 & 1 & 1 & 1\end{array}$ EMISFACT 224UA_2 MHRDOW7 0000000000

** Month $=$ November; Day of Week = Monday

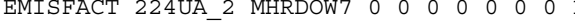

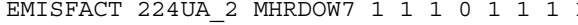

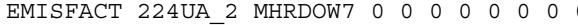

* Month $=$ December; Day of Week = Monday EMISFACT 224UA_2 MHRDOW7 0000000000 EMISFACT 224UA

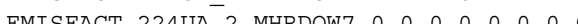

EMISFACT 224UA_2 MHRDOW7 $\begin{array}{llllllll}0 & 0 & 0 & 0 & 0 & 0 & 0 & 0\end{array}$ * Month = January; Day of Week = Tuesday EMISFACT 2240A_2 MHRDOW7 $0 \begin{array}{cccccc}0 & 0 & 0 & 0 & 0 & 0\end{array}$

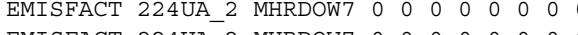
EMISFACT 224UA_2 MHRDOW7 00000000000

$*$ Month = February; Day of Week = Tuesday EMISFACT 224UA 2 MHRDOW7 $00 \begin{array}{lllllll}0 & 0 & 0 & 0 & 0 & 0\end{array}$ EMISFACT 224UA 2 MHRDOW7 $00 \begin{array}{lllllll}0 & 0 & 0 & 0 & 0 & 0 & 0\end{array}$ EMISFACT 224UA_2 MHRDOW7 $00 \begin{array}{lllllll}0 & 0 & 0 & 0 & 0 & 0 & 0\end{array}$ * Month $=$ March; Day of Week = Tuesday

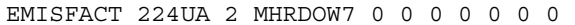


EMISFACT 224UA_2 MHRDOW7 0000000000000 EMISFACT 224UA_2 MHRDOW7 000000000000

** Month $=$ April; ${ }^{-}$Day of Week = Tuesday EMISFACT 224UA 2 MHRDOW7 $00 \begin{array}{lllllll}0 & 0 & 0 & 0 & 0 & 0 & 0\end{array}$ EMISFACT 224UA 2 MHRDOW7 00000000000 EMISFACT 224UA 2 MHRDOW7 $00 \begin{array}{llllllll} & 0 & 0 & 0 & 0 & 0 & 0 & 0\end{array}$

** Month = May; Day of Week = Tuesday

EMISFACT 224UA_2 MHRDOW7 $00 \begin{array}{llllllll}0 & 0 & 0 & 0 & 0 & 0 & 0\end{array}$ EMISFACT 224UA_2 MHRDOW7 000000000000 EMISFACT 224UA_2 MHRDOW7 0000000000

** Month $=$ June; Day of Week = Tuesday

EMISFACT 224UA_2 MHRDOW7 $00 \begin{array}{lllllll}0 & 0 & 0 & 0 & 0 & 0 & 0\end{array}$ EMISFACT 224UA_2 MHRDOW7 $00 \begin{array}{lllllll}0 & 0 & 0 & 0 & 0 & 0 & 0\end{array}$ EMISFACT 224UA_2 MHRDOW7 $00 \begin{array}{lllllll}0 & 0 & 0 & 0 & 0 & 0 & 0\end{array}$

** Month $=$ July; Day of week = Tuesday EMISFACT 224UA_2 MHRDOW7 000000000000

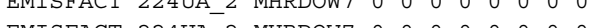
BMISFACT 224UA_2 MHRDOW7 $0 \begin{array}{lllllll}0 & 0 & 0 & 0 & 0 & 0 & 0\end{array}$

** Month = August; $\quad$ Day of Week = Tuesday EMISFACT 224UA_2 MHRDOW7 00000000000

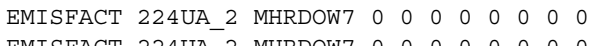
EMISFACT 224UA_2 MHRDOW7 00000000000

* Month $=$ September; Day of Week = Tuesday EMISFACT 224UA_2 MHRDOW7 0000000000 EMISFACT 224UA_2 MHRDOW7 $00 \begin{array}{lllllll}0 & 0 & 0 & 0 & 0 & 0 & 0\end{array}$

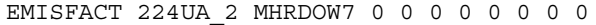

* Month = October; Day of Week = Tuesday EMISFACT 224UA 2 MHRDOW7 000000000 EMISFACT 224UA 2 MHRDOW7 1110

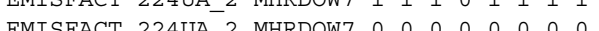

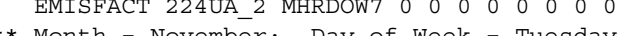
** Month = November; Day of Week = Tuesday

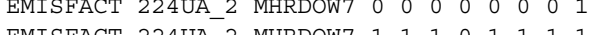
EMISFACT 224UA_2 MHRDOW7 $1 \begin{array}{lllllllll}1 & 1 & 1 & 0 & 1 & 1 & 1 & 1\end{array}$ EMISFACT 224UA_2 MHRDOW7 00000000000 * Month $=$ December; Day of Week = Tuesday

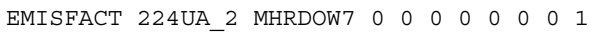

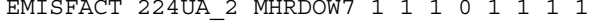

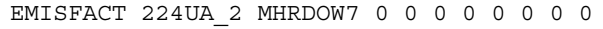

** Month $=$ January; Day of week $=$ Wednesday EMISFACT 224UA_2 MHRDOW7 000000000000

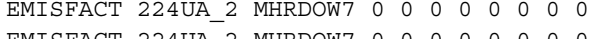

* Month = February; Day of week = Wednesday $\begin{array}{lllllllll}\text { Month }=\text { February; Day of Week } & = & \text { Wednesday } \\ \text { EMISFACT 224UA_2 MHRDOW7 } & 0 & 0 & 0 & 0 & 0 & 0 & 0 & 0\end{array}$

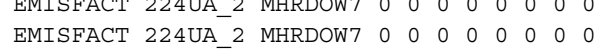
EMISFACT 224UA_2 MHRDOW7 00000000000

** Month $=$ March; Day of Week $=$ Wednesday EMISFACT 224UA_2 MHRDOW7 00000000000

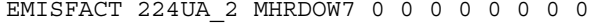

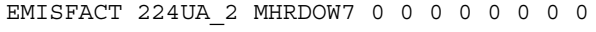

** Month $=$ April $;$ Day of Week = Wednesday

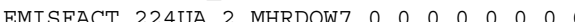


EMISFACT 224UA_2 MHRDOW7 00000000000

** Month $=$ May; Day of Week $=$ Wednesday

EMISFACT 224UA_2 MHRDOW7 000000000000 EMISFACT 224UA 2 MHRDOW7 00000000000 EMISFACT 224UA 2 MHRDOW7 00000000000

** Month = June; Day of Week = Wednesday

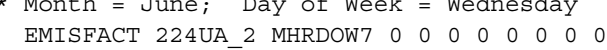

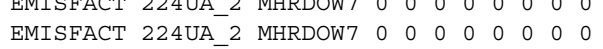
EMISFACT 224UA_2 MHRDOW7 00000000000

* Month $=$ July; Day of Week $=$ Wednesday EMISFACT 224UA_2 MHRDOW7 00000000000 EMISFACT 224UA_2 MHRDOW7 $00 \begin{array}{lllllllll}0 & 0 & 0 & 0 & 0 & 0 & 0 & 0\end{array}$ EMISFACT 224UA_2 MHRDOW7 00100000000

** Month $=$ August; Day of Week $=$ Wednesday EMISFACT 224UA 2 MHRDOW7 0000000000

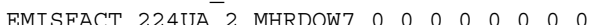

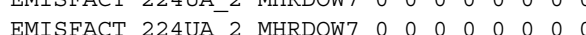

** Month = September; Day of Week = Wednesday

MMISFACT 224UA_2 MHRDOW7 $0 \begin{array}{lllllllll}0 & 0 & 0 & 0 & 0 & 0 & 0\end{array}$

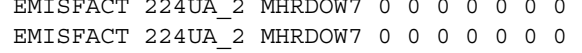
EMISFACT 224UA 2 MHRDOW7 $00 \begin{array}{lllllll}0 & 0 & 0 & 0 & 0 & 0\end{array}$

** Month $=$ October; Day of Week = Wednesday

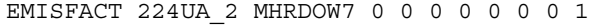
EMISFACT 224UA 2 MHRDOW7 $11110 \begin{array}{llllll}1 & 1 & 0 & 1 & 1 & 1\end{array}$ EMISFACT 224UA_2 MHRDOW7 00000000000

** Month $=$ November $;$ Day of Week $=$ Wednesday EMISFACT 224UA_2 MHRDOW7 00000000001

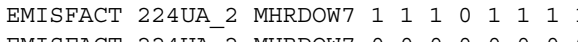

EMISFACT 224UA_2 MHRDOW7 000000000000

* Month $=$ December $;$ Day of Week = Wednesday EMISFACT 224UA 2 MRDOW7 110000 EMISFACT 224UA_2 MHRDW $1 \begin{array}{llllllll}1 & 1 & 1 & 0 & 1 & 1 & 1 & 1\end{array}$ EMISFACT 224UA_2 MHRDOW7 00000000000 * Month = January; Day of Week = Thursday EMISFACT 224UA_2 MHRDOW7 00000000000

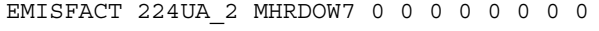

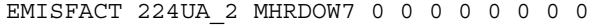

** Month $=$ February; Day of Week = Thursday EMISFACT 224UA_2 MHRDOW7 $00 \begin{array}{lllllll}0 & 0 & 0 & 0 & 0 & 0 & 0\end{array}$

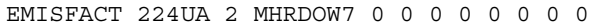
EMISFACT 224UA 2 MHRDOW7 00000000

* Month = March _ Month = Marchi Day of Week = Thursday DMISFACT 224UA- MHRDOW7 00000000000000 EMISFACT 224UA_2 MHRDW7 $\begin{array}{llllllllllllllll}0 & 0 & 0 & 0 & 0 & 0 & 0\end{array}$

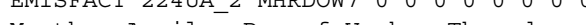
* Month $=$ April; Day of Week = Thursday EMISFACT 224UA_2 MHRDOW7 000000000000 EMISFACT 224UA_2 MHRDOW7 $0 \begin{array}{llllllll}0 & 0 & 0 & 0 & 0 & 0 & 0 & 0\end{array}$

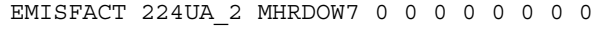

** Month = May; Day of Week = Thursday EMISFACT 224UA_2 MHRDOW7 000000000000 EMISFACT 224UA 2 MHRDOW7 
** Month $=$ June; Day of Week = Thursday EMISFACT 224UA 2 2 MHRDOW7 00000000000

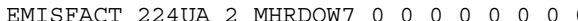
TMISFACT 224UA 2 MHRDOW7 $0 \begin{array}{lllllllllllllllll}0 & 0 & 0 & 0 & 0 & 0 & 0 & 0\end{array}$

* Month = July; Day of Week = Thursday

$\begin{array}{lllllllll}\text { MMISFACT 224UA } 2 \text { MHRDOW7 } & 0 & 0 & 0 & 0 & 0 & 0 & 0 & 0\end{array}$

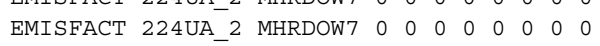
EMISFACT 224UA_2 MHRDOW7 $00 \begin{array}{lllllll}0 & 0 & 0 & 0 & 0 & 0 & 0\end{array}$

* Month $=$ August; Day of Week $=$ Thursday EMISFACT 224UA_2 MHRDOW7 00000000000 EMISFACT 224UA_2 MHRDOW7 $00 \begin{array}{lllllll}0 & 0 & 0 & 0 & 0 & 0 & 0\end{array}$ EMISFACT 224UA_2 MHRDOW7 $000 \begin{array}{lllllll}0 & 0 & 0 & 0 & 0 & 0 & 0\end{array}$

** Month $=$ September; Day of week $=$ Thursday EMISFACT 224UA_2 MHRDOW7 001000000000

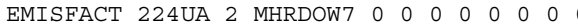
EMISFACT 224UA 2 MHRDOW7 000000000

* Month = 2240. * Month = October; Day of Week = Thursday EMISFACT 224UA_2 MHRDOW7 $0 \begin{array}{lllllllllll}0 & 0 & 0 & 0 & 0 & 0 & 1\end{array}$ EMISFACT 224UA_2 MHRDOW7 $0 \begin{array}{lllllll}1 & 1 & 0 & 1 & 1 & 1 & 1\end{array}$ EMISFACT 224UA_2 MHRDOW7 00000000000 * Month = November $;$ Day of Week = Thursday EMISFACT 224UA_2 MHRDOW7 000000000

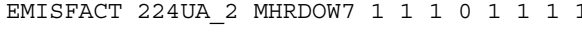
EMISFACT 224UA_2 MHRDOW7 000000000000

** Month $=$ December; Day of Week = Thursday

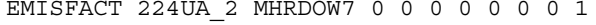
EMISFACT 224UA_2 MHRDOW7 $\begin{array}{llllllllll}1 & 1 & 1 & 0 & 1 & 1 & 1 & 1\end{array}$ EMISFACT 224UA 2 MHRDOW7 000000000000

** Month $=$ January; $;$ Day of Week = Friday EMISFACT 224UA 2 MHRDOW7 000000000000 EMISFACT 224UA 2 MHRDOW7 000000000000 EMISFACT 224UA_2 MHRDOW7 000000000000

$\begin{array}{lllllllll}\text { EMISFACT 224UA_2 MHRDOW7 } & 0 & 0 & 0 & 0 & 0 & 0 & 0 \\ \text { Month }=\text { February; }\end{array}$ ** Month = February; Day of Week = Friday
EMISFACT 224UA_2 MHRDOW7 000000000 EMISFACT 224UA_2 MHRDOW7 00000000000 EMISFACT 224UA_2 MHRDOW7 00000000000

** Month $=$ March; Day of Week = Friday

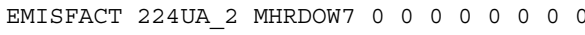

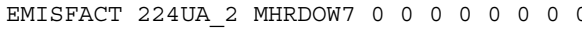

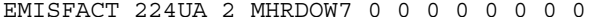

** Month $=$ April; $;$ Day of Week = Friday EMISFACT 224UA 2 MHRDOW7 0000000000 EMISFACT 224UA

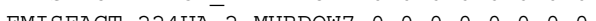

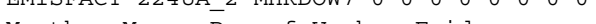
* Month = May; Day of Week = Friday EMISFACT 224UA_2 MHRDOW7 00000000000 EMISFACT 224UA_2 MHRDOW7 000000000000 EMISFACT 224UA_2 MHRDOW7 000000000000

$* *$ Month $=$ June; Day of Week $=$ Friday EMISFACT 224UA_2 MHRDOW7 00000000000

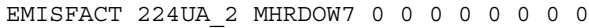
EMISFACT 224UA 2 MHRDOW7 000000000000

$* *$ Month $=$ July; Day of Week = Friday 
EMISFACT 224UA_2 MHRDOW7 000000000000 EMISFACT 224UA_2 MHRDOW7 000000000000

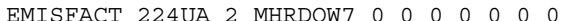

** Month = August $;$ Day of Week = Friday EMISFACT 224UA 2 MHRDOW7 000000000000 EMISFACT 224UA_2 MHRDOW7 000000000000 EMISFACT 224UA_2 MHRDOW7 000000000000

** Month $=$ September; Day of Week = Friday EMISFACT 224UA_2 MHRDOW7 000000000000

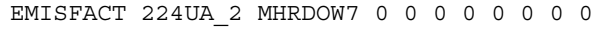

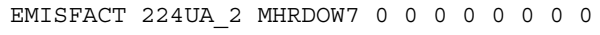

** Month = October; Day of Week = Friday

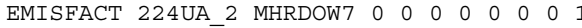
EMISFACT 224UA 2 MHRDOW7 $1 \begin{array}{llllllll}1 & 1 & 1 & 0 & 1 & 1 & 1 & 1\end{array}$

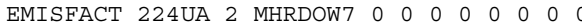

** Month $=$ NOVember;  PMISFACT 224UA- MRDOW7 1 EMSFACT 224UA_2 MHRDOW7 $\begin{array}{llllllll}1 & 1 & 1 & 0 & 1 & 1 & 1\end{array}$

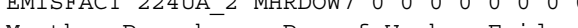

** Month $=$ December $;$ Day of Week = Friday EMISFACT 224UA_2 MHRDOW7 000000000 EMISFACT 224UA_2 MHRDOW7 $1 \begin{array}{llllllll}1 & 1 & 0 & 1 & 1 & 1 & 1\end{array}$ EMISFACT 224UA_2 MHRDOW7 00000000000

* Month = January; Day of Week = Saturday EMISFACT 224UA_2 MHRDOW7 00000000000 EMISFACT 224UA_2 MHRDOW7 $00 \begin{array}{lllllll}0 & 0 & 0 & 0 & 0 & 0 & 0\end{array}$ EMISFACT 224UA 2 MHRDOW7 00010000000

** Month = Februaryi Day of Week = Saturday EMISFACT 224UA 2 MHRDOW7 0000000000 EMTSEACT 224UA_2 MHRDOW7 00000000000

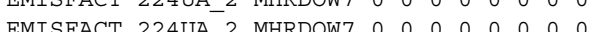

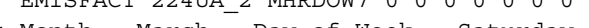
* Month = March; Day of Week = Saturday EMISFACT 224UA_2 MHRDOW7 000000000000

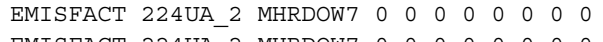
EMISFACT 224UA_2 MHRDOW7 00000000000

** Month $=$ April; $;$ Day of Week = Saturday EMISFACT 224UA_2 MHRDOW7 $00 \begin{array}{lllllll}0 & 0 & 0 & 0 & 0 & 0 & 0\end{array}$

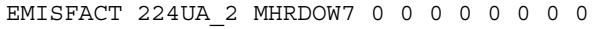

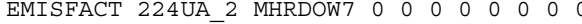

** Month = May; Day of Week = Saturday EMISFACT 224UA_2 MHRDOW7 000000000000 EMISFACT 224UA_2 MHRDOW7 00000 EMISTACI 224UA_2 MHRDOW7 00000000000

* Month = June; Day of Week = Saturday EMISFACT 224UA_2 MHRDOW7 $\begin{array}{lllllllll}0 & 0 & 0 & 0 & 0 & 0 & 0 & 0\end{array}$

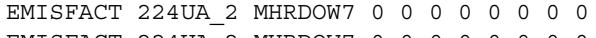
EMISFACT 224UA_2 MHRDOW7 00000000000

$* *$ Month $=$ July; Day of week = Saturday EMISFACT 224UA_2 MHRDOW7 $00 \begin{array}{lllllll}0 & 0 & 0 & 0 & 0 & 0 & 0\end{array}$ EMISFACT 224UA_2 MHRDOW7 $00 \begin{array}{llllllllllll}0 & 0 & 0 & 0 & 0 & 0 & 0\end{array}$

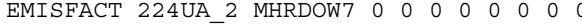

** Month $=$ August $;$ Day of Week = Saturday

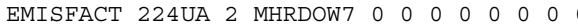


EMISFACT 224UA_2 MHRDOW7 000000000000 EMISFACT 224UA 2 MHRDOW7 00000000000

** Month = September; Day of Week = Saturday

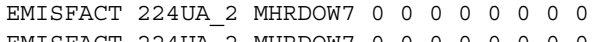
EMISTACT 2240_-2 MHRDOW7 $0 \begin{array}{llllllll}0 & 0 & 0 & 0 & 0 & 0 & 0\end{array}$

** Month = October; Day of Week = Saturday EMISFACT 224UA_2 MHRDOW7 00000000000

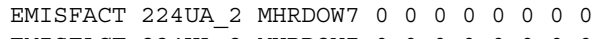
EMISFACT 224UA_2 MHRDOW7 0000000000

** Month $=$ November; $;$ Day of Week = Saturday

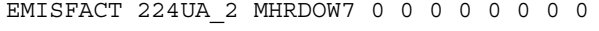
EMISFACT 224UA_2 MHRDOW7 $00 \begin{array}{lllllll}0 & 0 & 0 & 0 & 0 & 0 & 0\end{array}$ EMISFACT 224UA 2 MHRDOW7 000100000000

** Month $=$ December; Day of Week = Saturday EMISFACT 224UA 2 MHRDOW7 0000000000

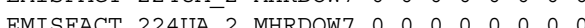

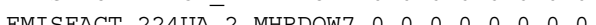

EMISEACT 224UA_2 MHRDOW $\begin{array}{cccccccc}0 & 0 & 0 & 0 & 0 & 0 & 0 & 0\end{array}$ * Month = January; Day of Week = Sunday EMISFACT 224UA_2 MHRDOW7 $0 \begin{array}{llllllll}0 & 0 & 0 & 0 & 0 & 0 & 0\end{array}$ EMISFACT 224UA_2 MHRDOW7 $0 \begin{array}{lllllllll}0 & 0 & 0 & 0 & 0 & 0 & 0 & 0\end{array}$ EMISFACT 224UA_2 MHRDOW7 000000000000

* Month = February; Day of Week = Sunday EMISFACT 224UA_2 MHRDOW7 00000000000 EMISFACT 224UA_2 MHRDOW7 $0 \begin{array}{llllllll}0 & 0 & 0 & 0 & 0 & 0 & 0 & 0\end{array}$ EMISFACT 224UA_2 MHRDOW7 $00 \begin{array}{ccccccc}0 & 0 & 0 & 0 & 0 & 0 & 0\end{array}$

** Month $=$ March; Day of Week = Sunday

EMISFACT 224UA_2 MHRDOW7 000000000000

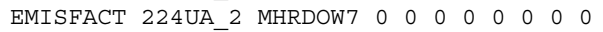
EMISFACT 224UA 2 MHRDOW7 000000000000

** Month = April; Day of Week = Sunday

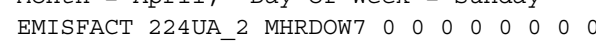
EMISFACT 224UA 2 MHRDOW7 00000000000 EMISFACT 224UA_2 MHRDOW7 $00 \begin{array}{llllllll}0 & 0 & 0 & 0 & 0 & 0 & 0\end{array}$

* Month = May; Day of Week = Sunday

EMISFACT 224UA_2 MHRDOW7 00000000000

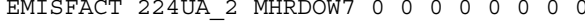

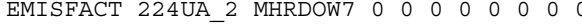

** Month $=$ June; Day of Week = Sunday

EMISFACT 224UA_2 MHRDOW7 000000000000

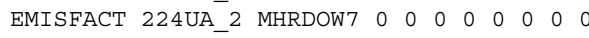

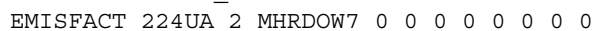

** Month $=$ July; Day of week = Sunday

EMISFACT 224UA 2 MHRDOW7 00000000000 EMISFACT 224UA_2 MHRDOW7 0010.000100000 EMISFACT 224UA_2 MHRDOW7 00000000000

$* *$ Month $=$ August $;$ Day of week $=$ Sunday EMISFACT 224UA_2 MHRDOW7 00000000000

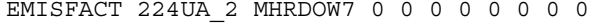
EMISFACT 224UA_2 MHRDOW7 $00 \begin{array}{lllllll}0 & 0 & 0 & 0 & 0 & 0 & 0\end{array}$

** Month $=$ September; Day of Week $=$ Sunday EMISFACT 224UA 2 MHRDOW7 0000000000 EMISFACT 224UA 2 MHRDOW7 00 
EMISFACT 224UA_2 MHRDOW7 0000000000

** Month $=$ October; Day of Week = Sunday

EMISFACT 224UA_2 MHRDOW7 000000000000 EMISFACT 224UA 2 MHRDOW7 $00 \begin{array}{llllllll}0 & 0 & 0 & 0 & 0 & 0 & 0\end{array}$

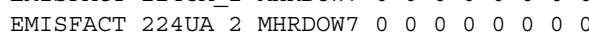

** Month = November; Day of week = Sunday EMISFACT 224UA_2 MHRDOW7 0000000000 EMISFACT 224UA_2 MHR EMISFACT 224UA_2 MHRDOW7 00000000000

* Month = December; Day of Week = Sunday EMISFACT 224UA_2 MHRDOW7 00000000000 EMISFACT 224UA_2 MHRDOW7 $00 \begin{array}{llllllll}0 & 0 & 0 & 0 & 0 & 0 & 0\end{array}$

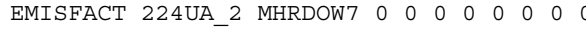

** Month $=$ January; Day of Week $=$ Monday EMISFACT 224UA 3 MHRDOW7 000000000

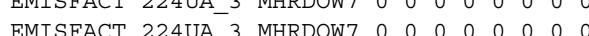
FMISFACT 224UA 3 MHRDOW7 00000000

BMSEACT 224UA_ MHRDOW7 0000000 * Month = February; Day of Week = Monday EMISFACT 2240A_3 MHRDOW7 $0 \begin{array}{ccccccc}0 & 0 & 0 & 0 & 0 & 0 & 0\end{array}$ EMISFACT 2240A_3 MHRDOW7 0 O 0000000000 EMISFACT 224UA_3 MHRDOW7 $000 \begin{array}{llllll}0 & 0 & 0 & 0 & 0 & 0\end{array}$

* Month $=$ March; Day of Week = Monday

EMISFACT 224UA_3 MHRDOW7 000000000

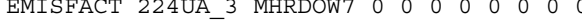

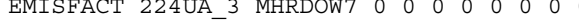

** Month $=$ April; Day of Week = Monday

EMISFACT 224UA_3 MHRDOW7 000000000000

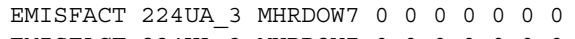

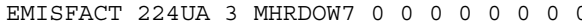

** Month = May; Day of Week = Monday

EMISFACT 224UA_3 MHRDOW7 00000000000 EMISFACT 224UA_3 MHRDOW7 000000000000 EMISFACT 224UA_3 MHRDOW7 0010000000000

* Month $=$ June; Day of Week = Monday

EMISFACT 224UA_3 MHRDOW7 $00 \begin{array}{lllllll}0 & 0 & 0 & 0 & 0 & 0 & 0\end{array}$

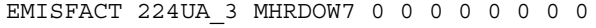

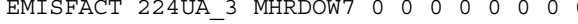

** Month $=$ July; Day of Week = Monday

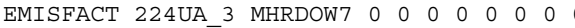

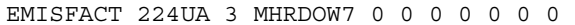
EMISFACT 224UA 3 MHRDOW7 0 a 000000

* Month = August; Day

Month = August; Day of Week = Monday

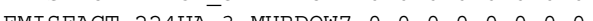

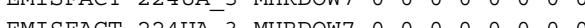
BMISFACT 224UA_ 3 MHDOW7 00000000 * Month = September; Day of Week = Monday EMISFACT 224UA_3 MHRDOW7 0000000000 EMISFACT 224UA_3 MHRDOW7 $00 \begin{array}{lllllll}0 & 0 & 0 & 0 & 0 & 0 & 0\end{array}$ EMISFACT 224UA_3 MHRDOW7 $0 \begin{array}{llllllll}0 & 0 & 0 & 0 & 0 & 0\end{array}$

** Month = October; Day of Week = Monday EMISFACT 224UA_3 MHRDOW7 0000000000 EMISFACT 224UA 3 MHRDOW7 $1 \begin{array}{llllllllllll}1 & 1 & 0 & 1 & 1 & 1 & 1\end{array}$ EMISFACT 224UA 3 MHRDOW7 00 
** Month $=$ November $;$ Day of week = Monday EMISFACT 224UA_3 MHRDOW7 000000000001 EMISFACT 224UA 3 MHRDOW7 $1 \begin{array}{llllllll}1 & 1 & 0 & 1 & 1 & 1\end{array}$ EMISFACT 224UA 3 MHRDOW7 $0 \begin{array}{lllllllllll}0 & 0 & 0 & 0 & 0 & 0 & 0\end{array}$

** Month $=$ December; Day of week = Monday EMISFACT 224UA_3 MHRDOW7 00000000000 EMISFACT 224UA_3 MHRDOW7 $1 \begin{array}{ccccccc}1 & 1 & 1 & 0 & 1 & 1 & 1\end{array}$

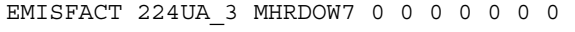

** Month $=$ January; Day of Week = Tuesday EMISFACT 224UA_3 MHRDOW7 00000000000 EMISFACT 224UA_3 MHRDOW7 00000000000

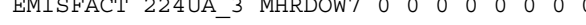

* Month $=$ February; Day of Week $=$ Tuesday EMISFACT 224UA 3 MHRDOW7 00000000000 EMISFACT 224UA 3 MHRDOW7 00000000000

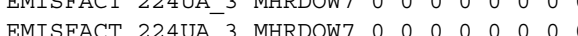

** Month = March; Day of Week = Tuesday

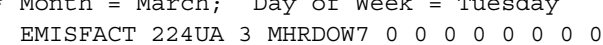
EMISFACT 224UA 3 MHRDOW7 000000000000 EMISFACT 224UA_3 MHRDOW7 $\begin{array}{llllllllllllll}0 & 0 & 0 & 0 & 0 & 0 & 0 & 0 \\ \text { EMISFACT 224UA } 3 \text { MHRDOW7 } & 0 & 0 & 0 & 0 & 0 & 0 & 0 & 0\end{array}$

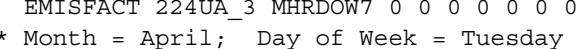

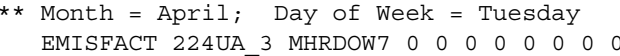

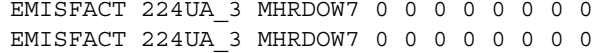

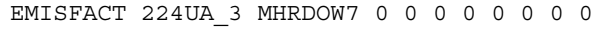

** Month = May; Day of Week = Tuesday

EMISFACT 224UA 3 MHRDOW7 0 O 000000000

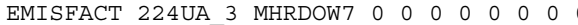

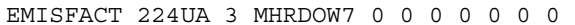

* Month $=$ June; Day of Week = Tuesday

EMISFACT 224UA 3 MHRDOW7 000000000000 EMISFACT 224UA_3 MHRDOW7 00000000000 EMISFACT 224UA 3 MHRDOW7 00000000000

** Month = July; Day of Week = Tuesday

EMISFACT 224UA_3 MHRDOW7 0000000000 EMISFACT 224UA_3 MHRDOW7 $000 \begin{array}{lllllll}0 & 0 & 0 & 0 & 0 & 0 & 0\end{array}$ EMISFACT 224UA_3 MHRDOW7 000000000000

* Month $=$ August $;$ Day of Week = Tuesday EMISFACT 224UA_3 MHRDOW7 00000000000

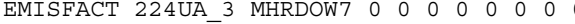

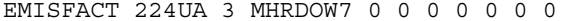

** Month $=$ Sept Month EMTSEACT 224UA 3 MHRDOW7 000400000000

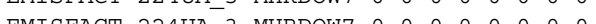

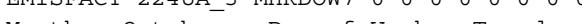
* Month = October; Day of Week = Tuesday EMISFACT 224UA_3 MHRDOW7 0000000001

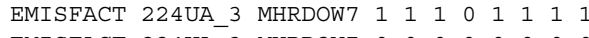

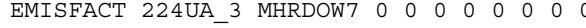

* Month $=$ November; Day of Week = Tuesday EMISFACT 224UA 3 MHRDOW7 $0 \begin{array}{lllllll}0 & 0 & 0 & 0 & 0 & 0\end{array}$

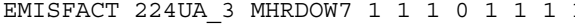

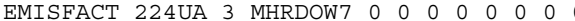

** Month $=$ December $;$ Day of Week $=$ Tuesday 


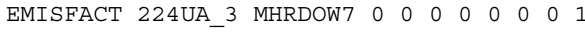

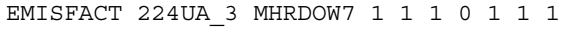

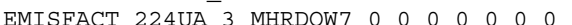

** Month = Januaryi Day of Week = Wednesday

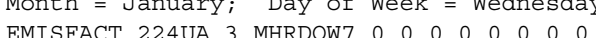
EMISFACT 224UA_ 3 MHROW $0 \begin{array}{llllllllllll}0 & 0 & 0 & 0 & 0 & 0\end{array}$

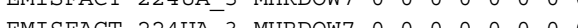

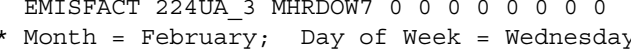
Month = February; Day of Week $=$ Wednesday
EMISFACT 224UA_3 MHRDOW7 00000000000 EMISFACT 224UA_3 MHRDOW7 $00 \begin{array}{lllllll}0 & 0 & 0 & 0 & 0 & 0 & 0\end{array}$ EMISFACT 224UA_3 MHRDOW7 00000000000

** Month $=$ March; Day of Week $=$ Wednesday EMISFACT 224UA_3 MHRDOW7 00010000000

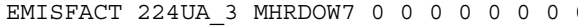

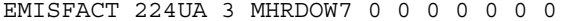

** Month = April; Day of Week = Wednesday TMISFACT 2240 ,

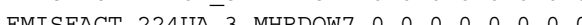

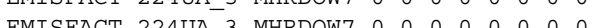
EMISFACT 2240A_3 MHRDOW7 000000000 * Month = May; Day of Week = Wednesday EMISFACT 224UA_3 MHRDOW7 $0 \begin{array}{lllllll}0 & 0 & 0 & 0 & 0 & 0 & 0\end{array}$ EMISFACT 224UA_3 MHRDOW7 000000000000 EMISFACT 224UA_3 MHRDOW7 00000000000

** Month $=$ June; Day of Week $=$ Wednesday EMISFACT 224UA_3 MHRDOW7 00000000000 EMISFACT 224UA_3 MHRDOW7 $00 \begin{array}{lllllll}0 & 0 & 0 & 0 & 0 & 0 & 0\end{array}$

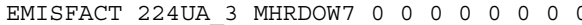

** Month = Julyi Day of Week

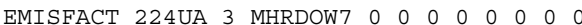
FMTSFACT 224UA 3 MHRDOW7 $00 \begin{array}{llllllllllllll}0 & 0 & 0 & 0 & 0 & 0 & 0\end{array}$

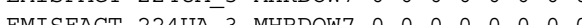

EMISFACT 2240A-3 MHRDOW7 $0 \begin{array}{llllllll}0 & 0 & 0 & 0 & 0 & 0 & 0 & 0\end{array}$

* Month = August $;$ Day of Week = Wednesday

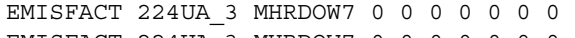

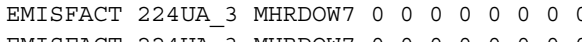
EMISFACT 224UA_3 MHRDOW7 000000000000

** Month = September; Day of Week = Wednesday EMISFACT 224UA_3 MHRDOW7 00000000000

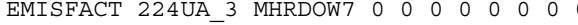

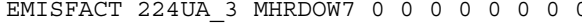

** Month $=$ October; Day of Week = Wednesday EMISFACT 224UA 3 MHRDOW7 0000000 EMISFACT 224UA 3 MHRDOW7 111001 EMTSEACT 224UA 3 MHRDON7 0000000

Month = November; Day of Week = Wednesday Month = November; Day of week $=$ Wednesday
EMISFACT 224UA_3 MHRDOW7 0000000001 $\begin{array}{lllllllll}\text { EMISFACT 224UA_3 MHRDDW7 } & 0 & 0 & 0 & 0 & 0 & 0 & 0 \\ \text { EMISFACT } & 224 \mathrm{UA} 3 \\ \text { MHRDOW7 } & 1 & 1 & 1 & 0 & 1 & 1 & 1\end{array}$ $\begin{array}{lllllllllll}\text { EMISFACT 224UA_3 } & \text { MHRDOW7 } & 1 & 1 & 1 & 0 & 1 & 1 & 1 & 1 \\ \text { EMISFACT 224UA_3 } & \text { MHRDOW7 } & 0 & 0 & 0 & 0 & 0 & 0 & 0 & 0\end{array}$

* Month $=$ December; Day of Week = Wednesday

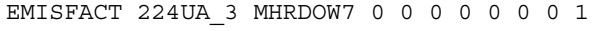
EMISFACT 224UA 3 MHRDOW7 $1 \begin{array}{lllllllllll}1 & 1 & 1 & 0 & 1 & 1 & 1 & 1\end{array}$ EMISFACT 224UA_3 MHRDOW7 $00 \begin{array}{lllllll}0 & 0 & 0 & 0 & 0 & 0 & 0\end{array}$

* Month = January; Day of Week = Thursday EMISFACT 224UA 3 MHRDOW7 0 - $\begin{array}{lllllllll}0 & 0 & 0 & 0 & 0 & 0 & 0\end{array}$ 
EMISFACT 224UA_3 MHRDOW7 000000000000 EMISFACT 224UA_3 MHRDOW7 000000000000

** Month $=$ February; Day of Week = Thursday EMISFACT 224UA 3 MHRDOW7 00000000000 EMISFACT 224UA_3 MHRDOW7 00000000000 EMISFACT 224UA_3 MHRDOW7 $00 \begin{array}{llllllll}0 & 0 & 0 & 0 & 0 & 0 & 0\end{array}$ * Month = March; Day of Week = Thursday EMISFACT 224UA_3 MHRDOW7 00000000000 EMISFACT 224UA_3 MHRDOW7 $0 \begin{array}{lllllllll}0 & 0 & 0 & 0 & 0 & 0 & 0\end{array}$ EMISFACT 224UA_3 MHRDOW7 00000000000

** Month $=$ April; ${ }^{3}$ Day of week = Thursday EMISFACT 224UA_3 MHRDOW7 $00 \begin{array}{lllllll}0 & 0 & 0 & 0 & 0 & 0 & 0\end{array}$ EMISFACT 224UA_3 MHRDOW7 $00 \begin{array}{lllllll}0 & 0 & 0 & 0 & 0 & 0 & 0\end{array}$ EMISFACT 224UA 3 MHRDOW7 $000 \begin{array}{llllll}0 & 0 & 0 & 0 & 0 & 0\end{array}$

** Month $=$ May; Day of Week = Thursday EMISFACT 224UA_3 MHRDOW7 000000000000 EMISFACT 224UA_3 MHRDOW7 $000 \begin{array}{lllllll}0 & 0 & 0 & 0 & 0 & 0 & 0\end{array}$

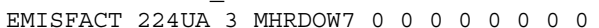

** Month = June; Day of Week = Thursday EMISFACT 224UA_3 MHRDOW7 $0 \begin{array}{lllllll}0 & 0 & 0 & 0 & 0 & 0 & 0\end{array}$ EMISFACT 224UA_3 MHRDOW7 000000000000 EMISFACT 224UA_3 MHRDOW7 00000000000

$* *$ Month $=$ July; Day of Week = Thursday

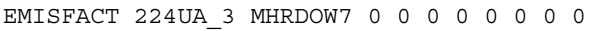

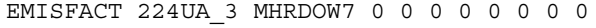
EMISFACT 224UA_3 MHRDOW7 $0 \begin{array}{lllllll}0 & 0 & 0 & 0 & 0 & 0 & 0\end{array}$

* Month = August; Day of Week = Thursday EMISFACT 224UA 3 MHRDOW7 0000000000 EMISFACT 224UA 3 MHRDOW7 0000000000

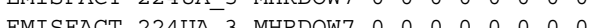

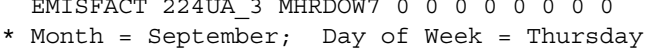
$\begin{array}{lllllllll}\text { Month }=\text { September; Day } & \text { EMISFACT 224UA } 3 \text { MHRDOW7 } 0 & 0 & 0 & 0 & 0 & 0 & 0 & 0\end{array}$

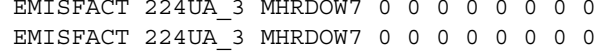
EMISFACT 224UA_3 MHRDOW7 00000000000 * Month = October; Day of Week = Thursday

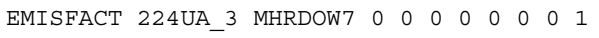
EMISFACT 224UA_3 MHRDOW7 $11110 \begin{array}{lllllll}1 & 0 & 1 & 1 & 1 & 1\end{array}$

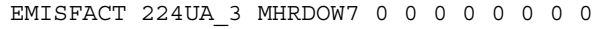

** Month $=$ November; Day of Week $=$ Thursday

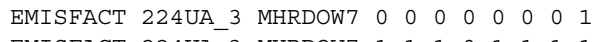
EMISFACT 224UA_3 MHRDOW7 111110011101 EMISFACT 224UA 3 MHRDOW7 00000000000

** Month = December; Day of Week = Thursday EMISFACT 224UA_3 MHRDOW7 000000000001 EMISFACT 224UA_3 MHRDOW7 $1 \begin{array}{llllllll}1 & 1 & 1 & 0 & 1 & 1 & 1 & 1\end{array}$ EMISFACT 224UA_3 MHRDOW7 00000000000

** Month = January; Day of Week = Friday EMISFACT 224UA_3 MHRDOW7 00000000000 EMISFACT 224UA_3 MHRDOW7 $0 \begin{array}{llllllllllll}0 & 0 & 0 & 0 & 0 & 0 & 0\end{array}$ EMISFACT 224UA_3 MHRDOW7 $00 \begin{array}{llllllll}0 & 0 & 0 & 0 & 0 & 0 & 0\end{array}$

** Month = February; Day of week = Friday EMISFACT 224UA_ 
EMISFACT 224UA_3 MHRDOW7 00000000000

** Month $=$ March; Day of Week = Friday

EMISFACT 224UA_3 MHRDOW7 000000000000 EMISFACT 224UA_3 MHRDOW7 00000000000 T 224UA 3 MHRDOW7 00000000000

** Month $=$ April; $;$ Day of Week = Friday

EMISFACT 224UA_3 MHRDOW7 $0 \begin{array}{llllllll}0 & 0 & 0 & 0 & 0 & 0 & 0\end{array}$ EMISFACT 224UA_3 MHRDOW7 $0 \begin{array}{lllllllll}0 & 0 & 0 & 0 & 0 & 0 & 0 & 0\end{array}$ EMISFACT 224UA_3 MHRDOW7 $00 \begin{array}{llllllllllllll}0 & 0 & 0 & 0 & 0 & 0 & 0\end{array}$

* Month = May; Day of Week = Friday

EMISFACT 224UA_3 MHRDOW7 00000000000 EMISFACT 224UA_3 MHRDOW7 $0 \begin{array}{lllllll}0 & 0 & 0 & 0 & 0 & 0 & 0\end{array}$

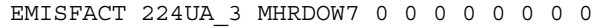

** Month $=$ June; Day of Week = Friday

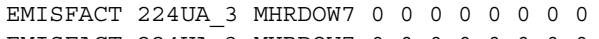
EMISFACT 224UA_3 MHRDOW7 $00 \begin{array}{llllllllll}0 & 0 & 0 & 0 & 0 & 0 & 0\end{array}$ MHRDOW7 $00 \begin{array}{lllllll}0 & 0 & 0 & 0 & 0 & 0 & 0\end{array}$

* Month $=$ July; Day of Week = Friday

EMISFACT 224UA_3 MHRDOW7 00000000000 EMISFACT 224UA_3 MHRDOW7 $\begin{array}{lllllllll}0 & 0 & 0 & 0 & 0 & 0 & 0 & 0\end{array}$ EMISFACT 224UA_3 MHRDOW7 00000000000

* Month $=$ August; Day of Week = Friday

EMISFACT 224UA_3 MHRDOW7 0000000000 EMISFACT 224UA_3 MHRDOW7 $0 \begin{array}{llllllllllllll}0 & 0 & 0 & 0 & 0 & 0 & 0\end{array}$

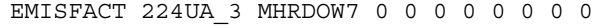

* Month $=$ September; Day of Week $=$ Friday EMISFACT 224UA 3 MHRDOW7 00000000000

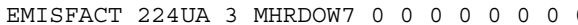
EMISFACT 224UA 3 MHRDOW7 00000000

* Month = OCtober, DaY of Week = Friday Month = October; Day of Week = Friday EMISFACT 224UA_3 MHRDOW7 $0 \begin{array}{lllllllllllll}0 & 0 & 0 & 0 & 0\end{array}$ EMISFACT 224UA_3 MHRDOW7 $1 \begin{array}{lllllll}1 & 1 & 1 & 0 & 1 & 1 & 1\end{array}$ EMISFACT 224UA_3 MHRDOW7 000000000

* Month $=$ November; Day of Week = Friday EMISFACT 224UA_3 MHRDOW7 0000000000 EMISFACT 224UA_3 MHRDOW7 1111100111 EMISFACT 224UA_3 MHRDOW7 $0 \begin{array}{llllllllll}0 & 0 & 0 & 0 & 0 & 0 & 0 & 0\end{array}$

** Month $=$ December $;$ Day of Week = Friday EMISFACT 224UA_3 MHRDOW7 0000000000 EMISFACT 224UA 3 MHRDOW7 $1 \begin{array}{llllllll}1 & 1 & 1 & 0 & 1 & 1 & 1\end{array}$ EMISFACT 224UA 3 MHRDOW7 0 C 0 O

* Month = January; Day of Week = Saturday Monthe January; Day or Week = Saturday

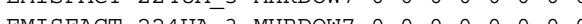

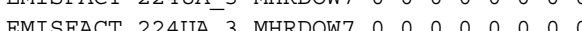
EMISFACI 2240A_3 MHRDOW7 00000000000 * Month = February; Day of week = Saturday EMISFACT 224UA_3 MHRDOW7 00000000000

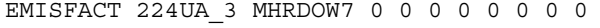
EMISFACT 224UA_3 MHRDOW7 $0 \begin{array}{lllllll}0 & 0 & 0 & 0 & 0 & 0 & 0\end{array}$

** Month $=$ March; Day of Week = Saturday

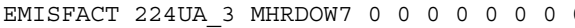
EMISFACT 224UA 3 MHRDOW7 $00 \begin{array}{llllllll}0 & 0 & 0 & 0 & 0 & 0 & 0\end{array}$ EMISFACT 224UA 3 MHRDOW7 
** Month $=$ April; Day of Week = Saturday EMISFACT 224UA_3 MHRDOW7 0000000000000

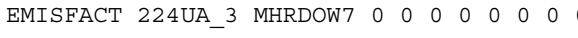

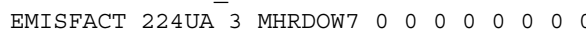

** Month = May; Day of Week = Saturday EMISFACT 224UA_3 MHRDOW7 $0 \begin{array}{lllllll}0 & 0 & 0 & 0 & 0 & 0 & 0\end{array}$ EMISFACT 224UA_3 MHRDOW7 $0 \begin{array}{llllllllllllllll}0 & 0 & 0 & 0 & 0 & 0 & 0\end{array}$

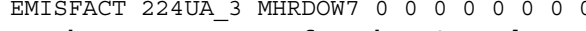

** Month $=$ June; Day of Week = Saturday EMISFACT 224UA_3 MHRDOW7 000000000000 EMISFACT 224UA_3 MHRDOW7 $00 \begin{array}{lllllll}0 & 0 & 0 & 0 & 0 & 0 & 0\end{array}$

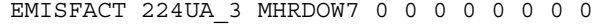

** Month $=$ July; Day of Week = Saturday

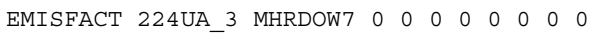

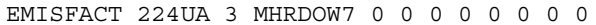
EMISFACT 224UA 3 MHRDOW7 0000

* EMISTACT 224UA_3 MHRDOW7 $00 \begin{array}{lllllll}0 & 0 & 0 & 0 & 0 & 0 & 0\end{array}$ ** Month = August; Day of Week = Saturday EMISFACT 224UA_3 MHRDOW7 $0 \begin{array}{llllllll}0 & 0 & 0 & 0 & 0 & 0 & 0\end{array}$

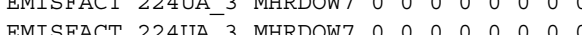

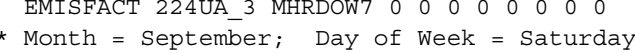

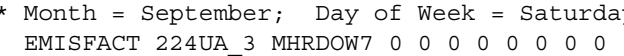

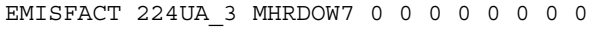

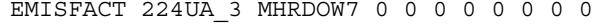

** Month = October; Day of Week = Saturday EMISFACT 224UA_3 MHRDOW7 00000000000 EMISFACT 224UA 3 MHRDOW7 $00 \begin{array}{lllllll}0 & 0 & 0 & 0 & 0 & 0 & 0\end{array}$

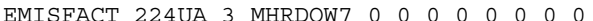

** Month $=$ November; Day of Week = Saturday EMISFACT 224UA_3 MHRDOW7 000000000000 EMISFACT 224UA_3 MHRDOW7 00000000000 EMISFACT 224UA 3 MHRDOW7 0000000000

** Month $=$ December $; \quad$ Day of Week = Saturday EMISFACT 224UA_3 MHRDOW7 $0 \begin{array}{lllllll}0 & 0 & 0 & 0 & 0 & 0 & 0\end{array}$

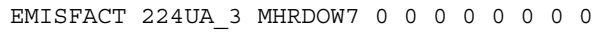

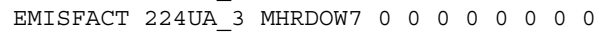

* Month = January; Day of Week = Sunday EMISFACT 224UA_3 MHRDOW7 00000000000

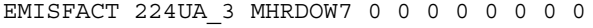

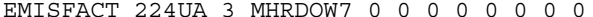

* Month = Februaryi EMISFACT 224 UA MHRDOW7 0 o 0 Sunday EMISFACT 224UA-3 MHRDOW7 000000000

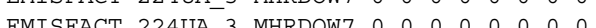

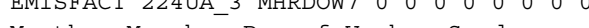
* Month $=$ March; Day of Week = Sunday EMISFACT 224UA_3 MHRDOW7 0000000000 EMISFACT 224UA_3 MHRDOW7 000000000000 EMISFACT 224UA_3 MHRDOW7 00000000000

* Month $=$ April; $;$ Day of Week = Sunday EMISFACT 224UA_3 MHRDOW7 00000000000 EMISFACT 224UA_3 MHRDOW7 $00 \begin{array}{lllllll}0 & 0 & 0 & 0 & 0 & 0 & 0\end{array}$ EMISFACT 224UA 3 MHRDOW7 000000000000

$* *$ Month $=$ May; Day of week $=$ Sunday 
EMISFACT 224UA_3 MHRDOW7 000000000000 EMISFACT 224UA_3 MHRDOW7 000000000000 EMISFACT 224UA 3 MHRDOW7 0 O

** Month $=$ June; Day of Week = Sunday

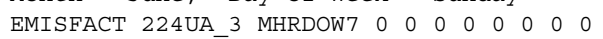
EMISFACT 224UA_3 MHRDOW7 $00 \begin{array}{lllllllllllllll} & 0 & 0 & 0 & 0 & 0 & 0\end{array}$ EMISFACT 224UA_3 MHRDOW7 0000000000

** Month $=$ July; Day of Week = Sunday

EMISFACT 224UA_3 MHRDOW7 $00 \begin{array}{lllllll}0 & 0 & 0 & 0 & 0 & 0 & 0\end{array}$

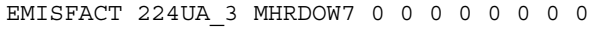

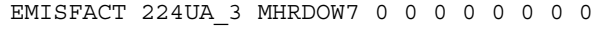

** Month $=$ August $; \quad$ Day of Week $=$ Sunday

EMISFACT 224UA_3 MHRDOW7 00000000000 EMISFACT 224UA_3 MHRDOW7 $00 \begin{array}{lllllll}0 & 0 & 0 & 0 & 0 & 0 & 0\end{array}$ EMISFACT 224UA 3 MHRDOW7 0001000000

** Month = September; Day of Week = Sunday Month = Septent

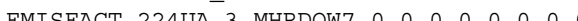

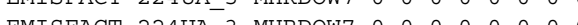
EMISFACT 2240A-3 MHRDOW7 0 * Month = October; Day of Week = Sunday EMISFACT 224UA_3 MHRDOW7 0000000000 EMISFACT 224UA_3 MHRDOW7 000000000000 EMISFACT 224UA_3 MHRDOW7 00000000000

* Month = November; Day of Week = Sunday EMISFACT 224UA_3 MHRDOW7 00000000000 EMISFACT 224UA 3 MHRDOW7 $00 \begin{array}{lllllll}0 & 0 & 0 & 0 & 0 & 0 & 0\end{array}$ EMISFACT 224UA 3 MHRDOW7 00000000000 FMTSFAT

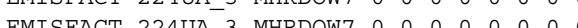

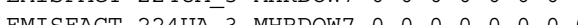

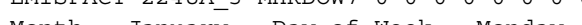
* Month = January; Day of Week = Monday EMISFACT 224UA_4 MHRDOW7 00000000000 EMISFACT 224UA_4 MHRDOW7 000000000000 EMISFACT 224UA_4 MHRDOW7 0000000000

$*$ Month $=$ February; Day of week $=$ Monday EMISFACT 224UA_4 MHRDOW7 00000000000 EMISFACT 224UA_4 MHRDOW7 00000000000

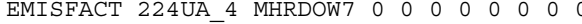

** Month $=$ March; Day of Week = Monday EMISFACT 224UA 4 MHRDOW7 0000000000

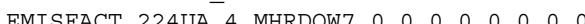
EMTSFACT 224UA- MHRDOW7 00000000

* Month = April; Day of Week = Monday

* * Month = April; $;$ Day of Week = Monday

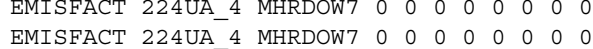
EMISFACT 224UA_4 MHRDOW7 000000000000

* Month = May; Day of Week = Monday

EMISFACT 224UA_4 MHRDOW7 00000000000 EMISFACT 224UA 4 MHRDOW7 $00 \begin{array}{lllllll}0 & 0 & 0 & 0 & 0 & 0 & 0\end{array}$

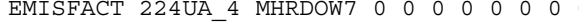
$* *$ Month $=$ June; Day of Week = Monday EMISFACT 224UA_4 MHRDOW7 000000000000 
EMISFACT 224UA_4 MHRDOW7 0000000000000 EMISFACT 224UA 4 MHRDOW7 000000000000

** Month $=$ July; Day of Week = Monday

EMISFACT 224UA_4 MHRDOW7 $00 \begin{array}{lllllll}0 & 0 & 0 & 0 & 0 & 0\end{array}$ EMISFACT 224UA 4 MHRDOW7 00000000000 EMISFACT 224UA 4 MHRDOW7 00000000000

** Month = August; Day of Week = Monday

EMISFACT 2240 EMISFACT 224UA_4 MHRDOW7 $00 \begin{array}{llllllll}0 & 0 & 0 & 0 & 0 & 0 & 0\end{array}$ EMISFACT 224UA_4 MHRDOW7 00000000000

* Month $=$ September; Day of Week = Monday

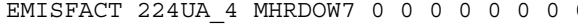

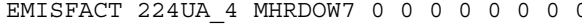

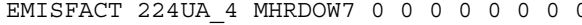

** Month $=$ October $;$ Day of Week = Monday EMTSFACT 224UA 4 MHRDOW7 0000000

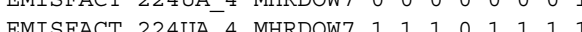
TMTSFACT 224UA_4 MHRDOW7 00000000

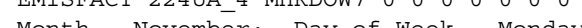
* Month = November; Day of Week = Monday EMISFACT 224UA_4 MHRDOW7 $\begin{array}{lllllll}0 & 0 & 0 & 0 & 0 & 0 & 0\end{array}$ EMISFACT 224UA_4 MHRDOW7 $11 \begin{array}{lllllll}1 & 1 & 0 & 1 & 1 & 1\end{array}$ EMISFACT 224UA_4 MHRDOW7 0000000000

* Month $=$ December; Day of Week = Monday EMISFACT 224UA_4 MHRDOW7 $00 \begin{array}{lllllll}0 & 0 & 0 & 0 & 0 & 0\end{array}$

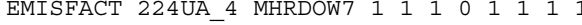

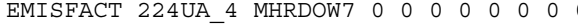

* Month = January; Day of Week = Tuesday EMISFACT 224UA 4 MHRDOW7 0000000000 EMISFACT 224UA MTISFACT 224UA

* EMISFACT 2240_4 MHRDOW7 $\begin{array}{lllllllll}0 & 0 & 0 & 0 & 0 & 0 & 0\end{array}$ * Month = February; Day of Week = Tuesday

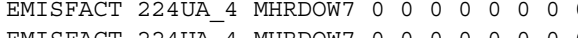
EMISFACT 224UA_4 MHRDOW7 $00 \begin{array}{lllllllll}0 & 0 & 0 & 0 & 0 & 0 & 0\end{array}$ EMISFACT 224UA_4 MHRDOW7 0000000000

* Month $=$ March; Day of Week = Tuesday EMISFACT 224UA_4 MHRDOW7 00000000000 EMISFACT 224UA_4 MHRDOW7 $00 \begin{array}{llllllll}0 & 0 & 0 & 0 & 0 & 0 & 0\end{array}$

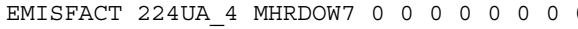

** Month $=$ April; Day of Week = Tuesday EMISFACT 224UA_4 MHRDOW7 000000000000 EMISFACT 224UA_4 MHRDOW7 000000000000

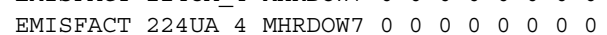

* Month = May; Day of Week = Tuesday

EMISFACT 224UA 4 MHRDOW7 $00 \begin{array}{ccccccc}0 & 0 & 0 & 0 & 0 & 0 & 0\end{array}$

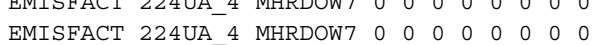

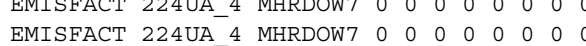

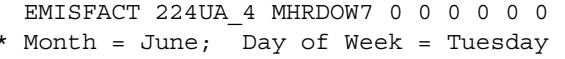
EMISFACT 224UA_4 MHRDOW7 00000000000

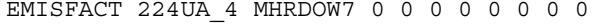

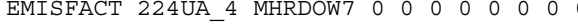

** Month $=$ July; Day of Week $=$ Tuesday

EMISFACT 224UA_4 MHRDOW7 000000000000 EMISFACT 224UA 4 MHRDOW7 0 C. 
EMISFACT 224UA_4 MHRDOW7 00000000000

** Month $=$ August $;$ Day of Week $=$ Tuesday

EMISFACT 224UA_4 MHRDOW7 00000000000 0

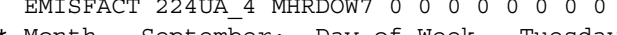

* Month = September; Day of Week = Tuesday EMISFACT 224UA_4 MHRDOW7 0000000000

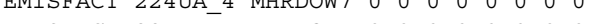
EMISFACT 224UA_4 MHRDOW7 $00 \begin{array}{lllllll}0 & 0 & 0 & 0 & 0 & 0 & 0\end{array}$

* Month = October; Day of Week = Tuesday EMISFACT 224UA_4 MHRDOW7 $000 \begin{array}{cccccc}0 & 0 & 0 & 0 & 0 & 1\end{array}$ EMISFACT 224UA_4 MHRDOW7 $1 \begin{array}{lllllllll}1 & 1 & 1 & 0 & 1 & 1 & 1 & 1\end{array}$

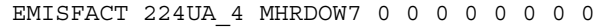

** Month $=$ November $; \quad$ Day of Week = Tuesday

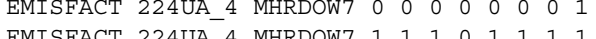

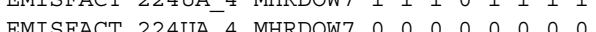

EMISFACT 2240A, MHROW 00 * Month = December; Day of Week = Tuesday EMISFACT 224UA_4 MHRDOW7 $0 \begin{array}{ccccccccc}0 & 0 & 0 & 0 & 0 & 0 & 1\end{array}$ EMISFACI 2240A_4 MHRDOW7 $\begin{array}{llllllllllllllllll}1 & 1 & 1 & 0 & 1 & 1 & 1 & 1\end{array}$

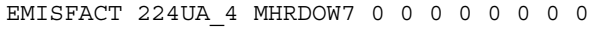

* Month $=$ January; Day of Week $=$ Wednesday

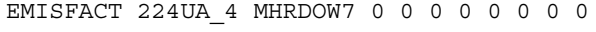

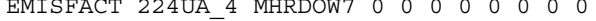

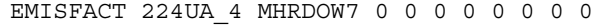

** Month $=$ February; Day of Week $=$ Wednesday EMISFACT 224UA_4 MHRDOW7 000000000000 EMISFACT 224UA_4 MHRDOW7 000000000000 EMISFACT 224UA 4 MHRDOW7 00000000000

** Month $=$ March; Day of Week = Wednesday EMISFACT 224UA_4 MHRDOW7 00000000000

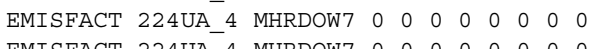

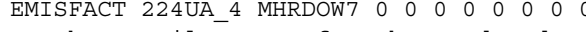

** Month $=$ April; $;$ Day of Week $=$ Wednesday EMISFACT 224UA_4 MHRDOW7 00000000000 EMISFACT 224UA_4 MHRDOW7 $000 \begin{array}{llllllll}0 & 0 & 0 & 0 & 0 & 0 & 0\end{array}$

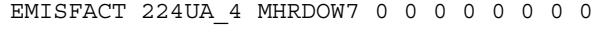

** Month $=$ May; Day of Week $=$ Wednesday EMISFACT 224UA_4 MHRDOW7 00000000000 EMISFACT 224UA 4 MHRDOW7 $00 \begin{array}{lllllll}0 & 0 & 0 & 0 & 0 & 0 & 0\end{array}$ EMISFACT 224UA_4 MHRDOW7 000000

* Month = Jun_Month = June;

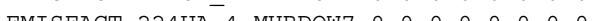
EMISFACT 224UA_4 MHRDOW7 $\begin{array}{llllllllllllllllll}0 & 0 & 0 & 0 & 0 & 0 & 0\end{array}$ EMISFACT 2240A_4 MHRDOW7 0 O 0000000000 * Month $=$ July; Day of Week $=$ Wednesday EMISFACT 224UA_4 MHRDOW7 0010000000000 EMISFACT 224UA_4 MHRDOW7 $0 \begin{array}{lllllllllll}0 & 0 & 0 & 0 & 0 & 0 & 0\end{array}$

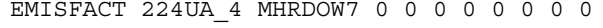

** Month = August; Day of Week $=$ Wednesday EMISFACT 224UA 4 MHRDOW7 00000000000

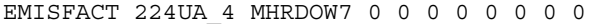

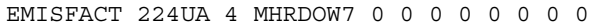


** Month $=$ September; Day of Week $=$ Wednesday EMISFACT 224UA_4 MHRDOW7 000000000000

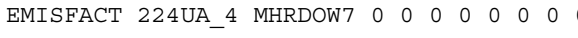
EMISFACT 224UA 4 MHRDOW7 00000000000

** Month $=$ October; $;$ Day of Week = Wednesday EMISFACT 224UA_4 MHRDOW7 000000000001 EMISFACI 2240A_4 MHRDOW7 $\begin{array}{cccccccc}1 & 1 & 1 & 0 & 1 & 1 & 1 & 1\end{array}$

** Month $=$ November; Day of Week = Wednesday EMISFACT 224UA_4 MHRDOW7 0000000000

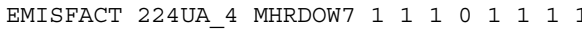
EMISFACT 224UA_4 MHRDOW7 $00 \begin{array}{lllllll}0 & 0 & 0 & 0 & 0 & 0 & 0\end{array}$

** Month $=$ December; Day of Week $=$ Wednesday

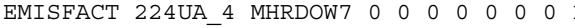
EMISFACT 224UA 4 MHRDOW7 $1 \begin{array}{lllllllllll}1 & 1 & 0 & 1 & 1 & 1 & 1\end{array}$ EMISFACT 224UA 4 MHRDOW7 0 O 0000000

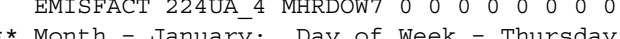
Month = January; Day of Week = Thursday EMISFACT 224UA - MHRDOW $0 \begin{array}{lllllllll}0 & 0 & 0 & 0 & 0 & 0\end{array}$ EMISFACT 224UA_4 MHRDW $0 \begin{array}{llllllll}0 & 0 & 0 & 0 & 0 & 0 & 0\end{array}$ EMISFACT 224UA_4 MHRDOW7 0 O 00000000000

* Month = February; Day of Week = Thursday EMISFACT 224UA_4 MHRDOW7 0000000000

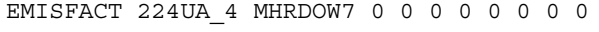
EMISFACT 224UA_4 MHRDOW7 $0 \begin{array}{lllllll}0 & 0 & 0 & 0 & 0 & 0 & 0\end{array}$

** Month $=$ March; Day of Week = Thursday EMISFACT 224UA_4 MHRDOW7 00010000000

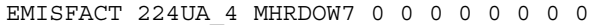
EMISFACT 224UA 4 MHRDOW7 000000000

* * Month = Aprili Day of Week = Thursday EMISEACT 224UA 4 MHRDOW7 00000000000 EMISFACT 224UA EMISFACT 224UA 4 MHRDOW7 0000000000

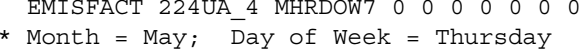

Month = May; Day of Week = Thursday
EMISFACT 224UA 4 MHRDOW7 0 00000000 $\begin{array}{llllllllll}\text { EMISFACT } & \text { 224UA_4 MHRDOW7 } & 0 & 0 & 0 & 0 & 0 & 0 & 0 & 0 \\ \text { EMISFACT } & \text { 224UA_4 MHRDOW7 } & 0 & 0 & 0 & 0 & 0 & 0 & 0 & 0\end{array}$ EMISFACT 224UA_4 MHRDOW7 00000000000

** Month $=$ June; Day of Week $=$ Thursday EMISFACT 224UA_4 MHRDOW7 00000000000 EMISFACT 224UA_4 MHRDOW7 00010000000

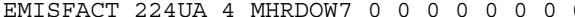

* Month = Julyi Day of Week = Thursday EMISFACT 224UA 4 MHRDOW7 000000 FMTSFACT 224UA 4 MHRDOW7 000000000 BMISFACT 224UA-4 MHRDOW7 $0 \begin{array}{lllllllllllllll}0 & 0 & 0 & 0 & 0 & 0 & 0\end{array}$ EMISFACT 2240A_4 MHRDOW7 0 o 000000 * Month = August $;$ Day of Week = Thursday EMISFACT 224UA_4 MHRDOW7 000000000000

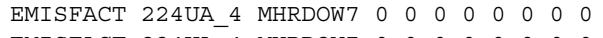
EMISFACT 224UA_4 MHRDOW7 00000000000

** Month $=$ September; Day of Week $=$ Thursday

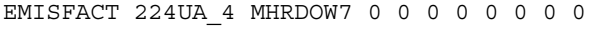
EMISFACT 224UA 4 MHRDOW7 $00 \begin{array}{lllllll}0 & 0 & 0 & 0 & 0 & 0 & 0\end{array}$

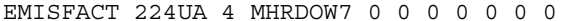

** Month $=$ October $;$ Day of Week $=$ Thursday 
EMISFACT 224UA_4 MHRDOW7 $00 \begin{array}{lllllll}0 & 0 & 0 & 0 & 0 & 0 & 1\end{array}$ EMISFACT 224UA_4 MHRDOW7 1111100111

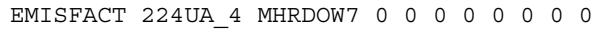

** Month $=$ November; $;$ Day of Week = Thursday

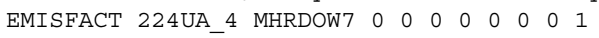

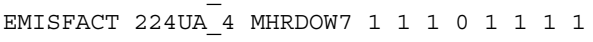
EMISFACT 224UA_4 MHRDOW7 $0 \begin{array}{llllllll}0 & 0 & 0 & 0 & 0 & 0 & 0\end{array}$

** Month $=$ December; Day of week = Thursday EMISFACT 224UA_4 MHRDOW7 $00 \begin{array}{lllllll}0 & 0 & 0 & 0 & 0 & 0 & 1\end{array}$ EMISFACT 224UA_4 MHRDOW7 $11110 \begin{array}{llllllll}1 & 1 & 1 & 1 & 1 & 1\end{array}$

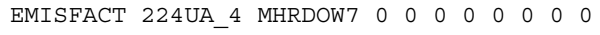

** Month = January; Day of Week = Friday EMISFACT 224UA_4 MHRDOW7 00010000000

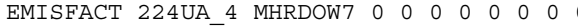

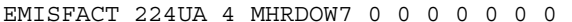

** Month = February; Day of Week = Friday

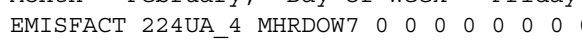
EMISFACT 224UA 4 MHRDOW7 $00 \begin{array}{lllllll}0 & 0 & 0 & 0 & 0 & 0 & 0\end{array}$ EMISFACT 224UA 4 MHRDOW7 001000000000

* Month $=$ March; $;$ Day of Week = Friday

** Month $=$ March; Day of Week = Friday

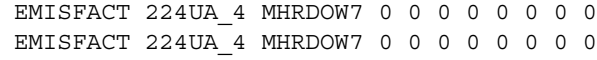
EMISFACT 224UA_4 MHRDOW7 0000000000

$* *$ Month $=$ April; $;$ Day of Week = Friday

EMISFACT 224UA_4 MHRDOW7 00000000000

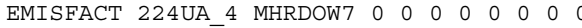

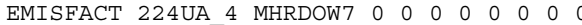

** Month $=$ May; Day of Week = Friday

EMISFACT 224UA 4 MHRDOW7 0000000000

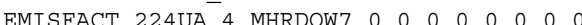

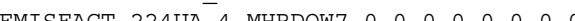

* Month $=$ June; Day of Week = Friday

EMISFACT 224UA_4 MHRDOW7 00000000000

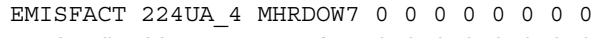

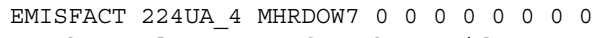

** Month $=$ July; Day of Week = Friday

EMISFACT 224UA_4 MHRDOW7 $00 \begin{array}{lllllll}0 & 0 & 0 & 0 & 0 & 0 & 0\end{array}$ EMISFACT 224UA_4 MHRDOW7 $00 \begin{array}{lllllll}0 & 0 & 0 & 0 & 0 & 0 & 0\end{array}$ EMISFACT 224UA_4 MHRDOW7 $00 \begin{array}{ccccccc}0 & 0 & 0 & 0 & 0 & 0 & 0\end{array}$

$* *$ Month $=$ August $;$ Day of week $=$ Friday EMISFACT 224UA_4 MHRDOW7 00000000000 EMISFACT 224UA 4 MHRDOW7 $00 \begin{array}{lllllll}0 & 0 & 0 & 0 & 0 & 0 & 0\end{array}$ EMISFACT 224UA 4 MHRDOW7 $00 \begin{array}{lllllll}0 & 0 & 0 & 0 & 0 & 0 & 0\end{array}$

* Month = September; Day of Week = Friday

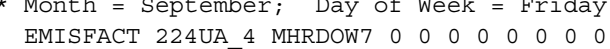

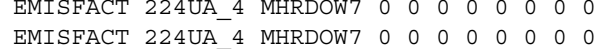
$\begin{array}{llllllllll}\text { EMISFACT 224UA_4 MHRDOW7 } & 0 & 0 & 0 & 0 & 0 & 0 & 0 & 0 \\ \text { EMISFACT } & \text { 224UA_4 MHRDOW7 } & 0 & 0 & 0 & 0 & 0 & 0 & 0 & 0\end{array}$

* Month = October; Day of Week = Friday

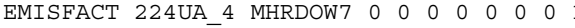

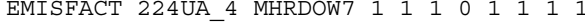
EMISFACT 224UA_4 MHRDOW7 00000000000 * Month $=$ November $;$ Day of Week = Friday EMISFACT 224UA 4 MHRDOW7 0 O 
EMISFACT 224UA_4 MHRDOW7 1111100111 EMISFACT 224UA_4 MHRDOW7 00000000000

** Month $=$ December $;$ Day of Week = Friday EMISFACT 224UA 4 MHRDOW7 0000000000 EMISFACT 224UA 4 MHRDOW7 $1 \begin{array}{llllllllll}1 & 1 & 0 & 1 & 1 & 1 & 1\end{array}$

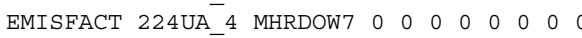

** Month = January; Day of Week = Saturday EMISFACT 224UA_4 MHRDOW7 0000000000 EMISFACT 224UA_4 MHRDOW7 0000000000000 EMISFACT 224UA_4 MHRDOW7 0000000000

$*$ Month $=$ February; Day of Week = Saturday

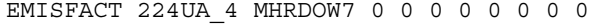
EMISFACT 224UA_4 MHRDOW7 $00 \begin{array}{lllllll}0 & 0 & 0 & 0 & 0 & 0 & 0\end{array}$ EMISFACT 224UA_4 MHRDOW7 000100000000

** Month $=$ March;

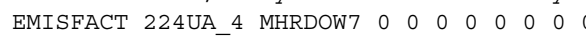
FMISFACT 224UA 4 MHRDOW7 0000000000

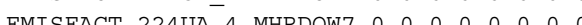

BMISEAC 2240_4 MHROW7 0 o 0 o 0000

* Month = April; Day of Week = Saturday EMISFACT 224UA_4 MHRDOW7 $\begin{array}{llllllll}0 & 0 & 0 & 0 & 0 & 0 & 0 & 0\end{array}$ EMISFACT 224UA_4 MHRDOW7 $00 \begin{array}{lllllll}0 & 0 & 0 & 0 & 0 & 0 & 0\end{array}$ EMISFACT 224UA_4 MHRDOW7 00000000000

* Month = May; Day of Week = Saturday

EMISFACT 224UA_4 MHRDOW7 $00 \begin{array}{lllllll}0 & 0 & 0 & 0 & 0 & 0 & 0\end{array}$ EMISFACT 224UA_4 MHRDOW7 $00 \begin{array}{lllllll}0 & 0 & 0 & 0 & 0 & 0 & 0\end{array}$

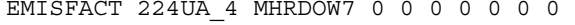

** Month = June; Day of Week = Saturday

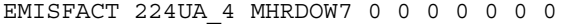

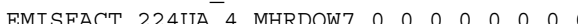

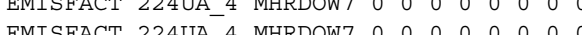

EMISFACT 2240_4 MHRDOW7 000000000

* Month $=$ July; Day of Week = Saturday EMISFACT 224UA_4 MHRDOW7 $0 \begin{array}{llllllllll}0 & 0 & 0 & 0 & 0 & 0 & 0 & 0\end{array}$

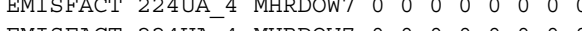
EMISFACT 224UA_4 MHRDOW7 00000000000 * Month = August; Day of Week = Saturday EMISFACT 224UA_4 MHRDOW7 00000000000 EMISFACT 224UA_4 MHRDOW7 $00 \begin{array}{llllllll}0 & 0 & 0 & 0 & 0 & 0 & 0\end{array}$

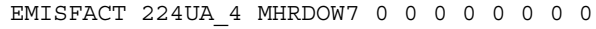

** Month $=$ September; Day of Week = Saturday EMISFACT 224UA_4 MHRDOW7 000000000000

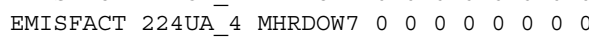
EMISFACT 224UA 4 MHRDOW7 0 O 0000000

EMT * Month = October; Day of Week = Saturday EMISFACT 224UA_4 MHRDOW7 00000000000 EMISFACT 224UA_ MHDOW7 $0 \begin{array}{lllllll}0 & 0 & 0 & 0 & 0 & 0 & 0\end{array}$

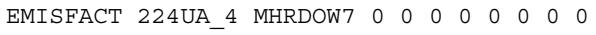

* Month $=$ November; Day of Week = Saturday

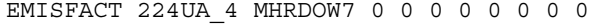
EMISFACT 224UA_4 MHRDOW7 $00 \begin{array}{llllllll}0 & 0 & 0 & 0 & 0 & 0 & 0\end{array}$ EMISFACT 224UA_4 MHRDOW7 $00 \begin{array}{llllllll}0 & 0 & 0 & 0 & 0 & 0 & 0\end{array}$

** Month = December; Day of Week = Saturday EMISFACT 224UA 4 MHRDOW7 0 
EMISFACT 224UA_4 MHRDOW7 0000000000

** Month $=$ January; Day of Week = Sunday

EMISFACT 224UA_4 MHRDOW7 00000000000 EMISFACT 224UA 4 MHRDOW7 $00 \begin{array}{llllllll} & 0 & 0 & 0 & 0 & 0 & 0 & 0\end{array}$

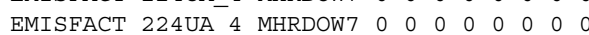

** Month = February; Day of week = Sunday EMISFACT 224UA_4 MHRDOW7 000000000 EMISFACT 2240_- MHRDOW7 00 EMISFACT 224UA_4 MHRDOW7 0000000000

* Month $=$ March; Day of Week = Sunday

EMISFACT 224UA_4 MHRDOW7 00000000000 EMISFACT 224UA_4 MHRDOW7 $00 \begin{array}{llllllll}0 & 0 & 0 & 0 & 0 & 0 & 0\end{array}$ EMISFACT 224UA_4 MHRDOW7 000000000000

** Month $=$ April $;$ Day of Week = Sunday

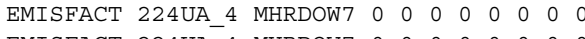
EMISFACT 224UA_4 MHRDOW7 $00 \begin{array}{llllllll}0 & 0 & 0 & 0 & 0 & 0 & 0\end{array}$

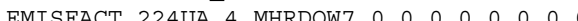

* Month = May; Day of Week = Sunday

Mon EMISFACT 2240A_4 MHRDOW7 $\begin{array}{lllllllllllllllll}0 & 0 & 0 & 0 & 0 & 0 & 0 & 0 & 0 & 0\end{array}$ EMISFACT 224UA_4 MHRDOW7 $00 \begin{array}{llllllllllllll}0 & 0 & 0 & 0 & 0 & 0 & 0\end{array}$

** Month $=$ June; Day of Week = Sunday

EMISFACT 224UA_4 MHRDOW7 000000000000 EMISFACT 224UA_4 MHRDOW7 $00 \begin{array}{lllllll}0 & 0 & 0 & 0 & 0 & 0 & 0\end{array}$ EMISFACT 224UA_4 MHRDOW7 $0 \begin{array}{lllllll}0 & 0 & 0 & 0 & 0 & 0 & 0\end{array}$

** Month $=$ July; Day of Week = Sunday

EMISFACT 224UA_4 MHRDOW7 000000000000 EMISFACT 224UA_4 MHRDOW7 $00 \begin{array}{lllllll}0 & 0 & 0 & 0 & 0 & 0 & 0\end{array}$ EMISFACT 224UA 4 MHRDOW7 $0 \begin{array}{llllllllllllllllll}0 & 0 & 0 & 0 & 0 & 0 & 0 & 0\end{array}$

** Month = August; Day of Week = Sunday EMISFACT 224UA 4 MHRDOW7 0000000000 ISFACT 224UA 4 MHRDOW7 $00 \begin{array}{lllllllll}-4 & 0 & 0 & 0 & 0 & 0 & 0\end{array}$ EMISFACT 224UA 4 MHRDOW7 $00 \begin{array}{lllllllll} & 0 & 0 & 0 & 0 & 0 & 0\end{array}$

* Month $=$ September; Day of Week $=$ Sunday EMISFACT 224UA_4 MHRDOW7 0000000000

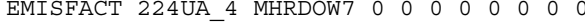
EMISFACT 224UA_4 MHRDOW7 $0 \begin{array}{lllllllllllll}0 & 0 & 0 & 0 & 0 & 0\end{array}$

* Month $=$ October; Day of Week = Sunday

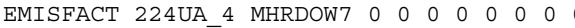
EMISFACT 224UA 4 MHRDOW7 $00 \begin{array}{llllllll}0 & 0 & 0 & 0 & 0 & 0 & 0\end{array}$ EMISFACT 224UA 4 MHRDOW7 000000

* Month = Movenberi Month = November; Day or Week = sunday DMTSFAC 224UA

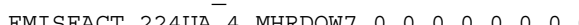

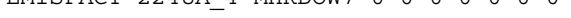
* Month $=$ December $;$ Day of Week = Sunday EMISFACT 224UA_4 MHRDOW7 0000000000 EMISFACT 224UA_4 MHRDOW7 $00 \begin{array}{lllllll}0 & 0 & 0 & 0 & 0 & 0 & 0\end{array}$

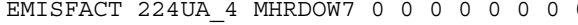

** Month = January; Day of Week = Monday

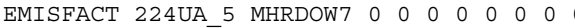

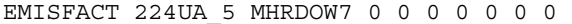
EMISFACT 224UA 5 MHRDOW7 
** Month $=$ February; $\quad$ Day of week = Monday EMISFACT 224UA 5 MHRDOW7 000000000000 UA_5 MHRDOW7 $00 \begin{array}{lllllllll} & 0 & 0 & 0 & 0 & 0 & 0 & 0\end{array}$

* Month = March; Day of Week = Monday

Month = March; Day of Week = Monday
EMISFACT 224UA 5 MHRDOW7 000000000 EMISFACT 224UA 5 MHRDOW7 000000000000 EMISFACT 224UA 5 MHRDOW7 0000000000

** Month $=$ April; ${ }^{-}$Day of Week = Monday

EMISFACT 224UA_5 MHRDOW7 00000000000 EMISFACT 224UA_5 MHRDOW7 $00 \begin{array}{lllllll}0 & 0 & 0 & 0 & 0 & 0 & 0\end{array}$ EMISFACT 224UA_5 MHRDOW7 $00 \begin{array}{lllllll}0 & 0 & 0 & 0 & 0 & 0 & 0\end{array}$

** Month = May; Day of Week = Monday

EMISFACT 224UA_5 MHRDOW7 0000000000000 EMISFACT 224UA_5 MHRDOW7 $0 \begin{array}{lllllll}0 & 0 & 0 & 0 & 0 & 0 & 0\end{array}$

** Month = June; Day of Week = Monday

$\begin{array}{llllllllll}\text { Month }=\text { June; } & \text { Day } \\ \text { EMISFACT 224UA 5 MHRDOW7 } & 0 & 0 & 0 & 0 & 0 & 0 & 0 & 0\end{array}$ EMISFACT 224UA 5 MHRDOW7 000000000000

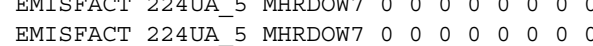

* Month $=$ July; Day of Week = Monday

EMISFACT 224UA_5 MHRDOW7 0000000000

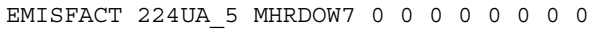
EMISFACT 224UA_5 MHRDOW7 $0 \begin{array}{llllllll}0 & 0 & 0 & 0 & 0 & 0 & 0\end{array}$

** Month $=$ August $;$ Day of Week $=$ Monday EMISFACT 224UA_5 MHRDOW7 000000000000 EMISFACT 224UA 5 MHRDOW7 $00 \begin{array}{lllllll}0 & 0 & 0 & 0 & 0 & 0 & 0\end{array}$

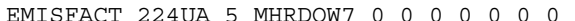

* * Month = September Month EMISFACT 224UA-5 MHRDOW7 $0 \begin{array}{llllllllll}0 & 0 & 0 & 0 & 0 & 0\end{array}$

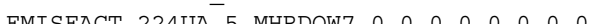
EMISFACT 2240A_5 MHRDOW7 $0 \begin{array}{lllllllllll}0 & 0 & 0 & 0 & 0 & 0 & 0\end{array}$ * Month = October; Day of Week = Monday

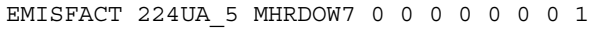

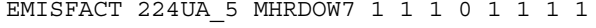
EMISFACT 224UA_5 MHRDOW7 001000000000

* Month $=$ November $;$ Day of Week $=$ Monday EMISFACT 224UA_5 MHRDOW7 0000000000

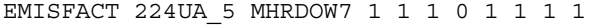

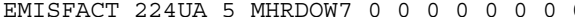

** Month $=$ Dece

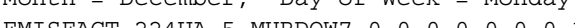

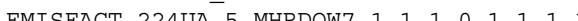

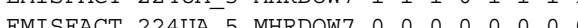
EMISFACT 2240A_5 MHRDOW7 $0 \begin{array}{lllllllll}0 & 0 & 0 & 0 & 0 & 0 & 0\end{array}$ * Month = January; Day of Week = Tuesday EMISFACT 224UA_5 MHRDOW7 00000000000 EMISFACT 224UA_5 MHRDOW7 000000000000 EMISFACT 224UA_5 MHRDOW7 00000000000

* Month $=$ February; Day of Week = Tuesday EMISFACT 224UA 5 MHRDOW7 $0 \begin{array}{lllllll}0 & 0 & 0 & 0 & 0 & 0\end{array}$ EMISFACT 224UA_5 MHRDOW7 $00 \begin{array}{lllllll}0 & 0 & 0 & 0 & 0 & 0 & 0\end{array}$ EMISFACT 224UA 5 MHRDOW7 00100000000

* Month $=$ March; Day of Week = Tuesday 
EMISFACT 224UA_5 MHRDOW7 000000000000 EMISFACT 224UA_5 MHRDOW7 000000000000 EMISFACT 224UA 5 MHRDOW7 0 O 0000000

** Month $=$ April; $;$ Day of Week = Tuesday EMISFACT 224UA 5 MHRDOW7 000000000000 EMISFACT 224UA_5 MHRDOW7 000000000000 EMISFACT 224UA_5 MHRDOW7 00000000000

** Month = May; Day of Week = Tuesday

EMISFACT 224UA_5 MHRDOW7 00000000000

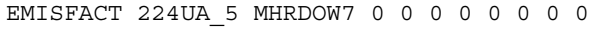
EMISFACT 224UA_5 MHRDOW7 0010000000000

** Month $=$ June; Day of Week = Tuesday

EMISFACT 224UA_5 MHRDOW7 00000000000

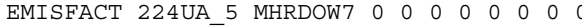

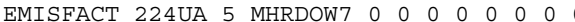

** Month $=$ July; Day of Week = Tuesday EMISFACT 224UA_5 MHRDOW7 000000000000 EMISFACT 224UA 5 MHRDOW7 000000000000 EMISFACT 224UA 5 MHRDOW7 00000000000

** Month = August $;$ Day of Week = Tuesday EMISFACT 224UA_5 MHRDOW7 0000000000 EMISFACT 224UA_5 MHRDOW7 000000000000 EMISFACT 224UA_5 MHRDOW7 000000000000

** Month = September; Day of Week = Tuesday EMISFACT 224UA_5 MHRDOW7 000000000000 EMISFACT 224UA_5 MHRDOW7 001000000000

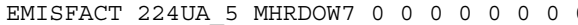
TMTSFACT 224UA 5 MURDOW7 00000000

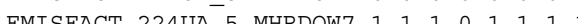

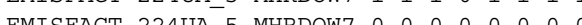

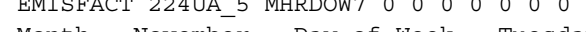
* Month = November $;$ Day of Week = Tuesda

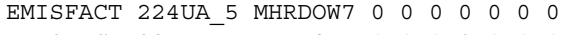
EMISFACT 224UA_5 MHRDOW7 $1 \begin{array}{cccccccc}1 & 1 & 1 & 0 & 1 & 1 & 1 & 1\end{array}$ EMISFACT 224UA_5 MHRDOW7 00000000000

* Month $=$ December; Day of Week $=$ Tuesday

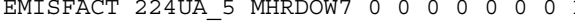

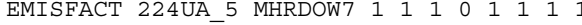
EMISFACT 224UA_5 MHRDOW7 $00 \begin{array}{lllllll}0 & 0 & 0 & 0 & 0 & 0 & 0\end{array}$

** Month $=$ January; Day of Week $=$ Wednesday EMISFACT 224UA_5 MHRDOW7 000000000000 EMISFACT 224UA 5 MHRDOW7 000000000000 EMISFACT 224UA 5 MHRDOW7 00000000000

** Month = February; Day of week = Wednesday $\begin{array}{lllllllll}\text { Month }=\text { February; } & \text { Day of } \\ \text { EMISFACT 224UA_5 MHRDOW7 } & 0 & 0 & 0 & 0 & 0 & 0 & 0 & 0\end{array}$

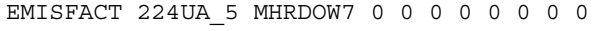
EMISFACT 224UA_5 MHRDOW7 00000000000

* Month $=$ March; Day of Week = Wednesday

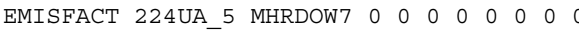
EMISFACT 224UA_5 MHRDOW7 $00 \begin{array}{lllllll}0 & 0 & 0 & 0 & 0 & 0 & 0\end{array}$

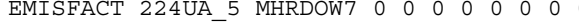
** Month $=$ April $;$ Day of Week $=$ Wednesday

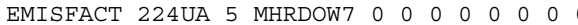


EMISFACT 224UA_5 MHRDOW7 000000000000 EMISFACT 224UA 5 MHRDOW7 $000 \begin{array}{llllll}0 & 0 & 0 & 0 & 0 & 0\end{array}$

** Month $=$ May; Day of Week = Wednesday FMISFACT 224UA 5 MHRDOW7 000000000 EMISFACT 224UA 5 MHRDOW7 00000000000 EMTSFACT 224UA 5 MHRDOW7 000000000

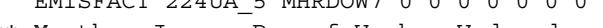
** Month = June; Day of Week = Wednesday EMISFACT 224UA_5 MHRDOW7 $\begin{array}{llllllllll}0 & 0 & 0 & 0 & 0 & 0 & 0 & 0\end{array}$ EMISFACT 224UA_5 MHRDOW7 $0 \begin{array}{lllllllll}0 & 0 & 0 & 0 & 0 & 0 & 0\end{array}$ EMISFACT 224UA_5 MHRDOW7 00000000000

** Month $=$ July; Day of Week = Wednesday EMISFACT 224UA_5 MHRDOW7 $00 \begin{array}{lllllll}0 & 0 & 0 & 0 & 0 & 0 & 0\end{array}$

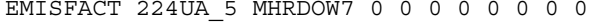
EMISFACT 224UA_5 MHRDOW7 $00 \begin{array}{lllllll}0 & 0 & 0 & 0 & 0 & 0 & 0\end{array}$

** Month $=$ August $; \quad$ Day of week $=$ Wednesday EMISFACT 224UA_5 MHRDOW7 00000000000 EMISFACT 224UA_ MHRDW 00000000000

* Month = September; Day of Week = Wednesday EMISFACT 224UA_5 MHRDOW7 00000000000 EMISFACT 224UA_5 MHRDOW7 00000000000 EMISFACT 224UA_5 MHRDOW7 00000000000

* Month $=$ October; Day of Week = Wednesday EMISFACT 224UA_5 MHRDOW7 00000000001 EMISFACT 224UA_5 MHRDOW7 $1 \begin{array}{cccccccc}1 & 1 & 1 & 0 & 1 & 1 & 1 & 1\end{array}$ EMISFACT 224UA_5 MHRDOW7 0000000000

$* *$ Month $=$ November; Day of Week $=$ Wednesday

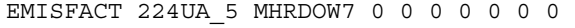
EMISFACT 224UA 5 MHRDOW7 1101000 MMTSEACT 224UA 5 MHRDOW7 00000000

$* *$ Month $=$ December; Day of Week $=$ Wednesday MMISFACT 224UA_5 MHRDOW7 00000000001

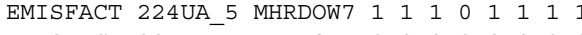
EMISFACT 224UA_5 MHRDOW7 00000000000

* Month = January; Day of Week = Thursday EMISFACT 224UA_5 MHRDOW7 00000000000

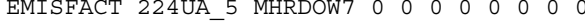
EMISFACT 224UA_5 MHRDOW7 000000000000

** Month $=$ February; Day of Week = Thursday EMISFACT 224UA_5 MHRDOW7 0000000000000 EMISFACT 224UA_5 MHRDOW7 001000000000 EMISFACT 224UA 5 MHRDOW7

** Month $=$ March; Day of Week = Thursday EMISFACT 224UA_5 MHRDOW7 0000000000 EMISFACT 224UA_5 MHRDOW7 0 O 00000000000 EMISFACT 224UA_5 MHRDOW7 000000000000

* Month $=$ April; $;$ Day of Week = Thursday EMISFACT 224UA_5 MHRDOW7 00000000000

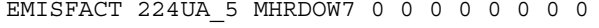

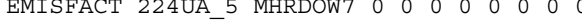

* Month $=$ May; Day of Week = Thursday

EMISFACT 224UA_5 MHRDOW7 000000000000 EMISFACT 224UA 5 MHRDOW7 000000000000 
EMISFACT 224UA_5 MHRDOW7 00000000000

** Month $=$ June; Day of Week = Thursday

EMISFACT 224UA_5 MHRDOW7 000000000000

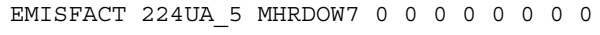
EMISFACT 224UA_5 MHRDOW7 00000000000

** Month $=$ July; Day of Week = Thursday EMISFACT 224UA_5 MHRDOW7 $000 \begin{array}{lllllll}5 & 0 & 0 & 0 & 0 & 0\end{array}$

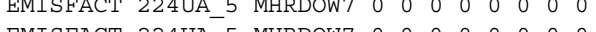
EMISFACT 224UA_5 MHRDOW7 000000000000

* Month = August; Day of Week = Thursday EMISFACT 224UA_5 MHRDOW7 00000000000 EMISFACT 224UA_5 MHRDOW7 $00 \begin{array}{lllllllll}0 & 0 & 0 & 0 & 0 & 0 & 0\end{array}$ EMISFACT 224UA_5 MHRDOW7 000000000000

** Month = September; Day of Week = Thursday EMISFACT 224UA_5 MHRDOW7 000000000000 EMISFACT 224UA_5 MHRDOW7 00000000000 EMISFACT 224UA 5 MHRDOW7 00000000000

** Month = October; Day of Week = Thursday EMISFACT 224UA_5 MHRDOW7 000000000001 EMISFACT 224UA_5 MHRDOW7 $1 \begin{array}{cccccccc}1 & 1 & 1 & 0 & 1 & 1 & 1 & 1\end{array}$

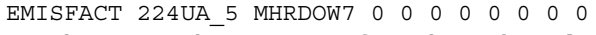

* Month $=$ November; Day of Week = Thursday EMISFACT 224UA_5 MHRDOW7 0000000001 EMISFACT 224UA 5 MHRDOW7 $1 \begin{array}{llllllll}1 & 1 & 0 & 1 & 1 & 1 & 1\end{array}$

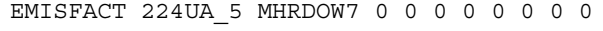

** Month $=$ December; $;$ Day of Week $=$ Thursday

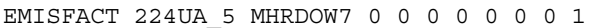

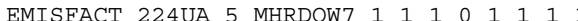
EMISFACT 224UA 5 MHRDOW7 $0 \begin{array}{llllllll}1 & 0 & 0 & 0 & 0 & 1 & 1 & 1\end{array}$

* Month = January Month = January; Day of Week = Friday EMISFACT 224UA_5 MHRDW7 $00 \begin{array}{ccccccc}0 & 0 & 0 & 0 & 0 & 0 & 0\end{array}$ EMISFACT 224UA_5 MHRDOW7 $0 \begin{array}{lllllllll}0 & 0 & 0 & 0 & 0 & 0 & 0\end{array}$ EMISFACT 224UA_5 MHRDOW7 000000000000 $*$ Month $=$ February; Day of Week = Friday

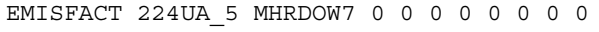

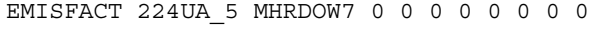
EMISFACT 224UA_5 MHRDOW7 $0 \begin{array}{llllllll}0 & 0 & 0 & 0 & 0 & 0 & 0 & 0\end{array}$

** Month $=$ March; Day of Week = Friday EMISFACT 224UA_5 MHRDOW7 0000000000000 EMISFACT 224UA_5 MHRDOW7 001000 C EMISFACT 224UA_5 MHRDOW7 0000000000 ** Month = April; Day of Week = Friday EMISFACT 224UA_5 MHRDOW7 $0 \begin{array}{cccccccc}0 & 0 & 0 & 0 & 0 & 0 & 0\end{array}$ EMISFACT 224UA_5 MHRDW7 $0 \begin{array}{lllllllllllllll}0 & 0 & 0 & 0 & 0 & 0 & 0\end{array}$

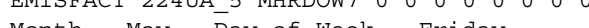
* Month = May; Day of Week = Friday EMISFACT 224UA_5 MHRDOW7 000000000 EMISFACT 224UA_5 MHRDOW7 $0 \begin{array}{llllllll}0 & 0 & 0 & 0 & 0 & 0 & 0\end{array}$

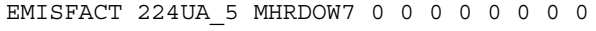

** Month $=$ June; Day of Week = Friday EMISFACT 224UA_5 MHRDOW7 0000000000000 EMISFACT 224UA 5 MHRDOW7 
** Month $=$ July; Day of week = Friday

EMISFACT 224UA_5 MHRDOW7 0000000000000

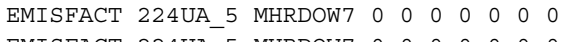

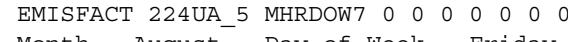

* Month = August; Day of Week = Friday EMISFACI 224UA_S MIRDOW7 $0 \begin{array}{lllllll}0 & 0 & 0 & 0 & 0 & 0 & 0\end{array}$ EMISFACT 224UA_5 MHROW7 0 C $00 \begin{array}{lllllll}0 & 0 & 0 & 0 & 0\end{array}$ EMISFACT 2240A_5 MHRDOW7 000000000000000

* Month = September; Day of Week = Friday EMISFACT 224UA_5 MHRDOW7 00000000000 EMISFACT 224UA_5 MHRDOW7 $00 \begin{array}{llllllll}0 & 0 & 0 & 0 & 0 & 0 & 0\end{array}$

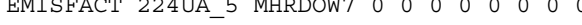

* Month = October; Day of Week = Friday EMISFACT 224UA_5 MHRDOW7 0000000000

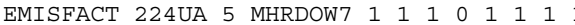
EMISFACT 224UA 5 MHRDOW7 0 O 000000

* Month = NOventer; Month $=$ November; $;$ Day of Week = Friday

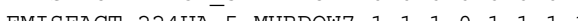
EMISFACT 224UA_5 MHRDOW7 $1 \begin{array}{lllllll}1 & 1 & 1 & 0 & 1 & 1 & 1\end{array}$ EMISFACT 224UA_5 MHRDOW7 0101000101010 * Month $=$ December; Day of Week = Friday EMISFACT 224UA_5 MHRDOW7 0000000000

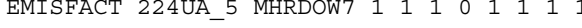

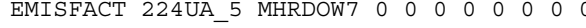

* Month = January; Day of Week = Saturday

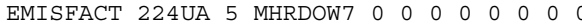
EMISFACT 224UA 5 MHRDOW7 $00 \begin{array}{lllllllllllllllll}0 & 0 & 0 & 0 & 0 & 0 & 0\end{array}$ EMISFACT 224UA 5 MHR

** Month = Februaryi Day of Week = Saturday TMISPA

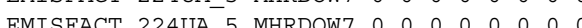
EMISFACT 224UA_5 MHRDOW7 0 O 000000 EMISFACT 2240A_5 MHRDOW7 $0 \begin{array}{lllllllllll}0 & 0 & 0 & 0 & 0 & 0 & 0\end{array}$ * Month = March; Day of Week = Saturday EMISFACT 224UA_5 MHRDOW7 0010000000000

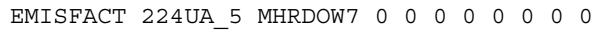
EMISFACT 224UA_5 MHRDOW7 0010000000000

** Month = April; Day of Week = Saturday EMISFACT 224UA 5 MHRDOW7 00010000000

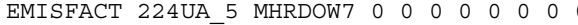

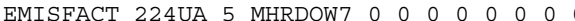

** Month $=$ May; Day of Week = Saturday EMISFACT 224UA 5 MHRDOW7 0000000000

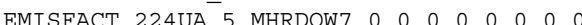

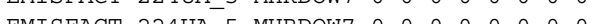

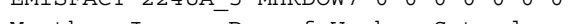
* Month = June; Day of Week = Saturday EMISFACT 224UA_5 MHRDOW7 $0 \begin{array}{llllllllllllll}0 & 0 & 0 & 0 & 0 & 0 & 0\end{array}$

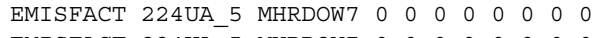

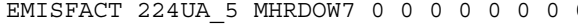

** Month $=$ July; Day of Week = Saturday

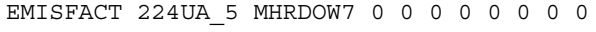

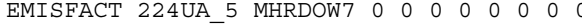
EMISFACT 224UA 5 MHRDOW7 $00 \begin{array}{ccccccc}0 & 0 & 0 & 0 & 0 & 0 & 0\end{array}$

* Month $=$ August $;$ Day of Week $=$ Saturday 
EMISFACT 224UA_5 MHRDOW7 000000000000 EMISFACT 224UA_5 MHRDOW7 000000000000 EMISFACT 224UA_5 MHRDOW7 00000000000

** Month $=$ September; $;$ Day of Week = Saturday EMISFACT 224UA_5 MHRDOW7 00000000000

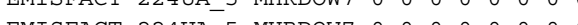
EMISFACT 2240A_5 MHRDOW7 $0 \begin{array}{llllllllllllllll}0 & 0 & 0 & 0 & 0 & 0 & 0\end{array}$

* Month = October; Day of Week = Saturday EMISFACT 224UA_5 MHRDOW7 00000000000 EMISFACT 224UA_5 MHRDOW7 $00 \begin{array}{llllllll}0 & 0 & 0 & 0 & 0 & 0 & 0\end{array}$ EMISFACT 224UA_5 MHRDOW7 000000000000

** Month $=$ November; Day of Week = Saturday EMISFACT 224UA_5 MHRDOW7 $00 \begin{array}{lllllll}0 & 0 & 0 & 0 & 0 & 0 & 0\end{array}$

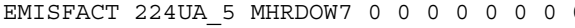

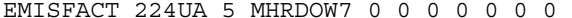

** Month = December; Day of Week Minth = Decemer, Day or Week = Saturday

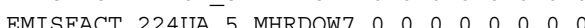
MTICT 2240 EMISFACT 2240A_5 MHRDOW7 0 O 00 o 000000 * Month = January; Day of Week = Sunday EMISFACT 224UA_5 MHRDOW7 00000000000 EMISFACT 224UA_5 MHRDOW7 0000000000000 EMISFACT 224UA_5 MHRDOW7 0010000000000

** Month $=$ February; Day of Week = Sunday EMISFACT 224UA 5 MHRDOW7 00000000000

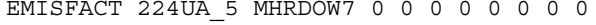
EMISFACT 224UA 5 MHRDOW7 0000000000

** Month = March; Day of Week = Sunday EMTSFACT 224UA 5 MHRDOW7 00000000000

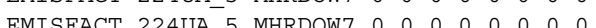

** Month = April; Day of Week = Sunday EMISFACT 224UA_5 MHRDOW7 $0 \begin{array}{lllllll}0 & 0 & 0 & 0 & 0 & 0 & 0\end{array}$ EMISFACT 224UA_5 MHRDOW7 $0 \begin{array}{llllllll}0 & 0 & 0 & 0 & 0 & 0 & 0 & 0\end{array}$ EMISFACT 224UA_5 MHRDOW7 001000000000

** Month = May; Day of Week = Sunday

EMISFACT 224UA_5 MHRDOW7 $00 \begin{array}{lllllll}0 & 0 & 0 & 0 & 0 & 0 & 0\end{array}$ EMISFACT 224UA_5 MHRDOW7 $00 \begin{array}{lllllll}0 & 0 & 0 & 0 & 0 & 0 & 0\end{array}$

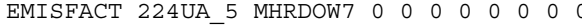

** Month $=$ June; Day of Week = Sunday

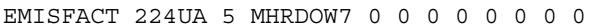
EMISFACT 224UA 5 MHRDOW7 00000000000

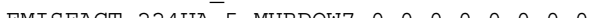

* Month = July; Day of Week = Sunday

* Month = July; Day of Week = Sunday

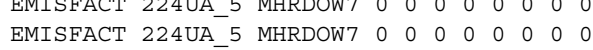
$\begin{array}{llllllllll}\text { EMISFACT 224UA_5 } & \text { MHRDOW7 } & 0 & 0 & 0 & 0 & 0 & 0 & 0 & 0 \\ \text { EMISFACT 224UA_5 MHRDOW7 } & 0 & 0 & 0 & 0 & 0 & 0 & 0 & 0\end{array}$

* Month $=$ August $;$ Day of Week $=$ Sunday EMISFACT 224UA_5 MHRDOW7 00000000000 EMISFACT 224UA_5 MHRDOW7 $00 \begin{array}{llllllll}0 & 0 & 0 & 0 & 0 & 0 & 0\end{array}$

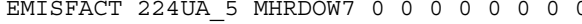
** Month $=$ September; Day of Week = Sunday

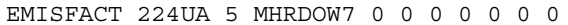


EMISFACT 224UA_5 MHRDOW7 000000000000 EMISFACT 224UA_5 MHRDOW7 000000000000

** Month $=$ October; $;$ Day of Week = Sunday EMISFACT 224UA 5 MHRDOW7 0000000000000 EMISFACT 224UA 5 MHRDOW7 $00 \begin{array}{llllllll}0 & 0 & 0 & 0 & 0 & 0 & 0\end{array}$ EMISFACT 224UA 5 MHRDOW7 000000000000

* Month = November; Day of Week = Sunday

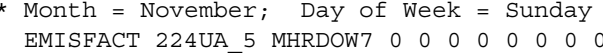
EMISFACT 224UA_5 MHRDDW EMISFACT 224UA 5 MHRDOW7 0000000000

* Month $=$ December; Day of Week = Sunday EMISFACT 224UA_5 MHRDOW7 00100000000 EMISFACT 224UA_5 MHRDOW7 $00 \begin{array}{lllllll}0 & 0 & 0 & 0 & 0 & 0 & 0\end{array}$

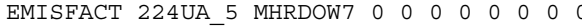

** Month $=$ January; $\quad$ Day of Week $=$ Monday EMISFACT 224UA_6 MHRDOW7 00000000000 EMISEACT 224 - 6 MHRDOW7 00000000000 EMTSFACT 224UA-6 MHRDOW 00000000000

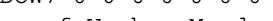
* * Month = February; Day of Week = Monday EMISFACT 224UA_6 MHRDOW7 0000000000 EMISFACT 224UA_6 MHRDOW7 $\begin{array}{lllllllllll}0 & 0 & 0 & 0 & 0 & 0 & 0\end{array}$ EMISFACT 224UA_6 MHRDOW7 0000000000

* Month $=$ March; Day of Week = Monday

EMISFACT 224UA_6 MHRDOW7 000000000000 EMISFACT 224UA_6 MHRDOW7 $00 \begin{array}{lllllllllllll}0 & 0 & 0 & 0 & 0 & 0 & 0\end{array}$ EMISFACT 224UA_6 MHRDOW7 $0 \begin{array}{lllllll}0 & 0 & 0 & 0 & 0 & 0 & 0\end{array}$

$\Omega \quad * *$ Month $=$ April; Day of week = Monday

EMISFACT 224UA_6 MHRDOW7 0000000000 EMISFACT 224UA 6 MHRDOW7 000000000000 EMTSEACT 224UA 6 MHRDOW7 00000000000

* Month = May; Day of Week = Monday

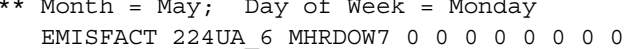

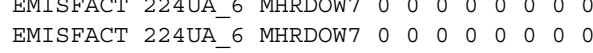
EMISFACT 224UA_6 MHRDOW7 00000000000

* Month $=$ June; Day of Week = Monday

EMISFACT 224UA_6 MHRDOW7 00000000000 EMISFACT 224UA_6 MHRDOW7 $00 \begin{array}{lllllll}0 & 0 & 0 & 0 & 0 & 0 & 0\end{array}$

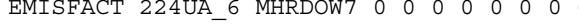

* Month $=$ July; Day of Week = Monday

EMISFACT 224UA_6 MHRDOW7 000000000000 EMISFACT 224UA_6 MHRDOW7 000000000000

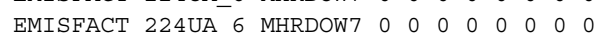

* Month = August; Day of Week = Monday

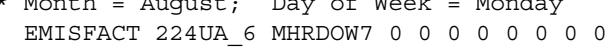
EMISFACT 224UA 6 MHRDOW7 000000000000 EMISFACT 224UA 6 MHRDOW7 00000000000 * Month = September; Day of Week = Monday EMISFACT 224UA_6 MHRDOW7 00000000000 EMISFACT 224UA_6 MHRDOW7 $00 \begin{array}{llllllll}0 & 0 & 0 & 0 & 0 & 0 & 0\end{array}$

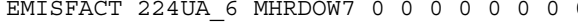

** Month = October; Day of Week = Monday EMISFACT 224UA 6 MHRDOW7 0000000000 EMISFACT 224UA 6 MHRDOW7 11 
EMISFACT 224UA_6 MHRDOW7 0000000000

* Month $=$ November; Day of Week = Monday EMISFACT 224UA_6 MHRDOW7 0000000000 EMISFACT 224UA 6 MHRDOW7 $1 \begin{array}{lllllllll}1 & 1 & 0 & 1 & 1 & 1\end{array}$ EMISFACT 224UA 6 MHRDOW7 000000000000

** Month = December; Day of week = Monday EMISFACT 224UA_6 MHRDOW7 000000000 EMISFACT 224UA_6 MHRDOW7 $1 \begin{array}{lllllll}1 & 1 & 1 & 0 & 1 & 1 & 1\end{array}$ EMISFACT 224UA_6 MHRDOW7 0000000000

* Month = January; Day of Week = Tuesday EMISFACT 224UA_6 MHRDOW7 000000000000

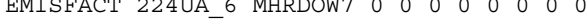
EMISFACT 224UA_6 MHRDOW7 00000000000

* Month $=$ February; Day of Week = Tuesday EMISFACT 224UA 6 MHRDOW7 000000000000 EMISFACT 224UA 6 MHRDOW7 0000000000

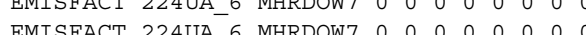

EMISTC 2240 - 6 MHRDOW7 0000000000

* Month $=$ March; $i$ Day of Week = Tuesday EMISFACT 224UA_6 MHRDOW7 00000000000

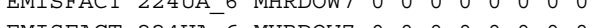
EMISFACT 224UA_6 MHRDOW7 00000000000

* Month $=$ April; Day of Week = Tuesday

EMISFACT 224UA_6 MHRDOW7 00000000000 EMISFACT 224UA_6 MHRDOW7 $0 \begin{array}{llllllll}0 & 0 & 0 & 0 & 0 & 0 & 0 & 0\end{array}$

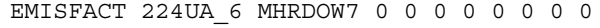

** Month = May; Day of Week = Tuesday

EMISFACT 224UA_6 MHRDOW7 000000000000

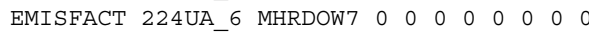

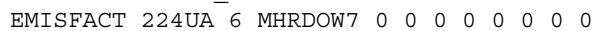

* Month $=$ June; Day of week = Tuesday EMISFACT 224UA 6 MHRDOW7 000000000000

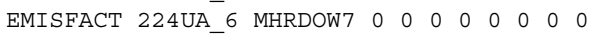
EMISFACT 224UA_6 MHRDOW7 00100000000000

** Month $=$ July; Day of Week = Tuesday EMISFACT 224UA_6 MHRDOW7 000000000000

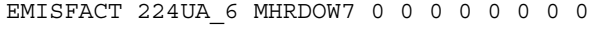
EMISFACT 224UA_6 MHRDOW7 $0 \begin{array}{lllllll}0 & 0 & 0 & 0 & 0 & 0 & 0\end{array}$

** Month $=$ August $;$ Day of Week = Tuesday EMISFACT 224UA_6 MHRDOW7 00000000000

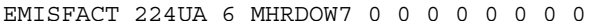
EMISFACT 224UA 6 MHRDOW7 0 O 000000

* Month = Septenber; Day of Week = Tuesday Month = September; Day of Week = Tuesday

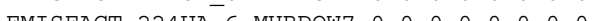

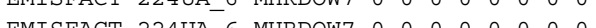
EMISFACT 2240A_6 MHRDOW7 0 O 0 O 0 0 0000000 * Month = October; Day of Week = Tuesday EMISFACT 224UA_6 MHRDOW7 000000000 EMISFACT 224UA_6 MHRDOW7 $1 \begin{array}{llllllll}1 & 1 & 1 & 0 & 1 & 1 & 1\end{array}$

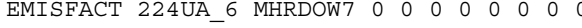

** Month $=$ November $;$ Day of Week = Tuesday

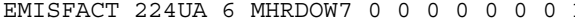

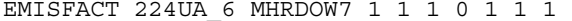
EMISFACT 224UA 6 MHRDOW7 0 
** Month $=$ December $;$ Day of Week = Tuesday EMISFACT 224UA_6 MHRDOW7 000000000001

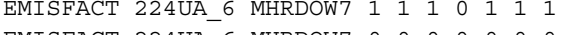

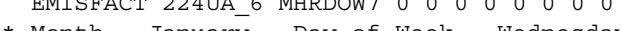

* Month = January; Day of Week = Wednesday EMISFACT 224UA_6 MHRDOW7 00000000 -

* Month $=$ February; Day of Week $=$ Wednesday

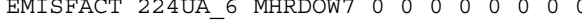

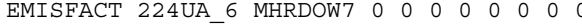

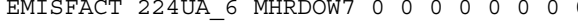

* Month $=$ March; Day of Week $=$ Wednesday EMISFACT 224UA 6 MHRDOW7 0 O 000000000

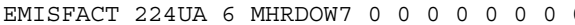

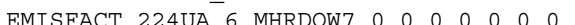

* Month = Aprili Day of Week = Wednesday Month $=$ April; $i$ Day of Week $=$ Wednesday

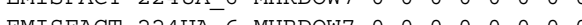
EMISFACT 224UA_6 MHRDOW7 $0 \begin{array}{llllllllllllll}0 & 0 & 0 & 0 & 0 & 0 & 0\end{array}$ EMISFACT 2240_6 MHRDOW7 00

* Month $=$ May; Day of Week = Wednesday EMISFACT 224UA_6 MHRDOW7 $00 \begin{array}{lllllll}0 & 0 & 0 & 0 & 0 & 0 & 0\end{array}$

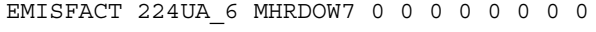

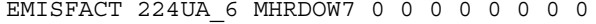

** Month $=$ June; Day of Week $=$ Wednesday EMISFACT 224UA_6 MHRDOW7 000100000000

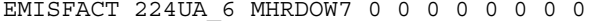

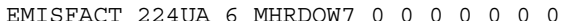

* Month = Julyi Day of Week $=$ Wednesday

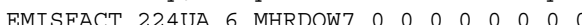
EMISFACT 224UA_6 MHRDOW7 $0 \begin{array}{cccccccc}0 & 0 & 0 & 0 & 0 & 0\end{array}$

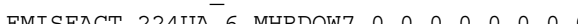
EMISFACT 224UA_6 MHRDOW7 00000000000

* Month = August; Day of Week = Wednesday EMISFACT 224UA_6 MHRDOW7 0000000000

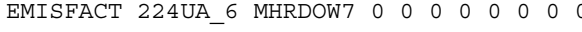
EMISFACT 224UA_6 MHRDOW7 000000000000

** Month = September; Day of Week = Wednesday EMISFACT 224UA_6 MHRDOW7 000000000000

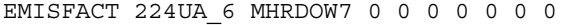
EMISFACT 224UA 6 MHRDOW7 $00 \begin{array}{lllllllllllll}0 & 0 & 0 & 0 & 0 & 0 & 0 & 0\end{array}$

** Month = October; Day of Week = Wednesday EMISFACT 224UA 6 MHRDOW7 00000000

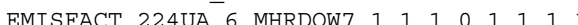

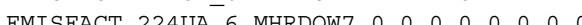

Month $=$ November; Day of Week $=$ Wednesday * Month = November; Day of Week $=$ Wednesday
EMISFACT 224UA 6 MHRDOW7 000000000 $\begin{array}{lllllllllll}\text { EMISFACT } & 224 \mathrm{UA} \_6 & \text { MHRDOW7 } & 0 & 0 & 0 & 0 & 0 & 0 & 0 & 1 \\ \text { EMISFACT } & 224 \mathrm{UA} & \text { MHRDOW7 } & 1 & 1 & 1 & 0 & 1 & 1 & 1 & 1\end{array}$ EMISFACT 224UA_6 MHRDOW7 000000000000

$* *$ Month $=$ December; Day of Week $=$ Wednesday

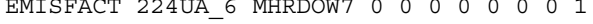

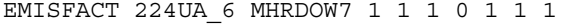

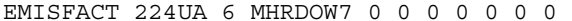

* Month $=$ January; Day of week $=$ Thursday 
EMISFACT 224UA_6 MHRDOW7 000000000000 EMISFACT 224UA_6 MHRDOW7 000000000000

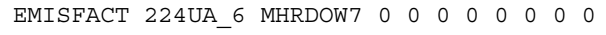

** Month = February; Day of Week = Thursday EMISFACT 224UA 6 MHRDOW7 000000000000 EMISFACT 224UA 6 MHRDOW7 000000000000 EMISFACT 224UA_6 MHRDOW7 00000000000

** Month $=$ March; Day of Week = Thursday EMISFACT 224UA_6 MHRDOW7 000000000000 EMISFACT 224UA_6 MHRDOW7 $000 \begin{array}{llllllll}0 & 0 & 0 & 0 & 0 & 0 & 0\end{array}$

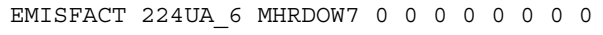

** Month $=$ April; Day of Week = Thursday EMISFACT 224UA_6 MHRDOW7 $00 \begin{array}{lllllll}0 & 0 & 0 & 0 & 0 & 0 & 0\end{array}$ EMISFACT 224UA_6 MHRDOW7 $00 \begin{array}{lllllll}0 & 0 & 0 & 0 & 0 & 0 & 0\end{array}$

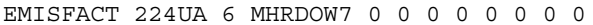

** Month = May; Day of Week = Thursday EMISEACT 224UA 6 MHRDOW7 000000000 EMISFACT 224IA- MHRDOW7 000000000

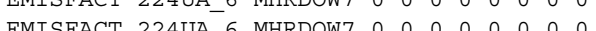

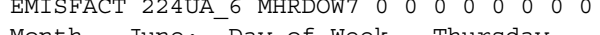
* Month $=$ June; Day of Week = Thursday EMISFACT 224UA_6 MHRDOW7 0000000000 EMISFACT 224UA_6 MHRDOW7 000000000000 EMISFACT 224UA_6 MHRDOW7 00000000000

* Month $=\mathrm{July} ;$ Day of Week = Thursday EMISFACT 224UA_6 MHRDOW7 00000000000

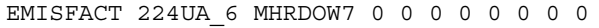

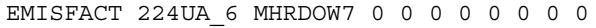
EMISFACT 224UA 6 MHRDOW7 0000000000 FMISFACT 2240 - 6 MHRDOW7 000000000

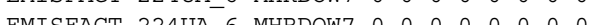

* Month = September; Day of Week = Thursday EMISFACT 224UA_6 MHRDOW7 0000000000 EMISFACT 224UA_6 MHRDOW7 000000000000 EMISFACT 224UA_6 MHRDOW7 00000000000

* Month $=$ October; $;$ Day of Week = Thursday

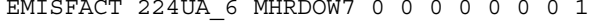

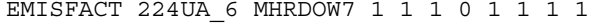

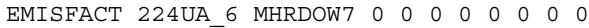

** Month $=$ November; Day of Week = Thursday

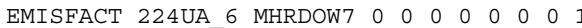

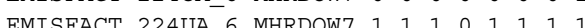
FMTSFACT 224UA- 6 MHRDOW7 00000000

EMISFACT 2240A-6 MHRDOW7 0 a 000000 ** Month = December $;$ Day of Week = Thursday EMISFACT 224UA_6 MHRDOW7 $0 \begin{array}{llllllllll}0 & 0 & 0 & 0 & 0 & 0 & 0 & 1\end{array}$ EMISFACT 224UA_6 MHRDOW7 $1 \begin{array}{llllllll}1 & 1 & 0 & 1 & 1 & 1 & 1\end{array}$ EMISFACT 224UA_6 MHRDOW7 00000000000

* Month = January; Day of Week = Friday EMISFACT 224UA 6 MHRDOW7 000000000000 EMISFACT 224UA_6 MHRDOW7 $00 \begin{array}{llllllll}0 & 0 & 0 & 0 & 0 & 0 & 0\end{array}$

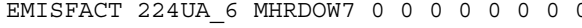

* Month $=$ February; Day of Week = Friday EMISFACT 224UA 6 MHRDOW7 000000000 
EMISFACT 224UA_6 MHRDOW7 000000000000 EMISFACT 224UA_6 MHRDOW7 00000000000

** Month $=$ March; ; Day of Week = Friday

EMISFACT 224UA 6 MHRDOW7 00000000000 EMISFACT 224UA 6 MHRDOW7 000000000000

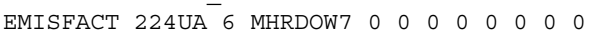

** Month = April; Day of Week = Friday

G MHRDOW7 $00 \begin{array}{llllllll} & 0 & 0 & 0 & 0 & 0 & 0 & 0\end{array}$ EMISFACT 224UA_6 MHRDOW7 $0 \begin{array}{lllllllll}0 & 0 & 0 & 0 & 0 & 0 & 0\end{array}$ EMISFACT 224UA_6 MHRDOW7 000000000000

$* *$ Month $=$ May; Day of Week = Friday

EMISFACT 224UA_6 MHRDOW7 000000000000 EMISFACT 224UA_6 MHRDOW7 $00 \begin{array}{lllllll}0 & 0 & 0 & 0 & 0 & 0 & 0\end{array}$

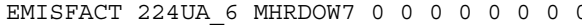

** Month $=$ June; Day of Week = Friday EMISFACT 224UA 6 MHRDOW7 00000000000 EMISEACT 224 - 6 MHRDOW7 00000000000 MTISEACT 224U-

* Month = July; Day of Week = Friday

* Month = July; Day of Week = Friday $\begin{array}{lllllllllll}\text { EMISFACT } & 224 \mathrm{UA} \text { - } 6 \text { MHRDOW7 } & 0 & 0 & 0 & 0 & 0 & 0 & 0 & 0 \\ \text { EMISFACT } & 224 \mathrm{UA} 6 \text { 6 MHRDOW7 } & 0 & 0 & 0 & 0 & 0 & 0 & 0 & 0\end{array}$

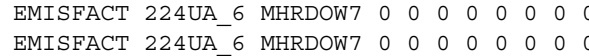

$* *$ Month $=$ August $;$ Day of Week = Friday EMISFACT 224UA_6 MHRDOW7 000000000000

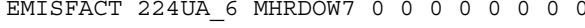

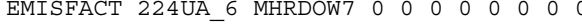

* Month $=$ September $;$ Day of Week $=$ Friday EMISFACT 224UA 6 MHRDOW7 0000000000

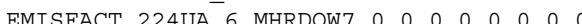
EMTSEACT 224UA 6 MHRDON7 00000000

EMISFACT 2240. * Month = October; Day of Week = Friday EMISFACT 224UA_6 MHRDOW7 00000000001

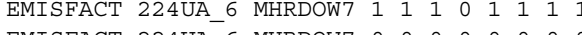
EMISFACT 224UA_6 MHRDOW7 0000000000 * Month = November; Day of Week = Friday EMISFACT 224UA_6 MHRDOW7 00000000001

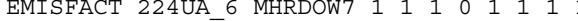

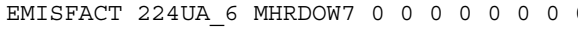

* Month $=$ December; Day of Week = Friday EMISFACT 224UA 6 MHRDOW7 0000000000

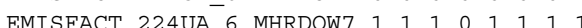
EMISEACT 224UA 6 MHRDOW7 00000000

* Month = Janua- 6 ; Day of Week = Saturday * Month = January; Day or Week = Saturday EMISFACT 224UA_6 MHRDOW7 00000000

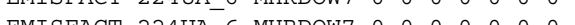
EMISFACT 224UA_6 MHRDOW7 0000000000

* Month = February; Day of Week = Saturday

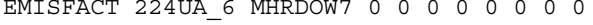
EMISFACT 224UA_6 MHRDOW7 $00 \begin{array}{llllllll}0 & 0 & 0 & 0 & 0 & 0 & 0\end{array}$

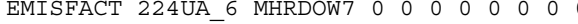

** Month $=$ March; Day of Week = Saturday

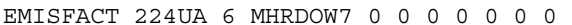
FMISFACT 224UA 6 MHRDOW7 0 - 0 - 0 - 0 
EMISFACT 224UA_6 MHRDOW7 0000000000

** Month $=$ April; Day of Week = Saturday

EMISFACT 224UA_6 MHRDOW7 000000000000 EMISFACT $224 \mathrm{UA} 6$ MHRDOW7 0 EMISFACT 224UA_6 MHRDOW7 001000000000 * Month = May; Day of Week = Saturday EMISFACT 224UA_6 MHRDOW7 $0 \begin{array}{cccccccc}0 & 0 & 0 & 0 & 0 & 0 & 0\end{array}$ EMISFACT 2240A_6 MHRDOW7 000000000000000 EMISFACT 224UA_6 MHRDOW7 00000000000 * Month = June; Day of Week = Saturday EMISFACT 224UA_6 MHRDOW7 00000000000 EMISFACT 224UA_6 MHRDOW7 $00 \begin{array}{llllllll}0 & 0 & 0 & 0 & 0 & 0 & 0\end{array}$

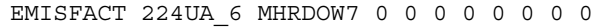

** Month $=$ July; Day of Week = Saturday EMISFACT 224UA_6 MHRDOW7 00000000000 EMISFACT 224UA_6 MHRDOW7 000000000000

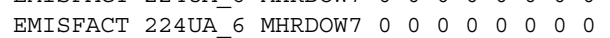

* Month = August $;$ Day of Week = Saturday $\begin{array}{lllllllll}\text { MMISFACT 224UA 6 MHRDOW7 } 0 & 0 & 0 & 0 & 0 & 0 & 0 & 0\end{array}$ EMISFACT 224UA_6 MHRDOW7 000000000000 EMISFACT 224UA 6 MHRDOW7 00000000000

* Month = September; Day of Week = Saturday EMISFACT 224UA_6 MHRDOW7 00000000000

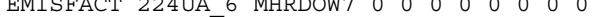
EMISFACT 224UA_6 MHRDOW7 $00 \begin{array}{lllllll}0 & 0 & 0 & 0 & 0 & 0 & 0\end{array}$

** Month = October; Day of week = Saturday EMISFACT 224UA 6 MHRDOW7 00000000000

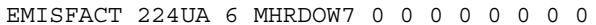

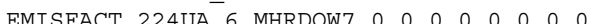

* Month = Noverberi Day of Week = Saturday Month = November, Day of Week = Saturday

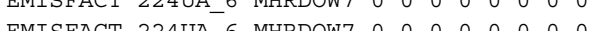
BMISFACT 224UA_6 MHRDOW7 $0 \begin{array}{llllllll}0 & 0 & 0 & 0 & 0 & 0\end{array}$

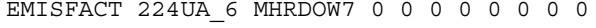

* Month $=$ December; Day of Week = Saturday EMISFACT 224UA_6 MHRDOW7 $00 \begin{array}{lllllll}0 & 0 & 0 & 0 & 0 & 0 & 0\end{array}$

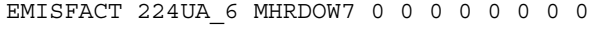
EMISFACT 224UA_6 MHRDOW7 $00 \begin{array}{lllllll}0 & 0 & 0 & 0 & 0 & 0 & 0\end{array}$

* Month $=$ January; Day of Week = Sunday EMISFACT 224UA_6 MHRDOW7 $00 \begin{array}{lllllll}0 & 0 & 0 & 0 & 0 & 0 & 0\end{array}$

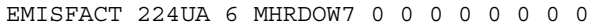

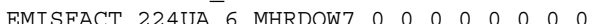

* Month = Februaryi Day of Week = Sunday Month = February; Day or Week = sunday

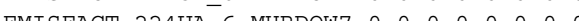
EMISFACT 224UA_6 MHRDOW7 0 C $00 \begin{array}{llllll}0 & 0 & 0 & 0 & 0 & 0\end{array}$ EMISFACT 2240A_6 MHRDOW7 0000000000000 * Month $=$ March; Day of Week = Sunday EMISFACT 224UA_6 MHRDOW7 000000000

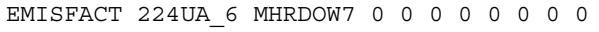

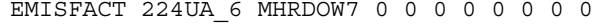

** Month = April; $\quad$ Day of Week = Sunday EMISFACT 224UA_6 MHRDOW7 0000000000000 EMISFACT 224UA 6 MHRDOW7 
** Month = May; Day of Week = Sunday

EMISFACT 224UA_6 MHRDOW7 000000000000 EMISFACT 224UA 6 MHRDOW7 000000000000

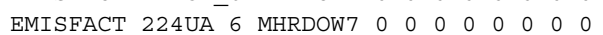

* Month = June; Day of week = Sunday

$\begin{array}{lllllllll}\text { Month }= & \text { June; } \\ \text { EMISFACT 224UA } 6 \text { MHRDOW7 } & 0 & 0 & 0 & 0 & 0 & 0 & 0 & 0\end{array}$ EMISFACT 224UA 6 MHRDOW7 000000000000

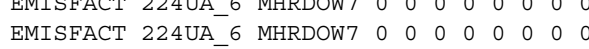

* Month $=$ July; Day of Week = Sunday

EMISFACT 224UA_6 MHRDOW7 00000000000

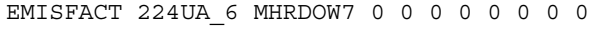

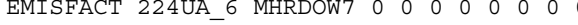

* Month $=$ August; Day of Week = Sunday

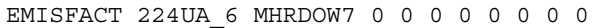

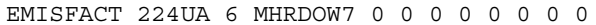
EMISFACT 224UA 6 MHRDOW7 0 O 000000

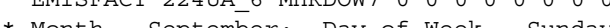
Month = September; $;$ Day of Week = Sunday MISACT 224UA_ EMISFACT 224UA_6 MHRDOW7 $\begin{array}{llllllllllllll}0 & 0 & 0 & 0 & 0 & 0 & 0\end{array}$ EMISFACT 2240A_6 MHRDOW7 000000000000 * Month = October; Day of Week = Sunday EMISFACT 224UA_6 MHRDOW7 0000000000

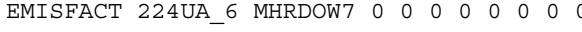

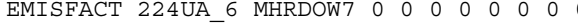

** Month $=$ November; Day of Week = Sunday EMISFACT 224UA_6 MHRDOW7 00000000000

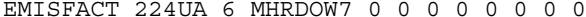
EMISFACT 224UA 6 MHRDOW7 0000

* Month $=$ December; Month Decembr, Day or week = sunday

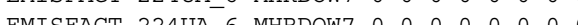

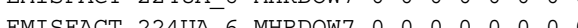
0 METHOD_2 224U_1 1

METHOD_2 224U_2 15

METHOD_2 224U 3115

METHOD_2 224U_4 15

METHOD_2 224U_5 15

METHOD_2 224U 615

METHOD_2 224U 7115

METHOD 2 224U 8 1 5

METHOD 2 224U 915

METHOD 2 224UA 1115

METHOD 2 224UA-1 15

METHOD 2 224UA 315

METHOD 2 224UA 4 - 5

METHOD 2240

(1)

पर

SRCGROÜP ALI

SO FINISHED

** AERMOD Receptor Pathway

$\star * * * * * * * * * * * * * * * * * * * * * * * * * * * * * * * * * * * * * * * * *$ 
RE STARTING

GRIDCART UCART1 STA

$\begin{array}{llllll}\text { XYINC 299049.99 } 50 \quad 5.00 & 5157806.84 & 50 & 5.00\end{array}$

RE FINISHED

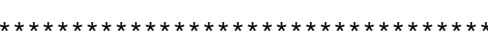

** AERMOD Meteorology Pathway

ME STARTING

SURFFILE C: \Jim\UDISPE 1 \HANDFO 1 $\backslash 2006 \backslash$ HAN19 06.SFC

PROFFILE C: \Jim\UDISPE 1 \HANDFO 1 $\backslash 2006 \backslash$ HAN19_06.PFL

SURFDATA 122192006

UAIRDATA $4106 \quad 2006$

PROFBASE 0 METERS

ME FINISHED

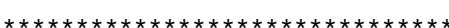

** AERMOD Output Pathway

OU STARTING

RECTABLE ALLAVE 1ST

RECTABLE 1 1ST

** Auto-Generated Plotfiles

PLOTFILE 1 ALL 1ST U UA BD3, AD $\backslash 01$ H1GALL. PLT

OU FINISHED

***************************************

*** SETUP Finishes Successfully $* * *$

*** AERMOD - VERSION $07026 * * * \quad * * *$ U/UA Building Demolition

*** Unit Release - Worst Case

**MODELOPTS :

CONC

DDEP

TOXICS FLAT

DRYDPL WETDPL

$* *$ Model Is Setup For Calculation of Average Concentration Values.

**Model Is Setup For Calculation of Dry DEPosition Values.

- DEPOSITION LOGIC --

**Model Uses DRY DEPLETION. DDPLETE $=\mathrm{T}$

$* *$ Model Uses WET DEPLETION. WDPLETE $=$ 
**NO GAS DRY DEPOSITION Data Provided.

**Model Uses RURAL Dispersion Only.

**Model Uses User-Specified Options:

1. Stack-tip Downwash.

2. Model Assumes Receptors on FLAT Terrain.

**other Options Specified:

TOXICS - Allows use of TOXICS option enhancements.

**Model Assumes No FLAGPOLE Receptor Heights.

**Model Calculates 1 Short Term Average(s) of: 1-HR

**This Run Includes: 15 Source(s); 1 Source Group(s); and 2500 Receptor(s)

**The Model Assumes A Pollutant Type of: U03

**Model set To Continue RUNning After the Setup Testing.

**output Options Selected:

Model Outputs Tables of Highest Short Term Values by Receptor (RECTABLE Keyword)

Model Outputs External File(s) of High Values for Plotting (PLOTFILE Keyword)

**NOTE: The Following Flags May Appear Following CONC Values: c for Calm Hours

m for Missing Hours

b for Both Calm and Missing Hour

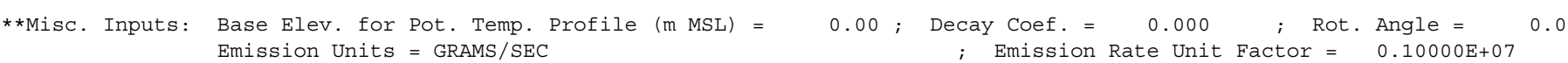

; Emission Rate Unit Factor $=0.10000 \mathrm{E}+07$

Output Units $=$ MICROGRAMS $/ \mathrm{M} * * 3$

**Approximate Storage Requirements of Model $=1.5 \mathrm{MB}$ of RAM

*** AERMOD - VERSION $07026 * * * \quad * * *$ U/UA Building Demolition **MODELOPTS

CONC
*** Unit Release - Worst Case

TOXICS FLAT $\begin{array}{ll}* * * & 02 / 09 / 09 \\ * * * & 10: 41: 11 \\ & \text { PAGE } 2\end{array}$

DRYDPL WETDPL

\section{*** POINT SOURCE DATA ***}

\begin{tabular}{|c|c|c|c|c|c|c|c|c|c|c|c|c|c|}
\hline SOURCE & $\begin{array}{c}\text { NUMBER } \\
\text { PART. }\end{array}$ & $\begin{array}{l}\text { EMISSION RATE } \\
\text { (GRAMS/SEC) }\end{array}$ & $\mathrm{X}$ & $Y$ & $\begin{array}{l}\text { BASE } \\
\text { ELEV }\end{array}$ & $\begin{array}{l}\text { STACK } \\
\text { HEIGHT }\end{array}$ & $\begin{array}{l}\text { STACK } \\
\text { TEMP. }\end{array}$ & $\begin{array}{l}\text { STACK } \\
\text { EXIT VEL. }\end{array}$ & $\begin{array}{l}\text { STACK } \\
\text { DIAMETER }\end{array}$ & $\begin{array}{l}\text { BLDG } \\
\text { EXISTS }\end{array}$ & $\begin{array}{l}\text { URBAN } \\
\text { SOURCE }\end{array}$ & $\begin{array}{l}\text { CAP/ } \\
\text { HOR }\end{array}$ & $\begin{array}{l}\text { EMIS RATE } \\
\text { SCALAR }\end{array}$ \\
\hline ID & CATS. & & (METERS) & (METERS) & (METERS) & (METERS) & (DEG.K) & $(\mathrm{M} / \mathrm{SEC})$ & (METERS) & & & & VARY BY \\
\hline - - & -- & - & - - - - & & - - - - & - - - - & - - - & $-\quad-\quad-$ & - - - - & - & & & \\
\hline
\end{tabular}




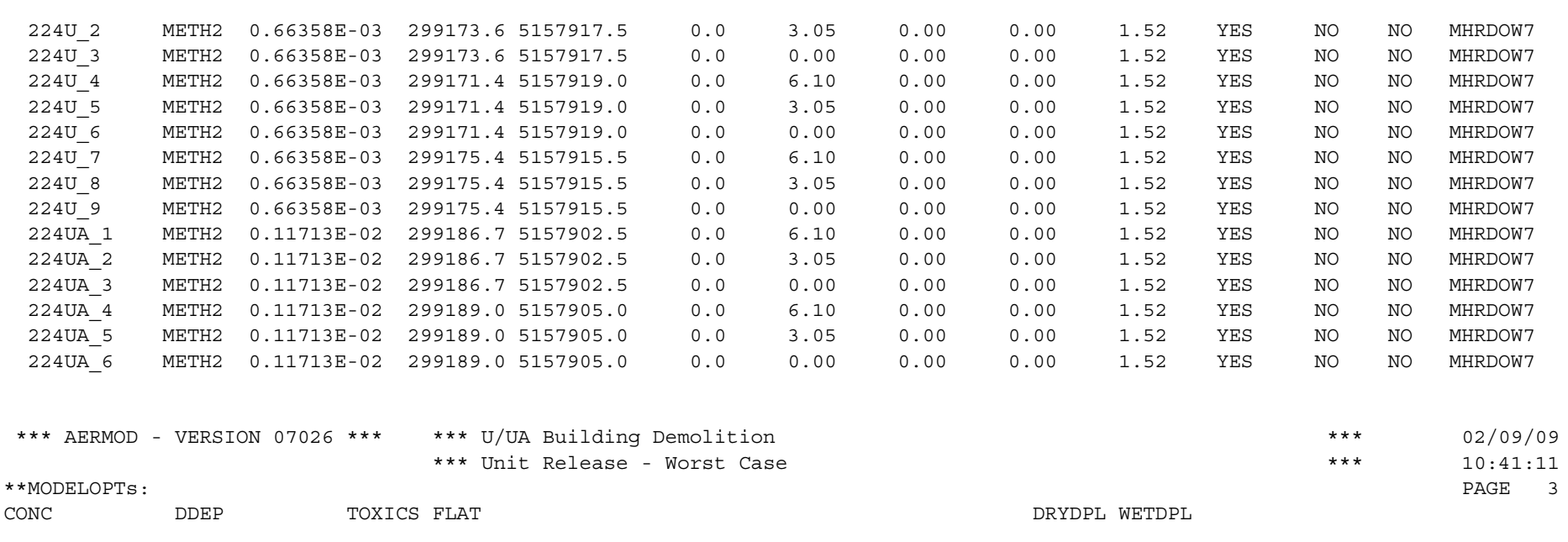

*** SOURCE IDS DEFINING SOURCE GROUPS ***

GROUP ID

SOURCE IDS

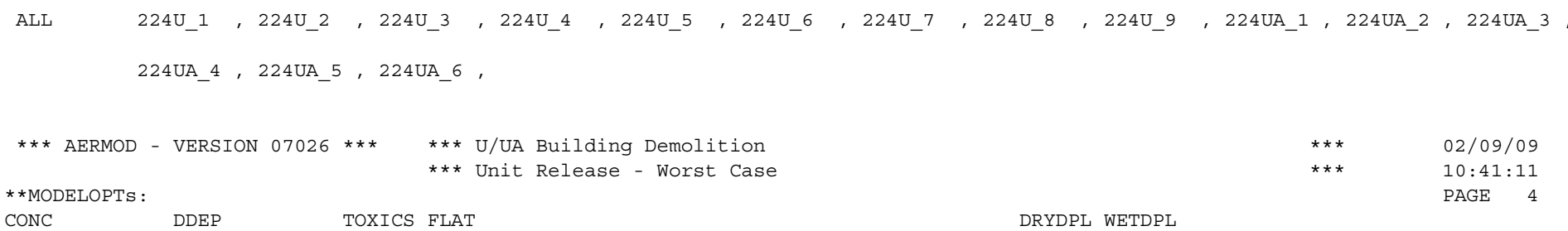

*** SOURCE PARTICULATE/GAS DATA ***

** SOURCE ID $=224 \mathrm{U}_{-} 1 ;$ SOURCE TYPE $=$ POINT $\quad$ ***

FINE PARTICLE MASS FRACTION $=$

1.00000

PARTICLE DIAMETER (MICRONS) =

5.00000 ,

PARTICLE DENSITY $(\mathrm{G} / \mathrm{CM} * * 3)=$ 
1.00000

** SOURCE ID $=224 \mathrm{U} \_2 ;$ SOURCE TYPE $=$ POINT FINE PARTICLE MASS FRACTION = 1.00000 ,

PARTICLE DIAMETER (MICRONS) = 5.00000

PARTICLE DENSITY $(\mathrm{G} / \mathrm{CM} * * 3)=$

1.00000 ,

** SOURCE ID $=224 \mathrm{U} 3 ;$; SOURCE TYPE $=$ POINT

FINE PARTICLE MASS FRACTION =

$$
1.00000,
$$

PARTICLE DIAMETER (MICRONS) =

$$
5.00000
$$

PARTICLE DENSITY $(\mathrm{G} / \mathrm{CM} * * 3)=$

1.00000 ,

FINE PARTICLE MASS FRACTION =

$$
1.00000
$$

PARTICLE DIAMETER (MICRONS) = 5.00000

PARTICLE DENSITY $(\mathrm{G} / \mathrm{CM} * * 3)=$

1.00000

$* *$ SOURCE ID $=224 \mathrm{U} \_5 ;$ SOURCE TYPE $=$ POINT

FINE PARTICLE MASS FRACTION =

$$
1.00000 \text {, }
$$


PARTICLE DIAMETER (MICRONS) =

5.00000 ,

PARTICLE DENSITY $(\mathrm{G} / \mathrm{CM} * * 3)=$

1.00000 ,

$* *$ SOURCE ID $=224 \mathrm{U} \_6 ;$ SOURCE TYPE $=$ POINT

FINE PARTICLE MASS FRACTION =

1.00000 ,

PARTICLE DIAMETER (MICRONS) =

5.00000 ,

PARTICLE DENSITY $(\mathrm{G} / \mathrm{CM} * * 3)=$

1.00000 ,

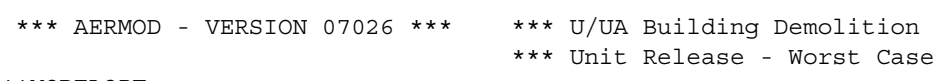

**MODELOPTS :

CONC

DDEP

TOXICS FLAT
*** $\quad 02 / 09 / 09$

$10: 41: 11$
PAGE 6

*** SOURCE PARTICULATE/GAS DATA ***

$* * *$ SOURCE ID $=224 \mathrm{U} 7 ;$ SOURCE TYPE $=$ POINT

FINE PARTICLE MASS FRACTION =

1.00000 ,

PARTICLE DIAMETER (MICRONS) =

5.00000 ,

PARTICLE DENSITY $(\mathrm{G} / \mathrm{CM} * * 3)=$

1.00000 ,

$* * *$ SOURCE ID $=224 \mathrm{U} 8 ;$ SOURCE TYPE $=$ POINT

FINE PARTICLE MASS FRACTION =

1.00000 ,

PARTICLE DIAMETER (MICRONS) =

5.00000 ,

PARTICLE DENSITY $(\mathrm{G} / \mathrm{CM} * * 3)=$

1.00000 , 
** SOURCE ID $=224 \mathrm{U} \_9 ;$ SOURCE TYPE $=$ POINT

FINE PARTICLE MASS FRACTION =

1.00000,

PARTICLE DIAMETER (MICRONS) =

5.00000 ,

PARTICLE DENSITY $(\mathrm{G} / \mathrm{CM} * * 3)=$

1.00000 ,

*** AERMOD - VERSION $07026 * * * \quad * * *$ U/UA Building Demolition

*** Unit Release - Worst Case

$\begin{array}{ll}* * * & 02 / 09 / 09 \\ * * * & 10: 41: 11\end{array}$

$\begin{array}{lll}\text { CONC } & \text { TOXEP }\end{array}$

DRYDPL WETDPL

$10: 41: 11$

*** SOURCE PARTICULATE/GAS DATA ***

$* *$ SOURCE ID $=224 \mathrm{UA} \_1 ;$ SOURCE TYPE $=$ POINT

***

FINE PARTICLE MASS FRACTION =

1.00000 ,

PARTICLE DIAMETER (MICRONS) = 5.00000 ,

PARTICLE DENSITY $(\mathrm{G} / \mathrm{CM} * * 3)=$

1.00000 ,

$\star \star *$ SOURCE ID $=224 \mathrm{UA} \_2 ;$ SOURCE TYPE $=$ POINT

FINE PARTICLE MASS FRACTION =

1.00000 ,

PARTICLE DIAMETER (MICRONS) = 5.00000 ,

PARTICLE DENSITY $(\mathrm{G} / \mathrm{CM} * * 3)=$ 1.00000 ,

** SOURCE ID $=224 \mathrm{UA} \_3 ;$ SOURCE TYPE $=$ POINT

FINE PARTICLE MASS FRACTION =

1.00000 ,

PARTICLE DIAMETER (MICRONS) = 
5.00000

PARTICLE DENSITY $(\mathrm{G} / \mathrm{CM} * * 3)=$

1.00000 ,

$\begin{array}{ll}* * * \text { AERMOD - VERSION } 07026 * * * \quad & * * * \text { U/UA Building Demolition } \\ & * * * \text { Unit Release - Worst Case }\end{array}$ **MODELOPTS

CONC

DDEP $\begin{array}{ll}* * * & 02 / 09 / 09 \\ * * * & 10: 41: 11\end{array}$

$10: 41: 11$
PAGE 8

** SOURCE ID $=224 \mathrm{UA} 4 ;$ SOURCE TYPE $=$ POINT

FINE PARTICLE MASS FRACTION =

1.00000 ,

PARTICLE DIAMETER (MICRONS) =

5.00000 ,

PARTICLE DENSITY $(\mathrm{G} / \mathrm{CM} * * 3)=$

1.00000 ,

$* *$ SOURCE ID $=224 \mathrm{UA} \_5 ;$ SOURCE TYPE $=$ POINT

FINE PARTICLE MASS FRACTION =

1.00000 ,

PARTICLE DIAMETER (MICRONS) =

5.00000

PARTICLE DENSITY $(\mathrm{G} / \mathrm{CM} * * 3)=$

1.00000 ,

$* *$ SOURCE ID $=224 \mathrm{UA} \_6 ;$ SOURCE TYPE $=$ POINT

FINE PARTICLE MASS FRACTION =

1.00000 ,

PARTICLE DIAMETER (MICRONS) =

5.00000,

PARTICLE DENSITY $(\mathrm{G} / \mathrm{CM} * * 3)=$

1.00000, 
$\star *$ MODELOPTS:

CONC

DDEP
*** Unit Release - Worst Case

TOXICS FLAT
DRYDPL WETDPL
** $10: 41: 11$
PAGE 9

*** DIRECTION SPECIFIC BUILDING DIMENSIONS ***

SOURCE ID: 224U_1

IFV BH BW BL XADJ YADJ IFV BH $\quad$ BW BL XADJ YADJ

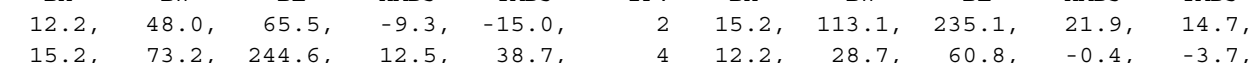

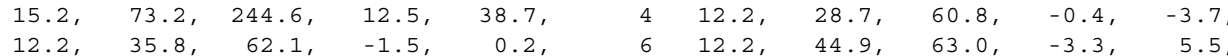

12.2, 52.6, 62.0, $-5.0, \quad 10.6, \quad 8 \quad 12.2, \quad 58.7, \quad 59.1, \quad-6.6, \quad 15.4$

12.2, 63.1, 54.4, -7.9, 19.7, $10 \quad 12.2, \quad 65.5, \quad 48.0, \quad-9.0, \quad 23.5$

12.2, 65.9, 40.2, $-9.9, \quad 26.5, \quad 12 \quad 12.2, \quad 64.4, \quad 31.2,-10.4, \quad 28.7$

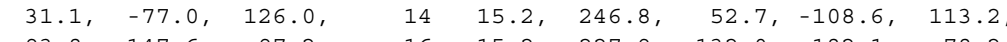

$15.2,241,0,93.8,-147.6,297.2, \quad 16,15.2,227.9,-132.0,-182.1,78.2$,

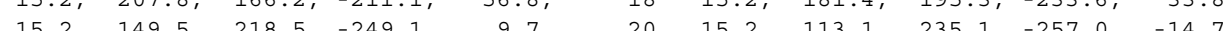

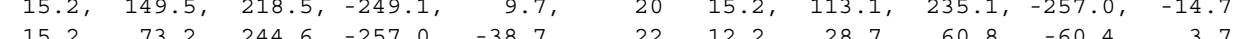

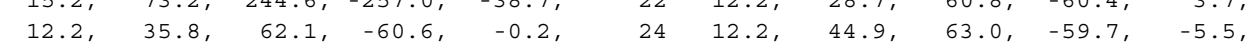

12.2, 52.6, 62.0, $-57.0,-10.6, \quad 26 \quad 12.2, \quad 58.7, \quad 59.1, \quad-52.5, \quad-15.4$

$12.2, \quad 63.1, \quad 54.4,-46.4,-19.7, \quad 28 \quad 12.2, \quad 65.5, \quad 48.0, \quad-39.0, \quad-23.5$

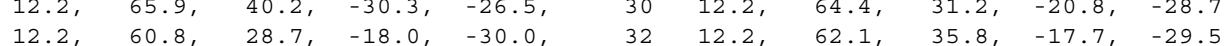

$12.2, \quad 63.0, \quad 44.9, \quad-17.0, \quad-28.2, \quad 34 \quad 12.2, \quad 62.0, \quad 52.6,-15.7,-26.0$

$\Omega \quad 3512.2,59.1, \quad 58.7,-14.0,-23.0$,

$36 \quad 12.2, \quad 54.4,63.1,-11.8,-19.3$,

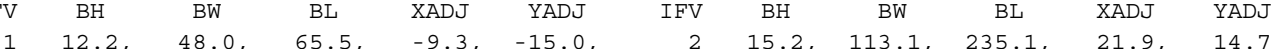

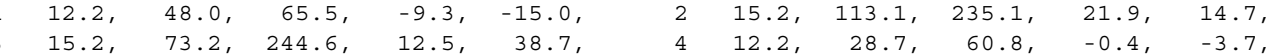

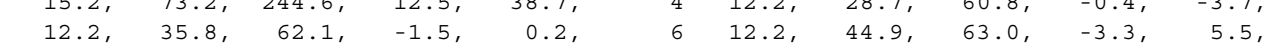

$712.2, \quad 52.6, \quad 62.0, \quad-5.0, \quad 10.6, \quad 8 \quad 12.2, \quad 58.7, \quad 59.1, \quad-6.6, \quad 15.4$

$912.2, \quad 63.1, \quad 54.4, \quad-7.9, \quad 19.7, \quad 10 \quad 12.2, \quad 65.5, \quad 48.0, \quad-9.0, \quad 23.5$

$15 \quad 15.2, \quad 241.0, \quad 93.8,-747.6, \quad \begin{array}{rrrrrrr}126.0, & 14 & 15.2, & 246.8, & 52.7, & -108.6, & 113.2 \\ 17 & 16 & 15.2, & 227.9, & 132.0, & -182.1, & 78.2\end{array}$

$17 \quad 15.2, \quad 207.8, \quad 166.2,-211.1, \quad 56.8, \quad 18$ 15.2, $181.4, \quad 195.3,-233.6, \quad 33.8$

$19 \quad 15.2, \quad 149.5,218.5,-249.1, \quad 9.7, \quad 20 \quad 15.2,113.1,235.1,-257.0,-14.7$

$21 \quad 15.2, \quad 73.2,244.6,-257.0,-38.7, \quad 22 \quad 12.2, \quad 28.7,60.8,-60.4,3.7$

$23 \quad 12.2, \quad 35.8, \quad 62.1, \quad-60.6, \quad-0.2, \quad 24 \quad 12.2, \quad 44.9, \quad 63.0, \quad-59.7, \quad-5.5$

$25 \quad 12.2, \quad 52.6, \quad 62.0, \quad-57.0, \quad-10.6, \quad 26 \quad 12.2, \quad 58.7, \quad 59.1, \quad-52.5, \quad-15.4$

$2712.2, \quad 63.1, \quad 54.4, \quad-46.4,-19.7, \quad 28-12.2, \quad 65.5, \quad 48.0, \quad-39.0, \quad-23.5$,

$29 \quad 12.2, \quad 65.9, \quad 40.2, \quad-30.3,-26.5, \quad 30 \quad 12.2, \quad 64.4, \quad 31.2, \quad-20.8, \quad-28.7$

$31 \quad 12.2, \quad 60.8, \quad 28.7, \quad-18.0, \quad-30.0, \quad 32 \quad 12.2, \quad 62.1, \quad 35.8, \quad-17.7, \quad-29.5$

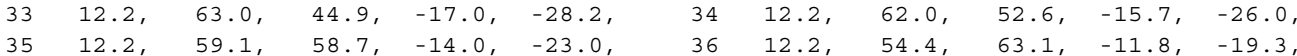

SOURCE ID: $224 \mathrm{U} 3$

BL XADJ YADJ

BW

YADJ 


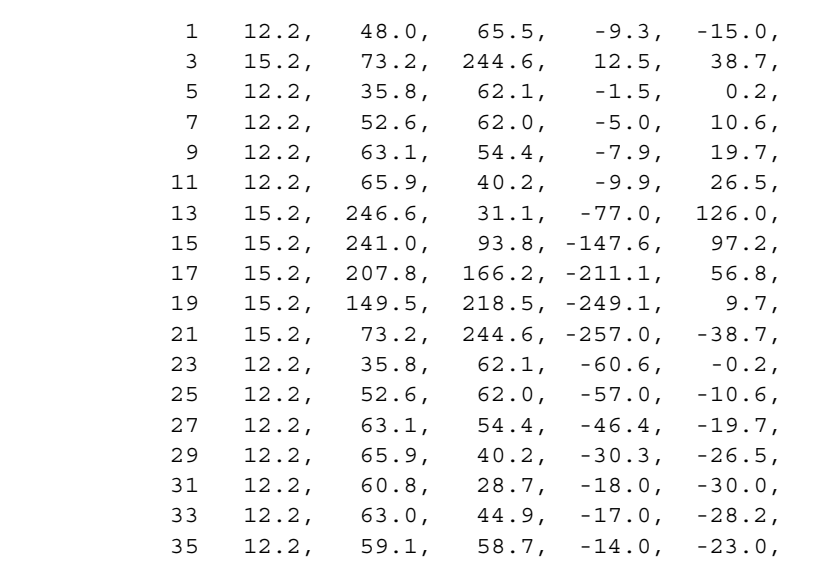

$\begin{array}{rrrrrr}2 & 15.2, & 113.1, & 235.1, & 21.9, & 14.7, \\ 4 & 12.2, & 28.7, & 60.8, & -0.4, & -3.7, \\ 6 & 12.2, & 44.9, & 63.0, & -3.3, & 5.5, \\ 8 & 12.2, & 58.7, & 59.1, & -6.6, & 15.4 \\ 10 & 12.2, & 65.5, & 48.0, & -9.0, & 23.5 \\ 12 & 12.2, & 64.4, & 31.2, & -10.4, & 28.7, \\ 14 & 15.2, & 246.8, & 52.7, & -108.6, & 113.2, \\ 16 & 15.2, & 227.9, & 132.0, & -182.1, & 78.2, \\ 18 & 15.2, & 181.4, & 195.3, & -233.6, & 33.8 \\ 20 & 15.2, & 113.1, & 235.1, & -257.0, & -14.7, \\ 22 & 12.2, & 28.7, & 60.8, & -60.4, & 3.7 \\ 24 & 12.2, & 44.9, & 63.0, & -59.7, & -5.5 \\ 26 & 12.2, & 58.7, & 59.1, & -52.5, & -15.4 \\ 28 & 12.2, & 65.5, & 48.0, & -39.0, & -23.5 \\ 30 & 12.2, & 64.4, & 31.2, & -20.8, & -28.7 \\ 32 & 12.2, & 62.1, & 35.8, & -17.7, & -29.5 \\ 34 & 12.2, & 62.0, & 52.6, & -15.7, & -26.0 \\ 36 & 12.2, & 54.4, & 63.1, & -11.8, & -19.3,\end{array}$

\begin{tabular}{|c|c|c|c|c|c|c|}
\hline & SOURC & ID: 2 & $4 \mathrm{U} \quad 4$ & & & \\
\hline & IFV & $\mathrm{BH}$ & BW & $\mathrm{BL}$ & XADJ & YADJ \\
\hline & 1 & 15.2 , & 149.5, & 218.5, & 29.1, & -12.1, \\
\hline & 3 & 15.2, & 73.2 , & 244.6, & 12.0, & 35.9 , \\
\hline & 5 & 12.2, & 35.8, & 62.1 & -1.0 , & -2.6, \\
\hline & 7 & 12.2 , & 52.6 & 62.0, & -3.6, & 8.2 , \\
\hline$\Omega$ & 9 & 12.2 , & 63.1, & 54.4, & -5.7, & 17.9, \\
\hline & 11 & 12.2, & 65.9, & 40.2, & -7.2, & 25.5, \\
\hline & 13 & 15.2, & 246.6 , & 31.1 , & -74.2, & 126.1, \\
\hline & 15 & 15.2, & 241.0, & 93.8, & -144.9, & 98.2, \\
\hline & 17 & 15.2 , & 207.8, & 166.2, & -208.9, & 58.7, \\
\hline & 19 & 15.2, & 149.5, & 218.5, & -247.7, & 12.1, \\
\hline & 21 & 15.2 , & 73.2 , & 244.6, & -256.6, & -35.9, \\
\hline & 23 & 12.2, & 35.8, & 62.1 & -61.1 , & 2.6 \\
\hline & 25 & 12.2, & 52.6, & 62.0, & -58.4, & -8.2, \\
\hline & 27 & 12.2, & 63.1, & 54.4, & -48.6, & -17.9, \\
\hline & 29 & 12.2, & 65.9, & 40.2 , & -33.0, & -25.5, \\
\hline & 31 & 12.2, & 60.8, & 28.7, & -20.9, & -30.0, \\
\hline & 33 & 12.2 , & 63.0 , & 44.9, & -19.6, & -29.2, \\
\hline & 35 & 12.2, & 59.1 , & 58.7, & -16.1 , & -24.8, \\
\hline
\end{tabular}

\begin{tabular}{|c|c|c|c|c|c|}
\hline IFV & BH & BW & BL & XADJ & YADJ \\
\hline 2 & 15.2 , & 113.1, & 235.1, & 20.9, & 12.0, \\
\hline 4 & 12.2 , & 28.7 & 60.8, & -0.4, & -6.6 , \\
\hline 6 & 12.2, & 44.9, & 63.0, & -2.3, & 2.8 \\
\hline 8 & 12.2, & 58.7, & 59.1, & -4.7, & 13.2, \\
\hline 10 & 12.2 , & 65.5, & 48.0, & -6.6 , & 22.0, \\
\hline 12 & 12.2 , & 64.4 & 31.2 , & -7.6 & 28.2, \\
\hline 14 & 15.2 , & 246.8, & 52.7, & -105.8, & 113.7, \\
\hline 16 & 15.2, & 227.9 , & 132.0, & -179.6, & 79.6 \\
\hline 18 & 15.2, & 181.4, & 195.3, & -231.8, & 36.0, \\
\hline 20 & 15.2, & 113.1 , & 235.1, & -256.0, & -12.0, \\
\hline 22 & 12.2 , & 28.7, & 60.8, & -60.5 & 6.6 \\
\hline 24 & 12.2, & 44.9, & 63.0, & -60.7, & -2.8, \\
\hline 26 & 12.2 , & 58.7, & 59.1, & -54.3, & -13.2, \\
\hline 28 & 12.2, & 65.5, & 48.0, & -41.5, & -22.0, \\
\hline 30 & 12.2 , & 64.4, & 31.2 , & -23.6, & -28.2, \\
\hline 32 & 12.2 , & 62.1, & 35.8, & -20.5, & -30.0, \\
\hline 34 & 12.2, & 62.0 , & 52.6, & -18.1, & -27.4, \\
\hline 36 & 12.2 & 54.4, & 63.1, & -13.6, & -21.5, \\
\hline
\end{tabular}

$* * *$ AERMOD - VERSION $07026 * * * \quad \begin{array}{r}* * \\ \text { U/UA Building Demolition } \\ * * * \text { Unit Release - Worst Case }\end{array}$ $* *$ MODELOPTS : CONC DDEP TOXICS FLAT $\begin{array}{ll}* * * & 02 / 09 / 09 \\ * * * & 10: 41: 11\end{array}$ $10: 41: 11$
PAGE $\quad 10$

*** DIRECTION SPECIFIC BUILDING DIMENSIONS *** 


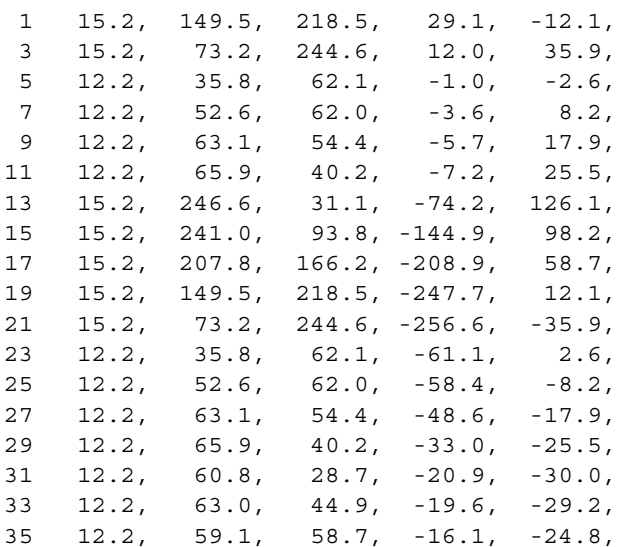

$\begin{array}{rrrrrr}2 & 15.2, & 113.1, & 235.1, & 20.9, & 12.0, \\ 4 & 12.2, & 28.7, & 60.8, & -0.4, & -6.6, \\ 6 & 12.2, & 44.9, & 63.0, & -2.3, & 2.8, \\ 8 & 12.2, & 58.7, & 59.1, & -4.7, & 13.2, \\ 0 & 12.2, & 65.5, & 48.0, & -6.6, & 22.0, \\ 2 & 12.2, & 64.4, & 31.2, & -7.6, & 28.2, \\ 4 & 15.2, & 246.8, & 52.7, & -105.8, & 113.7, \\ 6 & 15.2, & 227.9, & 132.0, & -179.6, & 79.6, \\ 8 & 15.2, & 181.4, & 195.3, & -231.8, & 36.0, \\ 0 & 15.2, & 113.1, & 235.1, & -256.0, & -12.0, \\ 2 & 12.2, & 28.7, & 60.8, & -60.5, & 6.6, \\ 4 & 12.2, & 44.9, & 63.0, & -60.7, & -2.8, \\ 6 & 12.2, & 58.7, & 59.1, & -54.3, & -13.2, \\ 8 & 12.2, & 65.5, & 48.0, & -41.5, & -22.0, \\ 0 & 12.2, & 64.4, & 31.2, & -23.6, & -28.2, \\ 3 & 12.2, & 62.1, & 35.8, & -20.5, & -30.0, \\ 34 & 12.2, & 62.0, & 52.6, & -18.1, & -27.4, \\ 6 & 12.2, & 54.4, & 63.1, & -13.6, & -21.5,\end{array}$

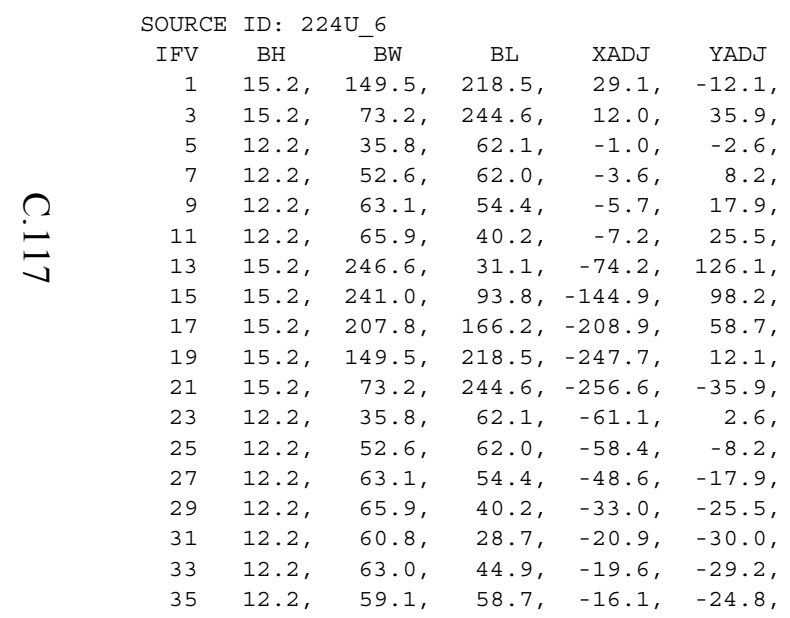

$\begin{array}{rrrrrr}\text { IFV } & \text { BH } & \text { BW } & \text { BL } & \text { XADJ } & \text { YADJ } \\ 2 & 15.2, & 113.1, & 235.1, & 20.9, & 12.0, \\ 4 & 12.2, & 28.7, & 60.8, & -0.4, & -6.6, \\ 6 & 12.2, & 44.9, & 63.0, & -2.3, & 2.8, \\ 8 & 12.2, & 58.7, & 59.1, & -4.7, & 13.2, \\ 10 & 12.2, & 65.5, & 48.0, & -6.6, & 22.0, \\ 12 & 12.2, & 64.4, & 31.2, & -7.6, & 28.2, \\ 14 & 15.2, & 246.8, & 52.7, & -105.8, & 113.7, \\ 16 & 15.2, & 227.9, & 132.0, & -179.6, & 79.6, \\ 18 & 15.2, & 181.4, & 195.3, & -231.8, & 36.0, \\ 20 & 15.2, & 113.1, & 235.1, & -256.0, & -12.0, \\ 22 & 12.2, & 28.7, & 60.8, & -60.5, & 6.6, \\ 24 & 12.2, & 44.9, & 63.0, & -60.7, & -2.8, \\ 26 & 12.2, & 58.7, & 59.1, & -54.3, & -13.2, \\ 28 & 12.2, & 65.5, & 48.0, & -41.5, & -22.0, \\ 30 & 12.2, & 64.4, & 31.2, & -23.6, & -28.2, \\ 32 & 12.2, & 62.1, & 35.8, & -20.5, & -30.0, \\ 34 & 12.2, & 62.0, & 52.6, & -18.1, & -27.4, \\ 36 & 12.2, & 54.4, & 63.1, & -13.6, & -21.5,\end{array}$

\section{SOURCE ID: 224U 7} $12.2,48.0,65.5, \quad-7.9,-12.8, \quad 2 \quad 15.2,113.1,235.1,22.9, \quad 17.1$ $3 \quad 15.2,73.2,244.6, \quad 13.1,41.1, \quad 4 \quad 12.2, \quad 28.7,60.8, \quad-0.3, \quad-17$ $512.2, \quad 35.8,62.1, \quad-1.8, \quad 2.8, \quad 6 \quad 12.2, \quad 44.9,63.0, \quad-4.1, \quad 7.9$ $7 \quad 12.2, \quad 52.6, \quad 62.0, \quad-6.2, \quad 12.9, \quad 8 \quad 12.2, \quad 58.7, \quad 59.1, \quad-8.1, \quad 17.5$ $9 \quad 12.2, \quad 63.1, \quad 54.4, \quad-9.8, \quad 21.5, \quad 10 \quad 12.2, \quad 65.5, \quad 48.0, \quad-11.2, \quad 24.9$ $11 \quad 12.2, \quad 65.9, \quad 40.2,-12.2, \quad 27.5, \quad 12 \quad 12.2, \quad 64.4, \quad 31.2,-12.9, \quad 29.3$, $13 \quad 15.2, \quad 246.6, \quad 31.1, \quad-79.5, \quad 126.2, \quad 14 \quad 15.2, \quad 246.8, \quad 52.7,-111.1,112.9$ 15 15.2, 241.0, 93.8, -150.0, 96.4, 16 15.2, 227.9, 132.0, -184.4, 77.0, 17 15.2, 207.8, 166.2, -213.1, 55.3, 18 15.2, 181.4, 195.3, -235.4, 31.9، 


\begin{tabular}{|c|c|c|c|c|c|c|c|c|c|c|c|}
\hline 19 & 15.2, & 149.5, & 218.5, & -250.5 & 7.5 & 20 & 15.2, & 113.1, & 235.1, & -258.0 & -17.1 , \\
\hline 21 & 15.2, & 73.2 & 244.6 , & -257.6 & -41.1 & 22 & 12.2, & 28.7 & 60.8 & -60.6 & 1.2, \\
\hline 23 & 12.2, & 35.8, & 62.1 & -60.3 & -2.8 & 24 & 12.2 & 44.9, & 63.0, & -58.9, & -7.9 \\
\hline 25 & 12.2, & 52.6, & 62.0, & -55.8 & -12.9 & 26 & 12.2 & 58.7, & 59.1, & -51.0, & -17.5, \\
\hline 27 & 12.2, & 63.1, & 54.4, & -44.6 & -21.5 & 28 & 12.2, & 65.5, & 48.0, & -36.8, & -24.9, \\
\hline 29 & 12.2, & 65.9, & 40.2 , & -28.0 & -27.5 & 30 & 12.2 & $64.4 ，$ & 31.2, & -18.3, & -29.3 \\
\hline 31 & 12.2, & 60.8, & 28.7, & -15.5 & -30.2, & 32 & 12.2, & 62.1, & 35.8, & -15.2, & -29.2, \\
\hline 33 & 12.2 , & 63.0 & 44.9, & -14.5 & -27.4 & 34 & 12.2 & 62.0, & 52.6, & -13.4, & -24.8 \\
\hline 35 & 12.2, & 59.1, & 58.7, & -11.9, & -21.4 & 36 & 12.2 & $54.4 ，$ & 63.1, & -10.1, & -17.4 , \\
\hline SOURC & CE ID: 2 & $24 \mathrm{U} \_8$ & & & & & & & & & \\
\hline IFV & $\mathrm{BH}$ & $\mathrm{BW}$ & $\mathrm{BL}$ & XADJ & YADJ & IFV & $\mathrm{BH}$ & BW & $\mathrm{BL}$ & XADJ & YADJ \\
\hline 1 & 12.2, & 48.0, & 65.5 & -7.9 & -12.8 & 2 & 15.2, & 113.1, & 235.1, & 22.9, & 17.1, \\
\hline 3 & 15.2, & 73.2 & 244.6, & 13.1 & 41.1 & 4 & 12.2 & 28.7, & 60.8 & -0.3 & -1.2, \\
\hline 5 & 12.2, & 35.8, & 62.1 & -1.8 & 2.8 & 6 & 12.2 & 44.9, & 63.0, & -4.1, & 7.9, \\
\hline 7 & 12.2, & 52.6 & 62.0 & -6.2, & 12.9, & 8 & 12.2, & 58.7, & 59.1, & -8.1, & 17.5, \\
\hline 9 & 12.2, & 63.1 & 54.4, & -9.8 & 21.5, & 10 & 12.2, & 65.5, & 48.0, & -11.2, & 24.9, \\
\hline 11 & 12.2, & 65.9, & 40.2, & -12.2 & 27.5 & 12 & 12.2, & 64.4 & 31.2, & -12.9 & 29.3, \\
\hline 13 & 15.2, & 246.6 & 31.1, & -79.5 & 126.2 , & 14 & 15.2 , & 246.8, & 52.7 & -111.1, & 112.9 \\
\hline 15 & 15.2, & 241.0, & 93.8, & -150.0 & 96.4 & 16 & 15.2, & 227.9 , & 132.0, & -184.4 & 77.0 \\
\hline 17 & 15.2， & 207.8, & 166.2, & -213.1 & 55.3, & 18 & 15.2, & 181.4, & 195.3, & -235.4 & 31.9, \\
\hline 19 & 15.2, & 149.5, & 218.5, & -250.5 & 7.5, & 20 & 15.2, & 113.1, & 235.1, & -258.0 & -17.1, \\
\hline 21 & 15.2, & 73.2 & 244.6 & -257.6 & -41.1 & 22 & 12.2, & 28.7 & 60.8 & -60.6 & 1.2, \\
\hline 23 & 12.2, & 35.8, & 62.1 & -60.3 & -2.8 & 24 & 12.2 & 44.9, & 63.0, & -58.9, & -7.9, \\
\hline 25 & 12.2, & 52.6 & 62.0, & -55.8 & -12.9, & 26 & 12.2 & 58.7, & 59.1, & -51.0 & -17.5 , \\
\hline 27 & 12.2, & 63.1, & 54.4, & -44.6 & -21.5 & 28 & 12.2, & 65.5, & 48.0, & -36.8, & -24.9, \\
\hline 29 & 12.2, & 65.9, & 40.2, & -28.0 & -27.5 & 30 & 12.2, & 64.4, & 31.2, & -18.3 & -29.3, \\
\hline 31 & 12.2, & 60.8 & 28.7, & -15.5 & -30.2, & 32 & 12.2 & 62.1, & 35.8, & -15.2, & -29.2, \\
\hline 33 & 12.2, & 63.0, & 44.9, & -14.5 & -27.4 & 34 & 12.2, & 62.0, & 52.6, & -13.4, & -24.8, \\
\hline 35 & 12.2, & 59.1, & 58.7, & -11.9, & -21.4 & 36 & 12.2, & 54.4, & 63.1, & -10.1, & -17.4, \\
\hline
\end{tabular}

\begin{tabular}{|c|c|c|c|c|c|c|c|c|c|c|c|}
\hline$* * *$ & ERMOD & - VERSION & 07026 & $* * *$ & $\begin{array}{l}* * * \text { U/UA } \\
\star * * \text { Unit }\end{array}$ & $\begin{array}{l}\text { Building D } \\
\text { Release - }\end{array}$ & $\begin{array}{r}\text { Demoli } \\
\text { Worst }\end{array}$ & $\begin{array}{l}\text { Eion } \\
\text { Case }\end{array}$ & & & \\
\hline$\approx$ MOD & LOPTS: & & & & & & & & & & \\
\hline $\mathrm{CONC}$ & & DDEP & & TOXICS & FLAT & & & & & & DRYDPI \\
\hline & & & & & $* * *$ & * DIRECTION & N SPEC & IFIC BUI & DING DI & MENSIONS & $\star * *$ \\
\hline SOURC & ID: 2 & 224U_9 & & & & & & & & & \\
\hline IFV & $\mathrm{BH}$ & BW & $\mathrm{BL}$ & XADJ & YADJ & IFV & BH & BW & BL & XADJ & YADJ \\
\hline 1 & 12.2 , & 48.0 & 65.5 & -7.9 & -12.8, & 2 & 15.2, & 113.1 & 235.1, & 22.9, & 17.1 \\
\hline 3 & 15.2, & 73.2, & 244.6 & 13.1 & 41.1 & 4 & 12.2, & 28.7 & 60.8 & -0.3 & -1.2, \\
\hline 5 & 12.2 , & 35.8, & 62.1 & -1.8 & 2.8 & 6 & 12.2, & 44.9, & 63.0, & -4.1 & 7.9, \\
\hline 7 & 12.2 , & 52.6 & 62.0, & -6.2 & 12.9, & 8 & 12.2, & 58.7, & 59.1, & -8.1 & 17.5, \\
\hline 9 & 12.2, & 63.1 & 54.4, & -9.8 & 21.5, & 10 & 12.2 & 65.5, & 48.0, & -11.2, & 24.9, \\
\hline 11 & 12.2 , & 65.9 & 40.2 , & -12.2 , & 27.5 & 12 & 12.2 & 64.4 & 31.2 , & -12.9 & 29.3, \\
\hline 13 & 15.2, & 246.6 & 31.1, & -79.5, & 126.2 , & 14 & 15.2, & 246.8, & 52.7 & -111.1 & 112.9 \\
\hline 15 & 15.2 , & 241.0 & 93.8, & -150.0 & 96.4 & 16 & 15.2, & 227.9 , & 132.0 & -184.4 & 77.0 , \\
\hline 17 & 15.2, & 207.8 & 166.2, & -213.1 & $55.3 ，$ & 18 & 15.2, & 181.4, & 195.3, & -235.4 & 31.9, \\
\hline
\end{tabular}

$\begin{array}{ll}* * * & 02 / 09 / 09 \\ * * * & 10: 41: 11\end{array}$ PAGE 11 


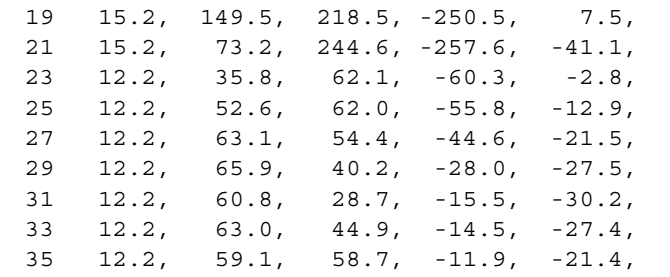

SOURCE ID: 224UA_1

$\begin{array}{rrrrrr}\text { IFV } & \text { BH } & \text { BW } & \text { BL } & \text { XADJ } & \text { YADJ } \\ 1 & 12.2, & 48.0, & 65.5, & 3.3, & 0.5, \\ 3 & 12.2, & 31.2, & 64.4, & 3.0, & 13.7, \\ 5 & 12.2, & 35.8, & 62.1, & -1.8, & 20.2, \\ 7 & 12.2, & 52.6, & 62.0, & -12.1, & 29.2, \\ 9 & 12.2, & 63.1, & 54.4, & -21.0, & 34.8, \\ 11 & 12.2, & 65.9, & 40.2, & -27.3, & 36.2, \\ 13 & 12.2, & 60.8, & 28.7, & -30.3, & 33.2, \\ 15 & 12.2, & 63.0, & 44.9, & -47.5, & 24.4, \\ 17 & 12.2, & 59.1, & 58.7, & -61.9, & 12.7, \\ 19 & 12.2, & 48.0, & 65.5, & -68.8, & -0.5, \\ 21 & 12.2, & 31.2, & 64.4, & -67.4, & -13.7, \\ 23 & 12.2, & 35.8, & 62.1, & -60.3, & -20.2, \\ 25 & 12.2, & 52.6, & 62.0, & -49.8, & -29.2, \\ 27 & 12.2, & 63.1, & 54.4, & -33.4, & -34.8, \\ 29 & 12.2, & 65.9, & 40.2, & -12.9, & -36.2, \\ 31 & 12.2, & 60.8, & 28.7, & 1.7, & -33.2, \\ 33 & 12.2, & 63.0, & 44.9, & 2.6, & -24.4, \\ 35 & 12.2, & 59.1, & 58.7, & 3.2, & -12.7,\end{array}$

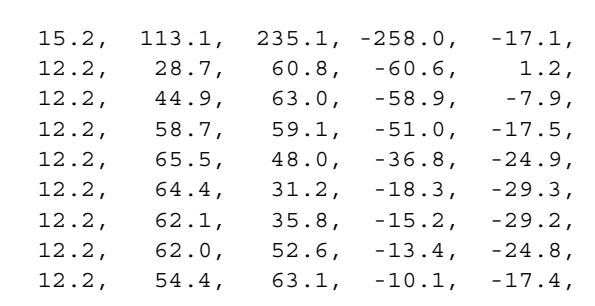

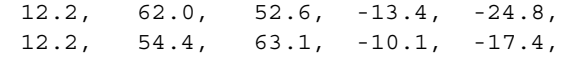

$\begin{array}{rccccc}\text { IFV } & \text { BH } & \text { BW } & \text { BL } & \text { XADJ } & \text { YADJ } \\ 2 & 12.2, & 40.2, & 65.9, & 3.2, & 7.2, \\ 4 & 12.2, & 28.7, & 60.8, & 2.8, & 16.0, \\ 6 & 12.2, & 44.9, & 63.0, & -7.1, & 25.1, \\ 8 & 12.2, & 58.7, & 59.1, & -16.8, & 32.5, \\ 10 & 12.2, & 65.5, & 48.0, & -24.5, & 36.0, \\ 12 & 12.2, & 64.4, & 31.2, & -29.2, & 35.2, \\ 14 & 12.2, & 62.1, & 35.8, & -38.1, & 29.2, \\ 16 & 12.2, & 62.0, & 52.6, & -55.6, & 18.9, \\ 18 & 12.2, & 54.4, & 63.1, & -66.3, & 6.2, \\ 20 & 12.2, & 40.2, & 65.9, & -69.1, & -7.2, \\ 22 & 12.2, & 28.7, & 60.8, & -63.6, & -16.0, \\ 24 & 12.2, & 44.9, & 63.0, & -55.9, & -25.1, \\ 26 & 12.2, & 58.7, & 59.1, & -42.2, & -32.5, \\ 28 & 12.2, & 65.5, & 48.0, & -23.5, & -36.0, \\ 30 & 12.2, & 64.4, & 31.2, & -1.9, & -35.2, \\ 32 & 12.2, & 62.1, & 35.8, & 2.3, & -29.2, \\ 34 & 12.2, & 62.0, & 52.6, & 2.9, & -18.9, \\ 36 & 12.2, & 54.4, & 63.1, & 3.3, & -6.2,\end{array}$

SOURCE ID: 224UA 2

\begin{tabular}{|c|c|c|c|c|c|c|c|c|c|c|c|}
\hline IFV & BH & BW & BI, & XADJ & YADJ & TFV & $\mathrm{BH}$ & RW & BT & דסמצ & YADJ \\
\hline 1 & 12.2, & 48.0, & 65.5, & 3.3 & 0.5 & 2 & 12.2, & 40.2, & 65.9, & 3.2, & 7.2 \\
\hline 3 & 12.2 , & 31.2 , & 64.4, & 3.0, & 13.7, & 4 & 12.2, & 28.7, & 60.8, & 2.8 & 16.0, \\
\hline 5 & 12.2, & 35.8, & 62.1, & -1.8, & 20.2, & 6 & 12.2, & 44.9, & 63.0, & -7.1 , & 25.1, \\
\hline 7 & 12.2 , & 52.6 & 62.0 & -12.1, & 29.2, & 8 & 12.2, & 58.7, & 59.1, & -16.8, & 32.5, \\
\hline 9 & 12.2 & 63.1, & 54.4, & -21.0, & 34.8, & 10 & 12.2, & 65.5, & 48.0 & -24.5, & 36.0, \\
\hline 11 & 12.2 , & 65.9, & 40.2 , & -27.3, & 36.2 , & 12 & 12.2, & 64.4, & 31.2, & -29.2, & 35.2 , \\
\hline 13 & 12.2 , & 60.8, & 28.7, & -30.3, & 33.2, & 14 & 12.2 , & 62.1, & 35.8, & -38.1, & 29.2, \\
\hline 15 & 12.2, & 63.0, & 44.9, & -47.5, & 24.4, & 16 & 12.2, & 62.0, & 52.6, & -55.6, & 18.9, \\
\hline 17 & 12.2 , & 59.1, & 58.7, & -61.9, & 12.7, & 18 & 12.2, & 54.4, & 63.1, & -66.3, & 6.2 \\
\hline 19 & 12.2 , & 48.0 & 65.5, & -68.8, & -0.5, & 20 & 12.2, & 40.2, & 65.9, & -69.1, & -7.2 , \\
\hline 21 & 12.2, & 31.2, & 64.4, & -67.4, & -13.7, & 22 & 12.2, & 28.7, & 60.8, & -63.6 , & -16.0, \\
\hline 23 & 12.2 , & 35.8, & 62.1, & -60.3, & -20.2, & 24 & 12.2, & 44.9, & 63.0, & -55.9, & -25.1, \\
\hline 25 & 12.2 , & 52.6 & 62.0, & -49.8, & -29.2, & 26 & 12.2, & 58.7, & 59.1 , & -42.2, & -32.5, \\
\hline 27 & 12.2, & 63.1, & 54.4, & -33.4, & -34.8, & 28 & 12.2, & 65.5, & 48.0, & -23.5, & -36.0 , \\
\hline 29 & 12.2 , & 65.9, & 40.2, & -12.9, & -36.2, & 30 & 12.2, & 64.4, & 31.2, & -1.9, & -35.2, \\
\hline 31 & 12.2 , & 60.8, & 28.7, & 1.7, & -33.2, & 32 & 12.2, & 62.1, & 35.8, & 2.3, & -29.2, \\
\hline 33 & 12.2 , & 63.0 , & 44.9, & 2.6, & -24.4, & 34 & 12.2, & 62.0, & 52.6 & 2.9, & -18.9 , \\
\hline 35 & 12.2 , & 59.1, & 58.7, & 3.2, & -12.7, & 36 & 12.2 , & 54.4, & 63.1, & 3.3, & -6.2 \\
\hline
\end{tabular}


SOURCE ID: 224UA_3

\begin{tabular}{|c|c|c|c|c|c|c|c|c|c|c|c|}
\hline IFV & $\mathrm{BH}$ & $\bar{B} W$ & $\mathrm{BL}$ & XADJ & YADJ & IFV & $\mathrm{BH}$ & BW & $\mathrm{BL}$ & XADJ & YADJ \\
\hline 1 & 12.2, & 48.0, & 65.5, & 3.3 & 0.5 & 2 & 12.2 , & 40.2 , & 65.9, & 3.2 & 7.2 , \\
\hline 3 & 12.2, & 31.2, & 64.4, & 3.0 , & 13.7, & 4 & 12.2 , & 28.7, & 60.8, & 2.8 & 16.0, \\
\hline 5 & 12.2, & 35.8, & 62.1, & -1.8, & 20.2 , & 6 & 12.2, & 44.9, & 63.0, & -7.1 , & 25.1, \\
\hline 7 & 12.2, & 52.6 & 62.0 , & -12.1, & 29.2, & 8 & 12.2, & 58.7, & 59.1 , & -16.8, & 32.5 , \\
\hline 9 & 12.2 , & 63.1, & 54.4, & -21.0, & 34.8, & 10 & 12.2 , & 65.5, & 48.0 , & -24.5, & 36.0 , \\
\hline 11 & 12.2, & 65.9, & 40.2 , & -27.3, & 36.2 , & 12 & 12.2, & 64.4, & 31.2 , & -29.2, & 35.2 , \\
\hline 13 & 12.2 , & 60.8, & 28.7, & -30.3, & 33.2, & 14 & 12.2, & 62.1 , & 35.8, & -38.1, & 29.2 , \\
\hline 15 & 12.2 , & 63.0, & 44.9, & -47.5, & 24.4, & 16 & 12.2, & 62.0 , & 52.6, & -55.6, & 18.9, \\
\hline 17 & 12.2, & 59.1, & 58.7, & -61.9, & 12.7, & 18 & 12.2 , & 54.4, & 63.1 , & -66.3, & 6.2 \\
\hline 19 & 12.2, & 48.0, & 65.5， & -68.8, & -0.5 & 20 & 12.2 , & 40.2 , & 65.9, & -69.1, & -7.2 , \\
\hline 21 & 12.2, & 31.2 , & 64.4, & -67.4, & -13.7, & 22 & 12.2 , & 28.7, & 60.8, & -63.6, & -16.0, \\
\hline 23 & 12.2 , & 35.8, & 62.1, & -60.3, & -20.2, & 24 & 12.2, & 44.9, & 63.0, & -55.9, & -25.1, \\
\hline 25 & 12.2, & 52.6, & 62.0, & -49.8, & -29.2, & 26 & 12.2 , & 58.7, & 59.1, & -42.2 & -32.5, \\
\hline 27 & 12.2 , & 63.1, & 54.4, & -33.4, & -34.8, & 28 & 12.2 , & 65.5， & 48.0 , & -23.5, & -36.0, \\
\hline 29 & 12.2, & 65.9, & 40.2 , & -12.9, & -36.2 , & 30 & 12.2 , & 64.4, & 31.2 , & -1.9, & -35.2, \\
\hline 31 & 12.2, & 60.8, & 28.7, & 1.7, & -33.2, & 32 & 12.2 , & 62. & 35.8, & 2.3 & -29.2, \\
\hline 33 & 12.2, & 63.0, & 44.9 , & 2.6, & -24.4, & 34 & 12.2, & 62.0, & 52.6, & 2.9, & -18.9, \\
\hline 35 & 12.2, & 59.1, & 58.7, & 3.2, & -12.7, & 36 & 12.2, & 54.4, & 63.1, & 3.3, & -6.2 , \\
\hline
\end{tabular}

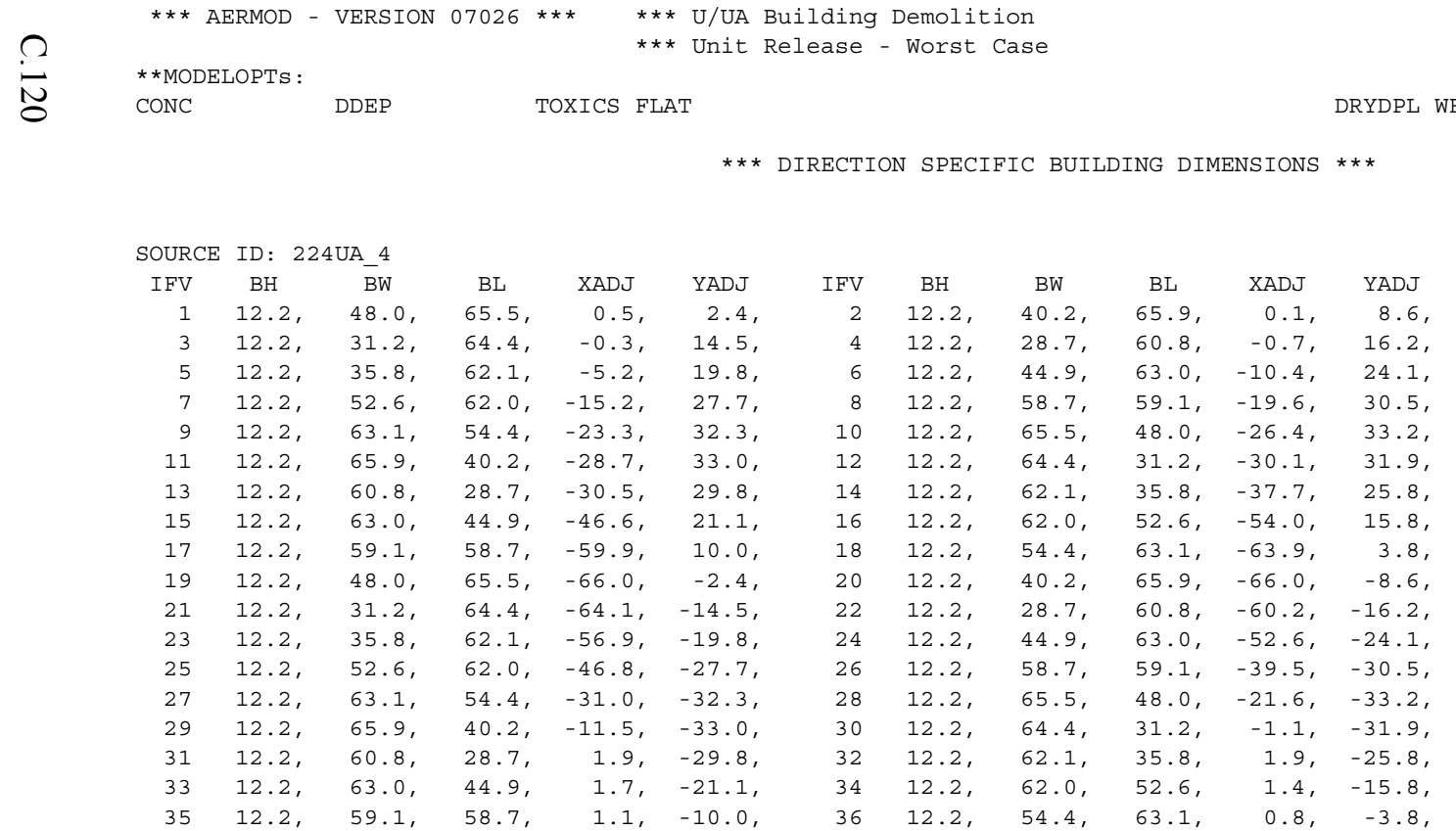




\begin{tabular}{|c|c|c|c|c|c|c|c|c|c|c|c|}
\hline SOURCE & ID: 2 & 224UA_5 & & & & & & & & & \\
\hline IFV & BH & $\bar{B} W$ & $\mathrm{BL}$ & XADJ & YADJ & IFV & $\mathrm{BH}$ & BW & $\mathrm{BL}$ & XADJ & YADJ \\
\hline 1 & 12.2, & 48.0, & 65.5, & 0.5 & 2.4 & 2 & 12.2 & 40.2, & 65.9, & 0.1 & 8.6 \\
\hline 3 & 12.2, & 31.2, & 64.4 & -0.3, & 14.5 & 4 & 12.2, & 28.7, & 60.8, & -0.7 & 16.2, \\
\hline 5 & 12.2, & 35.8, & 62.1 & -5.2, & 19.8, & 6 & 12.2, & 44.9, & 63.0, & -10.4 & 24.1, \\
\hline 7 & 12.2, & 52.6 & 62.0 & -15.2, & 27.7 & 8 & 12.2, & 58.7, & 59.1, & -19.6 & 30.5, \\
\hline 9 & 12.2, & 63.1, & 54.4, & -23.3 & 32.3 & 10 & 12.2 , & 65.5, & 48.0, & -26.4 & 33.2, \\
\hline 11 & 12.2, & 65.9 & 40.2 , & -28.7 & 33.0, & 12 & 12.2, & 64.4, & 31.2, & -30.1 & 31.9, \\
\hline 13 & 12.2, & 60.8 & 28.7 & -30.5 & 29.8 & 14 & 12.2 & 62.1, & 35.8, & -37.7 & 25.8, \\
\hline 15 & 12.2, & 63.0, & 44.9, & -46.6 & 21.1, & 16 & 12.2 & 62.0 & 52.6 & -54.0 & 15.8, \\
\hline 17 & 12.2, & 59.1, & 58.7, & -59.9 & 10.0, & 18 & 12.2, & 54.4 & 63.1, & -63.9 & 3.8 \\
\hline 19 & 12.2, & 48.0, & 65.5, & -66.0, & -2.4 & 20 & 12.2, & 40.2, & 65.9, & -66.0, & -8.6 \\
\hline 21 & 12.2, & 31.2, & 64.4, & -64.1, & -14.5 & 22 & 12.2, & 28.7, & 60.8, & -60.2, & -16.2 , \\
\hline 23 & 12.2, & 35.8, & 62.1 & -56.9, & -19.8 & 24 & 12.2 & 44.9, & 63.0, & -52.6 & -24.1 , \\
\hline 25 & 12.2, & 52.6 & 62.0 & -46.8 & -27.7 & 26 & 12.2 & 58.7, & 59.1, & -39.5 & -30.5 \\
\hline 27 & 12.2, & 63.1 & 54.4, & -31.0 & -32.3 & 28 & 12.2 & 65.5, & 48.0, & -21.6 & -33.2 \\
\hline 29 & 12.2, & 65.9 & 40.2, & -11.5 & -33.0 & 30 & 12.2 & 64.4, & 31.2, & -1.1 & -31.9 , \\
\hline 31 & 12.2, & 60.8 & 28.7, & 1.9 & -29.8 & 32 & 12.2, & 62.1, & 35.8, & 1.9, & -25.8 \\
\hline 33 & 12.2, & 63.0 & 44.9, & 1.7, & -21.1, & 34 & 12.2 & 62.0, & 52.6, & 1.4, & -15.8 \\
\hline 35 & 12.2, & 59.1, & 58.7, & 1.1, & -10.0 & 36 & 12.2, & 54.4, & 63.1, & 0.8, & -3.8 \\
\hline SOURCE & ID: 2 & $224 \mathrm{UA} \_6$ & & & & & & & & & \\
\hline IFV & BH & $\overline{\mathrm{B}} \mathrm{W}$ & $\mathrm{BL}$ & XADJ & YADJ & IFV & $\mathrm{BH}$ & BW & $\mathrm{BL}$ & XADJ & YADJ \\
\hline 1 & 12.2, & 48.0 & 65.5, & 0.5 & 2.4 & 2 & 12.2, & 40.2, & 65.9, & 0.1 & 8.6 \\
\hline 3 & 12.2, & 31.2, & 64.4 & -0.3, & 14.5 & 4 & 12.2, & 28.7, & 60.8, & -0.7 & 16.2, \\
\hline 5 & 12.2, & 35.8, & 62.1 & -5.2 & 19.8, & 6 & 12.2 & 44.9, & 63.0, & -10.4 & 24.1, \\
\hline 7 & 12.2, & 52.6 & 62.0 & -15.2, & 27.7 & 8 & 12.2 & 58.7, & 59.1, & -19.6 & 30.5, \\
\hline 9 & 12.2, & 63.1, & 54.4 & -23.3 & 32.3 & 10 & 12.2 & 65.5, & 48.0 & -26.4 & 33.2, \\
\hline 11 & 12.2, & 65.9, & 40.2 , & -28.7 & 33.0, & 12 & 12.2 , & 64.4, & 31.2, & -30.1 & 31.9, \\
\hline 13 & 12.2, & 60.8 & 28.7 & -30.5 & 29.8 & 14 & 12.2 & 62.1, & 35.8, & -37.7 & 25.8, \\
\hline 15 & 12.2, & 63.0 & 44.9 & -46.6 & 21.1 & 16 & 12.2, & 62.0 & 52.6 & -54.0 & 15.8, \\
\hline 17 & 12.2, & 59.1, & 58.7 & -59.9 & 10.0, & 18 & 12.2, & 54.4 & 63.1, & -63.9 & 3.8 \\
\hline 19 & 12.2, & 48.0, & 65.5, & -66.0, & -2.4 & 20 & 12.2 & 40.2, & 65.9, & -66.0 & -8.6 \\
\hline 21 & 12.2, & 31.2, & 64.4, & -64.1, & -14.5 & 22 & 12.2 , & 28.7, & 60.8, & -60.2, & -16.2 , \\
\hline 23 & 12.2, & 35.8, & 62.1 & -56.9, & -19.8 & 24 & 12.2, & 44.9, & 63.0, & -52.6 & -24.1, \\
\hline 25 & 12.2, & 52.6 & 62.0 & -46.8 & -27.7 & 26 & 12.2 & 58.7, & 59.1, & -39.5 & -30.5 \\
\hline 27 & 12.2, & 63.1 & 54.4 & -31.0 & -32.3 & 28 & 12.2 & 65.5, & 48.0 & -21.6 & -33.2 \\
\hline 29 & 12.2, & 65.9, & 40.2, & -11.5, & -33.0 & 30 & 12.2 & 64.4, & 31.2, & -1.1, & -31.9 , \\
\hline 31 & 12.2, & 60.8, & 28.7 & 1.9 & -29.8 & 32 & 12.2, & 62.1, & 35.8, & 1.9, & -25.8 \\
\hline 33 & 12.2, & 63.0, & 44.9 & 1.7, & -21.1, & 34 & 12.2 , & 62.0, & 52.6, & 1.4, & -15.8, \\
\hline 35 & 12.2, & 59.1, & 58.7, & 1.1 & -10.0 & 36 & 12.2 & 54.4, & 63.1, & 0.8, & -3.8 \\
\hline
\end{tabular}

\footnotetext{
*** AERMOD - VERSION $07026 * * * \quad * * *$ U/UA Building Demolition *** Unit Release - Worst Case **MODELOPTS : CONC DDEP TOXICS FLAT 


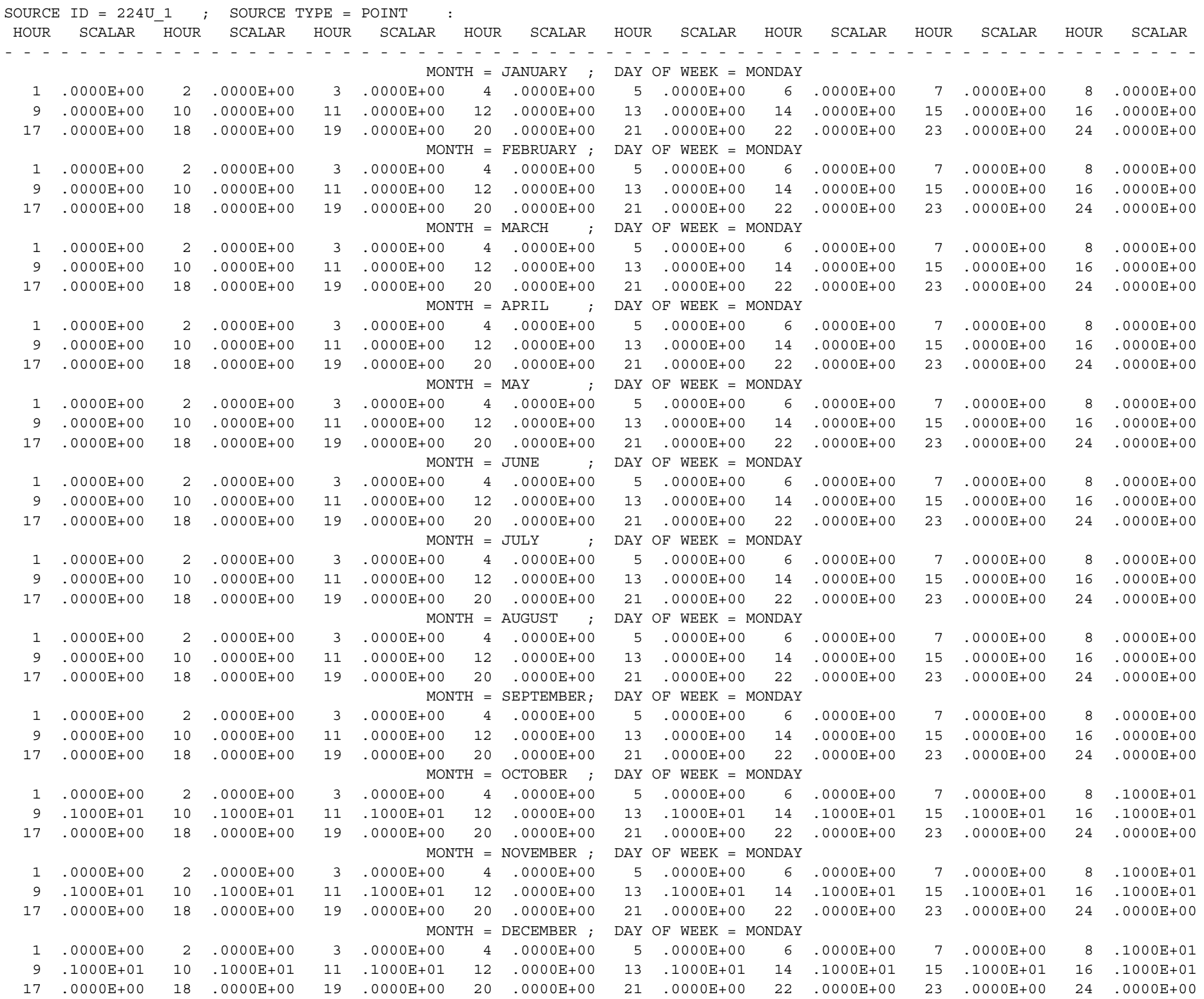




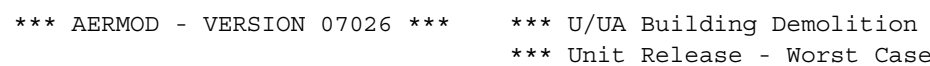
**MODELOPTS :

CONC $\begin{array}{ll}* * * & 02 / 09 / 09 \\ * * * & 10: 41: 11\end{array}$

$10: 41: 11$
PAGE $\quad 14$

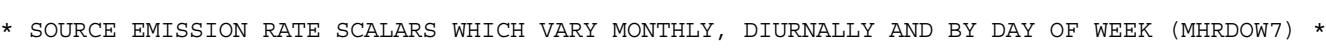

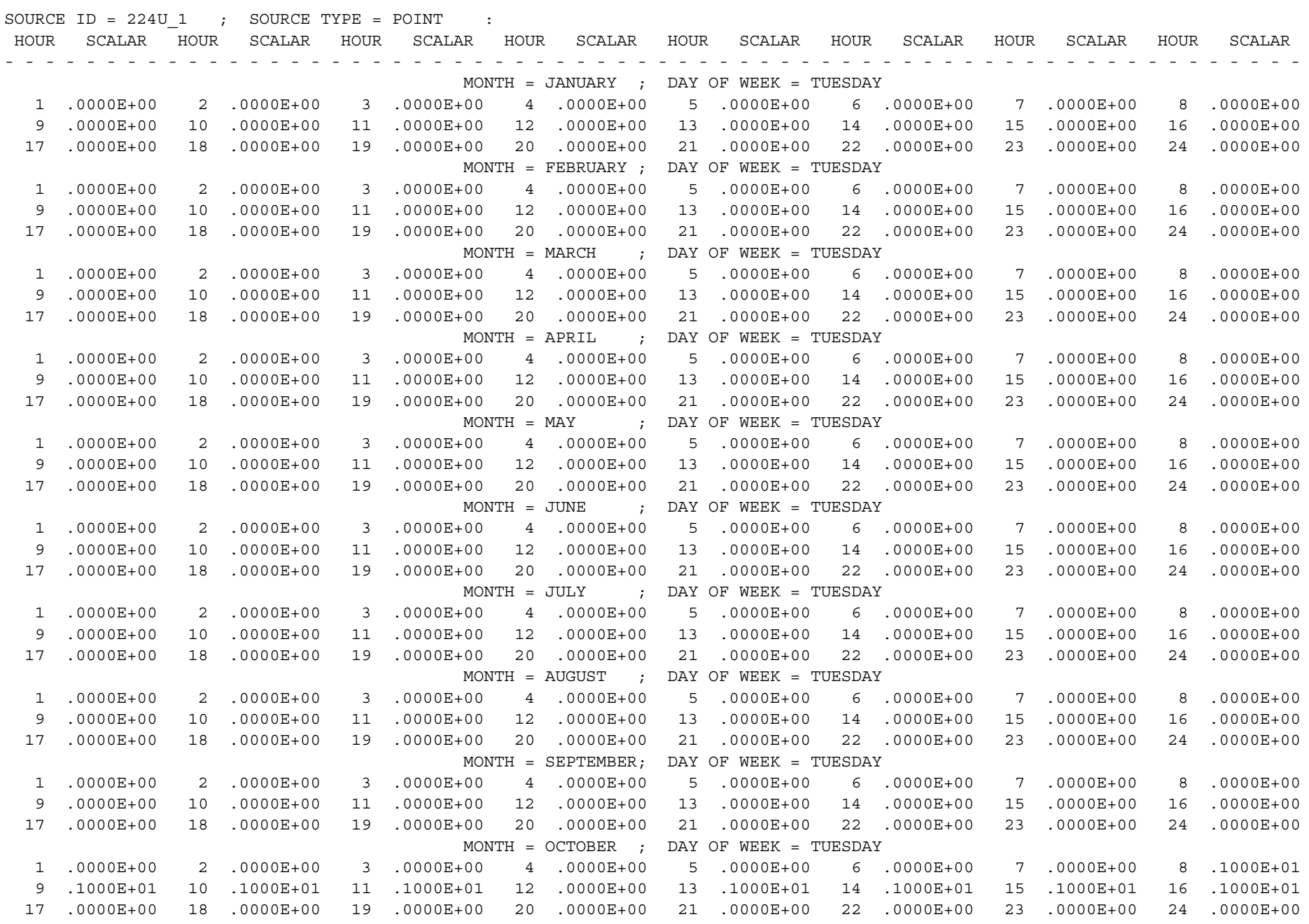

MONTH = NOVEMBER $;$ DAY OF WEEK = TUESDAY 


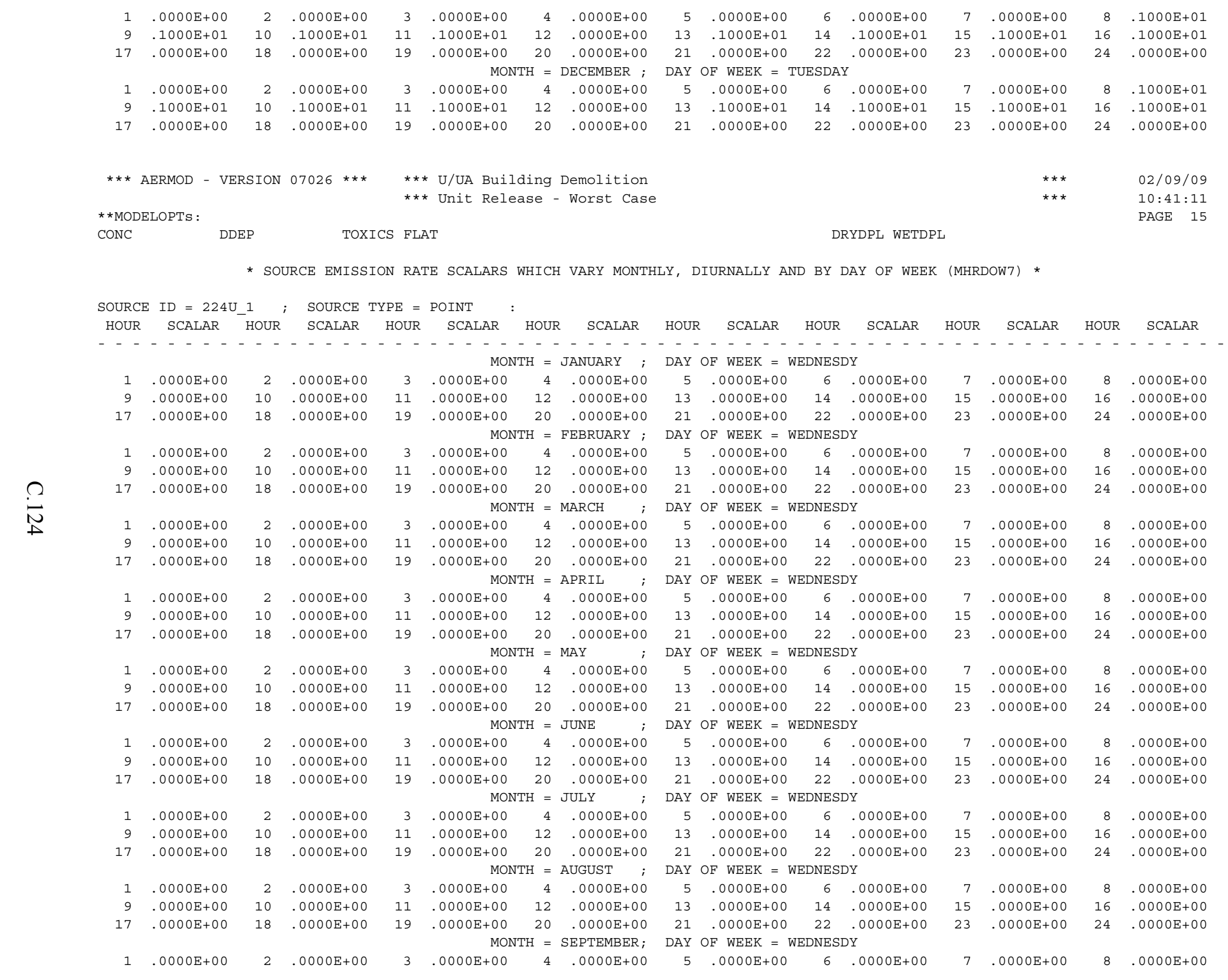




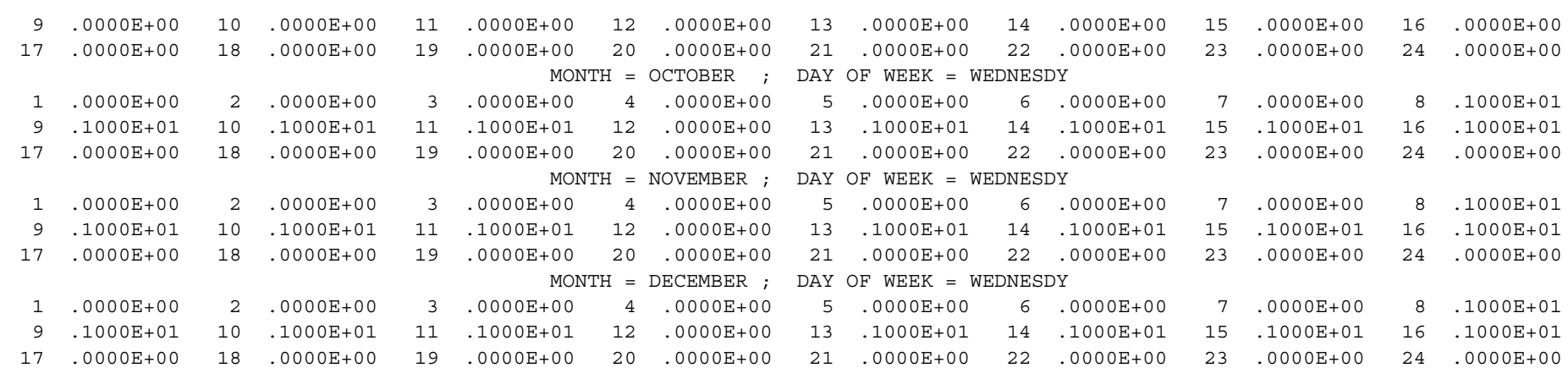

*** AERMOD - VERSION $07026 * * * \quad * * *$ U/UA Building Demolition

*** Unit Release - Worst Case

$\begin{array}{ll}* * * & 02 / 09 / 09 \\ * * * & 10: 41: 11\end{array}$ **MODELOPTS:

CONC

DDEP

TOXICS FLAT

DRYDPL WETDPL

$10: 41: 11$
PAGE 16

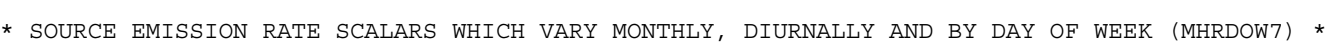

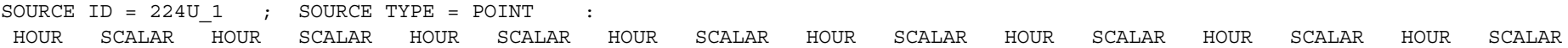

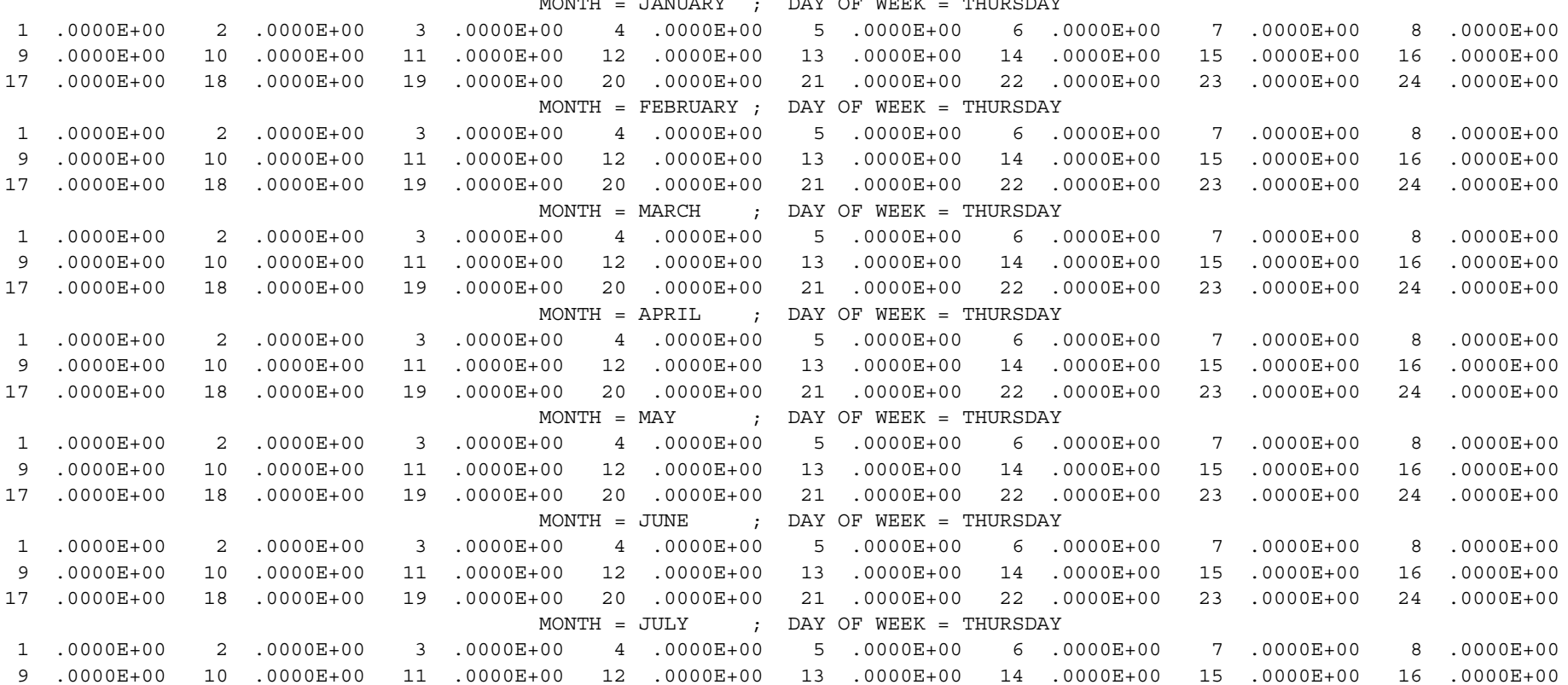




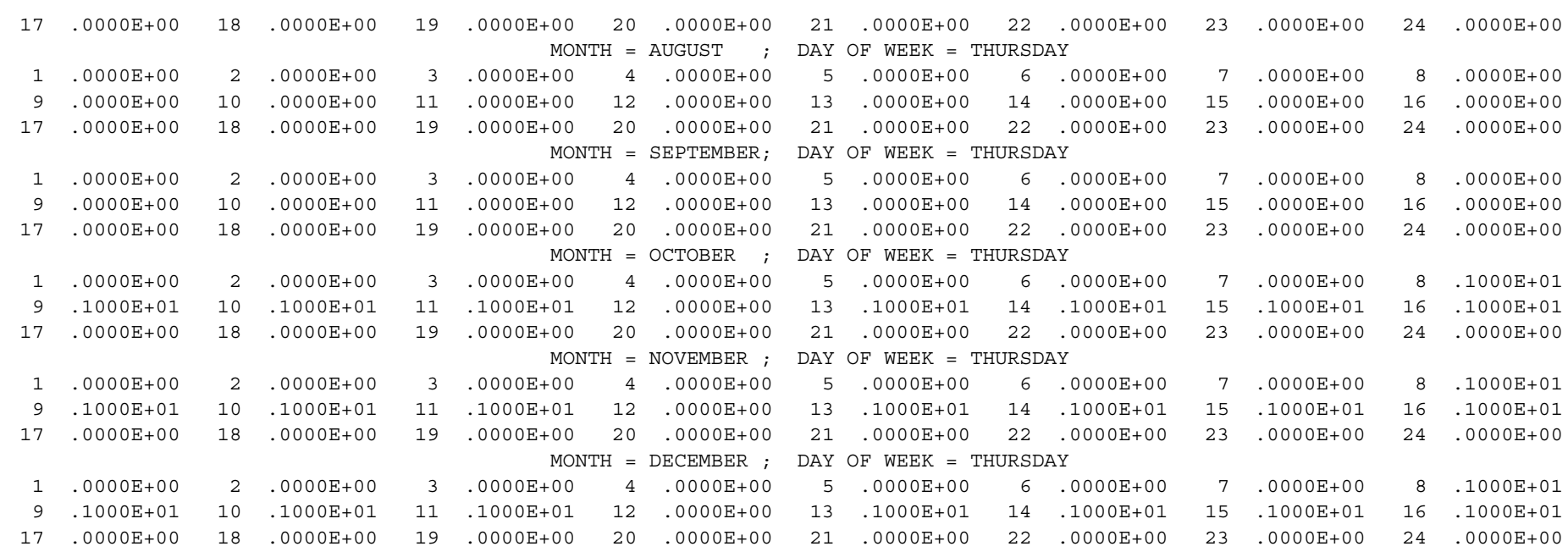

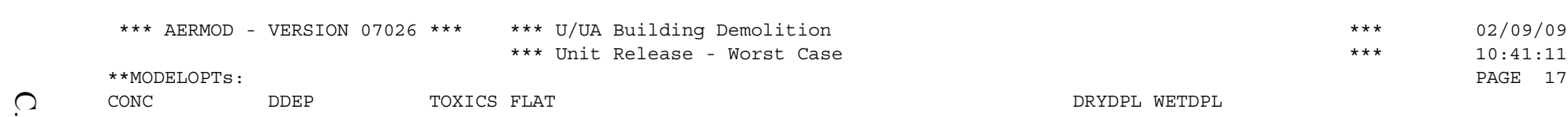

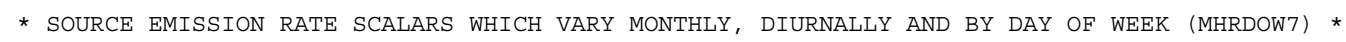

SOURCE ID $=224 \mathrm{U}_{-} 1 ; \quad$ SOURCE TYPE $=$ POINT

\begin{tabular}{|c|c|c|c|c|c|c|c|c|c|c|c|c|c|c|c|}
\hline $\begin{array}{l}\text { HOUR } \\
--\end{array}$ & $\begin{array}{l}\text { SCALAR } \\
----\end{array}$ & $\begin{array}{l}\text { HOUR } \\
-\quad-\quad-\end{array}$ & $\begin{array}{l}\text { SCALAR } \\
-\end{array}$ & $\begin{array}{l}\text { HOUR } \\
-\quad-\quad-\end{array}$ & $\begin{array}{c}\text { SCALAR } \\
---{ }^{-} \\
\text {MON }\end{array}$ & $\begin{array}{l}\text { HOUR } \\
---- \\
\mathrm{TH}=\end{array}$ & $\begin{array}{c}\text { SCALAR } \\
---\quad- \\
\text { JANUARY }\end{array}$ & $\begin{array}{c}\text { HOUR } \\
--- \\
\text { DAY }\end{array}$ & $\begin{array}{c}\text { SCALAR } \\
----1 \\
\text { OF } \text { WEEK = }\end{array}$ & $\begin{array}{r}\text { HOUR } \\
-\quad-- \\
\text { ERIDAY }\end{array}$ & $\begin{array}{l}\text { SCALAR } \\
-----\end{array}$ & $\begin{array}{l}\text { HOUR } \\
-\quad-\end{array}$ & $\begin{array}{l}\text { SCALAR } \\
-\end{array}$ & $\begin{array}{l}\text { HOUR } \\
--\end{array}$ & $\begin{array}{l}\text { SCALAR } \\
-\quad-\quad-\end{array}$ \\
\hline 1 & $.0000 \mathrm{E}+00$ & 2 & $.0000 \mathrm{E}+00$ & 3 & $.0000 \mathrm{E}+00$ & 4 & $.0000 \mathrm{E}+00$ & 5 & $.0000 \mathrm{E}+00$ & 6 & $.0000 \mathrm{E}+00$ & 7 & $.0000 \mathrm{E}+00$ & 8 & $.0000 \mathrm{E}+00$ \\
\hline 9 & $.0000 \mathrm{E}+00$ & 10 & $.0000 \mathrm{E}+00$ & 11 & $.0000 \mathrm{E}+00$ & 12 & $.0000 \mathrm{E}+00$ & 13 & $.0000 \mathrm{E}+00$ & 14 & $.0000 \mathrm{E}+00$ & 15 & $.0000 \mathrm{E}+00$ & 16 & $.0000 \mathrm{E}+00$ \\
\hline 1 & $.0000 \mathrm{E}+00$ & 2 & $.0000 \mathrm{E}+00$ & 3 & $.0000 \mathrm{E}+00$ & 4 & $.0000 \mathrm{E}+00$ & 5 & $.0000 \mathrm{E}+00$ & 6 & $.0000 \mathrm{E}+00$ & 7 & $.0000 \mathrm{E}+00$ & 8 & $.0000 \mathrm{E}+00$ \\
\hline 9 & $.0000 \mathrm{E}+00$ & 10 & $.0000 \mathrm{E}+00$ & 11 & $.0000 \mathrm{E}+00$ & 12 & $.0000 \mathrm{E}+00$ & 13 & $.0000 \mathrm{E}+00$ & 14 & $.0000 \mathrm{E}+00$ & 15 & $.0000 \mathrm{E}+00$ & 16 & $.0000 \mathrm{E}+00$ \\
\hline 17 & $.0000 \mathrm{E}+00$ & 18 & $.0000 \mathrm{E}+00$ & 19 & $.0000 \mathrm{E}+00$ & 20 & $.0000 \mathrm{E}+00$ & 21 & $.0000 \mathrm{E}+00$ & 22 & $.0000 \mathrm{E}+00$ & 23 & $.0000 \mathrm{E}+00$ & 24 & $.0000 \mathrm{E}+00$ \\
\hline 17 & $.0000 \mathrm{E}+00$ & 18 & $.0000 \mathrm{E}+00$ & 19 & $.0000 \mathrm{E}+00$ & 20 & $.0000 \mathrm{E}+00$ & 21 & $.0000 \mathrm{E}+00$ & 22 & $.0000 \mathrm{E}+00$ & 23 & $.0000 \mathrm{E}+00$ & 24 & $.0000 \mathrm{E}+00$ \\
\hline & & & & & MON & $\mathrm{TH}=$ & APRIL ; & DAY & OF WEEK = & FRIDAY & & & & & \\
\hline 1 & $.0000 \mathrm{E}+00$ & 2 & $.0000 \mathrm{E}+00$ & 3 & $.0000 \mathrm{E}+00$ & 4 & $.0000 \mathrm{E}+00$ & 5 & $.0000 \mathrm{E}+00$ & 6 & $.0000 \mathrm{E}+00$ & 7 & $.0000 \mathrm{E}+00$ & 8 & $.0000 \mathrm{E}+00$ \\
\hline 9 & $.0000 \mathrm{E}+00$ & 10 & $.0000 \mathrm{E}+00$ & 11 & $.0000 \mathrm{E}+00$ & 12 & $.0000 \mathrm{E}+00$ & 13 & .000 & 14 & .00 & 15 & .00 & 16 & .0000 \\
\hline 17 & $.0000 \mathrm{E}+00$ & 18 & $.0000 \mathrm{E}+00$ & 19 & $.0000 \mathrm{E}+00$ & 20 & $.0000 \mathrm{E}+00$ & 21 & $.0000 \mathrm{E}+00$ & 22 & $.0000 \mathrm{E}+00$ & 23 & $.0000 \mathrm{E}+00$ & 24 & $.0000 \mathrm{E}+00$ \\
\hline & & & & & MON & $\mathrm{TH}=$ & MAY ; & DAY & OF WEEK = & FRIDAY & & & & & \\
\hline 1 & $.0000 \mathrm{E}+00$ & 2 & $.0000 \mathrm{E}+00$ & 3 & $0000 \mathrm{E}+00$ & 4 & $.0000 \mathrm{E}+00$ & 5 & $.0000 \mathrm{E}+00$ & 6 & $.0000 \mathrm{E}+00$ & 7 & & & $.0000 \mathrm{E}+00$ \\
\hline
\end{tabular}




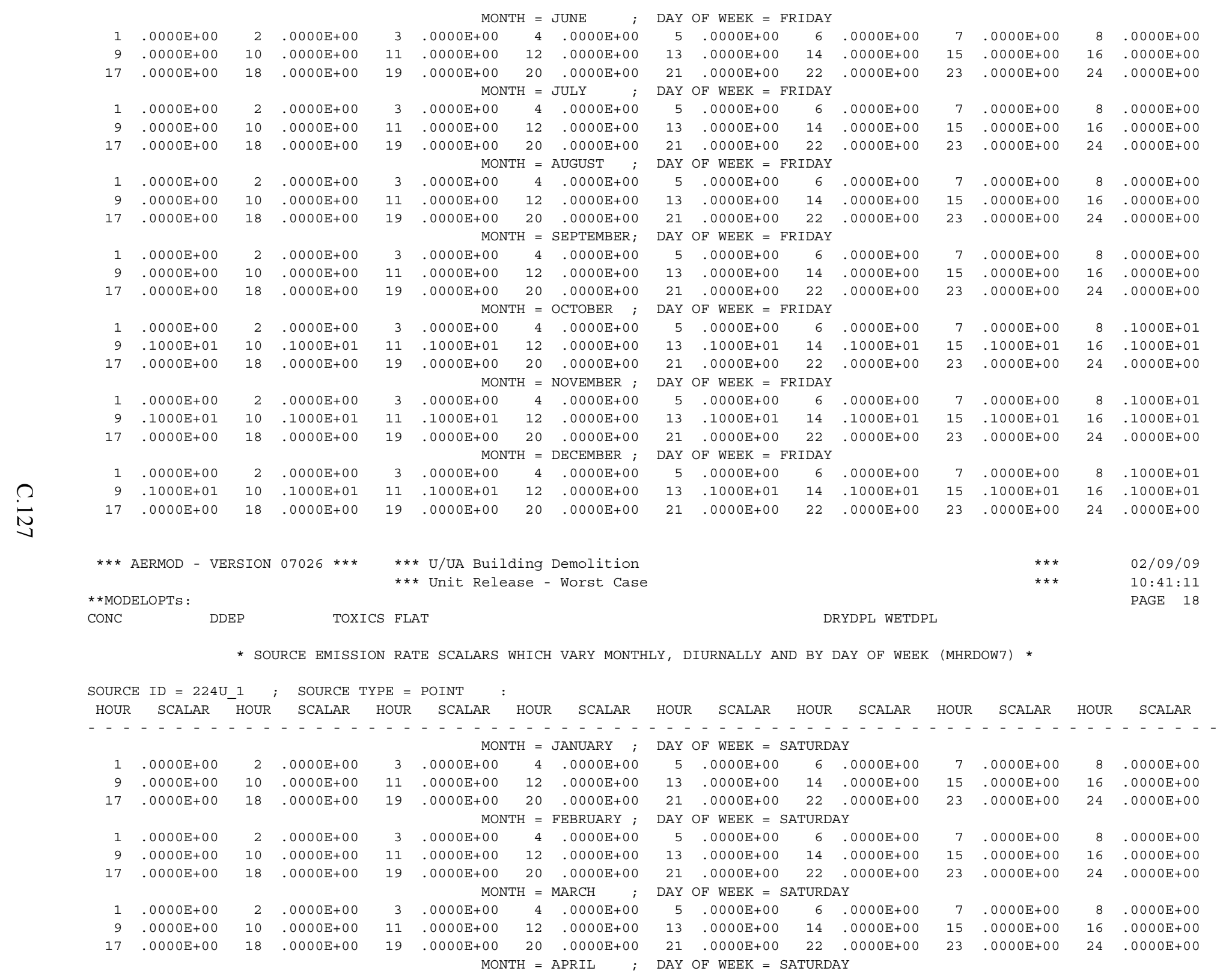




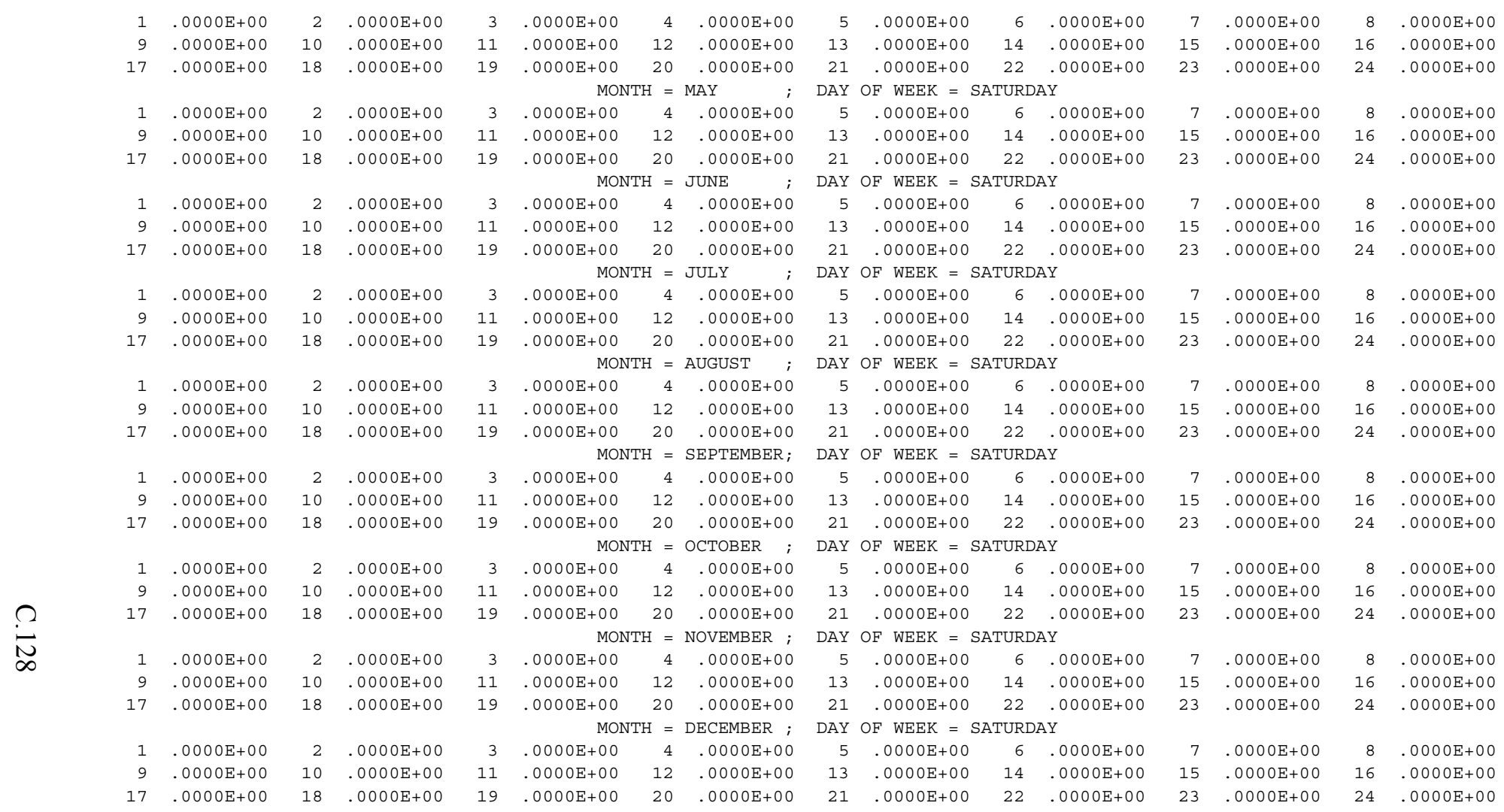

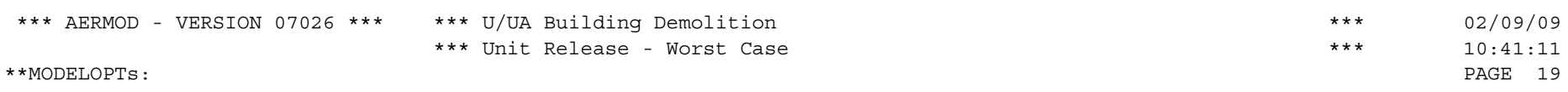

DDEP TOXICS FLAT

DRYDPL WETDPI

* SOURCE EMISSION RATE SCALARS WHICH VARY MONTHLY, DIURNALLY AND BY DAY OF WEEK (MHRDOW7) *

SOURCE ID $=224 \mathrm{U} 1 ;$ SOURCE TYPE $=$ POINT

HOUR SCALAR HOUR' SCALAR HOUR SCALAR HOUR SCALAR HOUR SCALAR HOUR SCALAR HOUR SCALAR HOUR SCALAR

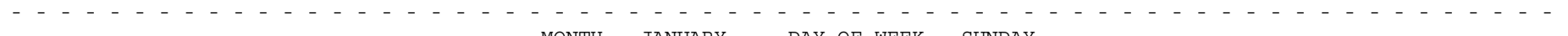

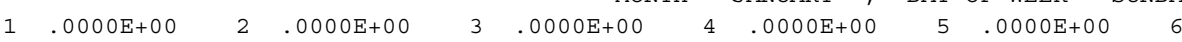

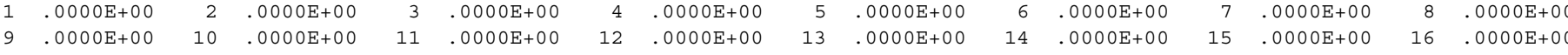

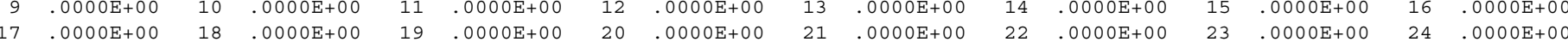

$1.0000+00$ MONTH = FEBRUARY $; \quad$ DAY OF WEEK = SUNDAY 


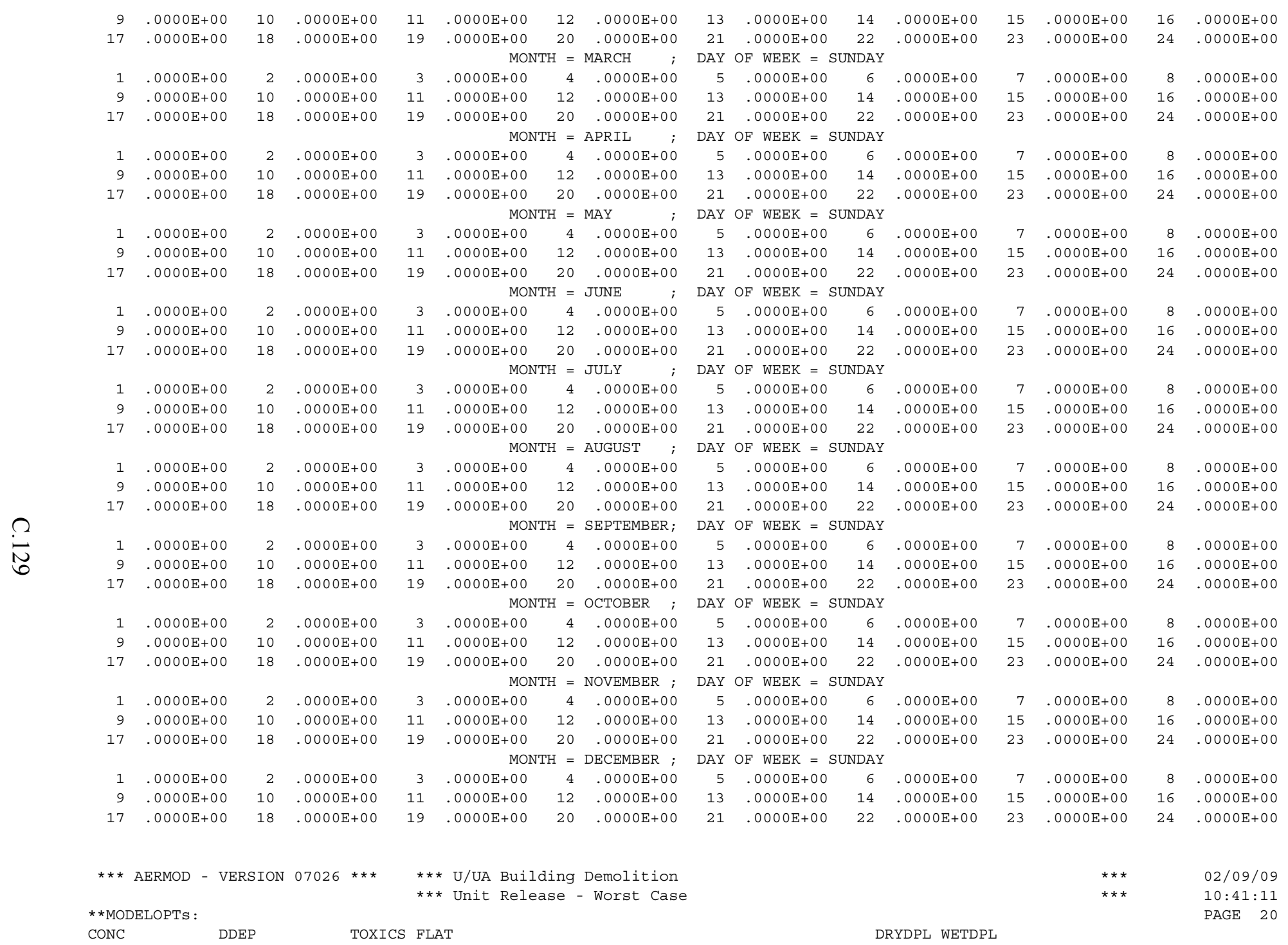

* source emission rate scalars which vary monthly, diURnally and by day of Week (MHrdow7) *

SOURCE ID $=224 \mathrm{U}_{-} 2 ;$ SOURCE TYPE $=$ POINT

HOUR SCALAR HOUR SCALAR HOUR SCALAR HOUR SCALAR HOUR SCALAR HOUR SCALAR HOUR SCALAR HOUR SCALAR 


\begin{tabular}{|c|c|c|c|c|c|c|c|c|c|c|c|c|c|c|c|}
\hline & & & & & & & JANUARY & & WEEK $=M$ & MONDAY & & & & & \\
\hline 1 & $.0000 \mathrm{E}+00$ & 2 & $.0000 \mathrm{E}+00$ & 3 & $.0000 \mathrm{E}+00$ & 4 & $.0000 \mathrm{E}+00$ & 5 & $.0000 \mathrm{E}+00$ & 6 & $.0000 \mathrm{E}+00$ & 7 & $.0000 \mathrm{E}+00$ & 8 & $.0000 \mathrm{E}+00$ \\
\hline 9 & $.0000 E+00$ & 10 & $.0000 E+00$ & 11 & $.0000 \mathrm{E}+00$ & 12 & $.0000 \mathrm{E}+00$ & 13 & $.0000 \mathrm{E}+00$ & 14 & $.0000 \mathrm{E}+00$ & 15 & $.0000 \mathrm{E}+00$ & 16 & $.0000 \mathrm{E}+00$ \\
\hline 17 & $.0000 \mathrm{E}+00$ & 18 & $.0000 \mathrm{E}+00$ & 19 & $.0000 \mathrm{E}+00$ & 20 & $.0000 \mathrm{E}+00$ & 21 & $.0000 \mathrm{E}+00$ & 22 & $.0000 \mathrm{E}+00$ & 23 & $.0000 \mathrm{E}+00$ & 24 & $.0000 \mathrm{E}+00$ \\
\hline & & & & & MON? & $\mathrm{H}=$ & FEBRUARY ; & DAY & OF WEEK $=M$ & MONDAY & & & & & \\
\hline 1 & $.0000 E+00$ & 2 & $.0000 \mathrm{E}+00$ & 3 & $.0000 \mathrm{E}+00$ & 4 & $.0000 \mathrm{E}+00$ & 5 & $.0000 \mathrm{E}+00$ & 6 & $.0000 \mathrm{E}+00$ & 7 & $.0000 \mathrm{E}+00$ & 8 & $.0000 \mathrm{E}+00$ \\
\hline 9 & $.0000 \mathrm{E}+00$ & 10 & $.0000 \mathrm{E}+00$ & 11 & $.0000 \mathrm{E}+00$ & 12 & $.0000 \mathrm{E}+00$ & 13 & $.0000 \mathrm{E}+00$ & 14 & $.0000 \mathrm{E}+00$ & 15 & $.0000 \mathrm{E}+00$ & 16 & $.0000 \mathrm{E}+00$ \\
\hline 17 & $.0000 \mathrm{E}+00$ & 18 & $.0000 \mathrm{E}+00$ & 19 & $.0000 \mathrm{E}+00$ & 20 & $.0000 \mathrm{E}+00$ & 21 & $.0000 \mathrm{E}+00$ & 22 & $.0000 \mathrm{E}+00$ & 23 & $.0000 \mathrm{E}+00$ & 24 & $.0000 \mathrm{E}+00$ \\
\hline & & & & & MONT & $\mathrm{H}=$ & MARCH ; & DAY & OF WEEK = M & MONDAY & & & & & \\
\hline 1 & $.0000 \mathrm{E}+00$ & 2 & $.0000 \mathrm{E}+00$ & 3 & $.0000 \mathrm{E}+00$ & 4 & $.0000 \mathrm{E}+00$ & 5 & $.0000 \mathrm{E}+00$ & 6 & $.0000 \mathrm{E}+00$ & 7 & $.0000 \mathrm{E}+00$ & 8 & $.0000 \mathrm{E}+00$ \\
\hline 9 & $.0000 \mathrm{E}+00$ & 10 & $.0000 \mathrm{E}+00$ & 11 & $.0000 \mathrm{E}+00$ & 12 & $.0000 \mathrm{E}+00$ & 13 & $.0000 \mathrm{E}+00$ & 14 & $.0000 \mathrm{E}+00$ & 15 & $.0000 \mathrm{E}+00$ & 16 & $.0000 \mathrm{E}+00$ \\
\hline 17 & $.0000 \mathrm{E}+00$ & 18 & $.0000 \mathrm{E}+00$ & 19 & $.0000 \mathrm{E}+00$ & 20 & $.0000 \mathrm{E}+00$ & 21 & $.0000 \mathrm{E}+00$ & 22 & $.0000 \mathrm{E}+00$ & 23 & $.0000 \mathrm{E}+00$ & 24 & $.0000 \mathrm{E}+00$ \\
\hline & & & & & MON & $\mathrm{H}=$ & APRIL ; & DAY & OF WEEK = M & MONDAY & & & & & \\
\hline 1 & $.0000 \mathrm{E}+00$ & 2 & $.0000 \mathrm{E}+00$ & 3 & $.0000 \mathrm{E}+00$ & 4 & $.0000 \mathrm{E}+00$ & 5 & $.0000 \mathrm{E}+00$ & 6 & $.0000 \mathrm{E}+00$ & 7 & $.0000 \mathrm{E}+00$ & 8 & $.0000 \mathrm{E}+00$ \\
\hline 9 & $.0000 \mathrm{E}+00$ & 10 & $.0000 \mathrm{E}+00$ & 11 & $.0000 \mathrm{E}+00$ & 12 & $.0000 \mathrm{E}+00$ & 13 & $.0000 \mathrm{E}+00$ & 14 & $.0000 \mathrm{E}+00$ & 15 & $.0000 \mathrm{E}+00$ & 16 & $.0000 \mathrm{E}+00$ \\
\hline 17 & $.0000 \mathrm{E}+00$ & 18 & $.0000 \mathrm{E}+00$ & 19 & $.0000 \mathrm{E}+00$ & 20 & $.0000 \mathrm{E}+00$ & 21 & $.0000 \mathrm{E}+00$ & 22 & $.0000 \mathrm{E}+00$ & 23 & $.0000 \mathrm{E}+00$ & 24 & $.0000 \mathrm{E}+00$ \\
\hline & & & & & MON & $\mathrm{H}=$ & MAY ; & DAY & OF WEEK = M & MONDAY & & & & & \\
\hline 1 & $.0000 \mathrm{E}+00$ & 2 & $.0000 \mathrm{E}+00$ & 3 & $.0000 \mathrm{E}+00$ & 4 & $.0000 \mathrm{E}+00$ & 5 & $.0000 \mathrm{E}+00$ & 6 & $.0000 \mathrm{E}+00$ & 7 & $.0000 \mathrm{E}+00$ & 8 & $.0000 \mathrm{E}+00$ \\
\hline 9 & $.0000 \mathrm{E}+00$ & 10 & $.0000 \mathrm{E}+00$ & 11 & $.0000 \mathrm{E}+00$ & 12 & $.0000 \mathrm{E}+00$ & 13 & $.0000 \mathrm{E}+00$ & 14 & $.0000 \mathrm{E}+00$ & 15 & $.0000 \mathrm{E}+00$ & 16 & $.0000 \mathrm{E}+00$ \\
\hline 17 & $.0000 \mathrm{E}+00$ & 18 & $.0000 \mathrm{E}+00$ & 19 & $.0000 \mathrm{E}+00$ & 20 & $.0000 \mathrm{E}+00$ & 21 & $.0000 \mathrm{E}+00$ & 22 & $.0000 \mathrm{E}+00$ & 23 & $.0000 \mathrm{E}+00$ & 24 & $.0000 \mathrm{E}+00$ \\
\hline & & & & & MON & $\mathrm{H}=$ & JUNE $\quad ;$ & DAY & OF WEEK $=M$ & MONDAY & & & & & \\
\hline 1 & $.0000 \mathrm{E}+00$ & 2 & $.0000 \mathrm{E}+00$ & 3 & $.0000 \mathrm{E}+00$ & 4 & $.0000 \mathrm{E}+00$ & 5 & $.0000 \mathrm{E}+00$ & 6 & $.0000 \mathrm{E}+00$ & 7 & $.0000 \mathrm{E}+00$ & 8 & $.0000 \mathrm{E}+00$ \\
\hline 9 & $.0000 \mathrm{E}+00$ & 10 & $.0000 \mathrm{E}+00$ & 11 & $.0000 E+00$ & 12 & $.0000 \mathrm{E}+00$ & 13 & $.0000 \mathrm{E}+00$ & 14 & $.0000 \mathrm{E}+00$ & 15 & $.0000 \mathrm{E}+00$ & 16 & $.0000 \mathrm{E}+00$ \\
\hline 17 & $.0000 \mathrm{E}+00$ & 18 & $.0000 \mathrm{E}+00$ & 19 & $.0000 \mathrm{E}+00$ & 20 & $.0000 \mathrm{E}+00$ & 21 & $.0000 \mathrm{E}+00$ & 22 & $.0000 \mathrm{E}+00$ & 23 & $.0000 \mathrm{E}+00$ & 24 & $.0000 \mathrm{E}+00$ \\
\hline & & & & & MON & $\mathrm{H}=$ & JULY ; & DAY & OF WEEK = M & MONDAY & & & & & \\
\hline 1 & $.0000 \mathrm{E}+00$ & 2 & $.0000 \mathrm{E}+00$ & 3 & $.0000 \mathrm{E}+00$ & 4 & $.0000 \mathrm{E}+00$ & 5 & $.0000 \mathrm{E}+00$ & 6 & $.0000 \mathrm{E}+00$ & 7 & $.0000 \mathrm{E}+00$ & 8 & $.0000 \mathrm{E}+00$ \\
\hline 9 & $.0000 \mathrm{E}+00$ & 10 & $.0000 \mathrm{E}+00$ & 11 & $.0000 \mathrm{E}+00$ & 12 & $.0000 \mathrm{E}+00$ & 13 & $.0000 \mathrm{E}+00$ & 14 & $.0000 \mathrm{E}+00$ & 15 & $.0000 \mathrm{E}+00$ & 16 & $.0000 \mathrm{E}+00$ \\
\hline 17 & $.0000 \mathrm{E}+00$ & 18 & $.0000 \mathrm{E}+00$ & 19 & $.0000 \mathrm{E}+00$ & 20 & $.0000 \mathrm{E}+00$ & 21 & $.0000 \mathrm{E}+00$ & 22 & $.0000 \mathrm{E}+00$ & 23 & $.0000 \mathrm{E}+00$ & 24 & $.0000 \mathrm{E}+00$ \\
\hline & & & & & MON & $\mathrm{H}=$ & AUGUST ; & DAY & OF WEEK $=M$ & MONDAY & & & & & \\
\hline 1 & $.0000 \mathrm{E}+00$ & 2 & $.0000 \mathrm{E}+00$ & 3 & $.0000 \mathrm{E}+00$ & 4 & $.0000 \mathrm{E}+00$ & 5 & $.0000 \mathrm{E}+00$ & 6 & $.0000 \mathrm{E}+00$ & 7 & $.0000 \mathrm{E}+00$ & 8 & $.0000 \mathrm{E}+00$ \\
\hline 9 & $.0000 \mathrm{E}+00$ & 10 & $.0000 \mathrm{E}+00$ & 11 & $.0000 \mathrm{E}+00$ & 12 & $.0000 \mathrm{E}+00$ & 13 & $.0000 \mathrm{E}+00$ & 14 & $.0000 \mathrm{E}+00$ & 15 & $.0000 \mathrm{E}+00$ & 16 & $.0000 \mathrm{E}+00$ \\
\hline 17 & $.0000 \mathrm{E}+00$ & 18 & $.0000 \mathrm{E}+00$ & 19 & $.0000 \mathrm{E}+00$ & 20 & $.0000 \mathrm{E}+00$ & 21 & $.0000 \mathrm{E}+00$ & 22 & $.0000 \mathrm{E}+00$ & 23 & $.0000 \mathrm{E}+00$ & 24 & $.0000 \mathrm{E}+00$ \\
\hline & & & & & MON & $\mathrm{H}=$ & SEPTEMBER ; & DAY & OF WEEK = M & MONDAY & & & & & \\
\hline 1 & $.0000 \mathrm{E}+00$ & 2 & $.0000 \mathrm{E}+00$ & 3 & $.0000 \mathrm{E}+00$ & 4 & $.0000 \mathrm{E}+00$ & 5 & $.0000 \mathrm{E}+00$ & 6 & $.0000 \mathrm{E}+00$ & 7 & $.0000 \mathrm{E}+00$ & 8 & $.0000 \mathrm{E}+00$ \\
\hline 9 & $.0000 \mathrm{E}+00$ & 10 & $.0000 \mathrm{E}+00$ & 11 & $.0000 \mathrm{E}+00$ & 12 & $.0000 \mathrm{E}+00$ & 13 & $.0000 \mathrm{E}+00$ & 14 & $.0000 \mathrm{E}+00$ & 15 & $.0000 \mathrm{E}+00$ & 16 & $.0000 \mathrm{E}+00$ \\
\hline 17 & $.0000 \mathrm{E}+00$ & 18 & $.0000 \mathrm{E}+00$ & 19 & $.0000 \mathrm{E}+00$ & 20 & $.0000 \mathrm{E}+00$ & 21 & $.0000 \mathrm{E}+00$ & 22 & $.0000 \mathrm{E}+00$ & 23 & $.0000 \mathrm{E}+00$ & 24 & $.0000 \mathrm{E}+00$ \\
\hline & & & & & MON & $\mathrm{H}=$ & OCTOBER ; & DAY & OF WEEK $=M$ & MONDAY & & & & & \\
\hline 1 & $.0000 \mathrm{E}+00$ & 2 & $.0000 \mathrm{E}+00$ & 3 & $.0000 \mathrm{E}+00$ & 4 & $.0000 \mathrm{E}+00$ & 5 & $.0000 \mathrm{E}+00$ & 6 & $.0000 \mathrm{E}+00$ & 7 & $.0000 \mathrm{E}+00$ & 8 & $.1000 \mathrm{E}+01$ \\
\hline 9 & $.1000 \mathrm{E}+01$ & 10 & $.1000 \mathrm{E}+01$ & 11 & $.1000 \mathrm{E}+01$ & 12 & $.0000 \mathrm{E}+00$ & 13 & $.1000 \mathrm{E}+01$ & 14 & $.1000 \mathrm{E}+01$ & 15 & $.1000 \mathrm{E}+01$ & 16 & $.1000 \mathrm{E}+01$ \\
\hline 17 & $.0000 \mathrm{E}+00$ & 18 & $.0000 \mathrm{E}+00$ & 19 & $.0000 \mathrm{E}+00$ & 20 & $.0000 \mathrm{E}+00$ & 21 & $.0000 \mathrm{E}+00$ & 22 & $.0000 \mathrm{E}+00$ & 23 & $.0000 \mathrm{E}+00$ & 24 & $.0000 \mathrm{E}+00$ \\
\hline & & & & & MON & $\mathrm{H}=$ & NOVEMBER ; & DAY & OF WEEK = M & MONDAY & & & & & \\
\hline 1 & $.0000 \mathrm{E}+00$ & 2 & $.0000 \mathrm{E}+00$ & 3 & $.0000 \mathrm{E}+00$ & 4 & $.0000 \mathrm{E}+00$ & 5 & $.0000 \mathrm{E}+00$ & 6 & $.0000 \mathrm{E}+00$ & 7 & $.0000 \mathrm{E}+00$ & 8 & $.1000 \mathrm{E}+01$ \\
\hline 9 & $.1000 \mathrm{E}+01$ & 10 & $.1000 \mathrm{E}+01$ & 11 & $.1000 \mathrm{E}+01$ & 12 & $.0000 \mathrm{E}+00$ & 13 & $.1000 \mathrm{E}+01$ & 14 & $.1000 \mathrm{E}+01$ & 15 & $.1000 \mathrm{E}+01$ & 16 & $.1000 \mathrm{E}+01$ \\
\hline 17 & $.0000 \mathrm{E}+00$ & 18 & $.0000 \mathrm{E}+00$ & 19 & $.0000 \mathrm{E}+00$ & 20 & $.0000 \mathrm{E}+00$ & 21 & $.0000 \mathrm{E}+00$ & 22 & $.0000 \mathrm{E}+00$ & 23 & $.0000 \mathrm{E}+00$ & 24 & $.0000 \mathrm{E}+00$ \\
\hline & & & & & MON & $\mathrm{H}=$ & DECEMBER ; & DAY & OF WEEK = M & MONDAY & & & & & \\
\hline 1 & $.0000 \mathrm{E}+00$ & 2 & $.0000 \mathrm{E}+00$ & 3 & $.0000 \mathrm{E}+00$ & 4 & $.0000 \mathrm{E}+00$ & 5 & $.0000 \mathrm{E}+00$ & 6 & $.0000 \mathrm{E}+00$ & 7 & $.0000 \mathrm{E}+00$ & 8 & $.1000 \mathrm{E}+01$ \\
\hline 9 & $.1000 \mathrm{E}+01$ & 10 & $.1000 \mathrm{E}+01$ & 11 & $.1000 \mathrm{E}+01$ & 12 & $.0000 \mathrm{E}+00$ & 13 & $.1000 \mathrm{E}+01$ & 14 & $.1000 \mathrm{E}+01$ & 15 & $.1000 \mathrm{E}+01$ & 16 & $.1000 \mathrm{E}+01$ \\
\hline 17 & $.0000 \mathrm{E}+00$ & 18 & $.0000 \mathrm{E}+00$ & 19 & $.0000 \mathrm{E}+00$ & 20 & $.0000 \mathrm{E}+00$ & 21 & $.0000 \mathrm{E}+00$ & 22 & $.0000 \mathrm{E}+00$ & 23 & $.0000 \mathrm{E}+00$ & 24 & $.0000 \mathrm{E}+00$ \\
\hline
\end{tabular}

*** AERMOD - VERSION $07026 * * * \quad * * *$ U/UA Building Demolition

*** Unit Release - Worst Case 
* SOURCE EMISSION RATE SCALARS WHICH VARY MONTHLY, DIURNALLY AND BY DAY OF WEEK (MHRDOW7) *

SOURCE ID $=224 \mathrm{U} 2 ;$ SOURCE TYPE $=$ POINT

HOUR SCALAR - HOUR' SCAIAR HOUR SCALAR' HOUR SCALAR HOUR SCALAR HOUR SCALAR HOUR SCALAR HOUR SCALAR HOUR

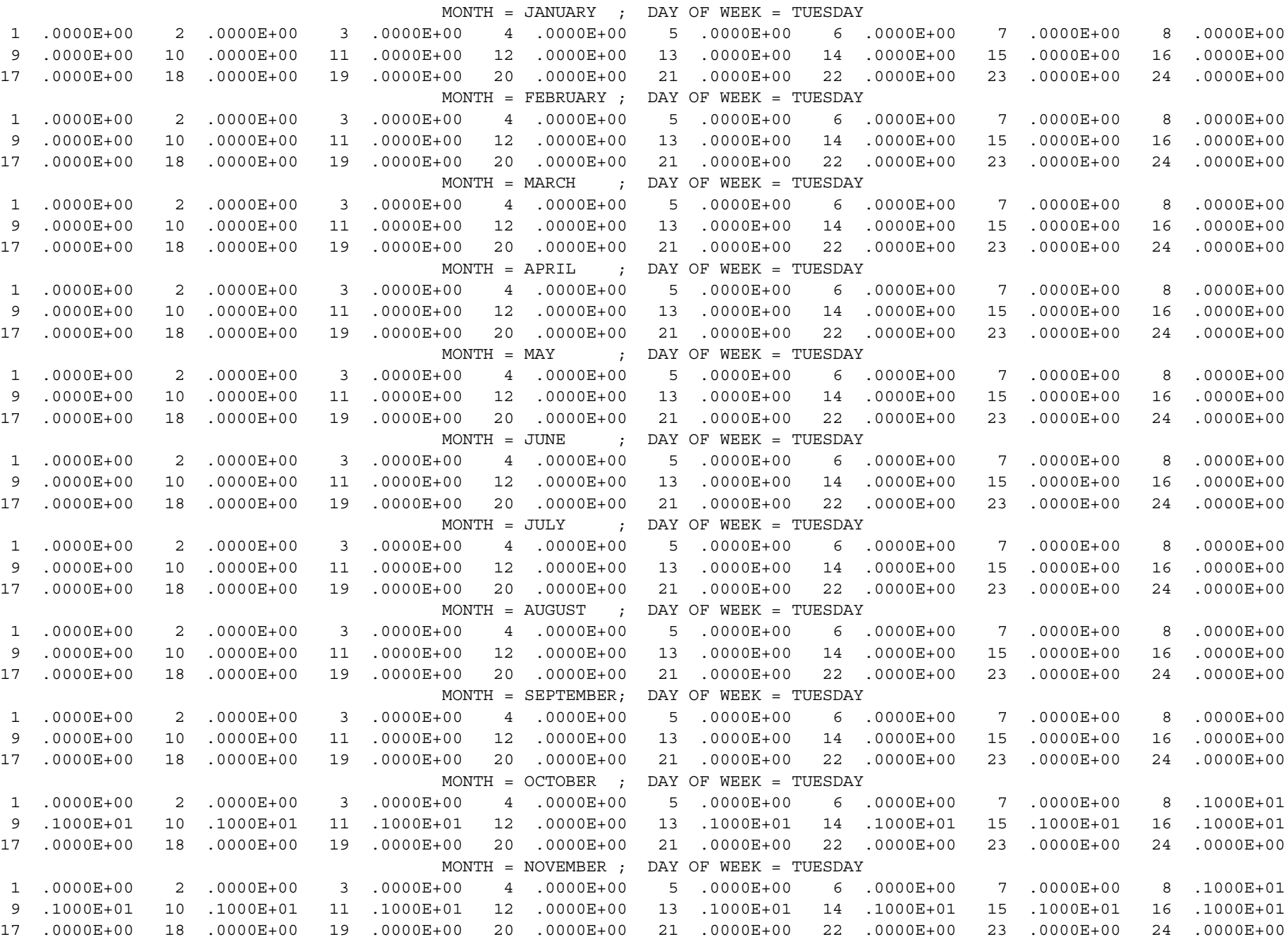
MONTH $=$ DECEMBER ; DAY OF WEEK = TUESDAY 


\begin{tabular}{|c|c|c|c|c|c|c|c|c|c|c|c|c|c|c|c|}
\hline 1 & $\mathrm{E}+00$ & 2 & $.0000 \mathrm{E}+00$ & 3 & $.0000 \mathrm{E}+00$ & 4 & $.0000 \mathrm{E}+00$ & 5 & $.0000 \mathrm{E}+00$ & 6 & $.0000 \mathrm{E}+00$ & 7 & $.0000 \mathrm{E}+00$ & 8 & $0 E+01$ \\
\hline 9 & $.1000 \mathrm{E}+01$ & 10 & $.1000 \mathrm{E}+01$ & 11 & $.1000 \mathrm{E}+01$ & 12 & $.0000 \mathrm{E}+00$ & 13 & $.1000 \mathrm{E}+01$ & 14 & $.1000 \mathrm{E}+01$ & 15 & $.1000 \mathrm{E}+01$ & 16 & $.1000 \mathrm{E}+01$ \\
\hline & $.0000 \mathrm{E}+00$ & 18 & $.0000 \mathrm{E}+00$ & 19 & $.0000 \mathrm{E}+00$ & 20 & $.0000 \mathrm{E}+00$ & 21 & $.0000 \mathrm{E}+00$ & 22 & $.0000 \mathrm{E}+00$ & 23 & $.0000 \mathrm{E}+00$ & 24 & $00 \mathrm{E}+00$ \\
\hline
\end{tabular}

$* * *$ AERMOD - VERSION $07026 * * * \quad \begin{aligned} & * * \text { U/UA Building Demolition } \\ & * * * \text { Unit Release - Worst Case }\end{aligned}$ $\star *$ MODELOPTS :

CONC $\begin{array}{ll}* * * & 02 / 09 / 09 \\ * * * & 10: 41: 11\end{array}$

DRYDPL WETDPL

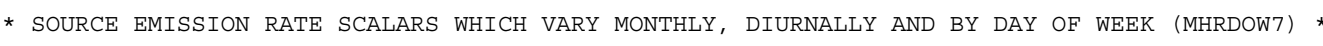

SOURCE ID $=224 \mathrm{U} \_2 ;$ SOURCE TYPE $=$ POINT

HOUR SCALAR HOUR SCALAR HOUR SCALAR HOUR SCALAR HOUR SCALAR HOUR SCALAR HOUR SCALAR HOUR SCALAR

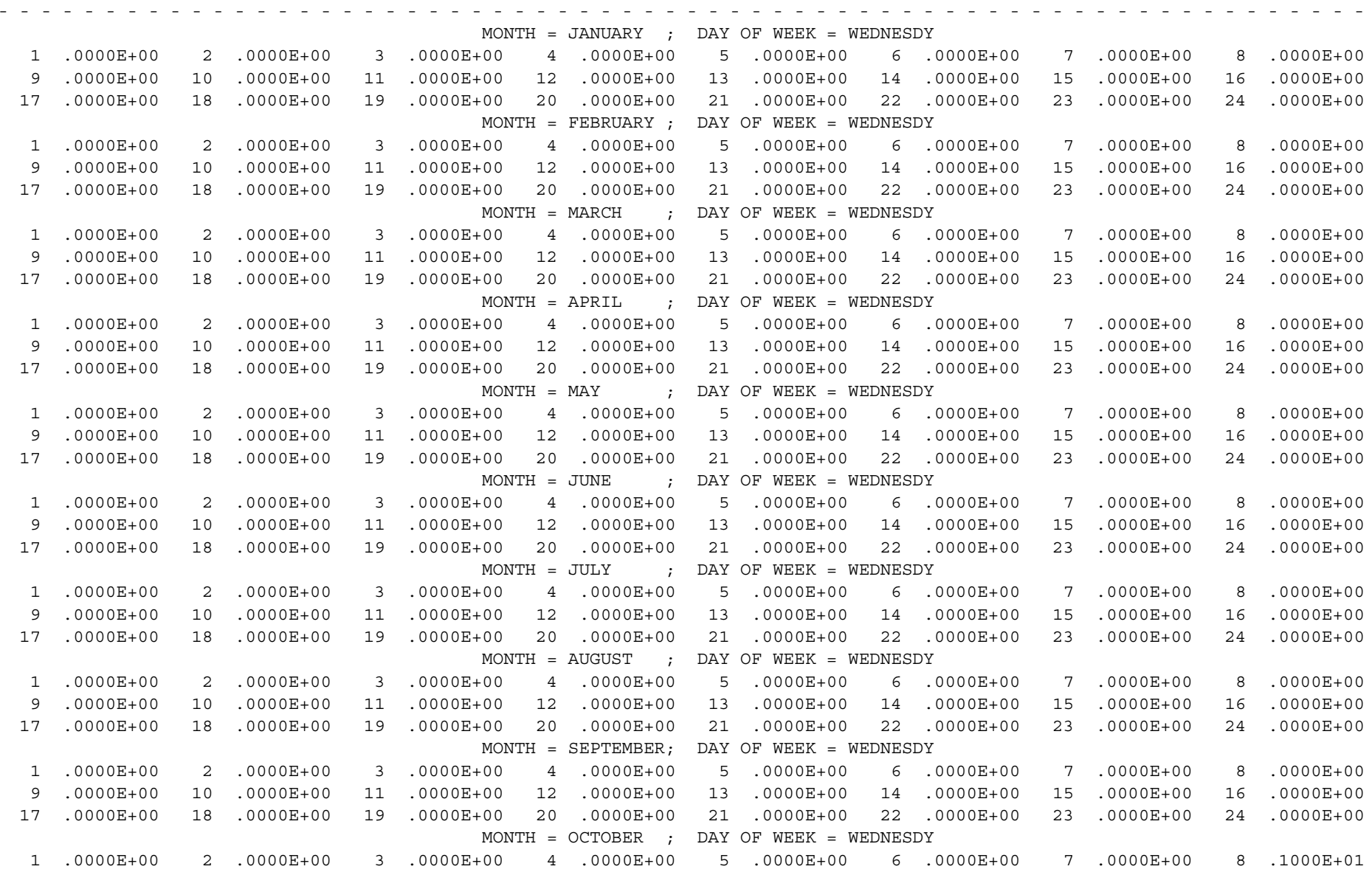




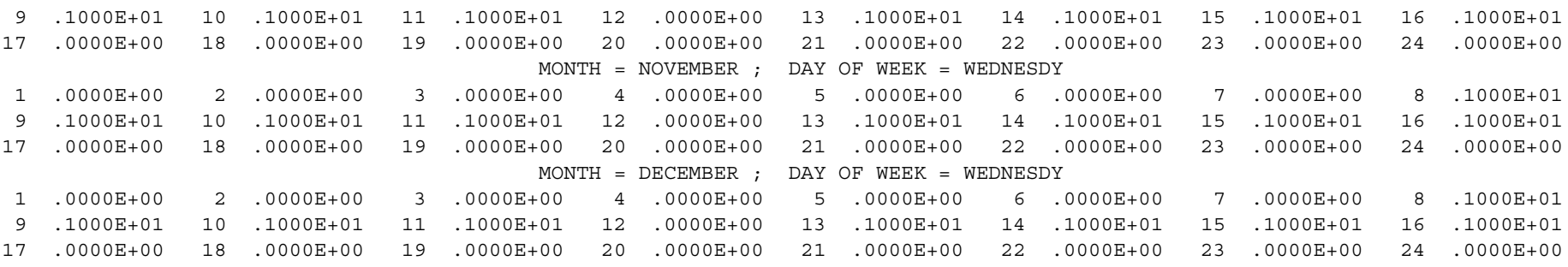

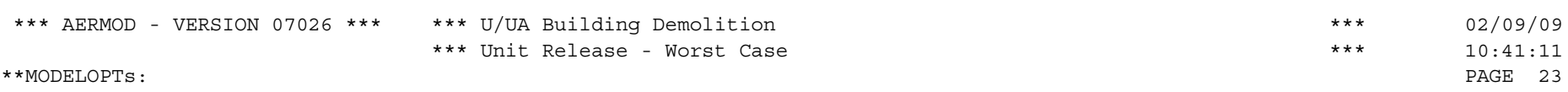
DDEP
TOXICS FLAT
DRYDPL WETDPL

CONC

PAGE 23

* SOURCE EMISSiOn RATE SCALARS WHICH VARY MONTHLY, DIURNALLY AND By DAY OF WEEK (MHRDOW7) *

SOURCE ID $=224 \mathrm{U}_{-} 2 ; \quad$ SOURCE TYPE $=$ POINT

HOUR SCALAR HOUR SCALAR HOUR SCALAR HOUR SCALAR HOUR SCALAR HOUR SCALAR HOUR SCALAR HOUR SCALAR

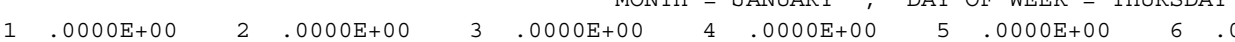

$9 \begin{array}{rrllllllllllrrr}.0000 \mathrm{E}+00 & 10 & .0000 \mathrm{E}+00 & 11 & .0000 \mathrm{E}+00 & 12 & .0000 \mathrm{E}+00 & 13 & .0000 \mathrm{E}+00 & 14 & .0000 \mathrm{E}+00 & 15 & .0000 \mathrm{E}+00 & 8 & .0000 \mathrm{E}+00\end{array}$

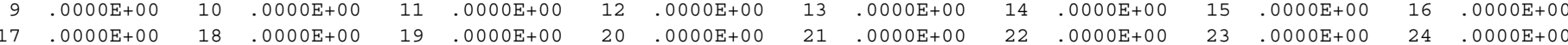

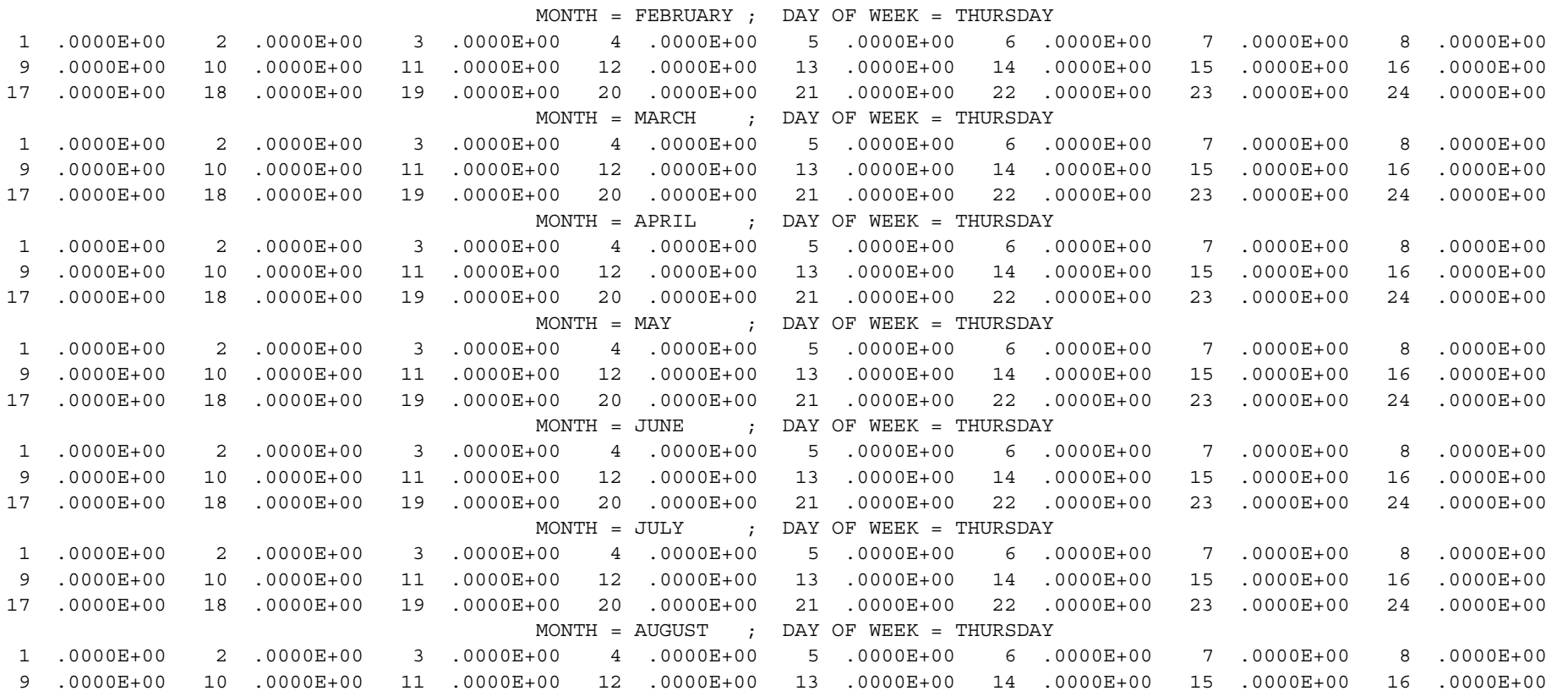




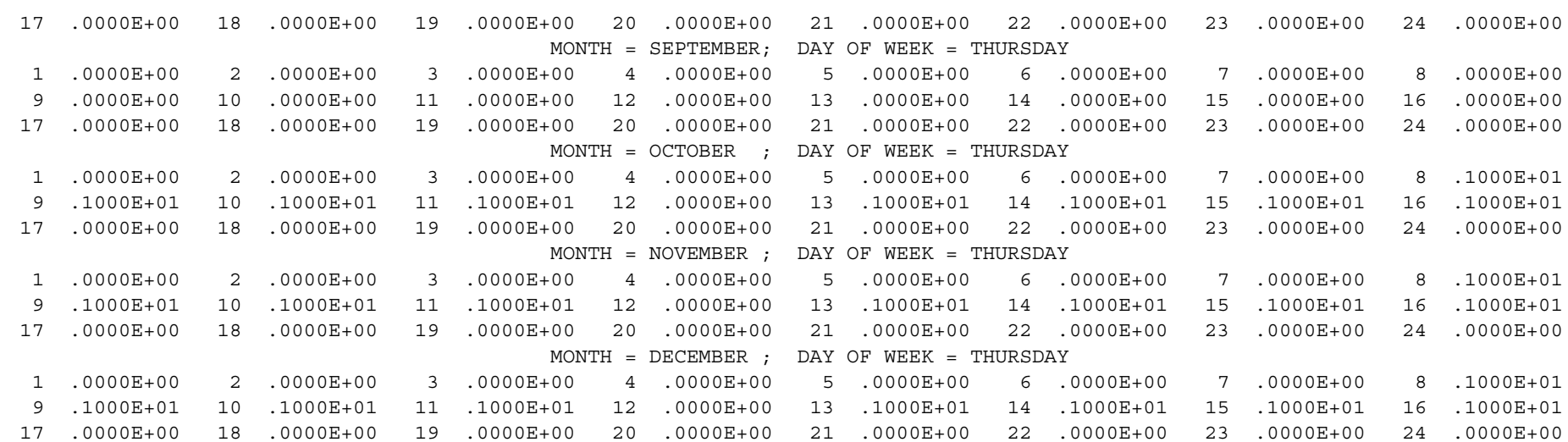

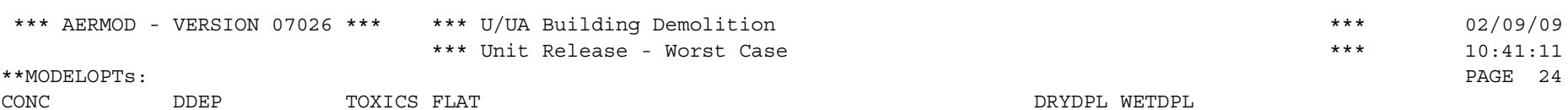
(anc

* SOURCE EMISSiON RATE SCALARS WHich VARY MONTHLy, DIURNALLy AND By DAY OF WEEK (MHRDOW7) *

SOURCE ID $=224 \mathrm{U}_{-} 2 \quad ; \quad$ SOURCE TYPE $=$ POINT

HOUR SCALAR HOUR SCALAR HOUR SCALAR HOUR SCALAR HOUR SCALAR HOUR SCALAR HOUR SCALAR HOUR SCALAR

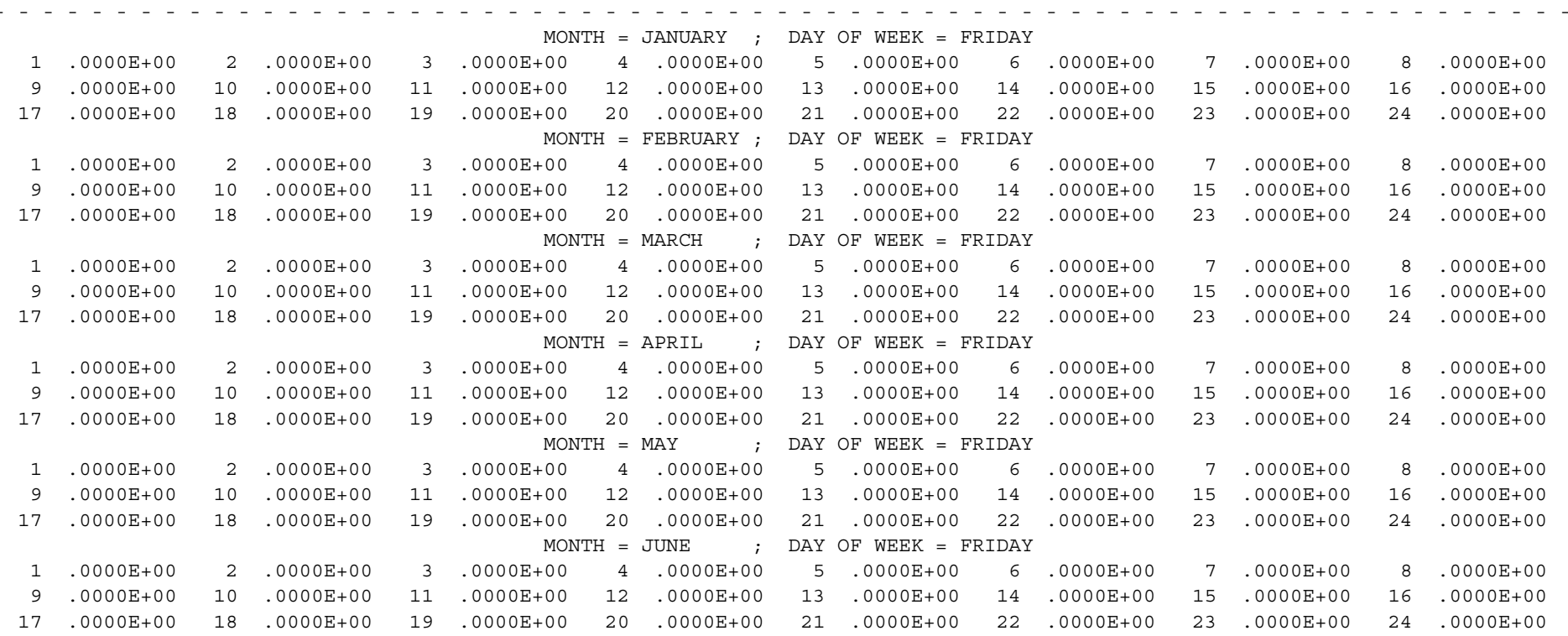




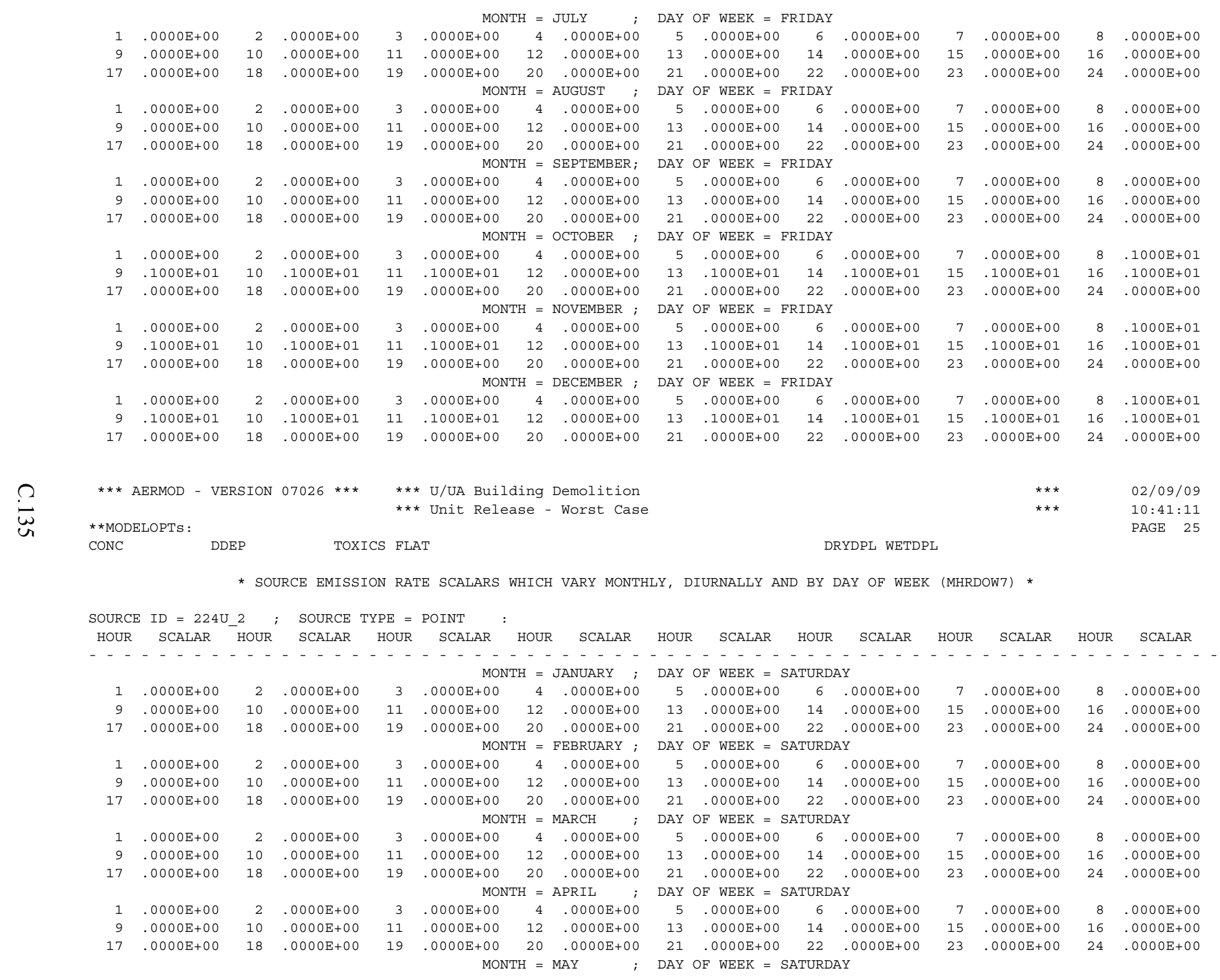




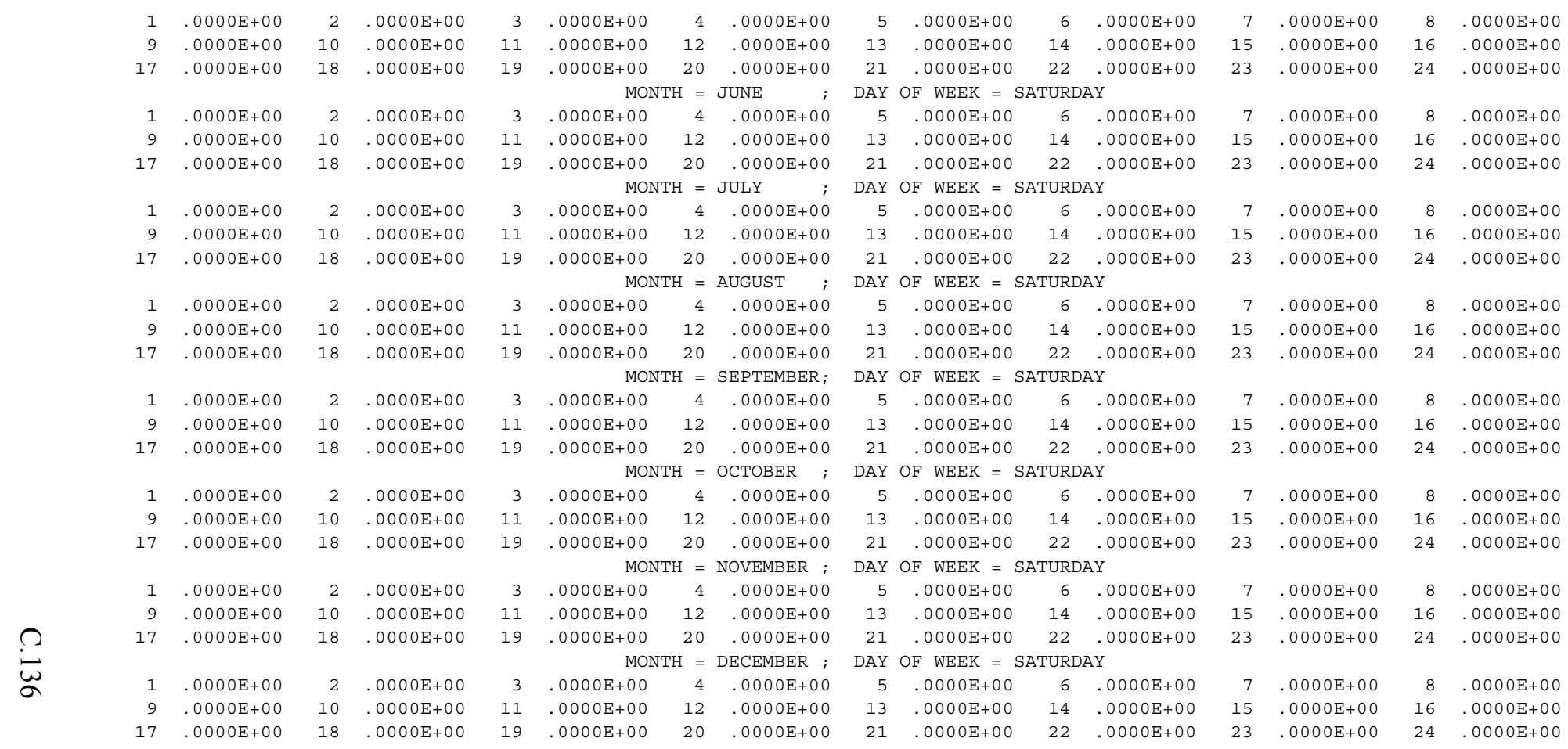

$* * *$ AERMOD - VERSION $07026 * * * \quad \begin{array}{r}* * \\ \text { U/UA Building Demolition } \\ * * * \text { Unit Release - Worst Case }\end{array}$ **MODELOPTS:

CONC
TOXICS FLAT $\begin{array}{ll}* * * & 02 / 09 / 09 \\ * * * & 10: 41: 11\end{array}$

PAGE 26

* SOURCE EMISSION RATE SCALARS Which VARY MONTHLY, DIURNALLY AND By DAY OF WEEK (MHRDOW7) *

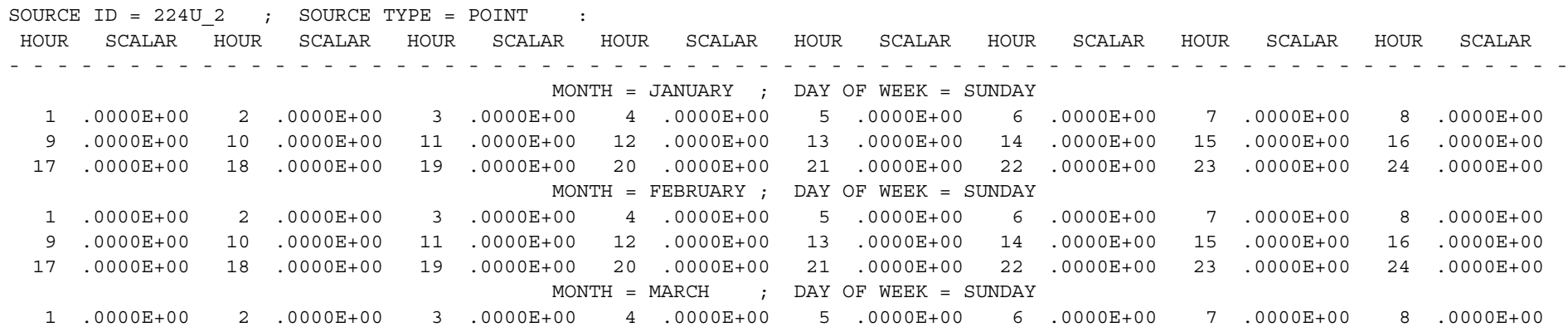




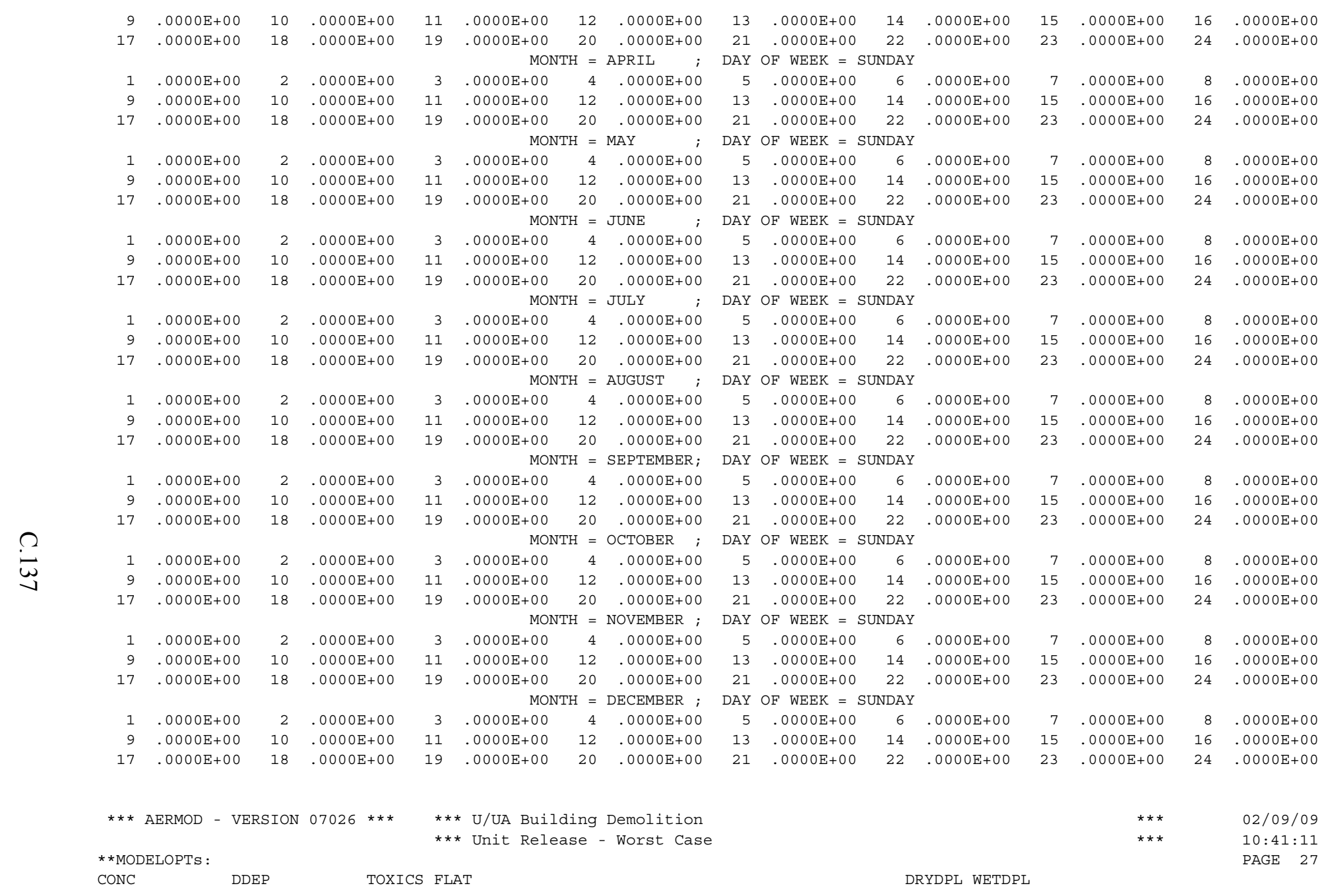

* SOURCE EMISSION RATE SCALARS WHICH VARY MONTHLY, DIURNALLY AND BY DAY OF WEEK (MHRDOW7) *

SOURCE ID $=224 U_{-} 3 ;$ SOURCE TYPE $=$ POINT;

HOUR SCALAR HOUR SCALAR HOUR SCALAR HOUR SCALAR HOUR SCALAR HOUR SCALAR HOUR SCALAR HOUR SCALAR

MONTH $=$ JANUARY; DAY OF WEEK = MONDAY
1

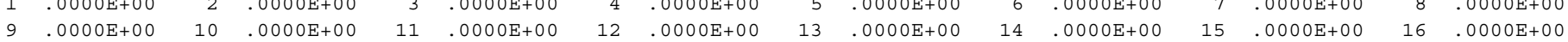




\begin{tabular}{|c|c|c|c|c|c|c|c|c|c|c|c|c|c|c|c|}
\hline 17 & $.0000 \mathrm{E}+00$ & 18 & $.0000 \mathrm{E}+00$ & 19 & $0000 \mathrm{E}+00$ & 20 & $.0000 \mathrm{E}+00$ & 21 & $.0000 \mathrm{E}+00$ & 22 & $.0000 \mathrm{E}+00$ & 23 & $.0000 \mathrm{E}+00$ & 24 & $.0000 \mathrm{E}+00$ \\
\hline & & & & & MONT & $\mathrm{H}=$ & FEBRUARY ; & DAY & OF $\mathrm{WEEK}=\mathrm{M}$ & MONDAY & & & & & \\
\hline 1 & $.0000 \mathrm{E}+00$ & 2 & $.0000 \mathrm{E}+00$ & 3 & $.0000 \mathrm{E}+00$ & 4 & $.0000 \mathrm{E}+00$ & 5 & $.0000 \mathrm{E}+00$ & 6 & $.0000 \mathrm{E}+00$ & 7 & $.0000 \mathrm{E}+00$ & 8 & $.0000 \mathrm{E}+00$ \\
\hline 9 & $.0000 \mathrm{E}+00$ & 10 & $.0000 \mathrm{E}+00$ & 11 & $.0000 \mathrm{E}+00$ & 12 & $.0000 \mathrm{E}+00$ & 13 & $.0000 \mathrm{E}+00$ & 14 & $.0000 \mathrm{E}+00$ & 15 & $.0000 \mathrm{E}+00$ & 16 & $.0000 \mathrm{E}+00$ \\
\hline 17 & $.0000 \mathrm{E}+00$ & 18 & $.0000 \mathrm{E}+00$ & 19 & $.0000 \mathrm{E}+00$ & 20 & $.0000 \mathrm{E}+00$ & 21 & $.0000 \mathrm{E}+00$ & 22 & $.0000 \mathrm{E}+00$ & 23 & $.0000 \mathrm{E}+00$ & 24 & $.0000 \mathrm{E}+00$ \\
\hline & & & & & MON? & & MARCH ; & DAY & OF WEEK $=M$ & MONDAY & & & & & \\
\hline 1 & $.0000 \mathrm{E}+00$ & 2 & $.0000 \mathrm{E}+00$ & 3 & $.0000 \mathrm{E}+00$ & 4 & $.0000 \mathrm{E}+00$ & 5 & $.0000 \mathrm{E}+00$ & 6 & $.0000 \mathrm{E}+00$ & 7 & $.0000 \mathrm{E}+00$ & 8 & $.0000 \mathrm{E}+00$ \\
\hline 9 & $.0000 \mathrm{E}+00$ & 10 & $.0000 \mathrm{E}+00$ & 11 & $.0000 \mathrm{E}+00$ & 12 & $.0000 \mathrm{E}+00$ & 13 & $.0000 \mathrm{E}+00$ & 14 & $.0000 \mathrm{E}+00$ & 15 & $.0000 \mathrm{E}+00$ & 16 & $.0000 \mathrm{E}+00$ \\
\hline 17 & $.0000 \mathrm{E}+00$ & 18 & $.0000 \mathrm{E}+00$ & 19 & $.0000 \mathrm{E}+00$ & 20 & $.0000 \mathrm{E}+00$ & 21 & $.0000 \mathrm{E}+00$ & 22 & $.0000 \mathrm{E}+00$ & 23 & $.0000 \mathrm{E}+00$ & 24 & $.0000 \mathrm{E}+00$ \\
\hline & & & & & MONT & $\mathrm{H}=$ & APRIL ; & DAY & OF $\mathrm{WEEK}=\mathrm{M}$ & MONDAY & & & & & \\
\hline 1 & $.0000 \mathrm{E}+00$ & 2 & $.0000 \mathrm{E}+00$ & 3 & $.0000 \mathrm{E}+00$ & 4 & $.0000 \mathrm{E}+00$ & 5 & $.0000 \mathrm{E}+00$ & 6 & $.0000 \mathrm{E}+00$ & 7 & $.0000 \mathrm{E}+00$ & 8 & $.0000 \mathrm{E}+00$ \\
\hline 9 & $.0000 \mathrm{E}+00$ & 10 & $.0000 \mathrm{E}+00$ & 11 & $.0000 \mathrm{E}+00$ & 12 & $.0000 \mathrm{E}+00$ & 13 & $.0000 \mathrm{E}+00$ & 14 & $.0000 \mathrm{E}+00$ & 15 & $.0000 \mathrm{E}+00$ & 16 & $.0000 \mathrm{E}+00$ \\
\hline 17 & $.0000 \mathrm{E}+00$ & 18 & $.0000 \mathrm{E}+00$ & 19 & $.0000 \mathrm{E}+00$ & 20 & $.0000 \mathrm{E}+00$ & 21 & $.0000 \mathrm{E}+00$ & 22 & $.0000 \mathrm{E}+00$ & 23 & $.0000 \mathrm{E}+00$ & 24 & $.0000 \mathrm{E}+00$ \\
\hline & & & & & MON? & $\mathrm{H}=$ & MAY ; & DAY & OF WEEK $=M$ & MONDAY & & & & & \\
\hline 1 & $.0000 \mathrm{E}+00$ & 2 & $.0000 \mathrm{E}+00$ & 3 & $.0000 \mathrm{E}+00$ & 4 & $.0000 \mathrm{E}+00$ & 5 & $.0000 \mathrm{E}+00$ & 6 & $.0000 \mathrm{E}+00$ & 7 & $.0000 \mathrm{E}+00$ & 8 & $.0000 \mathrm{E}+00$ \\
\hline 9 & $.0000 \mathrm{E}+00$ & 10 & $.0000 \mathrm{E}+00$ & 11 & $.0000 \mathrm{E}+00$ & 12 & $.0000 \mathrm{E}+00$ & 13 & $.0000 \mathrm{E}+00$ & 14 & $.0000 \mathrm{E}+00$ & 15 & $.0000 \mathrm{E}+00$ & 16 & $.0000 \mathrm{E}+00$ \\
\hline 17 & $.0000 \mathrm{E}+00$ & 18 & $.0000 \mathrm{E}+00$ & 19 & $.0000 \mathrm{E}+00$ & 20 & $.0000 \mathrm{E}+00$ & 21 & $.0000 \mathrm{E}+00$ & 22 & $.0000 \mathrm{E}+00$ & 23 & $.0000 \mathrm{E}+00$ & 24 & $.0000 \mathrm{E}+00$ \\
\hline & & & & & MONT & $\mathrm{H}=$ & JUNE $\quad ;$ & DAY & OF WEEK = M & MONDAY & & & & & \\
\hline 1 & $.0000 \mathrm{E}+00$ & 2 & $.0000 \mathrm{E}+00$ & 3 & $.0000 \mathrm{E}+00$ & 4 & $.0000 \mathrm{E}+00$ & 5 & $.0000 \mathrm{E}+00$ & 6 & $.0000 \mathrm{E}+00$ & 7 & $0 \mathrm{E}+00$ & 8 & $.0000 \mathrm{E}+00$ \\
\hline 9 & $.0000 \mathrm{E}+00$ & 10 & $.0000 \mathrm{E}+00$ & 11 & $.0000 \mathrm{E}+00$ & 12 & $.0000 \mathrm{E}+00$ & 13 & $.0000 \mathrm{E}+00$ & 14 & $.0000 \mathrm{E}+00$ & 15 & $.0000 \mathrm{E}+00$ & 16 & $.0000 \mathrm{E}+00$ \\
\hline 17 & $.0000 \mathrm{E}+00$ & 18 & $.0000 \mathrm{E}+00$ & 19 & $.0000 \mathrm{E}+00$ & 20 & $.0000 \mathrm{E}+00$ & 21 & $.0000 \mathrm{E}+00$ & 22 & $.0000 \mathrm{E}+00$ & 23 & $.0000 \mathrm{E}+00$ & 24 & $.0000 \mathrm{E}+00$ \\
\hline & & & & & MON? & $\mathrm{H}=$ & JULY ; & DAY & OF WEEK $=\mathrm{M}$ & MONDAY & & & & & \\
\hline 1 & $.0000 \mathrm{E}+00$ & 2 & $.0000 \mathrm{E}+00$ & 3 & $.0000 \mathrm{E}+00$ & 4 & $.0000 \mathrm{E}+00$ & 5 & $.0000 \mathrm{E}+00$ & 6 & $.0000 \mathrm{E}+00$ & 7 & $.0000 \mathrm{E}+00$ & 8 & $.0000 \mathrm{E}+00$ \\
\hline 9 & $.0000 \mathrm{E}+00$ & 10 & $.0000 \mathrm{E}+00$ & 11 & $.0000 \mathrm{E}+00$ & 12 & $.0000 \mathrm{E}+00$ & 13 & $.0000 \mathrm{E}+00$ & 14 & $.0000 \mathrm{E}+00$ & 15 & $.0000 \mathrm{E}+00$ & 16 & $.0000 \mathrm{E}+00$ \\
\hline 17 & $.0000 \mathrm{E}+00$ & 18 & $.0000 \mathrm{E}+00$ & 19 & $.0000 \mathrm{E}+00$ & 20 & $.0000 \mathrm{E}+00$ & 21 & $.0000 \mathrm{E}+00$ & 22 & $.0000 \mathrm{E}+00$ & 23 & $8+00$ & 24 & $E+00$ \\
\hline & & & & & MONT & $\mathrm{H}=$ & AUGUST ; & DAY & OF WEEK $=\mathrm{M}$ & MONDAY & & & & & \\
\hline 1 & $.0000 \mathrm{E}+00$ & 2 & $.0000 \mathrm{E}+00$ & 3 & $.0000 \mathrm{E}+00$ & 4 & $.0000 \mathrm{E}+00$ & 5 & $.0000 \mathrm{E}+00$ & 6 & $.0000 \mathrm{E}+00$ & 7 & $E+00$ & 8 & $.0000 \mathrm{E}+00$ \\
\hline 9 & $.0000 \mathrm{E}+00$ & 10 & $.0000 \mathrm{E}+00$ & 11 & $.0000 \mathrm{E}+00$ & 12 & $.0000 \mathrm{E}+00$ & 13 & $.0000 \mathrm{E}+00$ & 14 & $0 \mathrm{E}+00$ & 15 & $E+00$ & 16 & $.0000 \mathrm{E}+00$ \\
\hline 17 & $.0000 \mathrm{E}+00$ & 18 & $.0000 \mathrm{E}+00$ & 19 & $.0000 \mathrm{E}+00$ & 20 & $.0000 \mathrm{E}+00$ & 21 & $.0000 \mathrm{E}+00$ & 22 & $.0000 \mathrm{E}+00$ & 23 & $.0000 \mathrm{E}+00$ & 24 & $.0000 \mathrm{E}+00$ \\
\hline & & & & & MONT & $\mathrm{H}=$ & SEPTEMBER; & DAY & OF $\mathrm{WEEK}=\mathrm{M}$ & MONDAY & & & & & \\
\hline 1 & $.0000 \mathrm{E}+00$ & 2 & $.0000 \mathrm{E}+00$ & 3 & $.0000 \mathrm{E}+00$ & 4 & $.0000 \mathrm{E}+00$ & 5 & $.0000 \mathrm{E}+00$ & 6 & $.0000 \mathrm{E}+00$ & 7 & $.0000 \mathrm{E}+00$ & 8 & $.0000 \mathrm{E}+00$ \\
\hline 9 & $.0000 \mathrm{E}+00$ & 10 & $.0000 \mathrm{E}+00$ & 11 & $.0000 \mathrm{E}+00$ & 12 & $.0000 \mathrm{E}+00$ & 13 & $.0000 \mathrm{E}+00$ & 14 & $.0000 \mathrm{E}+00$ & 15 & $.0000 \mathrm{E}+00$ & 16 & $.0000 \mathrm{E}+00$ \\
\hline 17 & $.0000 \mathrm{E}+00$ & 18 & $.0000 \mathrm{E}+00$ & 19 & $.0000 \mathrm{E}+00$ & 20 & $.0000 \mathrm{E}+00$ & 21 & $.0000 \mathrm{E}+00$ & 22 & $.0000 \mathrm{E}+00$ & 23 & $.0000 \mathrm{E}+00$ & 24 & $.0000 \mathrm{E}+00$ \\
\hline & & & & & MONT & $\mathrm{H}=$ & OCTOBER ; & DAY & OF $\mathrm{WEEK}=\mathrm{M}$ & MONDAY & & & & & \\
\hline 1 & .0000 & 2 & $.0000 \mathrm{E}+00$ & 3 & $.0000 \mathrm{E}+00$ & 4 & $.0000 \mathrm{E}+00$ & 5 & $.0000 \mathrm{E}+00$ & 6 & & 7 & & 8 & $E+01$ \\
\hline 9 & .1000 & 10 & .100 & 11 & $.1000 \mathrm{E}+01$ & 12 & $00 \mathrm{E}+00$ & 13 & $0 E+01$ & 14 & $E+01$ & 15 & +01 & 16 & $E+01$ \\
\hline 17 & $.0000 \mathrm{E}+00$ & 18 & $.0000 \mathrm{E}+00$ & 19 & $.0000 \mathrm{E}+00$ & 20 & $.0000 \mathrm{E}+00$ & 21 & $.0000 \mathrm{E}+00$ & 22 & $.0000 \mathrm{E}+00$ & 23 & $.0000 \mathrm{E}+00$ & 24 & $.0000 \mathrm{E}+00$ \\
\hline & & & & & MON? & $\mathrm{H}=$ & NOVEMBER ; & DAY & OF $\mathrm{WEEK}=\mathrm{M}$ & MONDAY & & & & & \\
\hline 1 & $.0000 \mathrm{E}+00$ & 2 & $.0000 \mathrm{E}+00$ & 3 & $.0000 \mathrm{E}+00$ & 4 & $.0000 \mathrm{E}+00$ & 5 & $.0000 \mathrm{E}+00$ & 6 & $.0000 \mathrm{E}+00$ & 7 & $.0000 \mathrm{E}+00$ & 8 & $.1000 \mathrm{E}+01$ \\
\hline 9 & $.1000 \mathrm{E}+01$ & 10 & $.1000 \mathrm{E}+01$ & 11 & $.1000 \mathrm{E}+01$ & 12 & $.0000 \mathrm{E}+00$ & 13 & $.1000 \mathrm{E}+01$ & 14 & $.1000 \mathrm{E}+01$ & 15 & $.1000 \mathrm{E}+01$ & 16 & $.1000 \mathrm{E}+01$ \\
\hline 17 & $.0000 \mathrm{E}+00$ & 18 & $.0000 \mathrm{E}+00$ & 19 & $.0000 \mathrm{E}+00$ & 20 & $.0000 \mathrm{E}+00$ & 21 & $.0000 \mathrm{E}+00$ & 22 & $.0000 \mathrm{E}+00$ & 23 & $.0000 \mathrm{E}+00$ & 24 & $.0000 \mathrm{E}+00$ \\
\hline & & & & & MONT & & DECEMBER ; & DAY & OF WEEK $=\mathrm{M}$ & MONDAY & & & & & \\
\hline 1 & $.0000 \mathrm{E}+00$ & 2 & $.0000 \mathrm{E}+00$ & 3 & $.0000 \mathrm{E}+00$ & 4 & $.0000 \mathrm{E}+00$ & 5 & $.0000 \mathrm{E}+00$ & 6 & & 7 & & 8 & \\
\hline 9 & $.1000 \mathrm{E}+01$ & 10 & $.1000 \mathrm{E}+01$ & 11 & $.1000 \mathrm{E}+01$ & 12 & $.0000 \mathrm{E}+00$ & 13 & $.1000 \mathrm{E}+01$ & 14 & $.1000 \mathrm{E}+01$ & 15 & $.1000 \mathrm{E}+01$ & 16 & $.1000 \mathrm{E}+01$ \\
\hline 17 & $.0000 \mathrm{E}+00$ & 18 & $.0000 \mathrm{E}+00$ & 19 & $.0000 \mathrm{E}+00$ & 20 & $.0000 \mathrm{E}+00$ & 21 & $.0000 \mathrm{E}+00$ & 22 & $.0000 \mathrm{E}+00$ & 23 & $.0000 \mathrm{E}+00$ & 24 & $.0000 \mathrm{E}+00$ \\
\hline
\end{tabular}

*** AERMOD - VERSION $07026 * * * \quad * * *$ U/UA Building Demolition

$* * *$ Unit Release - Worst Cas $\begin{array}{ll}* * * & 02 / 09 / 09 \\ * * * & 10: 41: 11\end{array}$

PAGE 28

CONC

DDEP

TOXICS FLAT

DRYDPL WETDPL

* SOURCE EMISSION RATE SCALARS WHICH VARY MONTHLY, DIURNALLY AND BY DAY OF WEEK (MHRDOW7) * 


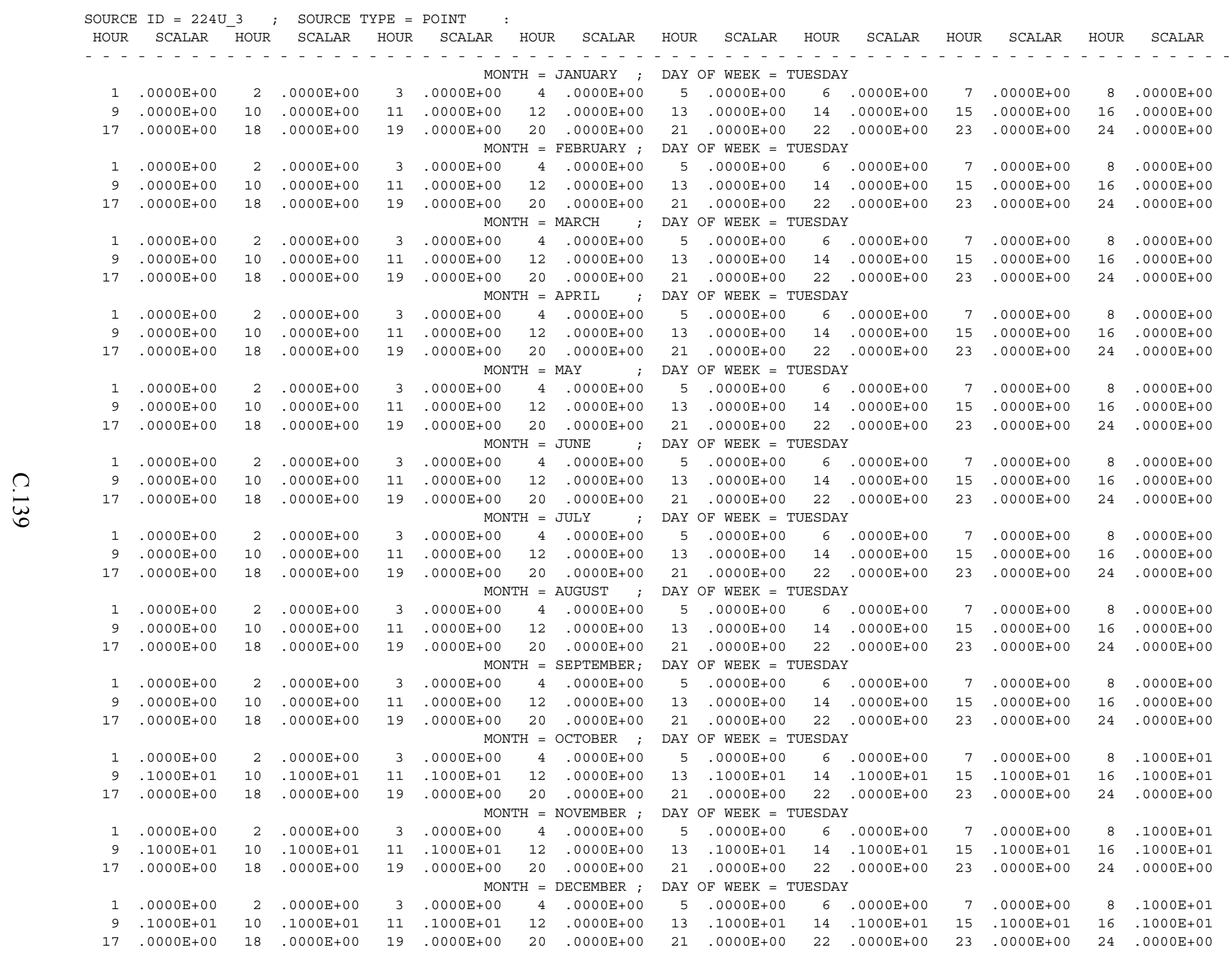




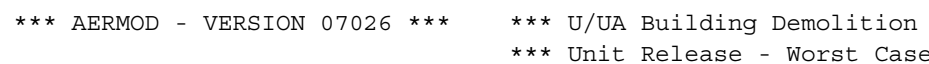
**MODELOPTS :

CONC $\begin{array}{ll}* * * & 02 / 09 / 09 \\ * * * & 10: 41: 11\end{array}$

$10: 41: 11$
PAGE $\quad 29$

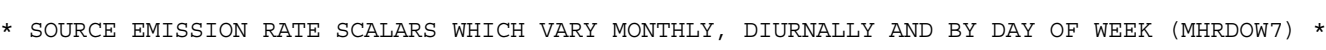

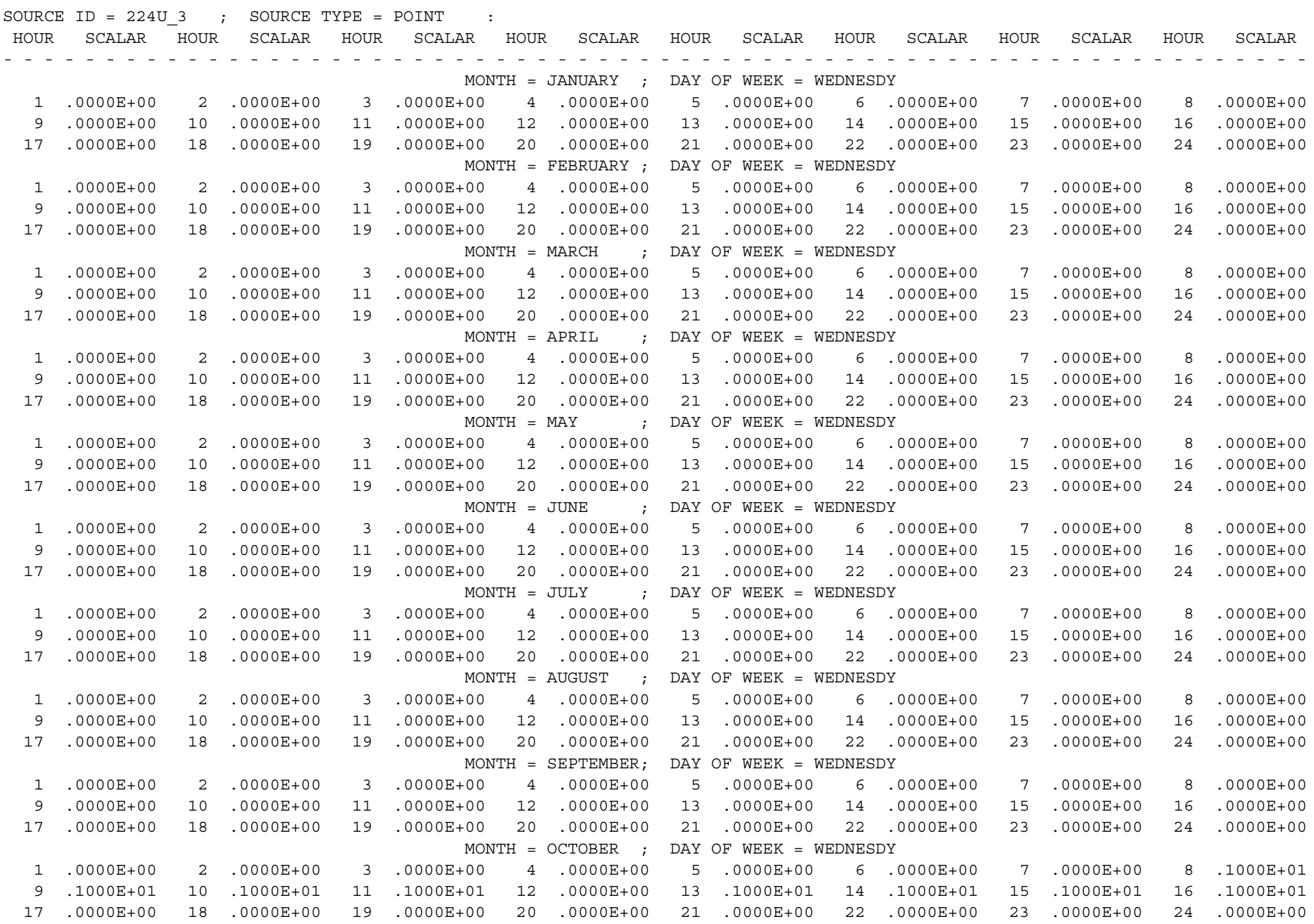

MONTH $=$ NOVEMBER $; \quad$ DAY OF WEEK $=$ WEDNESDY 


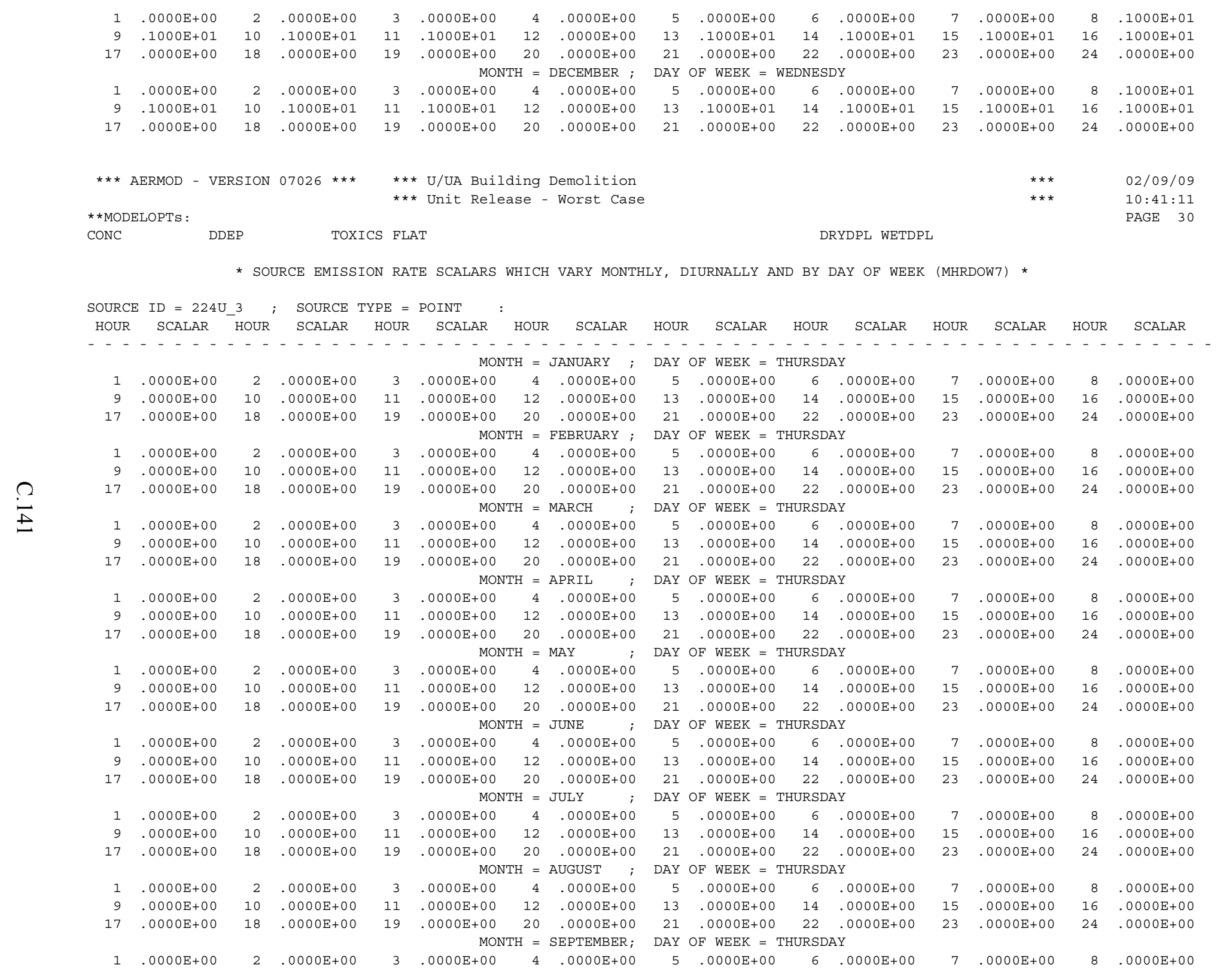




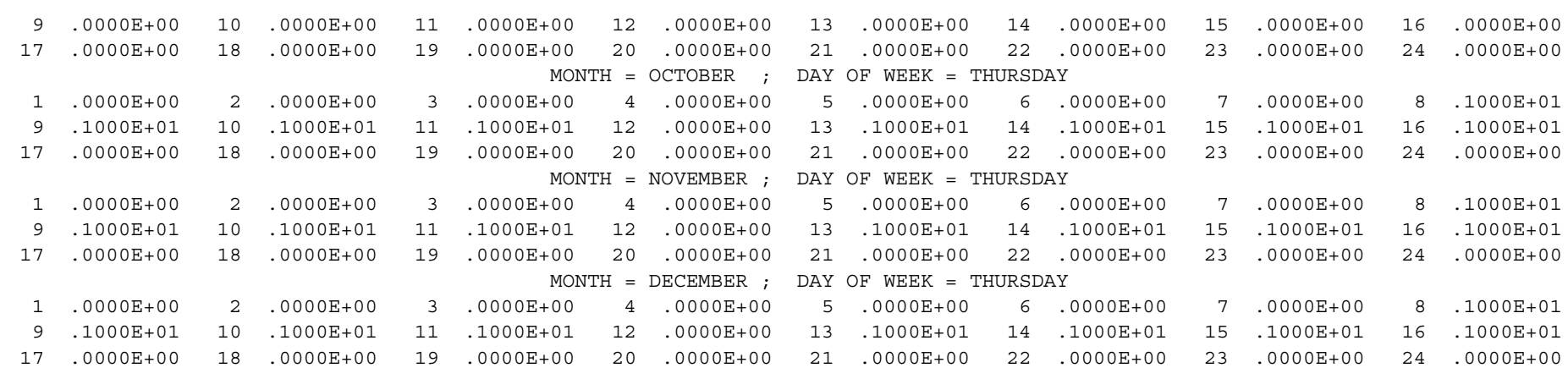

*** AERMOD - VERSION $07026 * * * \quad * * *$ U/UA Building Demolition

*** Unit Release - Worst Case

$\begin{array}{ll}* * * & 02 / 09 / 09 \\ * * * & 10: 41: 11 \\ & \text { PAGE } 31\end{array}$ **MODELOPTS:

CONC

DDEP

TOXICS FLAT

DRYDPL WETDPL

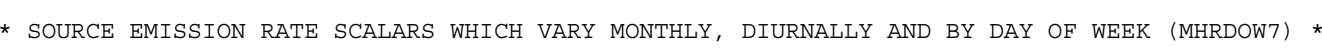

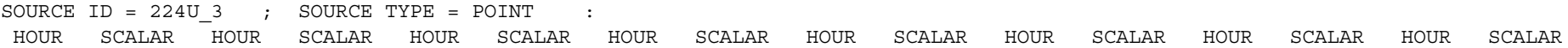

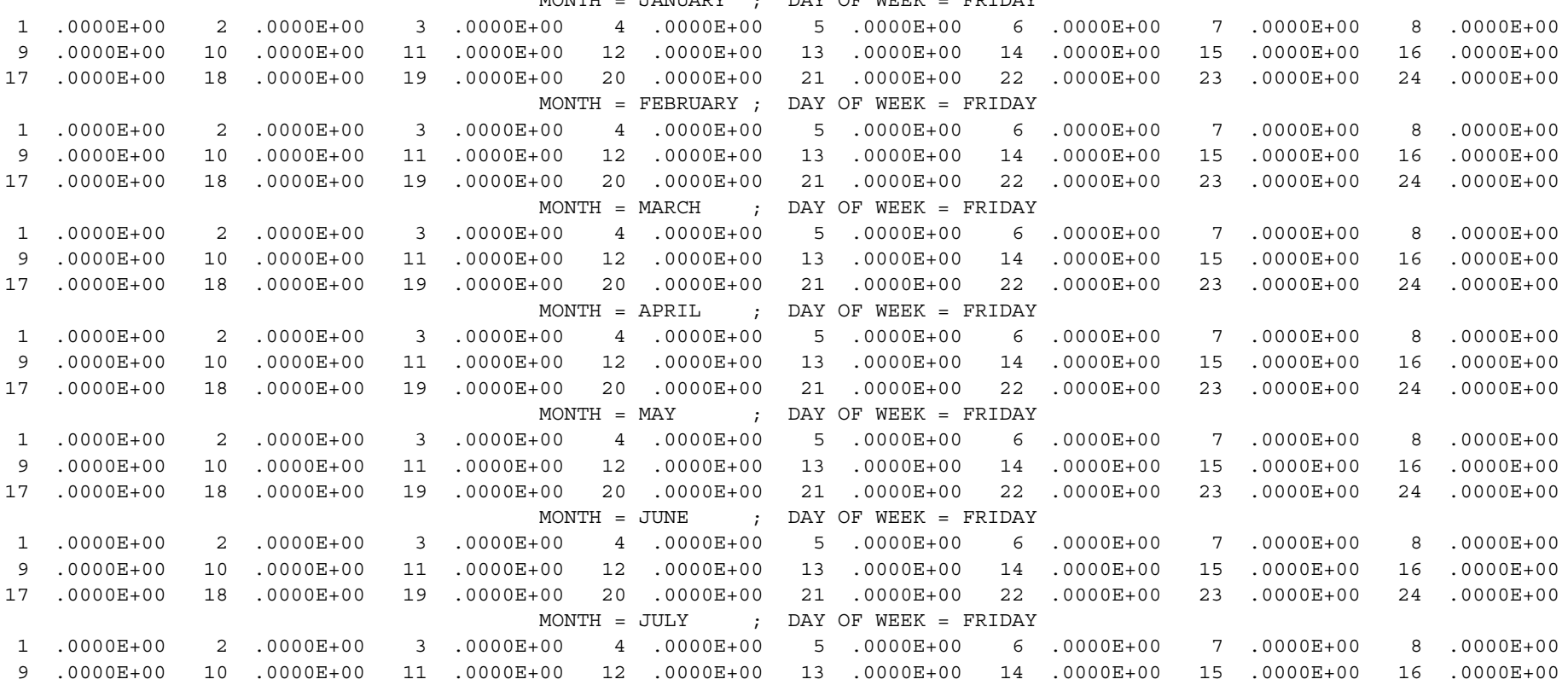




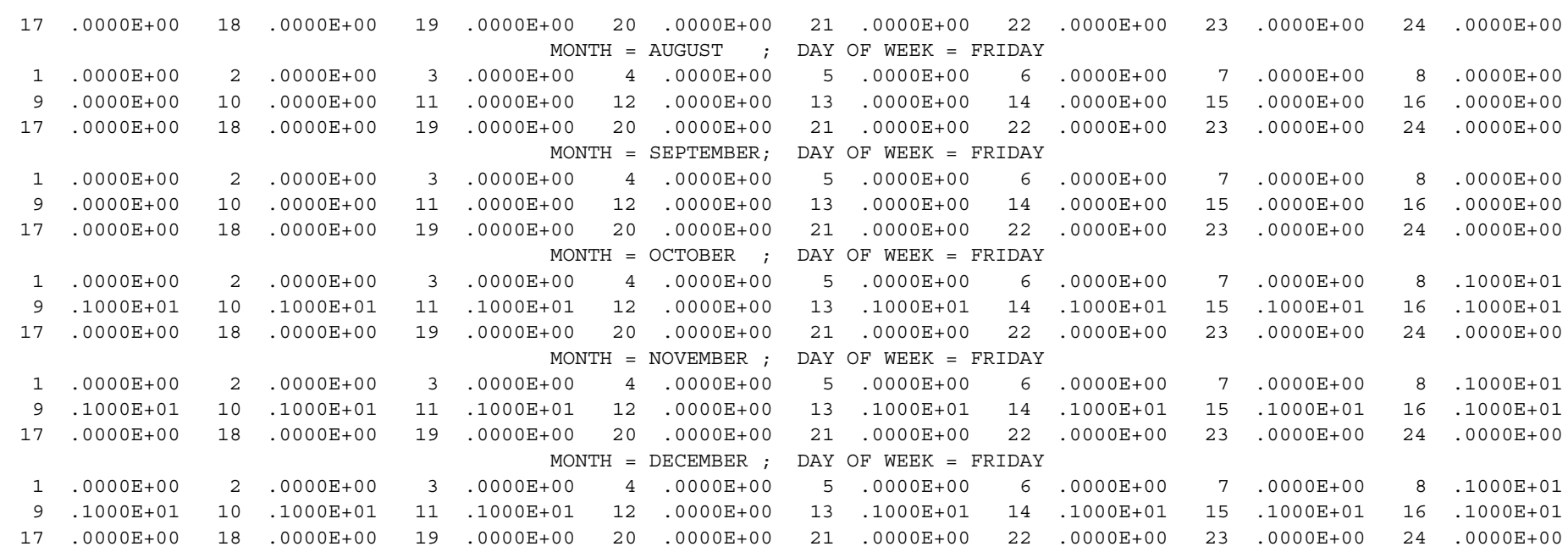

\footnotetext{
*** AERMOD - VERSION $07026 * * * \quad * * *$ U/UA Building Demolition

*** Unit Release - Worst Case

$\begin{array}{ll}* * * & 02 / 09 / 09 \\ * * * & 10: 41: 11\end{array}$

PAGE 32 **MODELOPTS :

DRYDPL WETDPL
}

* SOURCE EMISSION RATE SCALARS WHICH VARY MONTHLY, DIURNALLY AND BY DAY OF WEEK (MHRDOW7) *

SOURCE ID $=224 \mathrm{U}_{-} 3 ;$ SOURCE TYPE $=$ POINT

HOUR SCALAR HOUR SCALAR HOUR SCALAR HOUR SCALAR HOUR SCALAR HOUR SCALAR HOUR SCALAR HOUR SCALAR

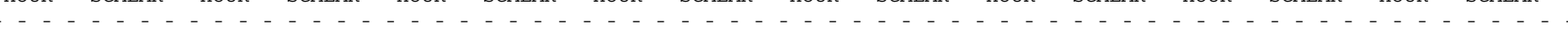

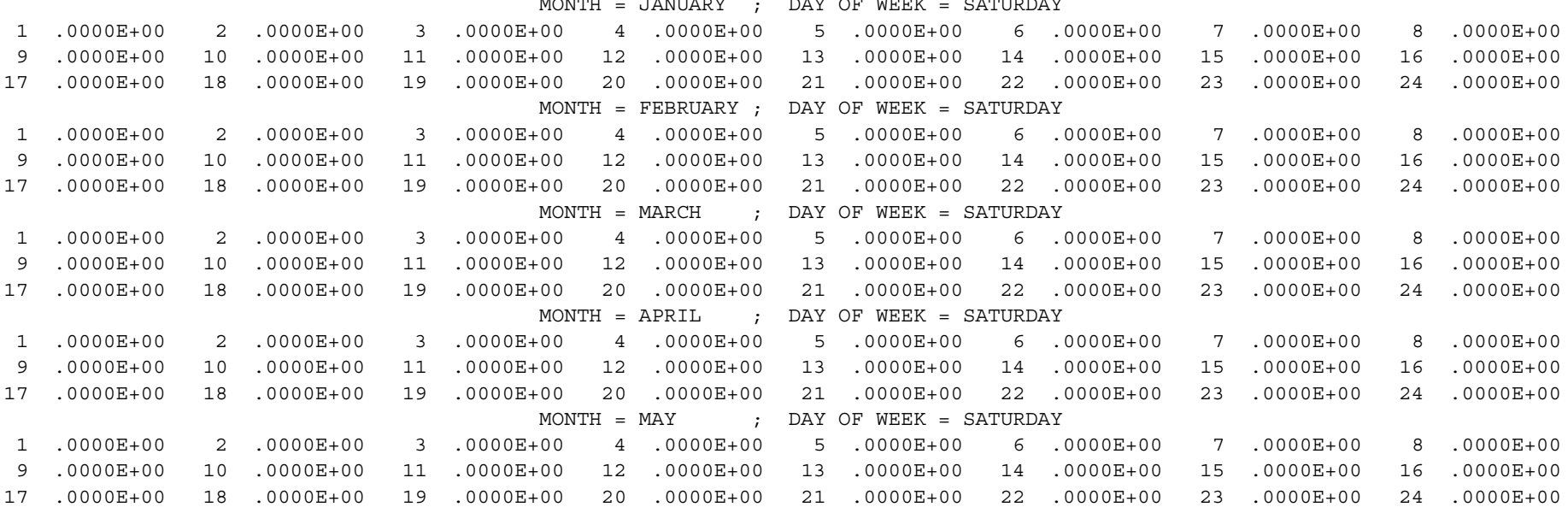




\begin{tabular}{|c|c|c|c|c|c|c|c|c|c|c|c|c|c|c|c|}
\hline & & & & & MONT & $\mathrm{TH}=$ & JUNE $\quad ;$ & DAY & OF $W E E K=S$ & ATURD & & & & & \\
\hline 1 & $.0000 \mathrm{E}+00$ & 2 & $.0000 \mathrm{E}+00$ & 3 & $.0000 \mathrm{E}+00$ & 4 & $.0000 \mathrm{E}+00$ & 5 & $.0000 \mathrm{E}+00$ & 6 & $.0000 \mathrm{E}+00$ & 7 & $.0000 \mathrm{E}+00$ & 8 & $.0000 \mathrm{E}+00$ \\
\hline 9 & $.0000 \mathrm{E}+00$ & 10 & $.0000 \mathrm{E}+00$ & 11 & $.0000 \mathrm{E}+00$ & 12 & $.0000 \mathrm{E}+00$ & 13 & $.0000 \mathrm{E}+00$ & 14 & $.0000 \mathrm{E}+00$ & 15 & $.0000 \mathrm{E}+00$ & 16 & $.0000 \mathrm{E}+00$ \\
\hline 17 & $.0000 \mathrm{E}+00$ & 18 & $.0000 \mathrm{E}+00$ & 19 & $.0000 \mathrm{E}+00$ & 20 & $.0000 \mathrm{E}+00$ & 21 & $.0000 \mathrm{E}+00$ & 22 & $.0000 \mathrm{E}+00$ & 23 & $.0000 \mathrm{E}+00$ & 24 & $.0000 \mathrm{E}+00$ \\
\hline & & & & & MONT & $\mathrm{TH}=$ & JULY ; & DAY & OF WEEK = S & ATURD & & & & & \\
\hline 1 & $.0000 \mathrm{E}+00$ & 2 & $.0000 \mathrm{E}+00$ & 3 & $.0000 \mathrm{E}+00$ & 4 & $.0000 \mathrm{E}+00$ & 5 & $.0000 \mathrm{E}+00$ & 6 & $.0000 \mathrm{E}+00$ & 7 & $.0000 \mathrm{E}+00$ & 8 & $.0000 \mathrm{E}+00$ \\
\hline 9 & $.0000 E+00$ & 10 & $.0000 \mathrm{E}+00$ & 11 & $.0000 \mathrm{E}+00$ & 12 & $.0000 \mathrm{E}+00$ & 13 & $.0000 \mathrm{E}+00$ & 14 & $.0000 \mathrm{E}+00$ & 15 & $.0000 \mathrm{E}+00$ & 16 & $.0000 \mathrm{E}+00$ \\
\hline 17 & $.0000 \mathrm{E}+00$ & 18 & $.0000 \mathrm{E}+00$ & 19 & $.0000 \mathrm{E}+00$ & 20 & $.0000 \mathrm{E}+00$ & 21 & $.0000 \mathrm{E}+00$ & 22 & $.0000 \mathrm{E}+00$ & 23 & $.0000 \mathrm{E}+00$ & 24 & $.0000 \mathrm{E}+00$ \\
\hline & & & & & MONT & $T H=$ & AUGUST ; & DAY & OF WEEK = S & ATURD & & & & & \\
\hline 1 & $.0000 \mathrm{E}+00$ & 2 & $.0000 \mathrm{E}+00$ & 3 & $.0000 \mathrm{E}+00$ & 4 & $.0000 \mathrm{E}+00$ & 5 & $.0000 \mathrm{E}+00$ & 6 & $.0000 \mathrm{E}+00$ & 7 & $.0000 \mathrm{E}+00$ & 8 & $.0000 \mathrm{E}+00$ \\
\hline 9 & $.0000 \mathrm{E}+00$ & 10 & $.0000 \mathrm{E}+00$ & 11 & $.0000 \mathrm{E}+00$ & 12 & $.0000 \mathrm{E}+00$ & 13 & $.0000 \mathrm{E}+00$ & 14 & $.0000 \mathrm{E}+00$ & 15 & $.0000 \mathrm{E}+00$ & 16 & $.0000 \mathrm{E}+00$ \\
\hline 17 & $.0000 \mathrm{E}+00$ & 18 & $.0000 \mathrm{E}+00$ & 19 & $.0000 \mathrm{E}+00$ & 20 & $.0000 \mathrm{E}+00$ & 21 & $.0000 \mathrm{E}+00$ & 22 & $.0000 \mathrm{E}+00$ & 23 & $.0000 \mathrm{E}+00$ & 24 & $.0000 \mathrm{E}+00$ \\
\hline & & & & & MONT & $\mathrm{TH}=$ & SEPTEMBER ; & DAY & OF WEEK = S & ATURD & & & & & \\
\hline 1 & $.0000 \mathrm{E}+00$ & 2 & $.0000 \mathrm{E}+00$ & 3 & $.0000 \mathrm{E}+00$ & 4 & $.0000 \mathrm{E}+00$ & 5 & $.0000 \mathrm{E}+00$ & 6 & $.0000 \mathrm{E}+00$ & 7 & $.0000 \mathrm{E}+00$ & 8 & $.0000 \mathrm{E}+00$ \\
\hline 9 & $.0000 \mathrm{E}+00$ & 10 & $.0000 \mathrm{E}+00$ & 11 & $.0000 \mathrm{E}+00$ & 12 & $.0000 \mathrm{E}+00$ & 13 & $.0000 \mathrm{E}+00$ & 14 & $.0000 \mathrm{E}+00$ & 15 & $.0000 \mathrm{E}+00$ & 16 & $.0000 \mathrm{E}+00$ \\
\hline 17 & $.0000 \mathrm{E}+00$ & 18 & $.0000 \mathrm{E}+00$ & 19 & $.0000 \mathrm{E}+00$ & 20 & $.0000 \mathrm{E}+00$ & 21 & $.0000 \mathrm{E}+00$ & 22 & $.0000 \mathrm{E}+00$ & 23 & $.0000 \mathrm{E}+00$ & 24 & $.0000 \mathrm{E}+00$ \\
\hline & & & & & MONT & $T H=$ & OCTOBER ; & DAY & OF WEEK = $S$ & ATURD & & & & & \\
\hline 1 & $.0000 \mathrm{E}+00$ & 2 & $.0000 \mathrm{E}+00$ & 3 & $.0000 \mathrm{E}+00$ & 4 & $.0000 \mathrm{E}+00$ & 5 & $.0000 \mathrm{E}+00$ & 6 & $.0000 \mathrm{E}+00$ & 7 & $.0000 \mathrm{E}+00$ & 8 & $.0000 \mathrm{E}+00$ \\
\hline 9 & $.0000 \mathrm{E}+00$ & 10 & $.0000 \mathrm{E}+00$ & 11 & $.0000 \mathrm{E}+00$ & 12 & $.0000 \mathrm{E}+00$ & 13 & $.0000 \mathrm{E}+00$ & 14 & $.0000 \mathrm{E}+00$ & 15 & $.0000 \mathrm{E}+00$ & 16 & $.0000 \mathrm{E}+00$ \\
\hline 17 & $.0000 \mathrm{E}+00$ & 18 & $.0000 \mathrm{E}+00$ & 19 & $.0000 \mathrm{E}+00$ & 20 & $.0000 \mathrm{E}+00$ & 21 & $.0000 \mathrm{E}+00$ & 22 & $.0000 \mathrm{E}+00$ & 23 & $.0000 \mathrm{E}+00$ & 24 & $.0000 \mathrm{E}+00$ \\
\hline & & & & & MONT & $\mathrm{TH}=$ & NOVEMBER ; & DAY & OF WEEK = S & ATURD & & & & & \\
\hline 1 & $.0000 \mathrm{E}+00$ & 2 & $.0000 \mathrm{E}+00$ & 3 & $.0000 \mathrm{E}+00$ & 4 & $.0000 \mathrm{E}+00$ & 5 & $.0000 \mathrm{E}+00$ & 6 & $.0000 \mathrm{E}+00$ & 7 & $.0000 \mathrm{E}+00$ & 8 & $.0000 \mathrm{E}+00$ \\
\hline 9 & $.0000 \mathrm{E}+00$ & 10 & $.0000 \mathrm{E}+00$ & 11 & $.0000 \mathrm{E}+00$ & 12 & $.0000 \mathrm{E}+00$ & 13 & $.0000 \mathrm{E}+00$ & 14 & $.0000 \mathrm{E}+00$ & 15 & $.0000 \mathrm{E}+00$ & 16 & $.0000 \mathrm{E}+00$ \\
\hline 17 & $.0000 E+00$ & 18 & $.0000 \mathrm{E}+00$ & 19 & $.0000 \mathrm{E}+00$ & 20 & $.0000 \mathrm{E}+00$ & 21 & $.0000 \mathrm{E}+00$ & 22 & $.0000 \mathrm{E}+00$ & 23 & $.0000 \mathrm{E}+00$ & 24 & $.0000 \mathrm{E}+00$ \\
\hline & & & & & MONT & $\mathrm{TH}=$ & DECEMBER ; & DAY & OF WEEK = S & ATURD & & & & & \\
\hline 1 & $.0000 \mathrm{E}+00$ & 2 & $.0000 \mathrm{E}+00$ & 3 & $.0000 \mathrm{E}+00$ & 4 & $.0000 \mathrm{E}+00$ & 5 & $.0000 \mathrm{E}+00$ & 6 & $.0000 \mathrm{E}+00$ & 7 & $.0000 \mathrm{E}+00$ & 8 & $.0000 \mathrm{E}+00$ \\
\hline 9 & $.0000 \mathrm{E}+00$ & 10 & $.0000 \mathrm{E}+00$ & 11 & $.0000 \mathrm{E}+00$ & 12 & $.0000 \mathrm{E}+00$ & 13 & $.0000 \mathrm{E}+00$ & 14 & $.0000 \mathrm{E}+00$ & 15 & $.0000 \mathrm{E}+00$ & 16 & $.0000 \mathrm{E}+00$ \\
\hline 17 & $.0000 \mathrm{E}+00$ & 18 & $.0000 \mathrm{E}+00$ & 19 & $.0000 \mathrm{E}+00$ & 20 & $.0000 \mathrm{E}+00$ & 21 & $.0000 \mathrm{E}+00$ & 22 & $.0000 \mathrm{E}+00$ & 23 & $.0000 \mathrm{E}+00$ & 24 & $.0000 \mathrm{E}+00$ \\
\hline$\star * * A$ & AERMOD - VEF & RSION & $07026 * * *$ & $* * *$ & * U/UA Build & ding & Demolition & & & & & & $\star \star *$ & & $02 / 09 / 09$ \\
\hline & & & & ** & * Unit Relea & ase - & - Worst Case & & & & & & $* * *$ & & $10: 41: 11$ \\
\hline$\star *$ MODE & DELOPTS : & & & & & & & & & & & & & & PAGE 33 \\
\hline CONC & DDF & EPP & TOXIC & CS FLA & & & & & & & RYDPL WETDPL & & & & \\
\hline & & * SOU & URCE EMISSIO & ON RAT & TE SCALARS W & $\mathrm{WHICH}$ & H VARY MONTHI & $L Y, D$ & IURNALLY AN & D BY I & DAY OF WEEK & (MHRD & Dow7) * & & \\
\hline SOURCE & $\mathrm{CE} I \mathrm{ID}=224 \mathrm{U}$ & I_3 ; & SOURCE TY & $\mathrm{YPE}=$ & POINT & & & & & & & & & & \\
\hline HOUR & SCALAR & ${ }^{-}$HOUR' & SCALAR & HOUR & SCALAR & HOUR & SCALAR & HOUR & SCALAR & HOUR & SCALAR & HOUR & SCALAR & HOUR & SCALAR \\
\hline & & & & & MONT & & $\begin{array}{c}-\cdots- \\
\text { JANUARY }\end{array}$ & $\begin{array}{c}-- \\
\text { DAY }\end{array}$ & $\begin{array}{l}-\cdots \\
\text { OF WEEK }=S\end{array}$ & $\begin{array}{l}-\cdots \\
\text { UNDAY }\end{array}$ & ---- & & $-\ldots$ & - & $-\ldots$ \\
\hline 1 & $.0000 \mathrm{E}+00$ & 2 & $.0000 \mathrm{E}+00$ & 3 & $.0000 \mathrm{E}+00$ & 4 & $.0000 \mathrm{E}+00$ & 5 & $.0000 \mathrm{E}+00$ & 6 & $.0000 \mathrm{E}+00$ & 7 & $.0000 \mathrm{E}+00$ & 8 & $.0000 \mathrm{E}+00$ \\
\hline 9 & $.0000 \mathrm{E}+00$ & 10 & $.0000 \mathrm{E}+00$ & 11 & $.0000 \mathrm{E}+00$ & 12 & $.0000 \mathrm{E}+00$ & 13 & $.0000 \mathrm{E}+00$ & 14 & $.0000 \mathrm{E}+00$ & 15 & $.0000 \mathrm{E}+00$ & 16 & $.0000 \mathrm{E}+00$ \\
\hline 17 & $.0000 \mathrm{E}+00$ & 18 & $.0000 \mathrm{E}+00$ & 19 & $.0000 \mathrm{E}+00$ & 20 & $.0000 \mathrm{E}+00$ & 21 & $.0000 \mathrm{E}+00$ & 22 & $.0000 \mathrm{E}+00$ & 23 & $.0000 \mathrm{E}+00$ & 24 & $.0000 \mathrm{E}+00$ \\
\hline & & & & & MONT & $T H=$ & FEBRUARY ; & DAY & OF WEEK $=\mathrm{S}$ & UNDAY & & & & & \\
\hline 1 & $.0000 \mathrm{E}+00$ & 2 & $.0000 \mathrm{E}+00$ & 3 & $.0000 \mathrm{E}+00$ & 4 & $.0000 \mathrm{E}+00$ & 5 & $.0000 \mathrm{E}+00$ & 6 & $.0000 \mathrm{E}+00$ & 7 & $.0000 \mathrm{E}+00$ & 8 & $.0000 \mathrm{E}+00$ \\
\hline 9 & $.0000 \mathrm{E}+00$ & 10 & $.0000 \mathrm{E}+00$ & 11 & $.0000 \mathrm{E}+00$ & 12 & $.0000 \mathrm{E}+00$ & 13 & $.0000 \mathrm{E}+00$ & 14 & $.0000 \mathrm{E}+00$ & 15 & $.0000 \mathrm{E}+00$ & 16 & $.0000 \mathrm{E}+00$ \\
\hline 17 & $.0000 \mathrm{E}+00$ & 18 & $.0000 \mathrm{E}+00$ & 19 & $.0000 \mathrm{E}+00$ & 20 & $.0000 \mathrm{E}+00$ & 21 & $.0000 \mathrm{E}+00$ & 22 & $.0000 \mathrm{E}+00$ & 23 & $.0000 \mathrm{E}+00$ & 24 & $.0000 \mathrm{E}+00$ \\
\hline & & & & & MONT & $T H=$ & MARCH ; & DAY & OF WEEK $=\mathrm{S}$ & UNDAY & & & & & \\
\hline 1 & $.0000 \mathrm{E}+00$ & 2 & $.0000 \mathrm{E}+00$ & 3 & $.0000 \mathrm{E}+00$ & 4 & $.0000 \mathrm{E}+00$ & 5 & $.0000 \mathrm{E}+00$ & 6 & $.0000 \mathrm{E}+00$ & 7 & $.0000 \mathrm{E}+00$ & 8 & $.0000 \mathrm{E}+00$ \\
\hline 9 & $.0000 \mathrm{E}+00$ & 10 & $.0000 \mathrm{E}+00$ & 11 & $.0000 \mathrm{E}+00$ & 12 & $.0000 E+00$ & 13 & $.0000 \mathrm{E}+00$ & 14 & $.0000 \mathrm{E}+00$ & 15 & $.0000 \mathrm{E}+00$ & 16 & $.0000 \mathrm{E}+00$ \\
\hline 17 & $.0000 \mathrm{E}+00$ & 18 & $.0000 \mathrm{E}+00$ & 19 & $.0000 \mathrm{E}+00$ & 20 & $.0000 \mathrm{E}+00$ & 21 & $.0000 \mathrm{E}+00$ & 22 & $.0000 \mathrm{E}+00$ & 23 & $.0000 \mathrm{E}+00$ & 24 & $.0000 \mathrm{E}+00$ \\
\hline
\end{tabular}

MONTH $=$ APRIL $;$ DAY OF WEEK $=$ SUNDAY 


\begin{tabular}{|c|c|c|c|c|c|c|c|c|c|c|c|c|c|c|c|}
\hline 1 & $.0000 \mathrm{E}+00$ & 2 & $.0000 \mathrm{E}+00$ & 3 & $.0000 \mathrm{E}+00$ & 4 & $.0000 \mathrm{E}+00$ & 5 & $.0000 \mathrm{E}+00$ & 6 & $.0000 \mathrm{E}+00$ & 7 & $.0000 \mathrm{E}+00$ & 8 & $.0000 \mathrm{E}+00$ \\
\hline 9 & $.0000 \mathrm{E}+00$ & 10 & $.0000 \mathrm{E}+00$ & 11 & $.0000 \mathrm{E}+00$ & 12 & $.0000 \mathrm{E}+00$ & 13 & $.0000 \mathrm{E}+00$ & 14 & $.0000 \mathrm{E}+00$ & 15 & $.0000 \mathrm{E}+00$ & 16 & $.0000 \mathrm{E}+00$ \\
\hline 17 & $.0000 \mathrm{E}+00$ & 18 & $.0000 \mathrm{E}+00$ & 19 & $.0000 \mathrm{E}+00$ & 20 & $.0000 \mathrm{E}+00$ & 21 & $.0000 \mathrm{E}+00$ & 22 & $.0000 \mathrm{E}+00$ & 23 & $.0000 E+00$ & 24 & $.0000 \mathrm{E}+00$ \\
\hline & & & & & MON? & & MAY ; & DAY & OF WEEK $=S$ & UNDAY & & & & & \\
\hline 1 & $.0000 \mathrm{E}+00$ & 2 & $.0000 \mathrm{E}+00$ & 3 & $.0000 \mathrm{E}+00$ & 4 & $.0000 \mathrm{E}+00$ & 5 & $.0000 \mathrm{E}+00$ & 6 & $.0000 \mathrm{E}+00$ & 7 & $.0000 \mathrm{E}+00$ & 8 & $.0000 \mathrm{E}+00$ \\
\hline 9 & $.0000 \mathrm{E}+00$ & 10 & $.0000 \mathrm{E}+00$ & 11 & $.0000 \mathrm{E}+00$ & 12 & $.0000 E+00$ & 13 & $.0000 \mathrm{E}+00$ & 14 & $.0000 \mathrm{E}+00$ & 15 & $.0000 \mathrm{E}+00$ & 16 & $.0000 \mathrm{E}+00$ \\
\hline 17 & $.0000 \mathrm{E}+00$ & 18 & $.0000 \mathrm{E}+00$ & 19 & $.0000 \mathrm{E}+00$ & 20 & $.0000 \mathrm{E}+00$ & 21 & $.0000 \mathrm{E}+00$ & 22 & $.0000 \mathrm{E}+00$ & 23 & $.0000 \mathrm{E}+00$ & 24 & $.0000 \mathrm{E}+00$ \\
\hline & & & & & MON' & $\mathrm{H}=$ & JUNE & DAY & $O F$ WEEK $=S$ & UNDAY & & & & & \\
\hline 1 & $.0000 \mathrm{E}+00$ & 2 & $.0000 \mathrm{E}+00$ & 3 & $.0000 \mathrm{E}+00$ & 4 & $.0000 \mathrm{E}+00$ & 5 & $.0000 \mathrm{E}+00$ & 6 & $.0000 \mathrm{E}+00$ & 7 & $.0000 \mathrm{E}+00$ & 8 & $.0000 \mathrm{E}+00$ \\
\hline 9 & $.0000 \mathrm{E}+00$ & 10 & $.0000 \mathrm{E}+00$ & 11 & $.0000 \mathrm{E}+00$ & 12 & $.0000 \mathrm{E}+00$ & 13 & $.0000 \mathrm{E}+00$ & 14 & $.0000 \mathrm{E}+00$ & 15 & $.0000 \mathrm{E}+00$ & 16 & $.0000 \mathrm{E}+00$ \\
\hline 17 & $.0000 \mathrm{E}+00$ & 18 & $.0000 \mathrm{E}+00$ & 19 & $.0000 \mathrm{E}+00$ & 20 & $.0000 \mathrm{E}+00$ & 21 & $.0000 \mathrm{E}+00$ & 22 & $.0000 \mathrm{E}+00$ & 23 & $.0000 \mathrm{E}+00$ & 24 & $.0000 \mathrm{E}+00$ \\
\hline & & & & & MON? & $\mathrm{H}=$ & JULY ; & DAY & OF WEEK $=S$ & UNDAY & & & & & \\
\hline 1 & $.0000 \mathrm{E}+00$ & 2 & $.0000 \mathrm{E}+00$ & 3 & $.0000 \mathrm{E}+00$ & 4 & $.0000 \mathrm{E}+00$ & 5 & $.0000 \mathrm{E}+00$ & 6 & $.0000 \mathrm{E}+00$ & 7 & $.0000 \mathrm{E}+00$ & 8 & $.0000 \mathrm{E}+00$ \\
\hline 9 & $.0000 \mathrm{E}+00$ & 10 & $.0000 \mathrm{E}+00$ & 11 & $.0000 E+00$ & 12 & $.0000 \mathrm{E}+00$ & 13 & $.0000 \mathrm{E}+00$ & 14 & $.0000 \mathrm{E}+00$ & 15 & $.0000 \mathrm{E}+00$ & 16 & $.0000 \mathrm{E}+00$ \\
\hline 17 & $.0000 \mathrm{E}+00$ & 18 & $.0000 \mathrm{E}+00$ & 19 & $.0000 \mathrm{E}+00$ & 20 & $.0000 \mathrm{E}+00$ & 21 & $.0000 \mathrm{E}+00$ & 22 & $.0000 \mathrm{E}+00$ & 23 & $.0000 \mathrm{E}+00$ & 24 & $.0000 \mathrm{E}+00$ \\
\hline & & & & & MON & $\mathrm{H}=$ & AUGUST ; & DAY & $O F$ WEEK $=S$ & UNDAY & & & & & \\
\hline 1 & $.0000 \mathrm{E}+00$ & 2 & $.0000 \mathrm{E}+00$ & 3 & $.0000 \mathrm{E}+00$ & 4 & $.0000 \mathrm{E}+00$ & 5 & $.0000 \mathrm{E}+00$ & 6 & $.0000 \mathrm{E}+00$ & 7 & $.0000 \mathrm{E}+00$ & 8 & $.0000 \mathrm{E}+00$ \\
\hline 9 & $.0000 \mathrm{E}+00$ & 10 & $.0000 \mathrm{E}+00$ & 11 & $.0000 \mathrm{E}+00$ & 12 & $.0000 \mathrm{E}+00$ & 13 & $.0000 \mathrm{E}+00$ & 14 & $.0000 \mathrm{E}+00$ & 15 & $.0000 \mathrm{E}+00$ & 16 & $.0000 \mathrm{E}+00$ \\
\hline 17 & $.0000 \mathrm{E}+00$ & 18 & $.0000 \mathrm{E}+00$ & 19 & $.0000 \mathrm{E}+00$ & 20 & $.0000 \mathrm{E}+00$ & 21 & $.0000 \mathrm{E}+00$ & 22 & $.0000 \mathrm{E}+00$ & 23 & $.0000 \mathrm{E}+00$ & 24 & $.0000 \mathrm{E}+00$ \\
\hline & & & & & MON & $\mathrm{H}=$ & SEPTEMBER; & DAY & $O F$ WEEK $=S$ & UNDAY & & & & & \\
\hline 1 & $.0000 \mathrm{E}+00$ & 2 & $.0000 \mathrm{E}+00$ & 3 & $.0000 \mathrm{E}+00$ & 4 & $.0000 \mathrm{E}+00$ & 5 & $.0000 \mathrm{E}+00$ & 6 & $.0000 \mathrm{E}+00$ & 7 & $.0000 \mathrm{E}+00$ & 8 & $.0000 \mathrm{E}+00$ \\
\hline 9 & $.0000 \mathrm{E}+00$ & 10 & $.0000 \mathrm{E}+00$ & 11 & $.0000 E+00$ & 12 & $.0000 \mathrm{E}+00$ & 13 & $.0000 \mathrm{E}+00$ & 14 & $.0000 \mathrm{E}+00$ & 15 & $.0000 \mathrm{E}+00$ & 16 & $.0000 \mathrm{E}+00$ \\
\hline 17 & $.0000 \mathrm{E}+00$ & 18 & $.0000 \mathrm{E}+00$ & 19 & $.0000 \mathrm{E}+00$ & 20 & $.0000 \mathrm{E}+00$ & 21 & $.0000 \mathrm{E}+00$ & 22 & $.0000 \mathrm{E}+00$ & 23 & $.0000 \mathrm{E}+00$ & 24 & $.0000 \mathrm{E}+00$ \\
\hline & & & & & MON & $\mathrm{I}=$ & OCTOBER ; & DAY & $O F$ WEEK $=S$ & UNDAY & & & & & \\
\hline 1 & $.0000 \mathrm{E}+00$ & 2 & $.0000 \mathrm{E}+00$ & 3 & $.0000 \mathrm{E}+00$ & 4 & $.0000 \mathrm{E}+00$ & 5 & $.0000 \mathrm{E}+00$ & 6 & $.0000 \mathrm{E}+00$ & 7 & $.0000 \mathrm{E}+00$ & 8 & $.0000 \mathrm{E}+00$ \\
\hline 9 & $.0000 \mathrm{E}+00$ & 10 & $.0000 \mathrm{E}+00$ & 11 & $.0000 E+00$ & 12 & $.0000 E+00$ & 13 & $.0000 \mathrm{E}+00$ & 14 & $.0000 \mathrm{E}+00$ & 15 & $.0000 \mathrm{E}+00$ & 16 & $.0000 \mathrm{E}+00$ \\
\hline 17 & $.0000 \mathrm{E}+00$ & 18 & $.0000 \mathrm{E}+00$ & 19 & $.0000 \mathrm{E}+00$ & 20 & $.0000 \mathrm{E}+00$ & 21 & $.0000 \mathrm{E}+00$ & 22 & $.0000 \mathrm{E}+00$ & 23 & $.0000 \mathrm{E}+00$ & 24 & $.0000 \mathrm{E}+00$ \\
\hline & & & & & MON & $\mathrm{H}$ & NOVEMBER ; & DAY & $O F$ WEEK $=S$ & UNDAY & & & & & \\
\hline 1 & $.0000 \mathrm{E}+00$ & 2 & $.0000 \mathrm{E}+00$ & 3 & $.0000 \mathrm{E}+00$ & 4 & $.0000 \mathrm{E}+00$ & 5 & $.0000 \mathrm{E}+00$ & 6 & $.0000 \mathrm{E}+00$ & 7 & $.0000 \mathrm{E}+00$ & 8 & $.0000 \mathrm{E}+00$ \\
\hline 9 & $.0000 \mathrm{E}+00$ & 10 & $.0000 \mathrm{E}+00$ & 11 & $.0000 \mathrm{E}+00$ & 12 & $.0000 \mathrm{E}+00$ & 13 & $.0000 \mathrm{E}+00$ & 14 & $.0000 \mathrm{E}+00$ & 15 & $.0000 \mathrm{E}+00$ & 16 & $.0000 \mathrm{E}+00$ \\
\hline 17 & $.0000 \mathrm{E}+00$ & 18 & $.0000 \mathrm{E}+00$ & 19 & $.0000 \mathrm{E}+00$ & 20 & $.0000 \mathrm{E}+00$ & 21 & $.0000 \mathrm{E}+00$ & 22 & $.0000 \mathrm{E}+00$ & 23 & $.0000 \mathrm{E}+00$ & 24 & $.0000 \mathrm{E}+00$ \\
\hline & & & & & MON & $1=$ & DECEMBER ; & DAY & $O F$ WEEK $=S$ & UNDAY & & & & & \\
\hline 1 & $.0000 \mathrm{E}+00$ & 2 & $.0000 \mathrm{E}+00$ & 3 & $.0000 \mathrm{E}+00$ & 4 & $.0000 \mathrm{E}+00$ & 5 & $.0000 \mathrm{E}+00$ & 6 & $.0000 \mathrm{E}+00$ & 7 & $.0000 \mathrm{E}+00$ & 8 & $.0000 \mathrm{E}+00$ \\
\hline 9 & $.0000 \mathrm{E}+00$ & 10 & $.0000 \mathrm{E}+00$ & 11 & $.0000 \mathrm{E}+00$ & 12 & $.0000 \mathrm{E}+00$ & 13 & $.0000 \mathrm{E}+00$ & 14 & $.0000 \mathrm{E}+00$ & 15 & $.0000 \mathrm{E}+00$ & 16 & $.0000 \mathrm{E}+00$ \\
\hline 17 & $.0000 \mathrm{E}+00$ & 18 & $.0000 \mathrm{E}+00$ & 19 & $.0000 \mathrm{E}+00$ & 20 & $.0000 \mathrm{E}+00$ & 21 & $.0000 \mathrm{E}+00$ & 22 & $.0000 \mathrm{E}+00$ & 23 & $.0000 \mathrm{E}+00$ & 24 & $.0000 \mathrm{E}+00$ \\
\hline
\end{tabular}

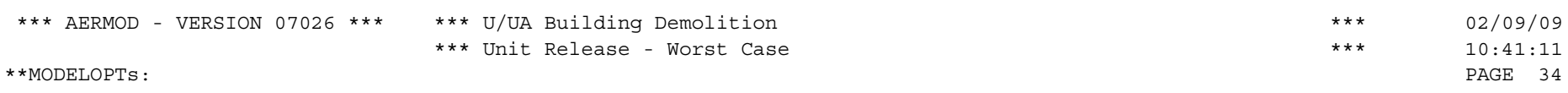

DDEP TOXICS FLAT

DRYDPL WETDPI

* SOURCE EMISSION RATE SCALARS WHICH VARY MONTHLY, DIURNALLY AND BY DAY OF WEEK (MHRDOW7) *

SOURCE ID $=224 U_{-} 4 ;$ SOURCE TYPE $=$ POINT

HOUR SCALAR HOUR SCALAR HOUR SCALAR HOUR SCALAR HOUR SCALAR HOUR SCALAR HOUR SCALAR HOUR SCALAR

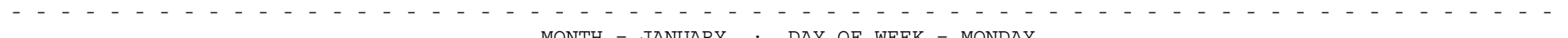

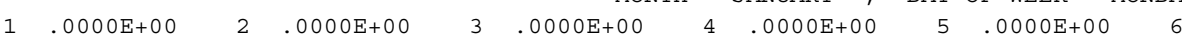

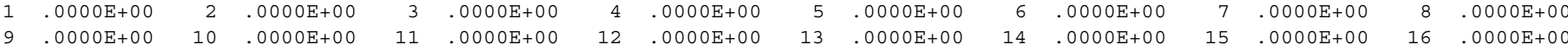

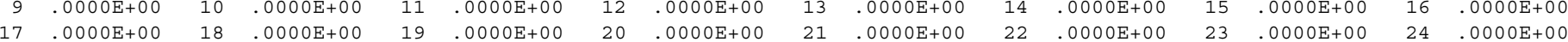

1 MONTH = FEBRUARY $; \quad$ DAY OF WEEK = MONDAY

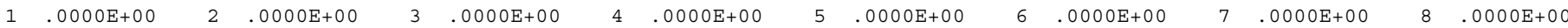




\begin{tabular}{|c|c|c|c|c|c|c|c|c|c|c|c|c|c|c|c|}
\hline 9 & $.0000 \mathrm{E}+00$ & 10 & $.0000 \mathrm{E}+00$ & 11 & $.0000 \mathrm{E}+00$ & 12 & $.0000 \mathrm{E}+00$ & 13 & $.0000 \mathrm{E}+00$ & 14 & $.0000 \mathrm{E}+00$ & 15 & $.0000 \mathrm{E}+00$ & 16 & $.0000 \mathrm{E}+00$ \\
\hline 17 & $.0000 \mathrm{E}+00$ & 18 & $.0000 \mathrm{E}+00$ & 19 & $.0000 \mathrm{E}+00$ & 20 & $.0000 \mathrm{E}+00$ & 21 & $.0000 \mathrm{E}+00$ & 22 & $.0000 \mathrm{E}+00$ & 23 & $.0000 \mathrm{E}+00$ & 24 & $.0000 \mathrm{E}+00$ \\
\hline & & & & & MONTH & $\mathrm{H}=$ & MARCH ; & DAY & OF WEEK = M & IONDAY & & & & & \\
\hline & $.0000 \mathrm{E}+00$ & 2 & $.0000 \mathrm{E}+00$ & 3 & $.0000 \mathrm{E}+00$ & 4 & $.0000 \mathrm{E}+00$ & 5 & $.0000 \mathrm{E}+00$ & 6 & $.0000 \mathrm{E}+00$ & 7 & $.0000 \mathrm{E}+00$ & 8 & $.0000 \mathrm{E}+00$ \\
\hline & $.0000 \mathrm{E}+00$ & 10 & $.0000 \mathrm{E}+00$ & 11 & $.0000 \mathrm{E}+00$ & 12 & $.0000 \mathrm{E}+00$ & 13 & $.0000 \mathrm{E}+00$ & 14 & $.0000 \mathrm{E}+00$ & 15 & $.0000 E+00$ & 16 & $.0000 \mathrm{E}+00$ \\
\hline 17 & $.0000 \mathrm{E}+00$ & 18 & $.0000 \mathrm{E}+00$ & 19 & $.0000 \mathrm{E}+00$ & 20 & $.0000 \mathrm{E}+00$ & 21 & $.0000 \mathrm{E}+00$ & 22 & $.0000 \mathrm{E}+00$ & 23 & $.0000 \mathrm{E}+00$ & 24 & $.0000 \mathrm{E}+00$ \\
\hline & & & & & MONTE & $H=$ & APRIL ; & DAY & OF $\mathrm{WEEK}=\mathrm{M}$ & ONDAY & & & & & \\
\hline & $.0000 \mathrm{E}+00$ & 2 & $.0000 \mathrm{E}+00$ & 3 & $.0000 \mathrm{E}+00$ & 4 & $.0000 \mathrm{E}+00$ & 5 & $.0000 \mathrm{E}+00$ & 6 & $.0000 \mathrm{E}+00$ & 7 & $.0000 \mathrm{E}+00$ & 8 & $.0000 \mathrm{E}+00$ \\
\hline & $.0000 \mathrm{E}+00$ & 10 & $.0000 \mathrm{E}+00$ & 11 & $.0000 \mathrm{E}+00$ & 12 & $.0000 \mathrm{E}+00$ & 13 & $.0000 \mathrm{E}+00$ & 14 & $.0000 \mathrm{E}+00$ & 15 & $.0000 \mathrm{E}+00$ & 16 & $.0000 \mathrm{E}+00$ \\
\hline 17 & $.0000 \mathrm{E}+00$ & 18 & $.0000 \mathrm{E}+00$ & 19 & $.0000 \mathrm{E}+00$ & 20 & $.0000 \mathrm{E}+00$ & 21 & $.0000 \mathrm{E}+00$ & 22 & $.0000 \mathrm{E}+00$ & 23 & $.0000 \mathrm{E}+00$ & 24 & $.0000 \mathrm{E}+00$ \\
\hline & & & & & MONTH & $H=$ & MAY ; & DAY & OF $\mathrm{WEEK}=\mathrm{M}$ & IONDAY & & & & & \\
\hline & $.0000 \mathrm{E}+00$ & 2 & $.0000 \mathrm{E}+00$ & 3 & $.0000 \mathrm{E}+00$ & 4 & $.0000 \mathrm{E}+00$ & 5 & $.0000 \mathrm{E}+00$ & 6 & $.0000 E+00$ & 7 & $.0000 \mathrm{E}+00$ & 8 & $.0000 \mathrm{E}+00$ \\
\hline & $.0000 \mathrm{E}+00$ & 10 & $.0000 \mathrm{E}+00$ & 11 & $.0000 \mathrm{E}+00$ & 12 & $.0000 \mathrm{E}+00$ & 13 & $.0000 \mathrm{E}+00$ & 14 & $.0000 \mathrm{E}+00$ & 15 & $.0000 \mathrm{E}+00$ & 16 & $.0000 \mathrm{E}+00$ \\
\hline 17 & $.0000 \mathrm{E}+00$ & 18 & $.0000 \mathrm{E}+00$ & 19 & $.0000 \mathrm{E}+00$ & 20 & $.0000 \mathrm{E}+00$ & 21 & $.0000 \mathrm{E}+00$ & 22 & $.0000 \mathrm{E}+00$ & 23 & $.0000 \mathrm{E}+00$ & 24 & $.0000 \mathrm{E}+00$ \\
\hline & & & & & MONTH & $\mathrm{H}=$ & JUNE ; & DAY & OF WEEK = M & IONDAY & & & & & \\
\hline & $.0000 \mathrm{E}+00$ & 2 & $.0000 \mathrm{E}+00$ & 3 & $.0000 \mathrm{E}+00$ & 4 & $.0000 \mathrm{E}+00$ & 5 & $.0000 \mathrm{E}+00$ & 6 & $.0000 \mathrm{E}+00$ & 7 & $.0000 \mathrm{E}+00$ & 8 & $.0000 \mathrm{E}+00$ \\
\hline & $.0000 \mathrm{E}+00$ & 10 & $.0000 \mathrm{E}+00$ & 11 & $.0000 \mathrm{E}+00$ & 12 & $.0000 \mathrm{E}+00$ & 13 & $.0000 \mathrm{E}+00$ & 14 & $.0000 \mathrm{E}+00$ & 15 & $.0000 \mathrm{E}+00$ & 16 & $.0000 \mathrm{E}+00$ \\
\hline 17 & $.0000 \mathrm{E}+00$ & 18 & $.0000 \mathrm{E}+00$ & 19 & $.0000 \mathrm{E}+00$ & 20 & $.0000 \mathrm{E}+00$ & 21 & $.0000 \mathrm{E}+00$ & 22 & $.0000 \mathrm{E}+00$ & 23 & $.0000 \mathrm{E}+00$ & 24 & $.0000 \mathrm{E}+00$ \\
\hline & & & & & MONTF & $\mathrm{H}=$ & JULY ; & DAY & OF $\mathrm{WEEK}=\mathrm{M}$ & IONDAY & & & & & \\
\hline & $.0000 \mathrm{E}+00$ & 2 & $.0000 \mathrm{E}+00$ & 3 & $.0000 \mathrm{E}+00$ & 4 & $.0000 \mathrm{E}+00$ & 5 & $.0000 \mathrm{E}+00$ & 6 & $.0000 \mathrm{E}+00$ & 7 & $.0000 \mathrm{E}+00$ & 8 & $.0000 \mathrm{E}+00$ \\
\hline & $.0000 \mathrm{E}+00$ & 10 & $.0000 \mathrm{E}+00$ & 11 & $.0000 \mathrm{E}+00$ & 12 & $.0000 \mathrm{E}+00$ & 13 & $.0000 \mathrm{E}+00$ & 14 & $.0000 \mathrm{E}+00$ & 15 & $.0000 \mathrm{E}+00$ & 16 & $.0000 \mathrm{E}+00$ \\
\hline 17 & $.0000 \mathrm{E}+00$ & 18 & $.0000 \mathrm{E}+00$ & 19 & $.0000 \mathrm{E}+00$ & 20 & $.0000 \mathrm{E}+00$ & 21 & $.0000 \mathrm{E}+00$ & 22 & $.0000 \mathrm{E}+00$ & 23 & $.0000 \mathrm{E}+00$ & 24 & $.0000 \mathrm{E}+00$ \\
\hline & & & & & MONTF & $\mathrm{H}=$ & AUGUST ; & DAY & OF $\mathrm{WEEK}=\mathrm{M}$ & IONDAY & & & & & \\
\hline & $.0000 \mathrm{E}+00$ & 2 & $.0000 \mathrm{E}+00$ & 3 & $.0000 \mathrm{E}+00$ & 4 & $.0000 \mathrm{E}+00$ & 5 & $.0000 \mathrm{E}+00$ & 6 & $.0000 \mathrm{E}+00$ & 7 & $.0000 \mathrm{E}+00$ & 8 & $.0000 \mathrm{E}+00$ \\
\hline & $.0000 \mathrm{E}+00$ & 10 & $.0000 \mathrm{E}+00$ & 11 & $.0000 \mathrm{E}+00$ & 12 & $.0000 \mathrm{E}+00$ & 13 & $.0000 \mathrm{E}+00$ & 14 & $.0000 \mathrm{E}+00$ & 15 & $.0000 \mathrm{E}+00$ & 16 & $.0000 \mathrm{E}+00$ \\
\hline 17 & $.0000 \mathrm{E}+00$ & 18 & $.0000 \mathrm{E}+00$ & 19 & $.0000 \mathrm{E}+00$ & 20 & $.0000 \mathrm{E}+00$ & 21 & $.0000 \mathrm{E}+00$ & 22 & $.0000 \mathrm{E}+00$ & 23 & $.0000 \mathrm{E}+00$ & 24 & $.0000 \mathrm{E}+00$ \\
\hline & & & & & MONTE & $H=$ & SEPTEMBER； & DAY & OF WEEK $=M$ & IONDAY & & & & & \\
\hline & $.0000 \mathrm{E}+00$ & 2 & $.0000 \mathrm{E}+00$ & 3 & $.0000 \mathrm{E}+00$ & 4 & $.0000 \mathrm{E}+00$ & 5 & $.0000 \mathrm{E}+00$ & 6 & $.0000 \mathrm{E}+00$ & 7 & $.0000 \mathrm{E}+00$ & 8 & $.0000 \mathrm{E}+00$ \\
\hline 9 & $.0000 \mathrm{E}+00$ & 10 & $.0000 \mathrm{E}+00$ & 11 & $.0000 \mathrm{E}+00$ & 12 & $.0000 \mathrm{E}+00$ & 13 & $.0000 \mathrm{E}+00$ & 14 & $.0000 \mathrm{E}+00$ & 15 & $.0000 \mathrm{E}+00$ & 16 & $.0000 \mathrm{E}+00$ \\
\hline 17 & $.0000 \mathrm{E}+00$ & 18 & $.0000 \mathrm{E}+00$ & 19 & $.0000 \mathrm{E}+00$ & 20 & $.0000 \mathrm{E}+00$ & 21 & $.0000 \mathrm{E}+00$ & 22 & $.0000 \mathrm{E}+00$ & 23 & $.0000 \mathrm{E}+00$ & 24 & $.0000 \mathrm{E}+00$ \\
\hline & & & & & MONTF & $\mathrm{H}=$ & OCTOBER ; & DAY & OF $\mathrm{WEEK}=\mathrm{M}$ & IONDAY & & & & & \\
\hline & $.0000 \mathrm{E}+00$ & 2 & $.0000 \mathrm{E}+00$ & 3 & $.0000 \mathrm{E}+00$ & 4 & $.0000 \mathrm{E}+00$ & 5 & $.0000 \mathrm{E}+00$ & 6 & $.0000 \mathrm{E}+00$ & 7 & $.0000 \mathrm{E}+00$ & 8 & $.1000 \mathrm{E}+01$ \\
\hline & $.1000 \mathrm{E}+01$ & 10 & $.1000 \mathrm{E}+01$ & 11 & $.1000 \mathrm{E}+01$ & 12 & $.0000 \mathrm{E}+00$ & 13 & $.1000 \mathrm{E}+01$ & 14 & $.1000 \mathrm{E}+01$ & 15 & $.1000 \mathrm{E}+01$ & 16 & $.1000 \mathrm{E}+01$ \\
\hline 17 & $.0000 \mathrm{E}+00$ & 18 & $.0000 \mathrm{E}+00$ & 19 & $.0000 \mathrm{E}+00$ & 20 & $.0000 \mathrm{E}+00$ & 21 & $.0000 \mathrm{E}+00$ & 22 & $.0000 \mathrm{E}+00$ & 23 & $.0000 \mathrm{E}+00$ & 24 & $.0000 \mathrm{E}+00$ \\
\hline & & & & & MONTH & $H=$ & NOVEMBER ; & DAY & OF $\quad \mathrm{WEEK}=\mathrm{M}$ & IONDAY & & & & & \\
\hline & $.0000 \mathrm{E}+00$ & 2 & $.0000 \mathrm{E}+00$ & 3 & $.0000 \mathrm{E}+00$ & 4 & $.0000 \mathrm{E}+00$ & 5 & $.0000 \mathrm{E}+00$ & 6 & $.0000 \mathrm{E}+00$ & 7 & $.0000 \mathrm{E}+00$ & 8 & $.1000 \mathrm{E}+01$ \\
\hline & $.1000 \mathrm{E}+01$ & 10 & $.1000 \mathrm{E}+01$ & 11 & $.1000 \mathrm{E}+01$ & 12 & $.0000 \mathrm{E}+00$ & 13 & $.1000 \mathrm{E}+01$ & 14 & $.1000 \mathrm{E}+01$ & 15 & $.1000 \mathrm{E}+01$ & 16 & $.1000 \mathrm{E}+01$ \\
\hline 17 & $.0000 \mathrm{E}+00$ & 18 & $.0000 \mathrm{E}+00$ & 19 & $.0000 \mathrm{E}+00$ & 20 & $.0000 \mathrm{E}+00$ & 21 & $.0000 \mathrm{E}+00$ & 22 & $.0000 \mathrm{E}+00$ & 23 & $.0000 \mathrm{E}+00$ & 24 & $.0000 \mathrm{E}+00$ \\
\hline & & & & & MONTH & $\mathrm{H}=$ & DECEMBER ; & DAY & OF $\mathrm{WEEK}=\mathrm{M}$ & IONDAY & & & & & \\
\hline & $.0000 \mathrm{E}+00$ & 2 & $.0000 \mathrm{E}+00$ & 3 & $.0000 E+00$ & 4 & $.0000 \mathrm{E}+00$ & 5 & $.0000 \mathrm{E}+00$ & 6 & $.0000 \mathrm{E}+00$ & 7 & $.0000 \mathrm{E}+00$ & 8 & $.1000 \mathrm{E}+01$ \\
\hline 9 & $.1000 \mathrm{E}+01$ & 10 & $.1000 \mathrm{E}+01$ & 11 & $.1000 \mathrm{E}+01$ & 12 & $.0000 \mathrm{E}+00$ & 13 & $.1000 \mathrm{E}+01$ & 14 & $.1000 \mathrm{E}+01$ & 15 & $.1000 \mathrm{E}+01$ & 16 & $.1000 \mathrm{E}+01$ \\
\hline 17 & $.0000 \mathrm{E}+00$ & 18 & $.0000 \mathrm{E}+00$ & 19 & $.0000 \mathrm{E}+00$ & 20 & $.0000 \mathrm{E}+00$ & 21 & $.0000 \mathrm{E}+00$ & 22 & $.0000 \mathrm{E}+00$ & 23 & $.0000 \mathrm{E}+00$ & 24 & $.0000 \mathrm{E}+00$ \\
\hline & AERMOD - VE & ION & $07026 * * *$ & $* * *$ & * U/UA Buildi & ing & Demolition & & & & & & $* * *$ & & $02 / 09 / 09$ \\
\hline & & & & & Unit Releas & & Worst Case & & & & & & $* \star *$ & & $10: 41: 11$ \\
\hline & DDELOPTS: & & & & & & & & & & & & & & PAGE 35 \\
\hline
\end{tabular}

* source emission rate scalars which vary monthly, diURnally and by day of Week (MHrdow7) *

SOURCE ID $=224 \mathrm{U}_{-} 4 ;$ SOURCE TYPE $=$ POINT

HOUR SCALAR HOUR SCALAR HOUR SCALAR HOUR SCALAR HOUR SCALAR HOUR SCALAR HOUR SCALAR HOUR SCALAR 


\begin{tabular}{|c|c|c|c|c|c|c|c|c|c|c|c|c|c|c|c|}
\hline & & & & & & & JANUARY ; & $\mathrm{AY}$ & OF WEEK = T & UESDAY & & & & & \\
\hline 1 & $.0000 \mathrm{E}+00$ & 2 & $.0000 \mathrm{E}+00$ & 3 & $.0000 \mathrm{E}+00$ & 4 & $.0000 \mathrm{E}+00$ & 5 & $.0000 \mathrm{E}+00$ & 6 & $.0000 \mathrm{E}+00$ & 7 & $.0000 \mathrm{E}+00$ & 8 & $.0000 \mathrm{E}+00$ \\
\hline 9 & $.0000 E+00$ & 10 & $.0000 E+00$ & 11 & $.0000 \mathrm{E}+00$ & 12 & $.0000 E+00$ & 13 & $.0000 \mathrm{E}+00$ & 14 & $.0000 \mathrm{E}+00$ & 15 & $.0000 E+00$ & 16 & $.0000 \mathrm{E}+00$ \\
\hline 17 & $.0000 \mathrm{E}+00$ & 18 & $.0000 \mathrm{E}+00$ & 19 & $.0000 \mathrm{E}+00$ & 20 & $.0000 \mathrm{E}+00$ & 21 & $.0000 \mathrm{E}+00$ & 22 & $.0000 \mathrm{E}+00$ & 23 & $.0000 \mathrm{E}+00$ & 24 & $.0000 \mathrm{E}+00$ \\
\hline & & & & & MON & $\mathrm{H}=$ & FEBRUARY ; & DAY & OF WEEK = T & UESDAY & & & & & \\
\hline 1 & $.0000 E+00$ & 2 & $.0000 \mathrm{E}+00$ & 3 & $.0000 \mathrm{E}+00$ & 4 & $.0000 \mathrm{E}+00$ & 5 & $.0000 \mathrm{E}+00$ & 6 & $.0000 \mathrm{E}+00$ & 7 & $.0000 E+00$ & 8 & $.0000 \mathrm{E}+00$ \\
\hline 9 & $.0000 \mathrm{E}+00$ & 10 & $.0000 \mathrm{E}+00$ & 11 & $.0000 \mathrm{E}+00$ & 12 & $.0000 \mathrm{E}+00$ & 13 & $.0000 \mathrm{E}+00$ & 14 & $.0000 \mathrm{E}+00$ & 15 & $.0000 \mathrm{E}+00$ & 16 & $.0000 \mathrm{E}+00$ \\
\hline 17 & $.0000 \mathrm{E}+00$ & 18 & $.0000 \mathrm{E}+00$ & 19 & $.0000 \mathrm{E}+00$ & 20 & $.0000 \mathrm{E}+00$ & 21 & $.0000 \mathrm{E}+00$ & 22 & $.0000 \mathrm{E}+00$ & 23 & $.0000 \mathrm{E}+00$ & 24 & $.0000 \mathrm{E}+00$ \\
\hline & & & & & MON & $\mathrm{H}=$ & MARCH ; & DAY & OF WEEK $=\mathrm{T}$ & UESDAY & & & & & \\
\hline 1 & $.0000 \mathrm{E}+00$ & 2 & $.0000 \mathrm{E}+00$ & 3 & $.0000 \mathrm{E}+00$ & 4 & $.0000 \mathrm{E}+00$ & 5 & $.0000 \mathrm{E}+00$ & 6 & $.0000 \mathrm{E}+00$ & 7 & $.0000 \mathrm{E}+00$ & 8 & $.0000 \mathrm{E}+00$ \\
\hline 9 & $.0000 \mathrm{E}+00$ & 10 & $.0000 \mathrm{E}+00$ & 11 & $.0000 \mathrm{E}+00$ & 12 & $.0000 \mathrm{E}+00$ & 13 & $.0000 \mathrm{E}+00$ & 14 & $.0000 \mathrm{E}+00$ & 15 & $.0000 \mathrm{E}+00$ & 16 & $.0000 \mathrm{E}+00$ \\
\hline 17 & $.0000 \mathrm{E}+00$ & 18 & $.0000 \mathrm{E}+00$ & 19 & $.0000 \mathrm{E}+00$ & 20 & $.0000 \mathrm{E}+00$ & 21 & $.0000 \mathrm{E}+00$ & 22 & $.0000 \mathrm{E}+00$ & 23 & $.0000 \mathrm{E}+00$ & 24 & $.0000 \mathrm{E}+00$ \\
\hline & & & & & MON & $\mathrm{H}=$ & APRIL ; & DAY & OF WEEK = T & UESDAY & & & & & \\
\hline 1 & $.0000 \mathrm{E}+00$ & 2 & $.0000 \mathrm{E}+00$ & 3 & $.0000 \mathrm{E}+00$ & 4 & $.0000 \mathrm{E}+00$ & 5 & $.0000 \mathrm{E}+00$ & 6 & $.0000 \mathrm{E}+00$ & 7 & $.0000 \mathrm{E}+00$ & 8 & $.0000 \mathrm{E}+00$ \\
\hline 9 & $.0000 \mathrm{E}+00$ & 10 & $.0000 \mathrm{E}+00$ & 11 & $.0000 \mathrm{E}+00$ & 12 & $.0000 \mathrm{E}+00$ & 13 & $.0000 \mathrm{E}+00$ & 14 & $.0000 \mathrm{E}+00$ & 15 & $.0000 \mathrm{E}+00$ & 16 & $.0000 \mathrm{E}+00$ \\
\hline 17 & $.0000 \mathrm{E}+00$ & 18 & $.0000 \mathrm{E}+00$ & 19 & $.0000 \mathrm{E}+00$ & 20 & $.0000 \mathrm{E}+00$ & 21 & $.0000 \mathrm{E}+00$ & 22 & $.0000 \mathrm{E}+00$ & 23 & $.0000 \mathrm{E}+00$ & 24 & $.0000 \mathrm{E}+00$ \\
\hline & & & & & MON & $\mathrm{H}=$ & MAY ; & DAY & OF WEEK = T & UESDAY & & & & & \\
\hline 1 & $.0000 \mathrm{E}+00$ & 2 & $.0000 \mathrm{E}+00$ & 3 & $.0000 \mathrm{E}+00$ & 4 & $.0000 \mathrm{E}+00$ & 5 & $.0000 \mathrm{E}+00$ & 6 & $.0000 \mathrm{E}+00$ & 7 & $.0000 \mathrm{E}+00$ & 8 & $.0000 \mathrm{E}+00$ \\
\hline 9 & $.0000 \mathrm{E}+00$ & 10 & $.0000 \mathrm{E}+00$ & 11 & $.0000 \mathrm{E}+00$ & 12 & $.0000 \mathrm{E}+00$ & 13 & $.0000 \mathrm{E}+00$ & 14 & $.0000 \mathrm{E}+00$ & 15 & $.0000 \mathrm{E}+00$ & 16 & $.0000 \mathrm{E}+00$ \\
\hline 17 & $.0000 \mathrm{E}+00$ & 18 & $.0000 \mathrm{E}+00$ & 19 & $.0000 \mathrm{E}+00$ & 20 & $.0000 \mathrm{E}+00$ & 21 & $.0000 \mathrm{E}+00$ & 22 & $.0000 \mathrm{E}+00$ & 23 & $.0000 \mathrm{E}+00$ & 24 & $.0000 \mathrm{E}+00$ \\
\hline & & & & & MON & $\mathrm{H}=$ & JUNE ; & DAY & OF WEEK $=T$ & UESDAY & & & & & \\
\hline 1 & $.0000 \mathrm{E}+00$ & 2 & $.0000 \mathrm{E}+00$ & 3 & $.0000 \mathrm{E}+00$ & 4 & $.0000 \mathrm{E}+00$ & 5 & $.0000 \mathrm{E}+00$ & 6 & $.0000 \mathrm{E}+00$ & 7 & $.0000 \mathrm{E}+00$ & 8 & $.0000 \mathrm{E}+00$ \\
\hline 9 & $.0000 \mathrm{E}+00$ & 10 & $.0000 \mathrm{E}+00$ & 11 & $.0000 \mathrm{E}+00$ & 12 & $.0000 \mathrm{E}+00$ & 13 & $.0000 \mathrm{E}+00$ & 14 & $.0000 \mathrm{E}+00$ & 15 & $.0000 E+00$ & 16 & $.0000 \mathrm{E}+00$ \\
\hline 17 & $.0000 \mathrm{E}+00$ & 18 & $.0000 \mathrm{E}+00$ & 19 & $.0000 \mathrm{E}+00$ & 20 & $.0000 \mathrm{E}+00$ & 21 & $.0000 \mathrm{E}+00$ & 22 & $.0000 \mathrm{E}+00$ & 23 & $.0000 \mathrm{E}+00$ & 24 & $.0000 \mathrm{E}+00$ \\
\hline & & & & & MON & $\mathrm{H}=$ & JULY ; & DAY & OF WEEK $=T$ & UESDAY & & & & & \\
\hline 1 & $.0000 \mathrm{E}+00$ & 2 & $.0000 \mathrm{E}+00$ & 3 & $.0000 \mathrm{E}+00$ & 4 & $.0000 \mathrm{E}+00$ & 5 & $.0000 \mathrm{E}+00$ & 6 & $.0000 \mathrm{E}+00$ & 7 & $.0000 \mathrm{E}+00$ & 8 & $.0000 \mathrm{E}+00$ \\
\hline 9 & $.0000 \mathrm{E}+00$ & 10 & $.0000 \mathrm{E}+00$ & 11 & $.0000 \mathrm{E}+00$ & 12 & $.0000 \mathrm{E}+00$ & 13 & $.0000 \mathrm{E}+00$ & 14 & $.0000 \mathrm{E}+00$ & 15 & $.0000 \mathrm{E}+00$ & 16 & $.0000 \mathrm{E}+00$ \\
\hline 17 & $.0000 \mathrm{E}+00$ & 18 & $.0000 \mathrm{E}+00$ & 19 & $.0000 \mathrm{E}+00$ & 20 & $.0000 \mathrm{E}+00$ & 21 & $.0000 \mathrm{E}+00$ & 22 & $.0000 \mathrm{E}+00$ & 23 & $.0000 \mathrm{E}+00$ & 24 & $.0000 E+00$ \\
\hline & & & & & MON & $\mathrm{H}=$ & AUGUST ; & DAY & OF WEEK $=T$ & UESDAY & & & & & \\
\hline 1 & $.0000 \mathrm{E}+00$ & 2 & $.0000 \mathrm{E}+00$ & 3 & $.0000 \mathrm{E}+00$ & 4 & $.0000 \mathrm{E}+00$ & 5 & $.0000 \mathrm{E}+00$ & 6 & $.0000 \mathrm{E}+00$ & 7 & $.0000 \mathrm{E}+00$ & 8 & $.0000 \mathrm{E}+00$ \\
\hline 9 & $.0000 \mathrm{E}+00$ & 10 & $.0000 \mathrm{E}+00$ & 11 & $.0000 \mathrm{E}+00$ & 12 & $.0000 \mathrm{E}+00$ & 13 & $.0000 \mathrm{E}+00$ & 14 & $.0000 \mathrm{E}+00$ & 15 & $.0000 \mathrm{E}+00$ & 16 & $.0000 \mathrm{E}+00$ \\
\hline 17 & $.0000 \mathrm{E}+00$ & 18 & $.0000 \mathrm{E}+00$ & 19 & $.0000 \mathrm{E}+00$ & 20 & $.0000 \mathrm{E}+00$ & 21 & $.0000 \mathrm{E}+00$ & 22 & $.0000 \mathrm{E}+00$ & 23 & $.0000 \mathrm{E}+00$ & 24 & $.0000 \mathrm{E}+00$ \\
\hline & & & & & MON & & SEPTEMBER ; & DAY & OF WEEK = T & UESDAY & & & & & \\
\hline 1 & $.0000 \mathrm{E}+00$ & 2 & $.0000 \mathrm{E}+00$ & 3 & $.0000 \mathrm{E}+00$ & 4 & $.0000 \mathrm{E}+00$ & 5 & $.0000 \mathrm{E}+00$ & 6 & $.0000 \mathrm{E}+00$ & 7 & $.0000 \mathrm{E}+00$ & 8 & $.0000 \mathrm{E}+00$ \\
\hline 9 & $.0000 \mathrm{E}+00$ & 10 & $.0000 \mathrm{E}+00$ & 11 & $.0000 \mathrm{E}+00$ & 12 & $.0000 \mathrm{E}+00$ & 13 & $.0000 \mathrm{E}+00$ & 14 & $.0000 \mathrm{E}+00$ & 15 & $.0000 \mathrm{E}+00$ & 16 & $.0000 \mathrm{E}+00$ \\
\hline 17 & $.0000 \mathrm{E}+00$ & 18 & $.0000 \mathrm{E}+00$ & 19 & $.0000 \mathrm{E}+00$ & 20 & $.0000 \mathrm{E}+00$ & 21 & $.0000 \mathrm{E}+00$ & 22 & $.0000 \mathrm{E}+00$ & 23 & $.0000 \mathrm{E}+00$ & 24 & $.0000 \mathrm{E}+00$ \\
\hline & & & & & MON & $\mathrm{H}=$ & OCTOBER ; & DAY & OF WEEK = T & UESDAY & & & & & \\
\hline 1 & $.0000 \mathrm{E}+00$ & 2 & $.0000 \mathrm{E}+00$ & 3 & $.0000 \mathrm{E}+00$ & 4 & $.0000 \mathrm{E}+00$ & 5 & $.0000 \mathrm{E}+00$ & 6 & $.0000 \mathrm{E}+00$ & 7 & $.0000 \mathrm{E}+00$ & 8 & $.1000 \mathrm{E}+01$ \\
\hline 9 & $.1000 \mathrm{E}+01$ & 10 & $.1000 \mathrm{E}+01$ & 11 & $.1000 \mathrm{E}+01$ & 12 & $.0000 \mathrm{E}+00$ & 13 & $.1000 \mathrm{E}+01$ & 14 & $.1000 \mathrm{E}+01$ & 15 & $.1000 \mathrm{E}+01$ & 16 & $.1000 \mathrm{E}+01$ \\
\hline 17 & $.0000 \mathrm{E}+00$ & 18 & $.0000 \mathrm{E}+00$ & 19 & $.0000 \mathrm{E}+00$ & 20 & $.0000 \mathrm{E}+00$ & 21 & $.0000 \mathrm{E}+00$ & 22 & $.0000 \mathrm{E}+00$ & 23 & $.0000 \mathrm{E}+00$ & 24 & $.0000 \mathrm{E}+00$ \\
\hline & & & & & MON & $\mathrm{H}=$ & NOVEMBER ; & DAY & OF WEEK $=T$ & UESDAY & & & & & \\
\hline 1 & $.0000 \mathrm{E}+00$ & 2 & $.0000 \mathrm{E}+00$ & 3 & $.0000 \mathrm{E}+00$ & 4 & $.0000 \mathrm{E}+00$ & 5 & $.0000 \mathrm{E}+00$ & 6 & $.0000 \mathrm{E}+00$ & 7 & $.0000 \mathrm{E}+00$ & 8 & $.1000 \mathrm{E}+01$ \\
\hline 9 & $.1000 \mathrm{E}+01$ & 10 & $.1000 \mathrm{E}+01$ & 11 & $.1000 \mathrm{E}+01$ & 12 & $.0000 \mathrm{E}+00$ & 13 & $.1000 \mathrm{E}+01$ & 14 & $.1000 \mathrm{E}+01$ & 15 & $.1000 \mathrm{E}+01$ & 16 & $.1000 \mathrm{E}+01$ \\
\hline 17 & $.0000 \mathrm{E}+00$ & 18 & $.0000 \mathrm{E}+00$ & 19 & $.0000 \mathrm{E}+00$ & 20 & $.0000 \mathrm{E}+00$ & 21 & $.0000 \mathrm{E}+00$ & 22 & $.0000 \mathrm{E}+00$ & 23 & $.0000 \mathrm{E}+00$ & 24 & $.0000 \mathrm{E}+00$ \\
\hline & & & & & MON & & DECEMBER ; & DAY & OF WEEK $=T$ & UESDAY & & & & & \\
\hline 1 & $.0000 \mathrm{E}+00$ & 2 & $.0000 \mathrm{E}+00$ & 3 & $.0000 \mathrm{E}+00$ & 4 & $.0000 \mathrm{E}+00$ & 5 & $.0000 \mathrm{E}+00$ & 6 & $.0000 \mathrm{E}+00$ & 7 & $.0000 \mathrm{E}+00$ & 8 & $.1000 \mathrm{E}+01$ \\
\hline 9 & $.1000 \mathrm{E}+01$ & 10 & $.1000 \mathrm{E}+01$ & 11 & $.1000 \mathrm{E}+01$ & 12 & $.0000 \mathrm{E}+00$ & 13 & $.1000 \mathrm{E}+01$ & 14 & $.1000 \mathrm{E}+01$ & 15 & $.1000 \mathrm{E}+01$ & 16 & $.1000 \mathrm{E}+01$ \\
\hline 17 & $.0000 \mathrm{E}+00$ & 18 & $.0000 \mathrm{E}+00$ & 19 & $.0000 \mathrm{E}+00$ & 20 & $.0000 \mathrm{E}+00$ & 21 & $.0000 \mathrm{E}+00$ & 22 & $.0000 \mathrm{E}+00$ & 23 & $.0000 \mathrm{E}+00$ & 24 & $.0000 \mathrm{E}+00$ \\
\hline
\end{tabular}

*** AERMOD - VERSION $07026 * * * \quad * * *$ U/UA Building Demolition

*** Unit Release - Worst Case 
* SOURCE EMISSION RATE SCALARS WHICH VARY MONTHLY, DIURNALLY AND BY DAY OF WEEK (MHRDOW7) *

SOURCE ID $=224 \mathrm{U} 4 ;$ SOURCE TYPE $=$ POINT

HOUR SCATAR - HOUR SCATAR HOUR SCALAR HOUR SCALAR HOUR SCALAR HOUR SCALAR HOUR SCALAR HOUR SCALAR HOUR SCALAR HOUR SCALAR HOUR SCALAR HOUR SCALAR HOUR SCALAR HOUR SCALAR HOUR SCALAR HOUR SCALAR

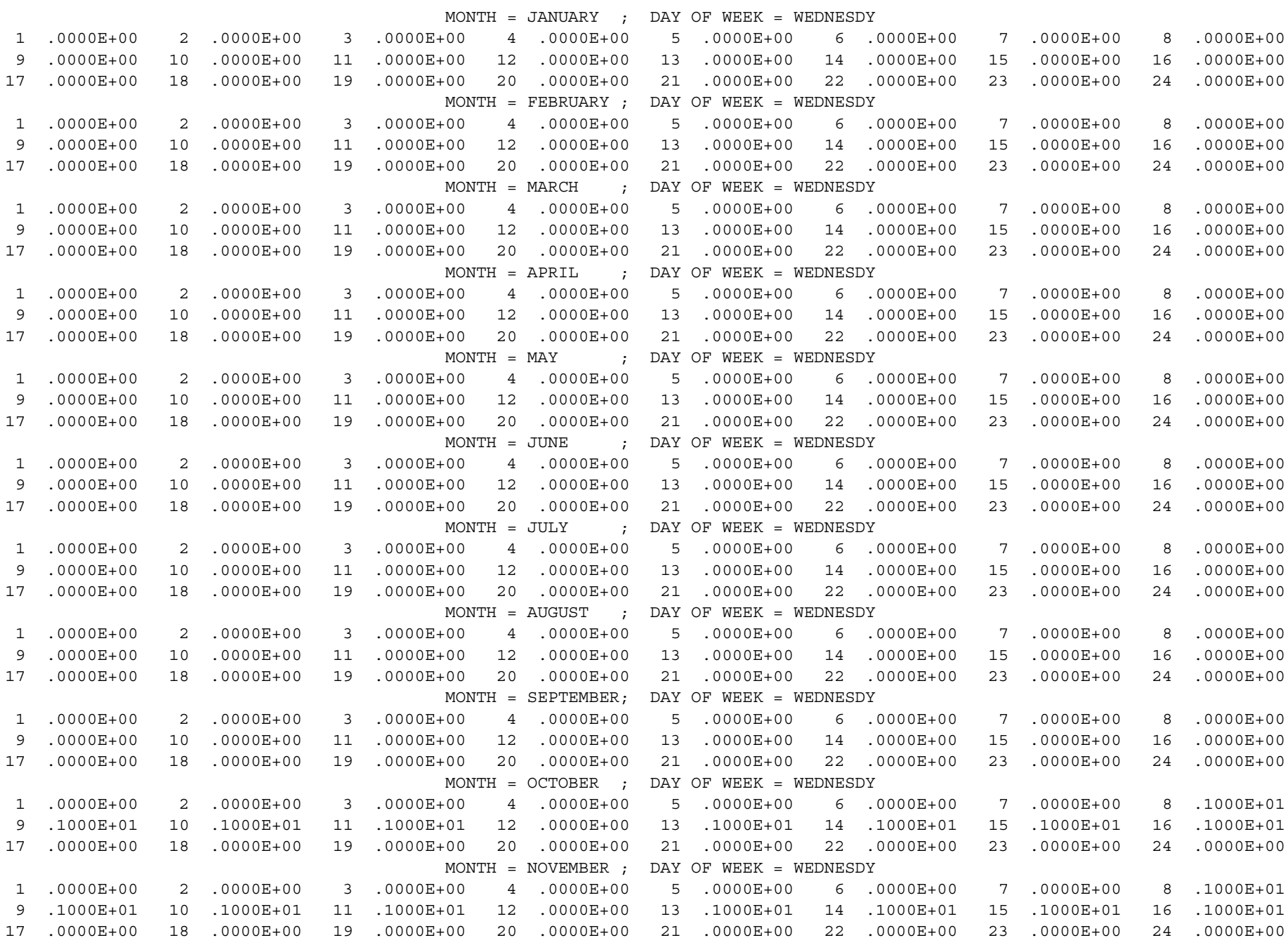
MONTH $=$ DECEMBER ; DAY OF WEEK $=$ WEDNESDY 


\begin{tabular}{|c|c|c|c|c|c|c|c|c|c|c|c|c|c|c|c|}
\hline 1 & $.0000 \mathrm{E}+00$ & 2 & $.0000 \mathrm{E}+00$ & 3 & $.0000 \mathrm{E}+00$ & 4 & $.0000 \mathrm{E}+00$ & 5 & $.0000 \mathrm{E}+00$ & 6 & $.0000 \mathrm{E}+00$ & 7 & $.0000 \mathrm{E}+00$ & 8 & $.1000 \mathrm{E}+01$ \\
\hline 9 & $.1000 \mathrm{E}+01$ & 10 & $.1000 \mathrm{E}+01$ & 11 & $.1000 \mathrm{E}+01$ & 12 & $.0000 \mathrm{E}+00$ & 13 & $.1000 \mathrm{E}+01$ & 14 & $.1000 \mathrm{E}+01$ & 15 & $.1000 \mathrm{E}+01$ & 16 & $.1000 \mathrm{E}+01$ \\
\hline & $.0000 \mathrm{E}+00$ & 18 & $.0000 \mathrm{E}+00$ & 19 & $.0000 \mathrm{E}+00$ & 20 & $.0000 \mathrm{E}+00$ & 21 & $.0000 \mathrm{E}+00$ & 22 & $.0000 \mathrm{E}+00$ & 23 & $.0000 \mathrm{E}+00$ & 24 & \\
\hline
\end{tabular}

$* * *$ AERMOD - VERSION $07026 * * * \quad \begin{aligned} & * * \text { U/UA Building Demolition } \\ & * * * \text { Unit Release - Worst Case }\end{aligned}$ $\star *$ MODELOPTS :

CONC $\begin{array}{ll}* * * & 02 / 09 / 09 \\ * * * & 10: 41: 11\end{array}$

DRYDPL WETDPL

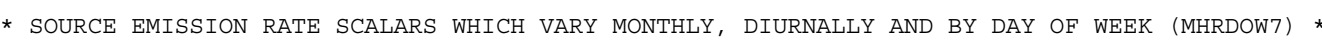

SOURCE ID $=224 U_{-} 4 ;$ SOURCE TYPE $=$ POINT

HOUR SCALAR HOUR SCALAR HOUR SCALAR HOUR SCALAR HOUR SCALAR HOUR SCALAR HOUR SCALAR HOUR SCALAR

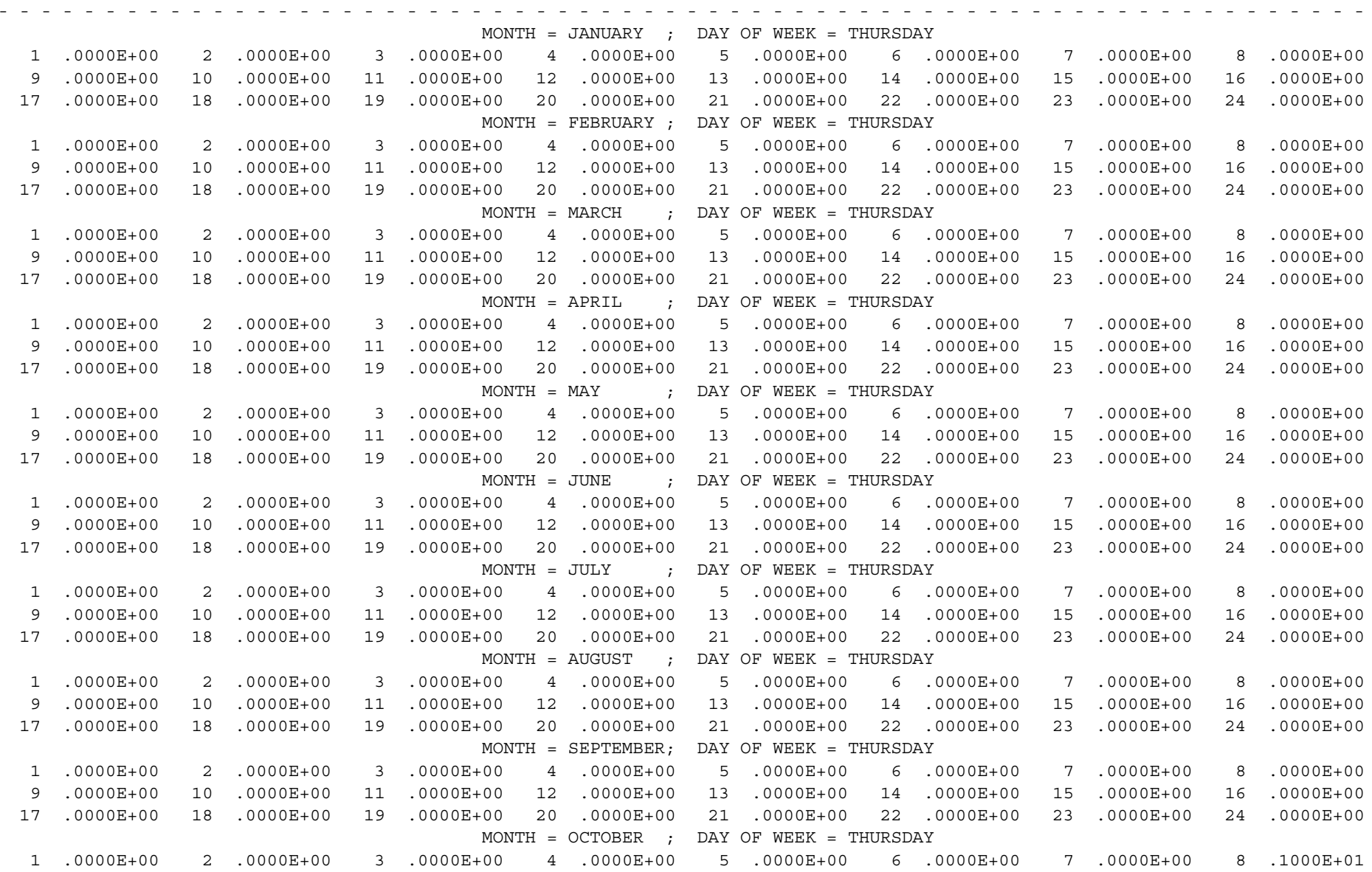




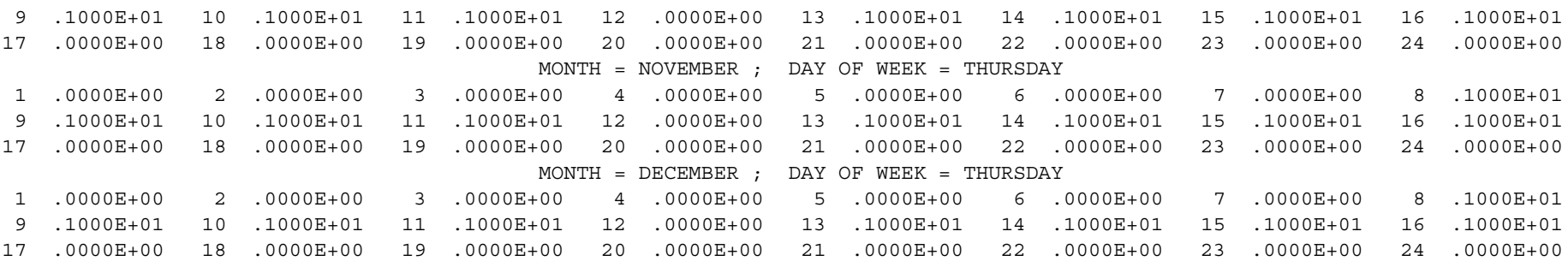

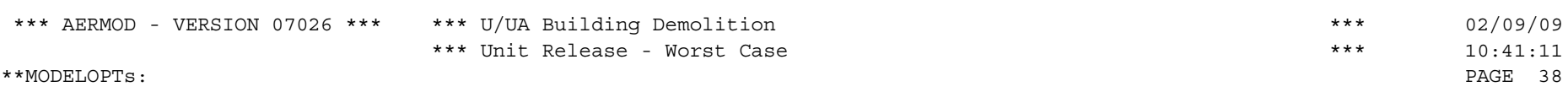
CONC
DDEP
TOXICS FLAT
DRYDPL WETDPL

$10: 41: 11$
PAGE 38

* SoURCe emission RAte scalars Which VARy monthly, DiURNALly AND by DAy of WEek (MHRDOW7) *

SOURCE ID $=224 \mathrm{U}_{-} 4 ; \quad$ SOURCE TYPE $=$ POINT :

HOUR SCALAR HOUR SCALAR HOUR SCALAR HOUR SCALAR HOUR SCALAR HOUR SCALAR HOUR SCALAR HOUR SCALAR

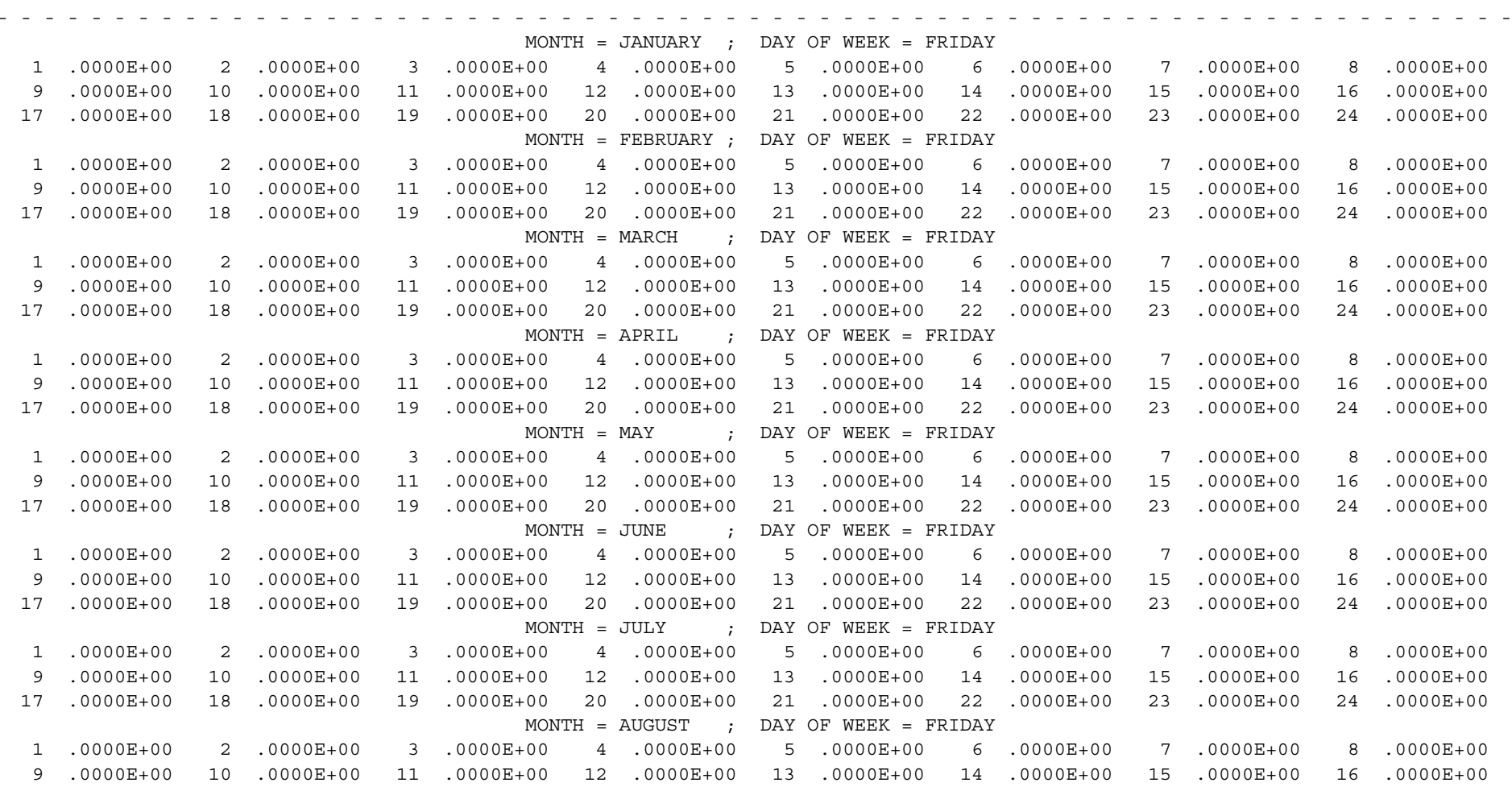




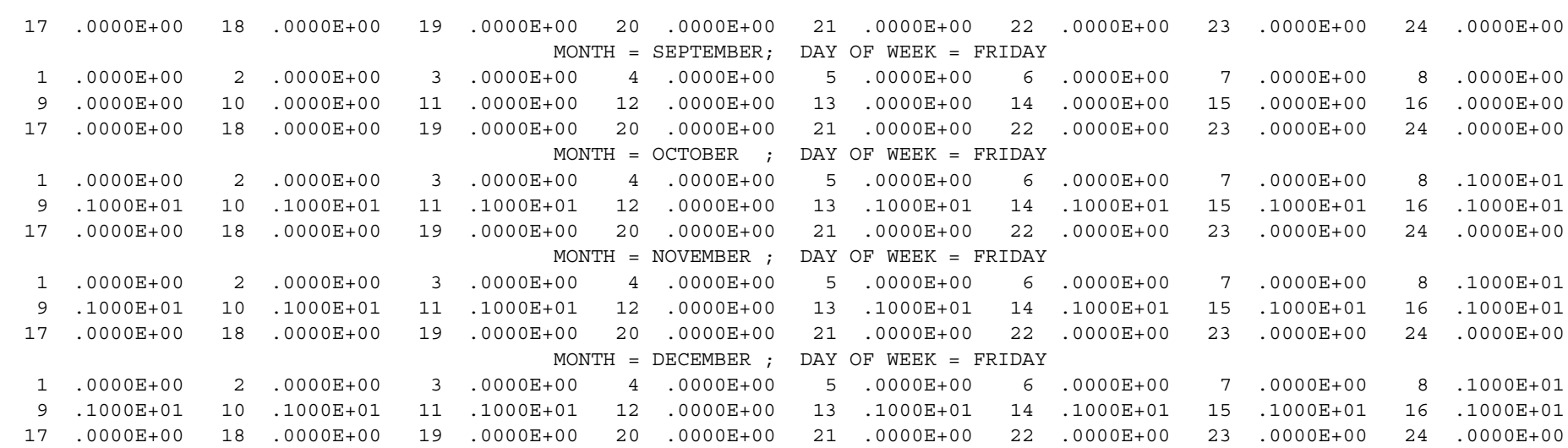

\begin{tabular}{|c|c|c|c|c|}
\hline *** AERMOD & - VERSION $07026 * \star *$ & $\begin{array}{l}* * * \text { U/UA Building Demolition } \\
* * * \text { Unit Release - Worst Case }\end{array}$ & $\begin{array}{l}* * * \\
* * *\end{array}$ & $\begin{array}{l}02 / 09 / 09 \\
10: 41: 11\end{array}$ \\
\hline & & & & PAGE $\quad 39$ \\
\hline
\end{tabular}

CONC

DDEP

DRYDPL WETDPL

PAGE 39

SOURCE ID $=224 \mathrm{U}_{-} 4 ;$ SOURCE TYPE $=$ POINT

HOUR SCALAR HOUR SCALAR HOUR SCALAR HOUR SCALAR HOUR SCALAR HOUR SCALAR HOUR SCALAR HOUR SCALAR

\begin{tabular}{|c|c|c|c|c|c|c|c|c|c|c|c|c|c|c|c|}
\hline 1 & $.0000 \mathrm{E}+00$ & 2 & $.0000 \mathrm{E}+00$ & 3 & $.0000 \mathrm{E}+00$ & 4 & $.0000 \mathrm{E}+00$ & $\begin{array}{r}\text { DAY } \\
5\end{array}$ & $.0000 \mathrm{E}+00$ & 6 & $.0000 \mathrm{E}+00$ & 7 & $.0000 \mathrm{E}+00$ & 8 & \\
\hline 9 & $.0000 \mathrm{E}+00$ & 10 & $.0000 \mathrm{E}+00$ & 11 & $.0000 \mathrm{E}+00$ & 12 & $.0000 \mathrm{E}+00$ & 13 & $.0000 E+00$ & 14 & $.0000 \mathrm{E}+00$ & 15 & $.0000 \mathrm{E}+00$ & 16 & $.0000 \mathrm{E}+00$ \\
\hline \multirow[t]{2}{*}{17} & $.0000 \mathrm{E}+00$ & 18 & $.0000 \mathrm{E}+00$ & 19 & $.0000 \mathrm{E}+00$ & 20 & $.0000 \mathrm{E}+00$ & 21 & $.0000 \mathrm{E}+00$ & 22 & $.0000 \mathrm{E}+00$ & 23 & $.0000 E+00$ & 24 & $.0000 \mathrm{E}+00$ \\
\hline & & & & & MON & & FEBRUARY ; & DAY & OF WEEK = & ATIRD & & & & & \\
\hline 1 & $.0000 \mathrm{E}+00$ & 2 & $.0000 \mathrm{E}+00$ & 3 & $.0000 E+00$ & 4 & $.0000 \mathrm{E}+00$ & 5 & $.0000 \mathrm{E}+00$ & 6 & $.0000 \mathrm{E}+00$ & 7 & $.0000 \mathrm{E}+00$ & 8 & $.0000 \mathrm{E}+00$ \\
\hline 9 & $.0000 \mathrm{E}+00$ & 10 & $.0000 \mathrm{E}+00$ & 11 & $0000 \mathrm{E}+00$ & 12 & $.0000 \mathrm{E}+00$ & 13 & $.0000 \mathrm{E}+00$ & 14 & $.0000 \mathrm{E}+00$ & 15 & $.0000 \mathrm{E}+00$ & 16 & $.0000 \mathrm{E}+00$ \\
\hline \multirow[t]{2}{*}{17} & $.0000 \mathrm{E}+00$ & 18 & $0000 E+00$ & 19 & $000 \mathrm{E}+00$ & 20 & $.0000 \mathrm{E}+00$ & 21 & $.0000 \mathrm{E}+00$ & 22 & & 23 & $E+00$ & 24 & $00 \mathrm{E}+00$ \\
\hline & & & & & MON & $\mathrm{TH}=$ & MARCH ; & DAY & OF WEEK = & ATURD & & & & & \\
\hline 1 & $.0000 \mathrm{E}+00$ & 2 & $.0000 \mathrm{E}+00$ & 3 & $.0000 \mathrm{E}+00$ & 4 & $.0000 \mathrm{E}+00$ & 5 & $.0000 \mathrm{E}+00$ & 6 & $.0000 \mathrm{E}+00$ & & $E+00$ & & $00 \mathrm{E}+00$ \\
\hline 9 & $.0000 \mathrm{E}+00$ & 10 & $.0000 \mathrm{E}+00$ & 11 & $.0000 E+00$ & 12 & $.0000 \mathrm{E}+00$ & 13 & $.0000 \mathrm{E}+00$ & 14 & .0000 & 15 & $10 \mathrm{E}+00$ & 16 & $.0000 \mathrm{E}+00$ \\
\hline \multirow[t]{2}{*}{17} & $.0000 \mathrm{E}+00$ & 18 & $.0000 \mathrm{E}+00$ & 19 & $.0000 \mathrm{E}+00$ & 20 & $.0000 \mathrm{E}+00$ & 21 & $.0000 \mathrm{E}+00$ & 22 & .0000 & 23 & $.0000 \mathrm{E}+00$ & 24 & $.0000 \mathrm{E}+00$ \\
\hline & & & & & MON & $\mathrm{TH}=$ & APRIL ; & DAY & OF WEEK = & ATURD & & & & & \\
\hline 1 & $.0000 \mathrm{E}+00$ & 2 & .000 & 3 & $.0000 \mathrm{E}+00$ & 4 & $.0000 \mathrm{E}+00$ & 5 & .0000 & 6 & $.0000 \mathrm{E}+00$ & & +00 & & $10 \mathrm{E}+00$ \\
\hline 9 & .0000 & 10 & 000 & 11 & $0000 \mathrm{E}+00$ & 12 & $.0000 \mathrm{E}+00$ & 13 & $.0000 \mathrm{E}+00$ & 14 & .00 & 15 & +00 & 16 & $.0000 \mathrm{E}+00$ \\
\hline \multirow{2}{*}{17} & $.0000 \mathrm{E}+00$ & 18 & $.0000 \mathrm{E}+00$ & 19 & $.0000 \mathrm{E}+00$ & 20 & $.0000 \mathrm{E}+00$ & 21 & $.0000 \mathrm{E}+00$ & 22 & $.0000 \mathrm{E}+00$ & 23 & $.0000 E+00$ & 24 & $.0000 \mathrm{E}+00$ \\
\hline & & & & & MON & & $\operatorname{MAY} \quad ;$ & DAY & OF WEEK = & CURD & & & & & \\
\hline 1 & $.0000 \mathrm{E}+00$ & 2 & $.0000 \mathrm{E}+00$ & 3 & $.0000 \mathrm{E}+00$ & 4 & $.0000 \mathrm{E}+00$ & 5 & $.0000 \mathrm{E}+00$ & 6 & $.0000 \mathrm{E}+00$ & 7 & $.0000 \mathrm{E}+00$ & 8 & $.0000 \mathrm{E}+00$ \\
\hline 9 & $.0000 \mathrm{E}+00$ & 10 & & 11 & $.0000 \mathrm{E}+00$ & 12 & $.0000 \mathrm{E}+00$ & 13 & & 14 & & 15 & & 16 & $0 \mathrm{E}+00$ \\
\hline \multirow[t]{2}{*}{17} & $.0000 \mathrm{E}+00$ & 18 & $1000 \mathrm{E}+00$ & 19 & $000 \mathrm{E}+00$ & 20 & $.0000 \mathrm{E}+00$ & 21 & $.0000 \mathrm{E}+00$ & 22 & $.0000 \mathrm{E}+00$ & 23 & $.0000 \mathrm{E}+00$ & 24 & $.0000 \mathrm{E}+00$ \\
\hline & & & & & MON & $\Gamma \mathrm{H}=$ & JUNE ; & DAY & OF WEEK = & ATURD & & & & & \\
\hline 1 & & 2 & & 3 & $.0000 \mathrm{E}+00$ & 4 & $.0000 \mathrm{E}+00$ & 5 & $.0000 \mathrm{E}+00$ & 6 & $.0000 \mathrm{E}+00$ & & & & \\
\hline 9 & $.0000 \mathrm{E}+00$ & 10 & \pm+00 & 11 & $000 \mathrm{E}+00$ & 12 & & 13 & & 14 & $.0000 \mathrm{E}+00$ & 15 & $.0000 E+00$ & 16 & $.0000 E+00$ \\
\hline 17 & $.0000 \mathrm{E}+00$ & 18 & $0000 \mathrm{E}+00$ & 19 & $0000 \mathrm{E}+00$ & 20 & $.0000 \mathrm{E}+00$ & 21 & $.0000 \mathrm{E}+00$ & 22 & $.0000 \mathrm{E}+00$ & 23 & $.0000 \mathrm{E}+00$ & 24 & $.0000 \mathrm{E}+00$ \\
\hline
\end{tabular}




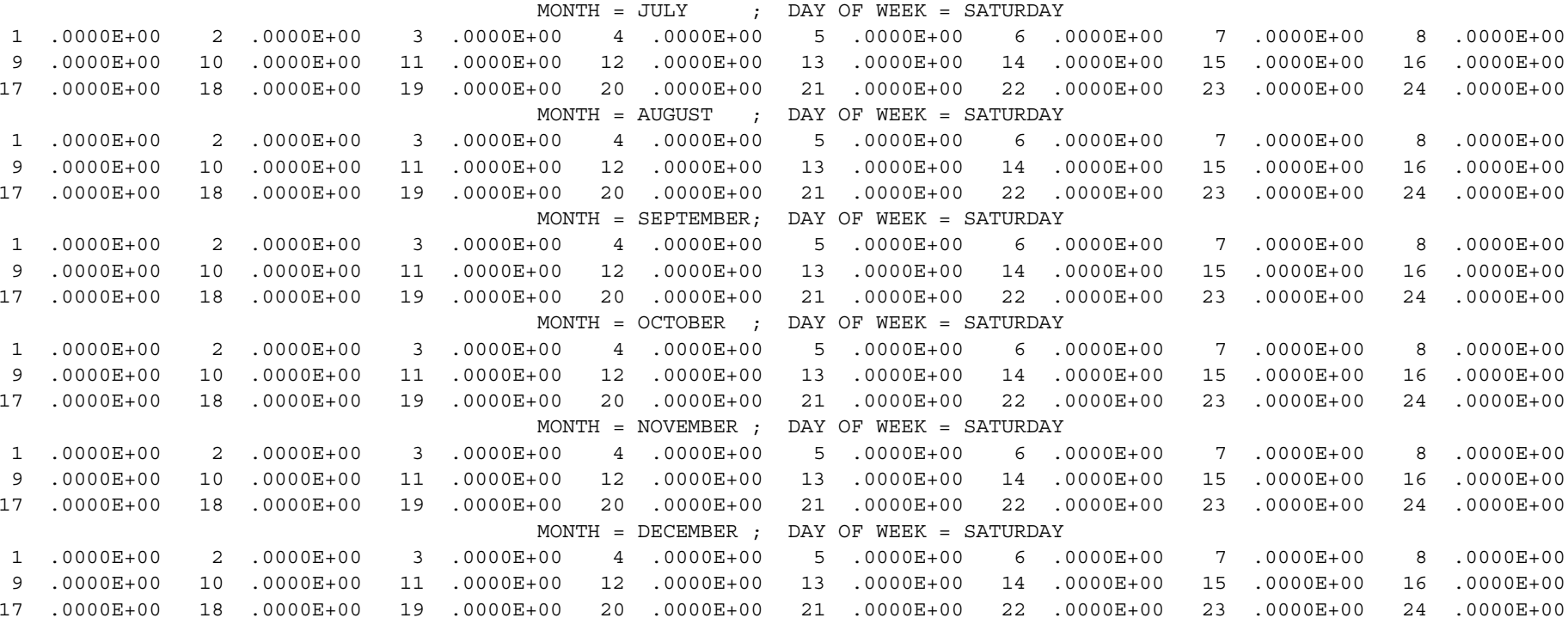

* SOURCE EMISSiOn RATE SCALARS WHich VARY MONTHLy, DIURNALLy AND by DAy OF WEEK (MHRDOW7) *

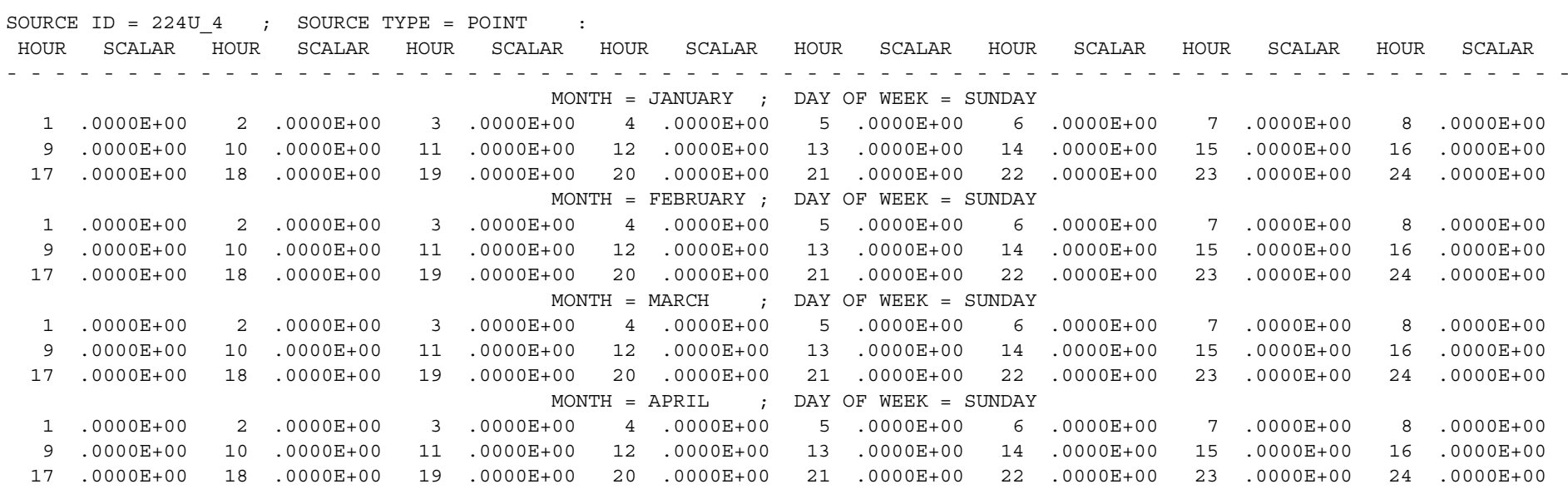




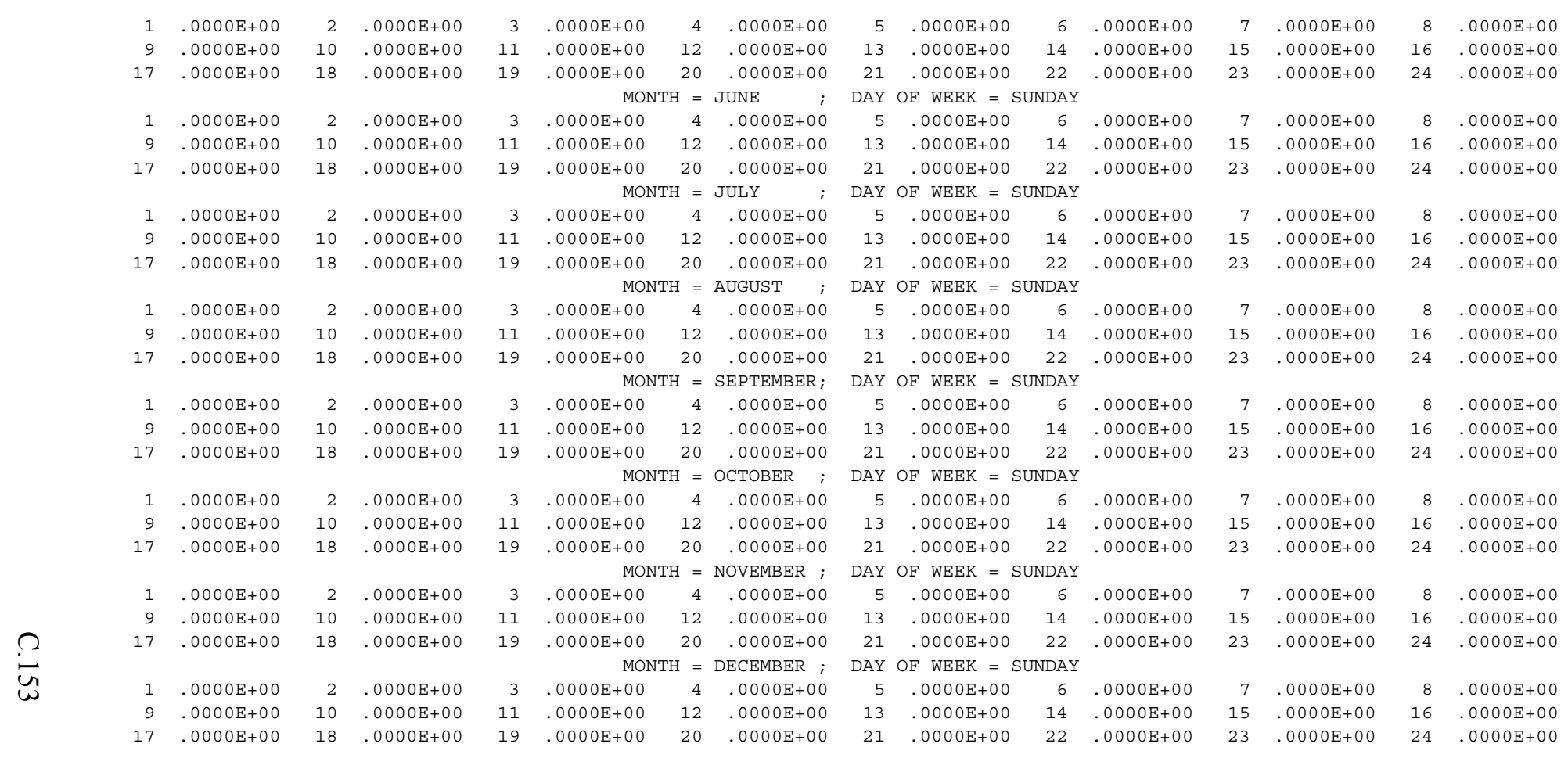

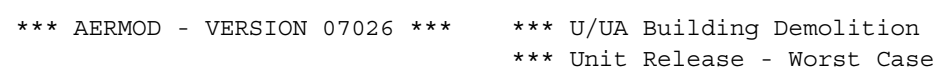

$\begin{array}{ll}* * * & 02 / 09 / 09 \\ * * * & 10: 41: 11\end{array}$ $* *$ MODELOPTS :

CONC

DDEP

TOXICS FLAT

DRYDPL WETDPL

* SOURCE EMISSION RATE SCALARS WHICH VARY MONTHLY, DIURNALLY AND BY DAY OF WEEK (MHRDOW7) *

\begin{tabular}{|c|c|c|c|c|c|c|c|c|c|c|c|c|c|c|c|}
\hline HOUR & SCALAR & HOUR' & SCALAR & HOUR & SCALAR & HOUR & SCALAR & HOUR & SCALAR & HOUR & SCALAR & HOUR & SCALAR & HOUR & SCALAR \\
\hline-- & $-\cdots--$ & -- & ---- & - & $\begin{array}{rrr}- & - \\
& \text { MON }\end{array}$ & & $\begin{array}{c}-\cdots- \\
\text { JANUARY }\end{array}$ & DAY & $\begin{array}{l}\text { OF WEEK } \\
\text { WF }\end{array}$ & $\begin{array}{l}--- \\
\text { MONDAY }\end{array}$ & $-\cdots-$ & -- & & -- & $-\quad-$ \\
\hline 1 & $.0000 E+00$ & 2 & $.0000 E+00$ & 3 & $.0000 \mathrm{E}+00$ & 4 & $.0000 \mathrm{E}+00$ & 5 & $.0000 \mathrm{E}+00$ & 6 & $.0000 \mathrm{E}+00$ & 7 & $.0000 \mathrm{E}+00$ & 8 & $.0000 \mathrm{E}+00$ \\
\hline 9 & $.0000 \mathrm{E}+00$ & 10 & $.0000 \mathrm{E}+00$ & 11 & $.0000 \mathrm{E}+00$ & 12 & $.0000 \mathrm{E}+00$ & 13 & $.0000 \mathrm{E}+00$ & 14 & $.0000 \mathrm{E}+00$ & 15 & $.0000 E+00$ & 16 & $.0000 \mathrm{E}+00$ \\
\hline & & & & & MON & $\mathrm{TH}=\mathrm{F}$ & FEBRUARY ; & DAY & $O F$ WEEK $=\mathrm{N}$ & MONDAY & & & & & \\
\hline 1 & $.0000 \mathrm{E}+00$ & 2 & $.0000 \mathrm{E}+00$ & 3 & $.0000 \mathrm{E}+00$ & 4 & $.0000 \mathrm{E}+00$ & 5 & $.0000 \mathrm{E}+00$ & 6 & $.0000 \mathrm{E}+00$ & 7 & $.0000 \mathrm{E}+00$ & 8 & $.0000 \mathrm{E}+00$ \\
\hline 9 & $.0000 \mathrm{E}+00$ & 10 & $.0000 \mathrm{E}+00$ & 11 & $.0000 \mathrm{E}+00$ & 12 & $.0000 \mathrm{E}+00$ & 13 & $.0000 \mathrm{E}+00$ & 14 & $.0000 \mathrm{E}+00$ & 15 & $.0000 \mathrm{E}+00$ & 16 & $.0000 \mathrm{E}+00$ \\
\hline 17 & $.0000 \mathrm{E}+00$ & 18 & $.0000 \mathrm{E}+00$ & 19 & $.0000 \mathrm{E}+00$ & 20 & $.0000 \mathrm{E}+00$ & 21 & $.0000 \mathrm{E}+00$ & 22 & $.0000 \mathrm{E}+00$ & 23 & $.0000 \mathrm{E}+00$ & 24 & $.0000 \mathrm{E}+00$ \\
\hline
\end{tabular}




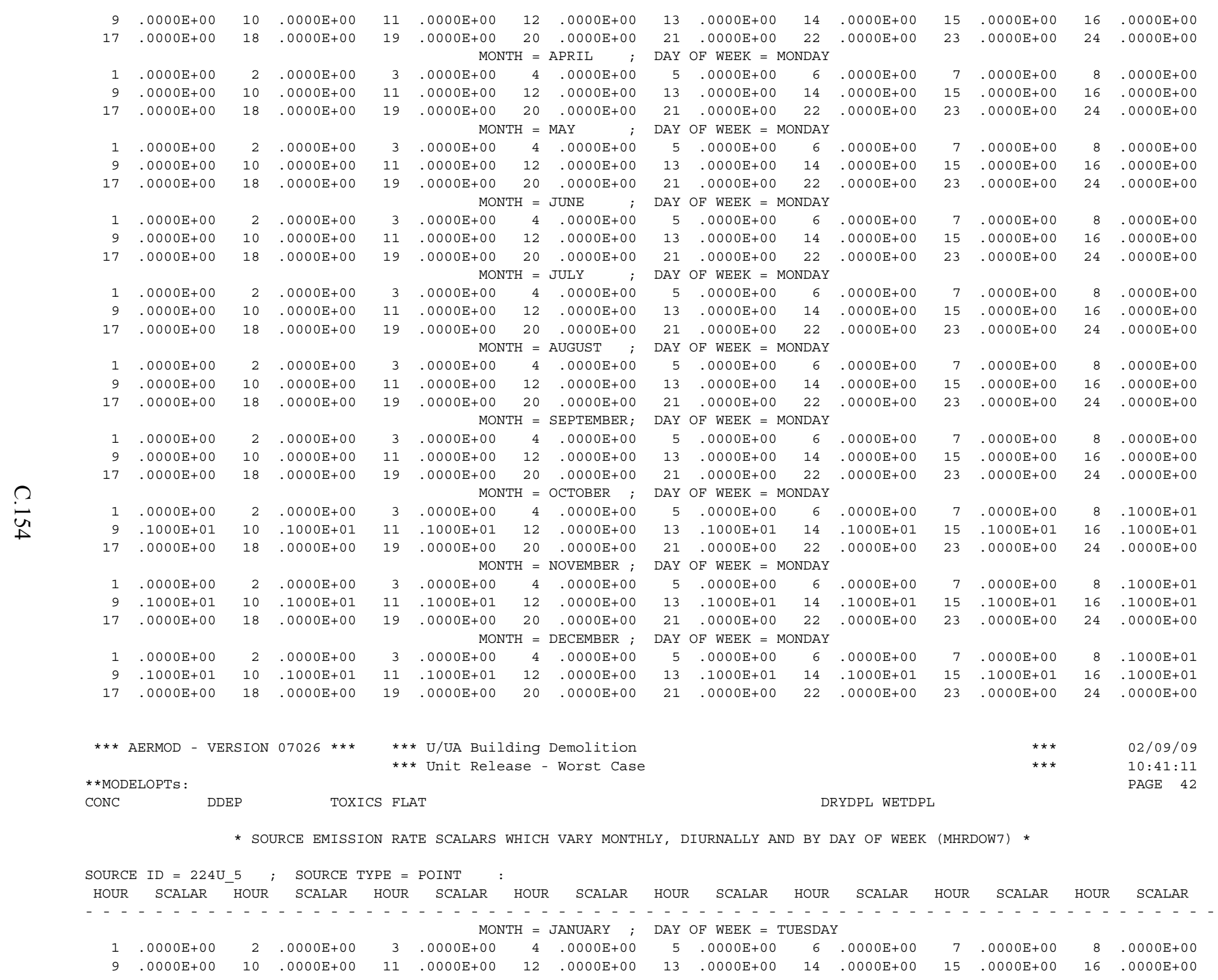




\begin{tabular}{|c|c|c|c|c|c|c|c|c|c|c|c|c|c|c|c|}
\hline 17 & $.0000 \mathrm{E}+00$ & 18 & $.0000 \mathrm{E}+00$ & 19 & $.0000 \mathrm{E}+00$ & 20 & $\begin{array}{l}.0000 E+00 \\
\text {. }\end{array}$ & 21 & $.0000 \mathrm{E}+00$ & 22 & $.0000 \mathrm{E}+00$ & 23 & $.0000 \mathrm{E}+00$ & 24 & $.0000 \mathrm{E}+00$ \\
\hline 1 & $.0000 \mathrm{E}+00$ & 2 & $.0000 \mathrm{E}+00$ & 3 & $.0000 \mathrm{E}+00$ & 4 & $.0000 \mathrm{E}+00$ & 5 & $.0000 \mathrm{E}+00$ & 6 & $.0000 \mathrm{E}+00$ & 7 & $.0000 \mathrm{E}+00$ & 8 & $.0000 \mathrm{E}+00$ \\
\hline 9 & $.0000 \mathrm{E}+00$ & 10 & $.0000 \mathrm{E}+00$ & 11 & $.0000 \mathrm{E}+00$ & 12 & $.0000 \mathrm{E}+00$ & 13 & $.0000 \mathrm{E}+00$ & 14 & $.0000 \mathrm{E}+00$ & 15 & $.0000 \mathrm{E}+00$ & 16 & $.0000 \mathrm{E}+00$ \\
\hline 17 & $.0000 \mathrm{E}+00$ & 18 & $.0000 \mathrm{E}+00$ & 19 & $.0000 \mathrm{E}+00$ & 20 & $.0000 \mathrm{E}+00$ & 21 & $.0000 \mathrm{E}+00$ & 22 & $.0000 \mathrm{E}+00$ & 23 & $.0000 \mathrm{E}+00$ & 24 & $.0000 \mathrm{E}+00$ \\
\hline & & & & & MONTH & $=$ & MARCH ; & DAY & OF WEEK = T & JESDAY & & & & & \\
\hline 1 & $.0000 \mathrm{E}+00$ & 2 & $.0000 \mathrm{E}+00$ & 3 & $.0000 \mathrm{E}+00$ & 4 & $.0000 \mathrm{E}+00$ & 5 & $.0000 \mathrm{E}+00$ & 6 & $.0000 \mathrm{E}+00$ & 7 & $.0000 \mathrm{E}+00$ & 8 & $.0000 \mathrm{E}+00$ \\
\hline 9 & $.0000 \mathrm{E}+00$ & 10 & $.0000 \mathrm{E}+00$ & 11 & $.0000 \mathrm{E}+00$ & 12 & $.0000 \mathrm{E}+00$ & 13 & $.0000 \mathrm{E}+00$ & 14 & $.0000 \mathrm{E}+00$ & 15 & $.0000 \mathrm{E}+00$ & 16 & $.0000 \mathrm{E}+00$ \\
\hline 17 & $.0000 \mathrm{E}+00$ & 18 & $.0000 \mathrm{E}+00$ & 19 & $.0000 \mathrm{E}+00$ & 20 & $.0000 \mathrm{E}+00$ & 21 & $.0000 \mathrm{E}+00$ & 22 & $.0000 \mathrm{E}+00$ & 23 & $.0000 \mathrm{E}+00$ & 24 & $.0000 \mathrm{E}+00$ \\
\hline & & & & & MONTH & $=$ & APRIL ; & DAY & OF WEEK = T & JESDAY & & & & & \\
\hline 1 & $.0000 \mathrm{E}+00$ & 2 & $.0000 \mathrm{E}+00$ & 3 & $.0000 \mathrm{E}+00$ & 4 & $.0000 \mathrm{E}+00$ & 5 & $.0000 \mathrm{E}+00$ & 6 & $.0000 \mathrm{E}+00$ & 7 & $.0000 \mathrm{E}+00$ & 8 & $.0000 \mathrm{E}+00$ \\
\hline 9 & $.0000 \mathrm{E}+00$ & 10 & $.0000 \mathrm{E}+00$ & 11 & $.0000 \mathrm{E}+00$ & 12 & $.0000 \mathrm{E}+00$ & 13 & $.0000 \mathrm{E}+00$ & 14 & $.0000 \mathrm{E}+00$ & 15 & $.0000 \mathrm{E}+00$ & 16 & $.0000 \mathrm{E}+00$ \\
\hline 17 & $.0000 \mathrm{E}+00$ & 18 & $.0000 \mathrm{E}+00$ & 19 & $.0000 \mathrm{E}+00$ & 20 & $.0000 \mathrm{E}+00$ & 21 & $.0000 \mathrm{E}+00$ & 22 & $.0000 \mathrm{E}+00$ & 23 & $.0000 \mathrm{E}+00$ & 24 & $.0000 \mathrm{E}+00$ \\
\hline & & & & & MONTH & $=$ & MAY ; & DAY & OF WEEK = T & JESDAY & & & & & \\
\hline 1 & $.0000 \mathrm{E}+00$ & 2 & $.0000 \mathrm{E}+00$ & 3 & $.0000 \mathrm{E}+00$ & 4 & $.0000 \mathrm{E}+00$ & 5 & $.0000 \mathrm{E}+00$ & 6 & $.0000 \mathrm{E}+00$ & 7 & $.0000 \mathrm{E}+00$ & 8 & $.0000 \mathrm{E}+00$ \\
\hline 9 & $.0000 \mathrm{E}+00$ & 10 & $.0000 \mathrm{E}+00$ & 11 & $.0000 \mathrm{E}+00$ & 12 & $.0000 \mathrm{E}+00$ & 13 & $.0000 \mathrm{E}+00$ & 14 & $.0000 \mathrm{E}+00$ & 15 & $.0000 \mathrm{E}+00$ & 16 & $.0000 \mathrm{E}+00$ \\
\hline 17 & $.0000 \mathrm{E}+00$ & 18 & $.0000 \mathrm{E}+00$ & 19 & $.0000 \mathrm{E}+00$ & 20 & $.0000 \mathrm{E}+00$ & 21 & $.0000 \mathrm{E}+00$ & 22 & $.0000 \mathrm{E}+00$ & 23 & $.0000 \mathrm{E}+00$ & 24 & $.0000 \mathrm{E}+00$ \\
\hline & & & & & MONTH & $=$ & JUNE ; & DAY & OF WEEK = T & JESDAY & & & & & \\
\hline 1 & $.0000 \mathrm{E}+00$ & 2 & $.0000 \mathrm{E}+00$ & 3 & $.0000 \mathrm{E}+00$ & 4 & $.0000 \mathrm{E}+00$ & 5 & $.0000 \mathrm{E}+00$ & 6 & $.0000 \mathrm{E}+00$ & 7 & $.0000 \mathrm{E}+00$ & 8 & $.0000 \mathrm{E}+00$ \\
\hline 9 & $.0000 \mathrm{E}+00$ & 10 & $.0000 \mathrm{E}+00$ & 11 & $.0000 \mathrm{E}+00$ & 12 & $.0000 \mathrm{E}+00$ & 13 & $.0000 \mathrm{E}+00$ & 14 & $.0000 \mathrm{E}+00$ & 15 & $.0000 \mathrm{E}+00$ & 16 & $.0000 \mathrm{E}+00$ \\
\hline 17 & $.0000 \mathrm{E}+00$ & 18 & $.0000 \mathrm{E}+00$ & 19 & $.0000 \mathrm{E}+00$ & 20 & $.0000 \mathrm{E}+00$ & 21 & $.0000 \mathrm{E}+00$ & 22 & $.0000 \mathrm{E}+00$ & 23 & $.0000 \mathrm{E}+00$ & 24 & $.0000 \mathrm{E}+00$ \\
\hline & & & & & MONTH & $=$ & JULY ; & DAY & OF WEEK = T & JESDAY & & & & & \\
\hline 1 & $.0000 \mathrm{E}+00$ & 2 & $.0000 \mathrm{E}+00$ & 3 & $.0000 \mathrm{E}+00$ & 4 & $.0000 \mathrm{E}+00$ & 5 & $.0000 \mathrm{E}+00$ & 6 & $.0000 \mathrm{E}+00$ & 7 & $.0000 \mathrm{E}+00$ & 8 & $.0000 \mathrm{E}+00$ \\
\hline 9 & $.0000 \mathrm{E}+00$ & 10 & $.0000 \mathrm{E}+00$ & 11 & $.0000 \mathrm{E}+00$ & 12 & $.0000 \mathrm{E}+00$ & 13 & $.0000 \mathrm{E}+00$ & 14 & $.0000 \mathrm{E}+00$ & 15 & $.0000 \mathrm{E}+00$ & 16 & $.0000 \mathrm{E}+00$ \\
\hline 17 & $.0000 \mathrm{E}+00$ & 18 & $.0000 \mathrm{E}+00$ & 19 & $.0000 \mathrm{E}+00$ & 20 & $.0000 \mathrm{E}+00$ & 21 & $.0000 \mathrm{E}+00$ & 22 & $.0000 \mathrm{E}+00$ & 23 & $.0000 \mathrm{E}+00$ & 24 & $.0000 \mathrm{E}+00$ \\
\hline & & & & & MONTH & $=$ & AUGUST ; & DAY & OF WEEK = T & JESDAY & & & & & \\
\hline 1 & $.0000 \mathrm{E}+00$ & 2 & $.0000 \mathrm{E}+00$ & 3 & $.0000 \mathrm{E}+00$ & 4 & $.0000 \mathrm{E}+00$ & 5 & $.0000 \mathrm{E}+00$ & 6 & $.0000 \mathrm{E}+00$ & 7 & $.0000 \mathrm{E}+00$ & 8 & $.0000 \mathrm{E}+00$ \\
\hline 9 & $.0000 \mathrm{E}+00$ & 10 & $.0000 \mathrm{E}+00$ & 11 & $.0000 \mathrm{E}+00$ & 12 & $.0000 \mathrm{E}+00$ & 13 & $.0000 \mathrm{E}+00$ & 14 & $.0000 \mathrm{E}+00$ & 15 & $.0000 \mathrm{E}+00$ & 16 & $.0000 \mathrm{E}+00$ \\
\hline 17 & $.0000 \mathrm{E}+00$ & 18 & $.0000 \mathrm{E}+00$ & 19 & $.0000 \mathrm{E}+00$ & 20 & $.0000 \mathrm{E}+00$ & 21 & $.0000 \mathrm{E}+00$ & 22 & $.0000 \mathrm{E}+00$ & 23 & $.0000 \mathrm{E}+00$ & 24 & $.0000 \mathrm{E}+00$ \\
\hline & & & & & MONTH & $=$ & SEPTEMBER; & DAY & OF $\mathrm{WEEK}=\mathrm{T}$ & JESDAY & & & & & \\
\hline & $.0000 \mathrm{E}+00$ & 2 & $.0000 \mathrm{E}+00$ & 3 & $.0000 \mathrm{E}+00$ & 4 & $.0000 \mathrm{E}+00$ & 5 & $.0000 \mathrm{E}+00$ & 6 & $.0000 \mathrm{E}+00$ & 7 & $.0000 \mathrm{E}+00$ & 8 & $.0000 \mathrm{E}+00$ \\
\hline 9 & $.0000 \mathrm{E}+00$ & 10 & $.0000 \mathrm{E}+00$ & 11 & $.0000 \mathrm{E}+00$ & 12 & $.0000 \mathrm{E}+00$ & 13 & $.0000 \mathrm{E}+00$ & 14 & $.0000 \mathrm{E}+00$ & 15 & $.0000 \mathrm{E}+00$ & 16 & $.0000 \mathrm{E}+00$ \\
\hline 17 & $.0000 \mathrm{E}+00$ & 18 & $.0000 E+00$ & 19 & $.0000 \mathrm{E}+00$ & 20 & $.0000 \mathrm{E}+00$ & 21 & $.0000 \mathrm{E}+00$ & 22 & $.0000 \mathrm{E}+00$ & 23 & $.0000 \mathrm{E}+00$ & 24 & $.0000 \mathrm{E}+00$ \\
\hline & & & & & MONTH & $=$ & OCTOBER ; & DAY & OF WEEK $=\mathrm{T}$ & JESDAY & & & & & \\
\hline 1 & $.0000 \mathrm{E}+00$ & 2 & $.0000 \mathrm{E}+00$ & 3 & $.0000 \mathrm{E}+00$ & 4 & $.0000 \mathrm{E}+00$ & 5 & $.0000 \mathrm{E}+00$ & 6 & $.0000 \mathrm{E}+00$ & 7 & $.0000 \mathrm{E}+00$ & 8 & $.1000 \mathrm{E}+01$ \\
\hline 9 & $.1000 \mathrm{E}+01$ & 10 & $.1000 \mathrm{E}+01$ & 11 & $.1000 \mathrm{E}+01$ & 12 & $.0000 \mathrm{E}+00$ & 13 & $.1000 \mathrm{E}+01$ & 14 & $.1000 \mathrm{E}+01$ & 15 & $.1000 \mathrm{E}+01$ & 16 & $.1000 \mathrm{E}+01$ \\
\hline 17 & $.0000 \mathrm{E}+00$ & 18 & $.0000 \mathrm{E}+00$ & 19 & $.0000 \mathrm{E}+00$ & 20 & $.0000 \mathrm{E}+00$ & 21 & $.0000 \mathrm{E}+00$ & 22 & $.0000 \mathrm{E}+00$ & 23 & $.0000 \mathrm{E}+00$ & 24 & $.0000 \mathrm{E}+00$ \\
\hline & & & & & MONTH & $\mathrm{H}=$ & NOVEMBER ; & DAY & OF $\mathrm{WEEK}=\mathrm{T}$ & JESDAY & & & & & \\
\hline 1 & $.0000 \mathrm{E}+00$ & 2 & $.0000 \mathrm{E}+00$ & 3 & $.0000 \mathrm{E}+00$ & 4 & $.0000 \mathrm{E}+00$ & 5 & $.0000 \mathrm{E}+00$ & 6 & $.0000 \mathrm{E}+00$ & 7 & $.0000 \mathrm{E}+00$ & 8 & $.1000 \mathrm{E}+01$ \\
\hline 9 & $.1000 \mathrm{E}+01$ & 10 & $.1000 \mathrm{E}+01$ & 11 & $.1000 \mathrm{E}+01$ & 12 & $.0000 \mathrm{E}+00$ & 13 & $.1000 \mathrm{E}+01$ & 14 & $.1000 \mathrm{E}+01$ & 15 & $.1000 \mathrm{E}+01$ & 16 & $.1000 \mathrm{E}+01$ \\
\hline 17 & $.0000 \mathrm{E}+00$ & 18 & $.0000 \mathrm{E}+00$ & 19 & $.0000 \mathrm{E}+00$ & 20 & $.0000 \mathrm{E}+00$ & 21 & $.0000 \mathrm{E}+00$ & 22 & $.0000 \mathrm{E}+00$ & 23 & $.0000 \mathrm{E}+00$ & 24 & $.0000 \mathrm{E}+00$ \\
\hline & & & & & MONT & $\mathrm{H}=$ & DECEMBER ; & DAY & OF $\mathrm{WEEK}=\mathrm{T}$ & JESDAY & & & & & \\
\hline 1 & $.0000 \mathrm{E}+00$ & 2 & $.0000 \mathrm{E}+00$ & 3 & $.0000 \mathrm{E}+00$ & 4 & $.0000 \mathrm{E}+00$ & 5 & $.0000 \mathrm{E}+00$ & 6 & $.0000 \mathrm{E}+00$ & 7 & $.0000 \mathrm{E}+00$ & 8 & $.1000 \mathrm{E}+01$ \\
\hline & $.1000 \mathrm{E}+01$ & 10 & $.1000 \mathrm{E}+01$ & 11 & $.1000 \mathrm{E}+01$ & 12 & $.0000 \mathrm{E}+00$ & 13 & $.1000 \mathrm{E}+01$ & 14 & $.1000 \mathrm{E}+01$ & 15 & $.1000 \mathrm{E}+01$ & 16 & $.1000 \mathrm{E}+01$ \\
\hline 17 & $.0000 \mathrm{E}+00$ & 18 & $.0000 \mathrm{E}+00$ & 19 & $.0000 \mathrm{E}+00$ & 20 & $.0000 \mathrm{E}+00$ & 21 & $.0000 \mathrm{E}+00$ & 22 & $.0000 \mathrm{E}+00$ & 23 & $.0000 \mathrm{E}+00$ & 24 & $.0000 \mathrm{E}+00$ \\
\hline *** & * AERMOD - VE & SION & $07026 * * *$ & $* * *$ & U/UA Buildi & ing & Demolition & & & & & & $* * *$ & & $02 / 09 / 09$ \\
\hline & & & & & " Unit Releas & & Worst Case & & & & & & $* * *$ & & $10: 41: 11$ \\
\hline & & & & & & & & & & & & & & & PAGE 4 \\
\hline CONC & & & TOXI & $5 \mathrm{FLZ}$ & & & & & & & & & & & \\
\hline
\end{tabular}

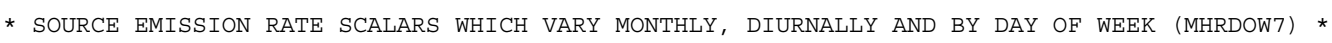




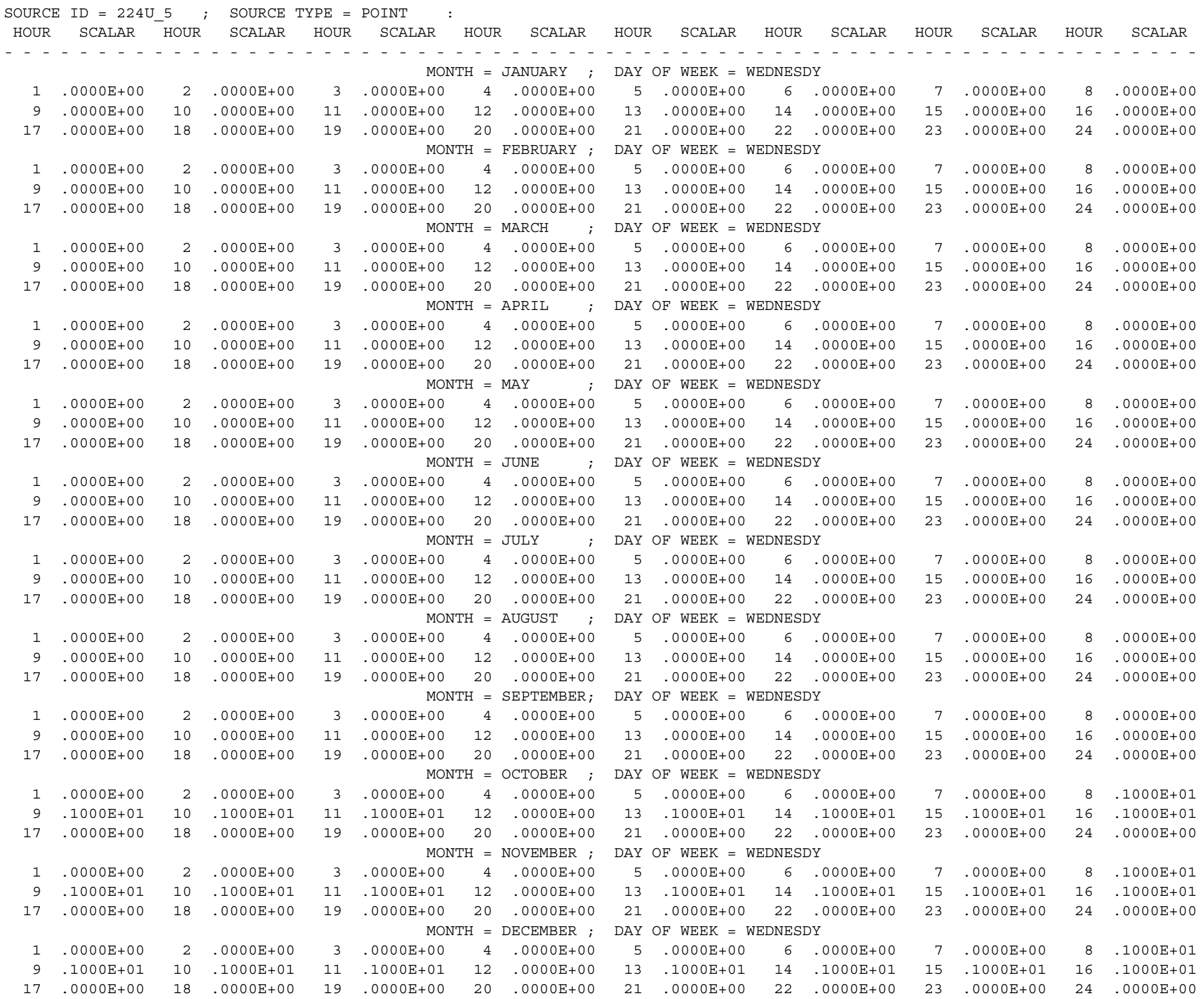




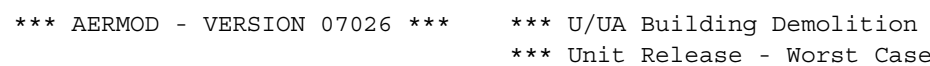
**MODELOPTS :

CONC
$* * * \quad 02 / 09 / 09$

$* * * \quad 10: 41: 11$
PAGE $\quad 44$

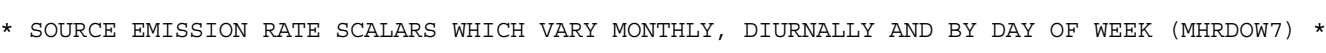

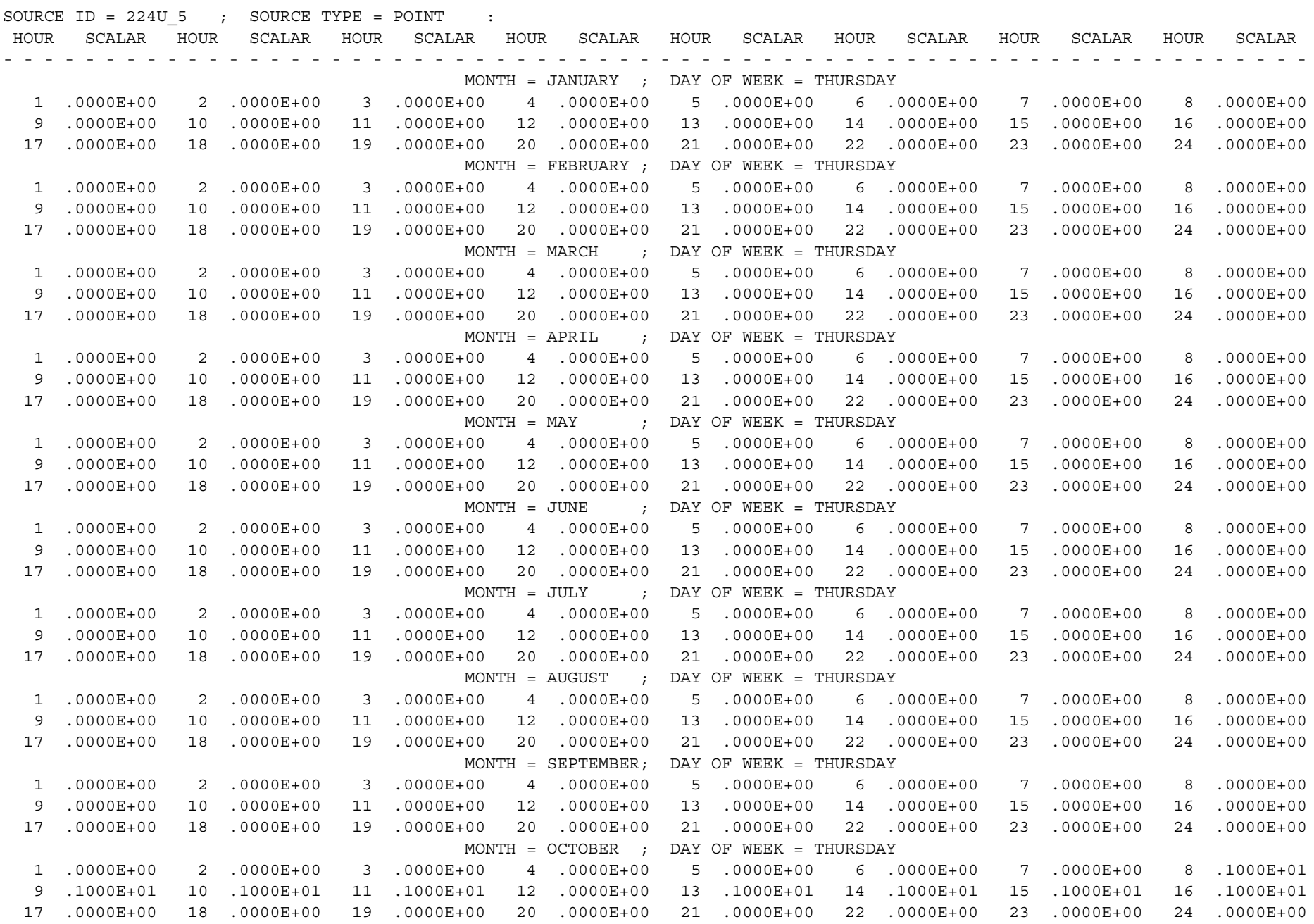

MONTH $=$ NOVEMBER $; \quad$ DAY OF WEEK $=$ THURSDAY 


\begin{tabular}{|c|c|c|c|c|c|c|c|c|c|c|c|c|c|c|c|}
\hline 1 & $.0000 \mathrm{E}+00$ & 2 & $.0000 \mathrm{E}+00$ & 3 & $.0000 \mathrm{E}+00$ & 4 & $.0000 \mathrm{E}+00$ & 5 & $.0000 \mathrm{E}+00$ & 6 & $.0000 \mathrm{E}+00$ & 7 & $.0000 \mathrm{E}+00$ & 8 & $1000 \mathrm{E}+01$ \\
\hline 9 & $.1000 \mathrm{E}+01$ & 10 & $.1000 \mathrm{E}+01$ & 11 & $.1000 \mathrm{E}+01$ & 12 & $.0000 \mathrm{E}+00$ & 13 & $.1000 \mathrm{E}+01$ & 14 & $.1000 \mathrm{E}+01$ & 15 & $.1000 \mathrm{E}+01$ & 16 & $.1000 \mathrm{E}+01$ \\
\hline 1 & $.0000 \mathrm{E}+00$ & 18 & $.0000 \mathrm{E}+00$ & 19 & $.0000 \mathrm{E}+00$ & 20 & $.0000 \mathrm{E}+00$ & 21 & $.0000 \mathrm{E}+00$ & 22 & $.0000 \mathrm{E}+00$ & 23 & $.0000 \mathrm{E}+00$ & 24 & $.0000 \mathrm{E}+00$ \\
\hline & & & & & MOI & & DECEMBER ; & DAY & OF WEEK = & URSI & & & & & \\
\hline 1 & $.0000 \mathrm{E}+00$ & 2 & $.0000 \mathrm{E}+00$ & 3 & $.0000 \mathrm{E}+00$ & 4 & $.0000 \mathrm{E}+00$ & 5 & $.0000 \mathrm{E}+00$ & 6 & $.0000 \mathrm{E}+00$ & 7 & $.0000 \mathrm{E}+00$ & 8 & $.1000 \mathrm{E}+01$ \\
\hline 9 & $.1000 \mathrm{E}+01$ & 10 & $.1000 \mathrm{E}+01$ & 11 & $.1000 \mathrm{E}+01$ & 12 & $.0000 \mathrm{E}+00$ & 13 & $.1000 \mathrm{E}+01$ & 14 & $.1000 \mathrm{E}+01$ & 15 & $.1000 \mathrm{E}+01$ & 16 & $.1000 \mathrm{E}+01$ \\
\hline & $.0000 \mathrm{E}+00$ & 18 & $.0000 \mathrm{E}+00$ & 19 & $.0000 \mathrm{E}+00$ & 20 & $.0000 \mathrm{E}+00$ & 21 & $0000 \mathrm{E}+00$ & 22 & $.0000 \mathrm{E}+00$ & 23 & $.0000 E+00$ & 24 & $0000 E+00$ \\
\hline
\end{tabular}

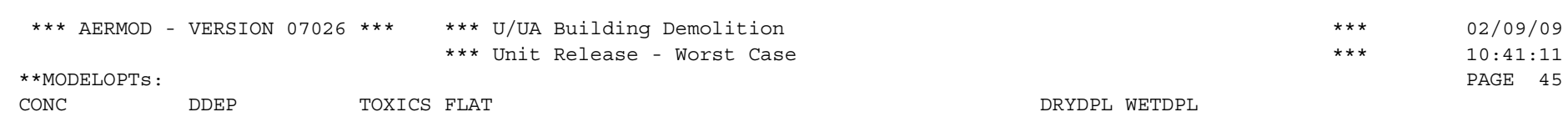

* source emission RAte scalars Which vary monthly, DiURNALly and by DAy of WeEk (MHRDOW7) *

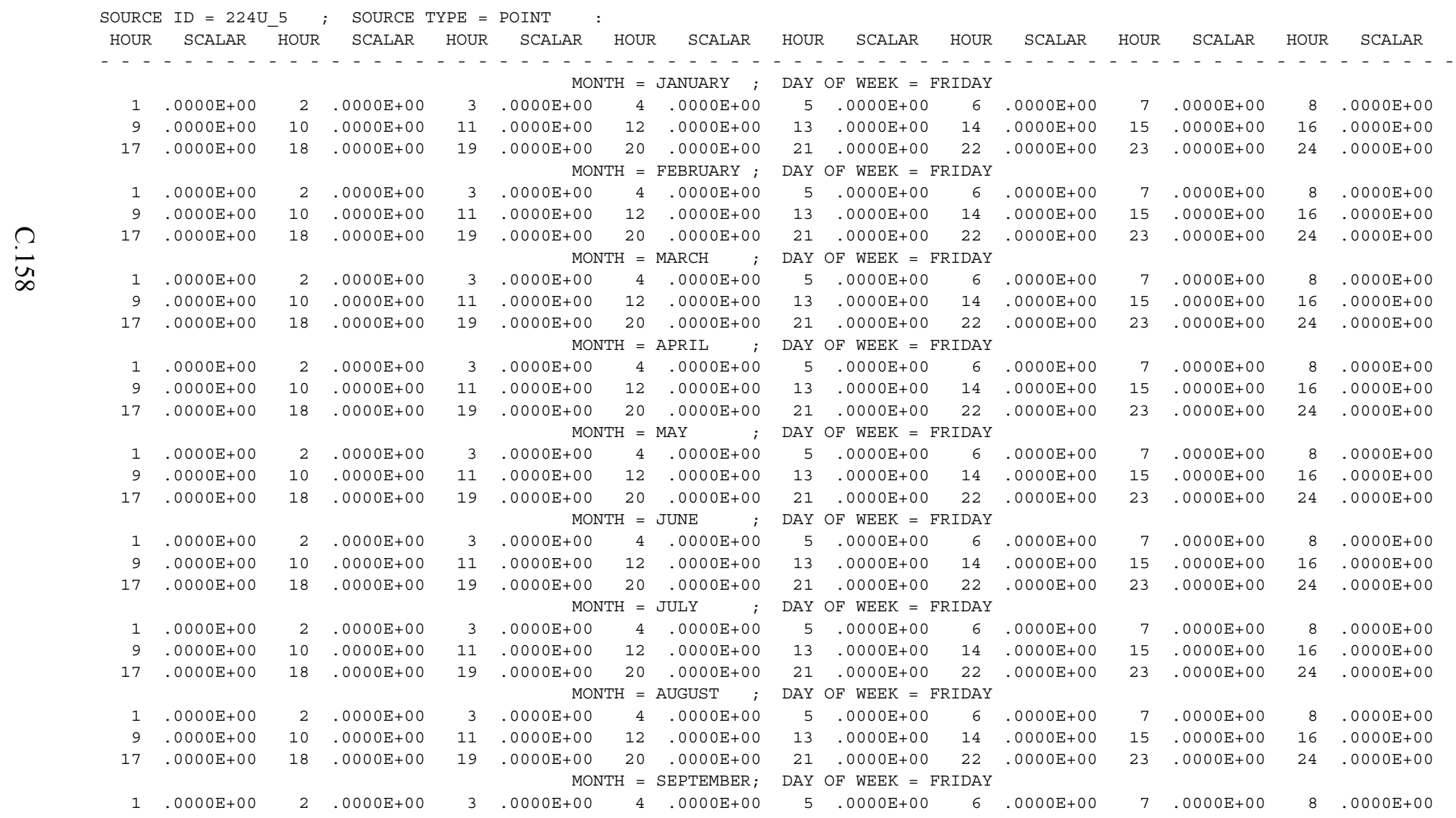




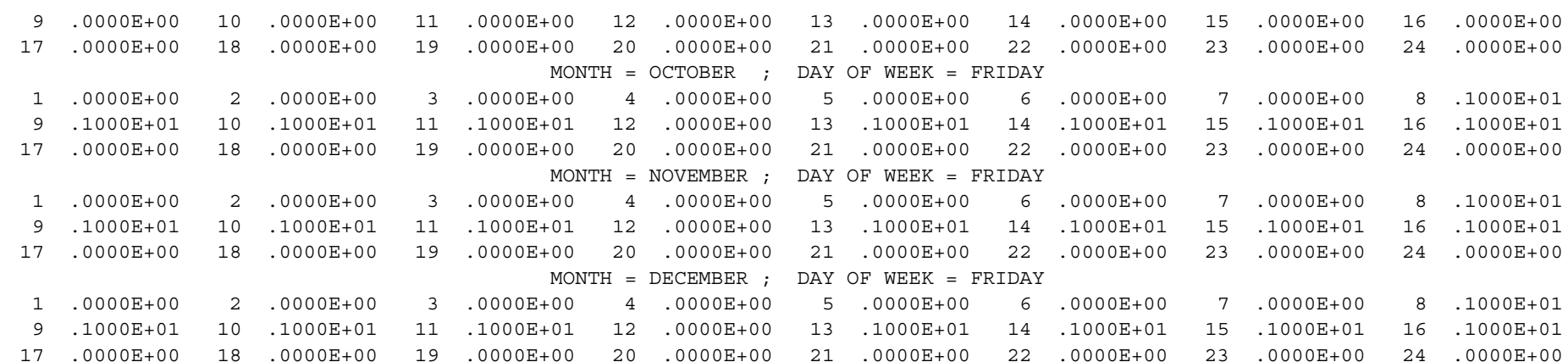

*** AERMOD - VERSION $07026 * * * \quad * * *$ U/UA Building Demolition

*** Unit Release - Worst Case

$\begin{array}{ll}* * * & 02 / 09 / 09 \\ * * * & 10: 41: 11\end{array}$ **MODELOPTS:

CONC

DDEP

TOXICS FLAT

DRYDPL WETDPL

$10: 41: 11$
PAGE $\quad 46$

* SOURCE EMISSion RATe SCALARS Which VARy MONTHLy, DIURNALly AND By DAy OF WEEK (MHRDOW7) *

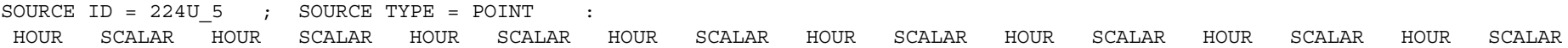

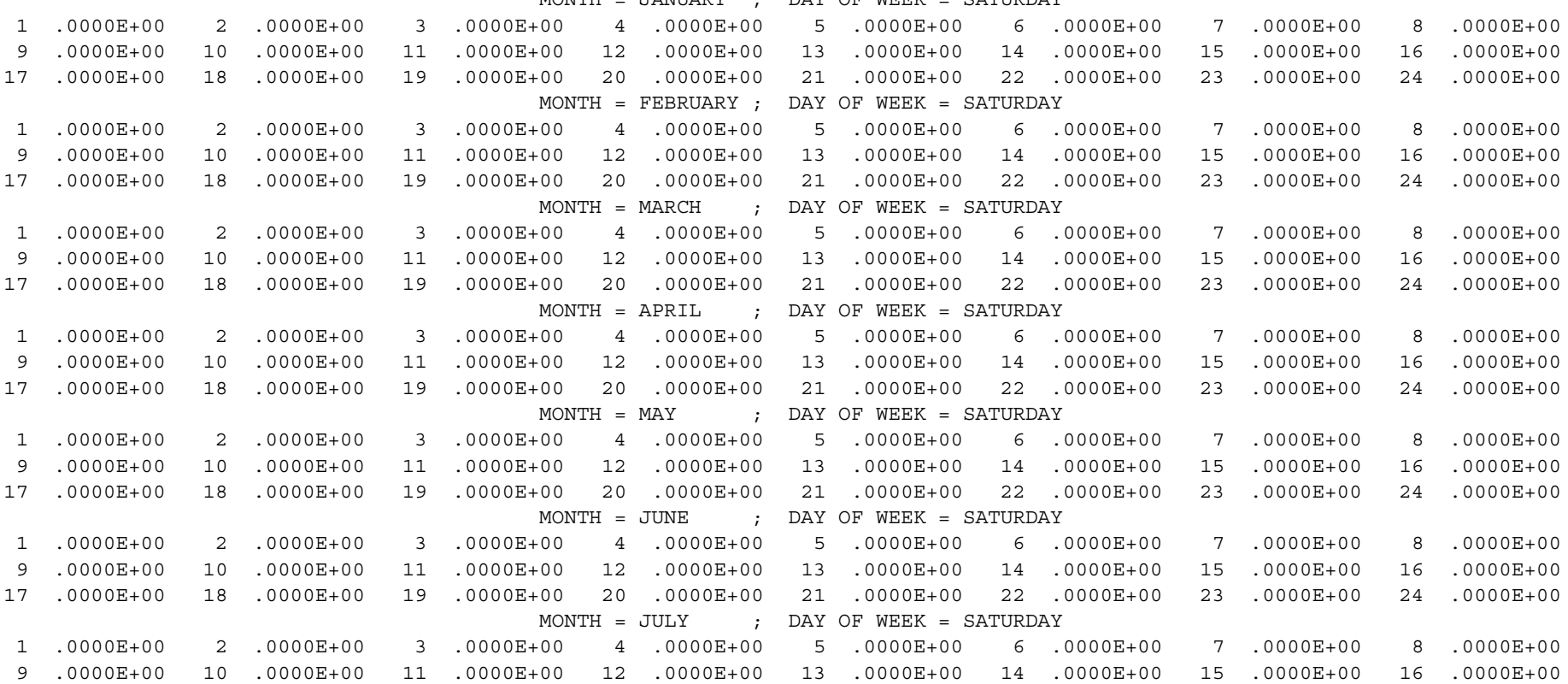




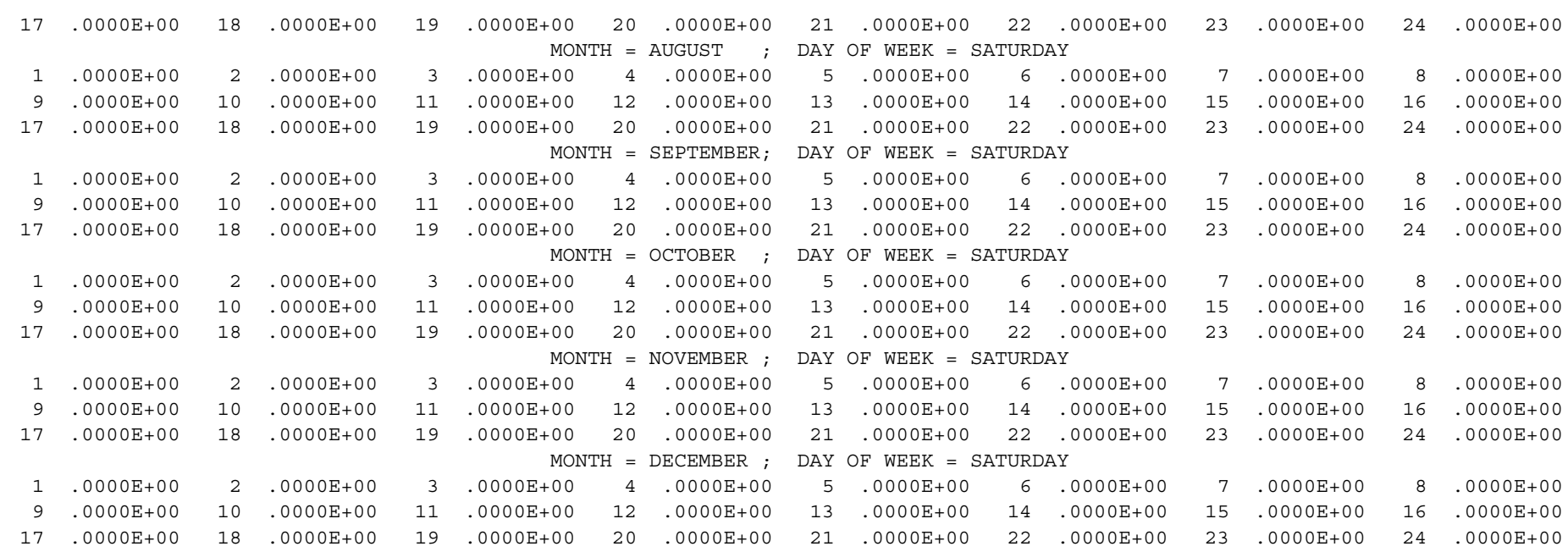

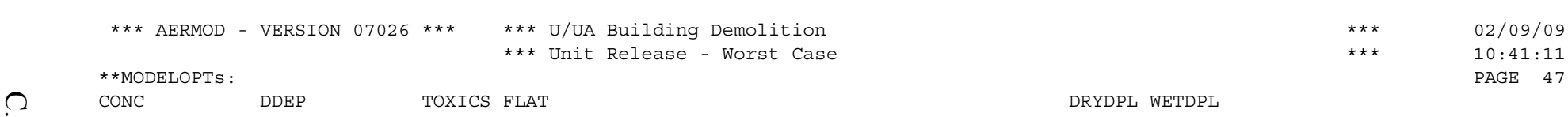

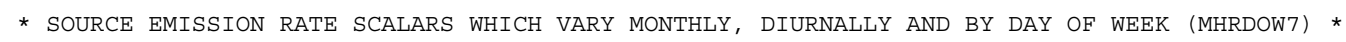

SOURCE ID $=224 \mathrm{U} \_5 ;$ SOURCE TYPE $=$ POINT

HOUR SCALAR HOUR' SCALAR HOUR SCALAR HOUR SCALAR HOUR SCALAR HOUR SCALAR HOUR SCALAR HOUR SCALAR HOUR SCALAR HOUR SCALAR HOUR SCALAR HOUR SCALAR HOUR SCALAR HOUR SCALAR HOUR SCALAR HOUR SCALAR

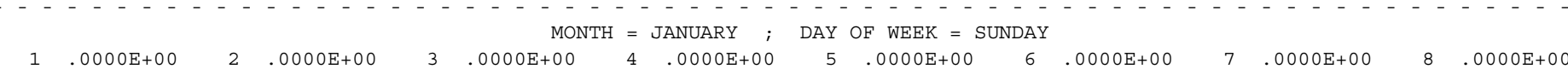

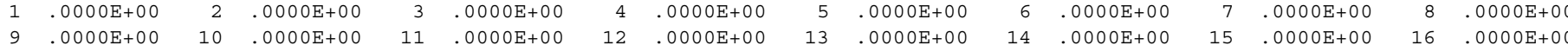

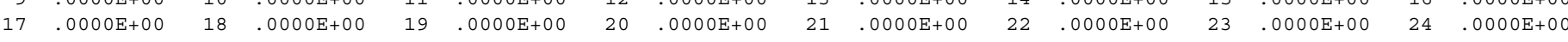
MONTH $=$ FEBRUARY; DAY OF WEEK = SUNDAY

$\begin{array}{rrrrrrrrrrrrr}9 & .0000 \mathrm{E}+00 & 4 & .0000 \mathrm{E}+00 & 5 & .0000 \mathrm{E}+00 & 6 & .0000 \mathrm{E}+00 & 7 & .0000 \mathrm{E}+00 & 8 & .0000 \mathrm{E}+00\end{array}$

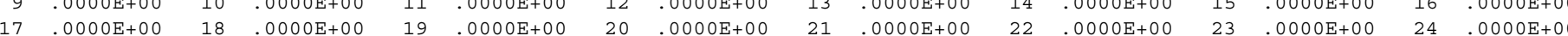

MONTH = MARCH ; DAY OF WEEK = SUNDAY

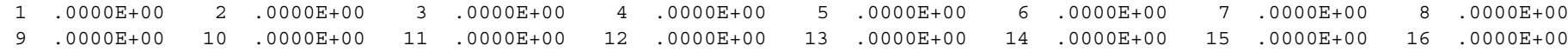

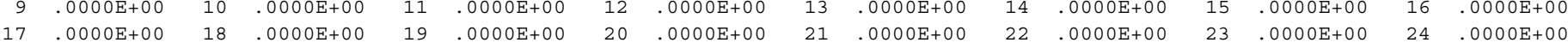

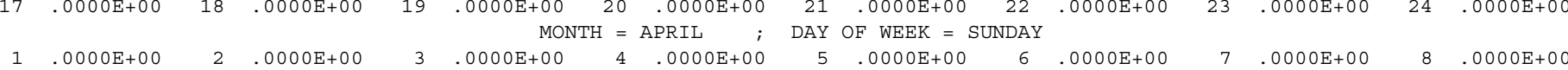

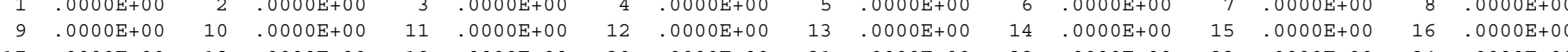

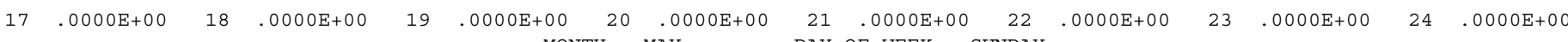

$\begin{array}{llllllllllllllll}1 & .0000 \mathrm{E}+00 & 2 & .0000 \mathrm{E}+00 & 3 & .0000 \mathrm{E}+00 & 4 & .0000 \mathrm{E}+00 & 5 & .0000 \mathrm{E}+00 & 6 & .0000 \mathrm{E}+00 & 7 & .0000 \mathrm{E}+00 & 8 & .0000 \mathrm{E}+00\end{array}$

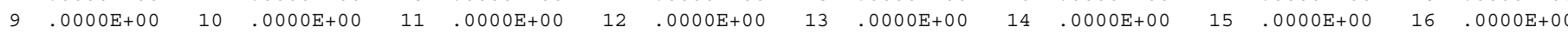

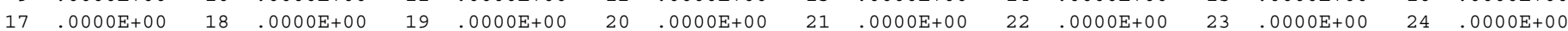




\begin{tabular}{|c|c|c|c|c|c|c|c|c|c|c|c|c|c|c|c|}
\hline & & & & & MONT & $\mathrm{TH}=$ & JUNE ; & DAY & OF WEEK = SL & UNDAY & & & & & \\
\hline 1 & $.0000 \mathrm{E}+00$ & 2 & $.0000 \mathrm{E}+00$ & 3 & $.0000 \mathrm{E}+00$ & 4 & $.0000 \mathrm{E}+00$ & 5 & $.0000 \mathrm{E}+00$ & 6 & $.0000 \mathrm{E}+00$ & 7 & $.0000 \mathrm{E}+00$ & 8 & $.0000 \mathrm{E}+00$ \\
\hline 9 & $.0000 \mathrm{E}+00$ & 10 & $.0000 \mathrm{E}+00$ & 11 & $.0000 \mathrm{E}+00$ & 12 & $.0000 \mathrm{E}+00$ & 13 & $.0000 \mathrm{E}+00$ & 14 & $.0000 \mathrm{E}+00$ & 15 & $.0000 \mathrm{E}+00$ & 16 & $.0000 \mathrm{E}+00$ \\
\hline 17 & $.0000 \mathrm{E}+00$ & 18 & $.0000 \mathrm{E}+00$ & 19 & $.0000 \mathrm{E}+00$ & 20 & $.0000 \mathrm{E}+00$ & 21 & $.0000 \mathrm{E}+00$ & 22 & $.0000 \mathrm{E}+00$ & 23 & $.0000 \mathrm{E}+00$ & 24 & $.0000 \mathrm{E}+00$ \\
\hline & & & & & MONT & $\mathrm{TH}=$ & JULY ; & DAY & OF WEEK = SL & UNDAY & & & & & \\
\hline 1 & $.0000 \mathrm{E}+00$ & 2 & $.0000 \mathrm{E}+00$ & 3 & $.0000 \mathrm{E}+00$ & 4 & $.0000 \mathrm{E}+00$ & 5 & $.0000 \mathrm{E}+00$ & 6 & $.0000 \mathrm{E}+00$ & 7 & $.0000 \mathrm{E}+00$ & 8 & $.0000 \mathrm{E}+00$ \\
\hline 9 & $.0000 E+00$ & 10 & $.0000 \mathrm{E}+00$ & 11 & $.0000 \mathrm{E}+00$ & 12 & $.0000 \mathrm{E}+00$ & 13 & $.0000 \mathrm{E}+00$ & 14 & $.0000 \mathrm{E}+00$ & 15 & $.0000 \mathrm{E}+00$ & 16 & $.0000 \mathrm{E}+00$ \\
\hline 17 & $.0000 \mathrm{E}+00$ & 18 & $.0000 \mathrm{E}+00$ & 19 & $.0000 \mathrm{E}+00$ & 20 & $.0000 \mathrm{E}+00$ & 21 & $.0000 \mathrm{E}+00$ & 22 & $.0000 \mathrm{E}+00$ & 23 & $.0000 \mathrm{E}+00$ & 24 & $.0000 \mathrm{E}+00$ \\
\hline & & & & & MONT & $T H=$ & AUGUST ; & DAY & OF WEEK $=S L$ & UNDAY & & & & & \\
\hline 1 & $.0000 \mathrm{E}+00$ & 2 & $.0000 \mathrm{E}+00$ & 3 & $.0000 \mathrm{E}+00$ & 4 & $.0000 \mathrm{E}+00$ & 5 & $.0000 \mathrm{E}+00$ & 6 & $.0000 E+00$ & 7 & $.0000 \mathrm{E}+00$ & 8 & $.0000 \mathrm{E}+00$ \\
\hline 9 & $.0000 \mathrm{E}+00$ & 10 & $.0000 \mathrm{E}+00$ & 11 & $.0000 \mathrm{E}+00$ & 12 & $.0000 \mathrm{E}+00$ & 13 & $.0000 \mathrm{E}+00$ & 14 & $.0000 \mathrm{E}+00$ & 15 & $.0000 \mathrm{E}+00$ & 16 & $.0000 \mathrm{E}+00$ \\
\hline 17 & $.0000 \mathrm{E}+00$ & 18 & $.0000 \mathrm{E}+00$ & 19 & $.0000 \mathrm{E}+00$ & 20 & $.0000 \mathrm{E}+00$ & 21 & $.0000 \mathrm{E}+00$ & 22 & $.0000 \mathrm{E}+00$ & 23 & $.0000 \mathrm{E}+00$ & 24 & $.0000 \mathrm{E}+00$ \\
\hline & & & & & MONT & $\mathrm{TH}=$ & SEPTEMBER ; & DAY & OF $\mathrm{WEEK}=\mathrm{SL}$ & UNDAY & & & & & \\
\hline 1 & $.0000 \mathrm{E}+00$ & 2 & $.0000 \mathrm{E}+00$ & 3 & $.0000 \mathrm{E}+00$ & 4 & $.0000 \mathrm{E}+00$ & 5 & $.0000 \mathrm{E}+00$ & 6 & $.0000 \mathrm{E}+00$ & 7 & $.0000 \mathrm{E}+00$ & 8 & $.0000 \mathrm{E}+00$ \\
\hline 9 & $.0000 \mathrm{E}+00$ & 10 & $.0000 \mathrm{E}+00$ & 11 & $.0000 \mathrm{E}+00$ & 12 & $.0000 \mathrm{E}+00$ & 13 & $.0000 \mathrm{E}+00$ & 14 & $.0000 \mathrm{E}+00$ & 15 & $.0000 \mathrm{E}+00$ & 16 & $.0000 \mathrm{E}+00$ \\
\hline 17 & $.0000 \mathrm{E}+00$ & 18 & $.0000 \mathrm{E}+00$ & 19 & $.0000 \mathrm{E}+00$ & 20 & $.0000 \mathrm{E}+00$ & 21 & $.0000 \mathrm{E}+00$ & 22 & $.0000 \mathrm{E}+00$ & 23 & $.0000 \mathrm{E}+00$ & 24 & $.0000 \mathrm{E}+00$ \\
\hline & & & & & MONT & $T H=$ & OCTOBER ; & DAY & OF $\mathrm{WEEK}=\mathrm{SL}$ & UNDAY & & & & & \\
\hline 1 & $.0000 \mathrm{E}+00$ & 2 & $.0000 \mathrm{E}+00$ & 3 & $.0000 \mathrm{E}+00$ & 4 & $.0000 \mathrm{E}+00$ & 5 & $.0000 \mathrm{E}+00$ & 6 & $.0000 \mathrm{E}+00$ & 7 & $.0000 \mathrm{E}+00$ & 8 & $.0000 \mathrm{E}+00$ \\
\hline 9 & $.0000 \mathrm{E}+00$ & 10 & $.0000 \mathrm{E}+00$ & 11 & $.0000 \mathrm{E}+00$ & 12 & $.0000 E+00$ & 13 & $.0000 \mathrm{E}+00$ & 14 & $.0000 \mathrm{E}+00$ & 15 & $.0000 \mathrm{E}+00$ & 16 & $.0000 \mathrm{E}+00$ \\
\hline 17 & $.0000 \mathrm{E}+00$ & 18 & $.0000 \mathrm{E}+00$ & 19 & $.0000 \mathrm{E}+00$ & 20 & $.0000 \mathrm{E}+00$ & 21 & $.0000 \mathrm{E}+00$ & 22 & $.0000 \mathrm{E}+00$ & 23 & $.0000 \mathrm{E}+00$ & 24 & $.0000 \mathrm{E}+00$ \\
\hline & & & & & MONT & $\mathrm{TH}=$ & NOVEMBER ; & DAY & $O F \quad W E E K=S L$ & UNDAY & & & & & \\
\hline 1 & $.0000 \mathrm{E}+00$ & 2 & $.0000 \mathrm{E}+00$ & 3 & $.0000 \mathrm{E}+00$ & 4 & $.0000 \mathrm{E}+00$ & 5 & $.0000 \mathrm{E}+00$ & 6 & $.0000 \mathrm{E}+00$ & 7 & $.0000 \mathrm{E}+00$ & 8 & $.0000 \mathrm{E}+00$ \\
\hline 9 & $.0000 \mathrm{E}+00$ & 10 & $.0000 \mathrm{E}+00$ & 11 & $.0000 \mathrm{E}+00$ & 12 & $.0000 \mathrm{E}+00$ & 13 & $.0000 \mathrm{E}+00$ & 14 & $.0000 \mathrm{E}+00$ & 15 & $.0000 \mathrm{E}+00$ & 16 & $.0000 \mathrm{E}+00$ \\
\hline 17 & $.0000 E+00$ & 18 & $.0000 E+00$ & 19 & $.0000 \mathrm{E}+00$ & 20 & $.0000 \mathrm{E}+00$ & 21 & $.0000 \mathrm{E}+00$ & 22 & $.0000 \mathrm{E}+00$ & 23 & $.0000 E+00$ & 24 & $.0000 \mathrm{E}+00$ \\
\hline & & & & & MONT & $\mathrm{TH}=$ & DECEMBER ; & DAY & $O F \quad W E E K=S L$ & UNDAY & & & & & \\
\hline 1 & $.0000 \mathrm{E}+00$ & 2 & $.0000 \mathrm{E}+00$ & 3 & $.0000 \mathrm{E}+00$ & 4 & $.0000 \mathrm{E}+00$ & 5 & $.0000 \mathrm{E}+00$ & 6 & $.0000 \mathrm{E}+00$ & 7 & $.0000 \mathrm{E}+00$ & 8 & $.0000 \mathrm{E}+00$ \\
\hline 9 & $.0000 \mathrm{E}+00$ & 10 & $.0000 \mathrm{E}+00$ & 11 & $.0000 \mathrm{E}+00$ & 12 & $.0000 \mathrm{E}+00$ & 13 & $.0000 \mathrm{E}+00$ & 14 & $.0000 \mathrm{E}+00$ & 15 & $.0000 \mathrm{E}+00$ & 16 & $.0000 \mathrm{E}+00$ \\
\hline 17 & $.0000 \mathrm{E}+00$ & 18 & $.0000 \mathrm{E}+00$ & 19 & $.0000 E+00$ & 20 & $.0000 E+00$ & 21 & $.0000 E+00$ & 22 & $.0000 \mathrm{E}+00$ & 23 & $.0000 E+00$ & 24 & $.0000 \mathrm{E}+00$ \\
\hline$\star * * A$ & AERMOD - VE & RSION & $07026 * * *$ & $\star * *$ & * U/UA Builo & ding & Demolition & & & & & & $\star \star *$ & & $02 / 09 / 09$ \\
\hline & & & & ** & * Unit Relea & ase - & - Worst Case & & & & & & $* * *$ & & $10: 41: 11$ \\
\hline$\star \star \mathrm{MODE}$ & DELOPTS : & & & & & & & & & & & & & & PAGE 48 \\
\hline $\mathrm{CONC}$ & DD & & TOXIC & CS FLA & & & & & & & RYDPL WETDPL & & & & \\
\hline & & * SOU & URCE EMISSIO & ON RAT & TE SCALARS W & $\mathrm{WHICH}$ & H VARY MONTHI & $L Y, D$ & IURNALLY ANI & D BY D & DAY OF WEEK & (MHRL & Dow7) * & & \\
\hline SOURCE & $\mathrm{CE} \quad \mathrm{ID}=224 \mathrm{U}$ & & SOURCE TY & $\mathrm{YPE}=$ & POINT & & & & & & & & & & \\
\hline HOUR & IR SCALAR & HOUR' & SCALAR & HOUR & SCALAR & HOUR & SCALAR & HOUR & SCALAR & HOUR & SCALAR & HOUR & SCALAR & HOUR & SCALAR \\
\hline & & & & & MONT & & $\begin{array}{c}-\cdots- \\
\text { JANUARY }\end{array}$ & $\begin{array}{c}-- \\
\text { DAY }\end{array}$ & $\begin{array}{l}---- \\
O F \text { WEEK }=\mathrm{MC}\end{array}$ & $\begin{array}{l}-\cdots \\
\text { ONDAY }\end{array}$ & ---- & - - - & $-\ldots$ & - & $-\ldots$ \\
\hline 1 & $.0000 \mathrm{E}+00$ & 2 & $.0000 \mathrm{E}+00$ & 3 & $.0000 \mathrm{E}+00$ & 4 & $.0000 \mathrm{E}+00$ & 5 & $.0000 \mathrm{E}+00$ & 6 & $.0000 \mathrm{E}+00$ & 7 & $.0000 \mathrm{E}+00$ & 8 & $.0000 \mathrm{E}+00$ \\
\hline 9 & $.0000 \mathrm{E}+00$ & 10 & $.0000 \mathrm{E}+00$ & 11 & $.0000 \mathrm{E}+00$ & 12 & $.0000 \mathrm{E}+00$ & 13 & $.0000 \mathrm{E}+00$ & 14 & $.0000 \mathrm{E}+00$ & 15 & $.0000 \mathrm{E}+00$ & 16 & $.0000 \mathrm{E}+00$ \\
\hline 17 & $.0000 \mathrm{E}+00$ & 18 & $.0000 \mathrm{E}+00$ & 19 & $.0000 \mathrm{E}+00$ & 20 & $.0000 \mathrm{E}+00$ & 21 & $.0000 \mathrm{E}+00$ & 22 & $.0000 \mathrm{E}+00$ & 23 & $.0000 \mathrm{E}+00$ & 24 & $.0000 \mathrm{E}+00$ \\
\hline & & & & & MONT & $T H=$ & FEBRUARY ; & DAY & OF WEEK $=\mathrm{M}$ & ONDAY & & & & & \\
\hline 1 & $.0000 \mathrm{E}+00$ & 2 & $.0000 \mathrm{E}+00$ & 3 & $.0000 \mathrm{E}+00$ & 4 & $.0000 \mathrm{E}+00$ & 5 & $.0000 \mathrm{E}+00$ & 6 & $.0000 \mathrm{E}+00$ & 7 & $.0000 \mathrm{E}+00$ & 8 & $.0000 \mathrm{E}+00$ \\
\hline 9 & $.0000 \mathrm{E}+00$ & 10 & $.0000 \mathrm{E}+00$ & 11 & $.0000 \mathrm{E}+00$ & 12 & $.0000 \mathrm{E}+00$ & 13 & $.0000 \mathrm{E}+00$ & 14 & $.0000 \mathrm{E}+00$ & 15 & $.0000 \mathrm{E}+00$ & 16 & $.0000 \mathrm{E}+00$ \\
\hline 17 & $.0000 \mathrm{E}+00$ & 18 & $.0000 \mathrm{E}+00$ & 19 & $.0000 \mathrm{E}+00$ & 20 & $.0000 \mathrm{E}+00$ & 21 & $.0000 \mathrm{E}+00$ & 22 & $.0000 \mathrm{E}+00$ & 23 & $.0000 \mathrm{E}+00$ & 24 & $.0000 \mathrm{E}+00$ \\
\hline & & & & & MONT & $T H=$ & MARCH ; & DAY & OF WEEK $=\mathrm{M}$ & ONDAY & & & & & \\
\hline 1 & $.0000 \mathrm{E}+00$ & 2 & $.0000 \mathrm{E}+00$ & 3 & $.0000 \mathrm{E}+00$ & 4 & $.0000 \mathrm{E}+00$ & 5 & $.0000 \mathrm{E}+00$ & 6 & $.0000 \mathrm{E}+00$ & 7 & $.0000 \mathrm{E}+00$ & 8 & $.0000 \mathrm{E}+00$ \\
\hline 9 & $.0000 \mathrm{E}+00$ & 10 & $.0000 \mathrm{E}+00$ & 11 & $.0000 \mathrm{E}+00$ & 12 & $.0000 E+00$ & 13 & $.0000 \mathrm{E}+00$ & 14 & $.0000 \mathrm{E}+00$ & 15 & $.0000 \mathrm{E}+00$ & 16 & $.0000 \mathrm{E}+00$ \\
\hline 17 & $.0000 \mathrm{E}+00$ & 18 & $.0000 \mathrm{E}+00$ & 19 & $.0000 \mathrm{E}+00$ & 20 & $.0000 \mathrm{E}+00$ & 21 & $.0000 \mathrm{E}+00$ & 22 & $.0000 \mathrm{E}+00$ & 23 & $.0000 \mathrm{E}+00$ & 24 & $.0000 \mathrm{E}+00$ \\
\hline
\end{tabular}

MONTH $=$ APRIL $;$ DAY OF WEEK $=$ MONDAY 


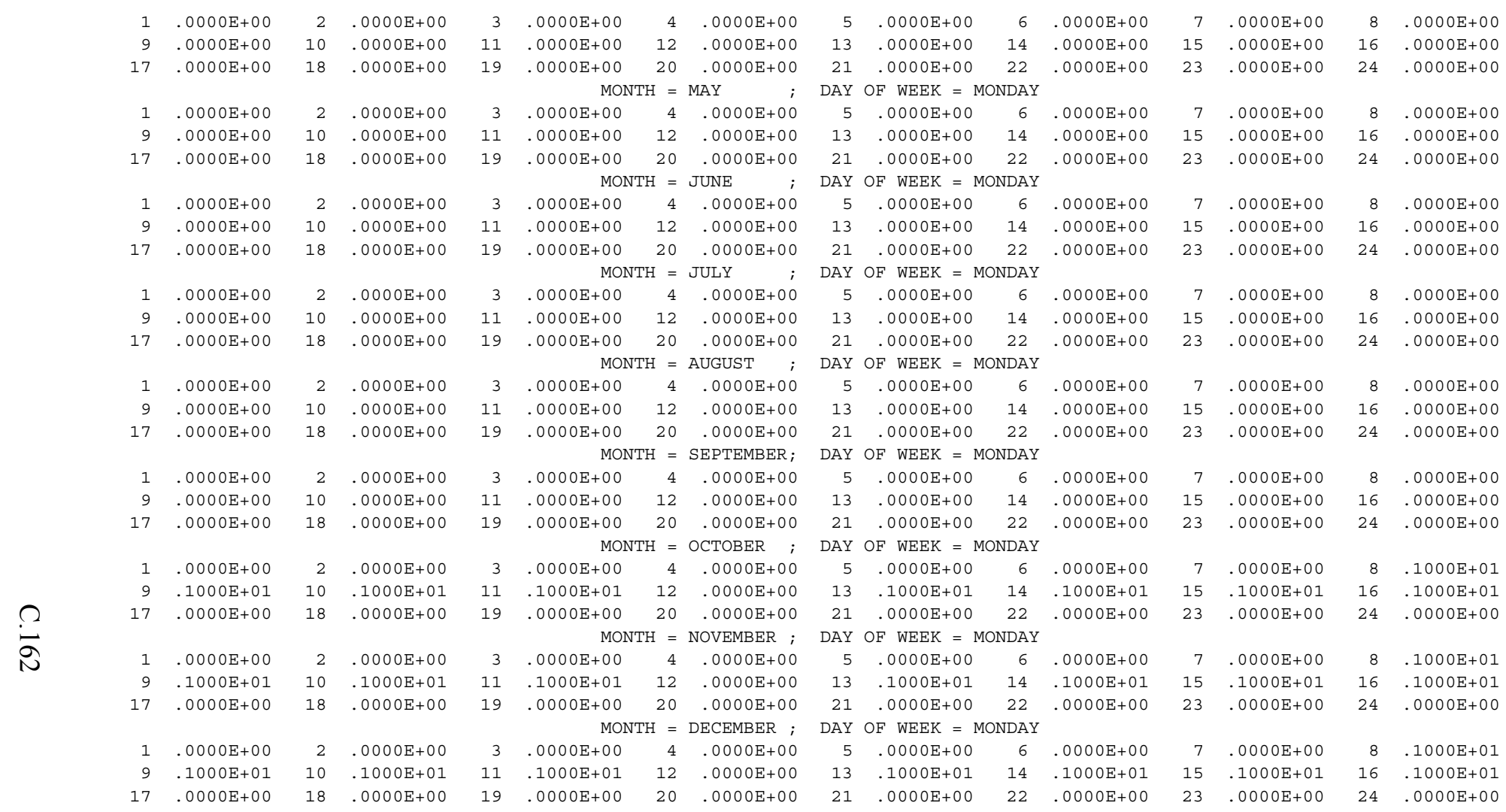

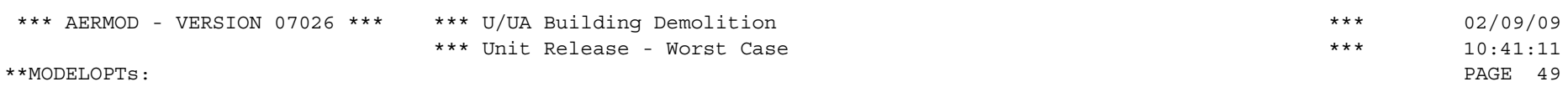

DDEP TOXICS FLAT

DRYDPL WETDPL

* SOURCE EMISSION RATE SCALARS WHICH VARY MONTHLY, DIURNALLY AND BY DAY OF WEEK (MHRDOW7) *

SOURCE ID $=224 U_{-} 6 ;$ SOURCE TYPE $=$ POINT

HOUR SCALAR HOUR SCALAR HOUR SCALAR HOUR SCALAR HOUR SCALAR HOUR SCALAR HOUR SCALAR HOUR SCALAR

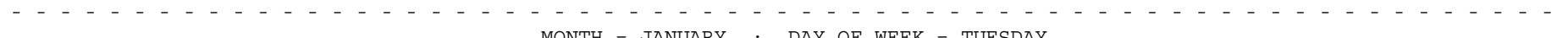

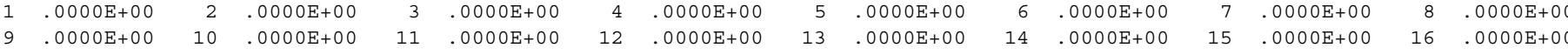

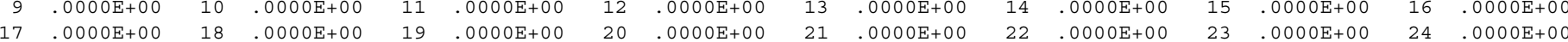

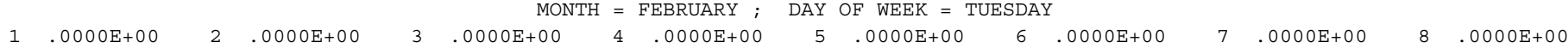




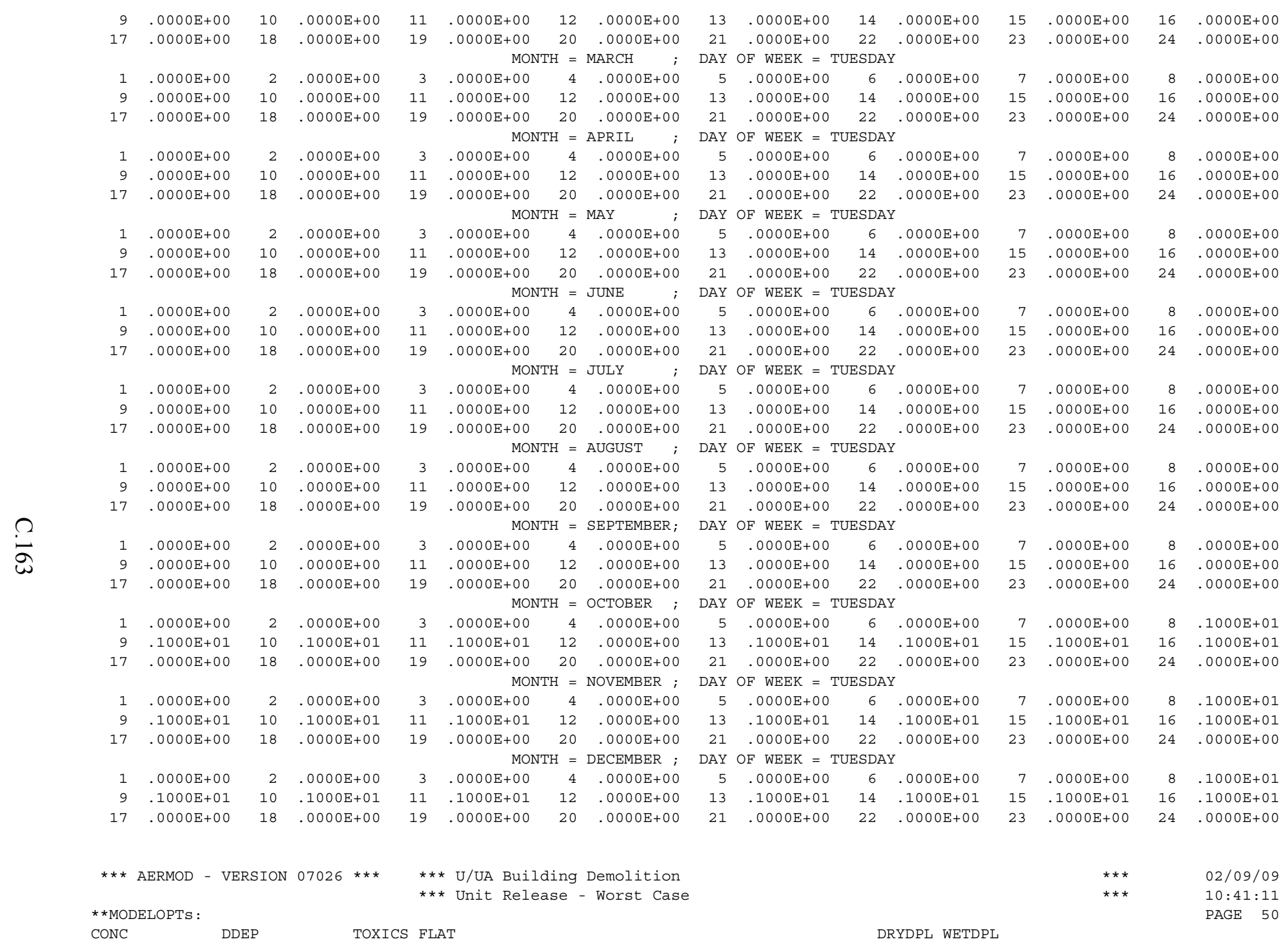

* SOURCE EMISSiON RATE SCALARS WHICH VARY MONTHLy, DIURNALly AND By DAY OF WEEK (MHRDOW7) *

SOURCE ID $=224 \mathrm{U}_{-} 6 ;$ SOURCE TYPE $=$ POINT

HOUR SCALAR HOUR SCALAR HOUR SCALAR HOUR SCALAR HOUR SCALAR HOUR SCALAR HOUR SCALAR HOUR SCALAR 


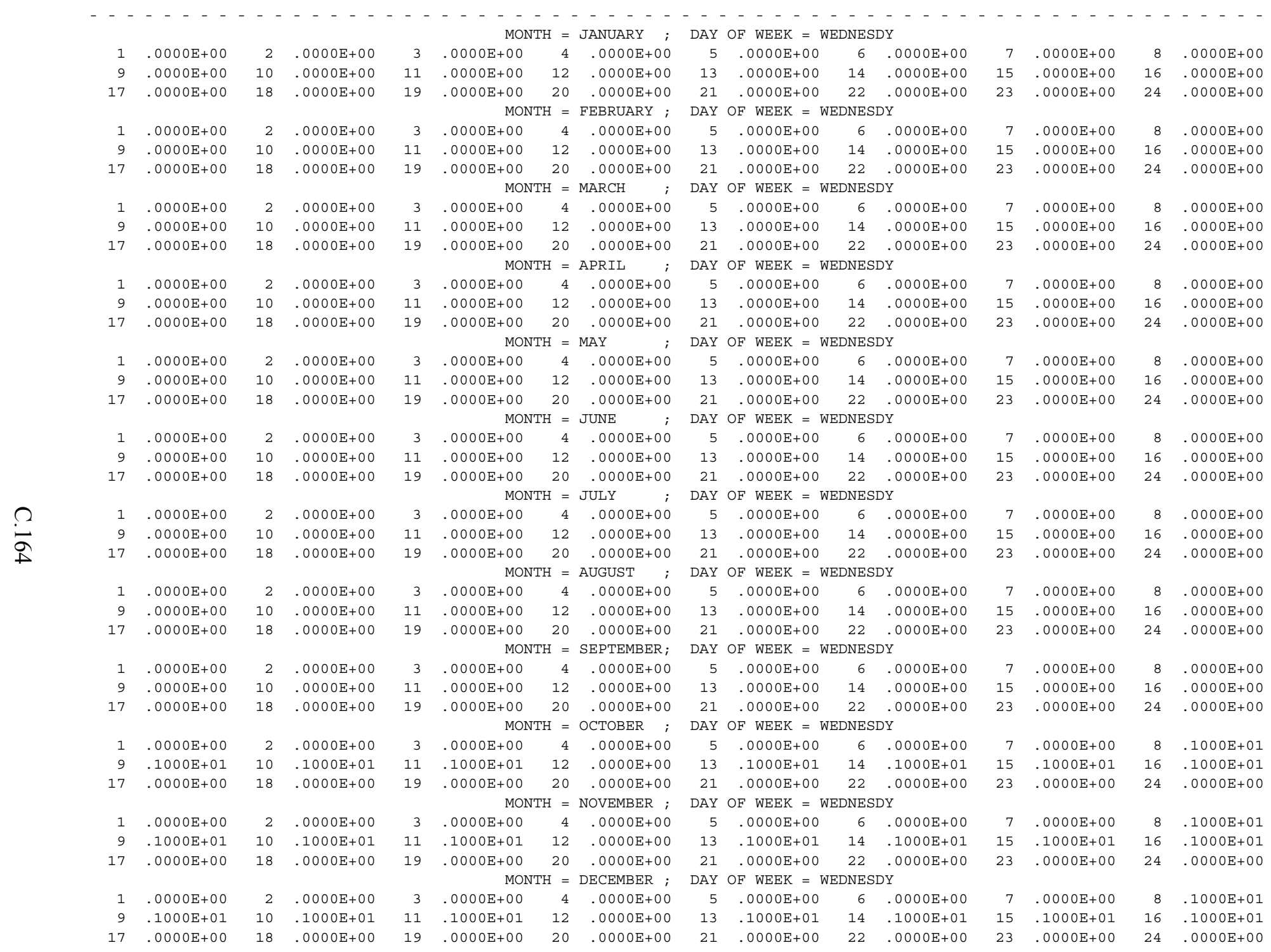

*** AERMOD - VERSION $07026 * * * \quad * * *$ U/UA Building Demolition

*** Unit Release - Worst Case 
* SOURCE EMISSION RATE SCALARS WHICH VARY MONTHLY, DIURNALLY AND BY DAY OF WEEK (MHRDOW7) *

SOURCE ID $=224 \mathrm{U} 6 ;$ SOURCE TYPE $=$ POINT

HOUR SCATAR - HOUR' SCATAR HOUR SCALAR' HOUR SCALAR HOUR SCALAR HOUR SCALAR HOUR SCALAR HOUR SCALAR

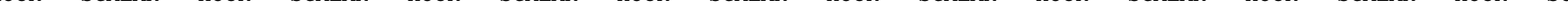

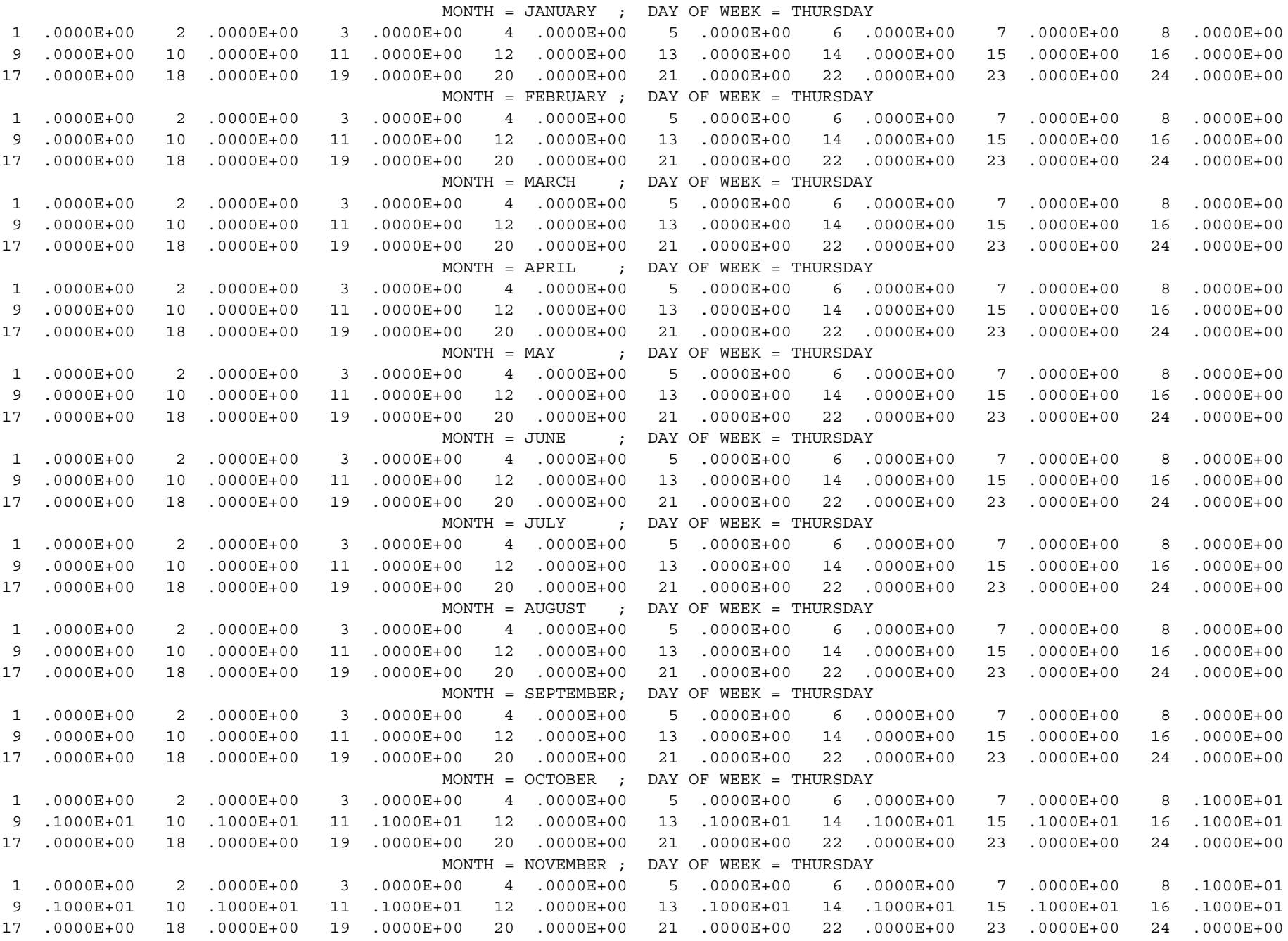
MONTH $=$ DECEMBER $;$ DAY OF WEEK $=$ THURSDAY 


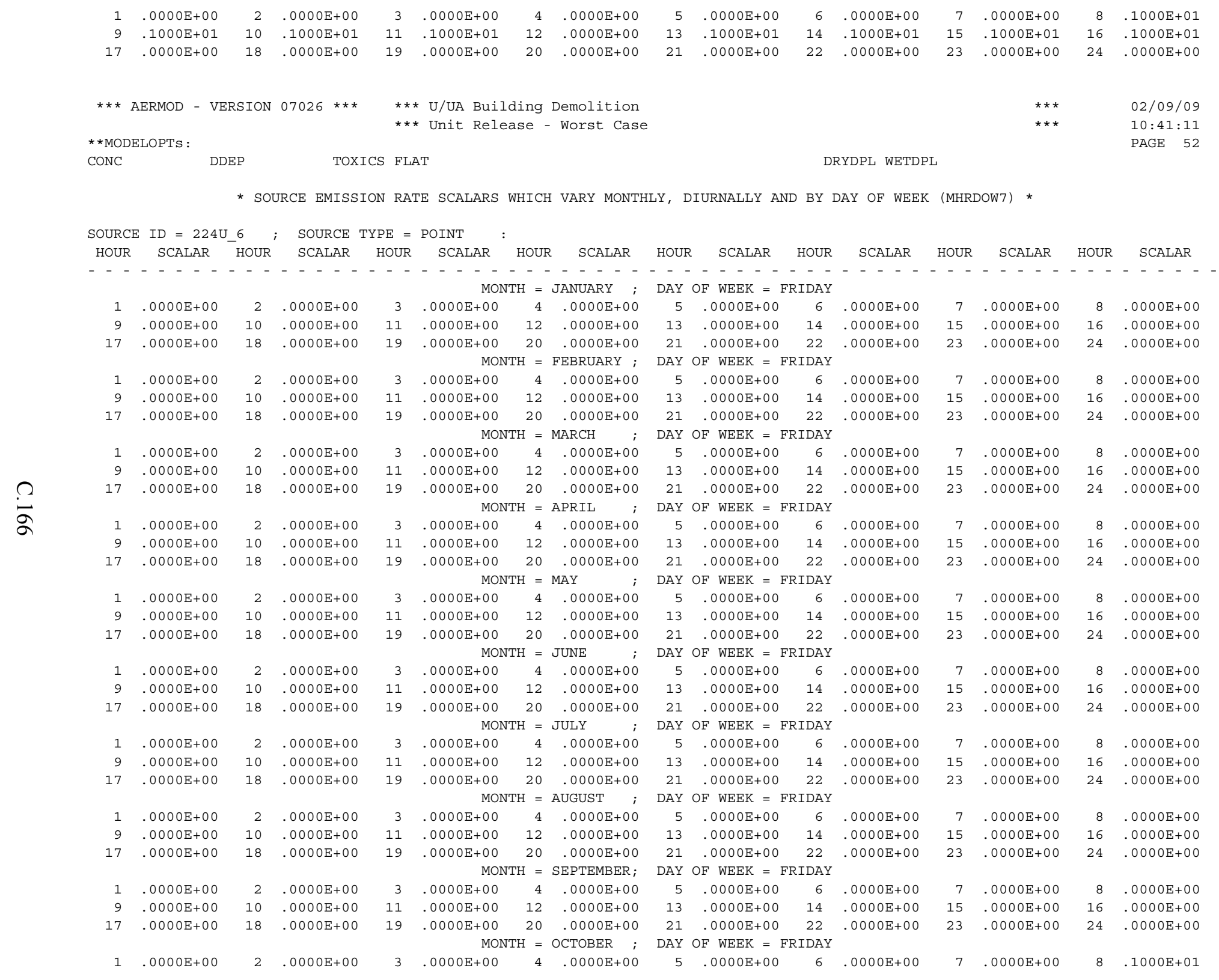




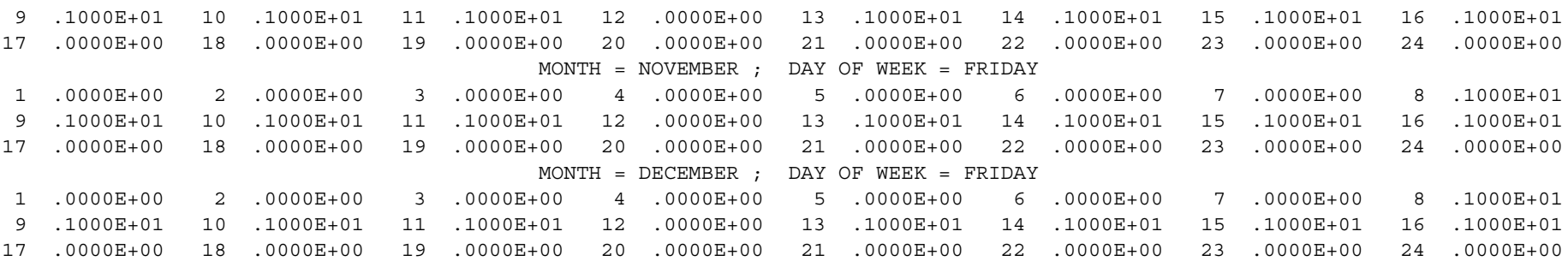

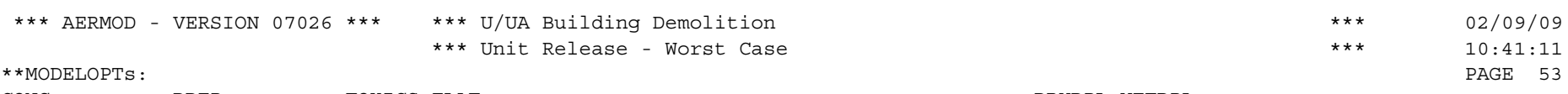
DDEP
TOXICS FLAT
DRYDPL WETDPL

CONC

PAGE 53

* SOURCE EMISSiOn RATE SCALARS WHICH VARY MONTHLY, DIURNALLY AND By DAY OF WEEK (MHRDOW7) *

SOURCE ID $=224 \mathrm{U}_{-} 6 ;$ SOURCE TYPE $=$ POINT

HOUR SCALAR HOUR SCALAR HOUR SCALAR HOUR SCALAR HOUR SCALAR HOUR SCALAR HOUR SCALAR HOUR SCALAR

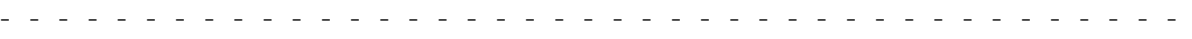

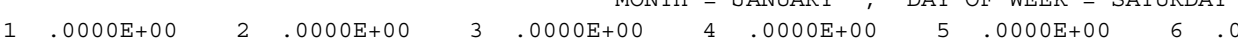

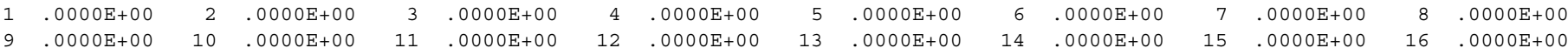

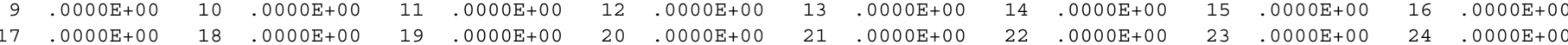

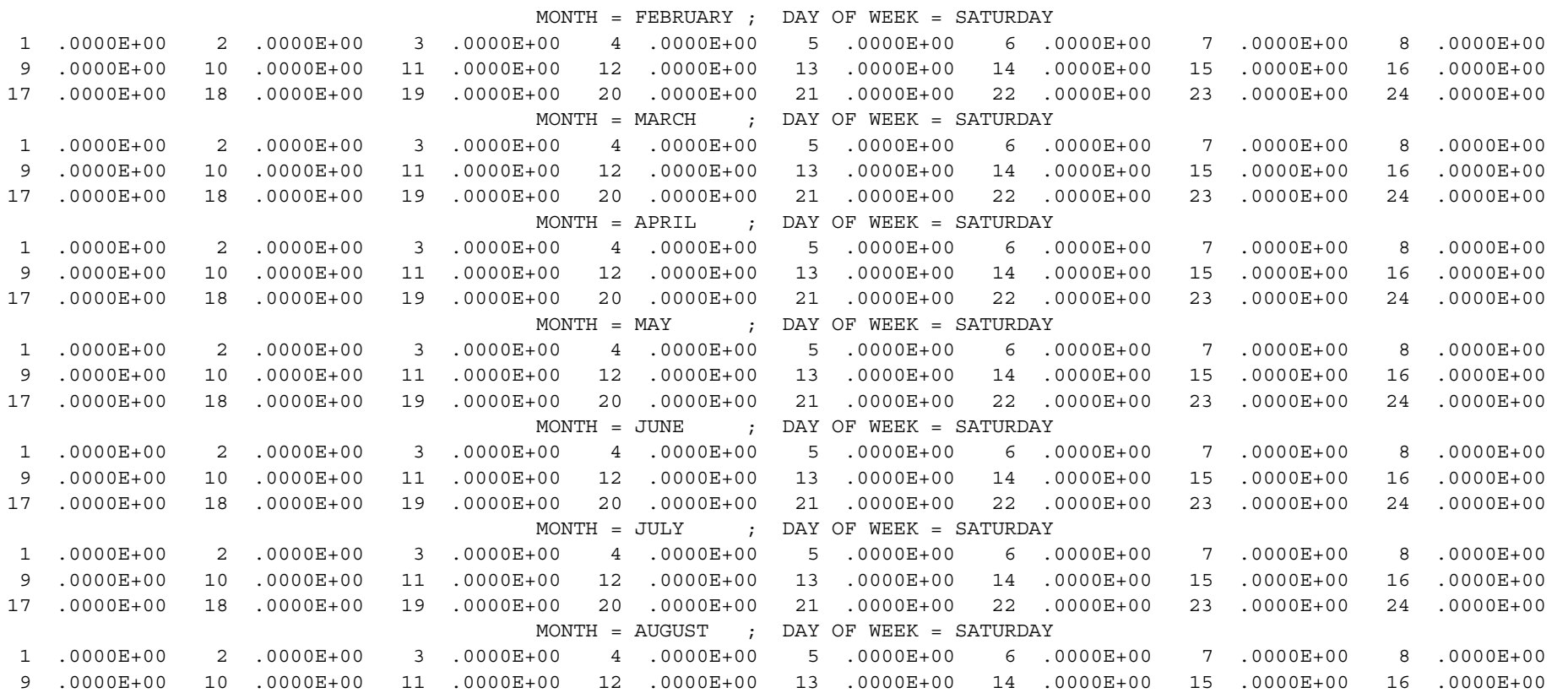




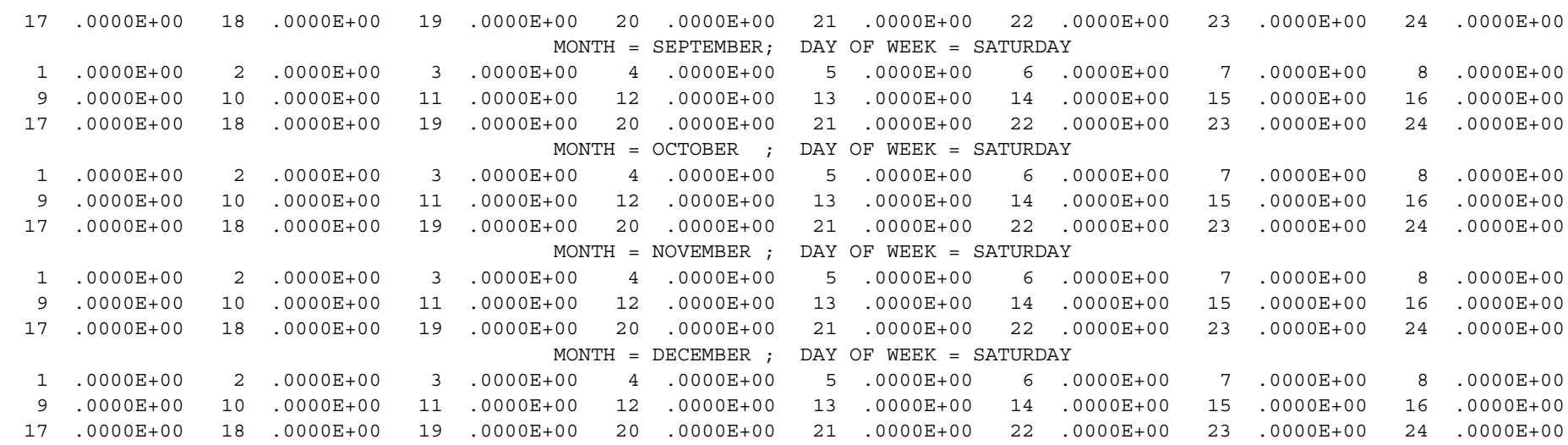

\begin{tabular}{|c|c|c|c|c|}
\hline *** AERMOD & - VERSION $07026 * \star *$ & $\begin{array}{l}* * * \text { U/UA Building Demolition } \\
* * * \text { Unit Release - Worst Case }\end{array}$ & $\begin{array}{l}* * * \\
* * *\end{array}$ & $\begin{array}{l}02 / 09 / 09 \\
10: 41: 11\end{array}$ \\
\hline & & & & PAGE $\quad 54$ \\
\hline
\end{tabular}

CONC

DDEP

DRYDPL WETDPL

PAGE 54

$\Omega$ SOURCE ID $=224 \mathrm{U}_{-} 6 ;$ SOURCE TYPE $=$ POINT

HOUR SCALAR HOUR SCALAR HOUR SCALAR HOUR SCALAR HOUR SCALAR HOUR SCALAR HOUR SCALAR HOUR SCALAR

\begin{tabular}{|c|c|c|c|c|c|c|c|c|c|c|c|c|c|c|c|}
\hline 1 & $.0000 \mathrm{E}+00$ & 2 & $.0000 \mathrm{E}+00$ & 3 & $\begin{array}{r}\text { MON } \\
.0000 \mathrm{E}+00\end{array}$ & $\begin{array}{r}\mathrm{H}= \\
4\end{array}$ & $\begin{array}{l}\text { JANUARY } \\
.0000 \mathrm{E}+00\end{array}$ & $\begin{array}{r}\text { DAY } \\
5\end{array}$ & $\begin{array}{r}\text { OF } \text { WEEK }= \\
0000 E+00\end{array}$ & $\begin{array}{c}\text { UNDAY } \\
6\end{array}$ & $.0000 \mathrm{E}+00$ & 7 & $.0000 \mathrm{E}+00$ & 8 & $.0000 \mathrm{E}+00$ \\
\hline 9 & $.0000 \mathrm{E}+00$ & 10 & $.0000 \mathrm{E}+00$ & 11 & $.0000 \mathrm{E}+00$ & 12 & $.0000 \mathrm{E}+00$ & 13 & $.0000 \mathrm{E}+00$ & 14 & $.0000 \mathrm{E}+00$ & 15 & $.0000 \mathrm{E}+00$ & 16 & $.0000 \mathrm{E}+00$ \\
\hline \multirow[t]{2}{*}{17} & $.0000 \mathrm{E}+00$ & 18 & $.0000 \mathrm{E}+00$ & 19 & $.0000 \mathrm{E}+00$ & 20 & $.0000 \mathrm{E}+00$ & 21 & $.0000 \mathrm{E}+00$ & 22 & $.0000 \mathrm{E}+00$ & 23 & $.0000 \mathrm{E}+00$ & 24 & $.0000 \mathrm{E}+00$ \\
\hline & & & & & MON & $\mathrm{H}=$ & FEBRUARY ; & DAY & OF WEEK = & UNDAY & & & & & \\
\hline 1 & $.0000 \mathrm{E}+00$ & 2 & $.0000 \mathrm{E}+00$ & 3 & $.0000 \mathrm{E}+00$ & 4 & $.0000 \mathrm{E}+00$ & 5 & $.0000 \mathrm{E}+00$ & 6 & $.0000 \mathrm{E}+00$ & 7 & $.0000 \mathrm{E}+00$ & 8 & $.0000 \mathrm{E}+00$ \\
\hline 9 & $.0000 \mathrm{E}+00$ & 10 & $.0000 \mathrm{E}+00$ & 11 & $.0000 \mathrm{E}+00$ & 12 & $.0000 \mathrm{E}+00$ & 13 & $.0000 \mathrm{E}+00$ & 14 & $.0000 \mathrm{E}+00$ & 15 & $.0000 \mathrm{E}+00$ & 16 & $.0000 \mathrm{E}+00$ \\
\hline \multirow[t]{2}{*}{17} & $.0000 \mathrm{E}+00$ & 18 & $.0000 \mathrm{E}+00$ & 19 & $.0000 \mathrm{E}+00$ & 20 & $.0000 \mathrm{E}+00$ & 21 & $.0000 \mathrm{E}+00$ & 22 & $.0000 \mathrm{E}+00$ & 23 & $.0000 \mathrm{E}+00$ & 24 & $.0000 \mathrm{E}+00$ \\
\hline & & & & & MON & $\mathrm{H}=$ & MARCH ; & DAY & OF WEEK $=$ & UNDAY & & & & & \\
\hline 1 & $.0000 \mathrm{E}+00$ & 2 & $.0000 \mathrm{E}+00$ & 3 & $.0000 \mathrm{E}+00$ & 4 & $.0000 \mathrm{E}+00$ & 5 & $.0000 E+00$ & 6 & $.0000 \mathrm{E}+00$ & 7 & $.0000 \mathrm{E}+00$ & 8 & $.0000 \mathrm{E}+00$ \\
\hline 9 & $.0000 \mathrm{E}+00$ & 10 & $.0000 \mathrm{E}+00$ & 11 & $.0000 \mathrm{E}+00$ & 12 & $.0000 \mathrm{E}+00$ & 13 & $.0000 \mathrm{E}+00$ & 14 & $.0000 \mathrm{E}+00$ & 15 & $.0000 \mathrm{E}+00$ & 16 & $.0000 \mathrm{E}+00$ \\
\hline \multirow[t]{2}{*}{17} & $.0000 \mathrm{E}+00$ & 18 & $.0000 \mathrm{E}+00$ & 19 & $.0000 \mathrm{E}+00$ & 20 & $.0000 \mathrm{E}+00$ & 21 & $.0000 \mathrm{E}+00$ & 22 & $.0000 \mathrm{E}+00$ & 23 & $.0000 \mathrm{E}+00$ & 24 & $.0000 \mathrm{E}+00$ \\
\hline & & & & & MON & $\mathrm{H}=$ & APRIL ; & DAY & OF WEEK = & SUNDAY & & & & & \\
\hline 1 & $.0000 \mathrm{E}+00$ & 2 & $.0000 \mathrm{E}+00$ & 3 & $.0000 \mathrm{E}+00$ & 4 & $.0000 \mathrm{E}+00$ & 5 & $.0000 \mathrm{E}+00$ & 6 & $.0000 \mathrm{E}+00$ & 7 & $.0000 \mathrm{E}+00$ & 8 & $.0000 \mathrm{E}+00$ \\
\hline 9 & $.0000 \mathrm{E}+00$ & 10 & $.0000 \mathrm{E}+00$ & 11 & $.0000 \mathrm{E}+00$ & 12 & $.0000 \mathrm{E}+00$ & 13 & $.0000 \mathrm{E}+00$ & 14 & $.0000 \mathrm{E}+00$ & 15 & $0 \mathrm{E}+00$ & 16 & $.0000 \mathrm{E}+00$ \\
\hline \multirow[t]{2}{*}{17} & $.0000 \mathrm{E}+00$ & 18 & $.0000 \mathrm{E}+00$ & 19 & $.0000 \mathrm{E}+00$ & 20 & $.0000 \mathrm{E}+00$ & 21 & $.0000 \mathrm{E}+00$ & 22 & $.0000 \mathrm{E}+00$ & 23 & $.0000 \mathrm{E}+00$ & 24 & $.0000 \mathrm{E}+00$ \\
\hline & & & & & MON & $\mathrm{H}=$ & MAY ; & DAY & OF WEEK $=$ & SUNDAY & & & & & \\
\hline 1 & $.0000 \mathrm{E}+00$ & 2 & $.0000 \mathrm{E}+00$ & 3 & $.0000 \mathrm{E}+00$ & 4 & $.0000 \mathrm{E}+00$ & 5 & $.0000 \mathrm{E}+00$ & 6 & $.0000 \mathrm{E}+00$ & 7 & $.0000 \mathrm{E}+00$ & 8 & $.0000 \mathrm{E}+00$ \\
\hline 9 & $.0000 \mathrm{E}+00$ & 10 & $.0000 \mathrm{E}+00$ & 11 & $.0000 \mathrm{E}+00$ & 12 & $.0000 \mathrm{E}+00$ & 13 & $.0000 \mathrm{E}+00$ & 14 & $.0000 \mathrm{E}+00$ & 15 & $.0000 \mathrm{E}+00$ & 16 & $.0000 \mathrm{E}+00$ \\
\hline \multirow[t]{2}{*}{17} & $.0000 \mathrm{E}+00$ & 18 & $.0000 \mathrm{E}+00$ & 19 & $.0000 \mathrm{E}+00$ & 20 & $.0000 \mathrm{E}+00$ & 21 & $.0000 \mathrm{E}+00$ & 22 & $.0000 \mathrm{E}+00$ & 23 & $.0000 \mathrm{E}+00$ & 24 & $.0000 \mathrm{E}+00$ \\
\hline & & & & & MON & $\mathrm{H}=$ & JUNE ; & DAY & OF WEEK = & UNDAY & & & & & \\
\hline 1 & $.0000 \mathrm{E}+00$ & 2 & $.0000 \mathrm{E}+00$ & 3 & $.0000 \mathrm{E}+00$ & 4 & $.0000 \mathrm{E}+00$ & 5 & $.0000 \mathrm{E}+00$ & 6 & $.0000 \mathrm{E}+00$ & 7 & $.0000 \mathrm{E}+00$ & 8 & $.0000 \mathrm{E}+00$ \\
\hline 9 & $.0000 \mathrm{E}+00$ & 10 & $.0000 \mathrm{E}+00$ & 11 & $.0000 \mathrm{E}+00$ & 12 & $.0000 \mathrm{E}+00$ & 13 & $.0000 \mathrm{E}+00$ & 14 & $.0000 \mathrm{E}+00$ & 15 & $.0000 \mathrm{E}+00$ & 16 & $.0000 \mathrm{E}+00$ \\
\hline 17 & $.0000 \mathrm{E}+00$ & 18 & $.0000 E+00$ & 19 & $.0000 \mathrm{E}+00$ & 20 & $.0000 \mathrm{E}+00$ & 21 & $.0000 \mathrm{E}+00$ & 22 & $.0000 \mathrm{E}+00$ & 23 & $.0000 \mathrm{E}+00$ & 24 & $.0000 \mathrm{E}+00$ \\
\hline
\end{tabular}




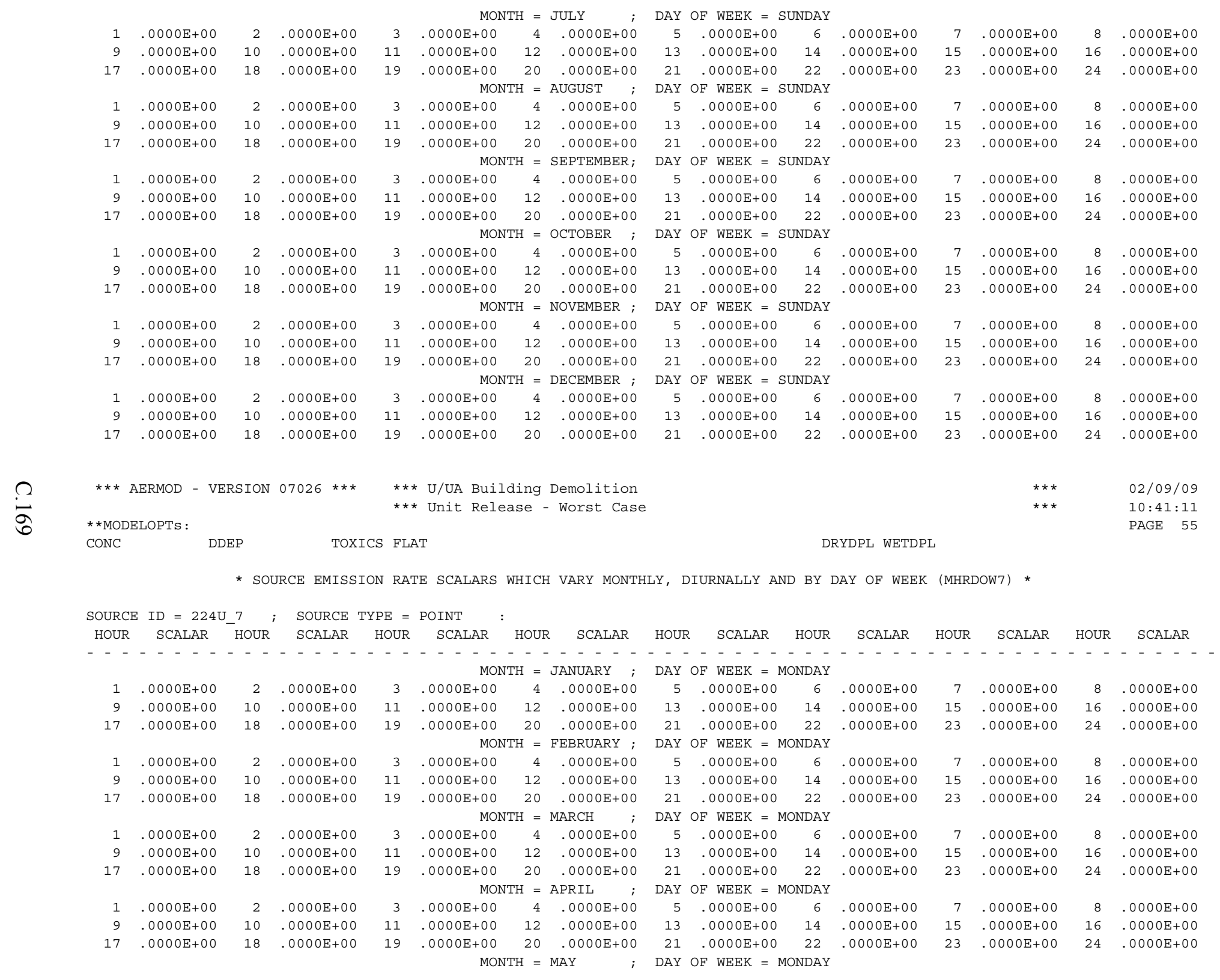




\begin{tabular}{|c|c|c|c|c|c|c|c|c|c|c|c|c|c|c|c|}
\hline 1 & $.0000 \mathrm{E}+00$ & 2 & $.0000 \mathrm{E}+00$ & 3 & $.0000 \mathrm{E}+00$ & 4 & $.0000 \mathrm{E}+00$ & 5 & $.0000 \mathrm{E}+00$ & 6 & $.0000 \mathrm{E}+00$ & 7 & $.0000 \mathrm{E}+00$ & 8 & $.0000 \mathrm{E}+00$ \\
\hline 9 & $.0000 \mathrm{E}+00$ & 10 & $.0000 \mathrm{E}+00$ & 11 & $.0000 \mathrm{E}+00$ & 12 & $.0000 \mathrm{E}+00$ & 13 & $.0000 \mathrm{E}+00$ & 14 & $.0000 \mathrm{E}+00$ & 15 & $.0000 \mathrm{E}+00$ & 16 & $.0000 \mathrm{E}+00$ \\
\hline 17 & $.0000 \mathrm{E}+00$ & 18 & $.0000 \mathrm{E}+00$ & 19 & $.0000 \mathrm{E}+00$ & 20 & $.0000 \mathrm{E}+00$ & 21 & $.0000 \mathrm{E}+00$ & 22 & $.0000 \mathrm{E}+00$ & 23 & $.0000 \mathrm{E}+00$ & 24 & $.0000 \mathrm{E}+00$ \\
\hline & & & & & MON & $\mathrm{H}=$ & JUNE ; & DAY & $O F \quad \mathrm{WEEK}=\mathrm{N}$ & DNDAY & & & & & \\
\hline 1 & $.0000 \mathrm{E}+00$ & 2 & $.0000 \mathrm{E}+00$ & 3 & $.0000 \mathrm{E}+00$ & 4 & $.0000 \mathrm{E}+00$ & 5 & $.0000 \mathrm{E}+00$ & 6 & $.0000 \mathrm{E}+00$ & 7 & $.0000 \mathrm{E}+00$ & 8 & $.0000 \mathrm{E}+00$ \\
\hline 9 & $.0000 \mathrm{E}+00$ & 10 & $.0000 \mathrm{E}+00$ & 11 & $.0000 \mathrm{E}+00$ & 12 & $.0000 \mathrm{E}+00$ & 13 & $.0000 \mathrm{E}+00$ & 14 & $.0000 \mathrm{E}+00$ & 15 & $.0000 \mathrm{E}+00$ & 16 & $.0000 \mathrm{E}+00$ \\
\hline 17 & $.0000 \mathrm{E}+00$ & 18 & $.0000 \mathrm{E}+00$ & 19 & $.0000 \mathrm{E}+00$ & 20 & $.0000 \mathrm{E}+00$ & 21 & $.0000 \mathrm{E}+00$ & 22 & $.0000 \mathrm{E}+00$ & 23 & $.0000 \mathrm{E}+00$ & 24 & $.0000 E+00$ \\
\hline & & & & & MON & $H=$ & JULY ; & DAY & OF $\mathrm{WEEK}=\mathrm{N}$ & DNDAY & & & & & \\
\hline 1 & $.0000 \mathrm{E}+00$ & 2 & $.0000 \mathrm{E}+00$ & 3 & $.0000 \mathrm{E}+00$ & 4 & $.0000 \mathrm{E}+00$ & 5 & $.0000 \mathrm{E}+00$ & 6 & $.0000 \mathrm{E}+00$ & 7 & $.0000 \mathrm{E}+00$ & 8 & $.0000 \mathrm{E}+00$ \\
\hline 9 & $.0000 \mathrm{E}+00$ & 10 & $.0000 \mathrm{E}+00$ & 11 & $.0000 \mathrm{E}+00$ & 12 & $.0000 \mathrm{E}+00$ & 13 & $.0000 \mathrm{E}+00$ & 14 & $.0000 \mathrm{E}+00$ & 15 & $.0000 \mathrm{E}+00$ & 16 & $.0000 \mathrm{E}+00$ \\
\hline 17 & $.0000 \mathrm{E}+00$ & 18 & $.0000 \mathrm{E}+00$ & 19 & $.0000 \mathrm{E}+00$ & 20 & $.0000 \mathrm{E}+00$ & 21 & $.0000 \mathrm{E}+00$ & 22 & $.0000 \mathrm{E}+00$ & 23 & $.0000 \mathrm{E}+00$ & 24 & $.0000 \mathrm{E}+00$ \\
\hline & & & & & MON & $H=$ & AUGUST ; & DAY & OF WEEK $=1$ & DNDAY & & & & & \\
\hline 1 & $.0000 \mathrm{E}+00$ & 2 & $.0000 \mathrm{E}+00$ & 3 & $.0000 \mathrm{E}+00$ & 4 & $.0000 \mathrm{E}+00$ & 5 & $.0000 \mathrm{E}+00$ & 6 & $.0000 \mathrm{E}+00$ & 7 & $.0000 \mathrm{E}+00$ & 8 & $.0000 \mathrm{E}+00$ \\
\hline 9 & $.0000 \mathrm{E}+00$ & 10 & $.0000 \mathrm{E}+00$ & 11 & $.0000 \mathrm{E}+00$ & 12 & $.0000 \mathrm{E}+00$ & 13 & $.0000 \mathrm{E}+00$ & 14 & $.0000 \mathrm{E}+00$ & 15 & $.0000 \mathrm{E}+00$ & 16 & $.0000 \mathrm{E}+00$ \\
\hline 17 & $.0000 \mathrm{E}+00$ & 18 & $.0000 \mathrm{E}+00$ & 19 & $.0000 \mathrm{E}+00$ & 20 & $.0000 \mathrm{E}+00$ & 21 & $.0000 \mathrm{E}+00$ & 22 & $.0000 \mathrm{E}+00$ & 23 & $.0000 \mathrm{E}+00$ & 24 & $.0000 \mathrm{E}+00$ \\
\hline & & & & & MON & $H=$ & SEPTEMBER； & DAY & OF $\mathrm{WEEK}=\mathrm{N}$ & DNDAY & & & & & \\
\hline 1 & $.0000 \mathrm{E}+00$ & 2 & $.0000 \mathrm{E}+00$ & 3 & $.0000 \mathrm{E}+00$ & 4 & $.0000 \mathrm{E}+00$ & 5 & $.0000 \mathrm{E}+00$ & 6 & $.0000 \mathrm{E}+00$ & 7 & $.0000 \mathrm{E}+00$ & 8 & $.0000 \mathrm{E}+00$ \\
\hline 9 & $.0000 \mathrm{E}+00$ & 10 & $.0000 \mathrm{E}+00$ & 11 & $.0000 \mathrm{E}+00$ & 12 & $.0000 \mathrm{E}+00$ & 13 & $.0000 \mathrm{E}+00$ & 14 & $.0000 \mathrm{E}+00$ & 15 & $.0000 \mathrm{E}+00$ & 16 & $.0000 \mathrm{E}+00$ \\
\hline 17 & $.0000 \mathrm{E}+00$ & 18 & $.0000 \mathrm{E}+00$ & 19 & $.0000 \mathrm{E}+00$ & 20 & $.0000 \mathrm{E}+00$ & 21 & $.0000 \mathrm{E}+00$ & 22 & $.0000 \mathrm{E}+00$ & 23 & $.0000 \mathrm{E}+00$ & 24 & $.0000 \mathrm{E}+00$ \\
\hline & & & & & MON & $H=$ & OCTOBER ; & DAY & OF WEEK $=1$ & DNDAY & & & & & \\
\hline 1 & $.0000 \mathrm{E}+00$ & 2 & $.0000 E+00$ & 3 & $.0000 \mathrm{E}+00$ & 4 & $.0000 \mathrm{E}+00$ & 5 & $.0000 \mathrm{E}+00$ & 6 & $.0000 \mathrm{E}+00$ & 7 & $.0000 \mathrm{E}+00$ & 8 & $.1000 \mathrm{E}+01$ \\
\hline 9 & $.1000 \mathrm{E}+01$ & 10 & $.1000 \mathrm{E}+01$ & 11 & $.1000 \mathrm{E}+01$ & 12 & $.0000 \mathrm{E}+00$ & 13 & $.1000 \mathrm{E}+01$ & 14 & $.1000 \mathrm{E}+01$ & 15 & $.1000 \mathrm{E}+01$ & 16 & $.1000 \mathrm{E}+01$ \\
\hline 17 & $.0000 \mathrm{E}+00$ & 18 & $.0000 \mathrm{E}+00$ & 19 & $.0000 \mathrm{E}+00$ & 20 & $.0000 \mathrm{E}+00$ & 21 & $.0000 \mathrm{E}+00$ & 22 & $.0000 \mathrm{E}+00$ & 23 & $.0000 \mathrm{E}+00$ & 24 & $.0000 \mathrm{E}+00$ \\
\hline & & & & & MON & $H=$ & NOVEMBER ; & DAY & OF $\mathrm{WEEK}=\mathrm{N}$ & DNDAY & & & & & \\
\hline 1 & $.0000 \mathrm{E}+00$ & 2 & .000 & 3 & $0 \mathrm{EE}+00$ & 4 & $.0000 \mathrm{E}+00$ & 5 & $.0000 \mathrm{E}+00$ & 6 & $E+00$ & 7 & $.0000 \mathrm{E}+00$ & 8 & $.1000 \mathrm{E}+01$ \\
\hline 9 & $.1000 \mathrm{E}+01$ & 10 & $.1000 \mathrm{E}+01$ & 11 & $.1000 \mathrm{E}+01$ & 12 & $.0000 \mathrm{E}+00$ & 13 & $.1000 \mathrm{E}+01$ & 14 & $.1000 \mathrm{E}+01$ & 15 & $.1000 \mathrm{E}+01$ & 16 & $.1000 \mathrm{E}+01$ \\
\hline 17 & $.0000 \mathrm{E}+00$ & 18 & $.0000 \mathrm{E}+00$ & 19 & $.0000 \mathrm{E}+00$ & 20 & $.0000 \mathrm{E}+00$ & 21 & $.0000 \mathrm{E}+00$ & 22 & $.0000 \mathrm{E}+00$ & 23 & $.0000 \mathrm{E}+00$ & 24 & $.0000 \mathrm{E}+00$ \\
\hline & & & & & MON & $I=$ & DECEMBER ; & DAY & OF $\mathrm{WEEK}=\mathrm{N}$ & ONDAY & & & & & \\
\hline 1 & $.0000 \mathrm{E}+00$ & 2 & $.0000 \mathrm{E}+00$ & 3 & $.0000 \mathrm{E}+00$ & 4 & $.0000 \mathrm{E}+00$ & 5 & $.0000 \mathrm{E}+00$ & 6 & $.0000 \mathrm{E}+00$ & 7 & $.0000 \mathrm{E}+00$ & 8 & $.1000 \mathrm{E}+01$ \\
\hline 9 & $.1000 \mathrm{E}+01$ & 10 & $.1000 \mathrm{E}+01$ & 11 & $.1000 \mathrm{E}+01$ & 12 & $.0000 \mathrm{E}+00$ & 13 & $.1000 \mathrm{E}+01$ & 14 & $.1000 \mathrm{E}+01$ & 15 & $.1000 \mathrm{E}+01$ & 16 & $.1000 \mathrm{E}+01$ \\
\hline 17 & .0000 & 18 & .00 & 19 & $0000 E+00$ & 20 & $.0000 \mathrm{E}+00$ & 21 & $.0000 \mathrm{E}+00$ & 22 & $E+00$ & 23 & $.0000 \mathrm{E}+00$ & 24 & $.0000 \mathrm{E}+00$ \\
\hline
\end{tabular}

$* * *$ AERMOD - VERSION $07026 * * * \quad \begin{aligned} & * * \text { U/UA Building Demolition } \\ & * * * \text { Unit Release - Worst Case }\end{aligned}$

$\begin{array}{ll}* * * & 02 / 09 / 09 \\ * * * & 10: 41: 11\end{array}$ **MODELOPTS :

CONC

DDEP

TOXICS FLAT

DRYDPL WETDPL

* SOURCE EMISSION RATE SCALARS WHICH VARY MONTHLY, DIURNALLY AND BY DAY OF WEEK (MHRDOW7) *

\begin{tabular}{|c|c|c|c|c|c|c|c|c|c|c|c|c|c|c|c|}
\hline HOUR & SCALAR & HOUR' & SCALAR & HOUR & SCALAR & HOUR & SCALAR & HOUR & SCALAR & HOUR & SCALAR & HOUR & SCALAR & HOUR & SCALAR \\
\hline & --- & $\cdots$ & - - - - & -- & $\begin{array}{r}-\cdots \\
\quad \text { MOI }\end{array}$ & $\begin{array}{l}-\cdots \\
\mathrm{THH}=\end{array}$ & $\begin{array}{c}---\cdots \\
\text { JANUARY }\end{array}$ & $\begin{array}{l}\cdots \\
\text { DAY }\end{array}$ & $\begin{array}{l}- \\
\text { OF } \mathrm{WEEK}=\end{array}$ & $\begin{array}{l}-\quad-- \\
\text { TUESDAY }\end{array}$ & - - - - & -- & - & -- & \\
\hline 1 & $.0000 E+00$ & 2 & $.0000 E+00$ & 3 & $.0000 \mathrm{E}+00$ & 4 & $.0000 \mathrm{E}+00$ & 5 & $.0000 \mathrm{E}+00$ & 6 & $.0000 \mathrm{E}+00$ & 7 & $.0000 \mathrm{E}+00$ & 8 & $.0000 \mathrm{E}+00$ \\
\hline 9 & $.0000 \mathrm{E}+00$ & 10 & $.0000 \mathrm{E}+00$ & 11 & $.0000 \mathrm{E}+00$ & 12 & $.0000 \mathrm{E}+00$ & 13 & $.0000 \mathrm{E}+00$ & 14 & $.0000 \mathrm{E}+00$ & 15 & $.0000 \mathrm{E}+00$ & 16 & $.0000 \mathrm{E}+00$ \\
\hline & & & & & MOI & $\mathrm{THH}=\mathrm{F}$ & FEBRUARY ; & DAY & OF WEEK = ? & TUESDAY & & & & & \\
\hline 1 & $.0000 \mathrm{E}+00$ & 2 & $.0000 \mathrm{E}+00$ & 3 & $.0000 \mathrm{E}+00$ & 4 & $.0000 \mathrm{E}+00$ & 5 & $.0000 \mathrm{E}+00$ & 6 & $.0000 \mathrm{E}+00$ & 7 & $.0000 \mathrm{E}+00$ & 8 & $.0000 \mathrm{E}+00$ \\
\hline 9 & $.0000 \mathrm{E}+00$ & 10 & $.0000 \mathrm{E}+00$ & 11 & $.0000 \mathrm{E}+00$ & 12 & $.0000 \mathrm{E}+00$ & 13 & $.0000 \mathrm{E}+00$ & 14 & & 15 & .000 & 16 & .000 \\
\hline 17 & $.0000 \mathrm{E}+00$ & 18 & $.0000 \mathrm{E}+00$ & 19 & $.0000 \mathrm{E}+00$ & 20 & $.0000 \mathrm{E}+00$ & 21 & $.0000 \mathrm{E}+00$ & 22 & $.0000 \mathrm{E}+00$ & 23 & $.0000 \mathrm{E}+00$ & 24 & $.0000 \mathrm{E}+00$ \\
\hline
\end{tabular}




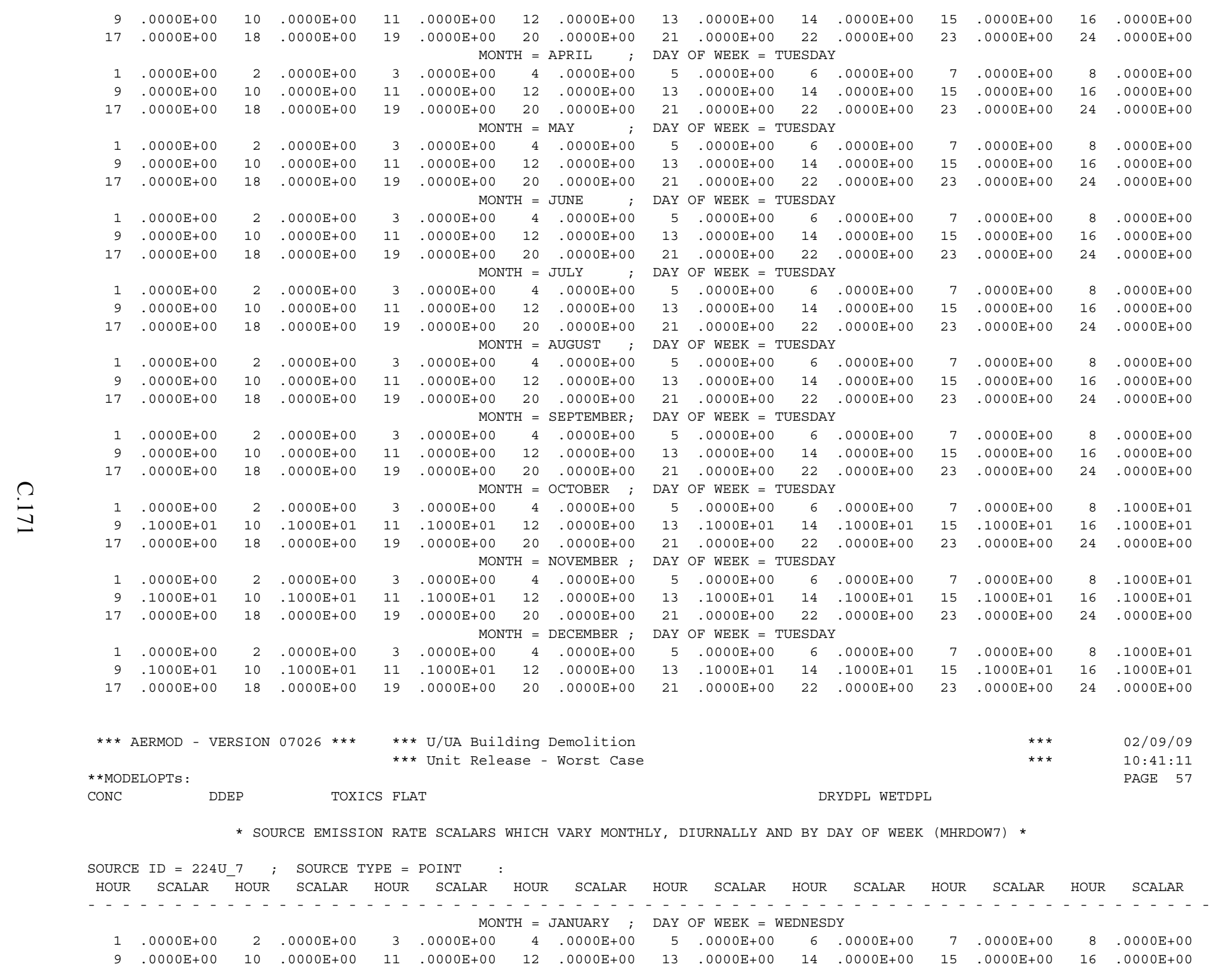




\begin{tabular}{|c|c|c|c|c|c|c|c|c|c|c|c|c|c|c|c|}
\hline 17 & $.0000 \mathrm{E}+00$ & 18 & $.0000 \mathrm{E}+00$ & 19 & $.0000 \mathrm{E}+00$ & 20 & $.0000 \mathrm{E}+00$ & $\begin{array}{r}21 \\
\end{array}$ & $.0000 \mathrm{E}+00$ & 22 & $.0000 \mathrm{E}+00$ & 23 & $.0000 \mathrm{E}+00$ & 24 & $.0000 \mathrm{E}+00$ \\
\hline & $.0000 \mathrm{E}+00$ & 2 & $.0000 \mathrm{E}+00$ & 3 & $.0000 \mathrm{E}+00$ & 4 & $.0000 \mathrm{E}+00$ & 5 & $.0000 \mathrm{E}+00$ & 6 & $.0000 \mathrm{E}+00$ & 7 & $.0000 E+00$ & 8 & $.0000 \mathrm{E}+00$ \\
\hline & $.0000 \mathrm{E}+00$ & 10 & $.0000 \mathrm{E}+00$ & 11 & $.0000 \mathrm{E}+00$ & 12 & $.0000 \mathrm{E}+00$ & 13 & $.0000 \mathrm{E}+00$ & 14 & $.0000 \mathrm{E}+00$ & 15 & $.0000 \mathrm{E}+00$ & 16 & $.0000 \mathrm{E}+00$ \\
\hline 17 & $.0000 \mathrm{E}+00$ & 18 & $.0000 \mathrm{E}+00$ & 19 & $.0000 \mathrm{E}+00$ & 20 & $.0000 \mathrm{E}+00$ & 21 & $.0000 \mathrm{E}+00$ & 22 & $.0000 \mathrm{E}+00$ & 23 & $.0000 \mathrm{E}+00$ & 24 & $.0000 \mathrm{E}+00$ \\
\hline & & & & & MONTH & $\mathrm{H}=$ & MARCH ; & DAY & $O F \quad \mathrm{WEEK}=W$ & DNES & & & & & \\
\hline & $.0000 \mathrm{E}+00$ & 2 & $.0000 \mathrm{E}+00$ & 3 & $.0000 \mathrm{E}+00$ & 4 & $.0000 \mathrm{E}+00$ & 5 & $.0000 \mathrm{E}+00$ & 6 & $.0000 \mathrm{E}+00$ & 7 & $.0000 \mathrm{E}+00$ & 8 & $.0000 \mathrm{E}+00$ \\
\hline & $.0000 \mathrm{E}+00$ & 10 & $.0000 \mathrm{E}+00$ & 11 & $.0000 \mathrm{E}+00$ & 12 & $.0000 \mathrm{E}+00$ & 13 & $.0000 \mathrm{E}+00$ & 14 & $.0000 \mathrm{E}+00$ & 15 & $.0000 \mathrm{E}+00$ & 16 & $.0000 \mathrm{E}+00$ \\
\hline 17 & $.0000 E+00$ & 18 & $.0000 \mathrm{E}+00$ & 19 & $.0000 \mathrm{E}+00$ & 20 & $.0000 \mathrm{E}+00$ & 21 & $.0000 \mathrm{E}+00$ & 22 & $.0000 \mathrm{E}+00$ & 23 & $.0000 \mathrm{E}+00$ & 24 & $.0000 \mathrm{E}+00$ \\
\hline & & & & & MONTH & $\mathrm{H}=$ & APRIL ; & DAY & OF $\mathrm{WEEK}=W$ & DNES & & & & & \\
\hline & $.0000 \mathrm{E}+00$ & 2 & $.0000 \mathrm{E}+00$ & 3 & $.0000 \mathrm{E}+00$ & 4 & $.0000 \mathrm{E}+00$ & 5 & $.0000 \mathrm{E}+00$ & 6 & $.0000 \mathrm{E}+00$ & 7 & $.0000 \mathrm{E}+00$ & 8 & $.0000 \mathrm{E}+00$ \\
\hline & $.0000 \mathrm{E}+00$ & 10 & $.0000 \mathrm{E}+00$ & 11 & $.0000 \mathrm{E}+00$ & 12 & $.0000 \mathrm{E}+00$ & 13 & $.0000 \mathrm{E}+00$ & 14 & $.0000 \mathrm{E}+00$ & 15 & $.0000 \mathrm{E}+00$ & 16 & $.0000 \mathrm{E}+00$ \\
\hline 17 & $.0000 \mathrm{E}+00$ & 18 & $.0000 \mathrm{E}+00$ & 19 & $.0000 \mathrm{E}+00$ & 20 & $.0000 \mathrm{E}+00$ & 21 & $.0000 \mathrm{E}+00$ & 22 & $.0000 \mathrm{E}+00$ & 23 & $.0000 \mathrm{E}+00$ & 24 & $.0000 \mathrm{E}+00$ \\
\hline & & & & & MONTH & $\mathrm{H}=$ & MAY & DAY & $O F \quad \mathrm{WEEK}=W$ & DNES & & & & & \\
\hline & $.0000 \mathrm{E}+00$ & 2 & $.0000 \mathrm{E}+00$ & 3 & $.0000 \mathrm{E}+00$ & 4 & $.0000 \mathrm{E}+00$ & 5 & $.0000 \mathrm{E}+00$ & 6 & $.0000 \mathrm{E}+00$ & 7 & $.0000 \mathrm{E}+00$ & 8 & $.0000 \mathrm{E}+00$ \\
\hline 9 & $.0000 \mathrm{E}+00$ & 10 & $.0000 \mathrm{E}+00$ & 11 & $.0000 \mathrm{E}+00$ & 12 & $.0000 \mathrm{E}+00$ & 13 & $.0000 \mathrm{E}+00$ & 14 & $.0000 \mathrm{E}+00$ & 15 & $.0000 \mathrm{E}+00$ & 16 & $.0000 \mathrm{E}+00$ \\
\hline 17 & $.0000 \mathrm{E}+00$ & 18 & $.0000 \mathrm{E}+00$ & 19 & $.0000 E+00$ & 20 & $.0000 \mathrm{E}+00$ & 21 & $.0000 \mathrm{E}+00$ & 22 & $.0000 \mathrm{E}+00$ & 23 & $.0000 \mathrm{E}+00$ & 24 & $.0000 \mathrm{E}+00$ \\
\hline & & & & & MONTH & $\mathrm{H}=$ & JUNE ; & DAY & OF $\mathrm{WEEK}=W$ & DNES & & & & & \\
\hline & $.0000 \mathrm{E}+00$ & 2 & $.0000 \mathrm{E}+00$ & 3 & $.0000 \mathrm{E}+00$ & 4 & $.0000 \mathrm{E}+00$ & 5 & $.0000 \mathrm{E}+00$ & 6 & $.0000 \mathrm{E}+00$ & 7 & $.0000 \mathrm{E}+00$ & 8 & $.0000 \mathrm{E}+00$ \\
\hline & $.0000 \mathrm{E}+00$ & 10 & $.0000 \mathrm{E}+00$ & 11 & $.0000 \mathrm{E}+00$ & 12 & $.0000 \mathrm{E}+00$ & 13 & $.0000 \mathrm{E}+00$ & 14 & $.0000 \mathrm{E}+00$ & 15 & $.0000 \mathrm{E}+00$ & 16 & $.0000 \mathrm{E}+00$ \\
\hline 17 & $.0000 \mathrm{E}+00$ & 18 & $.0000 \mathrm{E}+00$ & 19 & $.0000 \mathrm{E}+00$ & 20 & $.0000 \mathrm{E}+00$ & 21 & $.0000 \mathrm{E}+00$ & 22 & $.0000 \mathrm{E}+00$ & 23 & $.0000 \mathrm{E}+00$ & 24 & $.0000 \mathrm{E}+00$ \\
\hline & & & & & MONTH & $\mathrm{H}=$ & JULY ; & DAY & OF $\mathrm{WEEK}=W$ & DNES & & & & & \\
\hline & $.0000 \mathrm{E}+00$ & 2 & $.0000 \mathrm{E}+00$ & 3 & $.0000 \mathrm{E}+00$ & 4 & $.0000 \mathrm{E}+00$ & 5 & $.0000 \mathrm{E}+00$ & 6 & $.0000 \mathrm{E}+00$ & 7 & $.0000 \mathrm{E}+00$ & 8 & $.0000 \mathrm{E}+00$ \\
\hline & $.0000 \mathrm{E}+00$ & 10 & $.0000 \mathrm{E}+00$ & 11 & $.0000 \mathrm{E}+00$ & 12 & $.0000 \mathrm{E}+00$ & 13 & $.0000 \mathrm{E}+00$ & 14 & $.0000 \mathrm{E}+00$ & 15 & $.0000 \mathrm{E}+00$ & 16 & $.0000 \mathrm{E}+00$ \\
\hline 17 & $.0000 \mathrm{E}+00$ & 18 & $.0000 \mathrm{E}+00$ & 19 & $.0000 \mathrm{E}+00$ & 20 & $.0000 \mathrm{E}+00$ & 21 & $.0000 \mathrm{E}+00$ & 22 & $.0000 \mathrm{E}+00$ & 23 & $.0000 \mathrm{E}+00$ & 24 & $.0000 \mathrm{E}+00$ \\
\hline & & & & & MONTH & $\mathrm{H}=$ & AUGUST ; & DAY & $O F \quad \mathrm{WEEK}=W$ & DNES & & & & & \\
\hline & $.0000 \mathrm{E}+00$ & 2 & $.0000 \mathrm{E}+00$ & 3 & $.0000 \mathrm{E}+00$ & 4 & $.0000 \mathrm{E}+00$ & 5 & $.0000 \mathrm{E}+00$ & 6 & $.0000 \mathrm{E}+00$ & 7 & $.0000 \mathrm{E}+00$ & 8 & $.0000 \mathrm{E}+00$ \\
\hline 9 & $.0000 \mathrm{E}+00$ & 10 & $.0000 \mathrm{E}+00$ & 11 & $.0000 \mathrm{E}+00$ & 12 & $.0000 \mathrm{E}+00$ & 13 & $.0000 \mathrm{E}+00$ & 14 & $.0000 \mathrm{E}+00$ & 15 & $.0000 \mathrm{E}+00$ & 16 & $.0000 \mathrm{E}+00$ \\
\hline 17 & $.0000 \mathrm{E}+00$ & 18 & $.0000 \mathrm{E}+00$ & 19 & $.0000 \mathrm{E}+00$ & 20 & $.0000 \mathrm{E}+00$ & 21 & $.0000 \mathrm{E}+00$ & 22 & $.0000 \mathrm{E}+00$ & 23 & $.0000 \mathrm{E}+00$ & 24 & $.0000 \mathrm{E}+00$ \\
\hline & & & & & MONTH & $\mathrm{H}=$ & SEPTEMBER ; & DAY & OF $\mathrm{WEEK}=W$ & DNES & & & & & \\
\hline & $.0000 \mathrm{E}+00$ & 2 & $.0000 \mathrm{E}+00$ & 3 & $.0000 \mathrm{E}+00$ & 4 & $.0000 \mathrm{E}+00$ & 5 & $.0000 \mathrm{E}+00$ & 6 & $.0000 \mathrm{E}+00$ & 7 & $.0000 \mathrm{E}+00$ & 8 & $.0000 \mathrm{E}+00$ \\
\hline & $.0000 \mathrm{E}+00$ & 10 & $.0000 \mathrm{E}+00$ & 11 & $.0000 \mathrm{E}+00$ & 12 & $.0000 \mathrm{E}+00$ & 13 & $.0000 \mathrm{E}+00$ & 14 & $.0000 \mathrm{E}+00$ & 15 & $.0000 \mathrm{E}+00$ & 16 & $.0000 \mathrm{E}+00$ \\
\hline 17 & $.0000 E+00$ & 18 & $.0000 E+00$ & 19 & $.0000 \mathrm{E}+00$ & 20 & $.0000 \mathrm{E}+00$ & 21 & $.0000 \mathrm{E}+00$ & 22 & $.0000 \mathrm{E}+00$ & 23 & $.0000 \mathrm{E}+00$ & 24 & $.0000 \mathrm{E}+00$ \\
\hline & & & & & MONTH & $\mathrm{H}=$ & OCTOBER ; & DAY & $O F \quad \mathrm{WEEK}=W$ & DNES & & & & & \\
\hline & $.0000 \mathrm{E}+00$ & 2 & $.0000 \mathrm{E}+00$ & 3 & $.0000 \mathrm{E}+00$ & 4 & $.0000 \mathrm{E}+00$ & 5 & $.0000 \mathrm{E}+00$ & 6 & $.0000 \mathrm{E}+00$ & 7 & $.0000 \mathrm{E}+00$ & 8 & $.1000 \mathrm{E}+01$ \\
\hline & $.1000 \mathrm{E}+01$ & 10 & $.1000 \mathrm{E}+01$ & 11 & $.1000 \mathrm{E}+01$ & 12 & $.0000 \mathrm{E}+00$ & 13 & $.1000 \mathrm{E}+01$ & 14 & $.1000 \mathrm{E}+01$ & 15 & $.1000 \mathrm{E}+01$ & 16 & $.1000 \mathrm{E}+01$ \\
\hline 17 & $.0000 \mathrm{E}+00$ & 18 & $.0000 \mathrm{E}+00$ & 19 & $.0000 \mathrm{E}+00$ & 20 & $.0000 \mathrm{E}+00$ & 21 & $.0000 \mathrm{E}+00$ & 22 & $.0000 \mathrm{E}+00$ & 23 & $.0000 \mathrm{E}+00$ & 24 & $.0000 \mathrm{E}+00$ \\
\hline & & & & & MONTH & $\mathrm{H}=$ & NOVEMBER ; & DAY & OF $\mathrm{WEEK}=W$ & DNES & & & & & \\
\hline & $.0000 \mathrm{E}+00$ & 2 & $.0000 \mathrm{E}+00$ & 3 & $.0000 \mathrm{E}+00$ & 4 & $.0000 \mathrm{E}+00$ & 5 & $.0000 \mathrm{E}+00$ & 6 & $.0000 \mathrm{E}+00$ & 7 & $.0000 \mathrm{E}+00$ & 8 & $.1000 \mathrm{E}+01$ \\
\hline 9 & $.1000 \mathrm{E}+01$ & 10 & $.1000 \mathrm{E}+01$ & 11 & $.1000 \mathrm{E}+01$ & 12 & $.0000 \mathrm{E}+00$ & 13 & $.1000 \mathrm{E}+01$ & 14 & $.1000 \mathrm{E}+01$ & 15 & $.1000 \mathrm{E}+01$ & 16 & $.1000 \mathrm{E}+01$ \\
\hline 17 & $.0000 \mathrm{E}+00$ & 18 & $.0000 \mathrm{E}+00$ & 19 & $.0000 \mathrm{E}+00$ & 20 & $.0000 \mathrm{E}+00$ & 21 & $.0000 \mathrm{E}+00$ & 22 & $.0000 \mathrm{E}+00$ & 23 & $.0000 \mathrm{E}+00$ & 24 & $.0000 \mathrm{E}+00$ \\
\hline & & & & & MONTH & $\mathrm{H}=$ & DECEMBER ; & DAY & OF $\mathrm{WEEK}=W$ & DNES & & & & & \\
\hline & $.0000 \mathrm{E}+00$ & 2 & $.0000 \mathrm{E}+00$ & 3 & $.0000 \mathrm{E}+00$ & 4 & $.0000 \mathrm{E}+00$ & 5 & $.0000 \mathrm{E}+00$ & 6 & $.0000 \mathrm{E}+00$ & 7 & $.0000 \mathrm{E}+00$ & 8 & $.1000 \mathrm{E}+01$ \\
\hline & $.1000 \mathrm{E}+01$ & 10 & $.1000 \mathrm{E}+01$ & 11 & $.1000 \mathrm{E}+01$ & 12 & $.0000 \mathrm{E}+00$ & 13 & $.1000 \mathrm{E}+01$ & 14 & $.1000 \mathrm{E}+01$ & 15 & $.1000 \mathrm{E}+01$ & 16 & $.1000 \mathrm{E}+01$ \\
\hline 17 & $.0000 \mathrm{E}+00$ & 18 & $.0000 \mathrm{E}+00$ & 19 & $.0000 \mathrm{E}+00$ & 20 & $.0000 \mathrm{E}+00$ & 21 & $.0000 \mathrm{E}+00$ & 22 & $.0000 \mathrm{E}+00$ & 23 & $.0000 \mathrm{E}+00$ & 24 & $.0000 \mathrm{E}+00$ \\
\hline . & AERMOD - VE & SION & $07026 * * *$ & ** & U/UA Buildi & ing & Demolition & & & & & & $* * *$ & & $02 / 09 / 09$ \\
\hline & & & & & & & - Worst Case & & & & & & $* * *$ & & $10: 41: 11$ \\
\hline & & & & & & & & & & & & & & & PAGE 58 \\
\hline COIN & & & TOX & $\mathrm{FL}$ & & & & & & & & & & & \\
\hline
\end{tabular}

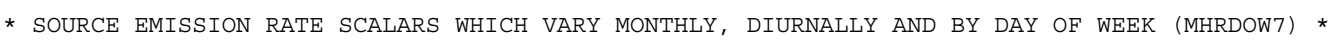




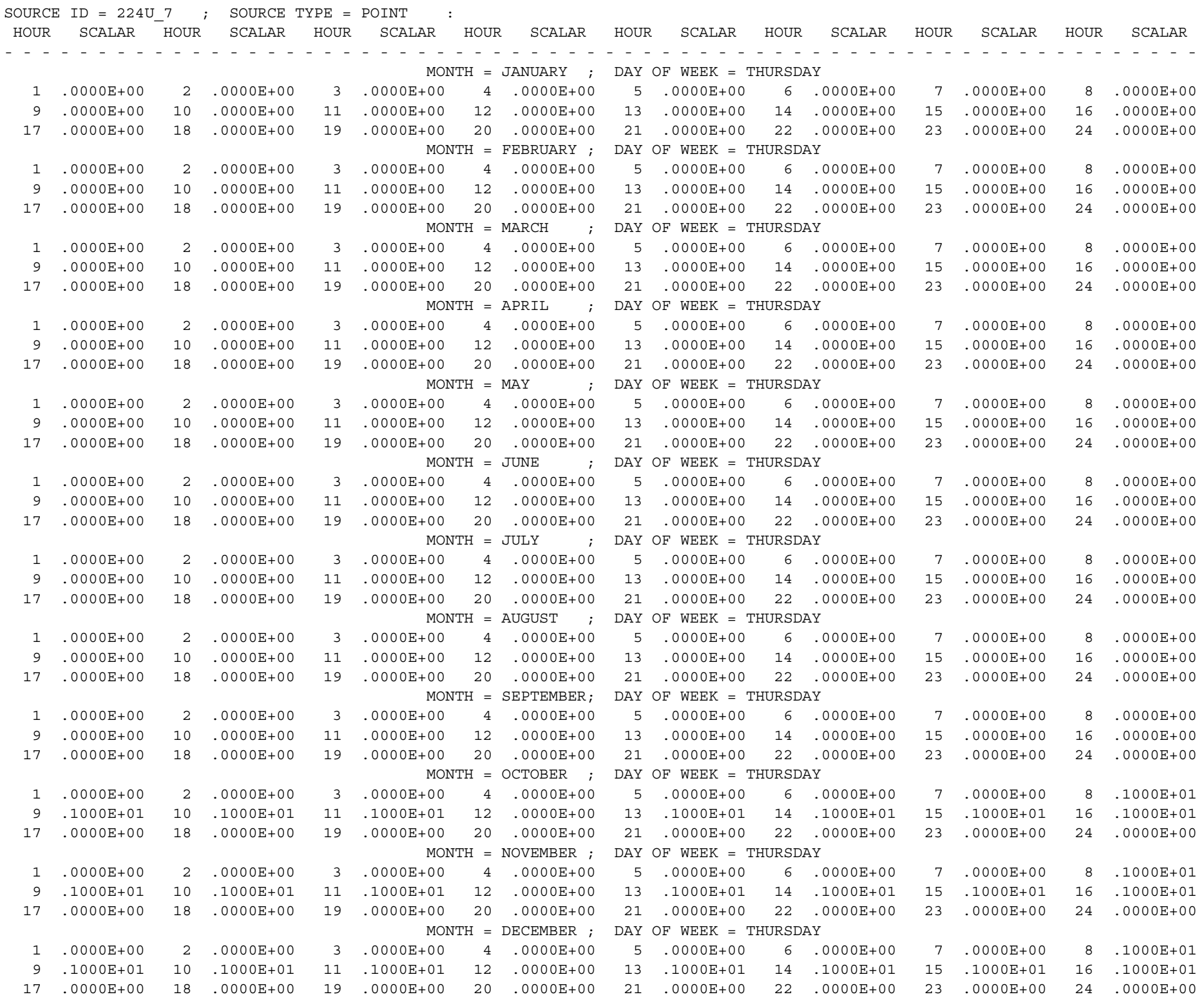




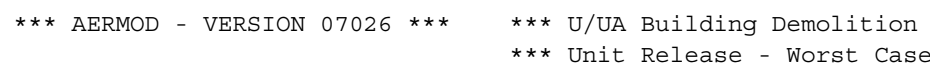
**MODELOPTS :

CONC $\begin{array}{ll}* * * & 02 / 09 / 09 \\ * * * & 10: 41: 11\end{array}$

$10: 41: 11$
PAGE $\quad 59$

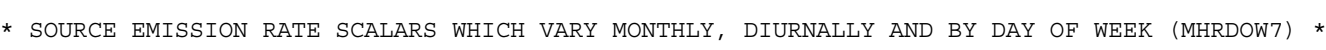

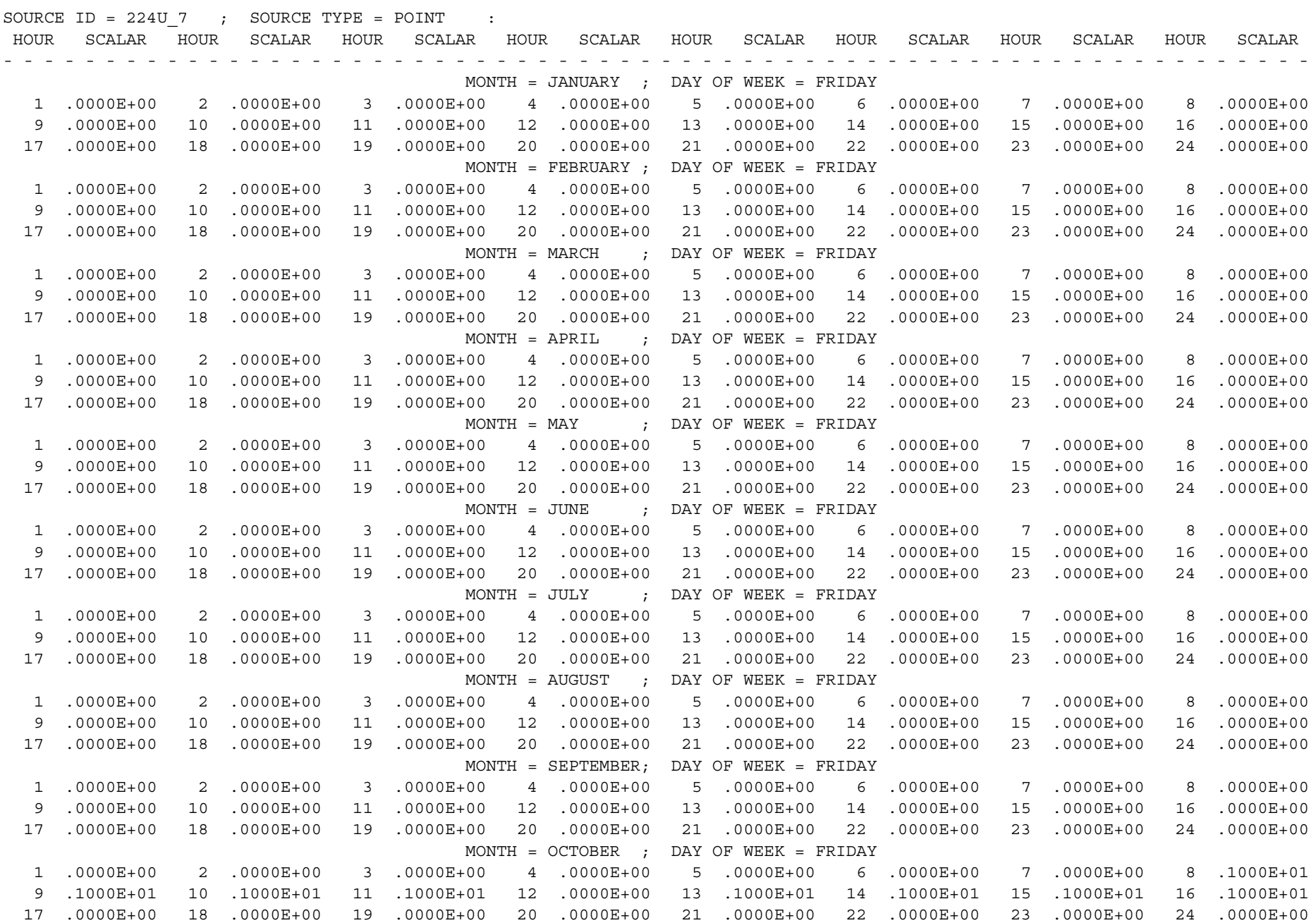
MONTH = NOVEMBER ; DAY OF WEEK = FRIDAY 


\begin{tabular}{|c|c|c|c|c|c|c|c|c|c|c|c|c|c|c|c|}
\hline 1 & $.0000 \mathrm{E}+00$ & 2 & $.0000 \mathrm{E}+00$ & 3 & $.0000 \mathrm{E}+00$ & 4 & $.0000 \mathrm{E}+00$ & 5 & $.0000 \mathrm{E}+00$ & 6 & $.0000 \mathrm{E}+00$ & 7 & $.0000 \mathrm{E}+00$ & 8 & $.1000 \mathrm{E}+01$ \\
\hline 9 & $.1000 \mathrm{E}+01$ & 10 & $.1000 \mathrm{E}+01$ & 11 & $.1000 \mathrm{E}+01$ & 12 & $.0000 \mathrm{E}+00$ & 13 & $.1000 \mathrm{E}+01$ & 14 & $.1000 \mathrm{E}+01$ & 15 & $.1000 \mathrm{E}+01$ & 16 & $.1000 \mathrm{E}+01$ \\
\hline \multirow[t]{2}{*}{17} & $.0000 \mathrm{E}+00$ & 18 & $.0000 \mathrm{E}+00$ & 19 & $.0000 \mathrm{E}+00$ & 20 & $.0000 \mathrm{E}+00$ & 21 & $.0000 \mathrm{E}+00$ & 22 & $.0000 \mathrm{E}+00$ & 23 & $.0000 \mathrm{E}+00$ & 24 & $.0000 \mathrm{E}+00$ \\
\hline & & & & & MOI & $I=$ & DECEMBER ； & DAY & OF WEEK = & RIDAY & & & & & \\
\hline 1 & $.0000 \mathrm{E}+00$ & 2 & $.0000 \mathrm{E}+00$ & 3 & $.0000 E+00$ & 4 & $.0000 \mathrm{E}+00$ & 5 & $.0000 \mathrm{E}+00$ & 6 & $.0000 \mathrm{E}+00$ & 7 & $.0000 \mathrm{E}+00$ & 8 & $.1000 \mathrm{E}+01$ \\
\hline 9 & $.1000 \mathrm{E}+01$ & 10 & $.1000 \mathrm{E}+01$ & 11 & $.1000 \mathrm{E}+01$ & 12 & $.0000 \mathrm{E}+00$ & 13 & $.1000 \mathrm{E}+01$ & 14 & $.1000 \mathrm{E}+01$ & 15 & $.1000 \mathrm{E}+01$ & 16 & $.1000 \mathrm{E}+01$ \\
\hline 17 & $.0000 \mathrm{E}+00$ & 18 & $.0000 \mathrm{E}+00$ & 19 & $.0000 \mathrm{E}+00$ & 20 & $.0000 \mathrm{E}+00$ & 21 & $.0000 \mathrm{E}+00$ & 22 & $.0000 \mathrm{E}+00$ & 23 & $.0000 \mathrm{E}+00$ & 24 & $.0000 \mathrm{E}+00$ \\
\hline
\end{tabular}

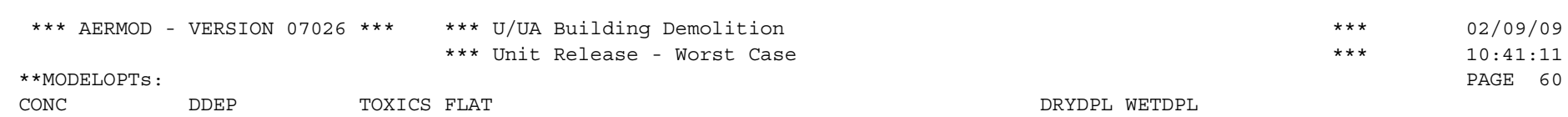

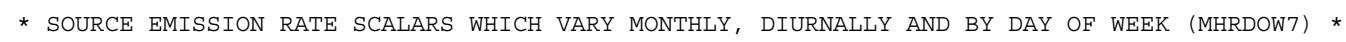

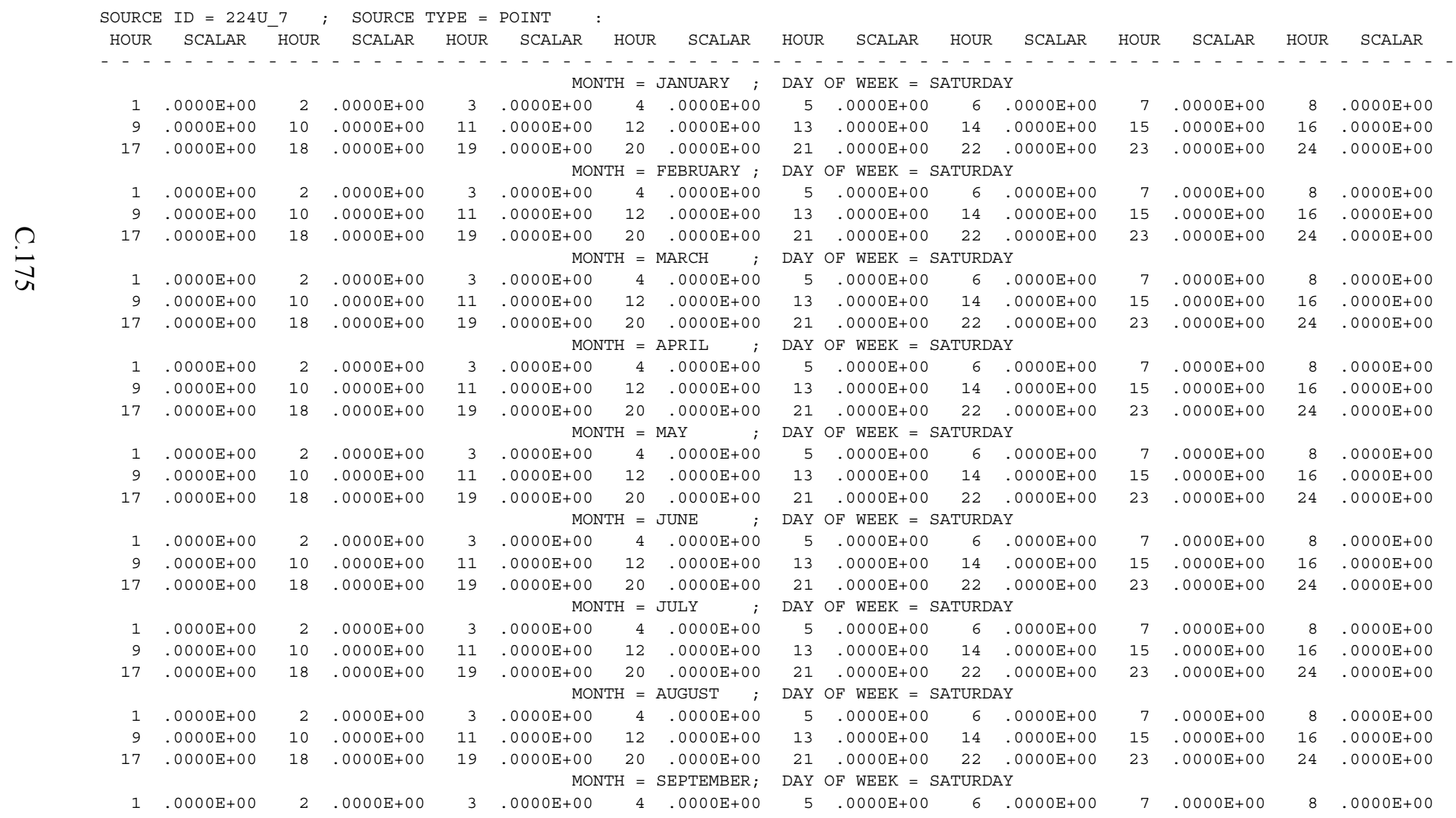




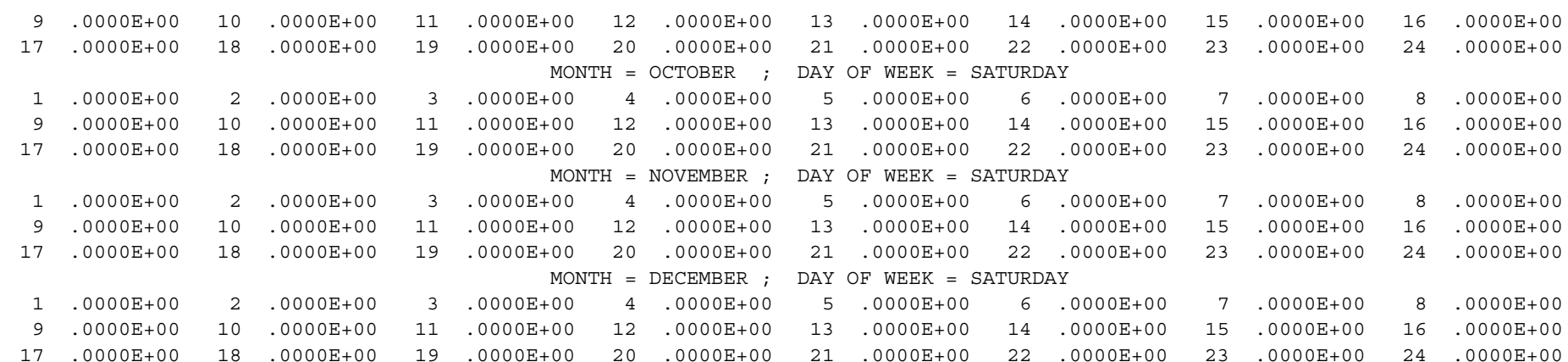

*** AERMOD - VERSION $07026 * * * \quad * * *$ U/UA Building Demolition

*** Unit Release - Worst Case

$\begin{array}{ll}* * * & 02 / 09 / 09 \\ * * * & 10: 41: 11 \\ & \text { PAGE } 61\end{array}$ **MODELOPTS:

CONC

DDEP

TOXICS FLAT

DRYDPL WETDPL

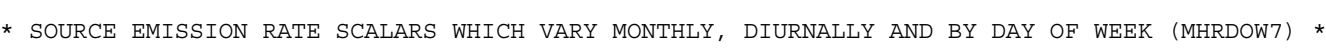

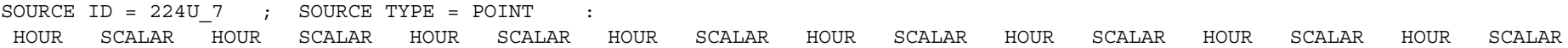

$\begin{array}{r}\text { MONTH = JANUARY } ; \text { DAY OF WEEK }=\text { SUNDAY } \\ \hline\end{array}$

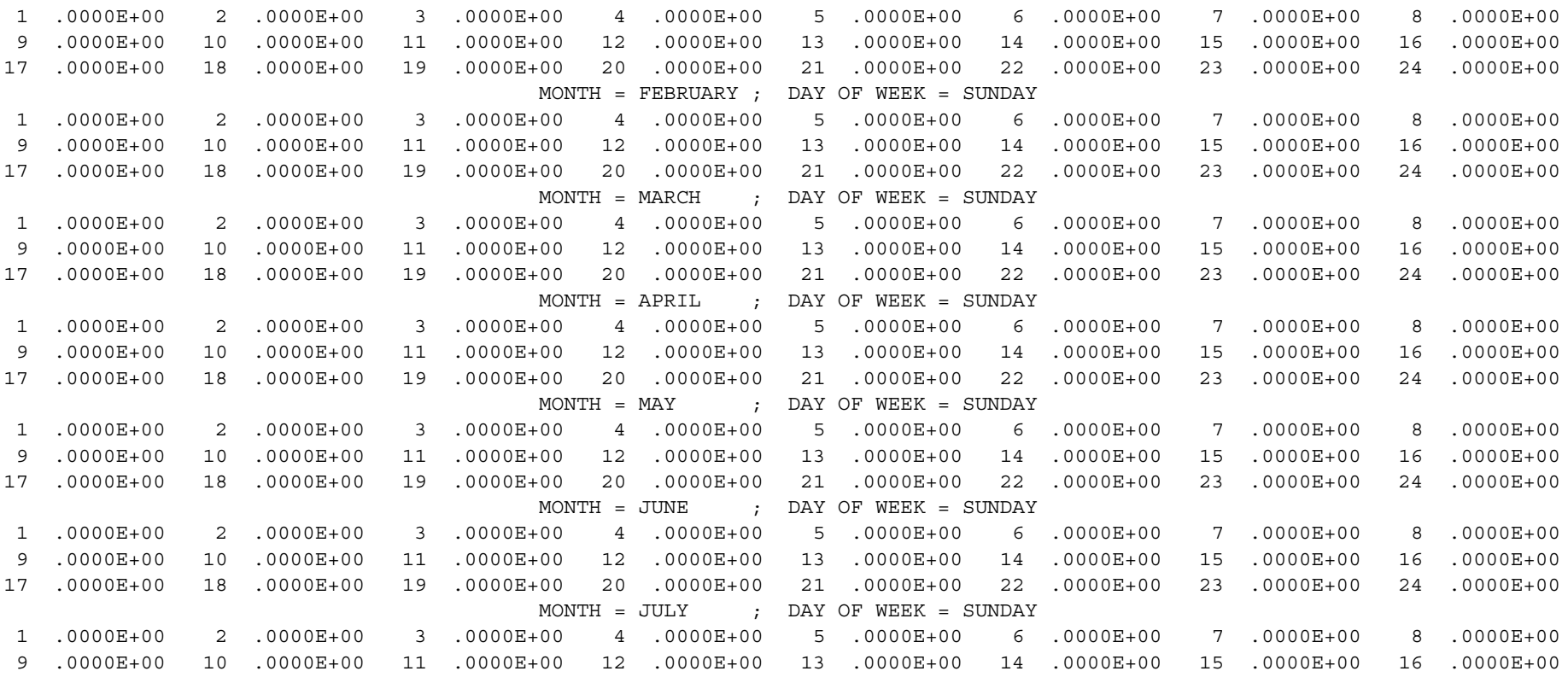




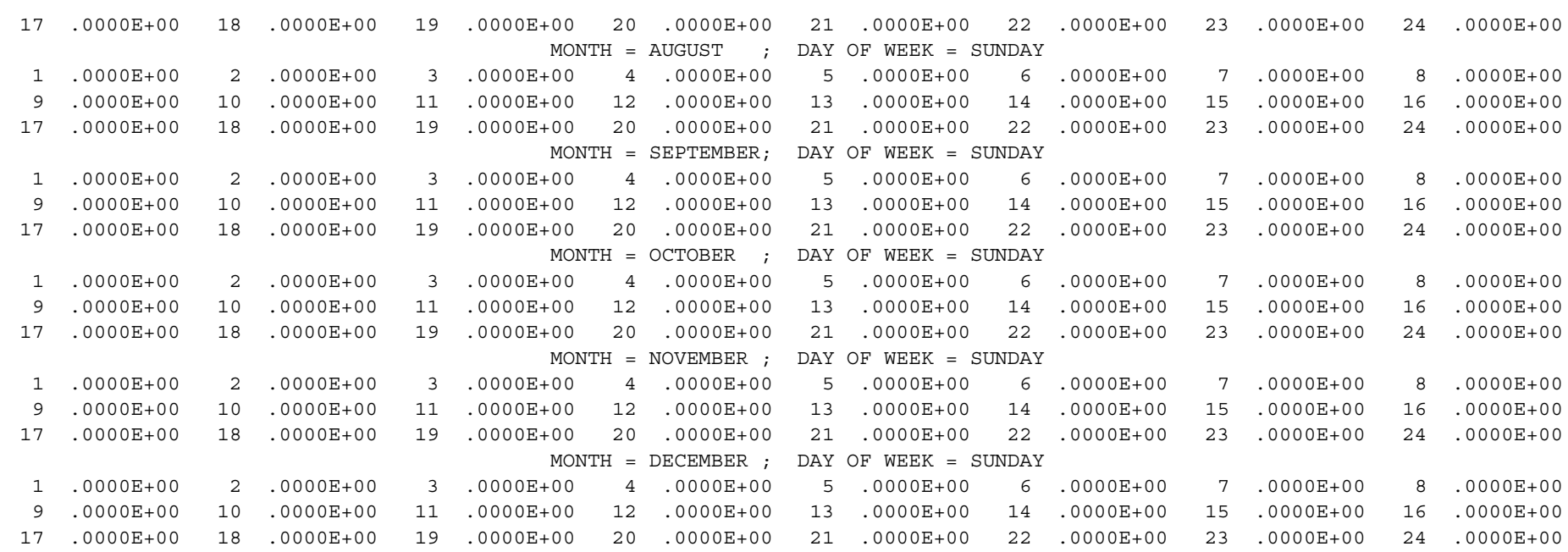

*** AERMOD - VERSION $07026 * * * \quad * * *$ U/UA Building Demolition

$\begin{array}{ll}* * * & 02 / 09 / 09 \\ * * * & 10: 41: 11\end{array}$

*** Unit Release - Worst Case

$10: 41: 11$
PAGE 62

$\star *$ MODELOPTS :

CONC

DDEP

TOXICS FLAT

DRYDPL WETDPL

* SOURCE EMISSION RATE SCALARS WHICH VARY MONTHLY, DIURNALLY AND BY DAY OF WEEK (MHRDOW7) *

SOURCE ID $=224 U_{-} 8 ;$ SOURCE TYPE $=$ POINT

HOUR SCALAR HOUR SCALAR HOUR SCALAR HOUR SCALAR HOUR SCALAR HOUR SCALAR HOUR SCALAR HOUR SCALAR

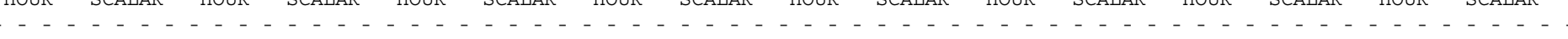

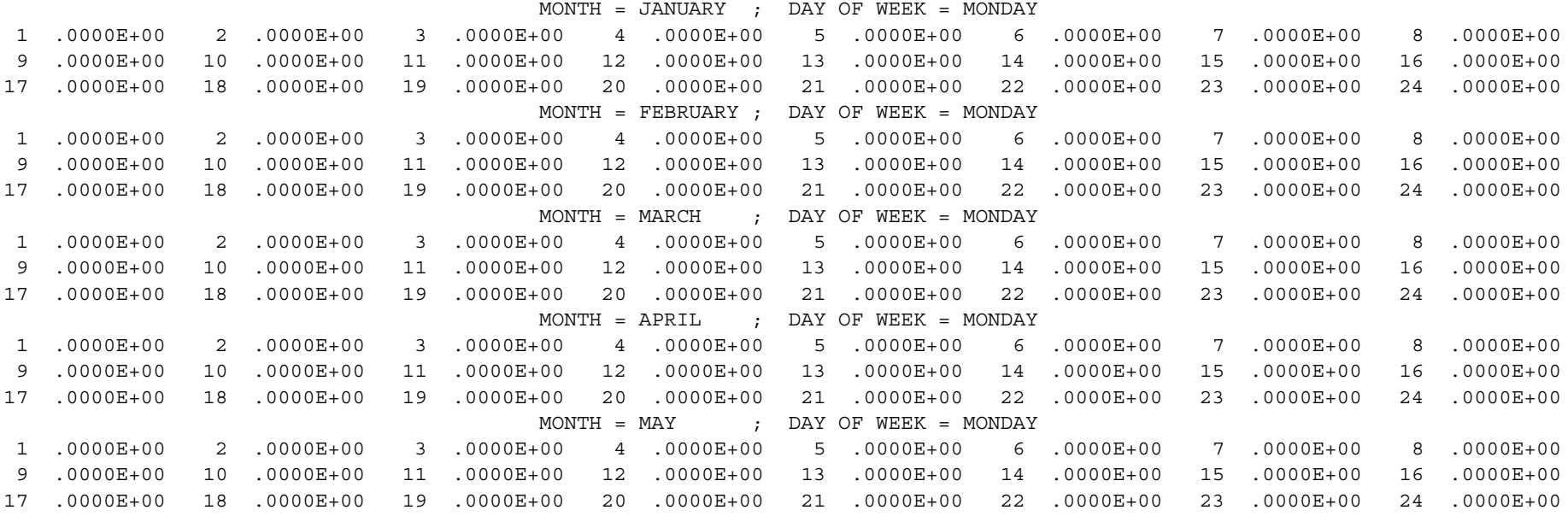




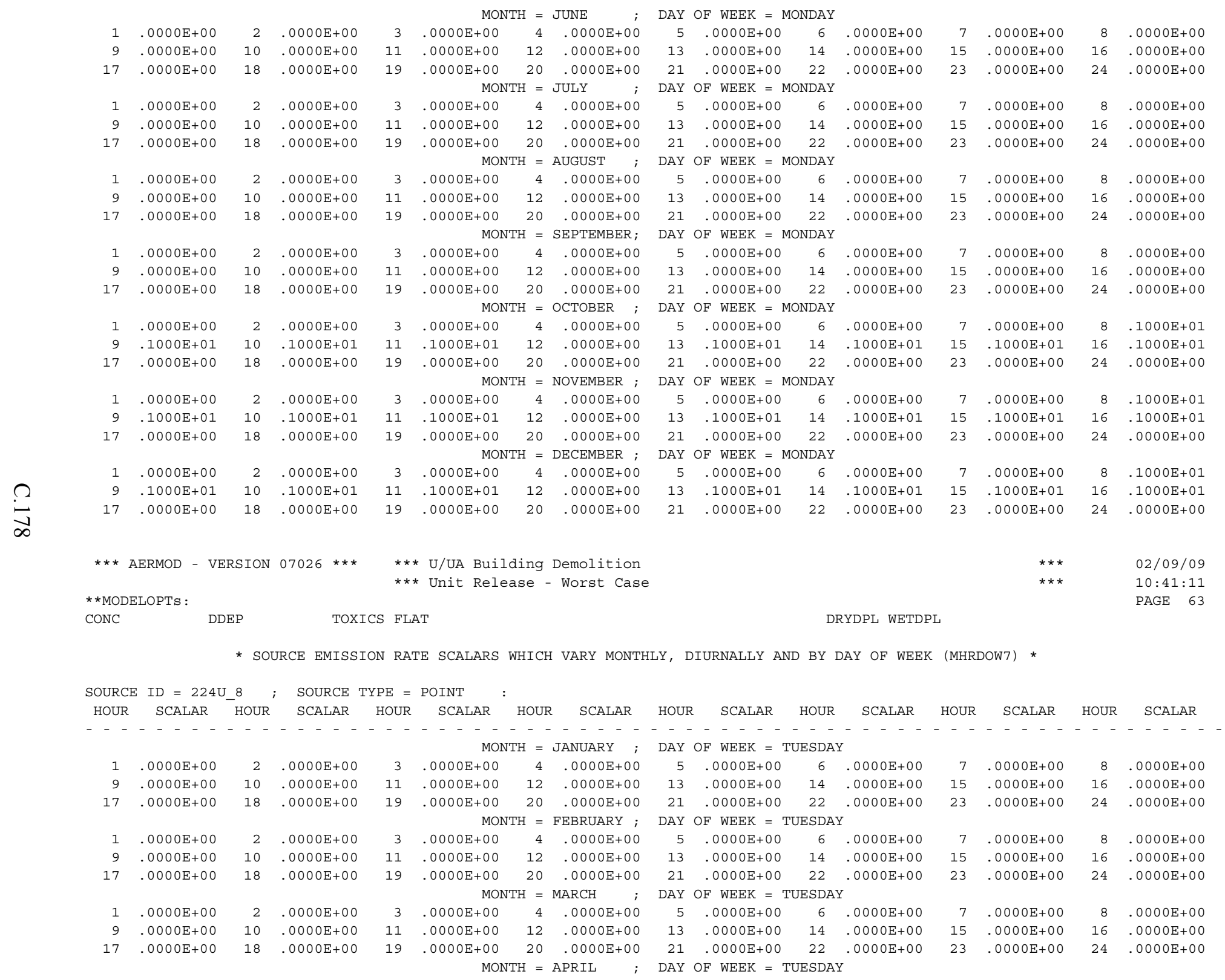




\begin{tabular}{|c|c|c|c|c|c|c|c|c|c|c|c|c|c|c|c|}
\hline 1 & $.0000 \mathrm{E}+00$ & 2 & $.0000 \mathrm{E}+00$ & 3 & $.0000 \mathrm{E}+00$ & 4 & $.0000 \mathrm{E}+00$ & 5 & $.0000 \mathrm{E}+00$ & 6 & $.0000 \mathrm{E}+00$ & 7 & $.0000 E+00$ & 8 & $.0000 E+00$ \\
\hline 9 & $.0000 \mathrm{E}+00$ & 10 & $.0000 \mathrm{E}+00$ & 11 & $.0000 \mathrm{E}+00$ & 12 & $.0000 \mathrm{E}+00$ & 13 & $.0000 \mathrm{E}+00$ & 14 & $.0000 \mathrm{E}+00$ & 15 & $.0000 \mathrm{E}+00$ & 16 & $.0000 \mathrm{E}+00$ \\
\hline 17 & $.0000 \mathrm{E}+00$ & 18 & $.0000 \mathrm{E}+00$ & 19 & $.0000 \mathrm{E}+00$ & 20 & $.0000 \mathrm{E}+00$ & 21 & $.0000 \mathrm{E}+00$ & 22 & $.0000 \mathrm{E}+00$ & 23 & $.0000 \mathrm{E}+00$ & 24 & $.0000 \mathrm{E}+00$ \\
\hline & & & & & MON & $\mathrm{H}=$ & MAY ; & DAY & OF WEEK $=T$ & JESDAY & & & & & \\
\hline 1 & $.0000 \mathrm{E}+00$ & 2 & $.0000 \mathrm{E}+00$ & 3 & $.0000 \mathrm{E}+00$ & 4 & $.0000 \mathrm{E}+00$ & 5 & $.0000 \mathrm{E}+00$ & 6 & $.0000 \mathrm{E}+00$ & 7 & $.0000 \mathrm{E}+00$ & 8 & $.0000 \mathrm{E}+00$ \\
\hline 9 & $.0000 \mathrm{E}+00$ & 10 & $.0000 \mathrm{E}+00$ & 11 & $.0000 \mathrm{E}+00$ & 12 & $.0000 \mathrm{E}+00$ & 13 & $.0000 \mathrm{E}+00$ & 14 & $.0000 \mathrm{E}+00$ & 15 & $.0000 \mathrm{E}+00$ & 16 & $.0000 \mathrm{E}+00$ \\
\hline 17 & $.0000 \mathrm{E}+00$ & 18 & $.0000 \mathrm{E}+00$ & 19 & $.0000 \mathrm{E}+00$ & 20 & $.0000 \mathrm{E}+00$ & 21 & $.0000 \mathrm{E}+00$ & 22 & $.0000 \mathrm{E}+00$ & 23 & $.0000 \mathrm{E}+00$ & 24 & $.0000 \mathrm{E}+00$ \\
\hline & & & & & MON & $\mathrm{H}=$ & JUNE ; & DAY & OF WEEK $=T$ & JESDAY & & & & & \\
\hline 1 & $.0000 \mathrm{E}+00$ & 2 & $.0000 \mathrm{E}+00$ & 3 & $.0000 \mathrm{E}+00$ & 4 & $.0000 \mathrm{E}+00$ & 5 & $.0000 \mathrm{E}+00$ & 6 & $.0000 \mathrm{E}+00$ & 7 & $.0000 \mathrm{E}+00$ & 8 & $.0000 \mathrm{E}+00$ \\
\hline 9 & $.0000 \mathrm{E}+00$ & 10 & $.0000 \mathrm{E}+00$ & 11 & $.0000 E+00$ & 12 & $.0000 \mathrm{E}+00$ & 13 & $.0000 \mathrm{E}+00$ & 14 & $.0000 \mathrm{E}+00$ & 15 & $.0000 \mathrm{E}+00$ & 16 & $.0000 E+00$ \\
\hline 17 & $.0000 \mathrm{E}+00$ & 18 & $.0000 \mathrm{E}+00$ & 19 & $.0000 \mathrm{E}+00$ & 20 & $.0000 \mathrm{E}+00$ & 21 & $.0000 \mathrm{E}+00$ & 22 & $.0000 \mathrm{E}+00$ & 23 & $.0000 \mathrm{E}+00$ & 24 & $.0000 \mathrm{E}+00$ \\
\hline & & & & & MON & $\mathrm{H}=$ & JULY ; & DAY & OF WEEK $=T$ & JESDAY & & & & & \\
\hline 1 & $.0000 \mathrm{E}+00$ & 2 & $.0000 \mathrm{E}+00$ & 3 & $.0000 \mathrm{E}+00$ & 4 & $.0000 \mathrm{E}+00$ & 5 & $.0000 \mathrm{E}+00$ & 6 & $.0000 \mathrm{E}+00$ & 7 & $.0000 \mathrm{E}+00$ & 8 & $.0000 \mathrm{E}+00$ \\
\hline 9 & $.0000 \mathrm{E}+00$ & 10 & $.0000 \mathrm{E}+00$ & 11 & $.0000 E+00$ & 12 & $.0000 \mathrm{E}+00$ & 13 & $.0000 \mathrm{E}+00$ & 14 & $.0000 \mathrm{E}+00$ & 15 & $.0000 \mathrm{E}+00$ & 16 & $.0000 E+00$ \\
\hline 17 & $.0000 \mathrm{E}+00$ & 18 & $.0000 \mathrm{E}+00$ & 19 & $.0000 \mathrm{E}+00$ & 20 & $.0000 \mathrm{E}+00$ & 21 & $.0000 \mathrm{E}+00$ & 22 & $.0000 \mathrm{E}+00$ & 23 & $.0000 \mathrm{E}+00$ & 24 & $.0000 \mathrm{E}+00$ \\
\hline & & & & & MON & $\mathrm{H}=$ & AUGUST ; & DAY & OF WEEK $=T$ & JESDAY & & & & & \\
\hline 1 & $.0000 \mathrm{E}+00$ & 2 & $.0000 \mathrm{E}+00$ & 3 & $.0000 \mathrm{E}+00$ & 4 & $.0000 \mathrm{E}+00$ & 5 & $.0000 \mathrm{E}+00$ & 6 & $.0000 \mathrm{E}+00$ & 7 & $.0000 \mathrm{E}+00$ & 8 & $.0000 \mathrm{E}+00$ \\
\hline 9 & $.0000 \mathrm{E}+00$ & 10 & $.0000 \mathrm{E}+00$ & 11 & $.0000 \mathrm{E}+00$ & 12 & $.0000 \mathrm{E}+00$ & 13 & $.0000 \mathrm{E}+00$ & 14 & $.0000 \mathrm{E}+00$ & 15 & $.0000 \mathrm{E}+00$ & 16 & $.0000 E+00$ \\
\hline 17 & $.0000 \mathrm{E}+00$ & 18 & $.0000 \mathrm{E}+00$ & 19 & $.0000 \mathrm{E}+00$ & 20 & $.0000 \mathrm{E}+00$ & 21 & $.0000 \mathrm{E}+00$ & 22 & $.0000 \mathrm{E}+00$ & 23 & $.0000 \mathrm{E}+00$ & 24 & $.0000 E+00$ \\
\hline & & & & & MON & $\mathrm{H}=$ & SEPTEMBER； & DAY & OF WEEK = T & JESDAY & & & & & \\
\hline 1 & $.0000 \mathrm{E}+00$ & 2 & $.0000 \mathrm{E}+00$ & 3 & $.0000 \mathrm{E}+00$ & 4 & $.0000 \mathrm{E}+00$ & 5 & $.0000 \mathrm{E}+00$ & 6 & $.0000 \mathrm{E}+00$ & 7 & $.0000 \mathrm{E}+00$ & 8 & $.0000 \mathrm{E}+00$ \\
\hline 9 & $.0000 \mathrm{E}+00$ & 10 & $.0000 \mathrm{E}+00$ & 11 & $.0000 \mathrm{E}+00$ & 12 & $.0000 \mathrm{E}+00$ & 13 & $.0000 \mathrm{E}+00$ & 14 & $.0000 \mathrm{E}+00$ & 15 & $.0000 \mathrm{E}+00$ & 16 & $.0000 \mathrm{E}+00$ \\
\hline 17 & $.0000 \mathrm{E}+00$ & 18 & $.0000 \mathrm{E}+00$ & 19 & $.0000 \mathrm{E}+00$ & 20 & $.0000 \mathrm{E}+00$ & 21 & $.0000 \mathrm{E}+00$ & 22 & $.0000 \mathrm{E}+00$ & 23 & $.0000 \mathrm{E}+00$ & 24 & $.0000 E+00$ \\
\hline & & & & & MON & $\mathrm{H}=$ & OCTOBER ; & DAY & OF WEEK = T & JESDAY & & & & & \\
\hline 1 & $.0000 \mathrm{E}+00$ & 2 & $.0000 \mathrm{E}+00$ & 3 & $.0000 \mathrm{E}+00$ & 4 & $.0000 \mathrm{E}+00$ & 5 & $.0000 \mathrm{E}+00$ & 6 & $.0000 \mathrm{E}+00$ & 7 & $.0000 \mathrm{E}+00$ & 8 & $.1000 \mathrm{E}+01$ \\
\hline 9 & $.1000 \mathrm{E}+01$ & 10 & $.1000 \mathrm{E}+01$ & 11 & $.1000 \mathrm{E}+01$ & 12 & $.0000 \mathrm{E}+00$ & 13 & $.1000 \mathrm{E}+01$ & 14 & $.1000 \mathrm{E}+01$ & 15 & $.1000 \mathrm{E}+01$ & 16 & $.1000 \mathrm{E}+01$ \\
\hline 17 & $.0000 \mathrm{E}+00$ & 18 & $.0000 \mathrm{E}+00$ & 19 & $.0000 \mathrm{E}+00$ & 20 & $.0000 \mathrm{E}+00$ & 21 & $.0000 \mathrm{E}+00$ & 22 & $.0000 \mathrm{E}+00$ & 23 & $.0000 \mathrm{E}+00$ & 24 & $.0000 \mathrm{E}+00$ \\
\hline & & & & & MON & $\mathrm{H}=$ & NOVEMBER ; & DAY & OF WEEK $=\mathrm{T}$ & ESDAY & & & & & \\
\hline 1 & $.0000 \mathrm{E}+00$ & 2 & $.0000 \mathrm{E}+00$ & 3 & $.0000 \mathrm{E}+00$ & 4 & $.0000 \mathrm{E}+00$ & 5 & $.0000 \mathrm{E}+00$ & 6 & $.0000 \mathrm{E}+00$ & 7 & $.0000 \mathrm{E}+00$ & 8 & $.1000 \mathrm{E}+01$ \\
\hline 9 & $.1000 \mathrm{E}+01$ & 10 & $.1000 \mathrm{E}+01$ & 11 & $.1000 \mathrm{E}+01$ & 12 & $.0000 \mathrm{E}+00$ & 13 & $.1000 \mathrm{E}+01$ & 14 & $.1000 \mathrm{E}+01$ & 15 & $.1000 \mathrm{E}+01$ & 16 & $.1000 \mathrm{E}+01$ \\
\hline 17 & $.0000 \mathrm{E}+00$ & 18 & $.0000 \mathrm{E}+00$ & 19 & $.0000 \mathrm{E}+00$ & 20 & $.0000 E+00$ & 21 & $.0000 \mathrm{E}+00$ & 22 & $.0000 \mathrm{E}+00$ & 23 & $.0000 E+00$ & 24 & $.0000 \mathrm{E}+00$ \\
\hline & & & & & MON & $\mathrm{H}=$ & DECEMBER ; & DAY & OF WEEK $=T$ & JESDAY & & & & & \\
\hline 1 & $.0000 \mathrm{E}+00$ & 2 & $.0000 \mathrm{E}+00$ & 3 & $.0000 \mathrm{E}+00$ & 4 & $.0000 \mathrm{E}+00$ & 5 & $.0000 \mathrm{E}+00$ & 6 & $.0000 \mathrm{E}+00$ & 7 & $E+00$ & 8 & $.1000 \mathrm{E}+01$ \\
\hline 9 & $.1000 \mathrm{E}+01$ & 10 & $.1000 \mathrm{E}+01$ & 11 & $.1000 \mathrm{E}+01$ & 12 & $.0000 \mathrm{E}+00$ & 13 & $.1000 \mathrm{E}+01$ & 14 & $.1000 \mathrm{E}+01$ & 15 & $.1000 \mathrm{E}+01$ & 16 & $.1000 \mathrm{E}+01$ \\
\hline 17 & $.0000 \mathrm{E}+00$ & 18 & $.0000 \mathrm{E}+00$ & 19 & $.0000 \mathrm{E}+00$ & 20 & $.0000 \mathrm{E}+00$ & 21 & $.0000 \mathrm{E}+00$ & 22 & $.0000 \mathrm{E}+00$ & 23 & $.0000 \mathrm{E}+00$ & 24 & $.0000 E+00$ \\
\hline
\end{tabular}

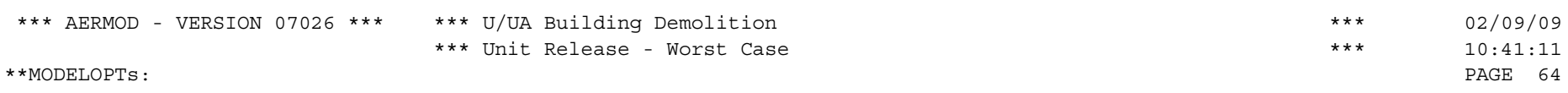

DDEP TOXICS FLAT

DRYDPL WETDPI

* SOURCE EMISSION RATE SCALARS WHICH VARY MONTHLY, DIURNALLY AND BY DAY OF WEEK (MHRDOW7) *

SOURCE ID $=224 \mathrm{U}_{-} 8 ;$ SOURCE TYPE $=$ POINT

HOUR SCALAR HOUR SCALAR HOUR SCALAR HOUR SCALAR HOUR SCALAR HOUR SCALAR HOUR SCALAR HOUR SCALAR

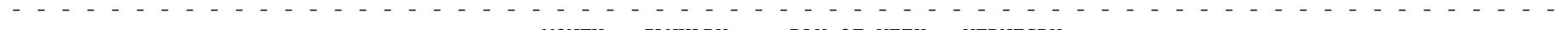

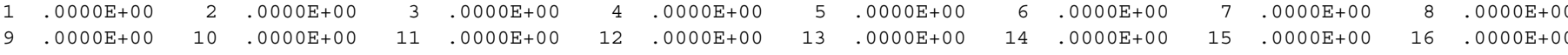

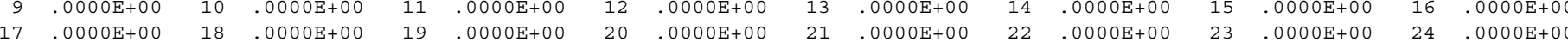

1. MONTH = FEBRUARY ; DAY OF WEEK = WEDNESDY

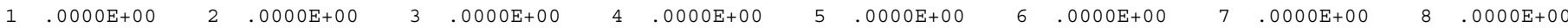




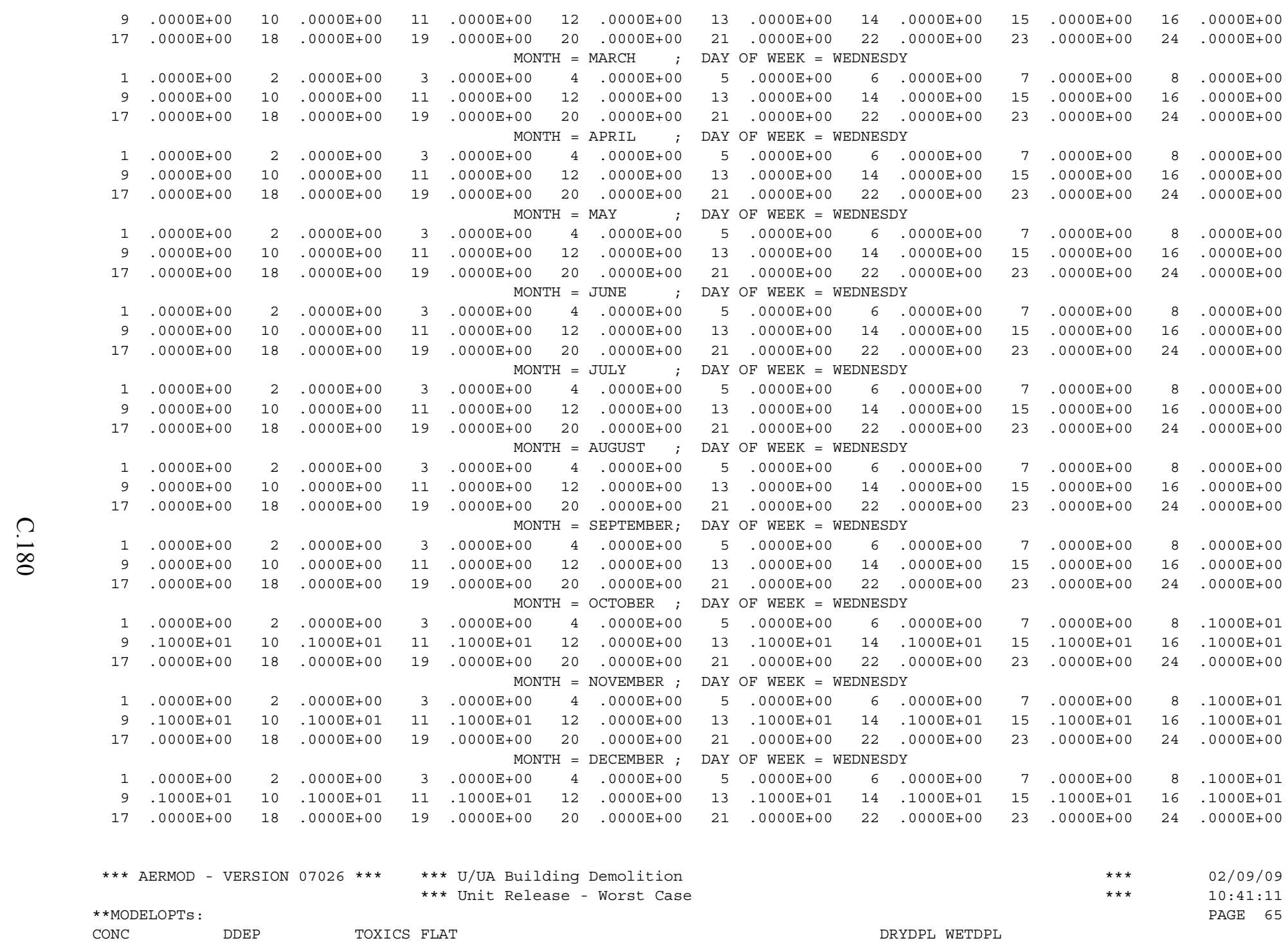

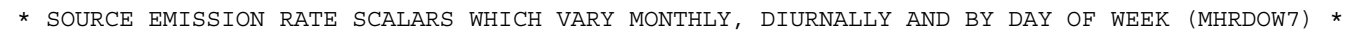

SOURCE ID $=224 \mathrm{U}_{-} 8 ;$ SOURCE TYPE $=$ POINT

HOUR SCALAR HOUR SCALAR HOUR SCALAR HOUR SCALAR HOUR SCALAR HOUR SCALAR HOUR SCALAR HOUR SCALAR 


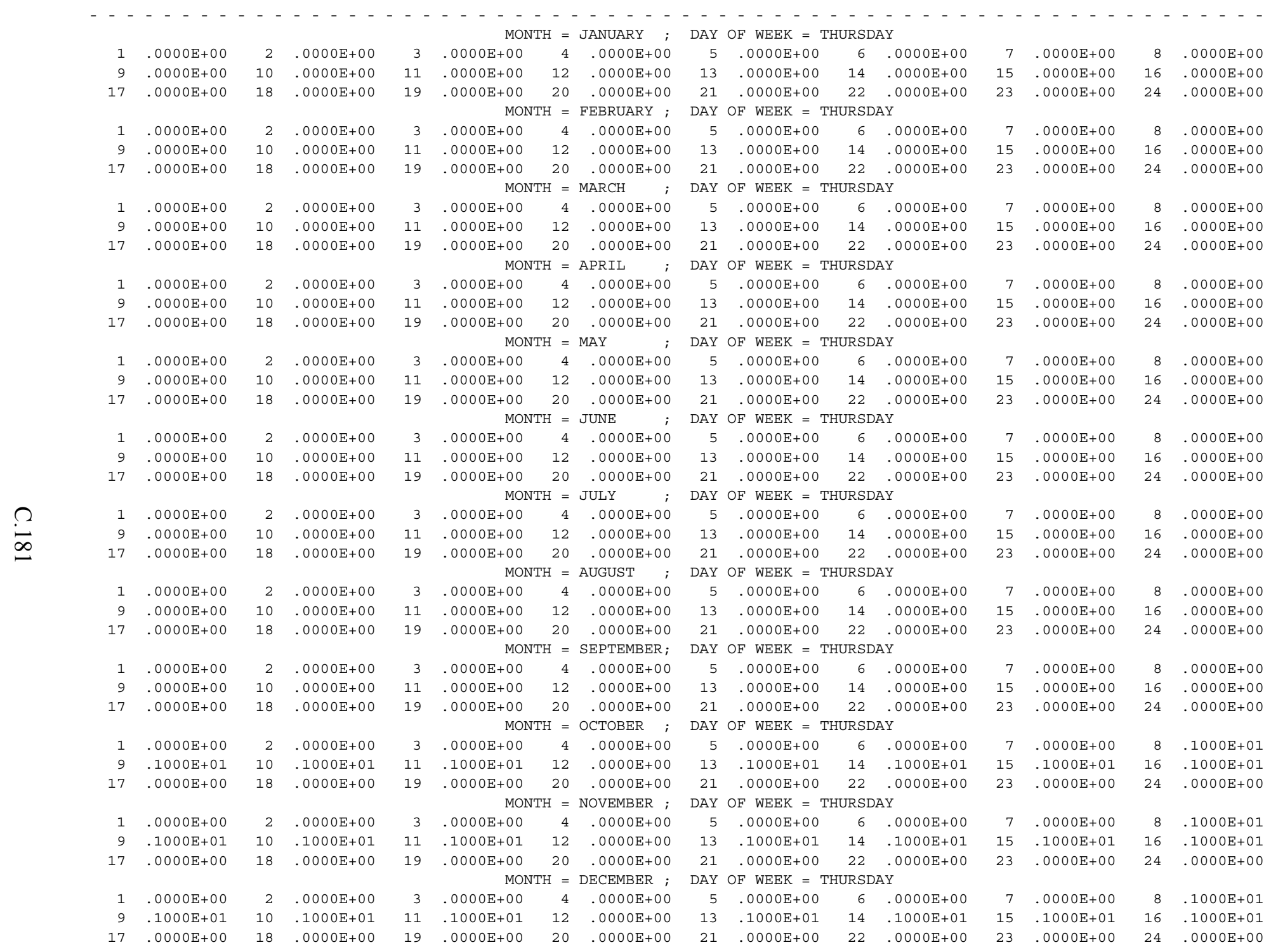

*** AERMOD - VERSION $07026 * * * \quad * * *$ U/UA Building Demolition

*** Unit Release - Worst Case 
* SOURCE EMISSION RATE SCALARS WHICH VARY MONTHLY, DIURNALLY AND BY DAY OF WEEK (MHRDOW7) *

SOURCE ID $=224 \mathrm{U} 8 ;$ SOURCE TYPE $=$ POINT

HOUR SCALAR - HOUR' SCAIAR HOUR SCALAR' HOUR SCALAR HOUR SCALAR HOUR SCALAR HOUR SCALAR HOUR SCALAR

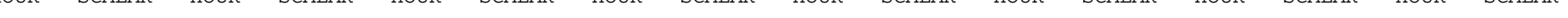

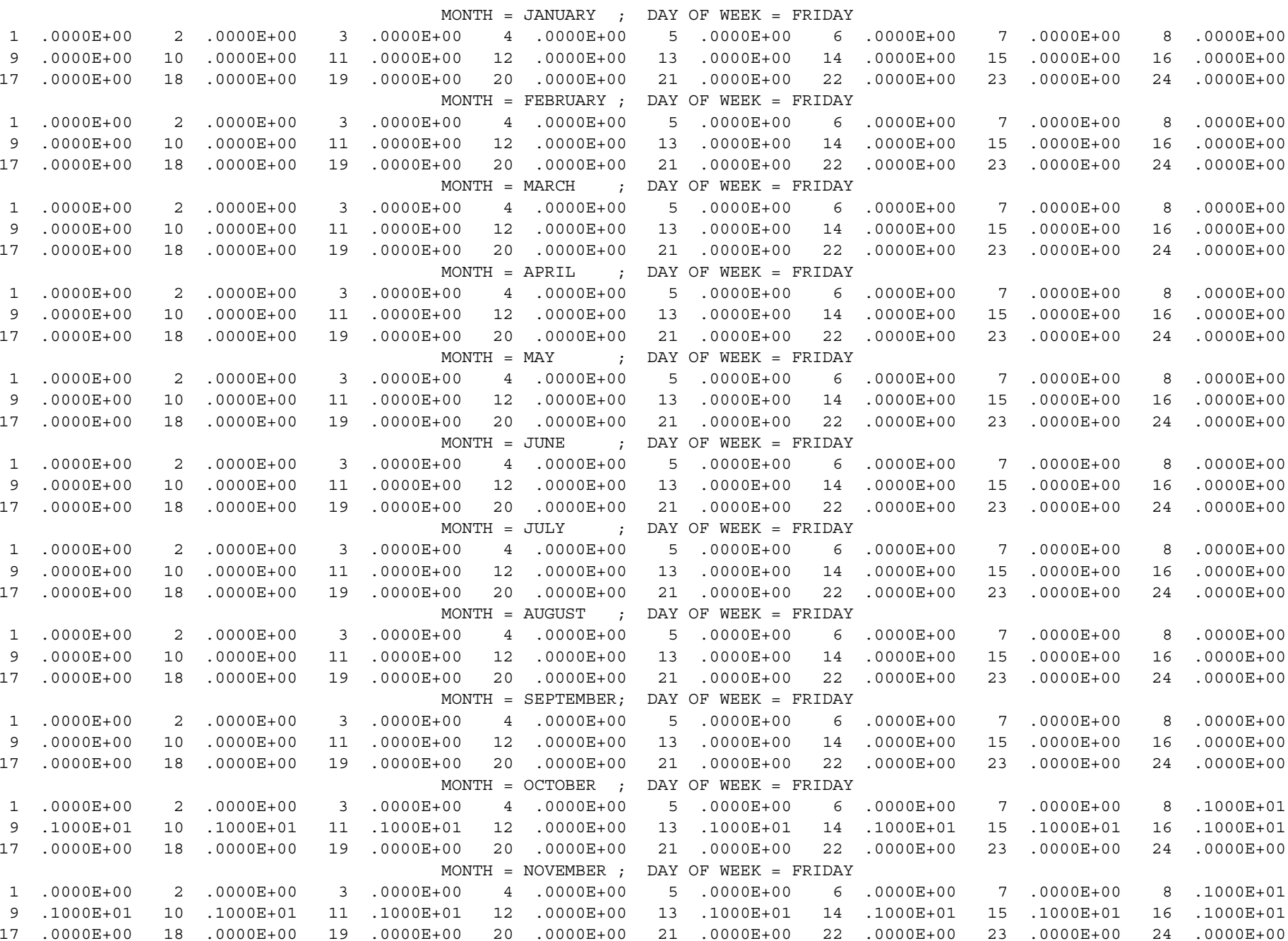
MONTH $=$ DECEMBER $;$ DAY OF WEEK = FRIDAY 


\begin{tabular}{|c|c|c|c|c|c|c|c|c|c|c|c|c|c|c|c|}
\hline 1 & $\mathrm{E}+00$ & 2 & $.0000 \mathrm{E}+00$ & 3 & $.0000 \mathrm{E}+00$ & 4 & $.0000 \mathrm{E}+00$ & 5 & $.0000 \mathrm{E}+00$ & 6 & $.0000 \mathrm{E}+00$ & 7 & $.0000 \mathrm{E}+00$ & 8 & $0 E+01$ \\
\hline 9 & $.1000 \mathrm{E}+01$ & 10 & $.1000 \mathrm{E}+01$ & 11 & $.1000 \mathrm{E}+01$ & 12 & $.0000 \mathrm{E}+00$ & 13 & $.1000 \mathrm{E}+01$ & 14 & $.1000 \mathrm{E}+01$ & 15 & $.1000 \mathrm{E}+01$ & 16 & $.1000 \mathrm{E}+01$ \\
\hline & $.0000 \mathrm{E}+00$ & 18 & $.0000 \mathrm{E}+00$ & 19 & $.0000 \mathrm{E}+00$ & 20 & $.0000 \mathrm{E}+00$ & 21 & $.0000 \mathrm{E}+00$ & 22 & $.0000 \mathrm{E}+00$ & 23 & $.0000 \mathrm{E}+00$ & 24 & $00 \mathrm{E}+00$ \\
\hline
\end{tabular}

$* * *$ AERMOD - VERSION $07026 * * * \quad \begin{aligned} & * * \text { U/UA Building Demolition } \\ & * * * \text { Unit Release - Worst Case }\end{aligned}$ $\star *$ MODELOPTS :

CONC $\begin{array}{ll}* * * & 02 / 09 / 09 \\ * * * & 10: 41: 11\end{array}$

DRYDPL WETDPL

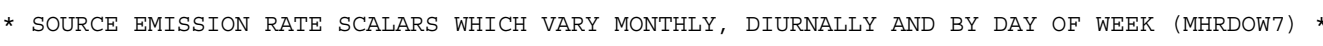

SOURCE ID $=224 \mathrm{U}_{-} 8 ;$ SOURCE TYPE $=$ POINT

HOUR SCALAR HOUR SCALAR HOUR SCALAR HOUR SCALAR HOUR SCALAR HOUR SCALAR HOUR SCALAR HOUR SCALAR

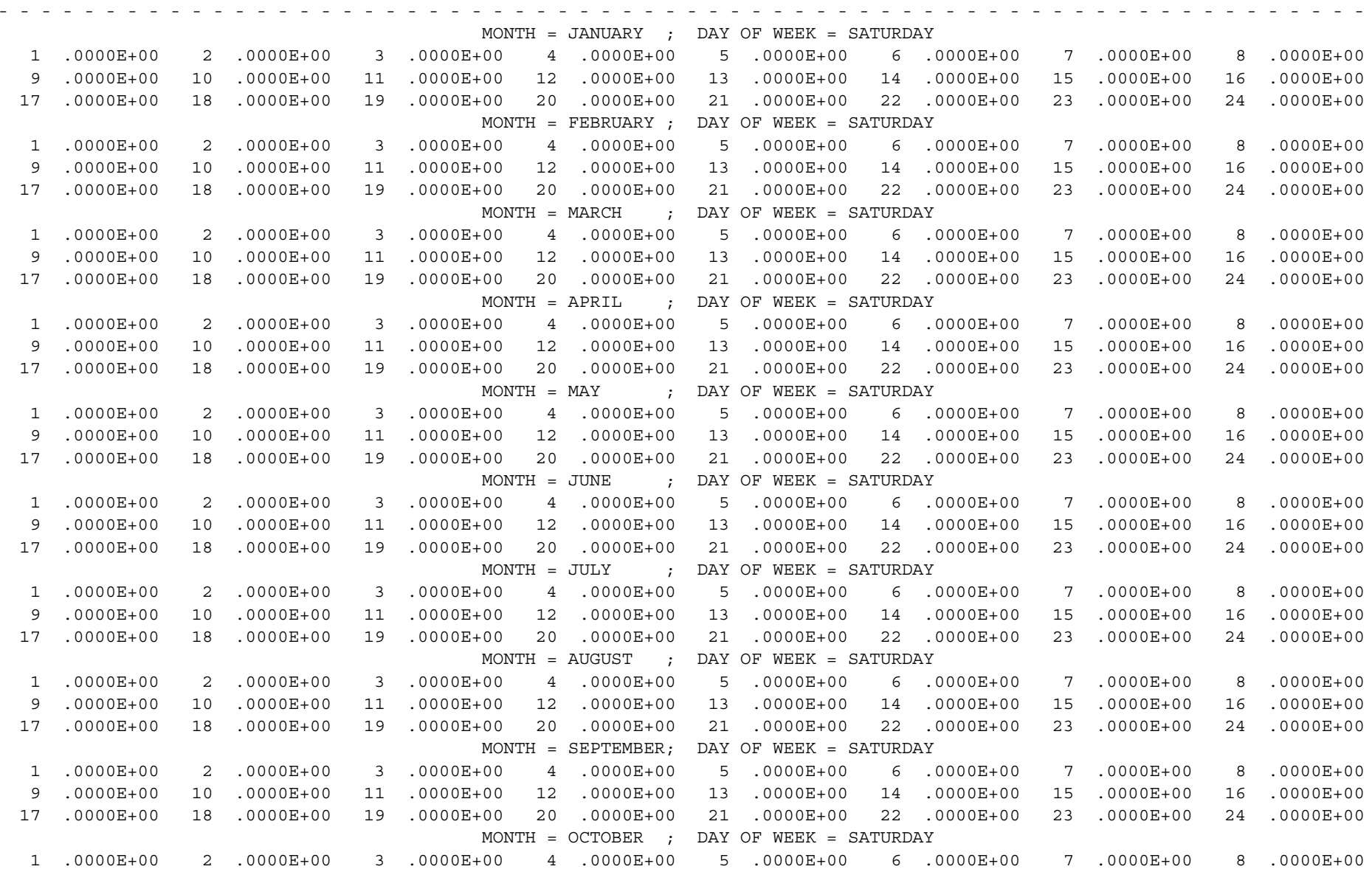




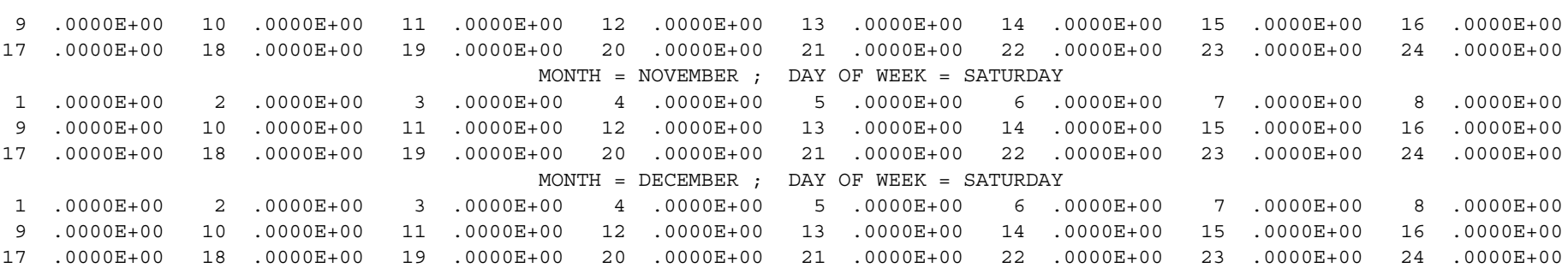

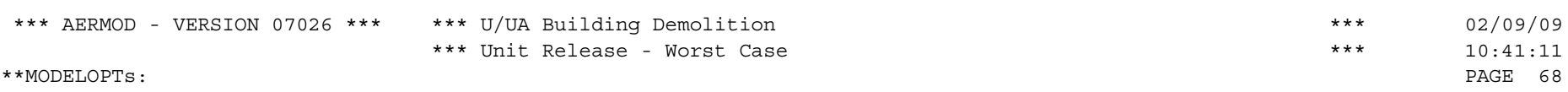
CONC
DDEP
TOXICS FLAT
DRYDPL WETDPL

PAGE 68

* SoURCe emission RAte scalars which VARy monthly, DiURNALly AND by DAy of WEek (MHRDOW7) *

SOURCE ID $=224 \mathrm{U}_{-} 8 ; \quad$ SOURCE TYPE $=$ POINT :

HOUR SCALAR HOUR SCALAR HOUR SCALAR HOUR SCALAR HOUR SCALAR HOUR SCALAR HOUR SCALAR HOUR SCALAR

_ . . . . . . -

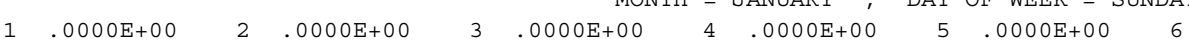

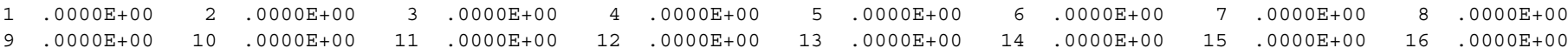

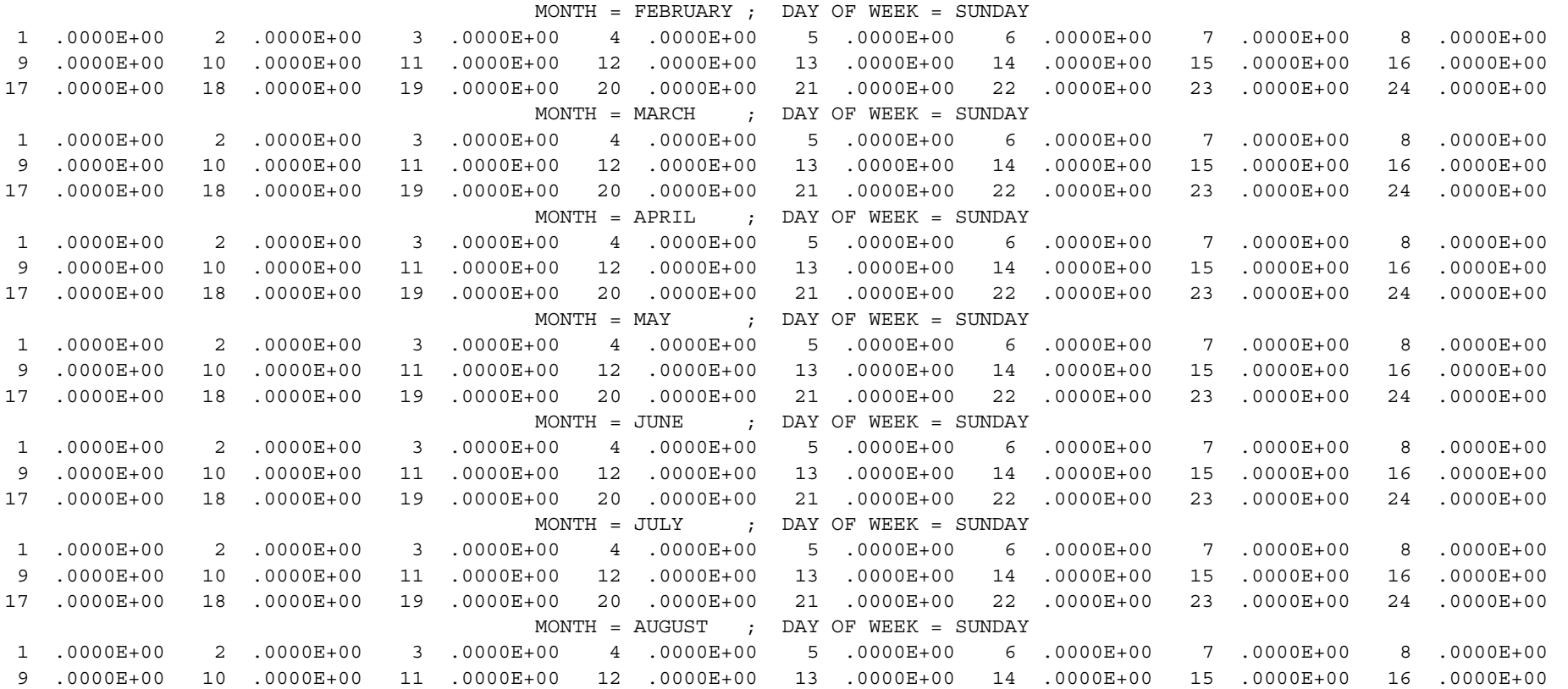




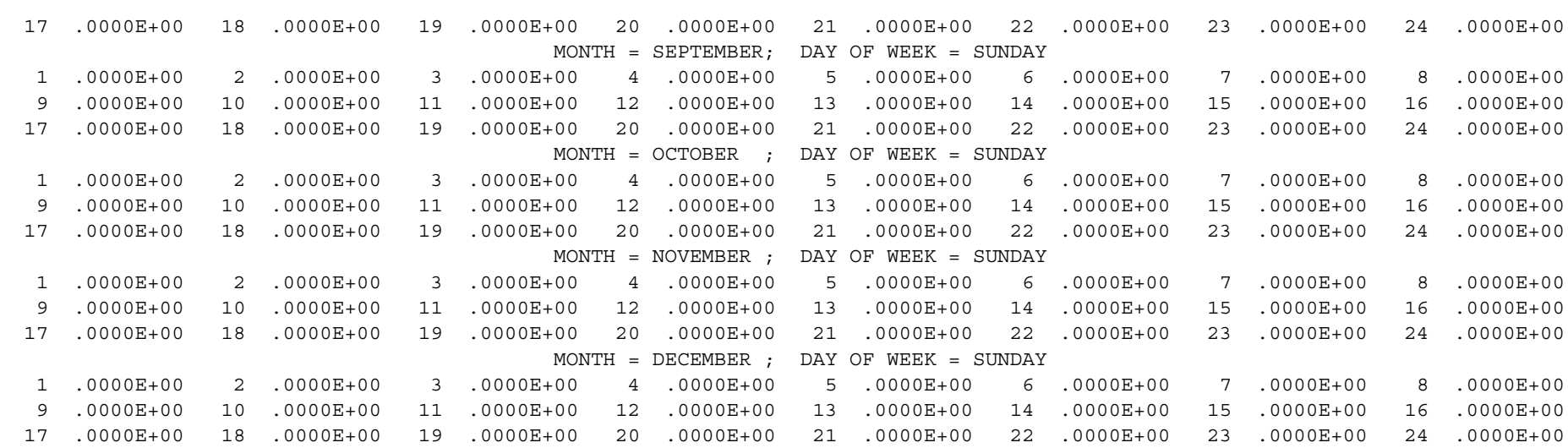

\begin{tabular}{|c|c|c|c|c|}
\hline *** AERMOD & - VERSION $07026 * \star *$ & $\begin{array}{l}* * * \text { U/UA Building Demolition } \\
* * * \text { Unit Release - Worst Case }\end{array}$ & $\begin{array}{l}* * * \\
* * *\end{array}$ & $\begin{array}{l}02 / 09 / 09 \\
10: 41: 11\end{array}$ \\
\hline & & & & PAGE $\quad 69$ \\
\hline
\end{tabular}

CONC

DDEP

DRYDPL WETDPL

PAGE 69

SOURCE ID $=$ 224U_9 $; \quad$ SOURCE TYPE $=$ POINT

HOUR SCALAR HOUR SCALAR HOUR SCALAR HOUR SCALAR HOUR SCALAR HOUR SCALAR HOUR SCALAR HOUR SCALAR

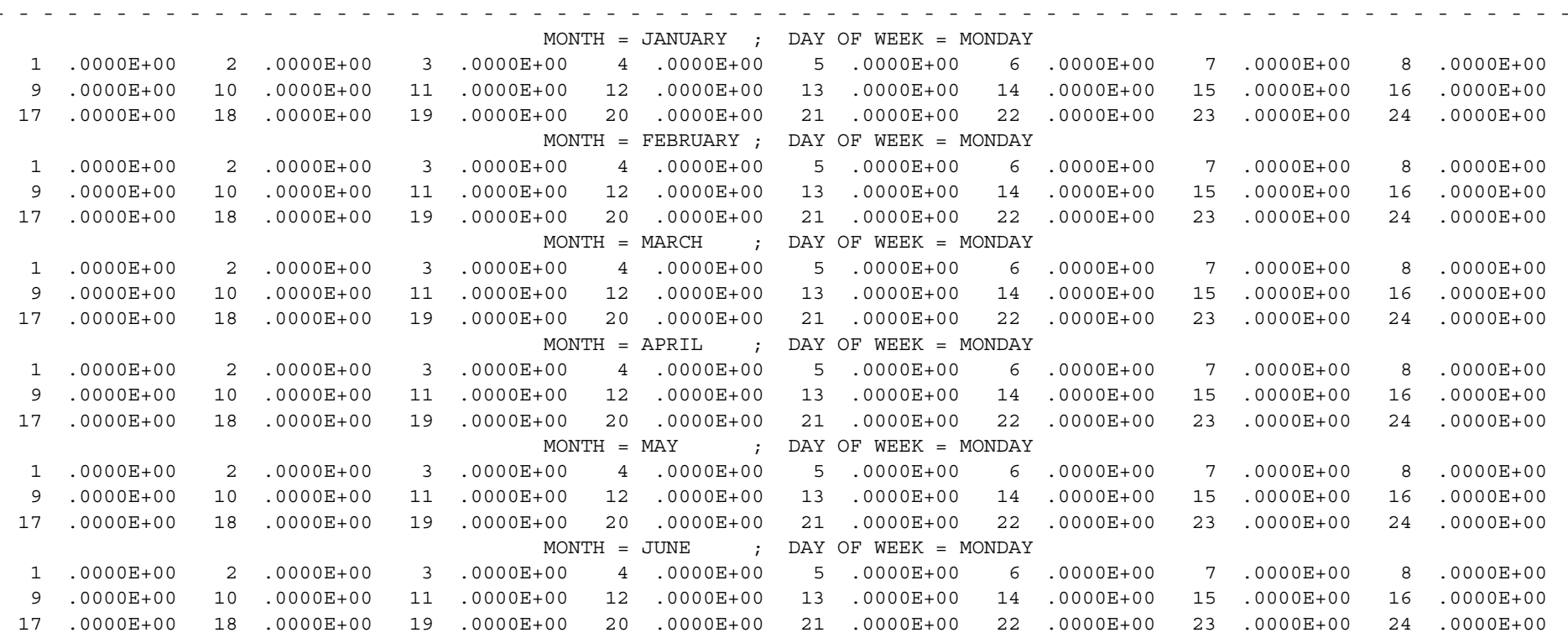




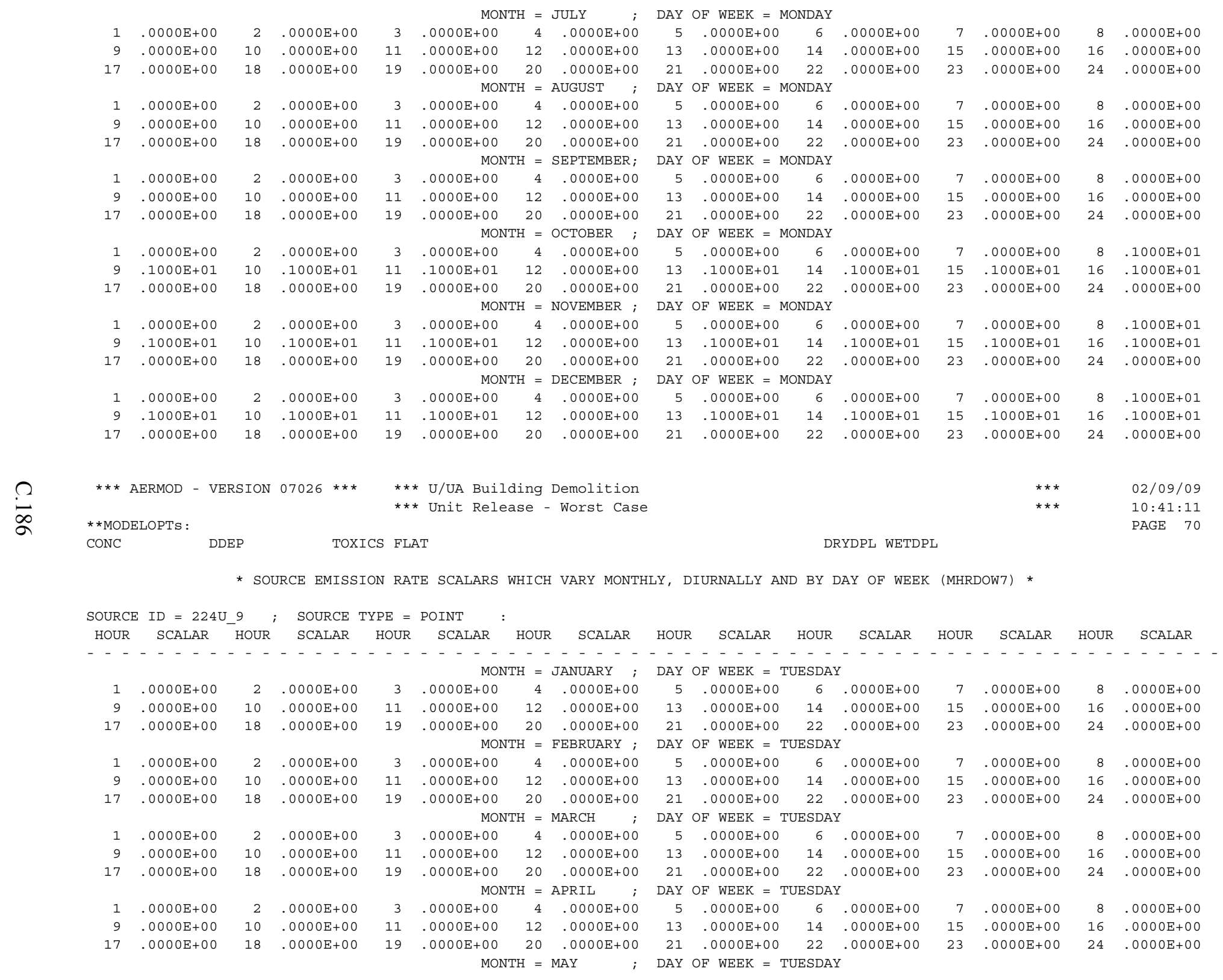




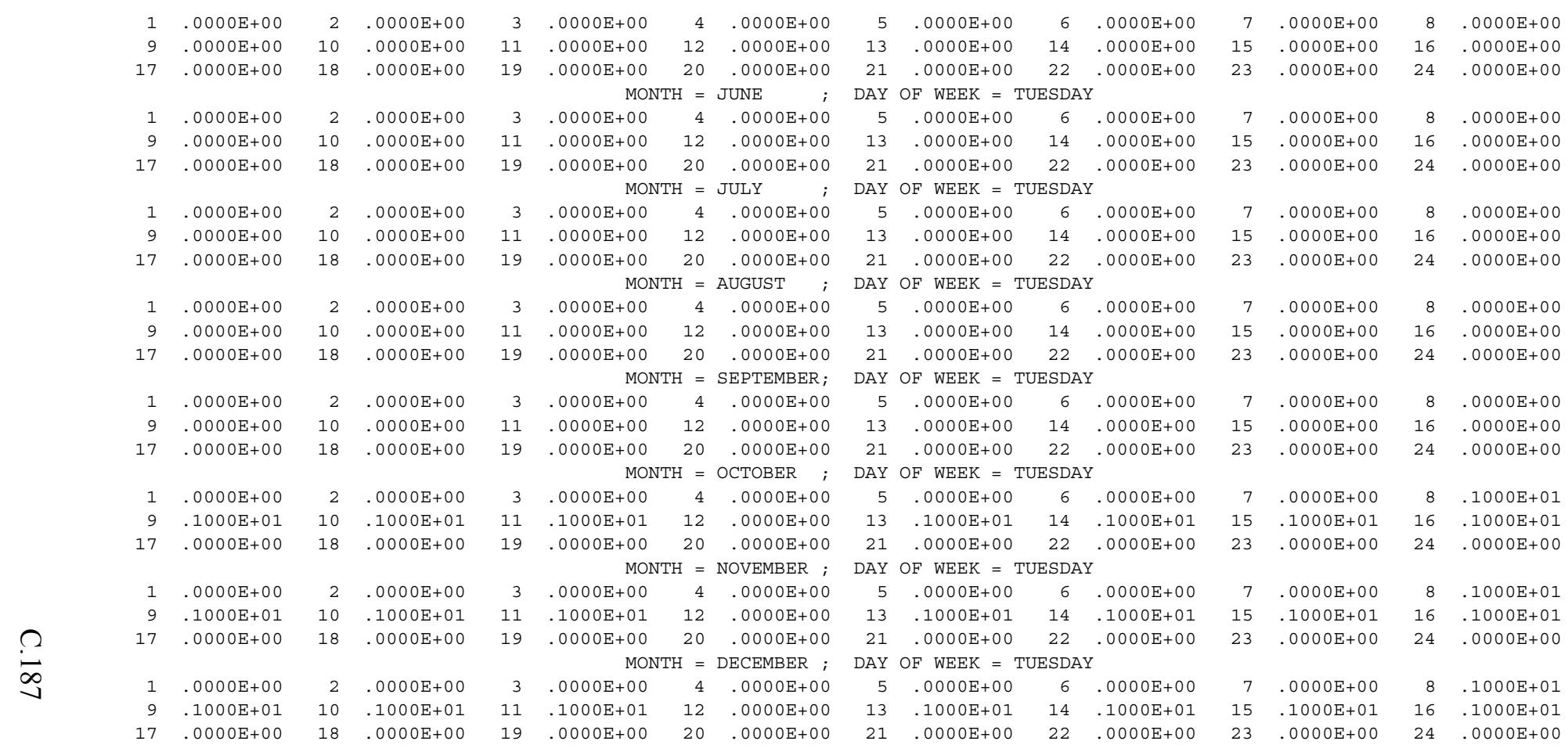

$* * *$ AERMOD - VERSION $07026 * * * \quad \begin{aligned} & * * \text { U/UA Building Demolition } \\ & * * * \text { Unit Release - Worst Case }\end{aligned}$

$\begin{array}{ll}* * * & 02 / 09 / 09 \\ * * * & 10: 41: 11\end{array}$ **MODELOPTS :

CONC

DDEP

TOXICS FLAT

DRYDPL WETDPL

* SOURCE EMISSION RATE SCALARS WHICH VARY MONTHLY, DIURNALLY AND BY DAY OF WEEK (MHRDOW7) *

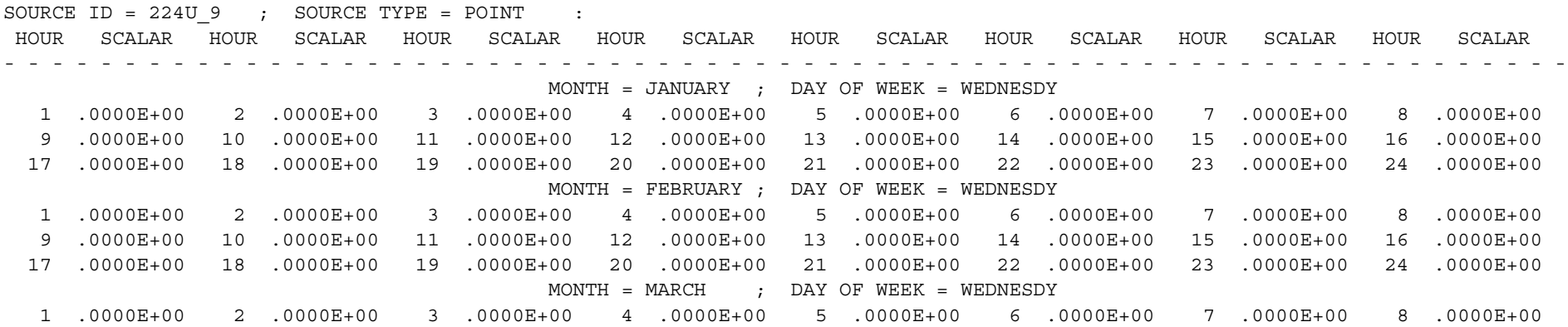




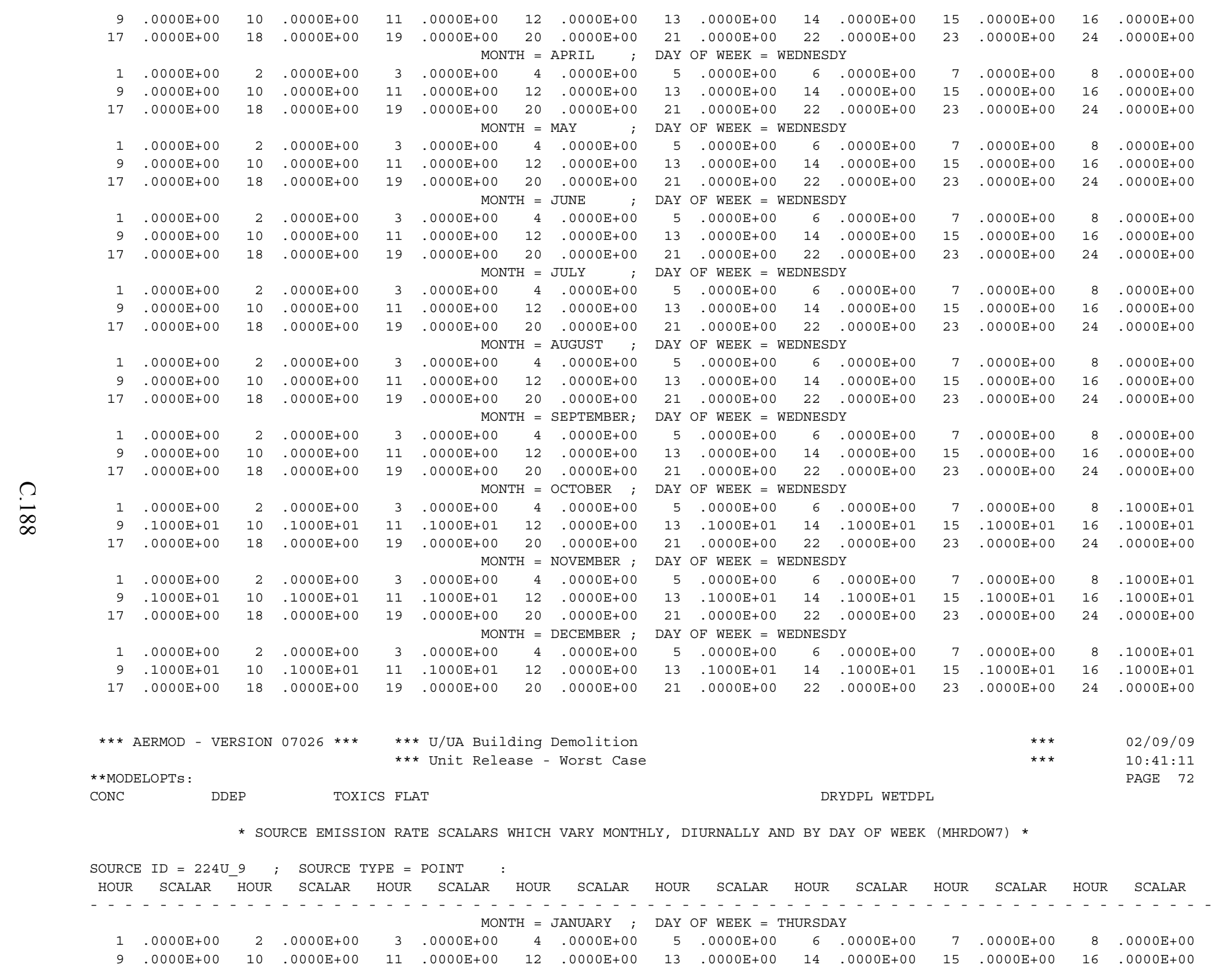




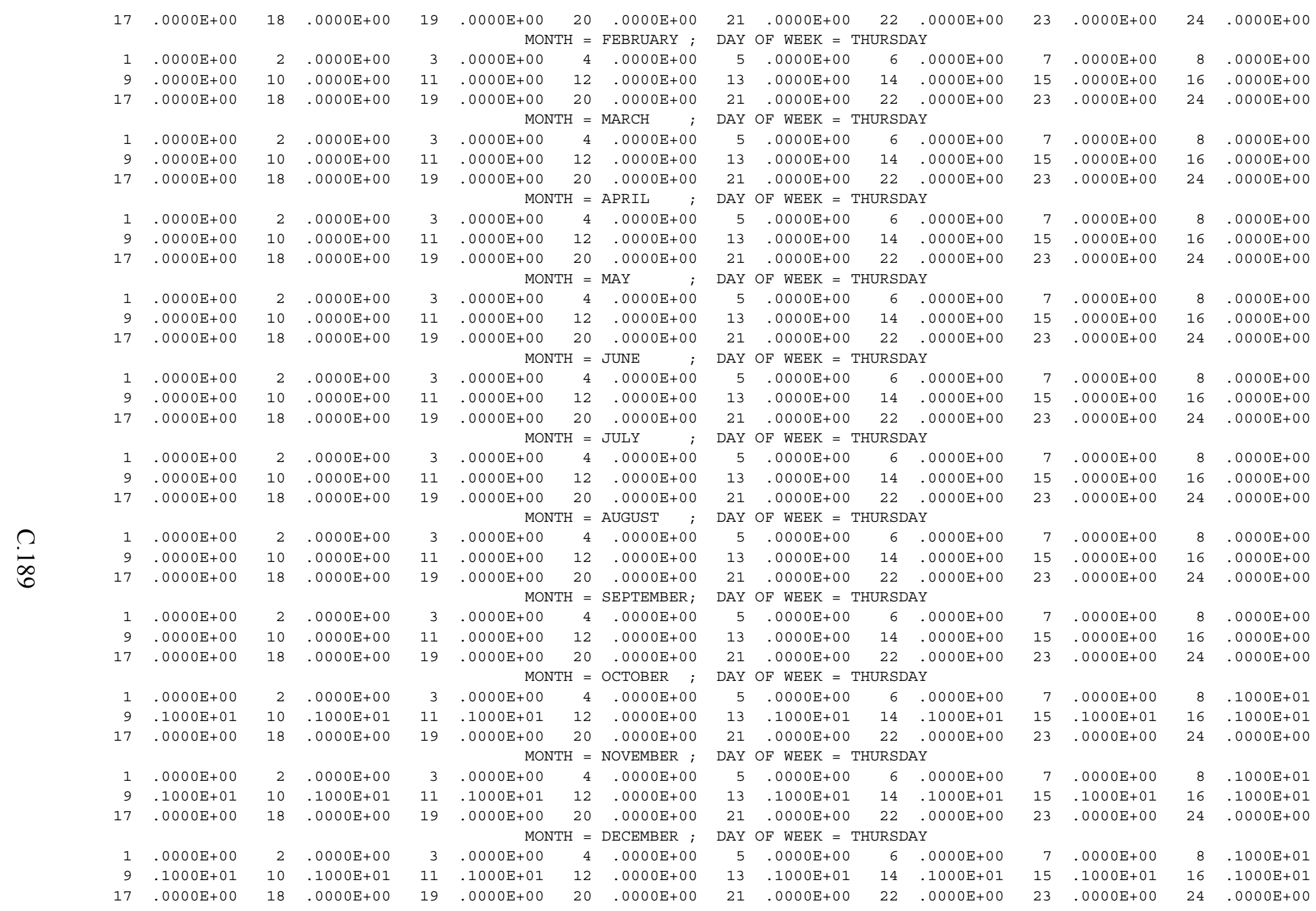

*** AERMOD - VERSION $07026 * * * \quad * * *$ U/UA Building Demolition

$* * *$ Unit Release - Worst Cas $\begin{array}{ll}* * * & 02 / 09 / 09 \\ * * * & 10: 41: 11\end{array}$

PAGE 73

CONC

DDEP

TOXICS FLAT

DRYDPL WETDPL

* SOURCE EMISSION RATE SCALARS WHICH VARY MONTHLY, DIURNALLY AND BY DAY OF WEEK (MHRDOW7) * 


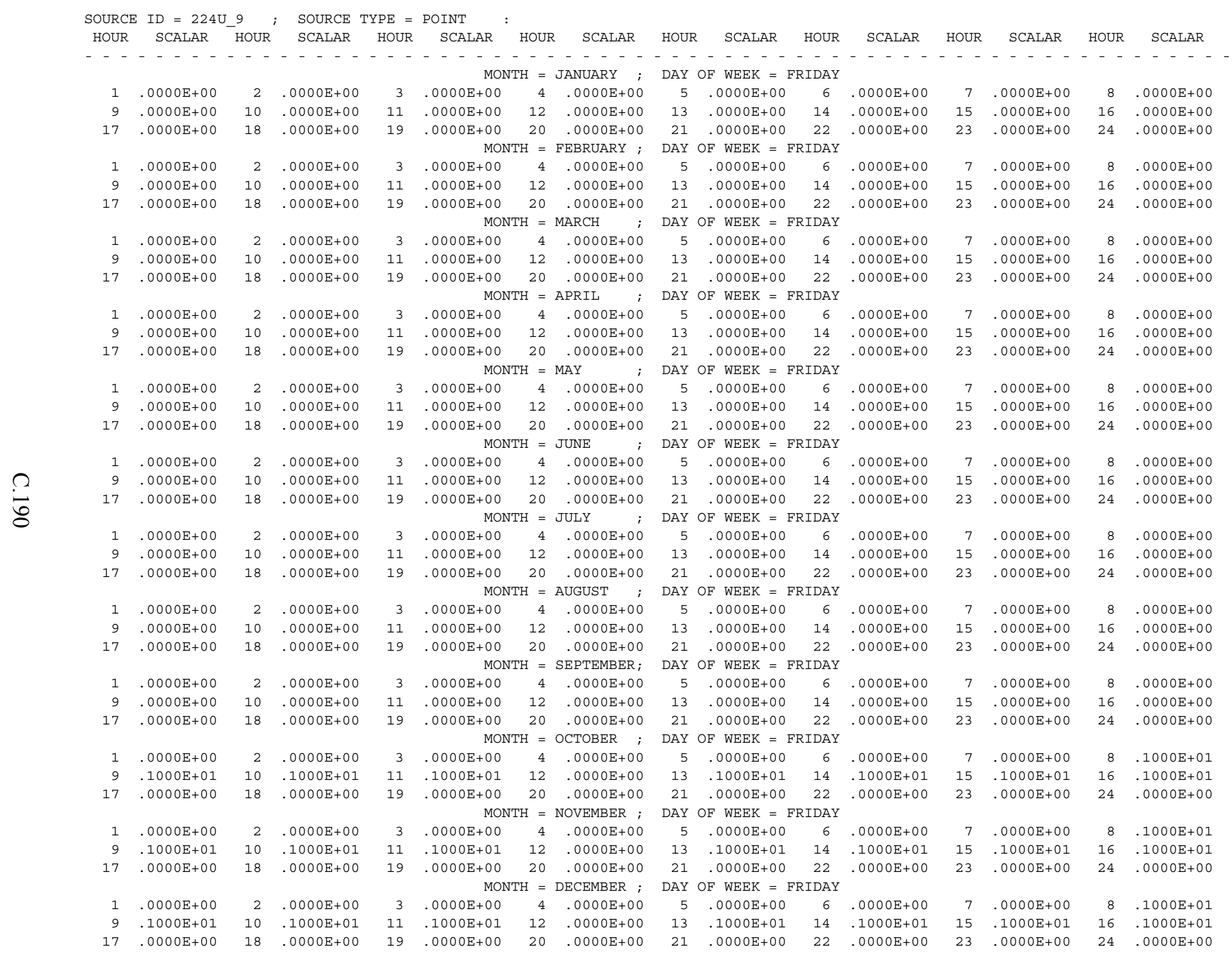




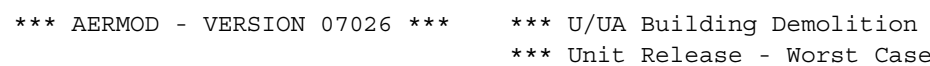
**MODELOPTS :

CONC
$* * * \quad 02 / 09 / 09$

$* * * \quad 10: 41: 11$
PAGE 74

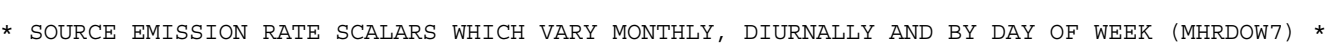

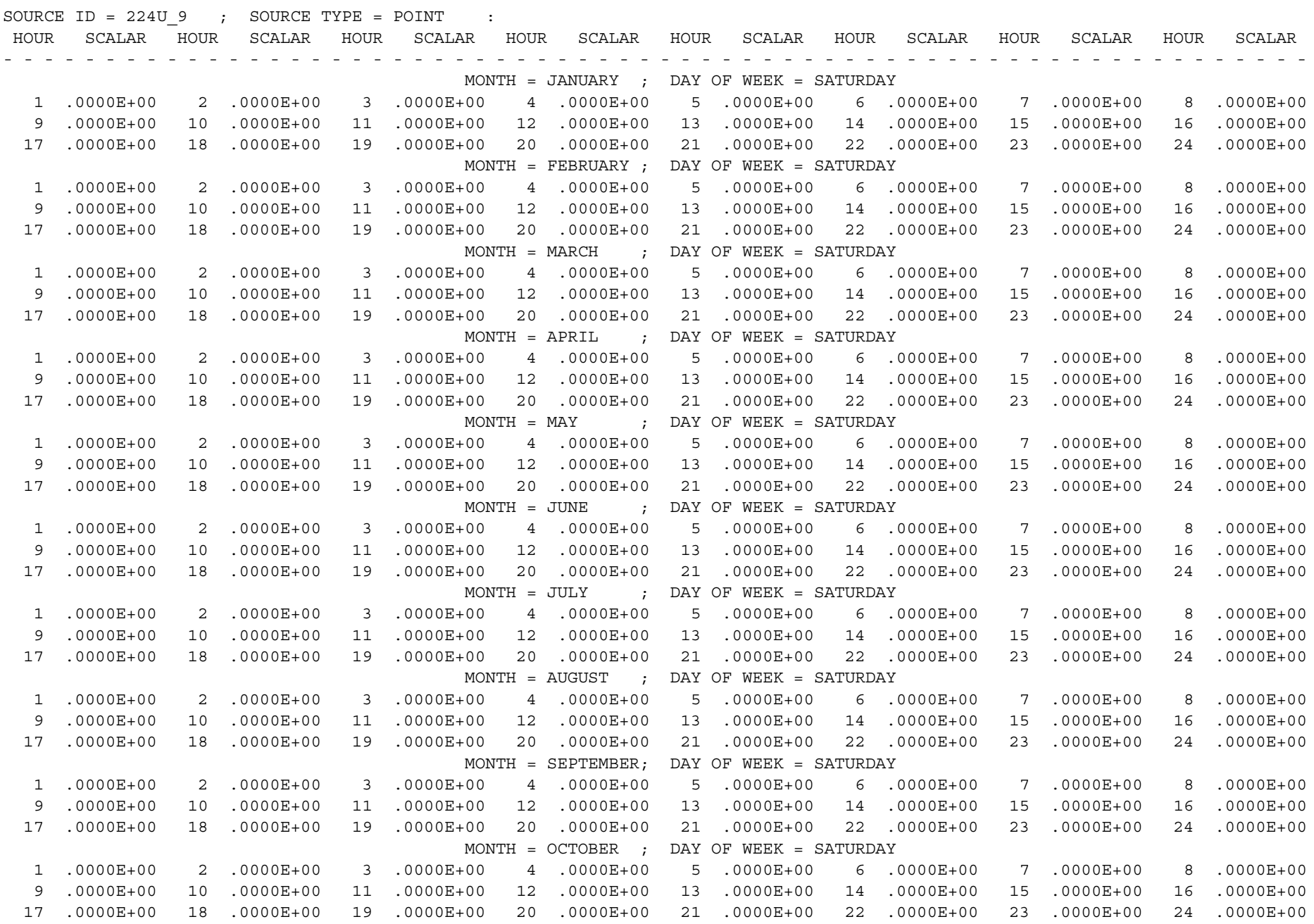

MONTH $=$ NOVEMBER $;$ DAY OF WEEK $=$ SATURDAY 


\begin{tabular}{|c|c|c|c|c|c|c|c|c|c|c|c|c|c|c|c|}
\hline 1 & $.0000 \mathrm{E}+00$ & 2 & $.0000 \mathrm{E}+00$ & 3 & $.0000 \mathrm{E}+00$ & 4 & $.0000 \mathrm{E}+00$ & 5 & $.0000 \mathrm{E}+00$ & 6 & $.0000 \mathrm{E}+00$ & 7 & $.0000 \mathrm{E}+00$ & 8 & $0000 \mathrm{E}+00$ \\
\hline 9 & $.0000 \mathrm{E}+00$ & 10 & $.0000 \mathrm{E}+00$ & 11 & $.0000 \mathrm{E}+00$ & 12 & $.0000 \mathrm{E}+00$ & 13 & $.0000 \mathrm{E}+00$ & 14 & $.0000 \mathrm{E}+00$ & 15 & $.0000 \mathrm{E}+00$ & 16 & $.0000 \mathrm{E}+00$ \\
\hline 17 & $.0000 \mathrm{E}+00$ & 18 & $.0000 \mathrm{E}+00$ & 19 & $.0000 \mathrm{E}+00$ & 20 & $.0000 \mathrm{E}+00$ & 21 & $.0000 \mathrm{E}+00$ & 22 & $.0000 \mathrm{E}+00$ & 23 & $.0000 \mathrm{E}+00$ & 24 & $.0000 \mathrm{E}+00$ \\
\hline & & & & & MOI & & DECEMBER ; & DAY & OF WEEK = & IURI & & & & & \\
\hline 1 & $.0000 \mathrm{E}+00$ & 2 & $.0000 \mathrm{E}+00$ & 3 & $.0000 \mathrm{E}+00$ & 4 & $.0000 \mathrm{E}+00$ & 5 & $.0000 \mathrm{E}+00$ & 6 & $.0000 \mathrm{E}+00$ & 7 & $.0000 \mathrm{E}+00$ & 8 & $.0000 \mathrm{E}+00$ \\
\hline 9 & $.0000 \mathrm{E}+00$ & 10 & $.0000 \mathrm{E}+00$ & 11 & $.0000 \mathrm{E}+00$ & 12 & $.0000 \mathrm{E}+00$ & 13 & $.0000 \mathrm{E}+00$ & 14 & $.0000 \mathrm{E}+00$ & 15 & $.0000 \mathrm{E}+00$ & 16 & $.0000 \mathrm{E}+00$ \\
\hline & $.0000 \mathrm{E}+00$ & 18 & $.0000 \mathrm{E}+00$ & 19 & $.0000 \mathrm{E}+00$ & 20 & $.0000 \mathrm{E}+00$ & 21 & $0000 \mathrm{E}+00$ & 22 & $.0000 \mathrm{E}+00$ & 23 & $.0000 E+00$ & 24 & $0000 E+00$ \\
\hline
\end{tabular}

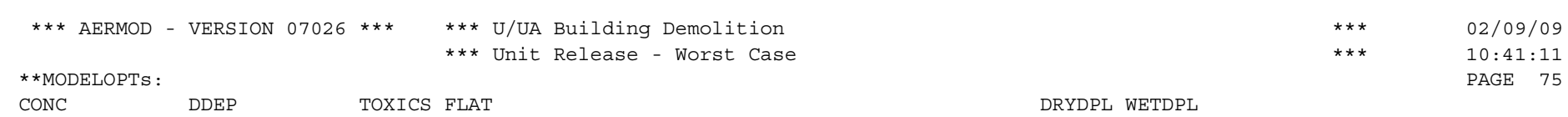

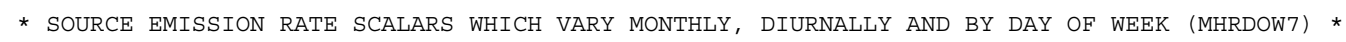

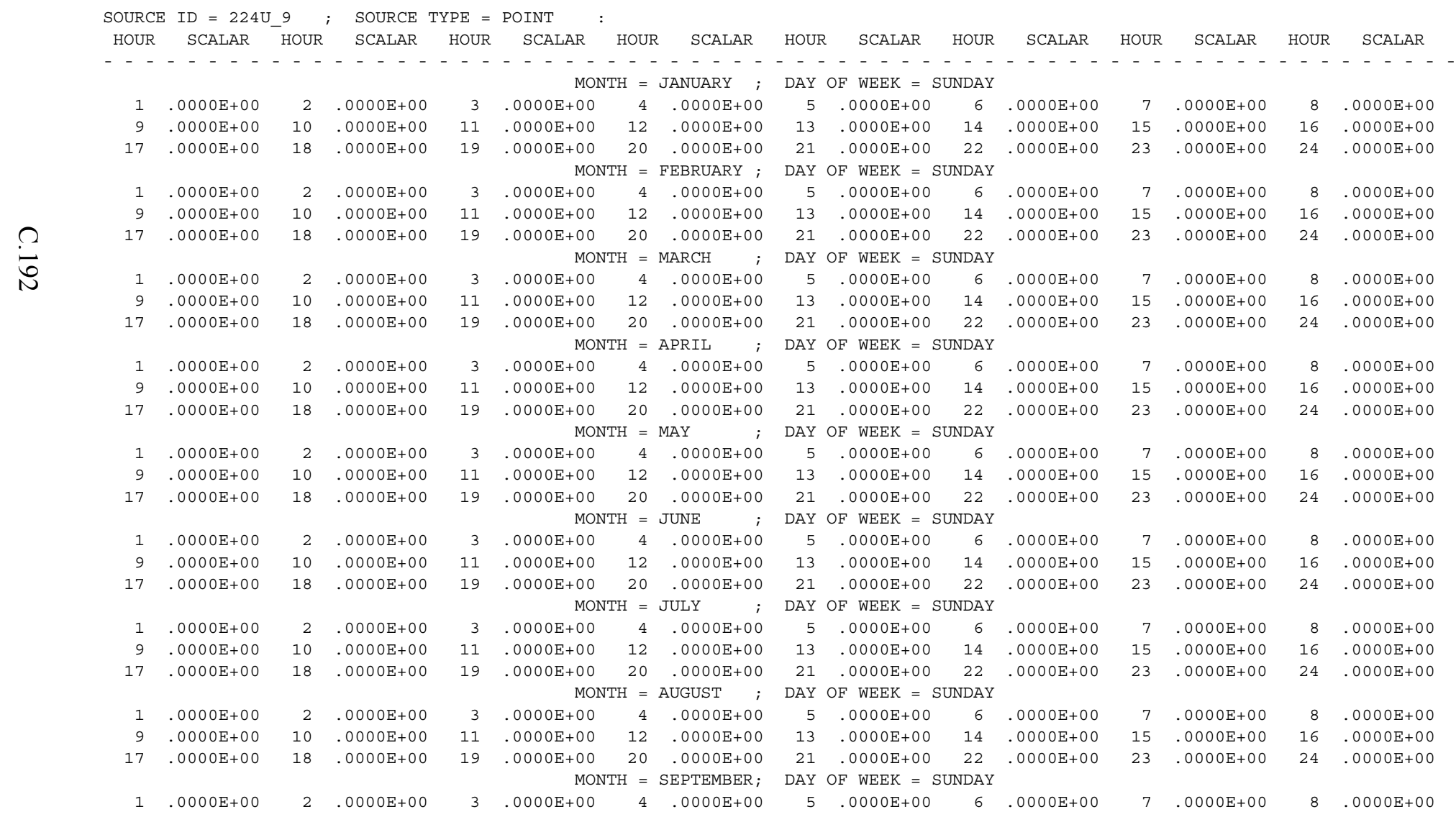




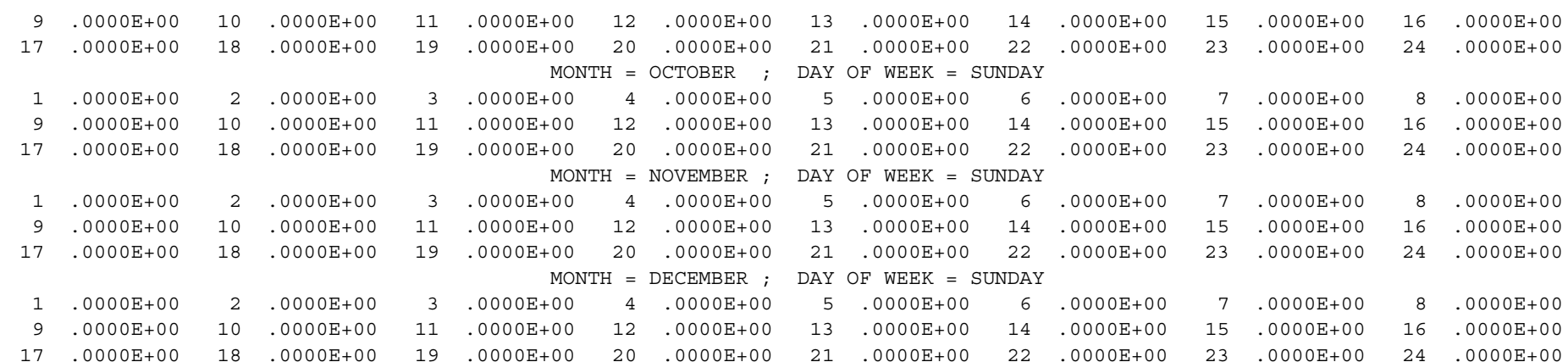

*** AERMOD - VERSION $07026 * * * \quad * * *$ U/UA Building Demolition

*** Unit Release - Worst Case

$\begin{array}{ll}* * * & 02 / 09 / 09 \\ * * * & 10: 41: 11 \\ & \text { PAGE } \quad 76\end{array}$ **MODELOPTS:

CONC

DDEP

TOXICS FLAT

DRYDPL WETDPL

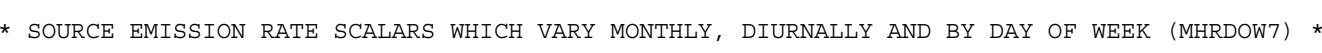

SOURCE ID $=224$ UA_ $1 ;$ SOURCE TYPE $=$ POINT

HOUR SCALAR HOUR SCALAR HOUR SCALAR HOUR SCALAR HOUR SCALAR HOUR SCALAR HOUR SCALAR HOUR SCALAR

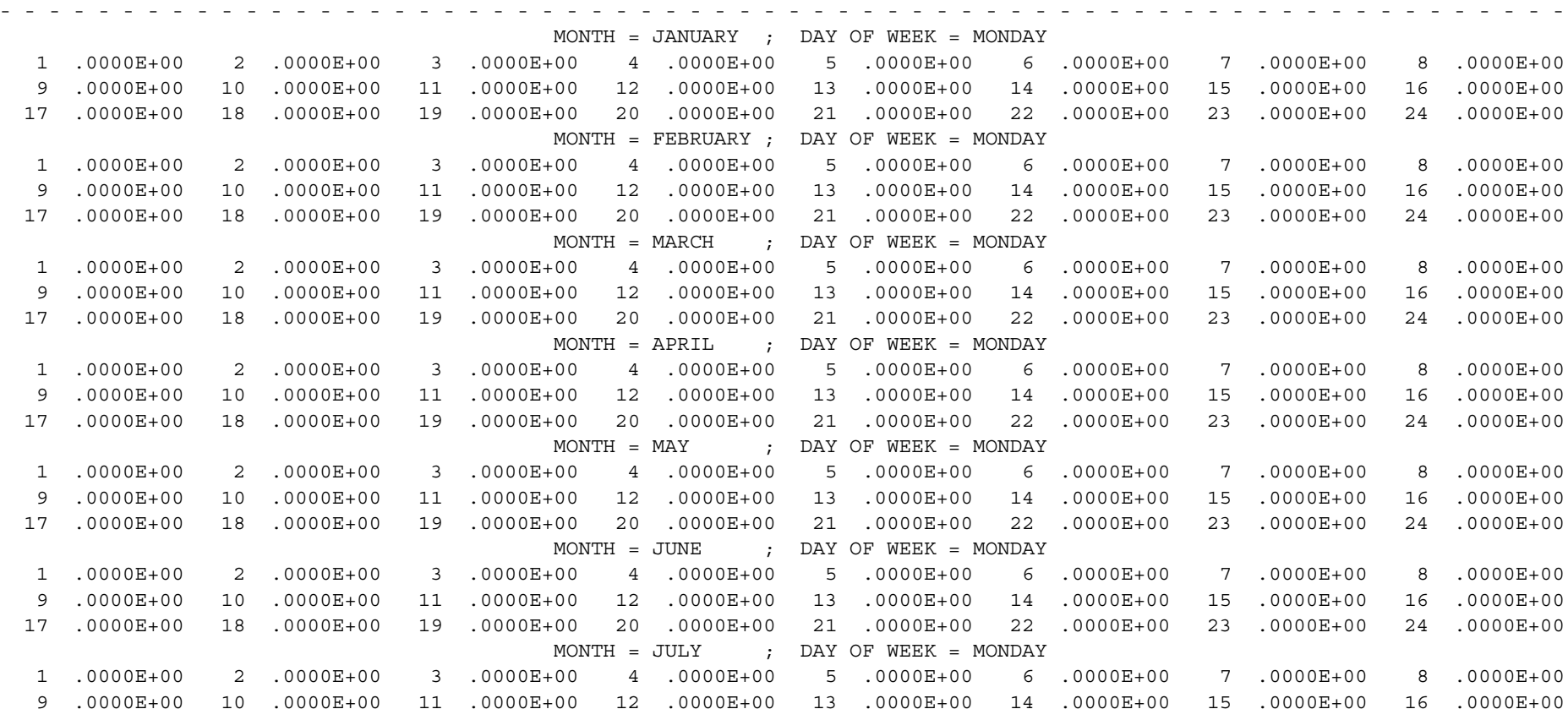




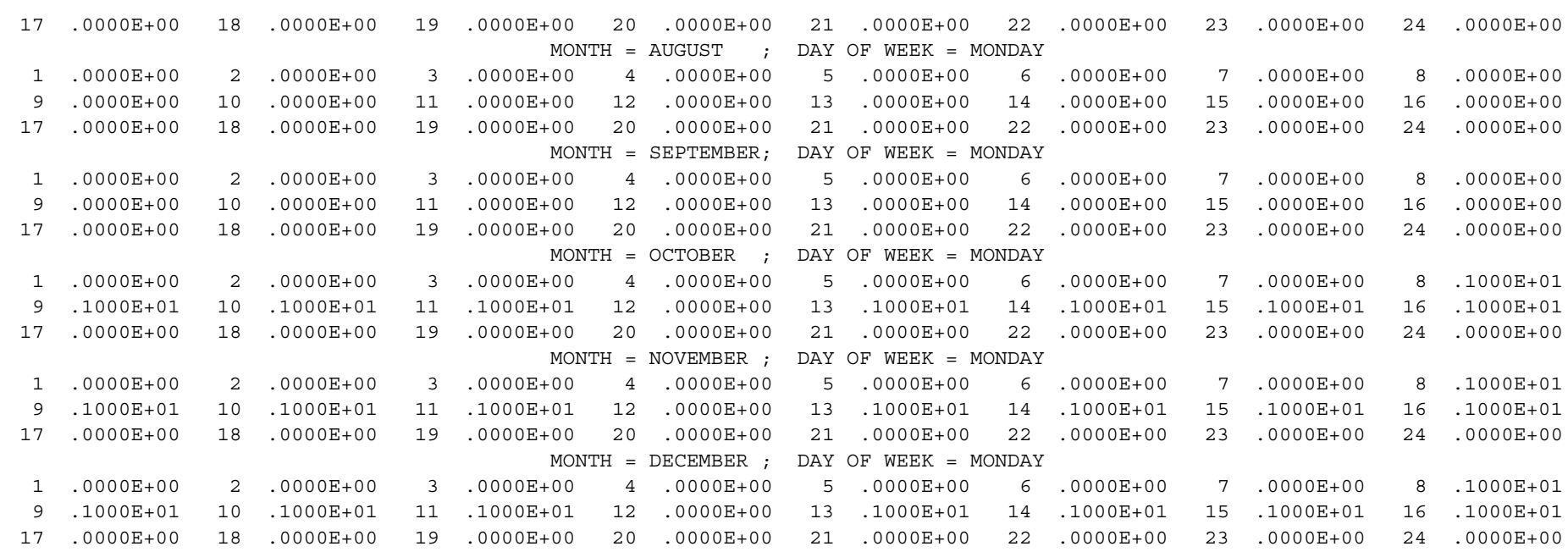

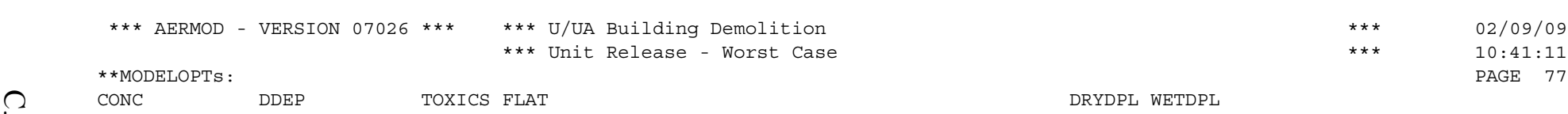

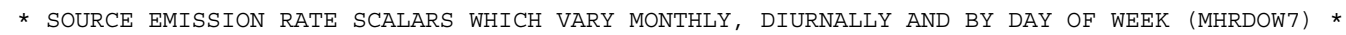

SOURCE ID $=224 \mathrm{UA}_{-} 1 ; \quad$ SOURCE TYPE $=$ POINT

HOUR SCALAR HOUR SCALAR HOUR SCALAR HOUR SCALAR HOUR SCALAR HOUR SCALAR HOUR SCALAR HOUR SCALAR

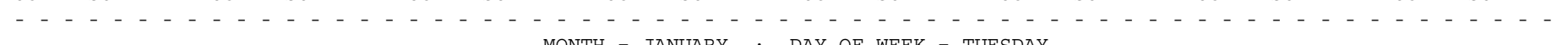

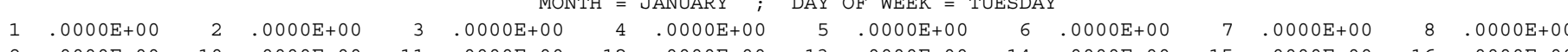

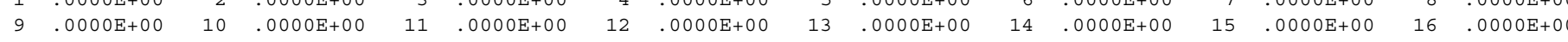

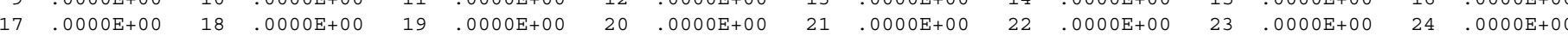

$\begin{array}{rrrrlrlllllllllll}1 & .0000 \mathrm{E}+00 & 2 & .0000 \mathrm{E}+00 & 3 & .0000 \mathrm{E}+00 & 4 & .0000 \mathrm{E}+00 & 5 & .0000 \mathrm{E}+00 & 6 & .0000 \mathrm{E}+00 & 7 & .0000 \mathrm{E}+00 & 8 & .0000 \mathrm{E}+00\end{array}$

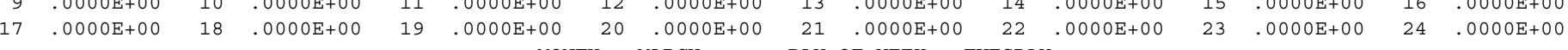

MONTH $=$ MARCH $; \quad$ DAY OF WEEK $=$ TUESDAY

$\begin{array}{rrrrrrrrrrrrrr}1.0000 \mathrm{E}+00 & 8 & .0000 \mathrm{E}+00\end{array}$

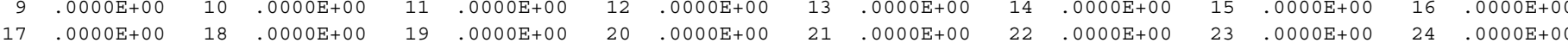

$\begin{array}{llllllllllllllll}\text { MONTH }=\text { APRIL } & ; & \text { DAY OF WEEK }=\text { TUESDAY } & \\ & .0000 \mathrm{E}+00 & 2 & .0000 \mathrm{E}+00 & 3 & .0000 \mathrm{E}+00 & 4 & .0000 \mathrm{E}+00 & 5 & .0000 \mathrm{E}+00 & 6 & .0000 \mathrm{E}+00 & 7 & .0000 \mathrm{E}+00 & 8 & .0000 \mathrm{E}+00\end{array}$

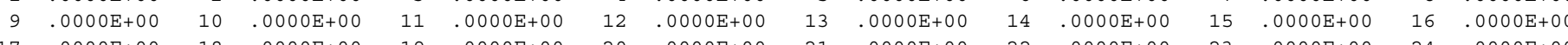

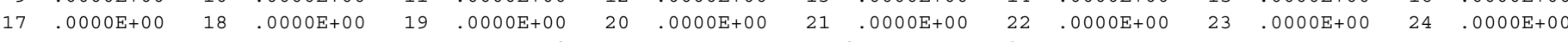

$\begin{array}{llllllcccccccccc}1 & .0000 \mathrm{E}+00 & 2 & .0000 \mathrm{E}+00 & 3 & .0000 \mathrm{E}+00 & 4 & .0000 \mathrm{E}+00 & 5 & .0000 \mathrm{E}+00 & 6 & .0000 \mathrm{E}+00 & 7 & .0000 \mathrm{E}+00 & 8 & .0000 \mathrm{E}+00\end{array}$

$\begin{array}{lllllllllllllll}.0000 \mathrm{E}+00 & 10 & .0000 \mathrm{E}+00 & 11 & .0000 \mathrm{E}+00 & 12 & .0000 \mathrm{E}+00 & 13 & .0000 \mathrm{E}+00 & 14 & .0000 \mathrm{E}+00 & 15 & .0000 \mathrm{E}+00 & 16 & .0000 \mathrm{E}+00\end{array}$

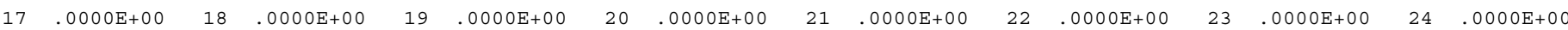




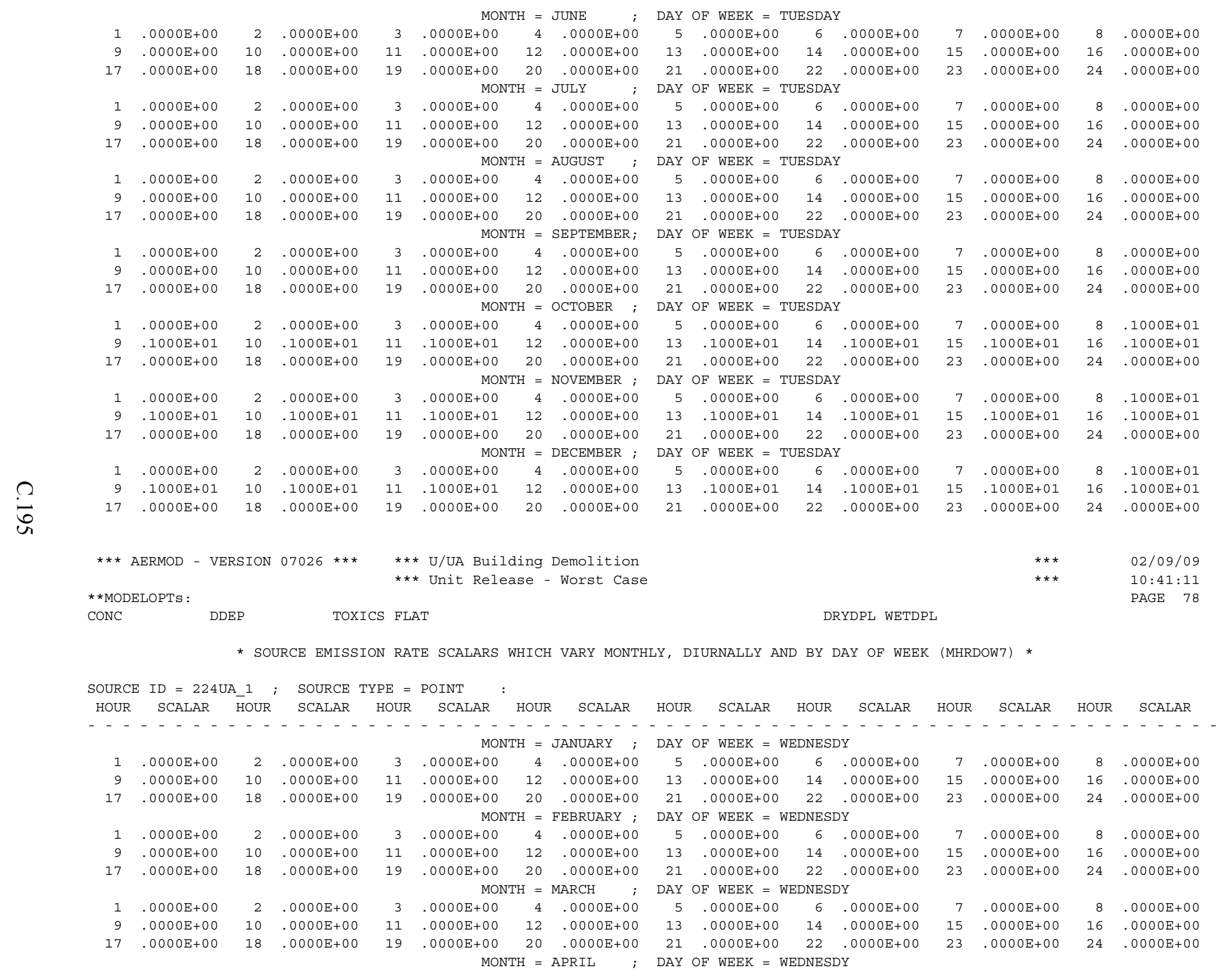




\begin{tabular}{|c|c|c|c|c|c|c|c|c|c|c|c|c|c|c|c|}
\hline 1 & $.0000 \mathrm{E}+00$ & 2 & $.0000 \mathrm{E}+00$ & 3 & $.0000 \mathrm{E}+00$ & 4 & $.0000 \mathrm{E}+00$ & 5 & $.0000 \mathrm{E}+00$ & 6 & $.0000 \mathrm{E}+00$ & 7 & $.0000 \mathrm{E}+00$ & 8 & $.0000 \mathrm{E}+00$ \\
\hline 9 & $.0000 \mathrm{E}+00$ & 10 & $.0000 \mathrm{E}+00$ & 11 & $.0000 \mathrm{E}+00$ & 12 & $.0000 \mathrm{E}+00$ & 13 & $.0000 \mathrm{E}+00$ & 14 & $.0000 \mathrm{E}+00$ & 15 & $.0000 \mathrm{E}+00$ & 16 & $.0000 \mathrm{E}+00$ \\
\hline 17 & $.0000 \mathrm{E}+00$ & 18 & $.0000 \mathrm{E}+00$ & 19 & $.0000 \mathrm{E}+00$ & 20 & $.0000 \mathrm{E}+00$ & 21 & $.0000 \mathrm{E}+00$ & 22 & $.0000 \mathrm{E}+00$ & 23 & $.0000 \mathrm{E}+00$ & 24 & $.0000 \mathrm{E}+00$ \\
\hline & & & & & MON & $\mathrm{H}=$ & MAY ; & DAY & OF WEEK = W & DNESI & & & & & \\
\hline 1 & $.0000 \mathrm{E}+00$ & 2 & $.0000 \mathrm{E}+00$ & 3 & $.0000 \mathrm{E}+00$ & 4 & $.0000 \mathrm{E}+00$ & 5 & $.0000 \mathrm{E}+00$ & 6 & $.0000 \mathrm{E}+00$ & 7 & $.0000 \mathrm{E}+00$ & 8 & $.0000 \mathrm{E}+00$ \\
\hline 9 & $.0000 \mathrm{E}+00$ & 10 & $.0000 \mathrm{E}+00$ & 11 & $.0000 \mathrm{E}+00$ & 12 & $.0000 \mathrm{E}+00$ & 13 & $.0000 \mathrm{E}+00$ & 14 & $.0000 \mathrm{E}+00$ & 15 & $.0000 \mathrm{E}+00$ & 16 & $.0000 \mathrm{E}+00$ \\
\hline 17 & $.0000 \mathrm{E}+00$ & 18 & $.0000 \mathrm{E}+00$ & 19 & $.0000 \mathrm{E}+00$ & 20 & $.0000 \mathrm{E}+00$ & 21 & $.0000 \mathrm{E}+00$ & 22 & $.0000 \mathrm{E}+00$ & 23 & $.0000 \mathrm{E}+00$ & 24 & $.0000 \mathrm{E}+00$ \\
\hline & & & & & MON & $\mathrm{H}=$ & JUNE ; & DAY & OF WEEK $=W$ & DNESI & & & & & \\
\hline 1 & $.0000 \mathrm{E}+00$ & 2 & $.0000 \mathrm{E}+00$ & 3 & $.0000 \mathrm{E}+00$ & 4 & $.0000 \mathrm{E}+00$ & 5 & $.0000 \mathrm{E}+00$ & 6 & $.0000 \mathrm{E}+00$ & 7 & $.0000 \mathrm{E}+00$ & 8 & $.0000 \mathrm{E}+00$ \\
\hline 9 & $.0000 \mathrm{E}+00$ & 10 & $.0000 \mathrm{E}+00$ & 11 & $.0000 \mathrm{E}+00$ & 12 & $.0000 \mathrm{E}+00$ & 13 & $.0000 \mathrm{E}+00$ & 14 & $.0000 \mathrm{E}+00$ & 15 & $.0000 \mathrm{E}+00$ & 16 & $.0000 E+00$ \\
\hline 17 & $.0000 \mathrm{E}+00$ & 18 & $.0000 \mathrm{E}+00$ & 19 & $.0000 \mathrm{E}+00$ & 20 & $.0000 \mathrm{E}+00$ & 21 & $.0000 \mathrm{E}+00$ & 22 & $.0000 \mathrm{E}+00$ & 23 & $.0000 \mathrm{E}+00$ & 24 & $.0000 \mathrm{E}+00$ \\
\hline & & & & & MON & $\mathrm{H}=$ & JULY ; & DAY & OF WEEK = W & DNESI & & & & & \\
\hline 1 & $.0000 \mathrm{E}+00$ & 2 & $.0000 \mathrm{E}+00$ & 3 & $.0000 \mathrm{E}+00$ & 4 & $.0000 \mathrm{E}+00$ & 5 & $.0000 \mathrm{E}+00$ & 6 & $.0000 \mathrm{E}+00$ & 7 & $.0000 \mathrm{E}+00$ & 8 & $.0000 \mathrm{E}+00$ \\
\hline 9 & $.0000 \mathrm{E}+00$ & 10 & $.0000 \mathrm{E}+00$ & 11 & $.0000 \mathrm{E}+00$ & 12 & $.0000 \mathrm{E}+00$ & 13 & $.0000 \mathrm{E}+00$ & 14 & $.0000 \mathrm{E}+00$ & 15 & $.0000 \mathrm{E}+00$ & 16 & $.0000 E+00$ \\
\hline 17 & $.0000 \mathrm{E}+00$ & 18 & $.0000 \mathrm{E}+00$ & 19 & $.0000 \mathrm{E}+00$ & 20 & $.0000 \mathrm{E}+00$ & 21 & $.0000 \mathrm{E}+00$ & 22 & $.0000 \mathrm{E}+00$ & 23 & $.0000 \mathrm{E}+00$ & 24 & $.0000 \mathrm{E}+00$ \\
\hline & & & & & MON & $\mathrm{H}=$ & AUGUST ; & DAY & OF WEEK = W & DNESI & & & & & \\
\hline 1 & $.0000 \mathrm{E}+00$ & 2 & $.0000 \mathrm{E}+00$ & 3 & $.0000 \mathrm{E}+00$ & 4 & $.0000 \mathrm{E}+00$ & 5 & $.0000 \mathrm{E}+00$ & 6 & $.0000 \mathrm{E}+00$ & 7 & $.0000 \mathrm{E}+00$ & 8 & $.0000 \mathrm{E}+00$ \\
\hline 9 & $.0000 \mathrm{E}+00$ & 10 & $.0000 \mathrm{E}+00$ & 11 & $.0000 \mathrm{E}+00$ & 12 & $.0000 \mathrm{E}+00$ & 13 & $.0000 \mathrm{E}+00$ & 14 & $.0000 \mathrm{E}+00$ & 15 & $.0000 \mathrm{E}+00$ & 16 & $.0000 \mathrm{E}+00$ \\
\hline 17 & $.0000 \mathrm{E}+00$ & 18 & $.0000 \mathrm{E}+00$ & 19 & $.0000 \mathrm{E}+00$ & 20 & $.0000 \mathrm{E}+00$ & 21 & $.0000 \mathrm{E}+00$ & 22 & $.0000 \mathrm{E}+00$ & 23 & $.0000 \mathrm{E}+00$ & 24 & $.0000 E+00$ \\
\hline & & & & & MON & $\mathrm{H}=$ & SEPTEMBER; & DAY & OF WEEK = W & DNESD & DY & & & & \\
\hline 1 & $.0000 \mathrm{E}+00$ & 2 & $.0000 \mathrm{E}+00$ & 3 & $.0000 \mathrm{E}+00$ & 4 & $.0000 \mathrm{E}+00$ & 5 & $.0000 \mathrm{E}+00$ & 6 & $.0000 \mathrm{E}+00$ & 7 & $.0000 \mathrm{E}+00$ & 8 & $.0000 \mathrm{E}+00$ \\
\hline 9 & $.0000 \mathrm{E}+00$ & 10 & $.0000 \mathrm{E}+00$ & 11 & $.0000 \mathrm{E}+00$ & 12 & $.0000 \mathrm{E}+00$ & 13 & $.0000 \mathrm{E}+00$ & 14 & $.0000 \mathrm{E}+00$ & 15 & $.0000 \mathrm{E}+00$ & 16 & $.0000 \mathrm{E}+00$ \\
\hline 17 & $.0000 \mathrm{E}+00$ & 18 & $.0000 \mathrm{E}+00$ & 19 & $.0000 \mathrm{E}+00$ & 20 & $.0000 \mathrm{E}+00$ & 21 & $.0000 \mathrm{E}+00$ & 22 & $.0000 \mathrm{E}+00$ & 23 & $.0000 \mathrm{E}+00$ & 24 & $.0000 \mathrm{E}+00$ \\
\hline & & & & & MON & $\mathrm{H}=$ & OCTOBER ; & DAY & OF WEEK = W & DNESD & & & & & \\
\hline 1 & $.0000 \mathrm{E}+00$ & 2 & $.0000 \mathrm{E}+00$ & 3 & $.0000 \mathrm{E}+00$ & 4 & $.0000 \mathrm{E}+00$ & 5 & $.0000 \mathrm{E}+00$ & 6 & $.0000 \mathrm{E}+00$ & 7 & $.0000 \mathrm{E}+00$ & 8 & $.1000 \mathrm{E}+01$ \\
\hline 9 & $.1000 \mathrm{E}+01$ & 10 & $.1000 \mathrm{E}+01$ & 11 & $.1000 \mathrm{E}+01$ & 12 & $.0000 \mathrm{E}+00$ & 13 & $.1000 \mathrm{E}+01$ & 14 & $.1000 \mathrm{E}+01$ & 15 & $.1000 \mathrm{E}+01$ & 16 & $.1000 \mathrm{E}+01$ \\
\hline 17 & $.0000 \mathrm{E}+00$ & 18 & $.0000 \mathrm{E}+00$ & 19 & $.0000 \mathrm{E}+00$ & 20 & $.0000 \mathrm{E}+00$ & 21 & $.0000 \mathrm{E}+00$ & 22 & $.0000 E+00$ & 23 & $.0000 \mathrm{E}+00$ & 24 & $.0000 \mathrm{E}+00$ \\
\hline & & & & & MON & $\mathrm{H}=$ & NOVEMBER ; & DAY & OF WEEK = W & DNESL & & & & & \\
\hline 1 & $.0000 \mathrm{E}+00$ & 2 & $.0000 \mathrm{E}+00$ & 3 & $.0000 \mathrm{E}+00$ & 4 & $.0000 \mathrm{E}+00$ & 5 & $.0000 \mathrm{E}+00$ & 6 & $.0000 \mathrm{E}+00$ & 7 & $.0000 E+00$ & 8 & $.1000 \mathrm{E}+01$ \\
\hline 9 & $.1000 \mathrm{E}+01$ & 10 & $.1000 \mathrm{E}+01$ & 11 & $.1000 \mathrm{E}+01$ & 12 & $.0000 \mathrm{E}+00$ & 13 & $.1000 \mathrm{E}+01$ & 14 & $.1000 \mathrm{E}+01$ & 15 & $.1000 \mathrm{E}+01$ & 16 & $.1000 \mathrm{E}+01$ \\
\hline 17 & $.0000 \mathrm{E}+00$ & 18 & $.0000 \mathrm{E}+00$ & 19 & $.0000 \mathrm{E}+00$ & 20 & $.0000 \mathrm{E}+00$ & 21 & $.0000 \mathrm{E}+00$ & 22 & $.0000 \mathrm{E}+00$ & 23 & $.0000 \mathrm{E}+00$ & 24 & $.0000 \mathrm{E}+00$ \\
\hline & & & & & MON & 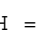 & DECEMBER ; & DAY & OF WEEK = & DNESD & & & & & \\
\hline 1 & $.0000 \mathrm{E}+00$ & 2 & $.0000 \mathrm{E}+00$ & 3 & $.0000 \mathrm{E}+00$ & 4 & $.0000 \mathrm{E}+00$ & 5 & $.0000 \mathrm{E}+00$ & 6 & $.0000 \mathrm{E}+00$ & 7 & $.0000 \mathrm{E}+00$ & 8 & $.1000 \mathrm{E}+01$ \\
\hline 9 & $.1000 \mathrm{E}+01$ & 10 & $.1000 \mathrm{E}+01$ & 11 & $.1000 \mathrm{E}+01$ & 12 & $.0000 \mathrm{E}+00$ & 13 & $.1000 \mathrm{E}+01$ & 14 & $.1000 \mathrm{E}+01$ & 15 & $.1000 \mathrm{E}+01$ & 16 & $.1000 \mathrm{E}+01$ \\
\hline 17 & $.0000 \mathrm{E}+00$ & 18 & $.0000 \mathrm{E}+00$ & 19 & $.0000 \mathrm{E}+00$ & 20 & $.0000 \mathrm{E}+00$ & 21 & $.0000 \mathrm{E}+00$ & 22 & $.0000 \mathrm{E}+00$ & 23 & $.0000 \mathrm{E}+00$ & 24 & $.0000 \mathrm{E}+00$ \\
\hline
\end{tabular}

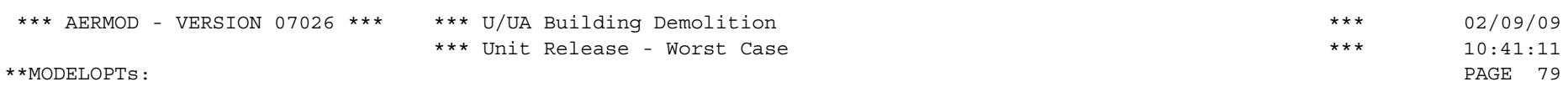

DDEP TOXICS FLAT

DRYDPL WETDPL

* SOURCE EMISSION RATE SCALARS WHICH VARY MONTHLY, DIURNALLY AND BY DAY OF WEEK (MHRDOW7) *

SOURCE ID $=224$ UA_1 $;$ SOURCE TYPE $=$ POINT

HOUR SCALAR HOUR SCALAR HOUR SCALAR HOUR SCALAR HOUR SCALAR HOUR SCALAR HOUR SCALAR HOUR SCALAR

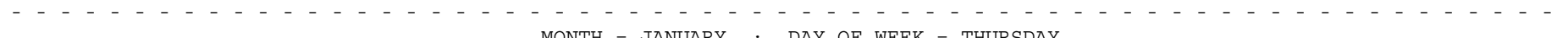

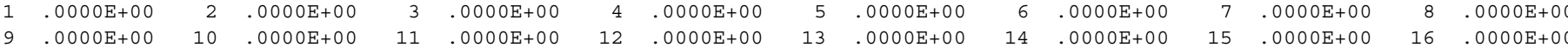

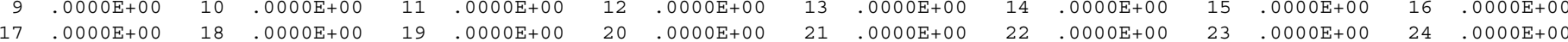

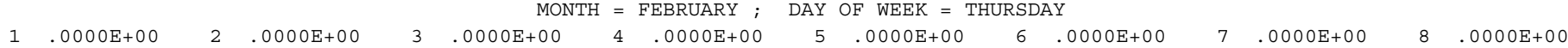




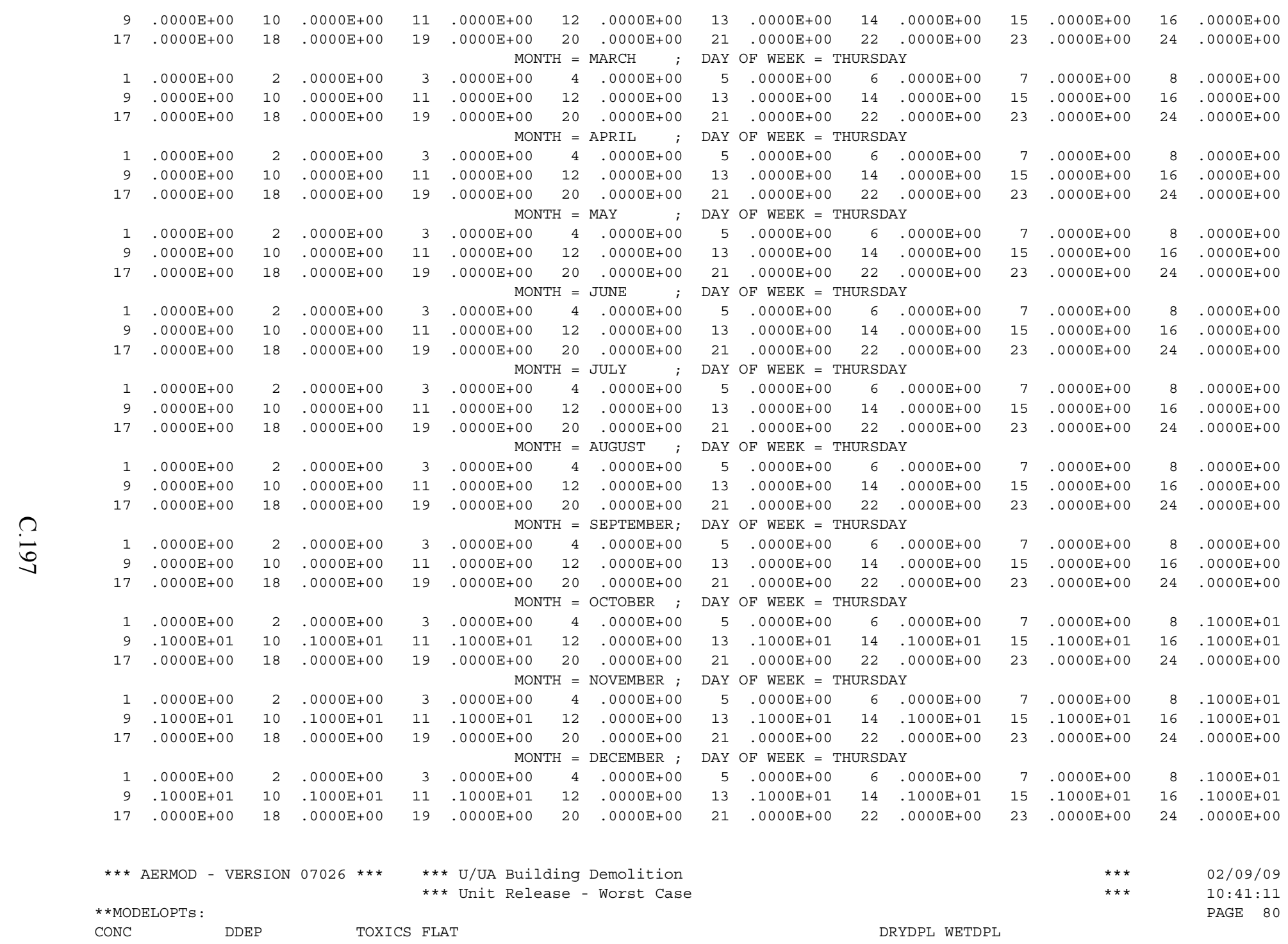

* source emission rate scalars which vary monthly, diURnally and by day of Week (MHrdow7) *

SOURCE ID $=$ 224UA_1 $;$ SOURCE TYPE $=$ POINT

HOUR SCALAR HOUR SCALAR HOUR SCALAR HOUR SCALAR HOUR SCALAR HOUR SCALAR HOUR SCALAR HOUR SCALAR 


\begin{tabular}{|c|c|c|c|c|c|c|c|c|c|c|c|c|c|c|c|}
\hline & & & & & & & JANUARY ; & & $F \quad W E E K=F$ & FRIDAY & & & & & \\
\hline 1 & $.0000 \mathrm{E}+00$ & 2 & $.0000 \mathrm{E}+00$ & 3 & $.0000 \mathrm{E}+00$ & 4 & $.0000 \mathrm{E}+00$ & 5 & $.0000 \mathrm{E}+00$ & 6 & $.0000 \mathrm{E}+00$ & 7 & $.0000 \mathrm{E}+00$ & 8 & $.0000 \mathrm{E}+00$ \\
\hline 9 & $.0000 E+00$ & 10 & $.0000 E+00$ & 11 & $.0000 \mathrm{E}+00$ & 12 & $.0000 \mathrm{E}+00$ & 13 & $.0000 \mathrm{E}+00$ & 14 & $.0000 \mathrm{E}+00$ & 15 & $.0000 \mathrm{E}+00$ & 16 & $.0000 \mathrm{E}+00$ \\
\hline 17 & $.0000 \mathrm{E}+00$ & 18 & $.0000 \mathrm{E}+00$ & 19 & $.0000 \mathrm{E}+00$ & 20 & $.0000 \mathrm{E}+00$ & 21 & $.0000 \mathrm{E}+00$ & 22 & $.0000 \mathrm{E}+00$ & 23 & $.0000 \mathrm{E}+00$ & 24 & $.0000 \mathrm{E}+00$ \\
\hline & & & & & MON & $\Gamma \mathrm{H}=$ & FEBRUARY ; & DAY & OF $\mathrm{WEEK}=\mathrm{F}$ & FRIDAY & & & & & \\
\hline 1 & $.0000 \mathrm{E}+00$ & 2 & $.0000 \mathrm{E}+00$ & 3 & $.0000 \mathrm{E}+00$ & 4 & $.0000 \mathrm{E}+00$ & 5 & $.0000 \mathrm{E}+00$ & 6 & $.0000 \mathrm{E}+00$ & 7 & $.0000 \mathrm{E}+00$ & 8 & $.0000 \mathrm{E}+00$ \\
\hline 9 & $.0000 \mathrm{E}+00$ & 10 & $.0000 \mathrm{E}+00$ & 11 & $.0000 \mathrm{E}+00$ & 12 & $.0000 \mathrm{E}+00$ & 13 & $.0000 \mathrm{E}+00$ & 14 & $.0000 \mathrm{E}+00$ & 15 & $.0000 \mathrm{E}+00$ & 16 & $.0000 \mathrm{E}+00$ \\
\hline 17 & $.0000 \mathrm{E}+00$ & 18 & $.0000 \mathrm{E}+00$ & 19 & $.0000 \mathrm{E}+00$ & 20 & $.0000 \mathrm{E}+00$ & 21 & $.0000 \mathrm{E}+00$ & 22 & $.0000 \mathrm{E}+00$ & 23 & $.0000 \mathrm{E}+00$ & 24 & $.0000 \mathrm{E}+00$ \\
\hline & & & & & MON & $\Gamma \mathrm{H}=$ & MARCH ; & DAY & OF WEEK $=F$ & FRIDAY & & & & & \\
\hline 1 & $.0000 \mathrm{E}+00$ & 2 & $.0000 \mathrm{E}+00$ & 3 & $.0000 \mathrm{E}+00$ & 4 & $.0000 \mathrm{E}+00$ & 5 & $.0000 \mathrm{E}+00$ & 6 & $.0000 \mathrm{E}+00$ & 7 & $.0000 \mathrm{E}+00$ & 8 & $.0000 \mathrm{E}+00$ \\
\hline 9 & $.0000 \mathrm{E}+00$ & 10 & $.0000 \mathrm{E}+00$ & 11 & $.0000 \mathrm{E}+00$ & 12 & $.0000 \mathrm{E}+00$ & 13 & $.0000 \mathrm{E}+00$ & 14 & $.0000 \mathrm{E}+00$ & 15 & $.0000 \mathrm{E}+00$ & 16 & $.0000 \mathrm{E}+00$ \\
\hline 17 & $.0000 \mathrm{E}+00$ & 18 & $.0000 \mathrm{E}+00$ & 19 & $.0000 \mathrm{E}+00$ & 20 & $.0000 \mathrm{E}+00$ & 21 & $.0000 \mathrm{E}+00$ & 22 & $.0000 \mathrm{E}+00$ & 23 & $.0000 \mathrm{E}+00$ & 24 & $.0000 \mathrm{E}+00$ \\
\hline & & & & & MON & $\Gamma \mathrm{H}=$ & APRIL ; & DAY & OF WEEK $=F$ & FRIDAY & & & & & \\
\hline 1 & $.0000 \mathrm{E}+00$ & 2 & $.0000 \mathrm{E}+00$ & 3 & $.0000 \mathrm{E}+00$ & 4 & $.0000 \mathrm{E}+00$ & 5 & $.0000 \mathrm{E}+00$ & 6 & $.0000 \mathrm{E}+00$ & 7 & $.0000 \mathrm{E}+00$ & 8 & $.0000 \mathrm{E}+00$ \\
\hline 9 & $.0000 \mathrm{E}+00$ & 10 & $.0000 \mathrm{E}+00$ & 11 & $.0000 \mathrm{E}+00$ & 12 & $.0000 \mathrm{E}+00$ & 13 & $.0000 \mathrm{E}+00$ & 14 & $.0000 \mathrm{E}+00$ & 15 & $.0000 \mathrm{E}+00$ & 16 & $.0000 \mathrm{E}+00$ \\
\hline 17 & $.0000 \mathrm{E}+00$ & 18 & $.0000 \mathrm{E}+00$ & 19 & $.0000 \mathrm{E}+00$ & 20 & $.0000 \mathrm{E}+00$ & 21 & $.0000 \mathrm{E}+00$ & 22 & $.0000 \mathrm{E}+00$ & 23 & $.0000 \mathrm{E}+00$ & 24 & $.0000 \mathrm{E}+00$ \\
\hline & & & & & MON & $\mathrm{TH}=$ & MAY ; & DAY & OF WEEK = F & FRIDAY & & & & & \\
\hline 1 & $.0000 \mathrm{E}+00$ & 2 & $.0000 \mathrm{E}+00$ & 3 & $.0000 \mathrm{E}+00$ & 4 & $.0000 \mathrm{E}+00$ & 5 & $.0000 \mathrm{E}+00$ & 6 & $.0000 \mathrm{E}+00$ & 7 & $.0000 \mathrm{E}+00$ & 8 & $.0000 \mathrm{E}+00$ \\
\hline 9 & $.0000 \mathrm{E}+00$ & 10 & $.0000 \mathrm{E}+00$ & 11 & $.0000 \mathrm{E}+00$ & 12 & $.0000 \mathrm{E}+00$ & 13 & $.0000 \mathrm{E}+00$ & 14 & $.0000 \mathrm{E}+00$ & 15 & $.0000 \mathrm{E}+00$ & 16 & $.0000 \mathrm{E}+00$ \\
\hline 17 & $.0000 \mathrm{E}+00$ & 18 & $.0000 \mathrm{E}+00$ & 19 & $.0000 \mathrm{E}+00$ & 20 & $.0000 \mathrm{E}+00$ & 21 & $.0000 \mathrm{E}+00$ & 22 & $.0000 \mathrm{E}+00$ & 23 & $.0000 \mathrm{E}+00$ & 24 & $.0000 \mathrm{E}+00$ \\
\hline & & & & & MON & $\mathrm{TH}=$ & JUNE ; & DAY & OF WEEK = F & FRIDAY & & & & & \\
\hline 1 & $.0000 \mathrm{E}+00$ & 2 & $.0000 \mathrm{E}+00$ & 3 & $.0000 \mathrm{E}+00$ & 4 & $.0000 \mathrm{E}+00$ & 5 & $.0000 \mathrm{E}+00$ & 6 & $.0000 \mathrm{E}+00$ & 7 & $.0000 \mathrm{E}+00$ & 8 & $.0000 \mathrm{E}+00$ \\
\hline 9 & $.0000 \mathrm{E}+00$ & 10 & $.0000 \mathrm{E}+00$ & 11 & $.0000 \mathrm{E}+00$ & 12 & $.0000 \mathrm{E}+00$ & 13 & $.0000 \mathrm{E}+00$ & 14 & $.0000 \mathrm{E}+00$ & 15 & $.0000 \mathrm{E}+00$ & 16 & $.0000 \mathrm{E}+00$ \\
\hline 17 & $.0000 \mathrm{E}+00$ & 18 & $.0000 \mathrm{E}+00$ & 19 & $.0000 \mathrm{E}+00$ & 20 & $.0000 \mathrm{E}+00$ & 21 & $.0000 \mathrm{E}+00$ & 22 & $.0000 \mathrm{E}+00$ & 23 & $.0000 \mathrm{E}+00$ & 24 & $.0000 \mathrm{E}+00$ \\
\hline & & & & & MON & $\Gamma \mathrm{H}=$ & JULY ; & DAY & OF WEEK = F & FRIDAY & & & & & \\
\hline 1 & $.0000 \mathrm{E}+00$ & 2 & $.0000 \mathrm{E}+00$ & 3 & $.0000 \mathrm{E}+00$ & 4 & $.0000 \mathrm{E}+00$ & 5 & $.0000 \mathrm{E}+00$ & 6 & $.0000 \mathrm{E}+00$ & 7 & $.0000 \mathrm{E}+00$ & 8 & $.0000 \mathrm{E}+00$ \\
\hline 9 & $.0000 \mathrm{E}+00$ & 10 & $.0000 \mathrm{E}+00$ & 11 & $.0000 \mathrm{E}+00$ & 12 & $.0000 \mathrm{E}+00$ & 13 & $.0000 \mathrm{E}+00$ & 14 & $.0000 \mathrm{E}+00$ & 15 & $.0000 \mathrm{E}+00$ & 16 & $.0000 \mathrm{E}+00$ \\
\hline 17 & $.0000 \mathrm{E}+00$ & 18 & $.0000 \mathrm{E}+00$ & 19 & $.0000 \mathrm{E}+00$ & 20 & $.0000 \mathrm{E}+00$ & 21 & $.0000 \mathrm{E}+00$ & 22 & $.0000 \mathrm{E}+00$ & 23 & $.0000 \mathrm{E}+00$ & 24 & $.0000 \mathrm{E}+00$ \\
\hline & & & & & MON & $\Gamma \mathrm{H}=$ & AUGUST ; & DAY & OF WEEK $=F$ & FRIDAY & & & & & \\
\hline 1 & $.0000 \mathrm{E}+00$ & 2 & $.0000 \mathrm{E}+00$ & 3 & $.0000 \mathrm{E}+00$ & 4 & $.0000 \mathrm{E}+00$ & 5 & $.0000 \mathrm{E}+00$ & 6 & $.0000 \mathrm{E}+00$ & 7 & $.0000 \mathrm{E}+00$ & 8 & $.0000 \mathrm{E}+00$ \\
\hline 9 & $.0000 \mathrm{E}+00$ & 10 & $.0000 \mathrm{E}+00$ & 11 & $.0000 \mathrm{E}+00$ & 12 & $.0000 \mathrm{E}+00$ & 13 & $.0000 \mathrm{E}+00$ & 14 & $.0000 \mathrm{E}+00$ & 15 & $.0000 \mathrm{E}+00$ & 16 & $.0000 \mathrm{E}+00$ \\
\hline 17 & $.0000 \mathrm{E}+00$ & 18 & $.0000 \mathrm{E}+00$ & 19 & $.0000 \mathrm{E}+00$ & 20 & $.0000 \mathrm{E}+00$ & 21 & $.0000 \mathrm{E}+00$ & 22 & $.0000 \mathrm{E}+00$ & 23 & $.0000 \mathrm{E}+00$ & 24 & $.0000 \mathrm{E}+00$ \\
\hline & & & & & MON & $\Gamma \mathrm{H}=$ & SEPTEMBER ; & DAY & OF WEEK = F & FRIDAY & & & & & \\
\hline 1 & $.0000 \mathrm{E}+00$ & 2 & $.0000 \mathrm{E}+00$ & 3 & $.0000 \mathrm{E}+00$ & 4 & $.0000 \mathrm{E}+00$ & 5 & $.0000 \mathrm{E}+00$ & 6 & $.0000 \mathrm{E}+00$ & 7 & $.0000 \mathrm{E}+00$ & 8 & $.0000 \mathrm{E}+00$ \\
\hline 9 & $.0000 \mathrm{E}+00$ & 10 & $.0000 \mathrm{E}+00$ & 11 & $.0000 \mathrm{E}+00$ & 12 & $.0000 \mathrm{E}+00$ & 13 & $.0000 \mathrm{E}+00$ & 14 & $.0000 \mathrm{E}+00$ & 15 & $.0000 \mathrm{E}+00$ & 16 & $.0000 \mathrm{E}+00$ \\
\hline 17 & $.0000 \mathrm{E}+00$ & 18 & $.0000 \mathrm{E}+00$ & 19 & $.0000 \mathrm{E}+00$ & 20 & $.0000 \mathrm{E}+00$ & 21 & $.0000 \mathrm{E}+00$ & 22 & $.0000 \mathrm{E}+00$ & 23 & $.0000 \mathrm{E}+00$ & 24 & $.0000 \mathrm{E}+00$ \\
\hline & & & & & MON & $\Gamma \mathrm{H}=$ & OCTOBER ; & DAY & OF WEEK $=F$ & FRIDAY & & & & & \\
\hline 1 & $.0000 \mathrm{E}+00$ & 2 & $.0000 \mathrm{E}+00$ & 3 & $.0000 \mathrm{E}+00$ & 4 & $.0000 \mathrm{E}+00$ & 5 & $.0000 \mathrm{E}+00$ & 6 & $.0000 \mathrm{E}+00$ & 7 & $.0000 \mathrm{E}+00$ & 8 & $.1000 \mathrm{E}+01$ \\
\hline 9 & $.1000 \mathrm{E}+01$ & 10 & $.1000 \mathrm{E}+01$ & 11 & $.1000 \mathrm{E}+01$ & 12 & $.0000 \mathrm{E}+00$ & 13 & $.1000 \mathrm{E}+01$ & 14 & $.1000 \mathrm{E}+01$ & 15 & $.1000 \mathrm{E}+01$ & 16 & $.1000 \mathrm{E}+01$ \\
\hline 17 & $.0000 \mathrm{E}+00$ & 18 & $.0000 \mathrm{E}+00$ & 19 & $.0000 \mathrm{E}+00$ & 20 & $.0000 \mathrm{E}+00$ & 21 & $.0000 \mathrm{E}+00$ & 22 & $.0000 \mathrm{E}+00$ & 23 & $.0000 \mathrm{E}+00$ & 24 & $.0000 \mathrm{E}+00$ \\
\hline & & & & & MON & $\Gamma \mathrm{H}=$ & NOVEMBER ; & DAY & OF WEEK = F & FRIDAY & & & & & \\
\hline 1 & $.0000 \mathrm{E}+00$ & 2 & $.0000 \mathrm{E}+00$ & 3 & $.0000 \mathrm{E}+00$ & 4 & $.0000 \mathrm{E}+00$ & 5 & $.0000 \mathrm{E}+00$ & 6 & $.0000 \mathrm{E}+00$ & 7 & $.0000 \mathrm{E}+00$ & 8 & $.1000 \mathrm{E}+01$ \\
\hline 9 & $.1000 \mathrm{E}+01$ & 10 & $.1000 \mathrm{E}+01$ & 11 & $.1000 \mathrm{E}+01$ & 12 & $.0000 \mathrm{E}+00$ & 13 & $.1000 \mathrm{E}+01$ & 14 & $.1000 \mathrm{E}+01$ & 15 & $.1000 \mathrm{E}+01$ & 16 & $.1000 \mathrm{E}+01$ \\
\hline 17 & $.0000 \mathrm{E}+00$ & 18 & $.0000 \mathrm{E}+00$ & 19 & $.0000 \mathrm{E}+00$ & 20 & $.0000 \mathrm{E}+00$ & 21 & $.0000 \mathrm{E}+00$ & 22 & $.0000 \mathrm{E}+00$ & 23 & $.0000 \mathrm{E}+00$ & 24 & $.0000 \mathrm{E}+00$ \\
\hline & & & & & MON & $\mathrm{H}=$ & DECEMBER ; & DAY & OF WEEK $=F$ & FRIDAY & & & & & \\
\hline 1 & $.0000 \mathrm{E}+00$ & 2 & $.0000 \mathrm{E}+00$ & 3 & $.0000 \mathrm{E}+00$ & 4 & $.0000 \mathrm{E}+00$ & 5 & $.0000 \mathrm{E}+00$ & 6 & $.0000 \mathrm{E}+00$ & 7 & $.0000 \mathrm{E}+00$ & 8 & $.1000 \mathrm{E}+01$ \\
\hline 9 & $.1000 \mathrm{E}+01$ & 10 & $.1000 \mathrm{E}+01$ & 11 & $.1000 \mathrm{E}+01$ & 12 & $.0000 \mathrm{E}+00$ & 13 & $.1000 \mathrm{E}+01$ & 14 & $.1000 \mathrm{E}+01$ & 15 & $.1000 \mathrm{E}+01$ & 16 & $.1000 \mathrm{E}+01$ \\
\hline 17 & $.0000 \mathrm{E}+00$ & 18 & $.0000 \mathrm{E}+00$ & 19 & $.0000 \mathrm{E}+00$ & 20 & $.0000 \mathrm{E}+00$ & 21 & $.0000 \mathrm{E}+00$ & 22 & $.0000 \mathrm{E}+00$ & 23 & $.0000 \mathrm{E}+00$ & 24 & $.0000 \mathrm{E}+00$ \\
\hline
\end{tabular}

*** AERMOD - VERSION $07026 * * * \quad * * *$ U/UA Building Demolition

*** Unit Release - Worst Case 
* SOURCE EMISSION RATE SCALARS WHICH VARY MONTHLY, DIURNALLY AND BY DAY OF WEEK (MHRDOW7) *

SOURCE ID $=$ 224UA $1 ;$ SOURCE TYPE $=$ POINT

HOUR SCALAR HOUR SCALAR HOUR SCALAR HOUR SCALAR HOUR SCALAR HOUR SCALAR HOUR SCALAR HOUR SCALAR HOUR SCALAR HOUR SCALAR HOUR SCALAR HOUR SCALAR HOUR SCALAR HOUR SCALAR HOUR SCALAR HOUR SCALAR

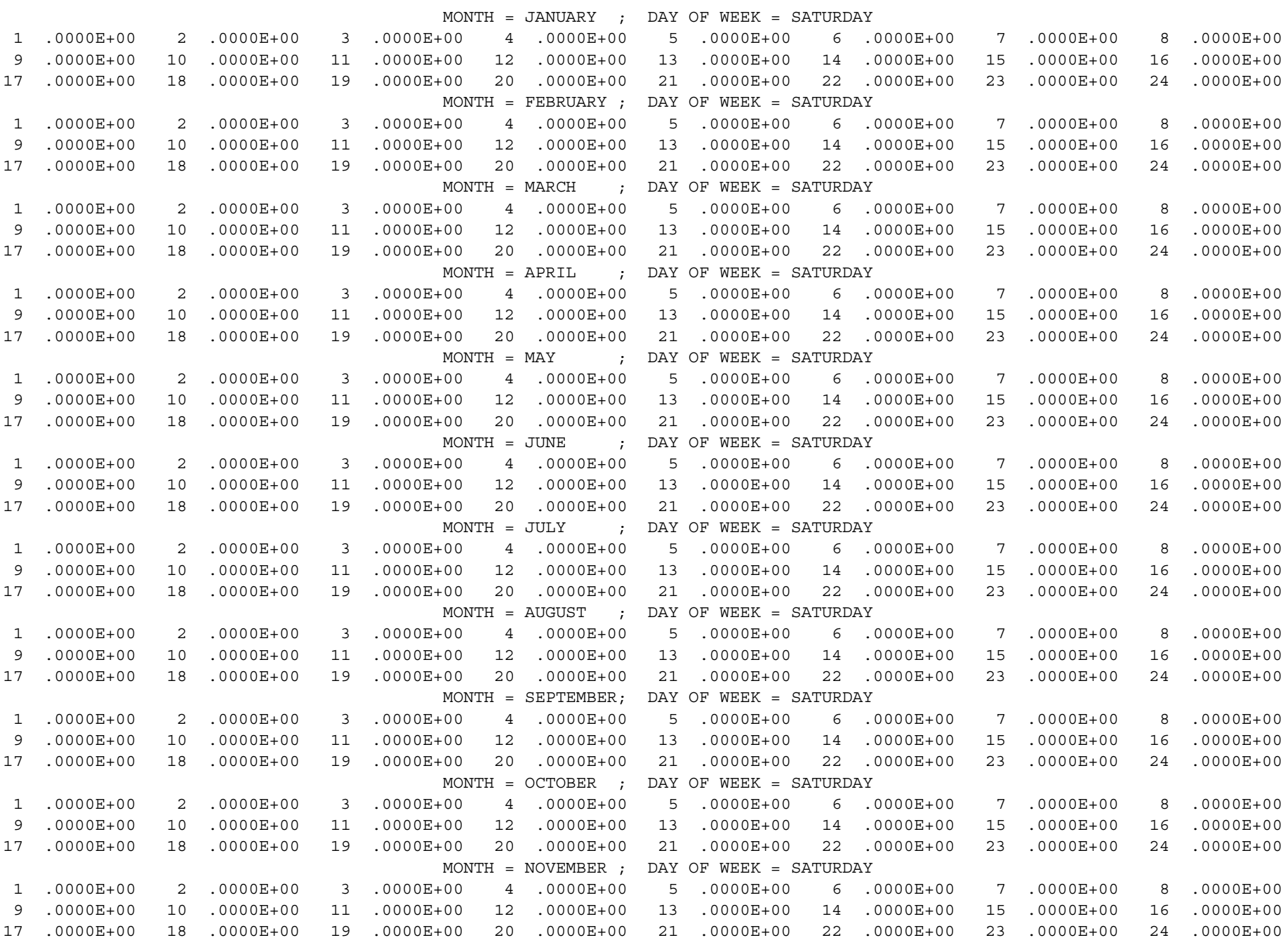
MONTH = DECEMBER ; DAY OF WEEK = SATURDAY 


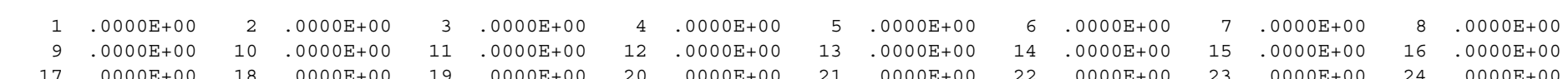

$\begin{array}{ll}* * * \text { AERMOD - VERSION } 07026 * * * \quad & * * * \text { U/UA Building Demolition } \\ & * * * \text { Unit Release - Worst Case }\end{array}$ $\star *$ MODELOPTS :

CONC $\begin{array}{ll}* * * & 02 / 09 / 09 \\ * * * & 10: 41: 11\end{array}$

DRYDPL WETDPL

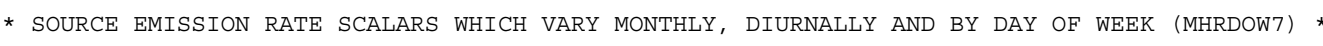

SOURCE ID $=224 \mathrm{UA} \_1 ;$ SOURCE TYPE $=$ POINT

HOUR SCALAR HOUR SCALAR HOUR SCALAR HOUR SCALAR HOUR SCALAR HOUR SCALAR HOUR SCALAR HOUR SCALAR

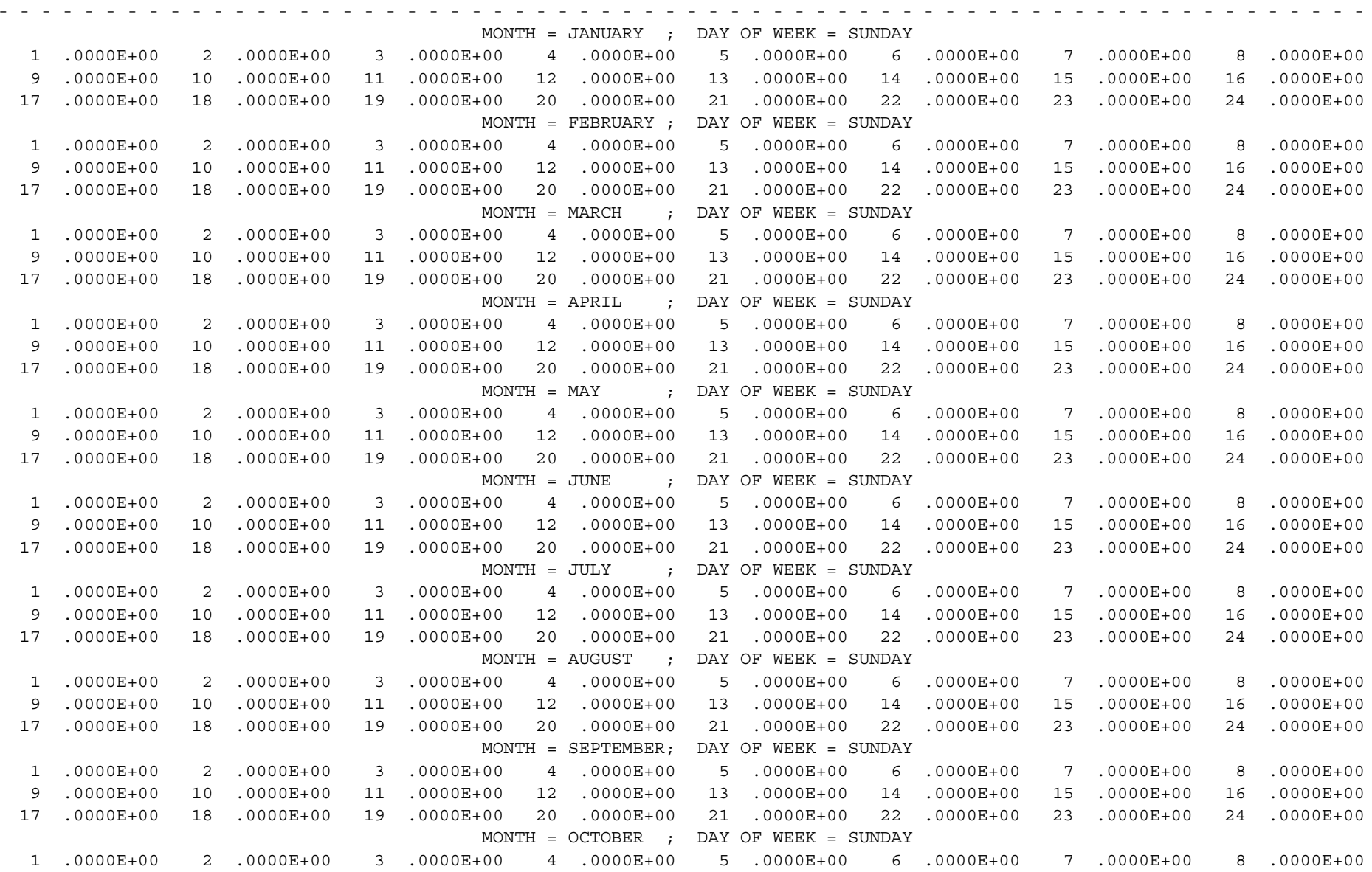




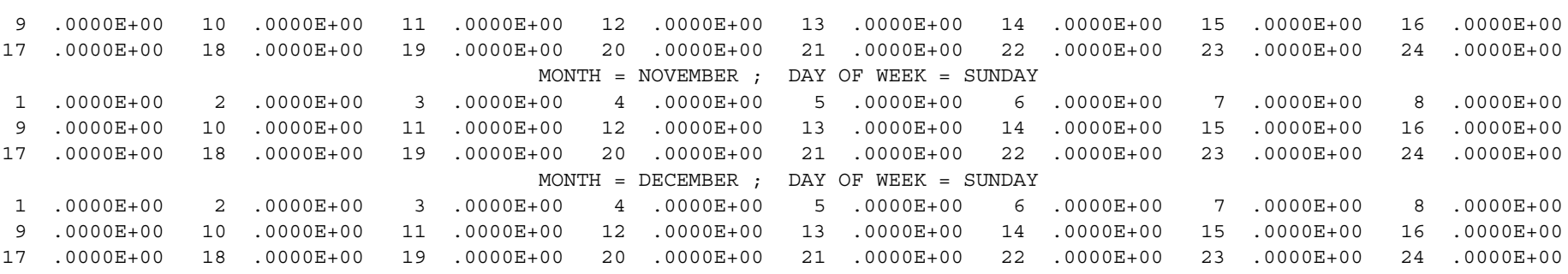

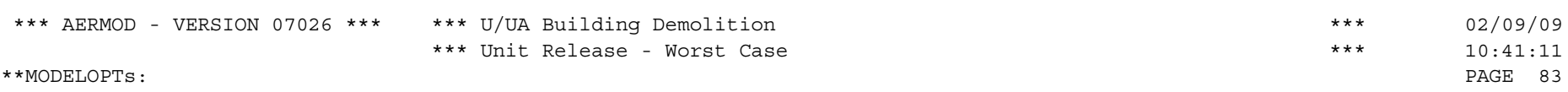
DDEP
TOXICS FLAT
DRYDPL WETDPL

CONC

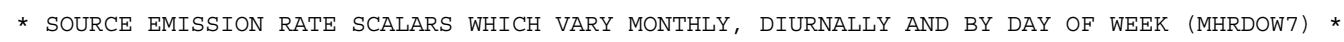

SOURCE ID $=224 \mathrm{UA} 2 ; \quad$ SOURCE TYPE $=$ POINT

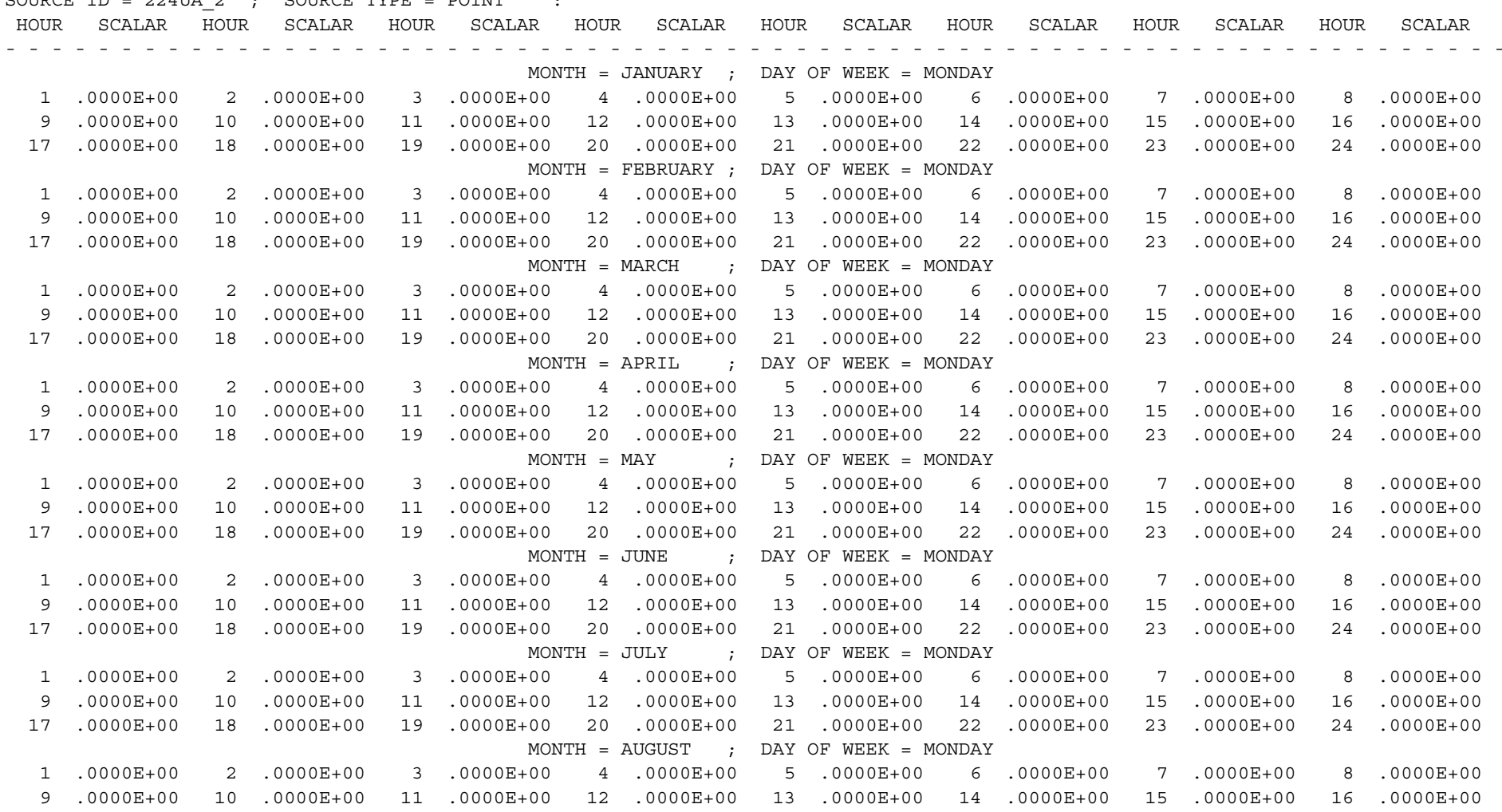




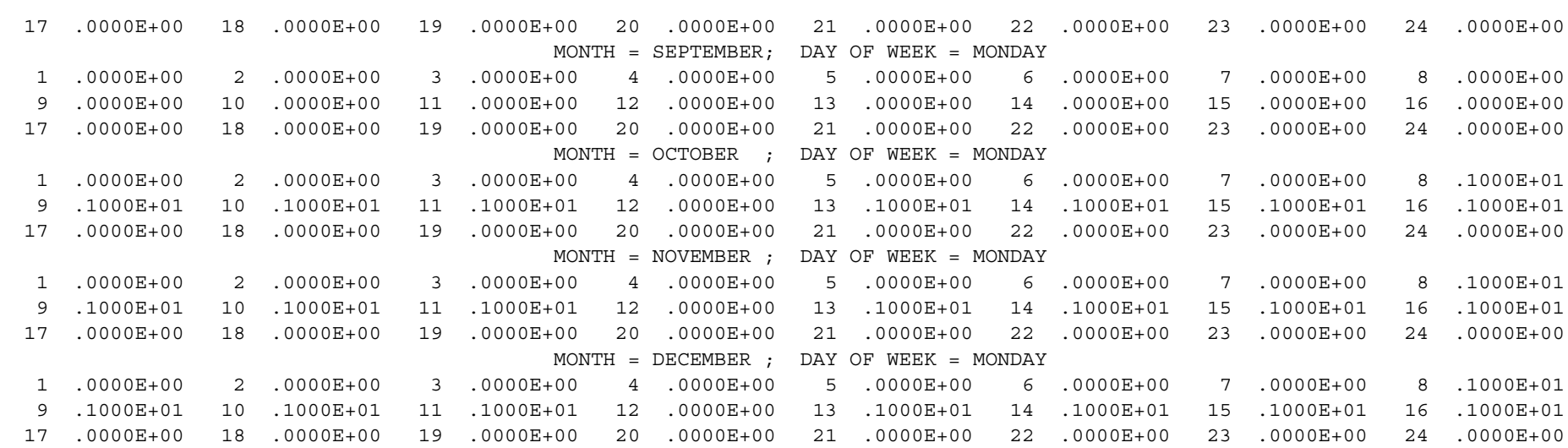

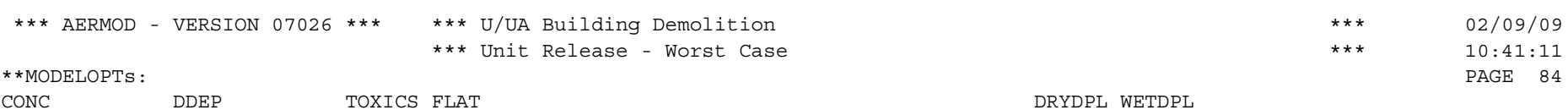

CONC

DDEP

DRYDPL WETDPL

PAGE 84

SOURCE ID $=$ 224UA_2 $;$ SOURCE TYPE $=$ POINT

HOUR SCALAR HOUR SCALAR HOUR SCALAR HOUR SCALAR HOUR SCALAR HOUR SCALAR HOUR SCALAR HOUR SCALAR

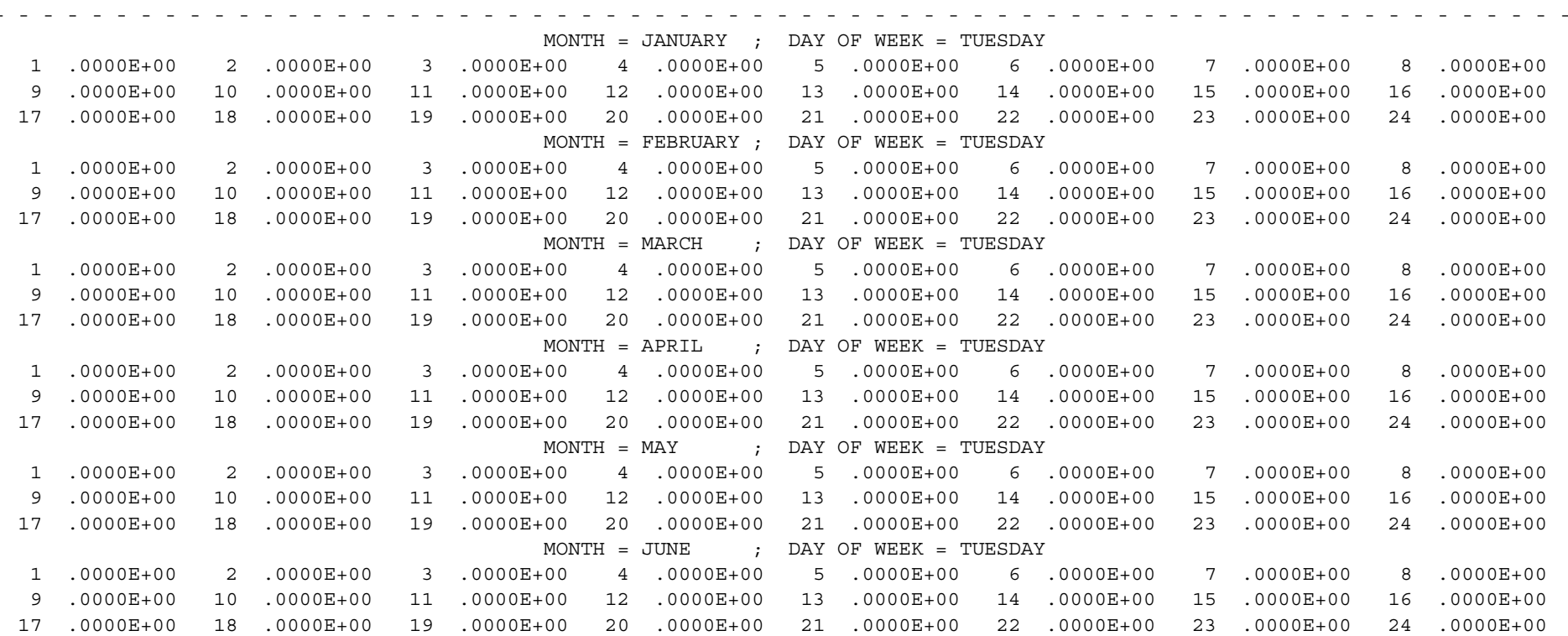




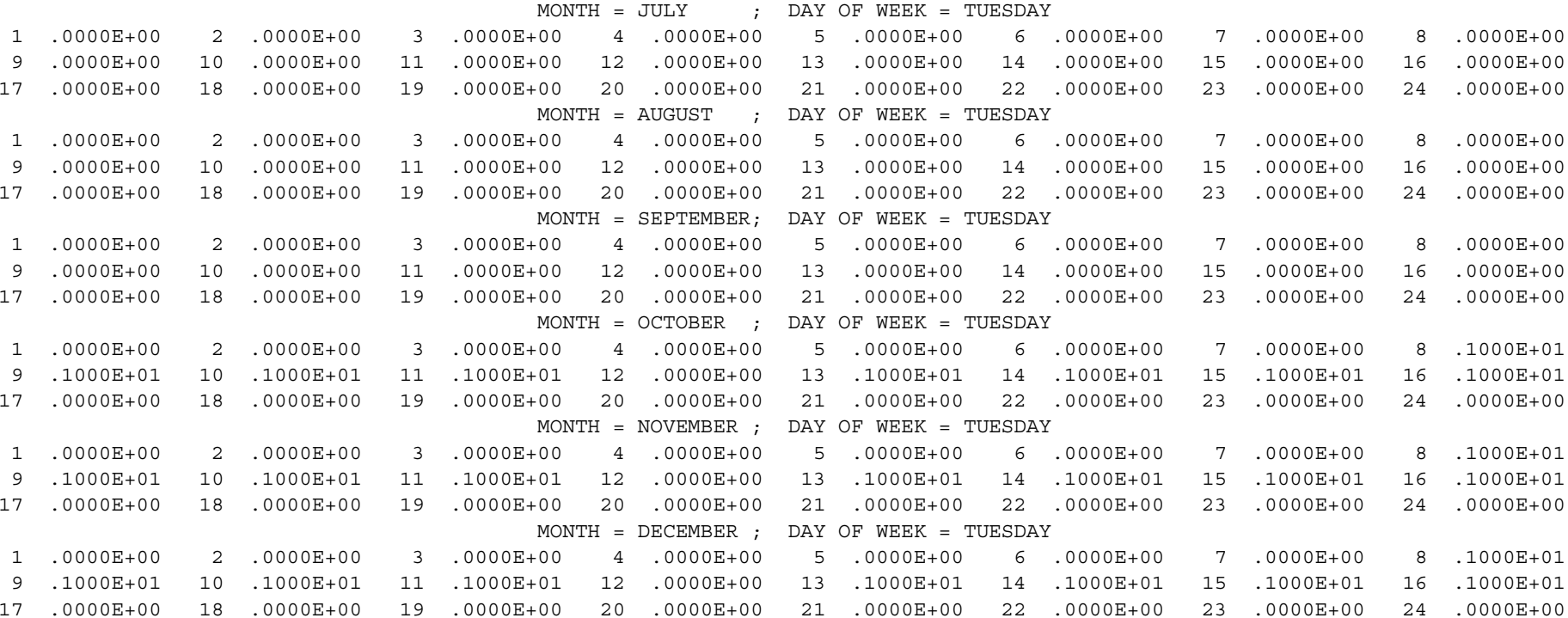

* SOURCE EMISSION RATE SCALARS WHICH VARY MONTHLY, DIURNALLY AND BY DAY OF WEEK (MHRDOW7) *

SOURCE ID $=224$ UA $2 ;$ SOURCE TYPE $=$ POINT

HOUR SCALAR HOUR SCALAR HOUR SCALAR HOUR SCALAR HOUR SCALAR HOUR SCALAR HOUR SCALAR HOUR SCALAR

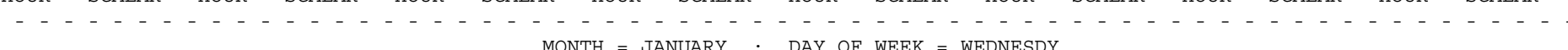

MONTH $=$ JANUARY $;$ DAY OF WEEK $=$ WEDNESDY

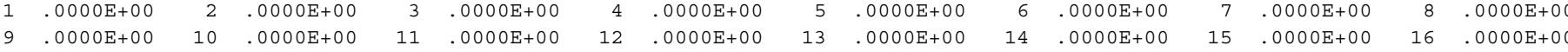

$\begin{array}{lllllll}16 & .0000 \mathrm{E}+00\end{array}$

MONTH $=$ FEBRUARY $; \quad$ DAY OF WEEK $=$ WEDNESDY

$\begin{array}{lllllllllllllll} & & & \end{array}$

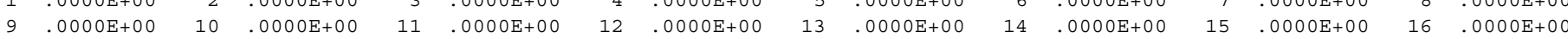

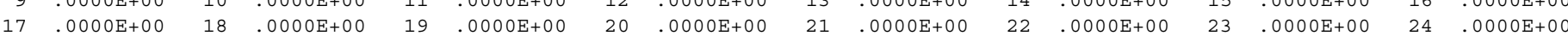

MONTH $=$ MARCH $;$ DAY OF WEEK $=$ WEDNESDY

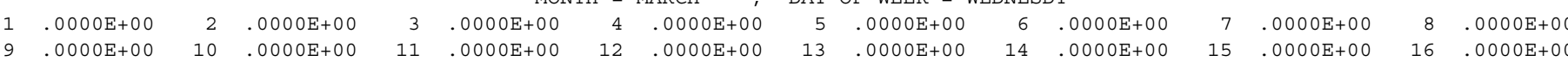

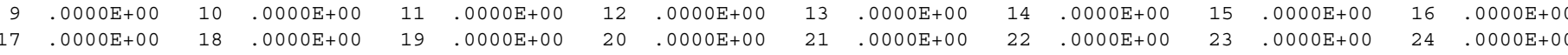

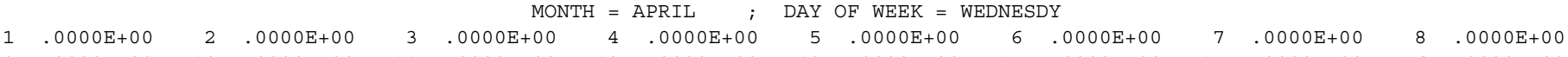

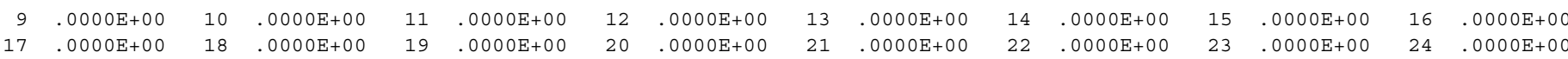

$$
\text { MONTH }=\text { MAY } \quad ; \quad \text { DAY OF WEEK }=\text { WEDNESDY }
$$




\begin{tabular}{|c|c|c|c|c|c|c|c|c|c|c|c|c|c|c|c|}
\hline 1 & $.0000 \mathrm{E}+00$ & 2 & $.0000 \mathrm{E}+00$ & 3 & $.0000 \mathrm{E}+00$ & 4 & $.0000 \mathrm{E}+00$ & 5 & $.0000 \mathrm{E}+00$ & 6 & $.0000 \mathrm{E}+00$ & 7 & $.0000 \mathrm{E}+00$ & 8 & $.0000 \mathrm{E}+00$ \\
\hline 9 & $.0000 \mathrm{E}+00$ & 10 & $.0000 \mathrm{E}+00$ & 11 & $.0000 \mathrm{E}+00$ & 12 & $.0000 \mathrm{E}+00$ & 13 & $.0000 \mathrm{E}+00$ & 14 & $.0000 \mathrm{E}+00$ & 15 & $.0000 \mathrm{E}+00$ & 16 & $.0000 \mathrm{E}+00$ \\
\hline 17 & $.0000 \mathrm{E}+00$ & 18 & $.0000 \mathrm{E}+00$ & 19 & $.0000 \mathrm{E}+00$ & 20 & $.0000 \mathrm{E}+00$ & 21 & $.0000 \mathrm{E}+00$ & 22 & $.0000 \mathrm{E}+00$ & 23 & $.0000 \mathrm{E}+00$ & 24 & $.0000 \mathrm{E}+00$ \\
\hline \multicolumn{16}{|c|}{ DAY OF WEEK = WEDNESDY } \\
\hline 1 & $.0000 \mathrm{E}+00$ & 2 & $.0000 \mathrm{E}+00$ & 3 & $.0000 \mathrm{E}+00$ & 4 & $.0000 \mathrm{E}+00$ & 5 & $.0000 \mathrm{E}+00$ & 6 & $.0000 \mathrm{E}+00$ & 7 & $.0000 \mathrm{E}+00$ & 8 & $.0000 \mathrm{E}+00$ \\
\hline 9 & $.0000 \mathrm{E}+00$ & 10 & $.0000 \mathrm{E}+00$ & 11 & $.0000 \mathrm{E}+00$ & 12 & $.0000 \mathrm{E}+00$ & 13 & $.0000 \mathrm{E}+00$ & 14 & $.0000 \mathrm{E}+00$ & 15 & $.0000 \mathrm{E}+00$ & 16 & $.0000 \mathrm{E}+00$ \\
\hline 17 & $.0000 \mathrm{E}+00$ & 18 & $.0000 \mathrm{E}+00$ & 19 & $.0000 \mathrm{E}+00$ & 20 & $.0000 \mathrm{E}+00$ & 21 & $.0000 \mathrm{E}+00$ & 22 & $.0000 E+00$ & 23 & $.0000 \mathrm{E}+00$ & 24 & $.0000 \mathrm{E}+00$ \\
\hline \multicolumn{16}{|c|}{ AY OF WEEK = WEDNESDY } \\
\hline 1 & $.0000 \mathrm{E}+00$ & 2 & $.0000 \mathrm{E}+00$ & 3 & $.0000 \mathrm{E}+00$ & 4 & $.0000 \mathrm{E}+00$ & 5 & $.0000 \mathrm{E}+00$ & 6 & $.0000 \mathrm{E}+00$ & 7 & $.0000 \mathrm{E}+00$ & 8 & $.0000 \mathrm{E}+00$ \\
\hline 9 & $.0000 \mathrm{E}+00$ & 10 & $.0000 \mathrm{E}+00$ & 11 & $.0000 \mathrm{E}+00$ & 12 & $.0000 \mathrm{E}+00$ & 13 & $.0000 \mathrm{E}+00$ & 14 & $.0000 E+00$ & 15 & $.0000 \mathrm{E}+00$ & 16 & $.0000 E+00$ \\
\hline 17 & $.0000 \mathrm{E}+00$ & 18 & $.0000 \mathrm{E}+00$ & 19 & $.0000 \mathrm{E}+00$ & 20 & $.0000 \mathrm{E}+00$ & 21 & $.0000 \mathrm{E}+00$ & 22 & $E+00$ & 23 & $.0000 \mathrm{E}+00$ & 24 & $.0000 \mathrm{E}+00$ \\
\hline \multicolumn{16}{|c|}{ MONTH $=$ AUGUST } \\
\hline 1 & $.0000 \mathrm{E}+00$ & 2 & $.0000 \mathrm{E}+00$ & 3 & $.0000 \mathrm{E}+00$ & 4 & $.0000 \mathrm{E}+00$ & 5 & $.0000 \mathrm{E}+00$ & 6 & $.0000 \mathrm{E}+00$ & 7 & $.0000 \mathrm{E}+00$ & 8 & $.0000 \mathrm{E}+00$ \\
\hline 9 & $.0000 \mathrm{E}+00$ & 10 & $.0000 \mathrm{E}+00$ & 11 & $.0000 \mathrm{E}+00$ & 12 & $.0000 \mathrm{E}+00$ & 13 & $.0000 \mathrm{E}+00$ & 14 & $.0000 \mathrm{E}+00$ & 15 & $.0000 \mathrm{E}+00$ & 16 & $.0000 \mathrm{E}+00$ \\
\hline 17 & $.0000 \mathrm{E}+00$ & 18 & $.0000 \mathrm{E}+00$ & 19 & $.0000 \mathrm{E}+00$ & 20 & $.0000 \mathrm{E}+00$ & 21 & $.0000 \mathrm{E}+00$ & 22 & $\mathrm{E}+00$ & 23 & $.0000 \mathrm{E}+00$ & 24 & $.0000 \mathrm{E}+00$ \\
\hline \multicolumn{16}{|c|}{ MONTH = SEPTEMBER; } \\
\hline 1 & $.0000 \mathrm{E}+00$ & 2 & $.0000 \mathrm{E}+00$ & 3 & $.0000 \mathrm{E}+00$ & 4 & $.0000 \mathrm{E}+00$ & 5 & $.0000 \mathrm{E}+00$ & 6 & $.0000 \mathrm{E}+00$ & 7 & $.0000 \mathrm{E}+00$ & 8 & $.0000 \mathrm{E}+00$ \\
\hline 9 & $.0000 \mathrm{E}+00$ & 10 & $.0000 \mathrm{E}+00$ & 11 & $.0000 \mathrm{E}+00$ & 12 & $.0000 \mathrm{E}+00$ & 13 & $.0000 \mathrm{E}+00$ & 14 & $.0000 \mathrm{E}+00$ & 15 & $.0000 \mathrm{E}+00$ & 16 & $.0000 \mathrm{E}+00$ \\
\hline 17 & $.0000 \mathrm{E}+00$ & 18 & $.0000 \mathrm{E}+00$ & 19 & $.0000 \mathrm{E}+00$ & 20 & $.0000 \mathrm{E}+00$ & 21 & $.0000 \mathrm{E}+00$ & 22 & $.0000 \mathrm{E}+00$ & 23 & $.0000 \mathrm{E}+00$ & 24 & $.0000 \mathrm{E}+00$ \\
\hline \multicolumn{16}{|c|}{ MONTH $=$ OCTOBER } \\
\hline 1 & $.0000 \mathrm{E}+00$ & 2 & $.0000 \mathrm{E}+00$ & 3 & $.0000 \mathrm{E}+00$ & 4 & $.0000 \mathrm{E}+00$ & 5 & $.0000 \mathrm{E}+00$ & 6 & $.0000 \mathrm{E}+00$ & 7 & $.0000 \mathrm{E}+00$ & 8 & $.1000 \mathrm{E}+01$ \\
\hline 9 & $.1000 \mathrm{E}+01$ & 10 & $.1000 \mathrm{E}+01$ & 11 & $.1000 \mathrm{E}+01$ & 12 & $.0000 \mathrm{E}+00$ & 13 & $.1000 \mathrm{E}+01$ & 14 & $.1000 \mathrm{E}+01$ & 15 & $.1000 \mathrm{E}+01$ & 16 & $.1000 \mathrm{E}+01$ \\
\hline 17 & $.0000 \mathrm{E}+00$ & 18 & $.0000 \mathrm{E}+00$ & 19 & $.0000 \mathrm{E}+00$ & 20 & $.0000 \mathrm{E}+00$ & 21 & $.0000 \mathrm{E}+00$ & 22 & $.0000 \mathrm{E}+00$ & 23 & $.0000 \mathrm{E}+00$ & 24 & $.0000 E+00$ \\
\hline \multicolumn{16}{|c|}{ OAY OF WEEK = W } \\
\hline 1 & $.0000 \mathrm{E}+00$ & 2 & $.0000 \mathrm{E}+00$ & 3 & $.0000 \mathrm{E}+00$ & 4 & $.0000 \mathrm{E}+00$ & 5 & $.0000 \mathrm{E}+00$ & 6 & $.0000 \mathrm{E}+00$ & 7 & $.0000 \mathrm{E}+00$ & 8 & $.1000 \mathrm{E}+01$ \\
\hline 9 & $.1000 \mathrm{E}+01$ & 10 & $.1000 \mathrm{E}+01$ & 11 & $.1000 \mathrm{E}+01$ & 12 & $.0000 \mathrm{E}+00$ & 13 & $.1000 \mathrm{E}+01$ & 14 & $.1000 \mathrm{E}+01$ & 15 & $.1000 \mathrm{E}+01$ & 16 & $.1000 \mathrm{E}+01$ \\
\hline 17 & $.0000 \mathrm{E}+00$ & 18 & $.0000 E+00$ & 19 & $.0000 \mathrm{E}+00$ & 20 & $.0000 \mathrm{E}+00$ & 21 & $.0000 \mathrm{E}+00$ & 22 & $.0000 \mathrm{E}+00$ & 23 & $.0000 \mathrm{E}+00$ & 24 & $.0000 \mathrm{E}+00$ \\
\hline \multicolumn{16}{|c|}{ MONTH $=$ DECEMBER } \\
\hline 1 & $.0000 \mathrm{E}+00$ & 2 & $.0000 \mathrm{E}+00$ & 3 & $.0000 \mathrm{E}+00$ & 4 & $.0000 \mathrm{E}+00$ & 5 & $.0000 \mathrm{E}+00$ & 6 & $.0000 \mathrm{E}+00$ & 7 & $.0000 \mathrm{E}+00$ & 8 & $.1000 E+01$ \\
\hline 9 & $.1000 \mathrm{E}+01$ & 10 & $.1000 \mathrm{E}+01$ & 11 & $.1000 \mathrm{E}+01$ & 12 & $.0000 \mathrm{E}+00$ & 13 & $.1000 \mathrm{E}+01$ & 14 & $.1000 \mathrm{E}+01$ & 15 & $.1000 \mathrm{E}+01$ & 16 & $.1000 \mathrm{E}+01$ \\
\hline 17 & $.0000 \mathrm{E}+00$ & 18 & $.0000 \mathrm{E}+00$ & 19 & $.0000 \mathrm{E}+00$ & 20 & $.0000 \mathrm{E}+00$ & 21 & $.0000 \mathrm{E}+00$ & 22 & $.0000 \mathrm{E}+00$ & 23 & $.0000 \mathrm{E}+00$ & 24 & $.0000 \mathrm{E}+00$ \\
\hline
\end{tabular}

$* * *$ AERMOD - VERSION $07026 * * * \quad \begin{array}{r}* * \\ \text { U/UA Building Demolition } \\ * * * \text { Unit Release - Worst Case }\end{array}$ **MODELOPTS:

CONC
TOXICS FLAT $\begin{array}{ll}* * * & 02 / 09 / 09 \\ * * * & 10: 41: 11\end{array}$

PAGE 86

* SOURCE EMISSION RATE SCALARS WHICH VARY MONTHLY, DIURNALLY AND BY DAY OF WEEK (MHRDOW7) *

SOURCE ID $=224 \mathrm{UA} \_;$SOURCE TYPE $=$POINT

HOUR SCALAR HOUR SCALAR HOUR SCALAR HOUR SCALAR HOUR SCALAR HOUR SCALAR HOUR SCALAR HOUR SCALAR

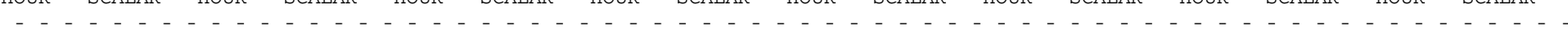

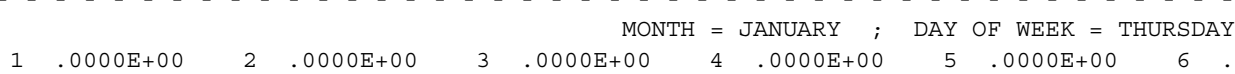

$\begin{array}{llllllllllllllll}1 & .0000 \mathrm{E}+00 & 2 & .0000 \mathrm{E}+00 & 3 & .0000 \mathrm{E}+00 & 4 & .0000 \mathrm{E}+00 & 5 & .0000 \mathrm{E}+00 & 6 & .0000 \mathrm{E}+00 & 7 & .0000 \mathrm{E}+00 & 8 & .0000 \mathrm{E}+00\end{array}$

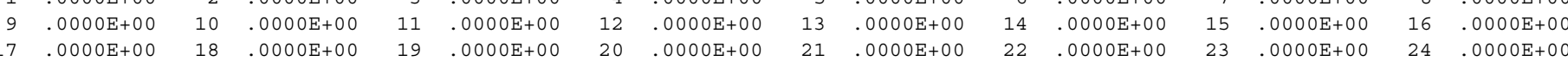

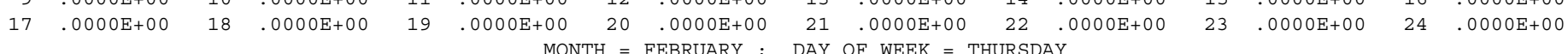

$\begin{array}{cccccccccccccccc}1 & .0000 \mathrm{E}+00 & 2 & .0000 \mathrm{E}+00 & 3 & .0000 \mathrm{E}+00 & 4 & .0000 \mathrm{E}+00 & 5 & .0000 \mathrm{E}+00 & 6 & .0000 \mathrm{E}+00 & 7 & .0000 \mathrm{E}+00 & 8 & .0000 \mathrm{E}+00\end{array}$

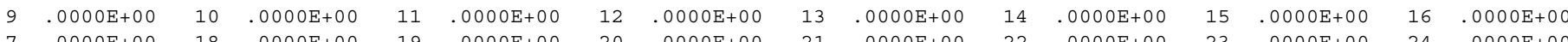

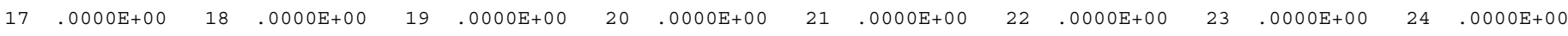

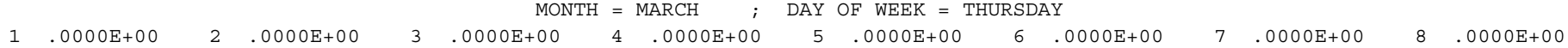




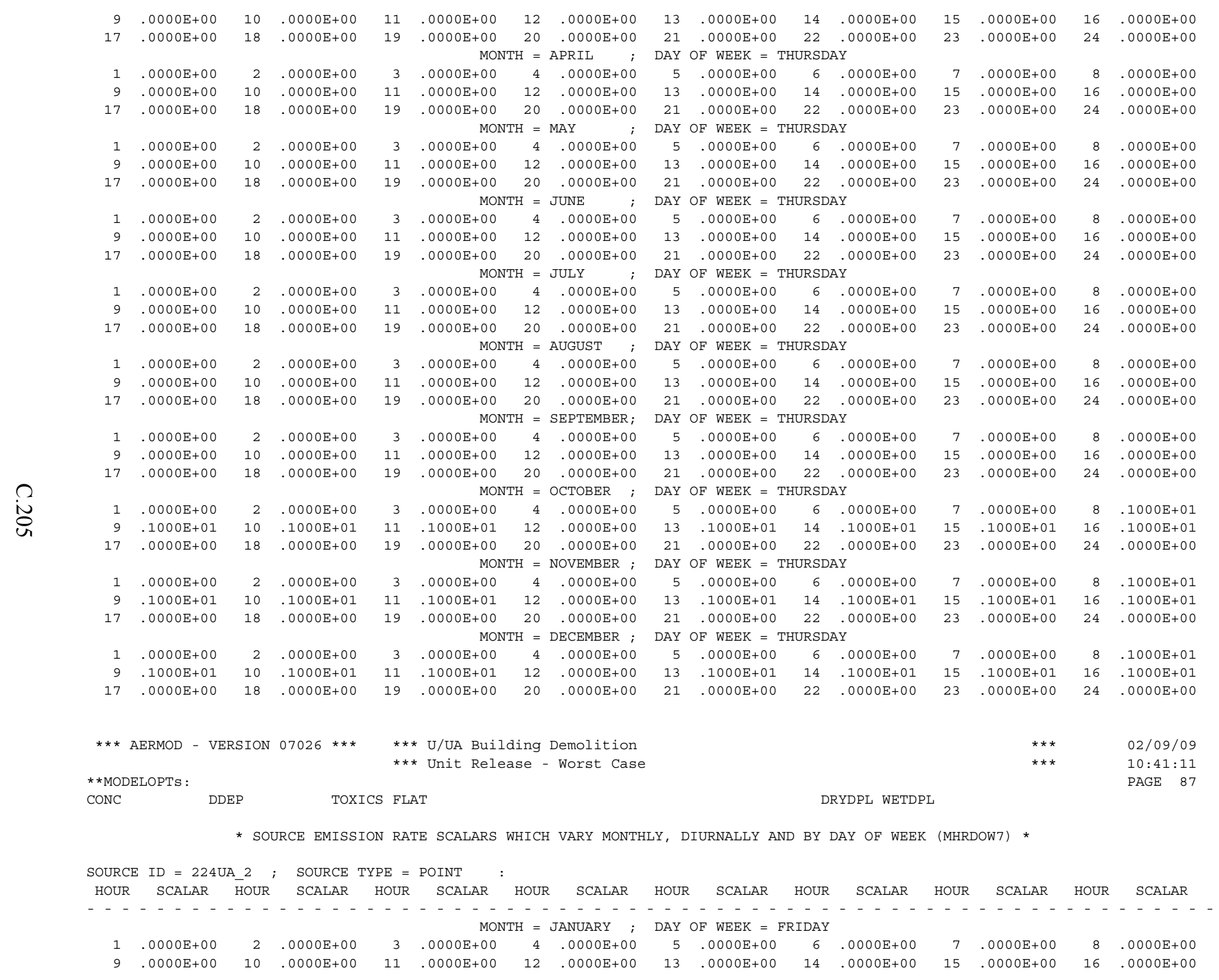




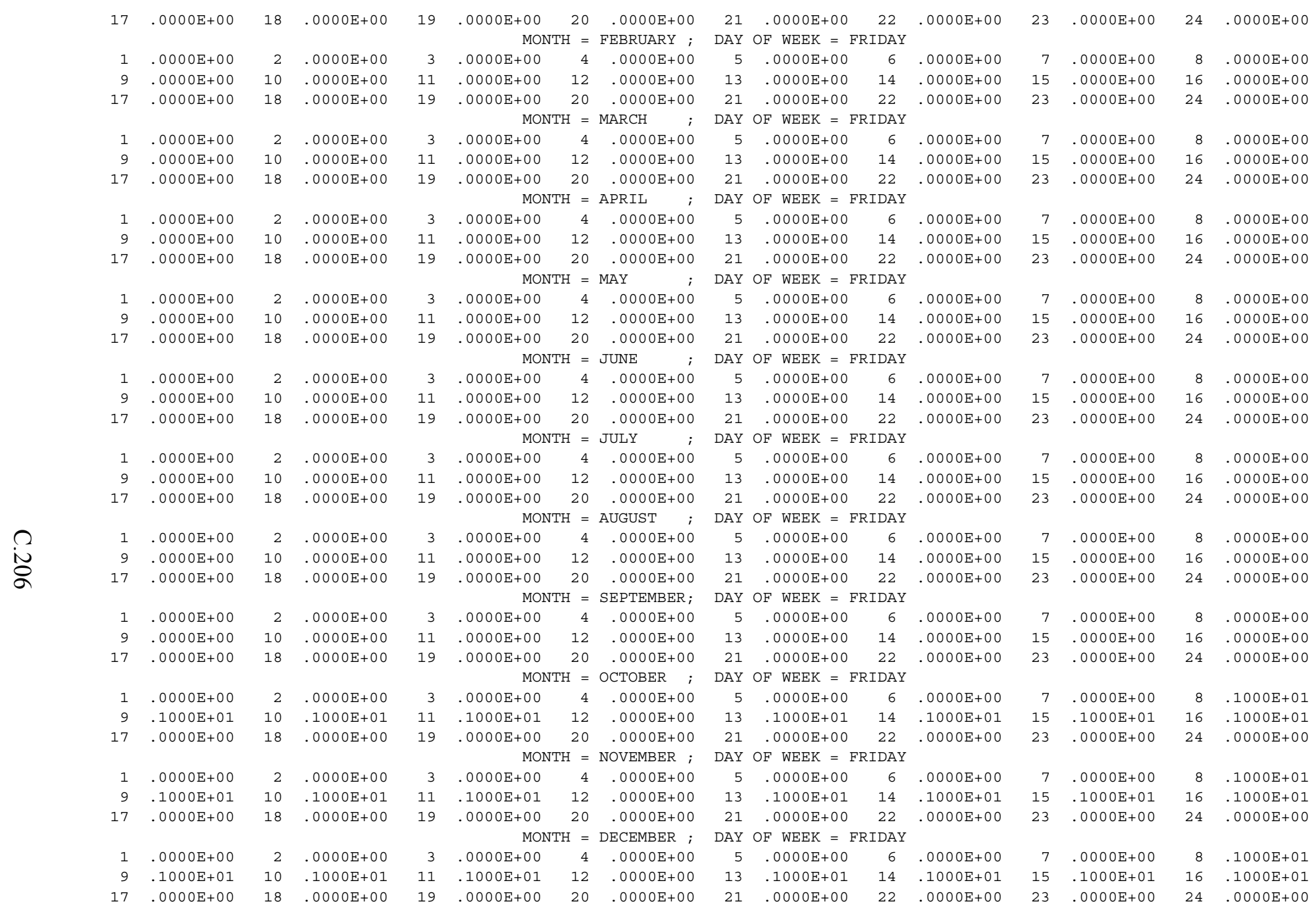

*** AERMOD - VERSION $07026 * * * \quad * * *$ U/UA Building Demolition

$* * *$ Unit Release - Worst Cas $\begin{array}{ll}* * * & 02 / 09 / 09 \\ * * * & 10: 41: 11\end{array}$

PAGE 88

CONC

DDEP

TOXICS FLAT

DRYDPL WETDPL

* SOURCE EMISSION RATE SCALARS WHICH VARY MONTHLY, DIURNALLY AND BY DAY OF WEEK (MHRDOW7) * 


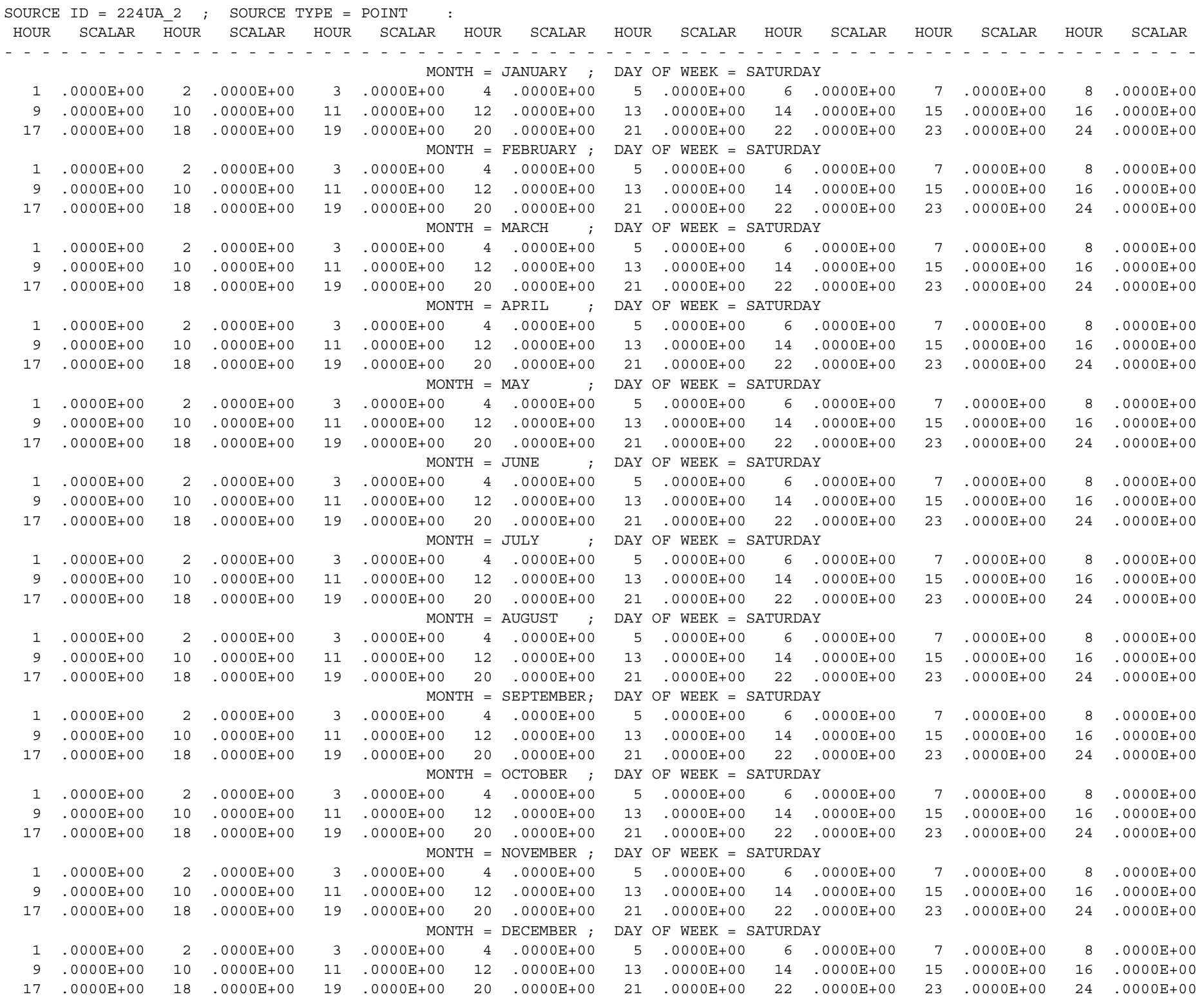




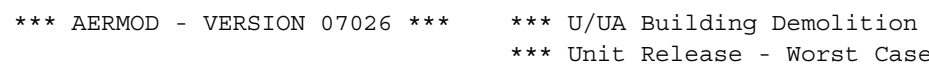
**MODELOPTS :

CONC
$* * * \quad 02 / 09 / 09$

$* * * \quad 10: 41: 11$
PAGE 89

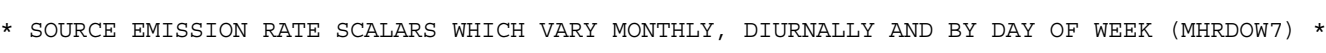

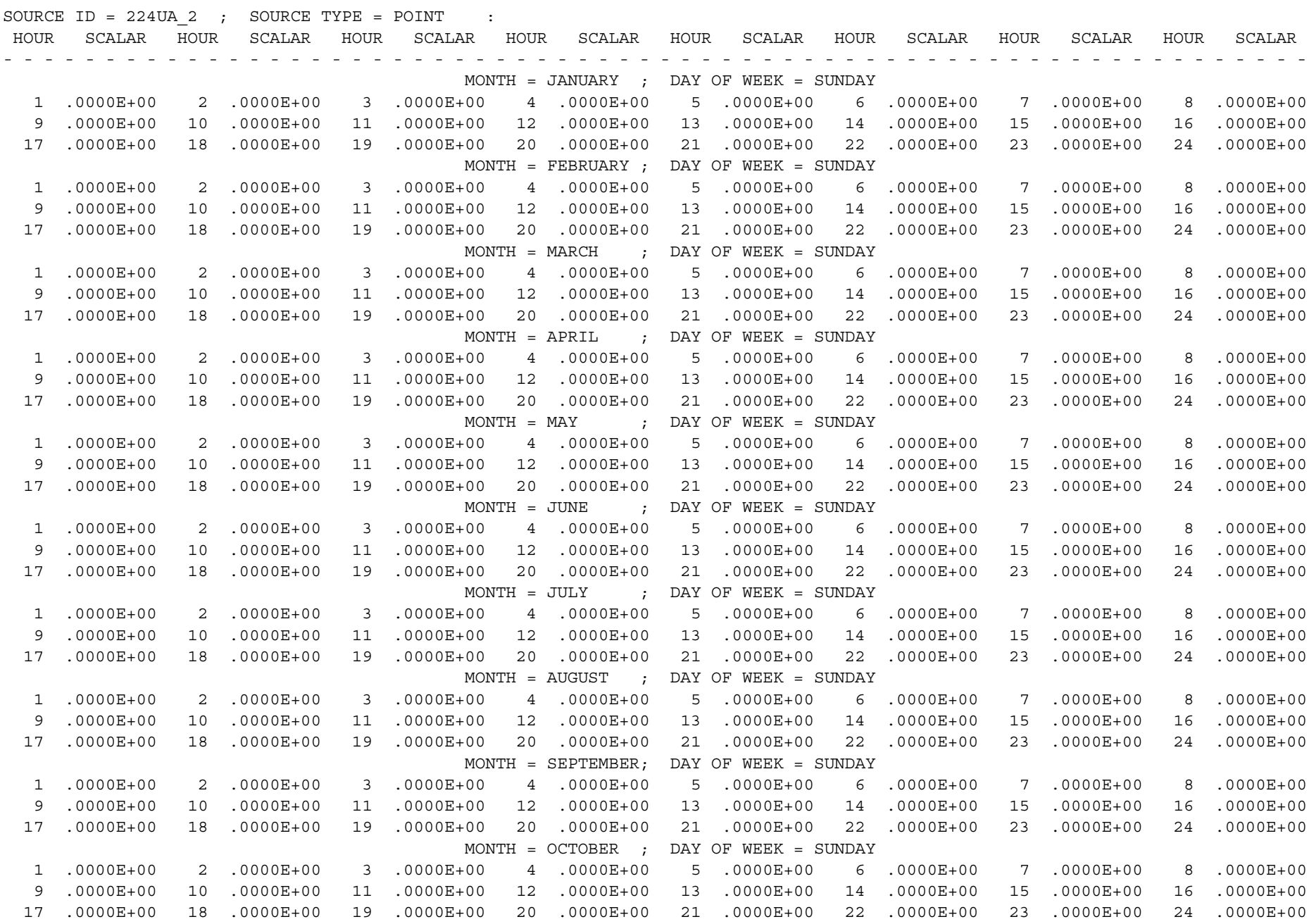
MONTH = NOVEMBER $;$ DAY OF WEEK = SUNDAY 


\begin{tabular}{|c|c|c|c|c|c|c|c|c|c|c|c|c|c|c|c|}
\hline 1 & $.0000 \mathrm{E}+00$ & 2 & $.0000 \mathrm{E}+00$ & 3 & $.0000 \mathrm{E}+00$ & 4 & $.0000 \mathrm{E}+00$ & 5 & $.0000 \mathrm{E}+00$ & 6 & $.0000 \mathrm{E}+00$ & 7 & $.0000 \mathrm{E}+00$ & 8 & $0000 \mathrm{E}+00$ \\
\hline 9 & $.0000 \mathrm{E}+00$ & 10 & $.0000 \mathrm{E}+00$ & 11 & $.0000 \mathrm{E}+00$ & 12 & $.0000 \mathrm{E}+00$ & 13 & $.0000 \mathrm{E}+00$ & 14 & $.0000 \mathrm{E}+00$ & 15 & $.0000 \mathrm{E}+00$ & 16 & $.0000 \mathrm{E}+00$ \\
\hline 1 & $.0000 \mathrm{E}+00$ & 18 & $.0000 \mathrm{E}+00$ & 19 & $.0000 \mathrm{E}+00$ & 20 & $.0000 \mathrm{E}+00$ & 21 & $.0000 \mathrm{E}+00$ & 22 & $.0000 \mathrm{E}+00$ & 23 & $.0000 \mathrm{E}+00$ & 24 & $.0000 \mathrm{E}+00$ \\
\hline & & & & & MOI & & DECEMBER ; & DAY & OF WEEK = & NDAY & & & & & \\
\hline 1 & $.0000 \mathrm{E}+00$ & 2 & $.0000 \mathrm{E}+00$ & 3 & $.0000 \mathrm{E}+00$ & 4 & $.0000 \mathrm{E}+00$ & 5 & $.0000 \mathrm{E}+00$ & 6 & $.0000 \mathrm{E}+00$ & 7 & $.0000 \mathrm{E}+00$ & 8 & $.0000 \mathrm{E}+00$ \\
\hline 9 & $.0000 \mathrm{E}+00$ & 10 & $.0000 \mathrm{E}+00$ & 11 & $.0000 \mathrm{E}+00$ & 12 & $.0000 \mathrm{E}+00$ & 13 & $.0000 \mathrm{E}+00$ & 14 & $.0000 \mathrm{E}+00$ & 15 & $.0000 \mathrm{E}+00$ & 16 & $.0000 \mathrm{E}+00$ \\
\hline & $.0000 \mathrm{E}+00$ & 18 & $.0000 \mathrm{E}+00$ & 19 & $.0000 \mathrm{E}+00$ & 20 & $.0000 \mathrm{E}+00$ & 21 & $0000 \mathrm{E}+00$ & 22 & $.0000 \mathrm{E}+00$ & 23 & $0000 \mathrm{E}+00$ & 24 & $0000 E+00$ \\
\hline
\end{tabular}

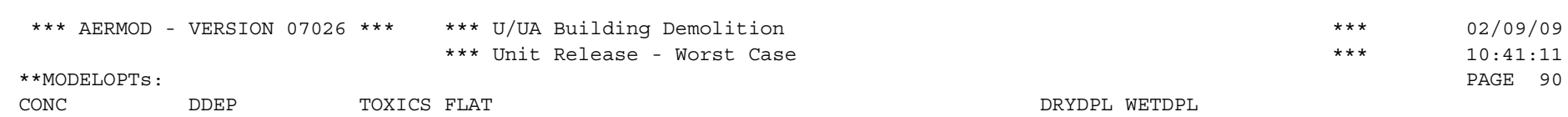

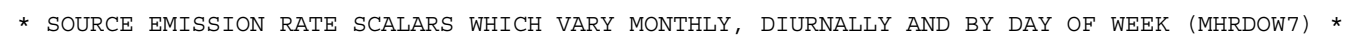

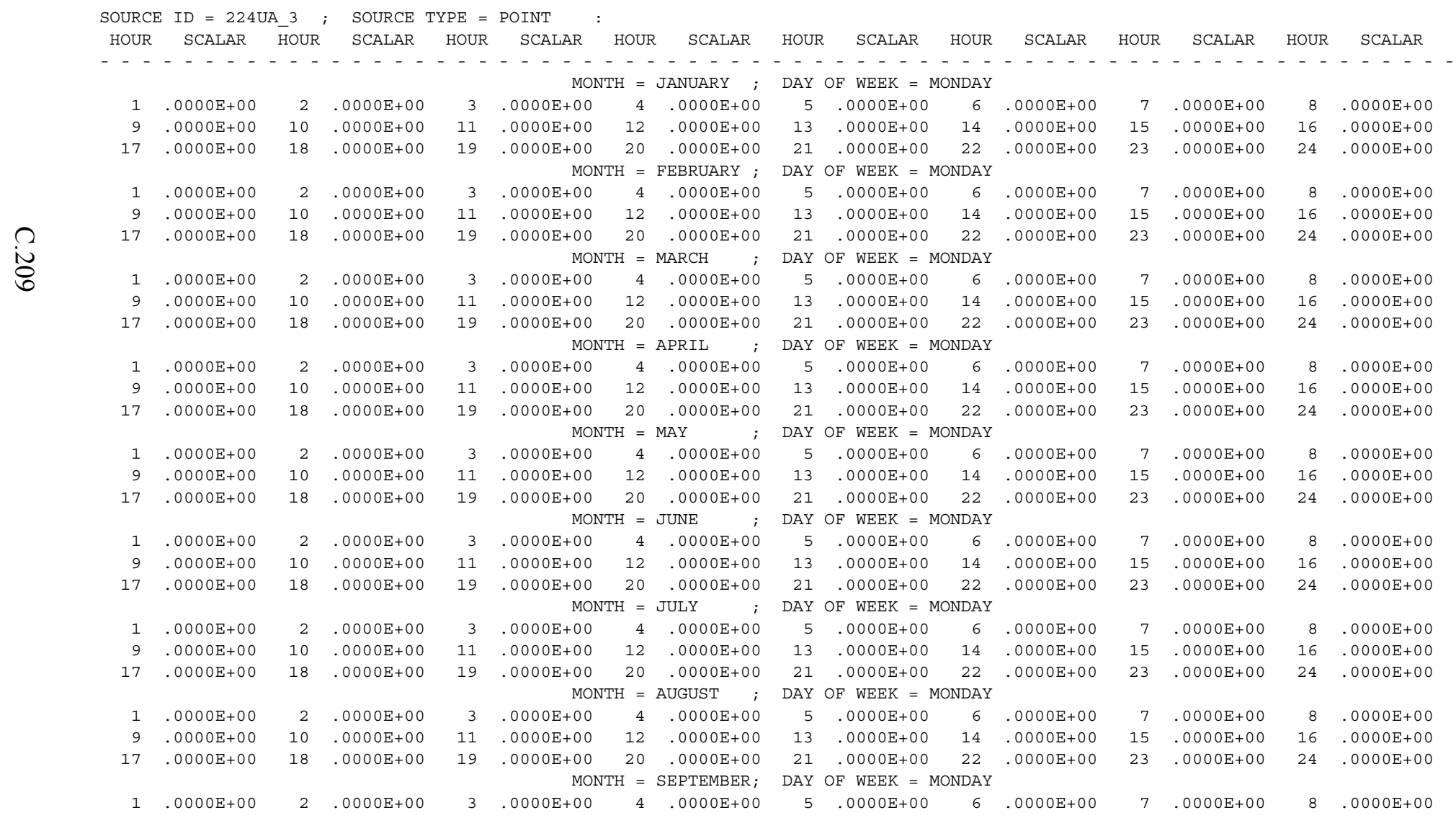




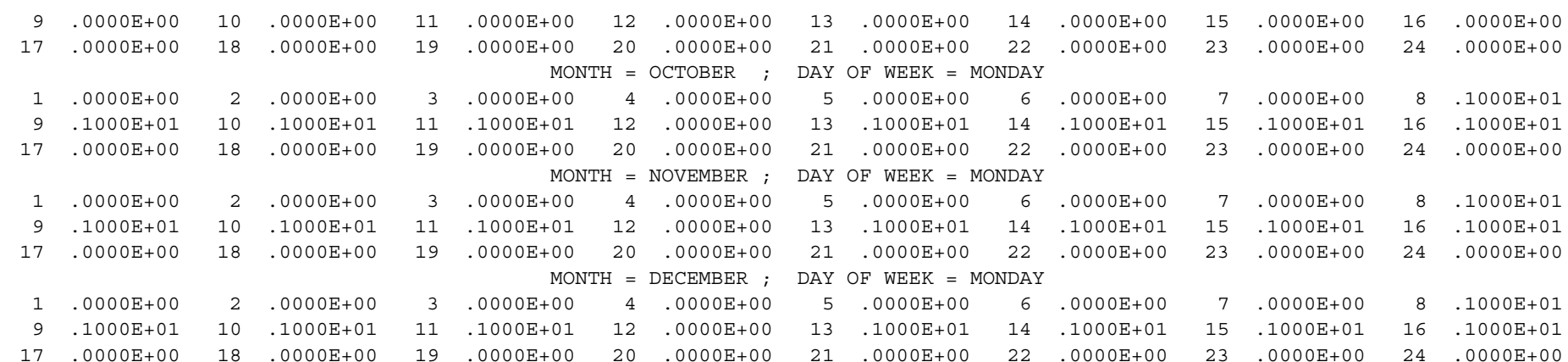

*** AERMOD - VERSION $07026 * * * \quad * * *$ U/UA Building Demolition

*** Unit Release - Worst Case

$\begin{array}{ll}* * * & 02 / 09 / 09 \\ * * * & 10: 41: 11 \\ & \text { PAGE } 91\end{array}$ **MODELOPTS:

CONC

DDEP

TOXICS FLAT

DRYDPL WETDPL

* SOURCE EMISSion RATe SCALARS Which VARy MONTHLy, DIURNALly AND By DAy OF WEEK (MHRDOW7) *

SOURCE ID $=224 \mathrm{UA} \_3 ;$ SOURCE TYPE $=$ POINT

HOUR SCALAR HOUR SCALAR HOUR SCALAR HOUR SCALAR HOUR SCALAR HOUR SCALAR HOUR SCALAR HOUR SCALAR

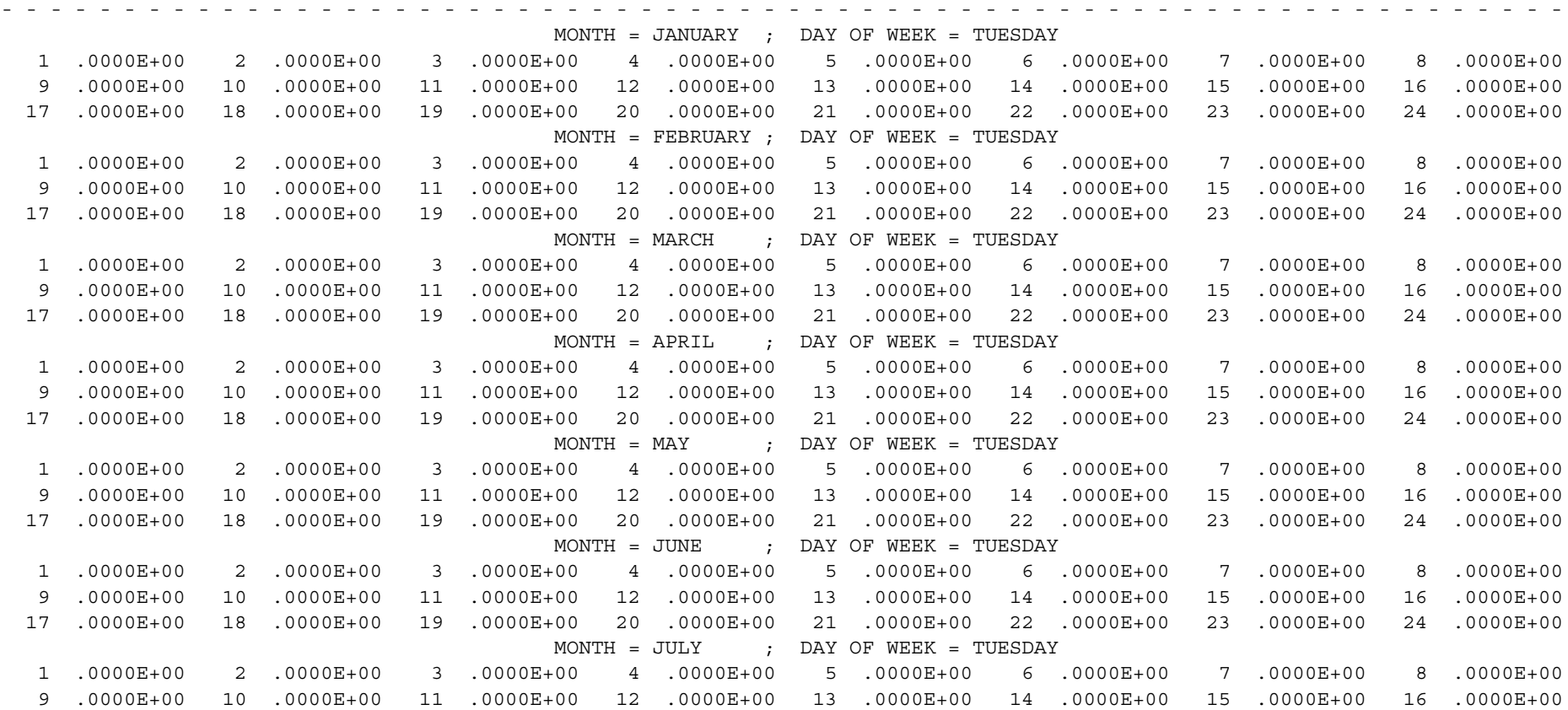




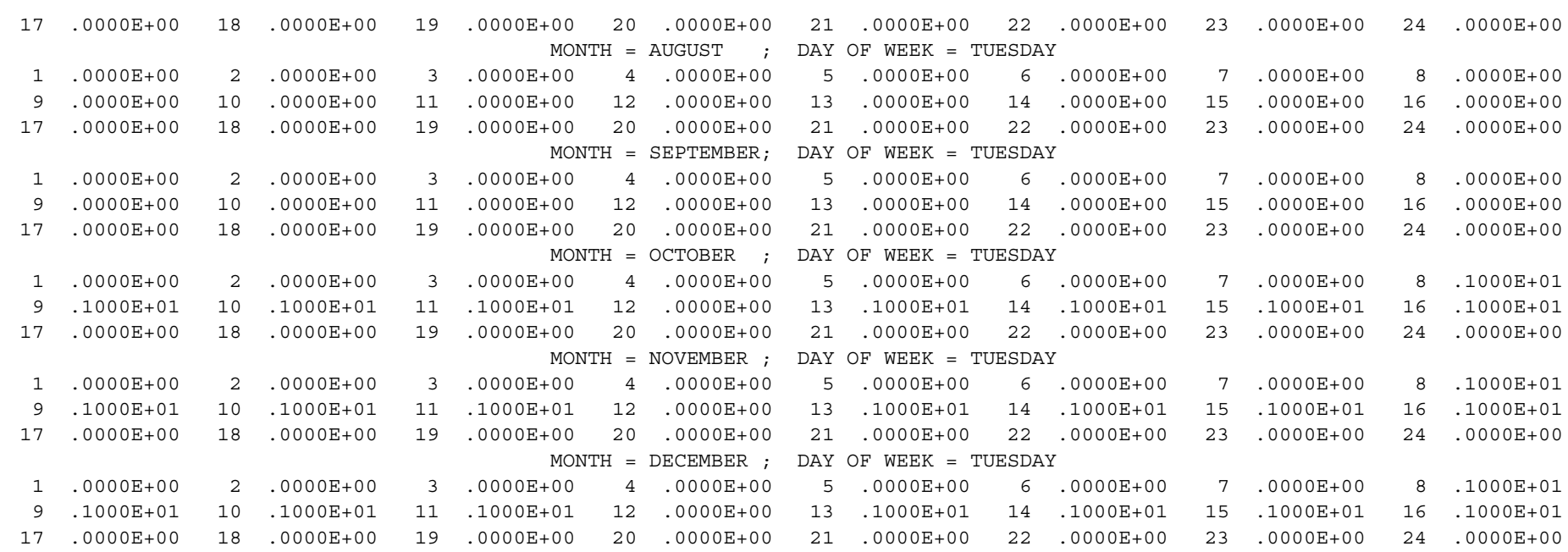

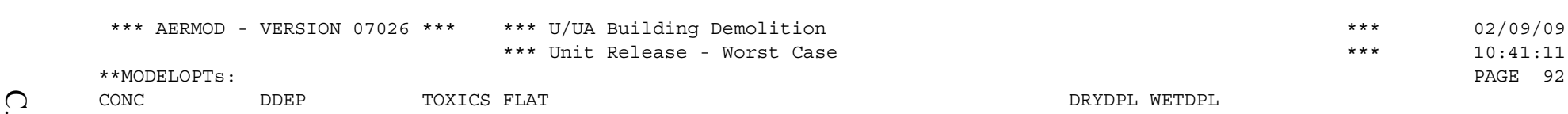

* SOURCE emission RATE SCALARS Which VARY MONTHLy, DIURNALLy AND by DAy OF WEek (MHRDOW7) *

SOURCE ID $=224 \mathrm{UA} 3 ; \quad$ SOURCE TYPE $=$ POINT

HOUR SCALAR HOUR SCALAR HOUR SCALAR HOUR SCALAR HOUR SCALAR HOUR SCALAR HOUR SCALAR HOUR SCALAR

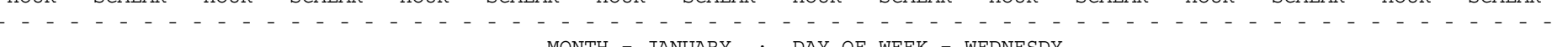

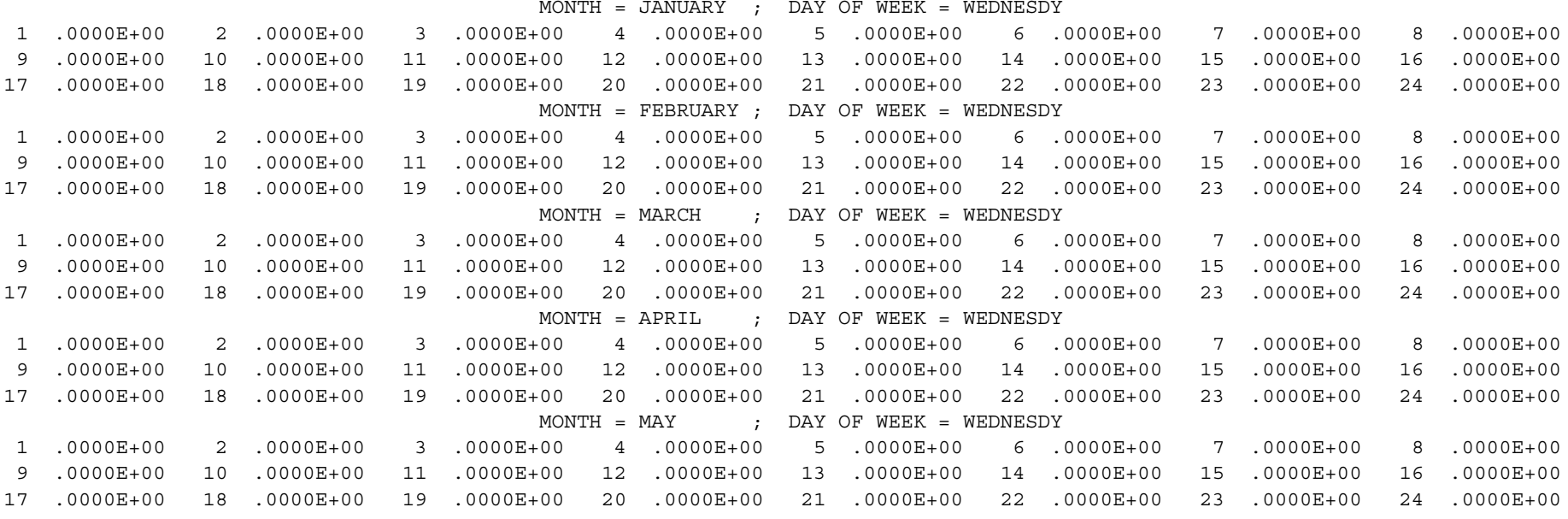




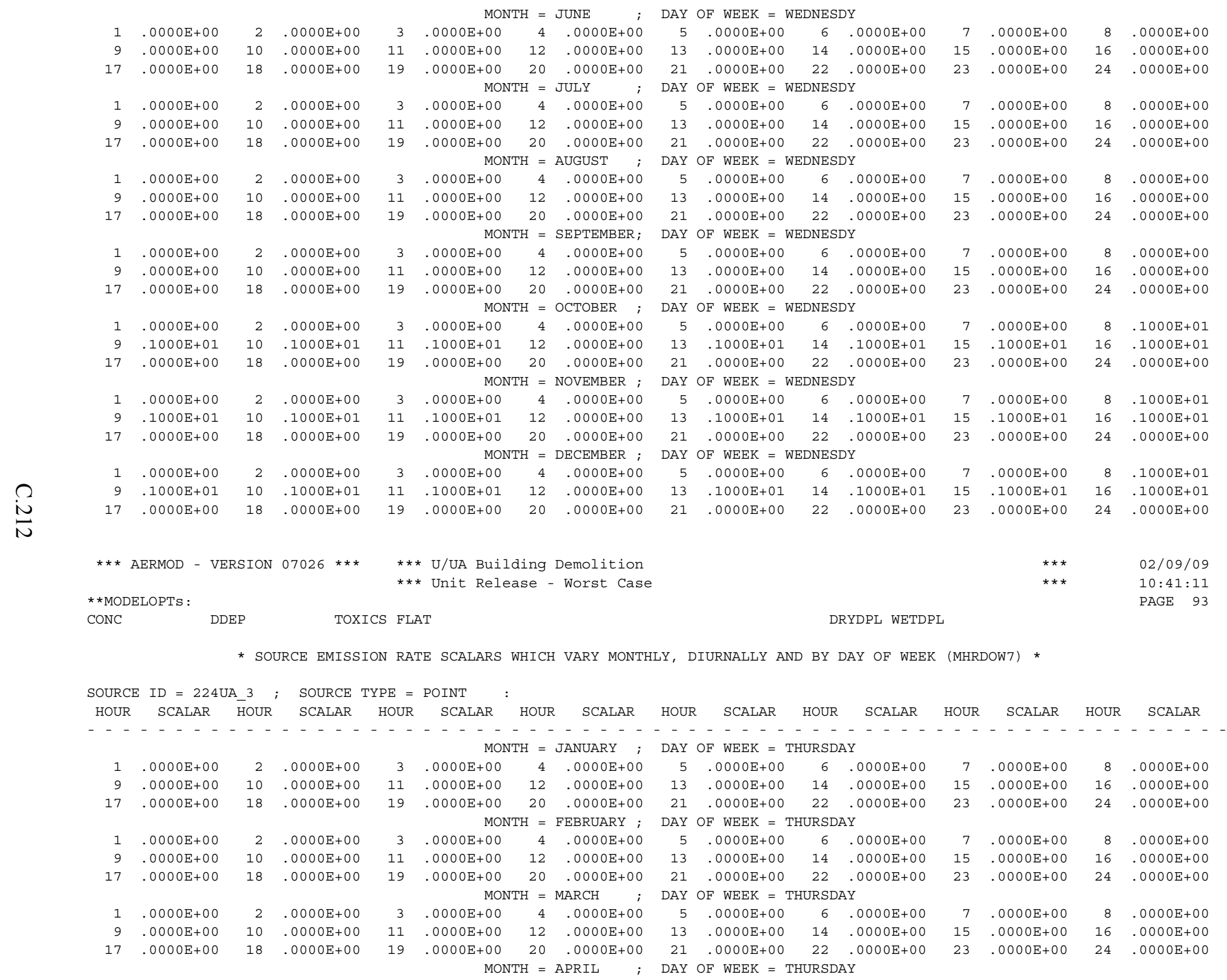




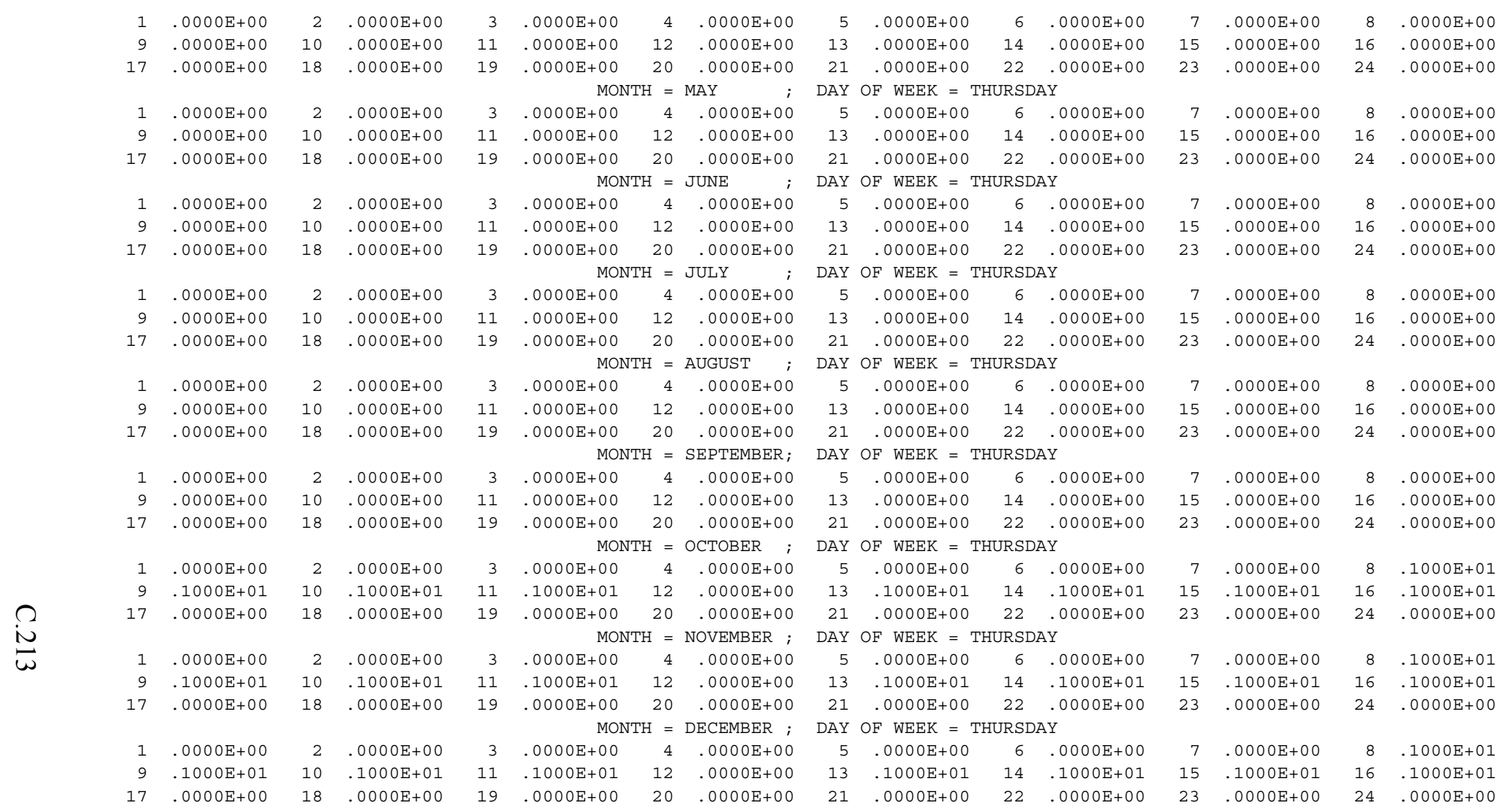

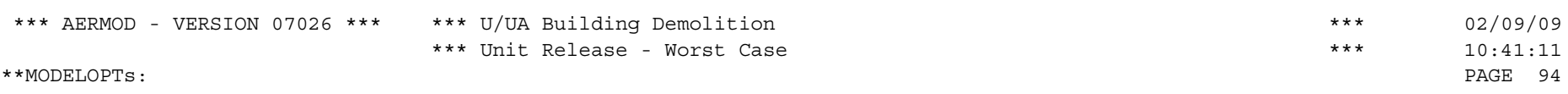

DDEP TOXICS FLAT

DRYDPL WETDPL

* SOURCE EMISSION RATE SCALARS WHICH VARY MONTHLY, DIURNALLY AND BY DAY OF WEEK (MHRDOW7) *

SOURCE ID $=224$ UA_3 $;$ SOURCE TYPE $=$ POINT

HOUR SCALAR HOUR SCALAR HOUR SCALAR HOUR SCALAR HOUR SCALAR HOUR SCALAR HOUR SCALAR HOUR SCALAR

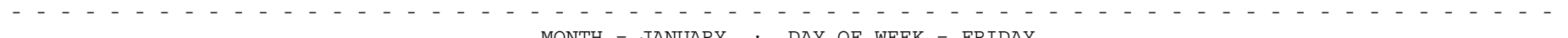

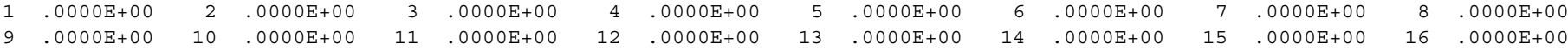

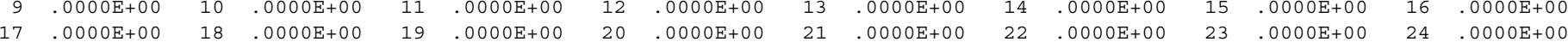

1. $.0000 \mathrm{E}+00$ MONTH = FEBRUARY ; DAY OF WEEK = FRIDAY

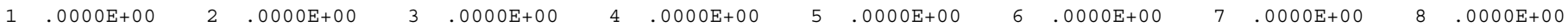




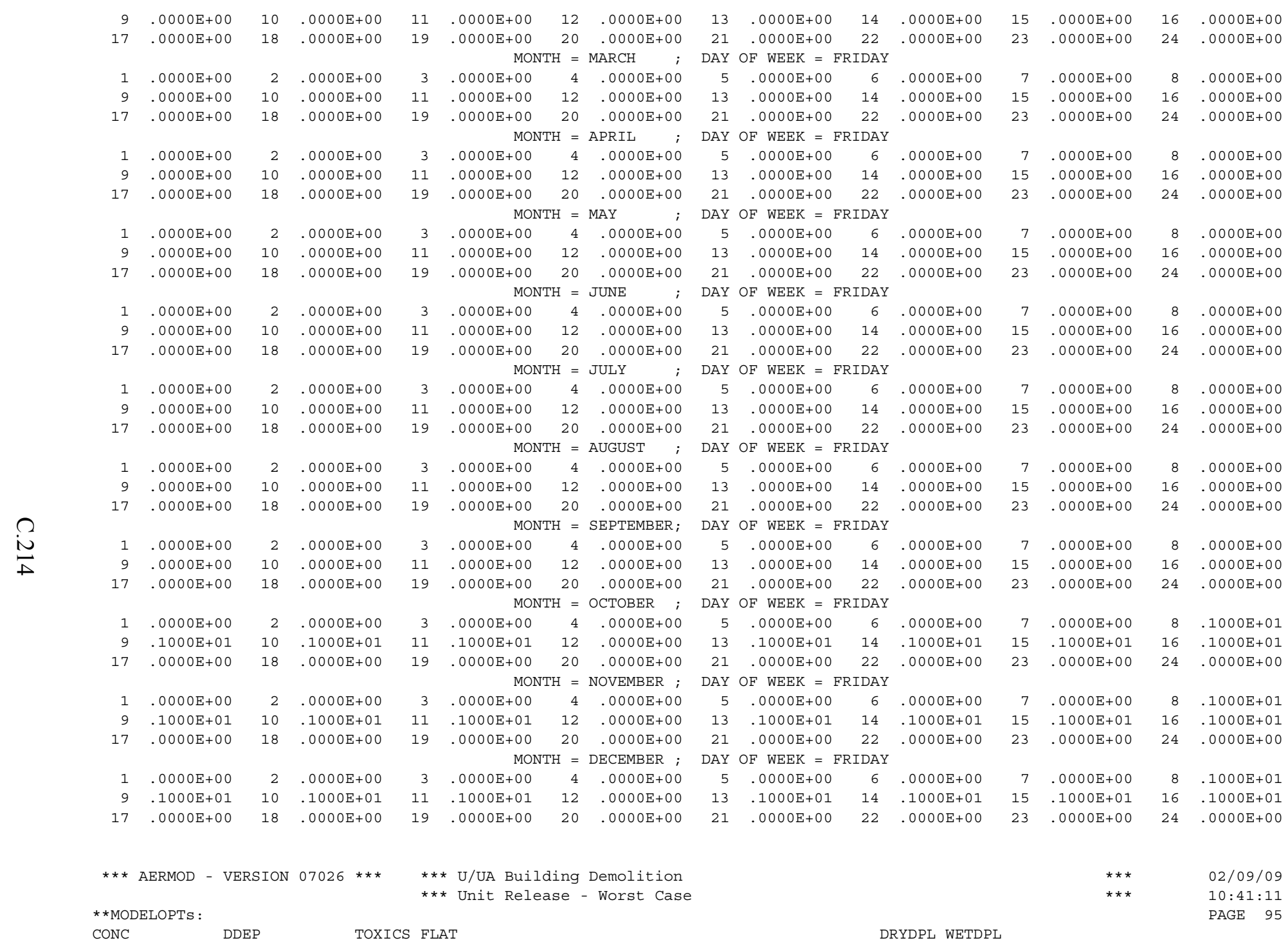

* source emission rate scalars which vary monthly, diURnally and by day of Week (MHrdow7) *

SOURCE ID $=224$ UA_3 $;$ SOURCE TYPE $=$ POINT

HOUR SCALAR HOUR SCALAR HOUR SCALAR HOUR SCALAR HOUR SCALAR HOUR SCALAR HOUR SCALAR HOUR SCALAR 


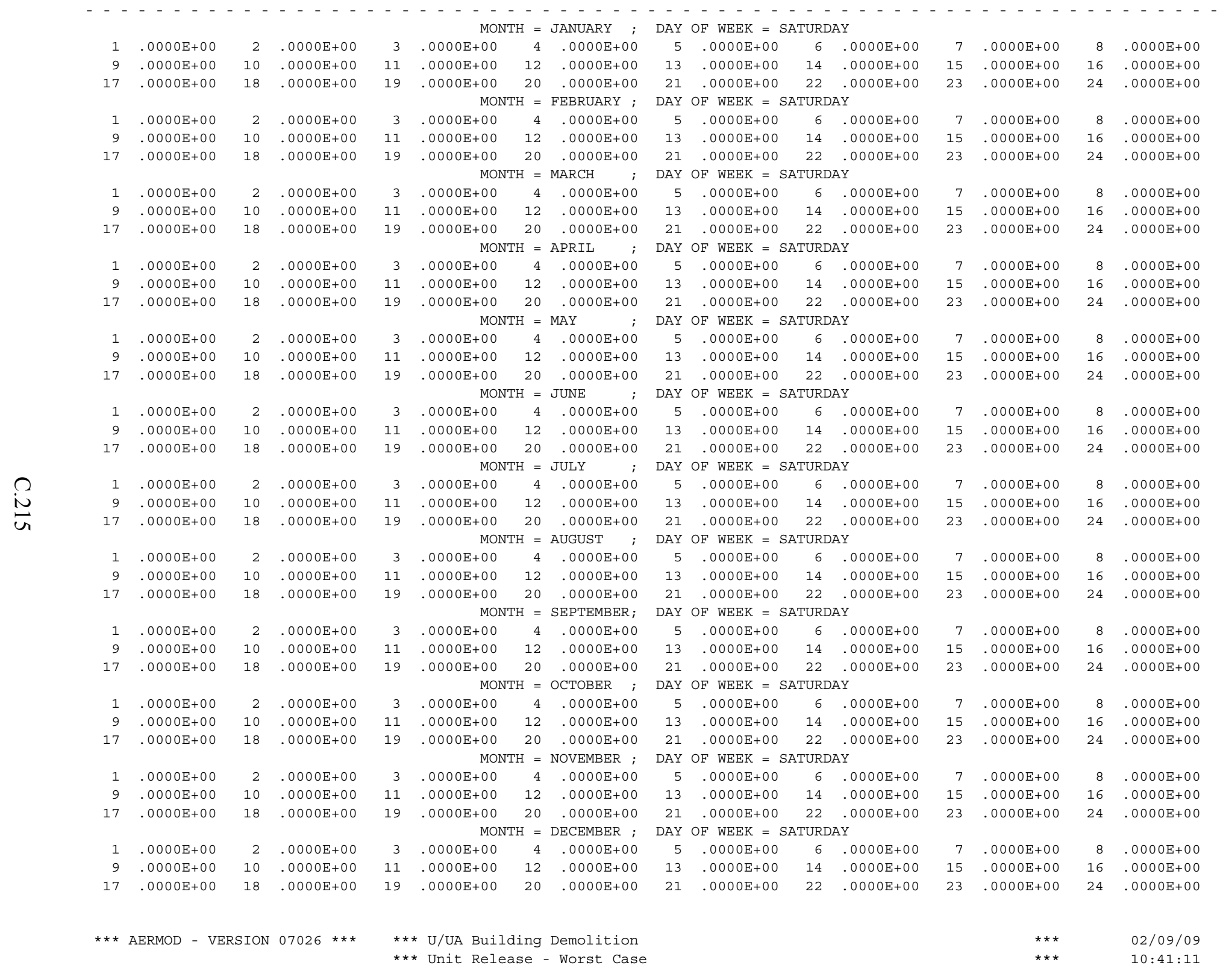


* SOURCE EMISSION RATE SCALARS WHICH VARY MONTHLY, DIURNALLY AND BY DAY OF WEEK (MHRDOW7) *

SOURCE ID $=224 \mathrm{UA} 3 ;$ SOURCE TYPE $=$ POINT

HOUR SCALAR 'OUUR' SCALAR HOUR SCALAR HOUR SCALAR HOUR SCALAR HOUR SCALAR HOUR SCALAR HOUR SCALAR WOUR

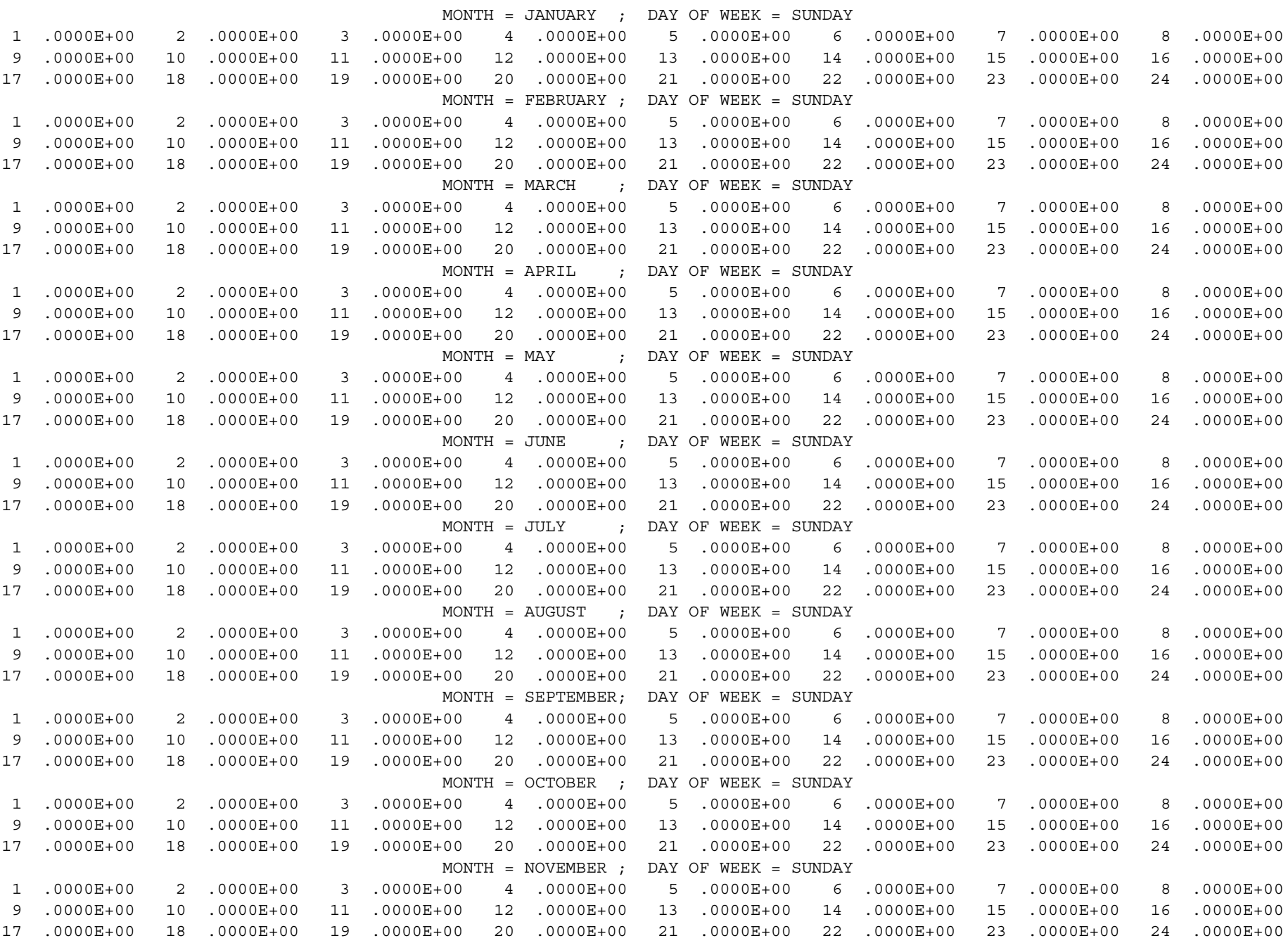
MONTH $=$ DECEMBER $;$ DAY OF WEEK $=$ SUNDAY 


\begin{tabular}{|c|c|c|c|c|c|c|c|c|c|c|c|c|c|c|c|}
\hline 1 & $E+00$ & 2 & $0 \mathrm{E}+00$ & 3 & $0 \mathrm{E}+00$ & 4 & $.0000 \mathrm{E}+00$ & 5 & $.0000 \mathrm{E}+00$ & 6 & $.0000 \mathrm{E}+00$ & 7 & $.0000 \mathrm{E}+00$ & 8 & \\
\hline 9 & $.0000 \mathrm{E}+00$ & 10 & $.0000 \mathrm{E}+00$ & 11 & $.0000 E+00$ & 12 & $.0000 \mathrm{E}+00$ & 13 & $.0000 \mathrm{E}+00$ & 14 & $.0000 E+00$ & 15 & $.0000 \mathrm{E}+00$ & 16 & $.0000 \mathrm{E}+00$ \\
\hline & $.0000 \mathrm{E}+00$ & 18 & $.0000 \mathrm{E}+00$ & 19 & $.0000 \mathrm{E}+00$ & 20 & $.0000 \mathrm{E}+00$ & 21 & $.0000 \mathrm{E}+00$ & 22 & $.0000 \mathrm{E}+00$ & 23 & $.0000 \mathrm{E}+00$ & 24 & $00 E+00$ \\
\hline
\end{tabular}

*** AERMOD - VERSION $07026 * * * \quad \begin{array}{r}* * \text { U/UA Building Demolition } \\ * * * \text { Unit Release - Worst Case }\end{array}$ $\star *$ MODELOPTS :

CONC $\begin{array}{ll}* * * & 02 / 09 / 09 \\ * * * & 10: 41: 11\end{array}$

DRYDPL WETDPL

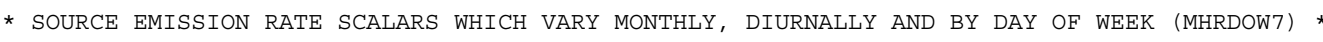

SOURCE ID $=224$ UA_4 $;$ SOURCE TYPE $=$ POINT

HOUR SCALAR HOUR SCALAR HOUR SCALAR HOUR SCALAR HOUR SCALAR HOUR SCALAR HOUR SCALAR HOUR SCALAR

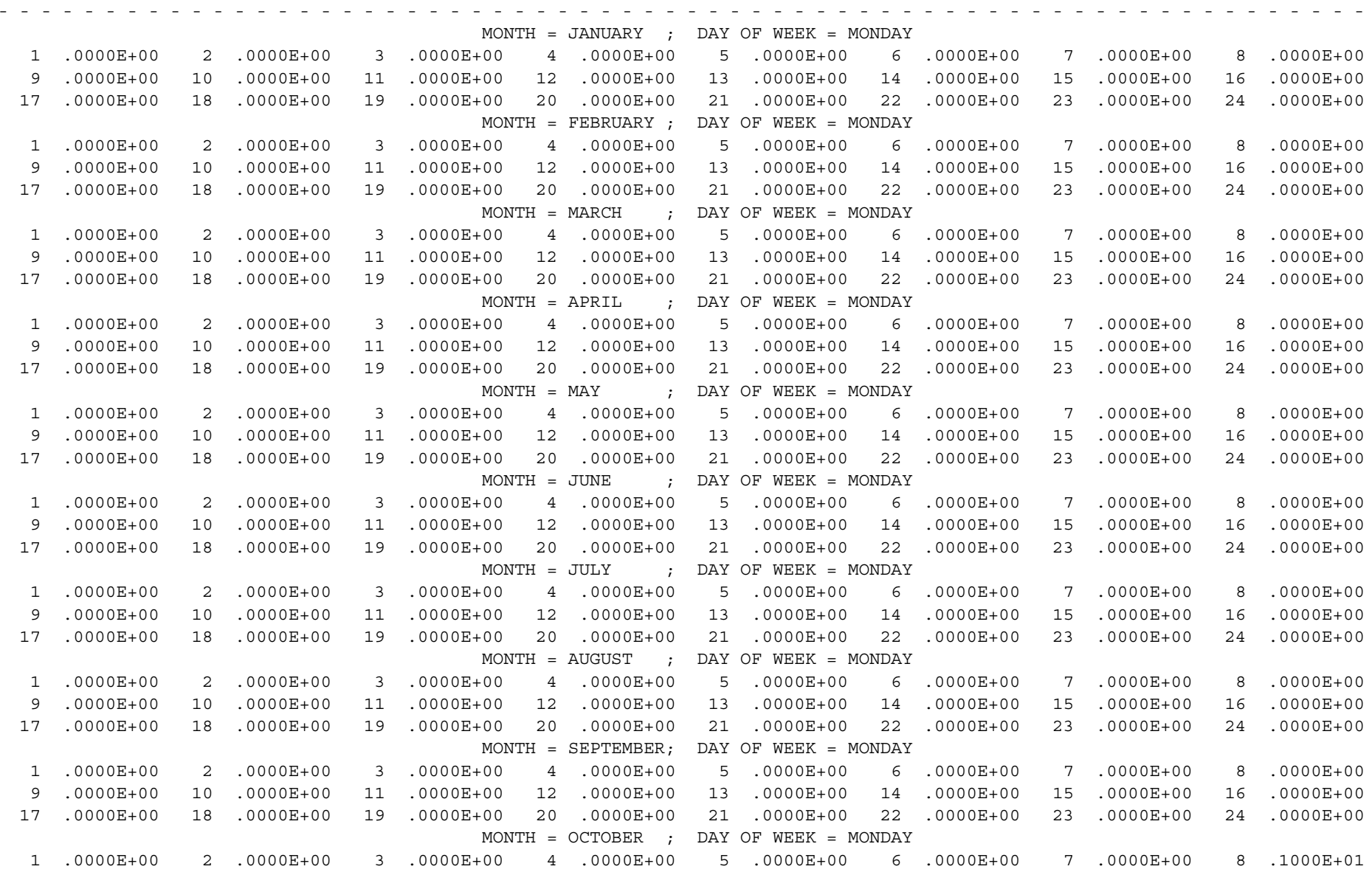




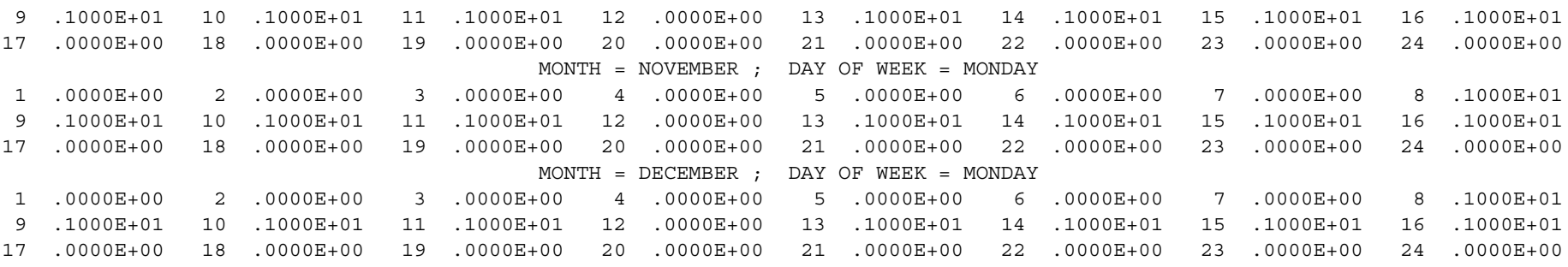

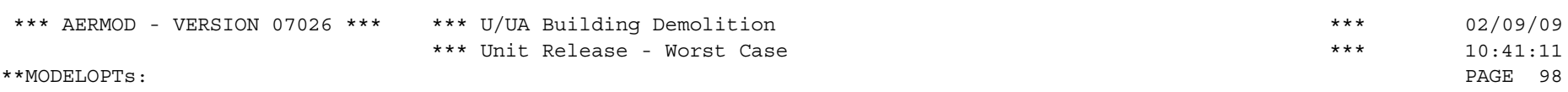
DDEP
TOXICS FLAT
DRYDPL WETDPL

CONC

PAGE 98

* SOURCE EMISSiOn RATE SCALARS WHICH VARY MONTHLY, DIURNALLY AND By DAY OF WEEK (MHRDOW7) *

SOURCE ID $=224 \mathrm{UA} \_4 ;$ SOURCE TYPE $=$ POINT

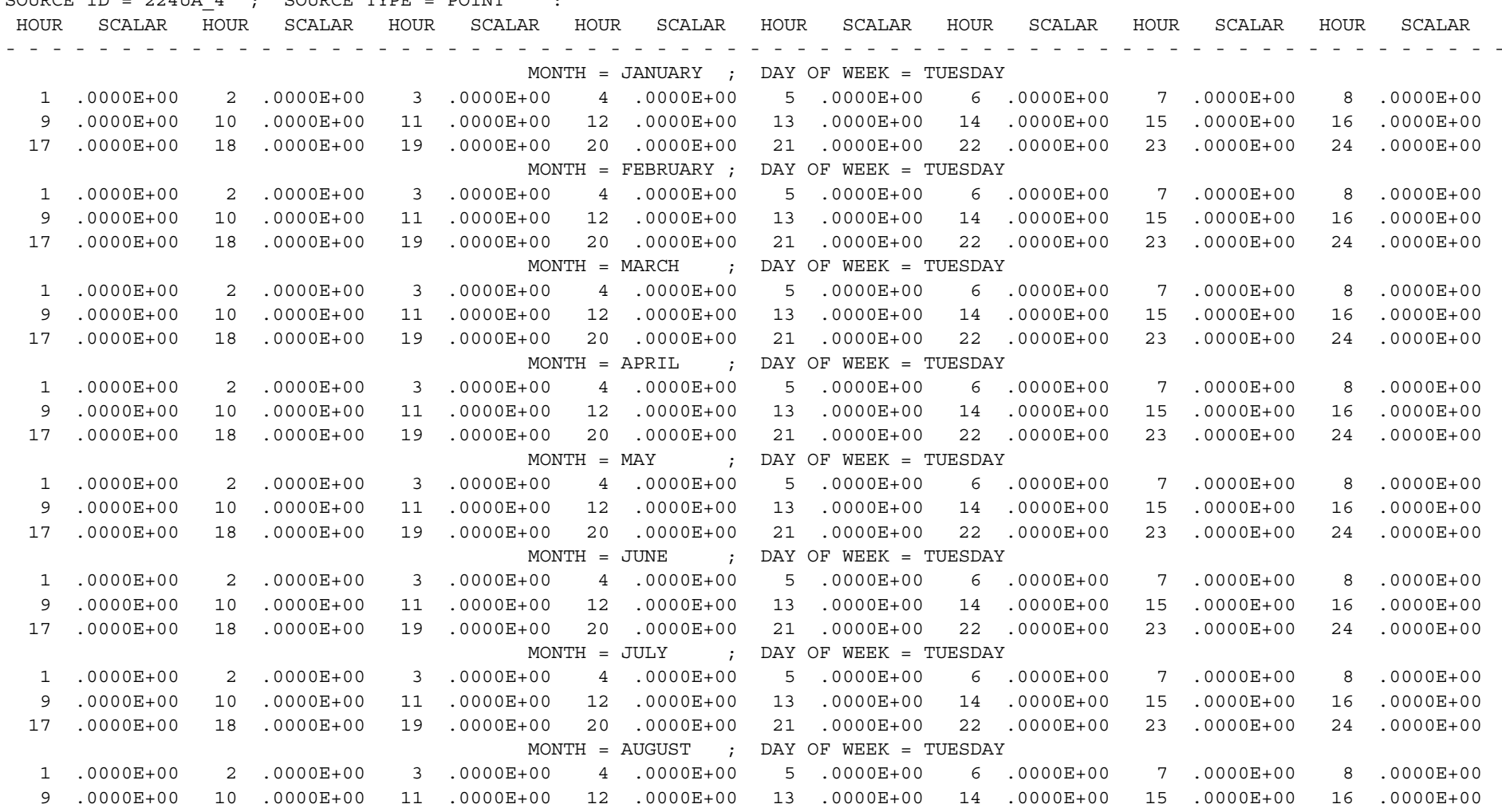




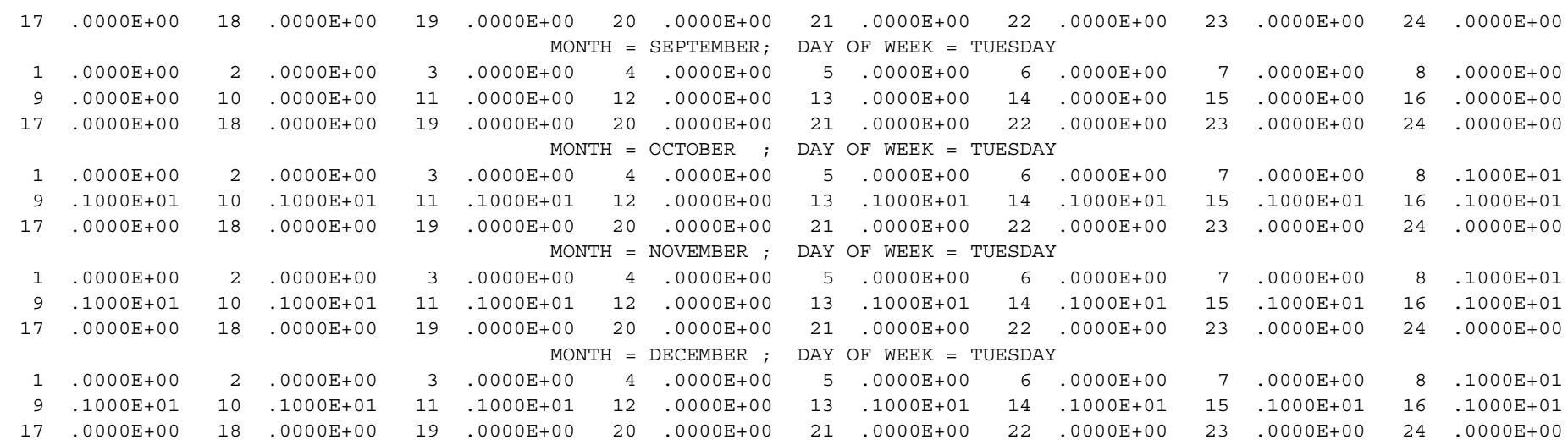

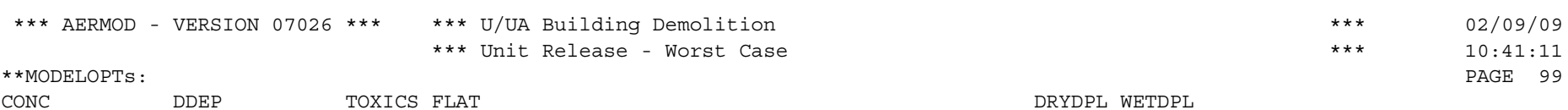

CONC

DDEP
DRYDPL WETDPL

PAGE 99

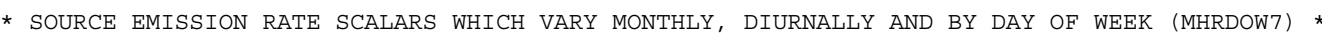

SOURCE ID $=$ 224UA $4 ;$ SOURCE TYPE $=$ POINT

HOUR SCALAR HOUR SCALAR HOUR SCALAR HOUR SCALAR HOUR SCALAR HOUR SCALAR HOUR SCALAR HOUR SCALAR

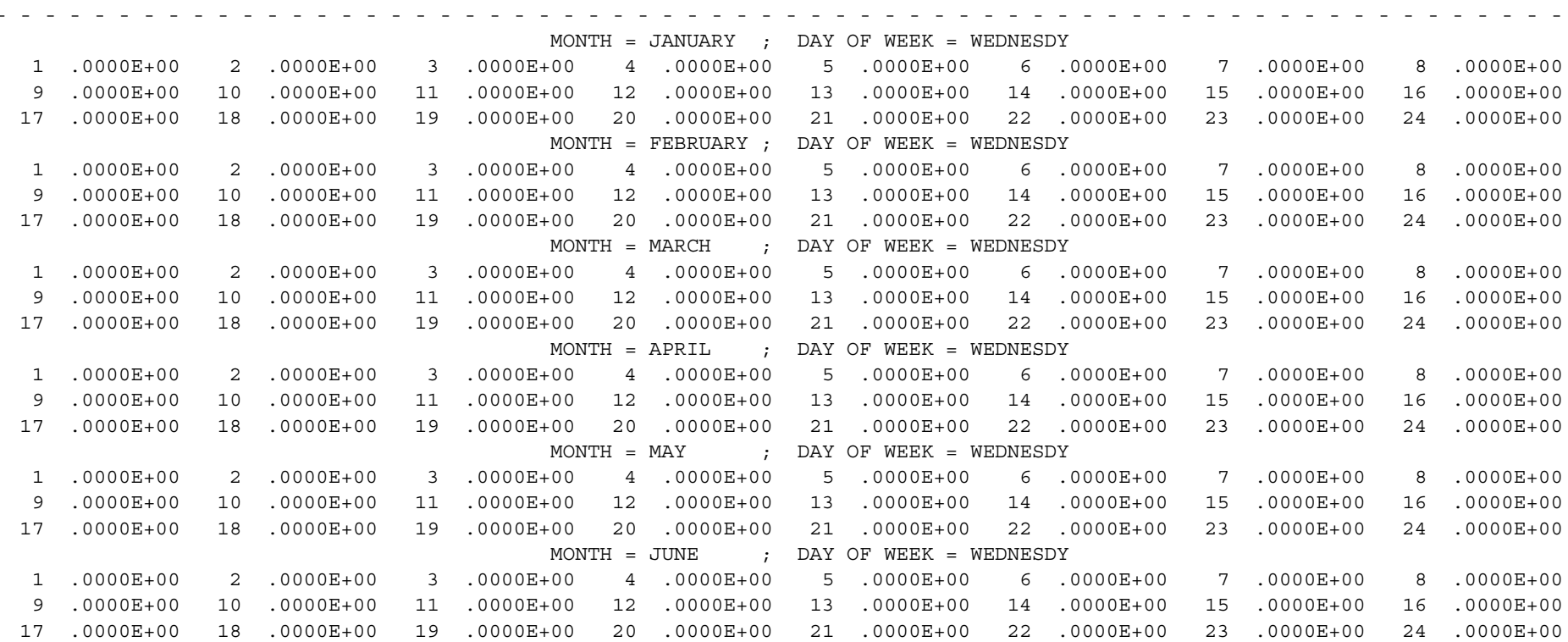




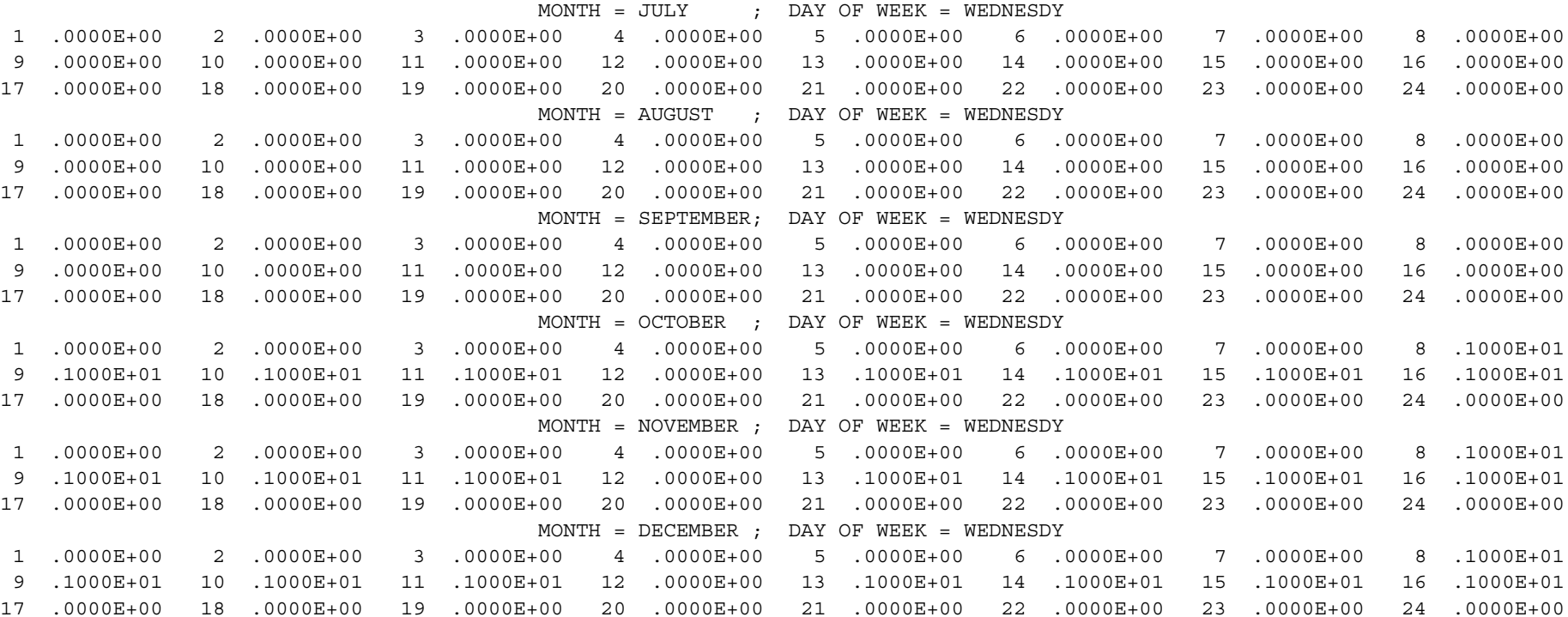

DRYDPL WETDPL

* SOURCE EMISSiON RATE SCALARS WHICH VARY MONTHLY, DIURNALLY AND BY DAY OF WEEK (MHRDOW7) *

SOURCE ID $=224 \mathrm{UA} \_4 ;$ SOURCE TYPE $=$ POINT

HOUR SCALAR HOUR SCALAR HOUR SCALAR HOUR SCALAR HOUR SCALAR HOUR SCALAR HOUR SCALAR HOUR SCALAR

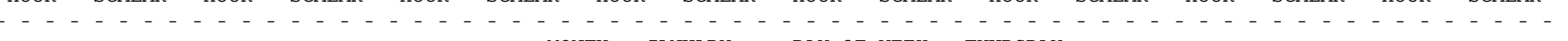

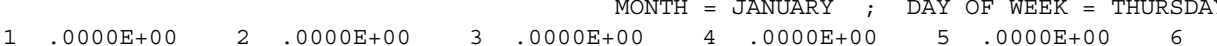

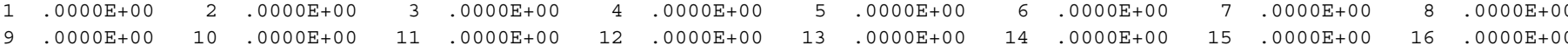

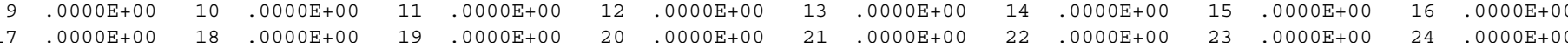

MONTH = FEBRUARY; DAY OF WEEK = THURSDAY

$\begin{array}{lllllllllllllllll}1 & .0000 \mathrm{E}+00 & 2 & .0000 \mathrm{E}+00 & 3 & .0000 \mathrm{E}+00 & 4 & .0000 \mathrm{E}+00 & 5 & .0000 \mathrm{E}+00 & 6 & .0000 \mathrm{E}+00 & 7 & .0000 \mathrm{E}+00 & 8 & .0000 \mathrm{E}+00\end{array}$

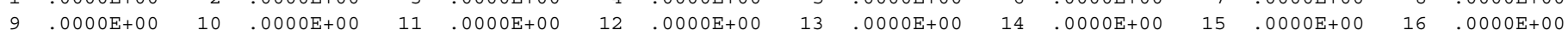

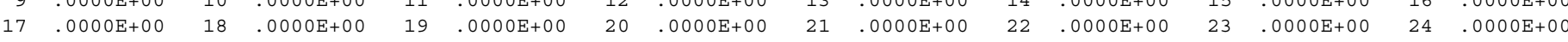

MONTH $=$ MARCH $; \quad$ DAY OF WEEK $=$ THURSDAY

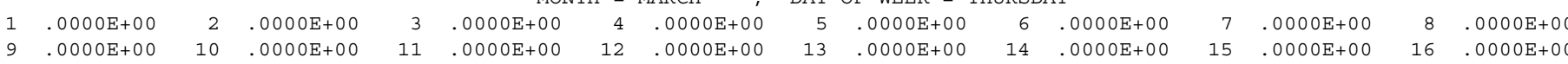

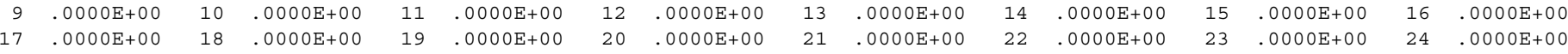

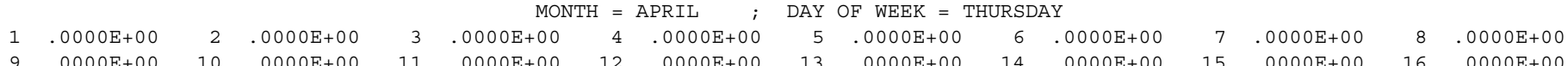

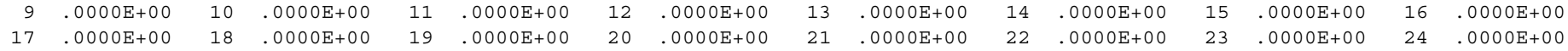

$$
\text { MONTH }=\text { MAY } \quad ; \quad \text { DAY OF WEEK }=\text { THURSDAY }
$$




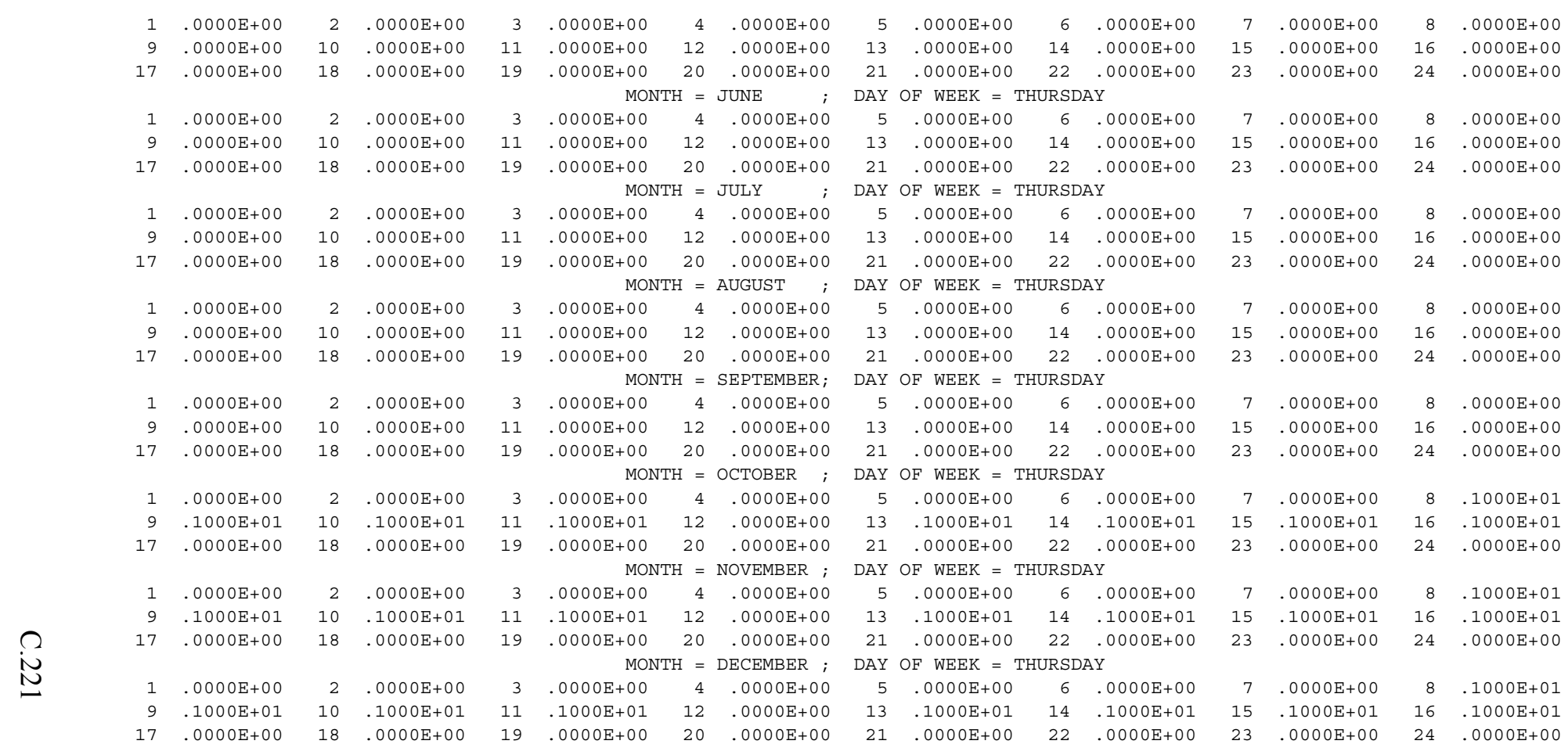
$* * *$ AERMOD - VERSION $07026 * * * \quad \begin{aligned} & * * \text { U/UA Building Demolition } \\ & * * * \text { Unit Release - Worst Case }\end{aligned}$

*** $\quad 02 / 09 / 09$

$* * * \quad 10: 41: 11$
PAGE 101

DDEP

TOXICS FLAT

DRYDPL WETDPL

* SOURCE EMISSION RATE SCALARS WHICH VARY MONTHLY, DIURNALLY AND BY DAY OF WEEK (MHRDOW7) *

SOURCE ID $=224 \mathrm{UA} \_4 ;$ SOURCE TYPE $=$ POINT

HOUR SCALAR HOUR SCALAR HOUR SCALAR HOUR SCALAR HOUR SCALAR HOUR SCALAR HOUR SCALAR HOUR SCALAR

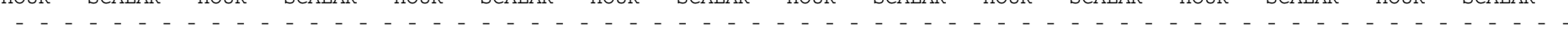

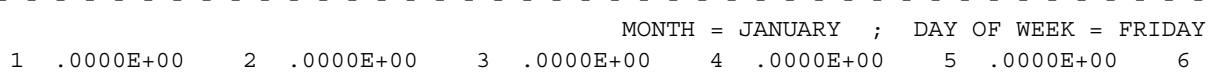

$\begin{array}{rrrrrrrrrrrrrrrr}1 & .0000 \mathrm{E}+00 & 2 & .0000 \mathrm{E}+00 & 3 & .0000 \mathrm{E}+00 & 4 & .0000 \mathrm{E}+00 & 5 & .0000 \mathrm{E}+00 & 6 & .0000 \mathrm{E}+00 & 7 & .0000 \mathrm{E}+00 & 8 & .0000 \mathrm{E}+00 \\ 9 & .0000 \mathrm{E}+00 & 10 & .0000 \mathrm{E}+00 & 11 & .0000 \mathrm{E}+00 & 12 & .0000 \mathrm{E}+00 & 13 & .0000 \mathrm{E}+00 & 14 & .0000 \mathrm{E}+00 & 15 & .0000 \mathrm{E}+00 & 16 & .0000 \mathrm{E}+00\end{array}$

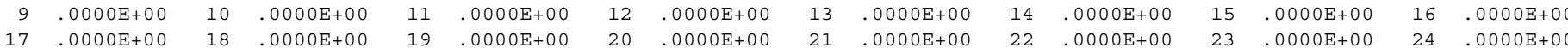

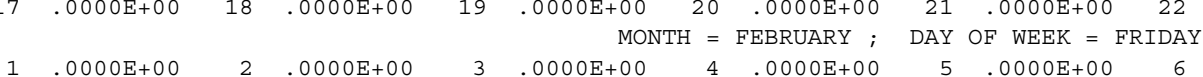

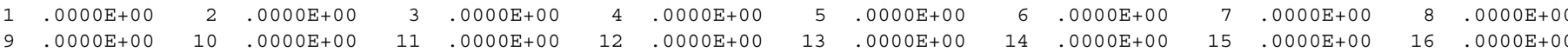

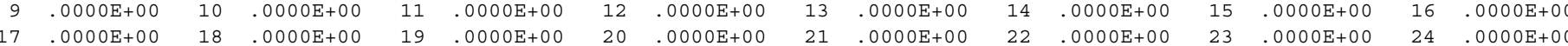

1. $.0000 \mathrm{E}+00 \quad$ MONTH $=$ MARCH $; \quad$ DAY OF WEEK $=$ FRIDAY

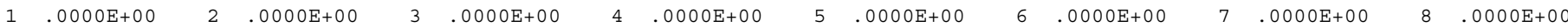




\begin{tabular}{|c|c|c|c|c|c|c|c|c|c|c|c|c|c|c|}
\hline 9 & $.0000 \mathrm{E}+00$ & 10 & $.0000 \mathrm{E}+00$ & 11 & $.0000 \mathrm{E}+00$ & $.0000 \mathrm{E}+00$ & 13 & $.0000 \mathrm{E}+00$ & 14 & $.0000 \mathrm{E}+00$ & 15 & $.0000 \mathrm{E}+00$ & 16 & $.0000 \mathrm{E}+00$ \\
\hline 17 & $.0000 \mathrm{E}+00$ & 18 & $.0000 \mathrm{E}+00$ & 19 & $.0000 \mathrm{E}+00 \quad 20$ & $.0000 \mathrm{E}+00$ & 21 & $.0000 \mathrm{E}+00$ & 22 & $.0000 \mathrm{E}+00$ & 23 & $.0000 \mathrm{E}+00$ & 24 & $.0000 \mathrm{E}+00$ \\
\hline & & & & & MONTH $=$ & APRIL $\quad ;$ & DAY & OF WEEK $=F$ & FIDAY & & & & & \\
\hline 1 & $.0000 \mathrm{E}+00$ & 2 & $.0000 \mathrm{E}+00$ & 3 & $.0000 E+00$ & $.0000 \mathrm{E}+00$ & 5 & $.0000 \mathrm{E}+00$ & 6 & $.0000 \mathrm{E}+00$ & 7 & $.0000 \mathrm{E}+00$ & 8 & $.0000 \mathrm{E}+00$ \\
\hline 9 & $.0000 \mathrm{E}+00$ & 10 & $.0000 \mathrm{E}+00$ & 11 & $.0000 \mathrm{E}+00$ & $.0000 \mathrm{E}+00$ & 13 & $.0000 \mathrm{E}+00$ & 14 & $.0000 \mathrm{E}+00$ & 15 & $.0000 \mathrm{E}+00$ & 16 & $.0000 \mathrm{E}+00$ \\
\hline 17 & $.0000 \mathrm{E}+00$ & 18 & $.0000 \mathrm{E}+00$ & 19 & $0000 \mathrm{E}+00$ & $.0000 \mathrm{E}+00$ & 21 & $.0000 \mathrm{E}+00$ & 22 & $.0000 \mathrm{E}+00$ & 23 & $.0000 \mathrm{E}+00$ & 24 & $.0000 \mathrm{E}+00$ \\
\hline & & & & & MONTH $=$ & MAY $\quad ;$ & DAY & $O F$ WEEK $=F$ & RIDAY & & & & & \\
\hline 1 & $.0000 \mathrm{E}+00$ & 2 & $.0000 \mathrm{E}+00$ & 3 & $.0000 E+00$ & $.0000 \mathrm{E}+00$ & 5 & $.0000 \mathrm{E}+00$ & 6 & $.0000 \mathrm{E}+00$ & 7 & $.0000 \mathrm{E}+00$ & 8 & $.0000 \mathrm{E}+00$ \\
\hline 9 & $.0000 E+00$ & 10 & $.0000 \mathrm{E}+00$ & 11 & $.0000 E+00$ & $.0000 \mathrm{E}+00$ & 13 & $.0000 E+00$ & 14 & $.0000 \mathrm{E}+00$ & 15 & $.0000 \mathrm{E}+00$ & 16 & $.0000 \mathrm{E}+00$ \\
\hline 17 & $.0000 \mathrm{E}+00$ & 18 & $.0000 \mathrm{E}+00$ & 19 & $.0000 \mathrm{E}+00 \quad 20$ & $.0000 \mathrm{E}+00$ & 21 & $.0000 \mathrm{E}+00$ & 22 & $.0000 \mathrm{E}+00$ & 23 & $.0000 \mathrm{E}+00$ & 24 & $.0000 \mathrm{E}+00$ \\
\hline & & & & & MONTH $=$ & JUNE $\quad ;$ & DAY & OF WEEK $=F$ & RIDAY & & & & & \\
\hline 1 & $.0000 \mathrm{E}+00$ & 2 & $.0000 \mathrm{E}+00$ & 3 & $.0000 \mathrm{E}+00$ & $.0000 \mathrm{E}+00$ & 5 & $.0000 \mathrm{E}+00$ & 6 & $.0000 \mathrm{E}+00$ & 7 & $.0000 \mathrm{E}+00$ & 8 & $.0000 \mathrm{E}+00$ \\
\hline 9 & $.0000 \mathrm{E}+00$ & 10 & $.0000 \mathrm{E}+00$ & 11 & $.0000 E+00$ & $.0000 \mathrm{E}+00$ & 13 & $.0000 \mathrm{E}+00$ & 14 & $.0000 \mathrm{E}+00$ & 15 & $.0000 \mathrm{E}+00$ & 16 & $.0000 \mathrm{E}+00$ \\
\hline 17 & $.0000 \mathrm{E}+00$ & 18 & $.0000 \mathrm{E}+00$ & 19 & $.0000 \mathrm{E}+00$ & $.0000 \mathrm{E}+00$ & 21 & $.0000 \mathrm{E}+00$ & 22 & $.0000 \mathrm{E}+00$ & 23 & $.0000 \mathrm{E}+00$ & 24 & $.0000 \mathrm{E}+00$ \\
\hline & & & & & MONTH $=$ & JULY ; & DAY & OF WEEK = F & RIDAY & & & & & \\
\hline 1 & $.0000 \mathrm{E}+00$ & 2 & $.0000 \mathrm{E}+00$ & 3 & $.0000 E+00$ & $.0000 \mathrm{E}+00$ & 5 & $.0000 \mathrm{E}+00$ & 6 & $.0000 \mathrm{E}+00$ & 7 & $.0000 \mathrm{E}+00$ & 8 & $.0000 \mathrm{E}+00$ \\
\hline 9 & $.0000 \mathrm{E}+00$ & 10 & $.0000 \mathrm{E}+00$ & 11 & $.0000 \mathrm{E}+00$ & $.0000 \mathrm{E}+00$ & 13 & $.0000 \mathrm{E}+00$ & 14 & $.0000 \mathrm{E}+00$ & 15 & $.0000 \mathrm{E}+00$ & 16 & $.0000 \mathrm{E}+00$ \\
\hline 17 & $.0000 \mathrm{E}+00$ & 18 & $.0000 \mathrm{E}+00$ & 19 & $.0000 E+00$ & $.0000 E+00$ & 21 & $.0000 \mathrm{E}+00$ & 22 & $.0000 E+00$ & 23 & $.0000 \mathrm{E}+00$ & 24 & $.0000 \mathrm{E}+00$ \\
\hline & & & & & MONTH $=$ & AUGUST ; & DAY & OF WEEK = F & RIDAY & & & & & \\
\hline 1 & $.0000 \mathrm{E}+00$ & 2 & $.0000 \mathrm{E}+00$ & 3 & $.0000 E+00$ & $.0000 \mathrm{E}+00$ & 5 & $.0000 \mathrm{E}+00$ & 6 & $.0000 \mathrm{E}+00$ & 7 & $.0000 \mathrm{E}+00$ & 8 & $.0000 \mathrm{E}+00$ \\
\hline 9 & $.0000 E+00$ & 10 & $.0000 \mathrm{E}+00$ & 11 & $.0000 \mathrm{E}+00$ & $.0000 \mathrm{E}+00$ & 13 & $.0000 E+00$ & 14 & $.0000 \mathrm{E}+00$ & 15 & $.0000 \mathrm{E}+00$ & 16 & $.0000 \mathrm{E}+00$ \\
\hline 17 & $.0000 \mathrm{E}+00$ & 18 & $.0000 \mathrm{E}+00$ & 19 & $.0000 \mathrm{E}+00$ & $.0000 \mathrm{E}+00$ & 21 & $.0000 \mathrm{E}+00$ & 22 & $.0000 \mathrm{E}+00$ & 23 & $.0000 \mathrm{E}+00$ & 24 & $.0000 \mathrm{E}+00$ \\
\hline & & & & & MONTH $=$ & SEPTEMBER; & DAY & OF WEEK = F & FIDAY & & & & & \\
\hline 1 & $.0000 \mathrm{E}+00$ & 2 & $.0000 \mathrm{E}+00$ & 3 & $.0000 \mathrm{E}+00$ & $.0000 \mathrm{E}+00$ & 5 & $.0000 \mathrm{E}+00$ & 6 & $.0000 \mathrm{E}+00$ & 7 & $.0000 \mathrm{E}+00$ & 8 & $.0000 \mathrm{E}+00$ \\
\hline 9 & $.0000 \mathrm{E}+00$ & 10 & $.0000 \mathrm{E}+00$ & 11 & $.0000 \mathrm{E}+00$ & $.0000 \mathrm{E}+00$ & 13 & $.0000 \mathrm{E}+00$ & 14 & $.0000 \mathrm{E}+00$ & 15 & $.0000 \mathrm{E}+00$ & 16 & $.0000 \mathrm{E}+00$ \\
\hline 17 & $.0000 \mathrm{E}+00$ & 18 & $.0000 \mathrm{E}+00$ & 19 & $.0000 \mathrm{E}+00 \quad 20$ & $.0000 \mathrm{E}+00$ & 21 & $.0000 \mathrm{E}+00$ & 22 & $.0000 \mathrm{E}+00$ & 23 & $.0000 \mathrm{E}+00$ & 24 & $.0000 \mathrm{E}+00$ \\
\hline$\Omega$ & & & & & MONTH $=$ & OCTOBER ; & DAY & OF WEEK = F & RIDAY & & & & & \\
\hline 1 & $.0000 \mathrm{E}+00$ & 2 & $.0000 \mathrm{E}+00$ & 3 & $.0000 E+00$ & $.0000 \mathrm{E}+00$ & 5 & $.0000 \mathrm{E}+00$ & 6 & $.0000 \mathrm{E}+00$ & 7 & $.0000 \mathrm{E}+00$ & 8 & $.1000 \mathrm{E}+01$ \\
\hline 9 & $.1000 \mathrm{E}+01$ & 10 & $.1000 \mathrm{E}+01$ & 11 & $1000 E+01$ & $.0000 \mathrm{E}+00$ & 13 & $.1000 \mathrm{E}+01$ & 14 & $.1000 \mathrm{E}+01$ & 15 & $.1000 \mathrm{E}+01$ & 16 & $.1000 \mathrm{E}+01$ \\
\hline 17 & $.0000 \mathrm{E}+00$ & 18 & $.0000 \mathrm{E}+00$ & 19 & $.0000 \mathrm{E}+00$ & $.0000 \mathrm{E}+00$ & 21 & $.0000 \mathrm{E}+00$ & 22 & $.0000 \mathrm{E}+00$ & 23 & $.0000 \mathrm{E}+00$ & 24 & $.0000 \mathrm{E}+00$ \\
\hline & & & & & MONTH $=$ & NOVEMBER ; & DAY & OF WEEK = F & FIDAY & & & & & \\
\hline 1 & $.0000 \mathrm{E}+00$ & 2 & $.0000 \mathrm{E}+00$ & 3 & $.0000 E+00$ & $.0000 \mathrm{E}+00$ & 5 & $.0000 \mathrm{E}+00$ & 6 & $.0000 \mathrm{E}+00$ & 7 & $.0000 \mathrm{E}+00$ & 8 & $.1000 \mathrm{E}+01$ \\
\hline 9 & $.1000 \mathrm{E}+01$ & 10 & $.1000 \mathrm{E}+01$ & 11 & $.1000 \mathrm{E}+01$ & $.0000 \mathrm{E}+00$ & 13 & $.1000 \mathrm{E}+01$ & 14 & $.1000 \mathrm{E}+01$ & 15 & $.1000 \mathrm{E}+01$ & 16 & $.1000 \mathrm{E}+01$ \\
\hline 17 & $.0000 \mathrm{E}+00$ & 18 & $.0000 \mathrm{E}+00$ & 19 & $.0000 E+00 \quad 20$ & $.0000 \mathrm{E}+00$ & 21 & $.0000 \mathrm{E}+00$ & 22 & $.0000 E+00$ & 23 & $.0000 \mathrm{E}+00$ & 24 & $.0000 \mathrm{E}+00$ \\
\hline & & & & & MONTH $=$ & DECEMBER ; & DAY & $O F$ WEEK $=F$ & RIDAY & & & & & \\
\hline 1 & $.0000 \mathrm{E}+00$ & 2 & $.0000 \mathrm{E}+00$ & 3 & $.0000 \mathrm{E}+00$ & $.0000 \mathrm{E}+00$ & 5 & $.0000 \mathrm{E}+00$ & 6 & $.0000 \mathrm{E}+00$ & 7 & $.0000 \mathrm{E}+00$ & 8 & $.1000 \mathrm{E}+01$ \\
\hline 9 & $.1000 \mathrm{E}+01$ & 10 & $.1000 \mathrm{E}+01$ & 11 & $.1000 \mathrm{E}+01$ & $.0000 \mathrm{E}+00$ & 13 & $.1000 \mathrm{E}+01$ & 14 & $.1000 \mathrm{E}+01$ & 15 & $.1000 \mathrm{E}+01$ & 16 & $.1000 \mathrm{E}+01$ \\
\hline 17 & $.0000 \mathrm{E}+00$ & 18 & $.0000 \mathrm{E}+00$ & 19 & $.0000 E+00$ & $.0000 \mathrm{E}+00$ & 21 & $.0000 \mathrm{E}+00$ & 22 & $.0000 \mathrm{E}+00$ & 23 & $.0000 \mathrm{E}+00$ & 24 & $.0000 \mathrm{E}+00$ \\
\hline$* * *$ & AERMOD - VE & SION & $07026 * * *$ & $\star \star \star *$ & U/UA Building & Demolition & & & & & & *** & & $02 / 09 / 09$ \\
\hline & & & & $* * *$ & Unit Release & - Worst Case & & & & & & $* * *$ & & $10: 41: 11$ \\
\hline$\star *$ MOD & DELOPTS : & & & & & & & & & & & & & PAGE 102 \\
\hline CONC & & & & FLAT & & & & & & & & & & \\
\hline
\end{tabular}

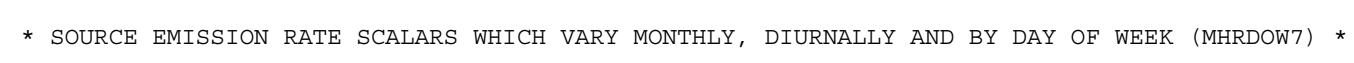

SOURCE ID $=224 \mathrm{UA} \_4 ;$ SOURCE TYPE $=$ POINT :

HOUR SCALAR HOUR SCALAR HOUR SCALAR HOUR SCALAR HOUR SCALAR HOUR SCALAR HOUR SCALAR HOUR SCALAR

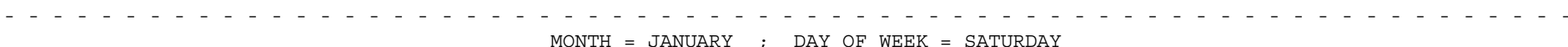

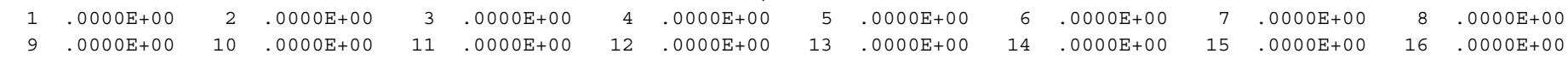




\begin{tabular}{|c|c|c|c|c|c|c|c|c|c|c|c|c|c|c|c|}
\hline 17 & $.0000 \mathrm{E}+00$ & 18 & $.0000 \mathrm{E}+00$ & 19 & $.0000 \mathrm{E}+00$ & 20 & $.0000 \mathrm{E}+00$ & 21 & $.0000 \mathrm{E}+00$ & 22 & $.0000 \mathrm{E}+00$ & 23 & $.0000 \mathrm{E}+00$ & 24 & $.0000 \mathrm{E}+00$ \\
\hline & & & & & MONT & $\mathrm{H}=\mathrm{I}$ & FEBRUARY ; & DAY & OF WEEK = S & SATURDA & & & & & \\
\hline 1 & $.0000 \mathrm{E}+00$ & 2 & $.0000 \mathrm{E}+00$ & 3 & $.0000 \mathrm{E}+00$ & 4 & $.0000 \mathrm{E}+00$ & 5 & $.0000 \mathrm{E}+00$ & 6 & $.0000 \mathrm{E}+00$ & 7 & $.0000 \mathrm{E}+00$ & 8 & $.0000 \mathrm{E}+00$ \\
\hline 9 & $.0000 \mathrm{E}+00$ & 10 & $.0000 \mathrm{E}+00$ & 11 & $.0000 \mathrm{E}+00$ & 12 & $.0000 \mathrm{E}+00$ & 13 & $.0000 \mathrm{E}+00$ & 14 & $.0000 \mathrm{E}+00$ & 15 & $.0000 \mathrm{E}+00$ & 16 & $.0000 \mathrm{E}+00$ \\
\hline 17 & $.0000 \mathrm{E}+00$ & 18 & $.0000 \mathrm{E}+00$ & 19 & $.0000 \mathrm{E}+00$ & 20 & $.0000 \mathrm{E}+00$ & 21 & $.0000 \mathrm{E}+00$ & 22 & $.0000 \mathrm{E}+00$ & 23 & $.0000 E+00$ & 24 & $.0000 \mathrm{E}+00$ \\
\hline & & & & & MONT & & MARCH ; & DAY & OF WEEK = $S$ & SATURDA & & & & & \\
\hline 1 & $.0000 \mathrm{E}+00$ & 2 & $.0000 \mathrm{E}+00$ & 3 & $.0000 \mathrm{E}+00$ & 4 & $.0000 \mathrm{E}+00$ & 5 & $.0000 \mathrm{E}+00$ & 6 & $.0000 \mathrm{E}+00$ & 7 & $.0000 \mathrm{E}+00$ & 8 & $.0000 \mathrm{E}+00$ \\
\hline 9 & $.0000 \mathrm{E}+00$ & 10 & $.0000 \mathrm{E}+00$ & 11 & $.0000 \mathrm{E}+00$ & 12 & $.0000 \mathrm{E}+00$ & 13 & $.0000 \mathrm{E}+00$ & 14 & $.0000 \mathrm{E}+00$ & 15 & $.0000 \mathrm{E}+00$ & 16 & $.0000 \mathrm{E}+00$ \\
\hline 17 & $.0000 \mathrm{E}+00$ & 18 & $.0000 \mathrm{E}+00$ & 19 & $.0000 \mathrm{E}+00$ & 20 & $.0000 \mathrm{E}+00$ & 21 & $.0000 \mathrm{E}+00$ & 22 & $.0000 \mathrm{E}+00$ & 23 & $.0000 \mathrm{E}+00$ & 24 & $.0000 \mathrm{E}+00$ \\
\hline & & & & & MONT & $\mathrm{H}=\mathrm{T}$ & APRIL ; & DAY & OF WEEK = S & SATURDA & & & & & \\
\hline 1 & $.0000 \mathrm{E}+00$ & 2 & $.0000 \mathrm{E}+00$ & 3 & $.0000 \mathrm{E}+00$ & 4 & $.0000 \mathrm{E}+00$ & 5 & $.0000 \mathrm{E}+00$ & 6 & $.0000 \mathrm{E}+00$ & 7 & $.0000 \mathrm{E}+00$ & 8 & $.0000 \mathrm{E}+00$ \\
\hline 9 & $.0000 \mathrm{E}+00$ & 10 & $.0000 \mathrm{E}+00$ & 11 & $.0000 \mathrm{E}+00$ & 12 & $.0000 E+00$ & 13 & $.0000 \mathrm{E}+00$ & 14 & $.0000 E+00$ & 15 & $.0000 \mathrm{E}+00$ & 16 & $.0000 \mathrm{E}+00$ \\
\hline 17 & $.0000 \mathrm{E}+00$ & 18 & $.0000 \mathrm{E}+00$ & 19 & $.0000 \mathrm{E}+00$ & 20 & $.0000 \mathrm{E}+00$ & 21 & $.0000 \mathrm{E}+00$ & 22 & $.0000 \mathrm{E}+00$ & 23 & $.0000 \mathrm{E}+00$ & 24 & $.0000 \mathrm{E}+00$ \\
\hline & & & & & MONT & $\mathrm{H}=\mathrm{I}$ & MAY ; & DAY & OF WEEK = $S$ & SATURDA & & & & & \\
\hline 1 & $.0000 \mathrm{E}+00$ & 2 & $.0000 \mathrm{E}+00$ & 3 & $.0000 \mathrm{E}+00$ & 4 & $.0000 \mathrm{E}+00$ & 5 & $.0000 \mathrm{E}+00$ & 6 & $.0000 \mathrm{E}+00$ & 7 & $.0000 \mathrm{E}+00$ & 8 & $.0000 \mathrm{E}+00$ \\
\hline 9 & $.0000 \mathrm{E}+00$ & 10 & $.0000 \mathrm{E}+00$ & 11 & $.0000 \mathrm{E}+00$ & 12 & $.0000 \mathrm{E}+00$ & 13 & $.0000 \mathrm{E}+00$ & 14 & $.0000 \mathrm{E}+00$ & 15 & $.0000 \mathrm{E}+00$ & 16 & $.0000 \mathrm{E}+00$ \\
\hline 17 & $.0000 \mathrm{E}+00$ & 18 & $.0000 \mathrm{E}+00$ & 19 & $.0000 \mathrm{E}+00$ & 20 & $.0000 \mathrm{E}+00$ & 21 & $.0000 \mathrm{E}+00$ & 22 & $.0000 \mathrm{E}+00$ & 23 & $.0000 \mathrm{E}+00$ & 24 & $.0000 \mathrm{E}+00$ \\
\hline & & & & & MONT & $\mathrm{H}=$ & JUNE ; ; & DAY & OF WEEK = S & SATURDA & & & & & \\
\hline 1 & $.0000 \mathrm{E}+00$ & 2 & $.0000 \mathrm{E}+00$ & 3 & $.0000 \mathrm{E}+00$ & 4 & $.0000 \mathrm{E}+00$ & 5 & $.0000 \mathrm{E}+00$ & 6 & $.0000 \mathrm{E}+00$ & 7 & $.0000 \mathrm{E}+00$ & 8 & $.0000 \mathrm{E}+00$ \\
\hline 9 & $.0000 \mathrm{E}+00$ & 10 & $.0000 \mathrm{E}+00$ & 11 & $.0000 \mathrm{E}+00$ & 12 & $.0000 \mathrm{E}+00$ & 13 & $.0000 \mathrm{E}+00$ & 14 & $.0000 \mathrm{E}+00$ & 15 & $.0000 \mathrm{E}+00$ & 16 & $.0000 \mathrm{E}+00$ \\
\hline 17 & $.0000 \mathrm{E}+00$ & 18 & $.0000 \mathrm{E}+00$ & 19 & $.0000 \mathrm{E}+00$ & 20 & $.0000 \mathrm{E}+00$ & 21 & $.0000 \mathrm{E}+00$ & 22 & $.0000 \mathrm{E}+00$ & 23 & $.0000 \mathrm{E}+00$ & 24 & $.0000 \mathrm{E}+00$ \\
\hline & & & & & MONT & $\mathrm{H}=\mathrm{s}$ & JULY ; & DAY & OF WEEK = $S$ & SATURDA & & & & & \\
\hline 1 & $.0000 \mathrm{E}+00$ & 2 & $.0000 \mathrm{E}+00$ & 3 & $.0000 \mathrm{E}+00$ & 4 & $.0000 \mathrm{E}+00$ & 5 & $.0000 \mathrm{E}+00$ & 6 & $.0000 \mathrm{E}+00$ & 7 & $.0000 \mathrm{E}+00$ & 8 & $.0000 \mathrm{E}+00$ \\
\hline 9 & $.0000 \mathrm{E}+00$ & 10 & $.0000 \mathrm{E}+00$ & 11 & $.0000 \mathrm{E}+00$ & 12 & $.0000 \mathrm{E}+00$ & 13 & $.0000 \mathrm{E}+00$ & 14 & $.0000 \mathrm{E}+00$ & 15 & $.0000 \mathrm{E}+00$ & 16 & $.0000 \mathrm{E}+00$ \\
\hline 17 & $.0000 \mathrm{E}+00$ & 18 & $.0000 \mathrm{E}+00$ & 19 & $.0000 \mathrm{E}+00$ & 20 & $.0000 \mathrm{E}+00$ & 21 & $.0000 \mathrm{E}+00$ & 22 & $.0000 \mathrm{E}+00$ & 23 & $.0000 \mathrm{E}+00$ & 24 & $.0000 \mathrm{E}+00$ \\
\hline & & & & & MONT & $\mathrm{H}=\mathrm{T}$ & AUGUST ; & DAY & OF WEEK = $S$ & SATURDA & & & & & \\
\hline 1 & $.0000 \mathrm{E}+00$ & 2 & $.0000 \mathrm{E}+00$ & 3 & $.0000 \mathrm{E}+00$ & 4 & $.0000 \mathrm{E}+00$ & 5 & $.0000 \mathrm{E}+00$ & 6 & $.0000 \mathrm{E}+00$ & 7 & $.0000 \mathrm{E}+00$ & 8 & $.0000 \mathrm{E}+00$ \\
\hline 9 & $.0000 \mathrm{E}+00$ & 10 & $.0000 \mathrm{E}+00$ & 11 & $.0000 \mathrm{E}+00$ & 12 & $.0000 \mathrm{E}+00$ & 13 & $.0000 \mathrm{E}+00$ & 14 & $.0000 \mathrm{E}+00$ & 15 & $.0000 \mathrm{E}+00$ & 16 & $.0000 \mathrm{E}+00$ \\
\hline 17 & $.0000 \mathrm{E}+00$ & 18 & $.0000 \mathrm{E}+00$ & 19 & $.0000 \mathrm{E}+00$ & 20 & $.0000 \mathrm{E}+00$ & 21 & $.0000 \mathrm{E}+00$ & 22 & $.0000 \mathrm{E}+00$ & 23 & $.0000 \mathrm{E}+00$ & 24 & $.0000 \mathrm{E}+00$ \\
\hline & & & & & MONT & $H=$ & SEPTEMBER ; & DAY & OF WEEK = S & SATURDA & & & & & \\
\hline 1 & $.0000 \mathrm{E}+00$ & 2 & $.0000 \mathrm{E}+00$ & 3 & $.0000 \mathrm{E}+00$ & 4 & $.0000 \mathrm{E}+00$ & 5 & $.0000 \mathrm{E}+00$ & 6 & $.0000 \mathrm{E}+00$ & 7 & $.0000 \mathrm{E}+00$ & 8 & $.0000 \mathrm{E}+00$ \\
\hline 9 & $.0000 \mathrm{E}+00$ & 10 & $.0000 \mathrm{E}+00$ & 11 & $.0000 \mathrm{E}+00$ & 12 & $.0000 \mathrm{E}+00$ & 13 & $.0000 \mathrm{E}+00$ & 14 & $.0000 \mathrm{E}+00$ & 15 & $.0000 \mathrm{E}+00$ & 16 & $.0000 \mathrm{E}+00$ \\
\hline 17 & $.0000 \mathrm{E}+00$ & 18 & $.0000 \mathrm{E}+00$ & 19 & $.0000 \mathrm{E}+00$ & 20 & $.0000 \mathrm{E}+00$ & 21 & $.0000 \mathrm{E}+00$ & 22 & $.0000 \mathrm{E}+00$ & 23 & $.0000 \mathrm{E}+00$ & 24 & $.0000 \mathrm{E}+00$ \\
\hline & & & & & MONT & $\mathrm{H}=$ & OCTOBER ; & DAY & OF WEEK = S & SATURDA & & & & & \\
\hline 1 & $.0000 \mathrm{E}+00$ & 2 & $.0000 \mathrm{E}+00$ & 3 & $.0000 \mathrm{E}+00$ & 4 & $.0000 \mathrm{E}+00$ & 5 & $.0000 \mathrm{E}+00$ & 6 & $.0000 \mathrm{E}+00$ & 7 & $.0000 \mathrm{E}+00$ & 8 & $.0000 \mathrm{E}+00$ \\
\hline 9 & $.0000 \mathrm{E}+00$ & 10 & $.0000 \mathrm{E}+00$ & 11 & $.0000 \mathrm{E}+00$ & 12 & $.0000 \mathrm{E}+00$ & 13 & $.0000 \mathrm{E}+00$ & 14 & $.0000 \mathrm{E}+00$ & 15 & $.0000 \mathrm{E}+00$ & 16 & $.0000 \mathrm{E}+00$ \\
\hline 17 & $.0000 \mathrm{E}+00$ & 18 & $.0000 \mathrm{E}+00$ & 19 & $.0000 \mathrm{E}+00$ & 20 & $.0000 \mathrm{E}+00$ & 21 & $.0000 \mathrm{E}+00$ & 22 & $.0000 \mathrm{E}+00$ & 23 & $.0000 \mathrm{E}+00$ & 24 & $.0000 \mathrm{E}+00$ \\
\hline & & & & & MONT & $H=$ & NOVEMBER； & DAY & OF WEEK = $S$ & SATURDA & & & & & \\
\hline 1 & $.0000 \mathrm{E}+00$ & 2 & $.0000 \mathrm{E}+00$ & 3 & $.0000 \mathrm{E}+00$ & 4 & $.0000 \mathrm{E}+00$ & 5 & $.0000 \mathrm{E}+00$ & 6 & $.0000 \mathrm{E}+00$ & 7 & $.0000 \mathrm{E}+00$ & 8 & $.0000 \mathrm{E}+00$ \\
\hline 9 & $.0000 \mathrm{E}+00$ & 10 & $.0000 \mathrm{E}+00$ & 11 & $.0000 \mathrm{E}+00$ & 12 & $.0000 \mathrm{E}+00$ & 13 & $.0000 \mathrm{E}+00$ & 14 & $.0000 \mathrm{E}+00$ & 15 & $.0000 \mathrm{E}+00$ & 16 & $.0000 \mathrm{E}+00$ \\
\hline 17 & $.0000 \mathrm{E}+00$ & 18 & $.0000 \mathrm{E}+00$ & 19 & $.0000 \mathrm{E}+00$ & 20 & $.0000 \mathrm{E}+00$ & 21 & $.0000 \mathrm{E}+00$ & 22 & $.0000 \mathrm{E}+00$ & 23 & $.0000 \mathrm{E}+00$ & 24 & $.0000 \mathrm{E}+00$ \\
\hline & & & & & MONT & $\mathrm{H}=$ & DECEMBER ; & DAY & OF WEEK = S & SATURDA & & & & & \\
\hline 1 & $.0000 \mathrm{E}+00$ & 2 & $.0000 \mathrm{E}+00$ & 3 & $.0000 \mathrm{E}+00$ & 4 & $.0000 \mathrm{E}+00$ & 5 & $.0000 \mathrm{E}+00$ & 6 & $.0000 \mathrm{E}+00$ & 7 & $.0000 \mathrm{E}+00$ & 8 & $.0000 \mathrm{E}+00$ \\
\hline 9 & $.0000 \mathrm{E}+00$ & 10 & $.0000 \mathrm{E}+00$ & 11 & $.0000 \mathrm{E}+00$ & 12 & $.0000 \mathrm{E}+00$ & 13 & $.0000 \mathrm{E}+00$ & 14 & $.0000 \mathrm{E}+00$ & 15 & $.0000 \mathrm{E}+00$ & 16 & $.0000 \mathrm{E}+00$ \\
\hline 17 & $.0000 \mathrm{E}+00$ & 18 & $.0000 \mathrm{E}+00$ & 19 & $.0000 \mathrm{E}+00$ & 20 & $.0000 \mathrm{E}+00$ & 21 & $.0000 \mathrm{E}+00$ & 22 & $.0000 \mathrm{E}+00$ & 23 & $.0000 \mathrm{E}+00$ & 24 & $.0000 \mathrm{E}+00$ \\
\hline & AERMOD - VE & ION & $07026 * * *$ & & U/UA Buil & ing & Demc & & & & & & *** & & $02 / 09 / 09$ \\
\hline & & & & & & & & & & & & & $* * *$ & & $10: 41: 11$ \\
\hline & & & & & & & & & & & & & & & PAGE 103 \\
\hline , & & & & & & & & & & & & & & & \\
\hline
\end{tabular}

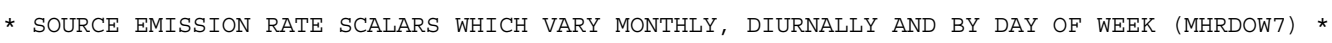




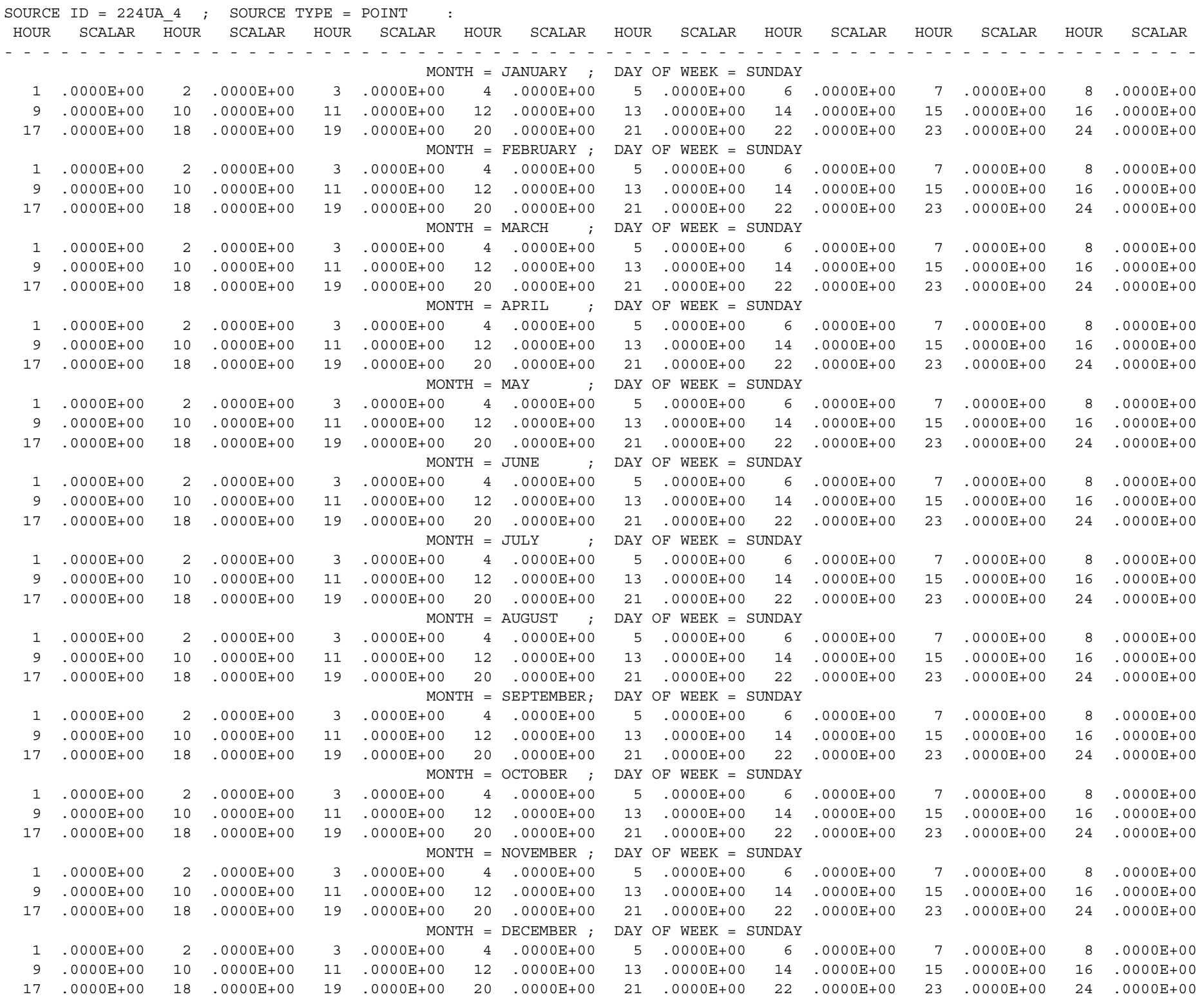




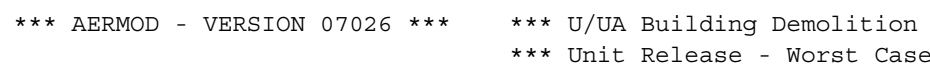
**MODELOPTS :

CONC

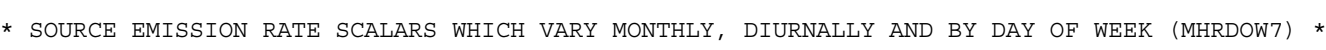

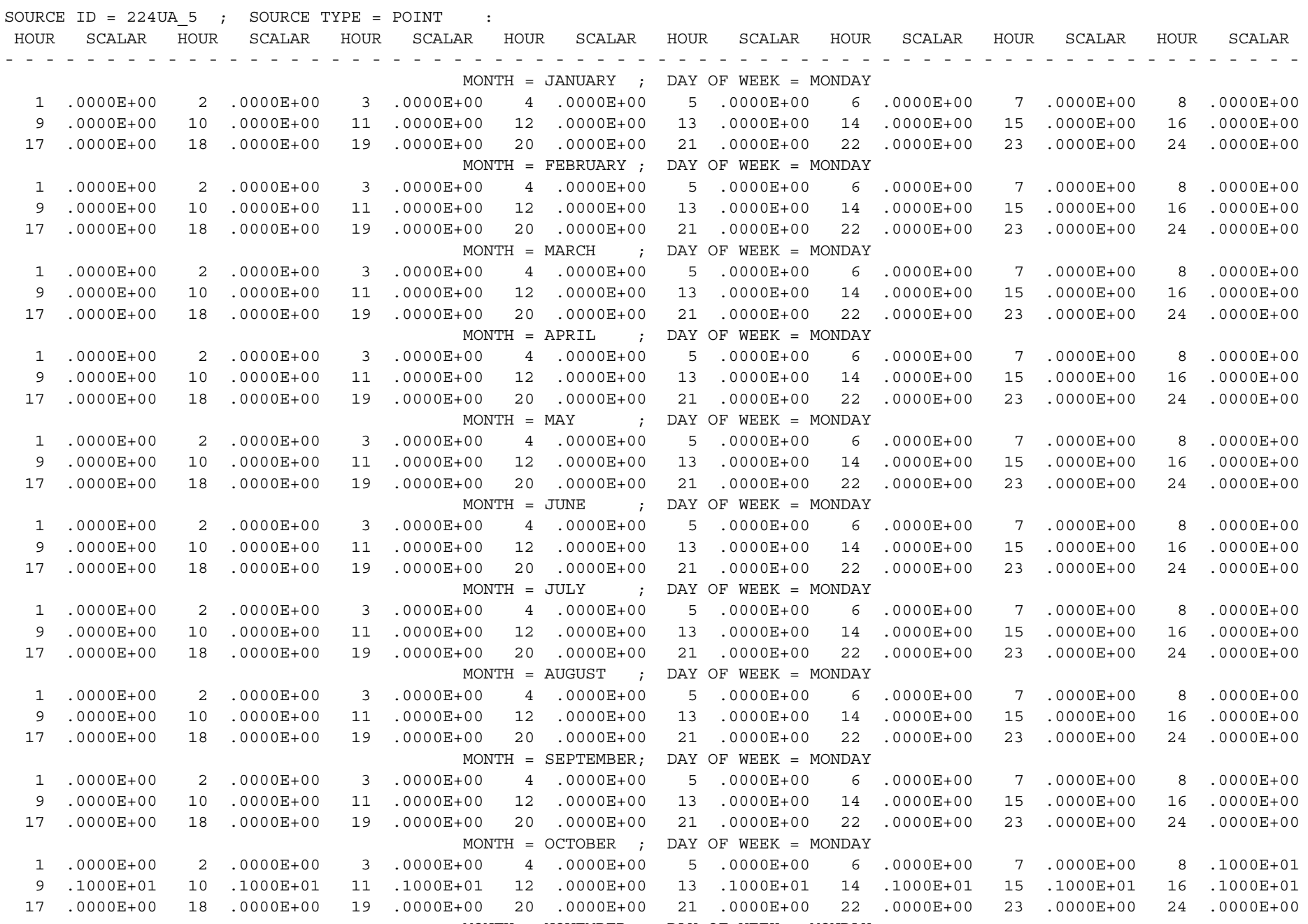

MONTH $=$ NOVEMBER $;$ DAY OF WEEK $=$ MONDAY 


\begin{tabular}{|c|c|c|c|c|c|c|c|c|c|c|c|c|c|c|c|}
\hline 1 & $.0000 \mathrm{E}+00$ & 2 & $.0000 \mathrm{E}+00$ & 3 & $.0000 \mathrm{E}+00$ & 4 & $.0000 \mathrm{E}+00$ & 5 & $.0000 \mathrm{E}+00$ & 6 & $.0000 \mathrm{E}+00$ & 7 & $.0000 \mathrm{E}+00$ & 8 & $1000 \mathrm{E}+01$ \\
\hline 9 & $.1000 \mathrm{E}+01$ & 10 & $.1000 \mathrm{E}+01$ & 11 & $.1000 \mathrm{E}+01$ & 12 & $.0000 \mathrm{E}+00$ & 13 & $.1000 \mathrm{E}+01$ & 14 & $.1000 \mathrm{E}+01$ & 15 & $.1000 \mathrm{E}+01$ & 16 & $.1000 \mathrm{E}+01$ \\
\hline 1 & $.0000 \mathrm{E}+00$ & 18 & $.0000 \mathrm{E}+00$ & 19 & $.0000 \mathrm{E}+00$ & 20 & $.0000 \mathrm{E}+00$ & 21 & $.0000 \mathrm{E}+00$ & 22 & $.0000 \mathrm{E}+00$ & 23 & $.0000 \mathrm{E}+00$ & 24 & $.0000 \mathrm{E}+00$ \\
\hline & & & & & MOI & & DECEMBER ; & DAY & OF $\mathrm{WEEK}=$ & NDAY & & & & & \\
\hline 1 & $.0000 \mathrm{E}+00$ & 2 & $.0000 \mathrm{E}+00$ & 3 & $.0000 \mathrm{E}+00$ & 4 & $.0000 \mathrm{E}+00$ & 5 & $.0000 \mathrm{E}+00$ & 6 & $.0000 \mathrm{E}+00$ & 7 & $.0000 \mathrm{E}+00$ & 8 & $.1000 \mathrm{E}+01$ \\
\hline 9 & $.1000 \mathrm{E}+01$ & 10 & $.1000 \mathrm{E}+01$ & 11 & $.1000 \mathrm{E}+01$ & 12 & $.0000 \mathrm{E}+00$ & 13 & $.1000 \mathrm{E}+01$ & 14 & $.1000 \mathrm{E}+01$ & 15 & $.1000 \mathrm{E}+01$ & 16 & $.1000 \mathrm{E}+01$ \\
\hline 1 & $.0000 \mathrm{E}+00$ & 18 & $.0000 \mathrm{E}+00$ & 19 & $.0000 \mathrm{E}+00$ & 20 & $.0000 \mathrm{E}+00$ & 21 & $0000 \mathrm{E}+00$ & 22 & $0000 E+00$ & 23 & $0000 \mathrm{E}+00$ & 24 & $0000 E+00$ \\
\hline
\end{tabular}

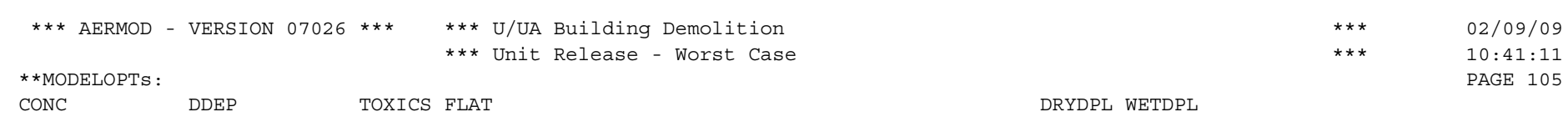

* source emission RAte scalars Which vary monthly, DiURNALly and by DAy of WeEk (MHRDOW7) *

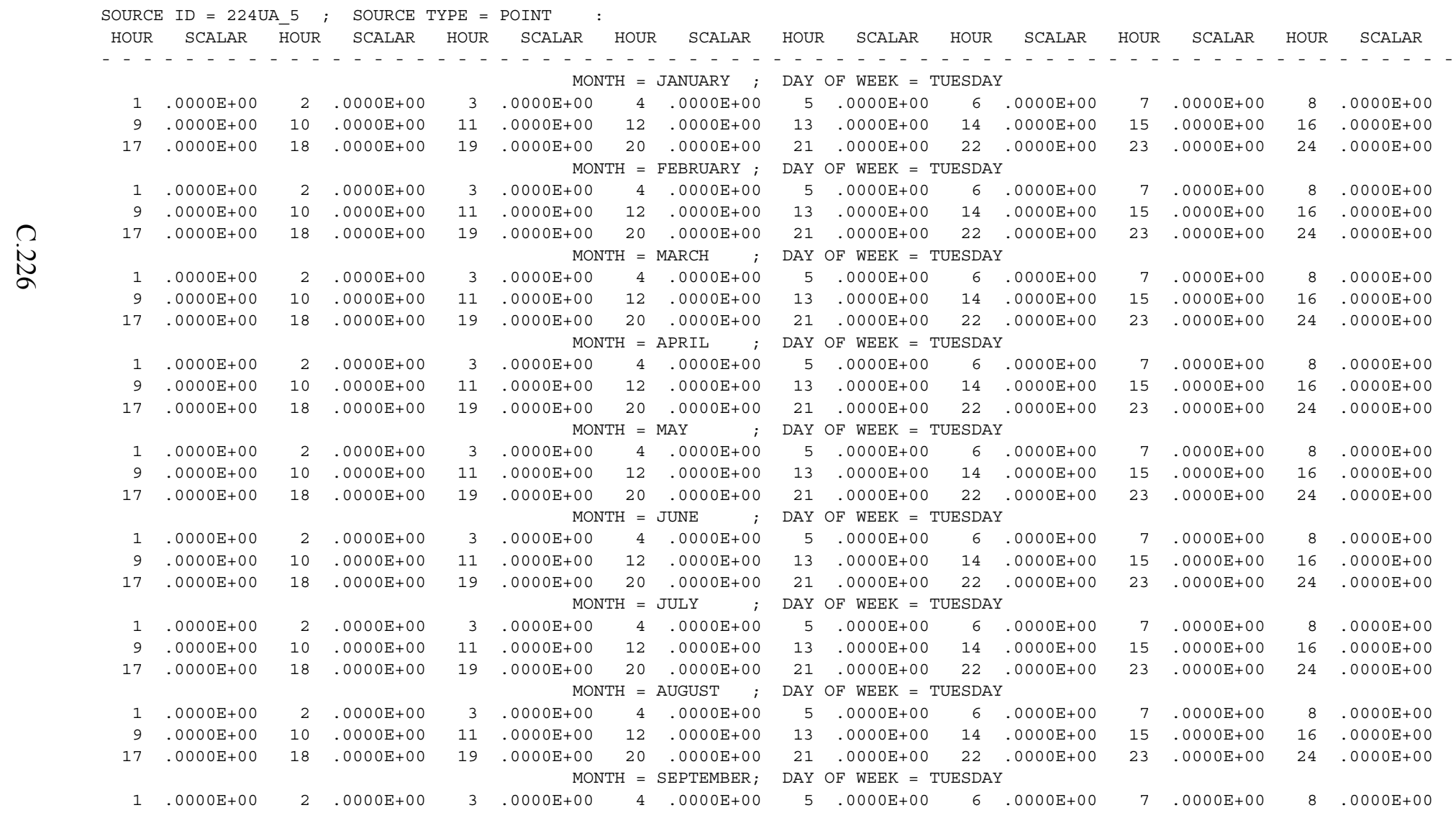




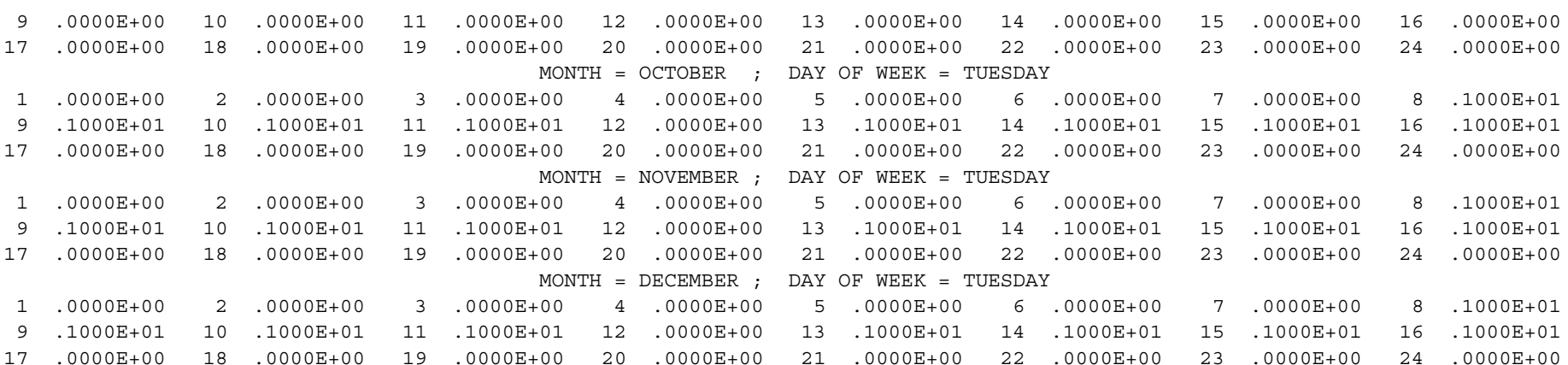

*** AERMOD - VERSION $07026 * * * \quad * * *$ U/UA Building Demolition

*** Unit Release - Worst Case

$\begin{array}{ll}* * * & 02 / 09 / 09 \\ * * * & 10: 41: 11 \\ & \text { PAGE } 106\end{array}$ **MODELOPTS :

CONC

DDEP

TOXICS FLAT

DRYDPL WETDPI

* SOURCE EMISsion RATE SCALARs Which VARY MONTHLy, DIURNALLy AND By DAY OF WEEK (MHRDOW7) *

SOURCE ID $=224 \mathrm{UA} \_5 ;$ SOURCE TYPE $=$ POINT

HOUR SCALAR HOUR SCALAR HOUR SCALAR HOUR SCALAR HOUR SCALAR HOUR SCALAR HOUR SCALAR HOUR SCALAR

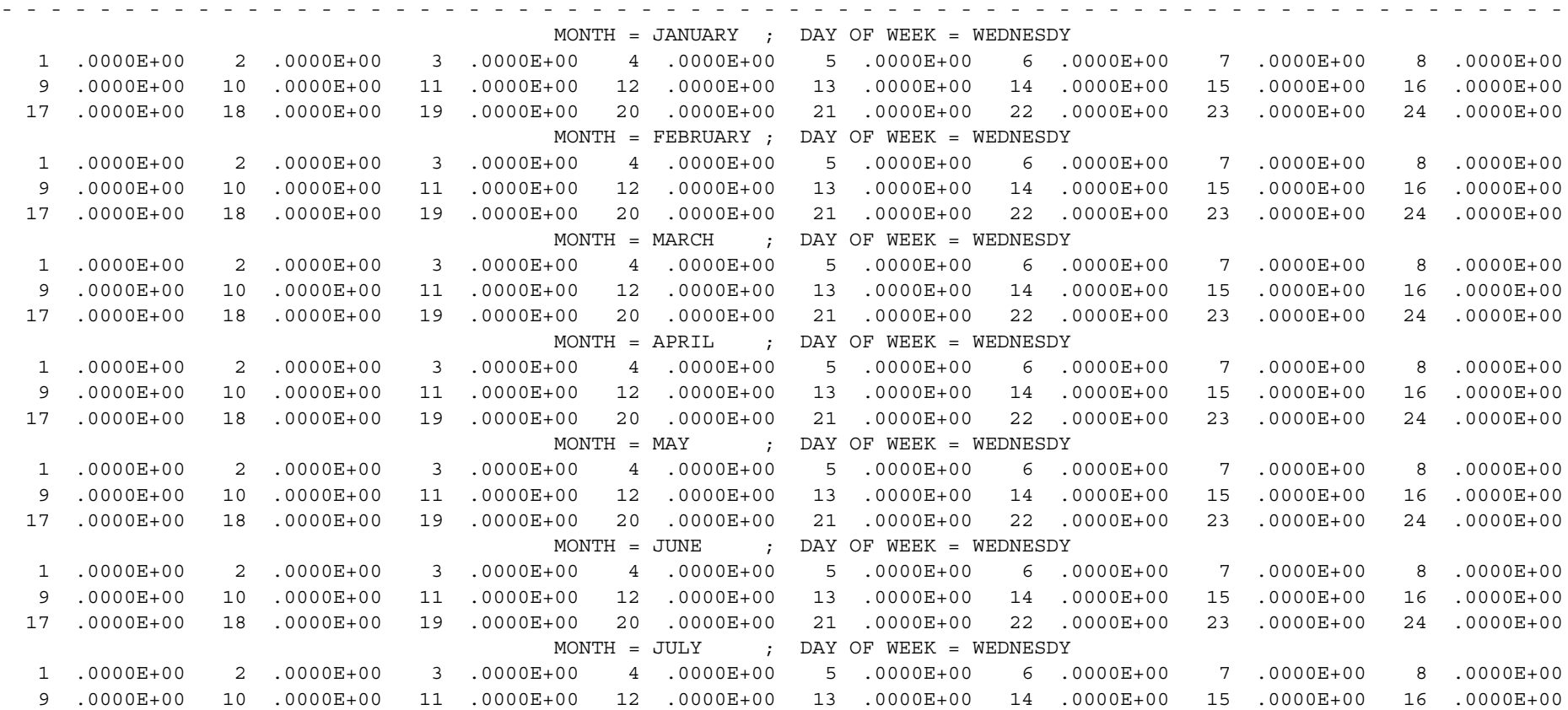




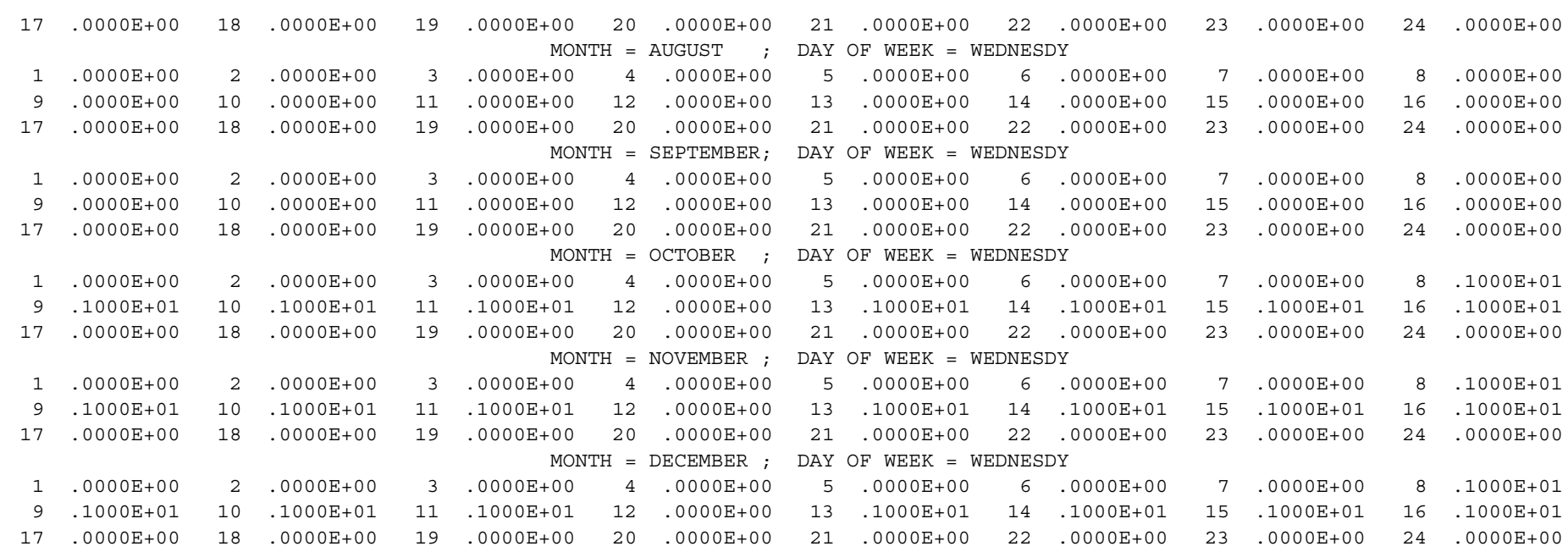

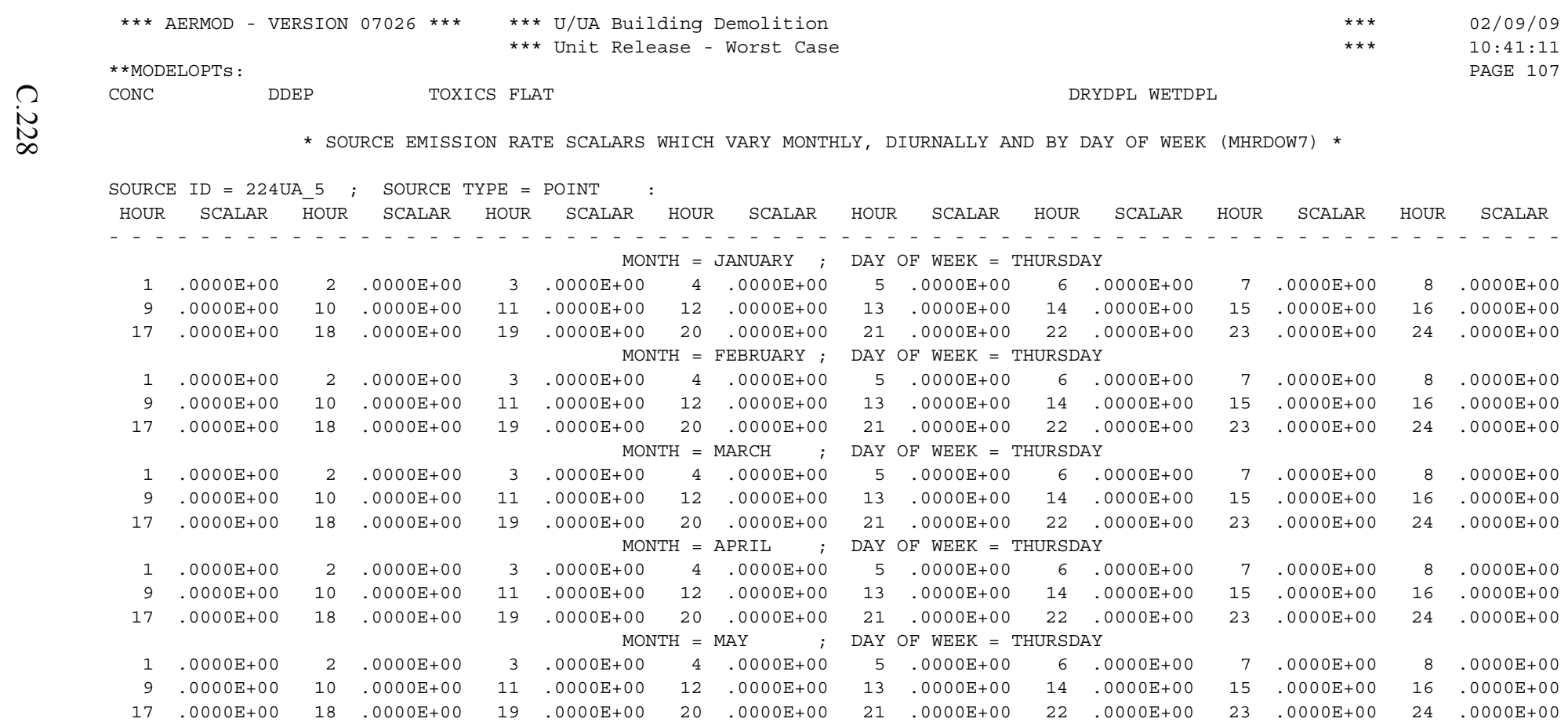




\begin{tabular}{|c|c|c|c|c|c|c|c|c|c|c|c|c|c|c|c|}
\hline & & & & & MON' & $\mathrm{TH}=$ & JUNE ; & DAY & OF $\mathrm{WEEK}=\mathrm{TH}$ & THURSDF & & & & & \\
\hline 1 & $.0000 \mathrm{E}+00$ & 2 & $.0000 \mathrm{E}+00$ & 3 & $.0000 \mathrm{E}+00$ & 4 & $.0000 \mathrm{E}+00$ & 5 & $.0000 \mathrm{E}+00$ & 6 & $.0000 \mathrm{E}+00$ & 7 & $.0000 \mathrm{E}+00$ & 8 & $.0000 \mathrm{E}+00$ \\
\hline 9 & $.0000 \mathrm{E}+00$ & 10 & $.0000 \mathrm{E}+00$ & 11 & $.0000 \mathrm{E}+00$ & 12 & $.0000 \mathrm{E}+00$ & 13 & $.0000 \mathrm{E}+00$ & 14 & $.0000 \mathrm{E}+00$ & 15 & $.0000 \mathrm{E}+00$ & 16 & $.0000 \mathrm{E}+00$ \\
\hline 17 & $.0000 \mathrm{E}+00$ & 18 & $.0000 \mathrm{E}+00$ & 19 & $.0000 \mathrm{E}+00$ & 20 & $.0000 \mathrm{E}+00$ & 21 & $.0000 \mathrm{E}+00$ & 22 & $.0000 \mathrm{E}+00$ & 23 & $.0000 \mathrm{E}+00$ & 24 & $.0000 \mathrm{E}+00$ \\
\hline & & & & & MON & $\mathrm{TH}=$ & JULY ; & DAY & OF $\mathrm{WEEK}=\mathrm{TE}$ & THURSDF & & & & & \\
\hline 1 & $.0000 \mathrm{E}+00$ & 2 & $.0000 \mathrm{E}+00$ & 3 & $.0000 \mathrm{E}+00$ & 4 & $.0000 \mathrm{E}+00$ & 5 & $.0000 \mathrm{E}+00$ & 6 & $.0000 \mathrm{E}+00$ & 7 & $.0000 \mathrm{E}+00$ & 8 & $.0000 \mathrm{E}+00$ \\
\hline 9 & $.0000 \mathrm{E}+00$ & 10 & $.0000 \mathrm{E}+00$ & 11 & $.0000 \mathrm{E}+00$ & 12 & $.0000 \mathrm{E}+00$ & 13 & $.0000 \mathrm{E}+00$ & 14 & $.0000 \mathrm{E}+00$ & 15 & $.0000 \mathrm{E}+00$ & 16 & $.0000 \mathrm{E}+00$ \\
\hline 17 & $.0000 \mathrm{E}+00$ & 18 & $.0000 \mathrm{E}+00$ & 19 & $.0000 \mathrm{E}+00$ & 20 & $.0000 \mathrm{E}+00$ & 21 & $.0000 \mathrm{E}+00$ & 22 & $.0000 \mathrm{E}+00$ & 23 & $.0000 \mathrm{E}+00$ & 24 & $.0000 \mathrm{E}+00$ \\
\hline & & & & & MON & $\mathrm{TH}=\mathrm{I}$ & AUGUST ; & DAY & OF $\mathrm{WEEK}=\mathrm{TH}$ & THURSDF & & & & & \\
\hline 1 & $.0000 \mathrm{E}+00$ & 2 & $.0000 \mathrm{E}+00$ & 3 & $.0000 \mathrm{E}+00$ & 4 & $.0000 \mathrm{E}+00$ & 5 & $.0000 \mathrm{E}+00$ & 6 & $.0000 \mathrm{E}+00$ & 7 & $.0000 \mathrm{E}+00$ & 8 & $.0000 \mathrm{E}+00$ \\
\hline 9 & $.0000 \mathrm{E}+00$ & 10 & $.0000 \mathrm{E}+00$ & 11 & $.0000 \mathrm{E}+00$ & 12 & $.0000 \mathrm{E}+00$ & 13 & $.0000 \mathrm{E}+00$ & 14 & $.0000 \mathrm{E}+00$ & 15 & $.0000 \mathrm{E}+00$ & 16 & $.0000 \mathrm{E}+00$ \\
\hline 17 & $.0000 \mathrm{E}+00$ & 18 & $.0000 \mathrm{E}+00$ & 19 & $.0000 \mathrm{E}+00$ & 20 & $.0000 \mathrm{E}+00$ & 21 & $.0000 \mathrm{E}+00$ & 22 & $.0000 \mathrm{E}+00$ & 23 & $.0000 \mathrm{E}+00$ & 24 & $.0000 \mathrm{E}+00$ \\
\hline & & & & & MON & $\mathrm{TH}=s$ & SEPTEMBER; & DAY & OF WEEK = TE & THURSDF & & & & & \\
\hline 1 & $.0000 \mathrm{E}+00$ & 2 & $.0000 \mathrm{E}+00$ & 3 & $.0000 \mathrm{E}+00$ & 4 & $.0000 \mathrm{E}+00$ & 5 & $.0000 \mathrm{E}+00$ & 6 & $.0000 \mathrm{E}+00$ & 7 & $.0000 \mathrm{E}+00$ & 8 & $.0000 \mathrm{E}+00$ \\
\hline 9 & $.0000 \mathrm{E}+00$ & 10 & $.0000 \mathrm{E}+00$ & 11 & $.0000 \mathrm{E}+00$ & 12 & $.0000 \mathrm{E}+00$ & 13 & $.0000 \mathrm{E}+00$ & 14 & $.0000 \mathrm{E}+00$ & 15 & $.0000 \mathrm{E}+00$ & 16 & $.0000 \mathrm{E}+00$ \\
\hline 17 & $.0000 \mathrm{E}+00$ & 18 & $.0000 \mathrm{E}+00$ & 19 & $.0000 \mathrm{E}+00$ & 20 & $.0000 \mathrm{E}+00$ & 21 & $.0000 \mathrm{E}+00$ & 22 & $.0000 \mathrm{E}+00$ & 23 & $.0000 \mathrm{E}+00$ & 24 & $.0000 \mathrm{E}+00$ \\
\hline & & & & & MON & $\mathrm{TH}=\mathrm{C}$ & OCTOBER ; & DAY & OF $\mathrm{WEEK}=\mathrm{TH}$ & THURSDF & & & & & \\
\hline 1 & $.0000 \mathrm{E}+00$ & 2 & $.0000 \mathrm{E}+00$ & 3 & $.0000 \mathrm{E}+00$ & 4 & $.0000 \mathrm{E}+00$ & 5 & $.0000 \mathrm{E}+00$ & 6 & $.0000 \mathrm{E}+00$ & 7 & $.0000 \mathrm{E}+00$ & 8 & $.1000 \mathrm{E}+01$ \\
\hline 9 & $.1000 \mathrm{E}+01$ & 10 & $.1000 \mathrm{E}+01$ & 11 & $.1000 \mathrm{E}+01$ & 12 & $.0000 \mathrm{E}+00$ & 13 & $.1000 \mathrm{E}+01$ & 14 & $.1000 \mathrm{E}+01$ & 15 & $.1000 \mathrm{E}+01$ & 16 & $.1000 \mathrm{E}+01$ \\
\hline 17 & $.0000 \mathrm{E}+00$ & 18 & $.0000 \mathrm{E}+00$ & 19 & $.0000 \mathrm{E}+00$ & 20 & $.0000 \mathrm{E}+00$ & 21 & $.0000 \mathrm{E}+00$ & 22 & $.0000 \mathrm{E}+00$ & 23 & $.0000 \mathrm{E}+00$ & 24 & $.0000 \mathrm{E}+00$ \\
\hline & & & & & MON & $\mathrm{TH}=\mathrm{I}$ & NOVEMBER ; & DAY & OF $\mathrm{WEEK}=\mathrm{TH}$ & THURSDF & & & & & \\
\hline 1 & $.0000 \mathrm{E}+00$ & 2 & $.0000 \mathrm{E}+00$ & 3 & $.0000 \mathrm{E}+00$ & 4 & $.0000 \mathrm{E}+00$ & 5 & $.0000 \mathrm{E}+00$ & 6 & $.0000 \mathrm{E}+00$ & 7 & $.0000 \mathrm{E}+00$ & 8 & $.1000 \mathrm{E}+01$ \\
\hline 9 & $.1000 \mathrm{E}+01$ & 10 & $.1000 \mathrm{E}+01$ & 11 & $.1000 \mathrm{E}+01$ & 12 & $.0000 E+00$ & 13 & $.1000 \mathrm{E}+01$ & 14 & $.1000 \mathrm{E}+01$ & 15 & $.1000 \mathrm{E}+01$ & 16 & $.1000 \mathrm{E}+01$ \\
\hline 17 & $.0000 \mathrm{E}+00$ & 18 & $.0000 \mathrm{E}+00$ & 19 & $.0000 \mathrm{E}+00$ & 20 & $.0000 \mathrm{E}+00$ & 21 & $.0000 \mathrm{E}+00$ & 22 & $.0000 \mathrm{E}+00$ & 23 & $.0000 \mathrm{E}+00$ & 24 & $.0000 \mathrm{E}+00$ \\
\hline & & & & & MON & $\mathrm{TH}=\mathrm{I}$ & DECEMBER ； & DAY & OF $\mathrm{WEEK}=\mathrm{TE}$ & THURSDF & & & & & \\
\hline 1 & $.0000 \mathrm{E}+00$ & 2 & $.0000 \mathrm{E}+00$ & 3 & $.0000 \mathrm{E}+00$ & 4 & $.0000 \mathrm{E}+00$ & 5 & $.0000 \mathrm{E}+00$ & 6 & $.0000 \mathrm{E}+00$ & 7 & $.0000 \mathrm{E}+00$ & 8 & $.1000 \mathrm{E}+01$ \\
\hline 9 & $.1000 \mathrm{E}+01$ & 10 & $.1000 \mathrm{E}+01$ & 11 & $.1000 \mathrm{E}+01$ & 12 & $.0000 \mathrm{E}+00$ & 13 & $.1000 \mathrm{E}+01$ & 14 & $.1000 \mathrm{E}+01$ & 15 & $.1000 \mathrm{E}+01$ & 16 & $.1000 \mathrm{E}+01$ \\
\hline 17 & $.0000 \mathrm{E}+00$ & 18 & $.0000 \mathrm{E}+00$ & 19 & $.0000 \mathrm{E}+00$ & 20 & $.0000 \mathrm{E}+00$ & 21 & $.0000 \mathrm{E}+00$ & 22 & $.0000 \mathrm{E}+00$ & 23 & $.0000 \mathrm{E}+00$ & 24 & $.0000 \mathrm{E}+00$ \\
\hline$* * *$ & AERMOD - VB & ERSION & $07026 * * *$ & $* * *$ & U/UA Builo & ding 1 & Demolition & & & & & & $\star * *$ & & $02 / 09 / 09$ \\
\hline & & & & $* * *$ & Unit Rele & ase - & Worst Case & & & & & & $* * *$ & & $10: 41: 11$ \\
\hline$\star \star$ MOD & DELOPTS : & & & & & & & & & & & & & & PAGE 108 \\
\hline $\mathrm{CONC}$ & & DEP & TOXICS & $S$ FLAT & & & & & & & RYDPL WETDPL & & & & \\
\hline & & * SOU & JRCE EMISSION & N RATE & E SCALARS & WHICH & I VARY MONTHI & LY, D & IURNALLY ANI & ND BY I & DAY OF WEEK & (MHRD & DOW7) * & & \\
\hline SOURC & $C C E I D=224 \mathrm{C}$ & UA_5 & SOURCE TYP & $P E=P$ & POINT & & & & & & & & & & \\
\hline HOUR & IR SCALAR & $\overline{\mathrm{HOUR}}$ & SCALAR $\quad \mathrm{H}$ & HOUR & SCALAR & HOUR & RCALAR & HOUR & SCALAR & HOUR & SCALAR & HOUR & SCALAR & HOUR & SCALAR \\
\hline & - - - - - - & $-\ldots$ & 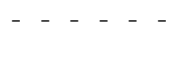 & $-\cdots$ & $\begin{array}{ccc}- & - & - \\
\text { MON }\end{array}$ & $\begin{array}{l}-\cdots \\
\mathrm{TH}=\end{array}$ & $\begin{array}{c}--\cdots \\
\text { JANUARY }\end{array}$ & $\begin{array}{c}-- \\
\text { DAY }\end{array}$ & $\begin{array}{l}\cdots \\
O F \text { WEEK }=\end{array}$ & $\begin{array}{l}-\quad- \\
\text { FRIDAY }\end{array}$ & 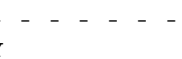 & -- & $-\cdots-$ & $-\quad-$ & ---- \\
\hline 1 & $.0000 \mathrm{E}+00$ & 2 & $.0000 \mathrm{E}+00$ & 3 & $.0000 \mathrm{E}+00$ & 4 & $.0000 \mathrm{E}+00$ & 5 & $.0000 \mathrm{E}+00$ & 6 & $.0000 \mathrm{E}+00$ & 7 & $.0000 \mathrm{E}+00$ & 8 & $.0000 \mathrm{E}+00$ \\
\hline 9 & $.0000 \mathrm{E}+00$ & 10 & $.0000 \mathrm{E}+00$ & 11 & $.0000 \mathrm{E}+00$ & 12 & $.0000 E+00$ & 13 & $.0000 E+00$ & 14 & $.0000 \mathrm{E}+00$ & 15 & $.0000 \mathrm{E}+00$ & 16 & $.0000 \mathrm{E}+00$ \\
\hline 17 & $.0000 \mathrm{E}+00$ & 18 & $.0000 \mathrm{E}+00$ & 19 & $.0000 \mathrm{E}+00$ & 20 & $.0000 \mathrm{E}+00$ & 21 & $.0000 \mathrm{E}+00$ & 22 & $.0000 \mathrm{E}+00$ & 23 & $.0000 \mathrm{E}+00$ & 24 & $.0000 \mathrm{E}+00$ \\
\hline & & & & & MON' & $\mathrm{TH}=\mathrm{H}$ & FEBRUARY ; & DAY & $O F \quad W E E K=F H$ & FRIDAY & & & & & \\
\hline 1 & $.0000 \mathrm{E}+00$ & 2 & $.0000 \mathrm{E}+00$ & 3 & $.0000 \mathrm{E}+00$ & 4 & $.0000 \mathrm{E}+00$ & 5 & $.0000 \mathrm{E}+00$ & 6 & $.0000 \mathrm{E}+00$ & 7 & $.0000 \mathrm{E}+00$ & 8 & $.0000 \mathrm{E}+00$ \\
\hline 9 & $.0000 \mathrm{E}+00$ & 10 & $.0000 \mathrm{E}+00$ & 11 & $.0000 \mathrm{E}+00$ & 12 & $.0000 \mathrm{E}+00$ & 13 & $.0000 \mathrm{E}+00$ & 14 & $.0000 \mathrm{E}+00$ & 15 & $.0000 \mathrm{E}+00$ & 16 & $.0000 \mathrm{E}+00$ \\
\hline 17 & $.0000 \mathrm{E}+00$ & 18 & $.0000 \mathrm{E}+00$ & 19 & $.0000 \mathrm{E}+00$ & 20 & $.0000 \mathrm{E}+00$ & 21 & $.0000 \mathrm{E}+00$ & 22 & $.0000 \mathrm{E}+00$ & 23 & $.0000 \mathrm{E}+00$ & 24 & $.0000 \mathrm{E}+00$ \\
\hline & & & & & MON' & $\mathrm{TH}=\mathrm{I}$ & MARCH $\quad ;$ & DAY & $O F \quad W E E K=F F$ & FRIDAY & & & & & \\
\hline 1 & $.0000 \mathrm{E}+00$ & 2 & $.0000 \mathrm{E}+00$ & 3 & $.0000 \mathrm{E}+00$ & 4 & $.0000 \mathrm{E}+00$ & 5 & $.0000 \mathrm{E}+00$ & 6 & $.0000 \mathrm{E}+00$ & 7 & $.0000 \mathrm{E}+00$ & 8 & $.0000 \mathrm{E}+00$ \\
\hline 9 & $.0000 \mathrm{E}+00$ & 10 & $.0000 \mathrm{E}+00$ & 11 & $.0000 \mathrm{E}+00$ & 12 & $.0000 E+00$ & 13 & $.0000 \mathrm{E}+00$ & 14 & $.0000 \mathrm{E}+00$ & 15 & $.0000 \mathrm{E}+00$ & 16 & $.0000 \mathrm{E}+00$ \\
\hline 17 & $.0000 \mathrm{E}+00$ & 18 & $.0000 \mathrm{E}+00$ & 19 & $.0000 \mathrm{E}+00$ & 20 & $.0000 \mathrm{E}+00$ & 21 & $.0000 \mathrm{E}+00$ & 22 & $.0000 \mathrm{E}+00$ & 23 & $.0000 \mathrm{E}+00$ & 24 & $.0000 \mathrm{E}+00$ \\
\hline
\end{tabular}

MONTH $=$ APRIL $;$ DAY OF WEEK $=$ FRIDAY 


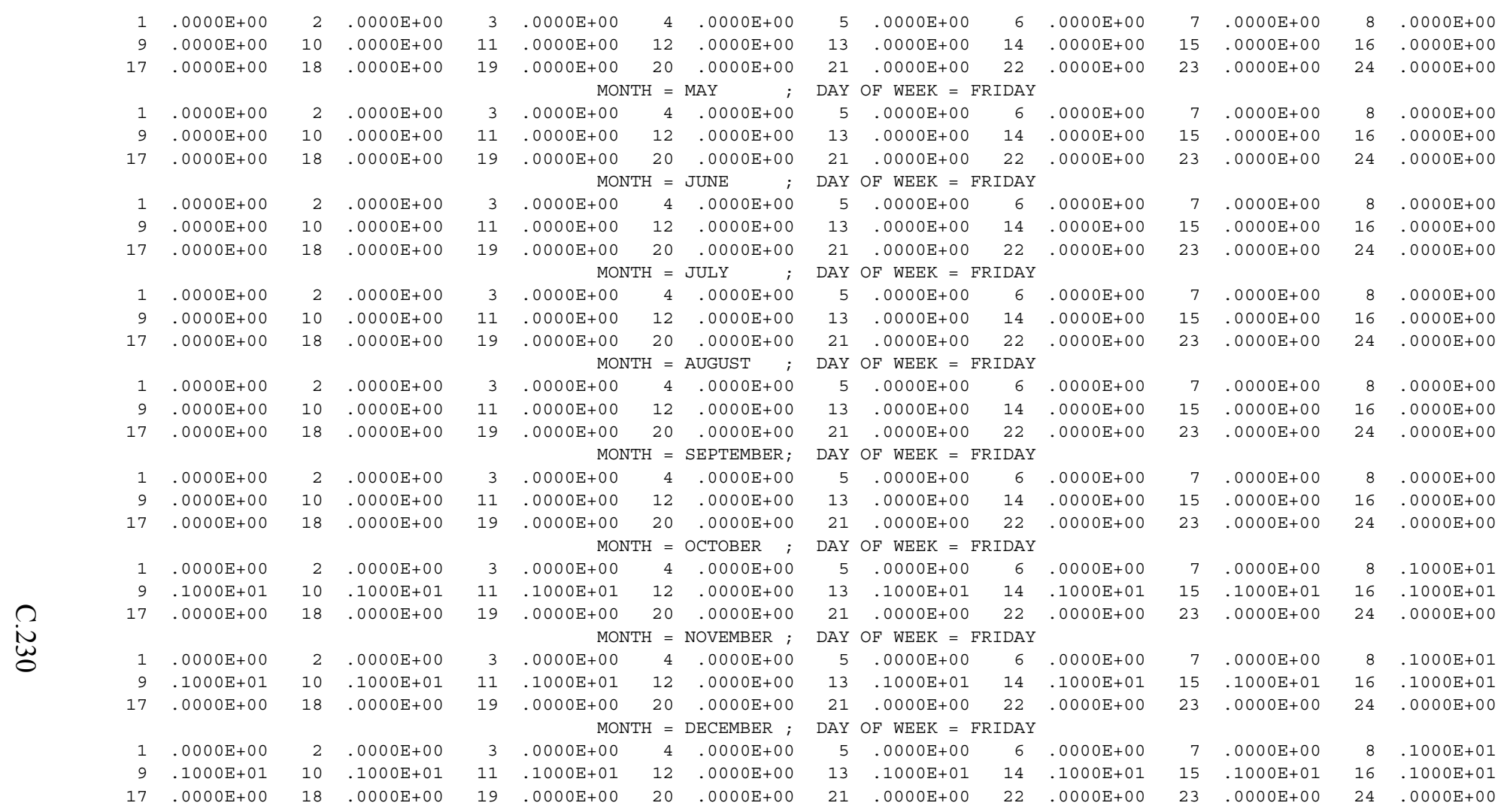

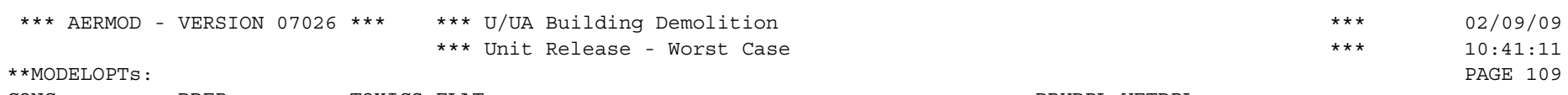

*** $\quad 10: 41: 11$

DDEP TOXICS FIAT

DRYDPL WETDPL

* SOURCE EMISSION RATE SCALARS WHICH VARY MONTHLY, DIURNALLY AND BY DAY OF WEEK (MHRDOW7) *

SOURCE ID $=224$ UA_5 $;$ SOURCE TYPE $=$ POINT

HOUR SCALAR HOUR SCALAR HOUR SCALAR HOUR SCALAR HOUR SCALAR HOUR SCALAR HOUR SCALAR HOUR SCALAR

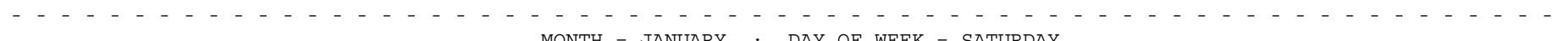

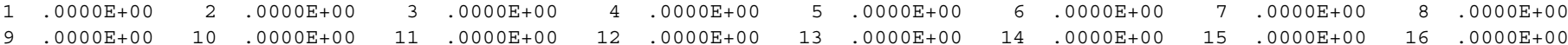

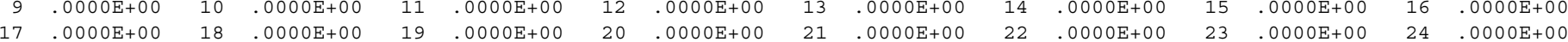

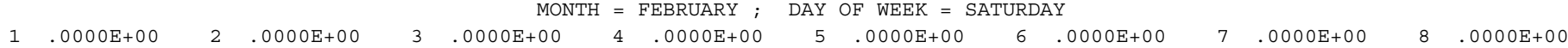




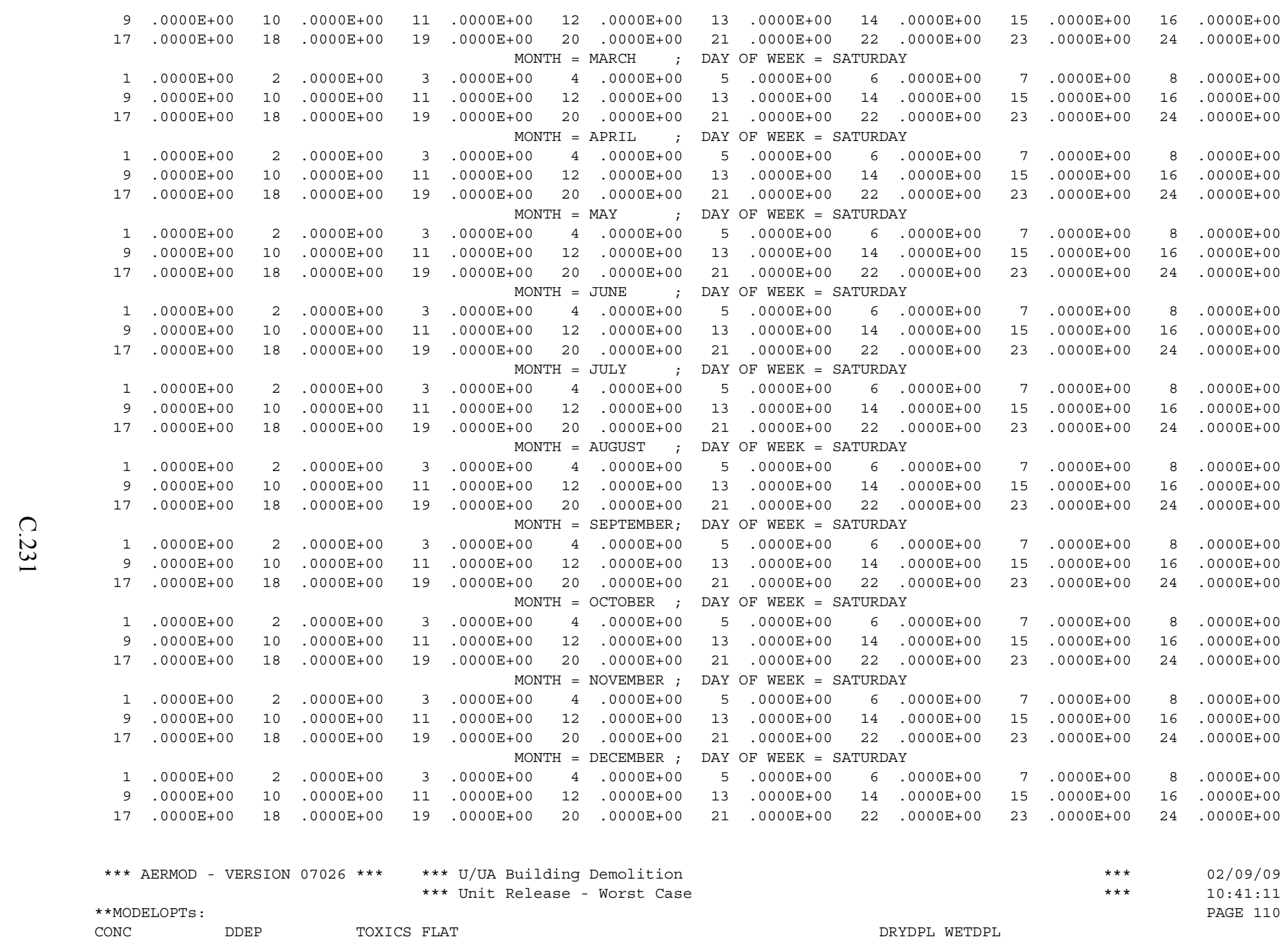

* source emission rate scalars which vary monthly, diURnally and by day of Week (MHrdow7) *

SOURCE ID $=224 \mathrm{UA} \_5 ;$ SOURCE TYPE $=$ POINT

HOUR SCALAR HOUR SCALAR HOUR SCALAR HOUR SCALAR HOUR SCALAR HOUR SCALAR HOUR SCALAR HOUR SCALAR 


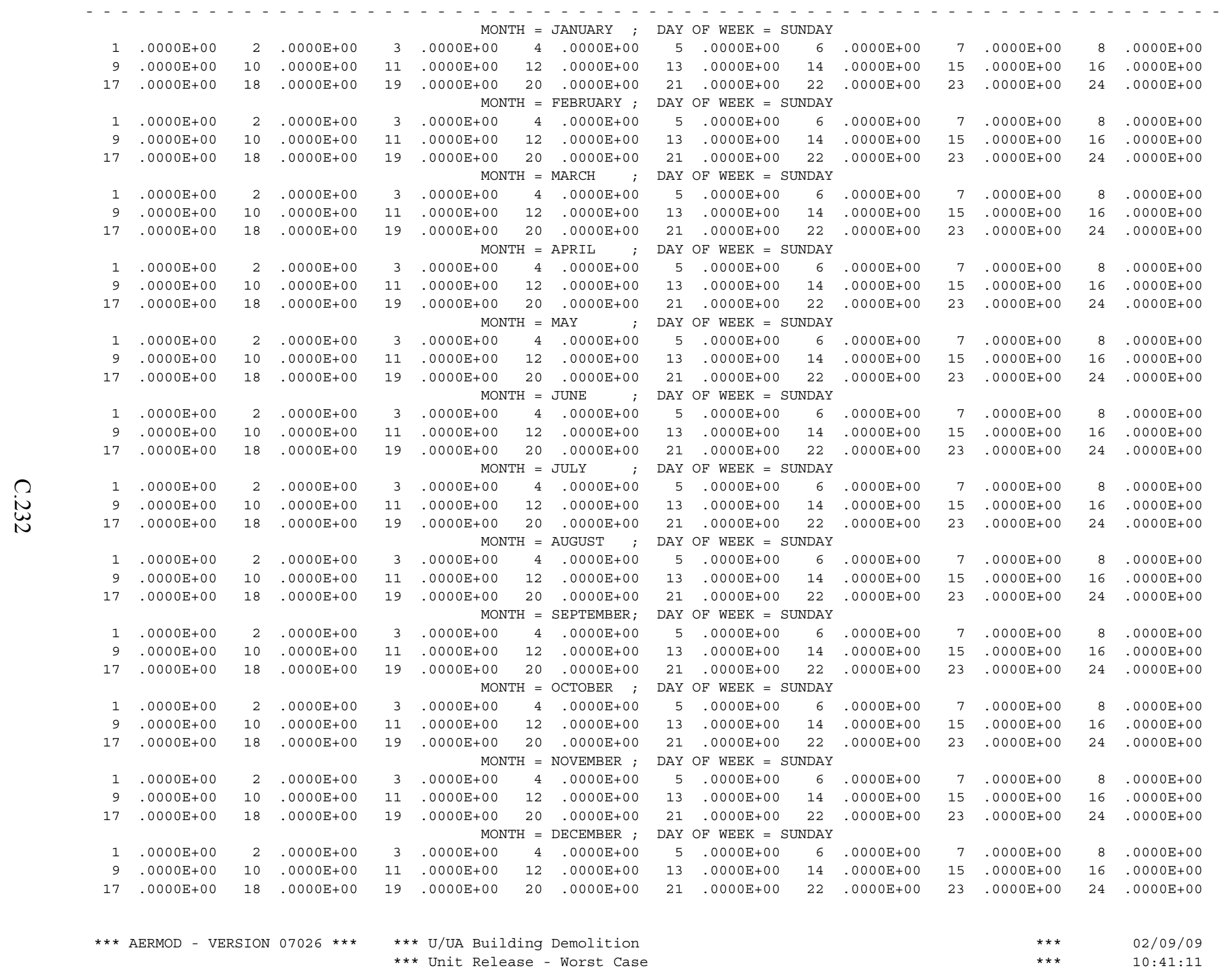


* SOURCE EMISSION RATE SCALARS WHICH VARY MONTHLY, DIURNALLY AND BY DAY OF WEEK (MHRDOW7) *

SOURCE ID $=$ 224UA $6 ;$ SOURCE TYPE $=$ POINT

HOUR SCATAR 'HOUR' SCALAR HOUR SCALAR HOUR SCALAR HOUR SCALAR HOUR SCALAR HOUR SCALAR HOUR SCALAR HOUR

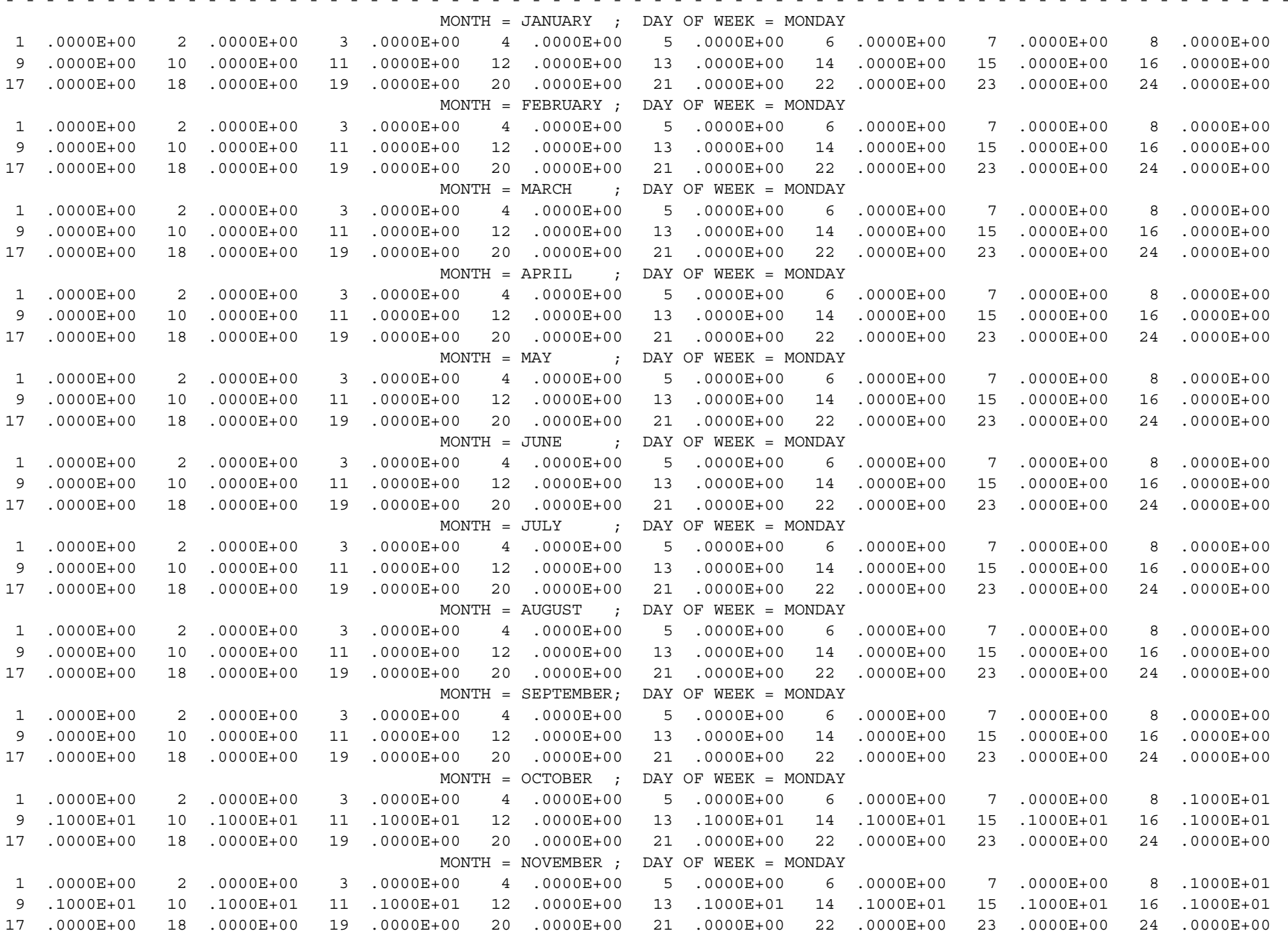
MONTH $=$ DECEMBER ; DAY OF WEEK = MONDAY 


\begin{tabular}{|c|c|c|c|c|c|c|c|c|c|c|c|c|c|c|c|}
\hline 1 & $\mathrm{E}+00$ & 2 & $.0000 \mathrm{E}+00$ & 3 & $.0000 \mathrm{E}+00$ & 4 & $.0000 \mathrm{E}+00$ & 5 & $.0000 \mathrm{E}+00$ & 6 & $.0000 \mathrm{E}+00$ & 7 & $.0000 \mathrm{E}+00$ & 8 & $0 E+01$ \\
\hline 9 & $.1000 \mathrm{E}+01$ & 10 & $.1000 \mathrm{E}+01$ & 11 & $.1000 \mathrm{E}+01$ & 12 & $.0000 \mathrm{E}+00$ & 13 & $.1000 \mathrm{E}+01$ & 14 & $.1000 \mathrm{E}+01$ & 15 & $.1000 \mathrm{E}+01$ & 16 & $.1000 \mathrm{E}+01$ \\
\hline & $.0000 \mathrm{E}+00$ & 18 & $.0000 \mathrm{E}+00$ & 19 & $.0000 \mathrm{E}+00$ & 20 & $.0000 \mathrm{E}+00$ & 21 & $.0000 \mathrm{E}+00$ & 22 & $.0000 \mathrm{E}+00$ & 23 & $.0000 \mathrm{E}+00$ & 24 & $00 \mathrm{E}+00$ \\
\hline
\end{tabular}

$* * *$ AERMOD - VERSION $07026 * * * \quad \begin{aligned} & * * \text { U/UA Building Demolition } \\ & * * * \text { Unit Release - Worst Case }\end{aligned}$ $\star *$ MODELOPTS :

CONC $\begin{array}{ll}* * * & 02 / 09 / 09 \\ * * * & 10: 41: 11\end{array}$

DRYDPL WETDPL

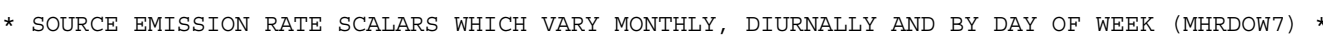

SOURCE ID $=224 \mathrm{UA} \_6 ;$ SOURCE TYPE $=$ POINT

HOUR SCALAR HOUR SCALAR HOUR SCALAR HOUR SCALAR HOUR SCALAR HOUR SCALAR HOUR SCALAR HOUR SCALAR

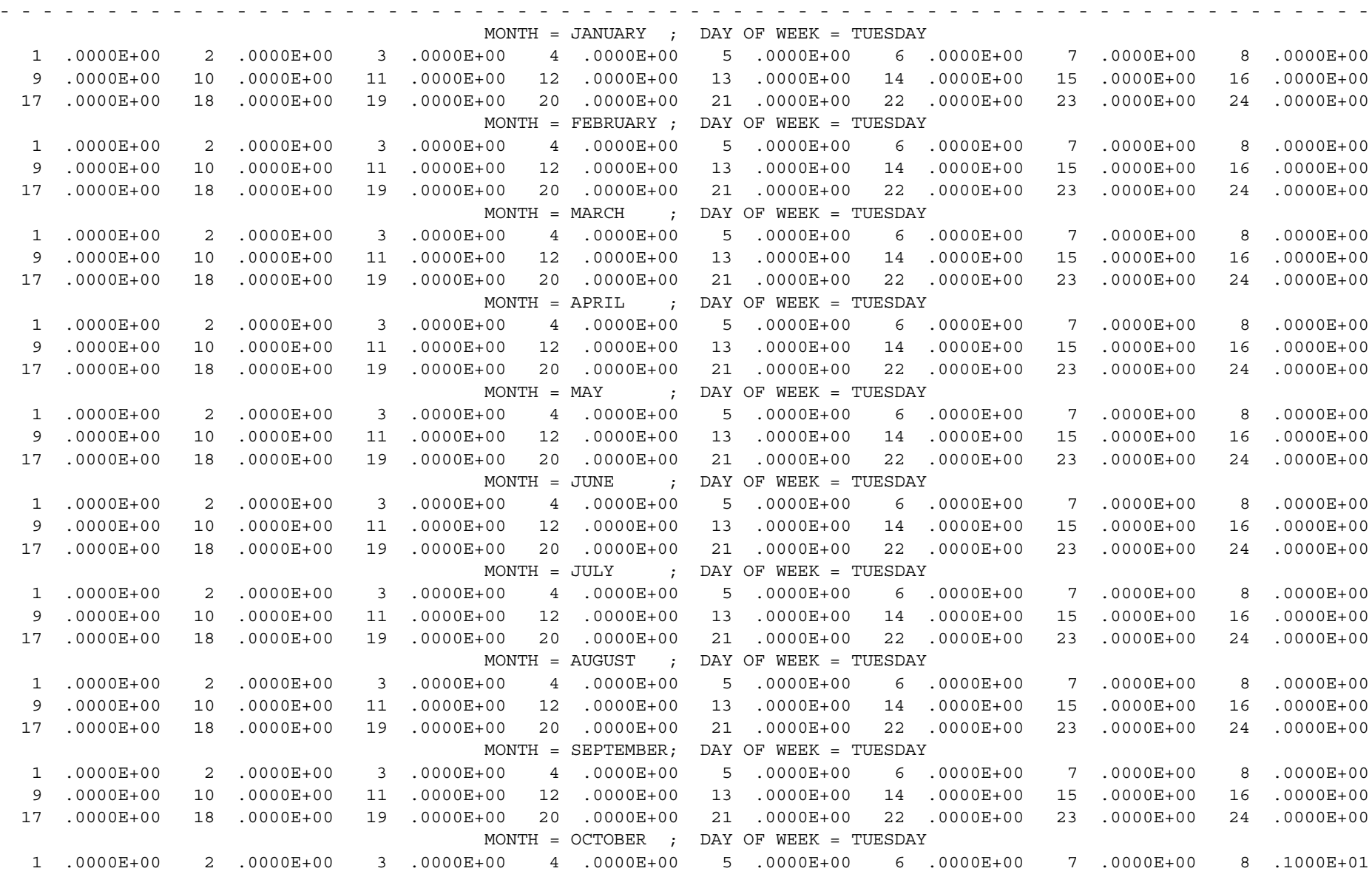




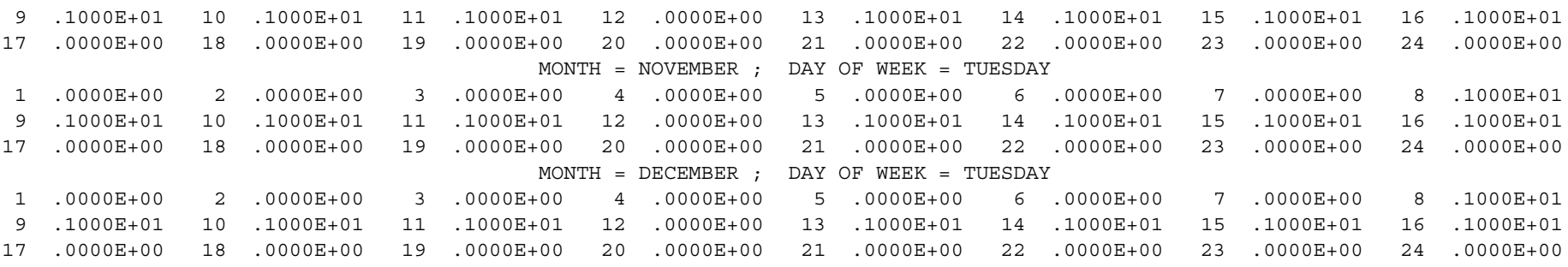

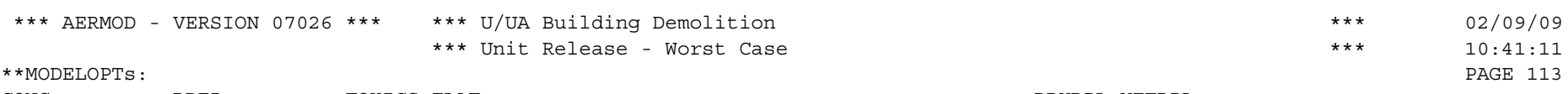

TOXICS FLAT

DRYDPL WETDPL

* SOURCE EMISSION RATE SCALARS WHICH VARY MONTHLY, DIURNALLY AND BY DAY OF WEEK (MHRDOW7) *

SOURCE ID $=224$ UA $6 ;$ SOURCE TYPE $=$ POINT

HOUR SCALAR HOUR SCALAR HOUR SCALAR HOUR SCALAR HOUR SCALAR HOUR SCALAR HOUR SCALAR HOUR SCALAR

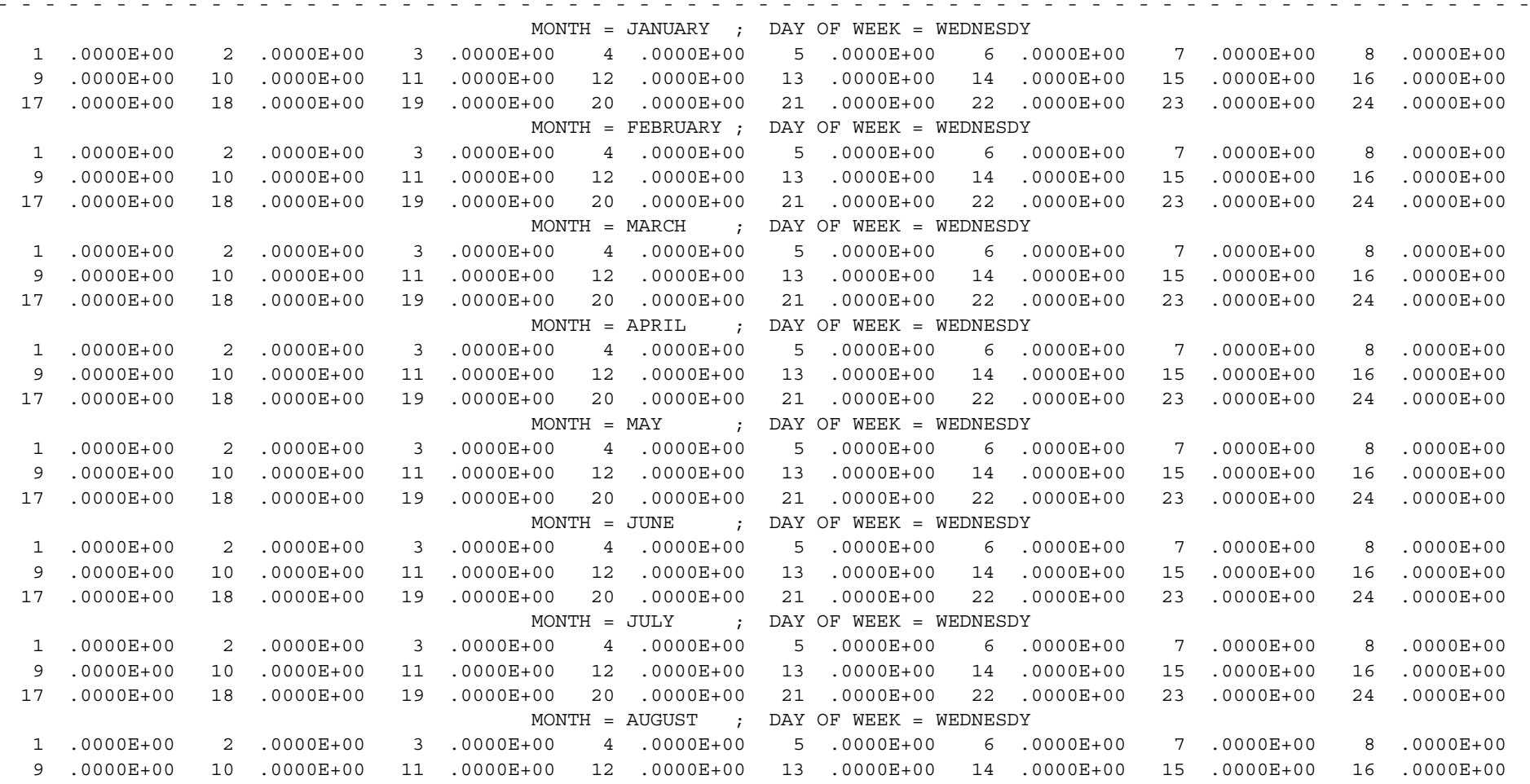




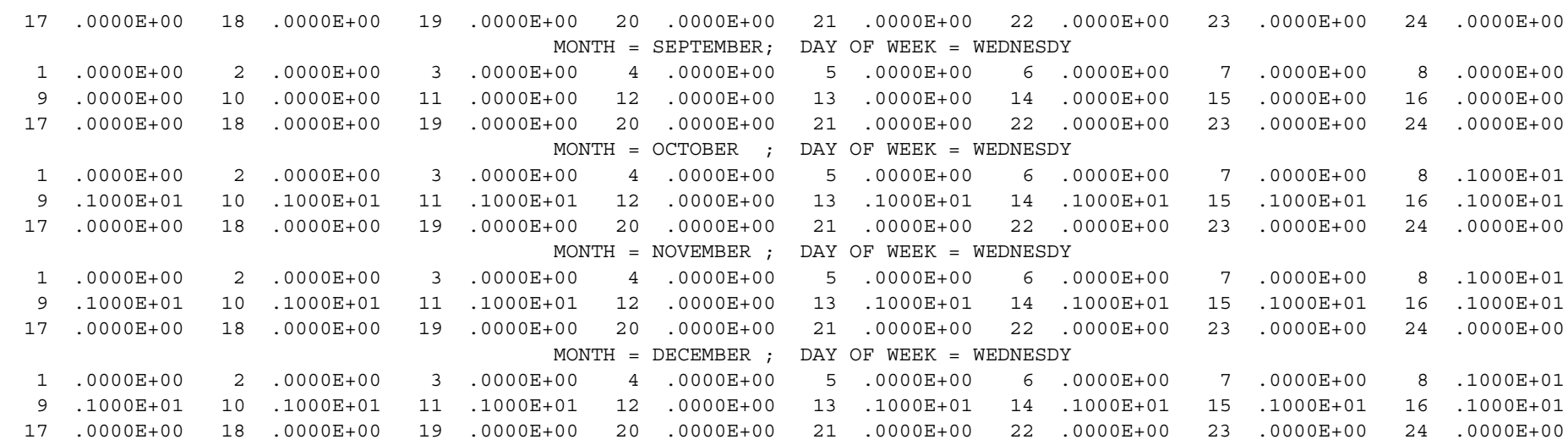

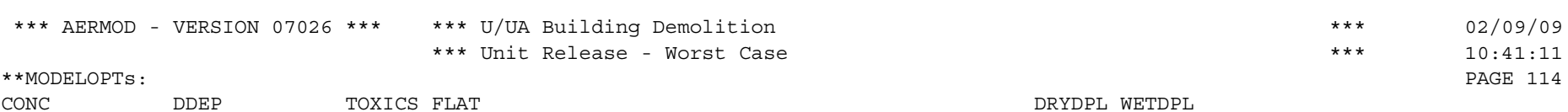
**MOD

* SOURCE EMISSION RATE SCALARS WHICH VARY MONTHLY, DIURNALLY AND BY DAY OF WEEK (MHRDOW7) *

SOURCE ID $=224 \mathrm{UA} \_6 ;$ SOURCE TYPE $=$ POINT

HOUR SCALAR HOUR SCALAR HOUR SCALAR HOUR SCALAR HOUR SCALAR HOUR SCALAR HOUR SCALAR HOUR SCALAR

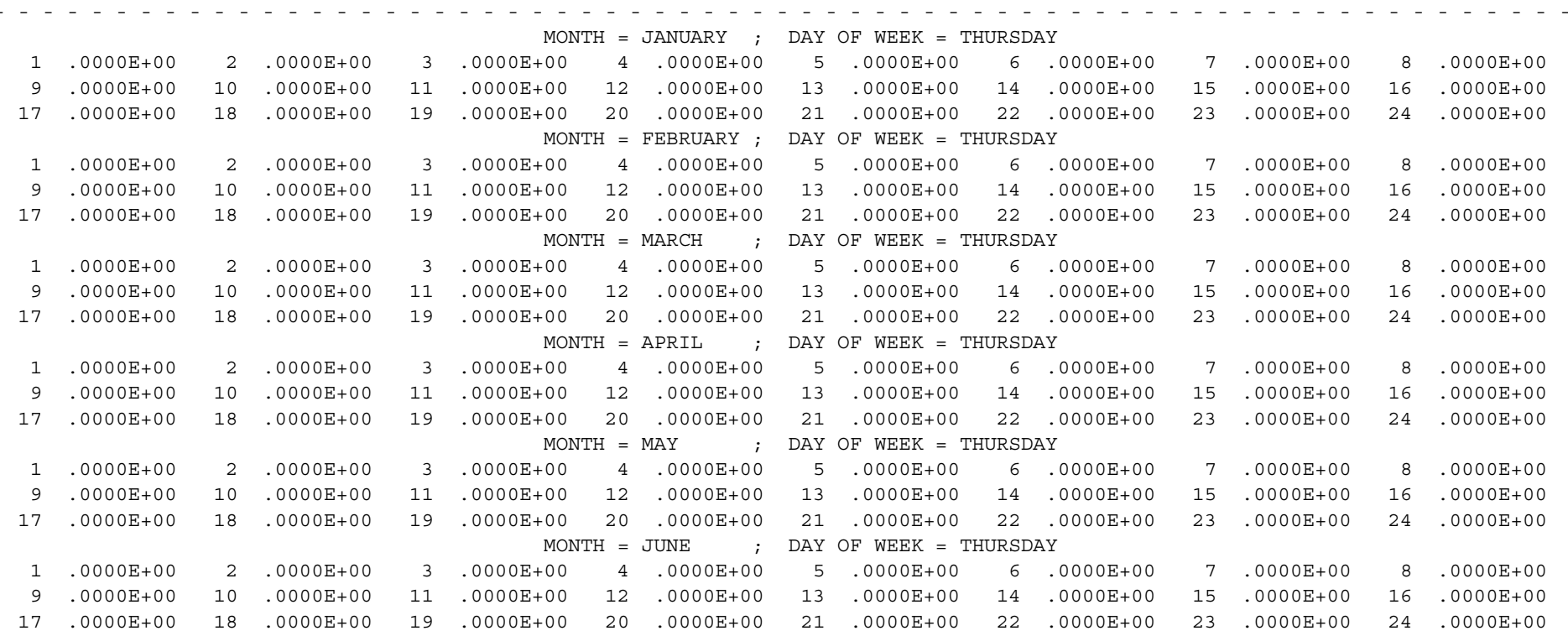




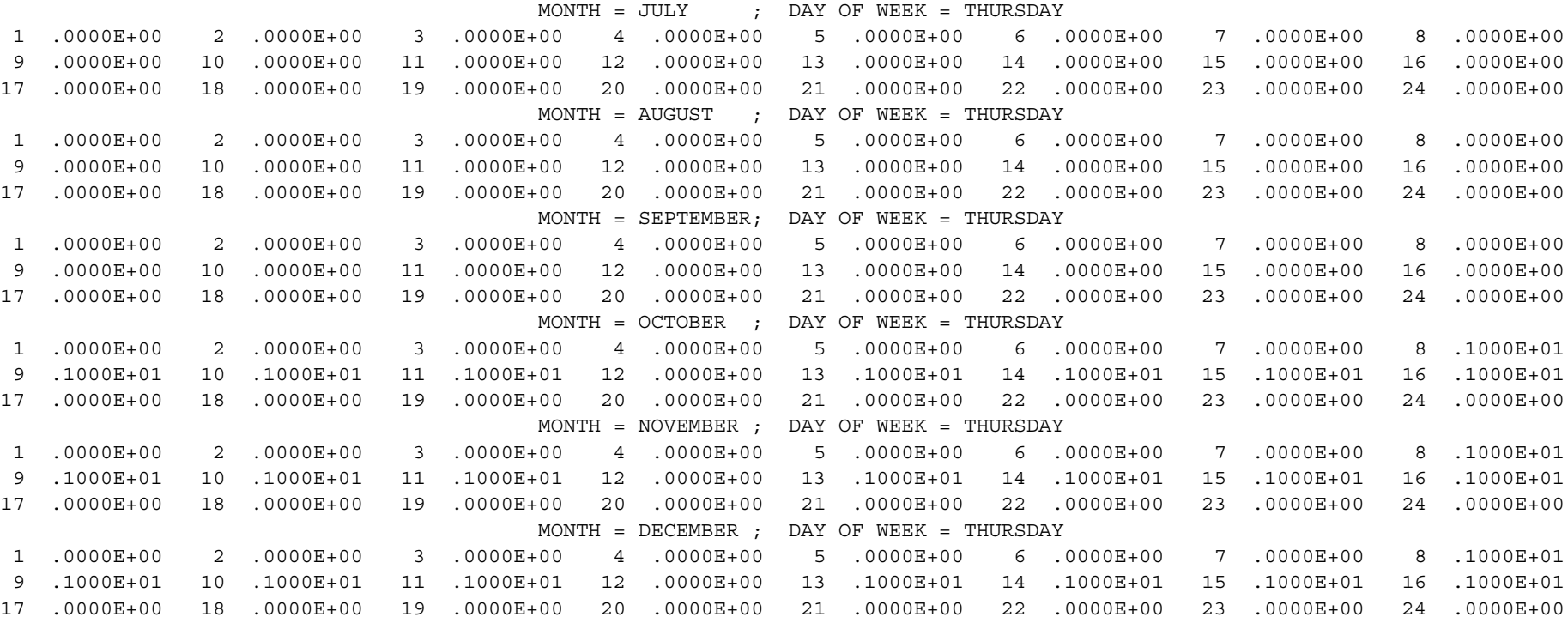

DRYDPL WETDPL

* SOURCE EMISSiOn RATE SCALARS WHich VARY MONTHLy, DIURNALLy AND by DAy OF WEEK (MHRDOW7) *

SOURCE ID $=224 \mathrm{UA} 6 ; \quad$ SOURCE TYPE $=$ POINT

HOUR SCALAR HOUR SCALAR HOUR SCALAR HOUR SCALAR HOUR SCALAR HOUR SCALAR HOUR SCALAR HOUR SCALAR

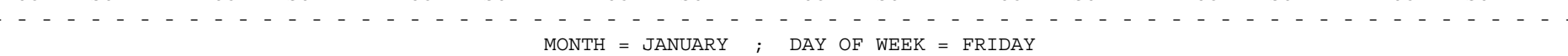

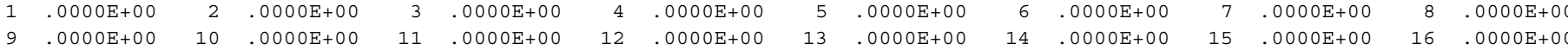

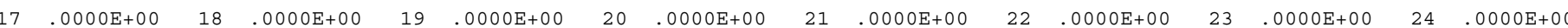

MONTH $=$ FEBRUARY ; DAY OF WEEK $=$ FRIDAY

$\begin{array}{lllllllllllll}3 & .0000 \mathrm{E}+00 & 4 & .0000 \mathrm{E}+00 & 5 & .0000 \mathrm{E}+00 & 6 & .0000 \mathrm{E}+00 & 7 & .0000 \mathrm{E}+00 & 8 & .0000 \mathrm{E}+00\end{array}$

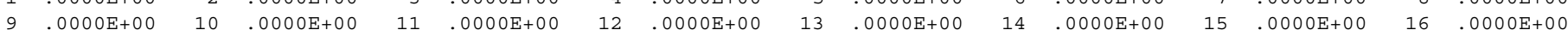

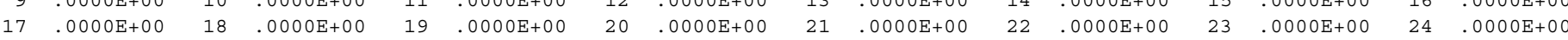

$1.0000 \mathrm{E}+00 \quad 2 \quad .0000 \mathrm{E}+00 \quad 3 \quad .0000 \mathrm{E}+00 \quad 4 \quad .0000 \mathrm{E}+00 \quad 5 \quad 5 \quad$ DAY OF WEEK $=$ FRIDAY

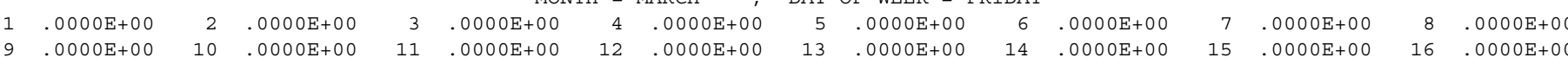

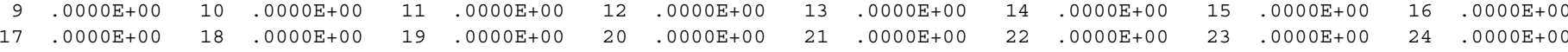

$\begin{array}{rrrrrrrrrrrrrrrrr}1 & .0000 \mathrm{E}+00 & 2 & .0000 \mathrm{E}+00 & 3 & .0000 \mathrm{E}+00 & 4 & .0000 \mathrm{E}+00 & 5 & .0000 \mathrm{E}+00 & 6 & .0000 \mathrm{E}+00 & 7 & .0000 \mathrm{E}+00 & 8 & .0000 \mathrm{E}+00\end{array}$

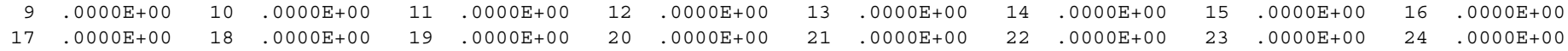

$$
\text { MONTH }=\text { MAY } \quad ; \quad \text { DAY OF WEEK }=\text { FRIDAY }
$$




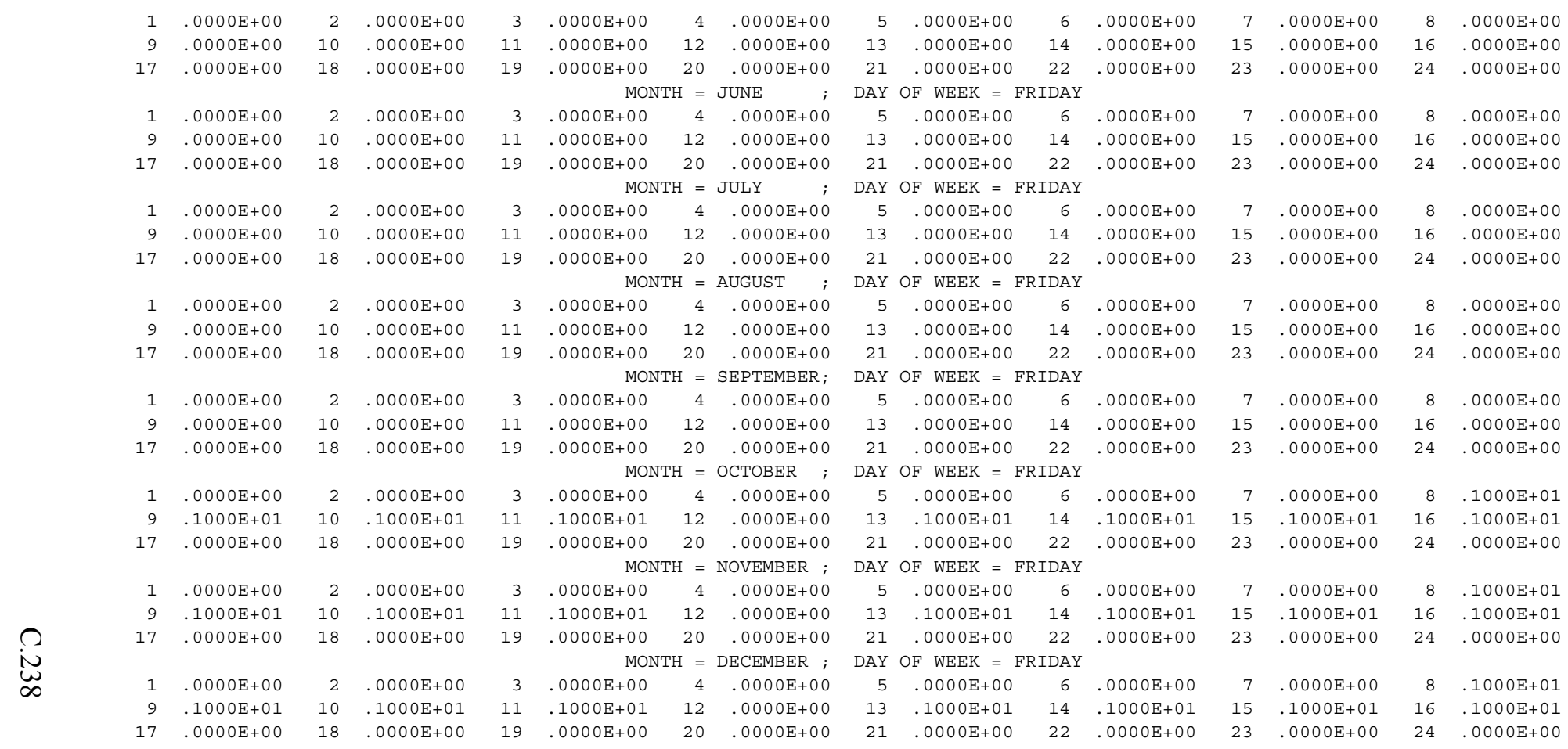
$* * *$ AERMOD - VERSION $07026 * * * \quad \begin{aligned} & * * \text { U/UA Building Demolition } \\ & * * * \text { Unit Release - Worst Case }\end{aligned}$

*** $\quad 02 / 09 / 09$

$10: 41: 11$
PAGE 116

DDEP

TOXICS FLAT

DRYDPL WETDPL

* SOURCE EMISSION RATE SCALARS WHICH VARY MONTHLY, DIURNALLY AND BY DAY OF WEEK (MHRDOW7) *

SOURCE ID $=224$ UA $6 ;$ SOURCE TYPE $=$ POINT

HOUR SCALAR HOUR SCALAR HOUR SCALAR HOUR SCALAR HOUR SCALAR HOUR SCALAR HOUR SCALAR HOUR SCALAR

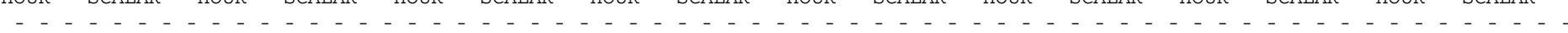
1. MONTH $=$ JANUARY ; DAY OF WEEK $=$ SATURDAY

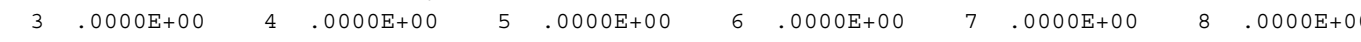

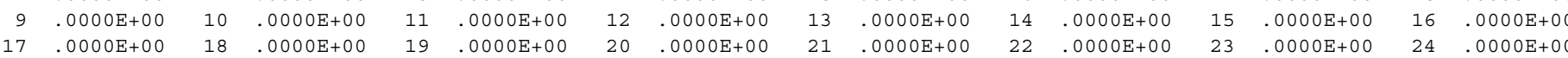

$\begin{array}{lllllllllllllll}18 & .0000 \mathrm{E}+00 & 19 & .0000 \mathrm{E}+00 & 20 & .0000 \mathrm{E}+00 & 21 & .0000 \mathrm{E}+00 & 22 & .0000 \mathrm{E}+00 & 23 & .0000 \mathrm{E}+00 & 24 & .0000 \mathrm{E}+00\end{array}$

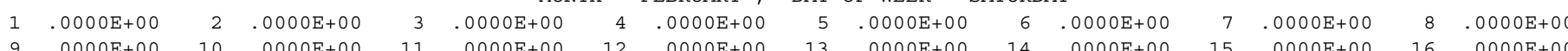

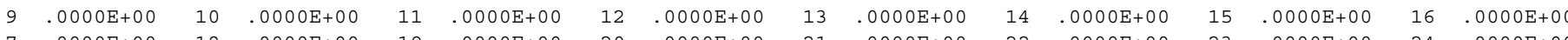

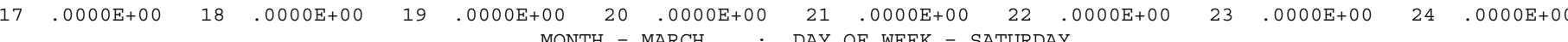

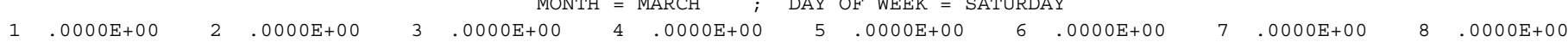




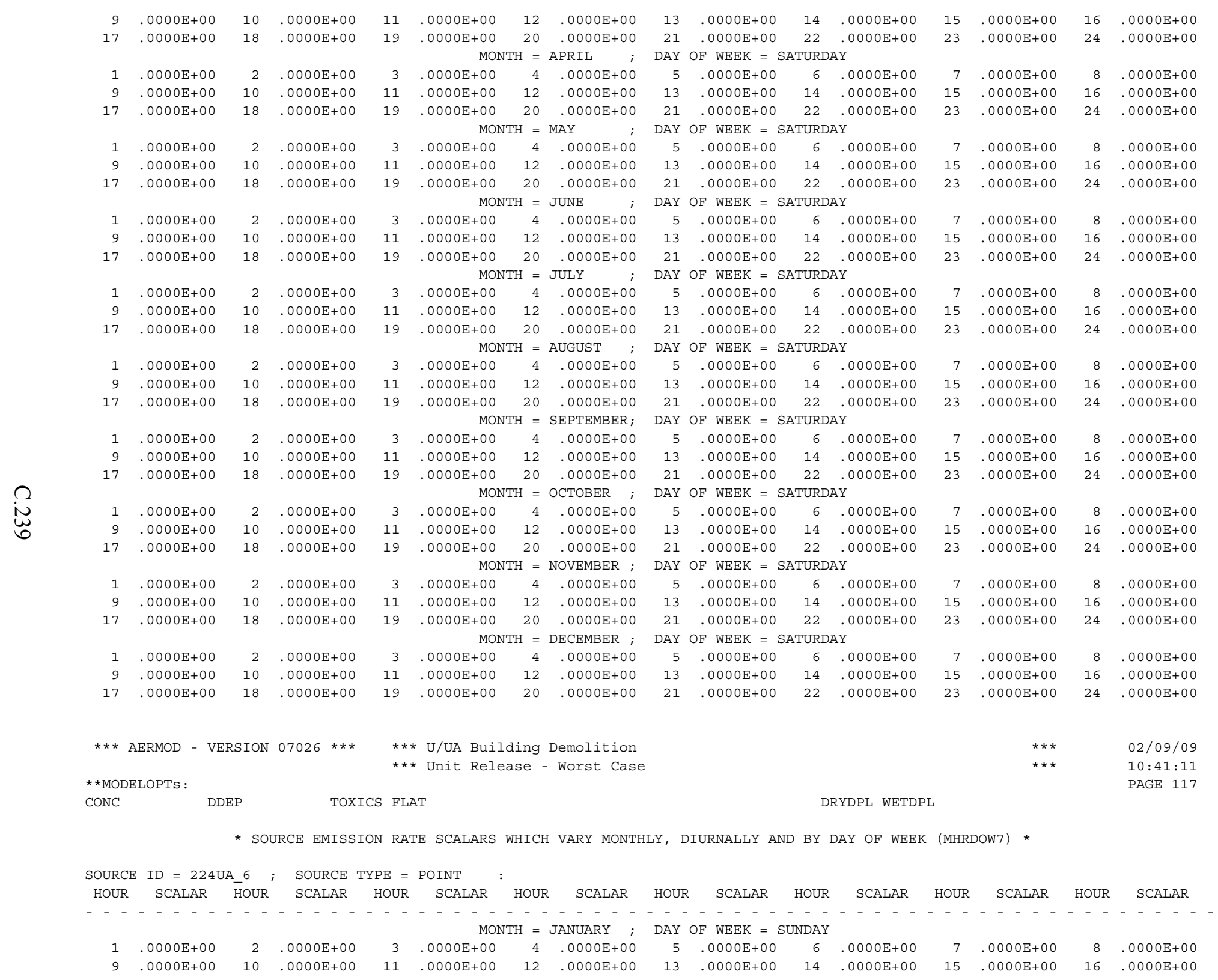




\begin{tabular}{|c|c|c|c|c|c|c|c|c|c|c|c|c|c|c|c|}
\hline 17 & $.0000 \mathrm{E}+00$ & 18 & $.0000 \mathrm{E}+00$ & 19 & $.0000 \mathrm{E}+00$ & 20 & & $\begin{array}{r}21 \\
0 \mathrm{P}\end{array}$ & & 22 & $.0000 \mathrm{E}+00$ & 23 & $.0000 \mathrm{E}+00$ & 24 & $.0000 \mathrm{E}+00$ \\
\hline 1 & $.0000 \mathrm{E}+00$ & 2 & $.0000 \mathrm{E}+00$ & 3 & $\begin{array}{r}\text { MON } \\
.0000 \mathrm{E}+00\end{array}$ & $\begin{array}{r}\mathrm{H}= \\
4\end{array}$ & $\begin{array}{l}\text { FEBRUARY ; } \\
.0000 E+00\end{array}$ & $\begin{array}{r}\text { DAY } \\
5\end{array}$ & $\begin{array}{l}\text { OF WEEK }=S \\
.0000 \mathrm{~S}+00\end{array}$ & $\begin{array}{c}\text { SUNDAY } \\
6\end{array}$ & $.0000 \mathrm{E}+00$ & 7 & $.0000 \mathrm{E}+00$ & 8 & $.0000 \mathrm{E}+00$ \\
\hline 9 & $.0000 \mathrm{E}+00$ & 10 & $.0000 \mathrm{E}+00$ & 11 & $.0000 \mathrm{E}+00$ & 12 & $.0000 \mathrm{E}+00$ & 13 & $.0000 \mathrm{E}+00$ & 14 & $.0000 E+00$ & 15 & $.0000 \mathrm{E}+00$ & 16 & $.0000 \mathrm{E}+00$ \\
\hline 17 & $.0000 \mathrm{E}+00$ & 18 & $.0000 \mathrm{E}+00$ & 19 & $.0000 \mathrm{E}+00$ & 20 & $.0000 \mathrm{E}+00$ & 21 & $.0000 \mathrm{E}+00$ & 22 & $.0000 \mathrm{E}+00$ & 23 & $.0000 \mathrm{E}+00$ & 24 & $.0000 \mathrm{E}+00$ \\
\hline & & & & & MON & $H=$ & MARCH ; & DAY & OF $W E E K=S$ & SUNDAY & & & & & \\
\hline 1 & $.0000 \mathrm{E}+00$ & 2 & $.0000 \mathrm{E}+00$ & 3 & $.0000 \mathrm{E}+00$ & 4 & $.0000 \mathrm{E}+00$ & 5 & $.0000 \mathrm{E}+00$ & 6 & $.0000 \mathrm{E}+00$ & 7 & $.0000 \mathrm{E}+00$ & 8 & $.0000 \mathrm{E}+00$ \\
\hline 9 & $.0000 \mathrm{E}+00$ & 10 & $.0000 \mathrm{E}+00$ & 11 & $.0000 \mathrm{E}+00$ & 12 & $.0000 \mathrm{E}+00$ & 13 & $.0000 \mathrm{E}+00$ & 14 & $.0000 \mathrm{E}+00$ & 15 & $.0000 \mathrm{E}+00$ & 16 & $.0000 \mathrm{E}+00$ \\
\hline 17 & $.0000 \mathrm{E}+00$ & 18 & $.0000 \mathrm{E}+00$ & 19 & $.0000 \mathrm{E}+00$ & 20 & $.0000 \mathrm{E}+00$ & 21 & $.0000 \mathrm{E}+00$ & 22 & $.0000 \mathrm{E}+00$ & 23 & $.0000 \mathrm{E}+00$ & 24 & $.0000 \mathrm{E}+00$ \\
\hline & & & & & MON & $\mathrm{H}=$ & APRIL ; & DAY & OF WEEK $=S$ & SUNDAY & & & & & \\
\hline 1 & $.0000 \mathrm{E}+00$ & 2 & $.0000 \mathrm{E}+00$ & 3 & $.0000 \mathrm{E}+00$ & 4 & $.0000 \mathrm{E}+00$ & 5 & $.0000 \mathrm{E}+00$ & 6 & $.0000 \mathrm{E}+00$ & 7 & $.0000 \mathrm{E}+00$ & 8 & $.0000 \mathrm{E}+00$ \\
\hline 9 & $.0000 \mathrm{E}+00$ & 10 & $.0000 \mathrm{E}+00$ & 11 & $.0000 \mathrm{E}+00$ & 12 & $.0000 \mathrm{E}+00$ & 13 & $.0000 \mathrm{E}+00$ & 14 & $.0000 \mathrm{E}+00$ & 15 & $.0000 \mathrm{E}+00$ & 16 & $.0000 \mathrm{E}+00$ \\
\hline 17 & $.0000 \mathrm{E}+00$ & 18 & $.0000 \mathrm{E}+00$ & 19 & $.0000 \mathrm{E}+00$ & 20 & $.0000 \mathrm{E}+00$ & 21 & $.0000 \mathrm{E}+00$ & 22 & $.0000 \mathrm{E}+00$ & 23 & $.0000 \mathrm{E}+00$ & 24 & $.0000 \mathrm{E}+00$ \\
\hline & & & & & MON & $H=$ & MAY ; & DAY & $O F \quad W E E K=S$ & SUNDAY & & & & & \\
\hline 1 & $.0000 \mathrm{E}+00$ & 2 & $.0000 \mathrm{E}+00$ & 3 & $.0000 \mathrm{E}+00$ & 4 & $.0000 \mathrm{E}+00$ & 5 & $.0000 \mathrm{E}+00$ & 6 & $.0000 \mathrm{E}+00$ & 7 & $.0000 \mathrm{E}+00$ & 8 & $.0000 \mathrm{E}+00$ \\
\hline 9 & $.0000 \mathrm{E}+00$ & 10 & $.0000 \mathrm{E}+00$ & 11 & $.0000 \mathrm{E}+00$ & 12 & $.0000 \mathrm{E}+00$ & 13 & $.0000 \mathrm{E}+00$ & 14 & $.0000 \mathrm{E}+00$ & 15 & $.0000 \mathrm{E}+00$ & 16 & $.0000 \mathrm{E}+00$ \\
\hline 17 & $.0000 \mathrm{E}+00$ & 18 & $.0000 \mathrm{E}+00$ & 19 & $.0000 \mathrm{E}+00$ & 20 & $.0000 \mathrm{E}+00$ & 21 & $.0000 \mathrm{E}+00$ & 22 & $.0000 \mathrm{E}+00$ & 23 & $.0000 \mathrm{E}+00$ & 24 & $.0000 \mathrm{E}+00$ \\
\hline & & & & & MON & $\mathrm{H}=$ & JUNE ; i & DAY & OF WEEK $=S$ & SUNDAY & & & & & \\
\hline 1 & $.0000 \mathrm{E}+00$ & 2 & $.0000 \mathrm{E}+00$ & 3 & $.0000 \mathrm{E}+00$ & 4 & $.0000 \mathrm{E}+00$ & 5 & $.0000 \mathrm{E}+00$ & 6 & $.0000 \mathrm{E}+00$ & 7 & $.0000 \mathrm{E}+00$ & 8 & $.0000 \mathrm{E}+00$ \\
\hline 9 & $.0000 \mathrm{E}+00$ & 10 & $.0000 \mathrm{E}+00$ & 11 & $.0000 \mathrm{E}+00$ & 12 & $.0000 \mathrm{E}+00$ & 13 & $.0000 \mathrm{E}+00$ & 14 & $.0000 \mathrm{E}+00$ & 15 & $.0000 \mathrm{E}+00$ & 16 & $.0000 \mathrm{E}+00$ \\
\hline 17 & $.0000 \mathrm{E}+00$ & 18 & $.0000 \mathrm{E}+00$ & 19 & $.0000 \mathrm{E}+00$ & 20 & $.0000 \mathrm{E}+00$ & 21 & $.0000 \mathrm{E}+00$ & 22 & $.0000 \mathrm{E}+00$ & 23 & $.0000 \mathrm{E}+00$ & 24 & $.0000 \mathrm{E}+00$ \\
\hline & & & & & MON & $H=$ & JULY ; & DAY & OF WEEK $=S$ & SUNDAY & & & & & \\
\hline 1 & $.0000 \mathrm{E}+00$ & 2 & $.0000 \mathrm{E}+00$ & 3 & $.0000 \mathrm{E}+00$ & 4 & $.0000 \mathrm{E}+00$ & 5 & $.0000 \mathrm{E}+00$ & 6 & $.0000 \mathrm{E}+00$ & 7 & $.0000 \mathrm{E}+00$ & 8 & $.0000 \mathrm{E}+00$ \\
\hline 9 & $.0000 \mathrm{E}+00$ & 10 & $.0000 \mathrm{E}+00$ & 11 & $.0000 \mathrm{E}+00$ & 12 & $.0000 \mathrm{E}+00$ & 13 & $.0000 \mathrm{E}+00$ & 14 & $.0000 \mathrm{E}+00$ & 15 & $.0000 \mathrm{E}+00$ & 16 & $.0000 \mathrm{E}+00$ \\
\hline 17 & $.0000 \mathrm{E}+00$ & 18 & $.0000 \mathrm{E}+00$ & 19 & $.0000 \mathrm{E}+00$ & 20 & $.0000 \mathrm{E}+00$ & 21 & $.0000 \mathrm{E}+00$ & 22 & $.0000 \mathrm{E}+00$ & 23 & $.0000 \mathrm{E}+00$ & 24 & $.0000 \mathrm{E}+00$ \\
\hline & & & & & MON & $\mathrm{H}=$ & AUGUST ; & DAY & OF WEEK = S & SUNDAY & & & & & \\
\hline 1 & $.0000 \mathrm{E}+00$ & 2 & $.0000 \mathrm{E}+00$ & 3 & $.0000 \mathrm{E}+00$ & 4 & $.0000 \mathrm{E}+00$ & 5 & $.0000 \mathrm{E}+00$ & 6 & $.0000 \mathrm{E}+00$ & 7 & $.0000 \mathrm{E}+00$ & 8 & $.0000 \mathrm{E}+00$ \\
\hline 9 & $.0000 \mathrm{E}+00$ & 10 & $.0000 \mathrm{E}+00$ & 11 & $.0000 \mathrm{E}+00$ & 12 & $.0000 \mathrm{E}+00$ & 13 & $.0000 \mathrm{E}+00$ & 14 & $.0000 \mathrm{E}+00$ & 15 & $.0000 \mathrm{E}+00$ & 16 & $.0000 \mathrm{E}+00$ \\
\hline 17 & $.0000 \mathrm{E}+00$ & 18 & $.0000 \mathrm{E}+00$ & 19 & $.0000 \mathrm{E}+00$ & 20 & $.0000 \mathrm{E}+00$ & 21 & $.0000 \mathrm{E}+00$ & 22 & $.0000 \mathrm{E}+00$ & 23 & $.0000 E+00$ & 24 & $.0000 \mathrm{E}+00$ \\
\hline & & & & & MON & $\mathrm{H}=$ & SEPTEMBER； & DAY & OF WEEK $=S$ & SUNDAY & & & & & \\
\hline 1 & $.0000 \mathrm{E}+00$ & 2 & $.0000 \mathrm{E}+00$ & 3 & $.0000 \mathrm{E}+00$ & 4 & $.0000 \mathrm{E}+00$ & 5 & $.0000 \mathrm{E}+00$ & 6 & $.0000 \mathrm{E}+00$ & 7 & $.0000 \mathrm{E}+00$ & 8 & $.0000 \mathrm{E}+00$ \\
\hline 9 & $.0000 \mathrm{E}+00$ & 10 & $.0000 \mathrm{E}+00$ & 11 & $.0000 \mathrm{E}+00$ & 12 & $.0000 E+00$ & 13 & $.0000 \mathrm{E}+00$ & 14 & $.0000 \mathrm{E}+00$ & 15 & $.0000 \mathrm{E}+00$ & 16 & $.0000 \mathrm{E}+00$ \\
\hline 17 & $.0000 \mathrm{E}+00$ & 18 & $.0000 \mathrm{E}+00$ & 19 & $.0000 \mathrm{E}+00$ & 20 & $.0000 \mathrm{E}+00$ & 21 & $.0000 \mathrm{E}+00$ & 22 & $.0000 \mathrm{E}+00$ & 23 & $.0000 \mathrm{E}+00$ & 24 & $.0000 \mathrm{E}+00$ \\
\hline & & & & & MON & $\mathrm{H}=$ & OCTOBER ; & DAY & OF WEEK $=S$ & SUNDAY & & & & & \\
\hline 1 & $.0000 \mathrm{E}+00$ & 2 & $.0000 \mathrm{E}+00$ & 3 & $.0000 \mathrm{E}+00$ & 4 & $.0000 \mathrm{E}+00$ & 5 & $.0000 \mathrm{E}+00$ & 6 & $.0000 E+00$ & 7 & $.0000 \mathrm{E}+00$ & 8 & $.0000 \mathrm{E}+00$ \\
\hline 9 & $.0000 \mathrm{E}+00$ & 10 & $.0000 \mathrm{E}+00$ & 11 & $.0000 \mathrm{E}+00$ & 12 & $.0000 \mathrm{E}+00$ & 13 & $.0000 E+00$ & 14 & $.0000 \mathrm{E}+00$ & 15 & $.0000 \mathrm{E}+00$ & 16 & $.0000 \mathrm{E}+00$ \\
\hline 17 & $.0000 \mathrm{E}+00$ & 18 & $.0000 \mathrm{E}+00$ & 19 & $.0000 \mathrm{E}+00$ & 20 & $.0000 \mathrm{E}+00$ & 21 & $.0000 \mathrm{E}+00$ & 22 & $.0000 \mathrm{E}+00$ & 23 & $.0000 E+00$ & 24 & $.0000 \mathrm{E}+00$ \\
\hline & & & & & MON & $H=$ & NOVEMBER ; & DAY & OF $W E E K=S$ & SUNDAY & & & & & \\
\hline 1 & $.0000 \mathrm{E}+00$ & 2 & $.0000 \mathrm{E}+00$ & 3 & $.0000 \mathrm{E}+00$ & 4 & $.0000 \mathrm{E}+00$ & 5 & $.0000 \mathrm{E}+00$ & 6 & $0 \mathrm{E}+00$ & 7 & $0 \mathrm{E}+00$ & 8 & $.0000 \mathrm{E}+00$ \\
\hline 9 & $.0000 \mathrm{E}+00$ & 10 & $.0000 \mathrm{E}+00$ & 11 & $.0000 \mathrm{E}+00$ & 12 & $.0000 E+00$ & 13 & $.0000 E+00$ & 14 & $E+00$ & 15 & $.0000 E+00$ & 16 & $E+00$ \\
\hline 17 & $.0000 \mathrm{E}+00$ & 18 & $.0000 \mathrm{E}+00$ & 19 & $.0000 \mathrm{E}+00$ & 20 & $.0000 \mathrm{E}+00$ & 21 & $.0000 \mathrm{E}+00$ & 22 & $.0000 \mathrm{E}+00$ & 23 & $.0000 \mathrm{E}+00$ & 24 & $.0000 \mathrm{E}+00$ \\
\hline & & & & & MON & $\mathrm{H}=$ & DECEMBER ; & DAY & OF WEEK $=\mathrm{S}$ & SUNDAY & & & & & \\
\hline 1 & $.0000 \mathrm{E}+00$ & 2 & $.0000 \mathrm{E}+00$ & 3 & $.0000 \mathrm{E}+00$ & 4 & $.0000 \mathrm{E}+00$ & 5 & $.0000 \mathrm{E}+00$ & 6 & $.0000 \mathrm{E}+00$ & 7 & $.0000 \mathrm{E}+00$ & 8 & $.0000 \mathrm{E}+00$ \\
\hline 9 & $.0000 \mathrm{E}+00$ & 10 & $.0000 \mathrm{E}+00$ & 11 & $.0000 \mathrm{E}+00$ & 12 & $.0000 \mathrm{E}+00$ & 13 & $.0000 \mathrm{E}+00$ & 14 & $.0000 \mathrm{E}+00$ & 15 & $.0000 \mathrm{E}+00$ & 16 & $.0000 \mathrm{E}+00$ \\
\hline 17 & $.0000 \mathrm{E}+00$ & 18 & $.0000 \mathrm{E}+00$ & 19 & $.0000 \mathrm{E}+00$ & 20 & $.0000 \mathrm{E}+00$ & 21 & $.0000 \mathrm{E}+00$ & 22 & $.0000 \mathrm{E}+00$ & 23 & $.0000 \mathrm{E}+00$ & 24 & $.0000 \mathrm{E}+00$ \\
\hline$\star * *$ & * AERMOD - VI & ION & $07026 * \star *$ & & U/UA Buil & ng & Demo & & & & & & *** & & $02 / 09 / 09$ \\
\hline & & & & & & & & & & & & & $* * *$ & & $10: 41: 11$ \\
\hline & & & & & & & & & & & & & & & PAGE 118 \\
\hline & & & & FL & & & & & & & & & & & \\
\hline
\end{tabular}


*** GRIDDED RECEPTOR NETWORK SUMMARY ***

*** NETWORK ID: UCART1; NETWORK TYPE: GRIDCART ***

*** X-COORDINATES OF GRID ***

$$
\text { (METERS) }
$$

299050.0, 299055.0, 299060.0, 299065.0, 299070.0, 299075.0, 299080.0, 299085.0, 299090.0, 299095.0,

$299100.0,299105.0,299110.0,299115.0,299120.0,299125.0,299130.0,299135.0,299140.0,299145.0$

299150.0, 299155.0, 299160.0, 299165.0, 299170.0, 299175.0, 299180.0, 299185.0, 299190.0, 299195.0,

299200.0, 299205.0, 299210.0, 299215.0, 299220.0, 299225.0, 299230.0, 299235.0, 299240.0, 299245.0,

299250.0, 299255.0, 299260.0, 299265.0, 299270.0, 299275.0, 299280.0, 299285.0, 299290.0, 299295.0,

\section{$\star * *$ Y-COORDINATES OF GRID ***}

(METERS)

$5157807.0,5157812.0,5157817.0,5157822.0,5157827.0,5157832.0,5157837.0,5157842.0,5157847.0,5157852.0$, $5157857.0,5157862.0,5157867.0,5157872.0,5157877.0,5157882.0,5157887.0,5157892.0,5157897.0,5157902.0$ $5157907.0,5157912.0,5157917.0,5157922.0,5157927.0,5157932.0,5157937.0,5157942.0,5157947.0,5157952.0$ $5157957.0,5157962.0,5157967.0,5157972.0,5157977.0,5157982.0,5157987.0,5157992.0,5157997.0,5158002.0$ $5158007.0,5158012.0,5158017.0,5158022.0,5158027.0,5158032.0,5158037.0,5158042.0,5158047.0,5158052.0$

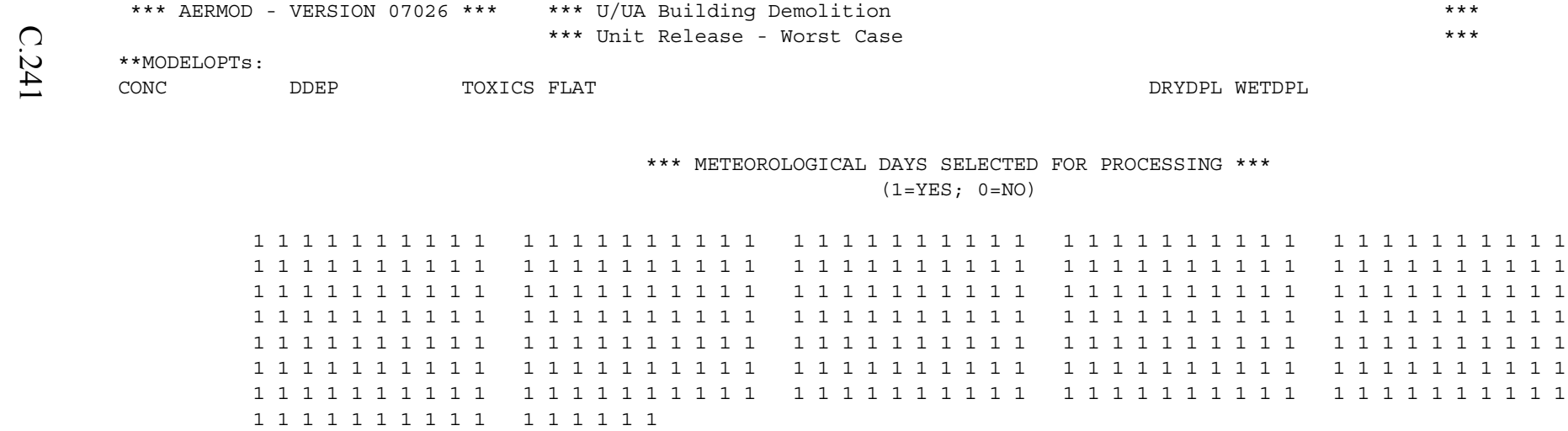

METEOROLOGICAL DATA PROCESSED BETWEEN START DATE: $\begin{array}{cccc}0 & 0 & 0 & 0\end{array}$

$$
\text { AND END DATE: } 9999999924
$$

NOte: METEOROLOGICAL DATA ACTUALLY PROCESSED WILL ALSO DEPEND ON WHAT IS INCLUDED IN the DATA FILE.

*** UPPER BOUND OF FIRST THROUGH FIFTH WIND SPEED CATEGORIES ***

(METERS/SEC) 


\begin{tabular}{|c|c|c|c|c|c|}
\hline *** AERMOD & - VERSION 07026 & $* * *$ & *** U/UA Building Demolition & $\star \star \star *$ & $\begin{array}{l}02 / 09 / 09 \\
10: 41: 11\end{array}$ \\
\hline MODELOPTS & & & & & PAGE 120 \\
\hline & DDEP & TOXICS & FLAT & & \\
\hline
\end{tabular}

*** UP TO THE FIRST 24 HOURS OF METEOROLOGICAL DATA ***

$\begin{array}{lll}\text { Surface file: } & \text { C: } \backslash \text { Jim } \backslash \text { UDISPE } ~ 1 \backslash \text { HANDFO } 1 \backslash 2006 \backslash \text { HAN19_06. SFC } \\ \text { Profile file: } & \text { C: }: \text { Jim } \backslash \text { UDISPE } 1 \backslash \text { HANDFO } 1 \backslash 2006 \backslash \text { HAN19 } 06 . \text { PFL }\end{array}$

Met Version: 06341

Surface format: ( $3(\mathrm{I} 2,1 \mathrm{X}), \mathrm{I} 3,1 \mathrm{X}, \mathrm{I} 2,1 \mathrm{X}, \mathrm{F} 6.1,1 \mathrm{X}, 3(\mathrm{~F} 6.3 \mathrm{3}, 1 \mathrm{X}), 2(\mathrm{~F} 5.0,1 \mathrm{X}), \mathrm{F} 8.1,1 \mathrm{X}, \mathrm{F} 6.3,1 \mathrm{X}, 2(\mathrm{~F} 6.2,1 \mathrm{X}), \mathrm{F} 7.2,1 \mathrm{X}, \mathrm{F} 5.0,3(1 \mathrm{X}, \mathrm{F} 6.1)$ )

Profile format: ( $4(\mathrm{I} 2,1 \mathrm{X}), \mathrm{F} 6.1,1 \mathrm{X}, \mathrm{I1}, 1 \mathrm{X}, \mathrm{F} 5.0,1 \mathrm{X}, \mathrm{F} 7.2,1 \mathrm{X}, \mathrm{F} 7.2,1 \mathrm{X}, \mathrm{F} 6.1,1 \mathrm{X}, \mathrm{F} 7.2)$

Surface station no.: $12219 \quad$ Upper air station no.: 4106

$$
\begin{array}{ll}
\text { Name: UNKNOWN } & \text { Name: UNKNOWN } \\
\text { Year: } 2006 & \text { Year: } 2006
\end{array}
$$

First 24 hours of scalar data
$\begin{array}{lllllllllll}\text { YR MO DY HR HO U* } & \text { W* DT/DZ ZICNV ZIMCH M-O LEN ZO BOWEN ALB REF WS WD HT REF TA HT IPCOD PRATE RH SFCP CCVR }\end{array}$

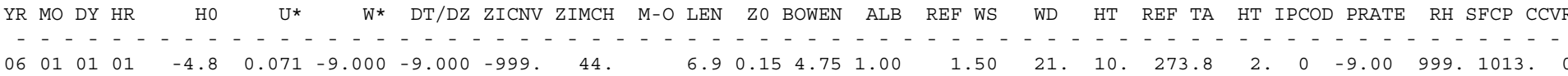

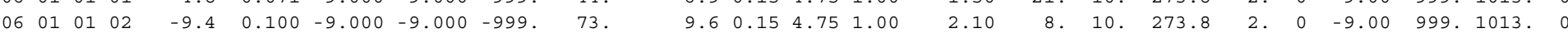

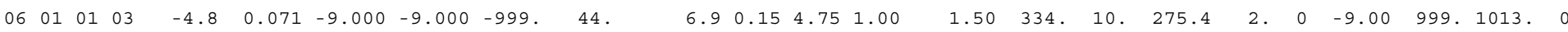

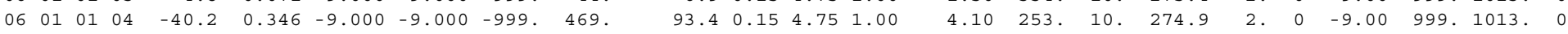

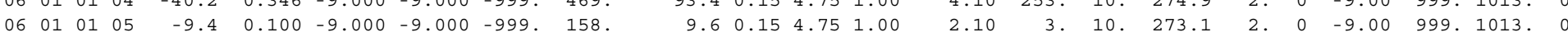

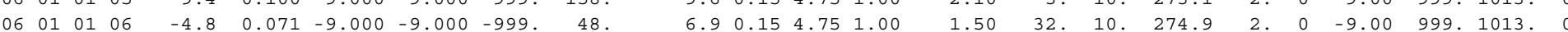

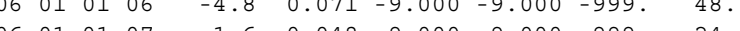

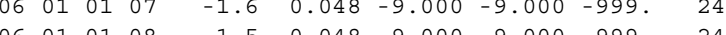

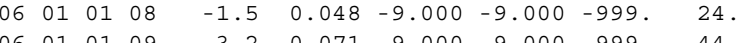

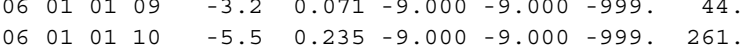

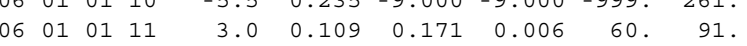

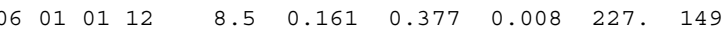
$\begin{array}{lllllllll}06 & 01 & 01 & 13 & 8.8 & 0.259 & 0.414 & 0.008 & 291.302 .\end{array}$ $\begin{array}{llllllllll}06 & 01 & 01 & 14 & 3.8 & 0.299 & 0.313 & 0.008 & 295 . & 377 .\end{array}$ $\begin{array}{llllllllll}06 & 01 & 01 & 15 & 4.0 & 0.253 & 0.321 & 0.008 & 299 . & 294 .\end{array}$ $\begin{array}{llllllllll}06 & 01 & 01 & 16 & -4.2 & 0.071 & -9.000 & -9.000 & -999 . & 87 .\end{array}$

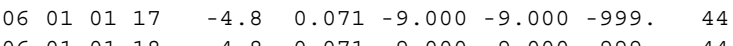
$\begin{array}{lllllllll}06 & 01 & 01 & 18 & -4.8 & 0.071 & -9.000 & -9.000 & -999.44\end{array}$

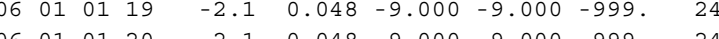

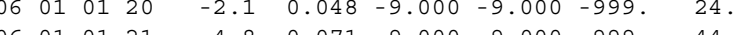

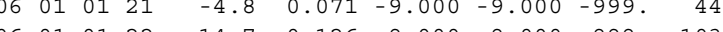

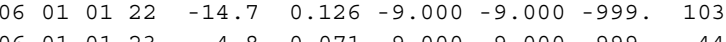
$\begin{array}{llllllllll}06 & 01 & 01 & 23 & -4.8 & 0.071 & -9.000 & -9.000 & -999 . & 44 \\ 06 & 01 & 01 & 24 & -4.8 & 0.071 & -9.000 & -9.000 & -999 . & 44\end{array}$ $\begin{array}{lllllllllllll}6.9 & 0.15 & 4.75 & 1.00 & 1.50 & 32 . & 10 . & 274.9 & 2 . & 0 & -9.00 & 999.1013 .0\end{array}$

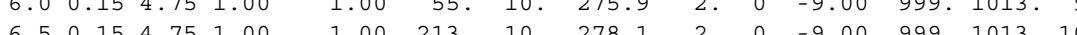

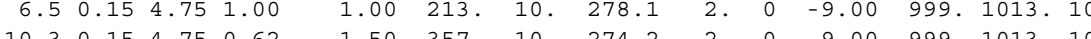

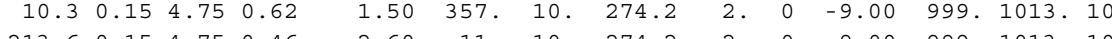
$\begin{array}{lllllllllllll}213.6 & 0.15 & 4.75 & 0.46 & 2.60 & 11 . & 10 . & 274.2 & 2 . & 0 & -9.00 & 999.1013 . & 10 \\ -38.7 & 0.15 & 4.75 & 0.40 & 1.00 & 14 . & 10 . & 275.4 & 2 . & 0 & -9.00 & 999.1013 . & 10\end{array}$ $\begin{array}{lllllllllllll}-44.8 & 0.15 & 4.75 & 0.38 & 1.50 & 356 . & 10 . & 275.4 & 2 . & 0 & -9.00 & 999.1013 .10\end{array}$ $\begin{array}{lllllllllllll}-178.1 & 0.15 & 4.75 & 0.38 & 2.60 & 3 . & 10 . & 275.9 & 2 . & 0 & -9.00 & 999.1013 .10\end{array}$ $\begin{array}{llllllllllll}-645.5 & 0.15 & 4.75 & 0.40 & 3.10 & 359 . & 10 . & 276.4 & 2 . & 0 & -9.00 & 999.1013 .10\end{array}$ $\begin{array}{lllllllllllll}-369.7 & 0.15 & 4.75 & 0.46 & 2.60 & 352 . & 10 . & 276.4 & 2 . & 0 & -9.00 & 999.1013 . & 0\end{array}$ $\begin{array}{llllllllllllll}7.9 & 0.15 & 4.75 & 0.61 & 1.50 & 344 . & 10 . & 275.9 & 2 . & 0 & -9.00 & 999.1013 . & 0\end{array}$ $\begin{array}{lllllllllllll}6.9 & 0.15 & 4.75 & 1.00 & 1.50 & 351 . & 10 . & 275.4 & 2 . & 0 & -9.00 & 999.1013 . & 0\end{array}$ $\begin{array}{llllllllllllll}6.9 & 0.15 & 4.75 & 1.00 & 1.50 & 337 . & 10 . & 275.4 & 2 . & 0 & -9.00 & 999.1013 . & 0\end{array}$ $\begin{array}{llllllllllllll}4.6 & 0.15 & 4.75 & 1.00 & 1.00 & 344 . & 10 . & 274.9 & 2 . & 0 & -9.00 & 999.1013 . & 0\end{array}$ $\begin{array}{llllllllllll}4.6 & 0.15 & 4.75 & 1.00 & 1.00 & 307 . & 10 . & 274.2 & 2 . & 0 & -9.00 & 999.1013 .0\end{array}$ $\begin{array}{llllllllllll}6.9 & 0.15 & 4.75 & 1.00 & 1.50 & 20 . & 10 . & 274.2 & 2 . & 0 & -9.00 & 999.1013 .\end{array}$

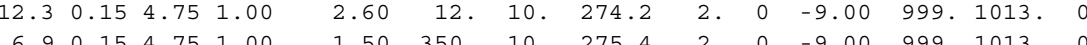
$\begin{array}{llllllllllllll}6.9 & 0.15 & 4.75 & 1.00 & 1.50 & 320 . & 10 . & 274.2 & 2 . & 0 & -9.00 & 999 . & 1013 . & 0\end{array}$

First hour of profile data

YR MO DY HR HEIGHT F WDIR WSPD AMB_TMP sigmaA sigmaW sigmaV

$\begin{array}{lllllllllllll}06 & 01 & 01 & 01 & 10.0 & 1 & 21 & 1.50 & 273.8 & 99.0 & -99.00 & -99.00\end{array}$

F indicates top of profile $(=1)$ or below $(=0)$ 
$* * *$ AERMOD - VERSION $07026 * * * \quad \begin{aligned} & * * \text { U/UA Building Demolition } \\ & * * * \text { Unit Release - Worst Case }\end{aligned}$

CONC

DDEP
TOXICS FLAT
*** $02 / 09 / 09$

$10: 41: 11$
PAGE 121

*** THE 1ST HIGHeSt 1-HR AVERAgE CONCENTRATION VALUES FOR SOURCE GROUP: ALL *** INCLUDING SOURCE (S) : $224 \mathrm{U}_{-} 1,224 \mathrm{U}_{-} 2,224 \mathrm{U} \_3,224 \mathrm{U} \_4,224 \mathrm{U} \_5,224 \mathrm{U} \_6,224 \mathrm{U} \_7$, $224 \mathrm{U}_{-} 8,224 \mathrm{U} \_9,224 \mathrm{UA} \_1,224 \mathrm{UA} \_2,224 \mathrm{UA} \_3,224 \mathrm{UA}_{-} 4,224 \mathrm{UA}_{-} 5,224 \mathrm{UA}_{-} 6{ }^{\prime}$,

*** NETWORK ID: UCART1; NETWORK TYPE: GRIDCART ***

** CONC OF UO3 IN MICROGRAMS $/ \mathrm{M} * * 3$

\begin{tabular}{l|l} 
Y-COORD \\
(METERS)
\end{tabular}

$5158052.0 \mid 12.10783(06121408)$

\begin{tabular}{r|r}
5158052.0 & $12.10783(06121408)$ \\
5158047.0 & $6.87362(06121408)$
\end{tabular}

\begin{tabular}{l|ll}
5158047.0 & 6.87362 & $(06121408)$ \\
5158042.0 & 6.56694 & $(06120816)$
\end{tabular}

\begin{tabular}{l|l}
5158037.0 & $8.74706(06120716)$
\end{tabular}

$11.29624(06120716)$

\begin{tabular}{l|ll}
5158027.0 & $14.05162(06120716)$
\end{tabular}

\begin{tabular}{l|l}
5158022.0 & $16.90654(06120716)$
\end{tabular}

\begin{tabular}{l|l}
5158017.0 & $19.76814 \quad(06120716)$
\end{tabular}

5158012.0
5158007.0

$24.97049(06120716)$

$26.67678(06120716)$

$25.69553(06120716)$

5757987.0

$26.24897(06120516)$

$27.61985(06120516)$

$27.20109(06120516)$

$24.74755(06120516)$

$20.75706(06120516)$

$20.24135(06122115)$

25.27271 (06122115)

$30.30183(06122115)$

$34.44835(06122115)$

$36.44756 \quad(06122115)$

$31.68346 \quad(06122115)$

$31.68346(06122115)$

5157922.0

$20.38744(06122115)$

$13.24276(06111716)$

$13.52547(06111716)$

13.34641 (06111716)

$12.71836(06111716)$

11.70367 (06111716)

11.04771 (06112908)

5157887.0
$14.03401 \quad(06121408)$

$12.71763(06121408)$

$7.25529(06121408)$

7.38609 (06120716)

$9.80311 \quad(06120716)$

$12.50172(06120716)$

$15.36911(06120716)$

$18.29876(06120716)$

$21.20027(06120716)$

$23.94717(06120716)$

26.25965 (06120716)

(27.61839 (06120716)

$25.63350 \quad(06120516)$

$27.73078(06120516)$

$28.30918(06120516)$

$26.79428(06120516)$

$23.32043(06120516)$

19.57389 (06122115)

$24.65737(06122115)$

$29.88729(06122115)$

$34.48245(06122115)$

$37.12381(06122115)$

$36.70826 \quad(06122115)$

$33.26920(06122115)$

7.89969 (06122115)

21.86253 (06122115)

$13.60127(06111716)$

13.89945 (06111716)

13.70958 (06111716)

13.04598 (06111716)

11.97661 (06111716)

11.68894 (06112908)

$13.06808(06112908)$
$15.76496(06121408)$

$14.65087(06121408)$

$13.34863(06121408)$

$9.09133(06121408)$

$8.35730(06120716)$

$10.94902 \quad(06120716)$

$13.78886(06120716)$

$16.75587 \quad(06120716)$

$19.74807(06120716)$

$22.67945(06120716)$

$25.39057(06120716)$

$27.50987(06120716)$

$27.50987(06120716)$

$27.48620(06120716)$

$27.42655(06120516)$

28. $93410(06120516)$

$28.48757(06120516)$

$25.80086 \quad(06120516)$

21.46911 (06120516)

$24.01762 \quad(06122115)$

$29.40454 \quad(06122115)$

$34.40667(06122115)$

$37.69928(06122115)$

$37.93862(06122115)$

34.88876 (06122115)

29. 59148 (06122115)

23.42241 (06122115)

17.35503 (06122115)

14.29707 (06111716)

14.09508 (06111716)

13.39246 (06111716)

12.26351 (06111716)

12.36823 (06112908)

13.74757 (06112908)
$17.16772-(06121408)$

$16.35625(06121408)$

$15.28370(06121408)$

$14.00150(06121408)$

$10.34632(06121408)$

$9.42436 \quad(06120716)$

5.15515 (06120716)

8.20974 (06120716)

(06120716)

$24.20563(06120716)$

$26.84624 \quad(06120716)$ $28.69114(06120716)$ $29.01904 \quad(06120716)$ 27.28459 (06120716) $9.06177(06120516)$ 9.71939 (06120516) $28.05804 \quad(06120516)$ $24.25476 \quad(06120516)$ 23.36188 (06122115) $8.86589(06122115)$ $34.22438(06122115)$ $38.16370 \quad(06122115)$ 39.12128 (06122115) 36.54007 (06122115) 6.54007 (06122115) 31.36092 (06122115) $25.07413(06122115)$ 8.77190 (06122115) 14.72191 (06111716) 14.50677 (06111716) $12.56739(06111716)$ $14.45580 \quad(06112908)$
$18.13393(06121408)$ 17.70067 (06121408) $16.95683(06121408)$ $15.93289 \quad(06121408)$ 14.67652 (06121408) $13.24969(06121408)$ $10.59010 \quad(06120716)$ $13.51362(06120716)$ 16.59949 (06120716) $19.72974 \quad(06120716)$ $22.82281 \quad(06120716)$ $28.7982(06120716)$ $28.30266(06120716)$ 29.37976 (06120716) $28.73522(06120516)$ $30.41468 \quad(06120516)$ 29.94537 (06120516) $26.98242 \quad(06120516)$ 22.69684 (06122115) $33.94274 \quad(06122115)$ $38.50549(06122115)$ $40.23248 \quad(06122115)$ $38.21072(06122115)$ $33.21359(06122115)$ 26.83204 (06122115) 20.29629 (06122115) 15.17721 (06111716) 14.94701 (06111716) 14.15422 (06111716) 12.88852 (06111716) 13.84896 (06112908) 15.19281 (06112908) 


\begin{tabular}{|c|c|c|c|c|c|c|c|c|c|c|}
\hline 5157882.0 & 13.54785 & $(06112908)$ & 14.16143 & $(06112908)$ & 14.78968 & (06112908) & 15.42952 & (06112908) & 16.07995 & $(06112908)$ \\
\hline 5157877.0 & 14.31577 & $(06112908)$ & 14.84275 & $(06112908)$ & 15.36660 & (06112908) & 15.88323 & (06112908) & 16.38933 & $(06112908)$ \\
\hline 5157872.0 & 14.62703 & $(06112908)$ & 15.02401 & $(06112908)$ & 15.39963 & (06112908) & 16.20304 & $(06122214)$ & 17.74322 & $(06122214)$ \\
\hline 5157867.0 & 14.43812 & $(06112908)$ & 15.68508 & $(06122214)$ & 17.13903 & $(06122214)$ & 18.67939 & (06122214) & 20.30118 & $(06122214)$ \\
\hline 5157862.0 & 16.53528 & $(06122214)$ & 17.97621 & $(06122214)$ & 19.48115 & $(06122214)$ & 21.03612 & $(06122214)$ & 22.61751 & $(06122214)$ \\
\hline 5157857.0 & 18.67656 & $(06122214)$ & 20.10315 & $(06122214)$ & 21.53856 & (06122214) & 22.94799 & $(06122214)$ & 24.28408 & $(06122214)$ \\
\hline
\end{tabular}

\begin{tabular}{|c|c|c|c|c|c|}
\hline *** AERMOD & VERSION 07026 & $* \star *$ & *** U/UA Building Demolition & $* * *$ & $02 / 09 / 09$ \\
\hline & & & *** Unit Release - Worst Case & $* * *$ & $10: 41: 11$ \\
\hline $\begin{array}{l}\star \star \text { MODELOPTS : } \\
\text { CONC }\end{array}$ & DDEP & TOXICS & FLAT & & PAGE 122 \\
\hline
\end{tabular}

*** THE 1ST HIGHEST 1-HR AVERAGE CONCENTRATION VALUES FOR SOURCE GROUP: ALL ***

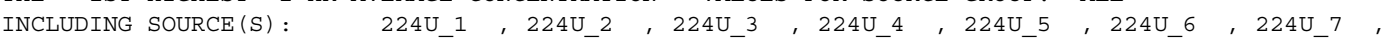
224U_8 , 224U_9, 224UA_1 $, 224 \mathrm{UA} \_2,224 \mathrm{UA} \_3,224 \mathrm{UA} \_4,224 \mathrm{UA} \_5,224 \mathrm{UA} \_6$,

*** NETWORK ID: UCART1 ; NETWORK TYPE: GRIDCART ***

** CONC OF UO3 IN MICROGRAMS $/ \mathrm{M} * 33$ **

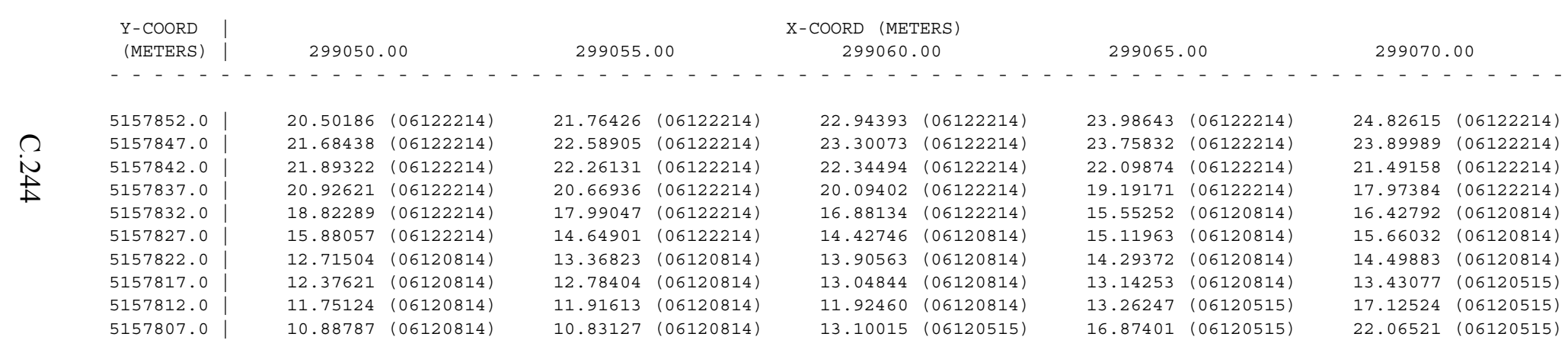
*** AERMOD - VERSION $07026 * * * \quad * * *$ U/UA Building Demolition
$* * *$ Unit Release - Worst Case
**MODELOPTS
DDEP TOXICS FLAT
DRYDPL WETDPL

*** THE 1ST HIGHeSt 1-HR AVERAge CONCENTRATION VALUES FOR SOURCE GROUP: ALL *** INCLUDING SOURCE $(\mathrm{S}): \quad 224 \mathrm{U}_{-} 1,224 \mathrm{U}_{-} 2,224 \mathrm{U}_{-} 3,224 \mathrm{U}_{-} 4,224 \mathrm{U}_{-} 5,224 \mathrm{U}{ }_{-} 6,224 \mathrm{U}_{-} 7$, $224 \mathrm{U} \_8,224 \mathrm{U} \_9,224 \mathrm{UA} \_1,224 \mathrm{UA} \_2,224 \mathrm{UA} \_3,224 \mathrm{UA}{ }_{-} 4,224 \mathrm{UA} \_5,224 \mathrm{UA} \_6$ ',

*** NETWORK ID: UCART1; NETWORK TYPE: GRIDCART ***

** CONC OF UO3 IN MICROGRAMS $/ \mathrm{M} * * 3$

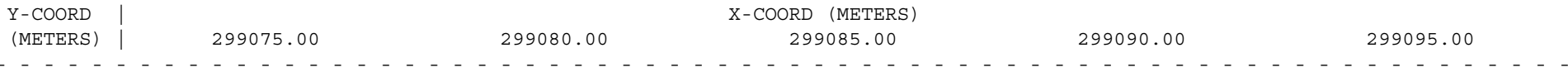


\begin{tabular}{l|ll}
5158052.0 & 18.59464 & $(06121408)$
\end{tabular} $5158047.0 \quad 18.58030(06121408)$ 5158042.0 | $18.23579(06121408)$ 5158032.0 | $16.56580(06121408)$ 515027.0 | 16.59781 (06121408) 5158027.0 | $15.37474(06121408)$ 5158022.0 . $\begin{array}{ll}5158017.0 & 11.85703(06120716)\end{array}$ $14.93218(06120716)$ $18.12218(06120716)$ $\begin{array}{ll}5158002.0 & 21.31901(06120716)\end{array}$ \begin{tabular}{l|l}
5157997.0 & $24.45469(06120716)$
\end{tabular} $\begin{array}{ll}5157992.0 & 27.39894(06120716)\end{array}$ \begin{tabular}{l|l}
5157987.0 & $29.73458(06120716)$ \\
5
\end{tabular} $5157982.0 \quad 30.67795(06120716)$ $5157977.0-29.44604(06120716)$ $5157972.0 \quad 30.55943 \quad(06120516)$ 5157967.0 I $30.55943(06120516)$ 5157962.0 | $29.49527(06120516)$ $5157962.0 \quad 1029.49527(06120516)$ 5157957.0
5157952.0 5157952.0
$57.67789(06122115)$ \begin{tabular}{l|l}
5157947.0 & $33.57515(06122115)$ \\
\hline
\end{tabular} \begin{tabular}{l|l}
5157942.0 & $38.70379(06122115)$ \\
\hline
\end{tabular} \begin{tabular}{l|ll}
5157937.0 & $41.23202(06122115)$ \\
5
\end{tabular} \begin{tabular}{l|ll}
5157932.0 & $39.87477(06122115)$
\end{tabular} $5157927.0 \quad 35.14628(06122115)$ \begin{tabular}{l|l}
5157922.0 & $28.70033(06122115)$ \\
5157917.0 & $21.93264(06122115)$
\end{tabular} 5157917.0 | $21.93264(06122115)$ 5157912.0 | 15.66789 (06111716) 5157907.0 | 15.42078 (06111716) 5157902.0 | 14.57521 (06111716) $5157897.0)(13.57521$ (06111716) 5157897.0
5157892.0 \begin{tabular}{l|l}
5157892.0 & $14.65349(06112908)$
\end{tabular} $15.95744(06112908)$ $16.73765(06112908)$ \begin{tabular}{l|ll}
5157882.0 & 16.73765 & $(06112908)$ \\
5157877.0 & 16.88028 & $(06112908)$
\end{tabular} \begin{tabular}{l|ll}
5157872.0 & 19.38560 & $(06122214)$
\end{tabular} $5157867.0 \quad 21.99203(06122214)$ $5157862.0 \quad 24.18955(06122214)$ $25.48484(06122214)$
$18.53236(06121408)$ $18.93573(06121408)$ $19.02234(06121408)$ $18.77236(06121408)$ $18.7235(06121408)$ $18.18425(06121408)$ $16.09777(06121408)$ $14.69736(06121408)$ $13.22843(06120716)$ $16.44253(06120716)$ $19.72210 \quad(06120716)$ 22.97959 (06120716) $29.06016(06120716)$ $31.10883(06120716)$ $31.37207(06120716)$ 30.20979 (06120516) $32.10225(06120516)$ $32.10225(06120516)$ $31.61495(06120516)$ $28.32717(06120516)$ 27.06091 (06122115) 38.77238 (06122115) 41.52125 (06122115) $23.69332(06122115)$ 16.19765 (06111716) 15.93140 (06111716) $15.93140 \quad(06111716)$ $14.24420(06112716)$ 15.50438 (06112908) 16.74956 (06112908) $17.39738(06112908)$ $18.34057 \quad(06122214)$ $21.12730(06122214)$ 23.73009 (06122214) $25.70118(06122214)$ $26.47508 \quad(06122214)$
18.41859 (06121810) $18.76010(06121408)$ $19.26760 \quad(06121408)$ $19.46040 \quad(06121408)$ $19.31135 \quad(06121408)$ 19.31135 (06121408) $18.81248(06121408)$ (06121408) 16.84618 (06121408) 15.46967 (06121408) 14.70469 (06120716) $18.04331 \quad(06120716)$ 21.40327 (06120716) $24.72042 \quad(06120716)$ $27.93494 \quad(06120716)$ $30.74936(06120716)$ $32.37459 \quad(06120716)$ (32.37459 (06120716) (31.78105 (06120716) 32.27095 (06120516) 33.16961 (06120516) 31.14619 (06120516) $26.49295(06120516)$ $32.65639(06122115)$ $38.72604 \quad(06122115)$ $42.87520 \quad(06122115)$ $43.12487(06122115)$ $39.22769(06122115)$ $32.79079(06122115)$ $25.58312(06122115)$ $16.77120 \quad(0611171$ $16.77120(06111716)$ 16.48301 (06111716) $15.55171(06112716)$ $15.39413(06112716)$ $16.40348(06112908)$ $17.56760 \quad(06112908)$ $18.05558(06112908)$ $20.08467 \quad(06122214)$ $22.95939(06122214)$ $25.47964 \quad(06122214)$ $27.08360 \quad(06122214)$ $27.16705 \quad(06122214)$
$18.10237 \quad(06121810)$ $18.68605(06121810)$ $19.01130(06121810)$ $19.59154(06121408)$ (9.89511 (06121408) (9.85238 (06121408) 19.85238 (06121408) $19.45052(06121408)$ 18.69687 (06121408) $17.62208 \quad(06121408)$ $16.27718(06121408)$ 16.28881 (06120716) $19.73672 \quad(06120716)$ 23.16945 (06120716) $26.55130 \quad(06120716)$ $29.79922(06120716)$ $33.46926(06120716)$ (31. 89116 (06120516) $31.89116(06120516)$ $3.55172(06120516)$ 29.86919 (06120516) 32.13982 (06122115) $38.58226(06122115)$ 43.51944 (06122115) 44.69228 (06122115) 41.36520 (06122115) 27.60661 (06122115) $20.33479 \quad(06122115)$ 17.08341 (06111716) 17.08341 (06111716) $16.69710(06112716)$ $17.35226(06112908)$ 18.40881 (06112908) $18.92082(06122214)$ $21.94694 \quad(06122214)$ 24.86256 (06122214) $27.18664 \quad(06122214)$ $28.24850(06122214)$ $27.46833 \quad(06122214)$
$17.31966 \quad(06121810)$ $18.22777(06121810)$ 18.92790 (06121810) $19.37863 \quad(06121810)$ (19.37863 (06121810) 19.90739 (06121408) 20.32626 (06121408) 20.10019 (06121408) 19.43490 (06121408) $8.42697(06121408)$ $17.12265(06121408)$ $17.98144 \quad(06120716)$ 21.52627 (06120716) $25.03271 \quad(06120716)$ $28.49164 \quad(06120716)$ $31.74990 \quad(06120716)$ 34.10786 (06120716) 34.30635 (06120716) (34. 24865 (06120516) (06120516) 35.33441 (06120516) (06120516) 31.59788 (06122115) 38.34769 (06122115) 44.04215 (06122115) $46.21214 \quad(06122115)$ 43.57067 (06122115) 29.77627 (06122115) 22.17552 (06122115) (18.15503 (06122115) 18.55803 (06112716) $18.41470 \quad(06112716)$ 16.74088 (06112908) $18.35220(06112908)$ 20.27228 (06112908) $23.92177 \quad(06122214)$ $26.80222 \quad(06122214)$ $28.77438(06122214)$ $27.29173 \quad(06122214)$
*** AERMOD - VERSION $07026 * * * \quad * * *$ U/UA Building Demolition **MODELOPTS :

CONC

OXICS FLAT $\begin{array}{ll}* * * & 02 / 09 / 09 \\ * * * & 10: 41: 11\end{array}$ PAGE 124

DRYDPL WETDPL

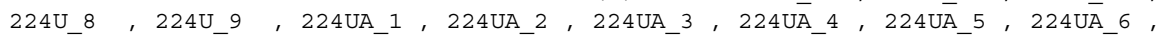

*** NETWORK ID: UCART1 ; NETWORK TYPE: GRIDCART *** 
** CONC OF UO3 IN MICROGRAMS $/$ M**3

\begin{tabular}{|c|c|c|c|c|c|c|c|c|c|c|}
\hline \multirow{3}{*}{$\begin{array}{c}\mathrm{Y}-\mathrm{COORD} \\
\text { (METERS) } \\
--\ldots-\end{array}$} & \multicolumn{10}{|c|}{$\mathrm{X}$-COORD (METERS) } \\
\hline & 299075 & & 299080 & .00 & 299085 & .00 & 299090 & & 299095 & 00 \\
\hline & $-\cdots$ & $\cdots \cdots$ & $-\cdots$ & $-\cdots-\cdots$ & $-\cdots$ & - - - - - & $-\cdots$ & $\cdots \cdots$ & $-\cdots$ & $\ldots-\cdots$ \\
\hline 5157852.0 & 25.39064 & $(06122214)$ & 25.60445 & $(06122214)$ & 25.39737 & $(06122214)$ & 24.71692 & $(06122214)$ & 24.46331 & $(06120814)$ \\
\hline 5157847.0 & 23.67064 & $(06122214)$ & 23.02813 & $(06122214)$ & 21.95493 & $(06122214)$ & 22.44424 & (06120814) & 23.83591 & $(06120814)$ \\
\hline 5157842.0 & 20.50963 & $(06122214)$ & 19.32329 & $(06120814)$ & 20.54814 & $(06120814)$ & 21.58867 & $(06120814)$ & 22.36100 & $(06120814)$ \\
\hline 5157837.0 & 17.83031 & $(06120814)$ & 18.78404 & $(06120814)$ & 19.54053 & $(06120814)$ & 20.03024 & $(06120814)$ & 20.18033 & $(06120814)$ \\
\hline 5157832.0 & 17.15522 & $(06120814)$ & 17.68369 & $(06120814)$ & 17.95793 & $(06120814)$ & 18.23545 & $(06120515)$ & 24.42479 & (06120515) \\
\hline 5157827.0 & 16.00767 & $(06120814)$ & 16.11870 & $(06120814)$ & 17.93923 & (06120515) & 23.90343 & (06120515) & 31.85743 & (06120515) \\
\hline 5157822.0 & 14.48812 & $(06120814)$ & 17.65665 & $(06120515)$ & 23.40897 & (06120515) & 31.00319 & (06120515) & 39.87147 & (06120515) \\
\hline 5157817.0 & 17.38623 & $(06120515)$ & 22.93985 & (06120515) & 30.20109 & (06120515) & 38.62120 & (06120515) & 46.79067 & (06120515) \\
\hline 5157812.0 & 22.49283 & $(06120515)$ & 29.44600 & $(06120515)$ & 37.45471 & $(06120515)$ & 45.20639 & (06120515) & 50.78735 & $(06120515)$ \\
\hline 5157807.0 & 28.73040 & $(06120515)$ & 36.36200 & (06120515) & 43.73162 & (06120515) & 49.06283 & $(06120515)$ & 50.66678 & $(06120515)$ \\
\hline
\end{tabular}

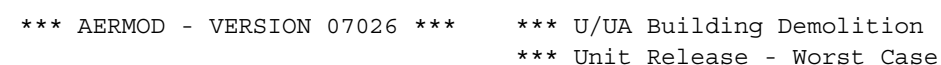
**MODELOPTS :

CONC

DRYDPL WETDPL

VALUES FOR SOURCE

THE 1ST HIGHEST 1-HR AVERAGE CONCENTRATION VALUES FOR SOURCE GROUP: ALL ***

INCLUDING SOURCE $(S): \quad 224 \mathrm{U}_{-} 1,224 \mathrm{U}_{-} 2,224 \mathrm{U}_{-} 3,224 \mathrm{U} \_4,224 \mathrm{U} \_5,224 \mathrm{U} \_6,224 \mathrm{U}_{-} 7$

$224 \mathrm{U} \_8,224 \mathrm{U} \_9,224 \mathrm{UA} \_1,224 \mathrm{UA} \_2,224 \mathrm{UA} \_3,224 \mathrm{UA} \_4,224 \mathrm{UA} \_5,224 \mathrm{UA} \_6$,

*** NETWORK ID: UCART1; NETWORK TYPE: GRIDCART ***

\begin{tabular}{|c|c|c|c|c|c|c|c|c|c|c|}
\hline \multirow{4}{*}{$\begin{array}{c}\text { Y-COORD } \\
\text { (METERS) } \\
-\quad----\end{array}$} & \multicolumn{4}{|c|}{ ** CONC OF UO3 } & \multicolumn{2}{|c|}{ N MICROGRAMS $/ \mathrm{M} * * 3$} & \multicolumn{2}{|r|}{ ** } & \\
\hline & & & & & -COORD (MET & ERS) & & & & \\
\hline & 299100. & & 299105 & & 299110. & .00 & 299115. & & 299120. & \\
\hline & ---- & ---- & & -2 & & -- & ---- & - - & ---- & - - - \\
\hline 5158052.0 & 18.58284 & $(06120409)$ & 21.89072 & (06120409) & 24.41767 & $(06120409)$ & 25.86089 & $(06120409)$ & 26.54718 & $(06120710)$ \\
\hline 5158047.0 & 17.31715 & $(06121810)$ & 20.71610 & (06120409) & 23.75303 & $(06120409)$ & 25.81004 & $(06120409)$ & 26.66408 & (06120409) \\
\hline 5158042.0 & 18.32803 & $(06121810)$ & 19.32641 & (06120409) & 22.80729 & (06120409) & 25.45570 & $(06120409)$ & 26.95347 & (06120409) \\
\hline 5158037.0 & 19.14541 & $(06121810)$ & 18.40345 & $(06121810)$ & 21.59503 & (06120409) & 24.79254 & $(06120409)$ & 26.94402 & (06120409) \\
\hline 5158032.0 & 19.72331 & $(06121810)$ & 19.33830 & $(06121810)$ & 20.15252 & $(06120409)$ & 23.82666 & (06120409) & 26.61397 & (06120409) \\
\hline 5158027.0 & 20.21757 & $(06121408)$ & 20.04642 & $(06121810)$ & 19.50852 & $(06121810)$ & 22.57829 & $(06120409)$ & 25.95691 & (06120409) \\
\hline 5158022.0 & 20.75597 & $(06121408)$ & 20.52229 & $(06121408)$ & 20.34817 & $(06121810)$ & 21.08079 & $(06120409)$ & 24.97558 & (06120409) \\
\hline 5158017.0 & 20.94660 & $(06121408)$ & 21.18453 & $(06121408)$ & 20.92394 & $(06121810)$ & 20.62997 & $(06121810)$ & 23.69130 & (06120409) \\
\hline 5158012.0 & 20.76206 & $(06121408)$ & 21.50200 & $(06121408)$ & 21.61387 & $(06121408)$ & 21.35186 & $(06121810)$ & 22.13601 & (06120409) \\
\hline 5158007.0 & 20.19511 & $(06121408)$ & 21.43760 & $(06121408)$ & 22.06388 & $(06121408)$ & 22.04461 & $(06121408)$ & 21.76391 & $(06121810)$ \\
\hline 5158002.0 & 19.26345 & $(06121408)$ & 20.97794 & $(06121408)$ & 22.12811 & $(06121408)$ & 22.63404 & $(06121408)$ & 22.48247 & $(06121408)$ \\
\hline 5157997.0 & 18.00911 & $(06121408)$ & 20.13286 & $(06121408)$ & 21.78569 & $(06121408)$ & 22.83690 & $(06121408)$ & 23.21993 & $(06121408)$ \\
\hline 5157992.0 & 19.78767 & $(06120716)$ & 18.93902 & $(06121408)$ & 21.03969 & $(06121408)$ & 22.62602 & $(06121408)$ & 23.57617 & $(06121408)$ \\
\hline 5157987.0 & 23.41862 & $(06120716)$ & 21.70970 & $(06120716)$ & 19.92114 & $(06121408)$ & 21.99595 & $(06121408)$ & 23.51054 & $(06121408)$ \\
\hline 5157982.0 & 27.00541 & $(06120716)$ & 25.42658 & $(06120716)$ & 23.75942 & $(06120716)$ & 21.97683 & $(06120716)$ & 23.01140 & $(06121408)$ \\
\hline 5157977.0 & 30.55757 & $(06120716)$ & 29.11518 & $(06120716)$ & 27.57311 & $(06120716)$ & 25.95800 & $(06120716)$ & 24.23624 & $(06120716)$ \\
\hline 5157972.0 & 33.78605 & $(06120716)$ & 32.77596 & $(06120716)$ & 31.39716 & (06120716) & 29.89132 & $(06120716)$ & 28.34504 & (06120716) \\
\hline
\end{tabular}


$5157967.0 \mid \quad 35.66922(06120716)$ 5157962.0 $5157957.0 \mid 36.35342(06120516)$ $5157952.0 \mid 35.84440 \quad(06120516)$ $31.67652 \quad(06120516)$ 5157942.0 | 38.03973 (06122115)

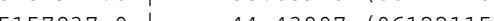
5157937.0 | 44.43807 (06122115) 5157932.0 $45.82677(06122115)$ $39.84997(06122115)$ $32.08785(06122115)$ \begin{tabular}{l|l}
5157917.0 & $32.08785(06122115)$ \\
5157912.0 & $24.16750(06122115)$
\end{tabular} \begin{tabular}{l|ll}
5157907.0 & $20.35139(06112716)$ \\
5
\end{tabular} $5157902.0 \quad 20.17262(06112716)$ $5157897.0 \quad 17.86064(06112908)$ \begin{tabular}{l|ll}
5157892.0 & $19.40724(06112908)$
\end{tabular} \begin{tabular}{l|l}
5157887.0 & $20.15427(06112908)$
\end{tabular} \begin{tabular}{l|l}
5157882.0 & $22.74293(06122214)$
\end{tabular} \begin{tabular}{l|l}
5157877.0 & $25.99345(06122214)$
\end{tabular} \begin{tabular}{l|ll}
5157872.0 & $28.72241(06122214)$
\end{tabular} \begin{tabular}{l|ll}
5157867.0 & $30.14153(06122214)$
\end{tabular} \begin{tabular}{l|ll}
5157867.0 & 30.14153 & $(06122214)$ \\
5157862.0 & $29.49969(06122214)$
\end{tabular} 5157857.0 | $26.58651(06120814)$
$35.88354 \quad(06120716)$ $37.04786 \quad(06120716)$ 36.58867 (06120516) $37.89633 \quad(06120516)$ (35.85633 (06120516) $35.32889(06120516)$ $37.69237(06122115)$ 44.71748 (06122115) 49.03173 (06122115) 48.13648 (06122115) $42.47463(06122115)$ $34.56686(06122115)$ $26.32486(06122115)$ 22.43807 (06112716) 22.21194 (06112716) $19.06625(06112908)$ $20.51665(06112908)$ $21.40722 \quad(06122214)$ 24.85537 (06122214) $5.85537(06122214)$ $28.13053(06122214)$ $31.16302 \quad(06122214)$ $29.36111(06122214)$ $28.91641(06120814)$
$35.16975 \quad(06120716)$ $37.98843(06120716)$ $38.07368 \quad(06120716)$ $39.05213 \quad(06120516)$ $38.05213(0612051$ $37.31539 \quad(06122115)$ 37.31539 (06122115) $40.88785(06122115)$ 50.31903 (06122115) 50.51514 (06122115) 45.28291 (06122115) 37.24991 (06122115) 28.67515 (06122115) $24.88906 \quad(06112716)$ 24.59840 (06112716) 20.36706 (06112908) $21.68539(06112908)$ $23.49224 \quad(06122214)$ 27. $09075 \quad(06122214)$ $30.27760 \quad(06122214)$ (30.27760 (06122214) $31.69814(06122214)$ $31.70681(06120814)$ $31.11561(06120814)$
$33.91578 \quad(06120716)$ $37.74358 \quad(06120716)$ $39.93453(06120716)$ $39.35386(06120516)$ $37.91507(06120516)$ (0612115) 51.50992 (06122115) 2.95815 (06122115) 48.29319 (06122115) $40.16069(06122115)$ $31.23905(06122115)$ 27.79928 (06112716) $23.03123(06103116)$ $22.51701 \quad(06103116)$ 22.91725 (06112908) $25.72730(06122214)$ (32.34532 (06122214) $33.36637(06122214)$ $4.59760(06120814)$ 34.63537 (06120814) $33.01382(06120814)$
$32.47318 \quad(06120716)$ $36.72811(06120716)$ $40.41023(06120716)$ $41.59093 \quad(06120716)$ $42.25944 \quad(06120516)$ 41.60057 (06120516) (4). 90643 (06122115) (52.60599 (06122115) $55.47733(06122115)$ $34.07830(06122115)$ $31.29875(06112716)$ $25.98374(06103116)$ $25.30570(06103116)$ $26.32937(06120814)$ $29.48279(06120814)$ $32.48263(06120814)$ $35.29273(06120814)$ $37.49922(06120814)$ $38.39821(06120814)$ $37.39192(06120814)$ $37.75800(06101208)$

*** THE 1ST HIGHEST 1-HR AVERAGE CONCENTRATION VALUES FOR SOURCE GROUP: ALI INCLUDING SOURCE (S) : $\quad 224 \mathrm{U}_{-} 1,224 \mathrm{U}_{-} 2,224 \mathrm{U}_{-} 3,224 \mathrm{U}_{-} 4,224 \mathrm{U}{ }_{-} 5,224 \mathrm{U}_{-} 6,224 \mathrm{U}_{-} 7$,

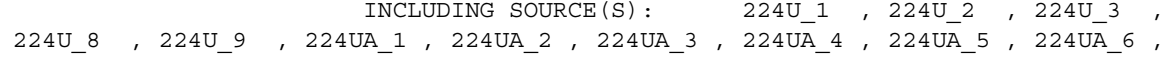

*** NETWORK ID: UCART1; NETWORK TYPE: GRIDCART ***

** CONC OF UO3 IN MICROGRAMS $/ \mathrm{M} * * 3$

$\mathrm{X}$-COORD (METERS)

299105.00 $299110.00 \quad 299115.00$

$\mathrm{Y}$-COORD 299100.00 **

(METERS)

$5157852.0 \mid \quad 26.28262(06120814)$ \begin{tabular}{l|l}
5157847.0 & $24.97489(06120814)$
\end{tabular} \begin{tabular}{l|l}
5157842.0 & $22.76946(06120814)$
\end{tabular} \begin{tabular}{l|l}
5157837.0 & $24.97935(06120515)$
\end{tabular} \begin{tabular}{l|ll}
5157832.0 & $32.77098(06120515)$ \\
5 & 41.21324 & $(06120515)$
\end{tabular} $41.21324 \quad(06120515)$ \begin{tabular}{l|l}
5157822.0 & $48.49773(06120515)$
\end{tabular} \begin{tabular}{l|l}
5157817.0 & $52.64336(06120515)$
\end{tabular} $5157812.0 \quad 52.37762(06120515)$ \begin{tabular}{l|l}
5157807.0 & $47.70892(06120515)$
\end{tabular}
$27.89066(06120814)$ $25.73933(06120814)$ $55.57051(06120515)$ $33.75112(06120515)$ $42.66306(06120515)$ $50.34377(06120515)$ $54.64423(06120515)$ $54.21512(06120515)$ $49.11153(06120515)$ $40.73046(06120515)$
$29.13928 \quad(06120814)$ $26.37387(06101208)$ $34.80849(06120515)$ $44.23386(06120515)$ $52.34809(06120515)$ $56.81476(06120515)$ $56.19501(06120515)$ $50.60571(06120515)$ $42.92287 \quad(06100308)$ $43.81057(06100308)$
$31.51704 \quad(06101208)$ $37.50670 \quad(06101208)$ $45.94708 \quad(06120515)$ 4.53509 (06120515) $59.17673(06120515)$ $58.33728(06120515)$ $52.20737(06120515)$ 46.05535 (06100308) $46.27639 \quad(06100308)$ 43.13091 (06100308)

299120.00

_ _ . . . . .

$44.66731 \quad(06101208)$ $49.20427(06101208)$ $56.93428 \quad(06120515)$ 61.76415 (06120515) $60.66594(06120515)$ $53.92966 \quad(06120515)$ $49.39399 \quad(06100308)$ 48.72371 (06100308) $44.35566 \quad(06100308)$ $37.51772(06100308)$ 
$* * *$ AERMOD - VERSION $07026 * * * \quad \begin{aligned} & * * \text { U/UA Building Demolition } \\ & * * * \text { Unit Release - Worst Case }\end{aligned}$

CONC

DDEP
TOXICS FLAT
*** $02 / 09 / 09$

$10: 41: 11$
PAGE 127

*** THE 1ST HIGHeSt 1-HR AVERAge CONCENTRATION VALUES FOR SOURCE GROUP: ALL *** INCLUDING SOURCE $(\mathrm{S}): \quad 224 \mathrm{U}{ }_{-1}, 224 \mathrm{U} \_2,224 \mathrm{U} \_3,224 \mathrm{U} \_4,224 \mathrm{U} \_5,224 \mathrm{U} \_6,224 \mathrm{U} \_7$, $224 \mathrm{U}_{-} 8,224 \mathrm{U} \_9,224 \mathrm{UA} \_1,224 \mathrm{UA} \_2,224 \mathrm{UA} \_3,224 \mathrm{UA}_{-} 4,224 \mathrm{UA}_{-} 5,224 \mathrm{UA}_{-} 6{ }^{\prime}$,

*** NETWORK ID: UCART1; NETWORK TYPE: GRIDCART ***

** CONC OF UO3 IN MICROGRAMS $/ \mathrm{M} * * 3$

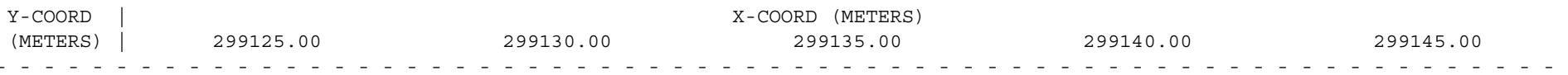

$5158052.0 \mid 27.83396(06120710)$

$27.83396(06120710)$
5158047.0

$5158047.0 \quad 27.97810(06120710)$

$5158042.0 \mid 27.91099(06120710)$

\begin{tabular}{l|ll}
5158037.0 & 27.81970 & $(06120409)$
\end{tabular}

$28.17637(06120409)$

\begin{tabular}{l|l}
5158027.0 & $28.21888(06120409)$ \\
5158022.0 & $27.92305(06120409)$
\end{tabular}

\begin{tabular}{l|ll}
5158022.0 & $27.92305(06120409)$
\end{tabular}

\begin{tabular}{l|ll}
5158017.0 & 27.27788 & $(06120409)$
\end{tabular}

\begin{tabular}{l|l}
5158012.0 & $26.28728 \quad(06120409)$
\end{tabular}

$24.96578 \quad(06120409)$

$23.34429(06120409)$

$22.93501(06121408)$

$23.83157(06121408)$

$23.83157(06121408)$

5157987.0
5157982.0

\begin{tabular}{l|l}
5157982.0 & $24.48515(06121408)$
\end{tabular}

$24.31285(06121408)$

$26.70857(06120716)$

$30.98522(06120716)$

$35.38327(06120716)$

\begin{tabular}{l|ll}
5157957.0 & $39.81934(06120716)$
\end{tabular}

$5157952.0 \quad 43.08970 \quad(06120716)$

$42.87242 \quad(06120716)$

$46.13735(06120516)$

$47.43983 \quad(06120516)$

$59.44130(06122115)$

$67.26194 \quad(06122115)$

5157922.0

$55.17099(06122115)$

$42.75137(06122115)$

$30.65316(06122115)$

$29.57597(06103116)$

$28.66343(06103116)$

$30.69018(06120814)$

5157887.0
$27.96395(06120710)$

$88.63412(06120710)$ $29.10987(06120710)$ $29.37365(06120710)$ $29.41300(06120710)$ $29.21678(06120710)$ $29.56219(06120409)$ $28.79432(06120409)$ $27.78138(06120409)$ $26.40778 \quad(06120409)$ (26.40778 (06120409) 24.72451 (06120409) $24.50317(06121408)$ $26.02662(06121408)$ $26.21811(06121408)$ 29.45796 (06120716) $3.19856(06120716)$ $56.24500 \quad(06120516)$ $65.94193 \quad(06120516)$ $72.57238 \quad(06122115)$ 5750 (06122115) $86.58157(06122115)$ $92.59264(06122115)$ $86.18866(06122115)$ $0.62805(06122115)$ $52.82055(06122115)$ $(06113015)$ 34.01025 (06103116) $32.75299(06103116)$ $38.98990 \quad(06120814)$
$26.93724 \quad(06120710)$

$8.9373(06120710)$

$29.07916(06120710)$

(06120710)

$30.51537(06120710)$

$30.91479(06120710)$

$31.07770 \quad(06120710)$

$30.99606 \quad(06120710)$

31.15371 (06120409)

$31.33745 \quad(06120409)$

$31.12904 \quad(06120409)$

$30.51350 \quad(06120409)$

29. $50690 \quad(06120409)$

(28.50690 (06120409)

$28.12508(06120409)$

$28.25114(06120710)$

29.82903 (06120710)

$28.47449(06121408)$

$32.53016 \quad(06120716)$

$46.48566 \quad(06120516)$

79.45309 (06120516)

88.23027 (06120516)

$87.48740-106$

112.65131 (06122115)

114. $72024(06122115)$

102.66364 (06122115)

$81.45026(06122115)$

$82.76256(06113015)$

$72.55362(06113015)$

$58.03580 \quad(06113015)$

$43.18808 \quad(06113015)$

$40.61528 \quad(06120814)$

44.90084 (06120814)
$25.31152 \quad(06122209)$

$26.41224 \quad(06120710)$

$27.83945(06120710)$

$29.15095(06120710)$

$30.31440(06120710)$

$31.30250 \quad(06120710)$

$32.09137(06120710)$

$32.98425(06120710)$

$33.06370 \quad(06120710)$

32.98818 (06120409)

$33.26483 \quad(06120409)$ $33.26483(06120409)$ $33.14318 \quad(06120409)$ $32.92455(06120710)$

$41.61358(06120710)$

$38.26603(06120710)$

$36.24850 \quad(06120409)$

$45.34499(06120516)$

.

$94.67013(06120716)$

$92.22504(06120716)$

$95.00898(06122115)$

$95.00898(06122115)$

$108.30492(06122115)$

87.71472 (06122115)

83.21507 (06113015)

90.75791 (06113015)

$76.34689(06113015)$

$57.73932(06113015)$

$46.52607 \quad(06120814)$

$52.28748(06120814)$
25.07856 (06122209)

$25.79459 \quad(06122209)$

$26.42052 \quad(06122209)$

$27.24461(06120710)$

$28.85285(06120710)$

$30.35394 \quad(06120710)$

$31.71675(06120710)$

32.91426 (06120710)

$33.91377 \quad(06120710)$

$35.24569(06120710)$

$35.53889 \quad(06120710)$

$35.57823 \quad(06120710)$

$35.57823(06120710)$

$50.76809(06120710)$

50.76809 (06120710)

$53.27847 \quad(06120710)$

$49.61718 \quad(06120409)$

$48.76971 \quad(06120409)$

$58.46348 \quad(06120716)$

$79.03596 \quad(06120716)$

$92.25015(06120716)$

$90.54031(06122115)$

$89.59745(06122115)$

$77.05166(06113015)$

$94.70149(0612215)$

94.70149 (06122115)

$81.99796 \quad(06113015)$

$77.15662(06102315)$

$75.80612(06113015)$

$57.78679(06113015)$

$56.88379(06101208)$

$82.71484(06101208)$ 


\begin{tabular}{|c|c|c|c|c|c|c|c|c|c|c|}
\hline 5157882.0 & 37.23143 & $(06120814)$ & 42.80085 & $(06120814)$ & 49.59567 & $(06120814)$ & 64.05501 & (06101208) & 101.04131 & $(06101208)$ \\
\hline 5157877.0 & 40.28503 & $(06120814)$ & 46.12931 & $(06120814)$ & 52.99449 & $(06120814)$ & 80.54646 & $(06101208)$ & 105.75711 & $(06101208)$ \\
\hline 5157872.0 & 42.29492 & $(06120814)$ & 47.54121 & $(06120814)$ & 65.84677 & (06101208) & 88.83582 & (06101208) & 98.48695 & $(06101208)$ \\
\hline 5157867.0 & 42.26361 & $(06120814)$ & 54.58201 & $(06101208)$ & 75.12579 & (06101208) & 87.94948 & (06101208) & 90.02835 & $(06101108)$ \\
\hline 5157862.0 & 45.34629 & $(06101208)$ & 63.31741 & $(06101208)$ & 77.39210 & $(06101208)$ & 80.02734 & $(06101208)$ & 81.24047 & $(06101108)$ \\
\hline 5157857.0 & 53.20776 & $(06101208)$ & 67.10064 & $(06101208)$ & 73.40158 & $(06101208)$ & 75.29838 & $(06120515)$ & 76.55746 & (06120515) \\
\hline
\end{tabular}

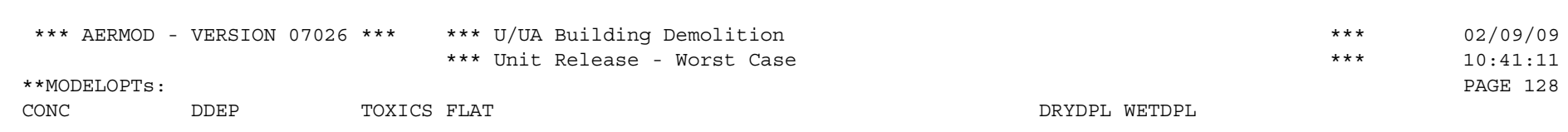

*** THE 1ST HIGHEST 1-HR AVERAGE CONCENTRATION VALUES FOR SOURCE GROUP: ALL ***

INCLUDING SOURCE $(\mathrm{S}): \quad 224 \mathrm{U} \_1,224 \mathrm{U} \_2,224 \mathrm{U} \_3,224 \mathrm{U} \_4,224 \mathrm{U} \_5,224 \mathrm{U} \_6,224 \mathrm{U} \_7$, 224U_8 , 224U_9, 224UA_1 $, 224 \mathrm{UA} \_2,224 \mathrm{UA} \_3,224 \mathrm{UA} \_4,224 \mathrm{UA} \_5,224 \mathrm{UA} \_6$,

*** NETWORK ID: UCART1; NETWORK TYPE: GRIDCART ***

** CONC OF UO3 IN MICROGRAMS $/ \mathrm{M} * 33$ **

Y-COORD
(METERS) | 299125.00

\begin{tabular}{l|llllllllll}
5157852.0 & 57.62557 & $(06101208)$ & 65.83209 & $(06101208)$ & 71.30095 & $(06120515)$ & 72.61015 & $(06120515)$ & 69.86571 & $(06100308)$ \\
5157847.0 & 59.58604 & $(06120515)$ & 67.77126 & $(06120515)$ & 69.12935 & $(06120515)$ & 64.41592 & $(06100308)$ & 66.97575 & $(06100308)$ \\
5157842.0 & 64.61459 & $(06120515)$ & 66.01363 & $(06120515)$ & 60.04571 & $(06120515)$ & 63.75525 & $(06100308)$ & 56.79057 & $(06100308)$ \\
5157837.0 & 63.21071 & $(06120515)$ & 57.82429 & $(06120515)$ & 60.21055 & $(06100308)$ & 56.27642 & $(06100308)$ & 43.38787 & $(06100308)$ \\
5157832.0 & 55.79428 & $(06120515)$ & 56.54596 & $(06100308)$ & 55.01138 & $(06100308)$ & 44.94002 & $(06100308)$ & 37.81384 & $(06102308)$ \\
5157827.0 & 52.90891 & $(06100308)$ & 53.21560 & $(06100308)$ & 45.68367 & $(06100308)$ & 35.59723 & $(06102308)$ & $37.23727(06102308)$ \\
5157822.0 & 51.07219 & $(06100308)$ & 45.74773 & $(06100308)$ & 34.96268 & $(06100308)$ & 35.59691 & $(06102308)$ & $36.29072(06102308)$ \\
5157817.0 & 45.26453 & $(06100308)$ & 36.32675 & $(06100308)$ & 33.47068 & $(06102308)$ & 35.24170 & $(06102308)$ & 34.97995 & $(06102308)$ \\
5157812.0 & 37.15831 & $(06100308)$ & 31.81688 & $(06121908)$ & 33.63387 & $(06102308)$ & 34.52397 & $(06102308)$ & $33.32626(06102308)$ \\
5157807.0 & 31.24272 & $(06102709)$ & 31.81514 & $(06121908)$ & 33.45672 & $(06102308)$ & 33.45717 & $(06102308)$ & 31.36849 & $(06102308)$
\end{tabular}

*** AERMOD - VERSION $07026 * * * \quad * * *$ U/UA Building Demolition

*** Unit Release - Worst Case

$\begin{array}{ll}* * * & 02 / 09 / 09 \\ * * * & 10: 41: 11\end{array}$

**MODELOPTS:

DRYDPL WETDPL

*** THE 1ST HIGHEST 1-HR AVERAge CONCENTRATION VALUES FOR SOURCE GROUP: ALL *** INCLUDING SOURCE(S): $224 \mathrm{U}_{-} 1,224 \mathrm{U}_{-}{ }^{2}, 224 \mathrm{U}_{-} 3,224 \mathrm{U}_{-} 4,224 \mathrm{U}_{-} 5,224 \mathrm{U}{ }^{6}, 224 \mathrm{U}_{-} 7$,

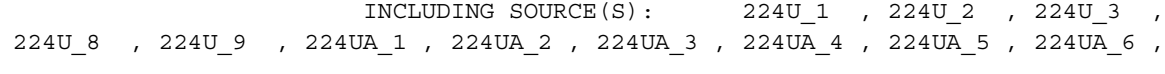

*** NETWORK ID: UCART1; NETWORK TYPE: GRIDCART ***

** CONC OF UO3 IN MICROGRAMS $/ \mathrm{M} * * 3$

**

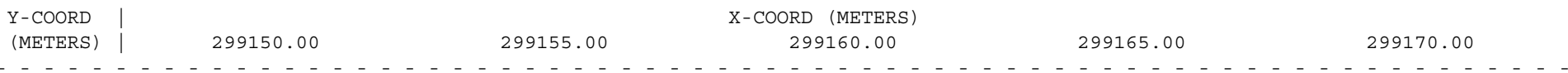


$5158052.0 \quad 23.76312 \quad(06122209)$ 5158047.0 5158042.0 5158037.0 5158032.0 5158027.0 5158022.0 5158017.0 5158012.0 5158007.0 5158002.0 5157997.0 5157992.0 5157987.0 5157987.0 5157982.0 5157972.0 157972.0 5157967.0 5157962.0 5157957.0 5157952.0 5157947.0 5157942.0 5157937.0 5157932.0 5157927.0 5157922.0 5157917.0 5157912.0 5757897.0 (5157897.0 5157892.0 5157887.0 5157882.0 5157877.0 5157872.0 5157867.0 5157857.0 $25.77727(06122209)$ 25.77727 (06122209) $26.68996(06122209)$ $27.52566(06122209)$ 28.26968 (06122209) $30.02380 \quad(06120710)$ $31.75667 \quad(06120710)$ $33.36875(06120710)$ $34.82726 \quad(06120710)$ $36.11073(06120710)$ $37.18816(06120710)$ $39.43425(06120710)$ $53.29895 \quad(06120710)$ $64.05196(06120710)$ $63.83174(06120710)$ $65.07378 \quad(06120409)$ $61.22173 \quad(0612$ $61.22173(06120409)$ (06120716) $91.74456 \quad(06120716)$ $91.54640 \quad(06120516)$ $91.05270 \quad(06120516)$ $88.47192(06122115)$ $95.20252(06122115)$ $84.42336(06113015)$ $85.87278 \quad(06102315)$ $87.72208 \quad(06102315)$ $85.94656 \quad(06102315)$ $85.94656 \quad(06102315)$ $76.83902(06101208)$ $110.94521(06101208)$ $130.94028(06101208)$ $127.36446(06101208)$ 112.68137 (06101108) $100.30024 \quad(06101108)$ $86.42428 \quad(06120515)$ $81.34300 \quad(06120515)$ $77.22717(06100308)$
$24.00200 \quad(06121916)$ $24.26080 \quad(06121916)$ $24.49578(06121916)$ $25.26652(06122209)$ (6.44939 (06122209) $26.44939(06122209)$ $28.65859(06122209)$ (28.65859 1061 $29.66384 \quad(06122209)$ $31.42916(06120710)$ $33.45007 \quad(06120710)$ $35.38489(06120710)$ $37.20292(06120710)$ $47.29894 \quad(06120710)$ $63.45821(06120710)$ $74.58569(06120710)$ $68.99601(06120409)$ $75.43503(06120409)$ $72.11536(06120409)$ $62.55339(06120716)$ $77.51595(06120716)$ $77.51595(06120716)$ $88.28444(06120716)$ $92.41232(06120716)$ $2.38016(06120516)$ $87.52885(06120516)$ $94.69034 \quad(06122115)$ 81.60123 (06122115) 88.11453 (06111609) 104.96597 (06103116) 96.11599 (06103116) $133.81183(06101208)$ $176.53302(06101208)$ $173.32050(06101208)$ 152.45840 (06101208) 133.24451 (06101108) 107.52735 (06101108) 97.71485 (06120515) $89.58924 \quad(06120515)$ $85.82629(06100308)$ $73.94920(06100308)$
$24.44075 \quad(06121916)$ $24.92050(06121916)$ $25.38460(06121916)$ 25.83679 (06121916) $26.27376 \quad(06121916)$ $26.27376 \quad(06121916)$ $26.69810 \quad(06121916)$ (27.13277 (06122209) $28.52176 \quad(06122209)$ 29.88331 (06122209) $31.20354 \quad(06122209)$ $33.18739(06120710)$ $39.07342(06121916)$ $53.72581 \quad(06120710)$ $60.06203 \quad(06122209)$ $66.02500(06122209)$ $63.01900(06120409)$ 58. 97022 (06120409) 54.77439 (06120409) 68.76771 (06120716) $68.46771(06120716)$ $82.20128(06120716)$ $90.70351(06120716)$ $91.98611(06120716)$ 90.93227 (06120516) 93.77922 (06122115) $96.67404 \quad(06120810)$ $122.26160(06120810)$ $33.05753 \quad(06120810)$ $127.08155 \quad(06111609)$ 179.25256 (06101208) $179.25256(06101208)$ $276.36020(06101208)$ 218.89668 (06101108) 106.77847 $06.77847 \quad(06101108)$ $113.65080 \quad(06120515)$ $109.01977 \quad(06122513)$ $95.37371(06100308)$

$77.13980(06100308)$ 54.17041 (06100308)
23.87909 (06121916) 24.55353 (06121916) $25.22448(06121916)$ $25.89805(06121916)$ $26.57012(06121916)$ 7..24382 (06121916) 7.92608 (06121916) 28.61335 (06121916) $29.31884(06121916)$ $30.05283(06121916)$ $32.68904 \quad(06121916)$ 45.80231 (06121916) $57.38789(06121916)$ $61.29078(06120710)$ 1.84559 (06120710) $65.91241 \quad(06120409)$ $6.97700 \quad(06120409)$ 64.75285 (06120409) $62.46471 \quad(06120409)$ $59.16055(06120409)$ 8.14081 (06120716) $2.08881(06120716)$ $92.36264 \quad(06120516)$ $93.47021(06120810)$ $145.03537(06120810)$ $228.47217(06122510)$ $269.98563(06122510)$ 303.83713 (06101208) 364.81409 (06101108) $280.12195(06101108)$ $189.40704 \quad(06101108)$ $152.49860 \quad(06120814)$ $137.79793(06120515)$ $135.81824 \quad(06122513)$ 106.72681 (06112111) $9.19173(06100308)$ $44.71314 \quad(06111009)$
$22.39453 \quad(06121916)$ $23.21454 \quad(06121916)$ $24.04560 \quad(06121916)$ 24.89557 (06121916) $25.76160 \quad(06121916)$ 25.64573 (06121916) (0.49587 (06121916) $29.47214 \quad(06121916)$ $30.50479 \quad(06121916)$ 36.49473 (06121916) $51.33615 \quad(06121916)$ $47.06080(06120710)$ $59.66666(06120710)$ $62.40732(06120409)$ $65.88141 \quad(06120409)$ 65.94478 (06120409) 64. $(06120716)$ (58.91476 (06113009) 206.93878 (06113009) $288.02667 \quad(06113009)$ 418.07626 (06113009) 1046.66663 (06103116) $2689.27490 \quad(06102708)$ 688.17645 (06110316) 83.01517 (06101108) $253.25491(06101108)$ $73.47037(06120814)$ $207.00839(06120814)$ $182.70633(06120515)$ $171.65268(06122513)$ $126.26097(06112111)$ $79.50050(06100308)$

$61.52109(06122516)$

$53.18835(06122516)$
*** AERMOD - VERSION $07026 * * * \quad * * *$ U/UA Building Demolition $\star *$ MODELOPTS

CONC

DDEP $\star * *$ U/UA Building Demolition
$\star * *$ Unit Release - Worst Case TOXICS FLAT

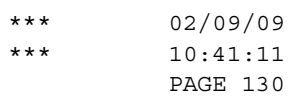

DRYDPL WETDPL

*** THE 1ST HIGHEST 1-HR AVERAGE CONCENTRATION VALUES FOR SOURCE GROUP: ALL *** INCLUDING SOURCE $(S): \quad 224 \mathrm{U}_{-} 1,224 \mathrm{U}_{-}{ }^{2}, 224 \mathrm{U}_{-}^{3}, 224 \mathrm{U}_{-} 4,224 \mathrm{U}_{-} 5,224 \mathrm{U}_{-} 6,224 \mathrm{U}_{-} 7$,

$224 \mathrm{U} \_8,224 \mathrm{U} \_9,224 \mathrm{UA} \_1,224 \mathrm{UA} \_2,224 \mathrm{UA} \_3,224 \mathrm{UA} \_4,224 \mathrm{UA} \_5,224 \mathrm{UA} \_6$,

*** NETWORK ID: UCART1 ; NETWORK TYPE: GRIDCART *** 
** CONC OF UO3 IN MICROGRAMS/M**3

\begin{tabular}{|c|c|c|c|c|c|c|c|c|c|c|}
\hline \multirow{3}{*}{$\begin{array}{l}\mathrm{Y} \text {-COORD } \\
\text { (METERS) } \\
-\quad-\quad-\quad-\end{array}$} & \multicolumn{10}{|c|}{ X-COORD (METERS) } \\
\hline & \multirow{2}{*}{\multicolumn{2}{|c|}{299150.00}} & \multicolumn{2}{|c|}{299155.00} & \multicolumn{2}{|c|}{299160.00} & \multicolumn{2}{|c|}{299165.00} & \multicolumn{2}{|c|}{299170.00} \\
\hline & & & ---- & $-\ldots$ & - & - & - & 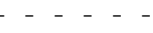 & - & \\
\hline 5157852.0 & 70.07700 & $(06100308)$ & 55.64323 & $(06100308)$ & 43.44194 & $(06102308)$ & 44.23166 & $(06111009)$ & 47.96000 & (06111009) \\
\hline 5157847.0 & 56.31091 & $(06100308)$ & 42.19220 & $(06102308)$ & 41.05851 & $(06102308)$ & 43.72822 & (06111009) & 46.69048 & (06111009) \\
\hline 5157842.0 & 40.92607 & $(06100308)$ & 40.62409 & $(06102308)$ & 38.93438 & (06111009) & 43.16992 & (06111009) & 45.29155 & (06111009) \\
\hline 5157837.0 & 39.51899 & $(06102308)$ & 38.68170 & $(06102308)$ & 38.88188 & $(06111009)$ & 42.53503 & (06111009) & 43.77418 & (06111009) \\
\hline 5157832.0 & 38.29227 & $(06102308)$ & 36.36853 & $(06102308)$ & 38.76634 & (06111009) & 41.79191 & $(06111009)$ & 42.12728 & (06111009) \\
\hline 5157827.0 & 36.69717 & $(06102308)$ & 34.43325 & $(06111009)$ & 38.57543 & (06111009) & 40.93185 & (06111009) & 40.38674 & (06111009) \\
\hline 5157822.0 & 34.74403 & $(06102308)$ & 34.63279 & (06111009) & 38.28672 & (06111009) & 39.94594 & (06111009) & 38.57627 & (06111009) \\
\hline 5157817.0 & 32.47909 & $(06102308)$ & 34.76252 & $(06111009)$ & 37.89684 & (06111009) & 38.83819 & (06111009) & 36.71888 & (06111009) \\
\hline 5157812.0 & 30.74072 & $(06111009)$ & 34.80832 & $(06111009)$ & 37.39029 & $(06111009)$ & 37.61634 & (06111009) & 34.83806 & (06111009) \\
\hline 5157807.0 & 31.09869 & $(06111009)$ & 34.76067 & $(06111009)$ & 36.76810 & $(06111009)$ & 36.29883 & (06111009) & 33.32795 & $(06112108)$ \\
\hline
\end{tabular}

\section{*** AERMOD - VERSION $07026 * * * \quad * * *$ U/UA Building Demolition **MODELOPTS

CONC

TOXICS FLAT

DRYDPL WETDPL

VALUES FOR SOURCE GROUP: ALL

INCLUDING SOURCE (S) : $\quad 224 \mathrm{U}_{-} 1,224 \mathrm{U}_{-} 2,224 \mathrm{U}_{-} 3,224 \mathrm{U}_{-} 4,224 \mathrm{U}{ }_{-} 5,224 \mathrm{U}{ }^{6}, 224 \mathrm{U}_{-} 7$, $224 \mathrm{U} \_8,224 \mathrm{U} \_9,224 \mathrm{UA} \_1,224 \mathrm{UA}{ }_{-} 2,224 \mathrm{UA} \_3,224 \mathrm{UA} \_4,224 \mathrm{UA}_{-} 5,224 \mathrm{UA}_{-} 6{ }^{\prime}$,

*** NETWORK ID: UCART1; NETWORK TYPE: GRIDCART ***

** CONC OF UO3 IN MICROGRAMS $/ \mathrm{M} * * 3$

\begin{tabular}{|c|c|c|c|c|}
\hline $\begin{array}{l}\text { Y-COORD } \\
\text { (METERS) }\end{array}$ & 299175 & & 299180 & \\
\hline & $-\cdots$ & & - - - & - \\
\hline 158052.0 & 20.16776 & $(06121916)$ & 21.89016 & $(06112108)$ \\
\hline 158047.0 & 21.06938 & $(06121916)$ & 23.54543 & $(06112108)$ \\
\hline 5158042.0 & 21.99722 & $(06121916)$ & 25.25170 & $(06112108)$ \\
\hline 5158037.0 & 22.95968 & $(06121916)$ & 27.00512 & $(06112108)$ \\
\hline 5158032.0 & 24.10831 & $(06112108)$ & 28.80162 & $(06112108)$ \\
\hline 5158027.0 & 25.89887 & $(06112108)$ & 30.63700 & $(06112108)$ \\
\hline 5158022.0 & 27.75527 & $(06112108)$ & 32.50698 & $(06112108)$ \\
\hline 5158017.0 & 29.67533 & $(06112108)$ & 34.40740 & $(06112108)$ \\
\hline 5158012.0 & 31.65689 & 10611210 & 36.33430 & $(06112108)$ \\
\hline 5158007.0 & 33.69797 & $(06112108)$ & 38.28419 & $(06112108)$ \\
\hline 5158002.0 & 39.04850 & (061219] & 40.25427 & $(06112108)$ \\
\hline 5157997.0 & 41.26534 & 10612 & 45.15772 & $(06113009)$ \\
\hline 5157992.0 & 50.14993 & $(06121916)$ & 50.43429 & $(06121916)$ \\
\hline 5157987.0 & 54.23477 & $(06122209)$ & 50.60843 & $(06121916)$ \\
\hline 157982.0 & 55.42260 & 1061222 & 50.74483 & $(06122209)$ \\
\hline 57977.0 & 56.85185 & $(06120710)$ & 52.58280 & $(06122209)$ \\
\hline & 59.04031 & & & \\
\hline
\end{tabular}

$\mathrm{X}$-COORD (METERS) 299185.00 299190.00 299195.00

$25.55946(06112108)$ $27.22268(06112108)$ $28.90564(06112108)$ $30.60283(06112108)$ $32.30872(06112108)$ $34.01790(06112108)$ $34.01790(06112108)$ $35.72523(06112108)$ $37.42596(06112108)$ $39.11589(06112108)$ $40.79168(06112108)$ $45.44331(06113009)$ $46.87290(06103116)$ $49.21605(06103116)$ $48.58232(06121916)$ $49.60632 \quad(06111009)$ $51.92809 \quad(06111009)$ 50.10162 (06121916)
27.98078 (06112108) 29.49954 (06112108) 31.00389 (06112108) $32.48810(06112108)$ $33.94678(06112108)$ 35.37498 (06112108) $36.76836(06112108)$ $38.12328(06112108)$ $39.43706(06112108)$ 40.82526 (06111009) $48.76196(06103116)$ 56.53268 (06103116) 46.46539 (06111009) $48.21162(06111009)$ 49.89358 (06111009) $51.52246(06111009)$ $48.67890 \quad(06112108)$
$28.66760(06112108)$ $29.91414(06112108)$ $32.27249(06112108)$ $33.37627 \quad(06112108)$ $35.06414(06112108)$ $35.06414 \quad(06111009)$ $36.77194 \quad(06111009)$ $38.40586(06111009)$ $39.95520 \quad(06111009)$ $41.41147 \quad(06111009)$ 52.96151 (06103116) 47.55817 (06103116) $48.60619 \quad(06103116)$ $49.55392 \quad(06103116)$ $50.39435 \quad(06103116)$ 51.12117 (06103116) 51.72881 (06103116) 
\begin{tabular}{l|ll}
5157967.0 & 62.26769 & $(06120409)$
\end{tabular} $64.58372(06120409)$ $5157957.0 \quad 65.84750(06120409)$ $5157952.0-65.96957(06120409)$ 5157952.0 . \begin{tabular}{l|l}
5157947.0 & $323.46503 \quad(06113009)$
\end{tabular} \begin{tabular}{l|l}
5157942.0 & $441.11893(06121808)$
\end{tabular} 5157937.0 | 1092.98779 (06113008) \begin{tabular}{l|l}
5157932.0 & $1146.04919(06113008)$
\end{tabular} \begin{tabular}{l|lll}
5157927.0 & 1955.42883 & $(06121809)$
\end{tabular} \begin{tabular}{l|lll}
5157922.0 & 3779.87378 & $(06121809)$
\end{tabular} \begin{tabular}{l|l}
5157917.0 & $6661.44092(06111708)$
\end{tabular} \begin{tabular}{l|lll}
5157912.0 & 2321.39746 & $(06122516)$
\end{tabular} \begin{tabular}{l|ll}
5157907.0 & 954.66290 & $(06122515)$
\end{tabular} \begin{tabular}{l|ll}
5157902.0 & $602.04736(06122515)$
\end{tabular} $\begin{array}{lll}5157897.0 & 417.94998 \quad(06122515) \\ 5 & 505.43289 & (06122515)\end{array}$ $5157892.0 \quad 305.43289 \quad(06122515)$ $232.55411(06122515)$ $150.82024(06122516)$ $150.82024(06122516)$ (105.18111 (06122516) \begin{tabular}{r|rr}
5157862.0 & $90.54697(06122516)$
\end{tabular} 5157857.0 | $81.07104(06122516)$

$55.95345(06120710)$ $58.85698(06120409)$ $62.12574 \quad(06120409)$ $64.52744(06112108)$ 64.52744 (06112108) 215.56760 (06121808) 287.14105 (06113008) 665.72650 (06111609) 812.71307 (06111609) 1680.39099 (06121809) 1365.19849 (06121809) 451.81482 (06111609) 2233.93359 (06122109) 1099.13428 (06120414) 680.21228 (06111008) $495.96692(06120515)$ $415.31061 \quad(06120608)$ $375.46988 \quad(06122515)$ 329.49631 (06122515) $283.27713 \quad(06122515)$ $236.31705(06122515)$ $188.23010(06122515)$ $149.78085(06122515)$ 125.89418 (06122515)
50.63816 (06111009) $55.67393 \quad(06111009)$ $61.13263(06111009)$ 67.00123 (06111009) 73.25395 (06111009) $8.25395(06111009)$ 80.14774 (06102308) $88.26582(06102308)$ $116.71205(06111609)$ 117.59891 (06111609) $128.07112(06111609)$ 149.38818 (06111609) 451.51617 (06111708) $826.70624 \quad(06110808)$ $3988.29956 \quad(06103116)$ $559.70233(06103116)$ 413.71808 (06111008) $297.01434 \quad(06111008)$ $53.60812(06120608)$ $254.50993(06120608)$ $226.94241(06120608)$ $92.67415(06122515)$ $172.66629(06122515)$ $159.58421(06122515)$
$49.38471 \quad(06111009)$ $54.85965(06111009)$ $61.34544 \quad(06102308)$ $68.20571(06102308)$ $75.20014 \quad(06102308)$ $82.08742(06102308)$ $88.58263(06102308)$ $94.01208(06102308)$ $97.99210 \quad(06102308)$ $101.27477(06111009)$ 106.37965 (06111009) 126.93841 (06120811) $1613.78845 \quad(06120409)$ 2093.27612 (06103116) $516.67456(06103116)$ $302.60483(06110108)$ $282.05792(06120414)$ 289.68530 (06111008) 241.32138 (06111008) $171.55412(06111008)$ 145.64931 (06120608) $141.95801(06120608)$ 138.42377 (06120608)
$49.70354 \quad(06102308)$ $67.34379(06102308)$ $73.02383(06102308)$ $78.16163(06102308)$ (8. (06121908) 96.26445 (06120810) 111.14811 (06120810) (16.60480 (06120810) $191.60512(06120516)$ $385.38690 \quad(06120710)$ $483.11469(06103116)$ $260.79895 \quad(06120416)$ $234.50209(06110108)$ (0).67615 (06120414) $12.81232(06111008)$ 212.66177 (06111008) $176.53482(06111008)$ $30.91585(06111008)$

98.91914 (06112716)

*** AERMOD - VERSION $07026 * * * \ldots *$ U/UA Building Demolition

*** Unit Release - Worst Case

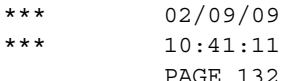

PAGE 132

DRYDPL WETDPI

CONC TOXICS FLAT

*** THE 1ST HIGHEST 1-HR AVERAGE CONCENTRATION VALUES FOR SOURCE GROUP: ALI

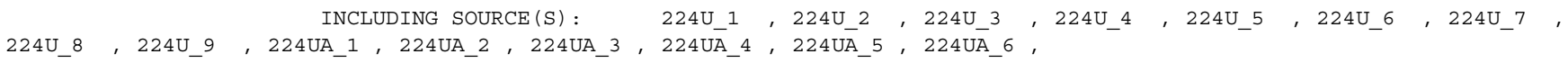

*** NETWORK ID: UCART1; NETWORK TYPE: GRIDCART ***

** CONC OF UO3 IN MICROGRAMS $/ \mathrm{M} * * 3$

**

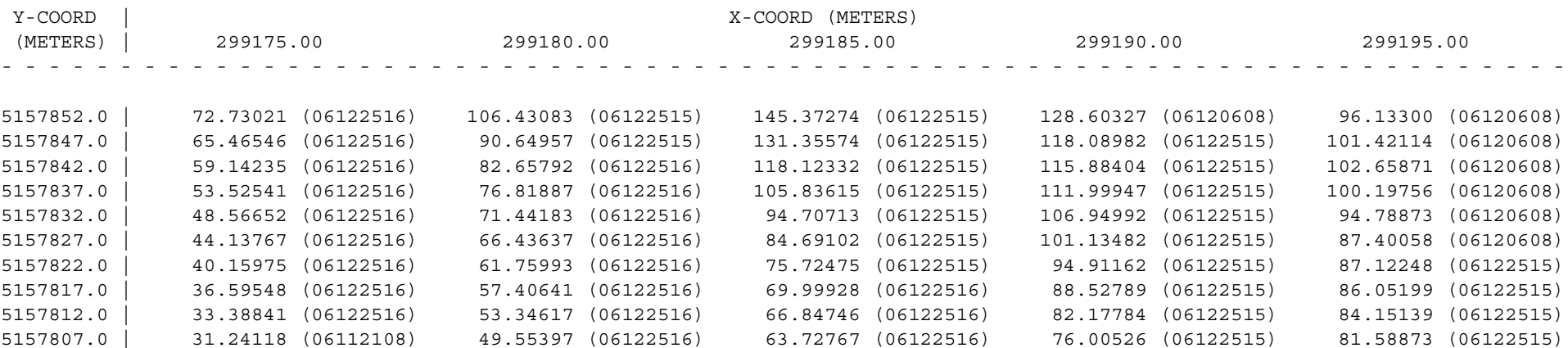


$* * *$ AERMOD - VERSION $07026 * * * \quad \begin{aligned} & * * \text { U/UA Building Demolition } \\ & * * * \text { Unit Release - Worst Case }\end{aligned}$

CONC

DDEP

TOXICS FLAT
*** $\quad 02 / 09 / 09$

$10: 41: 11$

PAGE 133

*** THE 1ST HIGHeSt 1-HR AVERAge CONCENTRATION VALUES FOR SOURCE GROUP: ALL *** INCLUDING SOURCE $(\mathrm{S}): \quad 224 \mathrm{U}_{-} 1,224 \mathrm{U}_{-} 2,224 \mathrm{U}_{-} 3,224 \mathrm{U}_{-} 4,224 \mathrm{U} \_5,224 \mathrm{U} \_6,224 \mathrm{U}_{-} 7$, $224 \mathrm{U}_{-} 8,224 \mathrm{U} \_9,224 \mathrm{UA} \_1,224 \mathrm{UA} \_2,224 \mathrm{UA} \_3,224 \mathrm{UA}_{-} 4,224 \mathrm{UA}_{-} 5,224 \mathrm{UA}_{-} 6{ }^{\prime}$,

*** NETWORK ID: UCART1; NETWORK TYPE: GRIDCART ***

** CONC OF UO3 IN MICROGRAMS $/ \mathrm{M} * * 3$

Y-COORD
(METERS) |

$5158052.0 \mid 28.05147(06111009)$

\begin{tabular}{l|ll}
5158047.0 & $29.65673(06111009)$
\end{tabular}

$5158042.0 \mid 31.20363(06111009)$

$5158037.0 \mid 32.67871(06111009)$

$5158032.0 \quad 34.06971(06111009)$

$5158027.0 \quad 35.36571(06111009)$

\begin{tabular}{l|l}
5158022.0 & $36.55766(06111009)$
\end{tabular}

$5158017.0 \quad 37.63855(06111009)$

$5158012.0 \quad 38.60389(06111009)$

$39.45193(06111009)$

$39.45193(06111009)$

$53.89642(06103116)$

5157997.0
$51.07557(06103116)$

5157992.0
5157987.0

\begin{tabular}{l|l}
5157987.0 & $52.77732(06103116)$ \\
$52.98309(06103116)$
\end{tabular}

$5157982.0 \quad 52.98309(06103116)$

$53.05611(06103116)$

$52.99562(06103116)$

$5157967.0 \quad 50.55452(06103116)$

\begin{tabular}{l|ll}
5157962.0 & $52.88959(06121908)$ \\
5157957.0 & $57.92242(06121908)$
\end{tabular}

$\begin{array}{lll}5157957.0 & 57.92242(06121908)\end{array}$

\begin{tabular}{l|l}
5157952.0 & $64.96648 \quad(06121809)$
\end{tabular}

$5157947.0-66.80423(06121908)$

$70.06940(06121908)$

72.19620 (06121908)

$30.73739(06120810)$

$106.32443(06120810)$

$127.36870(06120810)$

\begin{tabular}{l|l}
5157917.0 & 130.27951 \\
$(06120810)$
\end{tabular}

$124.74422(06121809)$

$176.98871(06112109)$

$\begin{array}{lll}5157902.0 & 229.02519 & (06111708)\end{array}$

\begin{tabular}{l|ll}
5157897.0 & 237.99274 & $(06111708)$
\end{tabular}

5157892.0

$311.81116(06110808)$

207.13770 (06120416)
$28.52713 \quad(06111009)$

$9.72827(06111009)$

$30.83116(06111009)$

$31.82714 \quad(06111009)$

$32.70942(06111009)$

$33.47334(06111009)$

$34.11649(06111009)$

$34.63899(06111009)$

35.07179 (06103116)

37.46257 (06102308)

51.46623 (06103116)

$51.69141 \quad(06103116)$

$52.92805(06103116)$

52.58261 (06103116)

52.10783 (06103116)

51.50707 (06103116)

50.78462 (06103116)

$52.91835 \quad(06121809)$ 62.67572 (06121809) 58.18091 (06121809) $56.79683(06121908)$ $58.15897(06121908)$ 58.31293 58.31293 (06121908) $81.23421(06120810)$ $107.00425(06120810)$ 123.66656 (06120810) 131.11124 (06120810) 138.20915 (06121915) (13.76895 (06120810) 143.04509 (06111708) (0.18077 (06110808) 252.22009 (06110808)
$29.03664 \quad(06103116)$

$29.03664(06103116)$

$30.30993(06103116)$

$.30993(06103116)$
$.93994(06103116)$
$.56520(06103116)$

$31.56520(06103116)$

32.19064 (06103116)

32.81798 (06103116)

$34.94897(06102308)$

$38.71806(06102308)$

46.23950 (06103116)

$46.50093 \quad(06103116)$

49.500934 (06103116)

49.00937 (06103116)

$47.94039(06103116)$

58.12955 (06121809)

$58.03004 \quad(06121809)$

$56.59483(06121809)$

52.18489 (06103108)

$48.28461 \quad(06121811)$

$48.79006 \quad(06121811)$

$48.70808 \quad(06112109)$

$45.88861 \quad(06112109)$

(5.8886 (06112109)

$70.02384(06120810)$

$70.92384 \quad(06120810)$

$94.10616 \quad(06120810)$

$114.54598 \quad(06120810)$

$127.90217(06120810)$

$131.01233(06120810)$

$123.10699(06120810)$

106.11813 (06120810)

$146.53810(06111708)$

167.93967 (06111708)

199.61510 (06110808)
$28.96621 \quad(06103116)$

$9.36922(06103116)$ $29.75286(06103116)$ $30.12034 \quad(06103116)$ $30.46740 \quad(06103116)$ 32.46029 (06102308) $34.27437(06102308)$ $35.79741 \quad(06102308)$ $36.98852(06102308)$ $38.05463 \quad(06121908)$ $39.24906 \quad(06103116)$ $14005(06103116)$ $3.35700 \quad(06121809)$ $2.84905(06121809)$ $51.05706(06121809)$ $58.73048 \quad(06103108)$ 46.12986 (06121811) 53.61082 (06121811) $5.13210(06121811)$ (06112109) $53.41049(06112109)$ 47.71400 (06112109) $57.40806(06120810)$ (50.22401 (06120810) $102.84464 \quad(06120810)$ $120.94566(06120810)$ 130.47734 (06120810) $129.12671 \quad(06120810)$ $117.22855(06120810)$ $106.37079(06111708)$ 138.40445 (06111708) $136.69052(06111708)$
$27.75481 \quad(06103116)$ $27.90614 \quad(06103116)$ $28.11623(06102308)$ $29.97546(06102308)$ 31.59915 (06102308) $32.94052 \quad(06102308)$ $33.96215(06102308)$ $35.10860 \quad(06121908)$ $36.08245 \quad(06121908)$ $36.62850 \quad(06121908)$ 36.73869 (06121908) $42.32148 \quad(06103116)$ $47.81700 \quad(06103116)$ 56.37637 (06121809) $55.71762(06103108)$ $47.26872(06121811)$ $52.69144 \quad(06121809)$ $52.79672 \quad(06121811)$ 57.77135 (06121811) $60.57559 \quad(06112109)$ 60.66961 (06112109) 55.79631 (06112109) 43.13272 (06102608) $52.86832(06122910)$ $66.29408(06120810)$ $89.50717(06120810)$ 10.86053 (06120810) $125.95988(06120810)$ $131.28760 \quad(06120810)$ $125.53116 \quad(06120810)$ 10.98139 (06120810) 117.37688 (06111708) 


\begin{tabular}{r|rlrllll}
5157882.0 & 187.06760 & $(06110108)$ & 168.39270 & $(06120416)$ & 210.41774 & $(06110808)$ \\
5157877.0 & 167.62366 & $(06110108)$ & 157.89363 & $(06110108)$ & 144.44641 & $(06120416)$ \\
5157872.0 & 148.81900 & $(06120414)$ & 151.88553 & $(06110108)$ & 126.22449 & $(06110108)$ \\
5157867.0 & 139.55734 & $(06111008)$ & 125.49722 & $(06110108)$ & 133.73109 & $(06110108)$ \\
5157862.0 & 145.73573 & $(06111008)$ & 109.12848 & $(06120414)$ & 121.66410 & $(06110108)$ \\
5157857.0 & 135.80872 & $(06111008)$ & 110.01987 & $(06111008)$ & 95.02576 & $(06110108)$
\end{tabular}

$175.26537 \quad(06110808)$ $170.16269(06110808)$ $126.31918 \quad(061204$ 104.33227 (06120416) $117.32599(06110108)$ 117.32599 (06110108)
$108.87243 \quad(06111708)$ $145.35393(06110808)$ 140.27922 (06110808) 114.90365 (06120416) $97.15274 \quad(06120416)$ 101.42816 (06110108)

\section{*** AERMOD - VERSION $07026 * * * \quad * * *$ U/UA Building Demolition **MODELOPTS

CONC

DDEP

OXICS FLAT

DRYDPL WETDPL

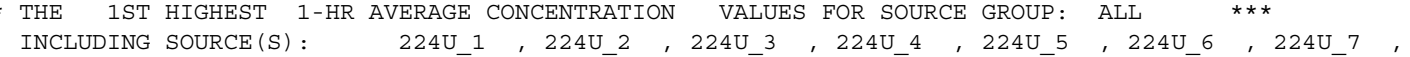
224U_8, 224U_9, 224UA_1 , 224UA_2, 224UA_3, 224UA_4 $, 224 \mathrm{UA} \_5,224 \mathrm{UA} \_6$,

*** NETWORK ID: UCART1; NETWORK TYPE: GRIDCART ***

** CONC OF UO3 IN MICROGRAMS $/$ M**3

Y-COORD
(METERS) | 299200.00
$87.82487 \quad(06120414)$ $98.99564(06111008)$ $97.45686(06111008)$ $87.45686(06111008)$ $88.81361(06111008)$ $70.16827(06112008)$

$\begin{array}{llrl}97.09904 & (06110108) & 106.55673 & (06110108) \\ 72.95721 & (06120414) & 96.72681 & (06110108) \\ 72.68594 & (06120414) & 76.57491 & (06110108) \\ 79.78236 & (06111008) & 62.65303 & (06120414) \\ 85.57850 & (06111008) & 62.02580 & (06111008) \\ 85.51586 & (06111008) & 70.37106 & (06111008) \\ 79.94086 & (06111008) & 75.48000 & (06111008) \\ 70.46040 & (06111008) & 76.24536 & (06111008) \\ 66.16528 & (06112008) & 72.64766 & (06111008) \\ 61.44008 & (06112008) & 65.66840 & (06111008)\end{array}$

$65.66840(06111008)$ $\begin{array}{llll}62.09046 & (06122509) & 75.81005 & (06111008) \\ 63.95454 & (06120608) & 63.70430 & (06112008) \\ 66.54883 & (06120608) & 56.01431 & (06112008) \\ 67.76134 & (06120608) & 53.56472 & (06122509)\end{array}$

$\begin{array}{llll}63.95454 & (06120608) & 63.70430 & (06112008) \\ 66.54883 & (06120608) & 56.01431 & (06112008) \\ 67.76134 & (06120608) & 53.56472 & (06122509)\end{array}$

$\begin{array}{llll}63.95454 & (06120608) & 63.70430 & (06112008) \\ 66.54883 & (06120608) & 56.01431 & (06112008) \\ 67.76134 & (06120608) & 53.56472 & (06122509)\end{array}$

$\begin{array}{llll}63.95454 & (06120608) & 63.70430 & (06112008) \\ 66.54883 & (06120608) & 56.01431 & (06112008) \\ 67.76134 & (06120608) & 53.56472 & (06122509)\end{array}$

\section{$\begin{array}{ll}* * * & 02 / 09 / 09 \\ * * * & 10: 41: 11\end{array}$ PAGE 135$$
\text { *** } \quad 10: 41: 11
$$

*** U/UA Building Demolition

DRYDPL WETDPL

*** THE 1ST HIGHEST 1-HR AVERAGE CONCENTRATION VALUES FOR SOURCE GROUP: ALL *** INCLUDING SOURCE (S) : $224 \mathrm{U}_{-1}{ }^{2}, 224 \mathrm{U}_{-} 2,224 \mathrm{U}_{-} 3,224 \mathrm{U}_{-} 4,224 \mathrm{U}_{-} 5,224 \mathrm{U}_{-} 6,224 \mathrm{U}_{-} 7$,

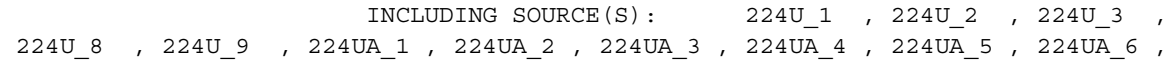

*** NETWORK ID: UCART1 ; NETWORK TYPE: GRIDCART ***

** CONC OF UO3 IN MICROGRAMS $/ \mathrm{M} * * 3$

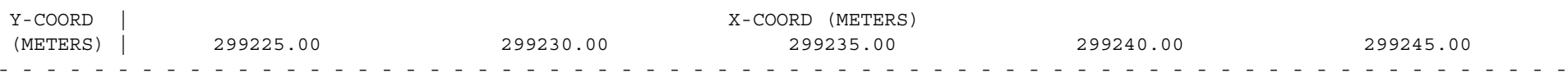


\begin{tabular}{l|ll}
5158052.0 & $25.79768(06102308)$
\end{tabular} \begin{tabular}{l|l}
5158047.0 & $27.48245(06102308)$
\end{tabular} $5158042.0+28.94030(06102308)$ 30.12809 (06102308) 5158032.0 ) $31.01184(06102308)$ 515032.0 | $32.01184(06102308)$ 515027.0 | 32.25984 (06121908) $33.14866(06121908)$ $5158017.0 \quad 33.63360(06121908)$ $33.70600(06121908)$ \begin{tabular}{l|l}
5158012.0 & 33.70600 \\
5158007.0 & $36.49086(06121908)$ \\
5158002.0 & $37.88338(06121809)$
\end{tabular} \begin{tabular}{l|l}
5158002.0 & $37.88338(06121809)$ \\
5157997.0 & $38.58580(06121809)$
\end{tabular} \begin{tabular}{l|ll}
5157997.0 & 38.58580 & $(06121809)$
\end{tabular} \begin{tabular}{l|ll}
5157992.0 & $39.33199(06121809)$
\end{tabular} \begin{tabular}{l|l}
5157987.0 & $55.27944(06103108)$
\end{tabular} $5157982.0 \mid 49.30345(06121809)$ $45.54523(06121809)$ $5157972.0 \quad 51.91048 \quad(06121811)$ 5157967.0 | 57.32225 (06121811) $5157962.0) \quad 57.32225(06121811)$ 5157962.0 | $59.50283(06112109)$ $5157957.0 \quad 63.79417(06112109)$ \begin{tabular}{l|l}
5157952.0 & $63.79417(06112109)$ \\
5 & $63.05759(06112109)$
\end{tabular} \begin{tabular}{l|l}
5157947.0 & $55.57904 \quad(06112109)$
\end{tabular} \begin{tabular}{l|l}
5157947.0 & 55.57904 \\
5157942.0 & $50.70305(06112109)$
\end{tabular} \begin{tabular}{l|ll}
5157937.0 & 45.60534 & $(06110208)$
\end{tabular} \begin{tabular}{l|l}
5157932.0 & $53.12477(06121915)$
\end{tabular} $5157927.0 \quad 54.03378(06122709)$

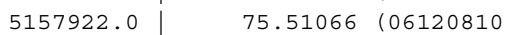
$98.50040-(06120810)$ 5157912.0 | $117.87013(06120810)$ 5157907.0 | 129.39151 (06120810) 5157902.0 | 130.29988 (06120810) 515702.0 | 130.29988 (06120810) 5157897.0 | $124.05071(06120810)$ $5157892.0 \quad 98.52784(06100508)$ $92.22055(06111708)$ $99.26784(06111708)$ $5157877.0 \quad 93.31033(06111708)$
5157872.0 \begin{tabular}{l|lll}
5157872.0 & 129.21310 & $(06110808)$
\end{tabular} \begin{tabular}{l|lll}
5157867.0 & 120.95768 & $(06110808)$
\end{tabular} $5157862.0 \quad 105.42683(06120416)$ $5157857.0 \quad 91.21018(06120416)$
$26.29020(06102308)$ $27.34990(06102308)$ $28.30687(06121908)$ $29.48349(06121908)$ $30.30768 \quad(06121908)$ $30.30768(06121908)$ 31.90491 (06121809) 31.90491 (06121809) $33.31192(06121809)$ $34.43731(06121809)$ $34.86591 \quad(06121809)$ $35.42630(06121809)$ $46.09493 \quad(06103108)$ 44.36696 (06103108) $50.56417 \quad(06121811)$ $50.97827(06121811)$ 56.00885 (06121811) $59.03794 \quad(06121811)$ $59.03794 \quad(06121811)$ $63.81312(06112109)$ 59.56921 (06112109) $51.32231(06112109)$ $43.59283(06110208)$ $47.31977 \quad(06121915)$ $50.10115(06102608)$ $53.36225(06102608)$ (61.74942 (06120810) $84.83464 \quad(06120810)$ $106.91770(06120810)$ $122.43841 \quad(06120810)$ $110.42452(06120810)$ $105.16257(06100508)$ 99.92177 (06100508) 84.45792 (06100508) $85.37474(06111708)$ 91.75359 (06111708) $82.41145(06111708)$ 116.07019 (06110808) $105.47050(06110808)$
$25.66043 \quad(06121908)$ 26.76291 (06121908) 27.53935 (06121908) $27.96484 \quad(06121908)$ $29.57275 \quad(06121809)$ (30.77367 (06121809) $31.71257 \quad(06121809)$ 32.35839 (06121809) 32.38015 (06121809) 41.16035 (06103108) $40.96646 \quad(06103108)$ $39.12896 \quad(06103108)$ 36.11816 (06103108) $35.36930(06122910)$ 43.42131 (06121811) $57.10739(06112109)$ $58.97510 \quad(06121811)$ 62.90205 (06122910) 62.90205 (06122910) 64.12376 (06112109) 61.18637 (06112109) 53.88438 (06112109) 49.29528 (06121811) 44.12683 (06110208) 49.37742 (06112109) $50.10115(06102608)$ $53.36225(06102608)$ $57.68046(06120509)$ $70.82438 \quad(06120810)$ $84.33929 \quad(06120810)$ 90.84859 (06100508) 0.8485 (06100508) (172 $(06100508)$ $94.64841(06100508)$ $84.35911(06100508)$ 67.69823 (06111708) 82.28028 (06111708) 84.34667 (06111708) $79.59144 \quad(06110808)$ 104.67596 (06110808) 93.24167 (06110808)
$24.83008 \quad(06121908)$ 26.39747 (06121809) 27.64395 (06121809) 28.68327 (06121809) 29.48196 (06121809) 0.01389 (06121809) 29.90416 (06121809) 36.65406 (06103108) $37.33748(06103108)$ $36.85137(06103108)$ 35.08784 (06103108) 32.46680 (06103108) $33.75043(06122910)$ 7.24837 (06122910) $0.17453(06122910)$ $50.81733(06112109)$ 64.05065 (06112109) 62.05065 (06112109) 62.47169 (06112109) 58.25038 (06121811) 9.29502 (06112109) (4.65160 (06112109) 45.78959 (06110208) $50.10115 \quad(06102608)$ $62.57193 \quad(06120509)$ $71.98969 \quad(06100508)$ $73.65490 \quad(06100508)$ $77.25057(06100508)$ $69.84603(06100508)$ $62.71391(06100508)$ $57.44934(06100508)$ 55.73666 (06100508) $67.90292(06111708)$ 78.78261 (06111708) $77.27264(06111708)$ $76.73618(06110808)$ $95.11480 \quad(06110808)$
$26.02055 \quad(06121809)$ 26.92978 (06121809) 27.61493 (06121809) $28.05869 \quad(06121809)$ 27.84053 (06121809) 27.84053 (06121809) 33.84182 (06103108) $34.13710 \quad(06103108)$ 33.34398 (06103108) 1.78077 (06103108) $9.47293(06122910)$ 32.72081 (06122910) $35.55964 \quad(06122910)$ 38.01965 (06122910) $39.74424 \quad(06122910)$ $40.44464 \quad(06122910)$ 55.66136 (06112109) $56.62948 \quad(06121811)$ 56.62948 (06121811) $58.41726 \quad(06121811)$ (06112109) 62.37997 (06112109) (06110208) $45.69240 \quad(06110208)$ $50.10115 \quad(06102608)$ $53.36225 \quad(06102608)$ 53.79466 (06120509) $61.58892 \quad(06120509)$ $62.51394 \quad(06120509)$ 72.84669 (06120509) 66.59373 (06120509) 66.59373 (06120509) $(06120509)$ $(06100508)$ 52.74660 (06100508) $54.38478(06100508)$ 52.31594 (06111708) 67.81427 (06111708) $75.20527(06111708)$
*** AERMOD - VERSION $07026 * * * \quad * * *$ U/UA Building Demolition *** Unit Release Worst Case **MODELOPTS :

CONC

DDEP TOXICS FLAT

*** THE 1ST HIGHeSt 1-HR AVERAGE CONCENTRATION VALUES FOR SOURCE GROUP: ALL *** INCLUDING SOURCE (S) : $\quad 224 \mathrm{U}_{-}{ }^{1}, 224 \mathrm{U}_{-} 2,224 \mathrm{U}_{-} 3,224 \mathrm{U}_{-} 4,224 \mathrm{U}_{-} 5,224 \mathrm{U}_{-} 6,224 \mathrm{U}_{-} 7$,

$224 \mathrm{U} \_8,224 \mathrm{U} \_9,224 \mathrm{UA} \_1,224 \mathrm{UA} \_2,224 \mathrm{UA} \_3,224 \mathrm{UA} \_4,224 \mathrm{UA} \_5,224 \mathrm{UA} \_6$,

*** NETWORK ID: UCART1 ; NETWORK TYPE: GRIDCART *** 
** CONC OF UO3 IN MICROGRAMS/M**3

\begin{tabular}{|c|c|c|c|c|c|c|c|c|c|c|}
\hline \multirow{3}{*}{$\begin{array}{c}\text { Y-COORD } \\
\text { (METERS) } \\
-\quad-\quad-\quad-\end{array}$} & \multicolumn{10}{|c|}{ X-COORD (METERS) } \\
\hline & \multirow{2}{*}{\multicolumn{2}{|c|}{$\begin{array}{l}299225.00 \\
-\quad-\quad-\end{array}$}} & \multicolumn{2}{|c|}{299230.00} & \multicolumn{2}{|c|}{299235.00} & \multicolumn{2}{|c|}{299240.00} & \multicolumn{2}{|c|}{299245.00} \\
\hline & & & --- & ---- & -- & ----1 & & -1 & & \\
\hline 5157852.0 & 86.81429 & (06110108) & 86.30596 & $(06120416)$ & 91.19057 & $(06120416)$ & 83.41129 & (06110808) & 86.99387 & $(06110808)$ \\
\hline 5157847.0 & 97.04881 & (06110108) & 73.69658 & (06110108) & 82.05756 & $(06120416)$ & 85.60352 & $(06120416)$ & 75.38702 & $(06110808)$ \\
\hline 5157842.0 & 94.09985 & $(06110108)$ & 86.95570 & $(06110108)$ & 62.92765 & $(06120416)$ & 78.30595 & $(06120416)$ & 80.69839 & $(06120416)$ \\
\hline 5157837.0 & 79.70537 & $(06110108)$ & 89.57453 & $(06110108)$ & 76.82033 & $(06110108)$ & 61.31487 & $(06120416)$ & 74.94685 & $(06120416)$ \\
\hline 5157832.0 & 59.67630 & $(06110108)$ & 80.82955 & (06110108) & 83.61607 & $(06110108)$ & 67.08696 & $(06110108)$ & 59.82761 & (06120416) \\
\hline 5157827.0 & 54.55461 & $(06120414)$ & 64.46863 & $(06110108)$ & 80.03782 & $(06110108)$ & 76.71069 & $(06110108)$ & 58.04495 & $(06110108)$ \\
\hline 5157822.0 & 56.02347 & (06111008) & 47.11279 & $(06120414)$ & 67.79771 & (06110108) & 77.55406 & (06110108) & 69.32652 & (06110108) \\
\hline 5157817.0 & 63.03164 & $(06111008)$ & 47.95618 & $(06120414)$ & 51.36496 & $(06110108)$ & 69.54164 & (06110108) & 73.69529 & $(06110108)$ \\
\hline 5157812.0 & 67.59599 & $(06111008)$ & 51.11157 & $(06111008)$ & 42.28660 & $(06120414)$ & 55.76444 & $(06110108)$ & 69.72182 & $(06110108)$ \\
\hline 5157807.0 & 68.82610 & $(06111008)$ & 57.12155 & $(06111008)$ & 42.43353 & $(06120414)$ & 40.45158 & $(06110108)$ & 59.03028 & $(06110108)$ \\
\hline
\end{tabular}

$* * *$ AERMOD - VERSION $07026 * * * \quad \begin{aligned} & * * \text { U/UA Building Demolition } \\ & * * * \text { Unit Release - Worst Case }\end{aligned}$ **MODELOPTS:

CONC

*** THE 1ST HIGHeSt 1-HR AVERAge CONCENTRATION VALUES FOR SOURCE GROUP: ALL *** INCLUDING SOURCE $(\mathrm{S}): \quad 224 \mathrm{U} \_1,224 \mathrm{U} \_2,224 \mathrm{U}{ }^{3} 3,224 \mathrm{U} \_4,224 \mathrm{U} \_5,224 \mathrm{U} \_6,224 \mathrm{U}_{-} 7$, $224 \mathrm{U} \_8,224 \mathrm{U} \_9,224 \mathrm{UA} \_1,224 \mathrm{UA} \_2,224 \mathrm{UA} \_3,224 \mathrm{UA} \_4,224 \mathrm{UA} \_5,224 \mathrm{UA} \_6$,'

*** NETWORK ID: UCART1; NETWORK TYPE: GRIDCART ***

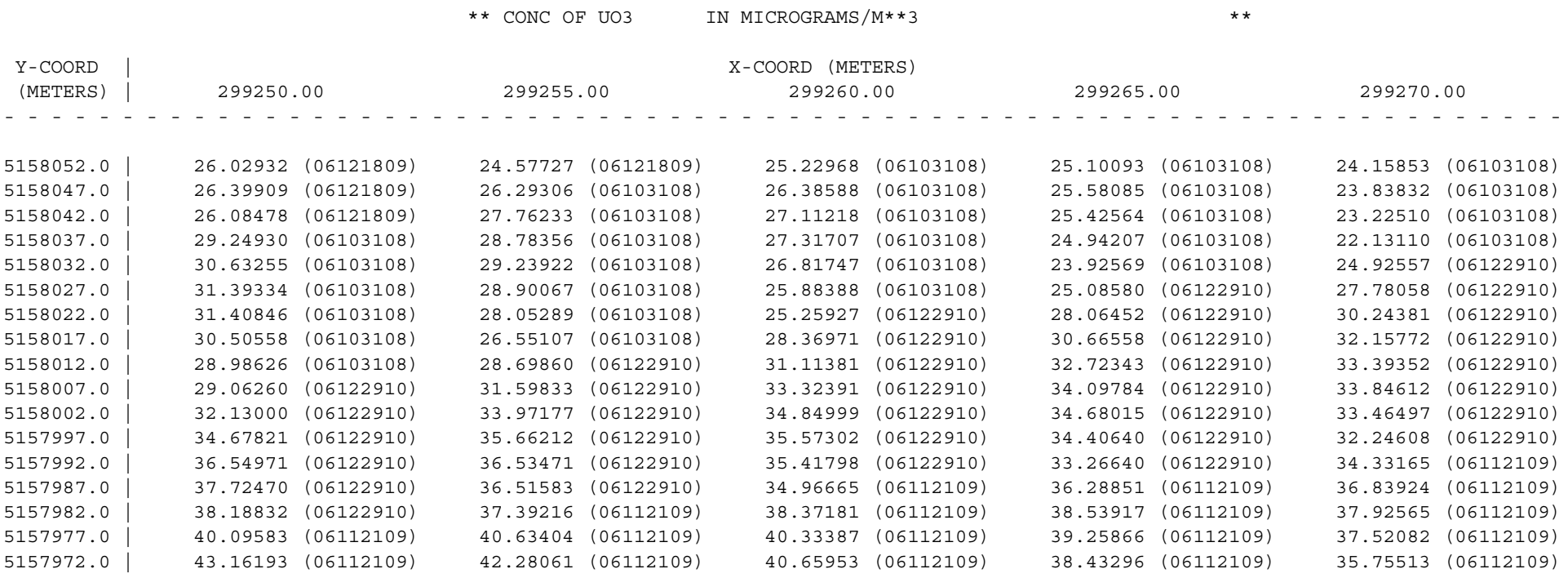


$5157967.0 \quad 51.25753$ (06112109) $5157962.0 \quad 60.53226 \quad(06112109)$ $5157957.0 \mid 62.28912(06112109)$ 5157952.0 - $53.62222(06112109)$ 5157947.0 5157942.0 | $45.30268(06110208)$ 5157937.0 | $50.48353(06122510)$ $5157932.0+50.10115(06122510)$ 5157927.0 $5157927.0 \quad 53.36225(06102608)$ $51.90894(06102608)$ \begin{tabular}{l|l}
5157917.0 & $65.52052(06102608)$
\end{tabular} \begin{tabular}{l|l}
5157917.0 & $65.52052(06102608)$ \\
5157912.0 & $59.12054(06102608)$
\end{tabular} \begin{tabular}{l|ll}
5157907.0 & $54.26292(06120509)$
\end{tabular} \begin{tabular}{l|l}
5157902.0 & $51.09654(06120509)$
\end{tabular}

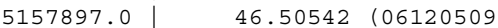
$5157892.0 \quad 44.11471(06100508)$ 5157887.0 (58.11471 (06100508) $5157882.0 \quad 52.10117 \quad(06100508)$ 5157877.0 ) 50.41277 (06100508) 515787.0 | 50.41277 (06100508) $54.25765(06111708)$ $5157862.0 \quad 67.24844(06111708)$ \begin{tabular}{l|ll}
5157867.0 & 67.24844 & $(06111708)$ \\
5157857.0 & $64.07627(06111708)$ \\
& &
\end{tabular}
42.19105 (06112109) (42.25589 (06112109) $45.02934 \quad(06122510)$ $43.13914 \quad(06122510)$ (3..13914 (06122510) $53.96838(06122510)$ $53.09013(06122510)$ (57.75261 (06110208) $57.75261(06110208)$ $54.45996 \quad(06110208)$ $55.28195(06102608)$ 54.11669 (06102608) $49.91380 \quad(06102608)$ 43.54210 (06102608) 37.15742 (06120509) $38.41438 \quad(06120509)$ $40.63947 \quad(06100508)$ $45.20415(06100508)$ $49.21820 \quad(06100508)$ $49.21820(06100508)$ $49.89035(06100508)$ $44.83698(06100508)$ $55.76432(06111708)$ $66.10987(06111708)$ $67.00948(06111708)$
39.39518 (06112109) 36.78410 (06112109) $33.33442 \quad(06112109)$ $37.59073(06122510)$ 4.45720 (06122510) $43.53467 \quad(06120809)$ $43.24487(06120809)$ 44.89290 (06110208) 44.41660 (06110208) 41.58659 (06110208) $39.40651 \quad(06102608)$ 38.10821 (06102608) $34.95110 \quad(06102608)$ $35.36426 \quad(06120509)$ $37.01263 \quad(06120509)$ $37.48074 \quad(06100508)$ $41.81115(06100508)$ $46.09528 \quad(06100508)$ $4.05078(06100508)$ 45.38078 (06100508)

45.98848 (06100508)

$56.77962(06111708)$ $64.40055(06111708)$
$36.25077 \quad(06112109)$ $33.05148 \quad(06112109)$ $29.76372(06122510)$ $30.84778 \quad(0612510)$ 30.95383 (06122510) 30.95383 (06122510) 32.11713 (06122510) $33.12180(06120809)$ $32.11892(06120809)$ 32.99274 (06110208) 32.27815 (06110208) $32.90375(06102608)$ $32.77386(06102608)$ $30.94343(06102608)$ $33.68419(06120509)$ $35.65373(06120509)$ $5.51425(06120509)$ $38.72543(06100508)$ (6. $21906(06100508)$ 6. 01757 (06100508) $0.78279(06100508)$ $45.64268(06111708)$ 57.24005 (06111708)
$32.91903 \quad(06112109)$ 29.38110 (06112109) $29.32678(06122510)$ 30.02027 (06122510) $29.75936 \quad(06122510)$ $28.55912(06122510)$ $28.55912(06122510)$ 28.36342 (06110208) $29.29280(06110208)$ 29.05314 (06110208) 30.45309 (06102608) 31.87132 (06102608) 31.76889 (06102608) $30.02112 \quad(06102608)$ $32.09013 \quad(06120509)$ $34.32473 \quad(06120509)$ 34.69049 (06120509) $35.87440 \quad(06100508)$ $40.02370 \quad(06100508)$ $43.72117(06100508)$ 43.72117 (06100508) $45.12502 \quad(06100508)$ 47.47864 (06111708)

*** AERMOD - VERSION $07026 * * * \quad * \star *$ U/UA Building Demolition

*** Unit Release - Worst Case

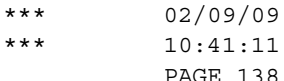

PAGE 138

DRYDPL WETDPI

CONC TOXICS FLAT

*** THE 1ST HIGHEST 1-HR AVERAGE CONCENTRATION VALUES FOR SOURCE GROUP: ALI

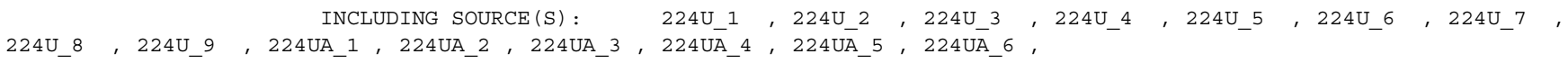

*** NETWORK ID: UCART1; NETWORK TYPE: GRIDCART ***

** CONC OF UO3 IN MICROGRAMS $/ \mathrm{M} * * 3$

**

\begin{tabular}{|c|c|c|c|c|c|c|c|c|c|c|}
\hline \multirow{3}{*}{$\begin{array}{l}\text { Y-COORD } \\
\text { (METERS) }\end{array}$} & \multicolumn{10}{|c|}{ X-COORD (METERS) } \\
\hline & 299250 & & 299255 & & 299260 & & 299265. & .00 & 299270. & \\
\hline & ---- & $-\ldots--$ & ---- & --- & ---- & ---- & --- & --- & ---- & \\
\hline 157852.0 & 70.51163 & $(06110808)$ & 57.87337 & $(06111708)$ & 62.54550 & $(06111708)$ & 62.17202 & $(06111708)$ & 57.12778 & (06111708) \\
\hline 157837.0 & 76.33867 & $(06120416)$ & 65.49041 & $(06120416)$ & 68.75459 & $(06110808)$ & 61.49862 & $(06110808)$ & 47.77344 & $(06110808)$ \\
\hline 5157832.0 & 71.89533 & $(06120416)$ & 72.42767 & $(06120416)$ & 61.84419 & $(06120416)$ & 64.12778 & $(06110808)$ & 58.75443 & $(06110808)$ \\
\hline$\$ 157827.0$ & 58.44054 & $(06120416)$ & 69.09696 & $(06120416)$ & 68.89635 & $(06120416)$ & 58.57608 & $(06120416)$ & 60.00810 & $(06110808)$ \\
\hline 157807.0 & 68.45448 & $(06110108)$ & 63.32517 & $(06110108)$ & 47.85564 & $(06110108)$ & 41.10772 & $(06120416)$ & 53.53741 & $(0612041$ \\
\hline
\end{tabular}




\begin{tabular}{l|llllllll}
5157882.0 & $37.22617(06100508)$ & 34.58010 & $(06100508)$ & 32.06364 & $(06100508)$ & 29.64695 & $(06100508)$ \\
5157877.0 & 41.08871 & $(06100508)$ & 38.46224 & $(06100508)$ & 35.91456 & $(06100508)$ & 33.46776 & $(06100508)$ \\
5157872.0 & 43.55296 & $(06100508)$ & 41.55712 & $(06100508)$ & 39.34126 & $(06100508)$ & 37.04070 & $(06100508)$ \\
5157867.0 & 42.64952 & $(06100508)$ & 42.22202 & $(06100508)$ & 41.13169 & $(06100508)$ & 39.57782 & $(06100508)$ \\
5157862.0 & 37.36264 & $(06100508)$ & 38.96877 & $(06100508)$ & 39.69400 & $(06100508)$ & 39.63863 & $(06100508)$ \\
5157857.0 & 37.24702 & $(06111708)$ & 34.72191 & $(06120810)$ & 34.44018 & $(06100508)$ & 36.15005 & $(06100508)$
\end{tabular}

$28.67496(06120509)$

$31.11548(06100508)$ $34.74137(06100508)$ $37.73228(06100508)$ $37.73228(06100508)$ $38.93919(06100508)$

\section{*** AERMOD - VERSION $07026 * * * \quad * * *$ U/UA Building Demolition \\ *** Unit Release - Worst Case}

$\begin{array}{ll}* * * & 02 / 09 / 09 \\ * * * & 10: 41: 11 \\ & \text { PAGE } 140\end{array}$

$* *$ MODELOPTS :
CONC

DRYDPL WETDPL

*** THE 1ST HIGHEST 1-HR AVERAGE CONCENTRATION VALUES FOR SOURCE GROUP: ALL ***

INCLUDING SOURCE $(\mathrm{S}): \quad 224 \mathrm{U} 1,224 \mathrm{U} 22,224 \mathrm{U} \_3,224 \mathrm{U} \_4,224 \mathrm{U} \_5 ， 224 \mathrm{U} \_6 ， 224 \mathrm{U} \_7$, 224U_8 , 224U_9 , 224UA_1 $, 224 \mathrm{UA} \_2,224 \mathrm{UA} \_3,224 \mathrm{UA} \_4,224 \mathrm{UA}_{-} 5,224 \mathrm{UA} \_6$,

*** NETWORK ID: UCART1 ; NETWORK TYPE: GRIDCART ***
** CONC OF UO3
IN MICROGRAMS $/ \mathrm{M} * * 3$

Y-COORD
(METERS)

\begin{tabular}{l|llllll}
5157852.0 & 48.89599 & $(06111708)$ & 39.41826 & $(06111708)$ & 33.15664 & $(06120810)$ \\
5157847.0 & 56.45490 & $(06111708)$ & 49.84426 & $(06111708)$ & 41.31897 & $(06111708)$ \\
5157842.0 & 56.43851 & $(06111708)$ & 55.25265 & $(06111708)$ & 50.29245 & $(06111708)$ \\
5157837.0 & 48.72239 & $(06111708)$ & 53.10318 & $(06111708)$ & 53.57592 & $(06111708)$ \\
5157832.0 & 47.00272 & $(06110808)$ & 44.24191 & $(06111708)$ & 49.57770 & $(06111708)$ \\
5157827.0 & 56.15202 & $(06110808)$ & 46.12381 & $(06110808)$ & 39.93421 & $(06111708)$ \\
5157822.0 & 56.34417 & $(06110808)$ & 53.69314 & $(06110808)$ & 45.16653 & $(06110808)$ \\
5157817.0 & 52.92027 & $(06120416)$ & 53.06115 & $(06110808)$ & 51.38509 & $(06110808)$ \\
5157812.0 & 60.01911 & $(06120416)$ & 50.45630 & $(06120416)$ & 50.09657 & $(06110808)$ \\
5157807.0 & 59.81064 & $(06120416)$ & 57.51912 & $(06120416)$ & 48.19444 & $(06120416)$
\end{tabular}

$\begin{array}{llll}33.20200 & (06120810) & 32.77552 & (06120810) \\ 32.59318 & (06111708) & 31.86893 & (06120810) \\ 42.89302 & (06111708) & 34.65422 & (06111708) \\ 50.23762 & (06111708) & 44.08052 & (06111708) \\ 51.48299 & (06111708) & 49.70023 & (06111708) \\ 45.95053 & (06111708) & 49.05070 & (06111708) \\ 35.85153 & (06111708) & 42.29333 & (06111708) \\ 44.15166 & (06110808) & 35.13293 & (06110808) \\ 49.22019 & (06110808) & 43.10457 & (06110808) \\ 47.41578 & (06110808) & 47.19020 & (06110808)\end{array}$

*** AERMOD - VERSION $07026 * * * \quad * * *$ U/UA Building Demolition

CONC TOXICS FLAT

DRYDPL WETDPL

*** THE 1ST HIGHEST 1 -HR DRY DEPOSITION VALUES FOR SOURCE GROUP: ALL *** INCLUDING SOURCE (S): $224 \mathrm{U}_{-} 1,224 \mathrm{U}_{-} 2,224 \mathrm{U}_{-} 3,224 \mathrm{U}_{-} 4,224 \mathrm{U}{ }^{5}, 224 \mathrm{U}_{-} 6,224 \mathrm{U}_{-} 7$, 224U_8 , 224U_9 $, 224 \mathrm{UA} \_1,224 \mathrm{UA} \_2,224 \mathrm{UA} \_3,224 \mathrm{UA} \_4,224 \mathrm{UA}_{-} 5,224 \mathrm{UA}_{-}{ }^{3} 6$,

*** NETWORK ID: UCART1; NETWORK TYPE: GRIDCART ***

** DEPO OF UO3 IN GRAMS $/ \mathrm{M} * * 2$

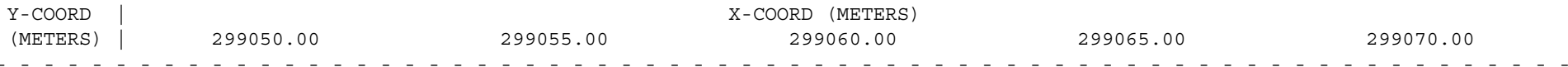




\begin{tabular}{|c|c|c|c|c|}
\hline 5158052.0 & 0.00010 & $(06111715)$ & 0.00011 & $(06102611)$ \\
\hline 5158047.0 & 0.00011 & $(06111715)$ & 0.00011 & $(06111715)$ \\
\hline 5158042.0 & 0.00011 & $(06111715)$ & 0.00012 & $(06111715)$ \\
\hline 5158037.0 & 0.00012 & $(06111715)$ & 0.00012 & $(06111715)$ \\
\hline 5158032.0 & 0.00012 & $(06111715)$ & 0.00012 & $(06111715)$ \\
\hline 5158027.0 & 0.00012 & $(06102613)$ & 0.00012 & (06111715) \\
\hline 5158022.0 & 0.00012 & $(06102613)$ & 0.00013 & $(06102613)$ \\
\hline 5158017.0 & 0.00012 & $(06102613)$ & 0.00013 & $(06102613)$ \\
\hline 5158012.0 & 0.00012 & $(06102711)$ & 0.00013 & $(06102613)$ \\
\hline 5158007.0 & 0.00012 & $(06102711)$ & 0.00013 & $(06102711)$ \\
\hline 5158002.0 & 0.00013 & $(06102711)$ & 0.00013 & $(06102711)$ \\
\hline 5157997.0 & 0.00014 & $(06100208)$ & 0.00014 & $(06100611)$ \\
\hline 5157992.0 & 0.00018 & $(06100208)$ & 0.00017 & $(06100208)$ \\
\hline 5157987.0 & 0.00023 & $(06100208)$ & 0.00021 & $(06100208)$ \\
\hline 5157982.0 & 0.00028 & $(06100208)$ & 0.00026 & $(06100208)$ \\
\hline 5157977.0 & 0.00033 & $(06100208)$ & 0.00031 & $(06100208)$ \\
\hline 5157972.0 & 0.00037 & $(06100208)$ & 0.00036 & $(06100208)$ \\
\hline 5157967.0 & 0.00040 & $(06100208)$ & 0.00040 & $(06100208)$ \\
\hline 5157962.0 & 0.00042 & $(06100208)$ & 0.00042 & $(06100208)$ \\
\hline 5157957.0 & 0.00042 & $(06100208)$ & 0.00043 & $(06100208)$ \\
\hline 5157952.0 & 0.00039 & $(06100208)$ & 0.00041 & $(06100208)$ \\
\hline 5157947.0 & 0.00035 & $(06100208)$ & 0.00037 & $(06100208)$ \\
\hline 5157942.0 & 0.00034 & $(06101008)$ & 0.00036 & $(06101008)$ \\
\hline 5157937.0 & 0.00033 & $(06101008)$ & 0.00035 & $(06101008)$ \\
\hline 5157932.0 & 0.00030 & $(06101008)$ & 0.00032 & $(06101008)$ \\
\hline 5157927.0 & 0.00026 & $(06101008)$ & 0.00028 & $(06101008)$ \\
\hline 5157922.0 & 0.00021 & $(06101008)$ & 0.00023 & $(06101008)$ \\
\hline 5157917.0 & 0.00023 & $(06101309)$ & 0.00024 & $(06101309)$ \\
\hline 5157912.0 & 0.00024 & $(06101309)$ & 0.00025 & (06101309) \\
\hline 5157907.0 & 0.00024 & $(06101309)$ & 0.00026 & (06101309) \\
\hline 5157902.0 & 0.00024 & $(06101309)$ & 0.00025 & $(06101309)$ \\
\hline 5157897.0 & 0.00022 & $(06101309)$ & 0.00023 & $(06101309)$ \\
\hline 5157892.0 & 0.00020 & $(06101309)$ & 0.00021 & $(06101309)$ \\
\hline 5157887.0 & 0.00017 & $(06101309)$ & 0.00018 & $(06101309)$ \\
\hline 5157882.0 & 0.00015 & $(06113015)$ & 0.00016 & $(06113015)$ \\
\hline 5157877.0 & 0.00014 & $(06113015)$ & 0.00015 & $(06113015)$ \\
\hline 5157872.0 & 0.00013 & $(06113015)$ & 0.00014 & $(06113015)$ \\
\hline 5157867.0 & 0.00012 & $(06113015)$ & 0.00013 & $(06113015)$ \\
\hline 5157862.0 & 0.00013 & $(06101109)$ & 0.00014 & (06101109) \\
\hline 5157857.0 & 0.00014 & (06101109) & 0.00015 & (06101109) \\
\hline
\end{tabular}

$\begin{array}{ll}0.00012 & (06102611) \\ 0.00012 & (06102611) \\ 0.00012 & (0611115) \\ 0.00012 & (06111715) \\ 0.00013 & (06111715) \\ 0.00013 & (06111715) \\ 0.00013 & (06102613) \\ 0.00014 & (06102613) \\ 0.00014 & (06102613) \\ 0.00014 & (06102711) \\ 0.00014 & (06102711) \\ 0.00015 & (06102711) \\ 0.0016 & (06100611) \\ 0.00019 & (06100208) \\ 0.00024 & (06100208) \\ 0.00030 & (06100208) \\ 0.00035 & (06100208) \\ 0.00039 & (06100208) \\ 0.00042 & (06100208) \\ 0.00044 & (06100208) \\ 0.00043 & (06100208) \\ 0.00040 & (06100208) \\ 0.00037 & (06101008) \\ 0.00037 & (06101008) \\ 0.00034 & (06101008) \\ 0.00030 & (06101008) \\ 0.00025 & (06101008) \\ 0.00026 & (06101309) \\ 0.00027 & (06101309) \\ 0.00027 & (06101309) \\ 0.00026 & (06101309) \\ 0.00025 & (06101309) \\ 0.00022 & (06101309) \\ 0.00019 & (06101309) \\ 0.00017 & (06113015) \\ 0.00016 & (06113015) \\ 0.00015 & (06113015) \\ 0.00014 & (06101109) \\ 0.00015 & (06101109) \\ 0.00016 & (06101109)\end{array}$

$0.00012 \quad(06102611)$ $0.00013 \quad(06102611)$

$0.00013 \quad(06102611)$

$0.00013(06111715)$

$0.00013(06111715)$

$0.00014(06111715)$

0.00014 (06111715)

$0.00015(06102613)$

$0.00015(06102613)$

$0.00015(06102613)$

0.00015 (06102711)

$0.00016 \quad(06102711)$

$0.00016(06102711)$

$0.00018(06100611)$

$0.00023(06100208)$

$0.00028 \quad(06100208)$

$0.00033(06100208)$

$0.00038(06100208)$

$0.00042(06100208)$

$0.00044(06100208)$

$0.00044 \quad(06100208)$

$0.00042(06100208)$

$0.00039(06101008)$

$0.00039(06101008)$

$0.00037(06101008)$

$0.00033(06101008)$

0.00027 (06101008)

0.00027 (06101309)

0.00027 (06101309)

$0.00029(06101309)$

$0.00029(06101309)$

$0.00028(06101309)$

$0.00026(06101309)$

$0.00023(06101309)$

0.00019 (06113015)

$0.00019(06113015)$

$0.00017(06113015)$

$0.00016(06113015)$

$0.00015(06101109)$

$0.00016(06101109)$

0.00017 (06101109)
$0.00013(06102611)$

$.00013 \quad(06102611)$

$.00013 \quad(06102611)$

$0.00013 \quad(06102611)$

$0.00013(06102611)$

0.00014 (06111715)

0.00015 (06111715)

$0.00016 \quad(06102613)$

$0.00016 \quad(06102613)$

$0.00016 \quad(06102613)$

$0.00017 \quad(06102711)$

$0.00018 \quad(06102711)$

$0.00018 \quad(06100611)$

$0.00026(06100208)$

$0.00026(06100208)$

$0.00032-(06100208)$

$0.00037(06100208)$

$0.00042(06100208)$

$0.00045(06100208)$

$0.00046(06100208)$

$0.00044 \quad(06100208)$

$0.00041 \quad(06101008)$

$0.00041(06101008)$

$0.00039(06101008)$

$0.00035(06101008)$

$0.00030(06101008)$

$0.00030 \quad(06101309)$

0.00031 (06101309)

0.00030 (06101309)

$0.00027(06101309)$

$0.00024 \quad(06101309)$

$0.00021 \quad(06113015)$

$0.00020 \quad(06113015)$

$0.00018 \quad(06113015)$

$0.00016 \quad(06113015)$

$0.00016(06101109)$

$0.00018 \quad(06101109)$

0.00018 (06101109)

\section{*** AERMOD - VERSION $07026 * * * \quad \begin{aligned} & * * \text { U/UA Building Demolition } \\ & * * * \text { Unit Release - Worst Case }\end{aligned}$} **MODELOPTS :

CONC TOXICS FLAT

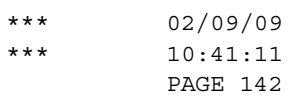

DRYDPL WETDPL

*** THE 1ST HIGHEST 1-HR DRY DEPOSITION VALUES FOR SOURCE GROUP: ALL ***

INCLUDING SOURCE $(\mathrm{S}): \quad 224 \mathrm{U}_{-} 1,224 \mathrm{U}_{-} 2,224 \mathrm{U}_{-}^{3}, 224 \mathrm{U}_{-} 4,224 \mathrm{U}_{-} 5,224 \mathrm{U}_{-} 6,224 \mathrm{U}_{-} 7$,

224U_8 , 224U_9 , 224UA_1 $, 224 \mathrm{UA} \_2,224 \mathrm{UA} \_3,224 \mathrm{UA} \_4,224 \mathrm{UA} \_5,224 \mathrm{UA} \_6$,'

*** NETWORK ID: UCART1 ; NETWORK TYPE: GRIDCART *** 


\begin{tabular}{|c|c|c|c|c|c|c|c|c|c|c|}
\hline \multirow{3}{*}{$\begin{array}{l}\text { Y-COORD } \\
(\text { METERS }) \\
-----\end{array}$} & \multicolumn{10}{|c|}{ X-COORD (METERS) } \\
\hline & \multicolumn{2}{|c|}{299050.00} & \multicolumn{2}{|c|}{299055.00} & \multicolumn{2}{|c|}{299060.00} & \multicolumn{2}{|c|}{299065.00} & \multicolumn{2}{|c|}{299070.00} \\
\hline & & $-\cdots$ & & $-\cdots-$ & & $-\ldots-$ & & -1 & & \\
\hline 5157852.0 & 0.00015 & (06101109) & 0.00016 & (06101109) & 0.00017 & (06101109) & 0.00018 & (06101109) & 0.00019 & $(06120814)$ \\
\hline 5157847.0 & 0.00016 & (06101109) & 0.00017 & (06101109) & 0.00017 & (06101109) & 0.00018 & $(06120814)$ & 0.00020 & $(06120814)$ \\
\hline 5157842.0 & 0.00017 & (06101109) & 0.00017 & (06101109) & 0.00018 & (06101109) & 0.00019 & $(06120814)$ & 0.00021 & $(06120814)$ \\
\hline 5157837.0 & 0.00017 & (06101109) & 0.00017 & (06101109) & 0.00018 & $(06120814)$ & 0.00020 & $(06120814)$ & 0.00021 & $(06120814)$ \\
\hline 5157832.0 & 0.00017 & $(06101109)$ & 0.00017 & $(06120814)$ & 0.00019 & $(06120814)$ & 0.00020 & $(06120814)$ & 0.00021 & (06120814) \\
\hline 5157827.0 & 0.00016 & (06101109) & 0.00017 & $(06120814)$ & 0.00018 & $(06120814)$ & 0.00019 & $(06120814)$ & 0.00020 & $(06120814)$ \\
\hline 5157822.0 & 0.00016 & $(06120814)$ & 0.00017 & $(06120814)$ & 0.00018 & $(06120814)$ & 0.00018 & $(06120814)$ & 0.00018 & $(06120814)$ \\
\hline 5157817.0 & 0.00016 & $(06120814)$ & 0.00016 & $(06120814)$ & 0.00017 & $(06120814)$ & 0.00017 & $(06120814)$ & 0.00017 & $(06120814)$ \\
\hline 5157812.0 & 0.00015 & $(06120814)$ & 0.00015 & $(06120814)$ & 0.00015 & $(06120814)$ & 0.00015 & $(06120814)$ & 0.00015 & (06120814) \\
\hline 5157807.0 & 0.00014 & $(06120814)$ & 0.00014 & $(06120814)$ & 0.00014 & $(06120814)$ & 0.00013 & $(06120814)$ & 0.00013 & (06102409) \\
\hline
\end{tabular}

\section{*** AERMOD - VERSION $07026 * * * \quad * * *$ U/UA Building Demolition **MODELOPTS

CONC

*** NETWORK ID: UCART1; NETWORK TYPE: GRIDCART ***

\section{** DEPO OF UO3 IN GRAMS $/ M * * 2$}

\section{$\mathrm{X}$-COORD (METERS)}

$\mathrm{Y}$-COORD
(METERS)

299075.00

299080.00 299085.00

$5158052.0 \mid \quad 0.00014(06102610)$

\begin{tabular}{l|l}
5158047.0 & $0.00014(06102610)$
\end{tabular}

\begin{tabular}{l|l}
5158042.0 & $0.00014(06102611)$
\end{tabular}

\begin{tabular}{c|cc}
5158037.0 & $0.00014(06102611)$
\end{tabular}

$5158032.0 \mid 0.00014(06102611)$

$5158027.0 \quad 0.00015(06111715)$

$0.00016(06111715)$

5158017.0 | $0.00016(06111715)$

\begin{tabular}{l}
5158017.00 | \\
\hline
\end{tabular}

5158012.0
5158007.0

$5158007.0 \quad 0.00017(06102613)$
5158002.0

5158002.0
5157907.0

\begin{tabular}{c|ccc}
5157997.0 & $0.00018(06102711)$ \\
\hline
\end{tabular}

\begin{tabular}{l|ll}
5157992.0 & $0.00019(06102711)$
\end{tabular}

\begin{tabular}{l|l}
5157987.0 & $0.00020(06102711)$
\end{tabular}

\begin{tabular}{l|l}
5157982.0 & $0.00021(06100611)$
\end{tabular}

\begin{tabular}{c|cc}
5157977.0 & $0.00024(06100208)$
\end{tabular}
$0.00016(06102610)$

$0.00016(06102610)$

$0.00015(06102610)$

$0.00015(06102611)$

$0.00015(06102611)$

$0.00015(06102611)$

$0.00016(06111715)$

$0.00017(06111715)$

$0.00018(06111715)$

$0.00018 \quad(06102613)$

$0.00019(06102613)$

$0.00019(06102613)$

$0.00020(06102711)$

$0.00021(06102711)$

$0.00022(06102711)$

$0.00023(06100611)$

$0.00028 \quad(06100208)$
$0.00017(06102610)$

$0.00017(06102610)$

$0.00017(06102610)$

$0.00017(06102610)$

$0.00016(06102610)$

$0.00016(06102611)$

$0.00016(06111715)$

$0.00017(06111715)$

$0.00018(06111715)$

$0.00019(06111715)$

$0.00020(06102613)$

$0.00020(06102613)$

$0.00021 \quad(06102711)$

$0.00023(06102711)$

$0.00024 \quad(06102711)$

0.00024 (06102711)

$0.00026(06122113)$
$0.00018(06102610)$ $0.00019(06102610)$

$0.00019(06102610)$

$0.00019(06102610)$

$0.00018(06102610)$

$0.00018 \quad(06102610)$

$0.00017 \quad(06102611)$

$0.00018(06111715)$

$0.00019(06111715)$

$0.00020(06111715)$

$0.00021(06102613)$

0.00022 (06102613)

$0.00022(06102613)$

0.00024 (06102711)

$0.00025 \quad(06102711)$

0.00027 (06102711)

0.00027 (06100611)
$0.00018 \quad(06102610)$ $0.00019 \quad(06102610)$

$0.00020(06102610)$

$0.00020(06102610)$

$0.00020 \quad(06102610)$

$0.00020(06102610)$

$0.00020(06102610)$

$0.00019 \quad(06102610)$

0.00019 (06111715)

0.00021 (06111715)

$0.00022(06111715)$

$0.00023 \quad(06102613)$

$0.00024 \quad(06102613)$

$0.00025(06102711)$

0.00027 (06102711)

$0.00029(06102711)$

$0.00030 \quad(06102711)$ 
\begin{tabular}{l|l}
5157967.0 & $0.00036(06100208)$
\end{tabular} $0.00041 \quad(06100208)$ $0.00045(06100208)$ $0.00047(06100208)$ 5157947.0 5157942.0 $0.00043(06101008)$ $515937.0 \quad 0.00043(06101008)$ $0.00041(06101008)$ $0.00038(06101008)$ $0.00031(06101309)$ 5157912.0
5157907.0 \begin{tabular}{l|l}
5157907.0 & $0.00033(06101309)$
\end{tabular} \begin{tabular}{l|ll}
5157902.0 & 0.00031 & $(06101309)$
\end{tabular} $\begin{array}{lll}5157897.0 & 0.00029 & (06101309)\end{array}$ $0.00025(06101309)$ $0.00023(06113015)$ 5157882.0 $0.00019(06113015)$ 5157877.0 | 10.00019 (06113015) 515782.0 |
5.00018 (06101211) \begin{tabular}{l|l}
5157862.0 & $0.00018(06101109)$ \\
5157857.0 & $0.00019(06101109)$
\end{tabular} 5157857.0 | $0.00020(06120814)$
$0.00034(06100208)$

$0.00040(06100208)$

$0.00045(06100208)$

$0.00048 \quad(06100208)$

$0.00048 \quad(06100208)$

$0.00046(06100208)$

$0.00044(06101008)$

$0.00040(06101008)$

0.00035 (06101008)

$0.00033(06101309)$

0.00035 (06101309)

$0.00035(06101309)$

$0.00033(06101309)$

$0.00030(06101309)$

$0.00025(06113015)$

$0.00023(06113015)$

$0.00021(06113015)$

$0.00019(06101109)$

$0.00020(06101109)$

$0.00021(06102710)$

$0.00022(06120814)$
$0.00033(06100208)$

$0.00039(06100208)$

$0.00045(06100208)$

$0.00049(06100208)$

0.00051 (06100208)

$0.00051(06100208)$

$0.00049(06100208)$

$0.00047(06101008)$

$0.00046(06101008)$

$0.00043(06101008)$

$0.00037(06101008)$

$0.00035 \quad(06101309)$

$0.00037(06101309)$

$0.00037 \quad(06101309)$

0.00035 (06101309)

$0.00032(06101309)$

$0.00028(06113015)$

$0.00027(06113015)$

$0.00024(06113015)$

0.00022 (06101211)

$0.00022(06101211)$

$0.00023(06102710)$

$0.00024(06102710)$

$0.00025(06102710)$
$0.00031(06100208)$ $0.00038(06100208)$ $0.00045(06100208)$ $0.00051 \quad(06100208)$ $0.00054(06100208)$ $0.00054(06100208)$ $0.00053(06100208)$ $0.00049(06100208)$ $0.00049(06101008)$ $0.00046(06101008)$ $0.00040 \quad(06101008)$ $0.00038(06100610)$ 0.00039 (06101309) $0.00039(06101309)$ $0.00037(06101309)$ $0.00029(06113015)$ $0.00026(06113015)$ $0.00024(06101109)$ 0.00024 (06101109) 0.00025 (06101109) $0.00028(06102710)$ $0.00029(06102710)$
$0.00032(06122113)$ $0.00038 \quad(06100208)$

$0.00046 \quad(06100208)$

$0.00053 \quad(06100208)$

$0.00057 \quad(06100208)$

$0.00057(06100208)$

$0.00059(06100208)$

$0.00055(06100208)$

$0.00049(06101008)$

$0.00044 \quad(06100610)$

$0.00043(06100610)$

$0.00042(06101309)$

$0.00042 \quad(06101309)$

$0.00040 \quad(06101309)$

$0.00035 \quad(06101309)$

$0.00033(06113015)$

$0.00031(06113015)$

0.00028 (06113015)

0.00028 (06101109)

$0.00029(06102710)$

$.00031(06102710)$

$0.00032(06102710)$

$0.00032(06102710)$

224U_8 $, 224 \mathrm{U} \_9,224 \mathrm{UA} \_1,224 \mathrm{UA} \_2,224 \mathrm{UA} \_3,224 \mathrm{UA} \_4,224 \mathrm{UA} \_5,224 \mathrm{UA} \_6$,

*** NETWORK ID: UCART1; NETWORK TYPE: GRIDCART ***

** DEPO OF UO3 IN GRAMS $/$ M**2

$\mathrm{X}$-COORD (METERS)

299080.00

299075.00

$299085.00 \quad 299090.00$

299095.00

\begin{tabular}{|c|c|c|c|c|}
\hline 57852.0 & 0.00021 & $(06120814)$ & 0.00024 & $(06120814)$ \\
\hline 5157847.0 & 0.00022 & $(06120814)$ & 0.00024 & $(06120814)$ \\
\hline 5157842.0 & 0.00023 & $(06120814)$ & 0.00025 & $(06120814)$ \\
\hline 5157837.0 & 0.00023 & $(06120814)$ & 0.00024 & $(06120814)$ \\
\hline 5157832.0 & 0.00022 & $(06120814)$ & 0.00022 & $(06120814)$ \\
\hline 5157827.0 & 0.00020 & $(06120814)$ & 0.00020 & $(06120814)$ \\
\hline 57822.0 & 0.00018 & $(06120814)$ & 0.00018 & $(06120814)$ \\
\hline 57817.0 & 0.00016 & $(06120814)$ & 0.00017 & $(06102409)$ \\
\hline 5157812.0 & 0.00015 & $(06102409)$ & 0.00019 & $(06101208)$ \\
\hline 157807.0 & 0.00017 & (06101208) & 0.00023 & $(06101208)$ \\
\hline
\end{tabular}

$0.00026(06120814)$

$.00027(06120814)$

$.00026(06120814)$

$0.00025(06120814)$

$0.00023(06120814)$

$0.00020(06120814)$

$0.00019(06101208)$

$0.00023(06101208)$

$0.00027 \quad(06101208)$

$0.00030(06101208)$
$0.00029(06120814)$

$0.00029(06120814)$

$0.00027(06120814)$

$0.00025(06120814)$

$0.00023(06120814)$

$0.00022(06101208)$

$0.00027(06101208)$

$0.00031(06101208)$

$0.00035(06101208)$

0.00037 (06101208) $0.00030 \quad(06120814)$ $0.00028(06120814)$ $.00026(06120814)$ $0.00026(06101208)$ $0.00032(06101208)$ $0.00036(06101208)$ $0.00040 \quad(06101208)$ $0.00043(06101208)$ $0.00044 \quad(06101208)$ 
$* * *$ AERMOD - VERSION $07026 * * * \quad \begin{aligned} & * * \text { U/UA Building Demolition } \\ & * * * \text { Unit Release - Worst Case }\end{aligned}$

**MODELOPTS :

CONC

DDEP
TOXICS FLAT
$* * * \quad 02 / 09 / 09$

$10: 41: 11$

PAGE 145

DRYDPL WETDPL

THE 1ST HIGHEST 1 -HR
INCLUDING SOURCE $(\mathrm{S}):$

$224 \mathrm{U}_{-} 8,224 \mathrm{U} \_9,224 \mathrm{UA} \_1,224 \mathrm{UA} \_2,224 \mathrm{UA} \_3,224 \mathrm{UA}_{-} 4,224 \mathrm{UA}_{-} 5,224 \mathrm{UA}_{-}{ }^{3} 6$,

** NETWORK ID: UCART1; NETWORK TYPE: GRIDCART ***

** DEPO OF UO3 IN GRAMS $/ M * * 2$

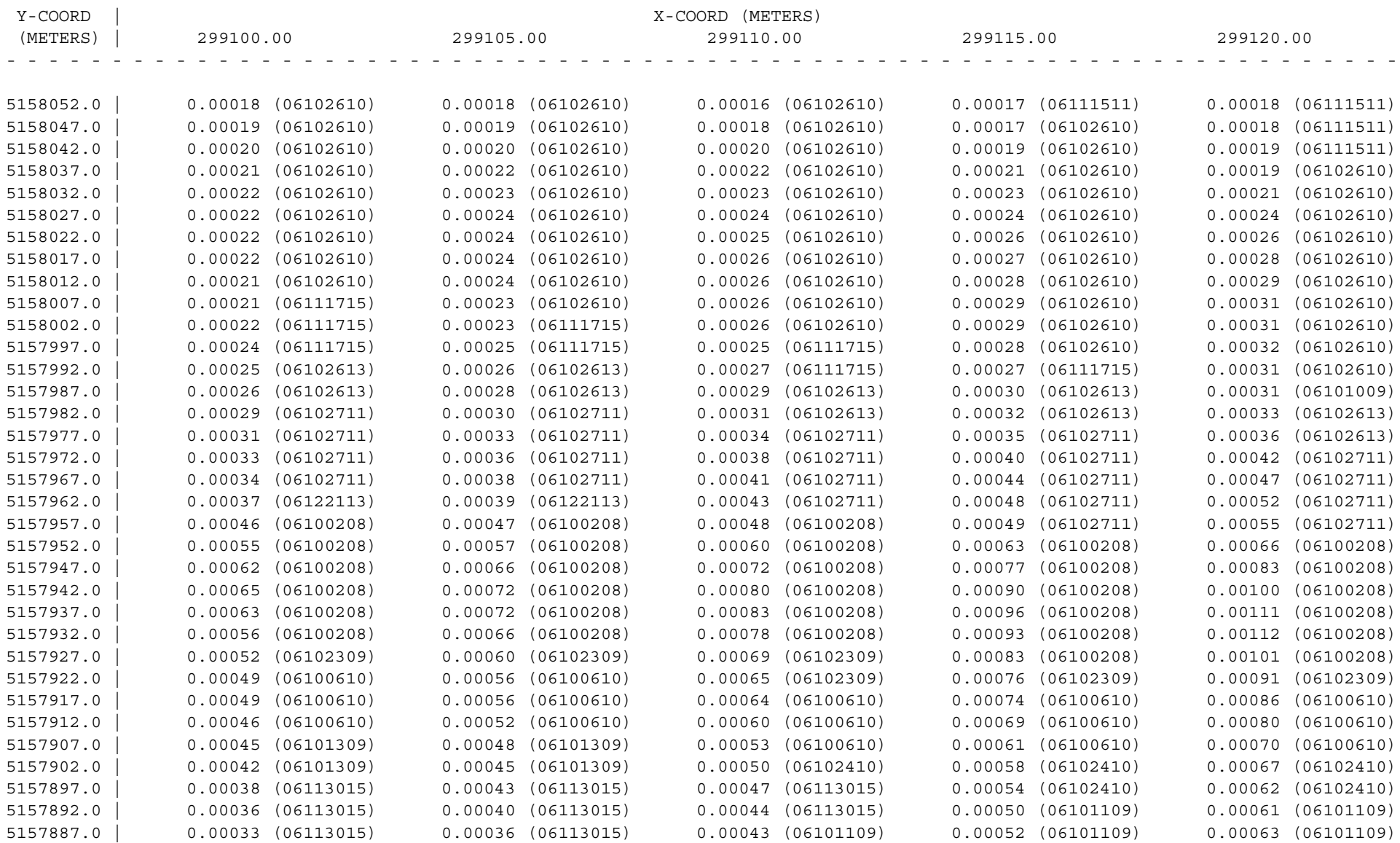




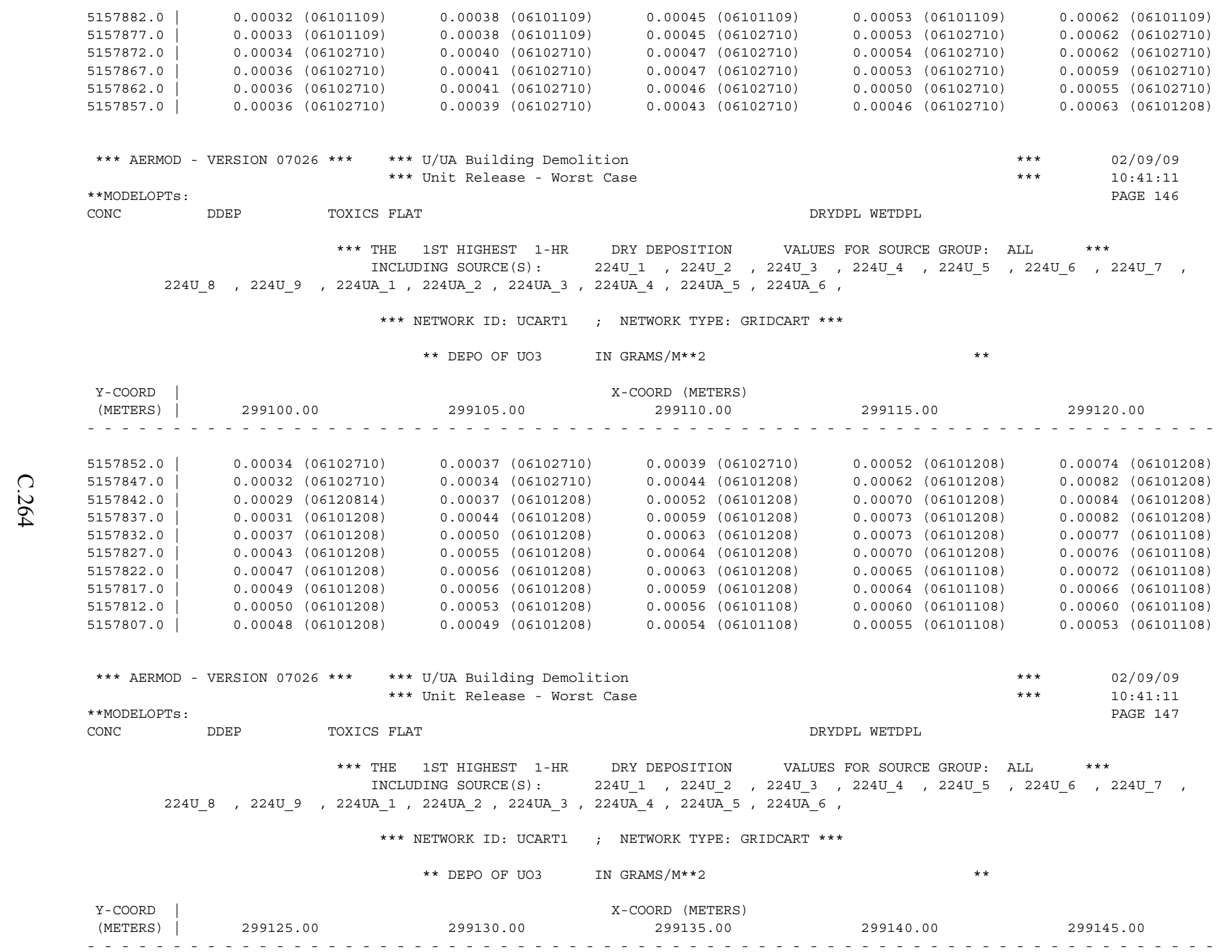




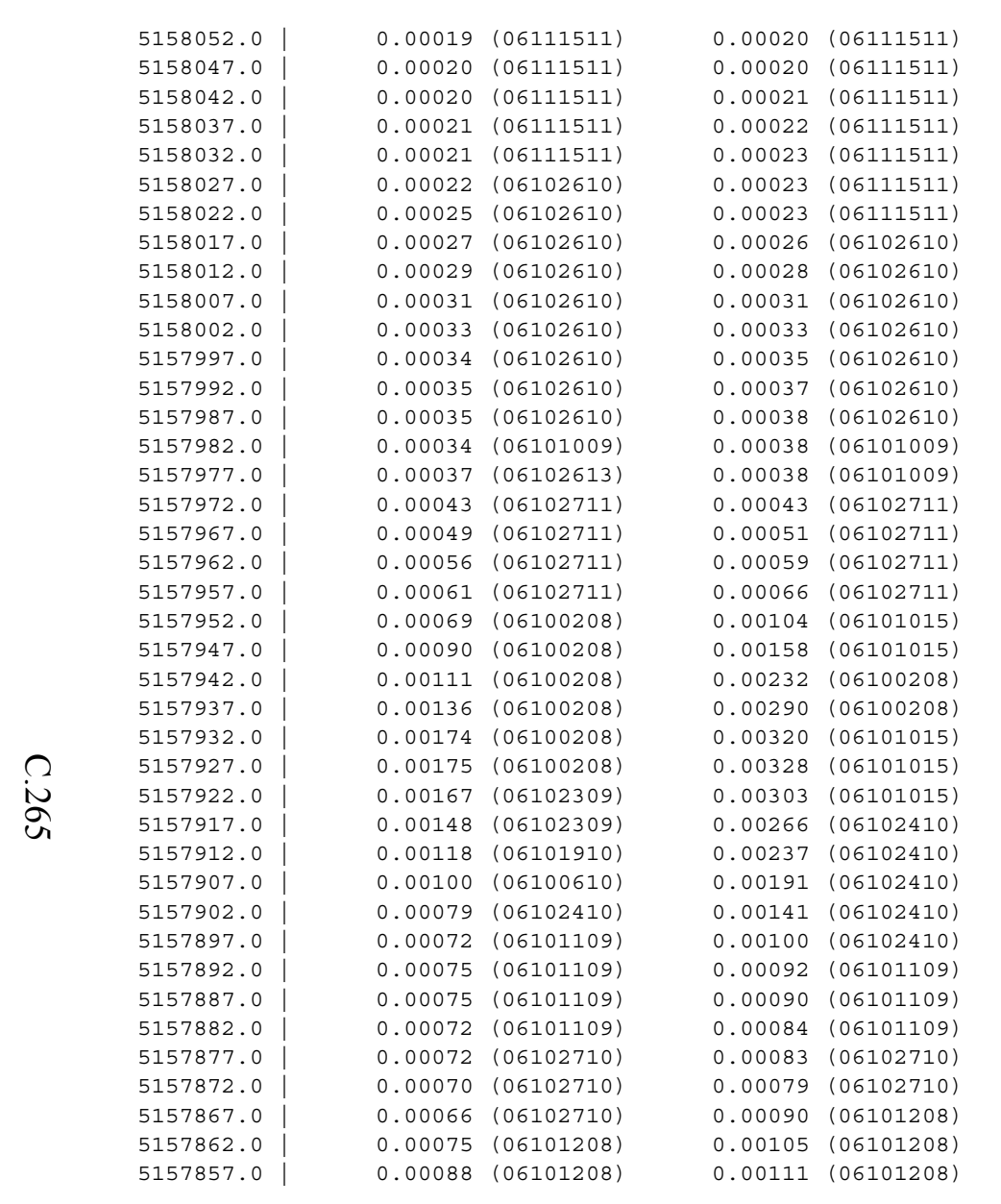

$\begin{array}{ll}0.00019 & (06111511) \\ 0.00020 & (06111511) \\ 0.00022 & (06111511) \\ 0.00023 & (06111511) \\ 0.00024 & (06111511) \\ 0.00025 & (06111511) \\ 0.00025 & (06111511) \\ 0.00026 & (06111511) \\ 0.00026 & (06101009) \\ 0.00029 & (06102610) \\ 0.00032 & (06102610) \\ 0.00035 & (06102610) \\ 0.00038 & (06102610) \\ 0.00040 & (06101009) \\ 0.00042 & (06101009) \\ 0.00043 & (06101009) \\ 0.00050 & (06101009) \\ 0.00056 & (06101009) \\ 0.00063 & (06101015) \\ 0.00125 & (06100208) \\ 0.00209 & (06100208) \\ 0.00305 & (06100208) \\ 0.00392 & (06100208) \\ 0.00449 & (06100208) \\ 0.00472 & (06102309) \\ 0.00482 & (06101015) \\ 0.00430 & (06102410) \\ 0.00435 & (06102410) \\ 0.00464 & (06102110) \\ 0.00387 & (06102410) \\ 0.00294 & (06102410) \\ 0.00206 & (06102410) \\ 0.00137 & (06102410) \\ 0.00107 & (06101109) \\ 0.00098 & (06102710) \\ 0.00095 & (06102710) \\ 0.00109 & (06101208) \\ 0.00124 & (06101208) \\ 0.00128 & (06101208) \\ 0.00128 & (06101108)\end{array}$

$0.00019 \quad(06120610)$

0.00021 (06111511)

0.00022 (06111511)

0.00022 (06111511)

0.00024 (06111511)

0.00025 (06111511)

0.00027 (06111511)

0.00028 (06111511)

0.00029 (06111511)

0.00031 (06101009)

$0.00038 \quad(06101009)$

$0.00053(06100510)$

$0.00060 \quad(06100510)$

0.00067 (06101009)

0.00067 (06101009)

0.00075 (06101009)

$0.00123(06101113)$

$0.00298(06100208)$

$0.00399(06100208)$

$0.00426 \quad(06100208)$

$0.00441 \quad(06101015)$

$0.00471(06101015)$

$0.00451 \quad(06101015)$

$0.00477(06102410)$

$0.00511(06102410)$

$0.00488 \quad(06102410)$

$0.00488(06102410)$

$0.00512(06102410)$

$0.00407(06102410)$

$0.00295(06102410)$

$0.00191 \quad(06102410)$

0.00126 (06101109)

$0.00115(06102710)$

$0.00133(06101208)$

$0.00147 \quad(06101208)$

$0.00150(06101108)$

$0.00153(06101108)$

0.00145 (06101108)
$0.00021 \quad(06120610)$

$0.00021 \quad(06120610)$

$0.00022(06120610)$

$0.00023 \quad(06120610)$

$0.00023(06120610)$

0.00023 (06111511)

$0.00025(06111511)$

0.00028 (06111511)

0.00029 (06111511)

$0.00030(06111511)$

$0.00032(06111511)$

$0.00033(06111511)$

$0.00044 \quad(06101316)$

$0.00062(06101316)$

$0.00074 \quad(06100510)$

$0.00080 \quad(06100510)$

0.00080 (06100510)

$0.00087(06101009)$

.00138 (06101114)

0.00211 (06101114)

$0.00274 \quad(06101010)$

$0.00330(06101010)$

$0.00381 \quad(06100208)$

$0.00422(06100208)$

$0.00433(06101015)$

$0.00470 \quad(06101015)$

$0.00457(06101015)$

$0.00486(06102410)$

$0.00511 \quad(06102410)$

$0.00572(06102315)$

$0.00558 \quad(06101109)$

$0.00463(06102315)$

$0.00381(06101109)$

$0.00225(06101109)$

0.00148 (06101109)

0.00167 (06101208)

$0.00175(06101208)$

$0.00182(06101108)$

$0.00176(06101108)$

$0.00159(06101108)$

0.00137 (06101108)

\section{*** AERMOD - VERSION $07026 * * * \quad * * *$ U/UA Building Demolition} **MODELOPTS :

CONC TOXICS FLAT

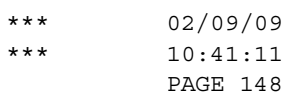

DRYDPL WETDPL

*** THE 1ST HIGHeSt 1-HR DRY DEPOSITION VALUES FOR SOURCE GROUP: ALL *** INCLUDING SOURCE $(S):$ : $224 \mathrm{U}_{-}{ }^{1}, 224 \mathrm{U}_{-}{ }^{2}, 224 \mathrm{U}_{-} 3,224 \mathrm{U}_{-} 4,224 \mathrm{U}_{-} 5,224 \mathrm{U}_{-} 6,224 \mathrm{U}_{-} 7$,

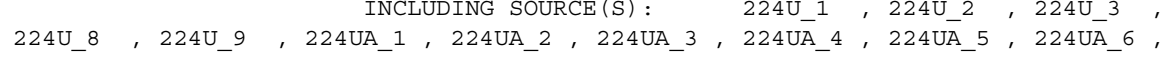

*** NETWORK ID: UCART1 ; NETWORK TYPE: GRIDCART *** 


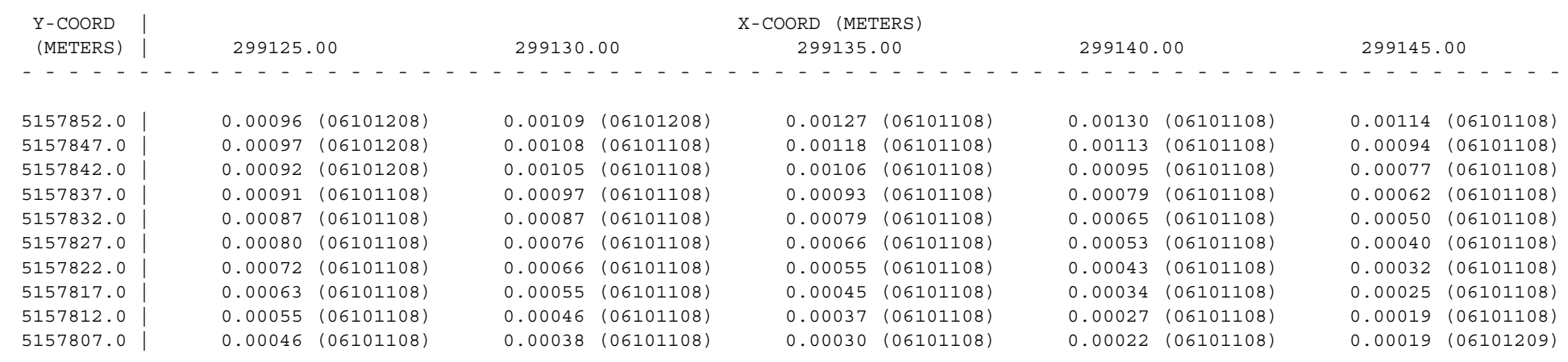

$\begin{array}{ll}* * \text { AERMOD - VERSION } 07026 * * * \quad \begin{array}{l}* * \text { U/UA Building Demolition } \\ * * \text { Unit Release - Worst Case }\end{array} & \end{array}$ **MODELOPTS

CONC

DDEP

TOXICS FLAT

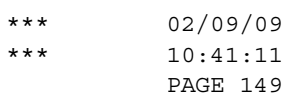

DRYDPL WETDPL

** THE 1ST HIGHEST 1-HR DRY DEPOSITION

VALUES FOR SOURCE GROUP: ALL

INCLUDING SOURCE $(\mathrm{S}): \quad 224 \mathrm{U}{ }_{-} 1,224 \mathrm{U} \_2,224 \mathrm{U} \_3,224 \mathrm{U} \_4,224 \mathrm{U} \_5,224 \mathrm{U} \_6,224 \mathrm{U} \_7$, $224 \mathrm{U} \_8,224 \mathrm{U} \_9,224 \mathrm{UA} \_1,224 \mathrm{UA} \_2,224 \mathrm{UA} \_3,224 \mathrm{UA} \_4,224 \mathrm{UA}{ }_{-} 5,224 \mathrm{UA}_{-} 6$,

*** NETWORK ID: UCART1; NETWORK TYPE: GRIDCART ***
** DEPO OF UO3
IN GRAMS $/ \mathrm{M} * * 2$

\begin{tabular}{|c|c|c|c|c|c|c|c|c|c|c|}
\hline $\mathrm{Y}-\mathrm{COORD}$ & & & & & DORD (ME? & ERS) & & & & \\
\hline (METERS) & 299150.0 & & 299155. & & 299160 & & 299165. & & 299170 . & \\
\hline & $-\quad--$ & $-\cdots$ & --- & - & & - & & 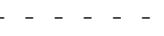 & & \\
\hline 5158052.0 & 0.00021 & $(06120610)$ & 0.00021 & $(06120610)$ & 0.00020 & $(06112210)$ & 0.00021 & $(06112210)$ & 0.00021 & $(06112210)$ \\
\hline 5158047.0 & 0.00022 & $(06120610)$ & 0.00022 & $(06120610)$ & 0.00021 & $(06120610)$ & 0.00022 & $(06112210)$ & 0.00022 & $(06112210)$ \\
\hline 5158042.0 & 0.00023 & $(06120610)$ & 0.00023 & $(06120610)$ & 0.00022 & $(06120610)$ & 0.00023 & $(06112210)$ & 0.00023 & $(06112210)$ \\
\hline 5158037.0 & 0.00024 & $(06120610)$ & 0.00024 & $(06120610)$ & 0.00024 & $(06120610)$ & 0.00024 & $(06112210)$ & 0.00024 & $(06112210)$ \\
\hline 5158032.0 & 0.00025 & $(06120610)$ & 0.00025 & $(06120610)$ & 0.00025 & $(06120610)$ & 0.00025 & $(06112210)$ & 0.00026 & $(06112210)$ \\
\hline 5158027.0 & 0.00025 & $(06120610)$ & 0.00026 & $(06120610)$ & 0.00026 & $(06120610)$ & 0.00026 & $(06112210)$ & 0.00027 & $(06112210)$ \\
\hline 5158022.0 & 0.00026 & $(06120610)$ & 0.00028 & $(06120610)$ & 0.00028 & $(06120610)$ & 0.00028 & $(06112210)$ & 0.00029 & $(06112210)$ \\
\hline 5158017.0 & 0.00027 & $(06111511)$ & 0.00029 & $(06120610)$ & 0.00029 & $(06120610)$ & 0.00029 & $(06112210)$ & 0.00030 & $(06112210)$ \\
\hline 5158012.0 & 0.00029 & (06111511) & 0.00030 & $(06120610)$ & 0.00031 & $(06120610)$ & 0.00031 & $(06112210)$ & 0.00032 & $(06112210)$ \\
\hline 5158007.0 & 0.00031 & (06111511) & 0.00031 & $(06120610)$ & 0.00032 & $(06120610)$ & 0.00032 & $(06112210)$ & 0.00034 & $(06112210)$ \\
\hline 5158002.0 & 0.00033 & $(06111511)$ & 0.00033 & $(06100510)$ & 0.00033 & $(06120610)$ & 0.00039 & $(06102614)$ & 0.00048 & $(06102614)$ \\
\hline 5157997.0 & 0.00036 & $(06101310)$ & 0.00046 & $(06101310)$ & 0.00060 & $(06101316)$ & 0.00077 & $(06101316)$ & 0.00082 & $(06101310)$ \\
\hline 5157992.0 & 0.00059 & $(06101316)$ & 0.00078 & $(06101316)$ & 0.00101 & $(06101316)$ & 0.00104 & $(06101316)$ & 0.00115 & $(06101316)$ \\
\hline 5157987.0 & 0.00077 & $(06101316)$ & 0.00098 & $(06100510)$ & 0.00124 & $(06100510)$ & 0.00150 & $(06100510)$ & 0.00134 & $(06100510)$ \\
\hline 5157982.0 & 0.00101 & $(06100510)$ & 0.00129 & $(06100510)$ & 0.00150 & $(06100510)$ & 0.00124 & $(06100510)$ & 0.00133 & $(06100510)$ \\
\hline 5157977.0 & 0.00103 & $(06100510)$ & 0.00127 & $(06100510)$ & 0.00147 & $(06101114)$ & 0.00177 & $(06101114)$ & 0.00149 & $(06101114)$ \\
\hline 5157972.0 & 0.00134 & (06101114) & 0.00188 & (06101114) & 0.00243 & (06101114) & 0.00228 & $(06101010)$ & 0.00181 & (06101114) \\
\hline
\end{tabular}


$5157967.0 \mid \quad 0.00229(06101114)$

$0.00310(06101010)$

5157957.0

$5157952.0) 0.00342(06101010)$

$0.00359(06100208)$

$0.00412(06100208)$

$0.00426(06100208)$

515737.0 | $0.00426(06100208)$

5157932.0 -

$0.00462(06101015)$

$0.00536(06102315)$

$0.00603(06102315)$

$\begin{array}{lll}5157907.0 & 0.00616(06102315)\end{array}$

\begin{tabular}{l|ll}
5157902.0 & $0.00618(06102315)$
\end{tabular}

\begin{tabular}{l|ll}
5157897.0 & $0.00602(06101109)$
\end{tabular}

\begin{tabular}{l|l}
5157892.0 & $0.00420(06101109)$
\end{tabular}

\begin{tabular}{l|l}
5157887.0 & $0.00266(06101109)$
\end{tabular}

\begin{tabular}{l|l}
5157882.0 & $0.00226(06101108)$
\end{tabular}

\begin{tabular}{l|l|l}
5157877.0 & $0.00226(06101108)$
\end{tabular}

\begin{tabular}{l|l}
5157872.0 & $0.00196(06101108)$
\end{tabular}

\begin{tabular}{l|l}
5157867.0 & $0.00166(06101108)$
\end{tabular}

\begin{tabular}{l|l}
5157867.0 & $0.00166(06101108)$ \\
5157862.0 & $0.00137(06101108)$
\end{tabular}

$5157857.0 \quad 0.00116(06102409)$
$0.00285(06101114)$

$0.00391(06101010)$

$0.00402(06101010)$

$0.00379(06101010)$

$0.00337(06101010)$

$0.00397(06100208)$

$0.00426(06100208)$

$0.00464(06101015)$

$0.00466(06101015)$

$0.00500(06102410)$

$0.00573(06102315)$

$0.00617(06102315)$

$0.00602(06102315)$

$0.00557(06101109)$

0.00378 (06101109)

$0.00260 \quad(06101108)$

$0.00210 \quad(06101108)$

$0.00170 \quad(06102409)$

$0.00162(06102409)$

$0.00140 \quad(06102409)$

$0.00111 \quad(06102409)$
$0.00336(06101010)$

$0.00378(06101010)$

$0.00401 \quad(06101010)$

$0.00400(06101010)$

$0.00376(06101010)$

$0.00379(06100208)$

$0.00421 \quad(06100208)$

$0.00460(06101015)$

$0.00469(06101015)$

$0.00530(06102315)$

$0.00600 \quad(06102315)$

$0.00617 \quad(06102315)$

0.00577 (06101109)

$0.00514 \quad(06101109)$

$0.00623(06101109)$

$0.00523(06101109)$

$0.00403 \quad(06101109)$

$0.00278 \quad(06101109)$

$0.00217 \quad(06102409)$

$0.00206(06102409)$

$0.00172(06102409)$

$0.00130(06102409)$

$0.00093(06102409)$
$0.00287(06101010)$

$0.00341(06101010)$

$0.00382(06101010)$

$0.00399(06101010)$

$.00372(06101010)$

0.00372 (06101010)

$0.00455(06101015)$

$0.00477(06102315)$

$0.00569(06102315)$

$0.00615(06102315)$

$0.00605(06102315)$

$0.00697(06101108)$

$0.00686(06101108)$

$0.00632(06101109)$

$0.00392(06101109)$

$0.00288(06102409)$

0.00272 (06102409)

$0.00215(06102409)$

$0.00215(06102409)$

$0.00094 \quad(06102409)$

$0.00060(06102310)$
$0.00234(06101010)$

$.00293(06101010)$

$0.00346(06101010)$

$0.00385(06101010)$

$0.00403(06101010)$

$0.00397(06101010)$

$0.00394(06100208)$

$0.00415(06101015)$

$0.00494 \quad(06102315)$

$0.00653 \quad(06110910)$

$0.01812 \quad(06110910)$

$0.01137(06121813)$

$0.00856 \quad(06101108)$

$0.00475(06101108)$

$0.00714 \quad(06101109)$

0.00583 (06101109)

$0.00419(06102409)$

$0.00379(06102409)$

$0.00273(06102409)$

$0.00273(06102409)$

$0.00087(06102310)$

$0.00074 \quad(06102310)$

$0.00063(06102310)$

*** NETWORK ID: UCART1; NETWORK TYPE: GRIDCART ***

** DEPO OF UO3 IN GRAMS $/ \mathrm{M} * * 2$

$\mathrm{X}$-COORD (METERS)

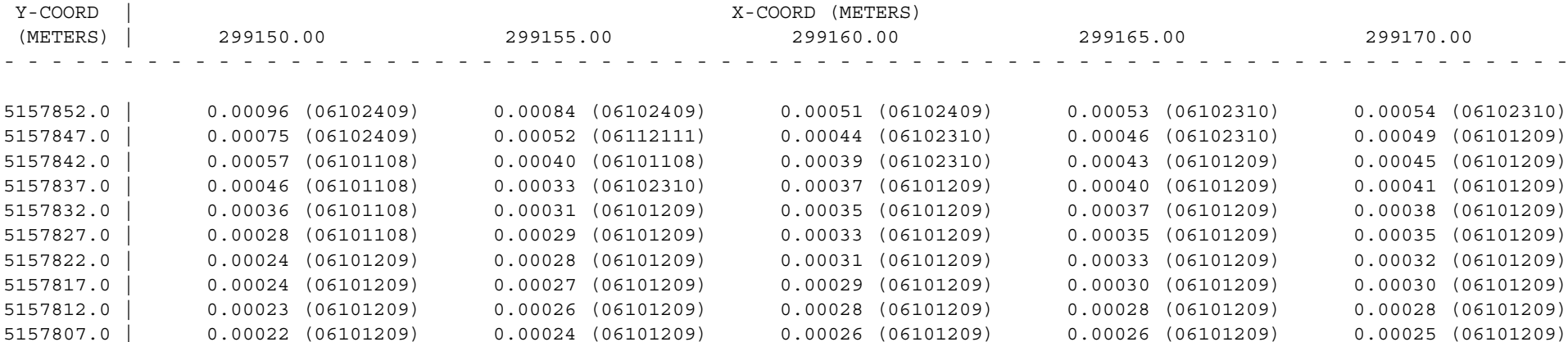

$299160.00 \quad 299165.00$ 
$* * *$ AERMOD - VERSION $07026 * * * \quad \begin{aligned} & * * \text { U/UA Building Demolition } \\ & * * * \text { Unit Release - Worst Case }\end{aligned}$

**MODELOPTS :

CONC

DDEP
TOXICS FLAT
*** $\quad 02 / 09 / 09$

$10: 41: 11$

PAGE 151

DRYDPL WETDPL

VALUES FOR SOURCE GROUP: ALL ***

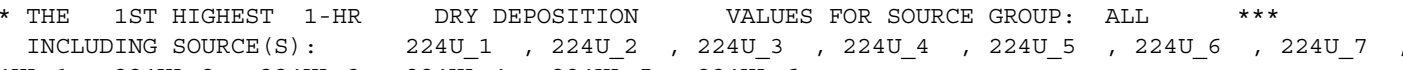

$224 \mathrm{U} \_8,224 \mathrm{U} \_9,224 \mathrm{UA} \_1,224 \mathrm{UA} \_2,224 \mathrm{UA} \_3,224 \mathrm{UA} \_4,224 \mathrm{UA} \_5,224 \mathrm{UA} \_6$,'

** NETWORK ID: UCART1; NETWORK TYPE: GRIDCART ***

** DEPO OF UO3 IN GRAMS $/ M * * 2$

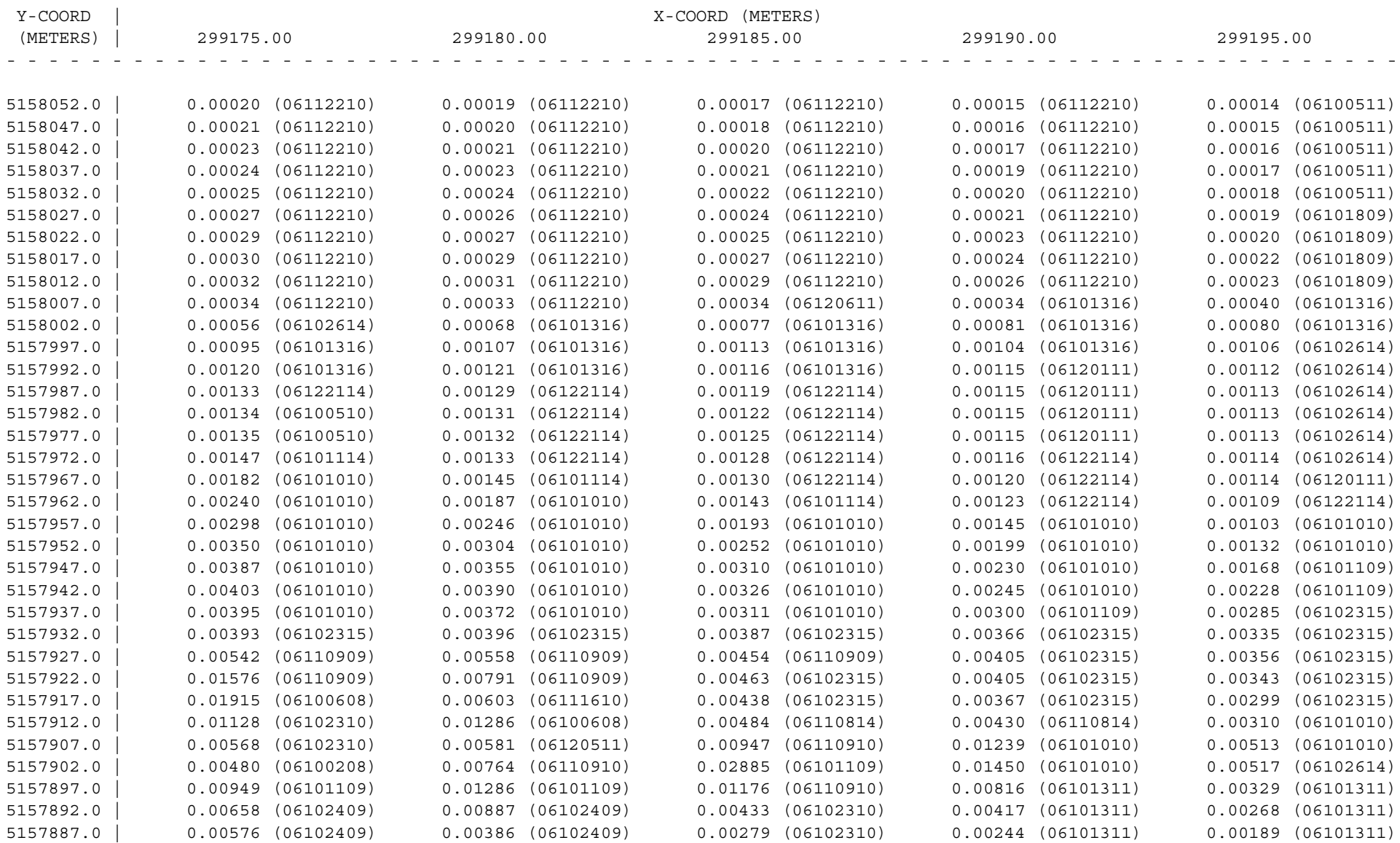




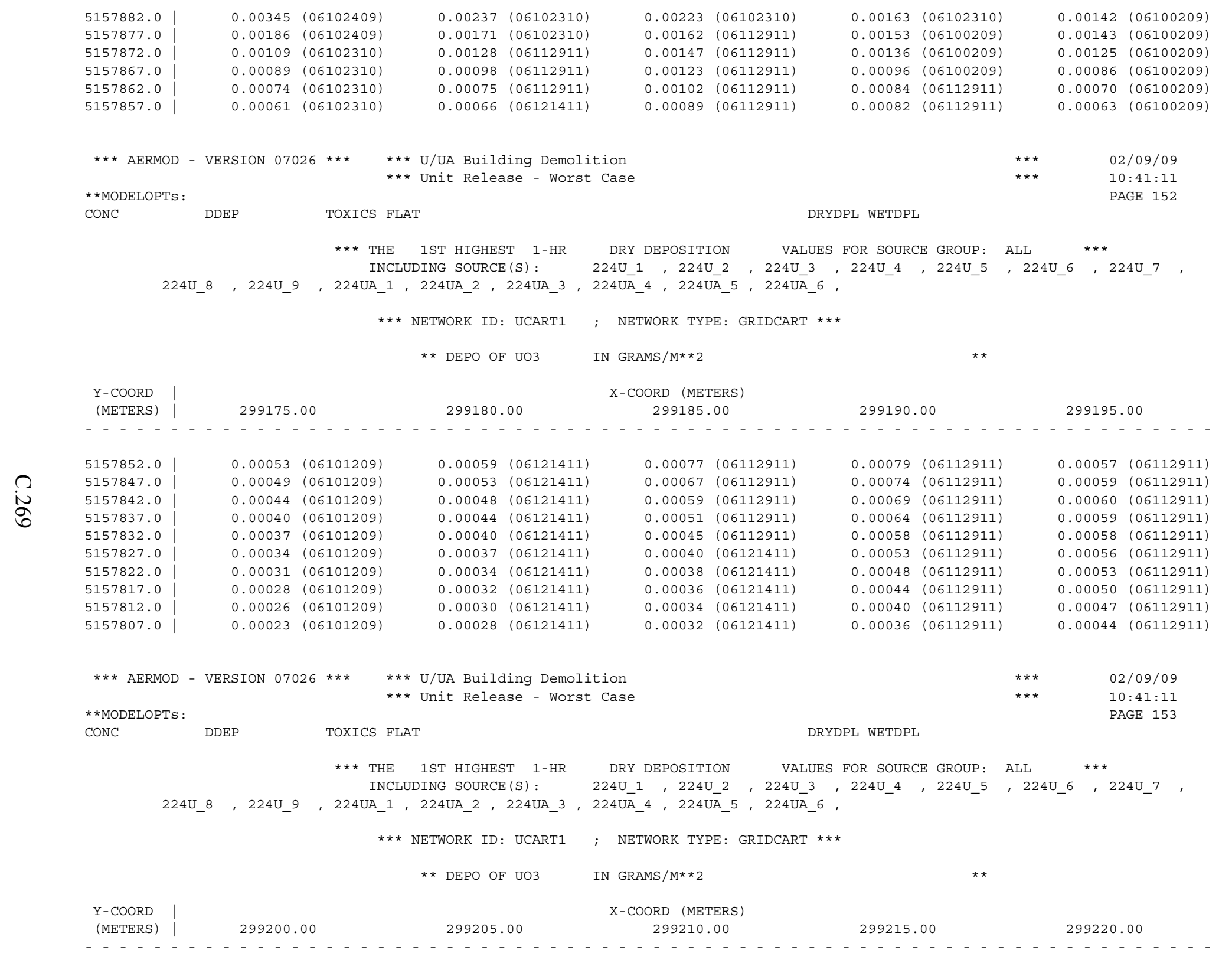




\begin{tabular}{|c|c|c|c|c|}
\hline 5158052.0 & 0.00013 & $(06101809)$ & 0.00013 & $(06113011)$ \\
\hline 5158047.0 & 0.00014 & $(06101809)$ & 0.00013 & $(06113011)$ \\
\hline 5158042.0 & 0.00015 & $(06101809)$ & 0.00014 & $(06113011)$ \\
\hline 5158037.0 & 0.00016 & $(06101809)$ & 0.00015 & $(06113011)$ \\
\hline 5158032.0 & 0.00017 & $(06101809)$ & 0.00016 & $(06113011)$ \\
\hline 5158027.0 & 0.00018 & $(06101809)$ & 0.00017 & $(06113011)$ \\
\hline 5158022.0 & 0.00019 & $(06101809)$ & 0.00018 & $(06113011)$ \\
\hline 5158017.0 & 0.00020 & $(06101809)$ & 0.00019 & $(06113011)$ \\
\hline 5158012.0 & 0.00022 & (06101809) & 0.00020 & $(06113011)$ \\
\hline 5158007.0 & 0.00041 & $(06101316)$ & 0.00040 & $(06101316)$ \\
\hline 5158002.0 & 0.00075 & $(06101316)$ & 0.00066 & $(06101316)$ \\
\hline 5157997.0 & 0.00106 & $(06102614)$ & 0.00100 & $(06102614)$ \\
\hline 5157992.0 & 0.00115 & $(06102614)$ & 0.00112 & $(06102614)$ \\
\hline 5157987.0 & 0.00115 & $(06102614)$ & 0.00111 & $(06102614)$ \\
\hline 5157982.0 & 0.00115 & $(06102614)$ & 0.00111 & $(06102614)$ \\
\hline 5157977.0 & 0.00115 & $(06102614)$ & 0.00110 & $(06102614)$ \\
\hline 5157972.0 & 0.00115 & $(06102614)$ & 0.00110 & $(06102614)$ \\
\hline 5157967.0 & 0.00114 & $(06102614)$ & 0.00108 & $(06102614)$ \\
\hline 5157962.0 & 0.00105 & $(06102614)$ & 0.00100 & $(06102614)$ \\
\hline 5157957.0 & 0.00095 & $(06102614)$ & 0.00091 & $(06102614)$ \\
\hline 5157952.0 & 0.00111 & $(06101109)$ & 0.00108 & $(06110909)$ \\
\hline 5157947.0 & 0.00161 & $(06101109)$ & 0.00144 & $(06101109)$ \\
\hline 5157942.0 & 0.00211 & (06101109) & 0.00185 & $(06102315)$ \\
\hline 5157937.0 & 0.00260 & $(06102315)$ & 0.00226 & $(06102315)$ \\
\hline 5157932.0 & 0.00295 & $(06102315)$ & 0.00248 & $(06102315)$ \\
\hline 5157927.0 & 0.00303 & $(06102315)$ & 0.00247 & $(06102315)$ \\
\hline 5157922.0 & 0.00281 & $(06102315)$ & 0.00243 & $(06100608)$ \\
\hline 5157917.0 & 0.00267 & $(06100608)$ & 0.00261 & $(06100608)$ \\
\hline 5157912.0 & 0.00266 & $(06100608)$ & 0.00268 & $(06100608)$ \\
\hline 5157907.0 & 0.00320 & $(06120114)$ & 0.00265 & $(06100608)$ \\
\hline 5157902.0 & 0.00433 & $(06110810)$ & 0.00291 & $(06110810)$ \\
\hline 5157897.0 & 0.00226 & $(06110811)$ & 0.00231 & $(06100608)$ \\
\hline 5157892.0 & 0.00295 & $(06100608)$ & 0.00202 & $(06100608)$ \\
\hline 5157887.0 & 0.00210 & $(06100608)$ & 0.00237 & $(06100608)$ \\
\hline 5157882.0 & 0.00130 & $(06100608)$ & 0.00176 & $(06100608)$ \\
\hline 5157877.0 & 0.00120 & $(06100209)$ & 0.00116 & $(06100608)$ \\
\hline 5157872.0 & 0.00103 & $(06120511)$ & 0.00076 & $(06100209)$ \\
\hline 5157867.0 & 0.00095 & $(06120511)$ & 0.00068 & $(06120511)$ \\
\hline 5157862.0 & 0.00080 & (06120511) & 0.00075 & $(06120511)$ \\
\hline 5157857.0 & 0.00063 & $(06120511)$ & 0.00073 & $(06120511)$ \\
\hline
\end{tabular}

\begin{tabular}{|c|c|c|c|c|c|}
\hline 0.00012 & (06113011) & 0.00011 & $(06113011)$ & 0.00010 & $(06113011)$ \\
\hline 0.00013 & $(06113011)$ & 0.00012 & $(06113011)$ & 0.00011 & $(06113011)$ \\
\hline 0.00013 & $(06113011)$ & 0.00012 & $(06113011)$ & 0.00011 & $(06113011)$ \\
\hline 0.00014 & (06113011) & 0.00013 & $(06113011)$ & 0.00012 & $(06113011)$ \\
\hline 0.00015 & (06113011) & 0.00014 & $(06113011)$ & 0.00012 & $(06113011)$ \\
\hline 0.00016 & (06113011) & 0.00014 & (06113011) & 0.00013 & $(06113011)$ \\
\hline 0.00017 & $(06113011)$ & 0.00015 & $(06113011)$ & 0.00013 & $(06113011)$ \\
\hline 0.00018 & (06113011) & 0.00016 & (06113011) & 0.00014 & $(06120611)$ \\
\hline 0.00019 & (06113011) & 0.00017 & (06113011) & 0.00015 & $(06120611)$ \\
\hline 0.00036 & $(06101316)$ & 0.00030 & $(06101316)$ & 0.00017 & $(06113010)$ \\
\hline 0.00054 & $(06102614)$ & 0.00045 & $(06102614)$ & 0.00035 & $(06120111)$ \\
\hline 0.00096 & $(06102614)$ & 0.00089 & $(06102614)$ & 0.00072 & $(06102614)$ \\
\hline 0.00104 & $(06102614)$ & 0.00098 & $(06102614)$ & 0.00090 & $(06102614)$ \\
\hline 0.00103 & $(06102614)$ & 0.00089 & $(06102614)$ & 0.00090 & $(06102614)$ \\
\hline 0.00102 & $(06102614)$ & 0.00088 & $(06102614)$ & 0.00080 & $(06102614)$ \\
\hline 0.00101 & $(06102614)$ & 0.00087 & $(06102614)$ & 0.00080 & $(06113010)$ \\
\hline 0.00099 & $(06102614)$ & 0.00086 & $(06102614)$ & 0.00089 & $(06110909)$ \\
\hline 0.00098 & $(06102614)$ & 0.00096 & (06110909) & 0.00086 & $(06110909)$ \\
\hline 0.00091 & $(06102614)$ & 0.00093 & $(06110909)$ & 0.00100 & $(06120114)$ \\
\hline 0.00098 & $(06110909)$ & 0.00098 & $(06101013)$ & 0.00115 & $(06120114)$ \\
\hline 0.00097 & $(06101013)$ & 0.00109 & $(06101013)$ & 0.00120 & $(06101013)$ \\
\hline 0.00117 & (06101109) & 0.00117 & (06110811) & 0.00127 & $(06101013)$ \\
\hline 0.00155 & $(06102315)$ & 0.00135 & (06110811) & 0.00145 & $(06110811)$ \\
\hline 0.00185 & $(06102315)$ & 0.00148 & $(06110811)$ & 0.00159 & $(06110811)$ \\
\hline 0.00199 & $(06102315)$ & 0.00153 & $(06110811)$ & 0.00165 & $(06110811)$ \\
\hline 0.00191 & $(06102315)$ & 0.00159 & $(06120713)$ & 0.00161 & $(06110811)$ \\
\hline 0.00213 & $(06100608)$ & 0.00193 & (06120713) & 0.00183 & (06120713) \\
\hline 0.00242 & $(06100608)$ & 0.00212 & $(06100608)$ & 0.00206 & (06120713) \\
\hline 0.00261 & $(06100608)$ & 0.00242 & $(06100608)$ & 0.00212 & $(06100608)$ \\
\hline 0.00268 & $(06100608)$ & 0.00261 & $(06100608)$ & 0.00242 & $(06100608)$ \\
\hline 0.00262 & $(06100608)$ & 0.00267 & $(06100608)$ & 0.00261 & $(06100608)$ \\
\hline 0.00246 & $(06100608)$ & 0.00260 & $(06100608)$ & 0.00266 & $(06100608)$ \\
\hline 0.00221 & $(06100608)$ & 0.00241 & $(06100608)$ & 0.00257 & $(06100608)$ \\
\hline 0.00237 & $(06100608)$ & 0.00240 & $(06100608)$ & 0.00214 & $(06100608)$ \\
\hline 0.00202 & $(06100608)$ & 0.00193 & $(06100608)$ & 0.00166 & $(06100608)$ \\
\hline 0.00141 & $(06100608)$ & 0.00144 & $(06100608)$ & 0.00122 & $(06100608)$ \\
\hline 0.00090 & $(06100608)$ & 0.00098 & $(06100608)$ & 0.00099 & $(06100608)$ \\
\hline 0.00052 & (06122011) & 0.00068 & $(06100608)$ & 0.00078 & $(06100608)$ \\
\hline 0.00049 & (06122011) & 0.00042 & $(06103110)$ & 0.00058 & $(06100608)$ \\
\hline 0.00058 & $(06120511)$ & 0.00043 & $(06122011)$ & 0.00038 & $(06103110)$ \\
\hline
\end{tabular}

\section{*** AERMOD - VERSION $07026 * * * \quad * * *$ U/UA Building Demolition} **MODELOPTS:

CONC TOXICS FLAT

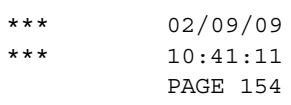

*** THE 1ST HIGHEST 1-HR DRY DEPOSITION VALUES FOR SOURCE GROUP: ALL *** INCLUDING SOURCE $(\mathrm{S}): \quad 224 \mathrm{U}_{-} 1,224 \mathrm{U}_{-}{ }^{2}, 224 \mathrm{U}_{-} 3,224 \mathrm{U}_{-} 4,224 \mathrm{U}_{-} 5,224 \mathrm{U}_{-} 6,224 \mathrm{U}_{-} 7$, $224 \mathrm{U} \_8,224 \mathrm{U} \_9,224 \mathrm{UA} \_1,224 \mathrm{UA} \_2,224 \mathrm{UA} \_3,224 \mathrm{UA} \_4,224 \mathrm{UA}{ }_{-} 5,224 \mathrm{UA}_{-} 6{ }^{\prime}$,

*** NETWORK ID: UCART1 ; NETWORK TYPE: GRIDCART *** 


\begin{tabular}{|c|c|c|c|c|c|c|c|c|c|c|}
\hline \multirow{3}{*}{$\begin{array}{c}\mathrm{Y}-\mathrm{COORD} \\
\text { (METERS) } \\
-\quad-\quad-\quad-\end{array}$} & \multicolumn{10}{|c|}{ X-COORD (METERS) } \\
\hline & \multicolumn{2}{|c|}{299200.00} & \multicolumn{2}{|c|}{299205.00} & \multicolumn{2}{|c|}{299210.00} & \multicolumn{2}{|c|}{299215.00} & \multicolumn{2}{|c|}{299220.00} \\
\hline & & $-\cdots$ & & $-\ldots-\cdots$ & & & & & & \\
\hline 5157852.0 & 0.00051 & $(06100209)$ & 0.00064 & (06120511) & 0.00061 & (06120511) & 0.00044 & $(06120511)$ & 0.00038 & (06122011) \\
\hline 5157847.0 & 0.00047 & $(06100209)$ & 0.00053 & (06120511) & 0.00060 & $(06120511)$ & 0.00050 & $(06120511)$ & 0.00036 & $(06122011)$ \\
\hline 5157842.0 & 0.00043 & $(06100209)$ & 0.00041 & (06120511) & 0.00054 & $(06120511)$ & 0.00052 & $(06120511)$ & 0.00040 & $(06120511)$ \\
\hline 5157837.0 & 0.00044 & $(06112911)$ & 0.00035 & $(06100209)$ & 0.00045 & (06120511) & 0.00051 & (06120511) & 0.00045 & $(06120511)$ \\
\hline 5157832.0 & 0.00046 & $(06112911)$ & 0.00032 & (06100209) & 0.00037 & $(06120511)$ & 0.00046 & $(06120511)$ & 0.00046 & $(06120511)$ \\
\hline 5157827.0 & 0.00047 & $(06112911)$ & 0.00034 & (06112911) & 0.00028 & $(06120511)$ & 0.00040 & $(06120511)$ & 0.00045 & $(06120511)$ \\
\hline 5157822.0 & 0.00047 & $(06112911)$ & 0.00036 & (06112911) & 0.00026 & (06100309) & 0.00033 & (06120511) & 0.00041 & $(06120511)$ \\
\hline 5157817.0 & 0.00047 & $(06112911)$ & 0.00037 & (06112911) & 0.00027 & $(06112911)$ & 0.00026 & $(06120511)$ & 0.00036 & (06120511) \\
\hline 5157812.0 & 0.00046 & $(06112911)$ & 0.00038 & (06112911) & 0.00029 & (06112911) & 0.00023 & (06100309) & 0.00030 & $(06120511)$ \\
\hline 5157807.0 & 0.00045 & $(06112911)$ & 0.00039 & (06112911) & 0.00030 & $(06112911)$ & 0.00022 & $(06112911)$ & 0.00025 & $(06120511)$ \\
\hline
\end{tabular}

\section{*** AERMOD - VERSION $07026 * * * \quad * * *$ U/UA Building Demolition **MODELOPTS

CONC

*** NETWORK ID: UCART1; NETWORK TYPE: GRIDCART ***

\section{** DEPO OF UO3 IN GRAMS $/ M * * 2$}

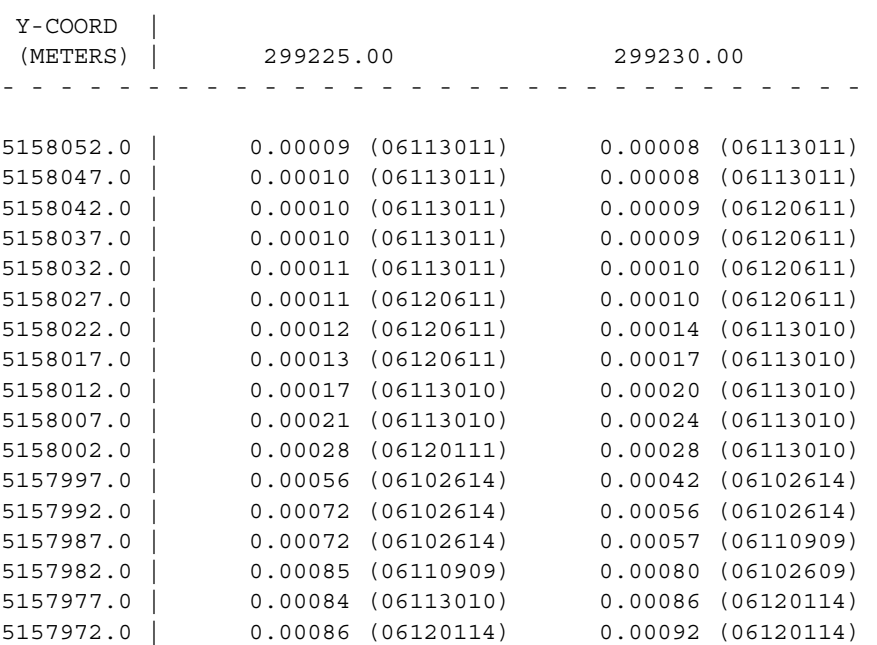

\section{$\mathrm{X}$-COORD (METERS)} 299235.00

$0.00007(06120611)$ $0.00008(06120611)$ $0.00008 \quad(06120611)$ $0.00008(06120611)$ $0.00009(06120611)$ $0.00014(06113010)$ $0.00017(06113010)$ $0.00020(06113010)$ $0.00023(06113010)$ $0.00023(06113010)$ $0.00027(06113010)$ $0.00035(06110909)$ $0.00041 \quad(06102614)$ $0.00041(06102614)$ $0.00063(06102609)$ $0.00097(06120114)$ $0.00106(06120114)$
0.00007 (06120611) 0.00007 (06110909) $0.00008(06110909)$ $0.00011(06113010)$ $0.00014 \quad(06113010)$ $0.00016(06113010)$ $0.00019(06113010)$ $0.00022(06113010)$ $0.00025(06113010)$ $0.00030 \quad(06110909)$ 0.00032 (06110909) $0.00035(06113010)$ $0.00037(06113010)$ $0.00042(06102609)$ 0.00049 (06101013) $0.00070 \quad(06120114)$ $0.00109(06120114)$
$0.00008 \quad(06112415)$ $0.00008 \quad(06110909)$ $0.00011 \quad(06113010)$

$0.00013(06113010)$

$0.00016 \quad(06113010)$

$0.00018(06113010)$ $0.00021(06113010)$ 0.00024 (06113010) $0.00028(06110909)$ $0.00029(06113010)$ $0.00032(06113010)$ $0.00034 \quad(06113010)$ $0.00038(06102609)$ $0.00043(06102609)$ $0.00049(06103114)$ $0.00068(06103114)$ $0.00087(06103114)$ 
\begin{tabular}{l|ll}
5157967.0 & 0.00094 & $(06120114)$
\end{tabular}

$5157962.0 \quad 0.00112(06120114)$

5157957.0 - $0.00123(06120114)$

5157952.0
5157947.0

0.00129 (06101013)

$0.00146(06110811)$

$5157937.0 \mathrm{I}=0.00146$ (06110811)

$50.00160(06110811)$

$5157927.0 \quad 0.00160(06110811)$

$0.00171(06120713)$

\begin{tabular}{l|l}
5157917.0 & $0.00200(06120713)$
\end{tabular}

\begin{tabular}{l|l}
5157912.0 & $0.00211(06120713)$ \\
5157907.0 & $0.00212(06100608)$
\end{tabular}

\begin{tabular}{l|l}
5157907.0 & $0.00212(06100608)$
\end{tabular}

\begin{tabular}{l|l}
5157902.0 & $0.00242(06100608)$
\end{tabular}

\begin{tabular}{c|c}
5157897.0 & $0.00259(06100608)$
\end{tabular}

\begin{tabular}{l|l}
5157892.0 & $0.00259(06100608)$
\end{tabular}

$0.00173(06100608)$

$0.00105(06100608)$

5157877.0 |

5157867.0

\begin{tabular}{l|l}
5157862.0 & $0.00082(06100608)$ \\
5157857.0 & $0.00066(06100608)$
\end{tabular}

$5157857.0 \mid 0.00050(06100608)$
$0.00108 \quad(06120114)$

$.00121(06120114)$

$0.00125(06120114)$

$0.00124(06101013)$

$0.00130(06101013)$

(5101013)

$00160(06110811)$

$0.00160(06110811)$

$0.00160 \quad(06110811)$

0.00158 (06120713)

$0.00192(06120713)$

$0.00209(06120713)$

$0.00206 \quad(06120713)$

$0.00241 \quad(06120713)$

$0.00225(06120713)$

$0.00148 \quad(06120713)$

0.00101 (06120713)

0.00094 (06100608)

$0.00094(06100608)$

0.00088 (06100608)

$0.00069(06100608)$

$0.00056(06100608)$
$0.00118 \quad(06120114)$

$0.00125(06120114)$

$0.00122(06120114)$

$0.00126(06101013)$

$0.00131 \quad(06101013)$

0.00147 (06110811)

0.00161 (06110811)

0.00165 (06110811)

0.00159 (06110811)

$0.00144(06120713)$

$0.00182(06120713)$

$0.00205(06120713)$

$0.00209 \quad(06120713)$

$0.00226(06120713)$

$0.00198 \quad(06120713)$

$0.00167(06120713)$

$0.00132(06120713)$

$0.00096 \quad(06120713)$

0.00084 (06100608)

$0.00080(06100608)$

$0.00075(06100608)$

$0.00068(06100608)$

$0.00059(06100608)$
$0.00124 \quad(06120114)$

$0.00124 \quad(06120114)$

0.00118 (06101013)

0.00131 (06101013)

0.00148 (06110811)

0.00161 (06110811)

0.00161 (06110811)

$0.00159(06110811)$

0.00143 (06110811)

$0.00158(06120713)$

$0.00165(06120713)$

$0.00180 \quad(06120713)$

$0.00184 \quad(06111010)$

$0.00107(06111010)$

0.00088 (06120713)

$0.00088(06120713)$

$0.00075(06100608)$

$0.00072(06100608)$

$0.00064(06100608)$

$0.00058(06100608)$
$0.00106 \quad(06103114)$

$0.00115(06120114)$

$0.00121(06101013)$

$0.00129(06101013)$

$0.00131(06101013)$

0.00149 (06110811)

$0.00162(06110811)$

0.00165 (06110811)

$0.00158 \quad(06110811)$

$0.00142 \quad(06110811)$

$0.00156(06110811)$

$0.00147(06111010)$

$0.00178 \quad(06111010)$

$0.00164(06111010)$

$0.00143 \quad(06111010)$

$0.00119(06111010)$

$0.00098(06120713)$

0.00098 (06120713)

$0.00066(06120713)$

$0.00066(06120713)$

$0.00064(06100608)$
$0.00055(06100608)$

*** AERMOD - VERSION $07026 * \star * \quad * * *$ U/UA Building Demolition

*** Unit Release - Worst Case

$$
\begin{array}{ll}
* * * & 02 / 09 / 09 \\
* * * & 10: 41: 11 \\
& \text { PAGE } 156
\end{array}
$$

DRYDPL WETDPL

ALUES FOR SOURCE GROUP: ALI

***

INCLUDING SOURCE (S) : $224 \mathrm{U}_{-} 1,224 \mathrm{U}_{-} 2,224 \mathrm{U}_{-} 3,224 \mathrm{U}_{-} 4,224 \mathrm{U}_{-} 5,224 \mathrm{U}_{-} 6,224 \mathrm{U}_{-} 7$

224U_8 $, 224 \mathrm{U} \_9,224 \mathrm{UA} \_1,224 \mathrm{UA} \_2,224 \mathrm{UA} \_3,224 \mathrm{UA} \_4,224 \mathrm{UA} \_5,224 \mathrm{UA}_{-}{ }^{3} 6{ }^{\prime}$,

*** NETWORK ID: UCART1; NETWORK TYPE: GRIDCART ***

** DEPO OF UO3 IN GRAMS $/ \mathrm{M} * * 2$

**

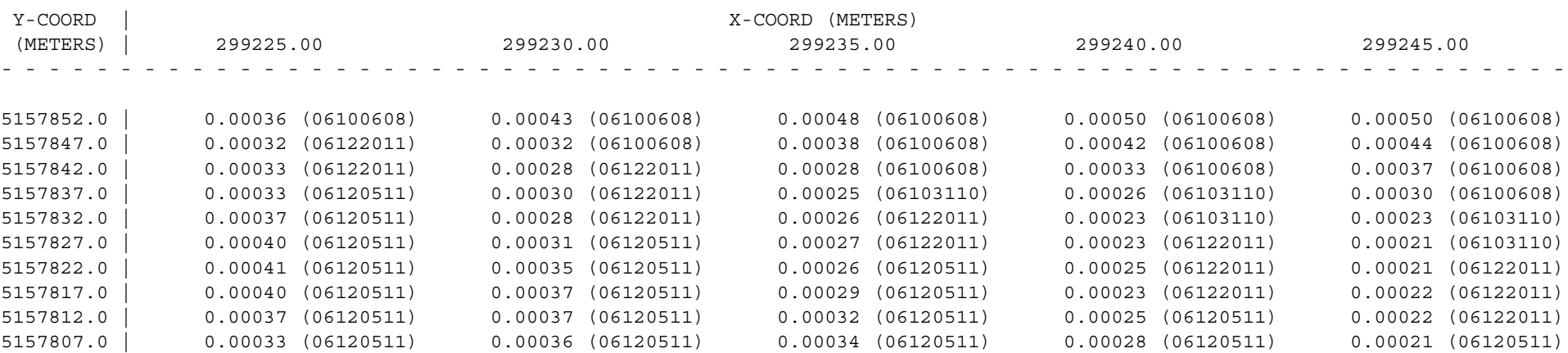


$* * *$ AERMOD - VERSION $07026 * * * \quad \begin{aligned} & * * \text { U/UA Building Demolition } \\ & * * * \text { Unit Release - Worst Case }\end{aligned}$

**MODELOPTS :

CONC

DDEP
TOXICS FLAT
$* * * \quad 02 / 09 / 09$

$10: 41: 11$

PAGE 157

DRYDPL WETDPL

VALUES FOR SOURCE GROUP: ALL ***

THE 1ST HIGHEST 1-HR
INCLUDING SOURCE $(\mathrm{S}):$

$224 \mathrm{U}_{-} 8,224 \mathrm{U} \_9,224 \mathrm{UA} \_1,224 \mathrm{UA} \_2,224 \mathrm{UA} \_3,224 \mathrm{UA}_{-} 4,224 \mathrm{UA}_{-} 5,224 \mathrm{UA}_{-}{ }^{3} 6$,

** NETWORK ID: UCART1; NETWORK TYPE: GRIDCART ***

** DEPO OF UO3 IN GRAMS $/ M * * 2$

\begin{tabular}{|c|c|c|c|c|c|c|c|c|c|c|}
\hline \multirow{3}{*}{$\begin{array}{c}\text { Y-COORD } \\
\text { (METERS) } \\
-\quad-\quad-\quad-\end{array}$} & \multicolumn{10}{|c|}{ X-COORD (METERS) } \\
\hline & \multicolumn{2}{|c|}{299250.00} & \multicolumn{2}{|c|}{299255.00} & \multicolumn{2}{|c|}{299260.00} & \multicolumn{2}{|c|}{299265.00} & \multicolumn{2}{|c|}{299270.00} \\
\hline & & $--_{-1}$ & & $-2--2-$ & & $-2-2--$ & & & & \\
\hline 5158052.0 & 0.00009 & (06110909) & 0.00011 & $(06113010)$ & 0.00012 & $(06113010)$ & 0.00013 & $(06113010)$ & 0.00020 & (06110909) \\
\hline 5158047.0 & 0.00011 & $(06113010)$ & 0.00013 & $(06113010)$ & 0.00014 & $(06113010)$ & 0.00020 & $(06110909)$ & 0.00021 & (06110909) \\
\hline 5158042.0 & 0.00013 & $(06113010)$ & 0.00014 & $(06113010)$ & 0.00016 & $(06113010)$ & 0.00021 & $(06110909)$ & 0.00021 & (06110909) \\
\hline 5158037.0 & 0.00015 & $(06113010)$ & 0.00017 & $(06113010)$ & 0.00022 & (06110909) & 0.00022 & $(06110909)$ & 0.00022 & (06110909) \\
\hline 5158032.0 & 0.00017 & $(06113010)$ & 0.00023 & (06110909) & 0.00023 & (06110909) & 0.00023 & $(06110909)$ & 0.00022 & (06110909) \\
\hline 5158027.0 & 0.00020 & $(06113010)$ & 0.00024 & (06110909) & 0.00024 & (06110909) & 0.00023 & $(06110909)$ & 0.00022 & (06110909) \\
\hline 5158022.0 & 0.00025 & (06110909) & 0.00025 & $(06110909)$ & 0.00024 & $(06110909)$ & 0.00023 & (06110909) & 0.00022 & (06110909) \\
\hline 5158017.0 & 0.00026 & (06110909) & 0.00026 & $(06110909)$ & 0.00025 & $(06113010)$ & 0.00024 & $(06113010)$ & 0.00025 & (06102609) \\
\hline 5158012.0 & 0.00027 & (06110909) & 0.00027 & $(06113010)$ & 0.00026 & $(06113010)$ & 0.00027 & $(06102609)$ & 0.00028 & (06102609) \\
\hline 5158007.0 & 0.00029 & $(06113010)$ & 0.00028 & $(06113010)$ & 0.00029 & $(06102609)$ & 0.00030 & $(06102609)$ & 0.00030 & (06102609) \\
\hline 5158002.0 & 0.00031 & $(06113010)$ & 0.00032 & $(06102609)$ & 0.00033 & (06102609) & 0.00033 & $(06102609)$ & 0.00032 & (06102609) \\
\hline 5157997.0 & 0.00035 & $(06102609)$ & 0.00036 & $(06102609)$ & 0.00036 & $(06102609)$ & 0.00035 & $(06102609)$ & 0.00034 & (06121811) \\
\hline 5157992.0 & 0.00039 & $(06102609)$ & 0.00039 & $(06102609)$ & 0.00038 & $(06102609)$ & 0.00036 & $(06121811)$ & 0.00035 & $(06121811)$ \\
\hline 5157987.0 & 0.00043 & $(06102609)$ & 0.00041 & $(06102609)$ & 0.00039 & $(06102609)$ & 0.00037 & $(06121811)$ & 0.00035 & $(06121811)$ \\
\hline 5157982.0 & 0.00045 & $(06102609)$ & 0.00043 & $(06102609)$ & 0.00039 & $(06102609)$ & 0.00037 & $(06121811)$ & 0.00034 & $(06121811)$ \\
\hline 5157977.0 & 0.00050 & $(06103114)$ & 0.00043 & $(06102609)$ & 0.00038 & $(06102609)$ & 0.00035 & $(06121811)$ & 0.00032 & $(06121811)$ \\
\hline 5157972.0 & 0.00073 & $(06103114)$ & 0.00041 & $(06102609)$ & 0.00037 & $(06102609)$ & 0.00033 & $(06121811)$ & 0.00029 & $(06121811)$ \\
\hline 5157967.0 & 0.00100 & $(06103114)$ & 0.00057 & $(06103114)$ & 0.00034 & $(06102609)$ & 0.00033 & $(06100908)$ & 0.00032 & $(06100908)$ \\
\hline 5157962.0 & 0.00113 & $(06103114)$ & 0.00080 & $(06103114)$ & 0.00038 & $(06100908)$ & 0.00037 & $(06100908)$ & 0.00036 & $(06100908)$ \\
\hline 5157957.0 & 0.00123 & $(06103114)$ & 0.00087 & $(06103114)$ & 0.00045 & $(06100908)$ & 0.00041 & $(06100908)$ & 0.00040 & $(06100908)$ \\
\hline 5157952.0 & 0.00129 & $(06101013)$ & 0.00107 & $(06103114)$ & 0.00062 & $(06103114)$ & 0.00045 & $(06100908)$ & 0.00042 & $(06100908)$ \\
\hline 5157947.0 & 0.00131 & $(06101013)$ & 0.00119 & $(06103114)$ & 0.00078 & $(06103114)$ & 0.00046 & $(06100908)$ & 0.00043 & $(06100908)$ \\
\hline 5157942.0 & 0.00142 & $(06110811)$ & 0.00115 & $(06103114)$ & 0.00088 & $(06103114)$ & 0.00052 & $(06100908)$ & 0.00043 & $(06100908)$ \\
\hline 5157937.0 & 0.00156 & $(06110811)$ & 0.00122 & $(06103114)$ & 0.00093 & $(06110810)$ & 0.00056 & $(06100908)$ & 0.00042 & $(06100908)$ \\
\hline 5157932.0 & 0.00161 & $(06110811)$ & 0.00116 & $(06101013)$ & 0.00093 & $(06110810)$ & 0.00056 & $(06110810)$ & 0.00040 & $(06100908)$ \\
\hline 5157927.0 & 0.00156 & (06110811) & 0.00115 & $(06110811)$ & 0.00085 & $(06110810)$ & 0.00056 & $(06110810)$ & 0.00031 & $(06100908)$ \\
\hline 5157922.0 & 0.00171 & (06110811) & 0.00110 & $(06110811)$ & 0.00072 & $(06110810)$ & 0.00053 & $(06110810)$ & 0.00033 & $(06110810)$ \\
\hline 5157917.0 & 0.00150 & $(06110811)$ & 0.00100 & $(06110811)$ & 0.00059 & $(06110810)$ & 0.00048 & $(06110810)$ & 0.00032 & $(06111010)$ \\
\hline 5157912.0 & 0.00128 & $(06111010)$ & 0.00087 & $(06110811)$ & 0.00051 & $(06101013)$ & 0.00045 & $(06111010)$ & 0.00039 & $(06111010)$ \\
\hline 5157907.0 & 0.00126 & $(06111010)$ & 0.00073 & $(06110811)$ & 0.00059 & $(06111010)$ & 0.00052 & $(06111010)$ & 0.00047 & $(06111010)$ \\
\hline 5157902.0 & 0.00118 & $(06111010)$ & 0.00073 & $(06111010)$ & 0.00066 & $(06111010)$ & 0.00059 & $(06111010)$ & 0.00053 & $(06111010)$ \\
\hline 5157897.0 & 0.00106 & $(06111010)$ & 0.00077 & $(06111010)$ & 0.00071 & $(06111010)$ & 0.00064 & $(06111010)$ & 0.00058 & $(06111010)$ \\
\hline 5157892.0 & 0.00092 & $(06111010)$ & 0.00081 & $(06120713)$ & 0.00073 & $(06120713)$ & 0.00066 & $(06111010)$ & 0.00060 & $(06111010)$ \\
\hline 5157887.0 & 0.00090 & $(06120713)$ & 0.00083 & $(06120713)$ & 0.00075 & $(06120713)$ & 0.00068 & $(06120713)$ & 0.00062 & $(06120713)$ \\
\hline
\end{tabular}




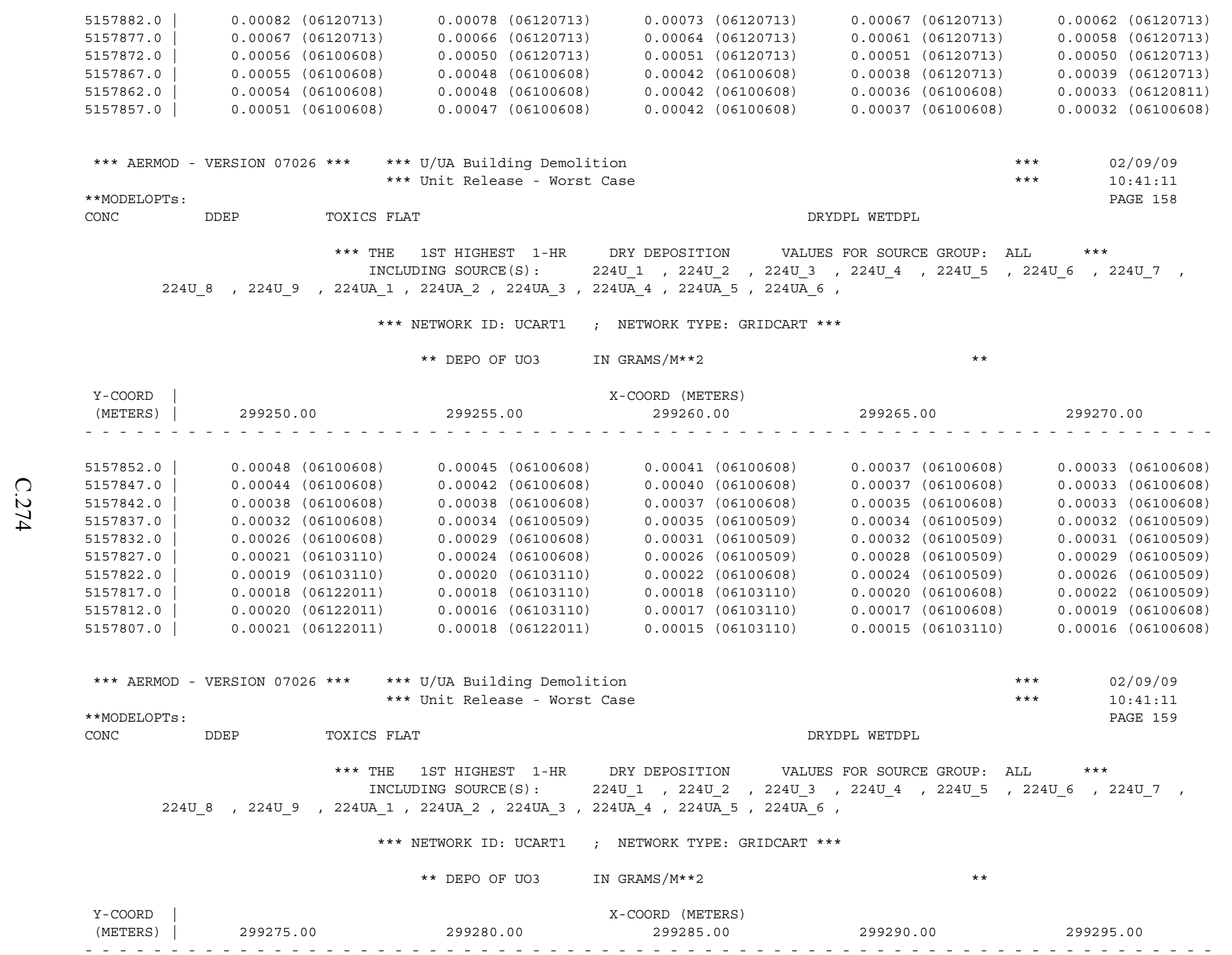


\begin{tabular}{l|ll}
5158052.0 & $0.00020(06110909)$
\end{tabular} $0.00021(06110909)$

$0.00021(06110909)$

5158037.0

5158032.0

$0.00021(06110909)$

515027.0 | $0.00023(06110909)$

5158022.0

$0.00026(06102609)$

$0.00030(06102609)$

$0.00031(06121811)$

$0.00033(06121811)$

$5157992.0 \quad 0.00033(06121811)$

$0.00032(06121811)$

$0.00030(06121811)$

5157977.0

$0.00028 \quad(06103109)$

5157967.0

$0.00035(06100908)$

$0.00035(06100908)$

$0.00038(06100908)$

$0.00040(06100908)$

$0.00040(06100908)$

$0.00040(06100908)$

\begin{tabular}{l|l}
5157942.0 & 0.00040 \\
5157937.0 & $0.00038(06100908)$
\end{tabular}

\begin{tabular}{l|l}
5157932.0 & $0.00035(06100908)$ \\
5 & $0.00028(06100908)$
\end{tabular}

\begin{tabular}{l|l}
5157927.0 & $0.00028(06100908)$
\end{tabular}

$0.00024(06100908)$

$0.00028(06111010)$

$0.00035(06111010)$

$0.00042(06111010)$

$0.00048(06111010)$

5757.0 .

515787.0 - $0.00053(06111010)$

$0.00055(06111010)$

$0.00056(06120713)$

$0.00057(06120713)$

$0.00055(06120713)$

\begin{tabular}{l|l}
5157872.0 & $0.00048(06120713)$
\end{tabular}

$5157867.0 \quad 0.00039(06120713)$

$5157862.0 \quad 0.00030(06120811)$

$0.00030 \quad(06120811)$
$0.00020(06110909)$

$0.00020(06110909)$

$0.00020(06110909)$

$0.00020 \quad(06110909)$

$0.00020 \quad(06110909)$

0.00021 (06102609)

$0.00024(06102609)$

$0.00026(06102609)$

$0.00027(06102609)$

$0.00029(06121811)$

$0.00030 \quad(06121811)$

$0.00031 \quad(06121811)$

$0.00030 \quad(06121811)$

$0.00029 \quad(06121811)$

$0.00025(06103109)$

0.00025 (06103109)

0.00031 (06100908)

0.00034 (06100908)

$0.00034(06100908)$

$0.00036(06100908)$

$.00037(06100908)$

$0.00037(06100908)$

$.00036(06100908)$

$0.00034(06100908)$

$0.00025(06100908)$

$0.00021(06100908)$

$0.00025(06111010)$

$0.00031(06111010)$

$0.00037(06111010)$

$0.00043 \quad(06111010)$

$0.00048(06111010)$

$0.00051 \quad(06111010)$

0.00051 (06111010)

$0.00052(06120713)$

$0.00051(06120713)$

$0.00047 \quad(06120713)$

$0.00039(06120713)$

$0.00030(06120713)$

0.00027 (06120811)
$0.00020 \quad(06110909)$

$0.00020(06110909)$

$.00020(06110909)$

0.00019 (06110909)

$0.00020 \quad(06102609)$

0.00022 (06102609)

$0.00022(06102609)$

$0.00024(06102609)$

$.00026 \quad(06102609)$

$0.00027 \quad(06121811)$

.00028 (06121811)

$0.00029(06121811)$

0.00028 (06121811)

$0.00027 \quad(06121811)$

$0.00026(06121811)$

$0.00023(06121811)$

$0.00025(06103109)$

0.00028 (06100908)

$0.00031(06100908)$

0.00031 (06100908)

$0.00035(06100908)$

$.00035(06100908)$

$.00035(06100908)$

$0.00033 \quad(06100908)$

$0.00031(06100908)$

$0.00025(06100908)$

$0.00022(06100908)$

$0.00019(06112710)$

$0.00022(06111010)$

$0.00027(06111010)$

$0.00033(06111010)$

$0.00033(06111010)$

$0.00043(06111010)$

$0.00047(06111010)$

$0.00047(06111010)$

$0.00048 \quad(06120713)$

$0.00048 \quad(06120713)$

$0.00045 \quad(06120713)$

$0.00039(06120713)$

$0.00031 \quad(06120713)$

$0.00024 \quad(06120811)$
$0.00019 \quad(06110909)$ 0.00019 (06110909)

$0.00018 \quad(06110909)$

0.00019 (06102609)

0.00019 (06102609)

$0.00023(06102609)$

0.00023 (06102609)

$0.00024(06102609)$

$0.00025(06121811)$

$0.00026(06121811)$

$.00027(06121811)$

$0.00022(06121811)$

0.00028 (06100908)

$0.00028(06100908)$

$0.00032(06100908)$

$0.00032(06100908)$

$0.00033(06100908)$

.

$.00032(06100908)$

$0.00030(06100908)$

$0.00028(06100908)$

$0.00022(06100908)$

$0.00018 \quad(06112710)$

$0.00024(06111010)$

$0.00024(06111010)$

$0.00030(06111010)$

$0.00039(06111010)$

$0.00043(06111010)$

$0.00044 \quad(06111010)$

$0.00044(06120713)$

$0.00044 \quad(06120713)$

$0.00038(06120713)$

$0.00031(06120713)$

$0.00024 \quad(06120713)$
$0.00018 \quad(06110909)$

$.00018 \quad(06110909)$

$0.00018 \quad(06102609)$

0.00018 (06102609)

$0.00021 \quad(06102609)$

$0.00023(06102609)$

$0.00023(06121811)$

$0.00025(06121811)$

$0.00025(06121811)$

0.00025 (06121811)

$0.00024 \quad(06121811)$

$0.00023 \quad(06121811)$

$0.00021 \quad(06121811)$

$0.00019 \quad(06121811)$

$0.00022(06100908)$

$0.00025(06100908)$

$0.00028(06100908)$

$0.00030(06100908)$

$0.00031(06100908)$

$0.00031(06100908)$

$0.00031(06100908)$

$0.00030 \quad(06100908)$

$0.00028 \quad(06100908)$

$0.00025(06100908)$

$0.00020(06100908)$

$0.00017(06100908)$

$0.00017(06112710)$

$0.00018(06112710)$

$0.00021(06111010)$

$0.00026(06111010)$

$0.00026(06111010)$

$0.00036(06111010)$

$0.00039(06111010)$

$0.00041(06111010)$

$0.00040(06111010)$

$0.00041 \quad(06120713)$

$0.00040 \quad(06120713)$

$0.00037(06120713)$

$0.00031(06120713)$

$0.00025(06120713)$
*** AFRMOD - VERSION $07026 * * * \quad * * *$ U/UA Building Demolition

*** Unit Builaing Demolition $\star *$ MODELOPTS

CONC

DDEP

TOXICS FLAT $\begin{array}{ll}* * * & 02 / 09 / 09 \\ * * * & 10: 41: 11\end{array}$

PAGE 160

DRYDPL WETDPL

INCLUDING SOURCE $(S):$ : $224 \mathrm{U}_{-1}{ }^{1}, 224 \mathrm{U}_{-}^{2}, 224 \mathrm{U}_{-}^{3}, 224 \mathrm{U}_{-} 4,224 \mathrm{U}_{-} 5,224 \mathrm{U}_{-} 6,224 \mathrm{U}_{-}{ }^{7}$,

*** NETWORK ID: UCART1 ; NETWORK TYPE: GRIDCART *** 


\begin{tabular}{|c|c|c|c|c|c|c|c|c|c|c|}
\hline \multirow{3}{*}{$\begin{array}{c}\mathrm{Y}-\mathrm{COORD} \\
\text { (METERS) } \\
-\quad-\quad-\quad-\end{array}$} & \multicolumn{10}{|c|}{ X-COORD (METERS) } \\
\hline & \multicolumn{2}{|c|}{299275.00} & \multicolumn{2}{|c|}{299280.00} & \multicolumn{2}{|c|}{299285.00} & \multicolumn{2}{|c|}{299290.00} & \multicolumn{2}{|c|}{299295.00} \\
\hline & & $-\cdots$ & & $-\ldots-\cdots$ & & & & & & \\
\hline 5157852.0 & 0.00029 & $(06120811)$ & 0.00027 & (06120811) & 0.00025 & (06120811) & 0.00023 & $(06120811)$ & 0.00020 & $(06120811)$ \\
\hline 5157847.0 & 0.00029 & $(06100608)$ & 0.00026 & (06120811) & 0.00025 & $(06120811)$ & 0.00023 & $(06120811)$ & 0.00021 & $(06120811)$ \\
\hline 5157842.0 & 0.00030 & $(06100608)$ & 0.00027 & (06100509) & 0.00024 & $(06100509)$ & 0.00023 & $(06120811)$ & 0.00021 & $(06120811)$ \\
\hline 5157837.0 & 0.00030 & $(06100509)$ & 0.00027 & $(06100509)$ & 0.00025 & (06100509) & 0.00023 & (06100509) & 0.00021 & $(06120811)$ \\
\hline 5157832.0 & 0.00030 & $(06100509)$ & 0.00028 & $(06100509)$ & 0.00026 & $(06100509)$ & 0.00023 & $(06100509)$ & 0.00021 & $(06100509)$ \\
\hline 5157827.0 & 0.00029 & $(06100509)$ & 0.00028 & (06100509) & 0.00026 & $(06100509)$ & 0.00024 & $(06100509)$ & 0.00022 & (06100509) \\
\hline 5157822.0 & 0.00027 & $(06100509)$ & 0.00026 & (06100509) & 0.00026 & (06100509) & 0.00024 & (06100509) & 0.00023 & $(06100509)$ \\
\hline 5157817.0 & 0.00024 & $(06100509)$ & 0.00024 & $(06100509)$ & 0.00024 & $(06100509)$ & 0.00024 & $(06100509)$ & 0.00023 & $(06100608)$ \\
\hline 5157812.0 & 0.00021 & $(06100608)$ & 0.00022 & $(06100509)$ & 0.00023 & (06100509) & 0.00023 & $(06100608)$ & 0.00023 & $(06100608)$ \\
\hline 5157807.0 & 0.00018 & $(06100608)$ & 0.00020 & $(06100608)$ & 0.00021 & $(06100608)$ & 0.00022 & $(06100608)$ & 0.00022 & $(06100608)$ \\
\hline
\end{tabular}

\section{*** AERMOD - VERSION $07026 * * * \quad * * *$ U/UA Building Demolition **MODELOPTS

CONC
*** RECEPTOR TYPES: GC $=$ GRIDCART $\mathrm{GP}=\mathrm{GRIDPOLR}$ $\mathrm{DC}=\mathrm{DISCCART}$ $\mathrm{DP}=\mathrm{DISCPOLR}$

$* * *$ AERMOD - VERSION $07026 * * * \quad * * *$ U/UA Building Demolition $\star *$ MODELOPTS : CONC DDEP
*** U/UA Building Demolition TOXICS FLAT

$\begin{array}{ll}* * * & 02 / 09 / 09 \\ * * * & 10: 41: 11 \\ & \text { PAGE } 162\end{array}$

$10: 41: 11$
PAGE 162
DRYDPL WETDPL

*** THE SUMMARY OF HIGHEST 1-HR RESULTS ***

** DEPO OF UO3 IN GRAMS $/$ M**2 
GROUP ID

DRY DEPO (YYMMDDHH)

RECEPTOR (XR，YR， ZELEV， ZHILL， ZFLAG)

OF TYPE GRID-ID

ALL HIGH 1ST HIGH VALUE IS

0.02885 ON 06101109: AT $(299185.00,5157902.00$,

0.00

0.00

$0.00)$ GC UCART1
*** RECEPTOR TYPES: GC $=$ GRIDCART
$\mathrm{GP}=\mathrm{GRIDPOLR}$
$\mathrm{DC}=\mathrm{DISCCART}$
$\mathrm{DP}=\mathrm{DISCPOL}$

$* * *$ AERMOD - VERSION $07026 * * * \quad \begin{aligned} & * * \text { U/UA Building Demolition } \\ & * * * \text { Unit Release - Worst Case }\end{aligned}$

**MODELOPTS

$\begin{array}{lll}* * * & 02 / 09 / 09 \\ * * * & 10: 41: 11\end{array}$

$\begin{array}{lll}\text { CONC DDEP } & \text { TOXICS FLAT }\end{array}$

DRYDPL WETDPL

PAGE 163

*** Message Summary : AERMOD Model Execution ***

-...-...- Summary of Total Messages -......

A Total of

A Total of 323 Informational Message(s)

$\Omega \quad$ A Total of

194 Calm Hours Identified

A Total of

129 Missing Hours Identified ( 1.47 Percent)

$* * * * * * *$ FATAL ERROR MESSAGES $* * * * * * * *$

$* * *$ NONE $* * *$

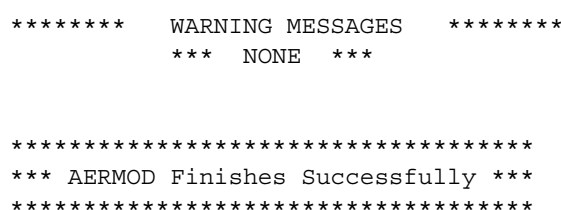

$\star * *$ A 


\section{Distribution}

No. of

Copies

8 CH2M Hill Plateau Remediation Contract

Derrick D. Alt

Gary T Chandler

Earl R Lloyd

Alex M Nazarali

Michael Stevens

Curtis B. Walker
$\mathrm{S} 2-42$

T4-35

T1-41

S2-42

T1-40

T4-35
No. of

\section{$\underline{\text { Copies }}$}

Robert E Wilkinson

T4-35

Lloyd C. Zinsli

$\mathrm{T} 1-41$

2 Local Distribution

Pacific Northwest National Laboratory James G Droppo (2) K9-30

Bruce A Napier

Jeremy Rishel

(PDF) 



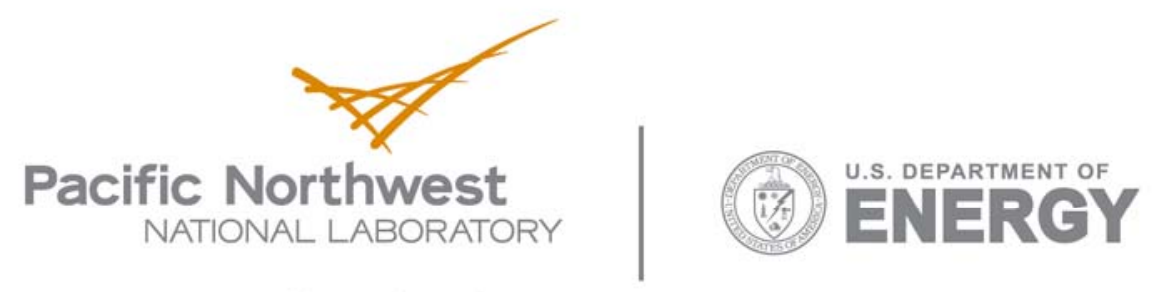

902 Battelle Boulevard

P.O. Box 999

Richland, WA 99352

1-888-375-PNNL (7665)

www.pnl.gov 